Angelika Wöllstein, Peter Gallmann, Mechthild Habermann und Manfred Krifka (Hrsg.)

Grammatiktheorie und Empirie in der germanistischen Linguistik 


\section{Germanistische Sprachwissenschaft um 2020}

Herausgegeben von

Albrecht Plewnia und Andreas Witt

\section{Band 1}




\section{Grammatiktheorie und Empirie in der germanistischen Linguistik}

Herausgegeben von Angelika Wöllstein, Peter Gallmann, Mechthild Habermann und Manfred Krifka 
Die Open-Access-Publikation dieses Bandes wurde gefördert vom Institut für Deutsche Sprache, Mannheim.

ISBN 978-3-11-049097-8

e-ISBN (PDF) 978-3-11-049099-2

e-ISBN (EPUB) 978-3-11-049104-3

\section{(c) BY}

Dieses Werk ist lizenziert unter der Creative Commons Attribution 4.0 Lizenz. Weitere Informationen finden Sie unter http://creativecommons.org/licenses/by/4.0/.

Bibliografische Information der Deutschen Nationalbibliothek

Die Deutsche Nationalbibliothek verzeichnet diese Publikation in der Deutschen Nationalbibliografie; detaillierte bibliografische Daten sind im Internet über http://dnb.dnb.de abrufbar.

(c) 2018 Angelika Wöllstein, Peter Gallmann, Mechthild Habermann und Manfred Krifka, publiziert von Walter de Gruyter GmbH, Berlin/Boston

Foto Einbandabbildung: (๑) Oliver Schonefeld, Institut für Deutsche Sprache, Mannheim Portrait Ludwig M. Eichinger, Seite V: () David Ausserhofer, Leibniz-Gemeinschaft Satz: Meta Systems Publishing \& Printservices GmbH, Wustermark Druck und Bindung: CPI books GmbH, Leck

www.degruyter.com 


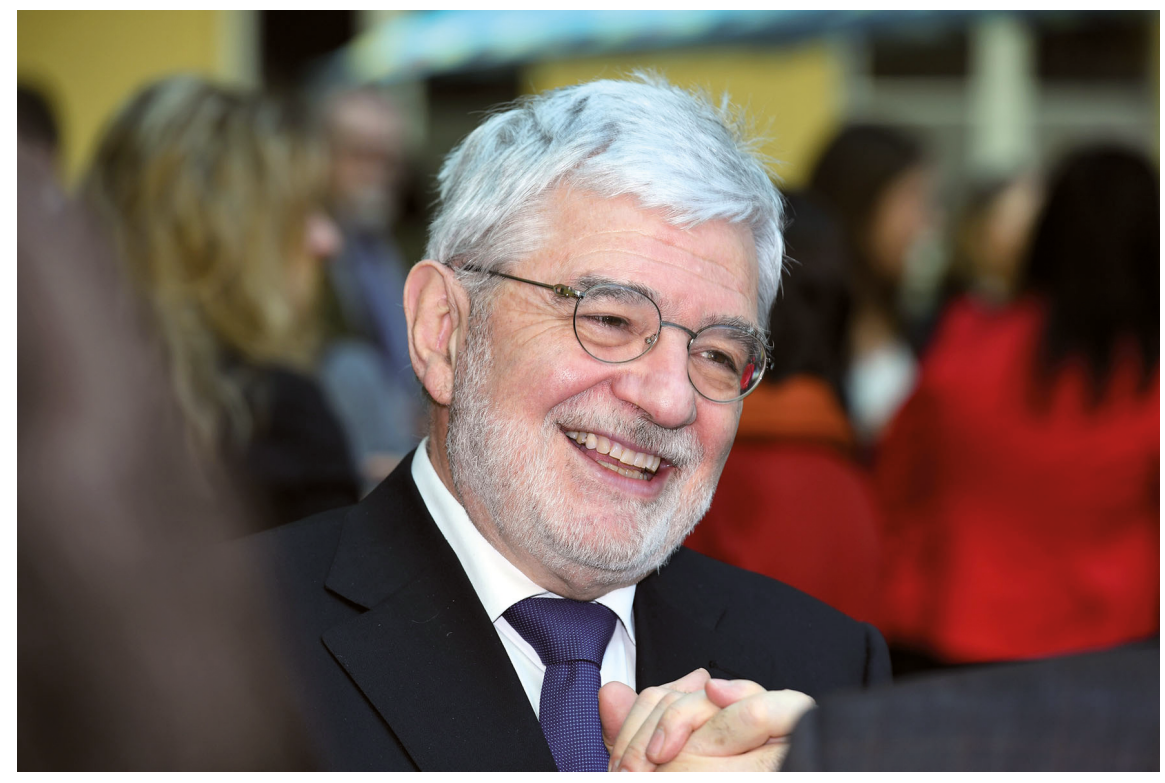

Ludwig M. Eichinger gewidmet 



\section{Vorwort}

Wo steht die germanistische Sprachwissenschaft aktuell? Der vorliegende Band mit dem Titel „Grammatiktheorie und Empirie in der germanistischen Linguistik“ ist der erste Teil einer auf sechs Bände angelegten Reihe, die eine zwar nicht exhaustive, aber doch umfassende Bestandsaufnahme derjenigen Themenfelder innerhalb der germanistischen Linguistik bieten will, die im Kontext der Arbeiten des Instituts für Deutsche Sprache in den letzten Jahren für das Fach von Bedeutung waren und in den kommenden Jahren von Bedeutung sein werden (und von denen nicht wenige auch vom Institut für Deutsche Sprache bedient wurden und werden). Jeder einzelne Band behandelt ein abgeschlossenes Themengebiet und steht insofern für sich; in der Zusammenschau aller Bände ergibt sich ein Panorama der „Germanistischen Sprachwissenschaft um 2020“.

Anlass des Erscheinens dieser Bände ist der Eintritt des langjährigen Direktors des Instituts für Deutsche Sprache, Ludwig M. Eichinger, in den Ruhestand. Ludwig M. Eichinger leitete das Institut von 2002 bis 2018. Seine akademische Laufbahn begann er als Wissenschaftlicher Assistent an der Universität Bayreuth; anschließend war er Heisenberg-Stipendiat an der LudwigMaximilians-Universität München. Ab 1990 hatte er eine Fiebiger-Professur für Deutsche Sprachwissenschaft an der Universität Passau inne, 1997 wurde er auf den Lehrstuhl für Deutsche Philologie an der Christian-Albrechts-Universität zu Kiel berufen. Mit seiner Ernennung zum Direktor des Instituts für Deutsche Sprache im Jahr 2002 wurde er auch Ordinarius für Germanistische Linguistik an der Universität Mannheim. Ludwig M. Eichinger ist Ehrendoktor der Pannonischen Universität Veszprém und der Universität Bukarest. Er ist Mitglied der Akademie der Wissenschaften und der Literatur zu Mainz und der Österreichischen Akademie der Wissenschaften; außerdem ist er Ständiger Gastprofessor an der Beijing Foreign Studies University.

Ludwig M. Eichinger hat das Institut in den Jahren seines Wirkens entscheidend geprägt; in Anerkennung und Dankbarkeit seien ihm diese Bände gewidmet.

Albrecht Plewnia und Andreas Witt - Reihenherausgeber - 



\section{Inhalt}

Vorwort - VII

Angelika Wöllstein, Peter Gallmann, Mechthild Habermann

und Manfred Krifka

Einleitung und inhaltliche Übersicht — 1

\section{Grammatiktheorie und Evidenz}

Beatrice Primus

1 Grammatiktheorie und Psycholinguistik - 9

Hubert Haider

2 Grammatiktheorien im Vintage-Look - Viel Ideologie, wenig Ertrag — 47

\section{Sprach- und Grammatikmodelle}

Thomas Ede Zimmermann

3 Intensionen, Typen und Modelle - 95

Stefan Müller

4 Und? Was läuft sonst so? - 117

III Grammatik, Korpus(linguistik) und Variation

Marek Konopka

5 Korpuslinguistik, Grammatiktheorie, Grammatikographie - 151

Eric Fuß

6 Sprachliche Variation -185

Stefan Th. Gries

7 Zur Identifikation von Mehrwortausdrücken: ein Algorithmus, seine Validierung und weiterführende Überlegungen - 225 
$\mathbf{X}$ Inhalt

Alexandra N. Lenz

8 Syntaktische Variation aus areallinguistischer Perspektive -241

IV Kontrastive Grammatik, Typologie und Wandel

Christiane von Stutterheim

9 Kontrastive Analyse 2020: Neue Horizonte - 281

Thomas Stolz

10 Deiktische Antworten auf räumliche Fragen - 309

Jürg Fleischer

11 Perspektiven der Grammatiktheorie: Sprachwandel - 331

\section{Grammatikographie}

Eva Breindl

12 Grammatikographie: Deskriptive Grammatik - 355

Mathilde Hennig

13 Wie funktional sind Grammatiken des Deutschen? - 383

Maria Thurmair

14 An der Schnittstelle von DaF und Germanistischer

Sprachwissenschaft -409

\section{Grammatik an den Schnittstellen}

Anke Holler

15 Textstrukturen: Was bleibt -435

Daniel Gutzmann und Petra B. Schumacher

16 Schnittstelle Semantik-Pragmatik — 471

Artemis Alexiadou und Gereon Müller

17 Externe Argumente und quantifikationale Variabilität im deutschen Passiv - 511 
Renate Raffelsiefen

18 Phonologische Abstraktheit und symbolische Repräsentation - 549

Nanna Fuhrhop

19 Graphematik des Deutschen im europäischen Vergleich — 587

Register -617

Autorinnen und Autoren -621 



\section{Angelika Wöllstein, Peter Gallmann, Mechthild Habermann und Manfred Krifka Einleitung und inhaltliche Übersicht}

Mit den Beiträgen der Kolleginnen und Kollegen in diesem Band haben die Herausgeberinnen und Herausgeber versucht, zum Gesamtvorhaben beizutragen mit dem Ziel, eine Positionsbestimmung der germanistischen Linguistik der Gegenwart unter der Perspektive Grammatiktheorie und Empirie in der germanistischen Linguistik vorzunehmen. Die Beiträge behandeln Themen, die in der jüngeren Zeit einer besonderen Dynamik und Diskussion unterlagen und in denen sich für das Fach wesentliche Entwicklungen sowohl andeuten wie auch vollziehen und perspektivisch neu ausgerichtet werden. Der Fokus liegt auf den folgenden Themen:

1. Grammatiktheorie und Evidenz,

2. Sprach- und Grammatikmodelle,

3. Grammatik, Korpus(linguistik) und Variation,

4. Kontrastive Grammatik, Typologie und Wandel,

5. Grammatikographie,

6. Grammatik an den Schnittstellen.

Die einzelnen Beiträge gehen Fragestellungen zu Standort- und Paradigmenbestimmungen nach, die einerseits heterogene Methoden und Teilziele durch die Fokussierung entweder auf den Sprachgebrauch oder auf die sprachliche Kompetenz verfolgen, andererseits jedoch dem allgemeinen Ziel des Erkenntnisgewinns über das Sprachsystem verpflichtet sind - wenn auch abhängig von Ergebnisorientiertheit, deskriptiver Vollständigkeit und systematischer empirischer Abdeckung oder dem Aufbau expliziter Modelle mit dem Ziel allgemeinerer Generalisierungen und trotz zum Teil scharfer Abgrenzungstendenzen

\footnotetext{
Angelika Wöllstein, Institut für Deutsche Sprache, R 5, 6-13, D-68161 Mannheim, E-Mail: woellstein@ids-mannheim.de

Peter Gallmann, Friedrich-Schiller-Universität Jena, Philosophische Fakultät, Institut für Germanistische Sprachwissenschaft, Fürstengraben 30, D-07743 Jena, E-Mail: Peter.Gallmann@uni-jena.de

Mechthild Habermann, Lehrstuhl für Germanistische Sprachwissenschaft, Friedrich-Alexander-Universität Erlangen-Nürnberg, Bismarckstraße 1, D-91054 Erlangen, E-Mail: Mechthild.Habermann@fau.de

Manfred Krifka, Leibniz-Zentrum Allgemeine Sprachwissenschaft, Schützenstr. 18, D-10117 Berlin, E-Mail: krifka@leibniz-zas.de

Ә Open Access. (c) 2018 Angelika Wöllstein, Peter Gallmann, Mechthild Habermann und Manfred Krifka, publiziert von De Gruyter. (cc) BY Dieses Werk ist lizenziert unter der Creative Commons Attribution 4.0 Lizenz.

https://doi.org/10.1515/9783110490992-001
} 
verschiedener grammatischer Theorien und Modelle. Bezüglich der Methoden(generierung) ist festzustellen, dass darin aktuell der Schlüssel zu sowohl deskriptiven als auch theoretischen Erkenntnisfortschritten gesehen wird. Außerdem bildet die Versammlung und Bewertung aller Fakten die Basis für das Maß des Erkenntnisfortschritts und damit den Maßstab für die Qualität der Disziplin - ob in der theoretischen oder in der sprachgebrauchsorientierten Forschung, ob kognitionslinguistisch motiviert, typologisch orientiert oder sprachdidaktisch rekonstruiert.

Zum Thema Grammatiktheorie und Evidenz diskutieren:

- Beatrice Primus „Grammatiktheorie und Psycholinguistik“

- Hubert Haider „Grammatiktheorien im Vintage-Look - Viel Ideologie, wenig Ertrag“.

Der Beitrag von Primus fokussiert multiperspektivisch im Hinblick auf generative Grammatik, Konnektionismus, frequenzbasierte Grammatik, Korpusanalyse und kognitive Sprachverarbeitung das kontrovers diskutierte Verhältnis zwischen Grammatiktheorie und Psycholinguistik und deren experimentelle Erträge für die Grammatiktheorie. Der Beitrag von Haider nimmt aus Perspektive wissenschaftstheoretischer Standards kritisch zum Minimalistischen Programm und zur Konstruktionsgrammatik Stellung. Zentrum der Auseinandersetzung bilden deren Heuristiken, Paradigmen sowie die empirische Adäquatheit und Bewährung der von den theoretischen Annahmen generierten Prädiktionen.

Zum Thema Sprach- und Grammatikmodelle diskutieren:

- Thomas Ede Zimmermann „Intensionen, Typen und Modelle“

- Stefan Müller „Und? Was läuft sonst so? Alternative Grammatiktheorien“.

Der Beitrag von Zimmermann wirft aus Perspektive Montagues einen Blick auf Ausgangspunkt und Veränderung dreier zentraler Begriffe der formalen Semantik. Der Beitrag von Müller stellt mit Dependenzgrammatik, Kategorialgrammatik, LFG, TAG, GPSG, HPSG und Konstruktionsgrammatik Alternativen zu Theorien im Rahmen der Government \& Binding-Theorie und des Minimalismus am zentralen Begriff der syntaktischen Valenz vor.

Zum Thema Grammatik, Korpus(linguistik) und Variation diskutieren:

- Marek Konopka „Korpuslinguistik, Grammatiktheorie, Grammatikographie“

- Eric Fuß „Sprachliche Variation“ 
- Stefan Th. Gries „Zur Identifikation von Mehrwortausdrücken: ein Algorithmus, seine Validierung und weiterführende Überlegungen“

- Alexandra N. Lenz „Syntaktische Variation aus areallinguistischer Perspektive“.

Der Beitrag von Konopka legt aus methodisch-korpuslinguistischer und grammatik-theoretischer Perspektive einen Entwurf für eine neue wissenschaftliche Grammatik des Deutschen vor, stellt mit Hinblick auf die Variation grammatischer Strukturen neue Forschungsfragen und zeigt auf, wie sie mit modernen korpuslinguistischen Methoden aufzuarbeiten sind. Hiermit werden Grundlagen für eine umfassende Theorie gelegt, in der Kompetenz und Performanz, Synchronie und Diachronie näher aneinanderrücken. Der Beitrag von Fuß diskutiert anhand von Kongruenzschwankungen Aspekte sprachlicher Variation. Es wird gezeigt, wie korpuslinguistisch grammatische Faktoren ermittelt werden können, die die Verteilung der Varianten steuern, und wie diese zu grammatiktheoretischen Erkenntnissen führen. Der Beitrag von Gries erörtert Aspekte datengetriebener Identifikation von Mehrwortausdrücken aus Korpora unter Einbezug von Validierungsstudien, kontrastiert sie mit konkurrierenden Methoden, überprüft die Prädiktivität für Spracherwerbsdaten und formuliert Desiderata für zukünftige Forschung. Der Beitrag von Lenz beleuchtet am Beispiel des Rezipientenpassivs syntaktische Variation aus Perspektive einer areallinguistisch orientierten Variationslinguistik. Dabei werden theoretische und methodische Fragestellungen diskutiert und das große Potential dieses Untersuchungskomplexes herausgearbeitet.

Zum Thema Kontrastive Grammatik, Typologie und Wandel diskutieren:

- Christiane v. Stutterheim „Kontrastive Analyse 2020: Neue Horizonte“

- Thomas Stolz „Deiktische Antworten auf räumliche Fragen“

- Jürg Fleischer „Perspektiven der Grammatiktheorie: Sprachwandel“.

Im Beitrag von v. Stutterheim wird für eine Weiterentwicklung der Kontrastiven Analyse unter Anwendung empirisch-experimenteller Methoden argumentiert, die die konzeptuelle Ebene als Basis des Sprachvergleichs heranzieht. Vorgeführt wird das anhand von Ergebnissen aus kontrastiven Studien zum Textaufbau, zur Konzeptualisierung von Bewegungsereignissen und zum Grad der Grammatikalisierung des Verbalaspekts. Der Beitrag von Stolz zeigt am Beispiel der Paradigmen räumlicher Interrogativa und ihrer lokal-deiktischen Äquivalente auf, wie sich die einzelsprachliche und kontrastive Forschung für die Sprachtypologie, theoretische Morphologie und Raum-Linguistik ertragreich erweist. Der Beitrag von Fleischer diskutiert an Beispielen wie Genitiv 
und Dativ Singular der althochdeutschen -iz-/-az-Stämme, dem Nebeneinander starker und schwacher Präterita und anhand des „Rückumlauts“ Aspekte von Sprachwandel als Reflex diachroner Entwicklungen und als synchroner Variation historisch „älterer“ und ,jüngerer“ Formen und fragt danach, welche Herausforderungen sich damit für die synchrone Grammatikographie ergeben.

Zum Thema Grammatikographie diskutieren:

- Eva Breindl „Grammatikographie: Deskriptive Grammatik“

- Mathilde Hennig „Wie funktional sind Grammatiken des Deutschen?“

- Maria Thurmair „An der Schnittstelle von DaF und Germanistischer Sprachwissenschaft - Bestandsaufnahme und Perspektiven“.

Der Beitrag von Breindl nimmt unter der Perspektive der Variationsbreite, der Konzeption von Gegenständen, Sprache, Grammatik und Standardsprache eine Bestandsaufnahme deskriptiver wissenschaftlicher Grammatiken des Deutschen vor und formuliert sich daraus ergebende Desiderata für die grammatikographische Praxis. Der Beitrag von Hennig thematisiert das Verhältnis von Form und Funktion als zentrale Frage für Grammatiktheorie und Grammatikschreibung und zeigt, wie Entwicklungen in der Korpuslinguistik die Rückbindung der Analyse grammatischer Formen an den Kontext ihres Gebrauchs ermöglichen. Der Beitrag von Thurmair beleuchtet am Beispiel von Text(sorten)linguistik und gesprochener Sprache und aus der Perspektive der unterrichtlichen Praxis, der Ausbildung sowie der Forschung, die Schnittstelle und die Berührungspunkte zwischen DaF und germanistischer Sprachwissenschaft und ermittelt daraus resultierende Desiderate.

Zum Thema Grammatik an den Schnittstellen diskutieren:

- Anke Holler „Textstrukturen: Was bleibt. Zu Phänomenen und Theorien des Textaufbaus“

- Daniel Gutzmann und Petra B. Schumacher „Schnittstelle Semantik/ Pragmatik“

- Artemis Alexiadou und Gereon Müller „Externe Argumente und quantifikationale Variabilität im deutschen Passiv“

- Renate Raffelsiefen „Phonologische Abstraktheit und symbolische Repräsentation“

- Nanna Fuhrhop „Graphematik des Deutschen im europäischen Vergleich“.

Der Beitrag von Holler spannt unter Berücksichtigung einschlägiger neuerer Theorien der Diskursmodellierung den weiten Bogen von den zentralen Fragestellungen der traditionellen Textlinguistik hin zu den aktuellen Themen der 
Textstrukturanalyse aus Perspektive der für die Textkonstitution einschlägigen Mittel als Basis für komplexere semantisch-pragmatische Diskursstrukturen, Informationssteuerung und -gewichtung. Er weist ausgewiesene textstrukturelle Phänomene und Theorien als verbindliche Standards aus und stellt einen Beitrag zur wissenschaftlichen Nachhaltigkeit im Bereich der Textmodellierung dar. Der Beitrag von Gutzmann und Schumacher stellt am Beispiel von unterbestimmten Bedeutungsaspekten sprachlich unterschiedlich komplexer Ausdrücke - ausgehend vom klassischen Grice'schen Ansatz - Grenzfälle für die Semantik/Pragmatik-Schnittstelle vor; diese werden dann sowohl aus der Perspektive neuerer theoretischer Ansätze als auch der experimentellen Forschung zum Verhältnis der Bedeutungsebenen diskutiert. Der Beitrag von Alexiadou und Müller thematisiert die alte wie rezente empirische Frage und die theoretischen Implikationen der syntaktischen Zugänglichkeit und Repräsentation externer Argumente in Passivkonstruktionen für andere syntaktische Prozesse. Der Beitrag von Raffelsiefen diskutiert am Phänomen der sogenannten Vokalopposition die symbolische Repräsentation sprachlicher Lautstruktur im Rahmen der Optimalitätstheorie sowie die Entwicklung geeigneter Verfahren zur Ermittlung einheitlicher und empirisch adäquater Abstraktionsgrade, basierend auf der Grundidee, wiederkehrendes Material trotz phonetischer Unterschiede als gleich aufzufassen und mit jeweils gleichen Zeichen $\mathrm{zu}$ assoziieren. Verschiedene Typen konvergierender empirischer Evidenz untermauern dabei die Annahme einer einzigen phonologisch relevanten Abstraktionsebene mit fünfzehn qualitativ unterschiedlichen Vollvokalen im Deutschen. Der Beitrag von Fuhrhop entwickelt Fragestellungen und Themen einer systematisch zu etablierenden Kontrastiven Graphematik des Deutschen, ausgehend von der Konzeption einer ,Grammatik des Deutschen im europäischen Vergleich‘. Exemplarisch werden u. a. das Phänomen der Schreibprinzipien, die Funktion der Doppelkonsonantenschreibung, die Distribution der Einheiten von Schreibdiphthongen auf Erst- oder Zweitbestandteil, die Schreibung von Funktionswörtern oder das Phänomen der graphematisch höheren Eindeutigkeit bei phonologisch höherer Mehrdeutigkeit kontrastiv diskutiert.

Die Bandherausgeber danken Saskia Ripp sehr für ihre Unterstützung und Sorgfalt bei den redaktionellen Arbeiten. 

I Grammatiktheorie und Evidenz 



\title{
1 Grammatiktheorie und Psycholinguistik
}

\begin{abstract}
Dieser Beitrag thematisiert das kontrovers diskutierte Verhältnis zwischen Grammatiktheorie und Psycholinguistik. Die im Strukturalismus entwickelte Idee einer autonomen Grammatik wird vertreten durch die generative Grammatiktheorie. Einflussreiche Gegenentwürfe einer Grammatik als emergente Folgeerscheinung der Sprachverwendung bieten Konnektionismus und frequenzbasierte Ansätze. Allerdings vernachlässigen auch sie die in psycholinguistischen Experimenten nachweisbare Prozessualität der kognitiven Sprachverarbeitung.
\end{abstract}

Keywords: frequenzbasierte Grammatik, generative Grammatik, kognitive Sprachverarbeitung, Konnektionismus, Korpusanalyse

\section{Einleitung}

Die Psycholinguistik befasst sich mit der Erforschung des menschlichen Spracherwerbs, den kognitiven und neuronalen Bedingungen für Sprachproduktion und Sprachverstehen sowie mit Sprachstörungen (vgl. Rickheit, Herrmann \& Deutsch 2003; Höhle 2010; Dietrich \& Gerwien 2017). Zu jedem dieser Bereiche gibt es kontrovers diskutierte grammatiktheoretische Positionen. Der vorliegende Beitrag kann aus Platzgründen nicht alle Bereiche thematisieren; er fokussiert die Sprachverarbeitung, besonders das Satzverstehen, und die Frage, wie verschiedene Forschungsparadigmen die Relation zwischen Grammatik und Sprachverarbeitung konzipieren. Aktuelle, international führende Grammatiktheorien oszillieren in ihrem Umgang mit psycholinguistischen Daten und Theorien zwischen zwei extremen Positionen. Gemäß der im Strukturalismus etablierten Auffassung einer strikten Trennung zwischen Grammatiksystem und seinem Gebrauch spielen psycho- und neurolinguistische $^{1}$ theoretische Konzepte und Daten für die generative Grammatiktheorie

1 Die Neurolinguistik wird oft unter Psycholinguistik subsumiert (vgl. Rickheit, Herrmann \& Deutsch 2003; Höhle 2010). Sie untersucht die neuronalen Prozesse im Gehirn mit dafür geeigneten Methoden wie etwa der Messung ereigniskorrelierter Potenziale (EKP) und der funk-

Beatrice Primus, Universität zu Köln, Institut für deutsche Sprache und Literatur I Sprachwissenschaft, Albertus Magnus Platz, D-50923 Köln, E-Mail: primus@uni-koeln.de

Ә Open Access. (C) 2018 Beatrice Primus, publiziert von De Gruyter. (c) BY Dieses Werk ist lizenziert unter der Creative Commons Attribution 4.0 Lizenz.

https://doi.org/10.1515/9783110490992-002 
Noam Chomskys keine oder allenfalls eine sekundäre Rolle. Der Hypothese des Primats eines autonomen Grammatiksystems gegenüber der Sprachverarbeitung widmet sich Abschnitt 2 dieses Beitrags. Eine entgegengesetzte Auffassung vertreten konnektionistische und frequenzbasierte Modelle, die grammatische Regeln als emergente Folgeerscheinungen der Sprachverwendung betrachten (Abschnitt 3). Allerdings vernachlässigen Vertreter dieser Modelle die Prozessualität der Sprachverarbeitung. Mit dem in Echtzeit ablaufenden, inkrementellen Satzverstehen beschäftigt sich Abschnitt 4. Der Schlussabschnitt 5 bietet eine zusammenfassende Betrachtung der verschiedenen Positionen. Um der Diskussion empirische Substanz zu verleihen, werden die verschiedenen Forschungsrichtungen durch zentrale Daten (Abschnitt 2) und empirische Studien (Abschnitt 3 und 4) illustriert. Bei Letzteren handelt es sich um den Majuskelgebrauch in einer künstlichen neuronalen Netzwerksimulation sowie um die Interaktion zwischen Agentivität und Telizität bei der Auxiliarwahl intransitiver Bewegungsverben, die von einem Forscherteam im Umfeld der Autorin mithilfe dreier Methoden - Korpuslinguistik, Akzeptabilitätsbefragung und Messung ereigniskorrelierter Gehirnpotenziale - untersucht wurde.

\section{Autonome Grammatik}

Das Verhältnis zwischen Grammatiktheorie und Psycholinguistik wurde bereits im frühen Strukturalismus durch die Annahme eines sprachlichen Dualismus geprägt. Sprache hat zwei Ausprägungen, die zwar unterschiedlich genannt werden, aber im Wesentlichen Ähnliches bezeichnen: Langue und Parole (vgl. Saussure 1916), System und Parole (vgl. Hjelmslev 1935) oder Kompetenz und Performanz (vgl. Chomsky 1965). Für Ferdinand de Saussure ist die Langue eine soziale Institution, die essentiell und homogen ist. Die Parole konstituiert sich im individuellen Akt des Sprechens, der heterogen und akzidentiell sein kann. Als natürliche Konsequenz dieser Auffassung bildet das Essentielle und Homogene, also die Langue, den zentralen Untersuchungsgegenstand strukturalistisch geprägter Sprachwissenschaft. Dabei vertritt Saussure eine moderate Auffassung über das Verhältnis von Langue und Parole: Langue und Parole hängen voneinander ab. Die Parole ist notwendig, um die Langue zu etablieren, die Langue ist notwendig, damit die Parole überindividuell verstanden

tionellen Magnetresonanztomographie (fMRT). Im vorliegenden Beitrag wird der weitere, die Neurolinguistik umfassende Psycholinguistikbegriff verwendet. 
werden kann. Demgegenüber vertritt Louis Hjelmslev eine radikalere Position: „Le système se définit comme une réalité abstraite et virtuelle [...] indépendant de la parole. Dans la parole on peut même constater des emplois qui ne sont pas possibles selon les exigences du système.“ (Hjelmslev 1935: 88). Diese Auffassung kulminiert in Noam Chomskys (1965: 3) viel zitierter Aussage: „Linguistic theory is concerned primarily with an ideal speaker-listener, in a completely homogeneous speech community [...] who is unaffected by such grammatically irrelevant conditions such as memory limitations." Da die generative Grammatiktheorie unter dem Einfluss Noam Chomskys eine prominente aktuelle Forschungsrichtung darstellt, orientieren sich die folgenden Ausführungen im vorliegenden Abschnitt an ihr.

In den Theorieversionen älteren Zuschnitts (bis ca. 1995) war das wichtigste Ziel generativer Grammatiktheorie, das ,logische Problem‘ (auch ,Induktionsproblem') des Erstspracherwerbs zu erklären: Wie kann ein Kind in so kurzer Zeit auf der Grundlage einer relativ kleinen Menge von Sprachdaten (engl. poverty of the stimulus) seine Muttersprache erwerben? Diese beeindruckende Leistung kann das Kind nicht durch datengetriebene Induktion, sondern durch den Zugriff auf eine angeborene Universalgrammatik bewerkstelligen, so die zentrale Hypothese dieser Forschungsrichtung (Nativismus). In der neuesten theoretischen Ausprägung, dem Minimalistischen Programm (ab ca. 1995), rückt die evolutionäre Fragestellung nach dem Ursprung der Sprache in den Mittelpunkt. Chomsky nimmt an, dass die Sprachfähigkeit im engeren Sinn auf eine spontane Mutation zurückgeht, die vor weniger als 100.000 Jahren unabhängig von der Evolution anderer soziokultureller und kognitiver Fähigkeiten stattfand (Berwick et al. 2013). Wenn sie durch spontane Mutation entstanden ist, muss die Universalgrammatik minimal sein. Die einzige Aufgabe der genetisch kodierten Universalgrammatik besteht darin, sprachliche Ausdrücke $\mathrm{zu}$ generieren, die phonetisch und semantisch in jeweils eigenen externen Systemen interpretiert werden können (vgl. Chomsky 2006; Berwick et al. 2013; Richards 2015). Die Sprachfähigkeit im engeren Sinn wird als ein Berechnungssystem verstanden (engl. computational system). Herzstück dieses Systems ist eine kontextfreie rekursive Verkettungsoperation (engl. external merge) und eine Kopieroperation (engl. internal merge bzw. copy). Erstere generiert komplexe sprachliche Ausdrücke aus einfacheren, z. B. Luise lacht aus Luise und lacht. Letztere sorgt dafür, dass sprachliche Elemente an verschiedenen Positionen im Satz auftreten können: Lacht Luise? vs. Luise lacht. Eine weitere Operation garantiert, dass nur Elemente mit zueinander passenden Merkmalen verkettet werden (engl. feature checking bzw. agree). So scheitert die Merkmalsüberprüfung beim komplexen Ausdruck der Junge helfen das Mädchen aufgrund inkompatibler Kongruenz- und Kasusmerkmale. Eine weitere 
Annahme ist, dass das Berechnungssystem rein syntaktischer Natur ist. Somit bildet die Syntax als zentrale Komponente der Universalgrammatik den Forschungsmittelpunkt (Syntaxzentriertheit, Formalismus). Das syntaktische Berechnungssystem interagiert zwar mit anderen kognitiven Systemen, ist allerdings diesen vorgelagert und arbeitet als autonomes Modul (Autonomie, Modularität). Die wichtigsten externen Performanzsysteme sind das artikulatorisch-perzeptuelle Lautsystem und das konzeptuell-intentionale Bedeutungssystem.

Um Sprachvariation zu erklären, werden die universellen Prinzipien durch Parameter ergänzt. Während in älteren Theorieversionen viele Prinzipien mit ausbuchstabierten Parametern nötig waren, um Spracherwerb zu erklären, nimmt der Minimalismus nur sehr wenige universell invariante Berechnungsoperationen wie die oben beschriebenen an. Der Großteil der grammatischen Erscheinungen einer Sprache, aber auch sprachliche Diversität und Varianz sind nicht Teil des Berechnungssystems. Zum einen zieht man dafür variierende grammatische Eigenschaften der Elemente im Lexikon heran. Zum anderen geht man von äußeren kognitiven Faktoren aus, wie etwa Grenzen des Kurzzeitgedächtnisses (vgl. Makuuchi et al. 2009; Phillips 2010 und Abschnitt 5 weiter unten). Damit überlässt die neuere generative Grammatiktheorie den größten und komplexesten Teil der Sprache den Schnittstellenwissenschaften, zu denen auch die Psycholinguistik gehört (vgl. u. a. Newmeyer 2005; Jackendoff 2007).

Zusammenfassend können wir mehrere aufeinander bezogene Charakteristika der ,internalisierten' Sprache, die durch die Sprachfähigkeit im engeren Sinne determiniert ist und den zentralen Forschungsgegenstand der generativen Grammatiktheorie ausmacht, herausarbeiten (vgl. Newmeyer 2003; Richards 2015). Sie enthält keine Variation zulassende, optionale Regeln und keine gradiente Kategorisierung und Akzeptabilität. Des Weiteren ist ,internalisierte‘ Sprache von hoher Systematizität, Redundanzfreiheit und Abstraktheit geprägt; Akzidentelles und Konstruktionsspezifisches gehört in den Bereich der Performanz oder des Lexikons. Zur Sprachfähigkeit im engeren Sinne gehört ausschließlich das formale Berechnungssystem der Syntax; funktionale Aspekte einschließlich der Semantik-Pragmatik, aber auch die MorphoPhonologie gehören nicht dazu. Schließlich befasst sich die generative Grammatiktheorie nicht mit dynamischen, d.h. in der Zeit ablaufenden, Sprachverarbeitungsprozessen, sondern mit statischen Strukturen und Repräsentationen (Marantz 2005). Als Gegenhypothese in dieser Forschungsrichtung ist Performanz und besonders der Gegenstand der Psycholinguistik durch Varianz, Gradienz, Akzidenz und Prozessualität charakterisiert.

Das Chomskysche Forschungsparadigma vertritt eine mentalistische Position. Sprachliche Kompetenz ist im Gehirn eines (jeden) individuellen Sprechers bzw. Hörers als Initialzustand genetisch kodiert und durch eine sehr kleine 
Zahl von Operationen erweitert. Von dieser so genannten ,internalisierten“ Sprache (engl. i-language) wird die ,externalisierte' Sprache (engl. e-language) unterschieden. Letztere manifestiert sich in der tatsächlichen Sprachverarbeitung und in der sozialen Kommunikation, zu der ein Individuum nicht jederzeit und in ihrem vollen Umfang Zugriff hat. Aufgrund dieser mentalistischen Position mag die Vernachlässigung mentaler Sprachverarbeitungsprozesse, wie sie die Psycholinguistik erforscht, verwunderlich erscheinen.

Für diese Distanziertheit ist, unter anderem, die von Chomsky angenommene Diskrepanz zwischen den wissenschaftlichen Methoden seiner Grammatiktheorie und der Psycholinguistik verantwortlich. Die Chomskysche Tradition beschreibt ihren wissenschaftlichen Stil als ,mathematisch“ oder ,Galileisch“, d.h. als ein nach mathematischer Perfektion in der Natur suchendes Verfahren (Freidin \& Vergnaud 2001; Richards 2015). Diese Methode wird als deduktiv charakterisiert: ,abstract theories and principled explanations and deductive chains of arguments from principles that do not look like descriptions of phenomena“ (Chomsky 2004: 70). In der Forschungslandschaft der Psycholinguistik dominieren dagegen experimentelle Vorgehensweisen (Höhle 2010: 11). Chomskys obiges Zitat sowie die scharfe Trennung zwischen ,experimentellem und ,mathematischem' Forschungsstil suggeriert, dass experimentelle Verfahren induktiv seien. Dieser Eindruck wird durch Aussagen experimentell arbeitender Linguisten wie die folgende verstärkt: „Data is a pre-condition for theory“ (Featherston 2007: 33). Am Ende des Abschnitts 3 dieses Beitrags werden wir auf diese methodologische Frage zurückkommen.

Wenn man den Forschungsstil und die Annahmen Chomskys akzeptiert, ist es müßig zu fragen, ob es für oder gegen eine genetisch kodierte Sprachfähigkeit und ihre Trennung von allgemeineren sprachbezogenen Fähigkeiten experimentelle psycholinguistische Evidenz gibt. Sie wäre wenig bedeutsam (vgl. Newmeyer 2003; Grewendorf 2007).

Nichtsdestoweniger soll hier nicht unerwähnt bleiben, dass es einschlägige experimentelle Untersuchungen gibt. Sie weisen darauf hin, dass eine den genetischen Sprachcode charakterisierende rekursive Verkettungsoperation, die sich bei selbsteinbettenden Strukturen wie die in (1) illustrierten manifestiert, von Menschen, nicht aber von nicht-menschlichen Primaten verarbeitet werden kann (Fitch \& Hauser 2004) und von Menschen in spezifischen neuronalen Bereichen prozessiert wird (Friederici et al. 2006; Makuuchi et al. 2009; Berwick et al. 2013).

(1) Rekursive, selbsteinbettende Struktur (engl. center-embedding) weil $\left[{ }_{\mathrm{A}}\right.$ die Frau $\left[{ }_{\mathrm{A}}\right.$ deren Nachbar $\left[{ }_{\mathrm{A}}\right.$ dessen Tochter studiert $\left.{ }_{\mathrm{B}}\right]$ gerne kocht $\left._{\mathrm{B}}\right]$ sich freut $_{\mathrm{B}}$ ]

Schematisch: $\left[{ }_{A}\left[{ }_{A}\left[\begin{array}{ll}B \\ B\end{array}\right]_{B}\right]\right.$ 
Nicht nur positive Evidenz für die angeborene Sprachfähigkeit zur strukturellen Selbsteinbettung, sondern auch negative Evidenz ist, soweit sie aus Performanzdaten stammt, in diesem Forschungsparadigma wenig bedeutsam. Die Seltenheit rekursiver Selbsteinbettung in Korpora (Karlsson 2007), ihre Absenz in mehreren Sprachen der Welt (Evans \& Levinson 2009) und die Schwierigkeiten bei ihrer Sprachverarbeitung (Vasishth et al. 2010) sind - wenn man Chomskys sprachlichen Dualismus akzeptiert - kein Gegenargument gegen eine genetisch kodierte Sprachfähigkeit (Fitch, Hauser \& Chomsky 2005: 203); an sprachlichen Performanzdaten kann man nämlich Sprachfähigkeit im Sinne Chomskys nicht ablesen. Sie ist - so die Kritiker - gegenüber empirischer Falsifizierung immunisiert (Evans \& Levinson 2009: 436, 443; Tomasello 2009: 471; St. Müller 2016: 455). Wichtig erscheint uns im Kontext psycholinguistischer Forschung auch ein anderer, bereits oben erwähnter Punkt. Die ,internalisierte، Sprache besteht nach Chomskys Auffassung aus wenigen Elementen. Alles andere - und das ist der größte und komplexeste Teil der Grammatik - entspricht der ,externalisierten' Sprache. Für die Erforschung der ,externalisierten“ Sprache müsste man - gerade wenn man dem sprachlichen Dualismus folgt Performanzdaten ernster nehmen als in der Praxis der generativen Grammatikbeschreibung mit wenigen Ausnahmen (z. B. Newmeyer 2005; Marantz 2005) bisher geschehen.

Diese allgemeine Darstellung der generativen Grammatik in ihrer von Noam Chomsky geprägten Version ist hinreichend, um auf diesem Hintergrund alternative Forschungspositionen, die im nächsten Abschnitt vorgestellt werden, einordnen zu können.

\section{Emergente Grammatik}

Widerstand gegen Chomskys Auffassung begann sich in der Linguistik früh zu regen. William Labov (1969) argumentierte mit statistischen Daten, die er dem aktuellen Sprachgebrauch verschiedener sozialer Gruppen entnahm, für variable grammatische Regeln. Noch näher im Forschungsumfeld von Noam Chomsky begann John Ross (1973) an der Wohldefiniertheit grammatischer Kategorien zu zweifeln und leistete der gradiente Kategorien propagierenden psycholinguistischen Prototypentheorie Vorschub (Rosch 1973). George Lakoff (1973) lehnte die Unterscheidung zwischen Kompetenz und Performanz rundum ab und zählt neben Ronald Langacker (1987) zu den Pionieren der Kognitiven Grammatik, der sich die funktional ausgerichtete Sprachtypologie anschloss. Beide Forschungsrichtungen verteidigen eine funktionale Grammatikauffassung, in der die Grammatik auf semantisch-pragmatische Erscheinun- 
gen zurückgeführt wird. Diese Richtung werden wir hier zugunsten psycholinguistisch geprägter Forschung nicht näher beschreiben.

\subsection{Konnektionismus}

Auch die Psycholinguistik erlebte eine Trendwende. Kognitive Prozesse sind in der einflussreichen Theorie von Jerry Fodor (1983) durch autonome syntaktische Regeln geleitete, sequenziell angeordnete Berechnungsprozesse über symbolische mentale Repräsentationen. Dieser Theorie ist auch das im vorigen Abschnitt skizzierte Grammatikmodell von Noam Chomsky verpflichtet (vgl. z. B. Marantz 2005). In den 1980er Jahren begannen alternative konnektionistische Ansätze Erfolge zu feiern, die das Computermodell für unrealistisch hielten und davon ausgingen, dass das menschliche Gehirn einige kognitive Leistungen gerade deshalb erbringen kann, weil es keine sequenzielle Symbolverarbeitung betreibt (z. B. Rumelhart \& McClelland 1986; Smolensky 1986; Elman et al. 1996). Im Konnektionismus werden künstliche neuronale Netzwerksimulationen verwendet. Das sind mathematische Modelle, die einfache Verarbeitungseinheiten (die sog. Neuronen) enthalten und Information in paralleler Weise prozessieren, ohne dass den vielen räumlich und zeitlich verteilten Verarbeitungseinheiten und -schritten ein bestimmter kognitiver Gehalt zugeordnet werden kann; man spricht deshalb auch von subsymbolischer Verarbeitung. Die künstlichen neuronalen Netzwerksimulationen eignen sich die erforderlichen Regeln und Repräsentationen aufgrund ihrer Verbindungsstruktur implizit selbst an. Ziel ist es, gewisse Gesetzmäßigkeiten, die für symbolische Systeme bestehen, als emergente Eigenschaften neuronaler Netze abzuleiten.

Um die Leistung künstlicher neuronaler Netzwerksimulationen besser beurteilen zu können, stellen wir im Folgenden den satzinternen Majuskelgebrauch in einer solchen Simulation vor und vergleichen diesen Ansatz mit der regelbasierten syntaktischen Rekonstruktion desselben Phänomens, die wir in (2) vorstellen:

(2) Der Kopf jeder Nominalphrase wird mit einer initialen Majuskel geschrieben. (z. B. Maas 1992; Fuhrhop 2009; Primus 2010)

Die syntaktische Rekonstruktion in (2) verwendet das syntaktische Konzept des Kopfes (auch Kern) einer Phrase (auch Wortgruppe), das in neueren Grammatiktheorien einschließlich des oben beschriebenen Minimalismus eine zentrale Rolle spielt. So enthält im Sinne des Minimalismus jede Verkettungsoperation (engl. merge) genau ein Element, das seine (z. B. kategorialen) Merkmale an die 
komplexe Einheit projiziert und ggf. die Merkmale der Schwesterkonstituente bestimmt (Adger 2003: 91). Jede Phrase hat einen Kopf. Dies wird bis zu einem gewissen Grad auch in der traditionellen Terminologie berücksichtigt: Verb Verbalphrase, Nomen - Nominalphrase, Adjektiv - Adjektivphrase usf. Des Weiteren bestimmt das Verknüpfungspotenzial des Kopfes, wodurch eine Phrase erweiterbar ist. Für den Kopf einer Nominalgruppe sind vorangestellte flektierte adjektivische Attribute (große Angst, robustes Ich) und artikelähnliche Wörter kennzeichnend (diese große Angst, etwas Unangenehmes). Diese nominalen Begleiter gehören deshalb zu den wichtigsten Kriterien für den Majuskelgebrauch in diesem Ansatz.

Man kann eine Kopfkategorie auch aufgrund der Kategorie der Phrase identifizieren. Wenn eine Nominalphrase vorliegt, dann muss sie auch einen nominalen Kopf haben, den man mit Initialmajuskel schreibt. In diesem $\mathrm{Zu}$ sammenhang steht das Kriterium der nominalen syntaktischen Funktion bzw. des nominalen Satzglieds wie Subjekt und Objekt, sofern sie durch eine Nominalphrase realisiert werden (vgl. Primus 2010). So haben wir nominale Köpfe in hat Angst und kriegt Angst, weil die betreffenden Verben an dieser Stelle nominale Objekte selegieren. Im Unterschied dazu liegen in mir ist angst und mir ist bange adjektivische Prädikative wie in mir ist kalt vor, weil das Verb sein in dieser unpersönlichen Konstruktion für die Prädikativposition keine Nominalphrase selegiert (vgl. die Konstruktion mit pronominalem das als Subjekt das/dich kennenzulernen ist mir ein Vergnügen vs. *das ist mir angst).

Kommen wir nun zum Majuskelgebrauch in Hans-Georg Müllers (2016) künstlicher neuronaler Netzwerksimulation. Wie bereits erwähnt, sind künstliche neuronale Netzwerke mathematische Modelle, die aus vielen kleinen Einheiten (so gen. Neuronen) bestehen. Diese sind in Schichten angeordnet und gewichtet miteinander verbunden. Müller verwendet den Netzwerktyp Pattern Associator, bei dem das neuronale Netz aus zwei Schichten, einer InputSchicht und einer Output-Schicht, besteht. Im Anwendungsfall Majuskelgebrauch repräsentiert der Input die grammatischen Eigenschaften einer schriftsprachlichen Entität. Der Output ist seine normgerechte Groß- bzw. Kleinschreibung. In Simulationsstudien mit einem Pattern Associator wird zwischen einer Trainings- und einer Testphase unterschieden. Innerhalb der Trainingsphase ist dem Netzwerk der Output vorgegeben und die Verbindungsgewichte werden nach Maßgabe der sog. Delta-Regel verändert. Dabei werden aktuell generierter und erwünschter Output verglichen und die Gewichte der Verknüpfungen zwischen den Neuronen so angepasst, dass sich das Netzwerk dem erwünschten Output annähert. In der anschließenden Testphase bleiben die Verbindungsgewichte konstant und das Netzwerk generiert für unbekannten Input entsprechende Outputmuster. Sofern dies adäquat gelingt, repräsentiert das Netzwerk die Muster des Inputs. 
Den Input des Pattern Associators in Müllers Studie bilden mehr als zwanzig grammatische Eigenschaften (H.-G. Müller 2016: 227), die in der Orthographiediskussion als mögliche Einflussfaktoren auf den Majuskel- vs. Minuskelgebrauch identifiziert wurden, wie etwa \pm Konkretum, \pm Subjekt, \pm Objekt, \pm Prädikat, \pm Kern einer NP, \pm Artikel, \pm attribuiert, \pm attribuierbar, \pm Wortart Nomen, \pm Wortart Verb usf. Die grammatischen Einflussfaktoren wurden, wie soeben gezeigt, in dichotomen Variablen gespeichert und jeweils einem InputNeuron zugewiesen. Das Trainingsmaterial für die Netzwerksimulation deckt den überwiegenden Teil der amtlichen Regelungen zur Groß- und Kleinschreibung durch jeweils mindestens sechs Beispiele ab. In den Beispielsätzen wurde die Verwendung potenzieller Einflussfaktoren auf den Majuskelgebrauch systematisch variiert.

Künstliche neuronale Netzwerke können Regelmäßigkeiten des Inputs als prototypische Muster abbilden, ohne die Bildungsregel des Musters im Vorhinein zu kennen. Nach erfolgreichem Lernprozess repräsentiert das Netzwerk die Regularitäten des Inputs durch die Struktur seiner Verbindungsgewichte. Das wird daran erkennbar, dass es adäquate Voraussagen für unbekannten, aber strukturähnlichen Input treffen kann, in Müllers Studie 98,7\% korrekte Schreibungen für unbekanntes Datenmaterial (H.-G. Müller 2016: 227-228).

Wir kommen nun zur vergleichenden Bewertung der regelgeleiteten syntaktischen und konnektionistischen Rekonstruktion der Majuskelverwendung. Der regelbasierte Ansatz verwendet möglichst wenige, abstrakte, d. h. an den Daten nicht unmittelbar ablesbare, Begriffe, wie etwa den Kopfbegriff. Im Konnektionismus arbeitet man bevorzugt mit mehreren oberflächennahen Eigenschaften, „um eine praktikable Operationalisierung zu ermöglichen“ (H.-G. Müller 2016: 210). Für den außerhalb des Konnektionismus arbeitenden Theoretiker bedeutet dies: Die zentrale Eigenschaft wird, viel Redundanz in Kauf nehmend, durch viele korrelierende Eigenschaften erweitert. Müllers Simulation demonstriert auf beeindruckende Weise, dass dieses Vorgehen das Resultat nicht kompromittiert. Wenn man nämlich das Merkmal Kern einer NP durch seine Epiphänomene ergänzt, kann man es eliminieren bzw. sein Gewicht reduzieren (so bei H.-G. Müller 2016: 228). Dies ist möglich, weil Netzwerksimulationen viel Redundanz im Input tolerieren. Regelbasierte Ansätze verlieren hingegen an Attraktivität, wenn ihre Regeln unnötig komplex sind.

Einer Erklärung des Majuskelgebrauchs kommt man anhand von Merkmalen, die mit dem nominalen Kopfbegriff lediglich korrelieren, nicht näher. Darüber hinaus ist eine Rekonstruktion eines Phänomens aufgrund von Epiphänomenen auch auf deskriptiver Ebene risikobehaftet. Verliert man das erklärende Konzept aus den Augen, so kann es vorkommen, dass bei der Wahl der Epiphänomene oder korrelierenden Merkmale einiges schiefläuft. Die Kritik 
an der inadäquaten Majuskelrekonstruktion in Rechtschreib- und Lehrwerken mittels eines Korrelats des nominalen Kopfbegriffs wie etwa Gegenständlichkeit verdeutlicht das Problem (vgl. z. B. Bredel 2010).

Generell erschwert wird die Erklärung eines Phänomens in Netzwerksimulationen dadurch, dass theoriegeleitete Generalisierungen nur verdeckt, z. B. wie in H.-G. Müller (2016) bei der Auswahl der Einflussfaktoren und des Trainingsmaterials, zum Zuge kommen. Im Netzwerk selbst ist das sprachbezogene Wissen nicht rekonstruierbar: Es ist in den einzelnen Neuronen und deren Verbindungsgewichten verteilt und mithin subsymbolischer Natur. Insoweit überrascht es nicht, dass diese grundsätzliche Kritik am Konnektionismus auch in den eigenen Kreisen fortbesteht (vgl. u. a. auch Busemann, Harbusch \& Wermter 1998; Mayor et al. 2014):

\footnotetext{
A main characteristic of mainstream connectionism is its eliminative character, i.e. the idea that the basic architecture of symbolism (including its crucial concepts such as representations, rules, compositionality, and modularity) has to be replaced by the concepts of neural networks [...]. In this way, the main advantage of traditional symbolism - the transparency and relative simplicity of descriptions and explanations - are likewise eliminated. (Blutner 2009: 53-54)
}

Als Reaktion auf solche Kritik wurden integrative Ansätze entwickelt, die sowohl symbolisches und regel- bzw. constraint-basiertes Wissen als auch parallelverteilte Informationsverarbeitung inkorporieren (z. B. Busemann, Harbusch \& Wermter 1998; Blutner 2009). Das einflussreichste Erbe des Konnektionismus ist die Übernahme einer parallelen (statt wie im Minimalismus seriellen) Grammatikarchitektur in mehreren Modellen, wie etwa der Optimalitätstheorie, der Head Driven Phrase Structure Grammar und der Lexical Functional Grammar (vgl. St. Müller 2016).

Hinsichtlich des Forschungsstils zeigt sich, dass H.-G. Müllers (2016) Netzwerksimulation nicht unabhängig von theoriegeleiteten Generalisierungen bei der Wahl der Einflussfaktoren und des Trainingsmaterials aufgestellt wurde. Insoweit trifft die allgemeine Kritik von Fodor und Pylyshyn (1988) auch auf diese Fallstudie zu: Konnektionismus kann Verhalten nicht wirklich erklären, bestenfalls liefert er eine Implementation eines vorgegebenen regelbasierten Symbolsystems. Dennoch ist der Wert einer Netzwerksimulation für die Überprüfung bestehender regelbasierter Theorien nicht $\mathrm{zu}$ unterschätzen, wie H.-G. Müllers Vergleich mehrerer fragmentierter Netzwerksimulationen für verschiedene regelbasierte Ansätze demonstriert (2016, Kap. 7.6).

Es gibt eine Eigenschaft, die der Konnektionismus mit regelbasierten Grammatiktheorien teilt: Die angenommenen künstlichen Neuronen haben wenig mit der neurophysiologischen Wirklichkeit gemeinsam, daher können sie 
auch wenig zur Erklärung mentalen Verhaltens beitragen. Beide Forschungsrichtungen erheben allerdings auch nicht den Anspruch, neurophysiologische Sprachverarbeitung direkt zu modellieren. Was mehrere Linguisten anstreben, ist ein Modell, das mit der neurophysiologischen Sprachverarbeitung kompatibel ist, wie etwa Culicover \& Jackendoff (2005) und Newmeyer (2005) (vgl. St. Müller 2016: 505-506 für Kriterien, die eine solche Grammatiktheorie erfüllen muss).

Im Sinne dieses Kompatibilitätsgebots könnte man kritisieren, dass theoretische Konstrukte im Allgemeinen und der Kopfbegriff, den der regelbasierte Majuskelgebrauchsansatz verwendet, im Besonderen abstrakte Begriffe sind, die der konkreten neurophysiologischen Sprachverarbeitung ferner stehen als die oberflächennäheren Variablen bzw. Hinweisreize (engl. cues), die man im Konnektionismus verwendet. Experimentelle Befunde lassen Zweifel an einer solchen Kritik aufkommen. Dass Phrasenköpfe intuitiv identifiziert und bei der Sprachverarbeitung genutzt werden, ist anhand neurophysiologischer Daten belegt (Friederici 2002; Bornkessel-Schlesewsky \& Schlesewsky 2009: 91). Näher an unserem Anwendungsfall sind die experimentellen Befunde von Jenny Saffran, die zeigen, dass ein Hinweisreiz auf einen nominalen Kopf und die damit ausgelöste Kopfidentifizierung und Verkettung von Wörtern zu Phrasen den Spracherwerb erleichtert (Saffran 2003: 112):

Do humans learn sequential structures better when they are organized into subunits such as phrases than when they are not? We identified a statistical cue to phrasal units, predictive dependencies (e.g., the presence of a word like the or $a$ predicts a noun somewhere downstream; the presence of a preposition predicts a noun phrase somewhere downstream), and determined that learners can use this kind of cue to locate phrase boundaries [...]. In a direct test of the theory that predictive dependencies enhance learnability, we compared the acquisition of two nonsense languages, one with predictive dependencies as a cue to phrase structure, and one lacking predictive dependencies [...]. We found better language learning in listeners exposed to languages containing predictive dependencies than in listeners exposed to languages lacking predictive dependencies.

\subsection{Frequenzbasierte Modelle}

Der Idee einer emergenten Grammatik ist nicht nur der Konnektionismus, sondern auch der einflussreiche gebrauchsbasierte Ansatz von Paul Hopper und Joan Bybee (Hopper 1987; Bybee \& Hopper 2001a, b) verpflichtet (vgl. Gries \& Divjak 2012; Adli, García García \& Kaufmann 2015; Behrens \& Pfänder 2015 u.v.m.). Folgende Aussagen verdeutlichen die Kernideen dieses Ansatzes:

[E]mergent structures are unstable and manifested stochastically ... From this perspective, mental representations are seen as provisional and temporary states of affairs that are 
sensitive, and constantly adapting themselves, to usage. 'Grammar' itself and associated theoretical postulates like 'syntax' and 'phonology' have no autonomous existence beyond local storage and real-time processing. (Bybee \& Hopper 2001b: 2-3)

Dass Form und Funktion sprachlicher Ausdrücke von ihrer Gebrauchsfrequenz beeinflusst werden, ist spätestens seit den Arbeiten von George Zipf (1935) bekannt. Gebrauchsfrequenz hat allerdings erst in den jüngeren gebrauchsbasierten Modellen, wie den oben erwähnten, einen zentralen Stellenwert erhalten. Auch in der Psycholinguistik sind frequenzbasierte Erklärungen für den Spracherwerb und die neuronale Sprachverarbeitung populär. Der Einfluss der Gebrauchsfrequenz in Spracherwerb und Sprachverarbeitung ist unbestritten. Viele Studien belegen, dass Kinder sehr früh distributionelle Muster und statistische Auftretenswahrscheinlichkeiten im sprachlichen Input, den sie erhalten, zuverlässig erkennen (Saffran 2003; Pelucchi, Hay \& Saffran 2009; Ellis 2015). Frequenz ist ein wichtiger Faktor auch in der neuronalen Sprachverarbeitung (z. B. MacDonald, Pearlmutter \& Seidenberg 1994; Jurafsky 1996; Crocker \& Brants 2000).

Auch im Rahmen der generativen Grammatiktheorie wird Frequenz, u. a. für die Erklärung diachroner Daten (vgl. Lightfoot 1991), als Faktor herangezogen. Der Unterschied zu frequenzbasierten Ansätzen ist dennoch sehr groß. Während frequenzbasierte Ansätze Gebrauchsfrequenz als zentralen erklärenden Faktor und Grammatikregeln als emergente Folgeerscheinungen behandeln, werden in der generativen Grammatiktheorie und von Theoretikern, die sich ihr in diesem Punkt anschließen, für die Erklärung menschlicher Sprachfähigkeit im engeren Sinne ausschließlich grammatische Prinzipien herangezogen. Gebrauchsfrequenz spielt nur auf der Ebene der Performanz eine Rolle. Geprägt wurde diese Auffassung von Noam Chomsky bereits in den 50er Jahren (Chomsky 1957). Eines seiner meist zitierten Argumente ist, dass Sätze wie Colorless green ideas sleep furiously, die wegen ihrer semantischen Anomalie gar nicht in Gebrauch sind, dennoch syntaktisch wohlgeformt sind. Seine diesbezügliche Annahme hat Generationen von Linguisten beeinflusst: „I think that we are forced to conclude that [...] probabilistic models give no particular insight into some of the basic problems of syntactic structure" (Chomsky 1957: 17). Gegen den Einfluss von Gebrauchsfrequenz auf die Grammatik argumentiert Jahrzehnte später auch Frederick Newmeyer:

The mental grammar contributes to an explanation of language use, but usage, frequency, and so on are not represented in the grammar itself. ... probabilistic information drawn from corpora is of the utmost value for many aspects of linguistic inquiry. But it is all but useless for providing insights into the grammar of any individual speaker. (Newmeyer 2003: 6, 20) 
Angesichts dieser divergierenden Positionen wollen wir im Folgenden die Möglichkeiten und Grenzen eines frequenzbasierten Ansatzes anhand einer Fallstudie illustrieren.

Die hier präsentierten Frequenzdaten wurden in Vorbereitung auf eine Akzeptabilitätsstudie und ein neurolinguistisches Experiment, die wir in diesem und nächsten Abschnitt referieren, erhoben (Graf et al. 2017; Philipp et al. 2017; Graf (in Vorb.)). Wie bereits erwähnt, verteidigen mehrere Arbeiten die Rolle von Gebrauchsfrequenz bei der Sprachverarbeitung, sodass dieser Faktor für die Erklärung unserer Akzeptabilitätsbefunde und ereigniskorrelierter Potenzial-Messungen (EKPs) in Betracht gezogen werden muss. Unsere Korpusstudie wurde für die anderen Studien als Vortest konzipiert und erhebt, insbesondere, was die geringe Datenmenge betrifft, nicht den Anspruch, rigorose korpuslinguistische Standards zu erfüllen. In diesem Beitrag dient sie lediglich dazu, die Frage zu illustrieren, welchen Erkenntnisgewinn wir aus Frequenzdaten auch im Vergleich zu Akzeptabilitäts- und EKP-Messungen - ziehen können. Darüber hinaus interessiert auch die im vorigen Abschnitt angeschnittene methodologische Frage, ob sich der Forschungsstil frequenz- und korpuslinguistischer Ansätze als rein induktiv und mithin dem Chomsky'schen deduktiven Vorgehen diametral entgegengesetzt charakterisieren lässt.

Unser Untersuchungsgegenstand ist die flexible Auxiliarwahl sein vs. haben. Um die Suche auf relevante Fälle einzugrenzen, extrahierten wir aus der Forschung die Hypothese, dass intransitive Bewegungsverben die Perfekttempora grundsätzlich sowohl mit haben als auch mit sein bilden können (z. B. Zifonun et al. 1997; Eisenberg 2013). Vgl. die Sätze (3a, b), deren relative Akzeptabilität weiter unten genauer besprochen wird:

(3) a. Der Pilot ist über der Stadt geflogen.

sein

b. Der Pilot hat über der Stadt geflogen.

haben

Unsere Korpusrecherche war also hypothesengeleitet. Aber auch ein induktives Vorgehen hätte ergeben, dass die von uns untersuchten Bewegungsverben eine variable Auxiliarwahl haben. Der Nachteil der induktiven Methode wäre ein größerer Aufwand bei der Suche und Mustererkennung gewesen. Auch waren wir - aus Gründen, die weiter unten erläutert werden - nicht an allen intransitiven Bewegungsverben mit variabler Auxiliarwahl interessiert, sondern suchten gezielt nach dem Perfektpartizip der sechs Verben fliegen, schwimmen, rollen, wirbeln, schweben und schlingern.

Da an unseren experimentellen Studien Kölner Studenten teilnahmen, haben wir auch für die Korpusuntersuchung den mittelrheinischen Sprachraum, zu dem auch Köln gehört, ausgewählt. Unsere Wahl fiel auf die Rhein Zeitung 
(Jahrgänge 1996-2012, Abk. RZ) aus dem Deutschen Referenzkorpus (Abk. DeReKo, Institut für Deutsche Sprache 2012), die vom Mittelrhein-Verlag mit Sitz in Koblenz herausgegeben wird.

Die Gebrauchshäufigkeit der untersuchten Verben schwankt erheblich. Die Suche nach geflogen in RZ ergab 6.728 Treffer; geschwebt und geschlingert waren in $\mathrm{RZ}$ so selten belegt, dass wir alle DeReKo-Korpora, die dem hochdeutschen Sprachraum zuzuordnen waren, heranzogen. Die erweiterte Suche ergab für geschwebt 105 Treffer und für geschlingert 44 Treffer. Für die häufigeren Verben (geflogen, geschwommen, gerollt, gewirbelt) wurden je 300 Belege exportiert. Diese und alle Belege mit geschwebt und geschlingert wurden manuell weiterverarbeitet. Eliminiert wurden Passive (z. B. hier wurde geschwommen, weil fast keine Strömung vorherrschte), nicht-wörtliche Lesarten (z. B. In der zweiten Halbzeit hat die Heimmannschaft zwischenzeitlich geschwommen) und Belege mit Akkusativ-NP (z. B. Endlich habe ich das Ding mal nach Hause geschwommen). Die verbleibenden 287 Belege wurden sortiert nach sein vs. haben. Vgl. Tabelle 1.1:

Tab. 1.1: Sein vs. haben bei geflogen, geschwommen, gerollt, gewirbelt, geschwebt, geschlingert.

\begin{tabular}{lrr}
\hline sein & 258 Belege von 287 & $89.90 \%$ \\
haben & 29 Belege von 287 & $10.10 \%$ \\
\hline
\end{tabular}

Zur inferenzstatistischen Analyse wurden von Tim Graf log-lineare Regressionsmodelle schrittweise in ihrer Komplexität erhöht und in Bezug darauf miteinander verglichen, wie gut sie die Streuung in den Daten erklären (Graf (in Vorb.)). Das Hinzufügen des Prädiktors Auxiliar erhöht gegenüber einem Modell ohne Prädikatoren die Erklärung der Varianz signifikant $(\Delta=87.900$, $p<.001)$. Unsere Pilotstudie bestätigt die Annahme der einschlägigen Forschung, dass sein gegenüber haben bei diesem Verbtyp präferiert wird (z. B. Randall et al. 2004; Legendre 2007). Allerdings ist der Erkenntnisgewinn dieser Frequenzdaten gering. Interessant wäre zu wissen, von welchen Faktoren die Auxiliarwahl bei diesem Verbtyp abhängt. Ein rein induktives Verfahren kommt schnell an seine Grenzen, denn ohne Rekurs auf bereits existierende Generalisierungen zu diesem Phänomen wäre die Suche nach Mustern offen und der Ausgang ungewiss. Es überrascht somit nicht, dass bei der Mustersuche in frequenzbasierten Ansätzen oft nur leicht auffindbare Merkmale herangezogen werden, wie hier sein vs. haben.

Um den Erkenntniswert zu steigern, haben wir unserer Frequenzstudie weitere Hypothesen zugrundegelegt. Eine weitere Hypothese, die wir der bis- 
herigen Forschung entnahmen, ist, dass die Auxiliarwahl von der Telizität der Sätze, in welche die Verben eingebettet sind, abhängt (z. B. Zifonun et al. 1997: 1862-1863; Engelberg 2000: 55-56; Eisenberg 2013: 99-100). Die von uns gewählten sechs Verben sind geeignet, diese Hypothese zu testen, weil sie sowohl eine telische als auch eine atelische Lesart haben können in Abhängigkeit davon, ob sie eine Lokativangabe, wie über der Stadt in (4a), oder ein Zielargument, wie ins Tal in (4b), zu sich nehmen (vgl. Lukassek et al. 2016). Die telische Lesart ist mit Zeitspannenadverbialen wie in zwei Stunden semantisch kompatibel, die atelische mit durativen Zeitadverbialen vom Typ zwei Stunden lang (Vendler 1957). Vgl. (4):

(4) a. Der Pilot flog zwei Stunden lang über der Stadt. ohne Zielargument atelisch

b. Die Staubwolke flog in zwei Stunden ins Tal. mit Zielargument telisch

Um unsere Belege gezielt nach Telizität $\mathrm{zu}$ sortieren, waren weitere Hypothesen nötig, die wir u. a. aus der Theorie von Vendler (1957) und Tenny (1994) herleiteten. Als telisch wurden Belege mit eindeutiger Ziel-Phrase (auch im Kontext) bewertet (z. B. nach Teheran geflogen). Ebenfalls als telisch eingestuft wurden Belege mit einer spezifischen Distanzangabe (z. B. unglaubliche 484 Kilometer weit geflogen). Als atelisch wurden Belege ohne Ziel- oder Distanz-Phrase im Satz oder Kontext bewertet. Ebenfalls als atelisch eingestuft wurden Belege mit einer Pfadangabe (z. B. durch Dieblich geschlingert), bei denen der Kontext eine telische Lesart unplausibel erscheinen lässt. Es blieben Zweifelsfälle, die wir protokolliert haben. Tabelle 1.2 fasst die Ergebnisse dieses Sortiervorgangs zusammen:

Tab. 1.2: Telizität bei den 258 Belegen mit sein.

\begin{tabular}{lll}
\hline telisch & atelisch & Telizität unklar \\
\hline 167 & 78 & 13 \\
$64.7 \%$ & $30.2 \%$ & $5.1 \%$ \\
\hline
\end{tabular}

Tab. 1.3: Telizität bei den 29 Belegen mit haben.

\begin{tabular}{lcl}
\hline telisch & atelisch & Telizität unklar \\
\hline 0 & 29 & 0 \\
$0 \%$ & $100 \%$ & $0 \%$ \\
\hline
\end{tabular}


Die Regressionsanalyse ergibt für die klaren Fälle, dass ein Modell mit den Prädiktoren Auxiliar und Telizität die Varianz in den Daten nochmals besser erklärt $(\Delta=13.666, p<.001)$. Wir können somit eine - zugegebenermaßen bescheidene - frequenzbasierte Evidenz dafür liefern, dass Telizität bei der Auxiliarwahl, wie von der Forschung vorhergesagt, tatsächlich eine Rolle spielt: sein wird in telischen und haben in atelischen Kontexten bevorzugt. Darüber hinaus führen unsere Korpusdaten zu einer neuen Beobachtung (vgl. auch Gillmann 2016): sein wird bei diesem Verbtyp in unserem Korpus in telischen wie atelischen Kontexten, haben nur in atelischen Kontexten gewählt. Die Regressionsanalyse zeigt hier, dass ein Modell mit der zusätzlichen Interaktion von Auxiliar und Telizität signifikant mehr Varianz erklärt $(\Delta=54.401, p<.001)$. Damit könnten wir unsere Korpusstudie beenden und hätten dabei eine Chance verpasst, zu weiteren Einsichten über das untersuchte Phänomen zu gelangen.

Den Weg zu weiteren Erkenntnissen ebnete uns die Unakkusativitätshypothese (Perlmutter 1978; Dowty 1991; Zaenen 1993; Keller \& Sorace 2003). Gemäß dieser These gibt es unakkusative und unergative intransitive Verben. Unakkusative Verben sind - so die Annahme - telisch, selegieren ein Patiens oder Thema als Subjekt und sein als Auxiliar. Unergative Verben sind atelisch, wählen ein Agens als Subjekt und haben als Auxiliar. Die von uns gewählten sechs Verben sind geeignet, diese Hypothese zu testen, weil sie sowohl mit belebten als auch mit unbelebten Subjekten kombinierbar sind. Belebte Subjektreferenten können kontrolliert handeln und werden somit per Default agentivisch interpretiert (vgl. Van Valin \& Wilkins 1996; Engelberg 2005), für unbelebte Subjektreferenten scheidet die Agensinterpretation aus. Vgl. (5):

(5) a. Die Staubwolke flog über der Stadt.

b. Der Pilot flog über der Stadt.

inanimat, Thema/Patiens animat, Agens

Keller \& Sorace (2003: 88) sagen voraus, dass für Verben wie die von uns untersuchten, die weder eindeutig unakkusativ noch eindeutig unergativ sind, Agentivität bzw. Belebtheit bei der Auxiliarselektion eine Rolle spielt. Solche Verben sollten haben für agentivische bzw. belebte Subjekte und sein für nichtagentivische bzw. unbelebte Subjekte präferieren.

Bei der Festlegung des Belebtheitswerts unserer Korpusbelege kamen aus der Forschung hergeleitete Generalisierungen über Belebtheit ins Spiel (z. B. Zaenen et al. 2006). Als animat haben wir Belege mit belebten, menschlichen Referenten eingestuft, als inanimat Belege mit unbelebten Referenten. Belege mit diesbezüglich unklaren Referenten, z. B. Tieren und Vereins- oder Firmennamen (z. B. Der VfL Wolfsburg ist nach München geflogen), haben wir als Zweifelsfälle eingeordnet. Die Tabellen 1.4 und 1.5 fassen die Ergebnisse dieses Sortiervorgangs zusammen: 
Tab. 1.4: Belebtheit bei den 258 Belegen mit sein.

\begin{tabular}{lll}
\hline animat & inanimat & Animatheit unklar \\
\hline 145 & 109 & 14 \\
$53.5 \%$ & $41.1 \%$ & $5.4 \%$ \\
\hline
\end{tabular}

Tab. 1.5: Belebtheit bei den 29 Belegen mit haben.

\begin{tabular}{lll}
\hline animat & inanimat & Animatheit unklar \\
\hline 14 & 12 & 3 \\
$48.3 \%$ & $41.4 \%$ & $10.3 \%$ \\
\hline
\end{tabular}

Entgegen der aus der Unakkusativitätshypothese hergeleiteten Voraussage von Keller \& Sorace (2003: 88) sind bei beiden Auxiliaren animate Subjekte häufiger als inanimate. Die statistische Analyse der klaren Fälle zeigt, dass ein Modell, welches Animatheit als zusätzlichen Prädiktor enthält, nochmals mehr Varianz erklärt $(\Delta=5.345, p=0.021)$. Die Inklusion der Interaktion von Auxiliar und Animatheit bringt jedoch keinen signifikanten Anstieg der Varianzerklärung $(\Delta=0.132, p=0.716)$. Zur Erinnerung: Verben, die weder eindeutig unergativ noch eindeutig unakkusativ sind, sollten haben für animate Subjekte und sein für inanimate Subjekte präferieren (Keller \& Sorace 2003: 88). Unabhängig vom Auxiliar werden aber bei den untersuchten Bewegungsverben belebte Subjekte gegenüber unbelebten bevorzugt. Dies entspricht der generellen Präferenz für belebte Subjekte im Deutschen (z. B. Bader \& Häusler 2010; Fischer 2013). Unsere kleine Korpusstudie widerlegt zwar die Voraussage von Keller und Sorace, bestätigt aber die Ergebnisse ihrer Akzeptabilitätsstudie (Keller \& Sorace 2003: 96): Sie ergab für die Auxiliarwahl anderer intransitiver Verben ebenfalls keine signifikanten Belebtheitseffekte. Ein leichter numerischer Trend in unseren Korpusdaten (vgl. Tab. 1.4 und 1.5) zeigt in eine andere Richtung als von der Unakkusativitätsthese vorausgesagt: Das Frequenzgefälle von animaten zu inanimaten Subjekten ist bei sein etwas größer als bei haben. Allerdings ist aufgrund der Präferenz für belebte Subjekte, der verbtypspezifischen Präferenz für sein (vgl. Tab. 1.1) und der kleinen Fallzahl von Belegen bei der Interpretation dieses numerischen Trends größte Vorsicht geboten.

Um Telizität und Animatheit als Einflussfaktoren auf die Auxiliarwahl näher zu untersuchen, haben wir eine Akzeptabilitätsstudie durchgeführt (Graf et al. 2017). Ein Experiment bietet den Vorteil, mutmaßliche Einflussfaktoren vollständig zu kreuzen und somit Bedingungsrelationen aufzudecken. Die Akzeptabilitätsstudie hat den Nachteil, dass die Experimentsituation nicht der 
realen Kommunikationssituation entspricht und die den Probanden gestellte Aufgabe, Sätze hinsichtlich ihrer Akzeptabilität zu bewerten, metasprachliche Kompetenzen abfragt. Das Material der Akzeptabilitätsstudie enthielt dieselben sechs Verben wie die Korpusstudie. Die Manipulation der Variablen SEIN/ HABEN, TELIZITÄT und ANIMATHEIT erfolgte in einem vollständig gekreuzten Design. Die Variablen und die syntaktische Struktur der Testitems werden in (6) illustriert und wurden anhand der Beispiele in (3)-(5) bereits erläutert:

(6) Itemstruktur für die Akzeptabilitäts- und EKP-Studie

Dass der Inlineskater / die Radkappe letzten Mittwoch auf dem Feldweg / zur Ampel gerollt ist / hat, verwunderte den Verkehrspolizisten.

Die 95 Probanden, deren Fragebögen analysiert wurden, waren Studierende an der Universität zu Köln (vgl. Graf et al. 2017 für weitere Details zur Durchführung und Auswertung des Experiments).

Die Ergebnisse der Akzeptabilitätsstudie sind mit denen der Korpusstudie kompatibel. Das Auxiliar sein wird für diesen Verbtyp signifikant besser beurteilt als haben. Es gibt eine zweifache Interaktion zwischen TELIZITÄT und SEIN/HABEN: Die Akzeptabilitätswerte für haben in der atelischen Bedingung sind signifikant besser als in der telischen, während für sein signifikant bessere Werte in der telischen (gegenüber der atelischen) Bedingung vorliegen. Auch wird haben in der telischen Bedingung schlechter bewertet als sein in der atelischen. Die dreifache Interaktion zwischen TELIZITÄT, SEIN/HABEN und ANIMATHEIT ergibt keinen statistisch signifikanten Unterschied. Es gibt nur einen numerischen Trend: In atelischen Kontexten mit haben werden unbelebte Subjektreferenten tendenziell besser bewertet als belebte Referenten.

Wir können die Ergebnisse beider Studien somit wie folgt zusammenfassen: Für intransitive Bewegungsverben, die hinsichtlich Auxiliarwahl, Belebtheit des Subjekts und Telizität (im oben spezifizierten Sinn) grundsätzlich flexibel sind, wird sein gegenüber haben generell präferiert, wobei sein in telischen und haben in atelischen Kontexten bevorzugt wird und die Dispräferenz für haben in telischen Kontexten größer ist als für sein in atelischen Kontexten. Belebtheit scheint die Auxiliarwahl nicht in einem signifikanten Umfang zu beeinflussen, aber beide Studien zeigen eine Tendenz, die der Unakkusativitätsthese widerspricht. In der Korpusstudie ist das Frequenzgefälle von animaten zu inanimaten Subjekten bei sein etwas größer als bei haben. In der Akzeptabilitätsstudie werden in atelischen Kontexten mit haben unbelebte Subjektreferenten tendenziell besser bewertet als belebte Referenten. Gemäß der aus der Unakkusativitätsthese hergeleiteten Prognose von Keller \& Sorace (2003: 88) sollte jedoch haben für agentivische bzw. belebte Subjektreferenten und sein 
für nicht-agentivische bzw. unbelebte Subjektreferenten präferiert sein. Unsere Befunde deuten auf einen entgegengesetzten Trend für den untersuchten Verbtyp hin, bei dem haben eher mit Unbelebtheit und sein eher mit Belebtheit harmoniert.

Die statistische Analyse lässt nur die angegebenen relativen graduellen Akzeptabilitätsangaben zu. Diese lassen sich vereinfachend wie folgt darstellen (angegeben sind die erreichten Mittelwerte ohne Angabe der Standardabweichung für belebte/unbelebte Subjektreferenten, maximaler Akzeptabilitätswert 4): sein-telisch $(3,17 / 3,12)>$ sein-atelisch $(2,69 / 2,59)>$ haben-atelisch $(2,19 /$ 2.46) > haben-telisch $(1,52 / 1,42)$. Bipolare absolute Einordungen als grammatisch vs. ungrammatisch oder akzeptabel vs. inakzeptabel, wie sie in vielen Grammatiktheorien, aber auch in normativen Grammatiken üblich sind, wären willkürliche Festlegungen. So kann man aus unserer Korpus- und Akzeptabilitätsstudie nicht zuverlässig ableiten, dass haben bei diesen Verben in der atelischen Bedingung (vgl. Bsp. (3b) weiter oben) nur marginal akzeptabel und in der telischen Bedingung völlig inakzeptabel ist. Auch die Korpusdaten rechtfertigen keine absolute (In)akzeptabilitätsangabe. Immerhin haben wir 29 Belege mit haben in atelischen Kontexten gefunden (vgl. Tabelle 3) und aus der Tatsache, dass haben in telischen Kontexten in unserem eher kleinen Korpus nicht belegt ist, lässt sich keine Inakzeptabilität ableiten. Nun könnte man dies gegen entsprechende Methoden der Datengewinnung in Anschlag bringen. Eine solche Kritik wäre unzeitgemäß, denn auch Referenzgrammatiken (z. B. Zifonun et al. 1997; Eisenberg 2013), die als Orientierung für ,gutes‘ Deutsch gelten, verzichten oft auf absolute Akzeptabilitätsangaben. Es reicht zu wissen, dass sein bei diesen Verben generell und insbesondere in telischen Kontexten gegenüber haben im untersuchten Sprachraum bevorzugt wird. Die daraus ableitbare Gegenhypothese ist, dass haben generell und insbesondere in telischen Kontexten gegenüber sein dispräferiert ist.

Neben der Tatsache, dass relative, gradiente Daten, die von einer Population von Muttersprechern elizitiert werden, nicht ohne weiteres in absolute (In)akzeptabilitäts- bzw. (Un)grammatikalitätsangaben übersetzt werden können, gibt es Weiteres zu bedenken. Erstens kann man aus einem solchen Datenpool das Verhalten individueller Muttersprecher, das kategorisch sein kann, nicht ableiten. Wenn jemand etwa den Satz (3b) weiter oben als völlig inakzeptabel einstuft und nie verwendet, wäre dies mit den gradienten Populationsdaten genauso kompatibel wie der entgegengesetzte Individualfall, dass (3b) ohne Bedenken verwendet wird. Zweitens erzwingen gradiente Populationsurteile keine gradient formulierten grammatischen Prinzipien oder Beschränkungen, wie sie in frequenzbasierten Ansätzen öfter vorkommen. Wenn „Gradienz ein Epiphänomen ist, das entsteht, weil mehrere Faktoren beteiligt sind, von 
denen einige stärkeren oder schwächeren Einfluss auf die Gesamtbeurteilung haben, dann ist die gradiente Formulierung eines Prinzips ein Missgriff. Sie verbaut geradezu den Weg zu einer präzisen Modellierung“ (Haider 2011: 42). Einen Weg, gradiente Populationsdaten durch eine gewichtete Interaktion kategorisch formulierter Beschränkungen zu erklären, schlägt die stochastische Optimalitätstheorie vor (vgl. z. B. Hayes 2001).

Dass Korpusfrequenzdaten und Akzeptabilitätsurteile - wie in unseren Untersuchungen - kompatible Ergebnisse liefern, stimmt mit den Ergebnissen mehrerer methodisch-komparativer Studien über andere Datenbereiche überein (vgl. Kempen \& Harbusch 2008; Bader \& Häusler 2010). Diese Studien zeigen allerdings, dass es für die dort untersuchten Phänomene auch Unterschiede gibt. Auch bei Übereinstimmung zwischen Korpusfrequenzdaten und Akzeptabilitätsurteilen, wie in unserer Fallstudie, wäre es voreilig, kausale Erklärungen zu bemühen. Die Ergebnisse unserer Akzeptabilitätsstudie könnte man genauso gut frequenzbasiert erklären wie die Frequenzdaten akzeptabilitätsbasiert und beide könnten auf einen dritten, noch unbekannten Faktor zurückgehen.

In unseren Auxiliar-Fallstudien haben wir keine induktive Methode $^{2}$ verwendet, sondern uns dem in der Psycholinguistik üblichen Verfahren bedient, aus theoretischen oder allgemeineren empirischen Hypothesen testbare spezifischere Voraussagen herzuleiten und anhand unserer Ergebnisse die allgemeineren Hypothesen zu stützen oder zu widerlegen:

Psycholinguistik versteht sich als empirische Wissenschaft. Es geht darum, theoretisch begründete Hypothesen über das Verhalten von Sprachbenutzern anhand von empirischen Beobachtungen zu stützen oder zu widerlegen, um so Entscheidungsgrundlagen für das Beibehalten oder Ablehnen der zugrunde liegenden Theorie zu erhalten. (Sichelschmidt \& Carbone 2003: 115)

Dieses Zitat verdeutlicht, dass Chomskys Trennung zwischen deduktiv-mathematischem Forschungsstil in der generativen Grammatiktheorie und induktivexperimentellem Forschungsstil in gebrauchsorientierten Ansätzen nicht der gängigen Forschungspraxis in der Psycholinguistik entspricht.

Der gebrauchsbasierte Ansatz von Bybee und Hopper geht davon aus, dass Grammatik keine autonome Existenz unabhängig von der in Echtzeit ablaufen-

2 Empirische Studien können allerdings Daten aufdecken, die noch nicht dokumentiert sind. Auf dieser Grundlage könnte man durch den induktiven Schritt der Generalisierung eine neue tentative Hypothese generieren, allerdings muss diese in weiteren empirischen Studien als Ausgangshypothese deduktiv eingesetzt werden, um sie als ernst zu nehmende Hypothese einzustufen. 
den Sprachverarbeitung hat. Wir erinnern uns an das Zitat zu Beginn dieses Unterabschnitts: „'Grammar’ itself and associated theoretical postulates like 'syntax' and 'phonology' have no autonomous existence beyond local storage and real-time processing “ (Bybee \& Hopper 2001b: 3). Allerdings erfassen weder quantitative Korpusstudien - die Hauptdatenquelle frequenzbasierter Ansätze (vgl. Bybee 2007: 18) - noch Akzeptabilitätsbefragungen die in der Zeit ablaufende Prozessualität der kognitiven Sprachverarbeitung. Beide Methoden untersuchen fertige Sprachprodukte und mithin das Endresultat von Sprachverarbeitungsprozessen. Aus diesem Grund werfen wir im nächsten Abschnitt einen Blick auf die in Echtzeit ablaufende Prozessualität der kognitiven Sprachverarbeitung.

\section{Inkrementelle Grammatik}

Bei allen kognitiven Vorgängen - wie etwa der Wahrnehmung von Sprache entstehen durch die Aktivität der Gehirnzellen elektrische Spannungsschwankungen (engl. potential shifts). Mithilfe der Elektroenzephalographie (EEG) kann man diese Spannungsschwankungen auf der Kopfoberfläche mit Elektroden messen und graphisch darstellen. Wenn die Potenziale parallel zu einem Sprachverstehensprozess aufgezeichnet werden, kann man einzelne Schritte der Verarbeitung in ihrer zeitlichen Abfolge erfassen und Potenziale zeitlich an ein spezifisches sprachliches Ereignis koppeln (ereigniskorrelierte Potenziale, Abk. EKP). Vergleiche zwischen zwei sich nur minimal voneinander unterscheidenden Bedingungen können zu einer Abweichung im EKP-Muster führen, wobei durch deren Charakter und die Art der Manipulation Rückschlüsse auf sprachverarbeitende Operationen möglich werden (Streb \& Rösler 2003). Vier Parameter eines EKP-Effekts sind für seine Klassifizierung ausschlaggebend: die negative oder positive Polarität, die Latenzzeit in Millisekunden (ms) nach dem Beginn des sprachlichen Stimulus, die Amplitude und die Topographie, die allerdings an der Kopfoberfläche gemessen nur einen indirekten Aufschluss über die neuronale Quelle gibt. So lässt sich bspw. der als N400 klassifizierte Effekt anhand seiner negativen Polarität, seiner Latenzzeit von ca. $400 \mathrm{~ms}$ und seiner zentro-parietalen Topographie charakterisieren. Der als P3 bezeichnete Effekt ist eine Positivierung mit einer variablen Latenzzeit von ca. 300-700 ms und einer posterioren Topographie. Mit diesen beiden Effekten werden wir uns weiter unten beschäftigen.

Bei der Interpretation zeitsensitiver EKP-Daten ist das inzwischen allgemein akzeptierte Prinzip der Inkrementalität der Verarbeitungsschritte beim Sprachverstehen maßgeblich (Crocker 1994; Bornkessel-Schlesewsky \& 
Schlesewsky 2009: 89-90). Es besagt, dass einzelne sprachliche Stimuli nicht nur voranschreitend wahrgenommen, sondern auch sofort mental weiterverarbeitet werden, z. B. indem ihnen eine Struktur und Interpretation mit den aktuell zur Verfügung stehenden Elementen und Informationen zugeordnet werden. Da im Verlauf des Sprachverstehens nicht zu jedem Zeitpunkt hinreichende Information zur Verfügung steht, werden bisweilen aufgrund unzureichender Information wie etwa kasusambiger Verbargumente Kategorien und Strukturen aufgebaut, die unter Umständen zu einem späteren Zeitpunkt revidiert werden müssen (Crocker 1994; Bornkessel-Schlesewsky \& Schlesewsky 2009: 93-94).

Um eine konkretere Vorstellung von der in Echtzeit ablaufenden Prozessualität der kognitiven Sprachverarbeitung zu gewinnen, werden wir im Folgenden unsere EKP-Studie zur Auxiliarwahl präsentieren (vgl. Philipp et al. 2017; Graf et al. 2017). ${ }^{3}$ Das Material dieser Untersuchung enthielt dieselben sechs Verben wie die Korpus- und Akzeptabilitätsstudie. Die Manipulation der Variablen SEIN/HABEN, TELIZITÄT und ANIMATHEIT erfolgte wie in der Akzeptabilitätsstudie in einem vollständig gekreuzten Design. Die Variablen und die syntaktische Struktur der Testitems wurden in (6) weiter oben illustriert und anhand der Beispiele in (3)-(5) erläutert. Die syntaktische Struktur der Testitems wurde so gewählt, dass bei der voranschreitenden visuellen Präsentation der einzelnen Wörter oder Phrasen (NPs und PPs wurden als Ganzes präsentiert) das Auxiliar sein vs. haben erst nach der Information zur Agentivität bzw. Belebtheit des Subjekts, zur Telizität (Zielargument vs. Lokalangabe) und zur Bedeutung des Verblexems zur Verfügung stand. Auf diese Weise war es möglich festzustellen, ob diese Faktoren einen Einfluss auf die Auxiliarwahl haben.

Die 28 Probanden, deren Daten analysiert wurden, waren Studierende an der Universität zu Köln, sodass das untersuchte Sprachgebiet in allen drei von uns durchgeführten Untersuchungen im Großen und Ganzen auf den mittelrheinischen Raum eingegrenzt werden konnte (vgl. Philipp et al. 2017 und Graf et al. 2017 für weitere Details zur Durchführung und Auswertung dieses Experiments). Die EKP-Befunde bei der Verarbeitung des Verbpartizips und des darauf folgenden Auxiliars sind im Kontext unserer oben beschriebenen korpuslinguistischen Untersuchung und Akzeptabilitätsbefragung besonders aufschlussreich und werden im Folgenden diskutiert.

3 Die in den Kapiteln 3.2 und 4 referierten experimentellen Studien wurden von der Deutschen Forschungsgemeinschaft im Rahmen des Projekts „Agentivity, Animacy and Telicity: Pragmatic Inferences in Intransitive Clauses“ im Schwerpunktprogramm 1727 „XPRAG - New Pragmatic Theories based on Experimental Evidence“ finanziell gefördert. 
Die EKP-Effekte auf dem Verbpartizip können aufgrund ihrer Polarität, Latenzzeit und Topographie als N400 klassifiziert werden. N400-Effekte spiegeln - nach den neueren Erkenntnissen über diesen viel untersuchten EKP-Effekt - erhöhte Aktivität im Langzeitgedächtnissystem während des Wiederauffindens des lexikalischen und konzeptuellen (im weitesten Sinn semantischen) Wissens, das mit einem Stimulus assoziiert wird (Kutas \& Federmeier 2011; Hoeks \& Brouwer 2014). In (7) zeigen wir, unter welchen Bedingungen erhöhte Aktivität bei der Verarbeitung des Verbpartizips in Form eines N400-Effekts gemessen werden konnte.

(7) N400-Effekte auf dem Verbpartizip in Abhängigkeit von Animatheit und Telizität (Philipp et al. 2017)

$\begin{array}{ll}\text { der Inlineskater ... auf dem Feldweg gerollt } & \text { N400 } \\ \text { der Inlineskater ... zur Ampel gerollt } & \text {-- } \\ \text { die Radkappe ... auf dem Feldweg gerollt } & \text {-- } \\ \text { die Radkappe ... zur Ampel gerollt } & \text { N400 }\end{array}$

Wie in (7) illustriert, bereiten zwei Konstellationen Probleme bei der Verblexemverarbeitung: belebte Referenten, die sich ziellos bewegen, gegenüber solchen, die sich zielgerichtet bewegen, und unbelebte Referenten, die sich zielgerichtet bewegen, gegenüber solchen, die sich ziellos bewegen. Diese Befunde widerlegen die Unakkusativitätsthese, der gemäß Agentivität und Telizität invers korrelieren (z. B. Zaenen 1993; Keller \& Sorace 2003) sowie dazugehörige semantische Rollentheorien, die Telizität und (räumliche) Zustandsveränderung als Patiensmerkmale einordnen (z. B. Dowty 1991). Sie bestätigen psycholinguistische Ansätze, die Zielorientierung als genuine Agenseigenschaft einordnen (z. B. Rakison \& Poulin-Dubois 2001; Carpenter, Call \& Tomasello 2005; Carey 2009).

Eine plausible Lösung für die widersprüchliche Einordnung einer zielgerichteten Positionsänderung als charakteristisches Agens- vs. Patiensmerkmal bietet Dowty (1991: 574). Er nimmt an, dass für die Einordnung von Bewegung (zu der auch eine Positionsänderung wie in unserem Anwendungsfall gehört) als Agens- oder Patienseigenschaft, Kausation Priorität hat. Unter dieser Annahme ist eine Positionsänderung mit einem spezifischen Ziel nur dann eine Patienseigenschaft, wenn sie von einem anderen Partizipanten oder extern verursacht wird. Wenn sie selbstinduziert ist (engl. autonomous movement), handelt es sich um eine Agenseigenschaft. Da unsere Verben nur einen Partizipanten aufweisen, ist die plausibelste Interpretation der Satzbestandteile bis zum Verbpartizip, dass die zielorientierte Positionsänderung bei belebten Referenten selbstverursacht ist. Selbstinduzierte Bewegung ist nicht nur für Dowty 
(1991), sondern auch für die oben erwähnte psycholinguistische Forschung ein Agensmerkmal. Belebte Referenten können sich selbsttätig bewegen, unbelebte Referenten, wie wir sie in unseren Testitems verwendet haben, nicht. Dieser Ansatz würde unsere Befunde erklären. Ein belebter Referent, der sich zielgerichtet bewegt, ist ein prototypischerer Agens als ein belebter Referent, der sich ziellos bewegt. Dementsprechend generierte nur die letzte Konstellation einen N400-Effekt. Umgekehrt ist ein unbelebter Referent, der sich ohne rekonstruierbare fremde Partizipation zielgerichtet bewegt, rollensemantisch schwerer einzuordnen als ein sich ziellos bewegender unbelebter Referent. Dementsprechend generierte nur die erste Konstellation einen N400-Effekt.

Für die Verarbeitung des Auxiliars, das in unseren Testsätzen dem Verblexem folgte, standen den Probanden alle relevanten Informationen zur Verfügung: Belebtheit des Subjekts, ziellose (atelische) vs. zielgerichtete (telische) Bewegung und Verblexembedeutung. Um zu gewährleisten, dass die Probanden bei der Verarbeitung der Testsätze ihre Sprachkompetenz einsetzen, werden bei EKP-Messungen sprachbezogene Aufgaben im Anschluss an die Präsentation eines Testsatzes gestellt. In unserer Studie sollten die Probanden den vorausgegangenen Testsatz auf einer binären Skala (Ja/Nein) hinsichtlich seiner Akzeptabilität bewerten. Da das Auxiliar die einzige Manipulation war, welche die Akzeptabilität stark beeinflusste, überraschte es nicht, dass ein P3Effekt gemessen werden konnte. Es handelt sich nach neueren Erkenntnissen um eine posterior verteilte Positivierung mit einer variablen Latenzzeit von 250-700ms, die eine aufgabenrelevante Entscheidung bzw. Stimulusidentifizierung indiziert (Roehm et al. 2007; Kretzschmar 2010). Bezogen auf sprachliche Stimuli konnte in anderen Studien festgestellt werden, dass eine frühe posteriore Positivierung (250-400ms) bei der Verarbeitung eines erwarteten aufgabenrelevanten Stimulus auftrat, wie etwa beim erwarteten Antonym in Das Gegenteil von schwarz ist weiß (vs. gelb, Roehm et al. 2007). Bei einem unerwarteten aufgabenrelevanten Stimulus, insbesondere im Kontext einer binären Entscheidungsoption wie auch in unserem Fall, ist die Latenzzeit dieser Positivierung länger (500-700ms, vgl. Roehm et al. 2007; Kretzschmar 2010).

Unsere EKP-Messungen auf dem Auxiliar stützen die weiter oben beschriebenen Ergebnisse unserer Korpus- und Akzeptabilitätsstudie. Im frühen Zeitfenster (250-400ms) war die P3-Amplitude für sein größer als für haben. Dies lässt sich damit erklären, dass sein das präferierte und somit erwartbare akzeptabilitätsentscheidende Auxiliar ist. Es gibt auch eine Interaktion zwischen TELIZITÄT und SEIN/HABEN für posterior platzierte Elektroden: haben generiert in atelischen Kontexten eine größere Positivierung als in telischen; umgekehrt induziert sein in telischen Kontexten eine größere Positivierung als in atelischen. Diese Ergebnisse stehen in Einklang mit der Forschungsliteratur und 
unseren anderen oben beschriebenen Studien: Das Auxiliar sein ist bei diesem Verbtyp gegenüber haben generell präferiert, wobei diese Präferenz in telischen Kontexten höher ist als in atelischen, während haben generell dispräferiert ist, in telischen Kontexten eher als in atelischen.

Im späteren Zeitfenster (500-700ms) erwarteten wir aufgrund der o. g. einschlägigen Forschung eine größere Positivierung für das dispräferierte Auxiliar und in der Tat fanden wir sie auf haben (gegenüber sein). Die zweifache Interaktion SEIN/HABEN $\times$ TELIZITÄT wurde durch eine größere Positivierung für die telischen Bedingungen mit haben ausgelöst. Dieser Befund steht in Einklang mit unseren Korpus- und Akzeptabilitätsdaten, die darauf hinweisen, dass haben in telischen Kontexten dispräferierter ist als sein in atelischen. Die statistisch signifikanten EKP-Befunde auf dem Auxiliar illustriert Tabelle 1.6:

Tab.1.6: Frühe und späte Positivierungen auf dem Auxiliar (nach Graf et al. 2017).

\begin{tabular}{ll}
\hline gerollt ist / hat & frühe größere Positivierung auf ist vs. hat (Haupteffekt) \\
\hline $\begin{array}{l}\text { zur Ampel / auf dem Feldweg } \\
\text { gerollt ist }\end{array}$ & $\begin{array}{l}\text { frühe größere Positivierung auf ist im Kontext von zur Ampel } \\
\text { vs. auf dem Feldweg (Interaktion SEIN/HABEN } \times \text { TELIZITÄT) }\end{array}$ \\
\hline $\begin{array}{l}\text { zur Ampel / auf dem Feldweg } \\
\text { gerollt hat }\end{array}$ & $\begin{array}{l}\text { frühe größere Positivierung auf hat im Kontext von auf dem } \\
\text { Feldweg vs. zur Ampel (Interaktion SEIN/HABEN } \times \text { TELIZITÄT) }\end{array}$ \\
\hline $\begin{array}{l}\text { gerollt ist / hat } \\
\text { zur Ampel / auf dem Feldweg } \\
\text { gerollt hat }\end{array}$ & $\begin{array}{l}\text { späte größere Positivierung auf hat vs. ist (Haupteffekt) } \\
\text { vs. auf dem Feldweg (Interaktion SEIN/HABEN } \times \text { TELIZITÄT) }\end{array}$ \\
\hline
\end{tabular}

Was Belebtheit betrifft, haben wir einen statistisch nicht signifikanten, aber als Tendenz feststellbaren Verarbeitungsnachteil für haben mit belebten Referenten in telischen Kontexten in Form einer späten P3 gefunden. Dass Belebtheit nur einen marginalen Einfluss auf die Auxiliarwahl hat, konnten wir auch in unserer Korpus- und Akzeptabilitätsstudie feststellen. Dieser marginale Einfluss weist in allen drei Studien darauf hin, dass haben eher mit Unbelebtheit und sein eher mit Belebtheit harmoniert. Er stellt die aus der Unakkusativitätsthese abgeleitete Vorhersage in Frage, dass haben eher mit Belebtheit bzw. Agentivität und sein eher mit Unbelebtheit bzw. Patienshaftigkeit harmoniert.

Dass Belebtheit die Auxiliarwahl direkt beeinflusst, könnte man aufgrund unserer zeitsensitiven EKP-Befunde in Frage stellen. Belebtheit beeinflusst direkt die Zuordnung semantischer Rollen, die auch von Telizität, wie oben beschrieben, abhängt. Belebtheit und Telizität spielen mithin eine zentrale Rolle bei der Integration rollensemantischer Information mit der Verbbedeu- 
tung. Unsere zeitsensitiven EKP-Befunde am Verbpartizip haben diese Annahme bestätigt: Es gibt eine statistisch signifikante Interaktion zwischen Belebtheit und Telizität, die bei der Verarbeitung des Auxiliars nicht signifikant und nur als schwache Tendenz erkennbar ist. Die weitergehende Forschungshypothese, dass Belebtheit und Telizität auch bei der Auxiliarwahl interagieren, konnten alle drei Studien, die wir durchgeführt haben, nicht bestätigen, allerdings auch nicht widerlegen. Es sind weitere experimentelle Studien nötig, um die Befundlage zu konsolidieren.

Hinsichtlich der Details der Interaktion zwischen Belebtheit, Telizität und Verbbedeutung bzw. Auxiliarwahl liefern alle drei Studien miteinander kompatible Ergebnisse. Belebte und mithin per Default als agentivisch interpretierte Referenten harmonieren bei den untersuchten Verben mit einer telischen Bewegung, unbelebte Referenten, die in unseren Studien nicht als Agens interpretierbar sind, harmonieren mit einer atelischen Bewegung. Diese Befunde stellen die in der theoretischen Diskussion bisher unangefochtene Unakkusativitätsthese, die von einer inversen Korrelation zwischen Agentivität und Telizität ausgeht, in Frage (u. a. Dowty 1991; Zaenen 1993; Keller \& Sorace 2003). Unsere Befunde stehen mit psycholinguistischen Ansätzen in Einklang, deren Erkenntnisse in der theoretischen Grammatikforschung wenig Beachtung erfahren haben (z. B. Rakison \& Poulin-Dubois 2001; Carpenter, Call \& Tomasello 2005; Carey 2009).

Bei der Erklärung unserer N400-Befunde am Verbpartizip (vgl. die Erläuterungen $\mathrm{zu}$ (7) weiter oben) haben wir eine prototypentheoretische Modellierung des Agenskonzepts bemüht. Ein belebter Referent, der sich selbsttätig zielgerichtet bewegt, ist ein prototypischerer Agens als ein belebter Referent, der sich ziellos bewegt. Dementsprechend verursachte nur die letzte Konstellation einen Verarbeitungsnachteil in Form eines N400-Effekts. Umgekehrt ist ein unbelebter Referent, der sich ohne rekonstruierbare fremde Partizipation zielgerichtet bewegt, rollensemantisch schwerer einzuordnen als ein sich ziellos bewegender unbelebter Referent. Dementsprechend verursachte nur die erste Konstellation einen Verarbeitungsnachteil in Form eines N400-Effekts. Diese Interpretation unserer Befunde steht in Einklang mit der neurolinguistischen Forschung, in welcher der N400-Effekt bei anderem Stimulusmaterial ebenfalls mit nicht-prototypischen semantischen Rollenkonstellationen in Verbindung gebracht wurde (z. B. Philipp et al. 2008; Nieuwland et al. 2013).

Es gibt allerdings eine Reihe anderer Einflussfaktoren auf die N400-Modulation (vgl. Streb \& Rössler 2003; Bornkessel-Schlesewsky \& Schlesewsky 2009; Kutas \& Federmeier 2011). Die Gebrauchsfrequenz, die in frequenzbasierten Ansätzen bei der Erklärung sprachlicher Phänomene eine zentrale Rolle spielt, ist einer der Faktoren. So verursachen seltene Stimuli erhöhte N400-Amplitu- 
den im Gegensatz zu vergleichbaren frequenten Stimuli (vgl. Van Petten \& Kutas 1990; Streb \& Rössler 2003; Bornkessel-Schlesewsky \& Schlesewsky 2009). Generell wird Frequenz als wichtiger Faktor in der neuronalen Sprachverarbeitung betrachtet (z. B. MacDonald, Pearlmutter \& Seidenberg 1994; Jurafsky 1996; Crocker \& Brants 2000). Einige unserer Akzeptabilitäts- und EKP-Befunde stimmen in der Tat mit unseren Korpusfrequenzdaten überein. So könnte der Verarbeitungsvorteil für sein gegenüber haben ein Reflex seiner höheren Gebrauchsfrequenz beim untersuchten Verbtyp sein (vgl. Tab. 1.1 weiter oben), genauso wie der höhere Verarbeitungsvorteil von sein in telischen gegenüber atelischen Kontexten frequenzbasiert erklärt werden könnte (vgl. Tab. 1.2). Umgekehrt könnte der Verarbeitungsnachteil für haben auf seine niedrigere Gebrauchsfrequenz zurückgehen (vgl. Tab. 1.1). Seine sehr seltene Verwendung in telischen Kontexten (in unserem Korpus gar nicht belegt, vgl. Tab. 1.3) könnte seinen erhöhten Verarbeitungsnachteil in diesen Kontexten erklären. Allerdings ist lediglich die Übereinstimmung zwischen Verarbeitungsdaten (EKPs und Akzeptabilität) und Korpusfrequenz durch unsere Studien gesichert. Eine kausale Erklärung kann man daraus nicht ableiten. Die Ergebnisse unserer Akzeptabilitäts- und EKP-Studie könnte man genauso gut frequenzbasiert erklären wie die Frequenzdaten durch Sprachverarbeitungspräferenzen und beide könnten auf einen dritten, noch unbekannten Faktor zurückgehen.

Die N400-Effekte am Verbpartizip, die eine Interaktion zwischen Belebtheit und Telizität indizieren, stimmen nicht mit Frequenzdaten überein. Da belebte Subjekte im Deutschen nicht nur beim untersuchten Verbtyp (vgl. Tab. 1.4 und 1.5), sondern generell frequenter sind als unbelebte Subjekte (vgl. Bader \& Häusler 2010; Fischer 2013), würde man in einem frequenzbasierten Ansatz N400-Effekte am Verbpartizip in den Bedingungen mit unbelebten Subjekten voraussagen. Diese Voraussage trifft auf unsere Befunde nicht zu. Unbelebte Subjekte sind in atelischen Kontexten im Vorteil und belebte Subjekte im selben Kontext im Nachteil (vgl. (7) weiter oben). Unsere Befunde lassen sich über Rollenprototypikalität angemessener erklären, wie oben erläutert. Auch prominente Vertreter frequenzbasierter Modelle erkennen an, dass Prototypikalität bei der Erklärung mancher sprachlicher Phänomene eine angemessenere Erklärung bietet als Frequenz (vgl. Ellis, O’Donnell \& Matthew 2014).

\section{Zusammenfassende Diskussion}

In diesem Beitrag haben wir das Verhältnis zwischen Grammatiktheorie und Psycholinguistik anhand von Grammatikmodellen diskutiert, die dieses Verhältnis sehr unterschiedlich konzipieren. Für die von Noam Chomsky beein- 
flusste Forschungsrichtung sind Grammatiksystem und Sprachgebrauch strikt getrennt (vgl. Abschnitt 2 dieses Beitrags). Chomsky nimmt an, dass die Sprachfähigkeit im Sinn einer genetisch kodierten Universalgrammatik auf eine spontane Mutation zurückgeht und daher minimal sein muss. Ihre einzige Aufgabe besteht darin, sprachliche Ausdrücke anhand eines sehr einfachen Berechnungssystems zu generieren, die phonetisch und semantisch in jeweils eigenen externen Systemen interpretiert werden (vgl. Chomsky 2006; Berwick et al. 2013; Richards 2015). Genetisch kodierte Sprachfähigkeit lässt sich durch konkrete Sprachdaten weder stützen noch widerlegen, da sie zum tatsächlichen Sprachgebrauch gehören. Daher sind Chomskys Annahmen über die genetisch verankerte Sprachfähigkeit gegenüber empirischer Falsifizierung immunisiert (Evans \& Levinson 2009; Tomasello 2009; St. Müller 2016).

Da die angeborene Universalgrammatik in den neuesten Versionen der generativen Grammatiktheorie minimal ist, gehört der Großteil der Grammatik zur ,externalisierten' Sprache. Die Einordnung des Kopfbegriffs, der im Abschnitt 3.2 besprochen wurde, und des viel untersuchten Kopfserialisierungsparameters ist aufschlussreich in dieser Hinsicht. In früheren Versionen der generativen Grammatiktheorie ging man davon aus, dass das Kopfprinzip (jede Phrase hat genau einen Kopf) mit dem Kopfserialisierungsparameter Teil der angeborenen Universalgrammatik ist. Diesem Parameter zufolge gehen die Phrasenköpfe ihren Schwesterkonstituenten konsistent entweder voran oder folgen ihnen. Im Minimalismus verbannt man diesen Parameter in das artikulatorisch-perzeptuelle Performanzsystem, das allerdings weiterhin mit den Mitteln der Syntax untersucht wird (Richards 2015: 834). Einschlägige Forschung aus der Domäne der kognitiven Sprachverarbeitung, die allgemeine Sprachverarbeitungsprinzipien für viele grundlegende grammatische Phänomene wie den genannten Kopfserialisierungsparameter bietet, wird oft vernachlässigt. Eine solche Kritik findet man auch bei prominenten Vertretern aus den eigenen Reihen, wie Frederick Newmeyer:

[...] the most compelling examples of function affecting form pertain to the shaping of grammars by on-line processing considerations. So take the tendency of heads to consistently precede complements or to follow complements. One might be tempted to simply declare a head-parameter provided by Universal Grammar and leave it at that. There very well might be a head parameter, as far as descriptively adequate grammars are concerned. But there is a lot more to the story ... As Hawkins 1994 has shown, all of these facts follow from performance-based pressure to shorten the recognition time for phrasal constituents. (Newmeyer 2003: 3-4)

Wir betonen hier, dass sich grammatische Strukturen an Sprachverarbeitungsbeschränkungen anpassen. In der Psycholinguistik ist allerdings unumstritten, dass sich auch das neuronale Sprachverarbeitungssystem eines Individuums 
an die Grammatikbeschränkungen seiner Muttersprache anpasst (vgl. u.a. Bornkessel-Schlesewsky \& Schlesewsky 2009; Vasishth et al. 2010).

Zusammenfassend halten wir fest: Folgt man der aktuellen, minimalistischen Version des sprachlichen Dualismus, ist ein minimales Berechnungssystem angeboren und der Großteil der Grammatik gehört zum Performanzsystem. Für diesen Großteil müsste man in diesem Paradigma neuere Erkenntnisse und Methoden der Performanzforschung - besonders die Fortschritte in der Psycholinguistik - ernster nehmen als bisher geschehen.

Als Gegenentwürfe zur Auffassung des Primats und der Autonomie einer abstrakten Grammatik haben wir den Konnektionismus (Abschnitt 3.1) und frequenzbasierte Ansätze (Abschnitt 3.2) besprochen und anhand von Fallstudien empirisch substantiiert. In diesen Forschungsparadigmen werden grammatische Regeln als emergente Folgeerscheinungen der Sprachverwendung betrachtet.

Die Netzwerksimulation des Majuskelgebrauchs von H.-G. Müller (2016), die wir als Fallstudie zur Illustration des Konnektionismus gewählt haben (Abschnitt 3.1), zeigt die Stärken, aber auch die Schwächen dieses Forschungsparadigmas gegenüber regelbasierten Ansätzen. Im Konnektionismus werden künstliche neuronale Netzwerke von einfachen Verarbeitungseinheiten angenommen, die in paralleler Weise prozessieren, ohne dass den vielen räumlich und zeitlich verteilten Verarbeitungseinheiten und -schritten ein bestimmter symbolischer Gehalt zugeordnet werden kann. Netzwerksimulationen können Regelmäßigkeiten des Inputs als prototypische Muster abbilden, ohne die Bildungsregel des Musters im Vorhinein zu kennen. Nach erfolgreichem Lernprozess repräsentiert H.-G. Müllers Netzwerk die Regularitäten des Minuskel- und Majuskelgebrauchs durch die Struktur seiner Verbindungsgewichte. Das wird daran erkennbar, dass es 98,7\% korrekte Schreibungen für unbekanntes Datenmaterial als Output hat. Den Input des Netzwerks bilden mehr als zwanzig, zum Teil eng miteinander korrelierende grammatische Eigenschaften, die in der Orthographiediskussion als Einflussfaktoren für den Majuskelgebrauch genannt werden. Der syntaktische, regelbasierte Ansatz zur Majuskelverwendung arbeitet hingegen nur mit dem Begriff des Kopfes einer Nominalphrase. Die Attraktivität regelbasierter Ansätze liegt an ihrer Redundanzfreiheit und der Transparenz, mit der sie symbolisches Sprachwissen abbilden. Netzwerksimulationen arbeiten hingegen mit viel Redundanz und können symbolisches sprachliches Wissen nicht direkt abbilden. Netzwerksimulationen liefern - so die Kritiker - lediglich eine Implementation vorgegebener regelbasierter Ansätze. Dennoch kann eine Netzwerksimulation für die Überprüfung bestehender regelbasierter Theorien eingesetzt werden, wie H.-G. Müllers Vergleich mehrerer fragmentierter Netzwerksimulationen für verschiedene regelbasierte Ansätze demonstriert (2016, Kap. 7.6). 
Der Hypothese einer dem Sprachgebrauch folgenden, emergenten Grammatik sind auch frequenzbasierte Ansätze in der Nachfolge von Paul Hopper und Joan Bybee verpflichtet (Abschnitt 3.2). Dass Form und Funktion sprachlicher Ausdrücke von ihrer Gebrauchsfrequenz beeinflusst werden, ist allgemein anerkannt. Gebrauchsfrequenz hat allerdings erst in den jüngeren gebrauchsbasierten Grammatik- und Psycholinguistikansätzen einen zentralen Stellenwert erhalten. Unsere drei Fallstudien (Abschnitt 3.2 und 4) zur flexiblen Auxiliarwahl bei Bewegungsverben im Deutschen zeigten eine hohe Übereinstimmung zwischen Korpusfrequenz einerseits und Akzeptabilitätsurteilen und neurophysiologischen Daten (ereigniskorrelierten Potenzialen (EKP)) andererseits. Man könnte daher geneigt sein, letztere als Frequenzeffekte zu erklären. So könnte der Verarbeitungsvorteil für sein gegenüber haben ein Reflex seiner höheren Gebrauchsfrequenz beim untersuchten Verbtyp sein, genauso wie der höhere Verarbeitungsvorteil von sein in telischen gegenüber atelischen Kontexten frequenzbasiert erklärt werden könnte. Umgekehrt könnte der Verarbeitungsnachteil für haben auf seine niedrigere Gebrauchsfrequenz zurückgehen. Seine sehr seltene Verwendung in telischen Kontexten (in unserem Korpus gar nicht belegt) könnte seinen erhöhten Verarbeitungsnachteil in diesen Kontexten erklären. Allerdings ist lediglich die Übereinstimmung zwischen Sprachverarbeitungsdaten und Korpusfrequenz durch unsere Studien gesichert. Eine kausale Erklärung kann man daraus nicht ableiten. Zur Einordnung von Frequenz als Ursache oder Wirkung äußert sich Joan Bybee (2007) allerdings optimistischer:

[...] the answer to the question of whether frequency is a cause or an effect is complex. On the one hand, frequency is just a tally, a pattern observable in texts, which is of course an effect. On the other hand, frequency or repetition of experiences has an impact on cognitive representations and in this way becomes a cause for the effects discussed in this book. (Bybee 2007: 18)

Bybees zitierte Annahme bezieht sich explizit auf kognitive domänenübergreifende Repräsentationen (2007: 88): Frequenz beeinflusst diese und über diesen Weg emergente grammatische Phänomene. Wie bereits erwähnt, spielt Frequenz für die kognitive Sprachverarbeitung zweifelsfrei eine Rolle (z. B. MacDonald, Pearlmutter \& Seidenberg 1994; Jurafsky 1996; Crocker \& Brants 2000). Damit ist aber noch nicht gesagt, dass sie der einzige oder zentrale Faktor ist und für bestimmte Phänomene nicht selbst durch andere Sprachverarbeitungsfaktoren determiniert wird (vgl. Bornkessel-Schlesewsky, Schlesewsky \& Friederici 2002; Phillips 2010; van Schijndel \& Schuler 2013). So nimmt Phillips (2010) an, dass probabilistische Verteilungen in der Grammatik, z. B. bei selbsteinbettenden Strukturen wie die in Abschnitt 2 besprochenen, durch die Gren- 
zen des Kurzzeitgedächtnisses determiniert werden. Passend dazu zeigen van Schijndel \& Schuler (2013) in einer experimentellen Studie, die Gedächtnis und Frequenz als Faktoren isoliert, dass Gedächtnisbeschränkungen Präferenzen bei selbsteinbettenden Strukturen tatsächlich besser erklären. Prototypikalität ist ein anderes - seit Elinor Rosch (1973) bekanntes - kognitives Prinzip, das sogar von Verteidigern frequenzbasierter Ansätze inzwischen als eigenständiger Faktor anerkannt wird (vgl. Ellis, O’Donnell \& Römer 2014). Sie spielte bei der Erklärung unserer EKP-Befunde am Verblexem eine wichtige Rolle.

Mehrere gebrauchsbasierte Ansätze gehen davon aus, dass Grammatik keine autonome Existenz unabhängig von der in Echtzeit ablaufenden Sprachverarbeitung hat. Allerdings können viele gängige empirische Methoden, wie quantitative Korpusstudien und Akzeptabilitätsbefragungen, die in der Zeit ablaufende Prozessualität der kognitiven Sprachverarbeitung nicht erfassen. Methoden wie diese untersuchen fertige Sprachprodukte und mithin das Endresultat von Sprachverarbeitungsprozessen. Aus diesem Grund haben wir im Abschnitt 4 einen Blick auf die in Echtzeit ablaufende Prozessualität der kognitiven Sprachverarbeitung anhand einer EKP-Studie zur flexiblen Auxiliarwahl geworfen. Diese zeitsensitive Methode ist in der Lage, die Verarbeitung des Verblexems (z. B. geflogen) und die des folgenden Auxiliars (ist vs. hat) gezielt zu messen. Die EKP-Messungen am Verblexem ergaben, dass beim untersuchten Verbtyp Telizität mit belebten und mithin agentivischen Subjektreferenten und Atelizität mit unbelebten bzw. patiensähnlichen Subjekten harmoniert und dass Belebtheit und Telizität bei der Integration semantischer Rolleninformation mit der Verblexembedeutung interagieren. Dieser Befund stellt die Unakkusativitätsthese, dass Atelizität mit Agentivität und Telizität mit Patienshaftigkeit harmonieren, in Frage. Die Interaktion zwischen Belebtheit und Telizität war bei der Verarbeitung des Auxiliars nur als schwache Tendenz erkennbar. Die weitergehende Forschungshypothese, dass Belebtheit und Telizität auch bei der Auxiliarwahl interagieren, konnten alle drei Studien, die wir durchgeführt haben, nicht bestätigen, allerdings auch nicht widerlegen. Es sind weitere experimentelle Studien nötig, um die Befundlage zu konsolidieren.

Der vorliegende Beitrag hat auch einige methodologische Fragen adressiert. Unsere gebrauchsbasierten empirischen Studien haben mehrere Forschungshypothesen bestätigt, die in theoretischen Arbeiten durch eine unsystematische Datenbeobachtung gestützt wurden. Dennoch haben vollfaktorielle experimentelle Designs den Vorteil, die Einflussfaktoren, welche Akzeptabilitätsurteile oder neuronale Sprachverarbeitungsprozesse determinieren, zuverlässiger $\mathrm{zu}$ isolieren als unsystematische Datenerhebungen (vgl. Featherston 2007). Ein weiterer Vorteil gegenüber unsystematischen Datenerhebungen ist, dass sie replizierbar, statistisch analysierbar und objektiv sind, letzteres insoweit, dass sie nicht auf der Introspektion des Sprachforschers basieren. 
Eine weitere methodologische Frage betrifft den Forschungsstil der Performanzforschung. Während Chomsky für sein Forschungsparadigma einen deduktiv-mathematischen Stil in Anspruch nimmt, suggeriert der sprachliche Dualismus die Gegenhypothese eines rein oder weitgehend induktiven Vorgehens in der Performanzforschung. Wir haben anhand mehrerer Fallstudien gezeigt, dass theoriegeleitetes deduktives Schließen - explizit oder implizit - üblich und die Annahme „Data is a pre-condition for theory“ (Featherston 2007: 33) nicht der gängigen Praxis in der Sprachgebrauchsforschung entspricht.

Schließlich haben wir darauf hingewiesen, dass gradiente, relative Datenurteile, die in einem psycholinguischen Experiment oder einer Korpusanalyse elizitiert werden, nicht ohne weiteres in absolute (In)akzeptabilitäts- bzw. (Un)grammatikalitätsangaben übersetzt werden können und dass ihre Beschreibung keine gradient formulierten Beschränkungen erzwingt.

\section{Literatur}

Adger, David (2003): Core syntax. A minimalist approach. Oxford: Oxford University Press.

Adli, Aria, Marco García García \& Göz Kaufmann (Hrsg.) (2015): Variation in language: System- and usage-based approaches. Berlin/New York: De Gruyter.

Bader, Markus \& Jana Häussler (2010): Toward a model of grammaticality judgments. Journal of Linguistics 46, 273-330.

Behrens, Heike \& Stefan Pfänder (Hrsg.) (2015): Experience counts: Frequency effects in language. Berlin: Mouton de Gruyter.

Berwick, Robert C. et al. (2013): Evolution, brain and the nature of language. Trends in Cognitive Sciences, 17 (2), 89-98.

Blutner, Reinhard (2009): Neural networks, penalty logic and Optimality Theory. ZAS Papers in Linguistics 51, 53-94.

Bornkessel-Schlesewsky, Ina, Matthias Schlesewsky \& Angela D. Friederici (2002): Grammar overrides frequency: Evidence from the online processing of flexible word order. Cognition 84, B21-B30.

Bornkessel-Schlesewksy, Ina \& Matthias Schlesewsky (2009): Processing syntax and morphology. A neurocognitive perspective. Oxford: Oxford University Press.

Bredel, Ursula (2010): Die satzinterne Großschreibung - System und Erwerb. In Ursula Bredel, Gabriele Hinney \& Astrid Müller (Hrsg.), Rechtschreib- und Schriftkompetenz: Graphematik, Didaktik, Empirie, 217-234. Tübingen: Niemeyer.

Busemann, Stephan, Karin Harbusch \& Stefan Wermter (Hrsg.) (1998): Hybride konnektionistische, statistische und regelbasierte Ansätze zur Verarbeitung natürlicher Sprache. Saarbrücken: Deutsches Forschungszentrum für künstliche Intelligenz (DFKI).

Bybee, Joan L. \& Paul J. Hopper (Hrsg.) (2001a): Frequency and the emergence of linguistic structure. Amsterdam: Benjamins.

Bybee, Joan L. \& Paul J. Hopper (2001b): Introduction. In Joan L. Bybee \& Paul Hopper (Hrsg.), Frequency and the emergence of linguistic structure, 1-24. Amsterdam: Benjamins. 
Bybee, Joan (2007): Frequency of use and the organization of language. Oxford: Oxford University Press.

Carey, Susan (2009): The origin of concepts. Oxford: Oxford University Press.

Carpenter, Malinda, Josep Call \& Michael Tomasello (2005): 12- and 18-month-olds copy actions in terms of goals. Developmental Science 8, F13-20.

Chomsky, Noam (1957): Syntactic structures. The Hague: Mouton.

Chomsky, Noam (1965): Aspects of the theory of syntax. Cambridge (Massachusetts): MIT Press.

Chomsky, Noam (2004): The generative enterprise revisited. Berlin: Mouton de Gruyter.

Chomsky, Noam (2006): Approaching UG from below. In Uli Sauerland \& Hans-Martin Gärtner, (Hrsg.), Interfaces + recursion = language? Chomsky's minimalism and the view from syntax-semantics, 1-29. Berlin/New York: Mouton de Gruyter.

Crocker, Matthew W. (1994): On the nature of the principle-based sentence processor. In: Charles Clifton, Lyn Frazier \& Keith Rayner (Hrsg.), Perspectives on sentence processing, 245-266. Hillsdale: Lawrence Erlbaum Associates.

Crocker, Matthew W. \& Thorsten Brants (2000): Wide-coverage probabilistic sentence processing. Journal of Psycholinguistic Research 29 (6), 647-669.

Culicover, Peter W. \& Ray Jackendoff (2005): Simpler syntax. Oxford: Oxford University Press.

Dietrich, Rainer \& Johannes Gerwien (2017): Psycholinguistik. Eine Einführung. 3. Aufl. Stuttgart: Metzler.

Dowty, David R (1991): Thematic proto-roles and argument selection. Language 67, 547-619.

Eisenberg, Peter (2013): Grundriss der deutschen Grammatik. Bd. 2: Der Satz. Unter Mitarb. von Rolf Thieroff. 4., aktual. und überarb. Aufl. Stuttgart: Metzler.

Ellis, Nick C., Matthew B. O’Donnell \& Ute Römer (2014): The processing of verb-argument constructions is sensitive to form, function, frequency, contingency, and prototypicality. Cognitive Linguistics 25 (1), 55-98.

Ellis, Nick C. (2015): Frequency in language learning and language change. In: Heike Behrens \& Stefan Pfänder (Hrsg.), Experience counts: Frequency effects in language, 239-256. Berlin: Mouton de Gruyter.

Elman, Jeffrey L. et al. (1996): Rethinking innateness: A connectionist perspective on development. Cambridge (Massachusetts): MIT Press.

Engelberg, Stefan (2000): Verben, Ereignisse und das Lexikon. Tübingen: Niemeyer.

Engelberg, Stefan (2005): Stativity, supervenience, and sentential subjects. In Claudia Maienborn \& Angelika Wöllstein (Hrsg.) Event arguments. Foundations and applications, 45-68. Tübingen: Niemeyer.

Evans, Nicholas \& Stephen Levinson (2009): The myth of language universals: Language diversity and its importance for cognitive science. Behavioral and Brain Sciences 32, 429-492.

Featherston, Sam (2007): Data in generative grammar: The stick and the carrot. Theoretical Linguistics 33 (3), 269-318.

Fischer, Klaus (2013): Satzstrukturen im Deutschen und Englischen. Typologie und Textrealisierung. Berlin: Mouton de Gruyter.

Fitch, Tecumseh W. \& Marc D. Hauser (2004): Computational constraints on syntactic processing in a nonhuman primate. Science 303, 377-380.

Fitch, Tecumseh W., Marc D. Hauser \& Noam Chomsky (2005): The evolution of the language faculty: Clarifications and implications. Cognition 97, 179-210.

Fodor, Jerry A. (1983): Modularity of mind: An essay on faculty psychology. Cambridge (Massachusetts): MIT Press. 
Fodor, Jerry A. \& Zenon W. Pylyshyn (1988): Connectionism and cognitive architecture: a critical analysis. Cognition 28, 3-71.

Freidin, Robert \& Jean-Roger Vergnaud (2001): Exquisite connections: Some remarks on the evolution of linguistic theory. Lingua 111, 639-666.

Friederici, Angela D. et al. (2006): The brain differentiates human and non-human grammars: Functional localization and structural connectivity. Proceedings of the National Academy of Sciences of the USA 103, 2458-2463.

Friederici, Angela D. (2002): Towards a neural basis of auditory sentence processing. Trends in Cognitive Sciences 6, 78-84.

Fuhrhop, Nanna (2009): Orthografie. 3., aktual. Aufl. Heidelberg: Universitätsverlag Winter.

Gillmann, Melitta (2016): Perfektkonstruktionen mit „haben“ und „sein“. Eine Korpusuntersuchung im Althochdeutschen, Altsächsischen und Neuhochdeutschen. Berlin: de Gruyter.

Graf, Tim (in Vorb.): Agentivität, Belebtheit und Telizität im Deutschen. Experimentelle Untersuchungen. Dissertation, Universität zu Köln.

Graf, Tim et al. (2017): The interaction between telicity and agentivity: Experimental evidence from intransitive verbs in German and Chinese. Lingua, doi: 10.1016/ j.lingua.2017.08.006.

Grewendorf, Günther (2007): Empirical evidence and theoretical reasoning in generative grammar. Theoretical Linguistics 33 (3), 369-380.

Gries, Stefan Th. \& Dagmar Divjak (Hrsg.) (2012): Frequency effects in language learning and processing. Berlin: Mouton de Gruyter.

Haider, Hubert (2011): Gradienz. Zeitschrift für Rezensionen zur germanistischen Sprachwissenschaft 3 (1), 39-46.

Hawkins, John A. (1994): A performance theory of order and constituency. Cambridge: Cambridge University Press.

Hayes, Bruce (2001): Gradient well-formedness in Optimality Theory. In Jost Dekkers et al. (Hrsg.), Optimality Theory. Phonology, syntax, and acquisition, 88-120. Oxford: Oxford University Press. 88-120.

Hjelmslev, Louis (1935): La catégorie des cas. Étude de grammaire générale. Teil I. Acta Jutlandica 7. Kopenhagen: Reitzels Forlag.

Hoeks, John C. J. \& Harm Brouwer (2014): Electrophysiological research on conversation and discourse processing. In Thomas M. Holtgraves (Hrsg.), The Oxford handbook of language and social psychology, 365-386. Oxford: Oxford University Press.

Höhle, Barbara (2010): Psycholinguistik. Berlin: Akademie-Verlag.

Hopper, Paul J. (1987): Emergent grammar. Berkeley Linguistics Society 13, 139-157.

Institut für Deutsche Sprache (2012): Deutsches Referenzkorpus. Archiv der Korpora geschriebener Gegenwartssprache 2012-II. Mannheim: Institut für Deutsche Sprache.

Jackendoff, Ray (2007): Language, consciousness, culture. Cambridge (Massachusetts): MIT Press.

Jurafsky, Daniel (1996): A probabilistic model of lexical and syntactic access and disambiguation. Cognitive Science 20, 137-194.

Karlsson, Fred (2007): Constraints on multiple center-embedding of clauses. Journal of Linguistics 43 (2), 365-392.

Keller, Frank \& Antonella Sorace (2003): Gradient auxiliary selection and impersonal passivization in German: An experimental investigation. Journal of Linguistics 39, 57-108. 
Kempen, Gerard \& Karin Harbusch (2008): Comparing linguistic judgements and corpus frequencies as windows on grammatical competence: A study of argument linearization in German clauses. In Anita Steube (Hrsg.), The discourse potential of underspecified structures. Berlin/New York: Mouton de Gruyter, 179-192.

Kretzschmar, Franziska (2010): The electrophysiological reality of parafoveal processing: On the validity of language-related ERPs in natural reading. Dissertation, University of Marburg. http://archiv.ub.uni-marburg.de/diss/z2011/0602/ (letzter Zugriff: 9.11. 2017).

Kutas, Marta \& Kara D. Federmeier (2011): Thirty years and counting: Finding meaning in the N400 component of the event-related brain potential (ERP). Annual Reviews in Psychology 62, 621-47.

Labov, William (1969): Contraction, deletion, and inherent variability of the English copula. Language 45, 716-762.

Lakoff, George (1973): Fuzzy grammar and the performance/competence terminology game. Proceedings of the Regional Meeting of the Chicago Linguistic Society 9, 271-291.

Langacker, Ronald (1987): Foundations of cognitive grammar. Theoretical prerequisites. Stanford: Stanford University Press.

Legendre, Géraldine (2007): On the typology of auxiliary selection. Lingua 117(9), 1522-1540.

Lightfoot, David W. (1991): How to set parameters: Arguments from language change. Cambridge (Massachusetts): MIT Press.

Lukassek, Julia et al. (2016): The semantic processing of motion verbs: Coercion or underspecification? Journal of Psycholinguistic Research, doi: 10.1007/s10936-016-9466-7.

Maas, Utz (1992): Grundzüge der deutschen Orthographie. Tübingen: Niemeyer.

MacDonald, Maryellen C., Neal J. Pearlmutter \& Mark S. Seidenberg (1994): The lexical nature of syntactic ambiguity resolution. Psychological Review 101, 676-703.

Makuuchi, Michiru et al. (2009): Segregating the core computational faculty of human language from working memory. Proceedings of the National Academy of Sciences U.S.A. 106, 8362-8367

Marantz, Alec (2005): Generative linguistics within the cognitive neuroscience of language. The Linguistic Review 22, 429-445.

Mayor, Julien, Pablo Gomez, Franklin Chang \& Gary Lupyan (2014): Connectionism coming of age: Legacy and future challenges. Frontiers in Psychology 5, Article 187.

Müller, Hans-Georg (2016): Der Majuskelgebrauch im Deutschen. Groß- und Kleinschreibung theoretisch, empirisch, ontogenetisch. Berlin: Mouton de Guyter.

Müller, Stefan (2016): Grammatical theory: From transformational grammar to constraintbased approaches. Berlin: Language Science Press.

Newmeyer, Frederick J. (2003): Grammar is grammar and usage is usage. Language 79, 682-707.

Newmeyer, Frederick J. (2005): Possible and probable languages: A generative perspective on linguistic typology. Oxford: Oxford University Press.

Nieuwland, Mante S., Andrea E. Martin \& Manuel Carreiras (2013): Event-related brain potential evidence for animacy processing asymmetries during sentence comprehension. Brain and Language 126 (2), 151-158.

Pelucchi, Bruna, Jessica F. Hay \& Jenny R. Saffran (2009): Statistical learning in a natural language by 8-month old infants. Child Development 80, 674-685.

Perlmutter, David M. (1978): Impersonal passives and the unaccusative hypothesis. In Proceedings of the 4th Annual Meeting of the Berkley Linguistic Society, 157-189.

Petten, Cyma van \& Marta Kutas (1990): Interactions between sentence context and word frequency in event-related brain potentials. Memory and Cognition 18 (4), 380-393. 
Philipp, Markus et al. (2008): The role of animacy in the real time comprehension of Mandarin Chinese: Evidence from auditory event-related brain potentials. Brain and Language 105, 112-133.

Philipp, Markus et al. (2017): Beyond verb meaning: Experimental evidence for incremental processing of semantic roles and event structure. Frontiers in Psychology, doi: 10.3389/ fpsyg.2017.01806.

Phillips, Collin (2010): Some arguments and nonarguments for reductionist accounts of syntactic phenomena. Language and Cognitive Processes 28, 156-187.

Primus, Beatrice (2010): Strukturelle Grundlagen des deutschen Schriftsystems. In Bredel, Ursula, Gabriele Hinney \& Astrid Müller (Hrsg.), Rechtschreib- und Schriftkompetenz: Graphematik, Didaktik, Empirie, 9-45. Tübingen: Niemeyer.

Rakison, David H. \& Diane Poulin-Dubois (2001): Developmental origin of the animateinanimate distinction. Psychological Bulletin 127 (2), 209-228.

Randall, Janet et al. (2004): Acquiring unaccusativity: A cross-linguistic look. In Alexiadou, Artemis, Elena Anagnostopoulou \& Martin Everaert (Hrsg.) The unaccusativity puzzle. Explorations of the syntax-lexicon interface, 332-353. Oxford: Oxford University Press.

Richards, Marc (2015): Minimalism. In Kiss, Tibor \& Artemis Alexiadou (Hrsg.), Syntax theory and analysis. An international handbook. 2., überarb. Aufl., 803-839. Berlin: Mouton de Gruyter.

Rickheit, Gert, Theo Herrmann \& Werner Deutsch (Hrsg.) (2003): Psycholinguistik: Ein internationales Handbuch. Berlin: Mouton de Gruyter.

Roehm, Dietmar et al. (2007): To predict or not to predict: Influences of task and strategy on the processing of semantic relations. Journal of Cognitive Neuroscience 19 (8), 1-16.

Rosch, Eleanor (1973): Natural categories. Cognitive Psychology 4, 532-547.

Ross, John R. (1973): Nouniness. In Fujimura, Osamu (Hrsg.), Three dimensions of linguistic theory. 137-258. Tokyo: TEC Company.

Rumelhart, David \& James McClelland (Hrsg.), (1986): Parallel distributed processing: Explorations in the microstructure of cognition, Vol. 1. Cambridge (Massachusetts): MIT Press.

Saffran, Jenny R. (2003): Statistical language learning: Mechanisms and constraints. Current Directions in Psychological Science 12, 110-114.

Saussure, Ferdinand de (1916): Cours de linguistique générale. Genève.

Schijndel, Marten van \& William Schuler (2013): An analysis of frequency- and memorybased processing costs. In Proceedings of the 2013 Conference of the North American of the Association for Computational Linguistics: Human Language Technologies.

Sichelschmidt, Lorenz \& Elena Carbone (2003): Experimentelle Methoden. In Rickheit, Gert, Theo Herrmann \& Werner Deutsch (Hrsg.), Psycholinguistik/Psycholinguistics.

Ein internationales Handbuch/An international Handbook, 115-124. Berlin/New York: Mouton de Gruyter.

Smolensky, Paul (1986): Information processing in dynamical systems: Foundations of Harmony Theory. In Rumelhart, David E. \& James L. McClelland (Hrsg.), Parallel Distributed Processing: Explorations in the microstructure of cognition, Vol. 1, 194-281. Cambridge, MA: MIT Press.

Streb, Judith \& Frank Rösler (2003): Spezielle Verfahren II: Elektrophysiologische Methoden. In Rickheit, Gert, Theo Herrmann \& Werner Deusch (Hrsg.), Psycholinguistik/

Psycholinguistics. Ein internationales Handbuch/An international Handbook, 168-181. Berlin/New York: Mouton de Gruyter.

Tenny, Carol (1994): Aspectual roles and the syntax-semantics interface. Dordrecht: Kluwer. 
Tomasello, Michael (2009): Universal Grammar is dead. The Behavioral and Brain Sciences 32 (5), 470-471.

Valin, Robert D. van \& Wendy Wilkins (1996): The case for 'effector': Case roles, agents, and agency revisited. In Shibatani, Masayoshi \& Sandra A. Thompson (Hrsg.), Grammatical constructions: Their form and meaning, 289-322. Oxford: Clarendon.

Vasishth, Shravan et al. (2010): Short-term forgetting in sentence comprehension: Crosslinguistic evidence from verb-final structures. Language and Cognitive Processes 25, 533-567.

Vendler, Zeno (1957): Verbs and times. The Philosophical Review 66, 143-160.

Zaenen, Annie (1993): Unaccusativity in Dutch: Integrating syntax and lexical semantics. In James Pustejovsky (Hrsg.), Semantics and the lexicon, 129-161. Dordrecht: Kluwer.

Zaenen, Annie et al. (2006): Animacy encoding in English: why and how. Proceedings of the 42nd Annual Meeting of the Association for Computational Linguistics, 2004, Workshop on Discourse Annotation, 118-125.

Zifonun, Gisela, Ludger Hoffmann, Bruno Strecker \& Joachim Ballweg (1997): Grammatik der deutschen Sprache. Bd 3. Berlin/New York: Mouton de Gruyter.

Zipf, Georg Kingsley (1935): The psycho-biology of language. Boston: Houghton Mifflin. 



\title{
Hubert Haider
}

\section{Grammatiktheorien im Vintage-Look - Viel Ideologie, wenig Ertrag}

\begin{abstract}
Construction grammar and the Minimalist Program occupy opposite, but equally polar, positions in the pre-scientific setting of studies that attempt to scientifically unravel the make-up of human languages. Both schools share a basic flaw. They favour outdated and inadequate strategies, primarily based on scientific-ideological preconceptions. Construction Grammar is - against better judgment - entangled in the quandaries of functional accounts that biology has conclusively found to be fatal for any scientific progress already a century ago. The MP has turned into a self-centred activity of playful tinkering with syntactic calculi, in a gated community that would let in nothing but hand-picked evidence. These two schools have lost their scientific orientation but their marketing is still superb. This paper is a 'profit warning' for young linguists. Investments into these companies are going to be investments à fonds perdu in the long run.

Der Beitrag ist wie folgt gegliedert. Der kurzen Einleitung folgen zwei methodenkritische Abschnitte. An die Kritik des Minimalistischen Programms (MP) im Abschnitt 2 schließt im Abschnitt 3 die Kritik an der Konstruktionsgrammatik $(\mathrm{CxG})$ an. Den zentralen Schwachpunkt beider Schulen bildet ihre negative Heuristik in Gestalt der jeweiligen ideologiegeleiteten Programmatik. Sie ist, wie der Titel suggeriert, rückwärtsgewandt. Gegenstand der Kritik ist die Theorie und Praxis der beiden Schulen im Verhältnis zu den etablierten wissenschaftstheoretischen Standards. Beide Schulen verfehlen sie. Daraus erklärt sich auch, warum beides unproduktive Paradigmen sind. Die von den theoretischen Annahmen generierten Prädiktionen bewähren sich empirisch nicht.
\end{abstract}

Keywords: generative Grammatik, Grammatiktheoretische Paradigmen, Konstruktionsgrammatik, Minimalistisches Programm, Wissenschaftstheorie

Anmerkung: Für die konstruktiv-kritische Befassung mit der Entwurfsversion und die vielen hilfreichen Anregungen danke ich Stefan Müller und dem Herausgebergremium, Peter Gallmann, Mechthild Habermann und Angelika Wöllstein. Verbliebene Fehler, Fehleinschätzungen und Polemik hat nur der Autor zu verantworten.

Hubert Haider, Universität Salzburg, Fachbereich Linguistik \& Centre for Cognitive Neuroscience UNIPARK Nonntal, A-5020 Salzburg, E-Mail: hubert.haider@sbg.ac.at

Ә Open Access. (C) 2018 Hubert Haider, publiziert von De Gruyter. (c) BY Dieses Werk ist lizenziert unter der Creative Commons Attribution 4.0 Lizenz.

https://doi.org/10.1515/9783110490992-003 


\section{Wissenschaftliche Ideologie in Konfrontation mit sprachlicher Realität}

Die letzten zwei Jahrzehnte boten zwei entgegengesetzten programmatischen Konzeptionen von Grammatiktheorie (zu) viel Raum. Die eine Konzeption präsentiert sich in einer degenerativen Phase, nämlich die Generative Grammatik in der Phase des Minimalistischen Programms (MP), während die andere davon profitiert, nämlich die Konstruktionsgrammatik $(\mathrm{CxG})$ in den diversen Ausformungen. Beide sind, wie das Datum von programmatischen Publikationen (vgl. Chomsky 1995a; Goldberg 1995) zeigt, nicht mehr taufrisch. Beide haben eine Schonfrist von zwei Jahrzenten hinter sich, allerdings ohne die erwarteten bahnbrechenden Erkenntnisse, zu denen die gewählte Theorie einen direkten Weg hätte weisen sollen.

Es sind Vintage-Modelle, denn sie gründen auf Annahmen aus der Tiefe des vorigen Jahrtausends. Das MP dreht die Zeit zurück bis zu ,Syntactic Structures' und die CxG propagiert eine Art von Induktivismus, wie ihn bereits Francis Bacon im Novum Organon von 1620 propagierte, in Kombination mit jener Art von Funktionalismus, den man in der Biologie nach lange geführten Debatten schon Mitte des neunzehnten Jahrhunderts endgültig als wissenschaftlich untauglich begrub.

Beiden Schulen ist gemeinsam, dass sie Maximen vor sich hertragen, deren Prädiktionen in den empirischen Befunden auf wenig Bestätigung, aber viel Widerstand treffen, mit dem zu erwartenden Versagen beim Erzielen von Erklärungskraft.

Wissenschaftsideologisch motivierte Einengungen - ,negative Heuristik', in Imre Lakatos (1978) Terminologie - sind zwar in der Gründungsphase jedes Paradigmas vonnöten, um dem Programm eine zielgerichtete Entwicklung in der initialen Unübersichtlichkeit zu gewähren. Sobald diese Vorannahmen sich aber im Wege von Gewöhnung petrifizieren, werden sie zum Problem, wenn man sie allen empirischen Widerständen zum Trotz als nicht-hinterfragbaren Kern des Paradigmas bedingungslos gegen Evidenz abschirmt, obwohl für eben diese Grundannahmen substantielle Revisionen geboten wären. So geht eine prolongierte Anfangsphase in eine Wir machen weiter, wie bisher-Phase über.

Die zwei Programme sind lohnende Studienobjekte. Ähnlich lang aktiv, ähnlich dogmatisch; das eine einer aristotelischen konzeptuell-analytischen Top-down-Strategie folgend und von den Erfolgen des Markennamens aus der Vergangenheit zehrend, während das andere induktivistisch und funktionalistisch eine Bottom-up-Vorgangsweise pflegt. Beides sind extreme Positionen und es wäre von Anfang an absehbar gewesen, dass man mit der gewählten Strategie nicht nachhaltig würde reüssieren können. 
Das MP ist die extreme Ausformung eines cartesianisch-idealistischen $\mathrm{Zu}$ gangs. Gesucht und postuliert werden die denknotwendigen (,virtually necessary'), minimalistischen Voraussetzungen, aus denen dann deduziert wird, wie die sprachliche Welt beschaffen sei, weil diese ja nicht anders sein könne als deren perfekte Implementierung. Sprache sei ein „perfect system, meeting external constraints as well as can be done." (Chomsky 1995b: 385-386). Chomsky bezeichnet das als „strong minimalist thesis“.

Im MP ist das sprachliche Material, scheint es, bloß der Rohstoff, mit dem bevorzugt vertrackte und möglichst verblüffende Analysethesen illustriert werden. Gegenstand der Zuwendung ist ein algebraisches Rätselspiel, bei dem es darum geht, sich für einen Satz eine lange Reihe von Ableitungsschritten auszudenken, die beliebig komplex sein mögen, solange sie nur den willkürlich gesetzten Rahmenbedingungen und Zielvorgaben genügen. Tun sie es nicht unmittelbar, ist das ein willkommener Anlass, Ad-hoc-Annahmen aus dem Ärmel zu schütteln, denen eine Deus-ex-machina-Funktion zukommt. Insgesamt sind es selbstsicher dargebrachte Übungen in spielerisch exerzierter Logik von algebraischer Syntax. Dass sie wenig mit der syntaktischen oder der neuro-kognitiven Realität zu tun haben, schmälert die Freude der Aktivisten nicht.

Die $\mathrm{CxG}$ propagiert eine primär induktive Forschungsstrategie, mit phänomenologisch-funktionaler Blickrichtung. „A 'what you see is what you get' [wysiwyg] $_{\mathrm{HH}}$ approach to syntactic form is adopted: no underlying levels of syntax or any phonologically empty elements are posited“, wobei gilt, „All levels of description are understood to involve pairings of form with semantic or discourse function.“ (Goldberg 2003: 219).

Vielleicht hat man nicht genügend reflektiert, was der Minimalkonsens ist, wenn man der Scientific Community von Wissenschaft im 21. Jahrhundert angehören möchte. Sonst würde man nicht das ignorieren, was seit Mitte des vorigen Jahrhunderts state-of-the-art in der Wissenschaftstheorie ist. Peter B. Medawar (1979: 84), Medizin-Nobelpreis 1960, formuliert es so: „The day-today business of science consists not in hunting for facts but in testing hypotheses - that is, ascertaining if they or their logical implications are statements about real life.“ Das heißt nicht, dass es nicht auf exakt geklärte Fakten ankäme, doch was ein relevantes Faktum ist, bestimmt nicht der indifferente Blick auf die Dinge, sondern der hypothesengeleitete prüfende Blick. Darwin hat das bekanntlich wie folgt beurteilt:

About 30 years ago there was much talk that Geologists ought only to observe \& not theorise; \& I well remember some one saying, that at this rate a man might as well go into a gravel-pit \& count the pebbles \& describe their colours. How odd it is that every one should not see that all observation must be for or against some view, if it is to be of any service. ${ }^{1}$

1 Brief an Henry Fawcett vom 18. September 1861. 
In der Wissenschaft ist häufig das, was man wahrzunehmen glaubt, nicht das, was der Fall ist. Eine CxG-Astronomie wäre ptolemäisch, denn die Sonne wandert ganz ,wysiwyg-offensichtlich ' um eine ruhende ${ }^{2}$ Erde, und eine CxG-Physik wäre aristotelisch. In ihr ist die Bewegung der Gegenstände, einschließlich der Himmelskörper, funktional gesteuert. Jeglicher Gegenstand, der sich nicht an seinem ,natürlichen“ Ort befindet, strebe diesem $\mathrm{zu}$, so Aristoteles. Jeder sprachliche Ausdruck strebt seiner ,natürlichen' Verbindung von Form und Funktion zu. Die Realität folgt dieser Vorstellung nicht.

Beide Paradigmen, CxG und MP, sind Vintage-Paradigmen. Die CxG beruft sich auf eine Tradition, die bis zu Aristoteles zurückreicht. „Constructions form and meaning pairings - have been the basis of major advances in the study of grammar since the days of Aristotle. “(Goldberg 2003: 219). Dem Satz könnte man sofort zustimmen, wenn ,advances‘ durch ,impediments` ersetzt würde.

Das MP forciert die Wiederbelebung der Ausgangsidee aus den 50erJahren, wonach Grammatik ein Algorithmus sei, der als Syntax-Algebra zu modellieren ist. ${ }^{3}$ Diese Idee ist nach einer Anfangseuphorie von der im Entstehen begriffenen Psycholinguistik wegen offenkundiger Untauglichkeit schnell wieder verworfen worden. ${ }^{4}$ Der Preis, den man heute für die Aufrechterhaltung zahlen muss, ist folgender. Man darf sich unbehelligt in die wunderbare platonische Welt von merge, copy \& paste, covert movement, roll-up, empty expletives etc. segregieren, angereichert mit einem Zoo seltsamer Features (z. B. EPP), von denen einige vor spell-out schnell eingefügt oder getilgt werden, denn man wird von den Nachbardisziplinen ohnehin nicht ernst genommen:

Unfortunately, to our knowledge, no experimental evidence has been offered to date that suggests that merge and move are real (in the same sense that the spatial frequency channels in human vision are). Generative linguists typically respond to calls for evidence for

2 Man bedenke, dass es in der geneigten Leserschaft wohl nicht viele sind, die aus dem Stand heraus ein einfaches und konklusives Experiment erläutern könnten, das nachweist, dass die Erde in Bewegung ist, wie z. B. das Foucaultsche Pendel.

3 Diese Idee ist verschwistert mit einer überholten Idee der Implementierung, nämlich eine zentralgesteuerte, sequentielle Rechenarchitektur. Informatiker und auch Neuro-Linguisten sind vor geraumer Zeit zu distribuiertem und parallelem Computing übergegangen (Gordon 1985), ohne dass das in der Generativen Grammatik Niederschlag gefunden hätte. Paralleles Computing fußt auf einer verteilten, simultan aktiven Rechenarchitektur. Von Phonologie bis Semantik wird interaktiv parallel gearbeitet. Worauf es ankommt, ist die Gleichzeitigkeit von Informationsverarbeitung und nicht ein technisch motiviertes Hintereinander.

4 Perfetti (1981: 153) fasst die endgültige Scheidung von generativer und experimenteller Psycho- und Neurolinguistik wie folgt zusammen: „As the 'psychological reality' of transformations became discredited, psychologists began to lose interest in linguistic structures, especially the more blatantly syntactic ones.“ 
the reality of their theoretical constructs by claiming that no evidence is needed over and above the theory's ability to account for patterns of grammaticality judgments elicited from native speakers. This response is unsatisfactory. (Edelman \& Christiansen 2003: 61)

\section{Das selbstverschuldete Scheitern der algebraischen Grammatik}

Die generative Grammatik ist ohne empirische Not oder Notwendigkeit zum Anfangspunkt von 1957 zurückgekehrt, nämlich zur Zielvorstellung eines komplett sequentiell-derivationellen Modells. Dieses charakterisiert grammatische Wohlgeformtheit eines Ausdrucks per Theorembeweis. Ein sprachlicher Ausdruck ist grammatisch wohlgeformt, wenn er aus den Axiomen unter Anwendung endlich vieler Derivationsschritte ableitbar ist. Ob das überhaupt algorithmisch-effizient ausführbar ist und ob das irgendeine plausible Beziehung zu menschlicher Sprachbeherrschung oder -verarbeitung hat, interessiert die Protagonisten nicht ernstlich. Grammatikforschung wird als Übung in spezieller Algebra und nicht als konsequente Erforschung eines empirisch gegebenen Gegenstandes begriffen. Es fehlt deutlich an dem, was Immanuel Kant das „rastlose Streben“ danach, „den Dingen auf den Grund zu gehen“, nannte.

Als aktiver Neo-Generativist befindet man sich im MP in einem permanenten Bastelmodus am Modell. Autoren sehen es als ihren kreativen Beitrag an, in Fällen von Gegenevidenz ungeprüfte Zusatzannahmen zu postulieren, um irgendeinen Ableitungsweg frei zu machen, der zum gesuchten Ziel führt, allerdings unter geflissentlicher Respektierung der vom Spiritus Rector gesetzten Axiome. Auf die wissenschaftliche Pflichtarbeit, nämlich die unverzichtbare, umfassende empirische Prüfung aller relevanten Konsequenzen, und auf die unverzichtbare Beibringung unabhängiger Evidenz wird gerne verzichtet. Daten werden aus dem Fundus so zusammensortiert, dass sie die vorgefasste Position bestätigen, und noch ergänzt mit der einen oder anderen Beobachtung, damit diese Raum für heroische Theorie-Rettungsaktionen biete.

Die tausendfüßlergleichen Derivationsschritte werden überdies zum erheblichen Teil ,undercover` zurückgelegt, auf verborgenen Wegen. Es entspricht ihnen jedenfalls keinerlei erkennbare Veränderung in der linearen Abfolge. Diese fiktiven Prozesse werden ,covert' genannt. Der Undercover-Modus macht es bequem, beliebig viele Derivationsschritte zu postulieren. Die UndercoverExistenz erlaubt es auch, umgestellte Ausdrücke in ihrer Originalposition komplett zu konservieren. Die terminalen Elemente werden nämlich multipliziert mittels ,copying' und die Originale an ihrem ,Heimatort' ihrer phonologischen Gestalt beraubt. Das lässt sich dann noch toppen mit einer absurd anmutenden 
These. Ihr Makel liegt nicht in der Anmutung von Absurdität - in jeder Wissenschaft gibt es viel ursprünglich absurd Erscheinendes, das sich später als richtig erweist -, sondern im totalen Ignorieren von massiver und unübersehbarer Gegenevidenz.

Man darf sich nämlich - bevorzugt mit SVO-Muttersprache - nach wie vor auf Konferenzen der MP-Community unwidersprochen als Anhänger der seinerzeit von Kayne (1994) intensiv propagierten Theorie outen, wonach alle Sprachen der Welt, insbesondere aber auch die SOV-Sprachen, nichts als verkapptes Englisch ${ }^{5}$ seien, nämlich strikte SVO-Sprachen. Alles, was in einer englischen VP hinter dem Verb steht, stünde im Deutschen ursprünglich auch dort, nur werde es anschließend vorangestellt ins Mittelfeld. Das betrifft im Fall von (1a) das Objekt, die Adverbiale, die Partikel des Partikelverbs und das vom Modalverb abhängige Hauptverb. Nennen wir das dem Proponenten zu Ehren K-Umstellungen. Die Grundabfolge wäre dann die in (1b). Sie entspricht der Abfolge im Englischen (1c). Deutsch wäre demnach bloß eine derivationelle Fortsetzung des Englischen. ${ }^{6}$

Jetzt kommt dazu, dass K-Umstellungen so wie alle Umstellungen im MP ein Ergebnis von copy \& paste sind. Dabei wird die Kopiervorlage auf stumm geschaltet, aber in der Repräsentation beibehalten. In (1d) sind das die durchgestrichenen Abschnitte.

Eine erste Klimax an Verwicklungen erzielt man bereits, wenn man auch noch das Vorfeld besetzt, wie in (1e). Die VP im Vorfeld ist als verschobene Phrase die Kopie eines Originals, das in (1f) durchgestrichen ist. Doch das Original ist ungrammatisch, wie (1g) illustriert, weil die rechte Klammer kein Nachfeldmaterial duldet. Somit ist der ganze Satz ungrammatisch und sollte daher unakzeptabel sein. Dem ist aber nicht so.

Konstellationen dieser Art gibt es viele, bekannt als ,movement paradoxes‘. Sie werden allerdings ignoriert, obwohl sie deutliche Gegenevidenz bilden. Schließlich muss man noch beide Umstellungen, nämlich die Ableitung des Vorfelds und die Ableitung der OV-Struktur per K-Umstellung, kombinieren (1h). Damit hat man dann endlich (1e) abgeleitet. Das wirkt nicht nur grotesk, es ist auch so, und es ist überdies falsch.

5 Kayne behauptet das nicht wörtlich, sondern im Ergebnis. Seine Behauptung lautet, dass syntaktische Strukturen ausschließlich und universell aus [Spec [head complement]]-Strukturen bestünden, in genau dieser Serialisierung. ,Zufällig‘ kommt Englisch dem ziemlich nahe.

6 Noch vor wenigen Jahrzehnten wurden die Sprachen nach dem Muster des Lateinischen analysiert. Die Genetiker präferieren zwar auch Fruchtfliegen als Modellorganismen, doch sie behaupten nicht im Handumdrehen, dass eine Fledermaus eine derivationell weiterentwickelte Fruchtfliege sei. 
(1) a. weil er im Deutschen alles ausnahmslos ins Mittelfeld umstellen muss

b. weil er muss stellen um alles ohne Ausnahme ins Mittelfeld im Deutschen

c. since he must move ahead everything invariably into the midfield in German

d. weil er im Deutschen alles ausnahmslos ins Mittelfeld umstellen muss stellen um alles ohne Ausnahme ins Mittelfeld im Deutschen

e. [Umstellen ins Mittelfeld] wird man im Deutschen alles müssen

f. [Umstellen ins Mittelfeld] wird man im Deutschen alles [umstellen ins Mittelfeld] müssen wird

g. ${ }^{\star}$ weil man im Deutschen alles umstellen ins Mittelfeld müssen wird

h. [Umstellen ins Mittelfeld] wird man im Deutschen alles [umstellen ins Mittelfeld] müssen wird müssen stellen um alles ins Mittelfeld im Deutschen

Erstens sind die zahlreichen unmotivierten Linksverfrachtungen, mit denen Deutsch in der K-Welt an die anglo-zentristische Grammatikwelt abgepasst wird, ptolemäische Syntax ${ }^{7}$ in Reinform. Sie entsprechen den zahlreichen unmotivierten ptolemäischen Epizykeln, mit denen die vermeintlichen geozentrischen Kreisbahnen der Planeten approximiert werden. Sie ist zwar, so wie die ptolemäische Astronomie, in hohem Maß sophistiziert, aber ohne prädiktive Kraft und produktiv nur bei der eigenen Widerlegung. So gut wie jede interessante Prädiktion des Modells ist falsch. Das letzte Kapitel von Haider (2013) ist dem gewidmet und präsentiert die nötigen Details. Selbst auf der Hand liegende Konsequenzen dieser Hypothese sind falsch. Alle Phrasen im Mittelfeld sollten qua Phrasen in Spec-Positionen Extraktion aus diesen Phrasen heraus strikt blockieren. Die Evidenz dagegen ist eindeutig (Haider 2010: 79-84; Haider 2013: 221).

Zweitens ist die Neuinterpretation von syntaktischer Umstellung als Fall von copy \& paste ebenfalls ohne empirische Substanz. Sie wurde nie einer sprachübergreifenden Evaluierung unterzogen. Selbst wenn es mit dem Englischen keine Probleme gäbe, besagt das nichts, denn die Probleme sind in Sprachen wie Deutsch unübersehbar. Das, was (1f) illustriert, ist bloß ein Fall von vielen (s. Haider 2016).

7 Liebermanns (2007: 435) Prophezeiung lautet: „In short [...] the linguistic enterprise, like the Ptolemaic astronomical theory, will in time be regarded as fruitless an exercise in logic and disjoint from reality." 
So willkürlich wie die typologische These ${ }^{8}$ über das Verhältnis von OV und VO ist, so deutlich ist auch der Widerspruch zwischen der Theorie und der Chomsky'schen These, wonach es in der Grammatiktheorie darum gehe, die menschliche Grammatikfähigkeit zu modellieren. Die Konzeption des Grammatiksystems im Minimalistischen Programm steht dazu in einem mit Händen greifbaren Widerspruch. Es gibt nicht das geringste psycholinguistische Indiz dafür, dass wir (1a) als (1d) verarbeiten oder (1f) als (1h). Hätte das MP Berührung mit der Realität, dann wäre ein auffälliges Merkmal menschlicher Rede ihre Interpunktion durch lange Pausen zwischen den Sätzen. Sie entstünden unvermeidlich dadurch, dass jeder Satz vom Ende her aufgebaut werden müsste und nach dem Ende der jeweils vorangegangenen Äußerung eine Pause entstünde, weil die nächste Äußerung vom Ende beginnend produziert werden muss, bis man das erste Wort artikulieren kann. Das geht so:

Laut MP startet die derivationelle Prozedur mit einer Auswahl der lexikalischen Atome, genannt Numeration. Diese Atome werden dann schrittweise aggregiert mittels einer binären Operation der Strukturerzeugung (,merge'). Gleichzeitig laufen darüber die zyklisch angewendeten Umstellungsoperationen ab (,copy \& merge'). Der Satz ist dann fertig generiert, wenn alle Prozesse konvergent am Wurzelknoten angelangt sind. In der Linearisierung ist das der Satzanfang. Da syntaktische Strukturen - zumindest phrasenintern - rechtsverzweigend ${ }^{9}$ sind, impliziert diese Vorgangsweise, dass jeder Satz so deriviert werden müsste, dass man mit der tiefsten Position beginnt und folglich sich von hinten nach vorne arbeitet. Der erste Schritt im folgenden Satz (2) wäre vermutlich - je nachdem, wo man Extraponiertes strukturell verortet - der Aufbau des Präpositionalausdruckes, from the rear' plus dessen Merger mit dem Verb ,start“ in (2a) bzw. ,beginnen müssen“ in (2b). Dabei muss man nun auch mitbedenken, dass wir Deutschsprachigen die Zusatzaufgabe hätten, auf dem Weg von (2a) nach (2b) auch noch alles nach links verfrachten zu müssen.

8 Es sei darauf hingewiesen, dass die von Kayne propagierte Ansicht nicht flächendeckend von allen MP-Anhängern beherzigt wird. Zustimmung findet sich aber u. a. selbst in den Niederlanden.

9 Rechtsverzweigend: ... [A $\left.\left[\mathrm{B}[\mathrm{C}]_{\mathrm{X}^{\prime}}\right]_{\mathrm{X}^{\prime}}\right]_{\mathrm{XP}}$... Linksverzweigend: ... [XP $\left.\left[\mathrm{X}^{\prime}[\mathrm{X}, \mathrm{C}] \mathrm{B}\right] \mathrm{A}\right] \ldots$ (Haider 2010: 27).

Beispielsweise ist die deutsche VP rechtsverzweigend (i) und kopf-final. Die englische VP ist kopf-initial und ebenfalls rechts-verzweigend (ii). Wäre sie linksverzweigend (iii), dann wäre die Struktur und die Abfolge der VP-internen Satzglieder das Spiegelbild von (i). Sprachen mit linksverzweigend strukturierten Phrasen sind unbekannt und existieren sehr wahrscheinlich nicht, zumal sie parser-unfreundlich wären (s. Haider 2013: 25-26).

i. [etwas [an Studenten [aushändigen $\left.\left.\mathrm{V}^{\circ}\right]\right]_{\mathrm{VP}}$

ii. $\quad\left[\text { hand }_{\mathrm{V}^{\circ}}\left[\text { something }\left[\left[-{ }^{-} \mathrm{V}^{\circ} \text { out }\right]_{\mathrm{V}^{\circ}} \text { to students }\right]\right]\right]_{\mathrm{VP}}$

iii. $*\left[\left[[\text { hand out }]_{\mathrm{V}^{\circ}} \text { to students] something }\right]_{\mathrm{VP}}\right.$ 
(2) a. How does this gentleman imagine that we manage to produce such a sentence without stumbling if we have to start it from the rear?

b. Wie stellt dieser Herr sich wohl vor, wie wir es schaffen, so einen Satz zu produzieren, ohne zu stolpern, wenn wir ihn von hinten beginnen müssen?

Nähme man das ernst, so folgte daraus, dass es im Gesprochenen kaum Probleme bei der Produktion eines Satzes mit dessen Ende geben könne, dass sich aber typischerweise die Probleme häufen, je näher man dem Anfang ist. Schließlich ist der Anfang des Satzes das Ende seiner Derivation, ab dem erst die Lippen geöffnet werden. Erfahrung bestätigt das Gegenteil. Das Anakoluth ist definitiv kein Satzanfangsphänomen. Deutsche Pausen wären überdies noch viel länger als englische, wegen der vielen K-Verfrachtungen.

Will man die Motivation hinter der Neukonzeption der Generativen Grammatik qua MP deuten, hilft dabei die - hoffentlich nicht universell gültige auf Wilhelm von Humboldt zielende Bemerkung, die im Roman „Die Vermessung der Welt“10 Carl Friedrich Gauß in den Mund gelegt bekommt: „Sprachwissenschaft. Gauß wiegte den Kopf. Das sei etwas für Leute, welche die Pedanterie zur Mathematik hätten, nicht jedoch die Intelligenz. Leute, die sich ihre eigene notdürftige Logik erfänden.“

Das MP verdankt sich zwar nicht einem Mangel an Intelligenz seitens seines Urhebers, wohl aber dessen „Pedanterie zur Mathematik“, insbesondere zu den formalen Grundlagen von Grammatiken. ${ }^{11}$ Grammatik wird als Kalkül gesehen. Jedem, der ein wenig Logik studiert hat, fällt sofort auf, dass das MP exakt so formuliert ist, wie man das im Logik-Proseminar gelernt hat: Ein Kalkül besteht erstens aus den atomaren Grundelementen, aus denen komplexere Ausdrücke zusammengesetzt werden. Im MP heißt dies ,Numeration'. Dazu kommen zweitens die Formationsregeln, mit denen festgelegt wird, wie die Bausteine zu komplexen Objekten, die auch wohlgeformte Formeln genannt werden, zusammengesetzt werden dürfen. Im MP ist das das rekursive ,Merging،. Drittens gibt es Transformationsregeln, die angeben, wie bestehende wohlgeformte Objekte des Kalküls umgeformt werden dürfen, um neue Objekte daraus zu erzeugen. Schließlich gibt es noch Axiome.

10 Daniel Kehlmann 2007. Hamburg: Rowohlt. S. 159.

11 Die theoretische Informatik ehrt Chomsky bekanntlich mit der Benennung „ChomskyHierarchie“ von formalen Grammatiken, gelegentlich auch Chomsky-Schützenberger-Hierarchie genannt. 
Das Rätselspiel Syntax gewinnt der, der einen (möglichst komplexen) Weg von der Numeration zum fertigen Satz zu argumentieren vermag, der den Axiomen und den Derivationsregeln genügt und dessen Abfolge terminaler Elemente einem halbwegs geläufigen Satz entspricht. Ein Hochleistungssport unter Kalkülspezialisten im Fach Logik ist es, zu beweisen, dass man den minimalen Kalkül gefunden habe. Das Minimalistische Programm wandelt auf diesen Pfaden, übersieht dabei aber, dass es nicht um einen Logikleistungskurs geht, sondern um einen empirischen Gegenstand, der einen großen Teil seiner Eigenschaften den evolutionsbedingt zufälligen Gegebenheiten eines sprachverarbeitenden Organs verdankt, nämlich unserem menschlichen Hirn.

Mit Kalkülen zu spielen erfreut das logische Gemüt und es braucht viel Intelligenz, deren vertrackte Ableitungswege $\mathrm{zu}$ verfolgen, um sie skizzieren zu können. Gleiches tut man im MP. Das generative Hirn ist ein kalkülverliebtes Hirn. Dass diese Vorliebe vielleicht auch Einsichten in die menschlichen Grammatikfähigkeiten abwerfen könnte, ist bloß ferne Hoffnung, die frucht- und erfolgslos genährt wird, indem man sich in ,Biolinguistik‘ umbenennt.

Der letztlich fatale Mangel aber ist die Sterilität des MP. Es ist ein unfruchtbares Paradigma. Zwei Jahrzehnte haben nicht gereicht, alte Probleme zu lösen oder Wege zu neuen Einsichten $\mathrm{zu}$ eröffnen. Es ist eine in sich gekehrte scholastische Beschäftigung von Menschen mit Pedanterie zu algebraischen Spielen geworden, die beiläufig den Eindruck erwecken, sie hätten mit Sprachwissenschaft $\mathrm{zu}$ tun.

Hier ist ein Beispiel: Ein mittlerweile klassisches, weil noch immer ungelöstes, Problem ist das der ,obligatorischen Kontrolle‘. Wie kommt es, dass der nicht lexikalisch repräsentierte Subjektsaktant des nicht-finiten Verbs in einer satzwertigen Infinitivkonstruktion stets abhängig interpretiert werden muss, obwohl auch die generische Interpretation verfügbar wäre? Das Subjekt des Infinitivsatzes (3a) wird exakt so als gebundenes Pronomen interpretiert, wie das explizite Subjekt in (3b). In (3c) wird das Subjekt des Infinitivsatzes als stummes generisches Pronomen interpretiert, so wie im finiten Gegenstück (3d). Der Problemfall ist (3e). Es ist nach wie vor unverstanden, weshalb dann, wenn es ein mögliches Bezugselement gibt, dieses gewählt werden muss. (3e) kann eben nicht als Paraphrase von (3f) interpretiert werden, obwohl, wie (3f) zeigt, diese Lesart eine plausible Lesart ergäbe.

(3) a. Jeder hoffte, als Erster den Durchbruch zu schaffen.

b. Jeder hoffte, dass er als Erster den Durchbruch schaffe.

c. Es ist möglich, als Erster den Durchbruch zu schaffen.

d. Es ist möglich, dass man als Erster den Durchbruch schafft. 

e. Ich hoffte, den Durchbruch zu schaffen.
(Infinitivsubjekt: ,ich', aber nicht ,man')
f. Ich hoffte, dass man den Durchbruch schafft.

Infinitivsätze haben ein obligatorisches Subjekt (ausführlicher in Abschnitt 2.1). Unter anderem verdankt die Apposition ,als Erster' in (3a-d) ihren NominativKasus $^{12}$ der Tatsache, dass sie sich auf das Subjekt, in diesem Fall auf das Infinitivsubjekt, bezieht. In der strukturellen Darstellung wird der Subjektsaktant von einem stummen Pronomen repräsentiert. Dieses kann entweder ein stummes Personalpronomen sein (3a, e) oder ein stummes generisches IndefinitPronomen, d.h. ,man'. Die ungeklärte Frage ist folgende. Was verbietet die generische Option in (3e), obwohl sie, wie (3f) zeigt, semantisch zulässig wäre?

Eine Antwort, wie man sie sich nur als Angehöriger der algebraischen Grammatikzunft vorzuschlagen erkühnen mag, hat Hornstein (2000) formuliert und intensiv beworben, mit Breitenwirkung in der Community. Man hätte sie auf der Stelle verwerfen müssen. Obligatorische Kontrolle erkläre sich daraus, dass der kontrollierende Ausdruck ,bigamistisch' existiere. Zuallererst sei er Subjekt des Infinitivsatzes und dann werde er, unter Zurücklassung seiner Kopie, derivationell in den Matrixsatz befördert, wo er eine Zweitexistenz als Matrix-Satzglied führe:

(4) a. Jeder hoffte, jeder als erster den Durchbruch zu schaffen.

b. Man hat Norbert nicht genug bedrängt, Norbert den Unfug bleiben zu lassen.

Klarerweise kann in Hornsteins Strukturierung von (3e) das Infinitivsubjekt nicht durch ein stummes ,man' repräsentiert werden, da ja das kontrollierende Element identisch ist mit dem kontrollierten und in zwei ,Kopien“ auftritt. Das Kontrollproblem ist damit allerdings gar nicht gelöst, denn die Fälle des Typs (3c) existieren nach wie vor. Es gibt satzwertige Infinitive mit generischer

12 Nominativ findet sich dann, wenn der Aktant, auf den sich eine Apposition oder ein nominales Prädikat bezieht, ein Subjektsaktant ist, unabhängig von dessen aktuellem Kasus (NullKasus bei Infinitivsätzen oder Akkusativ in AcI-Konstruktionen). Bei Letzteren ist neben Nominativ (i) auch Akkusativ (ii) eine Option.

i. Lass mich dein guter Herold sein (St. Zweig, Maria Antoinette)

ii. Wir lassen Gott einen lieben Mann sein.

iii. Es lässt die Männer ${ }_{\mathrm{Akk}}$ einer/einen nach dem anderen vortreten. 
Interpretation des Subjekts. Damit bleibt die Ausgangsfrage bestehen, was diese Option in (3a, e) ausschließt.

Die Folgeprobleme der Anhebungsanalyse sind noch übler und man sollte sie sich nicht ohne Not aufhalsen. Erstens hat Hornstein anscheinend die Debatte aus den siebziger Jahren aus seinem Gedächtnis verbannt. In jener Zeit versuchte man es mit Equi-NP-Deletion (Rosenbaum 1970) und wunderte sich, dass (4a) bedeutet, dass jeder hofft, dass er (selbst) gewinnt und nicht, gemäß der Equi-Analyse oder Hornsteins Bigamie-Verhältnis, dass jeder hofft, dass jeder gewinnt.

Zweitens haben die Algebraisten der Infinitivsyntax ein offensichtlich relevantes und bekanntes Datum komplett und persistierend mit Missachtung gestraft: Obligate Kontrolle gibt es auch bei Infinitivsätzen, die Teil von adverbial gebrauchten Phrasen sind (5a, b). Anhebung aus solchen Kontexten heraus ist aber zweifelsfrei unakzeptabel, was ebenfalls allgemein bekannt ist (5c, d). Das englische Gegenstück zu (5a) ist die extrem frequente ${ }^{13}$, in-order-to ${ }^{6}$ Konstruktion. Sie ist manifest unverträglich mit der Analyse und wird (deshalb?) in keinem einzigen Paper über Anhebung-an-Stelle-von-Kontrolle erwähnt, was man wohl als Indiz dafür werten darf, dass wissenschaftliche Maßstäbe im Algebraistenland extrem flach gehalten werden.

(5) a. Jeder/Er hat gewonnen, ohne etwas riskiert zu haben.

b. Die Bombe ist von britischen Streitkräften abgeworfen worden, ohne $\mathrm{zu}$ explodieren.

c. *Wen hat er die Wahl gewonnen, ohne -- überzeugt zu haben?

d. *Welcher Aufgabe hat jeder gefaulenzt, anstatt sich -- zu widmen?

Für alle, die sich erinnern, welche Vielzahl profunder Einsichten in die Bedingungen der Grammatik des Deutschen ab Mitte der 80er-Jahre aus der generativen Grammatik kamen, haben die letzten zwei Jahrzehnte den Vorteil, dass man sich die nicht unerhebliche Mühe guten Gewissens sparen kann, die nötig wäre, um sich in die algebraische Wunderwelt des MP einzuarbeiten. Es gibt keinen Ertrag, der deskriptiven Grammatikern des Deutschen ihre Mühe nur ansatzweise lohnen würde. Das ist betrüblich, da die grammatikschreibende Anwendung von einer soliden Grammatiktheorie geleitet sein sollte.

13 Googelt man ,in order to“, werden über eine Milliarde [sic! - 10] Hits angeboten. 
Das MP ist ein exemplarischer Fall einer Disziplin, für die Lakatos (1978) den Terminus ,degenerative Problemverschiebung prägte. In einer derartigen Phase der Paradigmenentwicklung ist das gesamte Augenmerk darauf gerichtet, die Kernannahmen des Modells durch Ad-hoc-Zusatzannahmen gegen Falsifikation zu immunisieren. Auch wenn diese einander widersprechen mögen, bewahren sie doch den „harten Kern“, wie Lakatos das nennt, vor unmittelbarer Widerlegung. Die degenerative Phase zeichnet sich daher dadurch aus, dass vorzugsweise bekannte Fakten reinterpretiert werden, um sie verträglich $\mathrm{zu}$ integrieren. Was fehlt, ist die produktive Qualität in Form von Vorhersagen bisher unbekannter Fakten.

Lakatos (1978: 6) formuliert das so: „The hallmark of empirical progress is not trivial verifications“, und Falsifizierbarkeit ist meist trivial gegeben, ,since all $\left[\right.$ research $\mathrm{H}_{\mathrm{H} . \mathrm{H}}$ ] programs grow in a permanent ocean of anomalies. What really counts are [...] unexpected, stunning predictions: a few of them are enough to tilt the balance."

Produktivität in Form von überraschenden Prädiktionen, die sich als empirisch prüfbar und richtig erweisen, ist das MP schuldig geblieben. In der realen Welt würde man das Zahlungsverzug oder gar -unfähigkeit nennen. Für das MP haben das Edelman \& Christiansen (2003: 60) bereits vor geraumer Zeit konstatiert, und es hat sich zwischenzeitlich nichts geändert. „Putting forward a theory is like taking out a loan, which must be repaid by gleaning an empirical basis for it; theories that fail to do so (or their successors that might have bought their debts) are declared bankrupt.“

Der Grund für den erkennbaren Bankrott des MP ist sein aristotelischer Grundtenor. Medawar (1979: 70-71) unterscheidet zwischen einem aristotelischen und einem galileischen Zugang. Ein aristotelischer Zugang „is contrived to demonstrate the truth of a preconceived idea. [...]. Joseph Glanvill, in common with many of his contemporary fellows of the Royal Society had the utmost contempt for Aristotle, whose teaching he regarded as major impediments to the advancement of learning: 'Aristotle ... did not use and imploy Experiments for the erecting of his theories: but having arbirtrarily pitch'd his Theories, his manner was to force Experience to suffragate, and yield countenance to his precarious Propositions'.“

Solange die Neo-Generative Grammatik nicht wieder die wissenschaftlichen Standards so, wie in den 80er-Jahren hochhält - penible Datenanalyse, sprachübergreifend abgesicherte Hypothesenbildung, Herleitung möglichst vieler kritischer Prädiktionen samt strenger empirischer Prüfung, mit Verzicht auf billige Ad-hoc-Rettungsmaßnahmen für Lieblingsideen - wird sie sich kontinuierlich marginalisieren, bis sich letztlich wohl kaum mehr jemand findet, den das algebraische Glasperlenspiel interessiert. 


\section{Konstruktionsgrammatik - Das Behagen in der Vorwissenschaftlichkeit}

Die Beschäftigung mit Grammatiken menschlicher Sprachen unter dem Blickwinkel von Konstruktionen und deren Gebrauchskontexten und kommunikativen Funktionen kann nicht verfehlt sein, mag man sich denken, denn das hat eine jahrhundertlange Tradition und „einen Sitz im Leben“. Das stimmt, aber sie hat keine wissenschaftlich gesicherten Einsichten in die Beschaffenheit des Wissenssystems erbracht, das diese Konstruktionen hervorbringt. Das ist der entscheidende Punkt. Es gibt genau solche Herangehensweisen mit jahrtausendlanger Tradition, die sich wissenschaftlich als nicht zielführend erwiesen haben, beispielsweise die tausendfünfhundert Jahre Astronomie von Claudius Ptolemäus an, von der uns erst Kepler und Newton befreiten.

Um Missverständnisse zu vermeiden, sei Folgendes festgehalten: Gegen die Beschäftigung mit Konstruktionen und deren sorgfältige Beschreibung ist nichts einzuwenden. Das ist das tägliche Brot der Linguistik. Doch das ist der Anfang, nicht das Ziel. Dass unter dem Markennamen CxG seriöse empirische Arbeiten erscheinen, wird nicht bestritten. Die hier vorzutragende Kritik richtet sich an das konstruktionsgrammatische Programm. Drei Hauptkritikpunkte seien explizit benannt. Der erste betrifft die Idee, dass es das Ziel der Grammatikforschung sei, Form-Funktions-Zusammenhänge zu studieren, d.h. Konstruktionen als Strukturen verknüpft mit Funktionen zu begreifen, ,pairings of form and function“ (Goldberg 2013: 15).

Der zweite Kritikpunkt zielt auf das Insistieren auf die gesamten, teilweise idiosynkratischen Eigenschaften von Konstruktionen in ihren kommunikativfunktionalen Einbettungen. Das macht die Herangehensweise letztlich explanativ untauglich. Die Beschreibung einer Konstruktion mag empirisch richtig oder falsch sein, sie macht aber keine testbare Prädiktion. Wissenschaft besteht in der Auffindung von Generalisierungen, die sich in überprüfbaren Prädiktionen niederschlagen. Die Existenz genau dieser Prädiktionen wird aber bestritten mit der Behauptung, dass sprachübergreifende grammatische Generalisierungen so gut wie nicht existierten; wenn, dann seien es Reflexe von domänenübergreifenden kognitiven Beschränkungen (Goldberg 2013: 16). Diese werden allerdings nicht präzisiert oder experimentell getestet.

Der dritte Kritikpunkt betrifft die Beziehung der beiden bereits genannten: Wenn man Formen nach ihren Funktionen sortiert, ergibt das eine inkohärente Sammlung, da das Sammelkriterium ,gemeinsame Funktion` keine Rücksicht auf strukturelle Kohärenz nimmt und die Formen nicht durch deren Funktion determiniert sind. Es sollte zu denken geben, dass die Biologie, die im achtzehnten Jahrhundert vor demselben Problem stand, mit guten Gründen Ab- 
stand davon nahm, die Funktionen als Beschreibungs- und Klassifikationskriterium für die strukturellen Explananda zu nehmen (s. Diskussion weiter unten). Die Form-Funktions-Zusammenhänge sind nicht kausal determiniert. Wer das nicht berücksichtigt, hat keine Chance zu wissenschaftlich akzeptablen Verallgemeinerungen und Erklärungen vorzudringen.

Betrachten wir zuerst das, was eine dezidierte Proponentin des Paradigmas als Maximen formuliert (Goldberg 1995, 2003, 2006, 2013). Die wesentlichen Eigenschaften von Konstruktionen werden wie folgt charakterisiert, wobei es unter Konstruktionisten unterschiedliche Grade der Obödienz gibt. Man erkennt unschwer, dass sich diese Liste wie der Negativabzug generativ-grammatischer Positionen liest. Doch, die bloße Aufhebung aller Paragraphen der generativen Hausordnung ergibt noch lange kein besseres Haus. Wenn es genügte, alle Annahmen einer insgesamt falschen Theorie einzeln zu negieren, um daraus eine richtige Theorie zu machen, dann wäre Wissenschaft ein Kinderspiel. Hier sind Leitmaximen der CxG, wie Goldberg (2003: 16) sie zusammenfasst:

(6) a. Konstruktionen sind Verknüpfungen von Form und Funktionen, die erlernt werden über die Verarbeitung des Inputs durch allgemeine kognitive Mechanismen, d.h. der Fähigkeit, Muster $\mathrm{zu}$ erkennen und $\mathrm{zu}$ verarbeiten.

b. Oberflächenbezogen: Die Semantik wird direkt von der ,Oberfläche‘ abgeleitet. Es gibt keine verborgenen Repräsentationsebenen und keine Leerelemente.

c. Sprachübergreifende Generalisierungen werden mittels sprachunspezifischer, allgemein kognitiver Prozesse erklärt oder über die Funktionen der beteiligten Konstruktionen.

d. Gebrauchsbasiert: Ob eine Konstruktion gebraucht wird (oder bloß gekannt wird) sowie ihre Frequenz ist maßgeblich für deren Systematik oder idiosynkratische Ausformung.

e. Kommunikativ funktional: Die primäre Funktion der Sprache ist Informationsübertragung. Formale Unterscheidungen sind daher nur insofern von Nutzen, als sie semantische oder pragmatische Unterscheidungen transportieren. ${ }^{14}$

Im Folgenden werden für jeden Punkt Fakten benannt, die im krassen Widerspruch zu diesen Annahmen stehen. Es sind alles keine in der Scientific

14 „The primary function of language is to convey information. Thus formal distinctions are useful to the extent that they convey semantic or pragmatic (including information theoretic) distinctions." (Goldberg 2013: 16). 
Community unbekannte oder etwa erst kürzlich entdeckte Sachverhalte. Das nährt den Verdacht, dass sich $\mathrm{CxG}$ genauso wie MP per selektiver Wahrnehmung und exklusiver Fokussierung auf bestätigende Sachverhalte vor Konfrontationen mit widersprechenden Fakten schützt. Das ist die als „Bestätigungsfehler“ (myside bias) gut bekannte menschliche Schwäche, die man im Alltag tolerieren mag, die aber in der Wissenschaft kontraproduktiv ist.

\subsection{Spracherwerb und Kognition}

Beginnen wir mit dem Thema der Erlernbarkeit von Konstruktionen allein durch allgemein kognitive (domain-general) Problemlösungsfertigkeiten, die uns unser Hirn anbietet. Diese allgemeinen kognitiven Fertigkeiten sind bekanntlich in jeder Population variabel ausgeprägt. Daher muss die Korrelationsthese (6a) konsequenterweise prädizieren, dass unterschiedlich hoch ausgeprägte kognitive mit ebenso unterschiedlich ausgeprägten Leistungsniveaus der Sprachverarbeitung kovariieren, da es ja angeblich zwei Seiten derselben Medaille sind. De facto gibt es aber Fälle von extremer Dissoziation, die es dann nicht geben dürfte, wenn Spracherwerb und -verarbeitung lediglich domain-general gesteuert ist.

Intelligenz ist normalverteilt, d.h. sie entspricht der Glockenkurve einer Gauß-Verteilung. Wenn (6a) zutrifft, muss die Virtuosität bzw. Minderleistung in der Sprachbeherrschung stark mit den allgemeinen Intelligenzfaktoren korrelieren. Insbesondere sollte der nonverbale und der verbale Teil von Intelligenztests in einer festen Relation stehen. Der verbale Teil von IQ-Tests ist allerdings nicht wirklich repräsentativ für das gesamte Spektrum der Sprachbeherrschung von Phonologie bis Pragmatik. Daher ist es ratsamer, psycholinguistisch etablierte Fakten heranzuziehen.

Es gibt zwei intensiv beforschte und dokumentierte Syndrome, für die eine extreme Dissoziation von allgemein kognitiven Leistungen und speziell sprachlichen Fertigkeiten charakteristisch ist, nämlich WBS (Williams-Beuren-Syndrom: Bellugi et al. 1994, 2000) im Vergleich zu Down-Syndrom (Schaner-Wolles 2004) und SLI (Specific language impairment; Rice 2016).

Menschen mit WBS liegen in ihren allgemeinen kognitiven Leistungen erheblich, d. h. durchschnittlich ein bis zwei Standardabweichungen unter der unauffälligen, alterstypischen Kontrollgruppe, sind aber sprachlich in einzelnen Bereichen der Grammatik ${ }^{15}$ alterstypisch kompetent und grammatisch signifi-

15 Es geht dabei nicht um die Gesamtleistung, sondern um die Beherrschung komplexer Teilbereiche, im Vergleich zu kognitiv gleichermaßen beeinträchtigten (s. Down-Syndrom) oder kognitiv unauffälligen Kindern und Jugendlichen mit (s. SLI) und ohne Sprachdefiziten. 
kant kompetenter als Menschen desselben kognitiven Niveaus, aber mit DownSyndrom. Auf Menschen mit SLI trifft das exakte Gegenteil zu. Selbst wenn sie allgemein-kognitiv im typischen Bereich sind, verfehlen die sprachlichen Leistungen den typischen Leistungsbereich um bis zu zwei Standardabweichungen, und das persistent, denn sprachliches Lernen ist erheblich beeinträchtigt, selbst bei spezieller Zuwendung. Wie lernt man als WBS-Betroffener mit völlig mangelhaften allgemein kognitiven Leistungen vertrackte grammatische Verhältnisse und weshalb versagt man mit SLI, obwohl man genug Intelligenz mitbringt, um Schulmathematik zu bewältigen? Weil es sich um eine domänen-spezifische Leistung handelt, die domänenspezifisch beeinträchtigt oder unbeeinträchtigt sein kann.

Es gibt noch sehr viel unwahrscheinlichere Indizien für domänen-spezifische Qualitäten der Sprachverarbeitung, ebenfalls gut bekannt und erforscht. Ein besonders beeindruckendes Faktum kommt aus der Sprach-Pathologie (s. Churchland 1986: 232) von Menschen mit visuellem Hemi-Neglekt. Diese blenden eine Hälfte des Gesichtsfeldes bei der Verarbeitung aus, und zwar nicht nur bei der Apperzeption von visuellem Input, sondern auch beim Wiederaufrufen abgespeicherter Information, was Bisiach \& Luzzatti (1978) herausfanden. ${ }^{16}$ Das wirklich Erstaunliche zeigt sich nun bei Gebärdensprechern: „Even severe hemispatial neglect does not seem to interfere substantially with normal sign language communication, either in terms of production or comprehension." (Bellugi et al. 2010: 6). Gebärden sind komplexe gestische und in der Rezeption daher visuell verarbeitete Abläufe. Genau dieser sprachlich-visuelle Input ist vom visuellen Hemi-Neglekt ausgespart. Offensichtlich ist die Verarbeitung von visuellem Input nicht domain-general. Die Verarbeitung von visuellem Input wird dann von der Störung ausgespart, wenn der zu einem Bereich gehört, für den es eine domänen-spezifische Verarbeitungskapazität gibt. Dieses domänenspezifische Netzwerk ist offenbar unbeeinträchtigt.

So wie MP ist auch CxG nicht auf experimentell-syntaktischer Forschung ${ }^{17}$ gegründet. Es gibt aber unabhängige, relevante Ergebnisse der psycho- und

16 Mailänder Patienten wurden aufgefordert, sich vorzustellen, mit dem Rücken zum Dom zu stehen, und den Platz zu beschreiben. Danach sollten sie sich mental auf die gegenüberliegende Platzseite begeben. In jeder der beiden Beschreibungen war die jeweils andere halbe Seite des Platzes für die Patienten nicht existent. Das geniale Design des Tests bewies aber, dass sie das Wissen über das Aussehen der beiden Seiten zur Verfügung hatten und dass es nicht um einen Fehler der basalen visuellen Wahrnehmung geht.

17 In der CxG beruft man sich auf Spracherwerbsforschung; vgl. Hoffmann \& Trousdale (2013), Abschnitt 4, zum Thema „Acquisition and Cognition“, mit Beiträgen von Holger Diessel und von Nick Ellis, und die dort zitierte Literatur. Dass im Spracherwerb Konstruktionen eine Rolle spielen, ist kompatibel mit CxG aber genauso mit anderen Ansätzen. Man kann in diesem 
neurolinguistischen Forschung. Bezöge man die konsequent ein, würde man von (6a) Abschied nehmen müssen. Dass die überschießende generative Angeborenheitsthese ebenfalls falsch ist, macht die CxG-These als deren Negation nicht allein deswegen schon richtig.

Die wirklich ironische Pointe ist ja folgende: Die allgemeinen kognitiven Kapazitäten unseres menschlichen Hirns sind allesamt angeborene Kapazitäten. In diesem Ensemble von angeborenen Verarbeitungsfertigkeiten gibt es Routinen, die sich für spezielle Verarbeitungskonstellationen eignen, ohne dass sie speziell dafür ,angefordert‘ oder ,bereitgestellt‘ wären. Sie werden aus dem Vorhandenen rekrutiert. Das ist empirisch bestätigt und zum Handbuchwissen geworden; siehe Goswami (2011). Dort formuliert es u. a. Melzoff (2011: 51) kurz und bündig: „We now know that there is a much richer innate state than posited by Freud, Piaget, and Skinner. The nativists won the battle over the new-born's mind."

Routinen, die für Sprachverarbeitung rekrutiert worden sind, erscheinen uns als domain-specific, solange keine andere kognitive Domäne diese Leistung mitbenutzt. Eine Konsequenz davon ist, dass Sprachen deswegen über bestimmte komplexe Eigenschaften verfügen können, weil das Hirn spezielle Berechnungskapazitäten dafür anbietet. Ein Affenhirn bietet sie nicht an und ist daher nicht in der Lage, menschliche Syntax zu bewältigen. Ein funktionalistischer Fehlschluss wäre es, anzunehmen, dass das Hirn die Kapazitäten hat, damit es bestimmte sprachliche Komplexitäten verarbeiten könne. Der nativistische Fehlschluss war, dass der Grammatikbau insgesamt angeboren sei.

Wie kommt es aber zu dem Zusammenspiel, das wir in den Grammatiken der Welt beobachten können? Hier ist eine Antwort, mit den Details dazu in Haider (2015b): Die speziellen Eigenschaften von Grammatiken sind das Ergebnis einer andauernden kognitiven Evolution grammatischer Struktursysteme, wobei die neuro-kognitiven Verarbeitungsgegebenheiten für Symbolverarbeitung das ,Sieb“ bilden, d.h. den Selektionsmechanismus. ${ }^{18}$ Jene Varianten gewinnen mehr spracherwerbende Hirne, die sich als günstiger für die vom Hirn angebotenen Verarbeitungsroutinen erweisen. Das ist ein Ergebnis von Evolution und die funktioniert substanzneutral, was schon Darwin (1871: 59) aufgefallen ist: „The formation of different languages and of distinct species, and the

multifaktoriell konstituierten Terrain jeweils vieles finden, wonach man sucht. Das gilt ebenso für die Spracherwerbsforschung im Generativen Lager; vgl. Guasti (2002) oder White (1989).

18 Der Mechanismus ist derselbe wie in der genetischen Evolution: Variation + Selektion ergibt Adaptation. In der kognitiven Evolution konkurrieren Repräsentationsvarianten für sprachliche Ausdrücke unter Selektion durch die sprachverarbeitenden Hirne darum, in einem sprachlernenden Hirn Platz zu nehmen. 
proofs that both have been developed through a gradual process, are curiously parallel.“

\subsection{Horror vacui}

Strukturen mit Leerelementen ${ }^{19}$ sind ,generativ ${ }^{6}$ und daher darf es sie nicht geben. Das mag die unausgesprochene Parole für die Motivationslage hinter deren Ablehnung sein. Ob es Leerstellen in der syntaktischen Repräsentation von Ausdrücken gibt, ist aber keine Frage von $\mathrm{Zu}$ - oder Abneigung. Es ist eine wissenschaftlich zu klärende Frage. Ist die empirisch adäquate Theorie eine mit oder ohne Heranziehung von Leerstellen? Die Frage wurde in den 80ern intensiv diskutiert. Man musste bloß an das Rasiermesser des Meisters von Ockham appellieren, um die Bringschuld bei den Proponenten zu erkennen. Wenn die aber liefern, was sie taten (siehe z. B. Koster \& May 1982), ist man als Leerstellenleugner selbst am Zug und muss nachweisen, dass beispielsweise die Analyse von Infinitivsätzen ohne Annahme eines stummen Subjekts oder von Komparativsätzen ohne stummes Vergleichselement derjenigen mit so einer Annahme empirisch überlegen ist. Das hat noch niemand aus dem CxG Lager konsequent nachgewiesen. ${ }^{20}$

Bei der folgenden Leerstellenverhandlung seien bloß zwei von mehreren Zeugen im Indizienprozess aufgerufen, die die Existenz von Nullpositionen bezeugen. Der eine Zeuge ist die satzwertige Infinitivkonstruktion, der andere die Komparativsatz-Konstruktion.

Infinitiv-Sätze, d. h. satzwertige Infinitivkonstruktionen mit dem Verb im zweiten Bechschen Status, haben ein Subjekt und das ist stumm. Es ist ein stummes Pronomen und es verhält sich auch wie ein Pronomen. So ermöglicht

19 Leerelemente, nämlich Null-Morpheme, wurden, worauf mich Mechthild Habermann dankenswerterweise hinweist, bereits in der Mitte des vorigen Jahrhunderts im amerikanischen Strukturalismus postuliert.

20 „Nullinstantiation“ (Fillmore \& Kay 1999) liefert jedenfalls keine adäquate Lösung. Wie im Folgenden gezeigt wird, ist in satzwertigen Infinitivkonstruktionen ein Subjekt strukturell präsent und nicht bloß erschließbar als Nullinstantiation eines impliziten Aktanten. Das zeigt sich unmittelbar daran, dass implizite Subjektsaktanten in finiten Sätzen möglich sind, nicht aber in satzwertigen Infinitiven, vgl. (9b) vs. (9c). Aufschlussreich ist auch der Vergleich der satzwertigen Konstruktion mit der nicht-satzwertigen. In letzterer liegt 'Nullinstantiation' vor. Die syntaktischen Unterschiede zwischen der kohärenten und der inkohärenten Konstruktionsvariante (10) liefern Evidenz dafür, dass Nullinstantiation für die satzwertige Konstruktion unzutreffend ist. 
es als Pronomen gespaltene Koreferenz ${ }^{21}$ (7a), wobei das stumme Pronomen als pluralisches Pronomen Bezugselement für ein Reziprokpronomen werden kann. Man vergleiche (7a) und (7b). Das Reziprokpronomen selbst erlaubt keinen gespaltenen Bezug, wie (7c) illustriert.

(7) a. Ich ${ }^{\mathrm{i}}$ habe $\mathrm{ihr}^{\mathrm{j}}$ vorgeschlagen, $\mathrm{PRO}^{\mathrm{i} \& \mathrm{j}}$ einander $^{\mathrm{i} \& \mathrm{j}}$ auf dem Laufenden $\mathrm{zu}$ halten.

b. Ich $^{\mathrm{i}}$ habe $i \mathrm{ih}^{\mathrm{j}}$ vorgeschlagen, dass wir ${ }^{\mathrm{i} \& \mathrm{j}}$ einander $^{\mathrm{i} \& \mathrm{j}}$ auf dem Laufenden halten.

c. ${ }^{\star}{ } \mathrm{Ich}^{\mathrm{i}}$ sollte $\mathrm{ihr} \mathrm{j}^{\mathrm{j}}$ einander ${ }^{\mathrm{i} \& \mathrm{j}}$ als Bürgen vorschlagen.

Noch deutlicher verrät sich das Pronomen unter seiner Tarnkappe dadurch, dass es sich als Ziel von Appositionen eignet und dass diese Apposition qua Subjekts-Apposition im Nominativkasus erscheint, und das in einem Kontext, in dem sonst kein Nominativ als Subjektskasus zuweisbar ist, denn in satzwertigen Infinitiven treten Nominativsubjekte nicht auf.

In $(8 \mathrm{a}, \mathrm{b})$ bezieht sich die Apposition auf das Infinitivsubjekt. Wie (8c, d) illustrieren, eignet sich ein impliziter Subjektsaktant, wie in der Passivkonstruktion, nicht als Bezugselement. Ferner zeigt (8e) im Vergleich zu (8f), dass sich ein Reziprokpronomen nicht auf ein Dativ-Objekt beziehen kann. In (8e) bezieht sich das Reziprokpronomen auf das stumme Infinitivsubjekt und erst dieses bezieht sich auf das Dativobjekt.

(8) a. Man hat mich ersucht, als erster den Saal zu verlassen.

b. Man hat uns dazu überredet, einer nach dem anderen in das Beiboot zu springen.

c. $\quad$ Damals wurde als erster gestreikt.

d. $\quad$ 'Damals wurde einer nach dem anderen geseufzt.

21 Voraussetzung für gespaltene Koreferenz ist natürlich ein Matrixverb, das sowohl Subjektwie Objektkontrolle zulässt. Eric Fuß danke ich für folgende positive Nachweise der Existenz der Konstruktion:

i. Er habe ihr vorgeschlagen, einander zu lieben (Hermann Hesse, Gertrud).

ii. Er bietet ihm an, einander zu verzeihen (Hermann Hesse, Gesammelte Werke. Frankfurt/M. Bd. 1, S. 234)

iii. [...] und schlägt ihr vor, einander besser kennenzulernen. (http://www.myfanbase.de/ teen-wolf/liebeskolumnen/?pid=22047 letzter Zugriff: 8.11. 2017) 

e. Ich habe ihnen geraten, einander nicht ins Gehege zu kommen.
f. $\quad$ *Ich habe ihnen einander vorgestellt.

Es gibt noch mehr zwingende Indizien für das Vorhandensein des stummen Gastes im Infinitivsatz. Subjektlose Infinitivsätze sind bekanntlich unakzeptabel. Der grammatische Grund ist, dass das „zu“ des damit präfigierten Verbs, dem im finiten Satz das finite Verb entspricht, obligat mit einem stummen Subjekt assoziiert ist. ${ }^{22}$

(9) a. Es ist möglich, damit zu argumentieren.

b. Es ist möglich, dass damit argumentiert wird.

c. ${ }^{\star}$ Es ist möglich, damit argumentiert zu werden.

Es gibt schließlich noch eine elegante Prädiktion, die unmittelbar mit dem strukturellen Vorhandensein oder der Abwesenheit eines stummen Subjekts einhergeht. Das geht so: Eine Teilmenge der Verben, die einen Infinitivsatz als Satzglied zu sich nehmen, erlaubt neben der satzwertigen, oder in Gunnar Bechs (1955/57) Diktion, inkohärenten Konstruktion auch alternativ eine nichtsatzwertige alias kohärente Konstruktion. Die kohärente Konstruktion bietet in der Satzstruktur keinen Raum für ein stummes Subjekt, da es ja bereits ein Nominativsubjekt im Satz gibt. Hier ist im Verbalkomplex, der sich in der rechten Klammer befindet und als komplexer verbaler Kopf der VP fungiert, die Identifikation der beiden Aktanten implementiert (Haider 2010: 257).

Die Prädiktion lautet wie folgt: Ein Reziprok- oder Reflexivpronomen, das sich in der satzwertigen Konstruktion auf ein Infinitiv-Subjekt bezieht, das sich wiederum auf ein Dativobjekt bezieht, führt in der kohärenten Konstruktion zu Ungrammatikalität, denn es müsste sich direkt auf den Dativ beziehen, was nicht akzeptabel ist. Wenn man daher die kohärente Konstruktion erzwingt, wird diese Konstellation deviant sein, und das ist sie auch, was (10c, d) im

22 Man beachte, dass das aus prinzipiellen Gründen (Haider 2010: 304-305) nur für jene Verben mit , $z u^{\prime}$ zutrifft, die den Kopf eines satzwertigen Infinitives bilden und nicht bloß im Verbalkomplex statusregiert werden, wie in (i) und (ii). Es müssen die Gegenstücke von finiten Verben sein, wie in (iii) und (iv):

i. dass ihm [zu grauen scheint]

ii. dass ihnen dabei nicht [applaudiert $z u$ werden pflegt]

iii. ^Es genügt, [ihm nicht zu grauen]

iv. ^Es ist üblich, [danach nicht applaudiert zu werden] 
Vergleich zur satzwertigen Konstruktion ${ }^{23}$ (10a, b) verdeutlicht. Der Unterschied ist ein struktureller. Das ist ein Problem für alle Modelle, in denen das Infinitivsubjekt nicht über der syntaktischen Struktur berechnet wird, sondern über der Argumentstruktur. Die ist ja in allen Fällen die gleiche.

Eine Voranstellung der Verben, wie in (10c), ist nur möglich, wenn sie derselben rechten Klammer angehören, und das ist nur bei kohärenter Konstruktion der Fall. Ebenso ist das Oberfeld bei der Ersatzinfinitivkonstruktion stets das Oberfeld ein und derselben rechten Klammer. Folglich ist die Infinitivkonstruktion in (10c, d) kohärent, also nicht satzwertig, und das Reziprokpronomen somit fehl am Ort. Ersetzt man in (10c, d) ,einander' durch ,die Kinder', sind die Sätze untadelig. Der Grund ist, dass ,einander ' in (10c, d) in Abwesenheit eines stummen Subjekts direkt auf den Dativ bezogen werden müsste, was in der deutschen Grammatik deviant ist. ${ }^{24}$

(10) a. Du hättest ihnen nicht erlauben sollen, --pRo einander $\mathrm{zu}$ fotografieren.

b. dass du ihnen [--pRo einander zu fotografieren] nicht hättest erlauben sollen

c. ${ }^{\star}$ dass $\mathrm{du}$ ihnen einander nicht [hättest $\mathrm{zu}$ fotografieren erlauben sollen]

d. *[Zu fotografieren erlauben sollen] hättest du ihnen einander nicht.

Zum Abschluss noch ein fehlendes Puzzlestück. ${ }^{25}$ Wer Leerstellen behauptet, muss auch zeigen, wie das Leere lernbar ist. Wie erkennt das kindliche sprachlernende Hirn im monolingualen Input, dass in Infinitiv-Sätzen ein leeres Subjekt vorhanden sein muss? Hier die Kürzestversion: Strukturverarbeitung funktioniert nicht algebraisch, sondern ,geometrisch'. Beim Verarbeiten - sei es produktiv oder rezeptiv - projizieren wir zumindest zweidimensionale Struktur-Schablonen (Schachtel-in-Schachtel-Strukturen als strukturelle ,prefabs $\left.{ }^{6}\right)^{26}$

23 Satzwertige Infinitive können im Deutschen sowohl im Nachfeld wie im Mittelfeld auftreten, im Niederländischen hingegen nur im Nachfeld. Bei umfänglicheren Exemplaren wird Nachstellung präferiert.

24 Niederländisch weist, wie erwartet, einen ähnlichen Kontrast auf, wie Henk van Riemsdijk (p.c.) bestätigt:

i. dat men hun niet zal beloven [elkaar te fotograferen $]_{\text {Inf.-Satz }}$

ii. *dat men hun elkaar niet [zal te fotograferen beloven $]_{\text {Verbalkomplex }}$

25 Dank an Stefan Müller, der mich auf diese Bringschuld hinwies.

26 Anders als in der CxG (cf. Goldberg 2013: 26) ist damit allerdings keine Form-FunktionsBeziehung verknüpft. Es sind Strukturschablonen, die auf lineare Abfolgen gepasst werden. 
über die jeweiligen Input- oder Outputketten. Die Schablonen für einfache Sätze sind für finite und für nicht-finite Sätze strukturell die gleichen. In nichtfiniten Sätzen bleibt aber, neben den Satzeinleitern, ${ }^{27}$ das Subjekt als syntaktischer Repräsentant des Subjektaktanten des Verbs leer. Das lernende Kind erkennt diese Lücke, da es das finite Gegenstück kennt. Die geforderte Intelligenzleistung ist nicht höher als die, die für die Vervollständigung von einfachen Figuren in Intelligenztests abgefragt wird.

Wenn nun die CxG-Leute alle die diskutierten Daten ${ }^{28}$ ohne Annahme eines stummen Subjekts empirisch adäquat $\mathrm{zu}$ modellieren vermöchten und auch noch eine korrekte unabhängige Prädiktion dieses Modells lieferten, die sich daraus ergibt, dass kein stummes Subjekt vorhanden sein könne, bin ich sofort bereit, das Ergebnis zu akzeptieren. Ich zweifle aber, dass diese Übung gelingen kann. Bis dahin werde ich mich im Recht fühlen, zu behaupten, dass die CxG für diese Phänomene keine empirisch akzeptable Lösung hat und dass die Analyse mit stummem Pronomen die elegantere und empirisch adäquate Analyse ist.

\subsection{Komparativsätze, und warum es auch dabei ohne Leerelemente nicht geht}

Eine Sternstunde der Generativen Grammatik in ihren guten Tagen war „On Wh-Movement“ (Chomsky 1977). Zum ersten Mal war es gelungen, eine Schar scheinbar verschiedener Konstruktionen als Mitglieder derselben Familie zu identifizieren. Dazu gehörten Fragesätze genauso wie Relativsätze, und, was besonders überraschend wirkte, Komparativsätze. Später stellte sich auch noch heraus, dass die Deklarativsätze aller germanischen Sprachen, außer Englisch, ebenfalls dazugehören, weil die sich alle aufgrund der V2-Satzstruktur durch ein variabel besetzbares Vorfeld auszeichnen. Das Markenzeichen dieser Konstruktionsfamilie ist die Besetzung der ersten Position im Satz durch eine vorangestellte Phrase. Diese kann auch aus einem eingebetteten Satz stammen. ${ }^{29}$ Dabei zeigen sich uniforme Beschränkungen, die nachweislich für alle Famili-

27 Lediglich im infiniten Finalsatz gibt es im Deutschen einen Satzeinleiter: „, [um [ein Beispiel zu geben]]“. Anstatt und ohne sind Präpositionen, die einen Infinitivsatz zu sich nehmen.

28 Diese Phänomene sind nicht nur für $\mathrm{CxG}$ problematisch, sondern für alle Modelle, die kein stummes Infinitivsubjekt annehmen und stattdessen die Argumentstruktur der beteiligten Verben heranziehen. Dazu gehört beispielsweise auch die HPSG.

29 Hermann Paul (1919: 321 ff.) hat das Phänomen schon vor hundert Jahren beobachtet, dafür den Terminus ,Satzverschlingung‘ geprägt und eine reiche Sammlung solcher Spezimina angelegt. 
enmitglieder gelten, und das nachweislich sprachübergreifend (s. Haider 2010, Kap. 3.1).

(11) a. Das Phänomen ist wichtiger [ ${ }_{\mathrm{PP}}$ als $\mathrm{P}_{\mathrm{P}}$ [dass man es einfach ignorieren könnte]]

b. Es gibt in diesem Satz mehr Dinge $\left[_{\mathrm{PP}}\right.$ als $\mathrm{P}_{\mathrm{P}}$ [die $\mathrm{CxG}$ glaubt, hier hören zu können]]

Im Deutschen sind Komparativsätze Sätze, die von der Präposition ,als`abhängen. Die ,als‘-PP ist mit einer komparativhaltigen Phrase als Antezedens assoziiert. Der PP-Status ist an (11a) zweifelsfrei erkennbar. Mit ,anstatt, dass`, ,ohne dass' und ,als dass' beginnt eine PP, die einen finiten Satz enthält. Der für das hier zu verhandelnde Argument kritische Fall aber ist der Typus (11b). Hier gibt es keine satzeinleitende Partikel und überdies fehlt ein Satzglied, nämlich jenes, das den Vergleichsgegenstand benennt.

Die erste und wichtigste Frage ist die: Wo ist das Objekt von ,hören“ in (11b)? Es gibt eines, denn es wird ja mit dem Objekt des Verbs ,geben“ verglichen und ,hören' ist in diesem Satz definitiv transitiv. Das Objekt fehlt im eingebetteten Infinitivsatz. Spricht man nun beispielsweise eine Varietät des bairischen Dialektraumes, dann weiß man sofort, was dahintersteckt. Was in (11b) nicht zu hören ist, ist das stumme Gegenstück des W-Pronomens ,wos‘ (was) von (12a). In der Standardvarietät des Deutschen ${ }^{30}$ bleibt das Pronomen stumm (12b), genauso wie ein Relativpronomen im englischen Relativsatz stumm bleiben kann (cf. The relative pronoun we should be able to hear in this clause is silent), um einen weiteren Kandidaten für eine Konstruktion mit pronominalem Leerelement zu erwähnen.

(12) a. Ea kend mi scho mea, ois wos ma liab sei kendd ${ }^{31}$ er kennt mich schon mehr als was mir lieb sein könnte

b. Es gibt in diesem Satz mehr Phrasen [als [ $\mathrm{O}_{\text {was }}$ die $\mathrm{CxG}$ glaubt, hier -zu hören]]

30 Man findet allerdings auch standardnahe Belege, wie etwa dieser aus einem Schweizer Schriftstück: [Das] „ist meistens mehr, als was mir möglich ist.“ (www.seval.ch/rapport/ annexe.pdf letzter Zugriff: 17. 8. 2017)).

31 Hans Kreiner, Im Gegenwind: Schrift und Mundart. Da Aungla. (S. 38). https://books. google.at/books?id=FuYq36Hh_AQC\&pg=PA38\&lpg=PA38\&dq=\%22mea+ois+wos $\% 22 \&$ source= bl\&ots=gy307iCUQ\&sig=NreK5VaYVj_YlTZo4v7Knj0eKOY\&hl=de\&sa=X\&ved= 0ahUKEwiRw536uv_UAhUHfxoKHdFVDFsQ6AEIKDAB\#v=onepage\&q= \%22mea\%20ois\%20wos\%22\&f=false (letzter Zugriff: 10.7. 2017) 
Und wenn man das Ganze ohne ein stummes Pronomen hinbekommen ${ }^{32}$ will? Dann gibt man erstens die offenkundige Parallele zwischen (12a) und (12b) auf und behauptet zwei verschiedene Mechanismen für engst verwandte Phänomene. Man muss aber die Tatsachen erfassen können, die nur bei Filler-GapKonstruktionen auftreten, und das hat bisher niemand überzeugend geschafft, der eine Filler-Gap-Konstellation deswegen nicht zulässt, weil er die Existenz eines stummen Filler-Elements dogmatisch verneint. (13b, d) sind genauso ungrammatisch wie ihre Pendants mit hörbaren Fillers (13e, f).

(13) a. Sie publiziert mehr Papers, als man bereit ist, -- zu lesen.

b. `Sie publiziert mehr Papers, als man ein Verbot, -- zu lesen, erlassen möchte.

c. Sie publiziert mehr Papers, als er sagt, dass man -- lesen müsse.

d. ${ }^{\star}$ Sie publiziert mehr Papers, als er sagt, warum man -- lesen sollte.

e. $\quad$ Solche Papers möchte er [ein Verbot -- zu lesen] erlassen.

f. *Welche Papers hat er gesagt, warum man -- lesen sollte?

Die Sache geht aber noch weiter. In Sprachen mit kopf-initialer VP, so wie im Englischen oder den skandinavischen oder romanischen Sprachen, sind Subjekte, weil präverbal, ebenfalls als Areale für Gaps von vorangestellten Fillers tabu (14a), wenn sich diese außerhalb befinden. Das gilt aber nicht für Objekte, weil diese postverbal sind (14b). In Sprachen mit kopf-finaler VP tritt diese Subjekt-Objekt-Asymmetrie systematisch nicht auf (14a, c):

(14) a. * She invited more people than [inviting --] was reasonable.

b. She invited more people than I would consider [inviting --].

c. Sie hat mehr Leute eingeladen als [-- dazu einzuladen] Sinn gemacht hätte.

Ferner gilt für jegliche Filler-Gap-Konstellationen, in denen sich der Filler in der satzinitialen Position (,Vorfeld`) befindet, dass die Lücke, zu der der Filler gehört, nicht in einer Nominalphrase eingebettet sein darf (13e) und sich auch nicht in einem indirekten Fragesatz befinden darf (13f). Er darf auch kein

32 Dass man theoretisch stets alles ,irgendwie hinbekommen kann, ist bekannt (s. DuhemQuine These in der Wissenschaftstheorie). Die entscheidende Frage ist bloß, was die empirisch adäquate Weise sei, d.h. wie es ,wirklich` ist. 
Koordinationsglied oder Teil eines Adverbialsatzes sein usw. Das alles trifft für V2-Deklarativsätze, für Fragesätze, für Relativsätze und eben auch für Komparativsätze zu. Bloß, der Filler in Komparativsätzen ist ein stummes Pronomen. Die CxG lehnt Tarnkappen-Pronomina ab. Sie steht daher in der Bringschuld. ${ }^{33}$

Die Bringschuld steigt noch, denn es steht ja auch der Anspruch zur Disposition, dass die Semantik strikt oberflächenbezogen konstruiert werde. Wo ist das Objekt von ,hören“ in (11b), jenes von ,lesen` in (13a, c), oder das von ,invite‘ in (14b) und das von ,einzuladen' in (14c)? Sie sind ganz offenkundig semantisch vorhanden und gehen in die kompositionale Konstruktion der Semantik dieses Satzes ein. Die Komparativsemantik stellt eine Beziehung her zwischen zwei Satzgliedern und daher müssen sie auch vorhanden sein, und sie sind es auch, selbst wenn man das nur im Dialekt hört. Die CxG will nur Hörbares gelten lassen, bleibt aber Erklärungen schuldig für die interessanten Fälle, in denen sie herausgefordert wäre. In den harten Fällen - bei den ,stunning

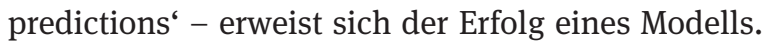

\subsection{Sprachübergreifende Generalisierungen}

Oberflächennah, also untief, wird die bereits im vorigen Abschnitt tangierte Frage nach sprachübergreifend gültigen syntaktischen Eigenschaften diskutiert, so z. B. in Goldberg (2013). Es zeugt von wenig Kenntnis in vergleichender Grammatik, wenn man meint, dass sich sprachübergreifende Konstanten wie folgt ergäben, wovon Goldberg (2013: 15-16) und die CxG Community offenbar ernsthaft ausgehen: „The cross-linguistic generalizations that do exist are explained by domain-general cognitive processes or by the functions of the constructions involved.“

Laut $\mathrm{CxG}$ gebe es kaum sprachübergreifende strukturelle Invarianten (s. Croft-Zitate in Abschnitt 3.5). Man kann wohl zur Annahme gelangen, dass es sie nicht gebe, wenn man nicht danach sucht oder sich im grammatischen Dschungel nicht zurechtfindet. Alle Vierbeiner sind bekanntlich verschieden, und alles, was habituell fliegt oder kreucht, ebenfalls. Das hat die Zoologen nicht davon abgehalten, nach gültigen, speziesübergreifenden Verallgemeinerungen zu suchen, und sie fanden sie, allerdings nicht beim Vergleich analoger

33 Hinweis: Das eben vorgetragene Argument behauptet nur, dass es Filler gibt, die phonetisch stumm sind. Ob in Filler-Gap-Konstellationen auch die Verrechnung der Gaps nur per Leerstellen funktioniere, ist eine andere Frage, die separat zu klären ist. In der Generalized Phrase Structure Grammar, beispielsweise, hat man seinerzeit dafür ein Pfadmarkierungsverfahren per Slash-Kategorien entwickelt, das Fehlpositionen verrechnet, ohne sie in der Struktur als Lücken einsetzen zu müssen. 
Strukturen, wie das die CxG tut, sondern im Vergleich homologer Strukturen. Man muss Strukturen mit Strukturen vergleichen und nicht Funktionen mit Strukturen.

Beginnen wir mit einem Phänomen, das von einer gern kolportierten und grundfalschen Legende begleitet wird, nämlich der ,freien“ Wortstellung in Tateinheit mit der morphologischen Identifizierbarkeit der Aktanten. Englisch oder die skandinavischen Sprachen hätten fixe Wortstellung (angenommen) und Deutsch eine variable (behalten), denn im Deutschen könne man anhand der Morphologie die Dinge auseinanderhalten. Bereits Jespersen (1894: §75) hat das in Abrede gestellt. Wenn fixe Wortstellung als Kompensation des Flexionsverlusts diente, dann müsste es ein grammatisches Interregnum gegeben haben: „We should have to imagine an intervening period in which the mutual relations of words were indicated in neither way; a period, in fact, in which speech would be unintelligible.“ Daraus folgert er zu Recht, „a fixed word order was the prius, or cause, and grammatical simplification, the posterus, or effect“". ${ }^{34}$

Die Vertauschung von Ursache und Wirkung ist der Kardinalfehler von Funktionalisten. Wer immer an die Legende glaubt, hat nämlich auch übersehen, dass im heutigen Englisch selbst ein Präpositionalobjekt seine starre Abfolge beibehält, obwohl es exzellent identifizierbar wäre. ${ }^{35}$

(15) a. They try to attribute every property to cognitive processes.

b. ${ }^{\star}$ They try to attribute to cognitive processes every property.

Für die Gegenprobe - starre Wortfolge trotz reicher Kasusmorphologie - muss man sich nur auf die Nachbarinseln begeben. Isländisch hat, so wie Englisch, kopf-initiale VPs, aber anders als Englisch hat es die altgermanische Kasusmorphologie nahezu konserviert. Doch die Abfolge der nominalen Satzglieder in der VP ist invariant. Dehé (2004: 94) berichtet, dass Ihre kontrolliert getesteten Informanten ausnahmslos die Umstellungsvarianten von (16), also Akkusativvor Dativobjekt, zurückgewiesen haben.

$34 \mathrm{Zu}$ dieser Legende gehört auch die Annahme, dass English deshalb zum SVO-Typ schwenkte, um das Subjekt klar identifizierbar zu machen. Das ist ein Non-sequitur. Man hätte bloß, wie in den kasusarmen westgermanischen Sprachen, auf das Scrambling von kasusambigen Aktanten zu verzichten brauchen, wie man das selbst im Deutschen tut und wie es im Afrikaans die Regel ist. Afrikaans hat so gut wie keine Flexion, ist aber SOV.

35 Das gesamte BNC (British National Corpus, 100 Millionen Wörter) und das gesamte CocA (Corpus of Contemporary American English, 500 Millionen Wörter) enthalten keinen einzigen Eintrag mit der Sequenz „attribute to it every“, wohl aber Einträge mit „attribute everything to“. Obwohl Pronomina sonst vorangestellt werden müssen (vgl. „look it up“ vs. * „look up it“), bleibt die Abfolge starr, nämlich V + Objekt + PP-Objekt. 
(16) a. Hann gaf konunginum ambáttina. ${ }^{36}$

Er gab König-DEF-DAT Zofe $_{\text {-DEF-ACC }}$

b. Pau sýndu foreldrunum krakkana. Sie zeigten Eltern-DEF-DAT Kinder-'DEF-ACC
- * Hann gaf ambáttina konunginum.

- * Pau sýndu krakkana foreldrunum.

Was ist die korrekte Generalisierung? In kopf-initialen Phrasen ist die Abfolge nominaler Satzglieder invariant; in kopf-finalen Phrasen ist die Abfolge variabel, sofern der Satzgliedstatus identifizierbar ist, und das sollte für jede SOV-Sprache gelten. ${ }^{37}$ Diese Generalisierung lässt sich selbst innerhalb einer einzigen Sprache gut erkennen. Deutsch reicht dafür völlig aus. Im Deutschen sind die Nominalphrasen kopf-initial, die Verbalphrasen aber kopf-final und überdies lässt sich aus jedem Verb ein Nomen gewinnen, durch InfinitivNominalisierung. Damit hat man eine ideale Minimalpaar-Situation. Die Abfolge in der Nominalphrase ist starr, die in der Verbalphrase variabel. Die deutsche NP verhält sich wie die englische, die deutsche VP tut das nicht, denn sie ist, anders als die Englische, kopf-final:
a. [einige Aufgaben an diese Behörde übertragen $]_{\mathrm{VP}}$
b. an diese Behörde einige Aufgaben übertragen
b. das [Übertragen einiger Aufgaben an diese Behörde $]_{\mathrm{NP}}$
c. *das Übertragen an diese Behörde einiger Aufgaben ${ }^{38}$

Es ist Achtlosigkeit, wenn man von „Sprachen“ mit freier Wortstellung spricht. Es ist keine holistische Eigenschaft einer Sprache, um die es geht; es ist die Struktur der Phrase, die relevant ist. Deutsch ist „frei“ in der VP und somit im Mittelfeld, aber „strikt“ in der NP, und das aus einem präzise benennbaren und nachprüfbaren Grund. Die eine Phrase ist kopf-final, die andere kopf-initial.

36 Dieses Beispiel sei hier zitiert, weil es häufig in der isländischen Syntaxliteratur zitiert wird, als möglicher Fall von variabler Abfolge. Die einzige Autorität dafür ist aber stets die Introspektion des jeweiligen Autors.

37 Dazu passt auch, dass Niederländisch mit seiner rechtsköpfigen VP genau die Abfolgevariation in (15) erlaubt, die dem linksköpfigen Englisch verboten ist (Haider 2010: 158). Gleiches trifft auf die finno-ugrischen Schwestersprachen Udmurtisch (OV) im Vergleich zum Finnischen zu (Schmidt 2016).

38 Das ist keine Stellungsrestriktion des Genitivs, denn sie betrifft ebenso die von-PPs (Haider 2013: 205):

i. *das Benützen im Brandfall des Lifts/von Liften.

ii. *das Vergleichen mit $\mathrm{CxG}$ von Konkurrenten 
Die grammatiktheoretische Herleitung dieses Faktums ist etwas umfänglicher (s. dazu Haider 2010, 2013, 2015a). Die Erklärung beruft sich natürlich nicht auf „domain-general cognitive processes or the functions of the constructions involved“, denn damit hat es nichts zu tun. Es sind Invarianten der menschlichen kognitiven ,Sprachsoftware‘, also domänen-spezifische Eigenheiten der Verarbeitung von Phrasenstrukturen, die unabhängig von Funktionsbezügen sind. Egal ob die NP einen Aktanten, ein Prädikat oder ein Adverbial repräsentiert, sie hat stets dieselben strukturellen Eigenschaften. Mit diesen Unterschieden korrelieren viele andere Eigenschaften, für die es weder allgemein-kognitive noch funktionale Motivationen gibt.

Nach diesem Einstieg noch ein etwas komplexer gelagertes Phänomen, das ebenfalls mit dem Faktor der Stellung des Kopfes der Phrase zu tun hat. Phrasen, die als Modifikatoren an eine NP oder VP treten - gewöhnlich als Attribut bzw. Adverbial bezeichnet - unterliegen folgender Beschränkung: Eine am linken Phrasenrand angefügte Modifikatorphrase einer linksköpfigen d.h. kopfinitialen Phrase, muss ihren eigenen Kopf unmittelbar am rechten Phrasenrand haben. Bei linken Modifikatoren von rechtsköpfigen Phrasen tritt dieser Effekt nicht auf. Wiederum verhält sich die deutsche NP (18a) so, wie die englische NP (18b). Die deutsche VP (18c), die bekanntlich kopf-final ist, verhält sich nicht so wie die englische (18d), und so wie im Deutschen verhält es sich mit allen Sprachen mit kopf-finaler VP, wie z. B. im Niederländischen.

(18) a. eine [sehr viel schlampigere ( ${ }^{\star}$ als ich erwartet hatte)] Analyse

b. a [much more sloppy ( ${ }^{\star}$ than I expected)] analysis

c. Man sollte die Wortstellung [viel sorgfältiger als die CxG Leute das tun] analysieren.

c. You should [much more carefully ( ${ }^{\star}$ than $C x G$ people do)] analyze word order.

Hier kommt nun eine petite complication, die eine Ingredienz einbringt, für die eine $\mathrm{CxG}$ keine Trennschärfe besitzt. Es ist der Faktor der „acceptable ungrammaticality“. Sprecher/innen verstoßen unbewusst gegen eine Grammatikrestriktion, weil das in der gegebenen Situation das geringere Übel ist, wenn sie die Produktion des Satzes nicht einfach abbrechen wollen, da sie mit grammatischen Gesetzen in Konflikt geraten. Man versuche sich an folgender Elizitationsübung. Gegeben sei als Vorbild (19a) und daraus soll eine Nominalphrase gemacht werden, wobei die prädikative Adjektivphrase von (19a) als Attribut einer NP fungiert. 
(19) a. Das Defizit ist [höher als erwartet].

b. *Das ist ein [höheres als erwartet] Defizit.

c. Das ist ein [höher als erwartetes] Defizit. ${ }^{39}$

d. ein [teurer als nötiges] Eingreifen ${ }^{40}$

Es ist ganz offensichtlich, dass in (19b, c) der Kopf des Attributs das Adjektiv ,höher' sein muss, und in (19d) ,teurer'. Was geschieht hier? Es wird stur der rechte Rand des Attributs kongruiert, wenn er sich denn lässt. Man schlägt mit der übergeordneten Grammatikregel zu, trifft aber das Falsche. Selbst Unflektierbares wird flektiert, wie (20) illustriert. ${ }^{41}$ Der Charme dieses speziellen Falls (20) ist, dass die Schreiber mit einer Singularität ringen. „Genug“ mit all seinen germanischen Kognaten ist eine Singularität. Das Wörtchen hat es über ein Millennium hinweg geschafft, seine ausnahmshafte Nachstellung zu behaupten. Damit kommt es aber der eben diskutierten Bedingung in die Quere. Anstatt auf „genügend“ umzuschwenken, welches ganz normal voranginge, flektieren diese grammatischen Ringkämpfer das aufmüpfige Wörtchen und wählen das vermeintlich kleinere Übel.
a. in fest genuger Zusammensetzung
(fest genug - genügend fest)
b. ein Taxi mit groß genugem Kofferraum (groß genug - genügend groß)
c. auf breit genugen Radwegen
(breit genug - genügend breit)

Wie in Haider (2011) dazu ausgeführt, liegt hier ein Fall von ,akzeptabler Ungrammatikalität vor (vgl. Frazier 2015). Das ist ein Phänomen, das sowohl in der deskriptiven Linguistik als auch in der Grammatiktheorie bisher nicht die verdiente Beachtung genießt. Die Beispiele (19b, c) und (20) sind Grammatikverstöße, aber ein unbefangener Proband neigt dazu, sie zu tolerieren. Es sind Fälle von grammatischen Täuschungen. So wie optische Täuschungen wirken sie akzeptabel, sind aber bei näherer Betrachtung Verstöße gegen die Gramma-

39 Selbst wenn man bei Google den Filter ,News‘ benutzt, findet man diese Beispiele. Hier ist eines aus Finanzen.net: ein deutlich höher als erwartetes Lagerplus.

40 Grüne schrecken auf der Bundestagsseite nicht vor dieser Ausdrucksweise zurück. https://www.gruene-bundestag.de/parlament/bundestagsreden/2011/oktober/gerhard-schickerrichtung-des-europaeischen-finanzaufsichtssystems.html (letzter Zugriff: 8. 11. 2017).

41 (20a) stammt von einer Dating-Seite, (20b) von einem Buchautor, (20c) aus einem WebChat unter Radlern. 
tik. Sie in die Grammatik integrieren zu wollen, würde die Grammatik inkonsistent machen. ${ }^{42}$

Die CxG mit ihrer Arbeitsmaxime, Gesagtes als gültig zu werten (d. h. usagebased), tut genau das nicht, was Wissenschaftler tun müssen. Schräge Rohdaten müssen geprüft werden, ob deren Zustandekommen nicht etwas Drittem geschuldet ist und sie deshalb gar nicht repräsentativ sind für die gesuchte oder zu prüfende Generalisierung. Genau das liegt hier vor. Als der Autor die oben genannte Generalisierung bei einer Tagung thematisierte, wurde er in der Diskussion auf Fälle wie (21a, b) hingewiesen, weil sie vermeintlich Gegenevidenz bilden. In der Tat widersprechen sie der Generalisierung. Es sind aber Fälle genau derselben Art von akzeptabler Ungrammatikalität wie im Deutschen, was (21c, d) zeigt. Man erkennt das bloß nicht so leicht, da im Englischen nicht morphologisch manifest kongruiert wird.

(21) a. a better than expected ...

(42 Belege in Coca, 16 in BNC, für alle Adjektive)

b. a higher than average ...

(69 Belege in Coca, 37 in BNC, für alle Adjektive)

c. ein besser als erwartetes ...

(über 1000 Google-Belege auf News-Seiten)

d. ein höher als durchschnittliches Einkommen

Sobald man den rechten Rand mit etwas besetzt, das nicht zum flektierten Ersatz-Kopf umgedeutet werden kann (22a, b), oder einen Kontext wählt, in dem der Kopf gar nicht flektiert wird (22c, d), löst sich der Spuk auf. Die großen Corpora enthalten keine Daten dieser Art und die Grammatik tritt in klarer Form wieder hervor:

(22) a. *a better than I expected result

b. *a higher than the median temperature

c. * She has better than expected solved the problem

d. * She has higher than average scored on this task

42 Es ist nicht ausgeschlossen, dass irgendwann in der Zukunft, abseits von Normierungszwängen, ,genug' flektierbar sein wird. Solange aber ,genuge‘ in (i) als deviant empfunden wird, ist das Ziel noch fern.

(i) Er hat eine genügende/*genuge Anzahl von Einwänden vorgebracht. 
Um sprachübergreifende Invarianten zu finden, braucht es mehr als den Vergleich von Konstruktionen. Der Autor hat mehr als drei Jahrzehnte darauf verwendet und gut ein Dutzend strukturell basierter Phänomene identifiziert, die direkt mit der Stellung des Kopfes in der Phrase korrelieren (Haider 2010, 2013, 2015a). Die germanischen Sprachen sind ein ideales Labor, weil sie sich in eine SOV- und eine SVO-Gruppe gespalten haben. Gleiches gilt übrigens für finnougrische Sprachen, mit Udmurtisch als OV-Sprache und der Schwestersprache Finnisch. Hier haben sich die Resultate, die an den germanischen Sprachen gewonnen wurden, unabhängig bestätigt (s. Schmidt 2016). Das alles erfordert intensive Arbeit „unter Tag“. Die Vogelperspektive des Darüberhinwegfliegens reicht jedenfalls nicht, weder zum Auffinden noch zum Bestreiten. Doch Goldberg (2013: 24) glaubt anscheinend, dass man Relevantes zur Thematik beisteuere, wenn man sich über den sogenannten Köpfigkeits-Parameter wie folgt äußert.

This "head-direction parameter" has long been used as an example of a purely syntactic generalization that requires an innate universal stipulation. [...] However, as is well known, this generalization is not without exceptions. Persian, for example, is a verb-final language, but has prepositions instead of postpositions.

Der Kopf-Parameter besagt, dass sich Sprachen darin unterscheiden, wie der Kopf einer Phrase kanonisch linearisiert ist. Es gibt strikt links-köpfige Systeme, wie Englisch, wo alle Phrasen linksköpfig sind. Es gibt strikt rechtsköpfige, wie Japanisch, und es gibt Systeme, die nach Kategorie differenzieren, wie Deutsch oder Niederländisch oder eben Persisch. Im Deutschen sind, wie in allen germanischen OV-Sprachen, die NP und die PP linksköpfig, die VP und die AP rechtsköpfig. Und dann gibt es überdies noch Sprachen, in denen die Köpfigkeitsrichtung nicht für alle Kategorien fixiert ist, wie in den slawischen Sprachen. Dies sind die Sprachen des ,dritten Typs' und werden stets oberflächlich mit SVO-Sprachen verwechselt (Szucsich \& Haider 2015).

Es gibt präzis formulierbare und empirisch bestätigte, rein syntaktische Korrelationen, die nichts mit Semantik oder Diskurs zu tun haben. Es ist eine triviale Tatsache, dass der eindimensionale akustische Kanal die Alternation von möglichen Kopfpositionen auf Voran- oder Hinterdreingehen reduziert. Über der linearen Abfolge wird eine syntaktische Struktur entfaltet. Syntax ist die spontan funktionierende kognitive Fertigkeit, aus der eindimensionalen phonologischen Präsentation die zumindest zweidimensionale Schachtel-inSchachtel-Struktur syntaktischer Phrasen zu errechnen. Dafür bedarf es jener $\mathrm{zu}$ erforschenden, domänenspezifisch gewordenen Verarbeitungsroutinen, die das Homo-sapiens-Hirn dafür zur Verfügung hat. Keiner anderen Spezies scheint das gegeben zu sein. 
Ob links- oder rechtsköpfig, alle Phrasen sind intern rechtsverzweigend ${ }^{43}$ aufgebaut (Details dazu in Haider 2015a). Bei kopf-initialen Phrasen führt, wie in Haider (2010, 2013, 2015a) gezeigt wird, die unvermeidliche Gegenläufigkeit zwischen Verzweigungsrichtung und Köpfigkeitsrichtung genau zu der Eigenschaft, die soeben illustriert worden ist, nämlich der starren Wortstellung in links-köpfigen Strukturen.

Aber es gibt Typologen, die Köpfigkeitsrichtung mit Verzweigungsrichtung verwechseln, wie Dryer (2009). ${ }^{44}$ Und dann gibt es auch noch welche, die überfordert sind, wie z. B. Whaley (1997: 103-106). In der funktionalistisch gehaltenen Typologieeinführung wird forsch kundgetan, Deutsch sei in seiner „basic constituent order“ SVO, da das „the most frequent order" und „the least marked order“ und the „pragmatically most neutral order“ sei, womit gleichzeitig einige irrelevante Eigenschaften zur Bestimmung von ,basic order‘ aufgezählt wären.

So viel Ahnungslosigkeit ist entwaffnend. Wäre die Autorin bloß auf dem Stand des Wissens der 80er-Jahre des 19. Jahrhunderts [sic!], dann wüsste sie, dass die Position des finiten Verbs in V2-Sprachen wie Deutsch nicht dessen Grundposition als Kopf der VP ist. Schließlich ist diese Einsicht in die V2-Struktur schon 1886 von Oskar Erdmann, in seiner Grammatik des Deutschen durchschaut, präzise erläutert und publiziert worden (Erdmann 1886: 181-184). ${ }^{45}$

Dass die germanistische Linguistik im deutschsprachigen Raum ab den zwanziger Jahren fast ein halbes Jahrhundert verfiel, bis die große Tradition völlig zunichte war, ist ein anderes Kapitel, und ein tragisches. Aber Whaley hätte zumindest Mallinson \& Blake (1981: 129) kennen müssen, die in ihrem Typologiebuch klipp und klar erklären: „The order used for a stylistically unmarked version of John saw Mary in German would be SVO, too, but to simply call German an SVO language would disguise the verb-second nature of its word order."

43 Siehe Fn. 9.

44 Mit dem in der funktionalen Typologie üblichen Hedging konstatiert er: „[...] languages tend towards being either consistently left-branching or consistently right-branching. "Was er meint, ist links- bzw. rechtsköpfig, denn ein Argument für die genannten gegenläufigen Verzweigungen liefert er nicht, und es würde ihm auch schwerfallen, konsistent linksverzweigende Sprachen zu finden. Sie existieren nicht, denn auch die konsistent links-köpfigen Sprachen haben rechtsverzweigende Strukturen (Haider 2015a).

45 Die Paragraphen 205-216 sollten unverzichtbare Pflichtlektüre für alle sein, die sich mit deutscher Satzstruktur beschäftigen. Darin steht das geschrieben, was erst hundert Jahre später neu entdeckt werden sollte. 


\subsection{Gebrauchsbasiert - gebrauchsverschmiert}

Bereits im vorigen Abschnitt (s. acceptable ungrammaticality) wurde eines der Probleme eines naiv-phänomenologischen Zugangs angeschnitten. Nicht alles, was gebraucht wird, ist grammatisch, verstanden als konform mit dem formalen Regelwerk der Sprache, und nicht alles, was grammatisch ist, wird gebraucht. Gebrauch ist ein Kuddelmuddel aus vielen interagierenden Bedingungssystemen - Grammatik, Parsing, Produktion, Interferenzen aus Multi-Dialektalität oder Mehrsprachigkeit, vermeintliche Normtreue oder intendierte Missachtung der Norm, idiosynkratische Präferenzen, und einiges mehr. Die direkte Modellierung von Kuddelmuddel ergibt ein Modell, das selbst Kuddelmuddel ist. Garbage in, garbage out, diagnostizieren Informatiker.

Das über alle Disziplinen hinweg etablierte Erfolgsmodell der Wissenschaft besteht in der Reduktion der Komplexität des Beobachteten, indem man es als berechenbares Resultat der Interaktion von weniger komplexen Teilsystemen darzustellen vermag. Dazu gehört auch, die Daten zu evaluieren und die systematischen Faktoren von den zufälligen zu trennen. Sie in einen großen Sack zu werfen, dem man die Etikette „wirklicher Gebrauch“ umhängt, wirft Dinge zusammen, die nicht zusammengehören, und man beraubt sich der Möglichkeit, sie analytisch separierend so zu durchdringen, dass sich am Ende ein klareres Bild ergibt.

Der Umstand, dass jemand Ausdrücke wie in (19b-d) oder (20) gebraucht, ist kein Grund, von einem Grammatikmodell zu verlangen, dass es Raum dafür bieten müsse, auch wenn sie gar nicht so selten vorkommen. Gebrauchsfrequenz ist kein Argument für Grammatikalität. ${ }^{46}$ Frequenz ist aber ein Faktor, der im Sprachwandel, beim Spracherwerb und in der Sprachproduktion eine Rolle spielt. Die Einbeziehung des Gebrauchs ist sinnvoll, um nicht zur mentalen Grammatik Gehöriges abtrennen zu können und um zu verstehen, wie es kommt, dass eine Sprache systemwidrige Ausnahmen konserviert, teilweise dadurch, dass etwas formelhaft und frequent verwendet wird und als syntaktische Gestalt dem deklarativen Gedächtnis eingemeindet wird, während Systematisches vom prozeduralen System verwaltet wird. Das hat im 18. Jahrhundert bereits der Physiker Georg Christoph Lichtenberg der Erwähnung wert gefunden. ${ }^{47}$

46 Hier ein extremes Beispiel: Auch Christen verwenden in ihrem Sprachgebrauch kein nachgestelltes Possessivum, obwohl sie es frequent benutzen, nämlich immer dann, wenn sie das „Vater-unser" sprechen.

47 „Diejenigen Verba, welche die Leute täglich im Munde führen, sind in allen Sprachen die irregulärsten; sum, sono, $\varepsilon \imath \mu$, ich bin, je suis, jag är, I am.“ 
Das wahrhaft Erstaunliche ist aber das Faktum, dass muttersprachliche Sprecher/innen auch bei total infrequenten Konstruktionen uniforme Urteile abliefern. Häufiger Gebrauch ist keine Bestätigung für Grammatikalität und infrequenter Gebrauch bewirkt keinen Mangel an Grammatikkompetenz. Die nicht negierte Aussage wäre übrigens eine der falschen Prädiktionen, die entstehen, wenn man die Existenz domänenspezifischer Kapazitäten dogmatisch ausschließt. Hier sind zwei Illustrationen eines Phänomens, das Gunnar Bech (1955) fast ${ }^{48}$ vollständig durchschaut hat.

(23) a. Er hat ja niemanden [zu stören versucht]

(= nicht versucht, jemanden zu stören)

b. Er hat [niemanden zu stören] ja versucht (= versucht, niemanden $\mathrm{zu}$ stören)

In (23a) ist das negierte Objekt des Infinitivs imstande, als Negator des Hauptsatzes zu fungieren, in (23b) ist es das nicht. Der Grund ist folgender. Alles, was zwischen den beiden Verben steht, also auch eine Partikel, eliminiert die Möglichkeit der kohärenten Konstruktion. Folglich liegt in (23b) ein eingebetteter Infinitivsatz vor. Der Skopus des negierten Objekts ist somit bloß der eingebettete Satz. De facto ist (23a) ambig zwischen der satzwertigen Konstruktion und der durch die Klammerung angegebenen nicht-satzwertigen, und damit auch der Negationsskopus, und deswegen alterniert in (24) die Kasuszuweisung zwischen Nominativ und Akkusativ. In der satzwertigen Konstruktion (24a, c) ist der Infinitivsatz das Objekt. Dessen Objekt ändert seinen Kasus natürlich nicht, wenn im Hauptsatz passiviert wird. In der kohärenten Konstruktion (24b, d) ist das Akkusativobjekt das Objekt des gesamten Verbalkomplexes des einfachen Satzes, was bei Passiv zu Nominativzuweisung führt. Das ist das sogenannte ,Fernpassiv‘. De facto ist es das gewöhnliche Passiv, aber in der kohärenten Konstruktion.

48 Was er übersehen hat ist die Tatsache, dass inkohärente Infinitivkonstruktionen auch im Mittelfeld auftreten und nicht nur, wie er meinte, im Nachfeld. Jedenfalls kommen in seinen Analysen satzwertige Infinitive im Mittelfeld nicht vor. Das wurde mir von seiner DoktoratsAbsolventin Cathrine Fabricius-Hansen (p.c.) bestätigt.

Hier ein Beleg: „Ich erzählte Dir, dass ich vor den Machinationen der Tante in Wien in die dunkelsten Gemächer dieser Dame zu fliehen mehrmals genötigt war.“ (Hackländer, Deutsche Romanbibliothek). 


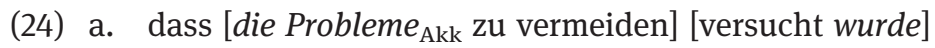

b. dass die Probleme $_{\mathrm{Nom}}$ [zu vermeiden versucht wurden]

c. dass [den Wagen zu betanken] [vergessen wurde]

d. dass der Wagen [zu betanken vergessen wurde]

Wenn irgendetwas zwischen dem $z u$-Infinitiv und dem Hauptverb Platz nimmt, erzwingt das die satzwertige Konstruktion und der Akkusativ ist dann mandatorisch. Erzwingt man andererseits die kohärente Konstruktion, indem man den Verbalkomplex ins Vorfeld stellt, wird der Nominativ ausgelöst. Über dieses implizite Wissen verfügt man als Sprecher/in ganz unabhängig davon, ob man so einen Satz je gehört hat, wie mir jährliche Tests an Syntax-Novizen bestätigen. Es empfiehlt sich, beim Nachtesten dafür unbedingt ein Elizitationsverfahren zu verwenden.

(25) a. dass die Probleme zu vermeiden ernsthaft versucht wurde/* wurden

b. $\mathrm{Zu}$ betanken vergessen wurde der Wagen/*den Wagen.

Der Gebrauch verursacht ausgetretene Trampelpfade durch das Gelände der Grammatik. Merkwürdigerweise sind Sprecherinnen aber auch mit den Teilen des Geländes vertraut, in denen sie nie zuvor gewandelt sind. Dabei vermeiden sie erstaunlicherweise Fehler, die durch bloße Analogiebildung entstehen müssten. ${ }^{49}$ Das ist das, was ein Grammatikmodell erklären können muss.

\subsection{Der Fehlschluss von der Funktion auf die Form}

Linguisten wollen, scheint es, vermeidbare Fehler gar nicht vermeiden. Die Funktionalismusdebatte haben nämlich die Biologen schon im 19. Jahrhundert konklusiv beendet. Die Berufung auf Funktionen ist in kausalen Erklärungen unzulässig, weil sie nicht valid ist. Hier die Zusammenfassung von Mayr (1982: 464). Man bemerke dabei die Erwähnung von Gebrauch (,utility'): „As Darwin

49 Goldberg (2013: 27) behauptet zwar „Particular languages are learned by generalizing over utterances that a learner has heard used.“ Das Geheimnis bleibt dabei bloß das Wie des ,Generalising'. Hier eines von vielen Beispielen zu irreführenden Generalisierungen: eine einfache Sache $\rightarrow$ Die Sache ist einfach; eine faszinierende/bezaubernde Person $\rightarrow$ die Person ist faszinierend/bezaubernd; ein staunender Leser $\rightarrow$ *Ein Leser ist staunend; ein gähnender/uns nervender Mensch $\rightarrow$ *Der Mensch ist gähnend/uns nervend. 
rightly said 'Nothing can be more hopeless than to attempt to explain the similarity of pattern in members of the same class, by utility or by the doctrine of final causes." "Mit ,final causes' ist die aristotelische Finalursache gemeint. Dabei wird der Zweck, d. h. die Funktion, als Ursache einer Form gedeutet.

Die anhaltend nicht-geführte Debatte in der Linguistik wäre dieselbe Debatte wie in der Biologie im 18. und 19. Jahrhundert. „The most important and widespread biological debate around the time of Darwin was not evolution versus creation, but biological functionalism versus structuralism.“ (Amundson 1998: 153). Was wurde aus dem biologischen Funktionalismus? Eine historische Episode einer Verirrung.

Linguistische Funktionalisten begehen exakt die gleichen methodischen Fehler. Funktionale Erklärungen sind keine validen Erklärungen. Es sind nicht die kommunikativen Funktionen, die erfordern, dass Grammatiken so sind, wie sie sind. Die sprachlichen Umsetzungen von kommunikativen Funktionen sind so, wie sie sind, weil die Grammatiken sie so ermöglichen, und die Grammatiken sind so, weil die spezifisch-sprachlichen Verarbeitungsroutinen, die das Gehirn zur Verfügung stellt, diese Strukturen begünstigen. Je nach einzelsprachlicher Grammatik können es völlig unterschiedliche Strukturen sein, die für denselben kommunikativen Zweck rekrutiert werden.

Hier ein einfaches Beispiel: Englisch ist die einzige germanische Sprache ohne Passiv von intransitiven Verben. Die CxG betrachtet das als bloße einzelsprachliche Eigenschaft einer Konstruktion. Doch die Passivierbarkeit von intransitiven Verben müsste doch auch im Englischen eine Funktion haben, und dennoch ignoriert die Grammatik das. Wie kann sie das, wenn sie funktionsgetrieben ist?

Wie kam es dazu? Durch einen Unfall. Englisch ist eine SVO-Sprache geworden und diese Sprachen haben, anders als die OV-Sprachen, eine obligate präverbale, strukturelle Subjektsposition, die nicht syntaktisch leer bleiben darf. Fehlt ein Subjekt, muss ein Expletiv her, oder der Satz ist deviant. So ist das in den nahverwandten skandinavischen Sprachen. Englisch aber fehlt ein geeignetes Expletiv, denn die zwei Kandidaten ,there' und ,it' sind bereits seit dem Mittelenglischen anderweitig ${ }^{50}$ vergeben (Haider 2017). Kommunikativer Bedarf hin oder her, die Grammatik bestimmt, was Sache ist, und subjektlose Sätze ohne Expletiv sind in SVO-Sprachen ungrammatisch.

50 ,It ist einerseits für semantische leere Subjekte (z. B. Wetterverben) reserviert und als Korrelat von nachgestellten Sätzen. ,There ' wäre der Kandidat der Wahl, doch im Englischen gibt es ein zwingendes Kongruenzerfordernis (vgl. There has emerged a problem; There have emerged several problems). Somit braucht ,there' stets einen kongruenzfähigen Partner. Den hat es bei Passivierung eines intransitiven Verbs nicht. Mangels Expletiv ist die Konstruktion daher ungrammatisch und somit ausgeschlossen. 
Ein ähnliches Schicksal begleitet die Mehrheit der romanischen Sprachen, nämlich jene mit der Null-Subjekt-Eigenschaft. Davon würde ein pronominales Expletiv geschluckt und daraus erklärt sich folgender Kontrast zwischen beispielsweise Italienisch und anderen romanischen Nullsubjekt-Sprachen einerseits und Französisch andererseits. Im Französischen werden pronominale Subjekte nicht nullifiziert, d. h. als Nullsubjekte behandelt. Es gibt ein Pronomen als Expletiv (26c, d). In den Null-Subjekt-Sprachen wäre ein pronominales Expletiv unentdeckbar, weil Null. Somit sind (26a, b) deviant, (26c, d) aber grammatisch. Im Dialekt des Venezianischen aber findet sich ein Expletiv aus der Lokaladverbklasse, und schon ist intransitives Passiv wieder möglich (26e). So ein Expletiv wird, da Adverbial, von der Null-Subjekt-Eigenschaft nicht tangiert.

(26) a. *[expl] E stato dormito bene in questo letto ist worden geschlafen gut in diesem Bett

b. *[expl] È stato tossito per il fumo ist worden gehustet wegen des Rauchs

c. Il a beaucoup été fumé dans cette salle Gaatone (1998: 124) es ist viel worden geraucht in diesem Raum

d. Il a été dormi dans ce lit Rivière (1981: 42) es ist worden geschlafen in diesem Bett

e. Z'è/Gh'è stà parlà de ti da ist worden gesprochen über dich

Die soeben erwähnten Fakten sollen an diesem exemplarischen Fall veranschaulichen, dass die Grammatik die Herrin des Hauses der Sprache ist, und die kommunikativen Bedürfnisse die Dienste in Anspruch nehmen dürfen, aber sie keineswegs diktieren können. Dafür verrenkt sich keine Grammatik. Eine Grammatik kann Funktionen verweigern und partiell kommunikativdysfunktional erscheinen. In diesem Fall müssen dann eben andere sprachliche Mittel eingesetzt werden. Was sich sagen lässt, lässt sich stets irgendwie sagen (s. Searles „Prinzip der sprachlichen Ausdrückbarkeit“ - „Principle of Expressibility“). ${ }^{51}$

51 Man kann im Englischen (i) oder (ii) zwar nicht direkt ausdrücken, aber man kann es etwas umständlich paraphrasieren: i. Damals wurde gestreikt. ii. Wer hat weshalb gestreikt? (s. Haider 2010: 119). 
Wenden wir uns jetzt kurz ${ }^{52}$ der grundsätzlichen Frage zu. Lässt sich aus Funktionszuschreibungen eine taugliche Kausalerklärung gewinnen? Die Antwort ist ein dezidiertes Nein. Das zentrale Problem ist das der funktionalen Äquivalenz, das andere das von unmöglicher Kausalwirkung aus der Zukunft in die Vergangenheit. Insgesamt gibt es keine valide Schlussfolgerung, die ausgehend von einer zu erfüllenden Funktion zur Entstehung einer grammatischen Eigenschaft führt, mit der diese Funktion umgesetzt wird.

Funktionale Äquivalenz ist im Bereich der Grammatik besonders leicht zu verstehen. Ein und dieselbe Funktion kann, je nach Sprache, mit völlig verschiedenen grammatischen Mitteln erzielt werden. Daher ist klar, dass eine bestimmte Funktion $\mathrm{F}$ das konkrete Mittel $\mathrm{M}_{\mathrm{i}}$ nicht determiniert, denn $\mathrm{F}$ könnte durch irgendeines der funktional äquivalenten Mittel $M_{1}$ bis $M_{n}$ erzielt werden. Die Funktion erklärt das Vorhandensein genau dieses Mittels $M_{i}$ nicht. Die Funktion determiniert $M_{i}$ nicht. Daher liefert sie keine Kausalerklärung für $M_{i}$.

Hier sind die klassischen Stellen, nämlich Hempel (1959: 310) und Nagel (1961: 403) zur Untauglichkeit funktionaler Erklärungen (Cummins 1975: 742): Wenn man behauptet, $\mathrm{M}_{\mathrm{i}}$ sei im System $\mathrm{S}$ als notwendige Bedingung vorhanden, um F zu garantieren, dann ist diese Prämisse empirisch falsch. F könnte auch von einem anderen, funktional äquivalenten Mittel $\mathrm{M}_{\mathrm{j}}$ getragen werden. Nimmt man für das Auftreten von $\mathrm{M}_{\mathrm{i}} \mathrm{F}$ als hinreichende Bedingung an, dann ist die Schlussfolgerung von $\mathrm{F}$ auf die Präsenz von $\mathrm{M}_{\mathrm{i}}$ logisch nicht gültig. Es ist ein abduktiver Schluss und als solcher logisch invalide, denn es ist der bekannte Fehlschluss aufgrund von , affirming the consequent ${ }^{*}{ }^{53}$

Die $\mathrm{CxG}$, die funktionale Typologie und die Linguistik schlechthin haben dasselbe Anliegen wie Biologen vor zweihundert Jahren. Sie möchten die unschwer erkennbaren adaptiven Eigenschaften eines Systems erklären. So wie anfangs die Biologie vermutet man in der $\mathrm{CxG}$, dass die Form durch die Funktion bedingt sei. Das ist ein Fehlschluss, wie Darwin nachdrücklich zeigte. Die Evolution zielt nicht aktiv auf Formverbesserung ab und die Funktion ist nicht kausal für die Formveränderung. ${ }^{54}$ Adaptation ist das Resultat von zufälliger Variation und konstanter, ,blinder' Selektion. Am Ende ist die Form an die Selektionsbedingungen adaptiert, und zwar deshalb, weil alle anderen, konkurrierenden, aber minder günstigen Formen von der Selektion ausgesiebt wurden.

52 Für eine ausführliche Auseinandersetzung sei auf Haider (2015b) verwiesen.

53 Logisch ungültiger Schluss: $[\mathrm{A} \rightarrow \mathrm{B}]$ \& B. Daher A.

54 Wright (1976: 20) charakterisiert teleologische Sichtweisen als anthropomorph und metaphorisch: They „represent dead anthropomorphic metaphors“. Wir sind gut darin, Vorgänge als intentional und zweckgeleitet zu verstehen. Wir sehen Absichten und Zwecke aber auch dort, wo es sie gar nicht gibt. 
Bei menschlichen Sprachen sind die Selektionsbedingungen nicht die Kommunikationsbedingungen. Es sind die Beschränkungen, die das sprachverarbeitende Hirn für die zu verarbeitenden Strukturen setzt, und es ist nicht das kollektive Engineering der Sprachgemeinschaft als unsichtbare Hand (s. Haider 2015b).

Im Handbook of Construction Grammar (Hoffmann \& Trousdale 2013) findet sich auf den fünfhundertsechsundachtzig Seiten keine einzige rechtfertigende Begründung dafür, warum man ein in der etablierten Wissenschaft nach intensiver Deliberation verworfenes Paradigma wiederauferstehen lassen zu dürfen glaubt. Das Register enthält weder „functional explanation“ noch „explanation“. Das wundert, denn Funktion und funktionale Erklärung sind für die CxG zentral. Croft (2013: 210) exponiert sich dazu wie folgt:

The basis of crosslinguistic comparison for grammatical structures must be their function, because of the great structural diversity of languages (the structural properties are essentially language-specific).

und an anderer Stelle:

Most typologists seek functional explanations for language universals. (Croft 2017: 52)

Explanations for language universals are usually functional in character, that is, in terms of how linguistic functions are encoded in linguistic form, in the context of communication and cognitive storage and processing. Because of this mode of explanation, the typological approach is sometimes called the functional-typological approach, and more recently has been subsumed under the more general category of functionalist approaches. (Croft 2009: 146)

Dass strukturelle Eigenschaften ,grundsätzlich“ sprachspezifisch seien, ist schlicht falsch, wie bereits die paar Phänomene, die weiter oben im Kontext OV vs. VO diskutiert wurden, illustrierten. Dass Croft sich irrt, wundert nicht. Wenn man Strukturen danach sortiert, wie sie in Funktionen eingebunden sind, kann man auf diese Idee kommen. Ein und dieselbe Funktion kann mit unterschiedlichsten Mitteln ausgefüllt werden. Dass diese Mittel keine Gemeinsamkeiten haben, darf nicht verwundern. Daraus aber zu folgern, dass es deswegen keine sprachübergreifenden Invarianten bei Strukturen gäbe, das muss verwundern.

Warum sortieren Biologen ,Konstruktionen“ nicht ,analog', d. h. nach Funktionen, sondern ,homolog‘, d.h. nach Strukturen? Bei Ersterem würde beispielsweise die Funktion des Fliegens diverseste Strukturen zusammenwürfeln, nämlich die von Insekten, von Vögeln, von Fledermäusen, Schlangen (Chrysopelea) und Fischen etwa. Was ist die strukturelle Gemeinsamkeit zwischen dem Flügel einer Stubenfliege und dem einer Fledermaus? Lediglich die, 
dass sie völlig unterschiedliche Lösungen für dasselbe physikalische Problem liefern. Die Frage führt zu keinen interessanten Generalisierungen. Die interessante Frage ist, was die strukturelle Gemeinsamkeit zwischen den Fledermausflügeln und den Händen, die diesen Text tippen, ist. Sie entgeht aber dem funktionsfixierten Typologen. Deswegen tun Biologen das nicht, bestimmte Linguisten aber schon. Sie bleiben bloß die Rechtfertigung dieses wissenschaftlich unfundierten Vorgehens schuldig. Konstruktionen penibel zu beschreiben ist eines, und es ist sicher nicht falsch. Diese strukturellen Daten aber mit Funktionen zu verquicken und danach zu sortieren, ist eine Haltung, die gut ins achtzehnte Jahrhundert passt.

Linguistischer Funktionalismus ist häufig auch noch krudester Panglossianismus. $\mathrm{Zu}$ einer Form wird eine Funktionalität hinzuphantasiert und diese flugs zum Grund für die Form gemacht. Wir amüsieren uns über Voltaires Pangloss, der auch noch einen linguistisch passenden Namen verpasst bekommen hat, aber sehen mitunter nicht, dass unsere fachliche Umgebung voller ebenso optimistischer Seelenverwandter ist. So unterhaltsam funktionale Linguistik ist, so untauglich ist sie wissenschaftlich. ${ }^{55}$ Sie kann keine validen Erklärungen liefern, die einer hinreichend strengen wissenschaftlichen Prüfung Stand hielten. Sie liefert keine belastbaren Prädiktionen.

\section{Fazit}

Sowohl MP wie CxG zeichnen sich mehr durch gutes Marketing als durch wissenschaftlich solide Programmatik aus. Sie stehen in einem Anti-Verhältnis zueinander, da sie polare Positionen besetzen. Hier die syntaktischen Algebraisten, die Sprache bevorzugt als Spielmaterial brauchen, dort die funktionsverhafteten Ganzheitler, die glauben, die Komplexität der menschlichen Grammatiken ließe sich im Partikularismus von Form-Funktions-Beschreibungen einfangen. Die einen sind Aristoteliker, die so tun, als ob sie Galileianer wären. Die anderen haben sich, ohne es zu wissen, in eine längst überholte Argumentationswelt, ganz ähnlich jener der Biologie der Rokoko-Zeit, verirrt. Beide zusammen sind hervorragend geeignet, die Grammatiktheorie noch längere Zeit auf der Stelle treten zu lassen.

55 Sogar Friedrich Engels (1896) outete sich als Funktionalist. Die sprachliche Welt und ihre Systeme hätten sich mit einem gewissen Determinismus zum bestmöglichen Zustand hin entwickelt, indem die Bedürfnisse sich ihr sprachliches Organ schufen, wie er das bekanntlich formulierte. 
Der Charme der Konstruktionsgrammatik liegt zum Teil darin, dass sie jedem Clubmitglied eine Spielwiese bietet, für die individuell bevorzugte CxG-Variante, von ,embodied' bis ,radical‘. Man beschreibt ein sprachliches Phänomen möglichst facettenreich und glaubt, damit seine Arbeit getan zu haben, hat man sich doch auch noch bemüht, zu erläutern, wofür man meint, dass die untersuchte Konstruktion gebraucht werde. Das ist sehr ähnlich dem, wie Biologie im siebzehnten und achtzehnten Jahrhundert zu Werke ging, als man die adaptiven Qualitäten in der Tier- und Pflanzenwelt erkannte. Jedes Tierchen ein Plaisierchen, jede Konstruktion eine Sensation.

Der Charme des MP hingegen ergibt sich daraus, dass man eher wenig von sprachübergreifenden Grammatikdaten wissen soll, um umso ungestörter die Freude am Derivationsbasteln generieren zu können. Teils rezykliert man Ergebnisse, die erbracht wurden, als generative Grammatik noch produktiv funktionierte, und fügt gewürzweise noch ein paar Daten hinzu, teils greift man einzelsprachliche Daten heraus und zwingt sie in das derivationelle Schema des Englischen. Wesentlich ist ein Talent für das Aushecken und Nachverfolgen von komplexen Deduktionspfaden.

Beide Paradigmen weisen einen Kardinaldefekt auf. Sie sind steril. Keines war bisher in der Lage, belastbare Gesetzmäßigkeiten aufzudecken, die selbst wieder zum Schlüssel für Entdeckungen im komplexen kognitiven Gebäude von Grammatiken menschlicher Sprachen würden.

Was ist die Lösung? Sie liegt auf der Hand. Will man Wissenschaftler sein, muss man die wissenschaftliche Methode verstehen, respektieren und bestmöglich umsetzen, und die ist ebenso einfach wie anspruchsvoll. Einer der Physik-Nobelpreisträger von 1965 beschreibt sie so:

In general we look for a new law by the following process. First we guess it. Then we compute the consequences of the guess to see what would be implied if this law that we guessed is right. Then we compare the result of the computation to nature, with experiment or experience, compare it directly with observation, to see if it works. If it disagrees with experiment it is wrong. In that simple statement is the key to science.

It does not make any difference how beautiful the guess is. It does not make any difference how smart you are, who made the guess, or what his name is - if it disagrees with experiment it is wrong. That is all there is to it (Feynman 1967: 156)

Uns Linguisten, deren Experimente ja zu einem großen Teil die Natur selbst durchgeführt hat, in Form der Existenz tausender verschiedener Sprachen, schreibt er Folgendes ins Stammbuch:

It is usually said when this is pointed out 'When you are dealing with psychological matters things can't be defined so precisely'. Yes, but then you cannot claim to know anything about it. (Feynman 1967: 159) 
So sieht man das in der Wissenschaft. Man hat die Wahl, Wissenschaft zu betreiben oder in unserer akademischen linguistischen Bubble vergnüglichem, wenn auch von außerhalb völlig zurecht kaum beachtetem Zeitvertreib nachzugehen, und dabei so zu tun, als ob man Wissenschaft betreibe. Richard Feynman hat auch dafür das passende Konzept: cargo cult science. ${ }^{56}$

\section{Literatur}

Amundson, Ron (1998): Typology reconsidered: Two doctrines on the history of evolutionary biology. Biology and Philosophy 13, 153-177.

Bech, Gunnar (1955/1957): Studien zum Deutschen Verbum infinitum. Historisks-filologiske Meddelelser. Bd. 35 (2) und 36 (6) [reprint 1983. Tübingen: Niemeyer].

Bellugi, Ursula, Edward S. Klima \& Gregory Hickok (2010): Brain organization: Clues from deaf signers with left or right hemisphere lesions. In Luis Clara (Hrsg.) Gesture and word, 1-17. Lisbon: Editorial Caminho.

Bellugi Ursula, L. Lichtenberger, W. Jones, Z. Lai \& M. St. George (2000): The neurocognitive profile of Williams Syndrome: a complex pattern of strengths and weaknesses. Journal of Cognitive Neuroscience 12, Suppl 1, 7-29.

Bellugi, Ursula, P. P. Wang \& T. L. Jernigan (1994): Williams syndrome: An unusual neuropsychological profile. In Sarah H. Broman and Jordan Grafman (Hrsg.), Atypical cognitive deficits in developmental disorders: Implications for brain function, 23-56. Hillsdale, NJ: Erlbaum.

Bisiach, Eduardo \& Claudio Luzzatti (1978): Unilateral neglect of representational space. Cortex 14, 129-133.

Chomsky, Noam (1977): On wh-movement. In Peter Culicover, Tom Wasow and Adrian Akmajian (Hrsg.), Formal syntax, 71-132. New York: Academic Press.

Chomsky, Noam (1995a): The Minimalist Program. Cambridge MA: MIT Press.

Chomsky, Noam (1995b): Bare phrase structure. In Gert Webelhuth (Hrsg.), Government and binding theory and the Minimalist Program, 383-439. Oxford: Blackwell.

Churchland, Patricia Smith (1986): Neurophilosophy: Toward a unified science of the mindbrain. Cambridge, MA: MIT Press.

Croft, William (2009): Methods for finding language universals in syntax. In Sergio Scalise, Elisabetta Magni, Antonietta Bisetto (Hrsg.), Universals of language today, 145-164. New York: Springer.

Croft, William (2013): Radical construction grammar. In Hoffmann \& Trousdale (Hrsg.), 211-232.

Croft, William (2017): Typology and universals. In Mark Aronoff and Janie Rees-Miller (Hrsg.), The handbook of linguistics. 2nd Edition. Hoboken, NJ: John Wiley.

Cummins, Robert (1975): Functional analysis. The Journal of Philosophy 72, 741-765.

56 calteches.library.caltech.edu/51/2/CargoCult.htm (letzter Zugriff: 8.11. 2017) [Engineering and Science, Volume 37 (7), June 1974]. 
Darwin, Charles (1871): The descent of man, and selection in relation with sex. London: John Murray, Albemarle Street.

Dehé, Nicole (2004): On the order of objects in Icelandic double object constructions. UCL Working Papers in Linguistics 16, 85-108.

Dryer, Matthew S. (2009): The branching direction theory of word order correlations revisited. In Sergio Scalise, Elisabetta Magni, Antonietta Bisetto (Hrsg.), Universals of language today, 185-207. Berlin: Springer.

Edelman, Shimon \& Morten H. Christiansen (2003): How seriously should we take Minimalist syntax? Trends in Cognitive Science 7 (2), 60-61.

Engels, Friedrich (1896) (postum): Dialektik der Natur. Anteil der Arbeit an der Menschwerdung des Affen. In: Karl Marx \& Friedrich Engels. Werke. Bd. 20, 444-454. Berlin: Dietz.

Erdmann, Oskar (1886): Grundzüge der deutschen Syntax nach ihrer geschichtlichen Entwicklung. Stuttgart: Cotta.

Feynman, Richard (1967): The character of physical law. Cambridge MA: MIT Press.

Fillmore, Charles \& Paul Kay (1999): Construction grammar coursebook. Ling X20. Berkeley: University of California.

Frazier, Lyn (2015): Two interpretive systems for natural language? Journal of Psycholinguistic Research 44(1), 7-25. doi: 10.1007/s10936-014-9328-0

Gaatone, David (1998): Le passif en français. Paris/Bruxelles: Duculot.

Goldberg, Adele (1995): Constructions: A construction grammar approach to argument structure. Chicago: University of Chicago Press.

Goldberg, Adele (2003): Constructions: A new theoretical approach to language. Trends in Cognitive Sciences 7 (5), 219-224.

Goldberg, Adele (2006): Constructions at work. The nature of generalization in language. Oxford: Oxford University Press.

Goldberg, Adele (2013): Constructionist approaches. In Hoffmann \& Trousdale (Hrsg.), 15-31.

Gordon, Barry (1985): A minimal neurolinguistic model and its lessons. In Michael J. Ackerman (Hrsg.) Proceedings of the Ninth Annual Symposium on Computer Applications in Medical Care, 248-251. Washington, D.C.: IEEE Computer Society Press.

Goswami, Usha (Hrsg.) (2011): The Wiley-Blackwell handbook of childhood cognitive development (2nd ed.). Hoboken, NJ: John Wiley \& Sons.

Guasti, Maria Teresa (2002): Language acquisition. The growth of grammar. Cambridge, MA: MIT Press.

Haider, Hubert (2010): The syntax of German. Cambridge: Cambridge University Press.

Haider, Hubert (2011): Grammatische Illusionen. Zeitschrift für Sprachwissenschaft 30, 223-257.

Haider, Hubert (2013): Symmetry breaking in syntax. Cambridge: Cambridge University Press. Haider, Hubert (2015a): Head directionality - in syntax and morphology. In Antonio Fábregas, Jaume Mateu, Mike Putnam (Hrsg.) Contemporary linguistic parameters, 73-97. London: Bloomsbury Academic.

Haider, Hubert (2015b): "Intelligent design" of grammars - a result of cognitive evolution. In Aria Adli, Marco García García \& Göz Kaufmann (Hrsg.): Variation in language: Systemand usage-based approaches, 205-240. Berlin/New York: De Gruyter.

Haider, Hubert (2016): Scientific ideology in grammar theory. Mskr. Univ. Salzburg. [downloadable: https://www.academia.edu/20284524/Scientific_ideology_in_grammar_ theory_rev.v._April_17_2016_ (letzter Zugriff: 8.11. 2017)] 
Haider, Hubert (2017): In the absence of a subject. In Clemens Mayr \& Edwin Williams (Hrsg.) Festschrift für Martin Prinzhorn (wlg edition Nr. 82). 87-98. Wien: Institut für Sprachwissenschaft.

Hempel, Carl (1959): The logic of functional analysis. In Llewellyn Gross (Hrsg.) Symposium on sociological theory, 271-307. New York: Harper \& Row.

Hornstein, Norbert (2000): Move! A Minimalist theory of construal. Oxford: Wiley-Blackwell.

Hoffmann, Thomas \& Graeme Trousdale (Hrsg.) (2013): The Oxford handbook of construction grammar. Oxford University Press.

Jespersen, Otto (1894): Progress in language; with special reference to English. London: Swan Sonnenschein \& Co.

Kayne, Richard (1994): The antisymmetry of syntax. Cambridge MA: MIT Press.

Koster, Jan \& Robert May (1982): On the constituency of infinitives. Language 58 (1), 116-143.

Lakatos, Imre (1978): The methodology of scientific research programmes: Philosophical papers. Vol. 1. Cambridge: Cambridge University Press.

Liebermann, Philip (2007): The hermetic nature of linguistic research. The Linguistic Review 24, 431-435.

Mallinson, Graham \& Barry J. Blake (1981): Language typology: Cross-linguistic studies in syntax. (North-Holland Linguistic Series 46). Amsterdam: North-Holland.

Mayr, Ernest (1982): The growth of biological thought. Cambridge, MA: Harvard University Press:

Medawar, Peter B. (1979): Advice to a young scientist. New York: Basic Books.

Meltzoff, Andrew N. (2011): Social cognition and the origins of imitation, empathy, and theory of mind. In Goswami (2011), 49-75.

Nagel, Ernest (1961): The structure of science: Problems in the logic of scientific explanation. New York/Burlingame: Harcourt, Brace and World Inc.

Paul, Hermann (1919): Deutsche Grammatik. Vol. III, Teil IV: Syntax. Halle an der Saale: Niemeyer.

Perfetti, Charles A. (1981): Review of 'Rules and Representations' by Noam Chomsky. Philosophy of Science 48 (1), 153-154.

Rice, Mabel L. (2016): Specific language impairment, nonverbal IQ, attention-deficit/ hyperactivity disorder, autism spectrum disorder, cochlear implants, bilingualism, and dialectal variants: Defining the boundaries, clarifying clinical conditions, and sorting out causes. Journal of Speech, Language and Hearing Research 59 (1), 122-132.

Rivière, Nicole (1981): La construction impersonnelle en français contemporain. Documents de Linguistique Quantitative no. 41. St. Sulpice-de-Favière: Éditions Jean-Favard.

Rosenbaum, Peter (1970): A principle governing deletion in English sentential complementation. In Roderick Jacobs \& Peter Rosenbaum (Hrsg.), Readings in English transformational grammar, 20-29. Waltham, MA: Ginn.

Schaner-Wolles, Chris (2004): Spared domain-specific cognitive capacities? Syntax and morphology in Williams syndrome and Down syndrome. In Susanne Bartke \& Julia Siegmüller (Hrsg.), Williams syndrome across Languages, 93-124. Amsterdam: Benjamins.

Schmidt, Andreas (2016): Udmurt as an OV language and Finnish as a VO language. Masterarbeit am Institut für Linguistik der Univ. Potsdam.

Szucsich, Luka \& Hubert Haider (2015): Freie Wortstellung in slavischen Sprachen und die Vo/OV-Unterscheidung. In Elena Dieser (Hrsg.), Linguistische Beiträge zur Slavistik: 
XX. JungslavistInnen-Treffen in Würzburg, 22.-24. September 2011, 94-124. München: Otto Sagner.

Whaley, Lindsay (1997): Introduction to typology: The unity and diversity of language. Thousand Oaks, CA: Sage Publications.

White, Lydia (1989): Universal grammar and second language acquisition. Amsterdam: Benjamins.

Wright, Larry (1976): Teleological explanation. Berkeley: University of California Press. 
II Sprach- und Grammatikmodelle 



\title{
3 Intensionen, Typen und Modelle
}

\author{
Bemerkungen zu einigen Entwicklungen \\ in der formalen Semantik
}

\begin{abstract}
Dieser Beitrag wirft einen Blick auf drei zentrale Begriffe der formalen Semantik und einige weniger offensichtliche Veränderungen, denen sie seit Montagues English as a Formal Language unterworfen waren: Intensionen, Typen und Modelle.
\end{abstract}

Keywords: formale Semantik, Intensionen, Modelle, Montaguegrammatik, Typen

Am Nachmittag des 15. Oktober 1968 hielt Richard Montague einen Vortrag auf der Mailänder Tagung Linguaggi nella società e nella tecnica, der später zum ersten seiner drei klassischen Aufsätze zur Semantik der natürlichen Sprache werden sollte: Montague (1970a). Auch wenn die Formulierung der allgemeinen Theorie der (später so genannten) Montaguegrammatik noch bis Montague (1970b) warten musste und die breitere linguistische Öffentlichkeit vor ihrer Popularisierung in Partee (1975) kaum Notiz von ihr nahm, waren die Fundamente der modernen kompositionellen Semantik mit dem Erscheinen von English as a Formal Language gelegt. Und in den 50 Jahren, die seither vergangen sind, hat sich das Gebiet - Montagues eigener (gelinde gesagt) skeptischer Einstellung zur generativen Grammatik zum Trotz - fest in der Linguistik etabliert und eine wachsende Gemeinschaft von WissenschaftlerInnen hervorgebracht, die es hinsichtlich deskriptiver Breite wie auch theoretischer Tiefe weit über die mathematischen und philosophischen Anfänge ausgedehnt und die formale Semantik zu einer reifen empirischen Disziplin gemacht haben. Dennoch sind die Spuren der frühen Montaguegrammatik bis in die gegenwärtige Forschung hinein unübersehbar: man kann ohne Übertreibung sagen, dass die

\footnotetext{
Anmerkung: Dank gebührt Ramona Hiller, Jan Köpping und Johannes Wolf für Hilfe mit der Erstellung der LaTeX-Datei und Ivano Caponigro für die Angabe des genauen Datums von Montagues Mailänder Vortrag von English as a Formal Language.
}

Thomas Ede Zimmermann, Goethe-Universität Frankfurt, Institut für Linguistik, NorbertWollheim-Platz 1, D-60323 Frankfurt, E-Mail: T.E.Zimmermann@lingua.uni-frankfurt.de

Ә Open Access. (C) 2018 Thomas Ede Zimmermann, publiziert von De Gruyter. (c) BY Dieses Werk ist lizenziert unter der Creative Commons Attribution 4.0 Lizenz. 
derzeit einflussreichsten formalsemantischen Theorien die Kernmerkmale des Montagueschen Ansatzes bewahrt haben. Insbesondere wird das gesamte Gebiet der formalen Semantik üblicherweise als modelltheoretisch, typenlogisch und/oder intensional charakterisiert. Dabei haben die Rollen, der Intensionen, Typen und Modelle auf dem Weg von Montagues Schriften zur Gegenwartssemantik eine Reihe von subtilen, aber bemerkenswerten Veränderungen durchgemacht. Der vorliegende Beitrag wirft einen genaueren (und zugegebenermaßen subjektiven) Blick auf einige der weniger augenfälligen Aspekte dieser Entwicklung.

\section{Intensionen}

Die Gesamt-Architektur von Montagues Theorie der formalen Semantik ist vor allem Freges (1892) Sprachphilosophie verpflichtet und insbesondere seiner Unterscheidung zwischen Sinn und Bedeutung. Insofern mag es überraschen, dass das Fragment in Montague (1970a) auf einer einschichtigen Deutung von Denotationen basierte. Und tatsächlich nimmt Montagues (1970a: 218f.) Bemerkung (xi) zu seinem Fragment mit dem Hinweis auf die schiere Möglichkeit einer kompositionellen Deutung des Englischen ohne nicht-propositionale Intensionen ein Stück weit Kaplans (1975) systematische Reduktion der zweischichtigen Fregeschen auf eine einschichtige ,Russellsche“ Architektur vorweg. Doch in seiner in Montague (1970b) dargestellten allgemeinen Referenztheorie steht die Sinn-und-Bedeutungs-Architektur wieder im Mittelpunkt, wie die Benennung der zentralen Konstruktion seines modelltheoretischen Ansatzes belegt: Fregean interpretation. Dieser Umschwung mag auf dem vorgeblichen Parallelismus zwischen natürlicher Sprache und mathematischer Logik gegründet sein; vielleicht spiegelt er auch bereits die angebliche Unvermeidbarkeit von Individualbegriffen als nicht-propositionale Intensionen wider, auf die in Montague (1973) angespielt wird. Auf jeden Fall hat der Großteil der semantischen Gemeinde - mit prominenten Ausnahmen wie Cresswell (1973) offenbar Montagues Fregeschen Turn begrüßt und in der Folge die Unterscheidung zwischen Sinn und Bedeutung akzeptiert - oder genauer: die zwischen Extension und Intension. Denn in der Montaguegrammatik wird - wohl aufgrund einer in Montague (1969: 165) zum Ausdruck gebrachten allgemeinen theoretischen Präferenz - die Rolle von Freges semantischen Werten Carnaps Extensionen und Intensionen zugewiesen. Genauer gesagt wird Kripkes Mögliche-Welten-Semantik der Modallogik im Geiste von Montague (1968) adaptiert und verallgemeinert, um Carnapsche Extensionen und Intensionen zu re-modellieren. Ungeachtet der Vorbehalte Carnaps (1947: §29) gegen eine 
Identifikation von Intensionen mit Sinnen sah Montague offenbar genügend Gemeinsamkeiten zwischen den beiden Vorgehensweisen in der semantischen Analyse, um die Carnapsche Unterscheidung als eine Variante - oder gar eine mengentheoretische Rekonstruktion - der Fregeschen $\mathrm{zu}$ präsentieren. Insbesondere werden beide mit derselben heuristischen Strategie bestimmt:

- Die Extensionen von Sätzen und Individualtermen stimmen mit ihren (Fregeschen) Referenten (,Bedeutungen') überein.

- Häufig kann die Kompositionalität herangezogen werden, um immer komplexere funktionale Extensionen zu konstruieren, die auf einfachere Extensionen angewandt werden.

- Wann immer sich Extensionen nicht kompositionell verhalten, werden sie durch entsprechende Intensionen ersetzt.

Der erste Schritt führt, in der inzwischen gängigen Notation aus Montague (1970b), zu der Identifikation der Basis-Extensionen der Typen e und t. Durch Anwendung des zweiten Strategems entstehen funktionale Extensionen als Lösungen semantischer Gleichungen per Funktionalapplikation. So sollte etwa die Extension des Satzes Maria schläft und die seiner unmittelbaren Konstituenten - des Namens Maria an Subjektstelle und des Prädikats schläft - die folgende Kompositionalitätsgleichung erfüllen:

(1) $\mid$ Maria schläft $\mid=F(\mid$ Maria $|$,$| schläft \mid)$

wobei ,|...|' eine Abkürzung für ,die Extension von ...' und $F$ eine geeignete kompositionelle Operation ist, die die Extensionen von Subjekt und Prädikat kombiniert, um die Extension des resultierenden Satzes zu bilden. Da letztere der Wahrheitswert von Maria schläft ist und außerdem als Extension des Subjekts der Träger des Namens Maria fungiert, läuft (1) hinaus auf:

(2) $\vdash$ Maria schläft $\dashv=F(\mathrm{~m}, \mid$ schläft $\mid)$

wobei , $\vdash \ldots \dashv^{\natural} \mathrm{zu}$ lesen ist als: ,derjenige Wahrheitswert, der 1 ist gdw. ... Die Betrachtung ähnlicher Gleichungen mit anderen Namen $N$ an Subjektstelle ergibt, dass der Wahrheitswert des resultierenden Satzes systematisch davon abhängt, wer oder was Träger des Namens $N$ ist; ersterer ist genau dann 1 , wenn letzterer schläft:

(3) $\mid N$ schläft $\mid=F(|N|$,|schläft $\mid)$

Freges (1891) Heuristik zufolge kann nun (3) verwendet werden, um die Extension des Prädikats zu identifizieren als eine Vergegenständlichung der Exten- 
sion des Mutterknotens - des Wahrheitswerts v - von der Extension des Schwesterknotens des Prädikats - des Namensträgers x:

(4) $|\operatorname{schläft~}|(x)=\vdash x$ schläft $\dashv$

Da die Gleichung (4) für beliebige Individuen x gilt, kann sie als punktweise Definition der Extension von schläft als einer Funktion des Typs 〈e,t $\rangle$ aufgefasst werden. Auf Basis von (4) kann nun die kompositionelle Gleichung (3) gelöst werden, indem $F$ mit der Operation APP der Funktionalapplikation gleichgesetzt wird:

$$
\begin{aligned}
& \mid N \text { schläft } \mid \\
= & A P P(\mid \text { schläft }|,| N \mid) \\
= & \mid \text { schläft } \mid(|N|) \\
= & \vdash \mathrm{N} \text { schläft } \dashv
\end{aligned}
$$

Ausgehend von (4) und (5) lässt sich die gleiche Prozedur wiederholen, um weitere Extensionen von immer komplexeren Typen hervorzubringen. Insbesondere erhalten auf diese Weise transitive Verben und quantifikationelle nominale Extensionen die Typen $\langle e,\langle e, t\rangle\rangle$ bzw. $\langle\langle e, t\rangle, t\rangle$; darum geht es in den Kapiteln 2 und 6 von Heim \& Kratzer (1998). Und die Prozedur lässt sich auch verwenden, um zuvor gefundene Spezifikationen von Extensionen zu revidieren - etwa aus Einheitlichkeitsgründen; ein typisches Beispiel dafür ist Montagues (1970b) Behandlung der Eigennamen, auf die wir noch zurückkommen werden:

(6) $|N|(|P|)=|P|(\mathrm{x})$

wobei x der Träger des Namens $N$ ist, den (6) also von $N$ s Extension unterscheidet, in teilweisem Konflikt mit Freges erstem Strategem.

Nebenbei sei erwähnt, dass sich die (3) zugrunde liegende Kompositionalitätsannahme nicht allein unter Berufung auf Lernbarkeit rechtfertigen lässt; denn Sprecher kennen im allgemeinen nicht die Extensionen aller Subjekte, und schon gar nicht die Wahrheitswerte aller Prädikationen. Allerdings garantiert in einem an Carnap orientierten Rahmen die extensionale Kompositionalität, dass sich auch Intensionen kompositionell verhalten; und die Kenntnis der Intensionen ist plausiblerweise eine Sache der sprachlichen Kompetenz - sieht man einmal von Putnams (1975) Bedenken ab. Genauer gesagt lässt sich (5) zu (7) verallgemeinern, wenn die Intension $\llbracket X \rrbracket$ eines Ausdrucks $X$ als diejenige 
Funktion definiert wird, die jedem Welt-Zeit-Paar (oder Index) $\langle w, t\rangle$ die Extension $\llbracket X \rrbracket^{w, t}$ von $X$ unter den Umständen $w$ und zur Zeit $t$ zuweist:

$$
\begin{aligned}
& \llbracket N \text { schläft } \rrbracket^{w, t}=A P P\left(\llbracket \text { schläft } \rrbracket^{w, t}, \llbracket N \rrbracket^{w, t}\right) \\
= & \llbracket \text { schläft } \rrbracket^{w, t}\left(\llbracket N \rrbracket^{w, t}\right) \\
= & \vdash N \text { schläft in } w \text { zu } t \dashv
\end{aligned}
$$

wobei $w$ und $t$ beliebige mögliche Welten bzw. Zeitpunkte sind. Infolgedessen lässt sich die erste Zeile von (7) als punktweise Spezifikation der Intension der Prädikation auf Basis der Intensionen ihrer unmittelbaren Teile verstehen:

$$
\llbracket N \text { schläft } \rrbracket(w, t)=A P P(\llbracket \operatorname{schläft~} \rrbracket(w, t), \llbracket N \rrbracket(w, t))
$$

Auf ähnliche Weise zeigt man nun, dass jegliche kompositionelle Kombination von Extensionen zu intensionaler Kompositionalität führt. Dabei ist zu beachten, dass die Argumentation wesentlich von der mengentheoretischen Beschaffenheit der Carnapschen Intensionen abhängt, die sich vollständig auf die oben angedeutete punktweise Charakterisierung festlegen lassen. Es ist (zumindest mir) nicht klar, ob ein feinkörnigerer Zugang zur kompositionellen Bedeutung auf Grundlage Fregescher Sinne intensionale Kompositionalität ebenso als Folge extensionaler Kompositionalität garantieren kann.

Nicht immer verhalten sich die mit der skizzierten Fregeschen Heuristik bestimmten Extensionen kompositionell. Wo sie es nicht tun, kommt Freges drittes Strategem zu Hilfe. Ein einschlägiges (englisches) Beispiel stammt aus Montague (1970b: 396):

(9) a. Jones seeks a unicorn.

b. Jones seeks a horse such that it speaks.

Da es weder Einhörner noch sprechende Pferde gibt, ist die Extension des lexikalischen Nomens unicorn die leere Menge (bzw. ihre charakteristische Funktion); dasselbe gilt für die Extension des komplexen Nomens horse such that it speaks. Angesichts dieser extensionalen Äquivalenz müssten die beiden Sätze in (9) material äquivalent sein, wenn sich Extensionen kompositionell verhielten. Die Tatsache, dass (9a) und (9b) (in der wirklichen Welt, zum Zeitpunkt des Verfassens) nicht denselben Wahrheitswert zu haben brauchen, zeigt, dass die extensionale Kompositionalität in diesem Fall versagt. Um die Extension der Prädikate in (9) zu bestimmen, reicht es also nicht, die Extensionen von Verb und Objekt zu kombinieren. Natürlich mag die Kompositionalität als sol- 
che noch zutreffen, aber eben nicht auf der Ebene der Extensionen. Statt eines Rückzugs auf die Intensions-Ebene bietet nun allerdings die Fregesche Heuristik eine lokale Reparatur-Strategie an: schließlich widersetzen sich von den beiden Konstituenten, die die Prädikate in (9) bilden, nur die (direkten) Objekte einer Substitution salva veritate; Freges drittem Strategem zufolge kann dann die Extension des Prädikats immer noch aufgrund der Extension des Verbs seek bestimmt werden (was immer diese Extension sein mag), solange das Objekt seine Intension beiträgt. Schreiben wir wieder $F$ für die (noch unbekannte) Operation, die das (opake) Verb mit seinem (indefiniten) Objekt verbindet, können wir also die semantische Gleichung (10), die Anlass zu Substitutionsproblemen gab, durch die vorsichtigere Formulierung (11) ablösen:

$$
\llbracket \text { seek a unicorn } \rrbracket^{w, t}=F\left(\llbracket \text { seek } \rrbracket^{w, t}, \llbracket \text { a unicorn } \rrbracket^{w, t}\right)
$$

$$
\llbracket \text { seek a unicorn } \rrbracket^{w, t}=F\left(\llbracket \text { seek } \rrbracket^{w, t}, \llbracket \text { a unicorn } \rrbracket\right)
$$

Auch wenn (11) die Substitutionsprobleme vermeidet, in die sich (10) verstrickt, liefert die Gleichung natürlich keine Erklärung dafür, wie es $F$ gelingt, die Intension des direkten Objekts und die des transitiven Verbs so zu kombinieren, dass sich die Menge der Einhorn-Suchenden (bzw. die charakteristische Funktion dieser Menge) ergibt. Fürs erste halten wir jedoch fest, dass mit einem Argument im Stil von (8) gezeigt werden kann, dass Analysen intensionaler Konstruktionen im Stil von (11) - genau wie die extensionalen Konstruktionen auch intensionale Kompositionalität garantieren. Zudem überträgt sich die Fregesche Identifikation unbekannter Extensionen mit funktionalen Lösungen kompositioneller Gleichungen ebenfalls auf intensionale Konstruktionen. Im vorliegenden Fall ist das transitive Verb seek betroffen, dessen Extension zunächst recht mysteriös anmutet. Doch wie der Übergang von (3) zu (4) zeigt, kann $\llbracket s e e k \rrbracket^{w, t}$ mit einer Funktion gleichgesetzt werden, die Intensionen quantifzierender Nominale Prädikatsextensionen zuweist, so dass sich das $F$ in (11) als Intensionale Funktionalapplikation im Sinne von Heim \& Kratzer (1998: 308) entpuppt:

$$
\text { (12) } \llbracket \text { seek a unicorn } \rrbracket^{w, t}=\llbracket \operatorname{seek} \rrbracket^{w, t}(\llbracket \text { a unicorn } \rrbracket)
$$

Doch auch (12) erklärt nicht, wie genau die Funktion $\llbracket s e e k \rrbracket^{w, t}$ es bewerkstelligt, vermittels ihres Inputs die Menge der Einhorn-Suchenden zu bestimmen: die durch das $F$ in (11) gerissene Erklärungslücke wird lediglich ins Lexikon verschoben; in Abschnitt 3 werden wir auf sie zurückkommen.

Die Analyse in (12) setzt voraus, dass die Extension von seek eine Funktion ist, die Intensionen (quantifikationeller Nominale) als Argumente nimmt, und 
verlangt daher nach einer Erweiterung des Typensystems: irgendwie muss es Intensionen gestattet werden, in die kompositionelle Maschinerie der Extensionen einzudringen. Für die gegenwärtigen Zwecke würde es genügen, für jeden funktionalen Typ der Gestalt $\langle a, b\rangle$ einen entsprechenden Substitutionsresistenten Typ $\langle\langle s, a\rangle, b\rangle$ hinzuzufügen, wobei - wiederum in Montagues (1970b) Notation - das , s` an (Fregeschen) ,Sinn` erinnert und (Carnapsche) ,Intension“ meint. Doch wäre das kaum genug. Zum einen ist nämlich IntensionsSensititvität keineswegs auf die (strukturell) höchsten Argumente funktionaler Extensionen beschränkt, sondern kann ebenso gut etwa sowohl das direkte als auch das indirekte Objekt betreffen - wie möglicherweise im Fall des ditransitiven Verbs owe; vgl. Zimmermann (2005: 223 f.). Zum anderen können Intensions-sensitive Operatoren, zu denen traditionell Einstellungsverben wie glauben und Satzadverbien wie möglicherweise zählen, selbst wieder Argumente von Modifikatoren sein, womit eine entsprechend tiefere Einbettung der s-Markierung notwendig wird. Von daher ist eine flexiblere Erweiterung der Hierarchie kompositioneller Extensionen unvermeidlich. Es gibt verschiedene Wege, dies zu erreichen; die populärsten sind die folgenden beiden:

a) Auf Basis der Grundtypen e und t, werden immer wieder neue Typen geschaffen, indem man entweder (wie zuvor) Paare bildet - von gegebenen Typen a und b zum funktionalen Typ $\langle\mathrm{a}, \mathrm{b}\rangle$ - oder ihnen ein , $\mathrm{s}^{\mathbf{6}}$ hinzufügt: von gegebenen Typen a zum intensionalen Typ $\langle\mathrm{s}, \mathrm{a}\rangle$.

b) In einem allgemeineren System fügt man s als dritten Grundtyp (neben e und t) hinzu und behält die allgemeine Regel, dass Typen unter Paarbildung abgeschlossen sind: von gegebenen Typen a und b zum funktionalen Typ $\langle\mathrm{a}, \mathrm{b}\rangle$.

Der Unterschied zwischen den beiden Systemen zeigt sich in der Tatsache, dass b) neben s selbst auch $\langle s, s\rangle,\langle t, s\rangle$, etc. als Extensionstypen zulässt. Während Montague (1970b) Option a) wählt, entscheiden sich die meisten modernen typenbasierten semantischen Ansätze für b), oft unter Hinweis auf Gallin (1975), der (in Anlehnung an prädikatenlogische Standard-Terminologie) vom System der zweisortigen Typen spricht. Man beachte, dass nach b) intensionale Typen als Spezialfälle funktionaler Typen behandelt werden. Und obwohl in beiden obigen Typensystemen , $s$ für Indizes steht (die üblicherweise mit Welt-ZeitPunkten identifiziert werden), werden diese nur nach b) als Extensionen sui generis behandelt - als Referenten und nicht nur als Referenzpunkte, wie Montague (1970b) (in etwa) gesagt hat. Doch auch Montagues (1970b) sparsameres Typensystem umfasst eine sog. Intensionen-Hierarchie: für jeden Montagueschen Typ a gibt es einen Typ $\langle\mathrm{s}, \mathrm{a}\rangle$ - und folglich auch die Typen $\langle\mathrm{s},\langle\mathrm{s}, \mathrm{a}\rangle\rangle$, 
$\langle\mathrm{s},\langle\mathrm{s},\langle\mathrm{s}, \mathrm{a}\rangle\rangle\rangle$, etc. pp. Obwohl an sich kaum problematisch - schließlich könnten alle Objekte, Intensionen eingeschlossen, über den Logischen Raum hinweg variieren - werden die höheren Stufen der Hierarchie in der kompositionellen Semantik kaum jemals aktiviert - wobei Groenendijk \& Stokhofs (1982) Analyse der indirekten Fragen als (Äquivalenz-) Relationen des Typs $\langle\mathrm{s},\langle\mathrm{s}, \mathrm{t}\rangle\rangle$ eine bemerkenswerte Ausnahme darstellt.

So elegant und mächtig das sogenannte zweisortige System auch sein mag, so wenig verträgt es sich mit der Frege-Carnap-Montague-Doktrin der Extensionen und Intensionen, wonach letztere lediglich als Ersatzextensionen im Kompositionalitätsprozess auftreten. In der semantischen Literatur wurde jedoch verschiedentlich argumentiert, dass mehr Flexibilität benötigt wird, als die o. g. lokalen Reparaturen gestatten, bei denen Intensionen nur bemüht werden, um Substitutionsprobleme zu lösen. Das folgende, aus Bäuerle (1983) adaptierte Beispiel ist ein typischer Beleg:

(13) Hans berichtet, dass Maria denkt, dass jedes Mitglied der Fußballmannschaft rothaarig ist.

Der Satz (13) besitzt eine Lesart, nach der er wahr ist (in einer Welt, zu einer Zeit), wenn Marias Glauben eine Gruppe von Personen betrifft, die Hans, aber und das ist der Punkt - nicht Maria, als Fußballmannschaft identifiziert, ob zu Recht oder zu Unrecht. Einer (leicht vereinfachten) Frege-Carnap-Analyse zufolge und unter Vernachlässigung des Zeitparameters bei gleichzeitiger Identifikation von Mengen mit ihren charakteristischen Funktionen, ist demnach der Beitrag, den der zuoberst eingebettete Nebensatz zur Extension (in einer Welt $w_{0}$ ) leistet, die Menge der möglichen Welten $w$, für die gilt:

$$
\llbracket \text { denk- } \rrbracket{ }^{w}\left(\left\{w^{\prime} \mid \llbracket \text { jed- } \rrbracket^{w^{\prime}}\left(\llbracket \mathrm{MdF} \rrbracket^{w}\right)\left(\llbracket \text { rothaarig } \rrbracket^{w^{\prime}}\right)=1\right\}\right)(\mathrm{m})=1
$$

Das Problem besteht nun darin, dass das Argument der Einstellung «denk- $\rrbracket^{w}$ (gegeben eine Welt $w$ ) nicht die Proposition (15a) ist, die der eingebettete Satz ausdrückt, sondern vielmehr die Menge (15b) - wobei ein einziger Akzent den ganzen Unterschied ausmacht:
a. $\quad\left\{w^{\prime} \mid \llbracket\right.$ jed- $\rrbracket^{w^{\prime}}\left(\llbracket \mathrm{MdF} \rrbracket^{w^{\prime}}\right)\left(\llbracket\right.$ rothaarig $\left.\left.\rrbracket^{w^{\prime}}\right)=1\right\}$
b. $\quad\left\{w^{\prime} \mid \llbracket\right.$ jed $-\rrbracket^{w^{\prime}}\left(\llbracket \mathrm{MdF} \rrbracket^{w}\right)\left(\llbracket\right.$ rothaarig $\left.\left.\rrbracket^{w^{\prime}}\right)=1\right\}$

Natürlich besitzt (13) auch eine ,de dicto'-Lesart, derzufolge der kompositionelle Beitrag des betreffenden Nebensatzes tatsächlich (15a) wäre - aber das ist nicht die Lesart, um die es Bäuerle geht. Um letztere auf kompositionelle Weise her- 
zuleiten, muss offenbar der eingebettete Nebensatz - anstelle seiner gewöhnlichen Intension (15a) - die Menge (15b) der Extension von denk- als Argument zuführen dürfen. Bäuerle (1983) schließt, dass dafür ein flexibleres Framework als die Theorie der Frege-Carnap-Intensionen benötigt wird: anstatt Extensionen von einem einzigen Index abhängen $\mathrm{zu}$ lassen, ist gegebenenfalls eine ganze Latte von Indizes vonnöten, um den komposionellen Beitrag eines Ausdrucks in einer nicht-extensionalen Umgebung zu bestimmen. Seither wurden eine Reihe weiterer Konstellationen gefunden, die ebenfalls für eine derartige multiple Index-Abhängigkeit sprechen und sogar als Beleg dafür angeführt wurden, dass die korrekte Analyse intensionaler Konstruktionen einen ähnlich komplexen Apparat von Referenz und Quantifikation erfordert wie er traditionell für den nominalen Bereich angenommen wird; mehr zu dieser Perspektive findet man in Cresswell (1990), Percus (2000) und Schlenker (2006).

Dennoch sind Konstellationen wie die in (14) nicht zwangsläufig das Ende von Montagues (1970b) Geschichte von der Theorie von Extension und Intension. Prinzipiell bieten sich sogar gleich zwei alternative Techniken an, um Frege-Carnap-Montaguesche Orthodoxie mit multipler Index-Abhängigkeit zu vereinbaren:

(i) Es wird Substantiven und anderen Konstituenten gestattet, auf LF intensionalen Skopus zu nehmen.

(ii) In intensionalen Umgebungen dürfen kompositionelle Beiträge höher auf der Intensions-Hierarchie angesiedelt sein.

Hinter Option (i) steckt die Idee, Montagues (1973) Behandlung von Quantorenambiguitäten $\mathrm{zu}$ verallgemeinern, um insbesondere Substantiven flexiblen Skopus zu geben, indem sie (generativ gesprochen) auf LF angehoben werden können - wie in der Struktur (16), auf deren Basis Bäuerles (1983) Lesart von (13) mit Hilfe gängiger Interpretationsmechanismen hergeleitet werden kann (wenn auch nicht hier):

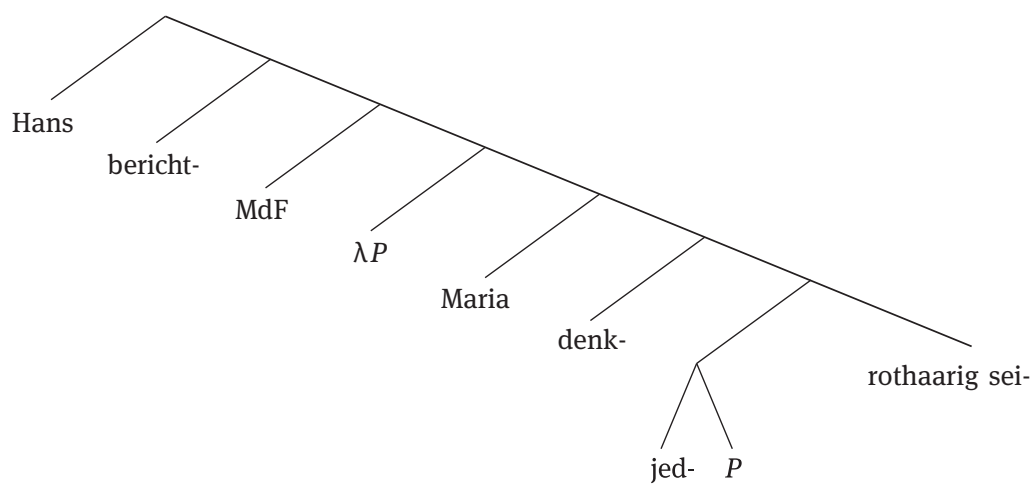


Analysen wie (16) wurden in Groenendijk \& Stokhof (1982: 199f.) entwickelt, um Ambiguitäten in indirekten Konstituentenfragen (mit which) zu erklären; aus Platz- und Lesbarkeitsgründen werden in der obigen Formulierung Konventionen der LF-Konstruktion nach Heim \& Kratzer (1998) verwendet.

Option (ii) geht auf eine Idee zurück, die häufig Frege (1892) zugeschrieben wird - vgl. allerdings Parsons (1981): multiple intensionale Einbettung verlangt indirekte Sinne. Genauer gesagt geht es um folgendes: während der unter das Einstellungsverb bericht- in (13) eingebettete Nebensatz seine Intension $p$ zur Extension des Satz-Prädikats beiträgt, scheint es, dass $p$ selbst aus der Intension von dessen Prädikat bestimmt werden muss, die ihrerseits durch Kombination der Intension von denk- mit dem kompositionellen Beitrag $q$ des unter diesem Verb eingebetteten Satzes hervorgeht. In der modernen formalen Semantik wird $q$ natürlich mit der gewöhnlichen Intension (15a) des eingebetteten Nebensatzes gleichgesetzt - was zu der langweiligen de-dicto-Lesart von (13) führt. Nach Frege jedoch sollte man erwarten, dass dieser Beitrag $q$ ein Objekt des Typs $\langle s,\langle s, t\rangle\rangle$ ist - die indirekte Intension des Satzes; denn das Einstellungsverb trägt bereits seine Intension bei und verlangt, dass sein satzwertiges Argument einen ,intensionaleren` Beitrag leistet. Freges (1892) Argumentation ist verschiedentlich (und m.E. zu Recht) für ihre Unvereinbarkeit mit grundlegenden Annahmen zur Lernbarkeit kritisiert worden, da nach ihr jeder lexikalische Ausdruck mit einer unendlichen Hierarchie von Sinnen assoziiert sein müsste; vgl. Davidson (1965). Doch wenn die vorgeblich obskuren indirekten Sinne von Ausdrücken systematisch aus ihren gewöhnlichen Intensionen hergeleitet werden könnten, ließe sich der Einwand abwehren. Wenn zudem insbesondere eine der systematisch ableitbaren indirekten Intensionen des besagten eingebetteten Nebensatzes die Funktion sein sollte, die jede Welt $w$ auf die Proposition (15b) abbildet, könnte Bäuerles (1983) Lesart (13) schließlich doch hergeleitet werden. Die detaillierte Ausführung dieses Programms einer Öffnung der Kompositionsmaschinerie für die Intensions-Hierarchie ist mühselig, aber sie ist zumindest grundsätzlich möglich und stellt insofern einen denkbaren Lösungsweg für die Probleme dar, die Beispiele wie (13) bereiten. Einen spezifischen Vorschlag in dieser Richtung findet man in Zimmermann (2018).

\section{Typen}

Die sichtbarsten Spuren, die die Montaguegrammatik auch noch in der zeitgenössischen Semantik hinterlassen hat, ist wohl in der Allgegenwart der funktionalen Typen zu sehen. Seit ihrer Verwendung in Heim \& Kratzers (1998) einflussreichem Lehrbuch ist Montagues e/t/s-Notation mit den spitzen Klammern 
geradezu zum Markenzeichen des führenden semantischen Paradigmas in der generativen Grammatik geworden. Die Rolle der Typen in der klassischen Montaguegrammatik ist allerdings kaum mit ihrer Stellung in der gegenwärtigen Semantik zu vergleichen. In Montague (1970a) waren sie gar nicht zu finden; in der allgemeinen Referenztheorie von Montague (1970b) waren sie lediglich ein taxonomisches Mittel zur Klassifikation von Denotaten nach ihrer mengentheoretischen Komplexität; und die Einteilung der kompositionellen Operationen in Montague (1973: 233f.) berührt die Typen allenfalls indirekt und entbehrt ohnehin jeglicher theoretischer Bedeutung. Der semantische Kompositionsprozess der klassischen Montaguegrammatik ist konstruktionsgesteuert, und Konstruktionsbedeutungen definieren sich nicht durch die Typen der Bedeutungen, die sie kombinieren, sondern durch die Art, in der diese Bedeutungen kombiniert werden. Insbesondere dürfen zwei unterschiedliche Konstruktionen denselben Input bekommen und sich dennoch in ihren Ouputs unterscheiden. Die sog. ,Tempusund Vorzeichen-Regeln“ (engl. Rules of tense and sign: ebd.) vermitteln einen sehr guten Eindruck davon, wie weit die klassische Montaguegrammatik von ihren typengesteuerten Nachfahren noch entfernt war. Nach diesen Regeln lässt sich das Verhältnis zwischen Subjekt und Prädikat positiv, negativ, zeitversetzt usw. auffassen. So werden den beiden Sätzen in (17) dieselben unmittelbaren Teile unterlegt, wobei die Bezeichnungen an den nicht-terminalen Knoten für syntaktische Operationen (oder Konstruktionen) stehen und nicht etwa für Kategorien:

(17) a. John slept.

b. John didn't sleep.

(18) a. POS-PAST

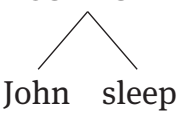

b. NEG-PAST

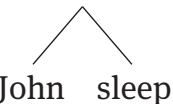

Die Strukturen in (18) stellen die semantisch relevante syntaktische AnalyseEbene $(\approx$ LF) dar, die den Input für die kompositionelle Deutung bildet; während (18a) als (17a) an die Oberfläche gelangt, liegt dem Satz (17b) die Struktur (18b) zugrunde. Bemerkenswerterweise unterscheiden sich die beiden Struk- 
turen lediglich in der verwendeten syntaktischen Konstruktion, aber nicht in den zugrundeliegenden oder (in Montagues Sprachgebrauch) disambiguierten Ausdrücken, auf die sie angewandt werden. Insbesondere bekommen also die mit (18a) und (18b) einhergehenden Bedeutungs-Kompositionen denselben Input, bringen aber unterschiedliche Outputs hervor. Etwas vereinfachend kann man sagen, dass nach (19a) die Extension des Subjekts $N$ auf die des Prädikats $V$ zu einem (nicht spezifizierten) früheren Zeitpunkt angewandt wird, während nach (19b) an allen früheren Zeitpunkten der Wahrheitswert verkehrt wird (wobei der Weltparameter konstant gehalten wird):

(19) a.

$$
P S T^{+}(\llbracket N \rrbracket)(\llbracket V \rrbracket)(w, t)=1
$$

gdw. $\llbracket N \rrbracket^{w, t^{\prime}}\left(\llbracket V \rrbracket^{w, t^{\prime}}\right)=1$ für irgendein $t^{\prime}$ vor $t$.

b. $\quad \operatorname{PST}^{-}(\llbracket N \rrbracket)(\llbracket V \rrbracket)(w, t)=1$

gdw. $\llbracket N \rrbracket^{w, t^{\prime}}\left(\llbracket V \rrbracket^{w, t^{\prime}}\right)=0$, für beliebige $t^{\prime}$ vor $t$.

(Man beachte, dass beide Operationen in (19) insofen (6) abdecken, als das Subjekt per Default als quantifikationell behandelt wird.) In einem typengesteuerten Ansatz à la Heim \& Kratzer (1998), nach dem die Denotate eines Mutterknotens durch die seiner Töchter determiniert werden, sind LFs wie (18) - und erst recht solche Deutungen wie (19) - ausgeschlossen. Stattdessen müssten die Strukturen in (18) umgeordnet werden, wobei Konstruktionen gegen zusätzliches funktionales Material ausgetauscht würden:

(20) a.

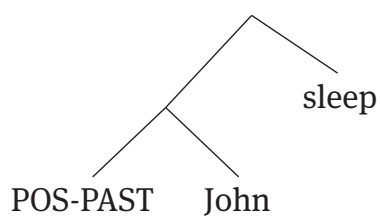

b.

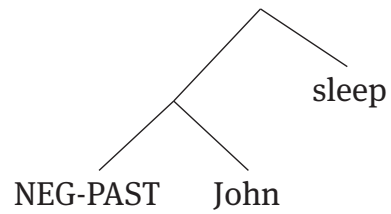

In (20) sind die nicht-terminalen Knoten unbeschriftet, da weder die Konstruktionen selbst noch ihr Effekt (auf die terminalen Ketten) im typengesteuerten Rahmen von Interesse sind. Wesentlich ist, dass die großgeschriebenen Ein- 
tragungen in (20) terminale Knoten okkupieren und daher nicht für syntaktische Konstruktionen, sondern für funktionale Morpheme stehen, die gerade die Operationen aus (19) bezeichnen:

(21) a. $\quad \llbracket \mathrm{POS}-\mathrm{PAST} \rrbracket^{w, t}(\llbracket N \rrbracket)(\llbracket V \rrbracket)=P S T^{+}(\llbracket N \rrbracket)(\llbracket V \rrbracket)(w, t)$

b. $\quad \llbracket N E G-P A S T \rrbracket^{w, t}(\llbracket N \rrbracket)(\llbracket V \rrbracket)=P S T^{-}(\llbracket N \rrbracket)(\llbracket V \rrbracket)(w, t)$

Da die in (21) definierten Operatoren vom Typ $\langle\langle s, q\rangle,\langle\langle s, p\rangle, t\rangle\rangle$ sind, wobei $q$ und p die Typen von Subjekt und Prädikat sind, kann die Deutung der Strukturen in (20) durch zwei aufeinanderfolgende Anwendungen eines vertrauten Prinzips der typengesteuerten Interpretation erfolgen: der Intensionalen Funktionalapplikation.

(20) stellt nicht die einzige Möglichkeit dar, die konstruktionsgesteuerten Analysen (18) mit typengesteuerten Mitteln zu erfassen. Eine gewissermaßen natürlichere Alternative geht von der Klammerung in (22) und einer naheliegenden Deutung (23) der funktionalen Morpheme als (zeit-abhängige) AussageOperatoren aus:

(22) a.

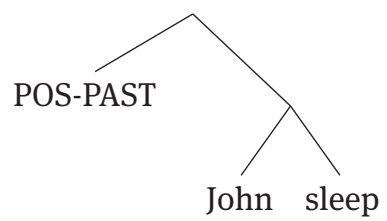

b.

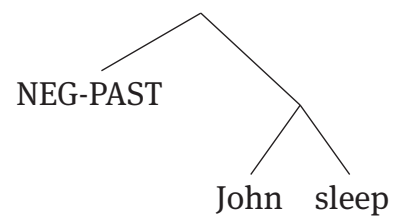

(23) a. $\quad \llbracket \mathrm{POS}-\mathrm{PAST} \rrbracket^{w, t}(\llbracket S \rrbracket)=1$ gdw. $\llbracket S \rrbracket^{w, t^{\prime}}=1$, für irgendein $t^{\prime}$ vor $t$.

b. $\quad \llbracket N E G-P A S T \rrbracket^{w, t}(\llbracket S \rrbracket)=1$ gdw. $\llbracket S \rrbracket^{w, t^{\prime}}=0$, für beliebige $t^{\prime}$ vor $t$.

Und schließlich könnte man die gemischten Operationen zerlegen, um die Effekte von Prädikation, Negation und Präteritum zu trennen - und potenzielle Skopusambiguitäten zu erhalten. Da dieser Schritt unabhängig von der typengesteuerten Strategie auch im konstruktionsgesteuerten Rahmen gemacht werden kann, werden wir ihn hier nicht weiter beachten. 
Der Übergang von der konstruktionsgesteuerten Analyse (19)-(20) zur typengesteuerten Version (21)-(22) lässt sich auf beliebige Bedeutungskompositionen verallgemeinern, soweit sich diese durch eine Funktion innerhalb der Typenhierarchie erfassen lassen. So kann jede extensionale oder intensionale Konstruktion durch Anwendung des Denotats eines entsprechenden grammatischen Morphems auf die Denotate der durch sie verbundenen Konstituenten simuliert werden. $\mathrm{Ob}$ damit alle grammatischen Konstruktionen überhaupt diesem Verfahren unterzogen werden können, hängt davon ab, ob es Bedeutungskompositionen gibt, die die funktionale Typenhierarchie übersteigen - also etwa Kontext-verschiebende oder Variablen-bindende Operationen. Aus Platzgründen muss hier der Hinweis genügen, dass erstere von Kaplans (1989) einflussreicher Theorie der Kontextabhängigkeit ausgeschlossen werden (vgl. ebd., 510 ff.), während letztere etwa im Rahmen von Jacobsons (1999) variablenfreier Semantik mit Mitteln der kombinatorischen Logik und verwandter Techniken umgangen werden können.

\section{Modelle}

VertreterInnen der formalen Semantik bezeichnen ihr Gebiet gern als modelltheoretisch, um es so von anderen - etwa strukturalistischen, kognitiven oder beweistheoretischen - Auffassungen der sprachlichen Bedeutung abzugrenzen. Die modelltheoretische Tradition in der linguistischen Semantik lässt sich direkt auf das Werk Richard Montagues zurückverfolgen, der Tarskis und Kripkes Paradigmen der mathematischen bzw. modalen Logik auf die kompositionelle Analyse der natürlichen Sprache übertragen hat. Die ursprünglich für logische Formelsprachen entwickelte Modelltheorie erfasst die zentralen semantischen Eigenschaften von und Beziehungen zwischen Formeln durch Abstraktion von den spezifischen ,materiellen' Anwendungen, um auf diese Weise ihre rein ,formale' semantische Struktur zu charakterisieren. In gewisser Hinsicht erinnern Modelle an mögliche Welten oder Indizes, die auch jeweils die Denotate aller Ausdrücke festlegen, wobei diese Denotate über die Klasse aller Modelle hinweg variieren, wie sie es ja auch von Index $\mathrm{zu}$ Index tun. Entgegen diesem ersten Eindruck darf man Modelle und Indizes aber keineswegs gleichsetzen. Sie auseinanderzuhalten und ihre grundsätzlich verschiedenen Rollen innerhalb der formalen Semantik anzuerkennen ist nämlich von größter Wichtigkeit. Um zu sehen, warum das so ist und um den Unterschied zu verstehen zwischen dem Modellraum - der Klasse (!) aller Modelle - und dem Logischen Raum - der Menge aller Indizes - lohnt es sich, ein spezifisches Beispiel zu betrachten, bei dem die beiden quasi in verschiedene Richtungen zerren. Jede 
seriöse semantische Analyse des Deutschen sollte den folgenden beiden Sätzen unterschiedliche Bedeutungen zuweisen:

(24) a. Der Präsident des größten Landes schläft.

b. Der Autor des längsten Romans ist wach.

Offensichtlich impliziert keiner der beiden Sätze den anderen, d. h. ihre Wahrheitswerte variieren frei. Und tatsächlich sieht es so aus, als liefere der Modellraum gerade diese Variation. Denn einigen Modellen zufolge sind beide Sätze in (24) wahr, weil etwa Hans das einzige Element in der Extension von Präsident des größten Landes und zudem ein Element (obwohl nicht unbedingt das einzige) der Extension von schläft ist und zugleich im selben Modell Fritz einziges Element der Extension von Autor des längsten Romans und zudem ein Element (obwohl nicht unbedingt das einzige) der Extension von ist wach ist, so dass sowohl (24a) als auch (24b) von derartigen Modellen als wahr bewertet werden. In anderen Modellen ist Hans nach wie vor einziges Element der Extension von Präsident des größten Landes und auch ein Element der Extension von schläft, während Otto, der (in dem Modell) das einzige Element von Autor des längsten Romans ist, nicht in der Extension von ist wach ist, wodurch (24a) und (24b) in derartigen Modellen als wahr bzw. falsch bewertet werden. Und so weiter, und so fort. Doch die Dinge - zumal die Modelle - verhalten sich komplizierter.

Zunächst enthalten die Modelle, die in der formalen Semantik (oder auch in der Logik) Verwendung finden, keine Personen wie Hans, Fritz und Otto. Modelle sind vielmehr mengentheoretische Objekte und bestehen demzufolge aus rein mengentheoretischem Material - und das heißt: aus Mengen. Wenn man sagt, dass in einem gegebenen Modell (oder diesem Modell zufolge) Hans Präsident ist, ist das allenfalls als eine metaphorische Aussage über eine bestimmte Menge zu verstehen, die die Person Hans repräsentiert (was immer das heißen mag). Ehe man ihn als pedantisch zurückweist, lohnt es sich, diesen Einwand unter die Lupe zu nehmen. Denn Modelle sind nicht nur frei von tatsächlichen Personen und konkreten Gegenständen; die Mengen, die sie stattdessen enthalten, sind auch in gewisser Weise vollkommen beliebig: ein Modell ist so gut wie das nächste, solange beide nur (nahezu) dieselbe mengentheoretische Struktur besitzen. Wenn also in einem Modell eine bestimmte Menge - sagen wir: die leere Menge $\emptyset$ - die Person Hans repräsentiert (was immer das heißt), dann muss es ein weiteres Modell geben, in dem diese Rolle von irgendeiner anderen Menge gespielt wird. Ja, zu jeder Menge $X$ im mengentheoretischen Universum muss es ein Modell geben, in dem $X$ dieselbe Rolle spielt wie $\emptyset$ im ursprünglichen Modell, also die Rolle von Hans. Gerade auf- 
grund dieser Eigenschaft der unbegrenzten Ersetzbarkeit - technisch: des Abschlusses unter mengentheoretischen Isomorphismen - ist die Gesamtheit aller Modelle eine echte Klasse und keine Menge.

Eine Folge der unbegrenzten Ersetzbarkeit betrifft Eigennamen und die Unterschiede zwischen ihnen. Die meisten SemantikerInnen scheinen mit Kripke (1972) davon überzeugt zu sein, dass Namen in dem Sinn konstante (oder starre) Intensionen besitzen, als ihre Referenten nicht über den Logischen Raum hinweg variieren dürfen. Andererseits gibt es haufenweise Leute namens Hans, Fritz oder Otto, und von daher kann keiner dieser Namen eine einzige Extension besitzen. Doch anstatt sie als Prädikate mit mengenartigen Extensionen zu analysieren, ziehen es zumindest einige SemantikerInnen vor, Eigennamen durch ihre Träger zu disambiguieren, auf die sie dann starr referieren können; mehr zu diesem Thema in Haas-Spohn (1995: Kap. 4). Im modelltheoretischen Rahmen kann jedoch dieser Träger nicht über die Extension des Namens identifiziert werden; denn wie jede Extension muss auch sie über den Modellraum hinweg variieren: des einen Modells Hans ist des nächsten Modells Otto. Auch wenn diese Beobachtung von fragwürdiger Relevanz für die semantische Analyse erscheinen mag - Wer will schon Namen nach ihren Trägern disambiguieren? -, offenbart sie doch, was Modelle nicht sind: Determinanten der Referenz; allenfalls determinieren sie die Repräsentanten von Referenten (was auch immer ...).

Trotz dieser Beobachtungen mag es scheinen, dass der Modellraum den Bedeutungsunterschied zwischen (24a) und (24b) auf angemessene Weise erfasst: im Gegensatz zu Personen können Wahrheitswerte über Modelle hinweg identifiziert werden, und in ihrem Wahrheitswert scheinen die beiden Sätze über den Modellraum hinweg zu variieren. Doch wieder einmal trügt der Schein. Denn die Modelle, die in der formalen Semantik (und anders als vielerorts in der Logik) Verwendung finden, legen nicht die Wahrheitswerte von Sätzen fest, sondern lediglich die Propositionen, die diese ausdrücken; ganz allgemein legt ein Modell Intensionen fest und keine Extensionen. Genauer gesagt ist jedes Modell mit einem vollen Logischen Raum von Indizes ausgestattet, die ihrerseits die Extensionen festlegen. Und da Modelle nun einmal mengentheoretische Gegenstände sind, sind auch diese Indizes Paare von beliebigen Mengen. Insbesondere wird kein Modell die wirkliche Welt zum Zeitpunkt des Verfassens oder Lesens dieses Aufsatzes als Index enthalten; allenfalls gibt es dort einen Index, der die Wirklichkeit zu einem gegebenen Zeitpunkt repräsentiert (...). Dass sich die Sätze in (24) über den Modellraum hinweg in ihrer Bedeutung unterscheiden, mag man also in dem Sinne verstehen, dass die Propositionen, die sie diesen Modellen gemäß ausdrücken, verschieden sind. Aber das muss nicht so sein. Schließlich gibt es haufenweise ,degenerierte‘ Modelle, 
um Rooths (1985) eingängigen Begriff zu benutzen, nach denen (24a) und (24b) ganz wörtlich dieselbe Proposition ausdrücken. Und das kann aus einer ganzen Reihe von Gründen so sein: in einigen Modellen gibt es ganz einfach keine Indizes, an denen auch nur einer der beiden Sätze wahr ist; in anderen sind beide notwendigerweise wahr; in manchen Modellen haben sogar die Subjekte der beiden Sätze dieselbe Intension - und ebenso die Prädikate; etc. pp. Natürlich ist keines dieser Modelle sonderlich ,realistisch“ - wie wieder Rooth (1985) sagen würde; doch zunächst einmal sind sie Teil des Modellraums. Um sie von dort $\mathrm{zu}$ vertreiben, müssen die SemantikerInnen Maßnahmen ergreifen. So kann man z. B. per Bedeutungspostulat Modelle ausschließen, in denen die Prädikate der beiden Sätze in (24) jemals überlappende Extensionen haben; oder man kann verfügen, dass der Logische Raum - die Gesamtheit der Indizes des Modells (die definitionsgemäß eine Menge bilden) - sehr groß ist und genügend Variation bietet, um die Intensionen von Sätzen wie denen in (24) wie auch die ihrer Konstituenten auseinanderzuhalten - solange das genannte Postulat dabei respektiert wird. Es ist (mir) nicht offensichtlich, wie dieses Resultat ohne Ad-Hockerei erreicht werden kann; vgl. dazu Zimmermann (2017). Doch zwei Effekte eines solchen Prozesses der erzwungenen Unabhängigkeit von (24a) und (24b) sollten in jedem Fall beachtet werden:

- Immer mehr ,degenerierte' Modelle werden eliminiert.

- Die Logischen Räume der verbleibenden ,realistischen“ Modelle bieten eine breite Variation der Extensionen (freilich innerhalb gewisser Grenzen).

Aufgrund des ersten Effekts wird der Modellraum immer kleiner, während infolge des zweiten Effekts Modelle mit un-,realistisch` kleinen Logischen Räumen verschwinden und in diesem Sinn der Logische Raum (sozusagen im Durchschnitt) immer größer wird. Insbesondere spielen also Modelle und Indizes ganz unterschiedliche Rollen in der formalen Semantik: um die Variation der Extensionen zu erfassen, muss man den Modellraum minimieren, während der Logische Raum maximiert wird.

Ein Grund, aus dem der Unterschied zwischen Welten und Modellen in unserem Fach selten anerkannt wird, kann darin liegen, dass er in der täglichen Arbeit der SemantikerInnen kaum jemals eine Rolle spielt, im Vertrauen darauf, dass ,unrealistische“ Modelle ausgeschlossen werden, wann immer man sie antrifft. Doch die Vernachlässigung des Unterschieds zwischen Logischem Raum und Modellraum läuft Gefahr, SemantikerInnen für die Art von Erklärungslücke blind zu machen, die wir in Abschnitt 1 gefunden haben, im Zusammenhang mit der Analyse von seek a unicorn, die hier um der Leserfreundlichkeit willen wiederholt wird:

(12) $\llbracket$ seek a unicorn $\rrbracket^{w, t}=\llbracket$ seek $\rrbracket^{w, t}(\llbracket$ a unicorn $\rrbracket)$ 
(12) war das Ergebnis einer Anwendung von (in erster Linie Fregeschen) Techniken zur Lösung eines Substitutionsproblems: nachdem sich die Extension des direkten Objekts in seiner Rolle als kompositioneller Beitrag als ungeeignet erwiesen hat, wurde sie (A) durch die Intension ersetzt, auf der (B) die Extension des Verbs operieren musste, um eine Prädikatsextension hervorzubringen. Es blieb jedoch das Rätsel, wie genau die Verbextension dieses Ergebnis erreicht. Um zu sehen, worum es dabei geht, mag man die Situation mit anderen Anwendungen der Fregeschen Heuristik (B) vergleichen. Wir hatten bereits einen einigermaßen trivialen Fall kennengelernt, nämlich die Konstruktion von Prädikatsextensionen im Übergang von (2) nach (3) und schließlich nach (4):

(2) $\vdash$ Maria schläft $\dashv=F(\mathrm{~m}$, $\mid$ schläft $\mid)$

(3) $\mid N$ schläft $\mid=F(|N|$,|schläft $\mid)$

(4) $|\operatorname{schläft~}|(x)=\vdash x$ schläft $\dashv$

(3) ist eine naheliegende Verallgemeinerung von (2): $\mathrm{m}$ ist die Referentin des Namens Maria und daher auch seine Extension. Der Knackpunkt ist, dass es im Fall von (2) offenkundig ist, worin der Beitrag der Extension des Namens besteht, die auf der rechten Seite der Gleichung explizit genannt wird: $\mid$ Maria $\mid=\mathrm{m}$. Die Annahme, dass sich andere Namen analog verhalten, ist daher nicht besonders gewagt. Und steht erst einmal (3), ist es nicht schwer, die systematische Abhängigkeit des Wahrheitswerts des Satzes vom Referenten des Subjekts zu verallgemeinern von Namensträgern $|N|$ auf beliebige Individuen x. Ein genauerer Blick auf die Herleitung der Extensionen quantifizierender Nominale mit derselben Methode würde zudem zeigen, dass der Wahrheitswert eines Satzes wie (25) auf einigermaßen durchsichtige Weise systematisch von der Prädikatsextension abhängt (wenn auch auf etwas weniger offensichtliche Weise als im vorangehenden Fall): er ist gerade dann 1, wenn die Prädikatsextension keine Person enthält.

(25) Niemand schläft.

Ausgehend von dieser Beobachtung ist es nicht schwer, die Quantorenextension (27) anzugeben, die schließlich den entscheidenden Beitrag zur Lösung der semantischen Gleichung (26) durch Gleichsetzung von F mit APP liefert:

(26) $\mid$ Niemand schläft $\mid=F(\mid$ niemand $|$,$| schläft \mid)$

(27) $\mid$ niemand $\mid(\mid$ schläft $\mid)=1 \mathrm{gdw}$. $\mid$ schläft $\mid=0$, für beliebige Personen $\mathrm{x}$. 
Ist jedoch in diesen und ähnlichen Fällen die funktionale Abhängigkeit der Extension des Mutterknotens von derjenigen der zuvor analysierten Schwester recht unproblematisch, so ist es doch alles andere als offensichtlich, dass die (12) erfüllende Funktion $\llbracket \operatorname{seek} \rrbracket^{w, t}$ ähnlich systematisch konstruierbar ist: wie soll denn diese Funktion die Menge der Personen, die ein Einhorn suchen, auf der Basis der Intension des (verallgemeinerten) Quantors bestimmen, den das Indefinitum a unicorn denotiert? Ja, es mag noch nicht einmal offenkundig sein (obwohl es durchaus zutrifft), dass es überhaupt so eine Funktion gibt. Es scheint, dass diese Art von Fragen typischerweise durch Anwendungen der Fregeschen Heuristik auf intensionale Konstruktionen aufgeworfen werden. Aus modelltheoretischer Perspektive scheint allerdings alles in Ordnung zu sein: führt die Extension des Objekts zu Substitutionsproblemen, so muss einfach ihr kompositioneller Beitrag in ihrer Intension bestehen. Jedes Modell, das die Gleichung (12) erfüllt (und analoge Gleichungen für andere Objekte) löst das Problem - und damit hat sich's. Am Ende wird die Extension von seek lediglich als Objekt eines gewissen Typs eingestuft, ohne darüber hinaus genauer charakterisiert $\mathrm{zu}$ werden; mit den Spezifikationen der Prädikats- und Nominal-Extensionen in (4) and (27) hat das nur wenig gemein. (Eine ähnliche Klage bringt übrigens Larson (2002) gegen Montagues (1970b) intensionale Analysen vor, wobei er allerdings die Schuld nicht in der dem modelltheoretischen Ansatz eigenen Unterspezifikation der Intensionen sieht, sondern in der Heranziehung von Intensionen nicht-satzwertiger Konstituenten im allgemeinen.) Natürlich kann auch eine modelltheoretische semantische Analyse über die bloße Identifikation der Extensionstypen hinausgehen - aber sie muss es nicht; und genau das macht sie zugleich attraktiv und gefährlich. In dieser Hinsicht sind semantische Ansätze, die den Versuchungen einer Fregeschen Faulheit nicht nachgeben, indem sie die modelltheoretische Analyse ablehnen, besser als ihr berühmter Vorläufer. Wenn das kein Fortschritt ist ... :-)

\section{Literatur}

Bäuerle, Rainer (1983): Pragmatisch-semantische Aspekte der NP-Interpretation. In Manfred Faust, Roland Harweg, Werner Lehfeldt \& Götz Wienold (Hrsg.), Allgemeine Sprachwissenschaft, Sprachtypologie und Textlinguistik, 121-131. Tübingen: Narr. Carnap, Rudolf (1947): Meaning and necessity. Chicago/London: University of Chicago Press. Cresswell, Maxwell J. (1973): Logics and languages. London: Methuen. Cresswell, Maxwell J. (1990): Entities and indices. Dordrecht, Boston, London: Kluwer. Davidson, Donald (1965): Theories of meaning and learnable languages. In Yehoshua BarHillel (Hrsg.), Logic, methodology, and philosophy of science, 383-394. Amsterdam: North-Holland Publishing Company. 
Frege, Gottlob (1891): Function und Begriff. Jena: Pohle.

Frege, Gottlob (1892): Über Sinn und Bedeutung. Zeitschrift für Philosophie und philosophische Kritik NF 100, 25-50.

Gallin, Daniel (1975): Intensional and higher-order modal logic. Amsterdam: North-Holland Publishing Company.

Groenendijk, Jeroen \& Martin Stokhof (1982): Semantic analysis of WH-complements. Linguistics and Philosophy 5(2), 175-233.

Haas-Spohn, Ulrike (1995): Versteckte indexikalität und subjektive bedeutung. Berlin: Akademie Verlag.

Heim, Irene \& Angelika Kratzer (1998): Semantics in generative grammar. Oxford: Blackwell Publishers Ltd.

Jacobson, Pauline (1999): Towards a variable-free semantics. Linguistics and Philosophy 22, 117-185.

Kaplan, David (1975): How to Russell a Frege-Church. Journal of Philosophy 72(19), 716-729.

Kaplan, David (1989): Demonstratives. An essay on the semantics, logic, metaphysics and epistemology of demonstratives and other indexicals. In J. Almog, John Perry \& Howard Wettstein (Hrsg.), Themes from Kaplan, 481-563. Oxford: Oxford University Press.

Kripke, Saul A. (1972): Naming and necessity. In Donald Davidson \& Gilbert Harman (Hrsg.), Semantics of natural language, 253-355. Dordrecht: Reidel.

Larson, Richard (2002): The grammar of intensionality. In Gerhard Preyer \& Georg Peter (Hrsg.), Logical form and language, 228-262. Oxford: Oxford University Press.

Montague, Richard (1968): Pragmatics. In Raymond Klibansky (Hrsg.), Contemporary philosophy. Volume I: Logic and the foundations of mathematics, 102-122. Florence: La Nuova Italia Editrice.

Montague, Richard (1969): On the nature of certain philosophical entities. The Monist 53(2), 159-195.

Montague, Richard (1970a): English as a formal language. In Bruno Visentini (Hrsg.), Linguaggi nella società e nella tecnica, 189-223. Milan: Edizioni di Comunitá.

Montague, Richard (1970b): Universal grammar. Theoria 36(3), 373-398.

Montague, Richard (1973): The proper treatment of quantification in ordinary English. In Jaakko Hintikka, Julius Moravcsik \& Patrick Suppes (Hrsg.), Approaches to natural language, 221-242. Dordrecht: Reidel.

Parsons, Terence (1981): Frege's hierarchies of indirect senses and the paradox of analysis. In P. French, T. Uehling \& H. Wettstein (Hrsg.), Midwest studies in philosophy VI: The foundations of analytic philosophy, 37-57. Minneapolis: University of Minnesota Press.

Partee, Barbara (1975): Montague grammar and transformational grammar. Linguistic Inquiry $\mathrm{VI}(2), 203-300$.

Percus, Orin (2000): Constraints on some other variables in syntax. Natural Language Semantics 9(1), 173-229.

Putnam, Hilary (1975): The meaning of 'meaning'. In Philosophical papers vol. 2: Mind, language, and reality, 215-271. Cambridge: Cambridge University Press.

Rooth, Mats (1985): Association with focus. University of Massachusetts at Amherst: Dissertation.

Schlenker, Philippe (2006): Ontological symmetry in language: A brief manifesto. Mind and Language 21(4), 504-539.

Zimmermann, Thomas Ede (2005): Coercion vs. indeterminacy in opaque verbs. In Reinhard Kahle (Hrsg.), Intensionality, 217-265. Wellesley, Mass.: AK Peters Ltd. 
Zimmermann, Thomas Ede (2017): Quantification over alternative intensions. Semantics and Pragmatics 10.

Zimmermann, Thomas Ede (2018): Fregean compositionality. In Derek Ball \& Brian Rabern (Hrsg.), The science of meaning, Oxford: Oxford University Press. 



\title{
Stefan Müller
}

\section{Und? Was läuft sonst so?}

\author{
Alternative Grammatiktheorien
}

\begin{abstract}
In diesem Aufsatz versuche ich auf engstem Raum die Alternativen zu Theorien im Rahmen der GB/des Minimalismus vorzustellen. Die besprochenen Theorien sind Dependenzgrammatik, Kategorialgrammatik, LFG, TAG, GPSG, HPSG und Konstruktionsgrammatik. Es wird darauf eingegangen, wie Valenz repräsentiert wird, und für einige der Phänomene, für die es transformationelle Analysen gibt, werden die Alternativen skizziert. Dazu gehört die Analyse von Aktiv/Passivalternationen, die Analyse der Verbstellung und Vorfeldbesetzung.
\end{abstract}

Keywords: Dependenzgrammatik, Government \& Binding, GPSG, HPSG, Konstituentenstellung, Konstruktionsgrammatik, LFG, Minimalismus, Passiv, Phrasenstrukturgrammatik, TAG, Transformation, V2

\section{Einleitung}

Unterhält man sich mit Wissenschaftlern, die im Rahmen der Government \& Binding-Theorie oder des Minimalismus arbeiten, über dominante Theorien oder Schulen, dann bestreiten diese mitunter, dass chomskysche Theorien dominant oder Mainstream seien. Dass man in einem Nicht-MainstreamFramework arbeitet, erkennt man aber daran, dass man bei Zeitschriftenartikeln immer einen allgemeinen Teil braucht, in dem erklärt wird, welche Voraussetzungen gemacht werden. Das heißt, man benötigt zwei bis fünf Seiten, um zu erklären, wie Konstituentenstrukturen lizenziert werden, wie Valenz repräsentiert wird usw. Veröffentlichungen in dominanten Frameworks verweisen einfach auf Chomsky (1981) oder Chomsky (1995) und beginnen mitten im Aufsatz.

Dieser Beitrag hat ein ähnliches Problem: Innerhalb von zwanzig Seiten sollen hier die Alternativen zum Mainstream vorgestellt werden, eigentlich bräuchte man aber pro alternativer Theorie zwanzig Seiten. Die Aufgabe kann

Stefan Müller, Institut für deutsche Sprache und Linguistik, Sprach- und literaturwissenschaftliche Fakultät, HU Berlin, E-mail: st.mueller@hu-berlin.de

Ә Open Access. (c) 2018 Stefan Müller, publiziert von De Gruyter. (c) BY Dieses Werk ist lizenziert unter der Creative Commons Attribution 4.0 Lizenz. 
hier also nur sein, der Leserin bzw. dem Leser Appetit zu machen und ihn dann zu Büchern weiterzuschicken, die die jeweiligen Theorien ausführlich besprechen und miteinander vergleichen.

Beim folgenden Überblick werde ich historisch vorgehen und erklären, aus welchen Gründen bestimmte Theorien entwickelt bzw. aufgegeben wurden und welche Ideen weitergeführt wurden. Die Dependenzgrammatik liegt etwas quer $\mathrm{zu}$ den anderen Theorien, die hier besprochen werden. Ich diskutiere sie kurz nach den einleitenden Bemerkungen über Phrasenstrukturgrammatiken, obwohl sie aus historischer Perspektive eigentlich als erste besprochen werden müsste.

\section{Phrasenstrukturgrammatiken und Transformationen}

Ausgangspunkt der historischen Betrachtungen sollen die Phrasenstrukturgrammatiken sein. Diese bilden einen wichtigen Bestandteil der Theorien, die im Folgenden diskutiert werden. Für die Analyse von Äußerungen mittels Phrasenstrukturgrammatiken muss man Äußerungen in Konstituenten zerlegen. Konstituenten werden in Distributionsklassen eingeteilt und bekommen eine entsprechende Kategorie. Den Satz in (1) kann man in zwei Nominalphrasen und ein Verb zerlegen. Die Nominalphrasen bestehen jeweils aus einem Determinator und einem Nomen.

(1) Der Mann streichelt den Hund.

Die Regeln, die man für die Analyse von Sätzen wie (1) in simplen Grammatiken annehmen würde, zeigt (2):

(2) $\mathrm{NP} \rightarrow \mathrm{D}, \mathrm{N}$

$$
\begin{aligned}
& \mathrm{S} \rightarrow \mathrm{NP}, \mathrm{V}, \mathrm{NP} \\
& \mathrm{V} \rightarrow \text { streichelt } \\
& \mathrm{D} \rightarrow \text { der } \\
& \mathrm{D} \rightarrow \text { den } \\
& \mathrm{N} \rightarrow \text { Mann } \\
& \mathrm{N} \rightarrow \text { Hund }
\end{aligned}
$$


Eine solche Grammatik ist zu ungenau, da man mit ihr auch Folgen wie den Mann streichelt den Hund analysieren könnte. Dieses Problem lässt sich leicht beheben, indem man die Symbole feiner differenziert und zum Beispiel N_nom, D_nom und NP_nom verwendet, um auch die Kasusunterschiede zu erfassen. Das würde dann aber zu einer Explosion der Kategoriesymbole und der Ersetzungsregeln führen. Statt also vier verschiedene Ersetzungsregeln für die Nominalphrase mit den jeweiligen Kasus anzunehmen, verwendet man komplexe, intern strukturierte Symbole und abstrakte Schemata. (3) zeigt das für die Nominalphrase:

(3) $\mathrm{NP}($ Kas $) \rightarrow \mathrm{D}$ (Kas), $\mathrm{N}($ Kas $)$

$$
\begin{aligned}
& \mathrm{D}(\text { nom }) \rightarrow \text { der } \\
& \mathrm{D}(\text { acc }) \rightarrow \text { den } \\
& \mathrm{N}(\text { nom }) \rightarrow \text { Mann } \\
& \mathrm{N}(\text { acc }) \rightarrow \text { Hund }
\end{aligned}
$$

Die Wortartinformation ist in diesen Grammatikregeln privilegiert. Sie bildet ein Gerüst für syntaktische Strukturen (in der Computerlinguistik wird das auch Phrasenstrukturrückgrat genannt). Es hindert einen jedoch nichts daran, auch von der Wortart zu abstrahieren. Wenn man das tut und für die Wortarten ebenfalls Variablen benutzt, bekommt man sehr abstrakte Schemata, die besagen: Kombiniere einen Kopf einer beliebigen Kategorie X mit seinen Komplementen, so dass eine komplexe Kategorie $X^{\prime}$ entsteht. Ein weiteres Schema kombiniert $\mathrm{X}^{\prime}$ mit einem so genannten Spezifikator $\mathrm{zu}$ einer XP. Solcherart abstrakte Schemata werden seit den 70er Jahren in der Mainstram Generative Grammar (MGG) verwendet (Jackendoff 1977; Chomsky 1970, 1981) und sind auch in alternativen Grammatiktheorien weit verbreitet. Chomsky ging ursprünglich nicht davon aus, dass das X-Schema für alle Sprachen gleichermaßen anwendbar ist, das hat sich aber im Laufe der Zeit geändert. In GPSG, LFG und HPSG werden ebenfalls $\bar{X}$-Strukturen angenommen, jedoch nicht für alle Sprachen.

Die Einführung von Merkmalen und die Verwendung von Regelschemata statt Regeln war ein großer Fortschritt, die ursprüngliche Kritik an Phrasenstrukturgrammatiken ist dadurch jedoch nicht hinfällig. Chomsky (1957) hat darauf hingewiesen, dass einfache Phrasenstrukturgrammatiken bestimmte Regelmäßigkeiten nicht erfassen können. So haben wir zum Beispiel in (4) eine Sammlung von Aktivsätzen und in (5) die dazugehörigen Passiväquivalente: 
(4) a. Er liest den Aufsatz.

b. Er gibt dem Mann den Topf.

c. Er bezichtigt den Mann des Mordes.

d. Er hilft dem Mann.

(5) a. Der Aufsatz wurde gelesen.

b. Der Topf wurde dem Mann gegeben.

c. Der Mann wurde des Mordes bezichtigt.

d. Dem Mann wurde geholfen.

Man könnte natürlich Phrasenstrukturregeln für diese Sätze aufschreiben, das Phänomen wäre dann aber nicht adäquat erfasst, denn die Passivregeln wären einfach Bestandteil der Grammatik, ohne dass klar wäre, in welcher Beziehung diese Regeln $\mathrm{zu}$ anderen stehen. Nirgendwo in der Grammatik wäre erfasst, was das Passiv eigentlich ausmacht: Im Passiv wird ein Subjekt mit Agenseigenschaften unterdrückt, wenn es im Aktiv ein Objekt im Akkusativ gibt, wird dieses zum Subjekt.

Weitere Beispiele für Zusammenhänge, die sich mit Phasenstrukturgrammatiken nicht gut erfassen lassen, sind die Beziehungen zwischen den Sätzen in (6), (7) und (8) sowie zwischen denen in (10) und (11):

(6) a. dass der Mann der Frau das Buch gibt

b. dass der Mann der Frau oft hilft

c. dass der Mann schläft

(7) a. Gibt der Mann der Frau das Buch?

b. Hilft der Mann der Frau oft?

c. Schläft der Mann?

(8) a. Der Frau gibt der Mann das Buch.

b. Oft hilft der Mann der Frau.

c. Der Mann schläft. 
In (6) und (7) haben wir Verbletztsätze und entsprechende Verberstsätze. In einer Phrasenstrukturgrammatik kann man zwar Regeln für all diese Abfolgen angeben, es wird aber nicht ausgedrückt, dass man im Deutschen zu (fast) jedem Verbletztsatz auch einen Verberstsatz bilden kann. (8) zeigt Verbzweitsätze. Diese haben im Prinzip den Aufbau der Verberstsätze, nur dass eine beliebige Konstituente nach vorn vor das finite Verb gestellt wurde. Wie das Beispiel in (9) zeigt, kann eine solche Voranstellung aus sehr tief eingebetteten Konstituenten erfolgen:

(9) [Um zwei Millionen Mark] $]_{i}$ soll er versucht haben, [eine Versicherung _i $\mathrm{zu}$ betrügen]. ${ }^{1}$

Als letztes Beispiel sei das Scrambling genannt. Im Deutschen können die Argumente des Verbs in beliebiger Reihenfolge angeordnet werden. Das gilt für zweistellige Verben wie in (10), aber auch für drei- und mehrstellige. Einige Beispiele für Umordnungen bei dreistelligen Verben sind in (11) angegeben:

(10) a. dass niemand diese Frau kennt

b. dass diese Frau niemand kennt

(11) a. dass niemand dieser Frau das Buch gibt

b. dass dieser Frau niemand das Buch gibt

c. dass dieser Frau das Buch niemand gibt

Die Umordenbarkeit ist eine allgemeine Eigenschaft des Deutschen und das muss irgendwie erfasst werden. Formuliert man für diese Stellungsmöglichkeiten einfach eigene Phrasenstrukturregeln, ist das Phänomen als solches nicht korrekt beschrieben.

Chomsky hat nun eine Erweiterung der Phrasenstrukturgrammatiken vorgeschlagen, die die Linguistik revolutionieren sollte: Transformationen. Die Idee, die auf Chomskys Doktorvater Harris zurückgeht, ist, dass es Prozesse gibt, die aus Syntaxbäumen andere Syntaxbäume erzeugen. Abbildung 4.1 zeigt das für das Passiv im Englischen. Transformationen wurden für die bisher genannten Phänomene auch in Transformationsgrammatiken des Deutschen angenommen (Bierwisch 1963; Reis 1974; Koster 1975; Thiersch 1978: Chapter 1;

1 taz, 4. 5. 2001, S. 20 (zitiert nach Müller 2007: 163). 

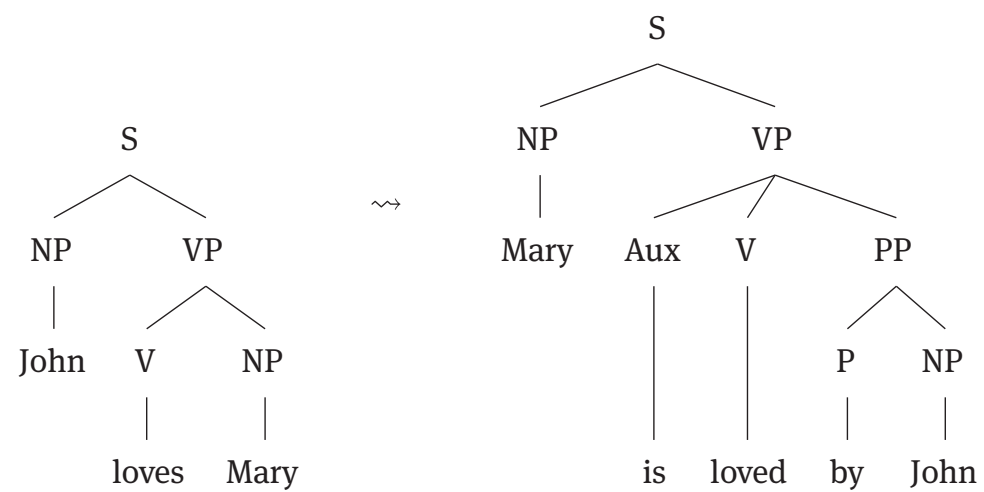

Abb. 4.1: Passivtransformation für das Englische.

den Besten 1983; Thiersch 1978; Grewendorf 1988; Frey 1993; Fanselow 1990, 1993).

Kurz nach der Veröffentlichung der ersten Arbeiten von Chomsky begannen Psycholinguisten nach Evidenz für die kognitive Realität von Transformationen zu suchen. Die Annahme war, dass man Transformationen nachweisen können müsste, wenn Sätze, deren Analyse Transformationen enthält, langsamer verarbeitbar sind als Sätze, die ohne Transformationen analysiert werden können. Zum Beispiel müssten Sätze im Passiv längere Verarbeitungszeiten benötigen, da man ja zuerst einen Aktivsatz aufbauen muss, den man dann in einen Passivsatz umwandelt. Die entsprechenden Theorien zur Verarbeitung liefen unter dem Namen Derivational Theory of Complexity und erste Arbeiten schienen diese Theorie zu bestätigen (Miller \& McKean 1964; Savin \& Perchonock 1965; Clifton \& Odom 1966), so dass Chomsky (1976: 249-250) noch 1968 von der psycholinguistischen Realität von Transformationen ausging. Die Arbeiten aus den 60er Jahren hielten aber späteren genaueren Überprüfungen nicht stand (Fodor, Bever \& Garrett 1974: 320-328). In aktuellen Arbeiten im Rahmen der MGG spricht man deshalb jetzt von Transformationen als Metaphern (Lohnstein 2014: 170; Chomsky 2001: Fn 4). Man fragt sich natürlich, wofür diese Metaphern stehen und ob man genau diese Metaphern verwenden sollte. In der GB-Zeit wurden Modelle entwickelt, die rein repräsentationell waren und ohne Transformationen auskamen. Hier ist es angebracht, von Transformationen und Bewegung und Umstellung als Metaphern zu sprechen, aber in jüngeren Arbeiten wird ernsthaft von bestimmten Verarbeitungsschritten und Reihenfolgen ausgegangen und nach allem, was man aus der Psycholinguistik weiß, sind diese Verarbeitungsmodelle nicht mit den Befunden aus der Psycholinguistik kompatibel. Entsprechende Minimalistische Theorien sind 
also entweder einfach falsch oder von der aus der Psycholinguistik kommenden Evidenz abgekoppelte Theorien, die nur indirekt mit dem menschlichen Sprachvermögen zu tun haben.

Die Theorien, die im Folgenden besprochen werden, sind nichttransformationelle Theorien. Ich möchte zeigen, wie sie die Phänomene behandeln, für die Transformationen nötig zu sein scheinen. Ich beginne mit der Dependenzgrammatik.

\section{Dependenzgrammatik}

Die Dependenzgrammatik wurde maßgeblich von Tesnière $(1959,2015)$ geprägt. Tesnières Arbeiten waren lange vor Chomskys ersten Veröffentlichungen fertig, wurden aber erst posthum veröffentlicht. Den wichtigsten Aspekt stellen, wie auch der Name des Frameworks schon sagt, die Abhängigkeiten dar. Das Verb bildet das Zentrum des Satzes, Adjunkte und Argumente hängen von ihm ab.

Da die Valenz von Verben im Aktiv anders ist als die Valenz von Verben im Passiv, ist klar, wie die Analyse des Passivs in der Dependenzgrammatik aussieht: Eine Lexikonregel setzt ein Verb mit Aktivvalenz zu einem mit Passivvalenz in Beziehung (Hellwig 2003: 629-630).

Viele Dependenzgrammatik-Analysen beschäftigen sich auch hauptsächlich mit den Dependenzen und sehen die Anordnung der Konstituenten als weniger wichtig an. Ein Beispiel für die Analyse eines Verbzweitsatzes als einfache alternative Anordnung der Konstituenten zeigt Abbildung 4.2 (Eroms 2000: Abschnitt 9.6.2; Groß \& Osborne 2009).

Gerade in jüngster Zeit wird immer wieder betont, dass die Dependenzgrammatik einfacher als die Phrasenstrukturgrammatik sei, weil die Analysen

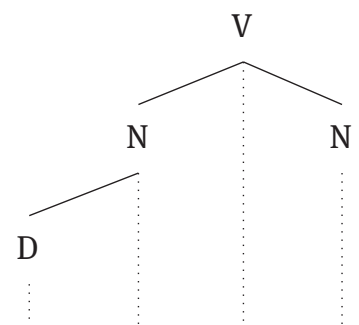

diesen Mann kennt jeder

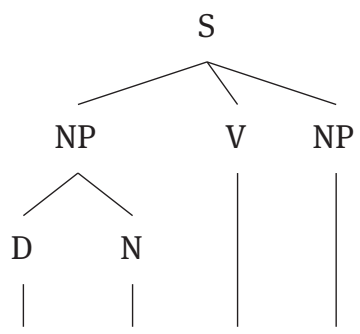

diesen Mann kennt jeder

Abb. 4.2: Analyse des Satzes Diesen Mann kennt jeder. in Dependenzgrammatik und Phrasenstrukturgrammatik. 
weniger Knoten benötigen würden (Groß \& Osborne 2009: 48). Dem ist aber nicht so. Es ist zwar richtig, dass die Analyse in Abbildung 4.2 nur einen Verbknoten enthält und nicht wie in den Phrasenstrukturgrammatikanalysen einen Knoten für das Verb und einen für den gesamten Satz, aber diese Einfachheit besteht nur, wenn man andere sprachliche Ebenen außer Acht lässt. So ist zum Beispiel die Bedeutung des gesamten Satzes nicht mit der Bedeutung des einzelnen Verbs identisch. Repräsentiert man diese Information irgendwo, so bekommt man dieselbe Komplexität, wie man sie in Phrasenstrukturgrammatiken hat. Dieser Punkt ist genauer in Müller (2016: Abschnitt 11.7.2.3) diskutiert. Dort wird der Ansatz von Hudson (2015) besprochen, der explizit zusätzliche Knoten für semantische Repräsentationen vorsieht.

Tesnière hat für die Analyse von Skopusphänomenen sogenannte Polygraphen benutzt (Osborne \& Kahane 2015: lix). Die Strukturen, die man dann für red cars that you saw yesterday bekommt, entsprechen genau den Strukturen, die man auch in der X-Analyse von NPs hat (Müller 2016: 379).

Die Behandlung von Fernabhängigkeiten in der DG ist problematisch, wenn diese über diskontinuierliche Konstituenten gemacht wird. Oft sind solche Ansätze nicht genügend ausgearbeitet und die Analysen lassen auch ungrammatische Äußerungen zu (Müller 2016: Abschnitt 11.7).

\section{Kategorialgrammatik}

Die Kategorialgrammatik wurde schon zwanzig Jahre vor Chomskys ersten Veröffentlichungen von dem polnischen Logiker Ajdukiewicz (1935) entwickelt. Sie ist insbesondere unter Semantikern beliebt, weil sie es erlaubt, Syntax und Semantik parallel zu führen (Montague 1974).

Im folgenden möchte ich die Grundmechanismen der Kategorialgrammatik vorstellen und darlegen, worin sie sich von den Phrasenstrukturgrammatiken unterscheidet und wie sie die von Chomsky aufgezeigten Herausforderungen meistert.

Im vorigen Abschnitt haben wir gesehen, dass simple Phrasenstrukturgrammatiken nicht genau genug sind und haben diese durch weitere Merkmale wie z. B. Kasus erweitert. Auch die Klassifizierung von Verben als V reicht nicht aus, denn wenn wir der Grammatik in (2) einen Lexikoneintrag für ein intransitives Verb wie lachen hinzufügen, könnte man mit der Grammatik Wortfolgen wie Peter lacht den Hund analysieren. Man muss also Valenzinformation an Verben im Lexikon spezifizieren. Die entsprechenden Regeln und Lexikoneinträge zeigt (12): 


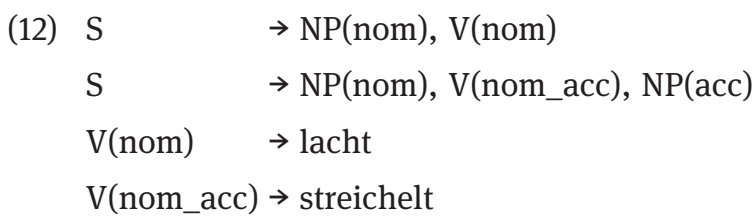

Ein Nachteil dieser Regeln ist, dass Valenzinformation im Prinzip doppelt kodiert ist: Zum einen steht beim Verb, in welche Valenzklasse es gehört, und zum anderen steht in der jeweiligen Regel, welche Argumente zu Verben einer bestimmten Valenzklasse gehören. Dieses Problem besteht in der Kategorialgrammatik nicht, denn dort sind die Kombinationsregeln ganz allgemein und Valenz ist komplett lexikalisch repräsentiert. Ein Verb, das noch links von sich eine NP braucht, um einen Satz zu bilden, bekommt die Kategorie s\np. Das Ergebnis der Kombination mit der NP ist dann ein s. Ergebnisse stehen immer auf der linken Seite der Schrägstriche. Ein zweistelliges Verb hat im Englischen die Kategorie (s\np)/np, denn es braucht rechts ein Objekt und dann links ein Subjekt. Die Regeln für die Kombination von Köpfen mit ihren Argumenten sind sehr abstrakt und denkbar einfach:

(13) a. Vorwärtsapplikation:

$$
\mathrm{X} / \mathrm{Y} * \mathrm{Y}=\mathrm{X}
$$

b. Rückwärtsapplikation:

$$
\mathrm{Y} * \mathrm{X} \backslash \mathrm{Y}=\mathrm{X}
$$

Es gibt zwei Varianten: Die eine kombiniert etwas, das etwas rechts von sich verlangt, mit dem Verlangten und die andere tut das gleiche nach links. Abbildung 4.3 zeigt die Analyse von (14) als Beispiel:

(14) The cat chased Mary.

$$
\underset{n p}{\stackrel{\frac{\text { the }}{n p / n} \underset{n}{\stackrel{c a t}{\longrightarrow}} \frac{\frac{\text { chased }}{(s \backslash n p) / n p} \stackrel{\text { Mary }}{n p}}{s \backslash n p}}{s}}
$$

Abb. 4.3: Analyse eines Satzes mit einem transitiven Verb in der Kategorialgrammatik.

Wie lässt sich nun das Passiv adäquat erfassen? Überlegt man sich, was das Ziel ist, so ist die Analyse verblüffend naheliegend: Da Ableitungen immer durch 
die beteiligten Lexikonelemente gesteuert werden, braucht man also für jedes Passivpartizip ein Lexikonelement, das für die entsprechenden Argumente subkategorisiert ist. Statt einer Transformation verwendet die Kategorialgrammatik also eine Lexikonregel (Dowty 1978: 412, 2003: Section 3.4). Dowty hat auch darauf hingewiesen, dass die Lexikonregeln lexically governed transformations sind. Sie sind also in gewissem Sinne das Gegenstück zu Transformationen in transformationslosen Theorien. Es gibt jedoch einen wichtigen Unterschied: Transformationen setzen Bäume zueinander in Beziehung, das heißt, Strukturen, die schon zusammengebaut worden sind. Lexikonregeln setzen dagegen Lexikonelemente zueinander in Beziehung. Lexikonelemente lizenzieren Mengen von Bäumen (oder anderen Strukturen). So kann ein zweistelliges Verb in den verschiedensten Sätzen vorkommen (mit und ohne Adjunkten, mit und ohne Voranstellungen, in Relativsätzen, Interrogativsätzen). Wenn ein zweistelliges Verb zu einem einstelligen passivierten Verb in Beziehung gesetzt wird, werden also Klassen von potenziellen Bäumen zueinander in Beziehung gesetzt und nicht fertige Bäume.

Für die Analyse der Verbstellung gibt es mehrere Vorschläge in der Literatur. Eine ist wieder, einen Lexikoneintrag (s/np)/np anzunehmen, der zum Lexikoneintrag (s\np)\np für die SOV-Stellung in Beziehung steht (Steedman 2000: 159).

Steedman (1989: Abschnitt 1.2.4) schlägt eine Analyse der Fernabhängigkeiten vor, die ohne Bewegung und ohne leere Elemente auskommt. Für Sätze wie die in (15) nimmt er an, dass die Kategorie von Harry must have been eating bzw. von Harry devours s/np ist.

(15) a. These apples, Harry must have been eating.

b. apples which Harry devours

Die vorangestellte NP these apples und das Relativpronomen which sind in der Analyse von (15) jeweils Funktoren, die s/np zum Argument nehmen.

Aus Platzgründen kann ich hier leider nicht darauf eingehen, wie die Kategorie für Harry must have been eating bestimmt wird. Dafür sind weitere Regeln für eine so genannte Typanhebung nötig.

Obwohl es die Kategorialgrammatik schon seit den 30er Jahren gibt, wurden erst in den 70er, 80er und 90er Jahren die Phänomene behandelt, die vorher transformationell behandelt wurden. Auch bei den phrasenstrukturbasierten Theorien hat es sehr lange gedauert, bis echte, transformationslose Alternativen wie die Lexikalisch Funktionale Grammatik (LFG), die Generalisierte Phrasenstrukturgrammatik (GPSG) und die Baumadjunktionsgrammatik (Tree Adjoining Grammar, TAG) entstanden. 


\section{Generalisierte Phrasenstrukturgrammatik}

Die GPSG wurde Ende der 1970er Jahre, Anfang der 1980er Jahre als transformationslose Alternative $\mathrm{zu}$ den chomskyschen Theorievarianten entwickelt. Die Kategorien waren Bündel von Merkmal-Wert-Paaren und die Regeln im Wesentlichen Phrasenstrukturregeln. Allerdings gab es eine wichtige Neuerung in Bezug auf die Regeln: Es wurde von der linearen Abfolge abstrahiert. Die Regeln standen nur noch für unmittelbare Dominanz (immediate dominance, ID), aber nicht mehr für die Abfolge der Konstituenten auf der rechten Regelseite (linear precedence, LP). Damit besteht die Möglichkeit, die Abfolgen in (10) und (11) mittels zweier Regeln zu erfassen: eine für die beiden Abfolgen der Argumente des zweistelligen Verbs kennt und eine für die sechs Abfolgen der Argumente des dreistelligen Verbs gibt und Verberst- und Verbletztstellung. Zu den Details einer solchen Analyse siehe Uszkoreit (1987). Mit der Ausklammerung der Linearisierungsregeln ist es dann auch möglich, allgemeine Stellungsregularitäten $\mathrm{zu}$ erfassen. So stellt Uszkoreit eine (verletzbare) Regel auf, die besagt, dass Pronomina vor Nicht-Pronomina angeordnet werden. Diese Regel ist unabhängig davon, ob das Verb zwei- oder dreistellig ist. In einfachen Phrasenstrukturgrammatiken müsste man die entsprechenden Beschränkungen über die Phrasenstrukturregeln formulieren, was eindeutig redundant wäre und die eigentliche Generalisierung nicht erfassen würde.

Eine weitere Innovation bei der GPSG waren die Metaregeln. Metaregeln sind Aussagen darüber, welche Regeln zu einer Grammatik gehören. So kann man ausdrücken, dass es immer, wenn es Regeln gibt, die bestimmte Eigenschaften aufweisen, auch Regeln geben muss, die bestimmte andere Eigenschaften aufweisen. Zum Beispiel kann eine entsprechende Metaregel für jede Regel, die einen Aktivsatz lizenziert, eine neue Regel definieren, die den entsprechenden Passivsatz lizenziert. Die Metaregel muss nur verlangen, dass in einer Regel ein Verb mit Subjekt vorkommt und kann dann festlegen, wie die entsprechende Passivregel ohne Subjekt aussieht. Das ähnelt den chomskyschen Transformationsgrammatiken, es gibt jedoch einen kleinen, aber feinen Unterschied: Es wird nicht erst ein Baum erzeugt, der dann transformiert wird, sondern es gibt zwei Regeln: eine für den Aktivsatz und eine für den Passivsatz. Liegt ein Passivsatz vor, wird dieser direkt mit der Passivregel analysiert. Die Aktiv- und die Passivregel sind aber über die Metaregel miteinander verbunden, so dass die Generalisierungen erfasst werden.

Mit Metaregeln und ID/LP-Format haben wir schon zwei Innovationen der GPSG kennengelernt, die größte Innovation war aber die Behandlung der Fernabhängigkeiten. Gazdar (1981) hat ein Verfahren entwickelt, wie man Information über eine lokal fehlende Konstituente (die Spur, die Lücke) lokal im Baum 
weitergeben kann, von Knoten zu Knoten bis zu der Stelle, wo sich eine vorangestellte Konstituente (der Füller) befindet. Auch hier braucht man also keine Transformation, die einen Baum auf einen anderen Baum abbildet, die gewünschten Zusammenhänge lassen sich auch durch lokale Weitergabe von Information transformationslos erfassen. (Die Analyse wird im folgenden Abschnitt im Rahmen der HPSG genauer erklärt werden.)

Eine interessante Eigenschaft der GPSG war, dass die computationelle Mächtigkeit des formalen Apparats genau der Mächtigkeit der kontextfreien Grammatiken entsprach, was man in den 70er und 80er Jahren noch wichtig fand. Mitte der 80er Jahre stellte sich aber heraus, dass es Sprachen gibt, die eine höhere Komplexität als kontextfrei besitzen (Shieber 1985; Culy 1985). Sucht man also nach Beschreibungsmitteln, die alle Sprachen beschreiben können, reicht GPSG in der damals vorgeschlagenen Variante nicht aus. Diese Ergebnisse waren ein Grund dafür, dass die Forschung im Rahmen der GPSG quasi über Nacht eingestellt wurde und sich die meisten der aktiven Wissenschaftler dem Nachfolgemodell HPSG zuwandten.

Ein anderer wesentlicher Nachteil der GPSG ist die Repräsentation von Valenz. Die Valenzinformation lebt nur in einer Zahl. In der Grammatik von Uszkoreit (1987) hatten Verben wie kennen die 6 und Verben wie helfen die 7. (16) zeigt entsprechende Regeln. H steht hier für Kopf (head), d. h. für das Verb.

$$
\begin{array}{ll}
\mathrm{V} 3 \rightarrow \mathrm{H}[6], \mathrm{N} 2[\mathrm{CASE} \text { nom], N2[CASE acc] } & \text { (lieben, suchen) } \\
\mathrm{V} 3 \rightarrow \mathrm{H}[7], \mathrm{N} 2[\mathrm{CASE} \text { nom], N2[CASE dat] } & \text { (helfen, vertrauen) }
\end{array}
$$

Erst wenn ein Element mit einer entsprechenden Zahl in eine Regel eingesetzt wird, ist klar, welche Argumente vorhanden sein müssen. Da es aber morphologische Prozesse gibt, die sensitiv dafür sind, ob es ein Akkusativobjekt gibt oder nicht (zum Beispiel die -bar-Derivation), ist die GPSG-Repräsentation von Valenz nicht ausreichend. Pauline Jacobson (1987), die im Rahmen der Kategorialgrammatik arbeitet, hat die Repräsentation von Valenz in ihrer Rezension des GPSG-Buches von 1985 ebenfalls kritisiert und weitere problematische Aspekte gut herausgearbeitet. Ein weiteres Problem für die Repräsentation der Valenz in GPSG sind partielle Voranstellungen. Im Deutschen ist es möglich, eine Teilmenge der Argumente eines Verbs zusammen mit diesem ins Vorfeld zu stellen:

(17) Ein Märchen erzählen wird er ihr wohl müssen.

Da die Valenz nur in einer Zahl kodiert ist, ist im Mittelfeld nicht klar, welche Argumente bereits im Vorfeld realisiert sind und welche noch im Mittelfeld 
realisiert werden müssen. Nerbonne (1986) und Johnson (1986) haben deshalb die Repräsentation der Valenz in GPSG in Richtung Kategorialgrammatik verändert (siehe auch Müller (2016: Abschnitt 5.5) zu Problemen mit Morphologie und der Voranstellung von Verbalphrasenteilen). Die HPSG, das Nachfolgemodell der GPSG, ist letztendlich eine Mischung von GPSG (Ivan Sag) und Kategorialgrammatik (Carl Pollard) und hat somit die GPSG-Probleme mit der Valenz nicht mehr. Die Stärken beider Ansätze konnten vereinigt werden.

\section{Head-Driven Phrase Structure Grammar}

Die HPSG ist die Nachfolgetheorie der GPSG (Pollard \& Sag 1987, 1994). Sie ist ein Best-Of aus verschiedenen Frameworks (Kategorialgrammatik, GPSG, Government \& Binding und Dependenzgrammatik) und insbesondere die Arbeiten von Ivan Sag waren ab 1995 stark durch die Konstruktionsgrammatik geprägt. Die HPSG-Varianten Constructional HPSG (Sag 1997) und Sign-Based Construction Grammar (Sag 2010, 2012) tragen den Konstruktionsbegriff auch im Namen.

Im Gegensatz zur GPSG ist die HPSG eine lexikalistische Theorie, d. h. Information über Valenz von Köpfen ist bei den Köpfen im Lexikon spezifiziert. (18) zeigt die Valenzlisten für kennen und helfen.
(18)
a. kennen, lieben, suchen: 〈 NP[nom], NP[acc $]\rangle$
b. helfen, vertrauen:
$\langle\mathrm{NP}[$ nom $], \mathrm{NP}[$ dat $]\rangle$

Wie bei der Kategorialgrammatik werden die Argumente des Kopfes eins nach dem anderen mit dem Kopf verbunden. ${ }^{2}$ Abbildung 4.4 zeigt das für einen einfachen Satz mit Verbletztstellung. Die Valenzlisten von Köpfen enthalten Beschreibungen der jeweiligen Argumente. Diese Beschreibungen werden mit den Beschreibungen der tatsächlich vorhandenen Elemente identifiziert. So wird zum Beispiel die Beschreibung des Akkusativobjektes von kennen mit der Beschreibung des Pronomens ihn identifiziert. Die Identifikation, die sogenannte Strukturteilung, wird durch Boxen mit entsprechenden Zahlen darin

2 Das ist nicht zwingend aus formalen Gründen so, für das Englische werden auch Strukturen angenommen, in denen ein Kopf mit all seinen Argumenten in einem Schritt verknüpft wird (Pollard \& Sag 1994; Ginzburg \& Sag 2000), aber für das Deutsche sind binär verzweigende Strukturen sinnvoll, da Adjunkte überall zwischen den Argumenten stehen können (Müller 2017a). Ich nehme auch für Sprachen wie Englisch binär verzweigende Strukturen an (Müller 2015, 2017b). 


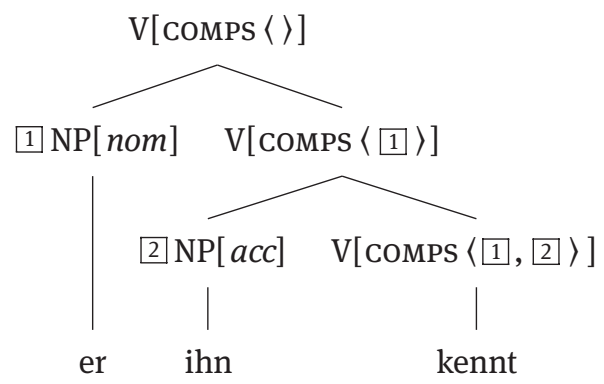

Abb. 4.4: Analyse eines Satzes mit Verbletztstellung.

angezeigt. Wenn ein Kopf mit einem Argument kombiniert wurde, wird dieses Argument nicht mehr in der Valenzliste des sich ergebenden linguistischen Objekts repräsentiert. Nach der Kombination von kennt mit seinem Akkusativobjekt enthält die Valenzliste von ihn kennt kein Akkusativobjekt mehr. ihn kennt braucht lediglich ein Subjekt und dieses wird dann auch im nächsten Schritt mit ihn kennt kombiniert, so dass letztendlich ein linguistisches Objekt entsteht, das eine leere comps-Liste hat.

HPSG benutzt für alle linguistischen Beschreibungsebenen (Phonologie, Morphologie, Syntax, Semantik, Informationsstruktur) Merkmal-Wert-Paare. Der Wert eines Merkmals kann atomar sein, wie zum Beispiel $s g$ und $p l$ als Werte des NUMERUs-Merkmals, oder er kann komplex sein. Als Beispiel für einen komplexen Wert sei hier der Wert des Merkmals HEAD erklärt. Der Wert von HEAD entspricht aller Information, die zwischen einem Kopf (einem Wort) und dessen Projektionen geteilt wird. So wird zum Beispiel der Kopfwert von gibt durch die Beschreibung in (19) beschrieben:

(19) $\left[\begin{array}{l}\text { verb } \\ \text { vFORM fin }\end{array}\right]$

verb ist dabei der Typ der Merkmalbeschreibung. Merkmalstrukturen vom Typ verb haben immer ein vFORM-Merkmal. Im konkreten Fall ist der Wert des VFORM-Merkmals fin. fin ist wieder ein Typ, es gehören jedoch keine weiteren Merkmale zu diesem Typ. Genau wie $s g$ und $p l$ ist fin ein atomarer Typ. Der Wert des Valenzmerkmals comps ist eine Liste. Es gibt noch ein weiteres Valenzmerkmal für die Selektion von Spezifikatoren, das hier jedoch aus Platzgründen nicht besprochen werden kann.

Abbildung 4.5 zeigt die Projektion von Kopfmerkmalen entlang der Projektionslinie. Der oberste Knoten hat eine leere ComPs-Liste und unter HEAD die Information, dass es sich um die Projektion eines finiten Verbs handelt. Diese 


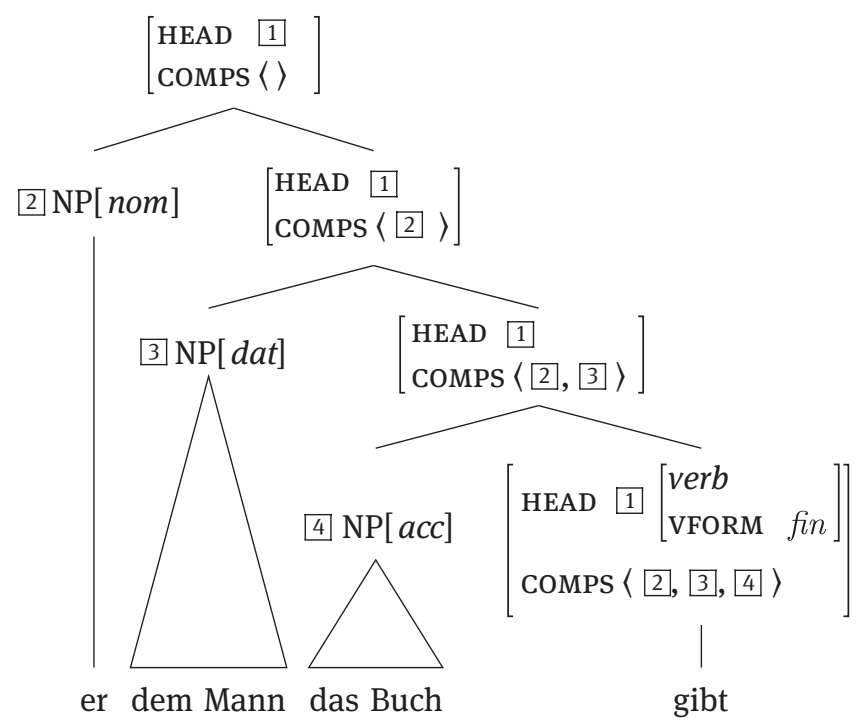

Abb 4.5: Projektion von Kopfmerkmalen eines Verbs.

komplexe Kategorie entspricht der Kategorie S in den einfachen Phrasenstrukturgrammatiken, die im Abschnitt 2 vorgestellt wurden. Die angenommenen Merkmalbeschreibungen sind teilweise sehr komplex, weil für die linguistischen Ebenen jeweils eigene Merkmalsnamen angenommen werden. So steht Information bezüglich phonologischer Eigenschaften unter PHON, syntaktische und semantische Information unter SYNSEM und innerhalb von SYNSEM wird dann in CAT (syntaktische Kategorie, enthält Wortart und Valenz) und coNT (semantischer Beitrag) unterteilt. Interessant ist, dass komplexe sprachliche Ausdrücke, d.h. solche, die aus mehreren Konstituenten bestehen, intern strukturiert sind. Phrasenstrukturregeln als solche gibt es in der HPSG nicht. Auch Mutter-TochterKonfigurationen werden mittels Merkmal-Wert-Paaren dargestellt. So wird zum Beispiel die komplexe Nominalphrase in Abbildung 4.6 mithilfe der Merkmalbeschreibung in (20) beschrieben:

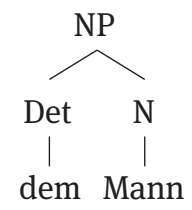

Abb. 4.6: Visualisierung der Struktur für dem Mann. 
(20)

$$
\left[\begin{array}{l}
\text { PHON }\langle\text { dem Mann }\rangle \\
\text { DTRS }\langle[\text { PHON }\langle\text { dem }\rangle],[\text { PHON }\langle\text { Mann }\rangle]\rangle
\end{array}\right]
$$

Die Töchter im Baum werden in den Merkmalbeschreibungen in einer Liste repräsentiert, die komplexe Beschreibungen der Töchter enthält. Die Töchter können selbst wieder Töchter enthalten. Die entsprechende Information wäre dann Bestandteil der Beschreibung der Töchter. Lexikonregeln sind Strukturen mit einem Mutterknoten und einem Tochterknoten und können dementsprechend genauso wie Syntaxbäume dargestellt werden (Meurers 2001). Schemata (Grammatikregeln), Lexikonregeln und Lexikoneinträge haben alle einen Typ, da jede Merkmalstrukutr von einem bestimten Typ sein muss. Die Typen sind in Hierarchien organisiert, so dass man Generalisierungen über Phrasen, über Lexikonregeln und über Lexikoneinträge gut erfassen kann. Viel mehr kann aus Platzgründen hier nicht zu den technischen Details gesagt werden. Der wichtige Punkt ist nur, dass alle Information in HPSG mit Merkmal-Wert-Paaren ausgedrückt wird.

Nach diesen Anmerkungen zur Repräsentation linguistischer Information mit Merkmal-Wert-Paaren möchte ich nun wieder zu Phänomenen kommen, die in der Transformationsgrammatik mithilfe von Transformationen analysiert werden. Dazu gehört das Passiv, die Verberststellung im Deutschen und die Voranstellung von Konstituenten (Verbzweitstellung im Deutschen).

Das Passiv wird wie auch in der Kategorialgrammatik mithilfe von Lexikonregeln analysiert: $\mathrm{Zu}$ jedem Verb gibt es einen Verbstamm, der entsprechend flektiert werden muss, um dann in syntaktischen Strukturen verwendet werden zu können.

Die Aktivformen haben dabei die im Lexikoneintrag vorangelegte Valenz, die Passivformen haben eine reduzierte Valenz (Heinz \& Matiasek 1994; Müller 2003). Es werden also nicht wie bei der GPSG Regeln in Beziehung zueinander gesetzt, die Aktiv- und Passivstrukturen lizenzieren können, sondern es werden Lexikonelemente zueinander in Beziehung gesetzt (ein Verbstamm zu einer Passivform). ${ }^{3}$

Die Analyse der Verbstellung baut auf Vorschlägen, die im Rahmen von GPSG und Kategorialgrammatik gemacht wurden, auf. Abbildung 4.7 zeigt die Analyse von (21):

3 Die Passivanalye kann hier nicht im Detail besprochen werden. Sie geht auf die Analyse von Haider (1986) zurück und unterscheidet auch zwischen lexikalischem und strukturellem Kasus. 
(21) Kennt jeder diesen Mann?

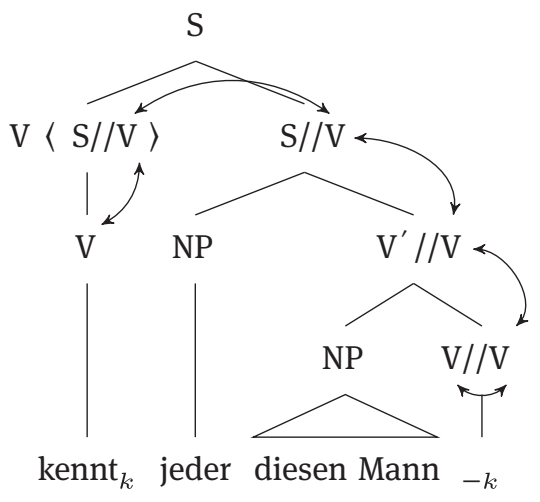

Abb. 4.7: Analyse der Verbstellung in HPSG.

Die Position des Verbs in Letztstellung wird durch ein phonologich leeres Element (eine Spur) besetzt, das aber ansonsten dieselben syntaktischen und semantischen Eigenschaften hat wie ein normales Verb. Die Information über diese Eigenschaften wird in der Verbspur mit dem Wert des Kopfmerkmals DSL (in der Abbildung durch zwei Striche ,// gekennzeichnet) geteilt. Da DSL ein Kopfmerkmal ist, ist es an allen Projektionen der Verbspur verfügbar. In der Erststellung befindet sich nun ein Verb, das ein S//V selegiert, d.h., es wird ein vollständiger Satz verlangt, dem genau das Verb fehlt, das in Initialposition steht. Das spezielle Lexikonelement für kennt, das ein S//V selegiert, wird mittels einer Lexikonregel aus dem Lexikonelement für kennt abgeleitet. Das spezielle Verb für die Verberststellung ähnelt einem Komplementierer wie z.B. dass: Beide verlangen einen Satz als Argument, der Komplementierer einen Satz mit overtem Verb, das vorangestellte Verb einen Satz mit covertem Verb, d. h. mit einer Verbspur. Die Parallelität zwischen Komplementierer und Verb in Erststellung ist erwünscht, da es viele Gemeinsamkeiten gibt (Höhle 2018).

Abbildung 4.8 zeigt nun die Analyse eines Verbzweitsatzes. Die Grundidee ist wieder die von Gazdar (1981): An der Stelle, an der ein Element normalerweise stehen könnte, wird die Information darüber, dass es lokal fehlt, im Baum nach oben gegeben. Das Merkmal, das in HPSG dafür benutzt wird, ist das SLASH-Merkmal. Im Baum ist das mit ,/ gekennzeichnet. Wie bei der Verbspur wird die Information bzgl. der fehlenden Konstituente im Baum nach oben gegeben. Für die Kombination von finitem Satz und extrahierter Konstituente gibt es dann ein spezielles Schema, das die vorangestellte Konstituente mit der Information über die Lücke identifiziert. Der Unterschied zwischen 


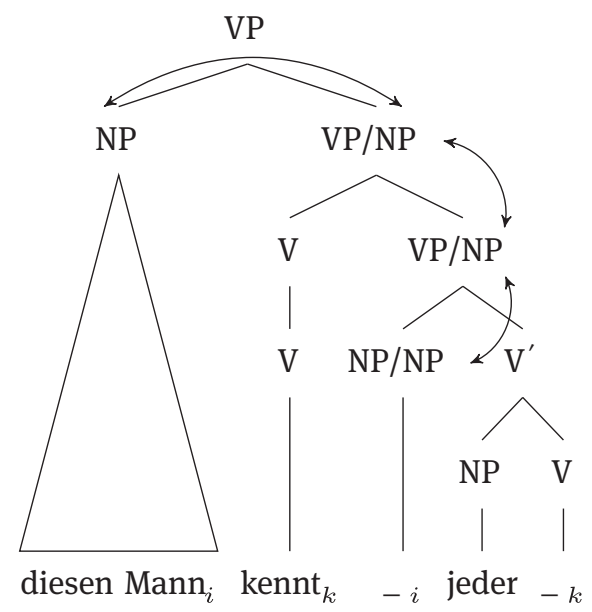

Abb. 4.8: Analyse von Fernabhängigkeiten in HPSG.

Kopfbewegung und Extraktion ist, dass die Kopfbewegung über das Merkmal DSL läuft und die Konstituentenbewegung über SLASH. DSL ist ein Kopfmerkmal, weshalb Verben nicht aus dem Satz herausbewegt werden können: Die Information über das fehlende Element kommt ja nur bis dahin, wo die Kopfinformation hinkommt. Die SLASH-Information wird hingegen auch über Satzgrenzen weitergegeben und somit ist es möglich, Extraktion aus Sätzen heraus $\mathrm{zu}$ analysieren.

\section{Tree Adjoining Grammar}

TAG unterscheidet sich radikal von allen anderen hier vorgestellten Theorien: In TAG sind die Lexikoneinheiten von Valenzträgern halb-fertige Bäume, die mit anderen Bäumen zu vollständigen Bäumen zusammengebaut werden können. In den lexikalischen Bäumen gibt es für gewöhnlich offene Stellen (Slots). Zum Beispiel zeigt die Abbildung 4.9 einen Baum für laughs, der einen offenen, durch $\downarrow$ markierten NP-Slot hat. In diesen Slot kann der Baum für John eingesetzt werden. Das Ergebnis ist dann ein vollständiger Baum für John laughs.

Neben der Substitution, d. h. der Einsetzung in entsprechende Slots, gibt es in TAG auch noch die Adjunktion. Dazu wird ein vorhandener Baum an wohldefinierten Stellen aufgebrochen und Material in der Mitte eingesetzt. Abbildung 4.10 zeigt die Adjunktion von always an die VP laughs. Der mit Stern markierte Knoten muss im Baum, an den adjungiert wird, vorhanden sein. Dort wird der Baum aufgespalten und das Adjunkt eingesetzt. 


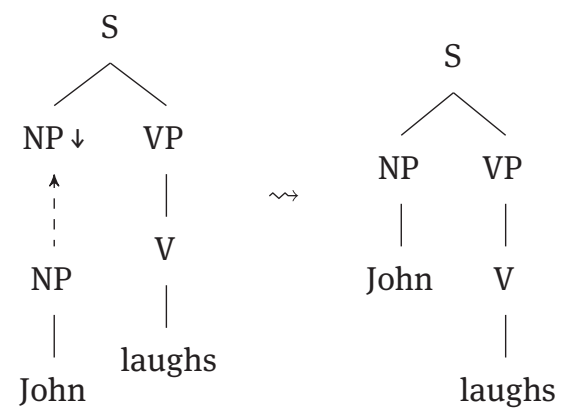

Abb. 4.9: Analyse von John laughs in TAG. Die NP wird in den Baum des Verbs eingesetzt.

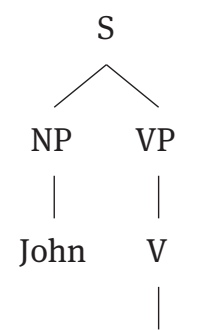

laughs

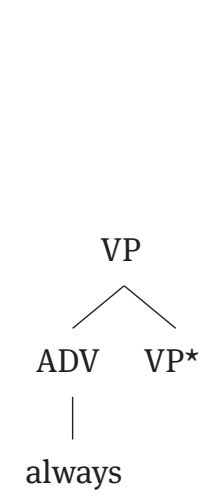

Abb. 4.10: Adjunktion eines Adverbs an eine VP.

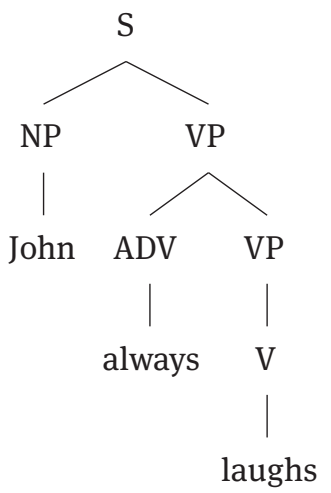

Die Aktiv-Passiv-Relation wird, wie in Kategorialgrammatik, LFG und HPSG auch, im Lexikon modelliert: $\mathrm{Zu}$ jedem Aktivbaum gibt es auch einen entsprechenden Passivbaum (Kroch \& Joshi 1985: 50-51). Das sieht nach Transformationen aus, unterscheidet sich aber fundamental von diesen: Es wird nicht eine komplette Phrase analysiert und dann mittels Transformationen umgewandelt, sondern es werden Lexikonelemente zueinander in Beziehung gesetzt. Bei der Verarbeitung von Sätzen werden die jeweils passenden Lexikonelemente direkt kombiniert.

Interessant ist auch die Analyse der Fernabhängigkeiten. Für Sätze wie (22) geht man davon aus, dass $w_{h o}$ that Bill likes ${ }_{-i}$ ein Baum ist, an den obligatorisch ein anderer Baum adjungiert werden muss.

(22) $\mathrm{Who}_{i}$ did John tell Sam that Bill likes ${ }_{i}$ ? 
who befindet sich also zuerst in einem lokalen Baum mit seinem Kopf likes und in diesen Baum muss dann beliebig komplexes weiteres Material eingesetzt werden.

TAG ist für die Analyse von Idiomen sehr interessant, da lexikalische Bäume ja beliebig komplex sein können. So kann man z. B. einen Baum für take advantage of mit entsprechenden offenen Slots definieren und hat alle Idiombestandteile vereint und die Relation, in der sie zueinander stehen, im Baum festgeschrieben (Abeillé \& Schabes 1989). Zu einer Analyse mit ähnlichen phrasalen Lexikoneinträgen in HPSG siehe Richter \& Sailer (2009).

Hier wurde nur die einfachste TAG-Variante besprochen. Es gibt noch Erweiterungen, die es möglich machen, bestimmte Verbalkomplexe zu analysieren (Joshi, Becker \& Rambow 2000). Außerdem gibt es die feature-based TAG, bei der Merkmalstrukturen statt atomarer Kategorien verwendet werden (VijayShanker \& Joshi 1988).

\section{Lexikalisch Funktionale Grammatik}

LFG verwendet Phrasenstrukturgrammatiken, wie sie im Abschnitt 2 vorgestellt wurden. Die Phrasenstrukturregeln sind für die Konsitutentenstrukutren von Äußerungen zuständig, die sogenannten c-Strukturen. Die c-Strukturregeln werden aber durch Annotationen ergänzt, die Symbole in den Regeln zu Merkmalstrukturen - den f-Strukturen - in Beziehung setzen. Abbildung 4.11 zeigt die Analyse des Beispielsatzes in (23):

(23) [dass] David den Apfel verschlingt

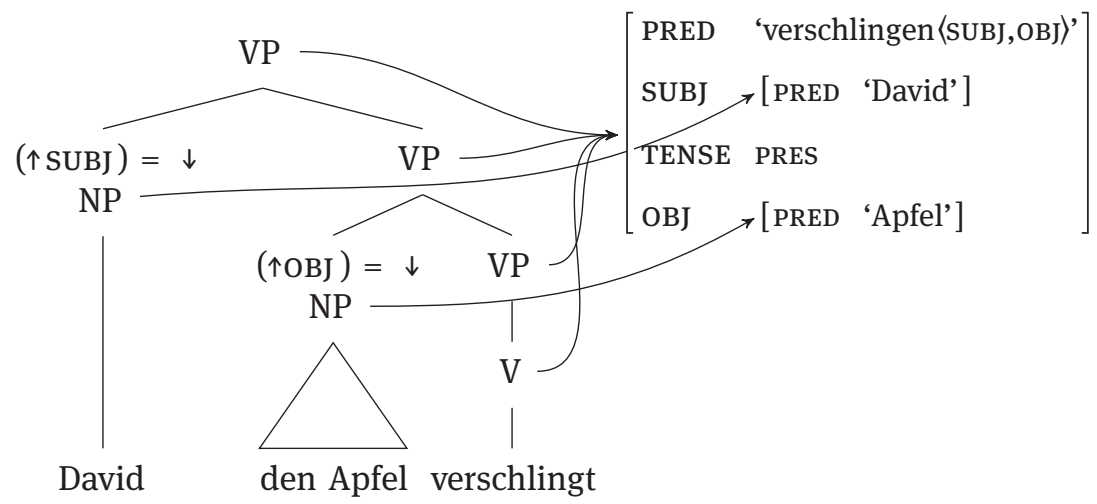

Abb. 4.11: Analyse der SOV-Stellung nach Berman (1996). 
Diese c-Struktur mit der dazugehörigen f-Struktur wird durch zwei Phrasenstrukturregeln lizenziert. Eine davon macht aus V eine VP, die andere kombiniert eine VP mit einer NP, wobei das Ergebnis der Kombination wieder eine VP ist. Diese Regel ist in (24) zu sehen:

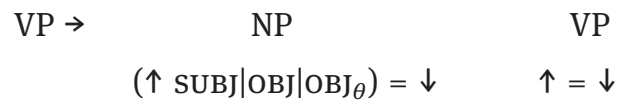

Unter den Kategoriesymbolen stehen Beschränkungen, die sich auf die f-Strukturen beziehen. $\uparrow=\downarrow$ bedeutet, dass die f-Struktur, die zu einem bestimmten Knoten gehört $(\downarrow)$, mit der f-Struktur des Mutterknotens $(\uparrow)$ identisch ist. Für die Regel in (24) bedeutet das, dass der VP-Knoten auf der rechten Regelseite zur selben f-Struktur gehört, wie der auf der linken Regelseite. In der Abbildung ist das dadurch dargestellt, dass von den VP-Knoten Pfeile zur selben f-Struktur gehen. Die Regel VP $\rightarrow$ V hat entsprechende Annotationen, so dass auch V und VP auf dieselbe f-Struktur abgebildet werden. Jedes Lexikonelement hat ein PRED-Merkmal. Der Wert kann komplex sein und grammatische Funktionen erwähnen. In unserem Beispiel haben wir als PRED-Wert verschlingen〈SUBJ, OBJ〉. Das ist die Valenzrepräsentation in LFG: verschlingen braucht ein Subjekt und ein Objekt. In LFG gibt es Wohlgeformtheitsprinzipien für f-Strukturen: Kohärenz und Vollständigkeit. Die Vollständigkeit verlangt, dass alle grammatischen Funktionen, die in PRED-Werten vorkommen, auch in den Merkmalstrukturen, in denen sich der PRED-Wert befindet, vorhanden sein müssen. Kohärenz verlangt, dass keine anderen grammatischen Funktionen dort auftreten. Bei einem einstelligen Verb mit Subjekt dürfte also kein oвJMerkmal in der f-Struktur auftauchen.

Wie in anderen lexikalistischen Theorien (Kategorialgrammatik, HPSG, L-TAG) wird das Passiv über Lexikonregeln analysiert (Bresnan 1982), d. h. eine Regel lizenziert zu passivierbaren Verben ein weiteres Lexikonelement, das entsprechend andere grammatische Funktionen verlangt. Die Passivtheorie wurde noch weiterentwickelt und so gibt es jetzt eine Sub-Theorie, die Lexical Mapping Theorie (Bresnan \& Kanerva 1989), die die Zuordnung von Argumenten $\mathrm{zu}$ grammatischen Funktionen regelt. Aus Platzgründen kann diese hier nicht besprochen werden.

Einige LFG-Varianten gehen davon aus, dass alle Elemente auf rechten Regelseiten optional sind. Dass dann in Äußerungen alles aufgeht und notwendige Äußerungsbestandteile vorhanden sind, wird über das Kriterium der Vollständigkeit sichergestellt. Dieses Setup erlaubt Analysen von Umstellungen, die ganz ohne leere Elemente auskommen. So kann man den Satz in (25) so analysieren, wie das Abbildung 4.12 zeigt. 
(25) Den Apfel verschlingt David.

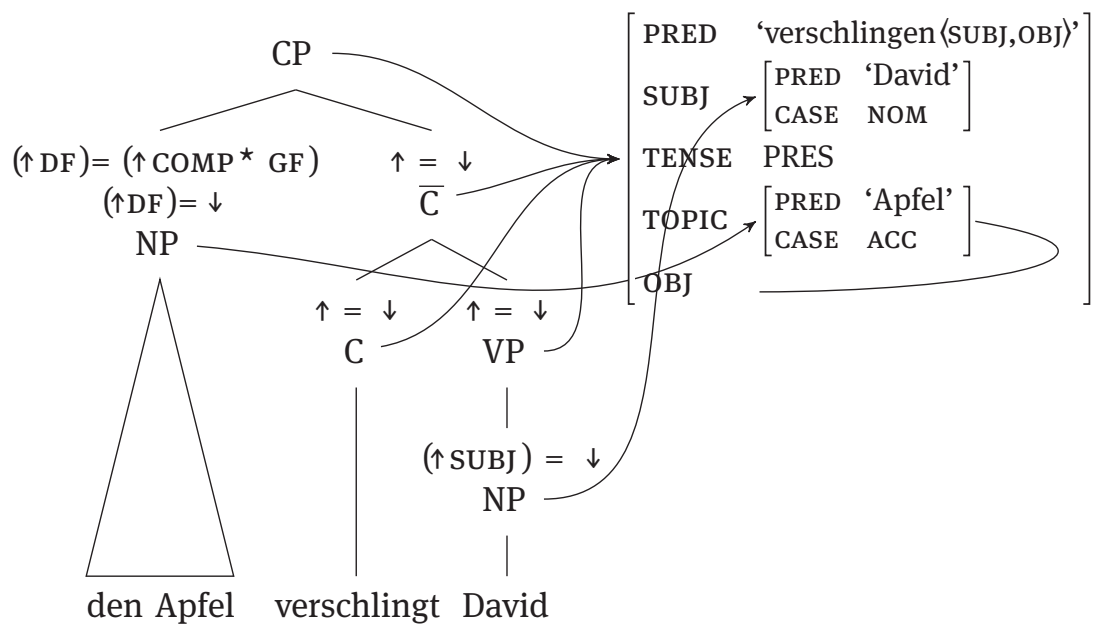

Abb. 4.12: Analyse der V2-Stellung nach Berman (1996).

Die VP enthält gar kein Verb, weil dieses optional ist und in der entsprechenden Regel nicht realisiert wurde. Ohne ein Verb gäbe es aber in der f-Struktur zwar ein SUBJ- aber kein PRED-Merkmal, d. h., es muss irgendwo anders ein Verb geben. Das Verb steht bei Verbzweitsätzen in der C-Position (wo ansonsten die Komplementierer stehen). Die $\overline{\mathrm{C}}$-Regel ist so annotiert, dass sowohl das C-Element als auch die VP zur selben f-Struktur beitragen. So kommen Subjekt und PRED des Verbs in dieselbe f-Struktur.

Interessant ist nun, wie das Element im Vorfeld zu der f-Struktur beiträgt, zu der das vorangestellte Element gehört. Schließlich befindet sich den Apfel ja nicht in der VP, in der es normalerweise stehen würde. Die Zuordnung des Vorfeldelements zu einer entsprechenden f-Struktur erfolgt wieder über Annotation der Regeln. Über das Vorfeldelement wird gesagt, dass es eine Diskursfunktion (Topic oder Focus) beisteuert. Diese Diskursfunktionen sind als DF zusammengefasst. Außerdem wird über das Vorfeldelement gesagt, dass die entsprechende Diskursfunktion mit einer beliebig tief eingebetteten grammatischen Funktion identisch sein muss. Grammatische Funktionen wie suBJ und OBJ sind unter GF zusammengefasst. Im Beispiel in Abbildung 4.12 ist den Apfel das Topic und wird als solches in die f-Struktur eingetragen. Außerdem ist das Topic mit der grammatischen Funktion овJ identisch, was durch die geschwungene Kurve in der Merkmalstruktur dargestellt wird. Diese geschwungenen Kurven entsprechen den durch Boxen mit Nummern gekennzeichneten Struktur- 
teilungen der HPSG. Durch die Identifizierung des Topics mit dem oBJ gibt es dann in der f-Struktur das vom PRED geforderte OBJ.

In Sätzen wie (26) bettet das Matrixverb ein Satzkomplement ein. Die entsprechende grammatische Funktion für Satzkomplemente ist comp. Der Wert von COMP ist eine f-Struktur, die dann das PRED von treffen und einen entsprechenden SUBJ- und oBJ-Wert enthält.

(26) Wen glaubst du, dass ich getroffen habe?

Das Vorfeldelement ist so annotiert, dass es sich eine grammatische Funktion sucht, die beliebig tief eingebettet sein kann. Das wird durch das comP* sichergestellt, was für eine Folge von beliebig vielen comp steht. Im in Abbildung 4.12 gegebenen Beispiel gibt es kein comp, aber es könnte auch eins, zwei oder mehr geben. Diese Unbestimmtheit wird funktionale Ungewissheit genannt (functional uncertainty). In der Analyse von (26) wird das Vorfeldelement mit сомр|овј identifiziert. Obwohl das Element im Vorfeld nicht in der lokalen Domäne von getroffen steht, kann es doch zu der entsprechenden f-Struktur beitragen und die Vollständigkeit kann lokal überprüft werden.

Diese Skizze hat einen anderen Weg gezeigt, Phrasenstrukturgrammatiken so zu erweitern, dass man die entsprechenden Phänomene ohne Transformationen erklären kann. Zu den Details siehe Dalrymple (2001), Bresnan, Asudeh, Toivonen \& Wechsler (2015) und im Vergleich zu anderen Theorien auch Müller (2016).

\section{Konstruktionsgrammatik}

Aus formaler Sicht gibt es zur Konstruktionsgrammatik nicht viel zu sagen. Die meisten Analysen sind nicht ausreichend formalisiert. Ausnahmen sind die Berkeley Construction Grammar (Fillmore 1988; Kay \& Fillmore 1999; Fried 2015) und die Sign-Based Construction Grammar (Sag 2012; Michaelis 2013). Die Grundannahmen der Berkeley Construction Grammar sind jedoch inkonsistent (Müller 2006), weshalb sie zugunsten von Sign-Based Construction Grammar aufgegeben wurde. Sign-Based Construction Grammar (SBCG) ist eine HPSGVariante (Sag 2010: 486) und dasselbe kann man auch von anderen formalen Konstruktionsgrammatiken wie zum Beispiel Embodied Construction Grammar (Bergen \& Chang 2005) sagen (Müller 2016: Kap. 10.6.3). Van Trijp (2013) hat sich bemüht zu zeigen, dass Fluid Construction Grammar fundamental anders ist als HPSG, ist aber in jedem einzelnen Punkt gescheitert (Müller 2017c). 
SBCG unterscheidet sich von anderen CxG-Varianten dadurch, dass sie explizit lexikalisch ist (Sag, Boas \& Kay 2012), d.h. Valenzinformation ist so wie in Abschnitt 6 dargestellt in Lexikoneinträgen repräsentiert. Das klassische Konstruktionsgrammatikbeispiel sind Resultativkonstruktionen wie die in (27), wo argumentiert wird, dass die Bedeutung des Gesamtausdrucks mehr als die Bedeutung der Teile enthält (nämlich die Kausativ-Relation: Sein Fischen bewirkt, dass der Teich leer wird).

(27) Er fischt den Teich leer.

In Theorien wie HPSG, Kategorialgrammatik und LFG geht man normalerweise davon aus, dass eine Lexikonregel ein weiteres Lexikonelement für fischt lizenziert, das dann ein Resultativprädikat, dessen Subjekt und ein eigenes Subjekt verlangt (Verspoor 1997, Wechsler 1997, Wechsler \& Noh 2001; Müller 2002: Kapitel 5; Simpson 1983). Die meisten Konstruktionsgrammatikvarianten nehmen dagegen an, dass es eine phrasale Konfiguration gibt, die die entsprechende Bedeutung beisteuert. Das ist letztendlich eine Rückkehr zu GPSG-Positionen mit all den damit verbundenen Problemen (Müller \& Wechsler 2014: Abschnitt 4.2; Müller 2018). Ich habe in den letzten Jahren immer wieder auf diese Probleme hingewiesen. Eine Lösung liegt bislang nicht vor und ist auch meiner Meinung nach nicht möglich: Valenz und Valenzalternationen sind lexikalische Eigenschaften und müssen als solche erfasst werden. Allerdings gibt es durchaus phrasale Konfigurationen, die auch, wie in der $\mathrm{CxG}$ vorgeschlagen, als phrasale Konstruktionen analysiert werden sollten (Jacobs 2008; Jackendoff 2008). Darauf komme ich im nächsten Abschnitt zurück.

\section{Minimalismus}

In diesem Beitrag wurde festgehalten, dass Ideen und Analysen aus der Transformationsgrammatik auch in andere Frameworks aufgenommen wurden. Insbesondere die Periode von Government \& Binding war, was das Deutsche angeht, sehr fruchtbar. In der GB-Zeit gab es repräsentationelle Modelle, die dem, was in HPSG und LFG gemacht wurde und wird, recht ähnlich waren. Mit dem Minimalistischen Programm (Chomsky 2001, 2008, 2013) hat sich Chomsky eindeutig wieder in Richtung derivationeller Analysen bewegt. Der ganze Unterbau mit Enumerationen, Workspaces, transderivationellen Beschränkungen, Ökonomie der Ableitungen (fewest steps), multiple spell out, Phasen, Feeding und Bleedeing usw. ist aus psycholinguistischer Sicht höchst fragwürdig. Dinge wie Labeling sind unnötige Spielereien, die keinerlei Gewinn oder Fort- 
schritt im Vergleich zu den 80er Jahren gebracht haben (Müller 2013). Lässt man diese ganzen Dinge weg, dann bleiben die Grundoperationen, die zur Verknüpfung von linguistischen Objekten angenommen werden. Diese sind Internal Merge und External Merge. External Merge kombiniert ein Element, das ein anderes Element selegiert, mit dem selegierten Element. External Merge entspricht der Funktionalapplikation aus der Kategorialgrammatik (Berwick \& Epstein 1995) bzw. entsprechenden HPSG-Schemata (Müller 2013). Internal Merge verknüpft ein Element, das in einer komplexen Struktur enthalten ist, mit dieser Struktur. Das heißt, ein Element wird aus einer Struktur entfernt und dann mit der Reststruktur kombiniert. Wie ich in Müller (2013) gezeigt habe, entspricht das ziemlich genau dem HPSG-Schema, das von Pollard \& Sag (1994) für die Analyse von Fernabhängigkeiten vorgeschlagen wurde.

Was im Minimalismus wie auch in der Dependenzgrammatik fehlt, ist die Möglichkeit, kopflose Strukturen mit mehreren Bestandteilen zu analysieren. Natürlich kann man immer stipulieren, dass ein bestimmtes Element der Kopf ist, aber mitunter ist das einfach nicht sinnvoll, wie Jackendoff (2008) gezeigt hat. Meiner Meinung nach ist sein überzeugendstes Beispiel die N-P-N-Konstruktion, die in Beispielen wie (28) vorliegt:

(28) a. Auge um Auge

b. Zeile für Zeile

Hier kann man nicht gut für den Kopfstatus eines der drei Elemente argumentieren. Die Wortgruppen verhalten sich wie NPs, ohne aber die interne Struktur einer NP zu haben. Es gibt keine Determinatoren und die Bildung von für Zeile ist außerhalb dieser Konstruktion deshalb nicht wohlgeformt.

Alternativ zur Festlegung eines der drei vorhandenen Elemente als Kopf bleibt natürlich immer die Annahme eines leeren Kopfes. Im Rahmen des Minimalismus wird gerne von leeren Köpfen Gebrauch gemacht, die alternativen Theorien verbieten leere Elemente jedoch per Dogma oder setzen sie bewusst sehr sparsam ein.

Was man also zu brauchen scheint, ist eine Theorie, die einfach kompositionale Verknüpfungen beschreiben kann (Funktionalapplikation in Kategorialgrammatik, Kombinationsregeln in HPSG und CxG-Varianten, External/ Internal Merge im Minimalismus) und außerdem noch in der Lage ist, idiosynkratische Kombinationen zu beschreiben (HPSG, CxG). 


\section{Zusammenfassung}

Die hier vorgestellten Alternativen zur Mainstream Generative Grammar zeichnen sich dadurch aus, dass sie keine Transformationen benutzen. In der Mainstream Generative Grammar gab es Phasen, in denen die Transformationen keine große Rolle mehr spielten und man konnte Zusammenhänge auch repräsentationell verstehen. Mit dem Minimalistischen Programm ist Chomsky in die entgegengesetzte, in die falsche Richtung gegangen. Die hier vorgestellten Theorien sind prinzipiell mit psycholinguistischen Befunden vereinbar, was für viele Minimalistische Analysen nicht gilt. Einsichten aus der GB-Zeit finden sich in HPSG- und LFG-Analysen wieder. Im Bereich der Nicht-MainstreamGrammatik ist wohl die Konstruktionsgrammatik am einflussreichsten, obwohl viele ihrer Varianten zu problematischen GPSG-ähnlichen Analysen zurückgekehrt sind, ohne dass das irgendwie theoretisch reflektiert würde.

In diesem Aufsatz habe ich die gängigsten der Alternativen zur Mainstream Generative Grammar vorgestellt. Aus Platzgründen konnte ich nur an der Oberfläche kratzen. Es gibt jedoch einige Bücher, die sich explizit dem Theorievergleich widmen und in denen auch die hier besprochenen Frameworks bzw. entsprechende Theorien ausführlicher besprochen werden. $\mathrm{Zu}$ nennen ist hier das Buch von Hagemann \& Staffeldt (2014), in dem ein Zeitungstext in verschiedenen Theorien analysiert wird. Kertész, Moravcsik \& Rákosi (2018) ist ein ähnliches Projekt in englischer Sprache. In meinen Büchern über Grammatiktheorie $(2010 ; 2016)$ vergleiche ich Theorien und erkläre, wie sie Kernbereiche des Deutschen analysieren (Verbstellung in Haupt- und Nebensätzen, V2, Scrambling, Passiv). Die englische Version des Buches enthält im Gegensatz zur deutschen Version zwei neue Kapitel über Minimalismus und Dependenzgrammatik. Wie ich auch in den Grammatiktheoriebüchern dargestellt habe, gibt es viele Gemeinsamkeiten zwischen den Theorien und es werden Bestandteile aus allen gebraucht, um Sprache adäquat zu beschreiben. (Phrasale) Konstruktionsgrammatik und Minimalismus sind gegenwärtig die beiden Extreme, die Wahrheit liegt wohl in der Mitte.

\section{Literatur}

Abeillé, Anne \& Yves Schabes (1989): Parsing idioms in Lexicalized TAG. In Harold Somers \& Mary McGee Wood (Hrsg.), Proceedings of the Fourth Conference of the European Chapter of the Association for Computational Linguistics, 1-9. Manchester, England: Association for Computational Linguistics.

Ajdukiewicz, Kazimierz (1935): Die syntaktische Konnexität. Studia Philosophica 1, 1-27. 
Bergen, Benjamin K. \& Nancy Chang (2005): Embodied Construction Grammar in simulationbased language understanding. In Jan-Ola Östman \& Mirjam Fried (Hrsg.), Construction Grammars: Cognitive Grounding and Theoretical Extensions, 147-190. Amsterdam/Philadelphia: Benjamins.

Berman, Judith (1996): Eine LFG-Grammatik des Deutschen. In Deutsche und französische Syntax im Formalismus der LFG (Linguistische Arbeiten 344), 11-96. Tübingen: Niemeyer.

Berwick, Robert C. \& Samuel David Epstein (1995): On the convergence of the 'Minimalist' Syntax and Categorial Grammar. In Anton Nijholt, Giuseppe Scollo \& Rene Steetskamp (Hrsg.), Algebraic Methods in Language Processing, 143-148. Enschede: University of Twente. http://eprints.eemcs.utwente.nl/9555/01/twlt10.pdf (letzter Zugriff: 27. 03. 2013).

Bierwisch, Manfred (1963): Grammatik des deutschen Verbs (studia grammatica 2). Berlin: Akademie.

Boas, Hans C. \& Ivan A. Sag (Hrsg.) (2012): Sign-based Construction Grammar (CSLI Lecture Notes 193). Stanford, CA: CSLI.

Bresnan, Joan (1982): The passive in lexical theory. In Joan Bresnan (Hrsg.), The Mental Representation of Grammatical Relations (MIT Press Series on Cognitive Theory and Mental Representation), 3-86. Cambridge, MA/London: MIT Press.

Bresnan, Joan, Ash Asudeh, Ida Toivonen \& Stephen Mark Wechsler (2015): Lexical-functional syntax, 2. Aufl. Oxford, UK/Cambridge, USA: Blackwell. doi: 10.1002/9781119105664.

Bresnan, Joan \& Jonni M. Kanerva (1989): Locative inversion in Chichewa: A case study of factorization in grammar. Linguistic Inquiry 20(1), 1-50.

Chomsky, Noam (1957): Syntactic structures (Janua Linguarum / Series Minor 4). The Hague/ Paris: Mouton.

Chomsky, Noam (1968): Language and the mind. Psychology Today 1(9), 48-68. Neudruck als: Chomsky (1976).

Chomsky, Noam (1970): Remarks on nominalization. In Roderick A. Jacobs \& Peter S. Rosenbaum (Hrsg.), Readings in English Transformational Grammar, Kap. 12, 184-221. Waltham, MA/Toronto/London: Ginn and Company.

Chomsky, Noam (1976): Language and the mind. In Diane D. Borstein (Hrsg.), Readings in the Theory of Grammar: From the 17th to the 20th Century, 241-251. Cambridge, MA: Winthrop. Neudruck von: Chomsky (1968).

Chomsky, Noam (1981): Lectures on government and binding. Dordrecht: Foris.

Chomsky, Noam (1995): The Minimalist Program (Current Studies in Linguistics 28). Cambridge, MA/London, England: MIT Press.

Chomsky, Noam (2001): Derivation by phase. In Michael Kenstowicz (Hrsg.), Ken Hale: A Life in Language, 1-52. Cambridge, MA: MIT Press.

Chomsky, Noam (2008): On phases. In Robert Freidin, Carlos P. Otero \& Maria Luisa Zubizarreta (Hrsg.), Foundational Issues in Linguistic Theory: Essays in Honor of Jean-Roger Vergnaud, 133-166. Cambridge, MA: MIT Press.

Chomsky, Noam (2013): Problems of projection. Lingua 130, 33-49.

Clifton, Charles Jr. \& Penelope Odom (1966): Similarity relations among certain English sentence constructions. Psychological Monographs: General and Applied 80(5), 1-35.

Culy, Christopher (1985): The complexity of the vocabulary of Bambara. Linguistics and Philosophy 8, 345-351.

Dalrymple, Mary (2001): Lexical Functional Grammar (Syntax and Semantics 34). New York: Academic Press. 
den Besten, Hans (1983): On the interaction of root transformations and lexical deletive rules. In Werner Abraham (Hrsg.), On the Formal Syntax of the Westgermania: Papers from the 3rd Groningen Grammar Talks, Groningen, January 1981 (Linguistik Aktuell/ Linguistics Today 3), 47-131. Amsterdam/Philadelphia: Benjamins.

Dowty, David R. (1978): Governed transformations as lexical rules in a Montague Grammar. Linguistic Inquiry 9(3), 393-426.

Dowty, David R. (2003): The dual analysis of adjuncts and complements in Categorial Grammar. In Ewald Lang, Claudia Maienborn \& Cathrine Fabricius-Hansen (Hrsg.), Modifying Adjuncts (Interface Explorations 4), 33-66. Berlin: Mouton de Gruyter.

Eroms, Hans-Werner (2000): Syntax der deutschen Sprache de Gruyter Studienbuch. Berlin: De Gruyter.

Fanselow, Gisbert (1990): Scrambling as NP-movement. In Günther Grewendorf \& Wolfgang Sternefeld (Hrsg.), Scrambling and Barriers (Linguistik Aktuell/Linguistics Today 5), 113-140. Amsterdam/Philadelphia: Benjamins.

Fanselow, Gisbert (1993): Die Rückkehr der Basisgenerierer. Groninger Arbeiten zur Germanistischen Linguistik 36, 1-74.

Fillmore, Charles J. (1988): The mechanisms of “Construction Grammar”. In Shelley Axmaker, Annie Jaisser \& Helen Singmaster (Hrsg.), Proceedings of the 14th Annual Meeting of the Berkeley Linguistics Society, 35-55. Berkeley, CA: Berkeley Linguistics Society.

Fodor, Jerry A., Thomas G. Bever \& Merrill F. Garrett (1974): The psychology of language: An introduction to psycholinguistics and Generative Grammar. New York: McGraw-Hill Book Co.

Frey, Werner (1993): Syntaktische Bedingungen für die semantische Interpretation: Über Bindung, implizite Argumente und Skopus (studia grammatica 35). Berlin: Akademie.

Fried, Mirjam (2015): Construction Grammar. In Tibor Kiss \& Artemis Alexiadou (Hrsg.), Syntax - Theory and analysis: An international handbook (Handbooks of Linguistics and Communication Science 42, 2. Aufl.), 974-1003. Berlin: Mouton de Gruyter.

Gazdar, Gerald (1981): Unbounded dependencies and coordinate structure. Linguistic Inquiry 12, 155-184.

Ginzburg, Jonathan \& Ivan A. Sag (2000): Interrogative investigations: The form, meaning, and use of English interrogatives (CSLI Lecture Notes 123). Stanford, CA: CSLI.

Grewendorf, Günther (1988): Aspekte der deutschen Syntax: Eine Rektions-Bindungs-Analyse (Studien zur deutschen Grammatik 33). Tübingen: Narr, jetzt Stauffenburg.

Groß, Thomas M. \& Timothy Osborne (2009): Toward a practical Dependency Grammar theory of discontinuities. SKY Journal of Linguistics 22, 43-90.

Hagemann, Jörg \& Sven Staffeldt (Hrsg.) (2014): Syntaxtheorien: Analysen im Vergleich (Stauffenburg Einführungen 28). Tübingen: Stauffenburg.

Haider, Hubert (1986): Fehlende Argumente: Vom Passiv zu kohärenten Infinitiven. Linguistische Berichte 101, 3-33.

Heinz, Wolfgang \& Johannes Matiasek (1994): Argument structure and case assignment in German. In John Nerbonne, Klaus Netter \& Carl J. Pollard (Hrsg.), German in Head-Driven Phrase Structure Grammar (CSLI Lecture Notes 46), 199-236. Stanford, CA: CSLI.

Hellwig, Peter (2003): Dependency Unification Grammar. In Vilmos Ágel, Ludwig M. Eichinger, Hans Werner Eroms, Peter Hellwig, Hans Jürgen Heringer \& Henning Lobin (Hrsg.), Dependenz und Valenz/Dependency and valency: Ein internationales Handbuch der zeitgenössischen Forschung/An international handbook of contemporary research (Handbücher zur Sprach- und Kommunikationswissenschaft 25.1), 593-635. Berlin: De Gruyter. 
Höhle, Tilman N. (1997): Vorangestellte Verben und Komplementierer sind eine natürliche Klasse. In Christa Dürscheid, Karl Heinz Ramers \& Monika Schwarz (Hrsg.), Sprache im Fokus: Festschrift für Heinz Vater zum 65.Geburtstag, 107-120. Tübingen: Niemeyer. Wiederveröffentlicht als Höhle (2018).

Höhle, Tilman N. (2018): Vorangestellte Verben und Komplementierer sind eine natürliche Klasse. In Stefan Müller, Marga Reis \& Frank Richter (Hrsg.), Beiträge zur Grammatik des Deutschen: Gesammelte Schriften von Tilman N. Höhle (Classics in Linguistics 5), Berlin: Language Science Press. DOI: 10.5281/zenodo.1145680. Zuerst veröffentlicht als Höhle (1997).

Hudson, Richard (2015): Pied piping in cognition. Manuskript London. http:// dickhudson.com/papers/ (letzter Zugriff: 24.7. 2015).

Jackendoff, Ray S. (1977): X syntax: A study of phrase structure. Cambridge, MA/London, England: MIT Press.

Jackendoff, Ray S. (2008): Construction after Construction and its theoretical challenges. Language 84(1), 8-28.

Jacobs, Joachim (2008): Wozu Konstruktionen? Linguistische Berichte 213, 3-44.

Jacobson, Pauline (1987): Review of Generalized Phrase Structure Grammar. Linguistics and Philosophy 10(3), 389-426.

Johnson, Mark (1986): A GPSG account of VP structure in German. Linguistics 24(5), 871- 882.

Joshi, Aravind K., Tilman Becker \& Owen Rambow (2000): Complexity of scrambling: A new twist to the competence-performance distinction. In Anne Abeille \& Owen Rambow (Hrsg.), Tree Adjoining Grammars: Formalisms, Linguistic Analysis and Processing (CSLI Lecture Notes 156), 167-181. Stanford, CA: CSLI.

Kay, Paul \& Charles J. Fillmore (1999): Grammatical Constructions and linguistic generalizations: The What's X Doing Y? Construction. Language 75(1), 1-33.

Kertész, András, Edith Moravcsik \& Csilla Rákosi (Hrsg.) (2018): Current approaches to syntax: A comparative handbook. Berlin: Mouton de Gruyter. In Vorbereitung.

Koster, Jan (1975): Dutch as an SOV language. Linguistic Analysis 1(2), 111-136.

Kroch, Anthony S. \& Aravind K. Joshi (1985): The linguistic relevance of Tree Adjoining Grammar. Tech. Rep. MS-CIS-85-16. University of Pennsylvania.

Lohnstein, Horst (2014): Artenvielfalt in freier Wildbahn: Generative Grammatik. In Hagemann \& Staffeldt (2014) 165-185.

Meurers, Walt Detmar (2001): On expressing lexical generalizations in HPSG. Nordic Journal of Linguistics 24(2), 161-217.

Michaelis, Laura A (2013): Sign-based construction grammar. In Thomas Hoffmann \& Graeme Trousdale (Hrsg.), The Oxford Handbook of Construction Grammar Oxford Handbooks, 133-152. Oxford: Oxford University Press.

Miller, George A. \& Kathryn Ojemann McKean (1964): A chronometric study of some relations between sentences. Quarterly Journal of Experimental Psychology 16(4), 297-308.

Montague, Richard (1974): Formal philosophy. New Haven: Yale University Press.

Müller, Stefan (2002): Complex predicates: Verbal complexes, resultative constructions, and particle verbs in German (Studies in Constraint-Based Lexicalism 13). Stanford, CA: CSLI.

Müller, Stefan (2003): Object-to-subject-raising and lexical rule: An analysis of the German passive. In Stefan Müller (Hrsg.), Proceedings of the 10th International Conference on Head-Driven Phrase Structure Grammar, Michigan State University, East Lansing, 278-297. Stanford, CA: CSLI.

Müller, Stefan (2006): Phrasal or lexical Constructions? Language 82(4), 850-883. doi: 10.1353/lan.2006.0213. 
Müller, Stefan (2007): Head-Driven Phrase Structure Grammar: Eine Einführung (Stauffenburg Einführungen 17). Tübingen: Stauffenburg Verlag.

Müller, Stefan (2010): Grammatiktheorie (Stauffenburg Einführungen 20). Tübingen: Stauffenburg.

Müller, Stefan (2013): Unifying everything: Some remarks on Simpler Syntax, Construction Grammar, Minimalism and HPSG. Language 89(4), 920-950.

Müller, Stefan (2015): The CoreGram project: Theoretical linguistics, theory development and verification. Journal of Language Modelling 3(1), 21-86. doi: 10.15398/jlm.v3i1.91.

Müller, Stefan (2016): Grammatical theory: From Transformational Grammar to constraintbased approaches (Textbooks in Language Sciences 1). Berlin: Language Science Press. doi: 10.17169/langsci.b25.167.

Müller, Stefan (2017a): German sentence structure: An analysis with special consideration of so-called multiple fronting Empirically Oriented Theoretical Morphology and Syntax. Berlin: Language Science Press. http://hpsg.fu-berlin.de/ stefan/Pub/gs.html (letzter Zugriff: 22.01. 2018). Revise and resubmit.

Müller, Stefan (2017b): Germanic syntax. Ms. Humboldt Universität zu Berlin, to be submitted to Language Science Press. http://hpsg.fu-berlin.de/ stefan/Pub/ germanic.html (letzter Zugriff: 22.01. 2018).

Müller, Stefan (2017c): Head-Driven Phrase Structure Grammar, Sign-Based Construction Grammar, and Fluid Construction Grammar: Commonalities and differences. Constructions and Frames 9(1), 139-174. doi: 10.1075/cf.9.1.05mul.

Müller, Stefan (2018): Phrasal constructions, derivational morphology, constituent structure and (cross-linguistic) generalizations: A discussion of template-based phrasal LFG approaches Conceptual Foundations of Language Science. Language Science Press. http://hpsg.huberlin.de/ stefan/Pub/phrasal-lfg.html (letzter Zugriff: 22. 01. 2018). erscheint.

Müller, Stefan \& Stephen Mark Wechsler (2014): Lexical approaches to argument structure. Theoretical Linguistics 40(1-2), 1-76. doi: 10.1515/tl-2014-0001.

Nerbonne, John (1986): 'Phantoms' and German fronting: Poltergeist constituents? Linguistics 24(5), 857-870.

Osborne, Timothy \& Sylvain Kahane (2015): Translators' introduction. In Tesnière (2015): Elements of structural syntax, xxix-lxxiii. Translated by Timothy Osborne \& Sylvain Kahane. Amsterdam/Philadelphia: Benjamins.

Pollard, Carl J. \& Ivan A. Sag (1987): Information-based syntax and semantics (CSLI Lecture Notes 13). Stanford, CA: CSLI.

Pollard, Carl J. \& Ivan A. Sag (1994): Head-Driven Phrase Structure Grammar Studies in Contemporary Linguistics. Chicago, IL/London: The University of Chicago Press.

Reis, Marga (1974): Syntaktische Hauptsatzprivilegien und das Problem der deutschen Wortstellung. Zeitschrift für Germanistische Linguistik 2(3), 299-327.

Richter, Frank \& Manfred Sailer (2009): Phraseological clauses as Constructions in HPSG. In Stefan Müller (Hrsg.), Proceedings of the 16th International Conference on Head-Driven Phrase Structure Grammar, University of Göttingen, Germany, 297-317. Stanford, CA: CSLI.

Sag, Ivan A. (1997): English relative clause constructions. Journal of Linguistics 33(2), 431- 484.

Sag, Ivan A. (2010): English filler-gap constructions. Language 86(3), 486-545.

Sag, Ivan A. (2012): Sign-Based Construction Grammar: An informal synopsis. In Boas \& Sag (2012) 69-202. 
Sag, Ivan A., Hans C. Boas \& Paul Kay (2012): Introducing Sign-Based Construction Grammar. In Boas \& Sag (2012) 1-29.

Savin, Harris B. \& Ellen Perchonock (1965): Grammatical structure and the immediate recall of English sentences. Journal of Verbal Learning and Verbal Behavior 4(5), 348-353.

Shieber, Stuart M. (1985): Evidence against the context-freeness of natural language. Linguistics and Philosophy 8(3), 333-343.

Simpson, Jane (1983): Resultatives. In Lori S. Levin, Malka Rappaport \& Annie Zaenen (Hrsg.), Papers in Lexical Functional Grammar, 143-157. Indiana: Indiana University Linguistics Club. Neudruck: Simpson (2005).

Simpson, Jane (2005): Resultatives. In Miriam Butt \& Tracy Holloway King (Hrsg.), Lexical Semantics in LFG, 149-161. Stanford, CA: CSLI.

Steedman, Mark J. (1989): Constituency and coordination in a Combinatory Grammar. In Mark R. Baltin \& Anthony S. Kroch (Hrsg.), Alternative Conceptions of Phrase Structure, 201-231. Chicago/London: The University of Chicago Press.

Steedman, Mark J. (2000): The syntactic process Language, Speech, and Communication. Cambridge, MA / London, England: MIT Press.

Tesnière, Lucien (1959): Eléments de syntaxe structurale. Paris: Librairie C. Klincksieck.

Tesnière, Lucien (2015): Elements of structural syntax. Amsterdam/Philadelphia: Benjamins. Übersetzt von Timothy Osborne and Sylvain Kahane.

Thiersch, Craig L. (1978): Topics in German syntax: M. I. T. Dissertation.

Uszkoreit, Hans (1987): Word order and constituent structure in German (CSLI Lecture Notes 8). Stanford, CA: CSLI.

van Trijp, Remi (2013): A comparison between Fluid Construction Grammar and Sign-Based Construction Grammar. Constructions and Frames 5(1), 88-116.

Verspoor, Cornelia Maria (1997): Contextually-dependent lexical semantics: University of Edinburgh Dissertation.

Vijay-Shanker, K. \& Aravind K. Joshi (1988): Feature structures based Tree Adjoining Grammars. In Dénes Vargha (Hrsg.), Proceedings of COLING 88, Band 1, 714-719. University of Budapest: Association for Computational Linguistics.

Wechsler, Stephen Mark (1997): Resultative predicates and control. In Ralph C. Blight \& Michelle J. Moosally (Hrsg.), Texas Linguistic Forum 38: The Syntax and Semantics of Predication: Proceedings of the 1997 Texas Linguistics Society Conference, 307-321. Austin, Texas: University of Texas, Department of Linguistics.

Wechsler, Stephen Mark \& Bokyung Noh (2001): On resultative predicates and clauses: Parallels between Korean and English. Language Sciences 23(4), 391-423. 

III Grammatik, Korpus(linguistik) und Variation 



\title{
Marek Konopka \\ 5 Korpuslinguistik, Grammatiktheorie, Grammatikographie
}

\begin{abstract}
Der Korpuslinguistik begegneten überwiegend introspektiv arbeitende Grammatiktheoretiker lange mit Misstrauen. Dabei kann sie, auch wenn sie selbst kein bestimmtes theoretisches Paradigma vorgibt, in sehr vielfältiger Weise zur Theoriebildung beitragen. Zum einen können mithilfe von Korpora theoretische Aussagen exemplifiziert und validiert werden. Zum anderen liefert die Korpuslinguistik große Mengen differenzierter Sprachdaten sowie Methoden, mit denen sie überschaut und analysiert werden können. Neue Daten müssen theoretisch in neuen Generalisierungen aufgearbeitet werden und auch die Datenvielfalt selbst rückt in den theoretischen Fokus. Die Grammatikforschung erfährt so eine empirische Wende, in der die Variation grammatischer Strukturen zu einem der zentralen Themen wird. Die theoretische Erfassung dieser Variation geht dabei weit über die Grenzen einer klassischen Theorie der Sprachkompetenz hinaus. Immer dringlicher wird damit eine neue wissenschaftliche Grammatik des Deutschen, die diese Entwicklung aufnimmt, sich den neuen Forschungsfragen stellt, sie mit modernen korpuslinguistischen Methoden untersucht und damit die Grundlagen für eine umfassende Theorie schafft, in der Kompetenz und Performanz (wie auch Synchronie und Diachronie) näher aneinanderrücken.
\end{abstract}

Keywords: Grammatikschreibung, Grammatiktheorie, Kompetenz, Korpuslinguistik, Performanz, Variation

\section{Einleitung}

Trägt die Korpuslinguistik etwas zur Grammatiktheorie bei? Diese Frage kann man sich gut stellen angesichts des seit über 30 Jahren andauernden

Anmerkung: Franziska Münzberg danke ich herzlich für das Gegenlesen und die hilfreichen Kommentare.

Marek Konopka, Institut für Deutsche Sprache, R5, 6-13, D-68161 Mannheim, E-Mail:konopka@ids-mannheim.de

Ә Open Access. (C) 2018 Marek Konopka, publiziert von De Gruyter. () BY Dieses Werk ist lizenziert unter der Creative Commons Attribution 4.0 Lizenz.

https://doi.org/10.1515/9783110490992-006 
Höhenflugs (moderner) linguistischer Korpusarbeit ${ }^{1}$ einerseits und einer sich immer mehr in der Abwehrhaltung befindlichen Grammatiktheorie klassischstrukturalistischer, vor allem generativer Prägung andererseits. Letztere hat vor Jahrzehnten entschieden, das Sprachwissen eines idealen Sprechers/ Hörers zu ihrem Gegenstand zu erklären, und ist seither überzeugt, auf dieses Wissen introspektiv ausreichend zugreifen zu können. ${ }^{2}$ Noam Chomsky, die Galionsfigur dieser Bewegung, antwortete in den 90er Jahren in einem Interview mit Baas Aarts auf die Frage „What is your view of modern corpus linguistics“ mit der Feststellung: „It doesn’t exist. If you have nothing, or if you are stuck, or if you are worried about Gothic, then you have no choice“ (Aarts 2000: 5). ${ }^{3}$

Um auf die Eingangsfrage dieses Beitrags differenziert einzugehen, muss man einige Klarheit über die Begriffe Korpuslinguistik und Grammatiktheorie erlangen. In dieser Einleitung soll der erstgenannte Begriff umrissen werden, während die für die Argumentation relevanten Vorstellungen von Grammatiktheorie erst nach und nach im Laufe des Artikels entwickelt werden.

Konstitutiv für die Korpuslinguistik ist die Arbeit mit Sprachkorpora - so viel ist klar. Darüber, was die Korpuslinguistik genau umfasst, gibt es allerdings unterschiedliche Meinungen. In verschiedenen Ansätzen kann ihr nahezu jeder Arbeitsabschnitt zugerechnet werden, der auf der Strecke von der Idee eines Korpus bis zur linguistischen Erkenntnis liegt. Dementsprechend umfassend ist Korpuslinguistik im Folgenden auch gemeint: Dazu gehören sollen also der Aufbau und die Bereitstellung von Korpora einschließlich verschiedenartiger linguistischer Annotationen, die Erarbeitung von Methoden und Werkzeugen, um die Korpora für linguistische Zwecke nutzbar zu machen, und die Analyse dieser Korpora im Hinblick auf konkrete linguistische Fragestellungen.

Korpuslinguistik ist nach ihrer Datenquelle benannt worden: der Sammlung authentischer sprachlicher Äußerungen, die in der Regel nicht gesteuert vom Korpuslinguisten entstanden sind und in Form von Texten oder Textausschnitten vorliegen. Grammatiktheorie braucht Sprachdaten, die die zu behandelnden grammatischen Strukturen enthalten. Um die Voraussetzungen für das Zusammenwirken der beiden scheint es also auf den ersten Blick bestens bestellt zu sein. Aber Sprachdaten können auch der Introspektion entstammen,

1 Zur Geschichte der modernen Korpuslinguistik vgl. etwa Facchinetti (2007: 11-86) mit dem bezeichnenden Titel „Corpus Linguistics 25 Years on“.

2 Über Ansätze dieser Art schreiben in diesem Band etwa Primus (in Abschnitt 2 allgemein umreißend), Haider (zum Minimalistischen Programm) und Fuß (mit einer konkreten Fallstudie). 3 Alternativlos seien Korpusuntersuchungen also nur dann, wenn die Sprache nicht gut bekannt ist und die Sprachdaten schwer zu konstruieren sind. Vgl. auch Tognini-Bonelli (2010: 14) mit einem weiteren einschlägigen Zitat von Chomsky. 
zufällige Funde sein, unsystematisch gesammelt, erfragt oder in einem Experiment induziert werden. Und danach werden sie introspektiv, durch Befragungen, Experimente oder eben mit korpuslinguistischen Methoden untersucht. Was die Arbeit mit Korpora der Introspektion einerseits und den anderen linguistischen Empiriearten wie Befragung und Experiment andererseits in jedem Fall voraushat, sind die (potenziell) große Menge und die Spontaneität der Sprachdaten. Die Korpuslinguisten können aber - wie man noch sehen wird - ins Hintertreffen geraten, wenn es um Fragen wie Akzeptabilität von Äußerungen im Allgemeinen und negative Evidenz im Besonderen oder auch um niedrigfrequente Phänomene geht.

Korpora werden heutzutage computerbasiert aufgebaut, aufbereitet, bereitgestellt, durchsucht und analysiert. Beginnend mit dem Siegeszug des allgemein verfügbaren Computers ist ihre Bedeutung für die linguistische Forschung enorm gestiegen, und Korpora sind jetzt immer mehr Interessierten zugänglich. Viele Debatten gab es und gibt es immer noch zu der Frage, ob Korpuslinguistik nur eine Methodologie ist oder ob ihr der Status einer Theorie zukommt. ${ }^{4}$ Hier soll dieses Problem nicht mehr diskutiert werden - interessieren soll vielmehr, was Korpuslinguistik speziell für die Grammatiktheorie leistet bzw. inwiefern sie zu theoretischen Überlegungen beiträgt, die auf die Erklärung von grammatischen Phänomenen abzielen. Veranschaulicht werden in den folgenden Abschnitten drei verschiedene Einsatzbereiche der Korpora. In diesen werden Korpora verwendet,

1. um theoretische Feststellungen $\mathrm{zu}$ exemplifizieren (Evidenz aus dem Korpus),

2. um theoretische Feststellungen zu überprüfen (Validierung am Korpus),

3. als Quelle für zu beschreibende und erklärungsbedürftige Phänomene (Induktion mithilfe des Korpus).

Während bei 1. im Korpus nach positiven Belegen gesucht wird, d. h. nach solchen, die eine Feststellung untermauern, wird bei 2. in der Regel nach negativen Belegen gesucht, $d$. h. solchen, die eine falsifizierbare Feststellung widerlegen. Bei 3. geht es schließlich nicht direkt um positive oder um negative Belege, sondern darum, eine adäquate Datenbasis für die Beschreibung konkreter Phänomene zu schaffen, bzw. darum, einen Datenbereich einzugrenzen, der beschrieben und erklärt werden soll. Diskutiert wird im Folgenden die von 1. bis 3. ansteigende Bedeutung der Korpuslinguistik für die theoretischen

4 Einige prominente Stimmen werden z.B. von Tognini-Bonelli (2010: v. a. 17 f.) und Gries (2011: 327 f.) zitiert. Bekannte Korpuslinguisten nehmen zu der Frage Stellung in Viana et al. (2011). 
Überlegungen zur Grammatik. Danach wird das Potenzial der Korpuslinguistik in einer Grammatikographie geprüft, die im spezifischen Kontext des Mannheimer Instituts für Deutsche Sprache (IDS) zu einer wissenschaftlichen Referenzgrammatik des Deutschen führen soll. Um die Argumentation zu stützen, werden immer wieder Ergebnisse einschlägiger grammatischer Untersuchungen herangezogen. Nur hier und da gestreift wird das Thema, wie man gezielt nach bestimmten grammatischen Phänomenen in annotierten oder nicht annotierten Korpora suchen kann, denn dies ist bereits ausführlich beschrieben worden (z. B. von Meurers 2005; Müller 2007; Meurers \& Müller 2009).

\section{Evidenz aus dem Korpus}

Sprachkorpora wurden wohl, seit es sie gibt, dazu benutzt, grammatische Theorien $\mathrm{zu}$ erläutern, $\mathrm{zu}$ veranschaulichen und durch (oft prestigehaltige) Sprachgebrauchsbeispiele zu untermauern. Diese Praxis bleibt manchmal der einzige Korpuseinsatzbereich, sie begleitet aber auch regulär die anderen, ,intensiveren` Arten der Korpusnutzung, die in Abschnitt 3 und 4 besprochen werden.

Bei der Evidenz aus dem Korpus geht es nicht etwa um die durchgehende Begleitung einer konsistenten Theorie durch einen Sprachgebrauchsextrakt, der die Theorie dann endgültig ,nachweist‘. Es geht nur um die Exemplifizierung von Theorieteilen, die in halbwegs autonomen theoretischen Feststellungen (im Weiteren ,Theoremen') bestehen, und zwar um deren Exemplifizierung mithilfe meist isolierter Korpusbeispiele. Ob die Theoreme deduktiv aus größeren theoretischen Zusammenhängen hergeleitet wurden, ob sie auf der Introspektion oder einer mehr oder weniger systematischen Empirie aufbauen, wird meist nicht thematisiert. Dafür scheint es mindestens bis in die 90er Jahre hinein in der auf Referenzwerke ausgerichteten wissenschaftlichen Grammatikographie keinen besonderen Bedarf gegeben zu haben. In der Grammatik der Deutschen Sprache (GDS, Zifonun et al. 1997) aus dem IDS ist Evidenz aus dem Korpus der einzige greifbare Einsatzbereich des Korpus. Dazu ein Beispiel:

Nachfeldstellung des Attributsatzes ist hier allenfalls dann möglich, wenn sein Bezugsnomen den Kopf der betreffenden Phrase bildet; von präpositionalen oder untergeordneten Phrasen (im Vorfeld) kann ein Attributsatz also nicht abgetrennt werden; man vergleiche: [...]

(15) Beim Tauziehen zwischen Sozialversicherung und öffentlicher Hand um die Frage, wer von beiden stärker zur Krankenhausfinanzierung herangezogen werden soll, scheint die Sozialversicherung am Boden gewonnen zu haben. (Welt, 4.2. 1966, 1) 
$\left(15^{\prime}\right){ }^{\star}$ Beim Tauziehen ... um die Frage scheint die Sozialversicherung am Boden gewonnen zu haben, wer von beiden stärker zur Krankenhausfinanzierung herangezogen werden soll. (Zifonun et al. 1997, Bd. 2: 1654)

Dabei ist die Evidenz aus dem Korpus interessanterweise mit einem konstruierten Beispiel verkettet, das als grammatisch nicht korrekt bewertet ist. Wohlgemerkt, die Feststellung der Nichtakzeptabilität kann nicht auf einer Korpusanalyse basieren, da negative Evidenz aus dem Korpus nicht möglich ist (dazu Abschnitt 3).

Über die Rolle des Korpus wird im Vorwort der GDS Folgendes geschrieben:

Besonderen Wert haben wir darauf gelegt, an zentralen Stellen der Argumentation BELEGE zu verwenden, authentische Sprachdaten aus Diskursen oder Texten. Es handelt sich um

- Material aus den (überwiegend am Rechner recherchierbaren) Korpora des IDS;

- Daten aus veröffentlichten Korpora oder Korpora der Autoren;

- von den Autoren in literarischen oder Gebrauchstexten gefundene Daten.

Die Belege sollen relativ direkt Sprachwirklichkeit repräsentieren.

(Zifonun et al. 1997, Bd. 1: 12) ${ }^{5}$

Unter der Überschrift „authentische Sprachdaten“ werden Korpora hier auf einer Ebene mit anders gearteten Sammlungen bzw. Funden behandelt. Die Autoren der GDS greifen zwar noch nicht systematisch auf authentische Sprachdaten zurück, um Theoreme zu evaluieren oder aus den Daten induktiv Hypothesen zu gewinnen. Das diesbezügliche Potenzial authentischer Daten bleibt ihnen aber keineswegs verborgen. Sie lassen es sich nicht nehmen, von der Erfahrung zu berichten, dass sie durch „, ungewöhnliche“ oder unerwartete Daten immer wieder zu neuen Überlegungen gezwungen und vor unzureichenden oder falschen Generalisierungen bewahrt“ worden seien (Zifonun et al. 1997, Bd. 1: 13).

\section{Validierung am Korpus}

Das evaluative Potenzial der Korpuslinguistik für die Grammatik ist bereits explizit reflektiert worden z. B. von Meurers (2005), Müller (2007) und Meurers \& Müller (2009). Meurers \& Müller (2009: 920) diskutieren, „how electronic corpora can be used in support of the creation and falsification of syntactic theories“.

5 Auch das kürzlich erschienene Werk „Grammatik des Deutschen im europäischen Vergleich. Das Nominal“ ist nicht korpusbasiert, d. h., Korpora werden darin in der Regel nur als Quellen für Belege benutzt (Gunkel et al. 2017, Bd. 1: $8 \mathrm{f}$.) 
Am Beispiel der Überprüfung der Vorfeldfähigkeit von Verbpartikeln, die von Müller (2007) und Meurers \& Müller (2009) durchgeführt wurde, soll hier das grundlegende Räsonnement bei der Validierung von Theoremen präsentiert werden.

Viele Grammatiker seien in der Vergangenheit davon ausgegangen, dass Verbpartikeln im Deutschen nicht ins Vorfeld gestellt werden können (Müller 2007). ${ }^{6}$ Derartige Annahmen sind selbstverständlich nicht mehr haltbar, wenn wohlgeformte Gegenbeispiele gefunden werden. Tatsächlich findet Müller mithilfe einer präzisen Abfrage in dem annotierten TIGER-Korpus ${ }^{7}$ Belege, in denen die Verbpartikel im Vorfeld steht wie in (1):

(1) Fest steht, daß dort 580 der insgesamt 4.650 Arbeitsplätze wegfallen. (aus Müller 2007: 75; Meurers \& Müller 2009: 928)

Das Ergebnis seiner Korpusrecherche zeigt damit, dass die Annahme der Nichtvorfeldfähigkeit von Verbpartikeln nicht korrekt ist. Validierung heißt hier Falsifikation bereits vorhandener Annahmen.

Der Beitrag von Korpusuntersuchungen zur grammatischen Theoriebildung besteht in solchen Fällen also darin, Theoreme, denen Übergeneralisierungen zugrunde liegen, als ungültig zu entlarven. $\mathrm{Zu}$ beachten ist dabei aber, dass es umgekehrt kein Beweis für die Gültigkeit eines Theorems ist, wenn keine Korpusbelege gefunden werden, die der ursprünglichen Annahme widersprechen. Dass eine Formulierung in einem Korpus fehlt, bedeutet nicht automatisch, dass sie nicht wohlgeformt oder sogar gebräuchlich sein könnte: Das Fehlen gilt nicht als negative Evidenz, weil ein Korpus einfach nicht alle Phänomene enthalten kann. Vielmehr sind darin nur relativ häufige Phänomene zuverlässig zu finden. An dieser Stelle muss man bedenken, dass nach dem Zipfschen Gesetz die Wahrscheinlichkeit des Auftretens einzelner Wörter im Korpus umgekehrt proportional zu ihrer Position innerhalb der Reihenfolge ist, die entsteht, wenn man die Wörter absteigend nach ihrer Häufigkeit ordnet. ${ }^{8}$

Mithilfe des Korpus können also zwar Theoreme falsifiziert, aber keine negative Evidenz für sprachliche Muster geliefert werden (damit können Theoreme anhand des Korpus auch kaum verifiziert werden ${ }^{9}$ ). Man könnte an dieser

\footnotetext{
6 Allerdings beschreibt schon die 1. Auflage von „Hauptschwierigkeiten der deutschen Sprache“ von 1965 Verbpartikeln im Vorfeld als „Wiederbelebung des Verbzusatzes als Satzglied in der Ausdrucksstellung“ (Duden 1965: 637, dazu auch Duden 2016a: 694).

7 Brants et al. 2004.

8 Dazu auch Meurers (2005: 1621) und Meurers \& Müller (2009: 920).

9 Als möglich erscheint die Verifizierung etwa im Falle von modalisierten positiven Aussagen wie „die Verbpartikel kann im Vorfeld stehen“, wenn die Aussagen durch eine ausreichende
} 
Stelle einwenden, dass mögliche Gegenbeispiele zu einem Theorem auch introspektiv konstruierbar sein müssten. ${ }^{10}$ Dabei würde man automatisch von einem konsistenten grammatischen System ausgehen, auf das idealerweise alle versierten Muttersprachler uneingeschränkten Zugriff haben. Wohl im Hinblick auf solche doch sehr verbreiteten Vorstellungen rechtfertigt Müller (2007) seine Korpusbenutzung zusätzlich mit dem Argument, „dass uns unsere grammatischen Fähigkeiten mitunter nicht zugänglich sind und dass wir uns deshalb misstrauen sollen“.

In ihren Überlegungen konzentrieren sich Meurers (2005), Müller (2007) und Meurers \& Müller (2009) besonders auf die Frage, wie man überhaupt aus einem Korpus Daten gewinnen kann, die für ein bestimmtes theoretisches Thema einschlägig sind. Die Autoren unterstreichen dabei die Bedeutung von Korpusannotationen - vor allem für die Suche nach komplexeren syntaktischen Phänomenen. Recherchen in einem nicht annotierten Korpus erforderten eine explizite Auflistung aller lexikalischen Realisierungen eines Phänomens, die oft einfach unmöglich sei. Die dann zur Anwendung kommenden Approximationspraktiken resultierten in einem Präzisions- und Recallverlust (Meurers \& Müller 2009: 922 ff.). Als Beispiel für ein solches komplexes syntaktisches Phänomen führen sie die „mehrfache Vorfeldbesetzung“ an.

Die traditionell stark verankerte Annahme, dass im Deutschen normalerweise nur eine Konstituente vor dem finiten Verb stehen kann, ist zwischenzeitlich zu einem Test der Konstituentenhaftigkeit weiterentwickelt und auch in Satzglieddefinitionen eingebaut worden. Wie Müller (2007: 79 ff.) aber zeigt, kann „die Vorfeldbesetzung allenfalls als Indiz für den Konstituentenstatus verwendet werden [...], keinesfalls jedoch als hinreichendes Kriterium“. Er führt Belege für die mehrfache Vorfeldbesetzung an wie (Beispiele aus Müller 2007):

(2) [Am schwersten] [mit der Selbstkritik] tat sich Jürgen Kocka.

(3) [Gezielt] [Mitglieder] [im Seniorenbereich] wollen die Kendoka allerdings nicht werben.

Eine systematische und effektive Suche nach derartigen Belegen sei nur in annotierten Korpora möglich. Identifizierbar sein solle dabei zumindest das finite Verb, gerne auch die unmittelbaren Konstituenten (hier reicht ja die Wortlemmatisierung alleine nicht aus, weil die Konstituenten öfter aus mehreren

Anzahl von Belegen bestätigt werden bzw. wenn sie durch Belege gestützt werden, die eindeutig als akzeptabel zu beurteilen sind (vgl. dazu weiter unten).

10 Pullum (2017: 283 ff.) kritisiert diese Position als „intuitional solipsism“. 
Wörtern bestehen). Müllers Darstellung legt letztlich die Nützlichkeit einer möglichst präzisen POS- und Baumstruktur-Annotation nahe, wie sie im TIGERKorpus vorliegt. Seine Suche in diesem Korpus führt dennoch nur zu sieben einschlägigen Belegen. Diese bestätigen zwar, dass es falsch wäre, zu behaupten, im Deutschen dürfe nur eine Konstituente vor dem finiten Verb stehen. Als Basis dafür, das Phänomen ,mehrfache Vorfeldbesetzung، genauer zu explorieren und anschließend sinnvolle Generalisierungen $\mathrm{zu}$ formulieren, reichte die Anzahl der Belege aber nicht aus.

Die letzte Feststellung enthielt bereits einen Vorgriff auf die Diskussion eines weiteren Aspekts des korpuslinguistischen Beitrags zur Grammatiktheorie. Es geht nicht mehr wie bisher um die Falsifikation vorhandener Theoreme, sondern (zwar nur in einem deduktiv eingeschränkten Bereich, aber immerhin) um die Induktion mithilfe der Korpusdaten. Das Korpus soll dabei die Quelle für erklärungsbedürftige Phänomene und eine empirische Basis für die Entstehung neuer Theoreme bilden. Bevor dies in den Kapiteln 4 und 5 genauer thematisiert wird, müssen aber die Überlegungen zur Theoremvalidierung am Korpus noch um eine wichtige Einsicht ergänzt und etwas genauer modelliert werden.

Weiter oben wurde behauptet, dass Theoreme, denen Korpusbelege widersprechen, nicht korrekt sind - das verlangt unbedingt nach einer Präzisierung. Ein im Korpus gefundenes grammatisches Muster, das einem Theorem zu widersprechen scheint, muss nicht akzeptabel sein, ${ }^{11}$ denn das Korpus kann auch Fehler enthalten. Das Problem spitzt sich zu, wenn es im Korpus nur ganz wenige dem Theorem widersprechende Belege gibt ${ }^{12}$ wie im zuletzt angeführten Fall der ,mehrfachen Vorfeldbesetzung‘. Was in solchem Fall notwendig ist, um Korpusfunde als Validierungsinstanzen bzw. als Falsifikatoren eines Theorems $\mathrm{zu}$ qualifizieren, sind die Urteile sprachlich versierter Muttersprachler, die die Wohlgeformtheit der Korpusfunde bewerten. Erst nachdem Sätze wie (2) und (3) weiter oben als wohlgeformt bewertet worden sind, ist das Theorem widerlegt. Es ist dann eventuell nicht präzise genug und muss an die Daten angepasst werden. Gibt es im Korpus wiederum viele Belege, die dem Theorem widersprechen, ist die Wahrscheinlichkeit, dass sie doch unakzeptabel sind, geringer.

Akzeptabilitätsurteile machen die Trias der für Validierung am Korpus wichtiger Gegebenheiten perfekt:

- das Theorem,

- die Korpusdaten,

- die Wohlgeformtheitsurteile.

11 Vgl. Meurers (2005: 1621).

12 Vgl. Conrad (2010: 237). 
Der Weg zur Validierung eines Theorems umfasst dann folgende Schritte:

1. Ein (wie auch immer zustande gekommenes) Theorem wird aufgestellt.

2. Es wird nach Sprachdaten gesucht, die dem Theorem widersprechen.

3. Wenn solche Daten gefunden werden, müssen sie als wohlgeformt oder nicht wohlgeformt qualifiziert werden.

Sind die dem Theorem widersprechenden Daten akzeptabel, muss das Theorem fallen gelassen werden, andernfalls kann es (zumindest bis zur nächsten Prüfung) bestehen bleiben. Letzteres gilt auch, wenn der Forscher bereits bei Schritt 2 scheitert, d. h., wenn keine Daten gefunden werden, die dem Theorem widersprechen. Damit ist der erste Entwurf einer Methodik gegeben, in der Hypothesen an Korpora evaluiert werden. Denjenigen Erscheinungen, die die alten Hypothesen falsifizieren, muss übrigens in neuen Hypothesen Rechnung getragen werden.

Mit dem Zusammenhang Theorem - Korpusdaten - Akzeptabilitätsurteil beschäftigt sich Pullum (2017: 296). Er zieht Parallelen zu der aus der Moralphilosophie bekannten Konzeption des Überlegungsgleichgewichts (,reflective equilibrium“):

What is needed for grammar is a triangular version of reflective equilibrium, involving linguistic behaviour (corpora), general constraints (grammars), and intuitive assessment of well-formedness (judgments). None of these is to be dismissed - yet crucially, none is sovereign.

Pullums Modell der grammatischen Theoriebildung soll hier durch Abbildung 5.1 illustriert werden.

[Daten +]Wohlgeformtheitsurteile

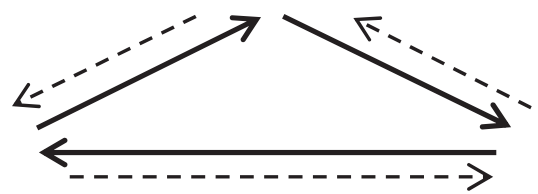

Korpusdaten

grammatische Theoreme

Abb. 5.1: Visualisierung von Pullums (2017) Konzeption des Überlegungsgleichgewichts in der grammatischen Theoriebildung.

Nach Pullums Vorstellung sollten anhand klarer Fälle, deren Wohlgeformtheit eindeutig bewertet werden kann, die ersten theoretischen Aussagen formuliert werden. Anschließend solle die Gültigkeit bzw. Vorhersagekraft dieser theore- 
tischen Aussagen mithilfe von Wohlgeformtheitsurteilen und Korpusdaten getestet werden. Die Relationen zwischen den Eckpunkten von Pullums Dreieck sind dabei reziprok konzipiert. Genauer heißt das: Wenn Wohlgeformtheitsurteile und theoretische Aussagen an einer bestimmten Stelle im Konflikt mit dem Korpus stehen, sei zu überlegen, ob das Korpus nicht einen Fehler enthält; wenn sowohl Wohlgeformtheitsurteile als auch das Korpus gegen die theoretischen Aussagen sprechen, sei wiederum eine Revision der theoretischen Aussagen zu erwägen. Darüber hinaus könne man bei einem gut bestätigten $^{13}$ theoretischen Prinzip auch überlegen, ob einige Wohlgeformtheitsurteile nicht falsch gewesen seien (vgl. Pullum 2017: 296 f.).

Etwas unklar bleibt, auf welche Sprachdaten sich in Pullums Modell die intuitiven Wohlgeformtheitsurteile beziehen. Sind diese Daten introspektiv entstanden oder können sie auch aus dem Korpus stammen? Einige Seiten zuvor begrüßt Pullum (2017: 286) introspektiv konstruierte Beispiele als Illustration von Phänomentypen (z. B. von einzelnen Satzarten) in Referenzgrammatiken. In seiner Argumentation zum Überlegungsgleichgewicht in der grammatischen Theoriebildung bringt er wiederum Wohlgeformtheitsurteile mit Korpusdaten in Verbindung, und zwar als die Validierungsinstanz für theoretische Aussagen. Dass sich die Wohlgeformtheitsurteile in Pullums Modell sowohl auf introspektiv als auch auf empirisch gewonnene Daten beziehen, ist also nicht auszuschließen.

Um Bisheriges zusammenzufassen: Korpusdaten können zum einen einer einfachen Exemplifizierung eines Phänomens (siehe Abschnitt 2) dienen. Sie können zum anderen für die Validierung eines Theorems benutzt werden und damit dessen Revision oder Präzisierung einleiten. Sie können wohl schließlich, wie es Zifonun et al. (1997) und Meurers (2005), Müller (2007) sowie Meurers \& Müller (2009) allerdings nur andeuten, die empirische Basis bilden, auf deren Grundlage mehr oder weniger induktiv Generalisierungen erarbeitet werden, die einen Ausschnitt der Grammatik genauer beschreiben bzw. erklären.

\section{Induktion mithilfe des Korpus?}

Auf die Falsifikation von Theoremen muss ihre Korrektur bzw. die Aufstellung neuer Theoreme folgen. Es fragt sich, was diesem in unseren bisherigen Überlegungen ersten konstruktiven Schritt der Theoriebildung zugrunde liegen soll. Wie bereits festgestellt, sind die Korpusdaten, die zur Falsifizierung alter Theo-

13 Ob Korpusdaten bei der Bestätigung eine Rolle spielen können, wird nicht klar. 
reme geführt haben, nicht immer ausreichend, um neue Generalisierungen zu ermöglichen, insbesondere wenn es sich nicht um triviale, sondern um wirklich komplexe Phänomene handelt: Solche Phänomene sind in der Regel selten und in der Konsequenz des Zipfschen Gesetzes - wenn überhaupt - nur in besonders großen Korpora enthalten, die dann unter Umständen nicht mehr präzise genug ${ }^{14}$ annotiert werden können, um die relevanten Daten $\mathrm{zu}$ finden (vgl. Meurers \& Müller 2009: 930).

Was tun? Wenn die Korpussuche nach relevanten Phänomenen, um ,verbesserte“ Theoreme aufzustellen, ergebnislos bleibt, kann man einerseits zu der Jahrhunderte alten Routine der linguistischen Forschung zurückkehren, d.h. die Introspektion bemühen und die weniger systematischen Empiriearten ${ }^{15}$ benutzen; Letzteres ist ein sehr aufwendiges Glücksspiel, Ersteres kann unzureichend sein, weil - wie schon feststellt - uns unsere grammatischen Fähigkeiten nicht immer zugänglich sind; so oder so hat man in der Theoriebildung höchstens einen sehr kleinschrittigen Fortschritt $\mathrm{zu}$ erwarten. Andererseits könnte man aber den traditionellen Pfad ,altes Theorem - Falsifizierung korrigiertes Theorem'verlassen. Man würde dann keinen Anschluss mehr an bisherige Theoreme, sondern einen richtigen Neuanfang mit dem Korpus suchen. Mit einem Schlag wäre man die Last der durch die Untersuchung klassischer Sprachen geprägten theoretischen Vorstellungen los, die so oft nicht auf den Untersuchungsgegenstand passten, und das könnte wirklich ein Meilenstein in der Linguistikgeschichte werden. So bahnt sich die Polarisierung der Korpuslinguistik in den Corpus-driven- und den Corpus-based-Ansatz ihren Weg.

Angesichts des Höhenflugs der Korpuslinguistik stellt Tognini-Bonelli beinahe enthusiastisch fest:

\footnotetext{
What started as a methodological enhancement but included a quantitative explosion (I am referring here to the quantity of data processed thanks to the aid of the computer) has turned out to be a theoretical and qualitative revolution in that it offered insights into the language that have shaken the underlying assumptions behind many well-established theoretical positions in the field. (Tognini-Bonelli 2010: 17)
}

Tognini-Bonelli spricht sich folgerichtig für einen radikalen Neuanfang in der Theoriebildung aus. Sie ist eine Fürsprecherin des Corpus-driven ${ }^{16}$-Ansatzes,

14 Nicht möglich sind dann vor allem ,manuelle‘ Annotationen.

15 Hier sind nicht Experimente und Befragungen gemeint, sondern nicht speziell vorbereitete Beobachtungen. Wenn die Korpora nicht ausreichend Daten liefern, verbleibt laut Müller (2007: 84 f.) als letzte Lösung, „die Technik zu benutzen, die schon in den letzten Jahrhunderten benutzt wurde: aufmerksam lesen“.

16 Gelegentliche Übersetzungen lauten korpusgesteuert, korpusgeleitet oder auch korpusbasiert (sic). Hier wird auf die Übersetzung verzichtet, um Missverständnisse zu vermeiden. 
in dem Korpusdaten unabhängig von bisherigen Theorien und vordefinierten Kategorien zu betrachten seien. Theoretische Aussagen sollen erst der Korpusbetrachtung entspringen, direkt auf die Korpusevidenz zurückgehen und ihr vollumfänglich Rechnung tragen (vgl. Tognini-Bonelli 2001: 84). Verwandte Positionen ${ }^{17}$ werden übrigens auch im IDS vertreten: Kupietz/Keibel (2009) entwickeln ein Forschungsprogramm, mit dem sie eine explanatorische Theoriebildung verfolgen wollen (vgl. auch Perkuhn, Keibel \& Kupietz 2012: 20 f.). Sie postulieren dabei die Notwendigkeit, sich der Sprache mit möglichst wenigen Vorannahmen zu nähern und sich konsequent von der Empirie leiten zu lassen, und werben für eine „emergentistische“ Perspektive auf Sprache, der zufolge alles Regelhafte und Konventionelle ein Epiphänomen des Sprachgebrauchs sei. ${ }^{18}$

Vertreter des Corpus-driven-Ansatzes sagen sich von der Introspektion los und setzen auf authentische Daten. Bei der Theoriebildung wird im Umgang mit den Daten die Induktion gegenüber der Deduktion so weit wie möglich priorisiert. Korpusannotationen etwa werden oft gänzlich abgelehnt. Die Methodologie, von der es sich abzugrenzen gelte, wird jetzt als corpus-based ${ }^{19}$ bezeichnet. Sie bediene sich der Korpora, nur um bereits vorgefasste korpusunabhängige Theorien und Beschreibungen zu erläutern, zu veranschaulichen oder zu testen (Tognini-Bonelli 2001: $65 \mathrm{f}$.).

Der Corpus-driven-Ansatz hat sich bisher nicht richtig etabliert. Das liegt hauptsächlich wohl daran, dass ein gänzlicher Verzicht auf bisherige Theoreme und traditionelle Kategorien kaum durchführbar ist (dazu auch Gries 2010: $328 \mathrm{ff}$.). Außerdem stecken in den bisherigen theoretischen Überlegungen auch wenn sich diese nicht direkt aus authentischen Sprachdaten gespeist haben - außerordentlich differenzierte Beobachtungen und viel linguistische Denkarbeit, und es wäre natürlich ein Rückschritt, alle Theoreme pauschal über Bord zu werfen. Einem gewaltsamen Rückfall in die Anfänge der Linguistik überhaupt ist vielmehr eine Forschungsperspektive vorzuziehen, in der die Korpuslinguistik nicht zerrissen zwischen dem Corpus-driven- und dem Corpus-based-Ansatz zu sehen ist, sondern in der die linguistischen Vorannahmen bewusst kontrolliert werden und die einzelnen Untersuchungen auf einem Kontinuum zwischen den beiden Ansätzen verortet werden können. Manche Untersuchungen haben nämlich einen ausgeprägten induktiven Anteil und nutzen doch die Vorteile der bisherigen Erkenntnis.

17 Weitere Vertreter des Corpus-driven-Ansatzes nennt Gries (2010: 328).

$18 \mathrm{Zu}$ ähnlichen Ansätzen vgl. Primus (i. d. Bd.: Abschnitt „Emergente Grammatik“).

19 Oft mit korpusgestützt übersetzt. 
So wurden am IDS verschiedenartige Untersuchungen zur Variation der starken Markierung des Nomens im Genitiv Singular (z. B. zwischen -s, -es, und $\emptyset$ wie in Wracks / Wrackes / [des] ?Wrack ${ }^{20}$ ) durchgeführt. In ihrem Rahmen konnten maschinell ca. 7 Mio. Sätze mit Genitivnomina aus dem Deutschen Referenzkorpus (DeReKo, Institut für Deutsche Sprache 2011) extrahiert werden (Bubenhofer et al. 2014b, Konopka \& Fuß 2016: 25 ff.). Dabei wurden mit einem Perl-Skript Taggerinformationen sowie formale und distributionelle Eigenschaften der Genitivnomina abgefragt, die in Voruntersuchungen festgelegt worden waren. Die Sätze mit den Genitivnomina wurden - um Metadaten ergänzt - in einer Datenbank (Bubenhofer et al. 2015) ${ }^{21}$ abgelegt und recherchierbar gemacht. Bei den Metadaten handelte es sich um jeweils bis zu 80 Angaben morphologischer, lexikalischer, prosodischer, phonologischer und extralinguistischer Art, die die Genitivnomina bzw. die sie enthaltenden Sätze unter verschiedensten Aspekten beschrieben. Die Metadaten gingen vor allem auf Korpusannotationen zurück oder wurden nach einem automatischen Abgleich der Genitivnomina mit dem deutschen Bestand der CELEX Lexical Database (Baayen et al. 1995) hinzugefügt. Die Identifizierung der Genitive im Korpus beruhte auf relativ unkomplizierten und unstrittigen Annahmen, was Genitive starker Nomina wie Vaters, Hauses, oder [des] Barock sind, und dennoch bildete sie wie auch die Hinzufügung der Metadaten einen theoriegeleiteten, von deduktiven Schlüssen abhängigen Schritt. In seinem Ergebnis entstand dann aber eine sehr breite und differenzierte Datenbasis aus Genitivfundstellen und Metadaten, die jetzt auch datengeleiteten Analysen zugänglich war, die zur Stärkung des induktiven Anteils an der Theoriebildung beitragen konnten. Eine solche Analyse wurde von Bubenhofer et al. (2014b) unternommen. Dabei wurde zur Auffindung relevanter Einflussfaktoren der Variation der Genitivmarkierung ein maschinelles Lernverfahren verwendet. Das Ziel war ein datengeleitet gewonnenes probabilistisches Modell der Variation. Das statistische Modell wurde als Entscheidungsbaum visualisiert, ${ }^{22}$ um das komplexe Zusammenspiel verschiedener Faktoren der Markierungsvariation linguistisch interpretierbar zu machen. In der Visualisierung fiel zuallererst die besondere Bedeutung der Lexemfrequenz auf, ${ }^{23}$ die in der bisherigen Forschung als Einflussfaktor der Genitivmarkierungswahl noch nicht etabliert war, im Entscheidungsbaum aber die bereits als wichtig bekannten Faktoren

20 Für entsprechende Belege vgl. Konopka/Fuß 2016: 18.

21 Siehe http://grammis.ids-mannheim.de/genitivdb (letzter Zugriff: 20.4. 2018).

22 Siehe http://grammis.ids-mannheim.de/korpusgrammatik/5032 (letzter Zugriff: 20.4. 2018).

23 Bei einsilbigen Nomina des Grundwortschatzes korrelierte hohe Lexemfrequenz mit der Endung -es (vgl. weiter unten). 
Silbenanzahl und Sonderwortschatzzugehörigkeit des Lexems in den Schatten stellte. Daneben wurde im Bereich des Grundwortschatzes die Relevanz des Faktors Wortausgang und - wiederum unerwartet - der zeitlichen und räumlichen Einordnung der Belege deutlich ${ }^{24}$ (Bubenhofer et al. 2014b: 415 ff.)

Vor dem Hintergrund bestimmter Fragestellungen kann also aus dem Korpus theoriegeleitet eine umfangreiche Datenbasis aus relevanten Sprachund potenziell relevanten Metadaten gewonnen werden, die im nächsten Schritt datengeleiteten Analysen unterzogen wird. In solchen Analysen werden die Daten möglichst nicht präjudizierend betrachtet, um den Anteil induktiver Schlüsse an der Theoriebildung hoch zu halten. Es scheint allerdings, dass im Kontext linguistischer Untersuchungen induktives Schließen ohne deduktive Vorbereitung nicht praktikabel ist. Wie elementar auch immer die am Anfang stehende theoriegeleitete Eingrenzung der Fragestellung und der relevanten Datenbasis ist, sie impliziert immer deduktives Schließen. Alles, was man tun kann, um die Induktion zu stärken, ist später mit Hypothesen zurückhaltend zu sein, damit eine Vielzahl von Korpusphänomenen eine Chance bekommt, in die Beobachtung einzugehen, die zum Theorem verallgemeinert wird. Die Prüfung des so entstandenen Theorems kann dann ohnehin, wie bereits weiter oben gezeigt, nur top-down erfolgen. Dennoch sind datengeleitete Untersuchungen nützlich, da sie zur Entdeckung von gänzlich neuen Aspekten einer Fragestellung führen können.

Letztendlich kann es sich also als fruchtbar erweisen, manche grundlegende theoretische Konzepte und elementare Aussagen nicht infrage zu stellen, dafür andere grundsätzlich zu hinterfragen. Das ist die Strategie, die zu schnellsten Erkenntnisfortschritten führt und uns nicht zuerst erheblich zurückwirft. Im Folgenden werden reine Corpus-driven-Ansätze in jedem Fall kein Thema mehr sein. Vielmehr wird diskutiert, wie Korpusdaten (trotz der in Abschnitt 3 genannten Einschränkungen) als Quelle für erklärungsbedürftige Phänomene zur Aufstellung neuer Theoreme beitragen können, daneben, wie die Datenfülle, der wir ausgesetzt sind, zur Entstehung neuer theoretisch interessanter Fragestellungen führt, die unsere Perspektiven auf Grammatik erweitern und uns helfen, ihr Regelwerk zu verstehen.

24 Die Einsilbigkeit und die Obstruenz am Wortausgang förderten im Bereich des Grundwortschatzes die Endung -es. Genitivnomina in Texten aus jüngerer Zeit tendierten stärker als sonst zu -s. Genitivnomina in Texten aus dem Südosten wiesen häufiger als sonst -es auf. Zum Einfluss der Sonderwortschatzzugehörigkeit vgl. weiter unten. 


\section{Forschungspraxis und neue Fragestellungen}

Um falsifizierte Theoreme $\mathrm{zu}$ korrigieren, alternative Theoreme aufzustellen oder neue Beschreibungen und Erklärungen der Sprachrealität zu konzipieren, bedarf es natürlichsprachlicher Daten, auf die sich die theoretischen Aussagen beziehen sollen. Wie bereits dargestellt, können solche Daten primär der Introspektion entspringen (und damit implizit oder explizit an Wohlgeformtheitsurteile gekoppelt sein), sowohl der Introspektion als auch dem Korpus (wie bei Meurers 2005; Müller 2007; Meurers \& Müller 2009 oder Pullum 2017) oder dem Korpus allein (wie gefordert von Tognini-Bonelli 2001, 2010). ${ }^{25}$ Im Fokus der folgenden Ausführungen stehen Korpusdaten unabhängig davon, ob sie von der Introspektion explizit begleitet werden oder nicht.

Viele betonen die Fülle der authentischen Sprachdaten, die die Forscher mit den großen Korpora und den modernen Analysemöglichkeiten beinahe auf einmal in den Blick bekommen. Viele stellen auch wie die Autoren der GDS (Zifonun et al. 1997) fest, dass Korpora immer wieder ,ungewöhnliche‘ oder unerwartete Daten bieten. Solche Daten müssen mit erfasst werden. Das heißt auch, dass die bisherigen grammatischen Beschreibungen und Erklärungen eventuell an sie angepasst werden müssen. So führt eine durch Korpora herbeigeführte Erweiterung der bekannten Aspekte des Beschreibungsgegenstandes Grammatik automatisch zur Weiterentwicklung der Theoriebildung, bzw. sie leitet diese direkt ein.

Die Datenfülle bringt also zum einen die Datenvielfalt mit sich. Zum anderen macht sie die Untersuchung der Frequenz grammatischer Phänomene interessant. Jetzt wird es sinnvoll, die Korpusdaten nicht nur qualitativ, sondern auch quantitativ zu betrachten und statistisch zu analysieren. Der (mittlerweile oft riesige) Umfang der Korpora ${ }^{26}$ verführt regelrecht zu der Annahme, dass sie für die Sprachvarietät, der die Korpustexte entstammen, repräsentativ sind. Es wird dann von den Verhältnissen im Korpus auf die Verhältnisse im Sprachgebrauch dieser Varietät im Allgemeinen geschlossen. Die Statistik wird auf diese Weise zum festen Bestandteil des linguistischen Instrumentariums, und das Korpus wird wie eine Stichprobe des Sprachgebrauchs behandelt (vgl. Baroni/ Evert 2009: 777). So kalkuliert Biber (1993: 253 f.) etwa, wie groß aus statistischer Sicht ein Korpus sein müsste, damit man ein bestimmtes grammatisches

25 Die Daten können selbstverständlich auch anderen Empiriearten entstammen wie Befragung oder Experiment. Dies ist aber für die weitere Argumentation, in der es speziell um die Bedeutung der Korpuslinguistik für die Grammatiktheorie geht, zweitrangig.

26 DeReKo etwa umfasste am 3.2. 201841 Mrd. Wörter. 
Phänomen (z. B. Relativsätze eines bestimmten Typs) in der englischen Sprache adäquat beschreiben kann.

Zurück zur Datenvielfalt: Die Vielfalt betrifft bei großen Korpora wie DeReKo, webbasierten Korpora in COW16 ${ }^{27}$, The Collins Corpus (The Bank of English $\left.{ }^{28}\right)$, Corpus of Contemporary American English $\left(\mathrm{COCA}^{29}\right)$ oder British National Corpus (BNC) sowohl den Dateninhalt selbst als auch die Umstände der Datenentstehung. ${ }^{30}$ Was den Dateninhalt angeht, wird nicht nur die Vielzahl verschiedener grammatischer Muster offenkundig, sondern auch die Tatsache, dass in bestimmten Situationen quasi eine Wahl zwischen verschiedenen grammatischen Mustern besteht.

So kann es bei einer nur generell festgelegten grammatischen Struktur immer wieder zu im Einzelnen abweichenden Realisierungen kommen. Conrad schreibt dazu:

The great contribution of corpus linguistics to grammar is that it increases researchers' ability to systematically study the variation in a large collection of text - produced by far more speakers and writers, and covering a far greater number of words, than could be analyzed by hand. (Conrad 2010: 228)

Variation ist das Schlüsselwort. Sie muss nicht nur deskriptiv festgehalten werden (das könnte vielleicht noch im Rahmen einer klassischen Kompetenztheorie geschehen), sondern sie verlangt auch danach, im Hinblick auf ihre Abhängigkeit von verschiedenen grammatikinternen und/oder -externen Einflussfaktoren erklärt $\mathrm{zu}$ werden. Dass damit Theoriebildung eingeleitet wird, steht außer Frage. Dass sich die zu formulierenden Theoreme in jedem Fall auf die Grammatik beziehen, kann auch nicht bestritten werden. Die Theorie soll hier aber nicht mehr nur das beschreiben, was man sich ursprünglich unter (grammatischer) Kompetenz vorgestellt hat, sondern auch die Wahlmöglichkeiten, die Performanzphänomene. Soll sie hier in eine Theorie der Kompetenz und eine Theorie der Performanz aufgespalten werden? Ist eine solche Trennung noch zweckdienlich?

Solche Fragen können im Rahmen dieses Beitrags nicht endgültig beantwortet werden. Aber schauen wir uns den sich unter Umständen anbahnenden Paradigmenwechsel in der Grammatiktheorie genauer an. Der Gegenstand der

27 Siehe http://corporafromtheweb.org/category/corpora/ (letzter Zugriff: 1.9. 2017).

28 Siehe https://collins.co.uk/page/The+Collins+Corpus (letzter Zugriff: 1.9. 2017).

29 Siehe https://corpus.byu.edu/coca/ (letzter Zugriff: 1.9. 2017).

$30 \mathrm{Im}$ Falle von BNC etwa wurden sie teils mithilfe festgelegter Auswahlkriterien gesteuert, teils nur festgehalten - siehe http://www.natcorp.ox.ac.uk/corpus/creating.xml (letzter Zugriff: 1.9. 2017). 
grammatischen Theorie in strukturalistischer Tradition ist so etwas wie ein idealisiertes, konsistentes grammatisches System, dessen Kern die Syntax ausmacht. Pullum, der eine gemäßigte strukturalistische Position generativer Prägung vertritt, ist der Meinung, dass dieses System normativer Natur ist:

The subject matter of syntax is neither past utterance production nor the functioning of inaccessible mental machinery; it is normative - a system of tacitly grasped constraints defining correctness of structure. (Pullum 2017: 283)

I take linguistics to have an inherently normative subject matter. The task of the syntactician is exact codification of a set of norms implicit in linguistic practice. (Pullum 2007: 41)

Die Normativität des Systems manifestiere sich in Wohlgeformtheitsurteilen über Äußerungen (vgl. Pullum 2017: 294). Der Schwerpunkt der Theorie liege darauf, welche Normen das idealisierte System konstituieren bzw. welche Äußerungen in diesem System möglich sind und welche nicht. Eine solche Perspektive ist dichotom - die Äußerungen werden als entweder korrekt oder nicht korrekt, akzeptabel oder nicht akzeptabel beurteilt (vgl. Conrad 2010: 227, darin auch weitere Literaturhinweise).

Diese dichotome Perspektive auf Sprache ist für die Untersuchungen und die theoretische Modellierung der Variation nicht ausreichend, denn bei dieser haben wir es mit zwei oder mehr Varianten zu tun, die alle wohlgeformt erscheinen. Die Perspektive verschiebt sich: Jetzt geht es darum, zunächst überhaupt die Variation zu diagnostizieren und dann zwischen in bestimmten Kontexten typischen und untypischen Varianten zu unterscheiden (vgl. Conrad 2010: 228). Die Dichotomien richtig/falsch, akzeptabel/nicht akzeptabel etc. sind nicht mehr zentral, sondern es geht um unter bestimmten Umständen mehr oder weniger Wahrscheinliches. Eine Problemstellung übrigens, die mittels Introspektion kaum angegangen werden kann, denn die Sprachbenutzer sind sich oft nicht bewusst, welche Wahl sie typischerweise treffen (Conrad 2010: 227).

Weiterhelfen können hingegen korpuslinguistische Techniken, die es erlauben, Varianten zu finden und Frequenzen von Varianten in bestimmten Kontexten miteinander zu vergleichen. Um feststellen zu können, ob wir es tatsächlich mit Varianten/Wahlmöglichkeiten zu tun haben und was die typische Variante/ Wahl in einer spezifischen Gebrauchskonstellation ist, müssen Gebrauchskonstellationen klassifiziert und im Korpus abfragbar gemacht werden. Das ist mit großen Korpora gut möglich - hier kommt der Annotation der Korpustexte eine große Bedeutung $\mathrm{zu}^{31}$

31 Vgl. Bubenhofer et al. (2014a: 66-77, 84-91). 
Die grammatischen Entscheidungen können mit lexikalischen Entscheidungen, grammatischer Organisation des Kotextes oder Aspekten des Sprachgebrauchs zusammenhängen, die über die Grammatik hinausgehen, ${ }^{32}$ wie etwa dem Medium, dem Register (der Textsorte) oder der regionalen Einbettung des Textes. ${ }^{33}$ Solche Begleitaspekte grammatischer Entscheidungen können aus einer anderen Perspektive als Einflussfaktoren der Variation betrachtet werden. Im Folgenden sollen drei Beispiele aus der Forschungspraxis vorgestellt werden, in denen lexikalische Faktoren, komplexe satzsemantische Faktoren und außergrammatische Faktoren der Variation exploriert wurden.

Eine Reihe lexikalischer Einflussgrößen wurde in den bereits erwähnten Korpusuntersuchungen von Konopka \& Fuß (2016) zur Variation der Markierung des starken Nomens im Genitiv Singular gefunden. Einen der wichtigsten Faktoren, die die Variation zwischen -es, -s und der Nullmarkierung (vor)bestimmen, stellt dort die Zugehörigkeit des Nomens zum Grundwortschatz oder zum Sonderwortschatz dar. Im ersteren Wortschatzbereich versammeln sich einheimische bzw. längst integrierte Appellativa, im letzteren in das (durch native Appellativa geprägte) Nomensystem schwach integrierte Wortgruppen wie Fremdwörter, Eigennamen (Personennamen, geographische Namen), Epochen- und Stilbezeichnungen oder Konversionen. Bei den Nomina des Grundwortschatzes kann es eigentlich nur die Wahl zwischen den Endungen -es und -s geben (die Nullmarkierung ist hier nur bei ca. 4,6\% Genitivtoken zu finden), bei den untersuchten Sonderwortschatzgruppen wiederum kommt als Alternative zur Nullmarkierung nur die Endung -s infrage (-es ist hier nur bei ca. 1,8\% Genitivtoken zu finden). Die Variantenauswahl wird somit durch die lexikalischen Eigenschaften der Nomina eingeschränkt, vgl. Tabelle 5.1.

Als weitere lexikalische Faktoren, die die Wahl der Genitivmarkierung beeinflussen, sind die Frequenz und das Alter eines Lexems hervorzuheben. Beide Faktoren sind eng miteinander verzahnt. Die Analysen im virtuellen DeReKo-Korpus aus Texten von 1955 bis 2011 weisen auf einen mit der Zeit voranschreitenden Schwa-Wegfall hin, also die Entwicklung hin zu -s. Dieser Entwicklung trotzen die fünf im Korpus häufigsten Nomina Jahr, Tag, Land, Kind, Mann, die in über 99\% der Fälle den Genitiv mit -es bilden. Bei diesen Erbwörtern scheint die alte Endung durch eine konstant sehr hohe Frequenz in besonderer Weise konserviert zu werden. Auch bei sonstigen Einsilbern des

32 Conrad (2010: $229 \mathrm{ff}$.) nennt als Faktoren, mit denen grammatische Entscheidungen assoziiert sind, etwas spezifischer: „(1) vocabulary, (2) grammatical co-text, (3) discourse-level factors, and (4) the context of the situation.“

33 Eine etwas andere Strukturierung und mehr individuumsbezogene Aspekte finden sich bei Fuß (i.d. Bd: Einleitung). 
Tab. 5.1: Lexikalische Faktoren der Genitivvariation des starken Nomens.

\begin{tabular}{lll}
\hline Keine Genitivendung & Endung $-s$ & Endung -es \\
\hline $\begin{array}{l}\text { Grundwortschatz } \\
\text { des Saal(e)s, des Zwang }(e) s, \text { des Blut(e)s, des Betrieb }(e) s\end{array}$ \\
Sonderwortschatz wie \\
Fremdwörter (des Internet(s), des Meeting(s)) \\
Eigennamen (des alten Rom(s), des jungen Werther(s)) \\
Epochen- und Stilbezeichnungen $($ des Punk(s), des Samba(s)) \\
Konversionen (des Rot(s), des Ich(s))
\end{tabular}

Grundwortschatzes beeinflusst die Kombination aus Lexemfrequenz und -alter die Wahl der Genitivmarkierung, und zwar gemäß der konservierenden Wirkung der Frequenz tendenziell in der Weise, dass -es umso wahrscheinlicher ist, je häufiger das Nomen gebraucht wird (Konopka \& Fuß 2016: $251 \mathrm{f}$.).

Frequenzdaten sind noch keine Erklärungen für Variationsphänomene. Sie weisen lediglich auf interessante Zusammenhänge hin, die erst theoretisch aufgearbeitet, sprich, genau beschrieben und erklärt werden müssen. Die Erklärungen können sich auf kognitive Prinzipien, historische Entwicklungen oder die pragmatische Funktion der grammatischen Wahl berufen (vgl. Conrad 2010: 228f.). Sie weisen dann meist über die Grenzen dessen hinaus, was aus generativer Perspektive als Grammatik betrachtet wird (vgl. Primus i.d. Bd., Fuß i.d. Bd.). Ein umfassender Versuch, die Wirkung der lexikalischen Faktoren wie Zugehörigkeit zum Grund- oder Sonderwortschatz, Frequenz und Wortalter auf die Wahl der Genitivmarkierung zu erklären, würde den Rahmen dieses Beitrags sprengen. Er ist aber in Konopka \& Fuß (2016: v. a. $250 \mathrm{ff}$.) nachzulesen.

Komplexe Faktoren, die der Syntax-Semantik-Schnittstelle entspringen, haben in der Untersuchung von Brandt (in Vorb., unter Mitwirkung von Bildhauer) einen Einfluss auf die Variation zwischen Infinitivkonstruktionen mit $z u$ - und dass-Sätzen in der Funktion eines Komplements, vgl.:

(4) „Doch ich habe geschworen, dass ich beim Malen sterbe", schreibt Paul Cézanne im Oktober 1906 an seinen Sohn.

(Neue Kronen-Zeitung, 17.1. 2000, S. 32)

(5) Ich hatte geschworen, die Wahrheit $z u$ suchen, aber mußte ich durch solch ein Labyrinth?

(Der Spiegel, 20. 9. 1993; Wunder sind mein Wesen) 
Brandt untersucht das Phänomen qualitativ sowie quantitativ mithilfe verschiedener statistischer Verfahren in einem über vier Mrd. Token großen Ausschnitt von DeReKo. Er konzentriert sich zunächst auf einen Faktor, dessen besonderer Einfluss auf die Wahl zwischen der zu- und der dass-Komplementierung in der Literatur schon öfter beobachtet wurde (vgl. Wöllstein 2015; Rapp et al. i. E.) die Koreferenz des Subjekts des dass-Satzes bzw. des impliziten Subjekts der Infinitivkonstruktion mit einem Element des Matrixsatzes (in (4) und (5) ist es ich/Ich). Die Alternation zwischen der dass- und der $z u$-Komplementierung soll insbesondere dann möglich sein, wenn das implizite Subjekt der Infinitivkonstruktion durch ein Argument des Matrixsatzes ,kontrolliert' werden kann. Brandt prüft, wie sich verschiedene Kontroll- bzw. Koreferenztypen genau auf die Alternation auswirken. Er unterscheidet je nach Funktion des koreferenten Elements des Matrixsatzes Koreferenz mit:

- Subjekt: siehe (4) und (5) weiter oben

- Akkusativobjekt: Der Mann hatte sie zuvor in der U-Bahn aufgefordert, das Rauchen zu unterlassen. (dpa, 17.4. 2008, „Münchner U-Bahnschläger wegen versuchten Mordes angeklagt")

- Dativobjekt: Für diesen Fall haben die Richter dem Gesetzgeber aufgetragen, Paritäten neu zu definieren. (Frankfurter Allgemeine, 1995)

- Element einer vom Verb abhängigen Präpositionalphrase (eines Präpositionalobjekts): Die Soldaten hätten von den Bewohnern gefordert, Barrikaden zu beseitigen und Waffen abzugeben. (dpa, 21. 6. 2010, „Tote bei neuen Unruhen in Kirgistan“) $)^{34}$

Eine logistische Regressionsanalyse ${ }^{35}$ anhand von 7.685 Belegen mit $z u$ - und dass-Komplementen zeigt auf der einen Seite an, dass jede Art von Koreferenzbeziehung zwischen einem Element im Matrixsatz und dem (expliziten oder impliziten) Subjekt des Komplements die Wahrscheinlichkeit erhöht, ein $z u$ Komplement vorzufinden (vgl. Abb. 5.2). Dabei steigt diese Wahrscheinlichkeit signifikant stärker an, wenn das koreferente Element im Matrixsatz die Funk-

34 Die Reihe wird komplettiert durch $z u$ - bzw. dass-Komplemente ohne koreferentes Element im Matrixsatz. Entsprechende Sätze mit $z u$-Komplementen sind verhältnismäßig selten, treten aber regulär bei obviativen Verben auf wie anordnen, billigen, gutheißen (z. B.: Vor einer Woche hatte Putin angeordnet, die Terroristen zu finden und zu vernichten, Rhein-Zeitung, 4. 7. 2006, „Gibt Moskau Agenten Lizenz zum Töten?“ - zu solchen Fällen vgl. Brandt, Trawinski \& Wöllstein 2016) und beim unpersönlichen Passiv im Matrixsatz (z. B.: Seit 20 Jahren wird versprochen, die Grundschulbildung zu verbessern, aber wenig ist passiert, Die Rheinpfalz, 16.4. 2009, S. 3, „Ein Milliardenvolk geht wählen“).

35 Zur Analyse vgl. Bildhauer \& Brandt: Alternation von zu- und dass-Komplementen - logistische Regressionsanalyse unter http://grammis.ids-mannheim.de/korpusgrammatik/6393 (letzter Zugriff: 14. 5. 2018). 


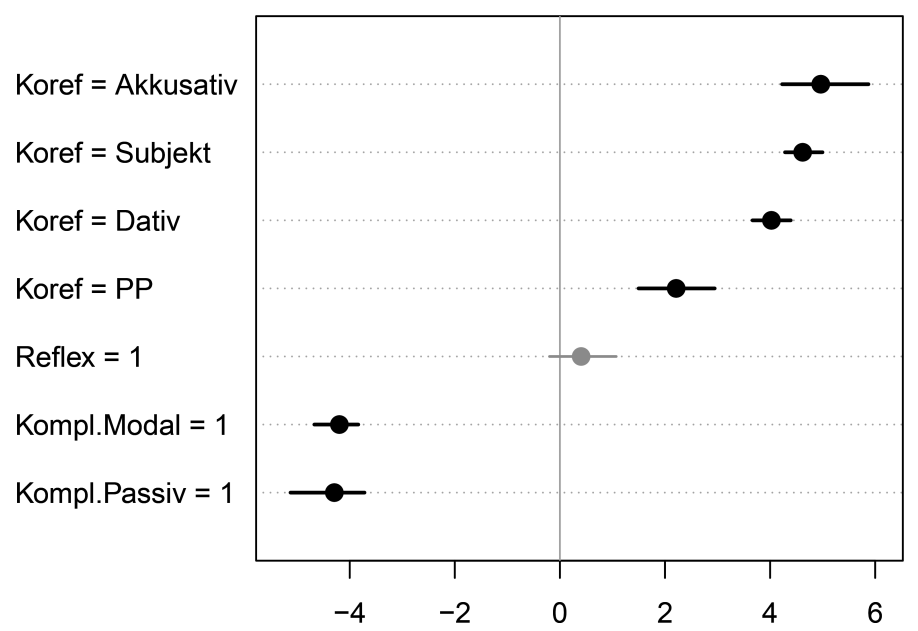

Abb. 5.2: Visualisierung der festen Effekte der Prädiktorvariablen auf die Wahrscheinlichkeit des zu-Komplements.

tion eines Subjekts, Akkusativobjekts oder Dativobjekts hat, als wenn es nur Teil einer Präpositionalphrase ist. Auf der anderen Seite fällt auf, dass die Anwesenheit eines Modalverbs oder des Passivs im Komplement (Infinitivkonstruktion oder dass-Satz) die Wahrscheinlichkeit seiner Realisierung als Infinitivkonstruktion deutlich senkt.

Erneut hat man es hier mit quantitativ gut begründeten Zusammenhängen zu tun, und die Wirkung der satzsemantischen Faktoren Koreferenz, Präsenz eines Modalverbs und Passiv im Komplement wartet jetzt darauf, theoretisch interpretiert $\mathrm{zu}$ werden. Eine Herausforderung dürfte dabei sein, dass man es hier nicht mit der Frage zu tun hat, wann eine Infinitivkonstruktion möglich ist, sondern mit der Frage, wann sie wie wahrscheinlich ist (zu theoretischen Vorschlägen vgl. Brandt i. Vorb.).

Münzberg \& Hansen-Morath (2018) untersuchen die Variation des Numerus des finiten Verbs bei zwei mit und koordinierten singularischen Nomina als Subjekt. Sie wird vor allem von einem syntaktischen Phänomen im Kontext beeinflusst. Bei koordinierten Nomina ohne zwingende Referenzidentität ${ }^{36}$ stehe das Verb normalerweise im Plural. Der Singular könne aber auch vorkommen, insbesondere wenn beide Nomina einen gewissen Abstraktheitsgrad

36 Hier scheiden Fälle wie der folgende aus: Die Sportwissenschaftlerin und Sportmedizinerin erklärt, dass bei solchen Extrembelastungen der Druck auf die Gefäße viel zu groß sei (Mannheimer Morgen, 22.3. 2004, „Statt Stress in der Muckibude lieber langsam laufen“). 
aufweisen. Die Autorinnen konzentrieren sich auf Sätze, in denen das erste Nomen mit dem definiten Artikel erscheint und das zweite zumindest nicht primär artikellos ist. ${ }^{37}$ Gerade ob das zweite Nomen mit oder ohne Artikel steht, könne als besonders starker Einflussfaktor bei der Variation des Numerus des finiten Verbs gesehen werden. Münzberg und Hansen-Morath betrachten Koordinationen von zwei Feminina, bei denen sich vier relevante Muster ergeben:

- Muster 1: Die Wucht und die Strömung war immens.

- Muster 2: Die Wucht und die Strömung waren immens.

- Muster 3: Die Wucht und Strömung war immens. ${ }^{38}$

- Muster 4: Die Wucht und Strömung waren immens.

Einschlägige Sätze werden aus einem Untersuchungskorpus extrahiert, das auf DeReKo (Institut für Deutsche Sprache 2014) zurückgeht und knapp 8 Mrd. Token umfasst. Nach manueller Annotation von mehreren für die Variation hypothetisch relevanten Merkmalen in 842 gültigen Belegen können die Autorinnen in statistischen Auswertungen zeigen, dass Sätze nach den Mustern 2 und 3 höchstsignifikant häufiger vorkommen als Sätze nach den Mustern 1 und 4. Das Fehlen des Artikels - in der Literatur üblicherweise als Artikelellipse bezeichnet $^{39}$ - unterstützt dementsprechend den Singular; in Sätzen ohne Artikelellipse überwiegt hingegen der Plural. Artikelellipse hat statistisch gesehen den größten Einfluss auf die Numerusentscheidung. Daneben stellen sich andere Faktoren wie der bereits angesprochene Abstraktionsgrad ${ }^{40}$ als relevant heraus. Unter ihnen findet sich auch eine außersprachliche Einflussgröße - die diatopische Einordnung des Textes.

Münzberg und Hansen-Morath machen von der Strategie Gebrauch, die behandelten Phänomene standardmäßig auf die Varianz innerhalb der Dimensionen Medium, Register (Textsorte), inhaltliche Domäne, Land und Region hin zu überprüfen - nach solchen Variationsdimensionen werden Korpustexte

37 Damit scheidet in jedem Fall etwa der folgende Satz aus, in dem das zweite Nomen primär artikellos ist: Die EU und Russland haben die letzten verbleibenden bilateralen Fragen für einen Beitritt Russlands zur Welthandelsorganisation WTO gelöst (NZZ, 22.10. 2011, S. 29, „Der WTOBeitritt Russlands rückt näher").

38 Dieses Muster entspricht dem Originalsatz: Die Wucht und Strömung war immens, die Pulosans wurden meilenweit ins offene Meer getrieben (Süddeutsche Zeitung, 19.12. 2011, S. 10, „Verheerende Fluten“).

39 Vgl. z. B. Hennig (2015: 59), Dammel (2015: 315-317).

40 Um den Einfluss der Kombination Abstraktionsgrad und Artikelellipse einzuschätzen, wurden mehrere logistische Regressionsmodelle berechnet und evaluiert. Als am besten hat sich das Modell mit beiden Faktoren und ohne Aufnahme der Interaktion zwischen ihnen erwiesen (detailliert in Münzberg \& Hansen-Morath 2018). 
nämlich im IDS-Projekt Korpusgrammatik annotiert, ${ }^{41}$ in dem die Autorinnen arbeiten. Sie stellen überraschenderweise fest, dass der Ellipseneffekt in den Schweizer Daten schwächer ausfällt, vgl. Abbildung 5.3:

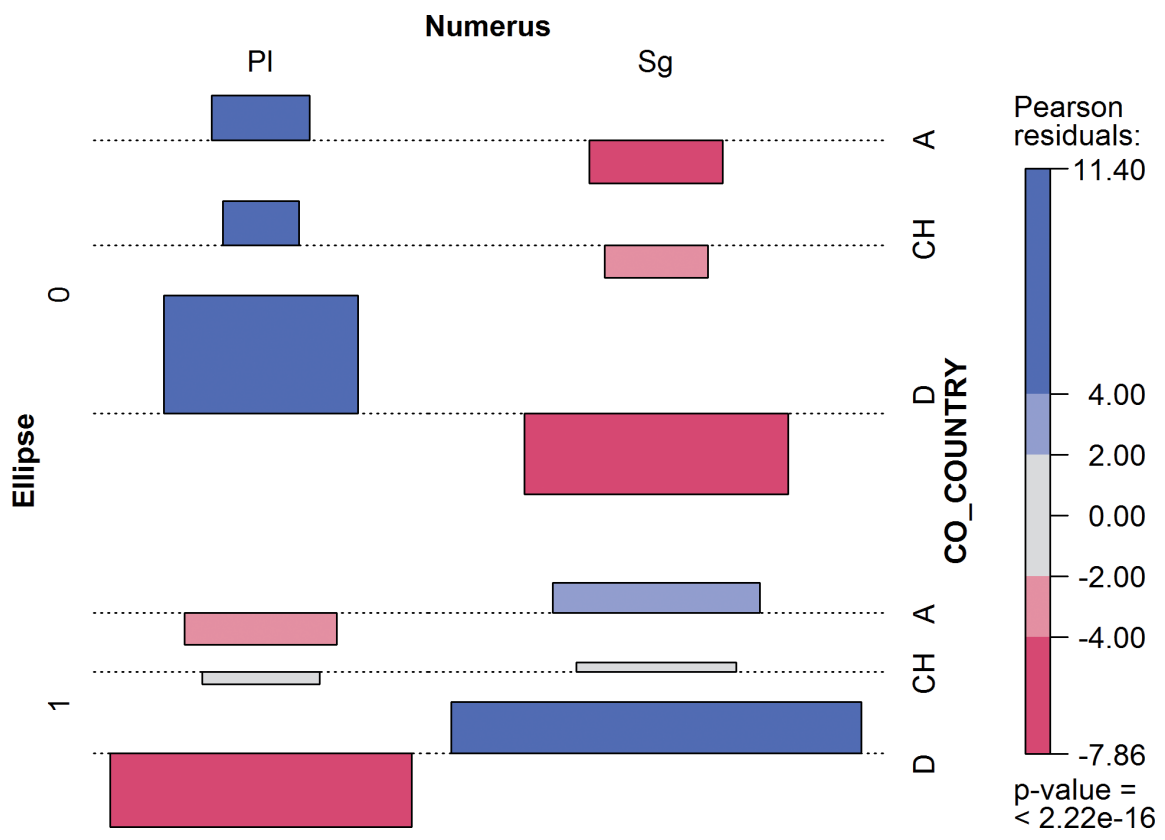

Abb. 5.3: Assoziationsplot ${ }^{42}$ zum Ellipseneffekt in Texten aus verschiedenen Ländern: ${ }^{43}$ starker Ellipseneffekt in Texten aus Deutschland (D) und Österreich (A), schwächerer Ellipseneffekt in den Texten aus der Schweiz (CH)

41 Vgl. Bubenhofer et al. (2014a: 62 ff.) bzw. - mit aktualisierten Daten - „Metadaten als Variabilitätsfaktoren“ unter http://grammis.ids-mannheim.de/korpusgrammatik/4755 (letzter Zugriff: 14. 5. 2018).

42 Der Assoziationsplot (vgl. Cohen 1980; Friendly 1992; Mayer et al. 2005) stellt die standardisierten Pearson-Residuen der Häufigkeiten von Singular und Plural in Abhängigkeit von den Faktoren ,Artikelellipse ' und ,Land“ dar. Balken oberhalb der gepunkteten Linie bedeuten, dass die Werte höher sind als erwartet, Balken unterhalb der Linie bedeuten, dass die Werte niedriger sind als erwartet. Die Breite der Balken spiegelt die erwartete Häufigkeit wider. Signifikante Pearson-Residuen werden rot bzw. blau eingefärbt.

43 Belastbare Zahlen gab es nur zu Deutschland, Österreich und der Schweiz. Ansonsten waren auch vereinzelt Belege aus Luxemburg zu finden. 
Ob der zweite Artikel fehlt oder erscheint, scheint für die Variation des Numerus des finiten Verbs in der Schweiz merklich weniger Bedeutung zu haben als in den beiden anderen Ländern. ${ }^{44}$ Tiefer gehende Untersuchungen zeigen, dass die schweizerische Schwäche des Ellipseneffekts ganz konkret darauf zurückgeht, dass in den entsprechenden Texten bei der Weglassung des Artikels unerwartet oft das Verb im Plural auftritt wie in:

(6) Die Erstellung und Bepflanzung kosten 70.000 Franken. (St. Galler Tagblatt, 12.4. 2010: 36; „St. Michael erhält einen Rebberg“)

Das ist eine Spur, der nachzugehen ist und die nach weiteren Analysen theoretisch aufgearbeitet werden muss. Neben einer assoziierten grammatischen Erscheinung, der Artikelellipse, und einem lexikalisch-semantischen Merkmal, dem Abstraktheitsgrad des Nomens, hat hier ein außergrammatischer Faktor, das Herkunftsland des Textes, den Zeiger ausschlagen lassen. Theoretisch herausfordernd ist $\mathrm{u}$. a. die Frage, ob hier noch von einem grammatischen Regelwerk bzw. von einem System auszugehen ist oder doch schon von verschiedenen diatopischen Varietäten.

Es ist Zeit für ein Zwischenfazit. Die modernen Korpora stellen große Datenmengen zur Verfügung und versorgen die Forscher u. U. mit ,neuen' Sprachphänomenen, die erst einmal zu erfassen und dann auch noch zu erklären sind. Dadurch wird der induktive Aspekt der Forschung gestärkt. Die Größe der modernen Korpora führt im Weiteren dazu, dass häufig von ihrer Repräsentativität für den Sprachgebrauch ausgegangen wird und vom Korpus auf die Verhältnisse in einem Sprachgebrauchsbereich oder gar in der Einzelsprache als Ganzes geschlossen wird. Die großen Datenmengen bringen große Datenvielfalt mit sich. Die Variation rückt in den Fokus und wird auch für Grammatiker zu einem großen Thema. Sie muss beschrieben und erklärt werden. Damit sind die zwei Pfeiler einer jeden Theorie - Deskription und Explanation bereits angelegt. Dass Korpusdaten und korpuslinguistische Methoden eine spezifische Art der Theoriebildung anregen, ist also nicht mehr von der Hand zu weisen. Es bleibt noch die Frage zu klären, welcher Art die Grammatiktheorien sein können, die die grammatische Variation im Korpus erklären. Die Antwort hängt natürlich erst einmal von der theoretischen Vorprägung ab. Ex negativo ist festzustellen, dass die Erkenntnisse aus den Korpora weit

44 Die Ergebnisse der für jedes Land einzeln durchgeführten Chi-Quadrat-Tests fallen alle höchst signifikant aus $(p<0,001)$. Die Effektstärke Phi ist in allen Fällen als groß einzustufen: Sie beträgt für Texte aus Deutschland und Österreich 0,69 bzw. 0,64 und für Texte aus der Schweiz 0,54. 
über das hinausgehen, was eine Theorie klassisch generativer Prägung gebrauchen kann (vgl. Fuß i.d. Bd.; Haider i.d. Bd.). So liefern Korpora etwa einer grammatischen Kompetenztheorie im Sinne Pullums zwar neue Daten, die beschrieben und erklärt werden müssen, spätestens dann aber, wenn Variationsphänomene in Augenschein genommen werden, deren Einflussfaktoren als außergrammatisch $\mathrm{zu}$ bewerten sind, wird eigentlich eine Theoriebildung angeregt, die für Strukturalisten (darunter auch Generativisten) auf die ungeliebte Theorie grammatischer Performanz hinauslaufen müsste. Es stellt sich die Frage, ob die Trennung in eine Theorie der Kompetenz und eine Theorie der Performanz jetzt noch zweckdienlich ist. Da die Beschreibung der Performanz immer präziser wird, strebt letztlich auch sie die Eliminierung des Konzepts der freien Variation zugunsten einer regelbasierten Theorie an. Ist es also nicht sinnvoll, das gesamte Sprachwissen als regelbasiert zu modellieren, das die Sprachteilhaber zur erfolgreichen Kommunikation befähigt? Dies könnte es zum Beispiel erleichtern, den Sprachwandel zu erklären. Alternativ zu einer regelbasierten Theorie, die Kompetenz und Performanz integriert, müsste man - wenn man sich unbedingt auf die Kompetenzbeschreibung beschränken wollte - zuerst für alle außergrammatischen Faktoren der Variation eine Taxonomie entwickeln und dann sehr viele verschiedene Kompetenzsysteme beschreiben, die sich auf die verschiedenen aus der Taxonomie abgeleiteten Varietäten beziehen.

So liefern Korpora einerseits mehr Daten, als eine Grammatiktheorie strukturalistischer Prägung brauchen oder verkraften kann, aber sie liefern trotzdem nicht genug. Was durch Datenmenge und -vielfalt nicht ausgeglichen werden kann, ist das Fehlen der Akzeptabilitätsurteile, da das Korpus keine negative Evidenz hervorbringen und nicht immer für gesicherte positive Evidenz sorgen kann. Die Akzeptabilitätsurteile werden also keineswegs überflüssig, wohl aber komplizierter, indem sie immer weiter von den Richtig/falsch-Grammatikalitätsurteilen über kontextfreie Strukturen wegrücken. Die Akzeptabilitätsurteile müssen jetzt probabilistisch gefasst werden, bzw. die Kontextfreiheit muss konsequent aufgegeben und die Spezifikation der extralinguistischen Situation präzise mit berücksichtigt werden. ${ }^{45}$

Schließlich soll hier auch festgehalten werden, dass die Korpusbenutzung andere Empiriearten nicht überflüssig macht. Korpus als Quelle für meist spontan entstandene Sprachdaten ist oft nicht genug, etwa wenn das zu untersuchende Phänomen sehr selten oder der Kontext zu spezifisch ist. In dem Fall können Befragungen oder Experimente das Mittel der Wahl sein.

45 Dazu auch Conrad 2010: $237 \mathrm{f}$. 


\section{Korpuslinguistik und die Grammatikographie des Deutschen}

Die technischen Möglichkeiten, große Datenmengen zu digitalisieren, zu speichern, allgemein zur Verfügung zu stellen und automatisch zu analysieren, mit denen der Höhenflug der modernen Korpuslinguistik untrennbar verbunden ist, formten auch die Fragestellungen der linguistischen Forschung. Die im Sprachgebrauch vorfindliche Vielfalt der Ausdrucksmöglichkeiten zog die Aufmerksamkeit der Forscher auf sich. Der Schwerpunkt der grammatischen Beschreibung bewegte sich von der Frage, was richtig sei, über die Einsicht, dass grammatische Strukturen variieren können, zu der Frage, was in der gegebenen Gebrauchssituation typisch ist. Grundsätzlich änderte sich an den Fragestellungen einer deskriptiven Grammatik nicht viel - es geht immer noch um die Beschreibung der in einer Sprache möglichen Strukturen und ihrer Distribution, nur soll diese Beschreibung jetzt noch einmal differenzierter werden. Angesichts der gegebenen Datenvielfalt ist sie vor allem hinsichtlich der Distribution, also der Bedingungen für das Auftreten von Strukturen, voranzutreiben. Das Interesse an Grammatik macht heute keinen Halt, wenn es darum geht, die - von der Grammatik her gesehen - freie Variation zu erklären und auch extralinguistische Bedingungen für das Erscheinen von Phänomenen, d. h. Einflussfaktoren wie Zeit, Raum, sozialen Hintergrund der Sprachteilhaber, Kommunikationssituation u. v. m. zu erläutern. Die Autoren wissenschaftlicher Grammatiken des Deutschen müssen sich diesem Bedarf stellen. Mit gutem Beispiel vorangegangen ist hier die Grammatikographie des Englischen (zur kritischen Diskussion vgl. Mukherjee 2006), die bereits vor längerer Zeit stärker korpusorientierte Referenzgrammatiken hervorgebracht hat wie Cambridge Grammar of English (Carter \& McCarthy 2006) und vor allem Longman Grammar of Spoken and Written English (Biber et al. 1999).

Unserer Introspektion können wir uns nicht immer sicher sein, denn schon ein Individuum aus unserem Umfeld kann manche grammatische Strukturen in Abhängigkeit von ihrem Präsentationsrahmen oder Informationsgehalt anders beurteilen als wir. Demgegenüber können wir in den Korpora unter Umständen das finden, was sehr viele Menschen gleichzeitig als akzeptabel betrachten (vgl. Conrad 2010: 237). Außerdem können uns Korpusdaten auf Strukturen aufmerksam machen, von denen wir vielleicht niemals gedacht haben, dass es sie gibt. Auch andere Empiriearten wie Befragungen, psycholinguistische Experimente oder - spezieller auch - neurolinguistische Messungen (vgl. Primus i.d.Bd.) können erst dann einsetzen, wenn die Datenmenge vorverarbeitet und die Datenvielfalt voranalysiert ist. Eine Grammatik des Deutschen, die auf einem umfangreichen Referenzkorpus basiert, ist heutzu- 
tage also ein immer dringender werdendes Forschungsdesiderat. Es sollte eine wissenschaftliche Grammatik sein, die die Nachvollziehbarkeit der Theoreme und die Replizierbarkeit der Ergebnisse von Einzeluntersuchungen sicherstellt, wie es sich die Forscher schon lange herbeisehnen. Eine solche Grammatik würde zur wissenschaftlichen Diskussion einladen und die Validierung vorgestellter Theoreme vereinfachen, was direkt ein Beitrag zum Fortschritt der Grammatiktheorie wäre.

Man kann sich fragen, welches Deutsch in einer solchen Grammatik beschrieben werden sollte, und dann auch, wie sich die Beschreibung zur linguistischen Theorie verhalten sollte. Zuerst zur ersten Frage: Auf der einen Seite erwarten die meisten heutzutage, dass eine Grammatik des Deutschen die Beschreibung einer Sprachform beinhaltet, die man üblicherweise als Standarddeutsch bezeichnet. Der Begriff ist auf der anderen Seite, wenn nicht intuitiv, dann zumindest sehr schwer operationalisierbar: ${ }^{46}$ Wo sind die Grenzen des Standarddeutschen? Ist es etwa eine einzige Varietät, die auf regionalspezifische, sozialgruppenspezifische, medienspezifische etc. Ausdrücke und Konstruktionen verzichtet und die in formellen und öffentlichen Kommunikationssituationen verwendet wird? Oder ist es eher ein Konglomerat von Varietäten, ${ }^{47}$ das diastratisch, diatopisch, diamesisch, diaphasisch etc. ausdifferenziert ist, um den meisten Kommunikationsanlässen in unserer differenzierten Gesellschaft gerecht zu werden? Wie auch immer man die Standardsprache definiert, jedem Grammatiker dürfte es schwerfallen, zu entscheiden, welche grammatischen Phänomene gerade noch zum Standarddeutsch gehören und welche nicht mehr. Zu rechnen ist dabei immer wieder auch mit Phänomenen, die im grammatischen Regelwerk nicht festgelegt sind und außergrammatisch modelliert werden müssen (Reis 2017).

Kommen wir zu der Frage nach der Rolle der linguistischen Theorie. Eine wissenschaftliche Referenzgrammatik sollte deskriptiv sein (dazu Zifonun 1997: 3f.). Ihre Domäne umfasst also zunächst einmal - je nach der Vorstellung von der zu beschreibenden Sprachform - alle grammatischen Strukturen und Phänomene, die im Standard möglich sind bzw. das gemeinsame grammatische Kernsystem aller Varietäten, die sich am Standard beteiligen. Von der grammatischen Theoriebildung klassisch strukturalistischer Prägung her gesehen (vgl. Abschnitt 3) gehört in den Zuständigkeitsbereich einer korpusgestützten Grammatik die Validierung der bisherigen Theoreme und die Lieferung von

46 Vgl. Bubenhofer et al. (2014a: 21 ff.) für eine ausführliche Diskussion.

47 Vgl. Löffler (2005) für eine ausführliche Diskussion solcher Fragen. Kleiner et al. sprechen im Duden (2015) in Bezug auf die gesprochene Sprache und Elspaß \& Dürscheid (2017) in Bezug auf die geschriebene Sprache von regional bzw. areal definierten Gebrauchsstandards. 
quantitativen Daten für verbesserte (datenkompatible) Theoreme. Da eine deskriptive Vollständigkeit hinsichtlich der Abdeckung der Grammatik einer ,Einzelsprache‘ bei einem wissenschaftlichen Werk, das ein hohes theoretisches Niveau und Aktualität anstreben muss, praktisch nicht möglich ist (vgl. Zifonun 1997: 3 f.), könnten seine Autoren sich unter Umständen auch nur auf die Validierung umstrittener Theoreme beschränken.

Über klassische Kompetenzbetrachtungen hinausgehend sollte eine innovative korpusgestützte Grammatik im Weiteren ausführlich die grammatische Variation behandeln. In Bezug auf einzelne Phänomene könnte dies folgendermaßen strukturiert werden:

1. Beschreibung grammatisch ,freier ' Variation im Standard/Kernbereich:

In allen Fällen, in denen keine grammatischen Bedingungen bekannt sind, die eine Auswahl weitgehend als ,kombinatorische Variation' aufklären, sollten die Häufigkeiten der Varianten im Verhältnis zueinander analysiert werden und nach Möglichkeit grammatische Faktoren ermittelt werden, die dieses Verhältnis beeinflussen.

2. Exploration der grammatisch ,freien' Variation entlang der außergrammatischen Variationsdimensionen:

a) eine routinemäßige Überprüfung (quantitative Exploration) der potenziellen Abhängigkeit der Variationsphänomene von Variationsdimensionen wie Medium, Register (Textsorte), Land, Region, Domäne, Zeit etc. etwa wie bei Münzberg \& Hansen-Morath (2018);

b) eine qualitative Explanation der Fälle, in denen eine Abhängigkeit von außergrammatischen Variationsdimensionen tatsächlich ermittelt wurde.

In Fällen, in denen (b) greift, begeben sich die Autoren automatisch in den Übergangsbereich zwischen dem zu beschreibenden Kern der Grammatik und seinen extralinguistisch markierten Erweiterungen. Sie können quantitative Daten und qualitative Argumentationen liefern, die zu einer genaueren Abgrenzung des Standards bzw. des den beteiligten Varietäten gemeinsamen Kernsystems und zur Konzeptualisierung solcher Vorstellungen erweitert werden können unter der Klärung der Frage, ob und evtl. wie viel extralinguistisch bedingte Variation zum Standard gehören kann.

Eine korpusgestützte Referenzgrammatik könnte, wie weiter oben erklärt, zur Theorie der Kompetenz im klassisch strukturalistischen Sinne beitragen. Bliebe man bei diesem Theorieverständnis, trüge der gesamte Punkt 2 aber nur zur Theorie der Performanz bei. Was die Zuordnung der Erträge der Grammatik zur Kompetenz oder Performanz angeht, wäre man sich bei Punkt 1 wiederum sehr unsicher. Hier sollten varietätenunabhängige ,Wahlfreiheiten“ ermittelt werden, die aufgrund nicht durchschlagender, aber immerhin nachweisbarer 
grammatischer Einflussfaktoren sich am Ende vielleicht doch als keine grammatischen Freiheiten erweisen. Derartige Überlegungen zeigen zum einen, dass eine moderne wissenschaftliche Grammatik (die sich ja der grammatischen Variation gegenüber positionieren muss) qua Textsorte und Lesererwartung zwangsläufig zwischen der Theorie der Kompetenz und der Theorie der Performanz oszilliert und dass es zwischen den beiden einen Übergangsbereich gibt, der nicht gerade droht, demnächst zu einer scharfen Grenze zu werden. Zum anderen wird klar, dass eine wissenschaftliche Vorgehensweise, die auf Evaluation und Aufstellung einzelner Theoreme ausgerichtet ist und nicht primär auf den Aufbau einer einzigen, bis ins Allerletzte kohärenten Theorie, sich idealerweise eines eklektischen Beschreibungsapparats bedienen muss, der eine tendenziell vollständige Deskription einer Einzelsprache sichert (dazu Mukherjee 2006). Da der Schwerpunkt der Grammatik zu einem nicht unbeträchtlichen Teil auf der Evaluation bisheriger Theorien läge, wäre die Übernahme der aus diesen stammenden Spezialterminologie vor dem Hintergrund eines Gerüsts aus der etablierten Terminologie neuerer umfangreicher deskriptiver Grammatikwerke ${ }^{48}$ naheliegend.

Der grammatisch basierten Theoriebildung im Allgemeinen würden bei der Entstehung des Grammatikwerkes unter Beachtung aller oben diskutierten Aspekte folgende Punkte zugutekommen:

- $\quad$ eine (durch die Textsorte einer Grammatik groß angelegte) Überprüfung von (strittigen) Theoremen,

- die Entwicklung von neuen Theoremen in den falsifizierten Bereichen bzw. die Bereitstellung der Daten und der ersten Hypothesen für eine solche Entwicklung,

- die Beschreibung und Explanation der Variation, mit dem Ziel eine Theoriebildung einzuleiten, in der sich Regeln, die die Kompetenz bestimmen, und präzise Beschreibungen der Performanz gegenseitig ergänzen,

- $\quad$ eine empirisch geleitete Problematisierung der theoretischen Konzepte der Variation, der Varietät, des Standards, des Konglomerats von Standardvarietäten, der Einzelsprache, des Kernsystems einer Einzelsprache etc. und ein Beitrag zur Modellierung des Übergangs entsprechender Sprachformen zu sog. Nonstandard-Varietäten.

Das wäre ein Ansatz für eine Theoriebildung, die die seit langem fragile Trennung zwischen einer Theorie der Kompetenz und einer Theorie der Performanz, wenn nicht überflüssig macht, dann zumindest in einer Gesamttheorie des grammatischen Wissens aufgehen lässt.

48 Man denke hier an GDS (Zifonun et al. 1997) oder Duden 2016b. 


\section{Zusammenfassung}

Die Eingangsfrage dieses Beitrags ließe sich etwas zugespitzt auch so formulieren: Ist die Korpuslinguistik als empirisches Forschungsparadigma theoriefeindlich? Klar ist sie das, sie ist die Feindin aller überarbeitungsbedürftigen Theorien. Sie hilft aber auch, die bestmöglichen hervorzubringen. So wurden in diesem Beitrag zunächst drei Korpuseinsatzbereiche diskutiert, die für die Grammatiktheorie relevant sind. Korpusdaten werden zum einen als Evidenz für theoretische Aussagen angeführt, zum anderen benutzt, um theoretische Aussagen zu validieren, und schließlich als Basis für die Entwicklung neuer Theoreme in einem zumindest teilweise induktiven Prozess eingesetzt. Die schon seit langem gebräuchliche Evidenz aus dem Korpus kann selbständig benutzt werden - etwa in Verbindung mit theoretischen Aussagen, die rein introspektiv basiert sind. Sie ist aber auch Teil der Validierung von Theoremen am Korpus und kann die Darstellung induktiv gewonnener Erkenntnisse begleiten. Die Validierung mithilfe des Korpus bedeutet meist den Versuch, theoretische Aussagen zu falsifizieren. Sie ist prinzipiell auf die Begleitung durch Akzeptabilitätsurteile angewiesen, denn einerseits sind Korpusdaten nicht per se akzeptabel und andererseits kann das Fehlen von Belegen für ein postuliertes Phänomen nicht als negative Evidenz gewertet werden. Die Validierung von Theoremen am Korpus ist inzwischen eine Strategie, deren Nutzen selbst für viele kompetenztheoretische Ansätze generativer Prägung eingesehen wird. Hingegen haben sich methodologische Ansätze, die auf die Induktion mithilfe der Korpusdaten setzen und die theoriegeleiteten Anteile an der Korpusforschung extrem minimieren wollen (die Corpus-driven-Ansätze), bisher nicht richtig etabliert. Vielversprechend erscheinen vielmehr gemischte Verfahren, in denen ein umfangreicher Datenbereich top down festgelegt und eventuell auch differenziert annotiert wird, um danach möglichst induktiv (datengeleitet) mit dem Ziel innovativer Theoriebildung exploriert zu werden.

Im Beitrag wurden weiter auch neue methodologische Entwicklungen und theoretische Problemstellungen umrissen, welche der Höhenflug der Korpuslinguistik und die Datenfülle, Datenvielfalt sowie Verfügbarkeit der Korpora mit sich bringen, wie die Popularisierung statistischer Methoden und vor allem das Aufkommen der Variation als zentralen Themas der grammatischen Korpusarbeit und großer Herausforderung für linguistische Theorien, die über klassische kompetenzfixierte Ansätze hinausgehen wollen. Beispiele aus der Forschungspraxis veranschaulichten neue, variationsorientierte Fragestellungen, verschiedene Typen von Einflussfaktoren grammatischer Variation und die Methoden ihrer korpuslinguistischen Exploration. 
Zum Schluss des Beitrags wurde argumentiert, dass die von der Entwicklung der Korpuslinguistik mitangeregte empirische Wende in der Grammatikforschung auch in einer neuen wissenschaftlichen Grammatik des Deutschen fruchten müsste, die auf einem umfangreichen Referenzkorpus basiert und sich den neuen Fragestellungen der Forschung stellt. Es wurden Probleme der Anlage einer solchen Grammatik diskutiert und die möglichen Lösungen erwogen. Es wurde auch überlegt, wie eine korpusgestützte Referenzgrammatik in theoretisch fundierter Weise die grammatische Variation zusammen mit ihren grammatischen und extralinguistischen Einflussfaktoren darstellen und damit zu einer grammatischen Theoriebildung beitragen könnte, die kompetenz- und performanzbezogene Überlegungen zu einer integrativen Gesamttheorie weiterentwickelt.

\section{Literatur}

Aarts, Bas (2001): Corpus linguistics, Chomsky and fuzzy tree fragments. In Christian Mair \& Marianne Hundt (Hrsg.), Corpus linguistics and linguistic theory, 5-13. Amsterdam: Rodopi.

Baayen, R. Harald, Richard Piepenbrock \& Leon Gulikers (1995): The CELEX Lexical Database (CD-ROM). Philadelphia: Linguistic Data Consortium.

Baroni, Marco \& Stefan Evert (2009): Statistical methods for corpus exploitation. In Anke Lüdeling \& Merja Kytö (Hrsg.), Corpus linguistics. An international handbook (Handbücher zur Sprach- und Kommunikationswissenschaft 29.2), 2. Bd., 777-803. Berlin/New York: De Gruyter.

Biber, Douglas (1993): Representativeness in corpus design. In Literary and Linguistic Computing 8 (4), 243-257.

Biber, Douglas, Stig Johansson, Geoffrey Leech, Susan Conrad \& Edward Finegan (1999): Longman grammar of spoken and written English. Harlow: Longman.

Brandt, Patrick (i. Vorb.): Alternation von zu- und dass- Komplementen: Kontrolle, Korpus, und Grammatik. IDS, Manuskript (unter Mitwirkung von Felix Bildhauer).

Brandt, Patrick, Beata Trawiński \& Angelika Wöllstein (2016): (Anti-)Control in German: Evidence from comparative, corpus- and psycholinguistic studies. In Ingo Reich \& Augustin Speyer (Hrsg.), Co- and subordination in German and other languages (Linguistische Berichte. Sonderheft 21), 77-98. Hamburg: Buske.

Brants, Sabine, Stefanie Dipper, Peter Eisenberg, Silvia Hansen-Schirra, Esther König, Wolfgang Lezius et al. (2004): TIGER: Linguistic interpretation of a German corpus. In Research on Language and Computation 2 (4), 597-620.

Bubenhofer, Noah, Sandra Hansen-Morath, Marek Konopka \& Roman Schneider (2015): GenitivDB 2.0 - Datenbank zur Genitivmarkierung (Release vom 01.09. 2015). Mannheim: Institut für Deutsche Sprache.

Bubenhofer, Noah, Marek Konopka \& Roman Schneider (2014a): Präliminarien einer Korpusgrammatik (Korpuslinguistik und interdisziplinäre Perspektiven auf Sprache 4). Tübingen: Narr. 
Bubenhofer, Noah, Sandra Hansen-Morath \& Marek Konopka (2014b): Korpusbasierte Exploration der Variation der nominalen Genitivmarkierung. In Zeitschrift für germanistische Linguistik 42 (3), 379-419.

Carter, Ronald \& Michael McCarthy (2006): Cambridge grammar of English. Cambridge: Cambridge University Press.

Cohen, Ayala (1980): On the graphical display of the significant components in two-way contingency tables. In Communications in Statistics - Theory and Methods 9 (10), $1025-1041$.

Conrad, Susan (2010): What can a corpus tell us about grammar? In Anne O'Keeffe \& Michael McCarthy (Hrsg.), The Routledge handbook of corpus linguistics (Routledge Handbooks in Applied Linguistics), 227-240. London/New York: Routledge.

Dammel, Antje (2015): One plus one make(s) - what? Determinants of verb agreement in German NP+NP coordination - A diachronic approach. In Jürg Fleischer, Elisabeth Rieken \& Paul Widmer (Hrsg.), Agreement from a diachronic perspective (Trends in Linguistics. Studies and Monographs 287), 287-326. Berlin: De Gruyter,

Duden (1965) = Grebe, Paul, Günther Drosdowski \& Wolfgang Müller (Bearb.): Der große Duden. Bd. 9. Hauptschwierigkeiten der deutschen Sprache. Mannheim:

Bibliographisches Institut.

Duden (2015) = Stefan Kleiner, Ralf Knöbl \& Max Mangold: Duden. Bd 6. Das Aussprachewörterbuch. Berlin: Dudenverlag und Mannheim: Institut für Deutsche Sprache.

Duden $(2016 a)=$ Dudenredaktion \& Mathilde Hennig (Hrsg.): Duden. Bd. 9. Das Wörterbuch der sprachlichen Zweifelsfälle. Berlin: Dudenverlag.

Duden (2016b) = Angelika Wöllstein \& Dudenredaktion (Hrsg.): Duden. Bd 4: Die Grammatik. Berlin: Dudenverlag.

Elspaß, Stephan \& Christa Dürscheid (2017): Areale grammatische Variation in den Gebrauchsstandards des Deutschen. In Marek Konopka \& Angelika Wöllstein, (Hrsg.), Grammatische Variation. Empirische Zugänge und theoretische Modellierung (Jahrbuch des Instituts für Deutsche Sprache 2016), 85-104. Berlin/Boston: De Gruyter.

Facchinetti, Roberta (Hrsg.) (2007): Corpus linguistics 25 years on. Amsterdam/New York: Rodopi.

Friendly, Michael (1992): Graphical methods for categorical data. Paper presented at the SAS SUGI 17 Conference, April, 1992. Online verfügbar unter www.math.yorku.ca/ SCS/sugi/sugi17-paper.html\#, (letzter Zugriff: 13.11. 2017).

Gries, Stefan Thomas (2010): Corpus linguistics and theoretical linguistics: A love-hate relationship? Not necessarily .... In International Journal of Corpus Linguistics 15 (3), 327-342.

Gries, Stefan Thomas (2011): Methodological and interdisciplinary stance in corpus linguistics. In Vander Viana, Sonia Zyngier \& Geoff Barnbrook (Hrsg.), Perspectives on corpus linguistics (Studies in Corpus Linguistics 48), 81-98. Amsterdam/Philadelphia: Benjamins.

Gunkel, Lutz, Adriano Murelli, Susan Schlotthauer, Bernd Wiese \& Gisela Zifonun (2017): Grammatik des Deutschen im europäischen Vergleich. Das Nominal (Schriften des Instituts für Deutsche Sprache 14). Unter Mitarbeit von Christine Günther und Ursula Hoberg. Berlin/Boston: De Gruyter.

Institut für Deutsche Sprache (2011): Deutsches Referenzkorpus/Archiv der Korpora geschriebener Gegenwartssprache 2011-I (Release vom 29.3. 2011). Mannheim: Institut für Deutsche Sprache. 
Hennig, Mathilde (2015): Explizite und elliptische Junktion in der Attribution: Eine Bestandsaufnahme. In Mathilde Hennig \& Robert Niemann (Hrsg.). Junktion in der Attribution. Ein Komplexitätsphänomen aus grammatischer, psycholinguistischer und praxistheoretischer Perspektive (Linguistik - Impulse \& Tendenzen 62), 21-84.

Konopka, Marek \& Eric Fuß (2016): Genitiv im Korpus. Untersuchungen zur starken Flexion des Nomens im Deutschen (Studien zur Deutschen Sprache 70). Tübingen: Narr.

Kupietz, Marc \& Holger Keibel (2009): Gebrauchsbasierte Grammatik: statistische Regelhaftigkeit. In Marek Konopka \& Bruno Strecker (Hrsg.), Deutsche Grammatik Regeln, Normen, Sprachgebrauch (Jahrbuch des Instituts für deutsche Sprache 2008), 33-50. Berlin/New York: De Gruyter.

Löffler, Heinrich (2005): Wieviel Variation verträgt die deutsche Standardsprache? Begriffsklärung: Standard und Gegenbegriffe. In Ludwig M. Eichinger \& Werner Kallmeyer (Hrsg.): Standardvariation. Wie viel Variation verträgt die deutsche Sprache? (Jahrbuch des Instituts für Deutsche Sprache 2004), 7-27. Berlin/New York: De Gruyter. Meurers, W. Detmar (2005): On the use of electronic corpora for theoretical linguistics. Case studies from the syntax of German. In Lingua 115, 1619-1639.

Meurers, W. Detmar \& Stefan Müller (2009): Corpora and syntax. In Anke Lüdeling, \& Merja Kytö (Hrsg.), Corpus linguistics. An international handbook (Handbücher zur Sprachund Kommunikationswissenschaft 29.2). 2. Bd., 920-933. Berlin/New York: De Gruyter. Müller, Stefan (2007): Qualitative Korpusanalyse für Grammatiktheorie. Introspektion vs. Korpus. In Werner Kallmeyer \& Gisela Zifonun (Hrsg.), Sprachkorpora - Datenmengen und Erkenntnisfortschritt (Jahrbuch des Instituts für Deutsche Sprache 2006), 70-90. Berlin: De Gruyter.

Münzberg, Franziska \& Sandra Hansen-Morath (2018): „Die Wucht und Strömung war immens“ - wie stark ist der Ellipseneffekt? In Eric Fuß, Marek Konopka, Beata Trawinski \& Ulrich Waßner, Grammar and corpora 2016, 73-95. Heidelberg: Heidelberg University Publishing.

Mukherjee, Joybrato (2006): Corpus linguistics and English reference grammars. In Antoinette Renouf \& Andrew Kehoe (Hrsg.), The changing face of corpus linguistics. Papers from the 24th International Conference on English Language Research on Computerized Corpora (ICAME 24), 337-354. Amsterdam: Rodopi.

Perkuhn, Rainer, Holger Keibel \& Marc Kupietz (2012): Korpuslinguistik. Paderborn: Fink (LIBAC).

Pullum, Geoffrey K. (2007): Ungrammaticality, rarity, and corpus use. In Corpus Linguistics and Linguistic Theory 3 (1), 33-47.

Pullum, Geoffrey K. (2017): Theory, data, and the epistemology of syntax. In Marek Konopka \& Angelika Wöllstein (Hrsg.): Grammatische Variation. Empirische Zugänge und theoretische Modellierung (Jahrbuch des Instituts für Deutsche Sprache 2016), 283-298. Berlin/Boston: De Gruyter.

Rapp, Irene, Ekaterina Laptieva, Alexander Koplenig \& Stefan Engelberg (i. E.): Lexikalischsemantische Passung und argumentstrukturelle Trägheit - eine korpusbasierte Analyse zur Alternation zwischen dass-Sätzen und zu-Infinitiven in Objektfunktion. In Deutsche Sprache.

Reis, Marga (2017): Grammatische Variation und realistische Grammatik. In Marek Konopka \& Angelika Wöllstein (Hrsg.), Grammatische Variation. Empirische Zugänge und theoretische Modellierung (Jahrbuch des Instituts für Deutsche Sprache 2016), 283-298. Berlin/Boston: De Gruyter. 
Tognini-Bonelli, Elena (2001): Corpus linguistics at work (Studies in Corpus Linguistics 6). Amsterdam: Benjamins.

Tognini-Bonelli, Elena (2010): Theoretical overview of the evolution of corpus linguistics. In Anne O'Keeffe \& Michael McCarthy (Hrsg.), The Routledge handbook of corpus linguistics (Routledge Handbooks in Applied Linguistics), 14-27. London/New York: Routledge.

Viana, Vander, Sonia Zyngier \& Geoff Barnbrook (Hrsg.) (2011): Perspectives on corpus linguistics (Studies in Corpus Linguistics, 48). Amsterdam/Philadelphia: Benjamins.

Wöllstein, Angelika (2015): Grammatik - explorativ. Hypothesengeleitete und -generierende Exploration variierender Satzkomplementationsmuster im standardnahen Deutsch. In Ludwig M. Eichinger (Hrsg.), Sprachwissenschaft im Fokus (Jahrbuch des Instituts für Deutsche Sprache 2014), 93-120. Berlin/Boston: De Gruyter.

Zifonun, Gisela, Ludger Hoffmann, Bruno Strecker et al. (1997): Grammatik der deutschen Sprache (Schriften des Instituts für deutsche Sprache 7). 3 Bde. Berlin/New York: De Gruyter. 


\title{
Eric Fuß \\ 6 Sprachliche Variation
}

\begin{abstract}
Der Beitrag diskutiert anhand von Kongruenzschwankungen im Zusammenhang mit Subjektreihungen verschiedene Aspekte sprachlicher Variation. Es wird gezeigt, wie mithilfe einer Korpusstudie grammatische Faktoren ermittelt werden können, die die Verteilung der Varianten steuern. Im Anschluss wird eine Analyse vorgestellt, die Variation darauf zurückführt, dass syntaktische Strukturen, die an der Schnittstelle zur Morphologie/Phonologie nicht vollständig interpretierbar sind, auf verschiedene Arten repariert werden können.
\end{abstract}

Keywords: First Conjunct Agreement, Intrasprechervariation, Kongruenz, Resolutionsregeln, Subjektreihungen

\section{Einleitung}

Es ist eine wesentliche Einsicht der modernen Linguistik, dass Sprache von Natur aus variabel ist. Im Mittelpunkt stand dabei zunächst die Beobachtung, dass abhängig vom Äußerungskontext oder gesellschaftlichen Status des Sprechers eine bestimmte linguistische Variable, d.h. eine bestimmte Bedeutung oder grammatische Funktion, durch verschiedene sprachliche Varianten realisiert werden kann. Die soziale Dimension von Sprache, die William Labov in seinen wegweisenden Arbeiten aus den frühen 1960er Jahren betonte, rückte in der zweiten Hälfte des 20. Jahrhunderts stärker in den Blickwinkel der Sprachwissenschaft, was dazu führte, dass sich die Soziolinguistik als Teildisziplin der modernen Linguistik etablieren konnte. ${ }^{1}$ Aus kognitionswissen-

1 Einer der ersten Grammatiker, der Variation im Sprachsystem als Phänomen erkannte und sie mit einem Begriff belegte (anyatarasyãm), war Pāninini (ca. 600 vor Chr.). Allerdings wurde dieser Aspekt von Pāninini’s Lehren von seinen Nachfolgern nicht aufgegriffen und geriet bald

Anmerkung: Ich möchte mich bei meinen Kollegen Felix Bildhauer, Patrick Brandt, Marek Konopka, Franziska Münzberg und Angelika Wöllstein sowie bei zwei anonymen Gutachtern für hilfreiche Änderungsvorschläge und Kommentare bedanken. Verbleibende Fehler und Unzulänglichkeiten liegen selbstverständlich in meiner Verantwortung.

Eric Fuß, Institut für Deutsche Sprache, R5, 6-13, D-68161 Mannheim, E-Mail: fuss@ids-mannheim.de

Ә Open Access. (C) 2018 Eric Fuß, publiziert von De Gruyter. (c) BY Dieses Werk ist lizenziert unter der Creative Commons Attribution 4.0 Lizenz. 
schaftlicher Sicht wurde dies so gedeutet, dass das sprachliche Wissen des Sprechers auch eine pragmatische Kompetenz beinhaltet, die sich darin äußert, dass Sprecher in der Regel dazu in der Lage sind, abhängig vom Äußerungskontext die jeweils angemessene sprachliche Variante $\mathrm{zu}$ wählen (vgl. z. B. Chomsky 1980: 92).

Weniger Aufmerksamkeit hat die Frage gefunden, wie sprachliche Variation - insbesondere solche, die nicht auf soziale Faktoren zurückgeführt werden kann - theoretisch modelliert werden kann. Dies liegt zumindest teilweise in der generellen Ausrichtung der modernen Linguistik im 20. Jahrhundert begründet, die seit Saussure primär an der Rekonstruktion struktureller Eigenschaften und Regeln von Sprache interessiert war und strikt zwischen langue und parole bzw. (im generativen Paradigma) Kompetenz und Performanz unterschied. In diesem Paradigma wurde Variation in der Regel lediglich als oberflächliches Nebenprodukt der Externalisierung von Sprache in der Gesellschaft betrachtet (d.h. als Bestandteil von parole bzw. Performanz). Während also die Frage, wie außergrammatische (insbes. soziale) Faktoren die Wahl linguistischer Varianten steuern, seit den 1960er Jahren intensiv untersucht wurde, stellt die grammatiktheoretische Beschreibung und Modellierung von sprachlicher Variation immer noch ein Forschungsdesiderat dar. ${ }^{2}$ Erst in der jüngeren

in Vergessenheit (vgl. Kiparsky 1979). Der römische Gelehrte Varro (116-27 vor Chr.) erkannte linguistische Variation als Eigenschaft der Mundarten bzw. der Volkssprache (consuetudo) und prägte den Ausspruch consuetudo loquendi est in motu ,die Volkssprache ist stets in Bewegung،. Auch er blieb jedoch ohne größeren Einfluss auf die Ideengeschichte der Linguistik (vgl. auch Chambers 2002: 6).

2 Dabei geht es auch um die generelle Frage, wie die inhärente Variabilität von Sprache theoretisch zu deuten ist; so wird sprachliche Variation zuweilen zum Anlass genommen, eine Abkehr von regel- und beschränkungsbasierten Ansätzen einzufordern (vgl. z. B. Hopper 1987). Solche alternativen gebrauchsbasierten Modelle verlieren aber oft die Tatsache aus dem Blick, dass nicht alle Bereiche der Grammatik in gleicher Weise variabel sind. So scheint die nominale Flexionsmorphologie im Deutschen generell variabler zu sein als die verbale Flexion (vgl. die Koexistenz von kurzen und langen Genitivformen wie (des) Verstands und (des) Verstandes, Konopka \& Fuß 2016, oder bekannte Unsicherheiten bei der Beugung von Adjektiven wie mit großem nachhaltigem/-en Erfolg). Gebrauchsbasierte Ansätze können auch nicht ohne Weiteres erklären, warum kompetente Sprecher in der Regel klare Intuitionen haben über gängige, mögliche und vor allem unmögliche Ausdrücke in einer Sprache - darunter auch viele, die ihnen selten oder gar nie zuvor begegnet sind. Es bleibt eine Kernaufgabe der Grammatiktheorie, dieses implizite sprachliche Wissen im Rahmen eines theoretischen Modells zu rekonstruieren, das es erlaubt, die Menge der wohlgeformten Sätze/Ausdrücke - und nur diese - in einer Sprache zu erfassen. Allerdings umfasst die Menge der wohlgeformten Ausdrücke einer Sprache auch Instanzen von sprachlicher Variation, d. h. unterschiedliche sprachliche Realisierungen einer bestimmten Bedeutung oder grammatischen Funktion. Ein adäquates regelbasiertes Modell muss entsprechende theoretische Instrumente zur Verfügung stellen, um solche 
Vergangenheit ist die Untersuchung linguistischer Variation auch in generativen Ansätzen stärker in den Vordergrund gerückt. Dabei lassen sich zwei Schwerpunkte ausmachen. Einschlägige Forschungsbemühungen konzentrieren sich zum einen auf die Schnittstelle von Syntax und Pragmatik, wobei insbesondere der Zusammenhang zwischen Diskursfunktion und Wortstellungsvariation untersucht wird. Besonders einflussreich war hier die Hypothese von Rizzi (1997), dass informationsstrukturelle Kategorien wie Topik oder Fokus ein direktes (morpho-)syntaktisches Korrelat in Form ausgezeichneter Positionen in der syntaktischen Struktur haben. Zum anderen wird der Zusammenhang zwischen Sprachwandel und linguistischer Variation thematisiert. Ausgehend von der Beobachtung, dass Sprachwandelsituationen in der Regel mit einem erhöhten Maß an Variation einhergehen, hat Kroch $(1989,1994)$ dafür argumentiert, dass entsprechende diachrone Übergangsstadien sich formal durch die Annahme erfassen lassen, dass Sprecher über mehrere internalisierte Grammatiken bzw. grammatische Optionen verfügen, die miteinander konkurrieren und die Produktion eigentlich inkompatibler Varianten erlauben (wie z. B. OV- und VO-Stellung), bevor sich eine Option gegen die andere durchsetzt. Diese beiden Forschungsrichtungen stellen dabei aus variationslinguistischer Sicht zwei gegenüberliegende Pole dar. Die Untersuchung informationsstruktureller Faktoren strebt die Eliminierung (scheinbarer) syntaktischer Variation an, indem die entsprechenden Wortstellungsalternationen als kombinatorische Varianten betrachtet werden, deren Verteilung letztlich vollständig durch grammatische Prinzipien bestimmt ist. Im Gegensatz dazu vertreten Arbeiten, die in der Tradition von Kroch (1989) stehen, die Auffassung, dass freie Variation (also Variation, die gerade nicht auf grammatische Faktoren zurückgeführt werden kann) eine notwendige Begleiterscheinung von Sprachwandelprozessen ist. Diesem Postulat liegt die Einsicht zugrunde, dass (interner) Sprachwandel nur dann möglich ist, wenn Sprachlerner nicht mehr in der Lage sind, die (grammatischen und außergrammatischen) Faktoren $\mathrm{zu}$ erkennen, die in der Zielgrammatik die Verteilung der Varianten regeln.

Zwischen diesen Polen, die als die beiden Endpunkte einer Skala zu denken sind, bewegen sich Fälle, in denen wir Faktoren identifizieren können, die zwar die Verteilung der Varianten in signifikanter Weise beeinflussen, aber nicht vollständig bestimmen. Bei der Untersuchung solcher Einflussgrößen, die oft lediglich Tendenzen ausdrücken, müssen verschiedene Typen sprachlicher Variation unterschieden werden, die sich anhand der Verteilung der Varianten und der Natur der relevanten Faktoren (grammatisch vs. außer-

konkreten Fälle von sprachlicher Variation zu beschreiben (vgl. z. B. Henry 2002). Vgl. Abschnitt 4 für einen entsprechenden Vorschlag. 
grammatisch) klassifizieren lassen. Eine wichtige Rolle spielt dabei die Unterscheidung zwischen Intersprechervariation und Intrasprechervariation:

- Intersprechervaration: Die verschiedenen Ausprägungen einer linguistischen Variable sind auf verschiedene Sprecher bzw. Sprechergruppen verteilt.

- Intrasprechervariation: Die verschiedenen Ausprägungen einer linguistischen Variable treten in der Sprachproduktion individueller Sprecher auf.

Traditioneller Gegenstand der Soziolinguistik sind dabei Fälle außergrammatisch determinierter Variation, ${ }^{3}$ die sowohl Sprechergruppen als auch individuelle Sprecher betreffen kann (vgl. die Übersicht in Tabelle 1). Variationsphänomene, die sich auf grammatische Faktoren zurückführen lassen, fallen hingegen in das Gebiet der allgemeinen Linguistik bzw. Grammatikforschung. Auch hier kann wiederum zwischen Intra- und Intersprechervariation unterschieden werden, wobei letztere für Sprachwandelprozesse charakteristisch ist, bei denen innerhalb einer Sprechergemeinschaft unterschiedliche grammatische Mittel zur Realisierung einer bestimmten Bedeutung oder grammatischen Funktion koexistieren: ${ }^{4}$

Tab. 6.1: Typen sprachlicher Variation.

\begin{tabular}{lll}
\hline & grammatisch bestimmt & außergrammatisch bestimmt \\
\hline Intersprechervariation & $\begin{array}{l}\text { abhängig von Diskursfunktion, } \\
\text { phonolog./morphosyntaktischem } \\
\text { Kontext, Satzprosodie etc. }\end{array}$ & $\begin{array}{l}\text { abhängig von Alter, } \\
\text { dialektalem Hintergrund, } \\
\text { Sozialstatus etc. }\end{array}$ \\
\hline \multirow{2}{*}{ Intrasprechervaration } & $\begin{array}{l}\text { abhängig von Diskursfunktion, } \\
\text { phonolog./morphosyntaktischem } \\
\text { Kontext, Satzprosodie etc. }\end{array}$ & $\begin{array}{l}\text { abhängig von Äußerungs- } \\
\text { kontext, Register 0. ä. }\end{array}$ \\
& \\
\hline
\end{tabular}

3 Zur außergrammatischen Variation mit dialektalem bzw. areallinguistischem Hintergrund, s. Lenz in diesem Band.

4 Vgl. Eichinger (2005) für entsprechende Variationsphänomene im Gegenwartsdeutschen, die darauf zurückzuführen sind, dass sich standardsprachliche Normen zugunsten von Sprachformen verschieben, die der strukturellen Mündlichkeit nahe stehen.

5 Einen interessanten Grenzfall stellt registergebundene Variation dar, die grammatische Eigenschaften betrifft (wie z. B. Abweichungen von der für Hauptsätze charakteristischen V2-Stellung im Kiezdeutschen, vgl. Wiese 2012). Wenn ein individueller Sprecher abhängig vom sozialen oder textuellen Kontext zu unterschiedlichen grammatischen Strukturen greift, scheint sich dies qualitativ von kontextabhängiger lexikalischer Variation zu unterscheiden (also z. B. unterschiedlichen Wörtern für den gleichen Begriff, Karre vs. Automobil). Bei näherer Betrachtung kann registergebundene morphosyntaktische Variation allerdings auf die gleiche Weise wie 
Wie bereits angedeutet ist dabei die Endphase von Sprachwandelprozessen oft von freier Variation geprägt, die sowohl Intersprecher- als auch Intrasprechervariation sein kann, d. h. Sprachwandel involviert in der Regel eine Übergangsphase, in der verschiedene sprachliche Varianten miteinander um die Realisierung einer bestimmten Bedeutung/Funktion konkurrieren. Dem Verlust einer der Varianten geht in der Regel ein Stadium voraus, in dem die Verteilung der Varianten nicht erkennbar durch soziale oder grammatische Faktoren gesteuert wird (vgl. Schäfer 2017 zur Variation zwischen Parallel- und Wechselflexion im Gegenwartsdeutschen bei Absenz einer Konjunktion/eines Kommas zwischen den involvierten Adjektiven).

Da die Untersuchung von Variationsphänomenen in der Regel einen Vergleich zwischen verschiedenen Sprechern und Sprechergruppen erfordert, ${ }^{6}$ wird in der Regel auf empirische Methoden wie Feldstudien, psycholinguistische Experimente (inkl. Akzeptabilitätsstudien) und Korpusuntersuchungen zurückgegriffen. Allerdings wurden insbesondere Korpusstudien bislang vor allem angewendet, um den Einfluss außergrammatischer Faktoren wie Textsorte, Register oder Region auf die Distribution grammatischer Varianten zu bestimmen. In der Regel (aber nicht ausschließlich) haben wir es dabei mit Formen der Intersprechervariation zu tun; dies gilt insbesondere für große Korpora, die die sprachlichen Produkte einer Vielzahl verschiedener Sprecher umfassen. In diesem Beitrag soll gezeigt werden, dass Korpusstudien aber auch durchaus zur Untersuchung grammatischer Einflussgrößen genutzt werden können (vgl. auch Brandt \& Fuß 2014, Konopka \& Fuß 2016, Fuß, Konopka \& Wöllstein 2017). ${ }^{7}$ Der empirische Gegenstand der vorliegenden Studie ist die Ausprägung der Subjekt-Verb-Kongruenz in Kombination mit Subjektreihungen der Art $d u$ und/oder $X$. In diesem Bereich herrscht insbesondere bei Reihungen aus zweiter und dritter Person eine gewisse Sprecherunsicherheit, die sich vor allem in Variation zwischen den Kongruenzoptionen 2. Pl. und 3. Pl. manifestiert (vgl. bereits Corbett 1983).

Der Beitrag ist wie folgt aufgebaut: In Abschnitt 2 wird der empirische Gegenstand des Aufsatzes eingeführt und es werden methodologische Aspekte

registergebundene lexikalische Variation erfasst werden: Wenn man davon ausgeht, dass die Wahl zwischen bestimmten syntaktischen Strukturen abhängig ist von der Wahl der lexikalischen und morphosyntaktischen Bausteine, aus denen sich diese Strukturen zusammensetzen, lässt sich auch (morpho)syntaktische Variation letztlich auf lexikalische Variation zurückführen.

6 Dies gilt allerdings nicht notwendig für die Untersuchung von Intrasprechervariation.

7 Die Frage, inwiefern es dabei auch möglich ist, Fälle von Intrasprechervariation eindeutig zu identifizieren, wird in Abschnitt 4 ausführlicher diskutiert. 
der durchgeführten Korpusstudie diskutiert. Abschnitt 3 präsentiert die Ergebnisse der Untersuchung und formuliert einschlägige deskriptive Generalisierungen; in 3.1 werden die Varianten und deren Verteilung vorgestellt, während 3.2 eine Auswahl an grammatischen Faktoren diskutiert, die die Verteilung der Varianten beeinflussen. In Abschnitt 4 werden die empirischen Befunde vor dem Hintergrund der Unterscheidung zwischen Inter- und Intrasprechervariation diskutiert. Es wird eine theoretische Beschreibung vorgeschlagen, die Variation als das Resultat konkurrierender Reparaturoptionen analysiert, die einen Merkmalskonflikt im Zusammenhang mit Subjektreihungen aus zweiter und dritter Person beseitigen. Abschnitt 5 fasst die Ergebnisse zusammen.

\section{Fallstudie: Verbkongruenz mit Subjektreihungen}

Im Zusammenhang mit komplexen gereihten Subjekten, die aus zwei koordinierten Elementen mit unterschiedlichen Werten für Person und ggf. Numerus bestehen (z. B. 2. Sg. + 3. Sg., du und er), entstehen in vielen Sprachen Kongruenzprobleme. Diese werden einzelsprachspezifisch auf zwei verschiedene Weisen gelöst: Entweder kongruiert das Verb lediglich mit einem der beiden Konjunkte, oder es werden die beiden konfligierenden Merkmalsmengen durch sog. Resolutionsregeln zu einer Merkmalsmenge zusammengeführt (vgl. z. B. Corbett 1983, 2000). Sprachübergreifend lassen sich dabei bestimmte Tendenzen beobachten: Numerusresolution führt typischerweise zu Pluralkongruenz am Verb (Sg. + Sg./Pl. = Pl.), während bei konfligierenden Werten für das Merkmal Person die markierteren Werte 1. Person/2. Person Vorrang vor der 3. Person erhalten (1./2. + 3. = 1./2.). Entsprechende Effekte lassen sich auch im Deutschen beobachten. So formuliert die aktuelle Auflage der Dudengrammatik (Wöllstein 2016, §1602; in der Folge „Dudengrammatik“) die folgende Kongruenzregel für Subjekte mit gereihten Subjektteilen (die gleiche Empfehlung spricht der Zweifelsfälle-Duden aus, vgl. Hennig 2016: 570):

(a) Die Reihung gilt gesamthaft als Plural, das finite Verb steht daher ebenfalls im Plural.

(b) Die 1. Person rangiert vor der zweiten Person, und die 2. Person rangiert vor der 3. Person.

Stimmen die Subjektteile im Merkmal Person überein, kommt es lediglich zur Numerusresolution. Relevante Fälle sind hier in der Regel auf die 3. Person beschränkt, das Verb steht dabei im Plural, vgl. (1). Hier lässt sich aber auch unter bestimmten Umständen Variation beobachten: Im Zusammenhang mit 
Abstrakta, formelhaften Wortpaaren wie Grund und Boden und Fällen, in denen eines der beiden Konjunkte das andere mit einschließt, ist auch Singularkongruenz zulässig (Dudengrammatik, §§1608-1610), vgl. die Beispiele in (2).

(1) Peter und Maria gehen/* geht ins Kino.

(2) a. Hass und Gewalt regiert die Welt.

b. Grund und Boden ist eine sichere Geldanlage.

c. Die Mannschaft und der ganze Verein ist verunsichert.

Liegt zusätzlich ein Konflikt zwischen den Werten für das Merkmal Person vor, ergeben sich nach der Dudengrammatik (vgl. §1604) die folgenden Resultate (man beachte, dass entsprechende Regeln auch für die Formen von Possessivpronomen und Reflexiva gelten):

(3) a. Du und ich freuen uns über unseren Erfolg. (2. Sg. + 1. Sg. = 1. Pl.)

b. Ich und ihr freuen uns über unseren Erfolg. (1. Sg. + 2. Pl. = 1. Pl.)

c. Wir und du freuen uns über unseren Erfolg. (1. Pl. + 2. Sg. = 1. Pl.)

d. Meine Freundin und ich freuen uns über unseren Erfolg.

(3. Sg. + 1. Sg. = 1. Pl.)

e. Du und er habt euch über euren Erfolg gewiss gefreut.

(2. Sg. + 3. Sg. = 2. Pl.)

f. Du und die anderen habt euch über euren Erfolg gewiss gefreut. (2. Sg. + 3. $\mathrm{Pl}$. = 2. $\mathrm{Pl}$.)

g. Sie und ihr habt euch über euren Erfolg gewiss gefreut.

(3. $\mathrm{Pl} .+$ 2. $\mathrm{Pl}$. = 2. $\mathrm{Pl}$.)

h. Ich, du und die anderen freuen uns über unseren Erfolg. (1. Sg. + 2. Sg. + 3. Pl. = 1. Pl. $)$

Introspektion und informelle Sprecherbefragungen scheinen aber nahe zu legen, dass sich Sprecher zumindest bei einem Teil der Fälle in (3) unsicher sind (auch die Dudengrammatik, §1604, räumt ein, dass diese Reihungen ,teilweise etwas hart" wirken). Besonders problematisch sind offenbar Kombinationen aus 2. und 3. Person. Hier kommt es zu grammatischer Variation, wie auch die Dudengrammatik feststellt (vgl. §1607), die allerdings lediglich Beispiele für Reihungen aus 2. Sg. und 3. Pl. wie in (4) zeigt: 
(4) a. du und deine angeblichen linguisten äussert euch auch so gut wie $\mathrm{zu}$ irgendwelchen fakten.

(WDD13/F13.52375: Diskussion:Florina, In: Wikipedia http://de.wikipedia.org/wiki/Diskussion:Florina: Wikipedia, 2013)

b. Dass du und andere das Semikolon schon häufig zur Textgliederung „missbraucht“ haben, macht diese Verwendung nicht sinnvoller. (WDD13/B38.13458: Diskussion:Bahnstrecke Düsseldorf-Solingen, In: Wikipedia - http://de.wikipedia.org/wiki/Diskussion:Bahnstrecke_ Düsseldorf-Solingen: Wikipedia, 2013)

Bereits Corbett (1983) weist allerdings darauf hin, dass diese Varianten auch bei Reihungen aus 2. Sg. und 3. Sg. auftreten: ${ }^{8}$

(5) a. Die Entscheidung, den Artikel solange zu sperren, bis du und Herrick euch hoffentlich beruhigt habt, ist absolut richtig. (WDD13/J14.24905: Diskussion:Judenfeindlichkeit/Archiv/1, In: Wikipedia - http://de.wikipedia.org/wiki/Diskussion: Judenfeindlichkeit/Archiv/1: Wikipedia, 2013)

b. Ich wundere mich immer wieder, dass du und Jim euch nicht verstehen!

(WDD13/I17.33247: Diskussion:Immer wieder Jim, In: Wikipedia http://de.wikipedia.org/wiki/Diskussion:Immer_wieder_Jim: Wikipedia, 2013)

Unter der Hypothese, dass die Kongruenzvarianten in (4) und (5) tatsächlich von ein und derselben Grammatik produziert werden, stellt sich natürlich die Frage, ob es sich um eine Art von freier Variation handelt oder ob sich Faktoren ermitteln lassen, die die Wahl der Varianten beeinflussen. Darüber hinaus muss geklärt werden, ob neben den o.g. Varianten noch weitere Optionen existieren.

In der Literatur zur Kongruenz mit Subjektreihungen werden in diesem Zusammenhang sowohl syntaktische als auch morphologische Faktoren genannt, deren Relevanz für die Alternation in (5) nachfolgend überprüft wird. Im Mittelpunkt stehen dabei Wortstellung, Art der Konjunktion (und vs. oder)

8 In Beispiel (5b) signalisieren die Formen für das Reflexivum (2. Pl.) und das finite Verb (3. Pl.) unterschiedliche Merkmalswerte. Dies kann als Hinweis darauf verstanden werden, dass Pronominalisierung und Verbkongruenz unterschiedliche Prozesse des Merkmalsabgleichs involvieren. 
und paradigmatische Eigenschaften der Verbflexion (insbesondere das Vorliegen von Synkretismen).

Sprachübergreifend scheint dabei insbesondere die Wortstellung einen wesentlichen Einfluss auf die Wahl der Kongruenzendung auszuüben (vgl. Corbett 2000, 2006; van Koppen 2005, 2006, 2012). So wird in Sprachen/ Varietäten, in denen das Verb lediglich mit einem der beiden Subjektteile kongruiert, die Form des Verbs häufig durch den Subjektteil bestimmt, der dem Verb am nächsten steht bzw. unmittelbar adjazent zum Verb ist. Relevante Phänomene treten z. B. in bairischen Varietäten auf, in denen die nebensatzeinleitende Konjunktion für Person und Numerus des Subjekts flektiert. ${ }^{9}$ In den entsprechenden Dialekten kongruiert die Konjunktion optional entweder mit dem ersten Subjektteil (sog. First Conjunct Agreement, FCA, Munn 1999) oder mit dem gesamten komplexen Subjekt (während für das satzfinale Verb nur letztere Option besteht), vgl. Bayer (2013), Fuß (2014); für Dialekte des Niederländischen vgl. van Koppen (2005): ${ }^{10}$

(6) a. dass-st [du und da Hans] noch Minga geh-ts (FCA)

b. dass-ts [du und da Hans] noch Minga geh-ts (Resolution)

Generell gilt dabei, dass Kongruenz mit nur einem Subjektteil einer Adjazenzbedingung unterliegt: Handelt es sich bei dem Pronomen um das zweite Konjunkt der Subjektreihung, muss die Konjunktion mit dem gesamten Subjekt kongruieren (vorausgesetzt, es existiert eine Flexion, um den resultierenden Merkmalsgehalt auszudrücken, Bayer 2013):

(7) dass-ts/*-st [da Hans und du] noch Minga geht-ts

Ein analoges Phänomen kann in Hauptsätzen mit Subjekt-Verb-Inversion beobachtet werden (vgl. Fuß 2008), vgl. (8a). In subjektinitialen Sätzen ist FCA offenbar generell ausgeschlossen, vgl. $(8 \mathrm{~b}-\mathrm{c})$ :

9 In den meisten bairischen Varietäten ist dieses Phänomen auf die 2. Person beschränkt, vgl. Bayer (1984) und Weiß (2005).

10 Man beachte, dass sich die Kongruenz an der Konjunktion von der Verbkongruenz unterscheidet, wenn die Konjunktion lediglich mit dem ersten Konjunkt kongruiert, vgl. (6a). Das Vorliegen von Pluralkongruenz am Verb zeigt zudem, dass FCA bei den vorliegenden Beispielen nicht auf die Koordination von Sätzen plus Ellipse zurückgeführt werden kann. Es handelt sich also um genuine Subjektreihungen. 
(8) a. Gesdan hoa-st/?hoab-ts [ du und da Hans] an Hauptpreis gwunna.

b. [Du und da Hans] hoab-ts/*hoa-st an Hauptpreis gwunna.

c. [Da Hans und du] hoab-ts/^hoa-st an Hauptpreis gwunna.

Hier spielt also sowohl die Position des Verbs (vor oder nach dem komplexen Subjekt) als auch die relative Abfolge der Subjektteile zueinander eine wesentliche Rolle. Ähnliche Effekte, d. h. Kongruenz mit nur einem Subjektteil, lassen sich auch im Standarddeutschen beobachten. Die Dudengrammatik unterscheidet dabei zwischen Subjektreihungen und koordinierten Sätzen, bei denen das finite Verb bzw. die Verbalphrase getilgt worden ist (vgl. §1602). Während bei Subjektreihungen ein einziges komplexes Subjekt vorliegt, handelt es sich bei Beispielen wie (9) um Ellipsen, bei denen Kongruenz mit dem Subjekt vorliegt, das dem finiten Verb am nächsten ist:

(9) a. Nicht nur Otto ist eingeladen, sondern auch du bist eingeladen.

b. Nicht nur du bist eingeladen, sondern auch Otto ist eingeladen.

Die Dudengrammatik (§1602) formuliert für entsprechende Fälle die folgende Kongruenzregel: „Bei zusammengezogenen Sätzen mit eingesparten finiten Verbformen zählt nur das Subjekt der ausformulierten finiten Verbform.“

Wie (9) zeigt, muss dabei die getilgte Verbform nicht notwendig die gleichen grammatischen Merkmale wie das sichtbare Verb aufweisen. Bei Kombinationen aus zwei Subjektteilen im Singular lassen sich die beiden Konstruktionstypen gut auseinanderhalten, wenn Pluralkongruenz am Verb vorliegt hier muss dann eine genuine Subjektreihung vorliegen. Weniger eindeutig sind Fälle, in denen das Verb Singularkongruenz zeigt (bzw. ein pluralischer Subjektteil vorliegt). Als weitere Quelle für Teilkongruenz nennt die Dudengrammatik (vgl. §1606) eine Konstellation, die FCA im Bairischen ähnelt: „Wenn eine Reihung mit und dem finiten Verb folgt, richtet sich das finite Verb zuweilen nur nach dem ersten Subjektteil, also wie in zusammengezogenen Sätzen“.

(10) Zwischen die drei Deutschen hatte sich nur der Schwede Kjell Sjöberg und der Russe Iwannikow geschoben.

(Dudengrammatik, §1606)

In Abschnitt 3 werde ich dafür argumentieren, dass Fälle, in denen das finite Verb nur mit dem ersten Teil von Reihungen aus 2. Sg. und 3. Sg. übereinstimmt, keine Ellipsen darstellen, sondern analog zu FCA im Bairischen zu analysieren sind. 
Einen großen Einfluss auf das Kongruenzverhalten übt zudem die Art der (nebenordnenden) Konjunktion aus. So stellt die Dudengrammatik fest (vgl. $\S 1616)$, dass in Subjektreihungen mit oder das finite Verb häufig mit lediglich einem der beiden Konjunkte kongruiert (in der Regel handelt es sich dabei um das Konjunkt, das näher zum Verb steht, vgl. auch Hennig 2016: 571). Daraus kann auch 3. Sg. Kongruenz am Verb resultieren: ${ }^{11}$

(11) Falls du oder jmd anderes mir eine verlässliche Internetquelle nennen kann, werd ich es im Artikel einfügen.

(WDD13/A40.82194: Diskussion:Andrés Iniesta, In: Wikipedia http://de.wikipedia.org/wiki/Diskussion:Andrés_Iniesta: Wikipedia, 2013)

Schließlich wird von Corbett (1983) für die Alternation zwischen 2. Pl. und 3. Pl. im Zusammenhang mit Subjektreihungen aus 2. Sg. und 3. Sg. ein morphologischer Faktor in Anschlag gebracht, der Bezug nimmt auf Eigenschaften des jeweiligen verbalen Flexionsparadigmas. Corbett vermutet, dass die 3. Pl.Endung -en der 2. Pl. auf - $t$ vorgezogen wird, da letztere für viele Verben mit der 3. Sg. zusammenfällt und somit nicht eindeutig Plural signalisiert. Mit anderen Worten, Corbett nimmt an, dass die Resolutionsregeln für das Merkmal Person ausnahmsweise zugunsten einer eindeutigen Numerusmarkierung (Plural) überschrieben werden. Dies legt eine Hierarchie von Merkmalen nahe, die bevorzugt markiert werden, und steht im Einklang mit der Beobachtung, dass in der Sprachgeschichte des Deutschen eine starke Tendenz zur Numerusprofilierung beobachtet werden kann (vgl. z. B. Polenz 2000: 155 f.). Diese Erklärung lässt erwarten, dass Resolution zugunsten von 3. Pl. bevorzugt mit Verben auftritt, bei denen 3. Sg. und 2. Pl. zusammenfallen, während 2. Pl. signifikant häufiger mit Verben erscheinen sollte, die keinen solchen Synkretismus aufweisen, also z. B. die Auxiliare haben und sein, Modalverben und starke Verben mit Umlaut bzw. e/i-Wechsel in der 3. Sg.:

(12) 3. Sg.: hat, ist, kann, spricht, tritt, fällt, schläft etc.

2. Pl.: habt, seid, könnt, sprecht, tretet, fallt, schlaft etc.

11 Allerdings können Fälle wie (11) auch als zusammengezogener Satz (d.h., als das Resultat von Koordination und Ellipse) analysiert werden. Eine trennscharfe Abgrenzung ist hier nicht immer möglich, siehe Abschnitt 3 für weitere Diskussion. 
Im Folgenden soll das Kongruenzverhalten im Zusammenhang mit Reihungen aus 2. und 3. Person im Rahmen einer Korpusstudie näher betrachtet werden. Im Mittelpunkt stehen dabei die folgenden Fragen:

1. Existieren neben den in der Literatur erwähnten Varianten noch weitere Optionen wie Kongruenz mit einem der beiden Konjunkte analog zum Bairischen?

2. Welche Faktoren steuern die Wahl zwischen den Varianten? Lässt sich der Einfluss syntaktischer und morphologischer Einflussgrößen gewichten?

3. Welchen Status hat die beobachtete grammatische Variation? Handelt es sich um Inter- oder Intrasprecher-Variation?

Um den Einfluss potenzieller außergrammatischer Faktoren soweit wie möglich eingrenzen zu können, liegt der nachfolgend beschriebenen Untersuchung eine Datenbasis zugrunde, die hinsichtlich Medium, Textsorte, Register und Erstellungszeitraum möglichst homogen ist, nämlich Wikipedia-Diskussionen aus dem Jahr 2013 aus dem Deutschen Referenzkorpus (DeReKo/WDD13). Die Wahl der Datengrundlage ist überdies dadurch motiviert, dass Subjektreihungen, die 2. Sg. involvieren, in anderen Teilkorpora des DeReKo (in denen vor allem Zeitungstexte dominieren) wesentlich seltener sind. Aus WDD13 wurden insgesamt 1.198 einschlägige Belege extrahiert (Suchmuster: du und ..., ... und $d u$, du oder ..., ... oder $d u$ ). Davon enthielten 859 Belege Subjektreihungen aus 2. Sg. und 3. Sg./3. Pl. (die anderen Datensätze entfallen auf Konjunkte der Art 1. Sg./1. Pl. und 2. Pl.). Die Belege wurden anschließend händisch für die folgenden Eigenschaften annotiert:

- Art der Konjunktion (und oder oder)

- Reihenfolge der Konjunkte ( $d u$... X, X ... du)

- Identität des zweiten Subjekts (er, irgendjemand, der Benutzer, Peter etc.)

- Art des zweiten Subjekts (Personalpronomen, Pronomen, NP, Eigenname)

- Belebtheit des zweiten Subjekts

- Verbkongruenz (Person und Numerus)

- Wortstellung (SV ..., ... VS ..., Verb-End)

- Linksversetzung/Aufnahme durch ein resumptives Pronomen

- Eigenschaften des verbalen Flexionsparadigmas (3. Sg. = 2. Pl.?)

\section{Ergebnisse}

\subsection{Die Häufigkeit der Varianten}

Die folgenden Tabellen und Abbildungen zeigen die Verteilung der Kongruenzvarianten in der erhobenen Stichprobe aus 859 Belegen (Subjektreihungen aus 
2. Sg. und 3. Sg./3. Pl.). Getrennt betrachtet werden dabei (a) Subjektreihungen mit 3. Pl. und (b) Fälle, in denen die beiden Subjektteile durch oder verknüpft werden. Letzteres ist dadurch motiviert, dass Verknüpfungen mit oder ein deutlich anderes Kongruenzverhalten als Subjektreihungen mit und zeigen, indem bevorzugt Kongruenz mit nur einem Subjektteil vorliegt, vgl. auch die Dudengrammatik, §1616. ${ }^{12}$ Die Muster werden jeweils im Anschluss an die Tabelle durch Beispiele illustriert.

Tab. 6.2: Subjektreihungen aus 2. Sg. und 3. Sg. (Verknüpfung durch und) Verteilung der Kongruenzvarianten.

\begin{tabular}{llllll}
\hline & 2. Pl. & 3. Pl. & 2. Sg. & 3. Sg. & Gesamt \\
\hline $\begin{array}{l}\text { du und 3. Sg./ } \\
\text { 3. Sg. und } d u\end{array}$ & $(37,6 \%)$ & $164(47,4 \%)$ & $43(12,4 \%)$ & $9(2,6 \%)$ & 346 \\
\hline
\end{tabular}

12 Subjektreihungen, die mehr als ein Element enthielten wie in (i) und (ii), wurden dabei nicht berücksichtigt (15 Belege bei 2. Sg. + 3. Sg.). Auch hier liegt Kongruenzvariation vor (wobei (ii) evtl. auch als zusammengezogener Satz im Sinne der Dudengrammatik, §1602 betrachtet werden kann):

(i) Und natürlich kannst du mit Pro abstimmen, auch wenn Pyrotechniker, Boris und du die Hauptautoren sind.

(WDD13/R01.50031: Diskussion:Rammstein/Archiv/1, In: Wikipedia - URL: http:// de.wikipedia.org/wiki/Diskussion:Rammstein/Archiv/1: Wikipedia, 2013)

(ii) Immerhin ein Ausspruch den ich, du und der ganze Rest Autoren zu „Charles Darwin“ eine angemessene Antwort schuldig bleibt. (WDD13/C36.13450: Diskussion: Charles Darwin/Archiv, In: Wikipedia - URL:http://de.wikipedia.org/wiki/Diskussion: Charles_Darwin/Archiv: Wikipedia, 2013)

13 Unter den 130 Fällen befinden sich auch 46 Belege, die morphologisch ambig sind, da bei den beteiligten Verben die Formen für 3. Sg. und 2. Pl. zusammenfallen. Aufgrund der Tatsache, dass eindeutige Fälle von 2. Pl. bei Subjektreihungen aus 2. Sg. und 3. Sg. aber wesentlich häufiger sind als eindeutige Fälle von 3. Sg. (85 vs. 9), habe ich mich entschlossen, ambige Fälle der 2. Pl. zuzuschlagen (bei Reihungen mit oder ist dies allerdings nicht ohne Weiteres möglich, s.u.). Dies betrifft aber auch einige Fälle, für die auch eine Interpretation als 3. Sg. nicht unplausibel zu sein scheint (insbesondere bei nicht-belebten Konjunkten). Hier ist mitunter auch eine Analyse als Ellipse möglich.

(i) Eine Tatsache, die die [sic] du und auch dein(?) Artikel verschweigt. (WDD13/G01.98428: Diskussion:Gender-Mainstreaming/Archiv, In: Wikipedia http://de.wikipedia.org/wiki/Diskussion:Gender-Mainstreaming/Archiv: Wikipedia, 2013)

(ii) Ich verstehe dich schon, aber auch du und alles was du schreibst unterliegt deiner Sichtweise.

(WDD13/T21.49504: Diskussion:Tabakrauchen/Archiv/1, In: Wikipedia http://de.wikipedia.org/wiki/Diskussion:Tabakrauchen/Archiv/1: Wikipedia, 2013) 


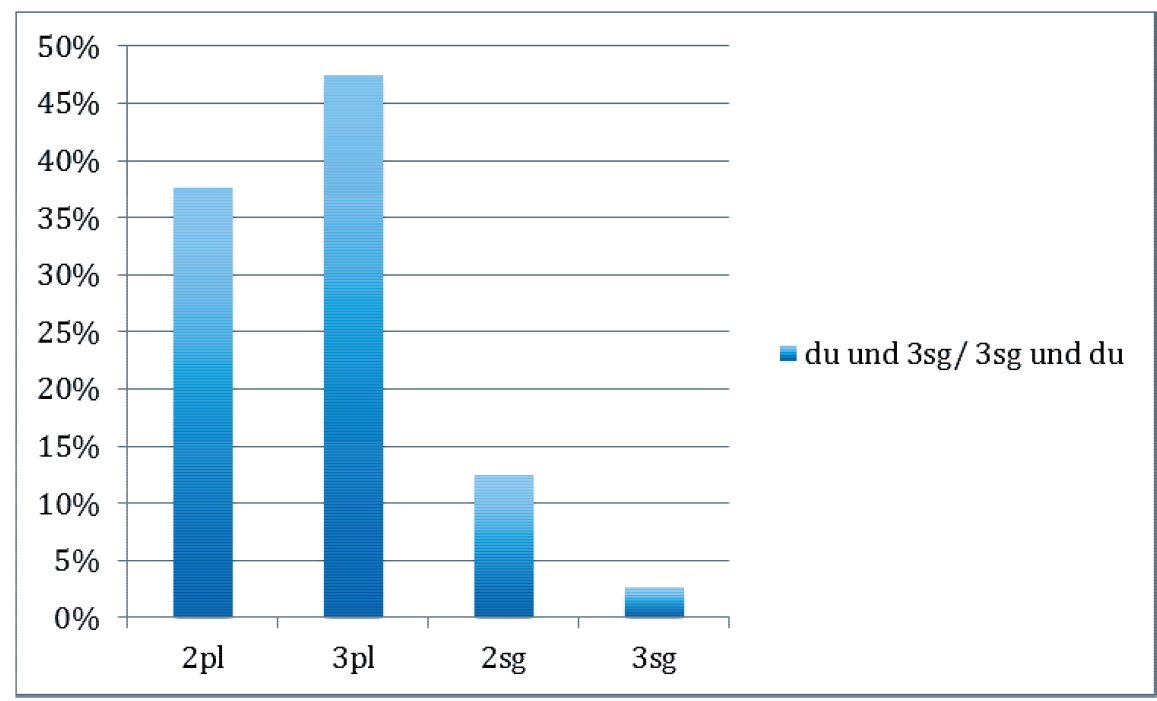

Abb. 6.1: Subjektreihungen aus 2. Sg. und 3. Sg. (Verknüpfung durch und) Verteilung der Kongruenzvarianten.

(13) 2. Pl.:

Ach ja: du und Danyalova seid euch sehr sehr sehr ähnlich.

(WDD13/T54.92245: Diskussion:Türken/Archiv/2007, In: Wikipedia http://de.wikipedia.org/wiki/Diskussion:Türken/Archiv/2007: Wikipedia, 2013)

(14) 3. Pl.:

Nur weil du und Sarrazin sich in einem Punkt einig sind (Verhältnis von Kritikern zu Befürwortern), heißt das noch lange nicht, dass ihr zwangsläufig richtig liegt.

(WDD13/D59.37433: Diskussion:Deutschland schafft sich ab/Archiv/4, In: Wikipedia - http://de.wikipedia.org/wiki/Diskussion:Deutschland_ schafft_sich_ab/Archiv/4: Wikipedia, 2013)

(15) 2. Sg.:

$\mathrm{Na}$ zumindest bist du und C. Berne euch darüber einig, das ihr Wikipedia „zum Kotzen“ findet.

(WDD13/E76.95741: Diskussion:Elisabeth Hering, In: Wikipedia http://de.wikipedia.org/wiki/Diskussion:Elisabeth_Hering: Wikipedia, 2013) 
(16) 3. Sg.:

Tun wir doch mal spaßeshalber so, als ob du und das was du schreibst ernst $\mathrm{zu}$ nehmen wäre.

(WDD13/F69.75386: Diskussion:Friedrich August von Hayek/Archiv/3, In: Wikipedia - http://de.wikipedia.org/wiki/Diskussion:Friedrich_ August_von_Hayek/Archiv/3: Wikipedia, 2013)

Die Befunde zeigen, dass alle theoretisch möglichen Varianten im Korpus vertreten sind. Allerdings sind Belege mit 3. Sg. recht selten; auffällig ist hierbei der recht große Anteil von nicht-belebten Subjektteilen (4 von 9 Belegen, vgl. auch (16)), die insgesamt nur einen Bruchteil der Belege ausmachen (27 von 346; 7,8\%). ${ }^{14}$ Die anderen drei Optionen sind aber robust belegt. Als erstes Ergebnis kann festgehalten werden, dass sich die weitverbreitete Auffassung, dass Subjektreihungen aus 2. Sg. und 3. Sg. bevorzugt 2. Pl.-Kongruenz am Verb auslösen sollten, nicht ohne Weiteres mit den empirischen Befunden vereinbaren lässt. Es handelt sich dabei lediglich um eines von drei möglichen Mustern, wobei 3. Pl. mit einem Anteil von annähernd $50 \%$ die häufigste Option darstellt. Überraschend ist auch, dass Kongruenz in der 2. Sg. relativ robust vertreten ist. Dabei handelt es sich fast ausschließlich um Beispiele wie (15), in denen ein komplexes Subjekt der Art $d u$ und $X$ dem finiten Verb in der linken Satzklammer nachfolgt, ähnlich wie bei den Fällen von First Conjunct Agreement im Bairischen (s. u. für weitere Diskussion und eine Abgrenzung zu elliptischen Strukturen).

Auch im Zusammenhang mit pluralischen Subjekten in der 3. Person sind alle theoretisch möglichen Varianten vertreten. Allerdings ist hier die Tendenz zu 3. Pl. (über $2 / 3$ aller Belege) noch wesentlich deutlicher als bei Subjekten in der 3. Sg. Dies entspricht nicht den Vorhersagen gängiger Darstellungen. Die Dudengrammatik (§1607) weist zwar darauf hin, dass hier Schwankungen zwischen 2. Pl. und 3. Pl. möglich sind, stellt aber 2. Pl. als Standardvariante

14 Ein Beleg wie (16) könnte auch als zusammengezogener Satz analysiert werden. Die Tatsache, dass Kongruenz in der 3. Sg. ein sehr seltenes Muster darstellt, scheint aber darauf hinzudeuten, dass dieser Konstruktionstyp im Zusammenhang mit und nur eine sehr marginale Rolle spielt. Darüber hinaus gibt es (freilich seltene) Beispiele wie (i), für die eine Analyse als Ellipse nicht möglich ist. Angesichts der geringen Zahl entsprechender Belege kann aber auch nicht gänzlich ausgeschlossen werden, dass es sich bei Fällen wie in (i) lediglich um Performanzfehler handelt.

(i) Bisher hat sich nur du und 20percent daran gestoßen, denn über viele Monate und Jahre hatte niemand etwas gegen die deutsche [sic] Namen einzuwenden.

(Diskussion:Pilsen/Archiv, In: Wikipedia - http://de.wikipedia.org/wiki/Diskussion: Pilsen/Archiv: Wikipedia, 2013) 
heraus. In WDD13 wird aber 2. Pl. sogar noch von der dritten Variante - 2. Sg. überflügelt.

Tab. 6.3: Subjektreihungen aus 2. Sg. und 3. Pl. (Verknüpfung durch und) Verteilung der Kongruenzvarianten.

\begin{tabular}{lllll}
\hline & 2. Pl. & 3. Pl. & 2. Sg. & Gesamt \\
\hline$d u$ und 3. Pl./3. Pl. und $d u$ & $24^{15}(12,1 \%)$ & $142(71,7 \%)$ & $32(16,2 \%)$ & 198 \\
\hline
\end{tabular}

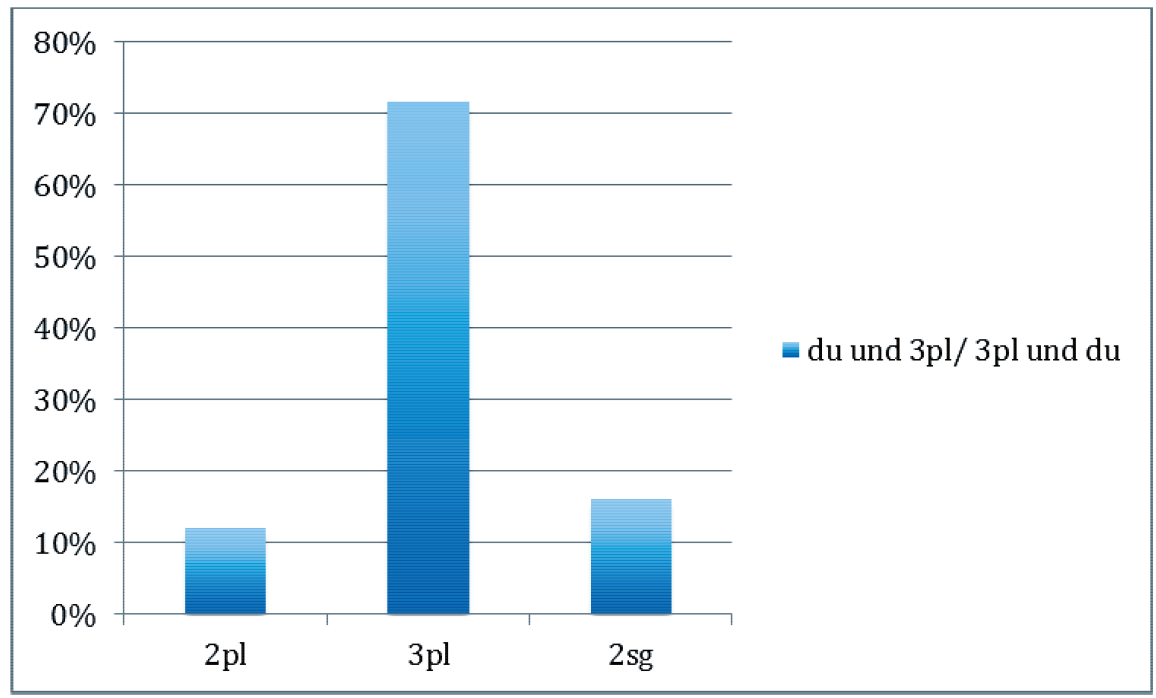

Abb. 6.2: Subjektreihungen aus 2. Sg. und 3. Pl. (Verknüpfung durch und) Verteilung der Kongruenzvarianten.

(17) 2. Pl.:

Wenn du und die anderen ein Zeichen setzen wollt, tut das. (WDD13/P61.63532: Diskussion:Pressefreiheit/Archiv, In: Wikipedia http://de.wikipedia.org/wiki/Diskussion:Pressefreiheit/Archiv: Wikipedia, 2013)

15 Auch hier wurden morphologisch ambige Formen (3. Sg./2. Pl.) als 2. Pl. gewertet. Dies ist bei Subjektreihungen mit 3. Pl. allerdings wesentlich unproblematischer, da hier 3. Sg. keine Kongruenzoption ist. Ferner wurden drei Belege mit mehrfacher Koordination ausgeschlossen. 
(18) 3. Pl.:

Ich hoffe, dass du und alle Wikipedianer, die sich um den Métroartikel bemühen, damit einverstanden sind. (WDD13/M02.07116: Diskussion:Métro Paris, In: Wikipedia http://de.wikipedia.org/wiki/Diskussion:Métro_Paris: Wikipedia, 2013)

(19) 2. Sg.:

Hast du und deine Mitstreiterinnen überhaupt gelesen um was es geht? (WDD13/H76.14109: Diskussion:Häusliche Gewalt/Archiv/2, In: Wikipedia - http:/de.wikipedia.org/wiki/Diskussion:Häusliche_ Gewalt/Archiv/2: Wikipedia, 2013)

Die Dominanz von Fällen, in denen das Verb in der 3. Pl. steht, kann nicht ohne Weiteres durch die Annahme erfasst werden, dass in Belegen wie (18) keine Subjektreihung, sondern ein zusammengezogener Satz (also Koordination + Ellipse) vorliegt. Wäre dies der Fall, dann würde man erwarten, dass diese Möglichkeit in gleicher Weise auch bei singularischen Subjektteilen besteht. Dort ist der Anteil von 3. Sg.-Kongruenz aber sehr gering (weniger als 3\%); im Umkehrschluss bedeutet dies, dass man auch für den vorliegenden Fall (Teilsubjekt in der 3. Pl.) davon ausgehen kann, dass elliptische Konstruktionen nur einen Bruchteil der Belege ausmachen und dass es sich in der Mehrzahl der Fälle tatsächlich um Subjektreihungen handelt (s. u. für den Status von Beispielen mit 2. Sg.-Kongruenz wie (19)).

Ein etwas anderes Bild ergibt sich, wenn man Subjektreihungen mit oder betrachtet. Auch hier beginnen wir mit Kombinationen aus 2. Sg. und 3. Sg. Der wesentliche Unterschied zu Reihungen mit und besteht darin, dass das Verb auch mit nur einem der beiden Subjektteile kongruieren kann (sowohl 2. Sg. als auch 3. Sg.). Daraus ergibt sich ein methodologisches Problem, da nun bei Verben, die 3. Sg. und 2. Pl. nicht unterscheiden, die Formen auf - $t$ echt ambig sind. In Tabelle 6.4 werden daher ambige Formen auch als solche ausgewiesen und in einer separaten Spalte aufgeführt: ${ }^{16}$

Tab. 6.4: Subjektreihungen aus 2. Sg. und 3. Sg. (Verknüpfung durch oder) Verteilung der Kongruenzvarianten.

\begin{tabular}{lllllll}
\hline & 2. Pl. & 3. Pl. & 2. Sg. & 3. Sg. & ambig: 3. Sg./2. Pl. & Gesamt \\
\hline du oder 3. Sg./ & 16 & 45 & 85 & 71 & $43(16,5 \%)$ & 260 \\
3. Sg. oder du & $(6,2 \%)$ & $(17,3 \%)$ & $(32,7 \%)$ & $(27,3 \%)$ & & \\
\hline
\end{tabular}

16 Ausgeschlossen wurden wiederum 21 Subjektreihungen mit mehr als zwei Subjektteilen. 


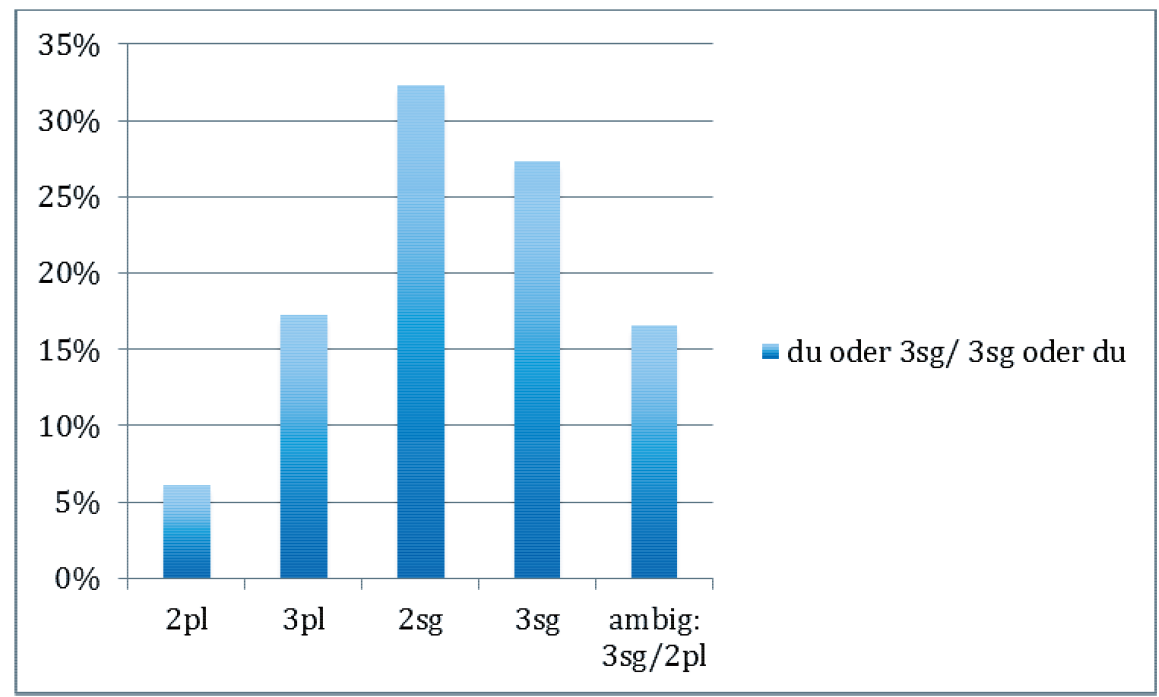

Abb. 6.3: Subjektreihungen aus 2. Sg. und 3. Sg. (Verknüpfung durch oder) Verteilung der Kongruenzvarianten.

(20) 2. Pl.:

Wenn du oder Bauernopfer Gegenargumente angeben könnt, dann bitteschön, aber doch nicht einfach wochenlang abwarten.

(WDD13/B08.01463: Diskussion:Backpropagation, In: Wikipedia http://de.wikipedia.org/wiki/Diskussion:Backpropagation: Wikipedia, 2013)

(21) 3. Pl.:

Deine Behauptung, du oder ein Blogschreiber haben es gehört, reicht nicht für die Darstellung in einem enzyklopädischen Artikel.

(WDD13/C13.05055: Diskussion:Christoph Butterwegge, In: Wikipedia http://de.wikipedia.org/wiki/Diskussion:Christoph_Butterwegge: Wikipedia, 2013)

(22) 2. Sg.:

Könntest du oder ein anderer den Abschnitt mal durchgehen?

(WDD13/C49.95088: Diskussion:Cocktail für eine Leiche, In: Wikipedia http://de.wikipedia.org/wiki/Diskussion:Cocktail_für_eine_Leiche: Wikipedia, 2013) 
(23) 3. Sg.:

Wenn du oder jemand anderes nun aber die Absicht hat, den Artikel merklich zu ergänzen, sieht die Sache für mich schon anders aus. (WDD13/G46.75394: Diskussion:Gäubahn (Stutgart-Hattingen)/Archiv, In: Wikipedia - http:/de.wikipedia.org/wiki/Diskussion: Gäubahn_(Stuttgart-Hattingen)/Archiv: Wikipedia, 2013)

(24) Ambig (3. Sg./2. Pl.):

a. Hallo sebmol, wenn du oder die Allgemeinheit in diesem Punkt nicht mit mir übereinstimt dann bleibts halt drin. (WDD13/A35.73886: Diskussion:Abdullah Öcalan/Archiv, In: Wikipedia - http://de.wikipedia.org/wiki/Diskussion:Abdullah_ Öcalan/Archiv: Wikipedia, 2013)

b. Wenn du oder jemand anders mir neue Daten mit Quelle vorlegt, können wir das gerne wieder aufnehmen. (WDD13/A12.38529: Diskussion:Atlantis/Archiv2, In: Wikipedia http://de.wikipedia.org/wiki/Diskussion:Atlantis/Archiv2: Wikipedia, 2013)

Aus Tabelle 6.4 und Abbildung 6.3 geht deutlich hervor, dass sich Subjektreihungen mit oder in Bezug auf die Wahl der verbalen Kongruenzendung anders verhalten als Reihungen mit und (was der Darstellung in der Dudengrammatik entspricht). Auffällig ist vor allem, dass in $60 \%$ aller Fälle Singularkongruenz, also Kongruenz mit einem der Subjektteile (2. Sg. oder 3. Sg.), auftritt und somit klar überwiegt. Dabei ist - vielleicht etwas überraschend - 2. Sg. die häufigste Variante (ca. 1/3 aller Belege), dicht gefolgt von 3. Sg. (27\%). Der relativ große Anteil von 3. Sg. kann möglicherweise darauf zurückgeführt werden, dass Beispiele mit oder leichter als das Resultat von Koordination plus Ellipse aufgefasst werden können. ${ }^{17}$ Diese Analyse kann allerdings nicht ohne Weiteres auf die überwiegende Zahl der Belege mit 2. Sg.-Kongruenz übertragen werden, da diese andere Eigenschaften aufweisen. Zum einen liegt in der Regel Kongruenz mit dem ersten Subjektteil vor, während bei 3. Sg. das Verb bevorzugt

17 Ich danke einem anonymen Gutachter für diesen Hinweis. Das unterschiedliche Verhalten von Reihungen mit und vs. oder entspricht auch den semantischen Unterschieden zwischen den beiden Konjunktionen (additiv vs. alternativenbildend). Vor diesem Hintergrund würde man zudem erwarten, dass die Wahl zwischen Singular- und Pluralkongruenz bei Konstruktionen mit oder von der Lesart der Konjunktion beeinflusst ist (inklusiv vs. exklusiv). Die Befunde sind allerdings nicht klar. So scheint in den Beispielen (20)-(24) und (27) trotz der Numerusunterschiede bei der Verbkongruenz eine inklusive Lesart stets verfügbar zu sein. 
mit dem zweiten Subjektteil kongruiert. Zum anderen tritt 2. Sg. - wie bereits oben angedeutet - vor allem in Inversionskontexten auf, vgl. (22). Eine Analyse solcher Fälle als Ellipse würde die Annahme einer komplexen Tilgungsoperation erforderlich machen, bei der im ersten Konjunktsatz die VP und im zweiten Konjunktsatz das finite Verb getilgt wird:

(25) [Könntest du den Abschnitt mal durchgehen] oder [könnte ein anderer den Abschnitt mal durchgehen]?

Darüber hinaus würde eine Tilgungsanalyse fälschlicherweise prognostizieren, dass 2. Sg.-Kongruenz ohne Weiteres auch in Fällen wie (26) möglich sein sollte, in denen das Verb in finaler Position erscheint. Zumindest mit und tritt dieses Muster im Korpus aber nicht auf. Wir können also festhalten, dass eine Tilgungsanalyse den besonderen Status von Inversionskontexten bei Fällen von 2. Sg.-Kongruenz nicht ausreichend berücksichtigt.

(26) [weil deine Freunde das Buch gelesen haben] und [du das Buch gelesen hast]

Kongruiert das Verb mit dem gesamten Subjekt, so liegt wiederum zumeist 3. Pl. vor (insgesamt 17\%), während es sich bei eindeutigen Fällen von 2. Pl. um eine Randerscheinung handelt, die auf lediglich $6 \%$ aller Fälle beschränkt ist. Dies kann so interpretiert werden, dass vermutlich in der überwiegenden Zahl aller ambigen Fälle von den Sprechern 3. Sg. intendiert ist - ganz im Gegensatz zu den Befunden, die wir für Reihungen mit und gewonnen haben. Der relativ große Anteil von 2. Sg. ist möglicherweise darauf zurückzuführen, dass bei Verknüpfungen mit oder zusätzlich zu den Mustern, bei denen ein Verb in der linken Satzklammer mit dem ersten nachfolgenden Subjektteil kongruiert (First Conjunct Agreement) 2. Sg. Kongruenz auch möglich ist, wenn das Verb (im Nebensatz) dem Subjekt folgt wie in dem folgenden Beispiel: ${ }^{18}$

18 Man beachte, dass in (27) keine Ellipse vorliegen kann, da das Verb nicht mit dem nächsten Subjekt kongruiert, sondern mit dem weiter entfernten $d u$. Es gibt keine Struktur, in der hast am Ende des Satzes erscheint und gleichzeitig Tilgung von hat im zweiten Konjunkt lizenziert ist. Eine entsprechende Koordination müsste wie in (i) aussehen. Hier steht hast zwar in finaler Position; die Konjunkte sind aber nicht wohlgeformt (du weiterführende Erkenntnisse bildet keine Konstituente; außerdem sind die beiden Konjunkte nicht hinreichend identisch), und hat sollte zudem nicht gelöscht werden können.

(i) Falls [du weiterführende Erkenntnisse] oder [jemand anders weiterführende Erkenntnisse hat] hast [...] 
(27) Falls du oder jemand anderes weiterführende Erkenntnisse/Quellen hast, wäre ich für eine Berichtigung oder Ergänzung ausdrücklich dankbar.

(WDD13/R66.72463: Diskussion:Reblin (Herscheid), In: Wikipedia http://de.wikipedia.org/wiki/Diskussion:Reblin_(Herscheid): Wikipedia, 2013)

Abschließend wollen wir noch einen Blick auf das letzte verbleibende Muster werfen, das Subjektreihungen aus 2. Sg. und 3. Pl. betrifft, die mit oder verknüpft sind (Tabelle 6.5 und Abbildung 6.4). ${ }^{19}$

Tab. 6.5: Subjektreihungen aus 2. Sg. und 3. Pl. (Verknüpfung durch oder) Verteilung der Kongruenzvarianten.

\begin{tabular}{lllll}
\hline & 2. Pl. & 3. Pl. & 2. Sg. & Gesamt \\
\hline du oder 3. Pl./3. Pl. oder $d u$ & $2(4,2 \%)$ & $39(81,2 \%)$ & $7(14,6 \%)$ & 48 \\
\hline
\end{tabular}

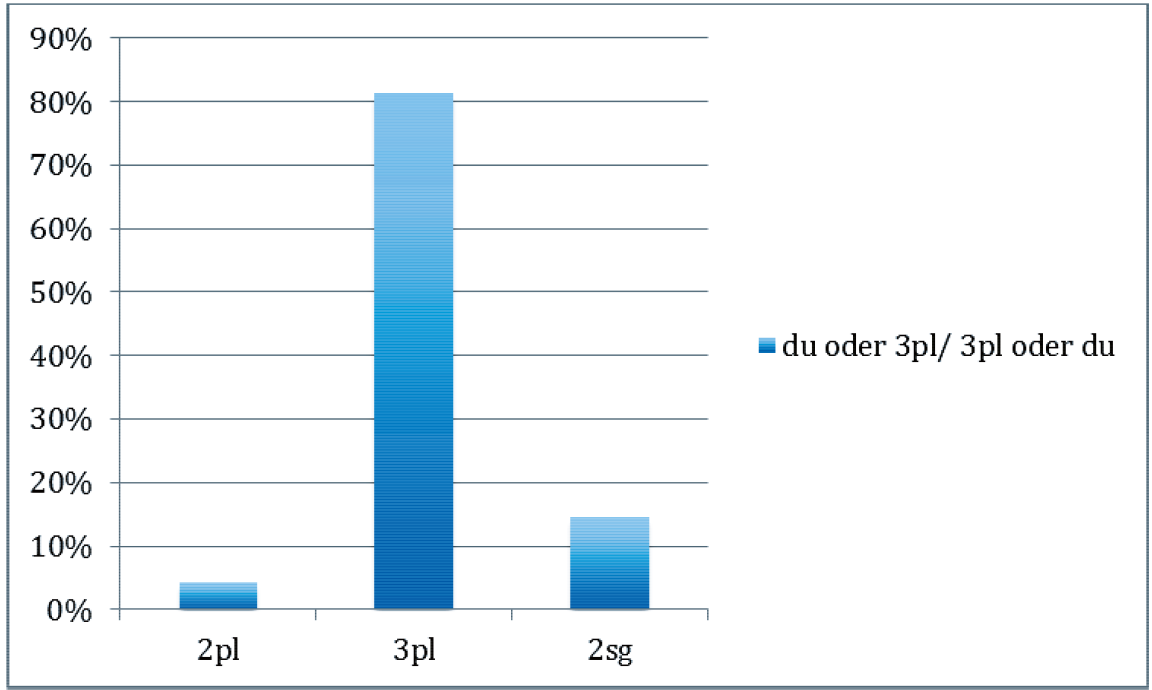

Abb. 6.4: Subjektreihungen aus 2. Sg. und 3. Pl. (Verknüpfung durch oder) Verteilung der Kongruenzvarianten.

19 Aussortiert wurden 2 Belege mit Subjektreihungen, die mehr als zwei Elemente enthalten. 
(28) 2. Pl.:

Falls du oder irgendwelche anderen Leser noch irgendetwas kürzen wollt, ohne $\mathrm{zu}$ viele Inhalte $\mathrm{zu}$ eliminieren, nur $\mathrm{zu}$.

(WDD13/A77.50782: Diskussion:Albert Schweitzer/Archiv, In: Wikipedia http://de.wikipedia.org/wiki/Diskussion:Albert_Schweitzer/Archiv: Wikipedia, 2013)

(29) 3. Pl.:

Wie du oder deine Bekannten es aussprechen, ist doch hier völlig irrelevant.

(WDD13/A71.60819: Diskussion:Amazon.com/Archiv/1, In: Wikipedia http://de.wikipedia.org/wiki/Diskussion:Amazon.com/Archiv/1: Wikipedia, 2013)

(30) 2. Sg.:

Dann könntest du oder andere ggf. mit ihm selbst spitzfindig darüber weiterdiskutieren.

(WDD13/A67.96255: Diskussion:American Pit Bull Terrier/Archiv, In: Wikipedia - http://de.wikipedia.org/wiki/Diskussion:American_Pit_ Bull_Terrier/Archiv: Wikipedia, 2013)

Offenbar gibt es bei Subjektreihungen mit oder einen deutlichen Kontrast zwischen solchen, die Subjekte in der 3. Sg., und solchen, die Subjekte in der 3. Pl. enthalten. Bei Letzteren ist 3. Pl. das absolut dominante Kongruenzmuster (ca. $80 \%$ aller Fälle), während 2. Sg. Kongruenz nur noch einen Anteil von knapp 15\% hat. Wiederum ist 2. Pl. mit einem Anteil von unter 5\% äußerst marginal. Die Befunde ähneln interessanterweise der Verteilung bei Subjektreihungen mit 3. $\mathrm{Pl}$. und und. Die starke Tendenz $\mathrm{zu}$ 3. Pl. ist möglicherweise darauf zurückzuführen, dass sowohl Resolution als auch Kongruenz mit dem pluralischen Subjektteil bevorzugt zu 3. Pl. führt.

Wir haben bislang gesehen, dass die Befunde der Korpusstudie zum Teil deutlich von den Darstellungen in einschlägigen Grammatiken abweichen. Besonders auffällig ist der starke Anteil von 3. Pl., das bis auf Subjektreihungen, die aus 2. Sg. und 3. Sg. mittels oder gebildet werden, das bevorzugte Kongruenzmuster darstellt. Ebenso unerwartet ist, dass 2. Sg. in allen Typen robust attestiert ist und z. T. sogar häufiger als 2. Pl. und 3. Pl. ist. Im Gegensatz dazu ist 2. Pl. überraschend schwach vertreten (recht häufig nur bei 2. Sg. + 3. Sg. verknüpft durch und), was nicht im Einklang mit gängigen Beschreibungen zu stehen scheint. Die Möglichkeit von 2. Sg.-Kongruenz kann dabei in vielen Fällen nicht darauf zurückgeführt werden, dass ein zusammengezogener Satz (d.h., eine elliptische Struktur) vorliegt. Vielmehr handelt es sich offenbar um echte Fälle von First Conjunct Agreement. Wie gängige Darstellun- 
gen in der Literatur bereits erwarten lassen, gibt es darüber hinaus einen deutlichen Kontrast zwischen Subjektreihungen mit und und solchen mit oder. Im Anschluss soll untersucht werden, welche weiteren grammatischen Faktoren die Verteilung der Kongruenzvarianten beeinflussen.

\subsection{Grammatische Faktoren}

Die Darstellung im vorangegangenen Abschnitt hat mit dem (semantischen) Unterschied zwischen den Konjunktionen und und oder bereits einen starken Faktor zu Tage gefördert, der die Verteilung der Kongruenzvarianten beeinflusst. In der Folge sollen weitere grammatische Einflussgrößen identifiziert und näher untersucht werden. Dabei werden sowohl morphologische als auch syntaktische Faktoren berücksichtigt. Insbesondere soll geprüft werden, ob sich die Verteilung der Kongruenzvarianten 2. Pl. und 3. Pl. durch die Hypothese von Corbett (1983) erklären lässt, die besagt, dass ein Zusammenfall von 3. Sg. und 2. Pl. die Wahl der Kongruenzendung bei komplexen Subjekten der Art 2. Sg. + 3. Sg. zugunsten von 3. Pl. beeinflusst. Ferner wird auch der Einfluss der Wortstellung untersucht - sowohl die relative Abfolge der Subjektteile als auch die Stellung des komplexen Subjekts relativ zum Verb.

Wie bereits zu Beginn des Abschnitts erwähnt, prognostiziert die Hypothese von Corbett (1983), dass bei Subjektreihungen aus 2. Sg. und 3. Sg. Kongruenzformen der 3. Pl. insbesondere mit Verben auftreten sollten, bei denen 3. Sg. und 2. Pl. zusammenfallen (um Numerusmarkierung $\mathrm{zu}$ gewährleisten). Um diese Vorhersage $\mathrm{zu}$ testen, wurden alle extrahierten Verbformen für das Vorliegen eines entsprechenden Synkretismus annotiert. ${ }^{20}$ Die Befunde sehen wie folgt aus. Zunächst betrachten wir Subjektreihungen aus 2. Sg. und 3. Sg. verknüpft durch und (insgesamt 294 Belege für die Kongruenzvarianten 2. Pl. und 3. Pl.):

Tab. 6.6: Einfluss des Zusammenfalls von 2. Pl. und 3. Sg. auf die Verteilung der Kongruenzvarianten 2. Pl. und 3. Pl. (Subjektreihungen aus 2. Sg. und 3. Sg., und).

\begin{tabular}{lll}
\hline & 2. Pl. & 3. Pl. \\
\hline 2. Pl. = 3. Sg. & $47(36,2 \%)$ & $70(42,7 \%)$ \\
2. Pl. $\neq$ 3. Sg. & $83(63,8 \%)$ & $94(57,3 \%)$ \\
\hline
\end{tabular}

20 3. Sg. und 2. Pl. fallen nur im Präsens Indikativ zusammen, während sie in anderen Tempora und Modi unterschieden werden. Bei vielen starken Verben (sowie den Hilfs- und Modalverben) sind zudem 3. Sg. und 2. Pl. auch im Präsens Indikativ distinkt (er/sie/es schläft, fährt, läuft vs. ihr schlaft, fahrt, lauft etc.). 
Wie man in Tabelle 6.6 erkennen kann, ist 3. Pl. die dominante Variante, unabhängig davon, ob die Verbformen für 3. Sg. und 2. Pl. zusammenfallen. Zwar ist der Anteil der Kongruenzvariante 2. Pl. größer, wenn die Formen für 3. Sg. und 2. Pl. distinkt sind. Eine inferenzstatistische Analyse zeigt jedoch, dass dieser Unterschied nicht signifikant ist. Der Chi-Quadrat-Test ergibt einen p-Wert von 0,31. Die Effektstärke ist mit 0,059 äußerst gering. Wir können also festhalten, dass sich der von Corbett prognostizierte Effekt in unseren Daten nicht nachweisen lässt. Falls sich keine anderen (grammatischen) Faktoren identifizieren lassen, deutet dies evtl. darauf hin, dass es sich bei den beobachteten Kongruenzschwankungen um einen Fall von freier Variation handelt, wie sie typischerweise in Sprachwandelsituationen auftritt (vgl. Abschnitt 4 für eine entsprechende Analyse). ${ }^{21}$

Ein etwas anderes Bild ergibt sich, wenn man Reihungen mit oder betrachtet. Hier sieht es zunächst so aus, als würde der Faktor \pm Synkretismus einen signifikanten Einfluss auf die Verteilung der Varianten haben ( $p=0,004$, Phi/ V-Koeffizient $=0,37$ ). Tatsächlich treten in der Stichprobe Belege mit 2. Pl. ausschließlich dann auf, wenn die Verbformen für 3. Sg. und 2. Pl. nicht zusammenfallen. Allerdings sind hier die Belegzahlen insgesamt zu gering, um von belastbaren Ergebnissen sprechen zu können (Tab. 6.7). ${ }^{22}$

Tab. 6.7: Einfluss des Zusammenfalls von 2. Pl. und 3. Sg. auf die Verteilung der Kongruenzvarianten 2. Pl. und 3. Pl. (Subjektreihungen aus 2. Sg. und 3. Sg., oder).

\begin{tabular}{lll}
\hline & 2. Pl. & 3. Pl. \\
\hline 2. Pl. = 3. Sg. & 0 & $19(43,2 \%)$ \\
2. Pl. $\neq$ 3. Sg. & $16(100 \%)$ & $25(56,8 \%)$ \\
\hline
\end{tabular}

21 Es ist jedoch durchaus denkbar, dass hier andere Einflussgrößen relevant sind und es sich tatsächlich nicht um völlig freie Variation handelt. So scheint 2. Pl. Kongruenz nicht ohne Weiteres möglich zu sein, wenn das zweite Subjekt nicht belebt ist. Die Korpusrecherche hat nur drei entsprechende Beispiele ergeben, wobei allerdings jeweils die Verbform ambig ist zwischen 3. Sg. und 2. Pl., sodass man nicht ausschließen kann, dass in Beispielen wie (i) 3. Sg. Kongruenz vorliegt:

(i) Eine Tatsache, die die du und auch dein(?) Artikel verschweigt.

(WDD13/G01.98428: Diskussion:Gender-Mainstreaming/Archiv, In: Wikipedia -

http://de.wikipedia.org/wiki/Diskussion:Gender-Mainstreaming/Archiv: Wikipedia, 2013)

22 Dies zeigt sich auch daran, dass bereits die Hinzunahme eines einzigen Belegs für 2. Pl. in der ersten Zelle ausreichen würde, um zu einem nicht-signifikanten Ergebnis zu führen. 
Tabelle 6.8 zeigt den Einfluss des Faktors \pm Synkretismus auf die Verteilung der Varianten, wenn man Subjekte der 2. Sg. und 3. Pl. durch und verknüpft. Auch hier ist der Effekt nicht signifikant. Tatsächlich liegen die Werte noch unter den Werten für Verknüpfungen mit 3. Sg. $(p=0,98$, Phi/V-Koeffizient $=0,002) .{ }^{23}$

Tab. 6.8: Einfluss des Zusammenfalls von 2. Pl. und 3. Pl. auf die Verteilung der Kongruenzvarianten 2. Pl. und 3. Pl. (Subjektreihungen aus 2. Sg. und 3. Pl., und).

\begin{tabular}{lll}
\hline & 2. Pl. & 3. Pl. \\
\hline 2. Pl. = 3. Sg. & $7(36,8 \%)$ & $57(40,1 \%)$ \\
2. Pl. $\neq$ 3. Sg. & $12(63,2 \%)$ & $85(59,9 \%)$ \\
\hline
\end{tabular}

Wir können also festhalten, dass sich die Prognosen von Corbett (1983) im Rahmen der vorliegenden Korpusstudie nicht bestätigen lassen. Zwar steigt der Anteil von 2. Pl.-Kongruenz an, wenn kein Synkretismus von 3. Sg. und 2. Pl. vorliegt; die Veränderung der Verhältnisse ist aber nicht signifikant. 3. Pl. ist stets die häufigere Kongruenzvariante - auch entgegen gängigen Darstellungen in der Literatur. Dabei sind die Belegzahlen für 2. Pl. insbesondere im Kontext von oder und bei Subjektreihungen mit 3. Pl. sehr niedrig. Dies weist möglicherweise darauf hin, dass hier auch Eigenschaften des komplexen Subjekts von Belang sind. In der Folge sollen daher die Faktoren Präsenz eines Subjektteils in der 3. Pl. und die Reihenfolge der Konjunkte kurz beleuchtet werden (einleitend haben wir bereits gesehen, dass die Wahl der Konjunktion ebenfalls das Kongruenzverhalten deutlich beeinflusst). Für den Einfluss von 3. Pl. haben wir die Fälle mit und und oder zusammengefasst (Subjektreihungen mit 3. Sg. und 3. Pl.). Die Ergebnisse sehen wie folgt aus:

Tab. 6.9: Einfluss der Präsenz eines Subjekteils in der 3. Pl. auf die Verbkongruenz.

\begin{tabular}{lll}
\hline & 2. Pl. & 3. Pl. \\
\hline Reihung enthält 3. Pl. & $26(15,1 \%)$ & $181(46,4 \%)$ \\
Reihung enthält nicht 3. Pl. & $146(84,9 \%)$ & $209(53,6 \%)$ \\
\hline
\end{tabular}

23 Auf eine Auswertung der Ergebnisse mit 3. Pl./oder habe ich an dieser Stelle verzichtet, da die Belegzahl noch deutlich unter den Werten in Tabelle 6.7 liegt. 
Aus Tabelle 6.9 geht hervor, dass der Anteil von 2. Pl. wesentlich geringer ausfällt, wenn die Reihung ein Subjekt in der 3. Pl. enthält. Der Effekt ist hochsignifikant ( $p=2.688 \mathrm{e}^{-12}$ ); der Phi/V-Koeffizient liegt mit 0,29 knapp unter der Schwelle für mittlere Effektstärken. Wir können also festhalten, dass die Präsenz eines Subjektteils in der 3. Pl. die Verteilung der Varianten deutlich zugunsten von 3. Pl. Markierung am Verb zu beeinflussen scheint.

Für die Fälle, in denen $d u$ der Konjunktion nachfolgt (insgesamt 93), ergibt sich die folgende Verteilung (und \& oder, 3. Sg. \& 3. Pl. als erstes Konjunkt). ${ }^{24}$ Zum Vergleich enthält Tabelle 6.10 auch die Angaben zur Verteilung der Varianten bei Subjektreihungen mit $d u$ als erstem Konjunkt.

Tab. 6.10: Einfluss der Abfolge in der Subjektreihung auf die Verbkongruenz.

\begin{tabular}{llcccc}
\hline & 2. Sg. & 2. Pl. & 3. Pl. & 3. Sg. & ambig: 3. Sg./2. Pl. \\
\hline$d u$ als zweites Konjunkt & - & 30 & 60 & 2 & 1 \\
$d u$ als erstes Konjunkt & 168 & 142 & 330 & 84 & 42 \\
\hline
\end{tabular}

Die relative Häufigkeit der Varianten 2. Pl. und 3. Pl. in Subjektreihungen mit $d u$ als zweitem Konjunkt unterscheidet sich anscheinend nicht wesentlich von den Befunden in anderen Kontexten. Bemerkenswert ist aber, dass die Kongruenzoption 2. Sg. in unserer Stichprobe nicht auftritt, wenn $d u$ der Konjunktion nachfolgt, obwohl sie in Fällen, in denen $d u$ das erste Konjunkt darstellt, die insgesamt zweithäufigste Variante ist. ${ }^{25}$ Dies deckt sich mit den Beobachtungen zum Bairischen, wo FCA (mit 2. Sg.) ebenfalls nicht möglich ist, wenn $d u$ das zweite Konjunkt ist (vgl. (7) oben). ${ }^{26}$ Da die Möglichkeit von 2. Sg. Kon-

24 Bei dem nicht-eindeutigen Beispiel lässt sich nicht mit Bestimmtheit sagen, ob am Verb 3. Sg. oder 2. Pl. vorliegt:

(i) Ja aber warum beleidigt WP oder du dann mich.

(WDD13/M41.92808: Diskussion:Muhabbet/Archiv/2, In: Wikipedia -

http://de.wikipedia.org/wiki/Diskussion:Muhabbet/Archiv/2: Wikipedia, 2013)

25 Die Stichprobe enthält allerdings ein Beispiel der Art $X$ oder $d u$, in dem 2. Sg. Kongruenz am Verb vorliegt. Allerdings handelt es sich bei dem ersten Konjunkt um ihr, sodass dieser Beleg nicht in die Auswertung eingegangen ist:

(i) Ihr, oder du, schreibst hier, dass Ehrlinger 1965 aus der Haft entlassen wurde.

(WDD13/E05.34741: Diskussion:Erich Ehrlinger, In: Wikipedia -

http://de.wikipedia.org/wiki/Diskussion:Erich_Ehrlinger: Wikipedia, 2013)

26 Dass 3. Sg. Kongruenz bei $d u$ als zweitem Konjunkt kaum auftritt, liegt darin begründet, dass entsprechende Belege mit oder (d.h., dem Kontext, in dem 3. Sg. Kongruenz in der Regel überhaupt möglich ist) recht selten sind (insgesamt nur 13 Fälle in der Stichprobe). 
gruenz offenbar von Eigenschaften der Wortstellung abhängig ist, soll nun abschließend untersucht werden, ob analog zum Bairischen (vgl. (8) oben) die Verfügbarkeit dieser Kongruenzoption ebenfalls von der Stellung des Subjekts relativ zum Verb beeinflusst wird.

Sollten die Befunde aus dem Bairischen auf das Kongruenzverhalten von Subjektreihungen im Standarddeutschen übertragbar sein, wäre Kongruenz mit dem ersten Subjektteil vor allem in Abfolgen zu erwarten, in denen der Kongruenzträger dem Subjekt vorangeht. Erste Beobachtungen zum Standarddeutschen scheinen dies zu bestätigen, vgl. die Beispiele (15), (19), (22) und (30) oben. In Tabelle 6.11 werden die einschlägigen Befunde aus WDD13 zusammengefasst:

Tab. 6.11: Einfluss der Wortstellung (Subjekt-Verb-Inversion) auf die 2. Sg. Kongruenz am Verb (du und 3. Sg./3. Pl.).

\begin{tabular}{llr}
\hline & 2. Sg. & \multicolumn{1}{l}{ Andere } \\
\hline Inversion & $56(74,7 \%)$ & $53(11,3 \%)$ \\
keine Inversion & $19(25,3 \%)$ & $416(88,7 \%)$ \\
\hline
\end{tabular}

Tabelle 6.11 zeigt, dass annähernd $75 \%$ aller verbalen 2. Sg.-Formen in Inversionskontexten auftreten. Andere Kongruenzoptionen sind hier mit $11 \%$ aller entsprechenden Belege relativ selten; allerdings ist dies auch dem Umstand geschuldet, dass Inversionsbelege insgesamt nur lediglich etwa ein Viertel aller Belege ausmachen. Ein auffälliger Kontrast ergibt sich auch, wenn man die Zeilen in Tabelle 6.11 betrachtet. So zeigt sich, dass in Inversionskontexten 2. Sg.-Formen etwa die Hälfte aller Belege ausmachen (56 von 109, 51,4\%), während sie bei Nicht-Inversion sehr selten sind (19 von 435, ca. 4\%). Ein Chi-Quadrat Test ergibt, dass die Verteilung der Kongruenzformen abhängig vom Faktor \pm Inversion hochsignifikant von einer gleichmäßigen Verteilung abweicht $\left(p<2.2 \mathrm{e}^{-16}\right)$. Die Effektstärke liegt dabei in einem Bereich, der gängigerweise als groß eingeschätzt wird (Phi/V-Koeffizient =0,54). Ein ähnliches Bild ergibt sich bei Reihungen mit oder:

Tab. 6.12: Einfluss der Wortstellung (Subjekt-Verb-Inversion) auf die 2. Sg. Kongruenz am Verb (du oder 3. Sg./3. Pl.).

\begin{tabular}{llc}
\hline & 2. Sg. & Andere \\
\hline Inversion & $68(73,9 \%)$ & $17(7,9 \%)$ \\
keine Inversion & $24(26,1 \%)$ & $199(92,1 \%)$ \\
\hline
\end{tabular}


Wieder ist das Ergebnis hochsignifikant $\left(p<2.2 \mathrm{e}^{-16}\right)$. Die Effektstärke liegt mit einem Phi/V-Koeffizienten von 0,67 sogar noch über dem Wert, der für den Einfluss des Faktors \pm Inversion für Reihungen mit und ermittelt wurde. Dies ist aber vor allem darauf zurückzuführen, dass andere Kongruenzoptionen bei Inversion deutlich seltener sind als bei Reihungen mit und und nur noch ca. 20\% (17 von 85) aller Inversionsbelege ausmachen - mit anderen Worten, 2. Sg. ist bei Subjekt-Verb-Inversion mit ca. $80 \%$ das absolut dominante Muster bei Reihungen mit oder (68 von 85 Belegen). Es ist denkbar, dass die Möglichkeit von FCA dadurch gestärkt wird, dass bei Reihungen mit oder Kongruenz mit nur einem Subjektteil generell eine Option darstellt (bzw., dass oder eine Konstruktion mit elliptischer Struktur leichter zulässt, s. o.). Die Verteilung von 2. Sg.-Formen bleibt dabei über die verschiedenen syntaktischen Kontexte recht konstant - wiederum entfallen ca. 3/4 aller einschlägigen Belege auf Inversionskontexte.

Es stellt sich allerdings sowohl bei Reihungen mit und als auch bei Reihungen mit oder die Frage, welchen Status Ausnahmen wie (31) haben, in denen 2. Sg.-Formen in Nicht-Inversionskontexten auftreten.

(31) a. Was du und deinesgleichen als „seriös“ betrachtest, kann ich mir lebhaft vorstellen.

(WDD13/A28.65153: Diskussion:Antifa/Archiv/2006, In: Wikipedia http://de.wikipedia.org/wiki/Diskussion:Antifa/Archiv/2006: Wikipedia, 2013)

b. Kleiner Hinwies [sic], bevor du und dein dicker Kumpel ninabot euch hier ein weiteres mal zum Affen machst:

(WDD13/G45.43881: Diskussion:Gargoyles - Auf den Schwingen der Gerechtigkeit/Archiv/1, In: Wikipedia - http://de.wikipedia.org/wiki/ Diskussion:Gargoyles_-Auf_den_Schwingen_der_Gerechtigkeit/ Archiv/1: Wikipedia, 2013)

Es handelt sich um 43 Belege, die immerhin ca. ein Viertel aller Belege mit 2. Sg. ausmachen. Auffällig ist, dass dabei stets eine Reihung der Art $d u$ und $X$ vorliegt und dass das finite Verb in 42 von 43 Beispielen in satzfinaler Position steht. Dieser Befund unterstreicht, dass auch Fälle von 2. Sg.-Kongruenz in Nebensätzen nicht auf eine elliptische Struktur zurückgeführt werden können, da diese bei Verbendstellung die umgekehrte Reihung ( $x$ und $d u$ ) erfordern würde (vgl. Fn. 18). Der einzige Beleg mit SV-Stellung im Hauptsatz ist in (32) wiedergegeben. Dieser ist allerdings so fehlerbehaftet, dass es fraglich ist, ob es sich um einen kompetenten Muttersprachler des Deutschen handelt. Wir 
können somit festhalten, dass - ganz analog zum Bairischen - FCA auch im Standarddeutschen in subjektinitialen Sätzen ausgeschlossen zu sein scheint.

(32) [...] ein „,von oben“ zentralistisch geführtes Projekt, sondern du und jeder andere kannst ganz demokratisch Information, die er für relevant hälst, ergänzen und korrigieren.

(WDD13/K11.67551: Diskussion:Klingenthal, In: Wikipedia http://de.wikipedia.org/wiki/Diskussion:Klingenthal: Wikipedia, 2013)

Zumindest meiner grammatischen Intuition zufolge sind allerdings auch Fälle wie (31a) äußerst marginal. Beispiele wie (31b), in denen sich das Reflexivum auf das Gesamtsubjekt bezieht (Resolution: 2. Pl.), während das Verb in der 2. Sg. steht, scheinen mir zumindest bei Verbendstellung gänzlich inakzeptabel zu sein. Dies weist vielleicht darauf hin, dass die Möglichkeit von 2. Sg.-Kongruenz mit einem nicht-invertierten komplexen Subjekt der Art du und/oder 3. Sg./ 3. Pl. einen Fall von Intersprechervariation darstellt, d. h. es gibt nur bestimmte Sprecher des Deutschen, deren Grammatik diese Option zulässt. ${ }^{27}$ Eine Überprüfung der Autorenschaft der entsprechenden Wikipediabelege hat ergeben, dass die 43 problematischen Belege von 40 verschiedenen Wikipedianutzern verfasst wurden. Unterstützung für die Hypothese, dass hier ein Fall von Intersprechervariation vorliegt, lässt sich aber möglicherweise aus der Beobachtung ableiten, dass vier der Belege von dem gleichen Nutzer stammen.

Die wesentlichen empirischen Befunde lassen sich wie folgt zusammenfassen:

- Bei Subjektreihungen aus 2. Sg. und 3. Sg. (verknüpft durch und) treten im Wesentlichen drei Arten von Kongruenzvariation auf: 2. Sg., 2. Pl., 3. Pl., wobei 3. Pl. das häufigste Muster ist.

- Bei Subjektreihungen, die durch oder verknüpft sind, tritt zusätzlich noch die Variante 3. Sg. auf (d. h., das Verb kann auch nur mit einem der beiden Subjektteile kongruieren).

- Bei Subjektreihungen aus 2. Sg. und 3. Pl. steht das Verb in der überwiegenden Zahl aller Belege (und: ca. $70 \%$, oder: ca. $80 \%$ ) in der 3. Pl.

- 3. Pl. ist insgesamt die dominante Kongruenzvariante. Die Hypothese (Corbett 1983), dass 3. Pl. vor allem im Zusammenhang mit Verben auftritt, bei denen 2. Pl. und 3. Sg. zusammenfallen, findet in der vorliegenden Stichprobe keine Unterstützung.

27 Alternativ könnte man auch vermuten, dass es sich bei Belegen wie (31b) schlicht um Performanzfehler handelt. 
- Die Kongruenzoption 2. Sg. (d.h., FCA) ist insgesamt ähnlich häufig wie 2. Pl., tritt aber vor allem in Kontexten mit Subjekt-Verb-Inversion auf, wobei $d u$ das erste Konjunkt sein muss.

\section{Diskussion und Analyse}

Die Untersuchung des Kongruenzverhaltens von Subjektreihungen hat gezeigt, wie im Rahmen einer Korpusstudie grammatische Faktoren ermittelt werden können, die die Verteilung von sprachlichen Varianten steuern. Für die grammatische Beschreibung und theoretische Analyse der Befunde stellt sich allerdings die Frage, welchen Status die im Korpus beobachtete linguistische Variation hat. Um zwischen Intersprechervariation und Intrasprechervariation klar unterscheiden zu können, müssten wir in der Lage sein, die linguistischen Belege einzelnen Sprechern zuordnen zu können; insbesondere bei sehr großen Korpora, bei denen sich die Datengrundlage aus den sprachlichen Produkten einer Vielzahl verschiedener Sprecher zusammensetzt, ist dies aber nicht immer ohne Weiteres möglich. Selbst wenn die Autorenschaft zweifelsfrei ermittelt werden kann, kann es bei eher seltenen Phänomenen wie der hier betrachteten Kongruenzvariation vorkommen, dass nicht alle Varianten, über die ein einzelner Sprecher verfügt, im Korpus enthalten sind, sodass der eindeutige Nachweis von Intrasprechervariation mitunter schwierig ist. Man kann aber auch nicht pauschal unterstellen, dass alle Sprecher alle Varianten besitzen bzw. akzeptieren. Ansonsten besteht die Gefahr, dass man ein System linguistischer Variation beschreibt, das zwar im Korpus auftritt, aber nicht der Grammatik einzelner Sprecher entspricht und somit möglicherweise kein reales oder $\mathrm{u}$. U. sogar ein unmögliches grammatisches System darstellt. ${ }^{28} \mathrm{Im}$ Extremfall könnte es auch sein, dass das Sprachverhalten und die Grammatik einzelner Sprecher invariant sind und jede Variante einer bestimmten Gruppe von Sprechern zuzuordnen ist. In diesem Fall würde eine Instanz von Intersprechervariation vorliegen, die dann aber nicht von einer einheitlichen grammatischen Beschreibung erfasst werden kann.

Vor dem Hintergrund dieser Überlegungen muss also zunächst geklärt werden, welcher Typ von Variation vorliegt, bevor wir uns der Frage zuwenden können, wie die Kongruenzalternation im Zusammenhang mit Subjektreihungen

28 Zudem kann man nicht vollständig ausschließen, dass es sich bei einigen (insbes. seltenen) Varianten um Performanzfehler handelt, die z. B. beim Redigieren durch das nachträgliche Einführen eines Subjekts (oder Subjektteils) zustande kommen. 
aus 2. Sg. und 3. Sg. analysiert werden kann. Dabei möchte ich die Arbeitshypothese verfolgen, dass den meisten Sprechern zumindest die Kongruenzvarianten 2. Pl. und 3. Pl. zur Verfügung stehen; zusätzlich hat eine Gruppe von Sprechern noch die Möglichkeit, dass in Inversionskontexten das Verb lediglich mit dem ersten Konjunkt - 2. Sg. in den hier untersuchten Reihungen kongruiert. Hinweise darauf, dass es sich bei dem vorliegenden Phänomen tatsächlich um eine Form von Intrasprechervariation handelt (dass also tatsächlich alle Varianten in einem System auftreten können und von einer Grammatik erzeugt werden können), liefert zum einen die Beobachtung, dass viele Sprecher unsicher sind, welche Verbform in Kombination mit Subjektreihungen aus 2. Sg. und 3. Sg. zu wählen ist und dass eine Tendenz dazu besteht, entsprechende Konstruktionen zu vermeiden (worauf auch bereits die Dudengrammatik hinweist). Darüber hinaus konnten in einigen Fällen verschiedene Varianten einem einzigen Sprecher zugeordnet werden. ${ }^{29}$ In dem Ausschnitt in (33) treten in Kombination mit dem komplexen Subjekt du und Hansele sowohl 2. Pl. als auch 3. Pl. am Verb auf; der Beleg entstammt einem Wikipedia-Diskussionsbeitrag, der von einem einzigen Wikipedianutzer verfasst wurde.

(33) Ah, du und Hansele meint eure These, also eure unbelegte Interpretation der letzten Wahlen. [...] Schon sehr symptomatisch, dass man immer wieder mit nur ein „paar“ Benutzern hier in Konflikt gerät (,und dazu gehören in erster Linie du und Hansele“).

(https://de.wikipedia.org/wiki/Diskussion:Evangelikalismus/Archiv/2006)

In dem Beispielpaar (34) finden sich die Kongruenzvarianten 3. Pl. und 2. Sg., die über den Wikipedia-Benutzernamen ebenfalls einem einzigen Sprecher zugeordnet werden können.

29 Allerdings ist die Zuordnung von Diskussionsbeitrag und Nutzername recht mühsam, da entsprechende Angaben nicht im Korpus verzeichnet sind; die Zuordnung ist nur über eine manuelle Suche auf den entsprechenden Wikipedia-Diskussionsseiten möglich. Aber auch dann kann die Identität des Sprechers nicht immer zweifelsfrei ermittelt werden. Manche Nutzer bleiben anonym; zudem ist es theoretisch möglich, dass ein Nutzername von mehreren Nutzern verwendet wird. Es scheint also, als könne die zentrale Unterscheidung zwischen Intersprecher- und Intrasprechervariation in einem rein korpusbasierten Ansatz nicht immer mit der notwendigen Trennschärfe gemacht werden; auf jeden Fall erscheint es ratsam, die korpusbasierte Untersuchung von linguistischer Variation durch andere empirische Methoden wie Introspektion und psycholinguistische Experimente zu komplementieren. 
(34) a. über die vor allem Du und SFF verfügen (https://de.wikipedia.org/wiki/Diskussion:Männerrechtsbewegung/ Archiv/007)

b. Wenn du oder Dinah es zitiert hättest, würde die Sache ganz anders aussehen, wenn aber dessen Autor auch den Inhalt der Onlineenzyklopädie bestimmen will, hat man ggf. nur einen neuen „Fall Ohff“, wo eine Auffassung so verbreitet wird, daß diese als einzig Wahre gilt. (WDD13/F12.84537: Diskussion:Fürst-Pückler-Eis, In: Wikipedia http://de.wikipedia.org/wiki/Diskussion:Fürst-Pückler-Eis: Wikipedia, 2013)

Die Beispiele in (33) und (34) zeigen, dass die Kongruenzvarianten offenbar von ein- und demselben grammatischen System erzeugt werden können. Die Tatsache, dass die Textsorte recht homogen ist, lässt zudem den Schluss zu, dass die Varianten auch nicht mit verschiedenen Registern assoziiert sind, die Teil der grammatischen Kompetenz ein- und desselben Sprechers sind. Vielmehr scheint ein genuiner Fall von grammatischer (Intrasprecher-)Variation vorzuliegen. Dieser Befund sagt allerdings zunächst nichts darüber aus, ob die Wahl zwischen den einzelnen Varianten möglicherweise von semantischpragmatischen Faktoren gesteuert wird. So wäre es unter Umständen denkbar, dass z.B. bei Subjektreihungen mit oder die Unterscheidung zwischen einer ein- bzw. ausschließenden Interpretation Einfluss auf das Kongruenzverhalten hat. $^{30}$ Ein weiterer möglicher pragmatischer Faktor könnte darin bestehen, dass 2. Pl. dann häufiger genutzt wird, wenn es sich im weitesten Sinne um direktive Sprechakte handelt, während Assertiva eher zu 3. Pl. tendieren. Es hat sich allerdings gezeigt, dass eine trennscharfe Annotation entsprechender z. T. subtiler Unterscheidungen im Rahmen einer Korpusstudie äußerst schwierig ist. Eine erste Durchsicht hat auch keine klaren Hinweise darauf geliefert, dass entsprechende Faktoren eine Rolle spielen. Sollte sich der Eindruck bestätigen, dass die verschiedenen Kongruenzvarianten keiner eindeutigen Diskursfunktion zugeordnet werden können, erscheint es naheliegend zu vermuten, dass es sich bei dem hier untersuchten Phänomen um einen Fall von freier Variation handelt, wie er für Sprachwandelprozesse charakteristisch ist. Dieser Schluss wird auch durch die Beobachtung gestützt, dass sich bei der Distribution der Varianten - mit Ausnahme von FCA/2. Sg. - keine eindeutigen Regulari-

30 Wie bereits in Fn. 17 erwähnt, lässt sich aber zumindest auf den ersten Blick kein klarer Zusammenhang zwischen den möglichen Lesarten von oder und der Art der Verbkongruenz (Singular vs. Plural) erkennen. 
täten erkennen lassen. Vielmehr liegen in der Regel lediglich Tendenzen vor, die aber keinen kategoriellen Charakter haben.

Abschließend möchte ich kurz skizzieren, wie diese Form von Variation theoretisch modelliert werden kann. Die Grundidee ist dabei, dass die Varianten das Resultat unterschiedlicher Reparaturoptionen sind, die zur Anwendung kommen, um einen Merkmalskonflikt in der syntaktischen Struktur aufzulösen. Ich werde mich dabei in der Folge auf das Kongruenzverhalten von Subjektreihungen konzentrieren, die durch und verknüpft sind. Bei oder besteht über die unten beschriebenen Möglichkeiten hinaus die Option, dass das Verb nur mit einem der beiden Subjektteile kongruiert, was zu einer größeren Bandbreite an Varianten führt (möglicherweise auch als Resultat einer elliptischen Konstruktion, die aber zumindest im vorliegenden Datensatz bevorzugt mit 3. Sg.-Kongruenz einhergeht).

Ausgangspunkt der Analyse ist die Annahme, dass die syntaktische Koordination von zwei Subjektteilen mit unterschiedlichem Merkmalsgehalt - im vorliegenden Fall 2. Sg. und 3. Sg. - zur Präsenz zweier Merkmalsmengen am komplexen Subjekt führen, die als geordnetes Paar wie in (35) dargestellt werden können: ${ }^{31}$

$$
<[\mathrm{M} 1],[\mathrm{M} 2]>
$$

Als Resultat einer Kongruenzregel wird dieses geordnete Paar anschließend an das finite Verb kopiert. Das Problem ist nun, dass diese komplexe Merkmalsstruktur nicht ohne Weiteres mit einem Kongruenzflexiv assoziiert werden kann, da letztere in der Regel nicht für geordnete Paare von Merkmalsmengen, sondern für einfache Merkmalsmengen wie [+2, -Plural] spezifiziert sind. Darüber hinaus enthält die Merkmalsstruktur in (35) im vorliegenden Fall konfligierende Merkmalswerte (2. Person vs. 3. Person). Um (35) auf eine Kongruenzendung abbilden zu können, muss die problematische Merkmalsstruktur, die aus der syntaktischen Ableitung resultiert, offenbar zunächst durch postsyntaktische Reparaturen weiter aufbereitet werden. ${ }^{32}$ Dies kann im Wesentlichen auf zwei verschiedene Weisen erreicht werden: Zum einen können die beiden

31 Die Entscheidung, die Merkmalsmengen als geordnetes Paar darzustellen, ist dadurch motiviert, dass gängigen Annahmen zufolge Koodinationsstrukturen einen asymmetrischen Charakter aufweisen, wobei das erste Konjunkt hierarchisch höher ist als das zweite (Munn 1993). Die Merkmalsnotation reflektiert somit die syntaktische Struktur.

32 Vgl. Brandt \& Fuß (2012) und Brandt (2016) für weitere Phänomene, die als das Resultat von Reparaturprozessen gedeutet werden können, die an den Schnittstellen zwischen der Syntax und den post-syntaktischen Komponenten der Grammatik operieren. 
Merkmalsmengen durch die Anwendung von Resolutionsregeln zu einer einzigen Merkmalsmenge reduziert werden (vgl. Corbett 1983; Sag et al. 1985; Dalrymple \& Kaplan 1997). Eine alternative Reparaturstrategie besteht darin, eine der beiden Merkmalsmengen zu tilgen. Letztere Option führt zu Kongruenz mit nur einem der beiden Subjektteile (wie z. B. bei FCA). Aus dieser Perspektive kann linguistische Variation dadurch zustande kommen, dass ein Sprecher Zugriff auf mehrere dieser Reparaturoperationen hat. In der Folge möchte ich diesen Vorschlag etwas weiter ausarbeiten, wobei ich mich zunächst den entsprechenden Resolutionsregeln zuwende.

Nach gängigen Annahmen bestehen Resolutionsregeln aus zwei Komponenten: Zunächst werden die beiden Merkmalsmengen vereinigt (Unifikation; vgl. Sag et al. 1985; Dalrymple \& Kaplan 1997). Anschließend werden die resultierenden Merkmalskonflikte aufgelöst. Vor diesem Hintergrund können die Resolutionsregeln für das Deutsche wie folgt beschrieben werden. Die Regel aus der Dudengrammatik (§1602) lässt sich wie in (36) ausdrücken:

(36) Resolutionsregel (Deutsch) I:

a. Vereinigung der Merkmalsmengen: $\langle[\mathrm{A}],[\mathrm{B}]>\rightarrow[\mathrm{A}] \cup[\mathrm{B}]$

b. $[+1],[+2] \rightarrow[+1]$

c. $[\alpha \mathrm{PL}],[\alpha /-\alpha \mathrm{PL}] \rightarrow[+\mathrm{PL}]$

Die Regel in (36b) legt fest, dass sich bei Koordination von erster und zweiter Person der Merkmalswert für erste Person durchsetzt. Wenn man ferner davon ausgeht, dass der „dritten“ Person kein separater Wert für das Merkmal [Person] entspricht, sondern dass die dritte Person vielmehr das Resultat der Absenz von Personmerkmalen darstellt (vgl. z. B. Benveniste 1966), ergibt sich ohne Weiteres, dass erste und zweite Person Vorrang vor der dritten Person haben: Da es kein Merkmal für dritte Person gibt, kann es auch keinen Merkmalskonflikt geben; erste und zweite Person bestimmen somit automatisch den entsprechenden Wert der reparierten Merkmalsmenge. (36c) bedient sich des Mittels der Alpha-Notation (Ersetzung unterschiedlicher Werte für ein Merkmal durch eine Variable, Chomsky \& Halle 1968), um zu gewährleisten, dass der resultierende Wert für das Merkmal Numerus (d.h., [ \pm Plural]) stets [+Plural] ist, unabhängig davon, welche Numeruswerte ([+Plural oder [-Plural]) in der Subjektreihung vorliegen. Die Oberflächeneffekte von (36) sind in (37) illustriert:

(37) a. dass du und der Peter willkommen seid $\Rightarrow<[+2,-\mathrm{PL}],[-\mathrm{PL}]\rangle \rightarrow[+2,+\mathrm{PL}]$ 
b. dass ich und du willkommen sind $\Rightarrow\langle[+1,-\mathrm{PL}],[+2,-\mathrm{PL}]\rangle \rightarrow[+1,+\mathrm{PL}]$

c. dass der Hans und der Peter willkommen sind $\Rightarrow<[-\mathrm{PL}],[-\mathrm{PL}]\rangle \rightarrow[+\mathrm{PL}]$

etc.

Wie kann nun aber erfasst werden, dass bei Subjektreihungen aus 2. Sg. und 3. Sg. die Kongruenzvariante 3. Pl. bevorzugt wird? Eine Möglichkeit, dies zu beschreiben, besteht in der Annahme, dass sich im Gegenwartsdeutschen eine vereinfachte Variante der Resolutionsregel in (36) ausbreitet, die sich dadurch auszeichnet, dass die Regel zur Personresolution ersetzt wird durch eine Tilgungsregel, die einen Konflikt zwischen unterschiedlichen Werten für das Merkmal Person dadurch beseitigt, dass das Merkmal Person vollständig gelöscht wird. Als Resultat davon kann im Zusammenhang mit Subjektreihungen (verknüpft durch und) nur noch die Pluralendung - $(e) n$ verwendet werden, die keine Personmerkmale signalisiert bzw. für das Merkmal [Person] unterspezifiziert ist. Die entsprechende Resolutionsregel ist in (38) wiedergegeben; die Effekte sind in (39) illustriert. ${ }^{33}$

(38) Resolutionsregel (Deutsch) II:

a. Vereinigung der Merkmalsmengen: $\langle[\mathrm{A}],[\mathrm{B}]\rangle \rightarrow[\mathrm{A}] \cup[\mathrm{B}]$

b. $[$ Person $] \rightarrow \emptyset$

c. $[\alpha \mathrm{PL}],[\alpha /-\alpha \mathrm{PL}] \rightarrow[+\mathrm{PL}]$

(39) a. dass du und der Peter willkommen sind $\Rightarrow\langle[+2,-\mathrm{PL}],[-\mathrm{PL}]\rangle \rightarrow[+\mathrm{PL}]$

b. dass ich und du willkommen sind $\Rightarrow<[+1,-\mathrm{PL}],[+2,-\mathrm{PL}]>\rightarrow[+\mathrm{PL}]$

c. dass der Hans und der Peter willkommen sind $\Rightarrow<[-\mathrm{PL}],[-\mathrm{PL}]\rangle \rightarrow[+\mathrm{PL}]$

etc.

33 Allerdings lässt diese Analyse zunächst offen, wie die Beobachtung erfasst werden kann, dass die Präsenz von 3. Pl. in der Subjektreihung die Wahl der Kongruenzendung zugunsten von 3. Pl. beeinflusst. 
Eine noch radikalere Reparatur kann darin bestehen, eine der beiden Merkmalsmengen vollständig zu tilgen. Hierbei haben wir beobachtet, dass diese Option bei Subjektreihungen aus 2. Sg. und 3. Sg. (bei Verknüpfungen mit und) in der Regel zu 2. Sg. Kongruenz am Verb führt, wobei das komplexe Subjekt dem Verb (in der Regel) nachfolgen muss. Diese Generalisierung kann durch die Tilgungsregel in (40) erfasst werden, die besagt, dass am Verb das zweite Glied des (geordneten) Merkmalsmengen-Paars getilgt wird, wenn sich in der gleichen (minimalen) prosodischen Domäne eine Merkmalsmenge befindet, die identisch ist mit dem ersten Glied des geordneten Paars:

$(40)<[\mathrm{M} 1],[\mathrm{M} 2]>\rightarrow[\mathrm{M} 1] /(\ldots[\mathrm{M} 1])$

Diese Analyse besagt, dass Kongruenz mit $d u$ alleine (also FCA) nur möglich sein sollte, wenn $d u$ direkt rechtsadjazent zum finiten Verb steht. Sie prognostiziert ferner, dass die Wahl zwischen FCA und Resolution von prosodischen Eigenschaften abhängig sein sollte. Normalerweise bildet das komplexe Subjekt eine eigenständige prosodische Domäne; alternativ kann auch das initiale Pronomen mit dem Verb in der linken Satzklammer eine prosodische Phrase bilden. Ist dies der Fall, kann die Regel in (40) greifen. Allerdings lassen sich auf der Basis schriftlicher Korpora kaum belastbare Aussagen zur prosodischen Phrasierung machen (vgl. aber Bayer 2013 für einschlägige Beobachtungen zu FCA im Bairischen). Interessanterweise liegt aber in den wenigen Fällen, in denen der zweite Subjektteil durch ein Komma abgetrennt ist, FCA vor, vgl. (41). Unter der Voraussetzung, dass die Kommasetzung in diesen Beispielen eine prosodische Grenze reflektiert, können diese Befunde als Unterstützung für die Existenz einer Regel wie (40) betrachtet werden.

(41) a. Im Übrigen schmeißt du, und auch andere, ständig die Begriffe Arzneimittel und Medizinprodukt durcheinander.

(WDD13/E68.73266: Diskussion:Elektrische Zigarette/Archiv/2, In: Wikipedia - http://de.wikipedia.org/wiki/Diskussion:Elektrische_ Zigarette/Archiv/2: Wikipedia, 2013)

b. Ausserdem musst du, und auch der ein oder andere hier mal verstehen, dass 01 nicht nur das Gerät an sich ist, sondern das da wesentlich mehr dahinter steckt ...

(WDD13/Y33.82965: Diskussion:Yamaha MT-01, In: Wikipedia http://de.wikipedia.org/wiki/Diskussion:Yamaha_MT-01: Wikipedia, 2013) 
Darüber hinaus kann (40) auch erklären, warum offenbar kein FCA möglich ist, wenn $d u$ das zweite Konjunkt darstellt: Folgt das Subjekt dem Verb nach, ist $d u \mathrm{zu}$ weit entfernt, um eine prosodische Phrase mit dem Verb bilden $\mathrm{zu}$ können. Befindet sich das komplexe Subjekt im Vorfeld, stellt es stets eine prosodische Einheit dar (d.h., eine Phrasierung des zweiten Subjektteils mit dem finiten Verb ist stets ausgeschlossen). Die Tatsache, dass einige Sprecher FCA auch produzieren, obwohl die Adjazenzbedingung nicht erfüllt ist (also in Verbendstrukturen), kann möglicherweise darauf zurückgeführt werden, dass sie die Regel in (40) soweit generalisiert haben, dass die Tilgung einer Teilmenge von Merkmalen auch dann möglich ist, wenn keine Adjazenz von Verb und Pronomen vorliegt.

\section{Fazit}

Die Bestimmung der Verbkongruenz im Zusammenhang mit Subjektreihungen aus zweiter und dritter Person stellt viele Sprecher des Deutschen vor Probleme. In diesem Beitrag habe ich versucht zu zeigen, wie im Rahmen eines korpusbasierten Ansatzes grammatische Faktoren identifiziert werden können, die die Wahl der entsprechenden morphologischen Varianten in der Grammatik individueller Sprecher beeinflussen. Eine Untersuchung von Wikipedia-Diskussionen, in denen entsprechende Reihungen besonders häufig auftreten, hat gezeigt, dass das tatsächliche Sprachverhalten z. T. stark von den Beschreibungen in einschlägigen Grammatiken des Deutschen abweicht, die prognostizieren, dass das Verb in der 2. Pl. stehen sollte. Tatsächlich handelt es sich dabei aber nur um eine von (mindestens) drei Optionen, die in den meisten Kontexten seltener ist als die Alternativen. Neben der dominanten Variante 3. Pl. ist hier vor allem die Möglichkeit zu nennen, dass das Verb in Inversionskontexten mit nur einem Subjektteil kongruiert (First Conjunct Agreement, FCA), was bei den hier untersuchten Reihungen zur Markierung von 2. Sg. am Verb führt. Die Korpusstudie hat zudem gezeigt, dass bisherige Erklärungsversuche, die die Möglichkeit von 3. Pl. Kongruenz mit dem Vorliegen eines Formenzusammenfalls von 3. Sg. und 2. Pl. in Verbindung bringen (Corbett 1983), die Fakten nicht zutreffend erfassen - 3. Pl. ist auch dann die häufigste Variante, wenn 3. Sg. und 2. Pl. nicht zusammenfallen. Die bei der Formenwahl auftretende Sprecherunsicherheit wurde als Hinweis darauf verstanden, dass die Varianten (mit wenigen Ausnahmen) nicht mit verschiedenen Gruppen von Sprechern assoziiert sind, sondern einen genuinen Fall von Intrasprechervariation darstellen, bei dem die konkurrierenden Kongruenzformen von ein und derselben Grammatik erzeugt werden können. Diese Hypothese wird auch durch die Beobachtung 
gestützt, dass im Rahmen der Korpusuntersuchung verschiedene Varianten in der Sprachproduktion einzelner Sprecher/Schreiber nachgewiesen werden konnten. Abschließend habe ich eine Analyse skizziert, die linguistische Variation auf grammatische Ursachen zurückführt. Aufbauend auf der Annahme, dass bei Subjektreihungen aus zweiter und dritter Person eine syntaktische Struktur vorliegt, die nicht ohne Weiteres von der morphologischen Komponente der Grammatik interpretiert werden kann, kommt linguistische Variation dadurch zustande, dass der Sprecher Zugriff auf eine Auswahl an Reparaturmechanismen hat - Resolutionsregeln oder Tilgung einer der beiden konfligierenden Merkmalsmengen - die jeweils zu unterschiedlichen Kongruenzvarianten führen (2. Sg., 2. Pl. oder 3. Pl.). Aus dieser Sicht ist die beobachtete Variation das Resultat unterschiedlicher Möglichkeiten, abstrakte linguistische Strukturen $\mathrm{zu}$ externalisieren, also mithilfe von einfachen und komplexen sprachlichen Zeichen und Zeichenketten (Silben/Wörter/Sätze/Äußerungen) lautlich oder gestisch zu realisieren. Eine Quelle für die Variabilität von Sprache liegt somit an der Schnittstelle zwischen abstrakten syntaktischen Strukturen und den Mechanismen, die diese Strukturen in phonologische Repräsentationen überführen.

\section{Literatur}

Bayer, Josef (1984): COMP in Bavarian syntax. The Linguistic Review 3, 209-274.

Bayer, Josef (2013): Klitisierung, Reanalyse und die Lizensierung von Nullformen:

zwei Beispiele aus dem Bairischen. In Werner Abraham \& Elisabeth Leiss (Hrsg.),

Dialektologie in neuem Gewand (Linguistische Berichte, Sonderheft 19), 29-45.

Hamburg: Helmut Buske.

Benveniste, Emile (1966): Problèmes de linguistique générale. Paris: Editions Gallimard.

Brandt, Patrick (2016): Discomposition redressed. Hidden change, modality, and comparison in German. Habilitationsschrift, Universität Mannheim.

Brandt, Patrick \& Eric Fuß (Hrsg.) (2012): Repairs. The added value of being wrong. Berlin: Mouton de Gruyter.

Brandt, Patrick \& Eric Fuß (2014): Most questionable pronouns: Variation between dasvs. was-relatives in German. Linguistische Berichte 239, 297-329.

Chambers, J. K. (2002): Studying language variation: An informal epistemology. In J. K. Chambers, Peter Trudgill \& Natalie Schilling (Hrsg.), The Handbook of language variation and change, 3-14. Oxford: Blackwell.

Chomsky, Noam (1980): Rules and representations. New York: Columbia University Press.

Chomsky, Noam \& Morris Halle (1968): The sound pattern of English. Cambridge, MA: MIT Press.

Corbett, Greville (1983): Resolution rules: Agreement in person, number, and gender. In G. Gazdar, E. Klein \& G. Pullum (Hrsg.), Order, concord and constituency, 175-206. Dordrecht: Foris. 
Corbett, Greville (2000): Number. Cambridge: Cambridge University Press.

Corbett, Greville (2006): Agreement. Cambridge: Cambridge University Press.

Dalrymple, Mary \& Ronald Kaplan (1997): A set-based approach to feature resolution. In Miriam Butt \& Tracy Halloway King (Hrsg.), Proceedings of the LFG 97 Conference. Stanford: CSLI. https://web.stanford.edu/group/cslipublications/cslipublications/LFG/ LFG2-1997/lfg97dalrymplekaplan.pdf

Eichinger, Ludwig. M. (2005): Standardnorm, Sprachkultur und die Veränderung der normativen Erwartungen. In Ludwig M. Eichinger \& Werner Kallmeyer (Hrsg.), Standardvariation. Wie viel Variation verträgt die deutsche Sprache?, 363-381. Berlin: De Gruyter.

Fuß, Eric (2008): Multiple agreement and the representation of inflection in the C-domain. Linguistische Berichte 213, 78-108.

Fuß, Eric (2014): Complementizer agreement (in Bavarian): Feature inheritance or feature insertion? In Günther Grewendorf \& Helmut Weiß (Hrsg.), Bavarian syntax. Contributions to the theory of syntax, 51-82. Amsterdam: Benjamins.

Fuß, Eric, Marek Konopka \& Angelika Wöllstein (2017): Perspektiven auf grammatische Variation. In Marek Konopka \& Angelika Wöllstein (Hrsg.), Grammatische Variation. Empirische Zugänge und theoretische Modellierung. Jahrbuch des Instituts für Deutsche Sprache 2016, 229-254. Berlin/Boston: De Gruyter.

Hennig, Mathilde (Hrsg.) (2016): Das Wörterbuch der sprachlichen Zweifelsfälle. Richtiges und gutes Deutsch. 8. vollst. überarb. Aufl. Mannheim/Leipzig/Wien/Zürich: Dudenverlag.

Henry, Alison (2002): Variation and syntactic theory. In J. K. Chambers, Peter Trudgill \& Natalie Schilling (Hrsg.), The handbook of language variation and change, 267-282. Oxford: Blackwell.

Hopper, Paul (1987): Emergent grammar. Berkeley Linguistics Society 13, 139-57.

Kiparsky, Paul (1979): Pāṇini as a variationist. Cambridge, MA: MIT Press.

Konopka, Marek \& Eric Fuß( 2016): Genitiv im Korpus. Untersuchungen zur starken Flexion des Nomens im Deutschen. Tübingen: Narr.

Koppen, Marjo van (2005): One probe - two goals: Aspects of agreement in Dutch dialects. Utrecht: LOT.

Koppen, Marjo van (2006): One probe, multiple goals: The case of first conjunct agreement. In Marjo van Koppen, Pepijn Hendriks, Frank Landsbergen, Mika Poss \& Jenneke van der Wal (Hrsg.), Special Issue of Leiden Papers in Linguistics 3 (2), 25-52. Leiden.

Koppen, Marjo van (2012): The distribution of phi-features in pronouns. Natural Language and Linguistic Theory 30, 135-177.

Kroch, Anthony (1989): Reflexes of grammar in patterns of language change. Journal of Language Variation and Change 1 (3), 199-244.

Kroch, Anthony (1994): Morphosyntactic variation. In K. Beals, J. Denton, B. Knippen, L. Melnar, H. Suzuki, \& E. Zeinfeld (Hrsg.), Proceedings of the Thirtieth Annual Meeting of the Chicago Linguistics Society. Vol. II, 180-201. Chicago: Chicago Linguistics Society.

Munn, Alan (1993): Topics in the syntax and semantics of coordinate structures. Doctoral dissertation, University of Maryland.

Munn, Alan (1999): First conjunct agreement: Against a clausal analysis. Linguistic Inquiry 30 (4), 643-668.

Polenz, Peter von (2000): Deutsche Sprachgeschichte vom Spätmittelalter bis zur Gegenwart, Band I. Berlin/New York: De Gruyter. 
Rizzi, Luigi (1997): The fine structure of the left periphery. In L. Haegeman (Hrsg.), Elements of grammar: Handbook in generative syntax, 281-337. Dordrecht: Kluwer.

Sag, Ivan, Ronald Kaplan, Lauri Karttunen, Martin Kay, Carl Pollard, Stuart Shieber \& Annie Zaenen (1985): Unification and grammatical theory. In Mary Dalrymple et al. (Hrsg.), Proceedings of the fifth West Coast Conference on Formal Linguistics, 238-254. Stanford: SLA, CSLI.

Schäfer, Roland (2017): Parallel- und Wechselflexion in obliken Adjektivreihen Eine empirische Vervollständigung. Vortrag, IDS Mannheim, 31.5. 2017.

Weiß, Helmut (2005): Inflected complementizers in Continental West Germanic Dialects. Zeitschrift für Dialektologie und Linguistik 72, 148-166.

Wiese, Heike (2012): Kiezdeutsch. Ein neuer Dialekt entsteht. München: C. H. Beck. Wöllstein, Angelika (Hrsg.) (2016): Duden: Die Grammatik. 9. Auflage. Mannheim/Leipzig/ Wien/Zürich: Dudenverlag. 
Stefan Th. Gries

\title{
7 Zur Identifikation von Mehrwortausdrücken: ein Algorithmus, seine Validierung und weiterführende Überlegungen
}

\begin{abstract}
In diesem Artikel diskutiere ich Aspekte der datengetriebenen Identifikation von Mehrwortausdrücken aus Korpora sowie einen einfachen neuen Ansatz und vier kurze Validierungsstudien; letztere verwenden verschiedene Methoden, kontrastieren den hier vorgestellten Ansatz mit einem konkurrierenden und überprüfen seine Prädiktivität für Spracherwerbsdaten. Abschließend bespreche ich einige Desiderata für zukünftige Forschung zu Mehrwortausdrücken, die bisher oft vernachlässigt wurden.
\end{abstract}

Keywords: Assoziation, Dispersion, Häufigkeit, Korpuslinguistik, Mehrwortausdrücke

\section{Einleitung}

Der vorliegende Beitrag beschäftigt sich mit der datengetriebenen Identifizierung von Kollokationen, Phraseologismen und Mehrwortausdrücken, ein viel untersuchtes Phänomen in der Korpuslinguistik. Zur Identifizierung solcher Ausdrücke sind in der Vergangenheit eine Reihe von Verfahren und verschiedenen statistischen Maßen vorgeschlagen worden, die erwartbarerweise auch unterschiedliche Ergebnisse lieferten. Die verwendeten Verfahren und Maße lassen sich „extern“ evaluieren, indem man etwa ihre Vorhersagen mit dem vergleicht, was Sprecher als Mehrwortausdrücke wahrnehmen. Auf diese Weise erhält man dann eine Einschätzung darüber, inwieweit ein gegebenes statistisches Maß (oder Verfahren) tatsächlich das Konzept („Mehrwortausdruck“) abbildet, für das man es einsetzt. In diesem Beitrag werden einige der vorgeschlagenen Verfahren und statistischen Maße problematisiert und Alternativen umrissen.

Stefan Th. Gries, University of California, Santa Barbara; Department of Linguistics, Santa Barbara, CA 93106-3100; U.S.A., E-Mail: stgries@linguistics.ucsb.edu

Ә Open Access. (C) 2018 Stefan Th. Gries, publiziert von De Gruyter. (C) BY Dieses Werk ist lizenziert unter der Creative Commons Attribution 4.0 Lizenz. 
Korpuslinguistik ist eine inhärent quantitative/statistische Disziplin, da Korpora streng genommen nur Häufigkeiten von Elementen als Datengrundlage anbieten, so dass alle anderen für Linguisten interessanten Begrifflichkeiten über (Kookkurrenz-)Häufigkeiten operationalisiert werden müssen. Der vorliegende Beitrag möchte einen Bereich fokussieren, der ein viel untersuchtes Problem in der Korpuslinguistik und für den Status statistischer Maße ist; behandelt wird die themenspezifische Relevanz statistischer Maße anhand der Identifikation von Kollokationen, Phraseologismen und Mehrwortausdrücken.

Mehrwortausdrücke sind aus mehreren Gründen relevant: Im Bereich der maschinellen Sprachverarbeitung sind Mehrwortausdrücke zum Beispiel wichtig, da sie oft keine kompositionelle Bedeutung haben und daher maschinelles Sprachverständnis oder Übersetzen erschweren können; im Bereich der angewandten Linguistik sind sie zum Beispiel relevant, da es für Sprachlerner oft schwierig ist, muttersprachliche Selektion (native-like selection; Pawley \& Syder 1983) zu erwerben; im Bereich der theoretischen Linguistik sind sie unter anderem relevant, da sie die Frage problematisieren, wie viel das mentale Lexikon enthält und wie viel erst ad hoc/bei Bedarf zusammengesetzt wird.

Kollokationsforschung hat sich für Jahrzehnte damit befasst, sogenannte Assoziationsmaße zu entwickeln, die die Kookkurrenz von zwei Einheiten quantifizieren; mittlerweile sind Dutzende solcher Maße vorgeschlagen worden, die üblicherweise verwendet werden, um potenzielle Kollokationen/Phraseologismen einer Rangfolge nach sortieren zu können, die ,Kollokationsstatus' widerspiegelt; intuitiv würde man wahrscheinlich erwarten, dass solch ein Ausdruck wie hermetisch verriegelt hohe Assoziationsmaße erzielt, während ein Ausdruck wie der Tisch nur vergleichsweise geringe Werte erzielt. Die allermeisten Assoziationsmaße basieren dabei auf einer Kreuztabelle von Häufigkeiten, wie sie in Tabelle 7.1 dargestellt ist. Die Kookkurrenzhäufigkeit der beiden Ausdrücke $x$ und $y$, deren Assoziation quantifiziert werden soll, ist in Zelle $a$, die Zellen $a+b$ sowie $a+c$ enthalten die Häufigkeiten der beiden Ausdrücke $x$ und $y$ im Korpus; die Summe $a+b+c+d$ ist die Korpusgröße.

Tab. 7.1: Schematische Kookkurenztabelle für die meisten korpuslinguistischen Assoziationsmaße.

\begin{tabular}{llll}
\hline & Ausdruck $y$ & andere Ausdrücke & Summe \\
\hline Ausdruck $x$ & $\begin{array}{l}\text { beobachtet: } a \\
\text { erwartet: }(a+b) \times(a+c) / n\end{array}$ & beobachtet: $b$ & $a+b$ \\
& erwartet: $(a+b) \times(b+d) / n$ & \\
\hline andere & beobachtet: $c$ & beobachtet: $d$ & $c+d$ \\
Ausdrücke & erwartet: $(c+d) \times(a+c) / n$ & erwartet: $(c+d) \times(b+d) / n$ & \\
\hline Summe & $a+c$ & $b+d$ & $a+b+c+d=n$ \\
\hline
\end{tabular}


Wie in Tabelle 7.1 dargestellt, werden oft diejenigen Häufigkeiten berechnet, die man gemäß der Nullhypothese erwarten würde, wenn Ausdruck $x$ und $y$ nicht kollokieren; danach werden üblicherweise Assoziationsmaße berechnet, von denen pointwise mutual information MI, $t$ und loglikelihood $G$ (siehe Dunning 1993) wohl die am weitesten verbreiteten Maße sind, dargestellt in (1).
(1) a. $M I=\log _{2} a_{\text {beobachtet }} / a_{\text {erwartet }}$
b. $t=\frac{a_{\text {beobachtet }}-a_{\text {erwartet }}}{\sqrt{a_{\text {erwartet }}}}$
c. $G=2 \times \sum_{a}^{d}$ beobachtet $\times \log \frac{\text { beobachtet }}{\text { erwartet }}$

Diese und andere Maße wurden in vielerlei Studien auf Zweiwort-Kollokationen (und teilweise auf die Kookkurrenz von Wörtern und/in Konstruktionen im konstruktionsgrammatischen Sinn) angewendet und auf Brauchbarkeit und Vorhersagekraft getestet, vgl. Krenn (2000), Evert \& Krenn (2001), Krenn \& Evert (2001), Evert (2004) und Pecina (2010) sowie Wiechmann (2008) für Wörter und Konstruktionen.

Im Vergleich zur Erforschung von Zweiwort-Kollokationen ist die korpuslinguistische bottom-up Identifikation von Mehrwortausdrücken weniger weit fortgeschritten. Während es inzwischen viele Studien in der angewandten Linguistik gibt, die sich mit N-Grammen - Sequenzen von $n$ Wörtern - beschäftigen, vgl. (2), so sind diese oft problematisch in der Hinsicht, dass die relevanten $N$ Gramme nicht komplett datengetrieben erhoben wurden, indem solche Studien beispielsweise nur bestimmte $N$-Gramm-Längen behandeln; viele Studien $\mathrm{zu}$ lexical bundles beispielsweise betrachten 4-Gramme. Dies ist problematisch, weil natürlich viele $N$-Gramme keine 4-Gramme sind: $N$ kann viele verschiedene Werte annehmen, mindestens die in (2) exemplifizierten (und diese berücksichtigen noch nicht die Möglichkeit von $N$-Grammen mit Lücken).
(2) a. because of
$n=2$
b. in spite of
$n=3$
c. on the other hand
$n=4$
d. be that as it may
$n=5$
e. the fact of the matter is
$n=6$

Das bedeutet: Selbst nur die Extraktion von Mehrwortausdrücken ist deutlich komplizierter als die von ,einfachen Kollokationen', weil zur Identifikation eines geeigneten Assoziationsmaßes die Fragen hinzukommen, (i) ob/wie das 
entsprechende Assoziationsmaß auf mehr als zwei Ausdrücke ausgedehnt werden kann, wie man entscheidet, was die optimale Länge eines angenommenen Mehrwortausdrucks ist und (ii) wie die vielen verschiedenen Kombinierungsmöglichkeiten von mehr als zwei Ausdrücken analysiert werden sollen. Ist in spite of bspw. eine Kombination von

(3) a. in und spite und of?

b. in spite und of

c. in und spite of

d. in of und spite?

Ein zentrales Ziel für die Erforschung von $\mathrm{N}$-Grammen ist daher, einen Algorithmus zu entwickeln, der $N$-gramme aus Korpora extrahieren kann, ohne dass die Länge der zu erhaltenden $N$-gramme von vorneherein festgelegt wird. In diesem Artikel verfolge ich zwei mit diesem Ziel verwandte Absichten: Nach einem kurzen und sehr selektiven Überblick über einige frühere Studien (Abschnitt 2) diskutiere ich zuerst einen neuen Algorithmus zur Identifikation von Mehrwortausdrücken und zeige, wie dieser in einigen kleinen Validierungsstudien abgeschnitten hat (Abschnitt 3). Zum anderen diskutiere ich zentrale problematische Eigenschaften von Algorithmen zur Identifikation von Mehrwortausdrücken und schlage Erweiterungen vor, die die Identifikation von Mehrwortausdrücken verbessern sollte (Abschnitt 4).

\section{Eine Auswahl bisheriger Studien}

Viele der bisherigen Studien zur Extraktion von Mehrwortausdrücken versuchen, sich der Komplexität des Problems über einen iterativen Ansatz anzunähern und auf diese Weise die Bestandteile langer N-Gramme zu bestimmen. In einer der ersten und einflussreichsten Studien schlug Jelinek (1990) vor:

1. einen Minimalwert $m$ für das $z u$ verwendende Assoziationsmaß zu definieren;

2. alle 2-Gramme eines Korpus zu extrahieren;

3. alle Kollokationen $\mathrm{zu}$ finden, deren Assoziationsmaß einen Wert von $m$ überschreitet und diese zu neuen Einheiten im Korpus zu kombinieren;

4. diesen Prozess iterativ $\mathrm{zu}$ wiederholen. 
Während sich spätere Arbeiten natürlich in vielen Details von Jelineks Ansatz unterscheiden, so haben viele doch eine ähnliche iterative Struktur. Eine hier wichtige Unterscheidung von verschiedenen Ansätzen ist, ob sie auf Tokenhäufigkeiten basieren oder auf Tokenhäufigkeiten und Assoziation; ich gebe in den folgenden Abschnitten einige Beispiele.

\subsection{Frequenz}

Wie Jelinek (1990) stellt auch die Studie von Kita et al. (1994) einen iterativen Ansatz vor. Dieser Ansatz basiert darauf zu berechnen, wieviel Aufwand das Prozessieren aller Formen eines Korpus benötigt. Im einfachsten Fall würde jedes Wort einen Aufwand in der Höhe von 1 verursachen, aber wenn $N$-Gramme mit $n>1$ als einfache Wörter rekonzeptualisiert werden, sinkt der Prozessieraufwand in Abhängigkeit zu der Häufigkeit des N-Gramms. Die Berechnung des dort vorgeschlagenen Kosten-Kriteriums (Kita's cost criterion) involviert nur die Multiplikation des Häufigkeitsunterschieds zweier $\mathrm{N}$-Gramme (von denen eines ein Ein-Wort kürzeres Subgramm des anderen ist) mit der Länge des kürzeren $N$-Gramms), wobei die Assoziationsstärke zwischen den Einheiten des $N$-Gramms keine Rolle spielt.

Die Studie von O’Donnell (2011) ist sehr ähnlich (zitiert Jelinek oder Kita et al. allerdings nicht). O’Donnell's Adjusted Frequency List (AFL) extrahiert erst alle $N$-Gramme bis zu einer bestimmten Länge aus einem Korpus und rechnet dann die Häufigkeiten von längeren $N$-Grammen sukzessive und rekursiv aus den Häufigkeiten von kürzeren $N$-Grammen heraus. Auch hier wird die Assoziationsstärke der Elemente, die in einen neuen Mehrwortausdruck eingehen, nicht berücksichtigt.

\section{2 (Frequenz und) Assoziation}

Die wahrscheinlich erste einflussreichere Diskussion zur Identifikation von Mehrwortausdrücken, die nicht nur Frequenz sondern auch Assoziationsstärke berücksichtigt, ist die bereits erwähnte Studie von Jelinek (1990), in der die Identifikation auf der Basis von $M I$ durchgeführt wird. Andere Studien gehen teilweise ähnlich vor, verwenden jedoch andere Assoziationsmaße. Ein Beispiel soll hier zunächst genügen, Wible et al. (2006) sowie Wible \& Tsao (2011). Diese Studien generieren keine Liste aller Mehrwortausdrücke in einem Korpus, sondern alle Mehrwortausdrücke mit einem bestimmten Suchwort (und verwenden außerdem Wortklasseninformation); das statistische Assoziationsmaß, das dort verwendet wird, ist ebenfalls $M I$. 
Ein sehr interessanter Ansatz, der leider viel zu wenig aufgegriffen wurde, ist Daudaravičius \& Murcinkevičienès (2004) Maß der lexical gravity G. Dieser Ansatz ist die Generalisierung eines ohnehin schon sehr innovativen Assoziationsmaßes, welches sich von allen anderen dahingehend unterscheidet, dass es nicht nur auf den Tokenfrequenzen a bis $d$ in Tabelle 7.1 basiert, sondern stattdessen auch die Anzahl an Typen berücksichtigt, die in den Zellen b und c repräsentiert sind. Lexical gravity $G$ wird wie folgt berechnet:

(4) Lexical Gravity $G=\log \frac{a \times t y p e \text { freq.von } a+b}{a+b}+\log \frac{a \times \text { type freq.von } a+c}{a+c}$

Wie aus (4) ersichtlich ist, nimmt dieses Maß zu, wenn $a$ und/oder die Typenfrequenz der Kollokate von Ausdruck $x$ und/oder die Typenfrequenz der Kollokate von Ausdruck y zunehmen; das Maß nimmt ab, wenn die Tokenfrequenzen $a+b$ und/oder $a+c$ zunehmen. Obwohl dieses Maß an sich schon als Assoziationsmaß sehr interessant ist, weil es als einziges nicht nur Token- sondern auch Typenfrequenz berücksichtigt, bauen Daudaravičius \& Murcinkevičienė es außerdem für $\mathrm{N}$-Gramme aus (von ihnen Kollokationsketten, collocational chains, genannt), indem sie vorschlagen, solche Ausdrücke als $N$-Gramm zu betrachten, die aus mehreren 2-Grammen bestehen, von denen jedes einen $G$-Wert hat, der > 5,5 ist, wobei die Motivation für genau diesen Grenzwert von 5,5 nicht offensichtlich ist.

Gries \& Mukherjee (2010) verwenden eine Variante von Daudaravičius \& Murcinkevičienės Maß, in der sie (i) für jedes $N$-Gramm den Durchschnitt der $G$-Werte berechnen und dann (ii) für alle $N$-Gramme, deren durchschnittlicher G-Wert > 5,5 ist, testen, ob es ein längeres $N$-Gramm gibt, dass das erstere enthält, aber einen höheren Durchschnitt von $G$-Werten hat. Wenn kein solches längeres $N$-Gramm existiert, wird das kürzere behalten, ansonsten das längere. Mit diesem ,Reinigungsprozess“ versuchen Gries \& Mukherjee, der Tatsache Rechnung zu tragen, dass auch $N$-Gramme mit hohen $G$-Werten theoretisch auch noch in weiteren längeren $\mathrm{N}$-Grammen mit ebenfalls hinreichender Assoziationsstärke vorkommen können.

Dieser Abschnitt konnte nur einen kurzen Überblick geben, sollte aber deutlich machen, dass (i) bisherige Arbeiten oft Jelinek (1990) folgen und iterativ vorgehen und dass (ii) existierende Arbeiten sich $u$. a. dahingehend unterscheiden, ob sie ausschließlich auf Häufigkeiten basieren oder auch Assoziationsstärke berücksichtigen; für weitere Studien vgl. auch das Sonderheft von Language Resources and Evaluation zu Mehrwortausdrücken sowie u. a. Nagao \& Mori (1994), Ikehara, Shirai, \& Uchino (1996), Shimohata, Sugio, \& Nagata (1997), da Silva et al. (1999), da Silva \& Lopez (1999). 


\section{MERGE und seine Validierung}

Der Ansatz, der hier vorgestellt wird, wird als MERGE bezeichnet (kurz für Multiword Expressions from the Recursive Grouping of Elements), siehe Wahl (2015), Wahl \& Gries (eingereicht a, b). MERGE basiert ebenfalls auf der Logik von Jelinek (1990) und beinhaltet folgende Schritte:

1. Ermittle die Häufigkeiten aller 1-Gramme eines Korpus (und damit die Korpusgröße);

2. ermittle alle 2-Gramme eines Korpus und ihre Häufigkeiten (diese können Lücken enthalten, in der Anwendung hier beschränke ich mich auf $N$-Gramme ohne Lücken);

3. berechne für jedes 2-Gramm das Assoziationsmaß loglikelihood ratio $G$, welches wie ein Signifikanztest sowohl auf Frequenz als auch auf Assoziation anspricht;

4. ermittle das 2-Gramm mit dem höchsten $G$-Wert und verwandle es in ein neues 1-Gramm;

5. aktualisiere das Korpus, indem alle Vorkommnisse des neu vereinigten 1-Gramms ein neues Wort werden, und aktualisiere alle Häufigkeitslisten entsprechend, und beginne erneut bei Schritt 1.

Das bedeutet, MERGE könnte z. B. in spite of dadurch finden, indem in einem Iterationsschritt in und spite zu in spite vereinigt werden (weil dies den höchsten $G$-Wert erzielt hat) und in einem späteren Schritt werden in spite und of zu in spite of verbunden. Dieser Prozess kann entweder enden, weil eine benutzerdefinierte Zahl von Iterationen durchlaufen wurde (beispielsweise 10.000) oder, weil ein benutzerdefinierter Schwellenwert von $G$ nicht mehr überschritten wird.

Genau wie andere Studien auch muss auch dieser methodologische Vorschlag sich die Frage gefallen lassen, ob er effektiv ist und valide Ergebnisse generiert. Um diese Frage zu beantworten, haben Wahl \& Gries (eingereicht a, b) mehrere kleine Validierungsstudien durchgeführt.

\subsection{Validierung 1: Mehrwortausdruckbewertungen linguistisch untrainierter Leser}

Wir wendeten MERGE auf die Kombination zweier Korpora an, das Santa Barbara Corpus of Spoken American English (ca. 250K Wörter, Du Bois, Chafe, Meyer \& Thompson 2000; Du Bois, Chafe, Meyer, Thompson \& Martey 2003; Du Bois \& Englebretson 2004; 2005) und den gesprochenen Teil des ICE- 
Canada (ca. 450K Wörter, Newman \& Columbus 2010). Die beiden Korpora wurden vorbereitet, indem Tags, Transkriptionszeichen und andere Annotationen entfernt wurden; danach wendeten wir MERGE auf das gemeinsame Korpus an (für 20.000 Iterationen). Dann wurden die ersten 40 und die letzten 40 Mehrwortausdrücke ermittelt und in unterschiedlichen (randomisierten) Auswertungsfragebogen zusammen mit einer Anleitung und weiteren konkreten Beispielen an 20 Studierende der University of California, Santa Barbara verteilt. Sie wurden gebeten, auf einer 7-stufigen Skala anzugeben, wie sehr jeder Mehrwortausdruck ein „common resuable chunk“ sei.

Die Wertungen wurden mit einem gemischten Regressionsmodell ausgewertet, das als zentralen Prädiktor die binäre Variable Rang (die ersten 40 vs. die letzten 40) enthielt, die Länge der Mehrwortausdrücke als Kontrollvariable und die größtmögliche Zufallsfaktorenstruktur (also Achsenabschnitte und Steigungen für Stimuli und Versuchspersonen). Das finale Modell ergab eine hoch signifikante Korrelation $\left(p<10^{-15}, R^{2}\right.$ marginal $\left.=0,64\right)$ und zeigte, dass die von MERGE als gute/frühe Mehrwortausdrücke generierten Ausdrücke in der Tat signifikant bessere Wertungen erhielten als schlechtere/spätere Mehrwortausdrücke, selbst wenn man Länge und Zufallsfaktoren kontrolliert; der Unterschied betrug 3,87 Punkte auf der 7-Punkt-Skala.

\subsection{Validierung 2: MERGE vs. AFL im Vergleich linguistisch untrainierter Leser}

In einem zweiten Schritt verglichen wir die Resultate von MERGE mit denen der AFL. Beide Algorithmen wurden auf dieselben Korpora wie oben angewendet; MERGE durchlief dieses Mal 1.000 Iterationen, für den AFL-Algorithmus setzten wir den erlaubten Minimalfrequenzwert auf 5 und nahmen nach dem Durchlauf des Algorithmus die häufigsten 1.000 Mehrwortausdrücke. Von beiden Listen ermittelten wir dann diejenigen Mehrwortausdrücke, die nur von einem der beiden Algorithmen gefunden wurden und zogen eine stratifizierte Zufallsstichprobe von je 180 Mehrwortausdrücken, die dann in randomisierten Fragebögen aufbereitet 20 weiteren Studierenden zur Beurteilung vorgelegt wurden.

Auch diese Beurteilungen wurden mit einem gemischten Regressionsmodell ausgewertet, das als zentralen Prädiktor die binäre Variable Quelle (MERGE vs. AFL) enthielt, die Länge der Mehrwortausdrücke als Kontrollvariable und die größtmöglichen Zufallsfaktorenstruktur (wie oben). Das finale Modell ergab eine signifikante, aber sehr schwache Korrelation ( $p_{\text {einseitig }}=0,022$, $R_{\text {marginal }}^{2}=0,02$ ). Sie zeigte allerdings, dass die von MERGE als Mehrwortausdrücke generierten Ausdrücke in der Tat signifikant bessere Wertungen erhiel- 
ten als die von der AFL generierten Mehrwortausdrücke, selbst dann, wenn man Länge und Zufallsfaktoren kontrolliert; der Unterschied betrug 0,6 Punkte auf der 7-Punkt-Skala. Dieses Ergebnis ist trotz der Schwäche des Effekts insofern interessant, als dass es zeigt, dass der Ansatz der Frequenz und Assoziation vereint - wie MERGE - besser abschneidet als AFL, der nur Frequenzinformationen verwendet.

\subsection{Validierung 3: MERGE vs. AFL für getaggte Mehrwortausdrücke im BNC}

In einer dritten Fallstudie wendeten wir MERGE und AFL auf alle gesprochenen Daten im BNC an, um zu testen, welcher der beiden Ansätze bessere Ergebnisse in der Auffindung derjenigen Mehrwortausdrücke zeigt, die die BNC-Kompilierer als Mehrwortausdrücke getaggt haben (mit dem ... Tag). Wir wendeten MERGE und AFL an und identifizierten die ersten 10.000 Mehrwortausdrücke beider Algorithmen. Dann prüften wir, wie viele der 388 Mehrwortausdrücke jeder der beiden Algorithmen identifiziert hatte: die AFL fand 93, MERGE 112, ein Unterschied von 20,4\%, der gemäß einseitiger Binomialtests (für beide Kontrastrichtungen) signifikant ist (beide $p_{\text {einseitig }}<0.018$ ); das bedeutet, die Performanz von MERGE ist signifikant besser als die der AFL.

\subsection{Validierung 4: Mehrwortausdrücke im Erstspracherwerb}

Die letzte Fallstudie untersucht, ob die Ergebnisse von MERGE damit korrelieren, welche $N$-Gramme Kinder anhand des elterlichen Inputs erwerben. Für diese Fallstudie verwendeten wir die Lara- und Thomas-Korpora (Rowland \& Fletcher 2006, Lieven et al. 2009). Beide Korpora wurden in ein Trainings- und ein Testkorpus im Verhältnis 2:1 aufgeteilt, danach wurden Kinder- und Erwachsenendaten getrennt. MERGE wurde angewendet auf die Erwachsenendaten des Trainingskorpus (bis der maximale $G$-Wert nicht mehr positiv war). Dann extrahierten wir alle 2-5-Gramme aus dem Testkorpus der beiden Kinder, löschten diejenigen $\mathrm{N}$-Gramme, die auch schon im Trainingskorpus der Kinder vorkamen und teilten die verbleibenden $N$-Gramme auf in (i) die, die die Erwachsenen verwendeten und die Kinder später auch und (ii) die, die die Erwachsenen verwendeten, die Kinder später jedoch nicht; dies wurde getan, damit wir testen konnten, ob die Mehrwortausdrücke, die die Kinder erworben hatten, sich durch höhere MERGE-Werte auszeichneten als die, die sie nicht erworben hatten. Wir gruppierten entsprechend die Mehrwortausdrücke in $G$-Wert-Klassen und berechneten für jede Klasse den Prozentsatz der Mehr- 
wortausdrücke, die das Kind gelernt hatte; dies war die abhängige Variable in unserer statistischen Analyse (wurzeltransformiert, um Verteilungsannahmen der Regression nicht zu verletzen). Diese Analyse war ein Regressionsmodell, in dem Kind (Lara vs. Thomas), G-Wert-Gruppe und die Länge der Mehrwortausdrücke sowie ihre potenziellen Interaktionen Prädiktoren waren.

Das finale Regressionsmodel ist hoch signifikant $\left(p<10^{-15}\right)$ und erklärt die Variabilität der Mengen an erworbenen $N$-Grammen sehr gut (adj. $R^{2}=0.7801$ ). Das Modell zeigt, dass die Dreifachinteraktion aller Prädiktoren signifikant ist, aber eine entsprechende Visualisierung zeigt, dass der wichtigste Befund der ist, dass Mehrwortausdrücke aus höheren $G$-Wert-Gruppen der Erwachsenen in der Tat die sind, die deutlich mehr von beiden Kindern erworben wurden.

\subsection{Zusammenfassung}

In vier Fallstudien konnte gezeigt werden, dass MERGE alles in allem vielversprechende Resultate erzielt:

1. Mehrwortausdrücke, die MERGE hoch bewertet, werden (i) von linguistisch untrainierten Lesern eher als solche wahrgenommen, (ii) besser bewertet als Mehrwortausdrücke, die MERGE niedrig bewertet oder die von dem AFLAlgorithmus erzeugt werden und (iii) werden häufiger erworben;

2. MERGE erzielt bessere Ergebnisse als AFL im Erkennen von Mehrwortausdrücken im BNC.

Diese Resultate zeigen meines Erachtens auch, welche verschiedenen Möglichkeiten zur Validierung Korpuslinguisten zur Verfügung stehen können, obwohl es bei der Identifikation von Mehrwortausdrücken noch Raum zur Verbesserung gibt. Der folgende letzte Abschnitt diskutiert hierzu einige Ideen.

\section{Vorschläge zur Verbesserung}

Ein meiner Ansicht nach besserer Ansatz zur Identifikation von Mehrwortausdrücken erfordert einige Änderungen, die ich in verschiedenen anderen Kontexten separat besprochen habe, die aber bisher wenig oder gar nicht in Verbindung mit der Identifikation von Mehrwortausdrücken gebracht wurden. Diese Änderungen haben mit Faktoren zu tun, auf denen nahezu jegliche korpuslinguistische Analyse oder datengetriebene Identifikation von ZweiwortKollokationen oder längeren Mehrwortausdrücken basieren: die Wahl des 
Assoziationsmaßes, die Rolle von Typenfrequenz und die Rolle von Dispersion (und andere, die ich hier nicht besprechen werde).

\subsection{Die Wahl des Assoziationsmaßes}

Zwei Faktoren sind relevant in Bezug auf die Wahl eines Assoziationsmaßes: erstens der Faktor Direktionalität. Nahezu alle Assoziationsmaße, die bisher verwendet wurden - für Kollokationsforschung, aber auch für die Identifikation von Mehrwortausdrücken - sind bidirektional, was bedeutet, dass sie die Assoziation zweier Einheiten $\mathrm{x}$ und y zueinander ausdrücken, aber nicht differenzieren, ob diese Assoziation wirklich bidirektional ist oder nur jeweils monodirektional vorliegt. Beispiele für 2-Gramme (im gesprochenen Teil des BNCs), die eine maximale bidirektionale Assoziation (gemessen durch $\Delta P$-Werte, i. e. die Differenzen von $p\left(\right.$ Wort $_{1} \mid$ Wort $\left._{2}\right)-p\left(\right.$ Wort $_{1} \mid \neg$ Wort $\left.\left._{2}\right)\right)$ haben, sind die folgenden Ausdrücke (aus bisher nicht veröffentlichten Daten von Gries 2013): papier mâché, fromage frais, spina bifida, tittle tattle, avant garde, higgledy piggledy, hocus pocus, lingua franca, modus operandi, rigor mortis, und terra firma; alle diese Ausdrücke bestehen aus zwei Teilen, die in diesem Korpus nur miteinander und niemals alleine sonst wo vorkommen: die Assoziation ist perfekt sowie bidirektional. Beispiele, bei denen das erste Wort das zweite sehr stark vorhersagt, aber nicht vice versa, sind: volte face, het up, insomuch as, insofar as, habeas corpus, upside down, etc. In den folgenden Beispielen hingegen verläuft die Assoziation vom zweiten zum ersten Ausdruck hin: de rigueur, al fresco, agent provocateur, super duper, ad hominem, ad infinitum, etc. Die allermeisten Assoziationsmaße unterscheiden Ausdrücke mit einer hohen Assoziation in einer Richtung nicht von Ausdrücken mit einer hohen Assoziation in der anderen Richtung. Es ist jedoch durchaus möglich, dass eine derartige Unterscheidung einen Einfluss auf die Resultate eines Mehrwortalgorithmus haben könnte, unabhängig davon, wie man die Information der beiden Assoziationsrichtungen bzw. $\Delta P$-Werte letztendlich mathematisch verwendet.

Der zweite relevante Faktor hat damit zu tun, wie ,konzeptuell rein' das verwendete Assoziationsmaß ist. Werte wie $t$ oder $G$ reflektieren nicht nur Assoziation, sondern auch Häufigkeit: Wenn man für Tabelle 7.1 aus Abschnitt 1 $t$ und $G$ errechnet, dann erhält man andere Resultate, als wenn man alle Werte in Tabelle 1 mit 10 multipliziert und wieder $t$ und $G$ errechnet; dies gilt nicht für Werte wie die Odds Ratio oder $\Delta P$. Es mag Situationen geben, in denen diese Verknüpfung von Assoziation und Häufigkeit in einen einzigen Wert vorteilhaft ist (siehe Gries 2012 im collostructional analysis-Kontext), aber dies muss von Fall zu Fall und auf der Basis von empirischen Daten entschieden werden; zur Identifikation von Mehrwortausdrücken gibt es m. E. noch keine 
systematischen Studien, die vergleichen, welche Art von Assoziationsmaß für einen bestimmten Zweck geeigneter ist. Mit anderen Worten, zukünftige Forschung sollten versuchen zu ermitteln,

(i) ob Assoziationsmaße, die Assoziation und Häufigkeit widerspiegeln, nützlicher sind als solche, die nur Assoziation widerspiegeln,

(ii) ob, falls wir Häufigkeit und Assoziation in einem einzigen Maß unterbringen, diese beiden Dimensionen gleich gewichtet werden sollten oder nicht oder

(iii) ob wir einen Ansatz zu Assoziationsmaßen brauchen, der beide Dimensionen verwendet, aber eventuell getrennt als 2-Tupel.

\subsection{Typenfrequenz}

Ein weiterer wichtiger Punkt wurde mit dem Assoziationsmaß lexical gravity G von Daudaravičius \& Marcinkevičienè (2004) bereits angesprochen. Die Frage ist, ob nicht nur die Tokenfrequenz von Kollokaten eine Rolle bei der Berechnung einer Assoziationsstärke spielen soll sondern auch die Typenfrequenz. Angesichts der Tatsache, dass Typenfrequenz generell ein wichtiges korpuslinguistisches Konzept ist, mit Auswirkungen auf Produktivität, Lernbarkeit und Lerngeschwindigkeit, Sprachwandel etc., erscheint es sinnvoll zu versuchen, Typenfrequenz bei der Einschätzung von Assoziation miteinzubeziehen. Die Tatsache, dass nach in spite fast ausschließlich of folgt, ist doch unzweifelhaft relevant und wahrscheinlich über Typenfrequenz direkter und intuitiver abgebildet als durch Tokenfrequenz.

\subsection{Dispersion im Korpus}

Ein vorerst letzter potenziell wichtiger Punkt ist Dispersion, die Verteilung von Mehrwortausdrücken im Korpus, die beispielsweise über das Maß DP (Deviation of Proportions; siehe Gries 2008; Biber et al. 2016) leicht gemessen werden kann. Auch wenn korpuslinguistische Arbeiten die Wichtigkeit von Dispersion noch immer unterschätzen, so gibt es doch inzwischen genügend Evidenz, dass die (Un-)Gleichmäßigkeit der Verteilung von Ausdrücken in einem Korpus einen massiven Einfluss auf alle möglichen quantitativen Resultate haben kann. Beispielsweise haben die Ausdrücke enormous und staining die gleiche Häufigkeit im Brown Korpus (37) und die gleiche Länge in Buchstaben. Sie sind dennoch unterschiedlich, wenn man betrachtet, was Häufigkeit oft operationalisieren soll, nämlich die Wahrscheinlichkeit, ein Wort zu sehen/hören: enormous kommt in 36 der 500 Teile des Brown Korpus vor, staining dagegen 
in 1, aber dort $37 \mathrm{Mal}$. Es ist daher wenig verwunderlich, dass Dispersion sich in einigen Studien als ein psycholinguistisch gesehen informativeres Konstrukt oder Messinstrument als Häufigkeit herausstellte (vgl. Adelman, Brown \& Quesada 2006; Gries 2010; Baayen 2010). Angesichts solcher Befunde ist es sinnvoll, sich zu fragen, ob Dispersion für die Identifikation von Mehrwortausdrücken ebenfalls eine Rolle spielt oder spielen sollte, und falls diese Frage bejaht wird, ist die nächste Frage, wie das geschehen sollte: wieder durch eine Kombination in einen einzigen Wert oder als ein weiterer Beitrag zu einem Assoziations-Tupel?

\subsection{Fazit}

Auch wenn viele Fragen noch ungeklärt bleiben, so sollte klar geworden sein, dass wir bei der korpuslinguistischen und datengetriebenen Erforschung von Mehrwortausdrücken eigentlich noch ziemlich am Anfang stehen: Wenige Ansätze gehen über die Verwendung von Tokenhäufigkeit und größtenteils bidirektionale Assoziationsmaße, die Tokenhäufigkeit enthalten, hinaus. Anderweitig oder sogar generell wichtige Begriffe wie direktionale Assoziation, Typenfrequenz und Dispersion sind für Mehrwortausdrücke so gut wie gar nicht behandelt worden. Auch wenn noch unklar ist, wie diese Begriffe im Detail zusammengeführt werden können - als ein Maß, als Tupel? - so ist doch klar, dass diese Arten von Fragen behandelt werden müssen. In Abwesenheit besserer Lösungen könnte zumindest versucht werden, alle obigen Dimensionen entweder als Tupel oder zunächst über Schwellenwerte zu verwenden. Wenn die Dispersion zu gering wird, wird ein Mehrwortausdruck nicht akzeptiert, eine Logik, die ja schon oft für Häufigkeit angewendet wird, indem Mindesthäufigkeiten postuliert werden. Wie auch immer, es bleibt viel zu tun und ich hoffe, dass dieser Artikel, wenn auch vielleicht keine Lösungen, so doch Lösungswege für zukünftige Studien skizzieren konnte.

\section{Literatur}

Adelman, James S., Gordon D. A. Brown \& Jose F. Quesada (2006): Contextual diversity, not word frequency, determines word-naming and lexical decision times. Psychological Science 19 (9), 814-823.

Baayen, R. Harald (2010): Demythologizing the word frequency effect: A discriminative learning perspective. The Mental Lexicon 5 (3), 436-461.

Biber, Douglas, Randi Reppen, Erin Schnur \& Romy Graham (2016): On the (non)utility of Juilland's D to measure lexical dispersion in large corpora. International Journal of Corpus Linguistics 21 (4), 439-464. 
Daudaravičius, Vidas \& Rūta Marcinkevičienè (2004): Gravity counts for the boundaries of collocations. International Journal of Corpus Linguistics 9 (2), 321-348.

Du Bois, John W., Wallace L. Chafe, Charles Meyers \& Sandra A. Thompson (2000): Santa Barbara corpus of spoken American English, part 1. Philadelphia: Linguistic Data Consortium.

Du Bois, John W., Wallace L. Chafe, Charles Meyers, Sandra A. Thompson \& Nii Martey (2003): Santa Barbara corpus of spoken American English, part 2. Philadelphia: Linguistic Data Consortium.

Du Bois, John W. \& Robert Englebretson (2004): Santa Barbara corpus of spoken American English, part 3. Philadelphia: Linguistic Data Consortium.

Du Bois, John W. \& Robert Englebretson (2005): Santa Barbara corpus of spoken American English, part 4. Philadelphia: Linguistic Data Consortium.

Dunning, Ted (1993): Accurate methods for the statistics of surprise and coincidence. Computational Linguistics 19 (1), 61-74.

Evert, Stefan 2004. The statistics of word cooccurrences: Word pairs and collocations. PhD dissertation, IMS, University of Stuttgart.

Evert, Stefan \& Brigitte Krenn (2001): Methods for the qualitative evaluation of lexical association measures. Proceedings of the 39th Annual Meeting of the Association for Computational Linguistics, 188-195.

Gries, Stefan Th. (2008): Dispersions and adjusted frequencies in corpora. International Journal of Corpus Linguistics 13 (4), 403-437.

Gries, Stefan Th. (2010): Dispersions and adjusted frequencies in corpora: Further explorations. In Stefan Th. Gries, Stefanie Wulff \& Mark Davies (Hrsg.), Corpus linguistic applications: Current studies, new directions, 197-212. Amsterdam: Rodopi.

Gries, Stefan Th. (2012): Frequencies, probabilities, association measures in usage-/ exemplar-based linguistics: Some necessary clarifications. Studies in Language 36 (3), 477-510.

Gries, Stefan Th. (2013): 50-something years of work on collocations: What is or should be next. International Journal of Corpus Linguistics 18 (1), 137-165.

Ikehara, Satoru, Satoshi Shirai \& Hajime Uchino (1996): A statistical method for extracting uninterrupted and interrupted collocations from very large corpora. Proceedings of the 16th Conference on Computational linguistics, Vol. 1, 574-579.

Jelinek, Frederick (1990): Self-organized language modeling for speech recognition. In Alex Waibel \& Kai-Fu Lee (Hrsg.), Readings in speech recognition, 450-506. San Mateo, CA: Morgan Kaufmann.

Kita, Kenji, Yasuhiko Kato, Takashi Omoto \& Yoneo Yano (1994): Automatically extracting collocations from corpora for language learning. Journal of Natural Language Processing 1 (1), 21-33.

Krenn, Brigitte (2000): The usual suspects: Data-oriented models for identification and representation of lexical collocations. PhD Thesis, Saarland University.

Krenn, Brigitte \& Stefan Evert (2001): Can we do better than frequency? A case study on extracting PP-verb collocations. Proceedings of the ACL Workshop on Collocations, 39-46.

Lieven, Elena, Dorothé Salomo \& Michael Tomasello (2009): Two-year-old children's production of multiword utterances: A usage-based analysis. Cognitive Linguistics 20 (3), 481-507.

Nagao, Makoto \& Shinsuke Mori (1994): A new method of n-gram statistics for large number of $\mathrm{n}$ and automatic extraction of words and phrases from large text data of Japanese. Proceedings of the 15th Conference on Computational Linguistics, 611-615. 
Newman, John and Georgie Columbus (2010): The International Corpus of English - Canada. Edmonton, Alberta: University of Alberta.

O’Donnell, Matthew Brook (2011): The adjusted frequency list: A method to produce clustersensitive frequency lists. ICAME Journal 35, 135-169.

Pawley, Andrew \& Frances H. Syder (1983): Two puzzles for linguistic theory: Nativelike selection and nativelike fluency. In Jack C. Richards \& Richard W. Schmidt (Hrsg.), Language and Communication, 191-225. London: Longman.

Pecina, Pavel (2010): Lexical association measures and collocation extraction. Language Resources and Evaluation 44 (1/2), 137-158.

Rayson, Paul, Scott Piao, Serge Sharoff, Stefan Evert and Begoña Villada Moirón (2010): Language Resources and Evaluation, volume 1-2.

Rowland, Caroline F. \& Sarah L. Fletcher (2006): The effect of sampling on estimates of lexical specificity and error rates. Journal of Child Language 33 (4), 859-877.

Shimohata, Sayori, Toshiyuki Sugio \& Junji Nagata (1997): Retrieving collocations by cooccurrences and word order constraints. Proceedings of the 35th Annual Meeting of the Association for Computational Linguistics, 476-481.

Silva, Joaquin F. da, Gaël Dias, Sylvie Guilloré \& José G. Pereira Lopes (1999): Using LocalMaxs Algorithm for the extraction of contiguous and non-contiguous multiword lexical units. Proceedings of the 9th Portuguese Conference on Artificial Intelligence: Progress in artificial intelligence, 113-132.

Silva, Joaquin da \& Gabriel Pereira Lopes (1999): A local maxima method and a dair dispersion normalization for extracting multi-word units from corpora. Proceedings of the 6th Meeting on the Mathematics of Language, 369-381.

Wahl, Alexander (2015): The distributional learning of multi-word expressions: A computational approach. Ph.D. dissertation, University of California, Santa Barbara.

Wahl, Alexander \& Stefan Th. Gries. (eingereicht a): Multi-word expressions: A novel computational approach to their bottom-up statistical extraction.

Wahl, Alexander \& Stefan Th. Gries. (eingereicht b): Computational extraction of formulaic sequences from corpora: Two case studies of a new extraction algorithm.

Wiechmann, Daniel (2008): On the computation of collostruction strength: Testing measures of association as expressions of lexical bias. Corpus Linguistics and Linguistic Theory 4 (2), 253-290.

Wible, David, Chin-Hwa Kuo, Meng-Chang Chen, Nai-Lung Tsao, and Tsung-Fu Hung (2006): A computational approach to the discovery and representation of lexical chunks. Paper presented at TALN. Leuven, Belgium.

Wible, David \& Nai-Lung Tsao (2011): Towards a new-generation of corpus-derived lexical resources for language learning. In Fanny Meunier, Sylvie De Cock, Gaetanelle Gilquin \& Magali Paquot (Hrsg.), A taste for corpora: In honor of Sylviane Granger, 237-255. Amsterdam/Philadelphia: Benjamins. 



\title{
8 Syntaktische Variation aus areallinguistischer Perspektive
}

\begin{abstract}
Der Beitrag setzt sich zum Ziel, syntaktische Variation aus Perspektive einer areallinguistisch orientierten Variationslinguistik zu beleuchten. Dabei werden theoretische und methodologische Herausforderungen, aber auch das Forschungspotenzial dieses Untersuchungskomplexes sowohl für die Variationslinguistik als auch die Grammatikforschung (wie -schreibung) herausgearbeitet. Dies geschieht unter Rückgriff auf Fragestellungen, Methoden und Ergebnisse aus jüngsten Studien im deutschsprachigen Raum.
\end{abstract}

Keywords: Areallinguistik, Korpora, Passiv, Syntax, Variation

\section{Zum Forschungsfeld „Areallinguistische Syntax" und zu diesem Beitrag}

Während die germanistische Dialektologie sowie die mit ihr eng verbundene Variationslinguistik in ihren Anfängen primär auf die Systemebenen der Phonetik \& Phonologie ausgerichtet waren, treten andere Systemebenen - und unter diesen gerade die Syntax - erst verspätet in den Untersuchungsfokus der Disziplinen. Die Erforschung syntaktischer Variation erfordert die Modifikation und die Ausweitung traditioneller theoretischer und methodischer Ansätze der Variationslinguistik, wie sie in einer Fülle von jüngsten Studien und Projekten erfolgreich umgesetzt wurden bzw. werden (s. Lenz \& Plewnia (Hrsg.) 2010; Lenz \& Patocka (Hrsg.) 2016). Vor dem Hintergrund des aktuellen Forschungsstands setzt sich der vorliegende Beitrag zum Ziel, syntaktische Variation aus Perspektive einer areallinguistisch orientierten Variationslinguistik zu beleuchten, das heißt den Faktor „Raum“ als sprachgeographische Größe in den Fokus der Syntaxforschung zu stellen. Dabei werden theoretische und methodologische Herausforderungen einer areallinguistischen Syntaxforschung diskutiert, aber auch das enorme Forschungspotential dieses Untersuchungs-

Alexandra N. Lenz, Universität Wien, Institut für Germanistik, Universitätsring 1, A-1010 Wien, E-Mail: alexandra.lenz@univie.ac.at

Ә Open Access. (c) 2018 Alexandra N. Lenz, publiziert von De Gruyter. (c) BY Dieses Werk ist lizenziert unter der Creative Commons Attribution 4.0 Lizenz.

https://doi.org/10.1515/9783110490992-009 
komplexes sowohl für die Variationslinguistik als auch die Grammatikforschung (und -schreibung) der Germanistik (und darüber hinaus) herausgearbeitet. Dies geschieht unter Rückgriff auf Fragestellungen, methodische Herangehensweisen und Ergebnisse aus jüngsten Studien im deutschsprachigen Raum.

Der Beitrag gliedert sich wie folgt: In einem nächsten Abschnitt werden zunächst Fragestellungen und Inhalte einer areallinguistischen Syntaxforschung vorgestellt und dabei zugleich die Vielfalt der theoretischen und methodischen Problemstellungen des Untersuchungsfelds skizziert (Abschnitt 2). Ausgewählte (und vor allem methodische) Fragestellungen werden dann auf Basis aktueller Analysen anhand eines speziell ausgewählten Phänomenkomplexes in Abschnitt 3 exemplarisch diskutiert, wobei auch empirische Innovationen der Disziplin (Stichwort „Sprachproduktionsexperimente“) Berücksichtigung finden. Der Beitrag schließt mit einer Zusammenfassung und einem Ausblick auf weiterführende Problemstellungen (Abschnitt 4), denen sich eine am Sprachgebrauch orientierte Syntaxforschung in der Zukunft stellen muss.

\section{Inhalte und Fragestellungen einer areallinguistischen Syntaxforschung}

Im Gegensatz zu rein dialektologischen Studien, die sich lediglich dem dialektalen Pol der Dialekt-Standard-Achse zuwenden, nimmt die moderne germanistische Areallinguistik das gesamte Spektrum arealer Variation auf der vertikal gedachten Dialekt-Standard-Achse in den Blick und wendet sich dabei auch „mittleren“ (regiolektalen) bis standardsprachlichen Ausschnitten des vertikalen Variationsspektrums zu (s. Auer 2005; Lenz 2010a; Schmidt \& Herrgen 2011). Trotz dieser „vertikalen“ Ausweitung des Untersuchungsfokus überwiegen in der syntaktisch orientierten Areallinguistik bislang noch immer Studien zum dialektalen Pol des vertikalen Spektrums. Doch auch was die seit Jahren boomende germanistische Dialektsyntax betrifft, sind großangelegte Projekte nach wie vor wenige im deutschsprachigen Raum angesiedelt. $\mathrm{Zu}$ nennen sind hier v. a. das Pionierprojekt „Dialektsyntax des Schweizerdeutschen“ (www.dialektsyntax.uzh.ch/de/projekt.html, letzter Zugriff: 15.11. 2017), das Projekt „Syntax des Alemannischen (SynAlm)“ (https://cms.unikonstanz.de/ling/syntax-des-alemannischen/, letzter Zugriff: 15.11. 2017) sowie das jüngst abgeschlossene und in Form einer Online-Informationsplattform (inkl. Online-Atlas) publizierte Projekt „Syntax hessischer Dialekte (SyHD)“ (www.syhd.info, letzter Zugriff: 15.11. 2017). Hinzu kommen auch Syntaxkarten bzw. Teilbände im Bayerischen Sprachatlas (z. B. Eroms [u. a.] 2006 für Niederbayern). 
Erst in jüngster Zeit wird auch der syntaktischen bzw. generell grammatischen Variation am anderen Extrempol der Dialekt-Standard-Achse Rechnung getragen. Im Hinblick auf areallinguistische Variation geschieht dies aktuell im Projekt „Variantengrammatik“ (www.variantengrammatik.net, letzter Zugriff: 15.11. 2017), das zum Ziel hat, arealgrammatische Variation aus pluriarealer Perspektive „in den Gebrauchsstandards des geschriebenen Deutsch zu ermitteln und in einem Kodex des Gebrauchsstandards zu präsentieren“ (Elspaß \& Dürscheid 2017: 93). ${ }^{1}$ Dabei finden alle Länder und Regionen des zusammenhängenden deutschsprachigen Raums Berücksichtigung. Die areale Dimension ist auch eine Variationsdimension, die - neben vielen anderen - im aktuellen IDS-Projekt „Korpusgrammatik“ im Hinblick auf Standardschriftsprache angegangen wird: „Aufgabe des Projekts sind systematische korpusorientierte Untersuchungen zu Variationsphänomenen in Morphologie und Syntax und die Dokumentation der Resultate in einer neuen, ,variationssensitiven' Grammatik des Standarddeutschen.“ (http://www1.ids-mannheim.de/gra/projekte/ korpusgrammatik.html, letzter Zugriff: 15.11. 2017). Die zunehmende Orientierung am Standardsprachgebrauch hat natürlich auch Auswirkungen auf die konzeptionelle Fassung des Standardsprachbegriffs sowie auf die grammatikographische Arbeit (s. hierzu etwa die Vorwörter der aktuellen Auflagen des Duden 4 (2016) und Duden 9 (2016)).

Im Hinblick auf areallinguistische Variation im „Zwischenbereich“ zwischen Dialekt und Standard stellt der „Atlas zur deutschen Alltagssprache (ADA)“ bislang das größte flächendeckende Projekt dar (http://www.atlas-alltags sprache.de/, letzter Zugriff: 15.11. 2017), dessen empirische Basis - entgegen der genannten korpuslinguistischen Standardsprachprojekte - vor allem Einschätzungsdaten bilden. Neben lautlichen und vor allem lexikalischen Phänomenen werden die ADA-Informanten mitunter auch bzgl. morphologischer und syntaktischer Phänomene befragt. Als Beispiel dienen etwa Passivsätze mit Dativpassivkonstruktionen (mit den Verben schenken, helfen, ersparen und den Auxiliarvarianten kriegen versus bekommen, s. www.atlas-alltagssprache.de/ runde-4/, letzter Zugriff: 15.11. 2017 und zur Interpretation der ADA-Karten Lenz (2013a: 176-180, 265-269)).

Während die zuvor genannten Projekte allesamt syntaktische (sowie teilweise auch morphologische) Variation in nur jeweils ausgewählten „Ausschnitten“ des vertikalen Variationsspektrums der Dialekt-Standard-Achse fokussieren, widmen sich jüngste areallinguistische Studien auch erstmals syntaktischer Variation im vertikalen Gesamtspektrum: Exemplarisch sei hier auf die Studie von Lenz 2013a verwiesen, die sich sämtlichen kriegen- und

1 Zum Konzept des Gebrauchsstandards s. Deppermann, Kleiner \& Knöbl 2013, Elspaß \& Dürscheid (2017: 89-93). 
bekommen-Konstruktionen in den Varietäten des deutschsprachigen Raums zuwendet, von den Dialekten über Regiolekte bis hin zum standardsprachlichen Spektrum, und zwar unter Berücksichtigung mündlicher wie schriftsprachlicher Daten. Dabei werden sowohl mehr „natürliche“ Sprachgebrauchsdaten aus Gesprächen, Chat-Kommunikationen und Zeitungstexten analysiert wie auch elizitierte Daten aus speziell entworfenen und stark standardisierten Sprachproduktionsexperimenten zum Dativpassiv. Sprachproduktionsexperimente zur Analyse syntaktischer Variation auf der „Vertikalen“ werden auch in der Studie von Kallenborn (2016) - zum moselfränkischen Ort Graach - sowie in der Wien-Studie von Breuer (2017) verwendet. In beiden lokalen „Tiefenbohrungen“ werden damit orale Daten zum „intendierten Standard“ sowie zum „intendierten Dialekt“ (s. Macha 1991; Lenz 2004) erhoben. Einen flächendeckenden Einsatz zur Analyse syntaktischer Variation in den vertikalen Gesamtspektren von Sprechenden erfahren Sprachproduktionsexperimente aktuell im SFB „Deutsch in Österreich. Variation - Kontakt - Perzeption“ (FWF F060, www.dioe.at, letzter Zugriff: 15.11. 2017) (s. Abschnitt 3).

Unabhängig von ihren „vertikalen Schwerpunktsetzungen“ belegen alle genannten Projekte und Studien die Bedeutung der arealen Variationsdimension bei der Analyse syntaktischer Phänomene. Doch auch wenn die bereits vorliegenden Ergebnisse einen wesentlichen Beitrag liefern, sind dennoch eine Fülle von Fragen der areallinguistischen Syntaxforschung noch nicht bzw. noch nicht erschöpfend beantwortet. $\mathrm{Zu}$ diesen Fragen gehören insbesondere die folgenden:

\section{Allgemein-theoretische Fragestellungen der Areallinguistik}

Was steuert syntaktische Variation überhaupt und welchen Anteil haben grammatikinterne Regularitäten bzw. generell linguistische Parameter einerseits und Arealität im Zusammenspiel mit anderen sprachexternen Faktoren wie Medialität, Register, Textsorte u.v.a. andererseits? Welche soziopragmatischen Funktionen erfüllen areal verortbare Syntaxvarianten (etwa im Rahmen von Shifting- und Switching-Prozessen)? Welche Unterschiede bzw. Parallelen decken areallinguistische Analysen syntaktischer Variation zwischen den verschiedenen linguistischen Systemebenen auf, d.h., wie ist das Verhältnis von syntaktischer Variation zu Variation auf anderen linguistischen Systemebenen wie der Phonologie, der Morphologie oder Lexik?

\section{Variablen- und varietätenlinguistische Fragestellungen}

Was macht eine syntaktische Variable im Sinne einer Menge von koexistierenden syntaktischen Alternativen aus und wie sind die syntaktischen Varianten einer Variable $\mathrm{zu}$ bestimmen und $\mathrm{zu}$ klassifizieren ${ }^{2}$ Wo ist eine syntaktische

2 Zum Problem der syntaktischen Variable s. auch die Diskussion in Lavandera 1978; Cheshire 1987 und 2005; Hasty 2014; Kallenborn 2016 und Lüdeling 2017. 
Variante vertikal-sozial auf der Dialekt-Standard-Achse einzuordnen, oder anders formuliert: Wo hört syntaktisch gesehen regionaler Non-Standard auf und fängt überregionaler bzw. großregionaler Standard an? Wieviel syntaktische Variation „verträgt“ die Standardsprache? ${ }^{3}$ In welchem Maße unterscheidet sich die Syntax arealsprachlicher Varietäten von der standardsprachlicher oder die Syntax nähesprachlicher/interaktionsorientierter Register von der distanzsprachlicher? Liefert die syntaktische Ebene Evidenzen für das Vorliegen von abgrenzbaren Varietäten der Vertikalen oder aber für ein vertikales Kontinuum zwischen Nonstandard und Standard (s. Auer 2005; Lenz 2003 und 2010a)? Lassen sich auf syntaktischer Ebene „Zwischenvarietäten“ (Regiolekte) zwischen Dialekt und Standard ausmachen? Welche Parallelen bzw. Unterschiede zeichnen sich auf syntaktischer Ebene hinsichtlich der vertikalen Gesamtstrukturen der Dialekt-Standard-Spektren in verschiedenen Räumen und Ländern des deutschsprachigen Raums ab?

\section{Sprachgeographisch-orientierte Fragestellungen}

In welchem Maße ist Syntax ein konstitutives Element für Sprachräume im Deutschen? Wie steht es generell um die areale Variation syntaktischer Merkmale und in welchen ,vertikalen Ausschnitten“ der Dialekt-Standard-Achse lässt sich welche areallinguistische Variation im Bereich der Syntax vorfinden, oder anders formuliert: Welche Parallelen und welche Unterschiede zeichnen sich hinsichtlich syntaktischer Variation zwischen Dialekten, Regiolekten und standardsprachlichen Varietäten ab? Welche Regionen oder Areale zeichnen sich auf syntaktischer Ebene im Raum ab, und in welchem Verhältnis stehen die auf syntaktischer Ebene gewonnenen geographischen Strukturen mit traditionellen Sprachraumgliederungen, die meist auf lautlicher Ebene basieren? In welchem Verhältnis stehen dialektale, regiolektale und standardsprachliche Raumstrukturen, wie sie sich auf syntaktischer Ebene eruieren lassen, zueinander? In welchem Maße korrelieren syntaktisch eruierte Sprachräume mit anderen extralinguistischen Parametern (z. B. geographischen, politischen, sozialen u. a.)?

\section{Sprachdynamische Fragestellungen}

Wie „funktioniert“ syntaktischer Wandel im Verhältnis zu Wandelprozessen auf anderen Systemebenen; lassen sich hier unterschiedliche Geschwindigkeitsgrade, Diffusionsprozesse oder andere Unterschiede bzw. Parallelen in der Art der Dynamik ausmachen? Welche areal-horizontalen syntaktischen Varian-

3 Die Formulierung ist angelehnt an den IDS-Jahresband 2004 „Standardvariation: Wie viel Variation verträgt die deutsche Sprache?“ (Eichinger \& Kallmeyer 2005). 
ten weisen besondere „Veränderungssensitivität“ (s. Lenz 2003) bzw. besondere Stabilität „im Raum“ auf? Können auch syntaktisch die auf phonetisch-phonologischer Ebene zu beobachtenden Tendenzen von Lokalität (Kleinräumlichkeit) hin zu Regionalität (Großräumigkeit) beobachtet werden? Welche Aussagen ermöglicht die synchrone Variation im Raum hinsichtlich diachroner Wandelprozesse?

\section{Syntaxtheoretische und (andere) interdisziplinäre Fragestellungen}

Welchen Beitrag leistet areallinguistische Syntaxforschung zu syntaxtheoretischen Fragestellungen und Herangehensweisen bzw. vice versa? Welche Konsequenzen bringen areallinguistische Befunde für syntax- bzw. allgemein sprachtheoretische Modellierungen mit sich? Welchen Beitrag leistet die areallinguistische Syntaxforschung zur Dialektologie bzw. Variationslinguistik im Allgemeinen? In welchem Verhältnis stehen (mehr) empirische und (mehr) theoretische Syntaxforschung zueinander, und wie können sie sich in Zukunft gegenseitig (mehr) befruchten? Welche interdisziplinären Hürden gilt es bei einer Annäherung der Paradigmen zu überwinden?

\section{Method(olog)ische Fragestellungen}

Mit welchen methodischen Herausforderungen sieht sich eine areallinguistisch orientierte Syntaxforschung konfrontiert? Welche Erhebungs- und Datenanalysemethoden haben welche Aussagekraft bzw. sind für welche Fragestellungen und welche konkreten syntaktischen Phänomene in welchem Maße geeignet? Welche Vor- und Nachteile bringen evozierte Daten im Vergleich zu spontansprachlichen Daten mit sich? Wie sieht ein optimales Korpus aus, das zur Analyse syntaktischer Variation aus areallinguistischer Perspektiver herangezogen werden kann?

\section{Attitudinal-perzeptive Fragestellungen}

Wie nehmen Hörende/Sprechende arealsprachliche Syntaxvarianten wahr und welche attitudinal-evaluativen Wertigkeiten (Stichwort „Salienz“, Lenz 2010b) werden diesen Varianten zugeschrieben? Welche Rolle spielen syntaktische Varianten in den „mental maps“ linguistischer Laien? Inwiefern fungieren auch syntaktische Varianten als soziale „Marker“?

\section{Grammatikographische Fragestellungen}

Welche arealsprachlichen Varianten haben das Potenzial als normkonform eingestuft zu werden bzw. als „Zweifelsfälle“ des Deutschen Fälle langandauernder Variabilität darzustellen? In welchem Maße kann und sollte auch in grammatischen Nachschlagewerken dem Faktor Arealität (neben anderen ext- 
ralinguistischen Steuerungsparametern) Rechnung getragen werden? Welche neuen Perspektivierungen auf Standardnorm(en) bringt die areallinguistische Perspektive mit sich? In welchem Maße können oder sollen auch attitudinalperzeptive Ergebnisse in die grammatikographische Arbeit Eingang finden?

Dies sind einige Fragen bzw. Fragekomplexe, die das Feld der areallinguistischen Syntaxforschung betreffen bzw. zu deren Beantwortung die Disziplin beitragen kann und will. Die Fülle und Komplexität der Fragen machen es unmöglich, sie im hier vorgegebenen Rahmen ausführlicher zu diskutieren. Stattdessen soll im Folgenden ein konkretes syntaktisches Phänomen aus areallinguistischer Perspektive exemplarisch analysiert werden, um einen Beitrag zu einigen ausgewählten Forschungsfragen zu leisten. Der Schwerpunkt der Diskussion wird dabei auf methodische und variablenlinguistische Aspekte sowie ihre Implikationen für die grammatikographische Arbeit gelegt.

\section{Das Dativpassiv als Beispielsphänomen}

\subsection{Zur Phänomenauswahl}

Das Ziel von Abschnitt 3 ist eine exemplarische Tiefenanalyse eines syntaktischen Phänomens aus areallinguistischer Perspektive. Als ein - aus mehreren Gründen (s.u.) - besonders aussagekräftiges syntaktisches Phänomen wird das Dativpassiv (alternativ Rezipienten-, Adressaten-, Benefizientenpassiv bzw. kriegen-/bekommen-/erhalten-Passiv) angesehen und deshalb ausgewählt. Während (vereinfacht formuliert) das Subjekt eines Vorgangspassivs (Der Orden wird ihm überreicht.) dem Akkusativaktanten eines entsprechenden Aktivsatzes entspricht (Der Vereinsvorsitzende überreicht ihm den Orden.), korrespondiert das Subjekt eines Dativpassivs (i. d. R.) mit dem Dativaktanten (valenzbedingter oder freier Dativ) des Aktivsatzes (Er bekommt den Orden überreicht.). Nach wie vor wird dem Dativpassiv in gegenwartssprachlichen Nachschlagewerken zur Standardsprache eine untergeordnete Rolle zugewiesen. So werden etwa im aktuellen Duden 9 (2016: 705) werden- und sein-Passive als „[d]ie beiden wichtigsten“ Passivformen „für die geschriebene Sprache“ aufgeführt. Was das Dativpassiv betrifft, ist es das bekommen-Passiv, dem (mittlerweile $)^{4}$ am ehesten standardsprachlicher Status zugeschrieben wird, mit dem Hinweis, dass es

4 Siehe hierzu den Überblick zur Dynamik der Artikeleinträge in den verschiedenen Auflagen der Duden-Grammatik in Lenz 2013b. 
fast ausschließlich von Verbkonstruktionen mit einem Akkusativ- und einem Dativaktanten gebildet [wird], in denen der Dativaktant eine für den Dativ typische semantische Rolle (Rezipient [Empfänger], Benefizient [Nutznießer], Possessor [Besitzer] usw. [...]) trägt. [...] Das bekommen-Passiv wird am häufigsten von prototypischen Verben mit Dativund Akkusativobjekt - Verben des Gebens, Nehmens, Mitteilens, Verbergens oder des Gegenteils - gebildet [...]. Etwas seltener entspricht das Subjekt im Passiv einem freie(re)n Dativaktanten [...]. (Duden 4 2016: 563 u. 564)

Bei einem Dativpassiv mit intransitiven dativregierenden Verben handelt es sich laut Duden-Grammatik (Duden 4 2016: 564) hingegen „um eine regionale, nicht allgemein akzeptierte Erscheinung, die im Wesentlichen in der gesprochenen Sprache begegnet“, wobei der angegebene Beispielsatz („,weil man immer geholfen kriegt“) ein kriegen-Passiv repräsentiert. Hinsichtlich der Auxiliarselektion werden v. a. „stilistisch[e]“ Steuerungsfaktoren angeführt bzw. wird bei erhalten auch auf den geringeren Grammatikalisierungsgrad hingewiesen: ${ }^{5}$

Erhalten begegnet bevorzugt im schriftsprachlichen, eher gehobenen Register und kriegen im mündlichen Register. Darüber hinaus scheint erhalten in höherem Ausmaß als bekommen bei Verben des Nehmens und ähnlichen „,negativen“ Vollverbkonstruktionen vermieden zu werden. (Duden 4 2016: 564)

Unklar lassen Duden 4 (2016) sowie Duden 9 (2016), was mit der Einordnung eines kriegen-Passivs als „regional“ bzw. „umgangssprachlich“ genau gemeint ist und in welchem Verhältnis die beiden Attribute zueinander stehen. Bezieht sich „umgangssprachlich“ eher auf eine medial-stilistische Ebene? Wenn es sich um ein areal begrenztes Phänomen handelt, ist es dann zumindest in besagter Region standardsprachlich akzeptiert? Um welche Regionen handelt es sich genau?

Neben der grammatikographischen Dynamik bzw. Unschärfe motivieren folgende Aspekte die Auswahl gerade dieses Phänomens, um Inhalte, Fragestellungen und Methoden der areallinguistischen Syntaxforschung in einer Zusammenschau zu präsentieren:

- Forschungsboom: Wenig andere syntaktische Konstruktionen haben innerhalb der letzten 20 Jahre mehr Interesse erfahren, sowohl aus Perspektive der areallinguistischen Variationslinguistik als auch aus Perspektive der Grammatikforschung außerhalb der Variationslinguistik. ${ }^{6}$ Dieses Interesse

5 Deutlich weniger aussagekräftig erscheinen hingegen die Informationen bzgl. der Auxiliarselektion im Duden 9 (2016: 706), in dem erhalten ohne weitere Angaben zu linguistischen oder extralinguistischen Steuerungsfaktoren lediglich genannt wird.

6 In Auswahl sei hier verwiesen auf Eroms 1978; Eroms 2000; Leirbukt 1997; Askedal 1984 und 2005; Abraham 1991 und 1985; Teuber (2005: 84 ff.); Glaser 2005; Zifonun [u.a.] (1997: 
scheint nicht nur für die Ergiebigkeit des Phänomens aus unterschiedlichsten linguistischen Forschungsrichtungen zu sprechen, sondern ermöglicht natürlich auch den Zugriff auf eine Fülle von bereits vorliegenden Forschungsergebnissen, die zudem aus verschiedenen theoretischen und methodischen Perspektiven gewonnen wurden. Im Fokus stehen im Folgenden die areallinguistisch $\mathrm{zu}$ interpretierenden Befunde.

- Komplexe areal-horizontale und sozial-vertikale Distribution: Nach allem, was areallinguistisch über das Dativpassiv bekannt ist, handelt es sich um eine Konstruktion, die im deutschsprachigen Raum (noch) sehr unterschiedlich weit grammatikalisiert ist. Die hoch spannenden sprachgeographischen Befunde lassen sich - in unterschiedlichem Maße - auf allen Ebenen des vertikalen Variationsspektrums nachvollziehen, von Dialekten bis hin zur Standardschriftsprache.

- Linguistische Komplexität: Hinzu kommen komplexe linguistische (syntaktisch-semantische) Steuerungsfaktoren, die „im Raum“ variieren und dabei synchron die fortschreitende Grammatikalisierung des Phänomens analysierbar machen.

- Hoher Dynamikgrad: Die Dynamik des Phänomens, die sich linguistisch und extralinguistisch synchron widerspiegelt, deckt sich mit Wandelprozessen, die auch über kurze Zeiträume hinweg nachgezeichnet werden können.

- Laienlinguistische Relevanz: Wenn auch die Passivkonstruktion an sich sicher nicht zu den salientesten ${ }^{7}$ Merkmalen auf laienlinguistischer Seite gehört, so sind doch die Auxiliare bzw. ihre lexikalischen Quellen häufiger Objekt metakommunikativer Diskurse. ${ }^{8}$

Die genannten Punkte stützen die These, dass der linguistischen wie soziolinguistischen Komplexität des areallinguistisch sehr interessanten Variationsphänomens nur durch eine multidimensionale empirische Herangehensweise Rechnung getragen werden kann. Dies soll im Folgenden geschehen, indem die bisherigen variationslinguistischen Befunde - die es zu unterschiedlichen Teilaspekten des Phänomens in unterschiedlichen Räumen des deutschsprachigen Raums und hier zu unterschiedlichen „Ausschnitten“ des vertikalen Spektrums auf der Dialekt-Standard-Achse gibt - zusammengetragen und durch aktuellste

1824 ff.); Molnárfi 1998; Cook 2004; Diedrichsen 2008 und 2012; Kasper 2017; Lenz 2009; 2012, 2013b, 2017.

7 Zum Salienzbegriff s. Lenz 2010b und Purschke 2014.

8 Siehe etwa die Forenzitate in Lenz (2013a: 406-417). 
Analysen erweitert werden, um anschließend vor allem Antworten geben zu können auf die oben formulierten methodischen Fragestellungen:

- Mit welchen methodischen Herausforderungen sieht sich eine areallinguistisch orientierte Syntaxforschung konfrontiert?

- Welche Erhebungs- und Datenanalysemethoden haben welche Aussagekraft bzw. sind für welche Fragestellungen und welche konkreten syntaktischen Phänomene in welchem Maße geeignet?

- Welche Vor- und Nachteile bringen evozierte Daten im Vergleich zu spontansprachlichen Daten mit sich?

- Wie sieht ein optimales Korpus aus, das zur Analyse syntaktischer Variation aus areallinguistischer Perspektive herangezogen werden kann?

\subsection{Areallinguistische Daten und ihre Befunde}

Die Daten bzw. Korpora, auf die sich die Analysen im Folgenden stützen, spiegeln die Breite an empirischen Herangehensweisen wider, wie sie in der areallinguistischen Syntaxforschung mittlerweile eingesetzt werden. Der Fokus wird hier explizit auf gesprochensprachliche Daten gelegt. Was areallinguistische Analysen zum Dativpassiv in medial-schriftlichen Korpora betrifft, sei auf Lenz 2013a verwiesen, wo sowohl Chat-Daten als auch Teilkorpora des „Deutschen Referenzkorpus (DeReKo)“ (s. http://www1.ids-mannheim.de/kl/projekte/ korpora/, letzter Zugriff: 15.11. 2017) ausgewertet wurden. Zu den im Folgenden herangezogenen oralsprachlichen Daten gehören stark kontrollierte elizitierte versus (mehr) spontansprachliche Daten bzw. (mehr) kompetenzorientierte versus (mehr) interaktionsorientierte Daten. Hinsichtlich der Daten zweiten Typs stellen die Korpora des IDS eine besonders ergiebige Quelle dar. Unter ihnen werden die Aufnahmen aus dem „Zwirner-“ und dem „Pfeffer-Korpus“ sowie dem im Aufbau befindlichen „Forschungs- und Lehrkorpus (FOLK)“ analysiert und kontrastiert, ${ }^{9}$ um Vor- und Nachteile freier(er) Gesprächsdaten im Hinblick auf areallinguistisch fokussierte Syntaxanalysen zu diskutieren. Während die FOLK-Daten (wenn auch noch in sehr unterschiedlichem Maße) den gesamten deutschsprachigen Raum abdecken, sind die Zwirner-Aufnahmen schwerpunktmäßig auf die Dialekte der alten Bundesrepublik beschränkt. Die genannten Korpora, die teilweise zu unterschiedlichen Zeiten in unterschiedlichen Räumen mit unterschiedlichen Methoden erhoben bzw. gesammelt wurden und zudem in unterschiedlichen Aufbereitungsstufen vorliegen, stellen

9 Die Gesprächskorpora sind online verfügbar über http://dgd.ids-mannheim.de, letzter Zugriff: 15.11. 2017. 
natürlich hinsichtlich ihrer Aussagekraft und Vergleichbarkeit eine Herausforderung dar. $\mathrm{Zu}$ generellen methodischen Problemen, die unabhängig von einer konkreten linguistischen Fragestellung auftreten, kommt hinsichtlich der Analyse syntaktischer Variation hinzu, dass sich syntaktische Phänomene auch unabhängig von soziolinguistischen Parametern - allein aufgrund ihrer komplexen semantisch-pragmatischen Steuerungsbedingungen in sehr unterschiedlichen Frequenzen auf Gesprächs- und Texttypen verteilen können (s. Lenz 2008, 2009; Kallenborn 2016). Als ein Ansatz zur Lösung dieses forschungspraktischen Problems werden den freien Gesprächsdaten hier die Ergebnisse eines speziell entwickelten „Sprachproduktionsexperiments“ gegenübergestellt, dessen Ziel es ist, quantitativ umfangreiche und auch qualitativ aussagekräftige Daten aus intraindividuell divergierenden, aber interindividuell vergleichbaren vertikalen Spektrenausschnitten $\mathrm{zu}$ evozieren. Die folgende Diskussion der Ergebnisse ist anhand der Datentypen strukturiert: Zu Beginn stehen die Auswertungen auf Basis freier Gesprächsdaten sowie der mit diesem Material verbundenen Vor- und Nachteile, die sich im Zusammenhang mit syntaktischen Variationsanalysen ergeben. Die Analysen auf Basis elizitierter Daten aus dem genannten Sprachproduktionsexperiment schließen sich in einem zweiten Analyseblock an.

\subsection{1 „Freie“ Gesprächsdaten (DGD)}

Im Folgenden werden drei große Korpora der „Datenbank Gesprochenes Deutsch“ (http://dgd.ids-mannheim.de/dgd/pragdb.dgd_extern.welcome, letzter Zugriff: 15.11. 2017) herangezogen, um - auf Basis des ausgewählten Beispielphänomens - der Ergiebigkeit, aber auch den Grenzen der (mehr oder weniger „freien“) Gesprächskorpora für areallinguistische Syntaxforschung nachzugehen:

- „Zwirner-Korpus“ (http://agd.ids-mannheim.de/ZW--_extern.shtml, letzter Zugriff: 15.11. 2017): Das Korpus besteht aus insgesamt 5.857 Tonaufnahmen mit einer durchschnittlichen Länge von jeweils ca. zwölf Minuten (insgesamt ca. 1.092 Aufnahmestunden). Die Inhalte umfassen dialektale (und seltener als „umgangssprachlich“ klassifizierte) Erzählungen und initiierte Monologe, die in der zweiten Jahreshälfte des letzten Jahrhunderts (vor allem zwischen 1959 und 1960) unter der Leitung von Eberhard Zwirner aufgezeichnet wurden. Bei den aufgenommenen Gewährspersonen handelt es sich in der Regel um drei autochthone Dialektsprecher aus drei Generationen, zu denen - falls möglich - drei Aussiedler aus den ehemaligen deutschen Ostgebieten hinzukamen. Das Ortsnetz des Zwirner-Korpus 
besteht aus ca. 1.000 ländlichen Ortspunkten in den alten Bundesländern, in Vorarlberg und Liechtenstein, im Elsass sowie in den Niederlanden. Die im Folgenden herangezogenen Daten stammen aus den Aufnahmen, deren Transkripte mit Ton aligniert online verfügbar sind. Unberücksichtigt bleiben die „Mundartgruppen“ Mecklenburgisch-Vorpommersch, Märkisch, Schleswigsch, Westthüringisch und Südbairisch, da die Anzahl ihrer verfügbaren Aufnahmen unter 20 Aufnahmen pro Mundartgruppe liegen, sowie die Aufnahmen aus den „außereuropäischen Sprachinseln“. Die Anzahl der ausgewerteten Zwirner-Aufnahmen beläuft sich damit auf 1.949.

- „Pfeffer-Korpus“ (http://agd.ids-mannheim.de/PF--_extern.shtml, letzter Zugriff: 15.11. 2017): Beim Pfeffer-Korpus handelt es sich um ein 1961 zur Dokumentation „städtischer Umgangssprachen“ erstelltes Korpus, das - in Ergänzung zum Zwirner-Korpus - Erzählungen und Dialoge von insgesamt 403 als „umgangssprachlich“ eingestuften Sprechern aus 57 Städten im gesamten deutschsprachigen Raum enthält. Unberücksichtigt bleiben hier aufgrund ihrer zu geringen Repräsentation alle Mundartgruppen mit weniger als zehn Aufnahmen im Korpus. Die Anzahl der ausgewerteten PfefferAufnahmen beläuft sich damit auf 368.

- „FOLK“ (http://agd.ids-mannheim.de/folk.shtml, letzter Zugriff: 15.11. 2017): Das im Entstehen begriffene „Forschungs- und Lehrkorpus Gesprochenes Deutsch“ beinhaltet „Gesprächsdaten aus unterschiedlichsten Bereichen des gesellschaftlichen Lebens (Arbeit, Freizeit, Bildung, öffentliches Leben, Dienstleistungen usw.) im deutschen Sprachraum“ aus den Jahren 2003 bis aktuell 2016. Die im FOLK enthaltenen Gesprächsaufnahmen repräsentieren unterschiedlichste Kommunikationssituationen mit heterogenen Formalitätsgraden und Inhalten (von z. B. „Familiengesprächen“ bis hin zu gesprochener Wissenschaftssprache). Wichtig zu betonen ist die Tatsache, dass dieses Korpus - im Gegensatz zu dem bald verfügbaren IDS-Korpus „Deutsch heute“ (http://prowiki.ids-mannheim.de/bin/view/ AADG, letzter Zugriff: 15. 11. 2017) keinen Anspruch auf regionale Flächendeckung oder eindeutige regionale Zuordnung der Aufgenommenen erhebt. Bei letzteren handelt es sich nicht selten um hochmobile Sprecher mit zahlreichen und wechselnden Aufenthaltsorten. Inwieweit das (ohne Frage höchst wertvolle) FOLK-Korpus dennoch (bereits heute) auch für areallinguistische Fragestellungen genutzt werden kann, wird am ausgewählten Beispielsphänomen getestet. Die FOLK-Aufnahmen haben aktuell (Stand: Juni 2017) eine Gesamtdauer von ca. 279,5 Stunden, wobei die einzelnen Aufnahmen in ihrer Länge deutlich variieren. 


\section{Pfeffer-und Zwirner-Analysen}

Die Abbildungen 8.1 und 8.2 kontrastieren zunächst die Ergebnisse, die sich aus den Analysen der 1.949 Zwirner- bzw. 368 Pfeffer-Aufnahmen ergeben. Da die hier visualisierten Befunde in Lenz 2007 zumindest teilweise ausführlicher dargestellt und interpretiert wurden, ${ }^{10}$ werden hier nur die wichtigsten Ergebnisse zusammengefasst. Bei einem Vergleich der beiden Diagramme ist zu bedenken, dass die „Mundartgruppen“ (DGD-Bezeichnung für die Dialektareale) durch das Zwirner-Korpus deutlich besser flächengedeckt sind als durch das Pfeffer-Korpus, in dem die Areale mitunter nur durch sehr wenige und dann eher städtische Ortspunkte repräsentiert werden. Die Frequenzen, denen 346 (Zwirner) bzw. 73 (Pfeffer) absolute Belege zugrunde liegen, beziehen sich auf die relativen Häufigkeiten von Dativpassiven in allen Aufnahmen pro Region. (Diese Berechnung stützt sich auf die Tatsache, dass die Zwirner- und PfefferAufnahmen alle eine vergleichbare Länge von jeweils ca. 12 Minuten umfassen und damit annähernd vergleichbar sind.) Die relativen Häufigkeiten von Dativpassiven in den berücksichtigten Zwirner- wie Pfeffer-Regionen betragen regionenübergreifend für die beiden Gesamtkorpora jeweils um die 18-20\%, was bedeutet, dass im Schnitt fast jede fünfte Aufnahme beider Korpora ein Dativpassiv enthält.

Wie die Balkendiagramme in Abbildung 8.1 und Abbildung 8.2 illustrieren, verteilen sich die Dativpassive allerdings bei weitem nicht gleichmäßig auf den Sprachraum, sondern ballen sich im westlichen Mitteldeutschen (Rhein- und Mittelfränkisch) und angrenzenden Westniederdeutschen, und das sowohl in den Dialekten wie auch den Regiolekten. Über diese areale Kernregion des Dativpassivs hinaus ist der Vergleich von Zwirner- und Pfeffer-Aufnahmen vor allem im Hinblick auf den oberdeutschen Sprachraum interessant. Die höheren Frequenzen des Dativpassivs in den Pfefferdaten liefern Evidenz für die These, dass das Dativpassiv in Teilen des oberdeutschen Sprachraums in „höheren“ Sprechlagen der vertikalen Dialekt-Standard-Achse schon frequenter bzw. weiter grammatikalisiert ist als in den Dialekten. Hier ist es somit ein syntaktisches Phänomen, das vertikal gesehen von „oben nach unten“ wandert (vgl. Bucheli Berger 2005; Glaser 2005: 54; Lenz 2013a).

Eine qualitative Analyse der Belege, die „hinter“ den Zahlen stehen, zeigt, dass wir es dialektal wie regiolektal nicht nur mit unterschiedlichen Häufigkeiten der Dativpassiv-Produktion zu tun haben, sondern dass in den Dialekten und Regiolekten des deutschsprachigen Raums auch variierende Selektions-

10 Minimale Differenzangaben ergeben sich dadurch, dass zwischenzeitlich die Tonaufnahmen noch einmal (zumindest stichprobenartig) geprüft und dabei Fehler in den Transkriptionen aufgedeckt wurden. 


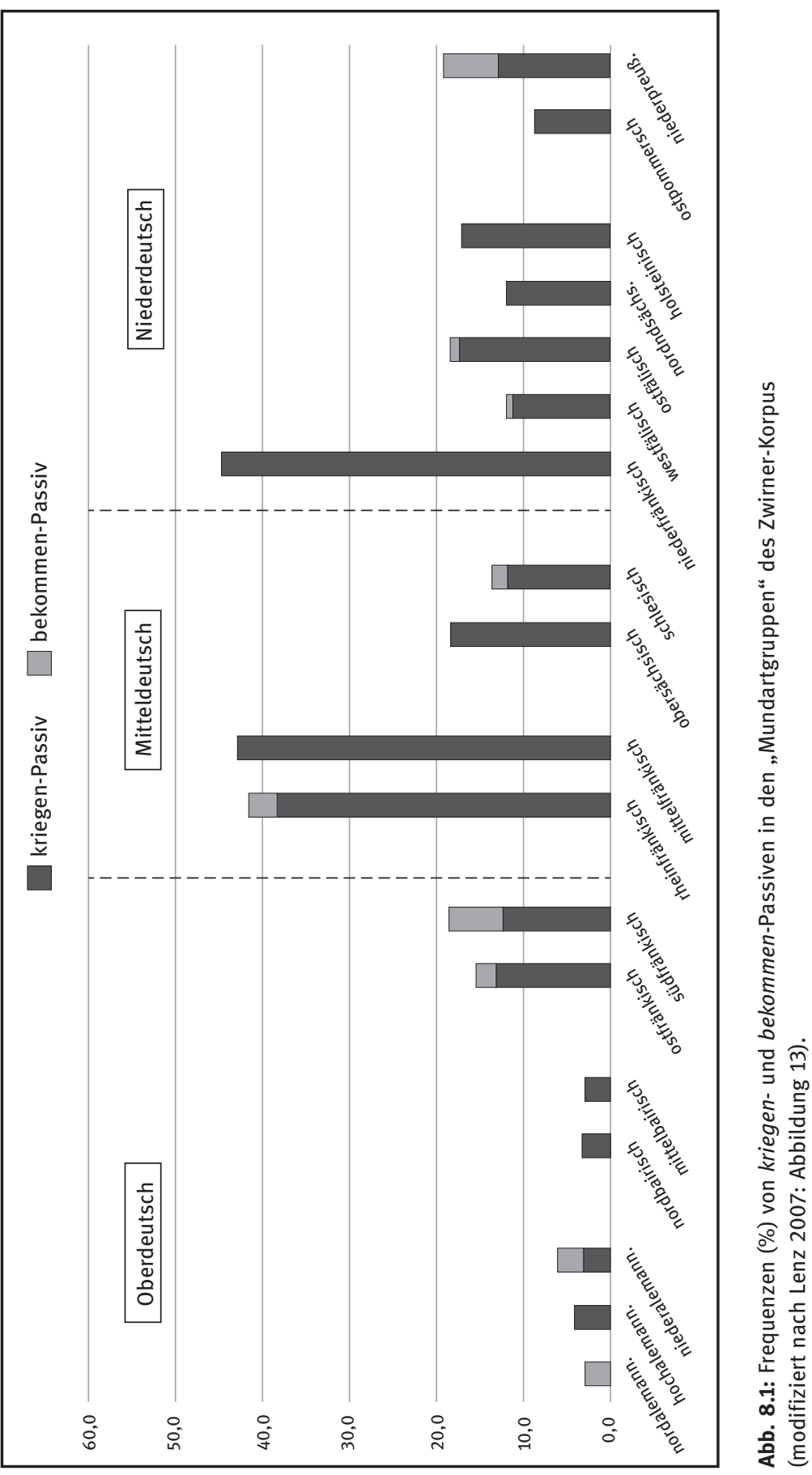




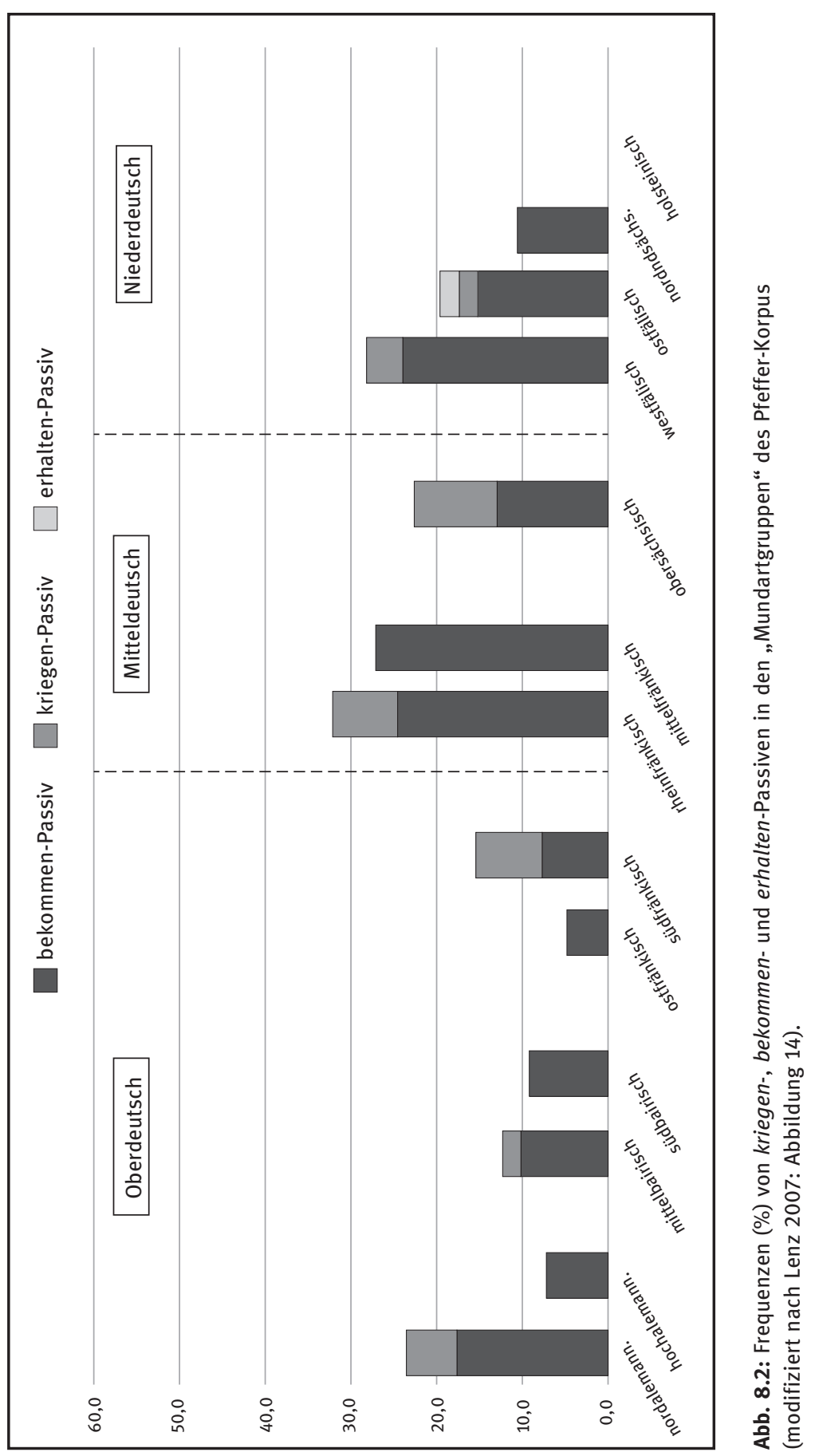


beschränkungen vorherrschen, denen die Konstruktion in den verschiedenen Arealen unterliegt. In den Regionen, in denen Dativpassive am häufigsten produziert werden, sind die syntakto-semantischen Selektionsbeschränkungen bereits deutlich mehr aufgeweicht als in den Regionen mit niedrigsten Frequenzen. Bezogen auf die Dialektdaten heißt das, dass dialektale kriegen-Passive mit Monotransitiva (nicht nur) des Schimpfens (wie schimpfen, schelten) ebenso wie mit deprivativen Ditransitiva (TAKE-Verben wie ab-/wegnehmen, entreißen, stehlen, klauen u.a.) fast ausschließlich in den westmitteldeutschen ZwirnerAufnahmen belegt sind. Damit zusammenhängend weisen die Subjektsreferenten von dialektalen Dativpassiven der weniger Dativpassiv-affinen Räume eher prototypische Rezipientenmerkmale auf (ein belebtes Subjekt, das den NachPossessor einer konkreten transferenziellen Handlung darstellt), während in der arealen Kernregion des Dativpassivs auch andere semantische Rollen (Quelle, Benefizient, Malefizient u. a.) vertreten sind. In den regiolektalen Pfeffer-Daten (und damit auch hinsichtlich des bekommen-Passivs) scheinen die Selektionsbeschränkungen auch in den Regionen mit höchsten Belegfrequenzen hingegen noch größere Gültigkeit zu besitzen: In diesem Korpus treten fast nur Dativpassive mit (mehr oder weniger prototypischem) Rezipienten einer (mehr oder weniger prototypischen) Transferaktion auf. Die wenigen, nämlich nur vier Belege mit TAKE-Verben (dreimal (jmdm. etw.) abnehmen, einmalig (jmdm. etw. wegreißen) verteilen sich mit drei Belegen auf das westliche Mittel- bzw. Niederdeutsche. Der vierte Beleg ist in einer ostfränkischen Pfeffer-Aufnahme zu finden.

Höchst ergiebig ist auch der Korpusvergleich im Hinblick auf die Auxiliare der Konstruktion: Während im dialektalen Zwirner-Korpus kriegen mit mehr als $91 \%$ eindeutig dominiert, werden die Dativpassive im Pfeffer-Korpus zu fast $82 \%$ mit bekommen und in einem Fall sogar mit erhalten gebildet. ${ }^{11}$ Interessant ist wiederum ein (aufgrund der nur relativ wenigen Belege vorsichtiger) Blick ins Alemannische, denn nur hier überwiegt das bekommen-Auxiliar bereits in den Dialektdaten. Im Gegensatz zu allen anderen Regionen ist es im Alemannischen das kriegen-Passiv, das den markierten Fall des oralen Nonstandards darstellt. Diese These wird auch durch den „Atlas zur deutschen Alltagssprache (ADA)“ gestützt (www.atlas-alltagssprache.de/runde-4/f22a-b/, letzter Zugriff: 15.11. 2017), in denen das kriegen-Passiv mit dem Ditransitivum schenken mit Ausnahme des schweizerdeutschen Raums in allen Regionen des deutschsprachigen Raums als „sehr üblich[es]“ Phänomen der „Alltagssprache“ eingestuft wird. Hingegen ist es „geschenkt bekommen“, das im

11 Siehe PF294 (ostfälisch): „Es war in, unter einem Zelt untergebracht, und von einer Küche erhielten wir unser Essen reichlich zugeteilt.“ 
Schweizerdeutschen häufiger als „sehr üblich“ bewertet wird. (Zur ausführlichen Interpretation der ADA-Karten „geholfen kriegen/bekommen“ und „Ärger erspart kriegen/bekommen“, s. Lenz 2013a: 176-180, 265-269). Diese schweizerdeutschen Befunde widersprechen den (in der Regel bundesdeutsch orientierten) grammatikographischen Angaben, nach denen das kriegen-Auxiliar per se und ohne weitere areale Differenzierung dem „mündlichen Register“ (Duden 4 2016: 564) zugeschrieben wird.

Trotz dieser interessanten Ergebnisse, die das Material explizit liefert, müssen die Befunde aus den (,relativ freien“) Aufnahmen vorsichtig interpretiert bzw. durch weitere Analysen ergänzt werden. ${ }^{12}$ Zwirner- und Pfeffer-Korpus liefern - wie Gesprächsdaten generell - lediglich positive Evidenzen für das in Rede stehende Phänomen, während das Nicht-Vorhandensein einer Konstruktion in einem Korpus nicht per se als ,systemlinguistisch unmöglich“ gedeutet werden kann. Denn die Realisierung eines Dativpassivs ist nicht nur an (auch regional bedingte) sprachexterne Faktoren gebunden, sondern es kommen komplexe semantisch-pragmatische Steuerungsfaktoren hinzu, die generell die Realisierung von Passivkonstruktionen steuern. Wird beispielsweise eine transferenzielle GEBEN-Handlung beschrieben, bei deren Verbalisierung gerade nicht der Rezipient perspektiviert werden soll, ist die Wahrscheinlichkeit, eine Alternativkonstruktion zum Dativpassiv zu produzieren, hoch, selbst in der arealen Kernregion des Phänomens. Im konkreten Fall des Dativpassivs können folglich in „freien“ Sprachdaten die Konkurrenzkonstruktionen nicht eindeutig ausgemacht und damit auch nicht quantifiziert werden. Deshalb sind in nicht-elizitierten Daten häufig auch lediglich die Frequenzen syntaktischer Phänomene auszumachen, ohne dass der Umfang ihres „Fehlens“ explizit gemacht werden könnte. Was also alles als Variable gezählt werden kann, die aus dem Dativpassiv und seinen Konkurrenzvarianten besteht, ist unklar.

\section{FOLK-Analysen}

Was die areale Verortung der FOLK-Aufnahmen betrifft, gibt es aktuell zwei Metadatentypen, die hierzu Informationen bereitstellen: erstens die Aufenthaltsorte der aufgenommenen Individuen sowie zweitens der Aufnahmeort. Beide Informationstypen sind - falls möglich - den Dialekträumen des deutschsprachigen Raums zugeordnet, wobei die Angaben häufig nicht miteinander korrelieren. Denn da viele FOLK-Sprecher bereits (und teilweise mehrfach) umgezogen sind, sind viele Aufnahmen mehr als einem Aufenthaltsort

12 Wie in Lenz 2013a deutlich gemacht wird, stellen etwa die großlandschaftlichen Dialektwörterbücher eine hervorragende Quelle dar, um die Zwirner-Analysen zu validieren und zu ergänzen. 
zugeordnet. Für unsere areallinguistischen Fragestellungen hilfreich ist dabei, dass verschiedene Aufenthaltsregionen einer FOLK-Gewährsperson häufig zum selben sprachlichen Großraum gehören (s. z. B. die Aufenthaltsorte „Rheinfränkische Sprachregion; Hessische Sprachregion“, die beide dem westmitteldeutschen Raum zugeordnet werden können).

In den FOLK-Aufnahmen treffen wir auf insgesamt 113 Dativpassive, unter denen sich 76 kriegen-Passive (67,3\%) und 37 bekommen-Passive (32,7\%) befinden. Die Dativpassiv-Belege verteilen sich auf 70 aufgenommene Individuen (von insgesamt 730), sodass folglich etwa jeder zehnte FOLK-Informant im Schnitt etwa 1,6 Dativpassive realisiert. 36 dieser Individuen können aufgrund ihrer Sesshaftigkeit lediglich genau einer Aufenthaltsregion zugeordnet werden; 27 übrige Sprecher sind mit bis zu sieben Aufenthaltsregionen (darunter auch „nicht dokumentierte“ Aufenthaltsorte) registriert. Sieben Informanten sind aufgrund fehlender Dokumentation überhaupt keiner Sprachregion zuzuordnen. Die 36 FOLK-Informanten, die sich aufgrund ihres eindeutigen Aufenthaltsorts einer Region zuordnen lassen, realisieren immerhin 57 und damit etwa die Hälfte aller FOLK-Dativpassive. Ihre Aufnahmen sind vor allem den folgenden Sprachräumen zuzuordnen: Ostfränkisch: 3 Belege; Schwäbisch: 4 Belege; Rheinfränkisch: 25 Belege; Obersächsisch: 5 Belege; Nordniederdeutsch: 6 Belege; Westfälisch: 11 Belege. Was darunter die 25 rheinfränkischen Belege betrifft, handelt es sich um zwei bekommen- und 22 kriegenPassive. Auf einen ersten Blick - und zunächst noch ohne Betrachtung der konkreten Auxiliare - decken sich diese FOLK-Befunde mit den Ergebnissen der Zwirner- und Pfeffer-Korpora: Die Dativpassivbelege, für die eine regionale Zuordnung aufgrund der geringen Mobilität der FOLK-Sprecher am ehesten möglich erscheint, stammen mit dem Rheinfränkischen und Westfälischen vor allem aus der dialektalen und regiolektalen Kernregion des Dativpassivs, dem Westmittel- und dem Westniederdeutschen. Angesichts der Tatsache, dass aber (mit 107 Ereignissen) die rheinfränkischen Aufnahmen insgesamt überproportional in den bisherigen FOLK-Daten repräsentiert sind, bedarf es weitergehender Evidenzen. Hierfür wird der Blick auf die 259 Sprachaufnahmen („Ereignisse“) der FOLK-Daten gelenkt, die in der DGD mit insgesamt 695 Aufenthaltsregionen ihrer Sprecher verknüpft sind. In Abbildung 8.3 ist jeder Dativpassiv-Beleg mit all den „seinem“ Ereignis zugeordneten Aufenthaltsregionen eingegangen. Frequenzen von 37,4 \% bzw. 12,1 \% für das Rheinfränkische entsprechen somit den relativen Häufigkeiten, in denen in einem (auch) dem Rheinfränkischen zugeordneten Ereignis (insgesamt 107 Ereignisse) ein kriegen- bzw. ein bekommen-Passiv vorkommt.

Eine Interpretation des gestapelten Balkendiagramms in Abbildung 8.3 muss vor dem Hintergrund der in den Daten vorliegenden areallinguistischen 


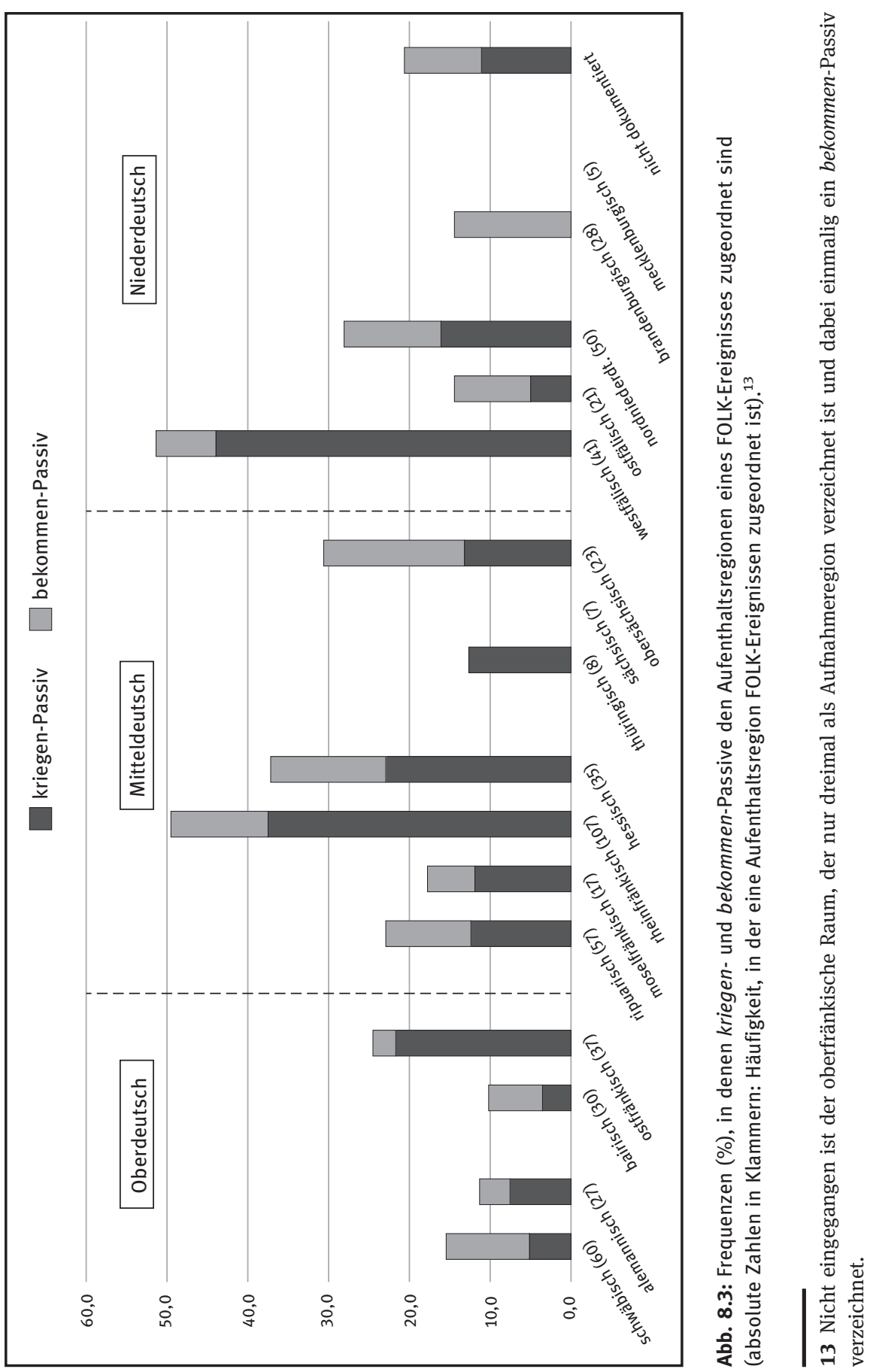


„Verzerrungen“ geschehen. Denn den FOLK-Ereignissen liegen nicht nur deutlich unterschiedliche Aufnahmelängen zugrunde, sondern in ein Ereignis gehen auch unterschiedlich viele Sprechende mit häufig jeweils variierenden Aufenthaltsregionen mit jeweils unterschiedlichen Aufenthaltslängen ein. (Als ein extremes Beispiel dient das Ereignis „FOLK_E_00121“, das Sprachaufnahmen von 14 Schülern in einer „alemannischen“ Unterrichtsstunde enthält, die außer im Alemannischen teilweise auch im Schwäbischen, Ostfränkischen und in nicht dokumentierten Regionen gelebt haben). Angesichts dieser areallinguistischen Verzerrungen ist es umso erstaunlicher, wie sich die Dativpassive auf die (häufig intraindividuell variierenden) Aufenthaltsregionen verteilen: Denn wie im Zwirner- und Pfeffer-Korpus, die mehr als 50 Jahre alte Sprachdaten von ,autochthonen“ Gewährspersonen enthalten, heben sich im FOLK-Korpus mit Rheinfränkisch (die bislang am häufigsten vertretene Aufenthaltsregion, die damit sicher auch die areallinguistisch verlässlichsten Ergebnisse liefert), Hessisch und Westfälisch gerade westmittel- und westniederdeutsche Aufenthaltsregionen als besonders Dativpassiv-affin ab. ${ }^{14}$

Die Vollverben, mit denen ein bekommen-Passiv im FOLK gebildet wird, sind die folgenden: prototypische Verben des Gebens (10×: v. a. schenken, (be)zahlen), Verben des Stellens/Setzens/Legens (8×: v. a. hin/vorlegen, unter/ hinstellen), Verben des Mitteilens (11×: v. a. sagen, erzählen) und Verben des Zeigens (7×: v. a. zeigen, vorführen). Nur einmalig tritt das Verb abfragen auf, das im weiteren Sinne als deprivatives Verb aufgefasst werden kann (FOLK S_00910 (Aufenthaltsregionen: 1. westfälisch, 2. nordniederdeutsch, 3. nicht dokumentiert): „Ich will einfach nur Fakten abgefragt bekommen und fertig.“). Hinsichtlich der kriegen-Passive im FOLK zeigen die Vollverben der Konstruktion hingegen größere semantische Breite. Neben (mehr oder weniger prototypischen) Verben des Gebens (36×: darunter v.a. schenken, (aus/be)zahlen, vererben) sind die folgenden Verbklassen vertreten: Verben der (körperlichen und verbalen) Aggression (9x: z. B. (eine) scheuern, (eine) verpassen, (eine) donnern), Verben des Stellens/Setzens/Legens (9×: z. B. stellen), dreimalig machen, Verben des Mitteilens (10x: v. a. sagen, erklären) und Verben des Zeigens ( $3 \times$ : beibringen, zeigen). Hinzu kommen immerhin sechs kriegen-Belege mit deprivativen Verben des Wegnehmens, die bei den bekommen-Passiven nur bedingt bis gar nicht auftreten. Die Aufenthaltsorte der fünf Sprecher mit diesen sechs kriegen-Belegen sind ausschließlich im Westmitteldeutschen (und hier dominierend im Rheinfränkischen) bzw. bei einem Sprecher im Westnieder-

$14 \mathrm{Zu}$ bedenken ist: Die „Mundartgruppe“ Mittelfränkisch der Zwirner- und Pfeffer-Daten entspricht den beiden FOLK-Regionen Ripuarisch und Moselfränkisch. Ebenso entspricht Rheinfränkisch der Zwirner- und Pfeffer-Daten den FOLK-Regionen Rheinfränkisch plus Hessisch. 
deutschen verankert. Folglich zeigen auch die qualitativen Befunde des FOLKKorpus in eine vergleichbare Richtung wie Zwirner- und Pfeffer-Korpora. Wie bei Pfeffer- und Zwirner-Daten stellt sich aber natürlich auch bei den FOLKDaten das Problem, dass sie lediglich positive, aber keine negativen Evidenzen für die postulierten Selektionsbeschränkungen liefern. Diesem forschungspraktischen Problem, das grundsätzlich mit freien Gesprächen einhergeht, wird weiter unten nachgegangen (Stichwort „Sprachproduktionsexperimente“).

Insgesamt lassen sich aus den drei herangezogenen oralsprachlichen Korpora offensichtlich parallele Tendenzen herausarbeiten, und das trotz ihrer unterschiedlichen Inhalte (divergierende Sprechergruppen, Gesprächstypen, Zielsetzungen $\mathrm{u}$.v. a.) und der mehr als 50 Jahre umfassenden Zeitspanne zwischen den Erhebungen. Alle drei Korpora liefern Evidenzen für die areal-horizontalen wie vertikal-sozialen Distributionen, nach denen es der westmittel- und westniederdeutsche Raum ist, in dem die Kernregion des genuin dialektal verankerten Dativpassivs innerhalb des deutschsprachigen Raums zu verankern ist. ${ }^{15}$ Während es sich im mittel- und niederdeutschen Raum areal-horizontal (in auch östlichere Regionen) sowie vertikal gesehen „nach oben“ in standardsprachnähere Registerausschnitte der vertikalen Dialekt-Standard-Achse ausbreitet bzw. (auch mit Blick auf die rezenten FOLK-Daten) schon ausgebreitet hat, steht es im oberdeutschen Raum - zumindest gesprochensprachlich - nach wie vor noch am Beginn seiner Grammatikalisierung. Besonders für den alemannischen Raum deuten ebenso Akzeptabilitätsurteile (s. ADA) darauf hin, dass die vertikale Dynamik der Konstruktion eher „,von oben nach unten“ verläuft und Dialekte erst sekundär erfasst werden. Dabei ist es das kriegen-Passiv, das regionen- und varietätenübergreifend - mit Ausnahme einiger oberdeutscher Regionen - schon weiter grammatikalisiert ist als das bekommen-Passiv. Erhalten-Passive sind gesprochensprachlich nach wie vor eine Seltenheit.

\subsubsection{Elizitierte orale Sprachdaten („Sprachproduktionsexperiment“)}

In einem nächsten Schritt werden die vorangehenden areallinguistischen Analysen „freier(er)“ gesprochensprachlicher Korpora durch stark kontrollierte Sprachdaten ergänzt. Hierzu werden die DGD-Analysen den Ergebnissen aus einem „Sprachproduktionsexperiment“ gegenübergestellt, das speziell zur Analyse des Dativpassivs konzipiert wurde und das schon mehrfach im deutschsprachigen Raum, aber auch im Niederländischen und Luxemburgischen, zum Ein-

15 Aus kontinentalwestgermanischer Perspektive ist es das westlich angrenzende Luxemburgische, in dem die Konstruktion bislang am weitesten grammatikalisiert ist, s. Lenz 2011. 
satz kam, um standardschriftsprachliche Daten bei Studierenden zu erheben. ${ }^{16}$ Im Rahmen dieses Experiments kommen kurze Videoclips zum Einsatz, in deren Zentrum ein und dieselbe (männliche) Hauptperson zu sehen ist, an der bestimmte Handlungen vollzogen werden, wie etwa einen Zahn ziehen, eine Brille auf die Nase setzen, die Haare kämmen u. a. Die Hauptperson ist somit in verschiedenen Rollen zu sehen, etwa als neutraler Empfänger einer Transferaktion, als Adressat einer Verbalaktion, als (mitunter auch als Pertinenzdativ realisierter) Benefizient einer angenehmen und positiven Handlung oder auch als Malefizient einer unangenehmen bis hin $\mathrm{zu}$ schmerzhaften Aktion. Von einer zweiten und agentiven Person, die die Handlungen an der Hauptperson ausübt, sind maximal Arme und Hände zu sehen. Die Aufgabe der Informanten besteht jeweils in der Beantwortung der Frage „Was passiert mit dem Mann?“; diese Beantwortung soll spontan und in einem einzigen, aber vollständigen Satz erfolgen. Durch die konkrete Frageformulierung sollte die Thema-Rhema-Gliederung der Informanten dahingehend beeinflusst werden, dass sie einen Antwortsatz formulieren, in dem die männliche Hauptperson als Subjektsreferent in Topikposition auftritt. Dass sich dieses Experiment über standardschriftsprachliche Analysen hinaus auch und besonders zur Elizitierung oralsprachlicher Daten aus verschiedenen vertikalen Registerausschnitten eignet, wurde und wird in drei jüngst abgeschlossenen bzw. aktuell laufenden Projekten nachgewiesen. $\mathrm{Zu}$ diesen gehört erstens das DFG-Projekt „Syntax hessischer Dialekte (www.syhd.info)“, ${ }^{17}$ bei dem neben umfangreichen indirekt eingeholten (schriftlichen) Fragebögen auch direkte Erhebungen zum Tragen kamen, in deren Rahmen mündliche Syntaxdaten zum dialektalen Pol älterer hessischer Dialektsprecher gesammelt wurden. Dabei wurden auch Dativpassivdaten auf Basis von sieben Videos bei 141 Sprechern an 140 dörflichen Ortspunkten Hessens evoziert. Die areallinguistischen Befunde zu den hessischen Basisdialekten, die im westmitteldeutschen Kernareal des Dativpassivs liegen, sind in Lenz 2016 und 2017 einzusehen. Sie belegen, dass es selbst innerhalb eines kleineren Sprachgebiets wie dem Hessischen möglich ist, auf Basis synchroner Arealstrukturen die Sedimentierungen verschiedener diachroner Grammatikalisierungsstufen einer Konstruktion nachvollziehbar zu machen. Im Folgenden wird der Fokus auf zwei weitere Studien bzw. Projekte gelegt, in deren Rahmen dasselbe Sprachproduktionsexperiment eingesetzt wurde bzw. wird, um neben Daten zum „intendierten Dialekt“ auch den „intendierten

16 Siehe Lenz 2009 und 2011.

17 Zum Projekt generell siehe Fleischer, Lenz \& Weiß 2017a und 2017b; Fleischer, Lenz \& Weiß 2015; Fleischer, Kasper \& Lenz 2012. Zum Vergleich indirekter und direkter Methoden bei SyHD, siehe Lenz 2016. 
Standard“ der befragten Individuen in den Blick zu nehmen. In beiden Studien/ Projekten kamen nicht nur ältere und prototypische Dialektsprecher, sondern auch Gewährspersonen einer jüngeren Generation zum Zuge, die hinsichtlich Bildungs- und Mobilitätsgrad variieren. Allen Gewährspersonen in beiden Projekten gemeinsam ist ihre subjektive Dialektkompetenz sowie ihre Autochthonie, die darin zum Ausdruck kommt, dass sie (wie auch mindestens ein Elternteil) den größten Teil ihres Lebens an ihrem Geburtsort verbracht haben.

- Kallenborn (2016): Bei der Wiener Dissertation von Tim Kallenborn handelt es sich um die erste im Paradigma der Marburger „Regionalsprachenforschung“ angesiedelte lokale Tiefenbohrung (s. Schmidt \& Herrgen 2011), die sich (sprech)sprachlicher Variation auf der gesamten Dialekt-StandardAchse mit einem Fokus auf Syntax widmet. Areal angesiedelt ist die Studie im moselfränkischen Ort Graach und damit im Kerngebiet des Dativpassivs. Das Dativpassiv stellt einen von insgesamt sieben Phänomenbereichen dar, die in der Studie auf Basis von freieren Gesprächsdaten sowie von (zum großen Teil eigens entwickelten) Sprachproduktionsexperimenten angegangen werden. Für die intersituative Analyse des Dativpassivs kamen insgesamt acht Videoclips (entnommen aus der Studie von Lenz 2008) zum Einsatz: Vier Videoclips wurden in einem standardsprachlichen Experiment-Durchgang eingesetzt, in dessen Rahmen die Informanten gebeten wurden, die (standardsprachlich eingesprochene) Frage „Was passiert mit dem Mann?“ auf „Hochdeutsch“ zu beantworten: „Haare schneiden“, „Blumentopf reichen“, „Banane wegnehmen“, „(aus dem Stuhl hoch)helfen“. Vier weitere und (halbwegs) vergleichbare Videoclips, die jeweils durch die (auf Band zu hörende) im Ortsdialekt gestellte identische Frage eingeleitet wurden, sollten hingegen im „Dialekt“ beschrieben werden: „Splitter (aus Finger) ziehen“, „Aschenbecher reichen“, „Hut (vom Kopf) wegziehen“, „(in den Mantel) helfen“. Durchgeführt wurden die Sprachproduktionsexperimente bei 30 Personen (15 älteren und 15 jüngeren Informanten).

- SFB „Deutsch in Österreich (DiÖ). Variation - Kontakt - Perzeption“ (FWF F060; www.dioe.at): Im Rahmen des Wiener SFB-Teilprojekts „PP03: Sprachrepertoires und Varietätenspektren“ erheben wir zurzeit an ländlichen Ortspunkten Österreichs und damit im Mittel- und Südbairischen sowie in einem kleinen alemannischen Gebiet (Vorarlberg) flächendeckend Sprachmaterial in insgesamt acht verschiedenen Erhebungssettings, in denen sowohl (mehr oder weniger) freie Gesprächsdaten (aus unterschiedlichen Kommunikationssituationen) wie auch stark elizitierte Daten aus Experimenten und Tests andererseits gewonnen werden. Aufbereitete Daten aus Sprachproduktionsexperimenten, die für den vorliegenden Beitrag 
zum Dativpassiv genutzt werden können, ${ }^{18}$ liegen bislang von 32 ,autochthonen“ Informanten (16 weiblich und 16 männlich) aus vier dörflichen Ortspunkten (unter 2.000 Einwohner) Österreichs vor: Neckenmarkt (Burgenland; mittelbairisch-südbairisches Übergangsgebiet), Allentsteig (Niederösterreich; ostmittelbairisch), Weißbriach (Kärnten: südbairisch) und Raggal (Vorarlberg; alemannisch). Eine ältere Generation (über 65 Jahre) besteht aus Rentnern, die Zeit ihres Lebens am Ort gelebt haben und ehemals einer manuellen Berufstätigkeit in unmittelbarer Nähe ihres Heimatortes nachgegangen sind. Ihnen zur Seite stehen Sprecher einer jüngeren Generation (zwischen 20 und 35 Jahre), die hinsichtlich Bildungsgrad, (regionaler) Mobilität und Art der Berufstätigkeit stärker stratifiziert ist. Die im Folgenden ausgewerteten Daten stammen aus den beiden Experimentdurchgängen, in deren Rahmen v. a. Daten zur syntaktischen Variation gesammelt werden, und zwar wie in der Kallenborn-Studie einmal im standardsprachlichen und einmal im dialektalen Spektrum der Gewährspersonen. Für den Phänomenkomplex „Dativpassiv“ kamen fünf Videoclips zum Einsatz, die die Hauptperson (und verschiedene ihrer Körperteile) im Zentrum verschiedener Handlungen (Transfer vs. Nicht-Transfer) und in unterschiedlichen semantischen Rollen zeigen. Die zu hörende Stimulusfrage „Was passiert mit dem Mann?“ wurde vorab von einem ORFNachrichtensprecher (Standard-Durchgang) bzw. von einer den jeweiligen lokalen Basisdialekt beherrschenden Person (Dialekt-Durchgang) eingesprochen. Sie wird den Informanten im Rahmen eines Experimentdurchgangs bei dem es insgesamt um verschiedene grammatische Phänomene geht, die in randomisierter Reihenfolge über einen Computer präsentiert werden - vor jedem Videoclip, der zu einer Dativpassiv-Aufgabe gehört, vorgespielt.

Bei einem Vergleich der Graacher und SFB-Daten sind folgende Dinge zu beachten: Im Korpus von Kallenborn 2016 gehen alte und junge Informanten mit jeweils 15 Personen gleich verteilt ein, während im SFB-Korpus, das ja noch im Aufbau begriffen ist, nach aktuellem Erhebungsstand die ältere Generation mit sieben und die jüngere mit 25 Personen vertreten ist. Während sich die Kallenborn-Studie auf einen einzigen Ort konzentriert, sind in die SFBDaten vier Ortspunkte aus verschiedenen Teilregionen des Bairischen bzw. Alemannischen eingegangen. Abbildung 8.4 und Abbildung 8.5 zeigen zunächst

18 Weitere syntaktische Phänomene, die bei den Sprachproduktionsexperimenten des SFB Berücksichtigung finden, sind etwa Complementizer Agreement, Relativsatzeinleitungen, Progressivkonstruktionen, „Artikelverdopplung“, Artikel vor Eigennamen, Possessivkonstruktionen und Infinitivkonstruktionen. 


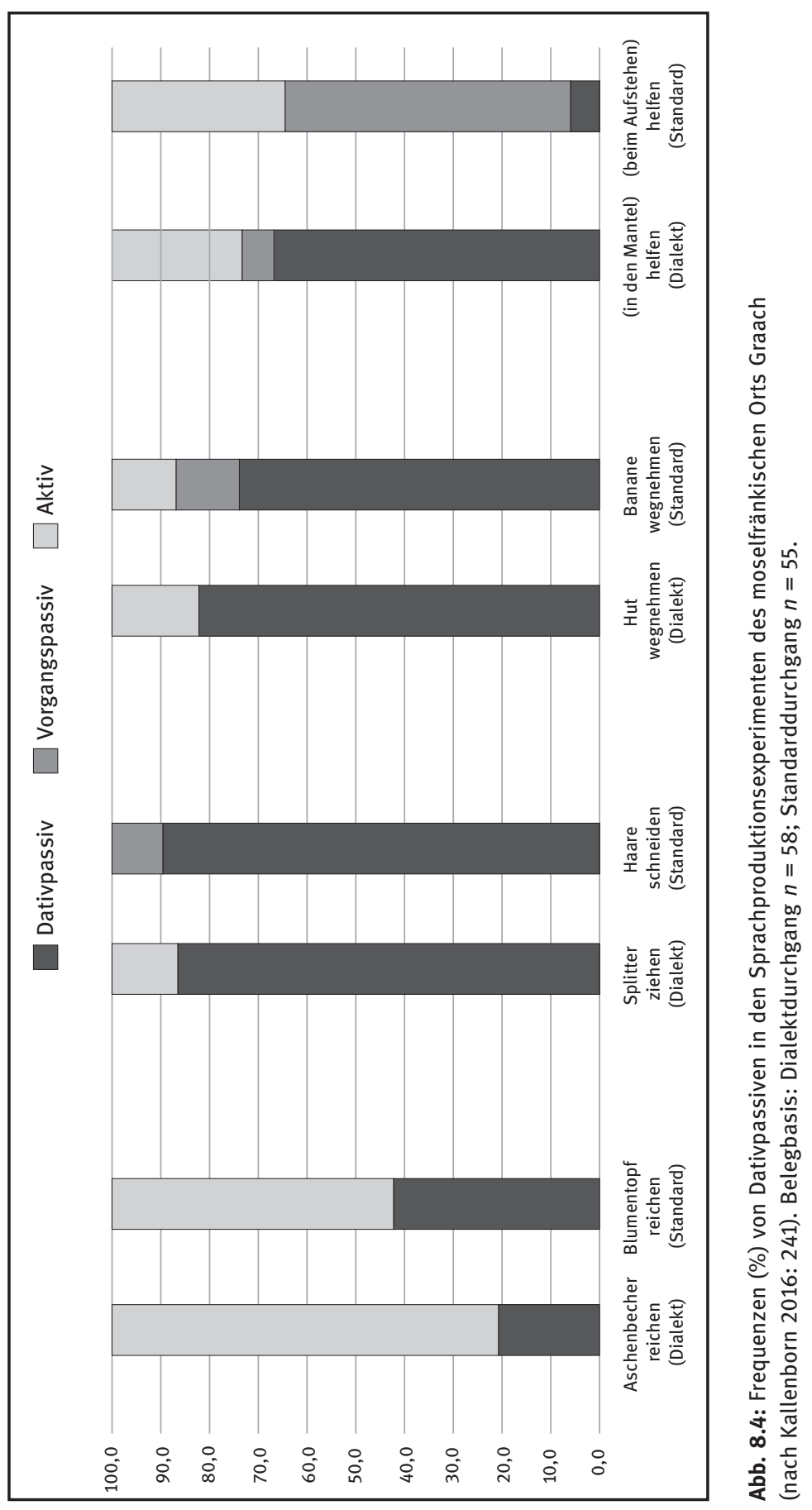




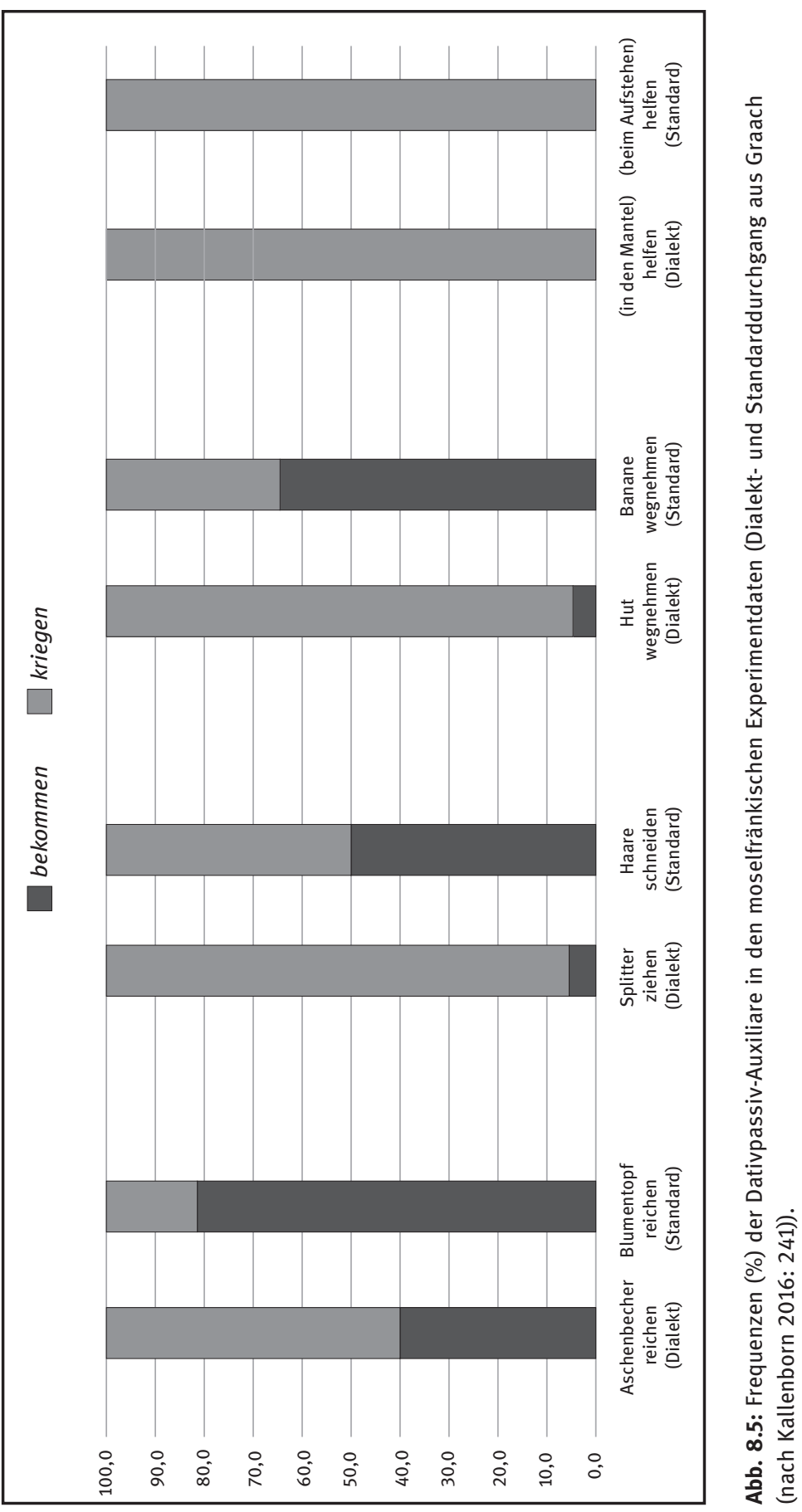


die Ergebnisse der Sprachproduktionsexperimente bei Kallenborn 2016. Dabei sind die Resultate von jeweils zwei inhaltlich vergleichbaren Videoclips in intersituativem Kontrast nebeneinander positioniert.

Wie die ersten beiden Videos fokussieren auch „Hut (vom Kopf) wegnehmen“ und „Banane (aus der Hand) wegnehmen“ eine Transferbewegung, die jedoch weg vom Vor-Possessor der transferierten Objekte geschieht und bei der der „Verlierer“ - nicht zuletzt durch Mimik und verbale Entrüstungszeichen auch eindeutig als Malefizient positioniert wird. Die beiden letzten Videos perspektivieren den Hauptdarsteller als Benefizienten einer nicht-transferenziellen Handlung. Wie in der Experimentanlage intendiert, wurden zur Beschreibung der ersten sechs abgebildeten Videos dominant ditransitive Verbkonstruktionen realisiert, während die beiden letzten Videos zur Evozierung des intransitiven Dativverbs helfen eingesetzt wurden. Die auf den ersten Blick erstaunlich niedrige Frequenz an Dativpassiven, die sich für die beiden ersten Videos ergeben (z.B. Er kriegt/bekommt einen Aschenbecher/Blumentopf über-/gereicht.), erklärt sich dadurch, dass zur Beschreibung dieser Videos vor allem Aktivkonstruktionen genutzt werden, die hier dieselben Funktionen erfüllen wie das (dann aber syntaktisch komplexere) Dativpassiv. ${ }^{19}$ Für unsere Fragestellungen interessanter sind die übrigen sechs Videoclips, aus denen folgende zentrale Ergebnisse abzulesen sind: Im Moselfränkischen sind Dativpassive mit deprivativen Verben des „Wegnehmens“ bereits voll grammatikalisiert, und zwar sowohl im Dialekt als auch im gesprochenen Standard, oder anders formuliert, sowohl mit kriegen- als auch mit bekommen-Auxiliar (s. Abb. 8.5). Ein Vergleich der Videos „Haare schneiden/Splitter ziehen“ mit den beiden helfen-Clips zeigt zudem, dass die Valenz der Vollverben auch im Moselfränkischen nach wie vor einen Einfluss auf die Frequenzen der Konstruktion hat: Obwohl in den vier Videos der Hauptdarsteller als Benefizient aus der Handlung hervorgeht, dominieren die Dativpassive intersituativ nur in Kombination mit ditransitiven Verbkonstruktionen (z. B. Er kriegt/bekommt einen Stachel aus dem Finger gezogen. bzw. Er kriegt/bekommt die Haare geschnitten.). Was intransitive Verben wie helfen betrifft, deuten die Daten auf eine bereits fortgeschrittene Grammatikalisierung des kriegen-Passivs hin (s. Dialektdaten), während kein einziger Beleg mit bekommen-Auxiliar plus Dativverb in den moselfränkischen Experiment-

19 Denn die am häufigsten realisierten Aktivkonstruktionen wie Er kriegt/bekommt einen Blumentopf. ermöglichen eine Argumentreduktion (Vernachlässigung eines Agensreferenten) sowie die gleichzeitige Übernahme der Subjektrolle, der Vorfeldposition sowie der Rolle als thematischer Gegenstand seitens des Rezipienten (vgl. Zifonun [u. a.] 1997: 1849f.). Vergleichbare Aktivkonstruktionen sind bei den übrigen Videoclips kaum möglich bzw. sie werden nur selten gebraucht (z. B. Er bekommt Hilfe.) 
daten auftritt. Hinsichtlich der Auxiliarverteilung deckt sich das Bild mit den Befunden aus den freie(re)n Gesprächsdaten der DGD-Korpora: Während kriegen das dominante Auxiliar der dialektaleren Register darstellt, überwiegt bekommen in standardsprachnäheren bzw. standardsprachlichen Kontexten, wobei die jüngeren Gewährspersonen aus Graach in dieser Varietätenzuordnung der Auxiliare weiter vorangeschritten sind als die ältere Generation (vgl. Kallenborn 2016: 246). Ein erhalten-Passiv ist in den Graacher Experimentdaten in beiden Generationen nicht belegt.

In Abbildung 8.6 werden die westmitteldeutschen Graach-Ergebnisse durch die SFB-Daten aus dem oberdeutschen Raum ergänzt. Auffallend sind zunächst einmal die insgesamt deutlich niedrigeren Frequenzen an Dativpassiven, die die Sprachproduktionsexperimente in Österreich im Vergleich zum Moselfränkischen evoziert haben. Diese deutlichen interregionalen Differenzen decken sich mit den Befunden, die aus den DGD-Korpora herausgearbeitet werden konnten. Innerhalb der SFB-Daten sind es lediglich die Videos mit Transferbewegungen in Richtung auf den benefaktiven Rezipienten, die höhere Frequenzen an Dativpassiven erreichen (z. B. Er kriegt/bekommt eine Brille aufgesetzt./Er kriegt/bekommt ein Glas Wasser eingeschenkt.) Patienshaltige Frames, in denen die Hauptperson zwar keinen prototypischen Rezipienten, aber zumindest einen Benefizienten repräsentiert, dem die Haare geschnitten werden bzw. dem ein Zahn gezogen wird, rufen nur vereinzelt Belege eines Dativpassivs hervor. Wie das Video „Banane wegnehmen“ andeutet, sind Dativpassive mit deprivativen Verben - im Gegensatz zum Westmitteldeutschen in Österreich noch nicht angekommen bzw. - mit Blick auf die geringe Datenlage vorsichtig formuliert - sehr selten. Bei den acht Videos, die österreichische Dativpassive evoziert haben, lassen sich quantitativ zwischen Dialekt- und Standarddurchgang keine signifikanten intersituativen Differenzen ablesen. Ein Blick ,hinter' die Frequenzen deckt allerdings auf, dass wir es im standardsprachlichen Durchgang lediglich mit bekommen-Passiven zu tun haben, während in den Dialektdaten ausschließlich kriegen-Passive realisiert werden. Die Varietätenzuordnung der Auxiliare scheint in den oberdeutschen Daten somit eindeutiger als in den westmitteldeutschen. Hinsichtlich der vier in die Daten eingegangenen SFB-Ortspunkte deuten sich interessante Parallelen zu den Zwirner- und Pfeffer-Ergebnissen an, die aber natürlich an einer größeren Datenmenge überprüft werden müssen: Während kriegen-Passive bislang nur in den bairischen Aufnahmeorten (Allentsteig, Neckenmarkt, Weißbriach) repräsentiert sind, kommen bekommen-Passive in den (standardsprachliche(re)n) Daten aller vier Aufnahmeorte vor, also auch im alemannischen Raggal.

Ergänzend $\mathrm{zu}$ den bisherigen Befunden soll ein Vergleich der mündlichen Sprachproduktionsdaten mit bereits schriftlich erhobenen Experimentdaten aus 


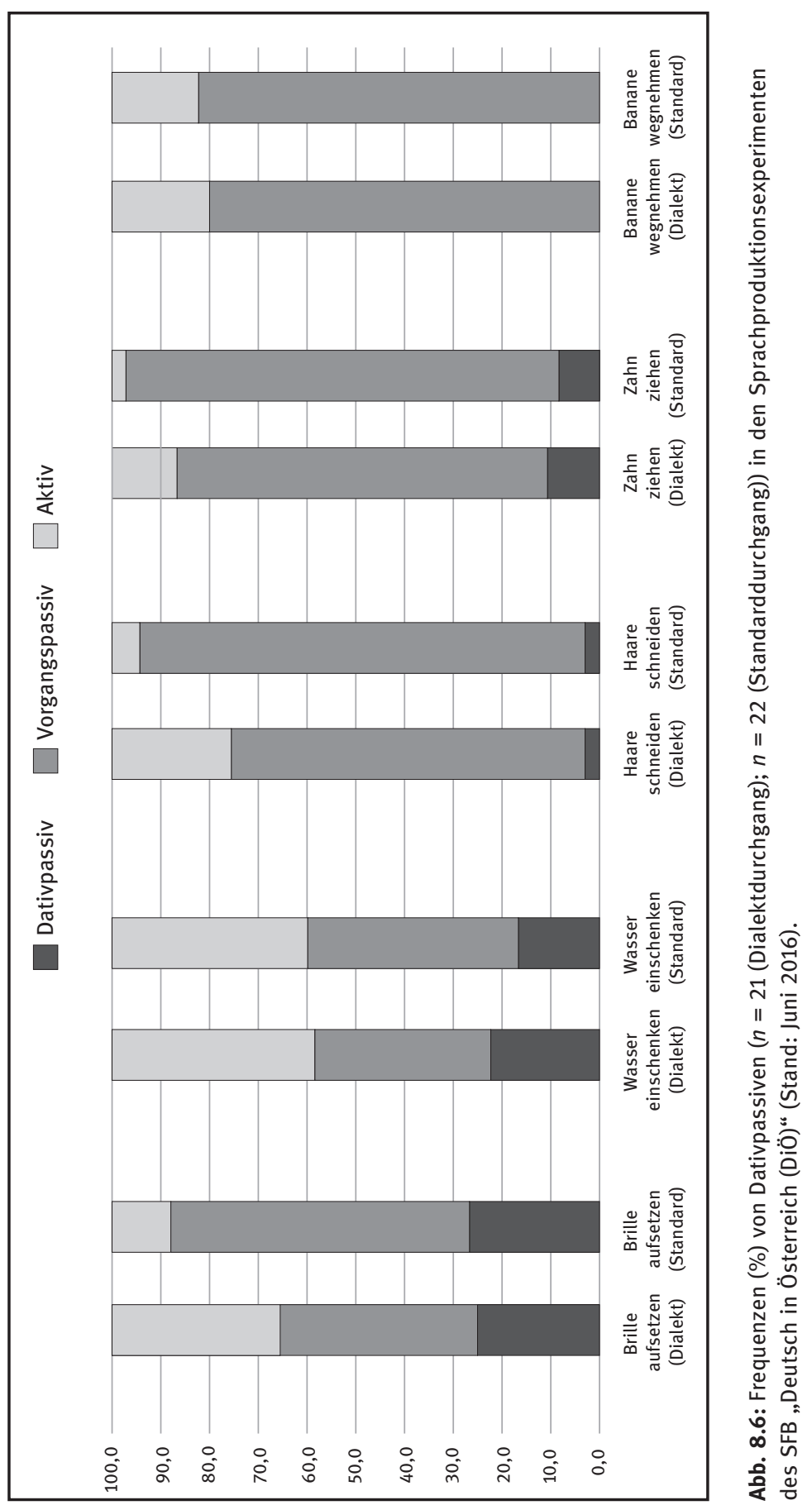


den beiden Regionen erfolgen. Hierzu werden die Ergebnisse aus Lenz 2009, die in mehreren Regionen des deutschsprachigen Raums (sowie im Luxem[dc10]?>burgischen und Niederländischen) eruiert wurden, herangezogen. Beim Vergleich der Ergebnisse ist $\mathrm{zu}$ bedenken, dass die standardschriftsprachlichen Österreich-Daten aus Lenz 2009 ausschließlich bei Studierenden der Universität Wien sowie die Moselfränkisch-Daten bei Studierenden der Universität Trier erhoben wurden, sodass hinsichtlich der soziodemographischen und arealen Kriterien Differenzen zu den aktuellen Graachund SFB-Daten bestehen. Der Vergleich in Abbildung 8.7 basiert lediglich auf den oralsprachlichen Standard-Durchgängen der Graach- und SFB-Experimente, denen die Ergebnisse aus den schriftsprachlichen Experimentdurchgängen aus Lenz 2009 gegenübergestellt sind. Das schriftsprachliche Experiment wurde auf Basis von Fragebogen durchgeführt, die den Studierenden im Rahmen von Lehrveranstaltungen vorgelegt wurden. Den Teilnehmern einer Lehrveranstaltung wurden die Videos gemeinsam vorgespielt. Die schriftlich auf den Fragebogen aufgeführte Frage „Was passiert mit dem Mann?“ musste von den Studierenden „spontan und in einem vollständigen Satz“ schriftlich beantwortet werden. In Lenz 2009 kamen insgesamt 18 Videoclips zum Einsatz, von den jeweils vier für einen Vergleich mit den gesprochensprachlichen SFB- und Graach-Daten genutzt werden können. Nicht abgefragt wurde im schriftsprachlichen Durchgang das Video „Zahn ziehen“, weswegen es für den Österreichvergleich nicht im Diagramm vertreten ist.

In beiden Regionen, moselfränkisch wie oberdeutsch, zeigen sich erstaunliche Parallelen zwischen standardsprechsprachlichen und standardschriftsprachlichen Befunden, was die Frequenzränge der Dativpassive bei den jeweils vergleichbaren Videos betrifft. Allerdings sind die Frequenzen in den schriftsprachlichen Daten insgesamt niedriger als in den gesprochensprachlichen Daten. ${ }^{20}$ Videoclips, die oralsprachlich nur geringe Frequenzen des Phänomens evoziert haben, weisen im Schriftsprachlichen noch weniger Realisierungen bzw. keine auf. Von den oralsprachlichen Graach-Daten bis hin zu den standardschriftsprachlichen Befunden im Ostoberdeutschen (Wiener Studierende) zeigt das Diagramm in Abbildung 8.7 einen kontinuierlichen Abbau der Frequenzen des Dativpassivs, der mit den konkreten Konstruktionsvarianten korreliert: Während etwa deprivative Verben des Wegnehmens standardsprechsprachlich westmitteldeutsch auftreten, sind sie schriftsprachlich auch in dieser Region zwar nachzuweisen, aber deutlich seltener. Im Ostoberdeut-

$20 \mathrm{Zu}$ den erklärbaren niedrigen Frequenzen an Dativpassiven für die Graacher Daten zum „Blumentopf“-Video s. oben. 

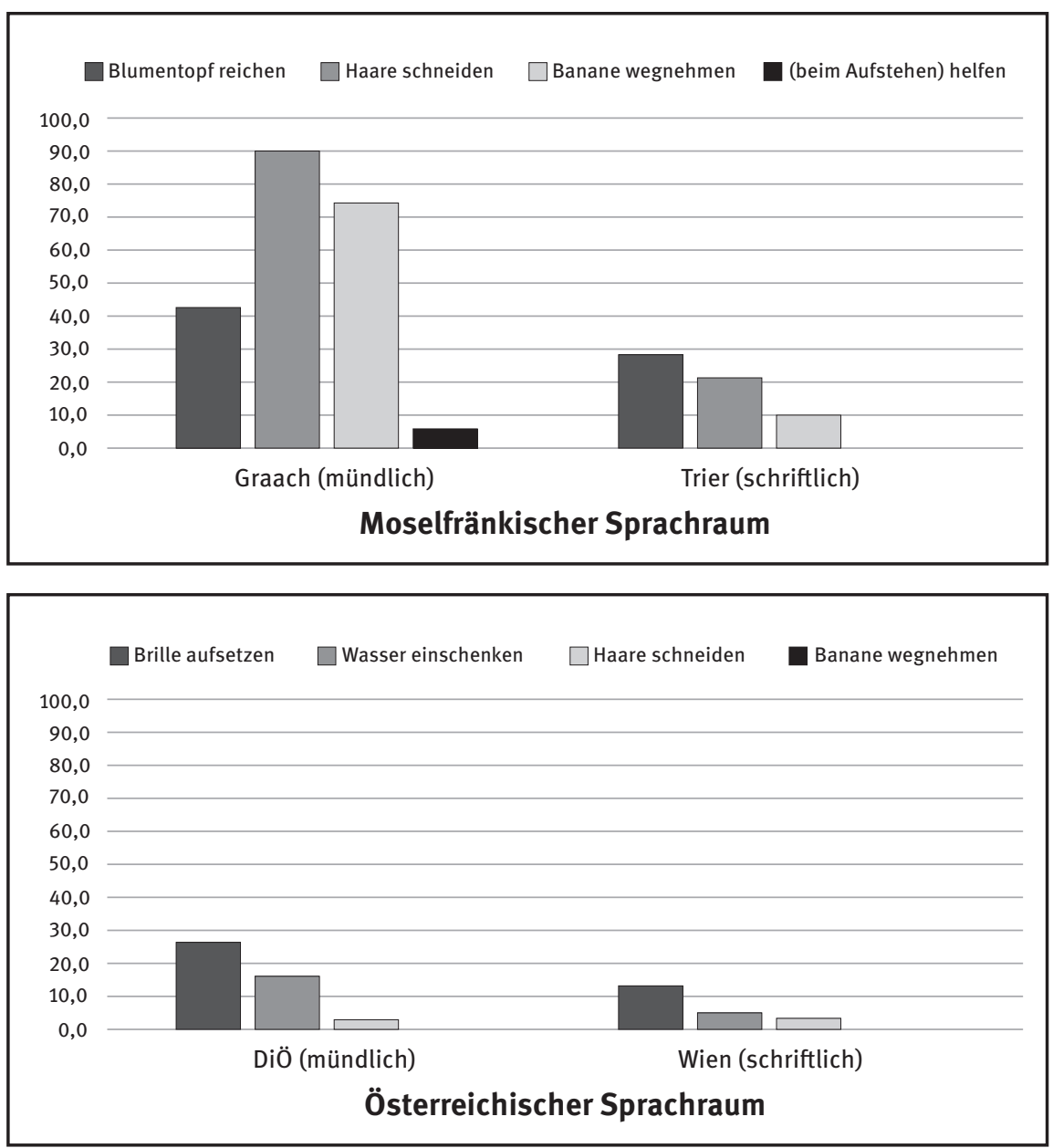

Abb. 8.7: Frequenzen (\%) von Dativpassiven im Vergleich standardsprechsprachlicher Sprachproduktionsexperimente (Kallenborn 2016 bzw. SFB-DiÖ) mit standardschriftsprachlichen Sprachproduktionsexperimenten (Lenz 2009). ${ }^{21}$

schen treten Dativpassive zwar auch schriftsprachlich auf, sie sind aber selbst auf der ersten Grammatikalisierungsstufe (mit Rezipient einer prototypischen Transferaktion) noch wenig vertreten. Dass diese Entwicklung allerdings in Zeitungstexten im oberdeutschen Raum schon weiter fortgeschritten ist,

21 Informantenanzahl: Graach (moselfränkisch): 30; Trier (moselfränkisch): 67; SFB „Deutsch in Österreich (DiÖ)“ (Österreich, bairisch und alemannisch): 32; Wien (mittelbairisch): 29. 
deuten die Analysen in Lenz 2013a auf Basis von Texten aus dem „Deutsche[n] Referenzkorpus (DeReKo)“ des IDS an. ${ }^{22}$

\section{Zusammenfassung und Ausblick}

Ziel des vorliegenden Beitrags war es, theoretische und empirische Herausforderungen einer areallinguistischen Syntaxforschung $\mathrm{zu}$ beleuchten und dabei die Ergiebigkeit dieses Forschungsfelds sowohl für die Variationslinguistik als auch für die Grammatikforschung (und -schreibung) der Germanistik (und darüber hinaus) herauszuarbeiten. Dies geschah unter Rückgriff auf Fragestellungen, methodische Herangehensweisen und Ergebnisse aus jüngsten Studien im deutschsprachigen Raum. Der Diskussion wurde mit dem Dativpassiv ein konkretes syntaktisches Phänomen zugrunde gelegt, dessen Auswahl u. a. durch seine variationsreiche areal-horizontale wie vertikal-soziale Distribution, seine linguistische Komplexität (s. syntakto-semantische Selektionsbeschränkungen) und seine „in vivo“ nachvollziehbare Dynamik motiviert wurde. Aus der Fülle an areallinguistisch relevanten Fragestellungen, die mit dem Dativpassiv einhergehen, wurden im vorliegenden Beitrag methodische Aspekte fokussiert, wobei der Schwerpunkt auf gesprochensprachlicher Variation lag. ${ }^{23}$ An zentralen Ergebnissen sind die folgenden festzuhalten:

Arealität ist ein zentraler extralinguistischer Parameter, der syntaktische Variation mitsteuert, sowohl was die Gebrauchshäufigkeiten und Akzeptabilitätsgrade eines Phänomens generell betreffen, als auch hinsichtlich der Selektionsbeschränkungen, denen ein Phänomen unterliegt und die arealhorizontal, vertikal-sozial wie medial-konzeptionell deutlich variieren können. Die Bedeutung der arealen Variationsdimension ist nicht nur für die Syntax der „klassischen“ areal verankerten Varietäten, der Dialekte, hervorzuheben, sondern ebenso für die regiolektale Syntax des mittleren Bereichs bis hin zu standardsprachlichen Registern der Oralität und Schriftlichkeit. Wie am

22 Doch auch wenn die Analysen der Niederösterreichischen Nachrichten sogar ähnlich hohe Frequenzen von bekommen-Passiven wie die Rhein-Zeitung erbringen (vgl. Lenz 2013a: 236), ähneln die qualitativen Befunde den Ergebnissen der obigen Analysen der DGD-Korpora: In den analysierten bekommen-Passiven der Niederösterreichischen Nachrichten aus dem Jahr 2009 treten ausschließlich bekommen-Passive mit ditransitiven Verben des Gebens, Mitteilens, Zeigens und Stellens/Setzens/Legens auf, während die Rhein-Zeitung auch Belege mit deprivativen Verben verzeichnet (s. Punkte abgezogen bekommen, 7.1. 2009; Punkte aberkannt bekommen, 5.1. 2009).

$23 \mathrm{Zu}$ Befunden in schriftsprachlichen Korpora (Chat-Korpus, DeReKo) sei auf Lenz 2013a verwiesen. 
Beispiel des Dativpassivs exemplifiziert werden konnte, lassen sich areale Strukturen im deutschsprachigen Raum über die gesamte vertikale DialektStandard-Achse hinweg aufspüren. Dabei ist die Richtung der Dynamik häufig, aber nicht immer, „von unten nach oben“, also von dialektaleren Registern in standardsprachliche hinein. Mitunter wandern Phänomene auch von der Standardsprache gestützt erst langsam „,von oben“ in andere „tiefere“ Varietäten des vertikalen Gesamtspektrums. Nicht immer ist es also die in grammatikographischen Werken häufiger angeführte (und leider in der Regel varietätenlinguistisch nicht näher differenzierte) „Umgangssprache“, in denen die Grammatikalisierung eines syntaktischen Phänomens schon weiter fortgeschritten ist als in der Standard(schrift)sprache. Nur durch flächendeckende und komplexe Analysen ist eine notwendige Modifikation und Konkretisierung von Attribuierungen wie „regional“, „umgangssprachlich“ oder „gesprochensprachlich“ im Hinblick auf die areal-horizontale, vertikal-soziale wie auch diamesische (konzeptionell-mediale) Variation möglich.

Um der areallinguistischen Variation eines syntaktischen Phänomens in seiner Komplexität Rechnung zu tragen, bedarf es multivariater Herangehensweisen: Im vorliegenden Beitrag wurde der Fokus auf die gesprochensprachliche Variation gelegt, wobei zwei verschiedene methodische Zugänge bzw. Datentypen gewählt wurden: Zum einen wurde der Variation des Dativpassivs (und seiner Konkurrenzvarianten) auf Basis dreier DGD-Korpora nachgegangen, die hinsichtlich ihrer Zielsetzungen, der konkreten Inhalte und sprechenden Individuen, der Zeitlichkeit und anderer Parameter deutlich differieren, deren kontrastiver Vergleich dennoch erstaunliche Parallelen bezüglich des analysierten Beispielphänomens aufdeckt. Eine Ergänzung korpuslinguistischer Analysen auf Basis freier Gesprächsdaten durch elizitierte Methoden, wie es im vorliegenden Beitrag durch den Einsatz von Sprachproduktionsexperimenten geschehen ist, wird nach wie vor nötig sein, um neben extralinguistischen Parametern auch den syntakto-semantischen Steuerungsfaktoren syntaktischer Phänomene auf den Grund gehen zu können. Die Kontrolle bestimmter soziolinguistischer, situativ-pragmatischer wie linguistischer Steuerungsfaktoren ermöglicht es zudem, die realisierten Konstruktionen einer Szenenbeschreibung als „echte“ Varianten einer syntaktischen Variable zu zählen. Dadurch wird es nicht nur möglich, die Gebrauchshäufigkeit eines Phänomens auf die Größe des zugrundeliegenden Datenmaterials zu beziehen, sondern auch im Verhältnis zu realisierten Konstruktionsalternativen zu berechnen. Damit leistet das skizzierte Sprachproduktionsexperiment auch einen wesentlichen Beitrag zur Modellierung syntaktischer Variablen.

Zusammengefasst schließe ich mit einem Plädoyer, die areale Variationsdimension innerhalb der Syntaxforschung und Grammatikographie in deutlich 
stärkerem Maße als bisher geschehen in den Blick zu nehmen und dabei multivariate Zugänge zu wählen.

\section{Literatur}

Abraham, Werner (1985): Grammatik von kriegen und bekommen. Osnabrücker Beiträge zur Sprachtheorie 30, 142-165.

Abraham, Werner (1991): Aktionsartsemantik und Auxiliarisierung im Deutschen. In Elisabeth Feldbusch, Reiner Pogarell \& Cornelia Weiss (Hrsg.), Neue Fragen der Linguistik. Akten des 25. Linguistischen Kolloquiums, Band I: Bestand und Entwicklung (Linguistische Arbeiten 270), 125-133. Paderborn 1990. Tübingen: Niemeyer.

$\mathrm{AdA}=$ Atlas zur deutschen Alltagssprache (2003 ff.). Von Stephan Elspass und Robert Möller. www.atlas-alltagssprache.de/ (letzter Zugriff: 15.11. 2017).

Askedal, John Ole (1984): Zum kontrastiven Vergleich des sogenannten „bekommen/ erhalten/kriegen-Passivs“ im Deutschen und entsprechender norwegischer Fügungen aus få und dem Partizip Perfekt. Norsk Lingvistisk Tidsskrift 2, 133-166.

Askedal, John Ole (2005): Grammatikalisierung und Persistenz im deutschen „RezipientenPassiv“ mit bekommen/kriegen/erhalten. In Torsten Leuschner, Tanja Mortelmans \& Sarah De Groodt (Hrsg.), Grammatikalisierung im Deutschen (Linguistik - Impulse und Tendenzen 9), 211-228. Berlin/New York: De Gruyter.

Auer, Peter (2005): Europe's sociolinguistic unity, or: A typology of European dialect/ standard constellations. In Nicole Delbecque, Johan van der Auwera \& Dirk Geeraerts (Hrsg.), Perspectives on variation: Sociolinguistic, historical, comparative (Trends in Linguistics, Studies and Monographs 163), 8-42. Berlin/New York: De Gruyter.

Breuer, Ludwig Maximilian (2017): Computers \& Coffee: Computergestützte Sprachproduktionstests zur syntaktischen Variation des „unbestimmten Artikels vor Massennomen“ im „Wienerischen“. In International Forum on Audio-Visual Research (Jahrbuch des Phonogrammarchivs 7), 86-111. Wien: Verlag der österreichischen Akademie der Wissenschaft.

Bucheli Berger, Claudia (2005): Passiv im Schweizerdeutschen. In Helen Christen (Hrsg.), Dialektologie an der Jahrtausendwende (Linguistik online 24, 3/05). www.linguistikonline.de/24_05/bucheli.html (letzter Zugriff: 15.11. 2017).

Cheshire, Jenny (1987): Syntactic variation, the linguistic variable, and sociolinguistic theory. Linguistics 25 (2), 257-282.

Cheshire, Jenny (2005): Syntactic variation and beyond: Gender and social class variation in the use of discourse-new markers. Journal of Sociolinguistics 9 (4), 479-508.

Cook, Philippa (2004): The datives that aren't born equal. Beneficiaries and the dative passive. In David Hole, André Meinunger \& Werner Abraham (Hrsg.), Datives and other cases. Between argument structure and event structure, 141-184. Amsterdam: Benjamins.

Deppermann, Arnulf, Stefan Kleiner \& Ralf Knöbl (2013): 'Standard usage’: Towards a realistic conception of spoken standard German. In Peter Auer, Javier Caro Reina \& Göz Kaufmann (Hrsg.), Language variation - European perspectives IV. Selected papers from the Sixth International Conference on Language Variation in Europe (ICLaVE 6), Freiburg, June 2011 (SILV 14), 83-116. Amsterdam/Philadelphia: Benjamins. 
Diedrichsen, Elke (2008): The grammaticalization of the bekommen-passive in a RRGperspective. In Rolf Kailuweit et al. (Hrsg.), New applications of role \& reference grammar: Diachrony, grammaticalization, Romance languages, 87-145. Cambridge: Cambridge Scholars Publishing.

Diedrichsen, Elke (2012): What you give is what you GET? On reanalysis, semantic extension and functional motivation with the German bekommen-passive construction. In Alexandra N. Lenz \& Gudrun Rawoens (Hrsg.), The Art of getting: GET verbs in European languages from a synchronic and diachronic point of view (Special Issue of Linguistics, volume 50/6), 1163-1204.

Duden 4 (2016) = Duden. Die Grammatik. Unentbehrlich für richtiges Deutsch. 9., vollständig überarbeitete und aktualisierte Auflage. Duden 4. Mannheim/Wien/Berlin: Dudenverlag.

Duden 9 (2016) = Duden. Das Wörterbuch der sprachlichen Zweifelsfälle. Richtiges und gutes Deutsch. Von „anscheinend/scheinbar“ bis „zumindest/mindestens“. 8., vollständig überarbeitete und erweiterte Auflage. Duden 9. Mannheim/Wien/Berlin: Dudenverlag.

Eichinger, Ludwig Maximilian \& Werner Kallmeyer (Hrsg.) (2005): Standardvariation: Wie viel Variation verträgt die deutsche Sprache? (Jahrbuch des Instituts für deutsche Sprache 2004). Berlin: De Gruyter.

Elspaß, Stephan \& Christa Dürscheid (2017): Areale Variation in den Gebrauchsstandards des Deutschen. In Marek Konopka \& Angelika Wöllstein (Hrsg.), Grammatische Variation. Empirische Zugänge und theoretische Modellierung (Jahrbuch des Instituts für Deutsche Sprache 2016), 85-104. Berlin/Boston: De Gruyter.

Eroms, Hans-Werner (1978): Zur Konversion der Dativphrasen. Sprachwissenschaft 3, 357405.

Eroms, Hans-Werner (2000): Syntax der deutschen Sprache. Berlin/New York: De Gruyter.

Eroms, Hans Werner, Birgit Röder \& Rosemarie Spannbauer-Pollmann (2006): Sprachatlas von Niederbayern (SNiB). Bd. 1: Einführung mit Syntaxauswertung. In Hans Werner Eroms (Hrsg.), Bayerischer Sprachatlas. Regionalteil V. Heidelberg: Winter.

Fleischer, Jürg, Simon Kasper, \& Alexandra N. Lenz (2012): Die Erhebung syntaktischer Phänomene durch die indirekte Methode: Ergebnisse und Erfahrungen aus dem Forschungsprojekt „Syntax hessischer Dialekte“ (SyHD). Zeitschrift für Dialektologie und Linguistik 79 (1), 1-42.

Fleischer, Jürg, Alexandra N. Lenz \& Helmut Weiß (2015): Das Forschungsprojekt „Syntax hessischer Dialekte (SyHD)“. In Roland Kehrein, Alfred Lameli \& Stefan Rabanus (Hrsg.), Regionale Variation des Deutschen - Projekte und Perspektiven, 261-287. Berlin/ Boston: De Gruyter.

Fleischer, Jürg, Alexandra N. Lenz \& Helmut Weiß (2017a): SyHD-atlas. Konzipiert von Ludwig M. Breuer unter Mitarbeit von Katrin Kuhmichel, Stephanie Leser-Cronau, Johanna Schwalm und Thomas Strobel. Marburg/Frankfurt am Main/Wien: dx.doi.org/10.17192/ es2017.0003

Fleischer, Jürg, Alexandra N. Lenz \& Helmut Weiß (2017b): SyHD-atlas: Einführung. In SyHD-atlas. www.syhd.info (letzter Zugriff: 30.11. 2017).

Glaser, Elvira (2005): Krieg und kriegen: zur Arealität der BEKOMMEN-Periphrasen. In Ulla Kleinberger Günther, Annelies Häcki Buhofer \& Elisabeth Piirainen (Hrsg.), „Krieg und Frieden "- Auseinandersetzung und Versöhnung in Diskursen, 43-64. Tübingen: Francke.

Hasty, Daniel J. (2014): We might should be thinking this way: Theory and practice in the study of syntactic variation. In Raffaella Zanuttini \& Laurence R Hirn. (Hrsg.), Microsyntactic variation in North American English (Oxford Studies in Comparative Syntax), 269-293. Oxford, NY: Oxford University Press. 
Kallenborn, Tim (2016): Regionalsprachliche Syntax: Horizontal-vertikal Variation im Moselfränkischen. Dissertation. Universität Wien.

Kasper, Simon (2017): Passiv, Possession und Belebtheit. In: SyHD-atlas. www.syhd.info/ apps/atlas/\#grammatikalisierungspfade-grade (letzter Zugriff: 15.11. 2017).

Lavandera, Beatrize R. (1978): Where does the sociolinguistic Variable stop? Language in Society 7 (2), 171-182.

Leirbukt, Oddleif (1997): Untersuchungen zum bekommen-Passiv im heutigen Deutsch (Reihe Germanistische Linguistik 177). Tübingen: Niemeyer.

Lenz, Alexandra N. (2003): Struktur und Dynamik des Substandards. Eine Studie zum Westmitteldeutschen (Wittlich/Eifel) (Zeitschrift für Dialektologie und Linguistik. Beihefte 125). Stuttgart: Steiner.

Lenz, Alexandra N. (2004): Zur Interpretation des Intendierten Ortsdialekts. In Alexandra N. Lenz, Edgar Radtke \& Simone Zwickl (Hrsg.), Variation im Raum. Variation and Space (VarioLingua 20), 113-131. Frankfurt am Main: Peter Lang.

Lenz, Alexandra N. (2007): Zur variationslinguistischen Analyse regionalsprachlicher Korpora. In Werner Kallmeyer \& Gisela Zifonun (Hrsg.), Sprachkorpora. Datenmengen und Erkenntnisfortschritt (Jahrbuch des Instituts für Deutsche Sprache 2006), 169-202. Berlin: De Gruyter.

Lenz, Alexandra N. (2008): Wenn einer etwas gegeben bekommt. Ergebnisse eines Sprachproduktionstests zum Rezipientenpassiv. In Franz Patocka \& Guido Seiler (Hrsg.), Morphologie und Syntax der Dialekte (Sammelband der Sektion „Morphologie und Syntax“ der IGDD-Jahrestagung 2006 in Wien), 155-178. Wien: Edition Präsens.

Lenz, Alexandra N. (2009): On the perspectivization of a recipient role - Crosslinguistic results from a speech production experiment. In Marc Fryd (Hrsg.), The passive in Germanic languages (Groninger Arbeiten zur germanistischen Linguistik (GAGL), Bd 49), 125-144. [http://gagl.eldoc.ub.rug.nl/root/2009-49/2009-49-06/]

Lenz, Alexandra N. (2010a): Emergence of varieties through restructuring and reevaluation. In Peter Auer \& Jürgen Erich Schmidt (Hrsg.), Language and space. An international handbook of linguistic variation. Volume 1: Theories and methods, 295-315. De Gruyter Mouton.

Lenz, Alexandra N. (2010b): Zum Begriff der Salienz und zum Nachweis salienter Merkmale. In Markus Hundt et al. (Hrsg.), Perceptual dialectology - Neue Wege der Dialektologie (Linguistik - Impulse \& Tendenzen), 89-110. Berlin/New York: De Gruyter.

Lenz, Alexandra N. (2011): Zum kréien-Passiv und seinen „Konkurrenten“ im schriftlichen und mündlichen Luxemburgischen. In Peter Gilles \& Melanie Wagner (Hrsg.), Linguistische und soziolinguistische Bausteine der Luxemburgistik (Mikroglottika 4), 5-27. Frankfurt am Main: Peter Lang.

Lenz, Alexandra N. (2012): On the genesis of the German recipient passive - Two competing hypotheses in the light of current dialect data. In Gunther de Vogelaer \& Guido Seiler (Hrsg.), The dialect laboratory. Dialects as a testing ground for theories of language change (SLCS128), 121-138. Amsterdam: Benjamins.

Lenz, Alexandra N. (2013a): Vom ,kriegen“ und ,bekommen‘. Kognitiv-semantische, variationslinguistische und sprachgeschichtliche Perspektiven (Linguistik - Impulse \& Tendenzen 53). Berlin/New York: De Gruyter.

Lenz, Alexandra N. (2013b): Three “competing auxiliaries" of a non-canonical passive On the German GET passive and its auxiliaries. In Alexiadou Artemis \& Florian Schäfer (Hrsg.), Non-canonical passives, 63-94. Amsterdam/Philadelphia: Benjamins. 
Lenz, Alexandra N. (2016): On eliciting dialect-syntactic data. Comparing direct and indirect methods. In Augustin Speyer \& Philipp Rauth (Hrsg.), Syntax aus Saarbrücker Sicht. Beiträge der SaRDiS-Tagung zur Dialektsyntax (Zeitschrift für Dialektologie und Linguistik. Beihefte 165), 187-219. Stuttgart: Steiner.

Lenz, Alexandra N. (2017): „Dativpassiv“ (kriegen-Passiv). In SyHD-atlas. www.syhd.info/ apps/atlas/\#dativpassiv (letzter Zugriff: 15.11. 2017).

Lenz, Alexandra N., Timo Ahlers \& Martina Werner (2015): Zur Dynamik bairischer Dialektsyntax - Eine Pilotstudie. Zeitschrift für Dialektologie und Linguistik 81 (1), $1-33$.

Lenz, Alexandra N. \& Patocka, Franz (Hrsg.) (2016): Syntaktische Variation. Areallinguistische Perspektiven (Wiener Arbeiten zur Linguistik 2). Göttingen: Vienna University Press.

Lenz, Alexandra N. \& Albrecht Plewnia (Hrsg.) (2010): Grammar between Norm and Variation (VarioLingua 40). Frankfurt am Main: Peter Lang.

Lüdeling, Anke (2017): Variationistische Korpusstudien. In Marek Konopka \& Angelika Wöllstein (Hrsg.), Grammatische Variation. Empirische Zugänge und theoretische Modellierung (Jahrbuch des Instituts für Deutsche Sprache 2016), 129-144. Berlin: De Gruyter.

Macha, Jürgen (1991): Der flexible Sprecher. Untersuchungen zu Sprache und Sprachbewußtsein rheinischer Handwerksmeister. Köln u. a.: Böhlau.

Molnárfi, Laszlo (1998): Kasusstrukturalitat und struktureller Kasus - zur Lage des Dativs im heutigen Deutsch. Linguistische Berichte 176, 535-580.

Purschke, Christoph (2014): „I remember it like it was interesting“: Zur Theorie von Salienz und Pertinenz. Linguistik Online 66 (4), 31-50. https://bop.unibe.ch/linguistik-online/ article/view/1571 (letzter Zugriff: 15.11. 2017).

Schmidt, Jürgen \& Joachim Herrgen (2011): Sprachdynamik. Eine Einführung in die moderne Regionalsprachenforschung (Grundlagen der Germanistik 49). Berlin: Erich Schmidt.

SyHD = Syntax hessischer Dialekte, s. oben Fleischer, Lenz \& Weiß (2017a)

Teuber, Oliver (2005): Analytische Verbformen im Deutschen. Syntax - Semantik Grammatikalisierung (Germanistische Linguistik. Monographien 18). Hildesheim u. a.: Olms.

Zifonun, Gisela et al. (1997): Grammatik der deutschen Sprache (Schriften des Instituts für deutsche Sprache 7.1-7.3). 3 Bände. Berlin/New York: De Gruyter. 

IV Kontrastive Grammatik, Typologie und Wandel 



\title{
Christiane von Stutterheim \\ 9 Kontrastive Analyse 2020: Neue Horizonte
}

\begin{abstract}
In dem Beitrag wird für eine Weiterentwicklung der Kontrastiven Analyse argumentiert, die unter Anwendung empirisch-experimenteller Methoden die konzeptuelle Ebene als Basis des Sprachvergleichs heranzieht. Kognitivorientierte kontrastive Analysen bereiten den Weg, um unser Wissen über die Funktionsweise einzelsprachlicher Systeme in unterschiedlichen Bereichen zu erweitern. Zielsetzungen, Methoden und Erkenntniswege werden durch Ergebnisse aus kontrastiven Studien zum Textaufbau, zur Konzeptualisierung von Bewegungsereignissen und zum Grad der Grammatikalisierung des Verbalaspekts illustriert.
\end{abstract}

Keywords: Kontrastive Analyse, psycholinguistische Methoden, Sprache und Kognition, Sprachvergleich, tertium comparationis

\section{Einführung}

Kontraste spielen in vielen Bereichen des menschlichen Wahrnehmens und Erkennens eine zentrale Rolle. Sie sind für die visuelle Wahrnehmung von entscheidender Bedeutung. Kontraste in Farbe, Oberfläche und Form bilden die Grundlage für das Erkennen von Gestalten. Auch in der auditiven Wahrnehmung ist das Erkennen von Kontrasten notwendig, um beispielsweise lautliche Zeichen $\mathrm{zu}$ unterscheiden. Wesentlich für die Identifikation von Kontrasten ist die Bestimmung einer Vergleichsdimension. So ist der kontrastierenden Betrachtung notwendig eine vergleichende vorausgesetzt. In der Sprachwissenschaft hat die vergleichende Methode eine lange Tradition. Im 19. Jahrhundert zielte der Sprachvergleich in erster Linie auf die Identifikation von Gemeinsamkeiten in der Vielfalt der sprachlichen Formen ab. Die historisch-vergleichenden Arbeiten eines Bopp (1816), Rask (1818) oder Grimm (1819) befassten sich mit zahlreichen europäischen Sprachen, um die synchron vorliegenden Unterschiede auf Gemeinsamkeiten historisch vorangehender

Christiane von Stutterheim, Universität Heidelberg, Institut für Deutsch als Fremdsprachenphilologie, Plöck 55, D-69117 Heidelberg, E-Mail: cvs@idf.uni-heidelberg.de

Ә Open Access. (C) 2018 Christiane von Stutterheim, publiziert von De Gruyter. (c) BY Dieses Werk ist lizenziert unter der Creative Commons Attribution 4.0 Lizenz.

https://doi.org/10.1515/9783110490992-010 
Sprachzustände zurückzuführen und so Prinzipien der Sprachentwicklung zu identifizieren. Die Vergleichsdimension war in dieser Phase der sich herausbildenden Sprachwissenschaft in erster Linie auf der lautlichen und morphologischen Ebene verankert. Während die historisch vergleichenden Studien ihren Fokus auf die Rekonstruktion von Gleichheiten legten, setzt Humboldt etwa zur gleichen Zeit die Methode des Sprachvergleichs ein, um auf der Grundlage empirischer Untersuchungen die Besonderheiten von Einzelsprachen zu ermitteln. Die Vergleichsdimensionen bleiben dabei weitgehend dieselben. Es geht um formale Eigenschaften auf den Ebenen der Phonologie, Morphologie und Syntax. Anders als die zeitgenössische sprachhistorische Forschung standen für Humboldt die Verschiedenheiten im Zentrum des Interesses. Dabei setzte er sich auch kritisch mit dem Problem eines klar bestimmten tertium comparationis auseinander.

Denn was der allgemeinen Sprachkunde noch vorzüglich abgeht, ist, dass man nicht hinlänglich in die Kenntnis der einzelnen Sprachen eingedrungen ist, da doch sonst die Vergleichung noch so vieler nur wenig helfen kann. Man hat genug zu thun geglaubt, wenn man einzelne abweichende Eigenthümlichkeiten der Grammatik anmerkte, und mehr, oder weniger zahlreiche Reihen von Wörtern mit einander verglich. (von Humboldt 19031936: 11).

Am Beispiel des Baskischen zeigt W. v. Humboldt (Hurch 2012), dass ein rein auf der Formebene verankerter Vergleich fehlgeleitet sein kann. Aus heutiger Sicht ist Humboldt seiner Zeit weit voraus, wenn er fordert, dass sich eine vergleichende Betrachtung nicht nur auf bestimmte formale Eigenschaften wie beispielsweise morphologische Kennzeichnungen unterschiedlicher Wortarten beschränken darf. Vielmehr nimmt er an, dass kategoriale Unterschiede auch auf der Ebene des Verstandes vorhanden sein können, ohne im sprachlichen Ausdruck ein Korrelat aufzuweisen. Als Beispiel führt er die Unterscheidung in Nomina und Adjektive an, die im Baskischen morphologisch nicht gekennzeichnet wird, die jedoch als Teil dessen, was das Wesen jeder Sprache ausmacht, in der Vorstellung vorhanden sein muss.

Sobald die Sprache sie aber nicht auch durch besondere charakteristische Merkmale wirklich bezeichnet, so sind sie nicht eigentlich in ihr, sondern nur in dem Verstande und der Einbildungskraft des Redenden $u$. Verstehenden vorhanden. (von Humboldt 2012: 215)

In moderner linguistischer Terminologie könnte man sagen, dass Humboldt mit dieser These die kognitive Ebene als maßgeblich für den Sprachvergleich bestimmt. Es sollte jedoch noch nahezu 200 Jahre dauern, bis diese Einsicht Eingang in die systematische sprachvergleichende Forschung fand.

Zunächst folgen wir der Entwicklung weiter chronologisch. Eine neue Bedeutung für die sprachwissenschaftliche Forschung gewinnt der Begriff des 
Kontrasts im Rahmen des Strukturalismus. Die Abkehr von der historischvergleichenden Methode hin zu einer synchronen Linguistik ist mit der Begrenzung des Forschungsgegenstandes auf die langue, das Sprachsystem an sich, verbunden (Saussure 1967). Gemäß dem strukturalistischen Programm ist es die Aufgabe der Sprachwissenschaft, diejenigen Einheiten auf den unterschiedlichen Ebenen zwischen Phonem und Satz zu identifizieren, die in dem jeweiligen Sprachsystem funktional sind. Die Methode, diese Einheiten zu ermitteln, ist die der Kontrastierung. Jeweils bezogen auf eine bestimmte Ebene des sprachlichen Systems - Phonologie, Morphologie, Syntax - werden Einheiten gegen andere ausgetauscht. Ergibt sich dadurch eine neue, in der Sprache akzeptable Einheit, so stehen diese in Kontrast oder auch Opposition zueinander. Die Vergleichsgröße ist in dieser Herangehensweise durch die Position der Einheiten innerhalb der syntagmatischen Kette gegeben.

Was zunächst auf einzelsprachliche Systeme bezogen wird, erfährt dann eine Erweiterung, indem unterschiedliche sprachliche Systeme miteinander kontrastiert werden. Dieser Ansatz, der mit dem Terminus Kontrastive Analyse (Contrastive Analysis) belegt wurde, erhielt seinen wesentlichen Impuls aus der Sprachlehrforschung. Lado (1957) entwickelte in seinem Werk Linguistics Across Cultures die Idee, dass Kontraste zwischen Sprachen zu Lernschwierigkeiten im Fremdsprachenerwerb führen sollten, während Übereinstimmung Lernerleichterung bedeuten sollte. In der Folge etablierte sich die Kontrastive Analyse als fester Bestandteil von fremdsprachendidaktischen Ansätzen. Sprachkontrastierende Untersuchungen dienten in diesem Kontext in erster Linie dem Ziel, Sprachlerner einer Sprache L2 mit Muttersprache L1 auf besondere Lernschwierigkeiten hinzuweisen. Die in diesem Rahmen entstandenen kontrastiven Analysen waren auf formale Unterschiede auf der sprachlichen Oberfläche ausgerichtet, wie, Sprache X verfügt über drei morphologische Kategorien in einem strukturellen Bereich, Sprache Y dagegen nur über zwei (z. B. in Bezug auf das Genussystem des Deutschen und des Französischen)‘. Eine weitergehende theoretische Analyse der jeweiligen sprachlichen Kategorien war nicht Ziel dieses Ansatzes. Durch diesen anwendungsbezogenen Blick wurde mit dem Begriff Kontrastive Analyse lange Zeit ein Forschungsfeld bezeichnet, das in der Sprachwissenschaft als theoretisch nicht wirklich relevant angesehen wurde. Diesem Blick auf die Kontrastive Analyse liegt eine irreführende Vermischung von Untersuchungsziel und Untersuchungsmethode zur Grunde, was hier gleich zu Beginn richtig gestellt werden muss. Die Gegenüberstellung von Sprachen, ihren Lautsystemen, ihren grammatischen Prinzipien, ihren lexikalischen Strukturen ist - wie bereits in der kurzen Einführung deutlich wurde - eine wesentliche Methode linguistischer Theoriebildung. Kaum ein Werk moderner Linguistik enthält keine Verweise auf Sprachunter- 
schiede. Im Sinne der grundlegenden Funktion der Kontrastierung dient der Blick auf unterschiedliche Strukturen der Schärfung der Konturen der Vergleichsobjekte. Mit welchem Ziel eine sprachvergleichende Methode eingesetzt wird, ist eine zweite Frage. Dies ergibt sich in keiner Weise aus dem Analyseansatz selbst (vgl. die Diskussion in Theisen 2016). In seiner Einführung in das Jahrbuch 2011, dessen Thema Deutsch im Sprachvergleich ist, führt Eichinger (2012) in einem knappen Überblick vor, wie die Methode der vergleichenden und kontrastierenden Sprachbetrachtung im Kontext linguistischer Theoriebildung zur Bearbeitung sehr unterschiedlicher Fragestellungen eingesetzt wurde. Im Folgenden sollen einige wesentliche Aspekte der kontrastiv-vergleichenden Forschung nachgezeichnet werden, um dann auf generelle Probleme dieser Methode einzugehen. Im Hauptteil des Beitrags wird dann anhand einiger Fallstudien dargelegt, welche Bedeutung der kontrastiven Methode für kognitiv orientierte Forschungsansätze zukommt und damit für eine Germanistik 2020.

\section{Kontrastive Sprachbetrachtung als Methode}

Die Methode der kontrastiven Sprachbetrachtung kann aus zwei komplementären Perspektiven eingesetzt werden. So kann zum einen eine Einzelsprache zum Ausgangspunkt gewählt werden, die Kontrastierung mit einer oder mehreren anderen Sprachen wird dann zur Erhellung bestimmter Phänomene herangezogen. E. König spricht in diesem Zusammenhang von ,kontrastiver Linguistik im Rahmen feinkörniger Sprachbetrachtung (2012: 30) und illustriert dieses Verfahren am Beispiel des Ausdrucks eigen. Als Ziel der kontrastiven Methode formuliert er,

zu zeigen, in welch vielfältiger Weise Eigenschaften dieses Ausdrucks [es geht hier um das Wort eigen, Anm. d. Verf.] durch einen Vergleich mit seinen Gegenstücken in anderen Sprachen und durch verschiedene vergleichende Perspektiven (historisch, typologisch, dialektal) deutlicher sichtbar werden, die sonst unbeachtet bleiben. (König 2012: 15)

Die kontrastive Methode ist unter dieser Perspektive als Heuristik anzusehen, um durch den Vergleich einzelner Komponenten sprachlicher Systeme auf Merkmale aufmerksam zu werden, die nicht durch eine overte Markierung in einer Einzelsprache erkennbar sind. Hier wirkt die Kontrastierung unterschiedlicher Sprachen in gewisser Weise als verlängerter Arm der strukturalistischen Idee der systeminternen Opposition.

Die komplementäre Perspektive verfolgt mit dem Sprachvergleich das Ziel, Gemeinsamkeiten und möglicherweise Universalien in der Varianz sprachlicher Strukturen zu ermitteln. Für diesen Ansatz hat sich der Begriff ,Sprach- 
typologie' etabliert. Was den Einsatz der kontrastiv-vergleichenden Methode angeht, so unterscheiden sich die beiden Perspektiven im Grunde nicht. Allerdings bedingt das Ziel sprachtypologischer Forschung, dass möglichst viele Sprachen in den Blick genommen werden, um das gesamte Variationsspektrum in einem bestimmten Bereich ausloten zu können und dann daraus Generalisierungen entwickeln zu können, in der Regel in der Form implikativer Universalien. Notwendigerweise können typologische Untersuchungen nicht so feinkörnig sein wie kontrastive Untersuchungen von wenigen Sprachen. Die bedeutenden typologischen Studien, die im Rahmen des Projekts Eurotyp durchgeführt wurden, sollen zur Verdeutlichung herangezogen werden (Dahl 2000). So werden beispielsweise in dem Themenbereich Tempus/Aspekt die Sprachen in ihrem möglichen Formeninventar erfasst. Unter der Kategorie des progressiven Aspekts finden sich z. B. für das Deutsche und das Niederländische vergleichbare Einträge (vgl. Bertinetto et al. 2000: 528). Untersuchungen mit der genannten feinkörnigen Zerlegung sprachlicher Strukturen lassen - im Unterschied zu weit gefassten typologischen Studien - erkennen, in welchen Kontexten bestimmte aspektuelle Markierungen eingesetzt werden. Für das Deutsche und das Niederländische ergeben sich mit diesem Ansatz dann ganz erhebliche Unterschiede in Bezug auf aspektuelle Markierungen (vgl. Behrens et al. 2013; Natale 2009).

Betrachtet man die heutige Forschungslandschaft, so lassen sich typologische und kontrastive Ansätze nicht mehr sinnvoll trennen. Arbeiten unter dem Titel ,Deutsch - typologisch“ (vgl. Lang \& Zifonun 1996) machen deutlich, dass der Versuch, kontrastive Linguistik und Typologie unterschiedlich zu verorten, wenig sinnvoll ist. Der Platz, den König der Kontrastiven Linguistik zuweist, ist letztlich nicht aus der Methode des Sprachvergleichs zu begründen. König nennt vier Aspekte, die nach seiner Auffassung für die Kontrastive Linguistik ${ }^{1}$ konstitutiv sind und sie von der typologischen Forschung abzugrenzen erlauben: a) synchrone Orientierung, b) feinkörniger Untersuchungsbereich (z. B. Einbeziehung sprachlicher Varietäten), c) Beschränkung auf zwei Sprachen, d) theoretische Offenheit (vgl. 2012: 36 ff.). Die von König dargelegten Beschränkungen ergeben sich aus gewissen Forschungstraditionen, sind aber in keiner Weise durch die kontrastive Methode bedingt. Wie bereits einführend dargelegt, lässt sich die kontrastive Sprachbetrachtung aus synchroner wie diachroner Perspektive fruchtbar anwenden. Die Feinkörnigkeit ist skalar zu bestimmen und findet in typologischen Untersuchungen ihre Grenzen lediglich an dem damit verbundenen Aufwand und der möglicherweise begrenzten Quellenlage.

1 Theissen greift diese Bestimmungen in seiner Arbeit Kontrastive Linguistik erneut auf und folgt ihnen mit einigen Erweiterungen (2016: 32). 
Der Skopus ist mit der Anzahl von zwei Sprachen willkürlich gesetzt, es gibt keine aus Gegenstand oder Fragestellung motivierte Begrenzung. Auch der letzte Punkt, die theoretische Offenheit, gilt für typologische Ansätze gleichermaßen. Die Diskussion um das Verhältnis von Kontrastiver Sprachanalyse und Sprachtypologie weiterzuführen scheint wenig fruchtbar. Wir werden im Folgenden die vergleichende und kontrastive Sprachanalyse als eine Methode und nicht als einen bestimmten Ansatz in dem von König vorgeschlagenen Sinne betrachten, eine Methode, die für sehr unterschiedliche Fragestellungen eingesetzt werden kann.

\section{Das tertium comparationis}

Ein Vergleich zwischen zwei Größen ist nur möglich über ein gemeinsames Drittes. Vergleicht man die Objekte Stuhl und Tisch im Hinblick auf ihre Materialeigenschaften, so mögen sie beide in eine Kategorie ,Holz' fallen. Vergleicht man sie im Hinblick auf ihre Funktionsmerkmale, so fallen sie in zwei unterschiedliche Kategorien. Der entscheidende Schritt für einen zielgerichteten Vergleich besteht in der Festlegung des tertium comparationis. Ein Blick in die Geschichte der sprachvergleichenden Forschung wirft viele Fragen bezüglich des jeweils gewählten tertium comparationis auf, sodass wir einige Gedanken hierzu den weiteren Ausführungen voranstellen. In seinem einleitenden Text zum Jahrbuch Deutsch im Sprachvergleich spricht Eichinger ein zentrales Dilemma an. Eichinger spricht von einer komplizierten Balance, hier bezogen auf vergleichende Grammatikbetrachtung:

Es ist eine Ebene des Vergleichs zu finden [...] die einerseits zu einer grammatischen Beschreibung des Deutschen passt, also Kategorien betrifft, die sich in einer grammatischen Beschreibung des Deutschen niederschlagen. Es ist die andere Seite einer ausgewogenen vergleichenden Beschreibung, dass die Ebene auch geeignet sein muss, die Verhältnisse in den Vergleichssprachen aus ihrem eigenen Blickwinkel zu beschreiben. (Eichinger 2012: VIII)

Es stellt sich die Frage, ob diese Balance überhaupt zu finden ist, wenn man die Vergleichsgröße auf der Ebene der sprachlichen Form festlegt. Die überwiegende Zahl früherer sowie gegenwärtiger sprachvergleichender Untersuchungen geht von einem formal festgelegten tertium comparationis aus. In den Anfängen der vergleichenden Sprachwissenschaft war dies ausschließlich der Fall. Vergleiche wurden in Bezug auf bestimmte morphologische Kategorien gezogen, wie beispielsweise die Tempora im Lateinischen, Griechischen und Deutschen. Betrachtet man in einem Zeitsprung neuere sprachvergleichende 
Arbeiten, so finden sich auch hier überwiegend formbezogen definierte Vergleichsgrößen (vgl. die Artikel in Gunkel \& Zifonun 2012 oder die Themen im Handbuch Typologie, Haspelmath et al. 2001). Kategorien wie Adjunkte, W(h)Clefts, Adnominale Adverbien, Konverben dienen zur Grundlage für sprachvergleichende und kontrastierende Studien. Lässt sich auf dieser Ebene die von Eichinger angesprochene Balance überhaupt herstellen? Bleiben wir für diese Überlegung bei der Betrachtung von Sprachen, die als gut beschrieben gelten, Englisch, Französisch und Deutsch. Alle drei Sprachen weisen morphologische Markierungen von Tempora auf. Vergleichen wir die Systeme anhand der etablierten Formkategorien Präsens, Präteritum, Perfekt, Plusquamperfekt etc., so ergibt sich eine weitgehende Übereinstimmung. Der Vergleich wird darüber hinaus zeigen, dass Unterschiede in der Anzahl der morphologischen Kategorien bestehen sowie sprachspezifische Interaktionen mit aspektuellen Markierungen. Wie viel erfahren wir aber über die Sprachen, wenn wir Gemeinsamkeiten und Unterschiede in dieser Form festhalten (vgl. die Diskussion in Klein \& Vater 1998)? Die formale Kategorie des Perfekts beispielsweise besitzt in diesen drei Sprachen grundsätzlich unterschiedliche semantische Eigenschaften. Um diese $\mathrm{zu}$ erfassen, kann das tertium comparationis nicht formal (i.S. v. formbezogen) definiert sein. Es muss der Schritt auf die semantische Ebene gemacht werden. Nehmen wir als Vergleichsgröße die temporalsemantische Grundlage der Tempora und beziehen die einzelsprachlichen Forminventare auf das semantische Verhältnis zwischen den Zeiteigenschaften einer Situation und der deiktisch gegliederten Zeitachse. Dieser Vergleichsansatz wird ergeben, dass das Perfekt im Englischen andere semantische Eigenschaften aufweist als das Perfekt im Deutschen und im Französischen, er wird auch zeigen, dass das deutsche Perfekt zwei Lesarten aufweist. Aber auch diese vergleichendkontrastive Betrachtung bleibt zu oberflächlich, um die Unterschiede zwischen den einzelsprachlichen Formen zu erfassen. So kann der Satz Ich bin dreimal in New York gewesen ohne weiteres mit I have been to New York three times wiedergegeben werden - der Satz Einstein hat dreimal Princeton besucht dagegen nicht mit *Einstein has visited Princeton three times (vgl. Klein 2000). Das bedeutet, dass wir auch über eine temporalsemantische, sprachformgebundene Kategorie hinausgehen müssen, um tatsächlich zu den Gemeinsamkeiten und Unterschieden zwischen Sprachen vorzudringen.

Betrachten wir noch ein zweites klassisches Beispiel der vergleichenden Sprachforschung: die Wortstellung. Mittlerweile füllen die vergleichenden Untersuchungen zu Wortstellungsmustern ganze Bibliotheken. Es gibt zahlreiche typologisch ausgerichtete Theorien, die sich mit Stellungsmustern im Sinne implikativer Universalien beschäftigt haben (Greenberg 1963; Hawkins 1983; Überblick in Dryer 2017). Die Vergleichsgrundlage in diesen Untersuchungen 
liegt auf der syntaktischen Ebene. Das tertium comparationis sind die grammatischen Funktionen Subjekt, Objekt, Verb. Gemeinsamkeiten und Unterschiede werden anhand eines basic word order (Hawkins 1983) pro Sprache ermittelt. Während wir durch diese Untersuchungen einen groben Überblick über Reihungsmöglichkeiten in Sprachen erhalten, in gewisser Weise eine Vermessung der Sprachenwelt, stellt sich wieder die Frage, wie viel wir tatsächlich über die Reihungseigenschaften einer einzelnen Sprache im Vergleich mit einer anderen erfahren. Dryer (2017) reflektiert einige zentrale Probleme in seiner enorm kenntnisreichen Übersicht im Weltatlas der Sprachen. Die erste Schwierigkeit liegt bereits darin, dass grammatische Kategorien wie Subjekt und Objekt nicht für alle Sprachen gleichermaßen zu definieren sind. Ein weiteres gravierendes Problem liegt in der Idee einer einzigen basic word order pro Sprache. Betrachten wir nur das Deutsche. Zwar ließen sich zwei syntaktisch bedingte Grundmuster bestimmen, SVO und SOV - was auch in zahlreichen typologischen Ansätzen so angenommen wird. Damit wird man den Stellungsvarianten im Deutschen jedoch keineswegs gerecht. Interessant für einen Sprachvergleich, durch den man erfährt, wann eine bestimmte Stellungsvariante in der einen Sprache angemessen ist und warum sie in einer anderen mit demselben basic word order aber keine angemessene Option darstellt, ist eine weitergehende Untersuchung, die die Funktionalität der jeweiligen Reihungsmuster mit in den Blick nimmt. Überlegungen, die sich durchaus auch in den ,klassischen“ Wortstellungstypologien finden (vgl. Dryer 2017), verweisen über das syntaktisch definierte tertium comparationis hinaus auf die semantische Ebene, indem Kategorien wie Agens und Patiens als Vergleichsgrößen zu Grunde gelegt werden, weiterführend auch auf die pragmatische Ebene, indem Kategorien wie Topic/Comment zur Grundlage für feinkörnigere Typologien herangezogen werden.

Die beiden Beispiele zeigen, wie weitgehend formbezogene Sprachvergleiche bestimmten Kategorisierungen verhaftet bleiben, die aus Eigenschaften spezifischer Einzelsprachen gewonnen wurden. Die Erkenntnisse, die durch diese Studien gewonnen wurden und werden, sollen hier keineswegs geschmälert werden. Es bleiben jedoch auf dem Weg, Gemeinsamkeiten und Unterschiede zwischen Sprachen verstehen zu wollen, noch viele Fragen offen. Im Folgenden wollen wir den Weg weitergehen und aufzeigen, wie durch eine Verschiebung des tertium comparationis von der sprachgebundenen auf die konzeptuelle Ebene weiterführende Einsichten gewonnen werden können. ${ }^{2}$

2 Vgl. die Festlegung extralinguistischer Kriterien wie Deixis, Textkohäsion oder Temporalität als Grundlagen für sprachtypologische Analysen (Haspelmath et al. 2001, Kap. VII). Allerdings nehmen Studien, die diesem Ansatz folgen, nur einen sehr kleinen Platz in dem Gesamtwerk zur Typologie ein (14 Kapitel von 125). 


\section{Sprachvergleich und Sprachkontraste auf der Grundlage konzeptueller Kategorien}

\subsection{Kognitive Kategorien als tertia comparationis}

Es ist eine Binsenweisheit, dass Sprachen gleiche Inhalte auf unterschiedliche Weise zum Ausdruck bringen. Sie drücken aber nicht nur gleiche Inhalte durch unterschiedliche formale Kategorien aus, beispielsweise durch Präpositionen in der einen Sprache, durch Partikel in der anderen. Sprachen unterscheiden sich auch darin, welche Gewichtung bestimmten begrifflichen Kategorien gegeben wird. Hier sei die Grammatikalisierung bestimmter Kategorien in der einen Sprache gegenüber fakultativer lexikalischer Darstellung in der anderen Sprache als Beispiel genannt. Zeitliche Einordnung, im Deutschen obligatorisch ausgedrückt durch Tempora, im Chinesischen fakultativ durch Adverbien, stellt einen solchen Kontrast dar. Eine weitere Kontrastdimension liegt in unterschiedlichen Differenzierungsgraden, die Sprachen für bestimmte kognitive Bereiche in ihrem Inventar repräsentieren. Zahlreiche vergleichende Studien haben gezeigt, wie das Basislexikon unterschiedlicher Sprachen bestimmte konzeptuelle Bereiche anhand unterschiedlicher Merkmale ausdifferenziert. Als Beispiel hierfür sind die Verblexika zum Ausdruck von Bewegungsereignissen illustrativ. Hier zeigt Slobin, dass beispielsweise das Englische und das Deutsche sehr viel differenzierter die Art der Bewegung kodieren als das Französische oder das Spanische. Diese wiederum weisen ein differenzierteres lexikalisches Repertoire im Bereich der Richtungsverben auf (Slobin 1996, 2000). Kontraste bestehen des Weiteren darin, wie bestimmte begriffliche Kategorien in einzelnen sprachlichen Ausdrucksformen zusammengeschlossen werden. Betrachten wir das Inventar kausativer Bewegungsverben, so verbindet das Deutsche mit der Kernsemantik eine konzeptuelle Kategorie, die sich aus den Objekteigenschaften ableitet: setzen, stellen, legen. Das Englische hat mit den Verben put oder place, das Französische mit mettre oder placer dieses konzeptuelle Cluster nicht in gleichem Maße lexikalisiert. Neben den sprachlich formalen Kontrasten bestehen Kontraste zwischen Sprachen im Hinblick auf die Salienz und Bündelung bestimmter konzeptueller Kategorien. Um diese Kontraste bzw. Gemeinsamkeiten zu identifizieren, muss das tertium comparationis auf einer der Einzelsprache vorausgesetzten Ebene - und zwar sowohl syntaktisch als auch semantisch - angesiedelt sein. Wählt man einen bestimmten kognitiven Bereich, wie beispielsweise die räumlichen Verhältnisse zwischen Objekten, so lässt sich durch empirische Untersuchungen ermitteln, wie Sprachen diese zur Darstellung bringen. Da keine Einschränkung bezüglich spezifischer Ausdrucksformen sowohl in syntaktischer als auch in semantischer 
Hinsicht besteht, lässt sich mit diesem Ansatz auch erkennen, wo bestimmte Sprachen keine Ausdrucksformen besitzen, wo unterschiedliche Differenzierungsgrade und konzeptuelle Clusterbildungen vorliegen.

Dieser kognitiv orientierte Ansatz wird ausgearbeitet und umgesetzt im Kontext großer sprachvergleichender Projekte, wie die raumkognitiven Arbeiten der Gruppe um S. Levinson (2003) oder die Textstrukturen vergleichenden Untersuchungen von Berman und Slobin (1994). Die Grundidee dieser Studien besteht darin, einen außersprachlich, in der Regel visuell gegebenen Inhalt von Sprechern unterschiedlicher Sprachen darstellen zu lassen. Die Perspektive wechselt damit von dem Vergleich sprachlicher Systeme auf den Vergleich der Verwendungsprinzipien sprachlicher Formen. Die Annahme ist, dass Menschen unabhängig von ihrer Muttersprache den visuell dargebotenen Stimulus in gleicher Weise erfahren. Die jeweils erzeugte sprachliche Darstellung ermöglicht Einblicke in Kontraste und Gemeinsamkeiten auf der Ebene des Sprachgebrauchs und auf dieser Grundlage Rückschlüsse auf die Eigenschaften sprachlicher Systeme im Hinblick auf alle oben genannten Aspekte.

Die Methoden, die in diesen Studien eingesetzt werden, sind grundsätzlich empirisch. Die Vielfalt der verwendeten Methoden erlaubt Vergleiche auf unterschiedlicher Granularitätsebene. Mit der Korngröße des gewählten kognitiven Feldes korrespondiert der Grad der experimentellen Kontrolle über den Versprachlichungsprozess. Allen Methoden ist gemeinsam, dass der auszudrückende Inhalt konstant gehalten sein muss. Der Inhalt kann komplex sein, beispielsweise eine ganze Ereigniskette, die in der Form eines Textes wiedergegeben wird. Der Inhalt kann minimal sein, wenn in Priming-Experimenten ${ }^{3}$ Objektklassenbildungsmuster über verschiedene Sprachen hinweg vergleichend ermittelt werden. Kontrastive Studien bedienen sich mittlerweile des gesamten Methodeninventars der modernen Psycholinguistik einschließlich bildgebender Verfahren.

Im Folgenden soll anhand von Fallbeispielen mit jeweils unterschiedlicher Korngröße des gewählten kognitiven Bereichs illustriert werden, welche Einsichten durch die Wahl eines sprachextern bestimmten tertium comparationis gewonnen werden können. Dabei sollen Eigenschaften der deutschen Sprache sowie im Ausblick Forschungsperspektiven einer Germanistischen Sprachwissenschaft $2020 \mathrm{im}$ Vordergrund stehen.

3 Vgl. das Fallbeispiel zur Priming-Methode unter 4.3. 


\subsection{Drei Fallstudien}

\subsubsection{Kontrastierung von Textstrukturen: Deutsch - Englisch}

In seinem Aufsatz The Optimization of Discourse Anaphora wählt Beaver einen kleinen Text zur Illustration informationsstruktureller Eigenschaften von Texten.

(1) John went to his favorite music store to buy a piano.

He had frequented the store for many years.

He was excited to be going to the store to actually buy a piano.

It was the biggest music store in the area.

It had just the piano that he wanted.

It was closing just as John arrived.

(Beaver 2004: 36)

Dieser Text dient ihm zur Verdeutlichung dessen, was als Topik gilt, sowie der Funktion der Topik in einem kohärenten Text. Die Schlussfolgerungen, die Beaver in seinem Aufsatz zieht, formuliert er als generelle Prinzipien der Informationsorganisation.

Ein kleines Experiment, in dem Studierende gebeten wurden, diesen Text auf Deutsch wiederzugeben, macht deutlich, dass das Kohärenzmuster des Englischen keineswegs für das Deutsche übernommen wurde. Eine typische Übersetzung ins Deutsche gibt das folgende Beispiel

(2) Hans ging zu seinem Lieblingsmusikgeschäft, um ein Klavier zu kaufen.

Schon seit Jahren war er dort hingegangen.

Er war aufgeregt, jetzt zu dem Laden zu gehen und tatsächlich ein Klavier zu kaufen.

Es war das größte Musikgeschäft in der Umgebung.

Dort gab es genau die Sorte Klavier, die er wollte.

Als er ankam, schloss der Laden gerade.

Es zeigt sich, dass die beiden Texte unterschiedliche konzeptuelle Bereiche für die Etablierung von Kohärenz wählen und dies, obwohl im Prinzip vergleichbare Ausdrucksmittel zur Verfügung stünden. Die Erklärung, es handele sich um unterschiedliche rhetorische Stile (z. B. Berman 1995) oder Diskurstraditionen (z. B. Kabatek 2011) kann nicht zufriedenstellen, vor allem dann nicht, wenn man in vergleichenden Studien feststellt, dass Sprachen sehr unterschiedlicher kultureller Einbindung ähnliche Muster aufweisen, während sehr eng verwandte, wie eben das Deutsche und Englische, in ihrem Textaufbau 
klar kontrastieren. ${ }^{4}$ Umfassende sprachvergleichende empirische Studien haben Einblicke in Prinzipien makrostruktureller Organisation geliefert, die sich als sprachspezifische Prinzipien der Informationsorganisation bestimmen lassen, die ihrerseits durch Unterschiede auf sprachstruktureller Ebene zu erklären sind (z. B. Fabricius-Hansen 2010; Carroll et al. 2008).

Zur Erforschung von Kontrasten und Gemeinsamkeiten auf der Ebene komplexer Informationsorganisation eignet sich der Ansatz der kognitiv orientierten kontrastiven Analyse. Wir illustrieren diesen Ansatz skizzenhaft anhand einer Fallstudie zur Textorganisation in narrativen Texten im Deutschen und Englischen (vgl. zur ausführlichen Darstellung der Studie v. Stutterheim \& Carroll 2018). Das Design sieht folgendermaßen aus: Sprecher der beiden Sprachen sehen denselben Stummfilm und haben dieselbe kommunikative Aufgabe zu lösen: Sie sollen mündlich darstellen, was in dem Film passiert ist. Die Vergleichsgrundlage bildet so der unter einer Erzählperspektive zu versprachlichende Inhalt. Gemeinsam ist damit ein makrostruktureller Referenzrahmen, in den einzelne Situationen der Filmwelt linearisiert eingebunden werden müssen. Dabei kommt der sequenziellen temporalen Verkettung eine zentrale Rolle für die Linearisierung der Informationen zu. Temporale Referenz hat in narrativen Texten globalen Topikstatus. ${ }^{5}$ In Ausarbeitung dieses Rahmens in Form kohärenter Äußerungsketten zeigen sich systematische Unterschiede zwischen den beiden Textgruppen. Diese Unterschiede betreffen $u$. a. die Wahl der kohärenzstiftenden Topikdomäne. Einige Ausschnitte verdeutlichen, worum es hier gehen soll:

(3) you see him land on a new plane

this plane is full of paper

(4) so now the man has dropped from the sky onto the next land

this land has a desert aspect

it just stretches to the horizon

\footnotetext{
4 Die sprachkontrastiven Arbeiten von Fabricius-Hansen zu norwegischen und deutschen Texten, in denen sie große Übersetzungskorpora im Hinblick auf informationsstrukturelle Muster und deren sprachliche Ausdrucksform untersucht, führen sie zu dem folgenden Schluss: „die Zusammenhänge zwischen sprachspezifischen syntaktischen Restriktionen und Textgestaltung bzw. Textverstehen sind jedoch noch zu wenig erforscht“ (2010: 195).

5 Vgl. für eine ausführliche Darstellung makrostruktureller Textorganisation und der damit verbundenen informationsstrukturellen Vorgaben Klein \& v. Stutterheim 1987, v. Stutterheim 1997.
} 
Wie bereits in dem Beispiel von Beaver zu beobachten war, wird von den englischen Sprechern als Topikelement der zweiten (3) bzw. zweiten und dritten (4) Äußerung eine Entität in der grammatischen Funktion des Subjekts gewählt. Bemerkenswert ist dabei, dass hier von der ersten zur folgenden Äußerung eine konzeptuelle Verschiebung von der Raumdomäne, ausgedrückt in Präpositionalphrasen, zur Domäne der Entitäten vorliegt. Kohärenz wird über einen konzeptuellen Wechsel (conceptual shift) hergestellt. In den deutschen Texten finden sich solche Strukturen nicht, vielmehr wird in vergleichbaren Kontexten eine Raumreferenz als kohärenzstiftende Topik gewählt:

(5) und das Männchen schlägt auf einer Oberfläche auf und auf dieser Oberfläche herrscht sehr großer Wind

(6) aus dem Sandloch raus fällt er in so ne Blätterwelt und da hört er dann wieder das Wasser tropfen

(7) jetzt sieht man eine Ebene überall fliegen auch Papierblätter durch die Luft

Dieser feinkörnige Vergleich verweist auf Gemeinsamkeiten wie auf Kontraste im Informationsmanagement deutscher und englischer Sprecher. Gemeinsam ist, dass die erste Position im Satz privilegiert ist als zentrale Topikposition und damit für die Etablierung von Kohärenz ausgezeichnet ist. Kontraste bestehen dahingehend, wie Kohärenz hergestellt wird. Im Unterschied zum Deutschen hat raumreferenzielle Information im Englischen keinen Zugang zur satzinitialen Position. Vielmehr erfolgt eine konzeptuelle Umkategorisierung in eine begriffliche Kategorie, die als Subjekt enkodierbar ist. Die im Englischen vorliegende Beschränkung kann einerseits auf die zentrale Funktion des Subjekts als Topik in satzinitialer Position zurückgeführt werden, andererseits auf die Tatsache, dass in narrativen Texten Raumreferenzen im Unterschied zu Zeitreferenzen keinen globalen Topikstatus besitzen. ${ }^{6}$ Die Anforderungen an eine kohärente Informationsorganisation in Einklang zu bringen mit den syntaktischen Bedingungen für die zentrale Topikposition wird im Englischen auch dadurch unterstützt, dass konzeptuelle Prozesse der Reifikation, der Vergegenständlichung von beispielsweise räumlichen und zeitlichen Konzepten, in

6 In beschreibenden Texten, in denen räumliche Relationen als globale kohärenzstiftende Größe fungieren, kann im Unterschied zu narrativen Texten raumreferenziellen Informationen die erste Position im Satz im Englischen zugänglich sein, wie folgendes Beispiel illustriert: there is a kiosk, to the left of that there are three children, to the right of the kiosk there is an old man with a beard ... (v. Stutterheim \& Carroll, i. Dr.). 
größerem Umfang möglich sind und genutzt werden als im Deutschen. Im Unterschied zum Englischen bestehen im Deutschen keine syntaktischen Beschränkungen für die Besetzung der ersten Position im Satz. Es bestehen an dieser Stelle grammatische Optionen für unterschiedliche Konstituenten und damit für unterschiedliche referentielle Verknüpfungen. Entitäten spielen für die Informationsorganisation im Deutschen keine privilegierte Rolle.

Was zeigen uns die Befunde aus diesem Ausschnitt einer textvergleichenden Studie? Sie zeigen zunächst sehr grundsätzlich, dass ein Sprecher, der die Kompetenz hat, Sätze zu erzeugen, damit noch lange nicht die Kompetenz hat, Texte zu erzeugen. Zur Produktion angemessener Texte muss die Sprachkompetenz des Weiteren Prinzipien makrostruktureller Organisation umfassen. Um diese in ihrer einzelsprachlichen Ausprägung zu erkennen, sind sprachvergleichende Analysen im Rahmen kognitionsorientierter Ansätze erforderlich. Vergleiche auf Satzebene können niemals Erkenntnisse über sprachliche Kontraste auf dieser Ebene ermöglichen. Wie dringend die Erforschung makrostruktureller Informationsorganisation unter sprachvergleichender Perspektive ist, zeigen Erfahrungen im Bereich des Fremdsprachenerwerbs wie auf dem Feld der Übersetzungen. Wie oft kann man bei Texten, die selbst von sehr fortgeschrittenen L2-Sprechern des Deutschen produziert worden sind, für die Erklärung von abweichenden Strukturen nicht viel mehr als seine Intuition ins Feld führen (vgl. v. Stutterheim 2016). Ebenso verlässt sich ein Übersetzer auf sein Sprachgefühl, wenn er bei der Übertragung von Texten in eine andere Sprache weitgehende Umorganisationen des informationsstrukturellen Aufbaus vornimmt, obwohl dafür auf Grund des sprachlichen Ausdrucksinventars kein Anlass bestünde. Die Prinzipien des Textaufbaus sind bisher nur in Ansätzen verstanden (s. dazu den Beitrag von Holler i. d. Bd.). Diese Prinzipien lassen sich als interfaces zwischen syntaktischen und lexikalischen Bedingungen der einzelnen Sprache und letztlich kommunikationsgetriebenen Anforderungen an eine makrostrukturelle Organisation von Informationen erfassen. Um diese $\mathrm{zu}$ identifizieren, müssen kontrastive Analysen auf der Grundlage eines konzeptuell definierten tertium comparationis konzipiert werden. Wie die kleine Fallstudie verdeutlicht hat, ließe sich durch eine Gegenüberstellung sprachlicher Ausdrucksmittel deren unterschiedliche Funktionalität niemals erkennen.

\subsubsection{Kontrastierung von Ereignisdarstellungen Deutsch - Französisch}

Bereits in der Anfangszeit der systematischen kontrastiven Analysen, die sich wie oben dargestellt - im Schwerpunkt mit dem Vergleich von Grammatiken beschäftigten, galt das Interesse, vor allem im Kontext germanistisch-ausgerich- 
teter Studien, unterschiedlichen Valenzeigenschaften von Verben in verschiedenen Sprachen. Zahlreiche vergleichende Valenzwörterbücher entstanden. Vergleichen wir einmal die Verben rouler und fahren im Hinblick auf ihre Valenzeigenschaften. Für fahren wird eine fakultative direktionale Ergänzung angenommen, er fährt nach Paris, für rouler dagegen nicht. *Il roule à Paris ist keine mögliche Wiedergabe des deutschen Satzes. Die Frage, die kontrastive Valenzwörterbücher nicht beantworten, ist die nach dem Warum. Wie kommt es, dass Verben, die semantisch übersetzungsäquivalent scheinen, unterschiedliche Valenzeigenschaften besitzen? Ein Weg, sich einen Einblick in die Hintergründe für diese Art grammatischer Kontraste $\mathrm{zu}$ verschaffen, liegt in dem Ansatz kognitiv orientierter Vergleiche im Kontext der Sprachverwendung. Die Frage lautet dann: Welche kognitiven Prozesse liegen der Wahl bestimmter sprachlicher Einheiten in unterschiedlichen Sprachen zu Grunde? In dem konkreten Fall: Finden sich unterschiedliche konzeptuelle Bedingungen für die Wahl von rouler für Sprecher des Französischen im Unterschied zu fahren durch deutsche Sprecher? Um dieser Frage nachzugehen, müssen kontrollierte, vergleichende Untersuchungen durchgeführt werden, die bei gleichen sprachexternen Gegebenheiten die sprachliche Umsetzung in den beiden Sprachen untersuchen. Richten wir unseren Blick zunächst auf die kognitive Raumtypologie, die sich seit Talmys bedeutenden Arbeiten mit Sprachkontrasten in der konzeptuellen Domäne der Bewegungsereignisse beschäftigt. Talmy (1985) unterscheidet zwei Typen von Sprachen: verb-framed und satellite-framed. Sie werden danach klassifiziert, wie die zentrale Information des Wegs (path) kategorial zum Ausdruck gebracht wird: entweder in Verben, wie beispielsweise in dem Verb überqueren, oder in einer anderen syntaktischen Kategorie wie Partikel, Adverben oder - in neueren Arbeiten mit in die Kategorie der Satelliten aufgenommen - auch Adjunkten, z. B. hin, geradeaus, in den Garten. Nach Talmys Typologie ist das Französische als verb-framed und das Deutsche als satellite-framed zu kategorisieren. Die Annahme ist daher, dass das Französische Bewegungsereignisse durch path-Verben wiedergibt, wobei weitere Komponenten, die ein Bewegungsereignis konstituieren wie beispielsweise die Art der Bewegung, unerwähnt bleiben können, während das Deutsche im Verb nicht die Bewegung kodiert, sondern die Art der Bewegung. Path wird durch andere syntaktische Konstituenten ausgedrückt.

(9) a. Une jeune fille entre la maison (en courant)

b. Ein Mädchen läuft ins Haus.

Beispiele dieser Art finden sich in zahlreichen kontrastiven Untersuchungen zum Deutschen bzw. zu den germanischen Sprachen und zum Französischen 
bzw. zu den romanischen Sprachen. Wie grob diese Klassifikation letztlich aber ist und wie wenig sie über die tatsächliche, unterschiedliche Konzeptualisierung von Bewegungsereignissen aussagt, zeigt ein Blick in die Empirie. Datenbasierte Vergleiche ergeben ein Bild, das zunächst nicht zu der typologischen Klassifikation zu passen scheint: Französische Sprecher verwenden für die Darstellung von Bewegungsereignissen in erheblichem Umfang ebenfalls Verben der Art und Weise. Betrachten wir eine umfassende kontrastive Studie zum Ausdruck von Bewegungsereignissen mit ihren Ergebnissen für das Deutsche und das Französische ${ }^{7}$ (vgl. Carroll et al. 2012; v. Stutterheim et al. 2012; Flecken et al. 2015; Gerwien \& v. Stutterheim eingereicht).

Das Design der Studie sieht folgendermaßen aus. Die Probanden sehen kurze Videoszenen, die Bewegungsereignisse in der realen Welt darstellen, z. B. ein Auto, das in eine Garage fährt, ein Mädchen, das auf ein Haus zuläuft. Die Videostimuli unterscheiden sich im Hinblick auf die räumlichen Eigenschaften der Ereignisse. Sie zeigen lange und kurze Wegverläufe mit möglichen Endpunkten, mit Richtungswechseln, mit Grenzüberschreitungen, mit Personen oder Gegenständen als sich bewegende Figuren. Das tertium comparationis bilden so die Komponenten der außersprachlichen Situation: die Figur, ihre Bewegungsart, Bewegungsausrichtung, der Weg mit Ausgangspunkt, Verlauf, Richtung und Ziel. Die Probanden werden aufgefordert, das dargestellte Geschehen mündlich zu versprachlichen. Die Audiodaten werden transkribiert und anhand formaler und inhaltlicher Kategorien analysiert. Für die Gruppen der französischen und deutschen Sprecher sind die folgenden Ergebnisse in dem vorliegenden Kontext von Bedeutung: ${ }^{8}$

\section{- Kontraste auf der Ebene der Segmentierung}

Französische und deutsche Sprecher segmentieren die dargestellten Szenen anhand unterschiedlicher Kriterien. Französische Sprecher segmentieren typischerweise dort, wo eine Veränderung der Orientierung der sich bewegenden Figur vorliegt bzw. dann, wenn sich bei einem längeren Weg ein mögliches Ziel erkennen lässt. Deutsche Sprecher dagegen drücken die dargestellten Situationen typischerweise in einer Äußerung aus.

\footnotetext{
7 In dieser groß angelegten sprachvergleichenden Studie werden Sprachen unterschiedlicher Sprachfamilien untersucht: romanische, germanische, slawische und semitische. Wir beschränken uns hier zum Zwecke der Illustration auf das Deutsche und das Französische. Befunde für weitere Sprachen sind unter den oben angegebenen Referenzen sowie in v. Stutterheim et al. (2012) zu finden.

8 Für die statistischen Auswertungen im Einzelnen sei der Leser auf die folgenden Artikel verwiesen (Carroll et al. 2012; Gerwien \& v. Stutterheim eingereicht). Im gegebenen Kontext geht es uns um die grundsätzliche Forschungsausrichtung und nicht um die umfassende Darstellung einer einzelnen Studie.
} 
Eine Situation, in der ein Mann eine Straße entlang läuft und dann in ein Haus geht, wird von französischen Sprechern als (10a) und von deutschen Sprechern als (10b) ausgedrückt:

(10) a. Un homme marche (dans la rue) et entre dans une maison

b. Ein Mann geht (die Straße entlang) in ein Haus (In Klammern werden typische, aber weniger häufige Muster angegeben.)

\section{- Kontraste auf der Ebene der Informationsauswahl}

Die Sprecher der beiden Sprachen selegieren unterschiedliche inhaltliche Komponenten der Bewegungsereignisse, und sie verknüpfen sie in unterschiedlicher Weise zu konzeptuellen Repräsentationen der Ereignisse. Französische Sprecher wählen in der Regel Informationen zur Art der Bewegung, wenn die Intention und Ausrichtung der Figur in Bezug auf ein bestimmtes Ziel nicht erkennbar oder nicht eindeutig ist. So entstehen Äußerungen wie un homme marche oder une voiture roule. Diese Konzeptualisierung kann mit einer Lokalreferenz verbunden sein:

(11) Une voiture roule sur la route.

Grundsätzlich tritt in dieser Art der Darstellung die gerichtete Bewegung und damit die Weginformation in den Hintergrund. Bewegungsverben der Art und Weise liefern im Französischen primär Informationen über bestimmte Eigenschaften der sich bewegenden Figur und nicht über eine sich auf einem Weg befindliche Entität. Dies ist vergleichbar einem deutschen Satz wie Der Mann humpelt, einer Konzeptualisierung, bei der nicht unbedingt ein Weg mitgedacht wird. Ist dagegen ein Ziel auch als klar erkennbare Ausrichtung der Figur wahrnehmbar oder eine Grenzüberschreitung gegeben, so wählen die französischen Sprecher diese für die Repräsentation des Bewegungsereignisses aus:

(12) Une femme s'approche d'une maison/entre dans une bâtiment.

Entscheidend für die Konzeptualisierung des Bewegungsereignisses ist die Orientierung der Figur (vgl. Carroll 2000, zum Begriff der figure-oriented spatial concepts). Die deutschen Sprecher orientieren sich in ihrer Informationsauswahl in Bezug auf die räumlichen Konzepte nicht an der sich bewegenden Figur, sondern an den Eigenschaften des Grundes, auf dem der Weg verläuft (ground-oriented spatial concepts). Der Situationstyp wird 
durch die Art und Weise der Bewegung bestimmt und im Verb ausgedrückt, Weginformationen müssen dann in anderen grammatischen Einheiten kodiert werden:

(13) Eine Frau rennt aus dem Haus über den Hof in ein gegenüberliegendes Gebäude.

Äußerungen mit Verben ohne Adjunkt - wie in den französischen Daten kommen in den deutschen Daten nicht vor. Ebenso finden sich so gut wie keine Lokalreferenzen in den deutschen Daten, während diese in den französischen Daten im Kontext von Verben der Art und Weise häufig sind. Die Ereignisschemata, die für die Interpretation und Repräsentation des außersprachlichen Inputs gewählt werden, unterscheiden sich für die beiden Sprechergruppen in den maßgeblichen konzeptuellen Größen.

Die Kontraste, die sich zwischen deutschen und französischen Sprechern im Kontext von Sprachproduktion zeigen, lassen sich durch weitere psycholinguistische Methoden überprüfen. So wurden in der vorliegenden Studie Blickbewegungsdaten erhoben. Geprüft wurde, wie sich die visuelle Aufmerksamkeit auf die sich bewegende Figur bzw. auf bestimmte Landmarken des Wegs, insbesondere auf einen potenziellen Zielpunkt, verteilt (vgl. Flecken et al. 2015). Auch mit dieser Methode zeigen sich Kontraste zwischen den beiden Sprechergruppen, die sich in Korrelation zu den sprachlichen Daten interpretieren lassen. Die Sprecher des Französischen widmen früher und länger ihre visuelle Aufmerksamkeit der sich bewegenden Figur. In einer weiteren Studie, die mit der gleichen Art Stimulusmaterial arbeitet, wurden die Probanden gebeten, die dargestellten Szenen in Ereigniseinheiten zu segmentieren. Sie sollten Ereignisgrenzen mit einem Tastendruck markieren. Diese non-verbale Aufgabe bestätigte die verbalen Befunde. Französische Probanden segmentierten die Videoclips häufiger als deutsche (vgl. Gerwien \& v. Stutterheim eingereicht) und an unterschiedlichen Stellen.

Fasst man die durch unterschiedliche Erhebungsmethoden gewonnenen Ergebnisse zusammen, so ergibt sich ein konvergierendes Bild. Sprecher des Französischen und des Deutschen folgen unterschiedlichen konzeptuellen Prinzipien, wenn sie Bewegungsereignisse repräsentieren. Diese Prinzipien lassen sich nicht durch den typologischen Kontrast zwischen verb-framed- und satellite-framed-Sprachen hinreichend erfassen. Vielmehr weisen sie jeweils den Weg- und den Artverben eine unterschiedliche Rolle $\mathrm{zu}$. Im Französischen ist die sich bewegende Figur die wichtigste Größe, sie steht an hierarchisch oberster Position für die konzeptuelle Repräsentation eines Bewegungsereig- 
nisses. Lassen sich keine Figur-bezogenen Raumkonzepte aus dem Input gewinnen, so wird zunächst nur die Art der Bewegung dargestellt. Sobald sich Hinweise für einen gerichteten Wegverlauf erkennen lassen, wird ein (neues) Bewegungsereignis aus der Perspektive der Figur konzeptualisiert. So erklären sich die Segmentierungsmuster und die Tatsache, dass die französischen Sprecher Bewegungsverläufe mit längeren Wegen in zwei oder mehr Äußerungen kodieren. Deutsche Sprecher sehen den Wegverlauf, so wie er durch die Eigenschaften des Grundes bestimmt ist, als zentrale Größe für die Konzeptualisierung von Bewegungsereignissen. Diese sind nicht - oder nur sehr begrenzt in Verben kodierbar. So dienen die Artverben zur Enkodierung des Bewegungsereignisses, dessen spezifischer Verlauf in Satelliten ausgedrückt wird. Hier kommt nun die Valenz ins Spiel. Die zu Beginn angeführten Beispiele rouler/ fahren in ihren unterschiedlichen Valenzmustern erklären sich durch den Unterschied in den wesentlichen semantischen Merkmalen. Fahren weist das Merkmal Direktionalität/Ortswechsel auf und verlangt daher eine Spezifikation des Wegs. Rouler weist dieses Merkmal nicht auf, es wird daher ohne Wegspezifikation als nacktes Verb oder mit einer Lokalangabe verbunden. ${ }^{9}$ Die kognitivorientierten Untersuchungen zeigen, wie dieser Kontrast eingebunden und motiviert ist durch unterschiedliche kognitive Strategien für die Repräsentation von Bewegungsereignissen. Kontraste, die auf der Ebene der Grammatik oder auch der form-orientierten Typologie beobachtet werden, können in differenzierter Weise auf die Ebene zurückgeführt werden, auf der sie begründet sind.

\subsubsection{Kontrastierung von temporaler Perspektivierung}

Ein notorisches Problem im Erwerb fremdsprachlicher Kompetenz stellen aspektuelle Markierungen dar. Wie in der Einführung bereits kurz angesprochen, besteht die Schwierigkeit weniger in dem Erlernen der Formen selbst, als vielmehr in dem angemessenen Einsatz unterschiedlicher Aspektformen. Sprachen die vollständig grammatikalisierte Aspektsysteme ausgebildet haben, wie das Englische, das Russische oder die arabischen Varietäten, weisen keineswegs Übereinstimmung darin auf, in welchen Kontexten eine Form, die für alle diese

\footnotetext{
9 Man wird vielleicht einwenden, dass der Satz, das Auto fährt durchaus akzeptabel ist und auch im Französischen gesagt werden kann, la voiture roule vers le village. Allerdings sind diese Verwendungsweisen in unterschiedlicher Weise beschränkt. Der deutsche Satz fokussiert auf die Eigenschaft und präsupponiert den Kontrast zu einer anderen Erwartung z. B. erstaunlich, dein Auto fährt ja. Die Verbindung von rouler mit Zieladjunkten ist eingeschränkt, rouler à mit Zielangabe ist keine mögliche Verbindung, Grenzüberschreitungen sind - wie immer wieder beschrieben mit dem Begriff border crossing constraint - generell nicht möglich.
} 
Sprachen beispielsweise als Imperfektiv bezeichnet wird, verwendet werden muss oder kann. Die Prinzipien, nach denen aspektuelle Markierungen gewählt werden, sind noch schwieriger zu bestimmen, wenn es sich um Sprachen handelt, die aspektuelle Kategorien nicht vollständig grammatikalisiert haben. Es handelt sich um Systeme, die unterschiedliche Übergangsstufen in der Grammatikalisierung von aspektuellen Markierungen repräsentieren. Als Beispiele sind das Italienische und das Niederländische zu nennen. Die Fragen, wie sich aspektuelle Systeme entwickeln, welche Kontexte bestimmte Markierungen anziehen und ob sich sprachübergreifende Prinzipien erkennen lassen, lassen sich nur empirisch klären. Ein kognitiv orientierter kontrastiver Ansatz ermöglicht, differenziert zu prüfen, welche konzeptuellen Grundlagen die Verwendung aspektueller Formen steuern.

Behrens et al. (2013) untersuchen in einer empirischen Studie die Verwendungsbedingungen für progressive Aspektformen in den Sprachen Niederländisch, Norwegisch und Deutsch. Alle drei Sprachen verfügen über verbale Konstruktionen, die eine Situation in Bezug auf einen zeitlichen Referenzpunkt als im Verlauf befindlich ausdrücken. Im Deutschen ist es die sogenannte Rheinische Verlaufsform er ist am/beim Lesen, im Niederländischen die vergleichbare Konstruktion hij is aan het lezen oder auch hij zit aan het lezen, das Norwegische weist zwei Konstruktionen auf: a) eine Pseudokoordination, bei der ein Positionsverb und ein Vollverb mit und verbunden werden en dame som sitter og perler et perlekjede (,eine Frau die sitzt und eine Perlenkette auffädelt'); b) eine Konstruktion mit Präposition und Infinitiv ähnlich wie in den anderen beiden Sprachen. Daneben existieren in allen drei Sprachen weitere Formen, die jedoch sehr wenig gebräuchlich sind. Keine der Sprachen hat einen grammatikalisierten Aspekt ausgebildet. Die aspektuell markierten Formen sind in keinem Kontext obligatorisch zu verwenden. Die aspektuell unmarkierten Formen stehen nicht in semantischer Opposition zu ihnen. Die Frage, der die Studie nachgeht, bezieht sich auf die Verwendungsbedingungen der Verlaufskonstruktionen. Folgen die Sprecher der drei Sprachen gleichen Prinzipien, d.h. folgen sie einem gemeinsamen Entwicklungspfad im Sinne der Grammatikalisierungstheorie (Bybee et al. 1993)? Die Untersuchung stützt sich auf kontrolliert erhobene Sprachproduktionsdaten. Auch in dieser Studie werden den Probanden kurze, realweltliche Videoclips gezeigt, die sie online versprachlichen sollen. Inhalt und Verwendungskontexte sind damit für die Sprecher der verschiedenen Sprachen vergleichbar. Die Situationen wurden anhand der folgenden Eigenschaften variiert:

1. +/- Zustandswechsel (herbeigeführt durch ein effiziertes oder affiziertes Objekt),

2. +/- Homogenität,

3. Bewegungsereignisse mit $+/-$ erreichtem Ziel (vgl. Behrends et al. 2013: 112). 
Betrachtet man die Ergebnisse über alle Typen von Stimuli hinweg, so ergibt sich folgendes Bild. Niederländer verwenden Verlaufsformen in 27,5\% der Fälle, Norweger in 14,5\% und Deutsche in 1,4\% $\%^{10}$. Betrachtet man nun differenziert, ob es Eigenschaften von Situationen gibt, die über die Sprachen hinweg Progressivmarkierung anziehen, wenn auch nur zu relativ geringem Prozentsatz, so zeigt sich, dass für alle Sprecher Situationen ohne Zustandswechsel die höchsten Frequenzen aufweisen (Niederländisch 65,8\%, Norwegisch 38,6\%, Deutsch 9,4\%). Niederländische Sprecher verwenden die Formen allerdings auch in Verbindung mit Situationen mit effizierten und affizierten Objekten $(62,1 \%$ und 52,5\%), während sie für Bewegungsereignisse nicht eingesetzt werden. Das Bild sieht etwas anders für die norwegischen Sprecher aus. Sie verwenden die Formen in Verbindung mit effizierten Objekten (21,8\%), weniger mit affizierten (8,7\%), dagegen in wenigen Fällen auch für die Darstellung von Bewegungsereignissen (5\%). In den deutschen Daten werden Bewegungsereignisse nie mit einer Verlaufsform ausgedrückt und auch bei Zustandswechselsituationen fällt der Gebrauch auf 1\%. Die Autoren der Studie interpretieren die Befunde in folgender Weise. ${ }^{11}$ Die drei Sprachen befinden sich auf unterschiedlichen Niveaus der Grammatikalisierung. Für das Deutsche lässt sich keine Tendenz in Richtung Grammatikalisierung erkennen. Das Niederländische und Norwegische unterscheiden sich zum einen in der Frequenz, zum anderen auch in der Formenvielfalt. Im Niederländischen besteht eine klare Präferenz für die periphrastische Form aan het $x$ zijn, während im Norwegischen keine der unterschiedlichen Formen in der Frequenz heraussticht. Beides führt zu der Annahme, dass das Niederländische sich auf dem Weg zur Grammatikalisierung einer aspektuellen Verbform befindet.

Aspekttypologisch zeigen die Ergebnisse, dass in allen drei germanischen Sprachen die Verlaufsform besonders durch Aktivitäten, die zu einem gegebenen Zeitpunkt gerade geschehen, angezogen wird: Störe mich nicht. Ich bin gerade am Telefonieren. Das bedeutet, es handelt sich hier nicht um einen Progressiv, als Ausdruck eines zu einem Umschlagpunkt ablaufenden Geschehens, sondern um einen imperfektiven Aspekt. Im Niederländischen hat sich die Verwendung auf weitere Kontexte ausgedehnt, von Aktivitäten auf Situationen mit Zustandswechsel. Die Befunde belegen so, dass von den drei germanischen Sprachen das Niederländische den höchsten Grad der Grammatikalisierung einer Aspektform repräsentiert. Die Bedeutung empirisch-experimenteller Studien für unser Verständnis der Funktionsweise von Sprachen heben die Autoren selbst hervor:

10 Hier sei angemerkt, dass die Probanden aus allen Teilen Deutschlands kamen.

11 Für eine differenzierte Diskussion der Ergebnisse sei auf Behrends et al. 2013 verwiesen. 
The present study illustrates the advantages of looking at actual language use based on identical input when analyzing the nature of the forms available in different languages. By correlating the use of linguistic means with the actual visual input, that is, properties manipulated on systematic terms in the situations presented, the informants' choice of expression can be compared across languages, allowing generalizations over the function of the different aspect markers on this basis. This would not be possible in a study based on corpus data. (Behrens et al. 2013: 133).

Wir bleiben abschließend bei dem Thema Aspekt und stellen in einem letzten Abschnitt dar, wie eng fokussierte, experimentelle Methoden der Psycholinguistik eingesetzt werden können, um über Verhaltensdaten auf Kontraste zwischen sprachlichen Systemen schließen zu können. Es wurde gerade verdeutlicht, dass eine interessante Frage, die sich im Zusammenhang mit strukturellen Veränderungsprozessen von Sprachen stellt, die nach dem Grad der Grammatikalisierung ist. Unter Aspekten der Sprachverarbeitung würde man annehmen, dass die Verwendung einer Struktur auf dem Wege der Grammatikalisierung immer weitergehend automatisiert wird und damit auch stabiler in Bezug auf unterschiedliche Kontexte und mögliche störende Einflüsse wird. Eine Methode, die grundsätzlich zur Überprüfung von Vernetzungsstrukturen innerhalb von sprachlichem Wissen eingesetzt wird, ist das Priming. Hierbei erhalten Probanden, bevor sie die eigentliche Aufgabe lösen (wie Sprechen, Verstehen, Bilder erkennen), einen Reiz. Dieser kann unterschiedliche Form haben und kann bewusst oder unbewusst von den Probanden aufgenommen werden. Gemessen wird der Einfluss dieses Prime-Elements auf das nachfolgende Verhalten (z. B. Reaktionszeiten, Fehlerhäufigkeit, Gebrauchsfrequenzen). Mit Manipulation des Prime-Elements lassen sich unterschiedliche Hypothesen über Vernetzungen in der kognitiven und sprachlichen Verarbeitung testen. Eingesetzt haben diese Methode Gerwien \& Flecken (2015), um den Grammatikalisierungsgrad der Aspektperiphrase im Niederländischen zu testen und zu prüfen, ob für deutsche Sprecher die periphrastische Verbform konzeptuell weniger fest verankert ist. Die häufigste aspektuelle Verbalkonstruktion im Niederländischen ist wie im Deutschen mit dem Gebrauch einer Präposition verbunden: Auxiliar + Präpostion (aan/an, bei) + definiter Artikel (het/dem) + nominalisierter Infinitiv. Die Präpositionen haben in dieser Funktion einen Prozess der Ausbleichung durchlaufen, tragen selbst keine spezifischen semantischen Merkmale zur Bedeutung bei. Daneben werden diese Präpositionen in ihrer Kernfunktion zum Ausdruck von räumlichen, zeitlichen und modalen Verhältnissen sowie zur Komplementmarkierung verwendet. Ausgehend von dieser Multifunktionalität der Präpositionen entwickeln Gerwien \& Flecken folgende Hypothese: Wenn für niederländische Sprecher der verbale Aspekt als eigenständige grammatikalische Kategorie mit einem eindeutigen konzep- 
tuellen Korrelat bereits fest im sprachlichen Wissen verankert ist, dann sollte keine Verbindung zu formal gleichen, aber funktional unterschiedlich belegten Formen hergestellt werden. Für Sprecher des Deutschen sollte diese Selbständigkeit der Verbalperiphrase nicht gegeben sein. Das Experiment sah folgendermaßen aus. ${ }^{12}$ Die Probanden erhielten die Aufforderung, einen kurzen Bildstimulus mit einem Satz zu beschreiben. Jeweils vor den kritischen Bildstimuli wurde den Probanden ein Prime-Satz präsentiert, in dem entweder eine aspektuell markierte Verbform verwendet wurde (14a) oder ein räumliches Adjunkt bzw. Dativobjekt in Form einer Präpositionalphrase (14b):

(14) a. De kleuter is een film aan het kijken.

(Das Kind ist einen Film am Schauen.)

b. Het kasteel staat aan het water.

(Das Schloss steht am Wasser.)

In einer neutralen Vergleichsbedingung wurde den Versuchsteilnehmern ein Satz ohne Präpositionalphrase präsentiert. Probanden waren eine Gruppe niederländische muttersprachliche Sprecher (L1) und eine Gruppe Deutsche mit sehr fortgeschrittener Kompetenz im Niederländischen (L2), die aus unterschiedlichen Regionen Deutschlands kamen. Ausgewertet wurde, wie häufig die Probanden die Aspektformen im Verhältnis zu den Prime-Bedingungen für die Beschreibung der Bilder verwendeten. Holzschnittartig zusammengefasst zeigen die Ergebnisse, dass bei den L1-Sprechern die aspektuell-markierten Prime-Sätze zu einer häufigeren Verwendung der Aspektform in der Szenenbeschreibung führten als die neutralen Prime-Sätze, die Prime-Sätze mit Lokativund Dativpräpositionalphrasen in Komplementfunktion dies jedoch nicht bewirkten. Für die L2-Sprecher ergab sich kein solcher Effekt. Man kann daraus schlussfolgern, dass die L1-Sprecher mit der aan-het-Verbalperiphrase die semantisch-kognitive Kategorie des Aspekts fest verbunden haben. Diese wird durch die Einführung einer entsprechenden temporalen Perspektive im PrimeSatz aktiviert und verstärkt die Tendenz, diese Form dann im Folgenden zu wählen. Formengleichheit allein stellt die Verbindung zu der konzeptuellen Kategorie des Aspekts nicht her. Für die deutschen L2-Sprecher lässt sich keine Beförderung der Gebrauchshäufigkeit durch Priming feststellen. Letzteres

12 Für Details der Studie sei der Leser an Gerwien \& Flecken (2015) verwiesen. Da es im vorliegenden Kontext darum geht, exemplarisch zu illustrieren, welche Erkenntnisse sich durch den Einsatz experimenteller Methoden für kontrastive Sprachanalysen gewinnen lassen, wird die Studie nicht im Einzelnen nachgezeichnet. 
könnte zwei Gründe haben: Entweder ist die Kompetenz in der L2 nicht groß genug, sodass die feste Verbindung zwischen Aspektform und semantischkognitivem Korrelat nicht etabliert ist. Entsprechend kann es keine Auswirkung im Sinne eines voraktivierten Konzepts geben. Die andere Möglichkeit wäre, dass deutsche Sprecher generell die entsprechende aspektuelle Kategorie nicht als Teil ihres (unbewussten) Sprachwissens ausgebildet haben und daher auch eine entsprechende Unterscheidung in einer Fremdsprache nicht treffen. Eine Fortführung, die eine entsprechende Priming-Studie für das Deutsche umsetzt, könnte hier weiteren Aufschluss geben.

Diese Studien zum Aspekt machen deutlich, dass wir unter Einsatz experimenteller Methoden, in denen die kognitive Ebene maßgeblich für die Wahl eines tertium comparationis ist, Einblicke in Sprachkontraste gewinnen können, die über Systemkontrastierung - egal auf welcher Ebene - nicht möglich wären. Empirische Methoden bieten uns feinkörnigere Aufschlussmöglichkeiten, deren Ergebnisse letztlich für die Erklärung eines angemessenen Sprachgebrauchs entscheidend sind. Auf dieser Grundlage lassen sich Fragen beantworten wie die nach der funktionalen Äquivalenz bzw. Unterschiedlichkeit oberflächlich ähnlich erscheinender Strukturen. Auf diesem Wege lassen sich auch Unterschiede im Status bestimmter Formen innerhalb verschiedener sprachlicher Systeme erkennen. Was man landläufig als Präferenzen, stilistische Variationen oder vielleicht sogar Idiosynkrasien einzelner Systeme beschreibt und damit im Grunde für nicht systematisch erklärbar hält, kann unter Bezug auf die kognitive Ebene möglicherweise auf einzelsprachliche Prinzipien zurückgeführt werden.

\section{Kontrastive Analyse 2020}

Kontraste schärfen den Blick und ermöglichen das Erkennen von Gestalten. Die drei Fallbeispiele haben gezeigt, dass eine Weiterentwicklung der kontrastiven Analyse in der Sprachwissenschaft Ebenen des sprachlichen Wissens erschließen kann, die bisher kaum in den Blick kontrastiver Betrachtungen genommen wurden. Auf der Grundlage des einzelsprachlichen Inventars an lexikalischen Formen und grammatischen Prinzipien haben sich Prinzipien der Sprachverwendung ausgebildet, die den Einsatz sprachlicher Formen für die Darstellung kommunikativer Inhalte steuern. Man kann sie als funktionale Prinzipien bezeichnen, die als Interface zwischen kognitiv repräsentierten Inhalten und sprachlichem Ausdrucksinventar fungieren. Diese Prinzipien zeigen sich in der Auswahl von inhaltlichen Komponenten, mit denen bestimmte außersprachliche Gegebenheiten repräsentiert werden. Sie zeigen sich in der Perspektiven- 
wahl und Strukturierung von Inhalten im Kontext komplexer Informationsvermittlung. Kontrastive Analysen, wie die in den drei Fallstudien dargestellten, geben Einblicke in die Wirkungsweise dieser Prinzipien auf den Ebenen des Textaufbaus ebenso wie der Satzkomposition. Untersuchungen dieser Art setzen eine Verschiebung des tertium comparationis von der Ebene der einzelsprachlichen Einheiten und Strukturen - die bisher in der Regel für kontrastive Studien maßgeblich war - auf die der einzelsprachlichen Form vorausgesetzte, kognitive Ebene voraus. Damit einher gehen neue Anforderungen an die Forschungsmethoden. Die drei Fallstudien haben für unterschiedliche Bereiche zwischen Text und verbalen Paradigmen illustriert, wie experimentelle Methoden eingesetzt werden können, um Kontraste $\mathrm{zu}$ ermitteln, die auf der Ebene der funktionalen Prinzipien zu verorten sind. Diese bilden den Ausgangspunkt um zu verstehen, wie und warum bestimmte Formen in den jeweiligen Einzelsprachen eingesetzt werden können und welchen Beschränkungen sie unterliegen. Kognitiv-orientierte kontrastive Analysen bereiten den Weg, um unser Wissen über die Funktionsweise einzelsprachlicher Systeme in unterschiedlichen Bereichen zu erweitern. Wie die Studie zum Aspekt verdeutlicht, können kognitiv-orientierte Studien genauer Auskunft über Eigenschaften und Status bestimmter Formen innerhalb eines einzelsprachlichen Systems geben. Die Untersuchung zur Ereignisrepräsentation zeigt, wie an der sprachlichen Oberfläche scheinbar vergleichbare Einheiten innerhalb eines Systems einen unterschiedlichen Wert besitzen können und so dieselben außersprachlichen Gegebenheiten in keineswegs inhaltlich äquivalenter Weise wiedergegeben werden. Die dritte Fallstudie verdeutlicht, dass Informationsaufbau und Informationsmanagement in Texten einzelsprachlichen Prinzipien unterliegt, die wiederum nicht durch Vergleiche auf der Ebene des verfügbaren Formeninventars zu erkennen sind.

Beschreiten wir diesen Weg weiter im Sinne von Eichinger, der den Forschungsansatz der vergleichenden und kontrastierenden Sprachanalyse mit der Entwicklung der linguistischen Theorien und Methoden eng verbunden sieht, so eröffnen sich Forschungsperspektiven für eine Germanistik 2020, die nicht nur unsere Kenntnisse über die deutsche Sprache erweitern werden, sondern darüber hinaus auch Einsichten liefern können, die für anwendungsbezogene Teildisziplinen wie die Fremd- und Zweitsprachendidaktik oder die Übersetzungswissenschaften fruchtbar gemacht werden können.

\section{Literatur}

Beaver, David I. (2004): The optimization of discourse anaphora, Linguistics and Philosophy 2004. 3-56. 
Behrens, Bergljot, Monique Flecken \& Mary Carroll (2013): Progressive attraction: On the use and grammaticalization of progressive aspect in Dutch, Norwegian, and German. In Journal of Germanic Linguistics 25 (2), 95-136.

Berman, Ruth A. (1995): Aspects of Hebrew/English contrastive rhetoric. In H. Shyldkrot \& L. Kupferman (Hrsg.), Tendances Récentes dans la linguiste française et générale, 4154. Amsterdam: Benjamins.

Berman, Ruth A. \& Dan I. Slobin (1994): Relating events in narrative. A crosslinguistic developmental study. Hillsdale N): Erlbaum.

Bertinetto, Pier M., Karen H. Ebert \& Casper de Groot (2000): The progressive in Europe. In Östen Dahl (Hrsg.), Tense and aspect in the languages of Europe, 517-558. Berlin: De Gruyter.

Bopp, Franz (1816): Über das Conjugationssystem der Sanskritsprache in Vergleichung mit jenem der griechischen, lateinischen, persischen und germanischen Sprache. Frankfurt am Main: Andreäsche Buchhandlung.

Bybee, Joan, Revere Perkins \& William Pagliuca (1993): The Evolution of grammar: Tense, aspect and modality in the languages of the world. Chicago: University of Chicago Press.

Carroll, Mary (2000): Representing path in language production in English and German: Alternative perspectives on figure and ground. In C. Habel \& C. v. Stutterheim (Hrsg.), Räumliche Konzepte und sprachliche Strukturen, 97-118. Tübingen: Niemeyer.

Carroll, Mary, Katja Weimar, Monique Flecken, Monique Lambert \& Christiane v. Stutterheim (2012): Tracing trajectories: Motion event construal by advanced L2 French-English and L2 French-German speakers. Language Interaction \& Acquisition 3 (2), 202-230.

Carroll, M., A. Roßdeutscher, M. Lambert \& C. v. Stutterheim (2008): Subordination in narratives and macrostructural planning. A comparative point of view. In C. FabriciusHansen \& W. Ramm (Hrsg.), Subordination versus coordination in sentence and text.), 161-186. Amsterdam: Benjamins.

Dahl, Östen (2000): Tense and aspect in the languages of europe. Berlin: De Gruyter. Dryer, Matthew S. (2017): Order of subject, object and verb. In Matthew S. Dryer,. \& Martin Haspelmath (Hrsg.), The world atlas of language structures online. Leipzig: Max Planck Institute for Evolutionary Anthropology. http://wals.info/chapter/81 (letzter Zugriff: 2. 9. 2017).

Eichinger, Ludwig M. (2012): Deutsch im Sprachvergleich. Grammatische Kontraste und Konvergenzen. In Lutz Gunkel \& Gisela Zifonoun (Hrsg.), Deutsch im Sprachvergleich. Grammatische Kontraste und Konvergenzen, VII-XVI. Berlin: De Gruyter.

Fabricius-Hansen, Cathrine (2010): Deutsch im Kontrast - textbezogen. Zeitschrift für Germanistische Linguistik, 171-199.

Flecken, Monique, Mary Carroll, Katja Weimar \& Christiane v. Stutterheim (2015): Driving along the road or heading for the village? Conceptual differences underlying motion event perception and description in French, German, and French-German L2 Users. Modern Language Journal, 100-122.

Gerwien, Johannes \& Monique Flecken (2015): There is no prime for time: The missing link between form and concept of progressive aspect in L2 production. International Journal of Bilingual Education and Bilingualism 18 (5), 561-587.

Gerwien, Johannes \& Christiane v. Stutterheim (eingereicht): Event segmentation: Crosslinguistic differences in verbal and non-verbal tasks. Universität Heidelberg.

Greenberg, Joseph (1963): Some universals of grammar with particular reference to the order of meaningful elements. In Joseph Greenberg (Hrsg.), Universals of language, 110-113. London: MIT Press. 
Grimm, Jacob (1919): Deutsche Grammatik. Göttingen: Dieterich.

Gunkel, Lutz \& Gisela Zifonoun (Hrsg.) (2012): Deutsch im Sprachvergleich. Grammatische Kontraste und Konvergenzen. Berlin: De Gruyter.

Haspelmath, Martin, Ekkehard König, Wulf Oesterreicher \& Wolfgang Raible (Hrsg.) (2001): Sprachtypologie und sprachliche Universalien. Ein Internationales Handbuch. HSK 20.2. Berlin: De Gruyter.

Hawkins, John A. (1983): Word order universals. New York: Academic Press.

Humboldt, Wilhelm v. (1903-1936): Über das vergleichende Sprachstudium in Beziehung auf die verschiedenen Epochen der Sprachentwicklung. In Albert Leitzmann (Hrsg.), Gesammelte Schriften. Bd. IV, 1-34. Berlin: Behr.

Humboldt, Wilhelm v. (2012): Vaskische Grammatik. In: Wilhelm von Humboldt: Baskische Wortstudien und Grammatik, hg. von Bernhard Hurch. Paderborn etc.: Schöningh, 201236.

Hurch, Bernard (Hrsg.) (2012): Wilhelm von Humboldt. Baskische Wortstudien und Grammatik. Paderborn: Schöningh.

Kabatek, Johannes (2011): Diskurstraditionen und Genres. In Sarah Dessí Schmid, Ulrich Detges, Paul Gévaudan, Wiltrud Mihatsch \& Richard Waltereit, Rahmen des Sprechens. Beiträge zu Valenztheorie, Varietätenlinguistik, Kreolistik, Kognitiver und Historischer Semantik, 89-100. Tübingen: Narr.

Klein, Wolfgang (2000): An analysis of the German perfect. Language 76, 58-382.

Klein, Wolfgang, \& Heinz Vater (1998): The perfect in English and German. In L. Kulikov \& H. Vater (Hrsg.), Typology of verbal categories. Papers presented to Vladimir Nedjalkov on the occasion of his 70th birthday, 215-235. Tübingen: Niemeyer.

Klein, Wolfgang \& Christiane v. Stutterheim (1987): Quaestio und referentielle Bewegung in Erzählungen. Linguistische Berichte 108, 163-183.

König, Ekkehard (2012): Zur Standortbestimmung der Kontrastiven Linguistik innerhalb der vergleichenden Sprachwissenschaft. In Lutz Gunkel \& Gisela Zifonun (Hrsg.), Deutsch im Sprachvergleich. Grammatische Kontraste und Konvergenzen, 13-40. Berlin: De Gruyter.

Rask, Rasmus (1818): Undersøgelse om det gamle Nordiske eller Islandske Sprogs Oprindelse. Kopenhagen: Gyldendalske Boghandlings Forlag.

Robert Lado (1957): Linguistics across cultures. Michigan: University of Michigan Press.

Lang, Ewald \& Gisela Zifonun (Hrsg.) (1996): Deutsch - typologisch. Institut für Deutsche Sprache, Jahrbuch 1995. Berlin: De Gruyter.

Levinson, Stephen C (2003): Space in language and cognition: Explorations in cognitive diversity. Cambridge: Cambridge University Press.

Natale, Silvia (2009): Gebrauchsdeterminanten der progressiven Verbalperiphrase stare + gerundio. Tübingen: Narr.

Saussure, Ferdinand de (1967): Grundfragen der Allgemeinen Sprachwissenschaft. Berlin: De Gruyter.

Slobin, Dan I. (1996): Two ways to travel: Verbs of motion in English and Spanish. In M. Shibatani \& S. C. A. Thompson (Hrsg.), Grammatical constructions: Their form and meaning, 195-220. Oxford: Clarendon Press.

Slobin, Dan I. (2000): Verbalized events: A dynamic approach to linguistic relativity and determinism. In S. Niemeier \& R. Dirven (Hrsg.), Evidence for linguistic relativity, 107-138. Berlin: Mouton de Gruyter.

Stutterheim, Christiane v. (1997): Einige Prinzipien des Textaufbaus. Tübingen: Niemeyer.

Stutterheim, Christiane v. (2016): Prinzipien der Sprachverwendung als Teil unseres Sprachwissens. Studien zum Sprachvergleich und zu fortgeschrittenen Lernervarietäten. In Jahrbuch des IDS 2016. 
Stutterheim, Christiane v., Martin Andermann, Mary Carroll, Monique Flecken \& Barbara Schmiedtova (2012): How grammaticized concepts shape event conceptualization in the early phases of language production. Insights from linguistic analysis, eye tracking data and memory performance. Linguistics 50 (4), 833-869.

Stutterheim, Christiane v. \& Mary Carroll (2018): Texts as answers to questions: Information structure and its grammatical underpinnings in narratives and descriptions in German and English (topic and anaphoric linkage) In A. Hübl \& M. Steinbach, Linguistic foundations of narration. Amsterdam/Philadelphia: Benjamins.

Talmy, Leonard (1985): Lexicalization patterns: Semantic structure in lexical forms. In T. Shopen (Hrsg.), Language typology and syntactic description, Vol. III., 57-149. Cambridge: Cambridge University Press.

Theisen, Joachim (2016): Kontrastive Linguistik. Tübingen: Narr.

Vollmann, Ralf (2000): Wilhelm von Humboldt und die Grammatik des Baskischen. In Michaela Ofitsch \& Christian Zinko (Hrsg), 125 Jahre Indogermanistik in Graz. Festband anläßlich des 125-jährigen Jubiläums in Graz (Arbeiten aus der Abteilung ,Vergleichende Sprachwissenschaft‘ Graz. Bd. 17), 487-503. Graz: Leykam. 


\title{
10 Deiktische Antworten auf räumliche Fragen
}

\begin{abstract}
Mit diesem Beitrag soll nachgewiesen werden, dass es sich für die Sprachtypologie, theoretische Morphologie, Raum-Linguistik als äußerst lohnenswert erweisen könnte, die Paradigmen räumlicher Interrogativa und ihrer lokal-deiktischen Äquivalente einzel- wie übereinzelsprachlich vergleichend zu untersuchen. Dies erfolgt zunächst innerhalb der Germania, bevor auf der Grundlage der im Sprachvergleich gemachten Beobachtungen vorläufige Schlüsse für den Grundriss eines künftigen sprachtypologischen Projekts gezogen werden sollen.
\end{abstract}

Keywords: Deixis, Morphologie, Raum-Linguistik, Sprachtypologie, Sprachvergleich

\section{Deutsche Verhältnisse als Einstiegsdroge}

Mit diesem Beitrag möchte ich den Nachweis antreten, dass es sich gleich für mehrere sprachwissenschaftliche Forschungsprogramme - Sprachtypologie, theoretische Morphologie, Raum-Linguistik - als äußerst lohnenswert erweisen könnte, die Paradigmen räumlicher Interrogativa (= RI) wie z.B. wo und

\footnotetext{
Anmerkung: Diese Arbeit ist im Rahmen des durch die Deutsche Forschungsgemeinschaft (DFG) im Zeitraum 2016-2018 geförderten Projekts (STO 186/19-2) Wo - Wohin - Woher. Räumliche Interrogativa und ihre lokaldeiktischen Entsprechungen entstanden. Ich danke Nataliya Levkovych, Julia Nintemann und Maja Robbers für ihre tatkräftige Unterstützung bei der Vorbereitung dieses Beitrags, der als Pilotstudie für die zweite Phase des von uns durchgeführten Projekts gedacht ist. Dankeschön möchte ich auch Ekkehard König und einem anonymen Gutachter bzw. einer anonymen Gutachterin der DFG sagen, die nachdrücklich darauf bestanden haben, das Projekt auch auf die Lokaldeiktika auszudehnen. Ein Dankeswort geht an die Herausgeberin dieser Festschrift, die es mir ermöglicht hat, unser Thema publik zu machen. Ungeachtet der technischen Hilfe durch mein Projektteam bleibt doch alles, was und wie ich es in diesem Aufsatz sage, ganz und gar in meiner alleinigen Verantwortung.
}

Thomas Stolz, Universität Bremen, FB 10: Linguistik, Universitäts-Boulevard 13, D-28359 Bremen, E-Mail: stolz@uni-bremen.de

Ә Open Access. (c) 2018 Thomas Stolz, publiziert von De Gruyter. (c) BY Dieses Werk ist lizenziert unter der Creative Commons Attribution 4.0 Lizenz.

https://doi.org/10.1515/9783110490992-011 
ihrer lokal-deiktischen Äquivalente (= LD) wie z. B. hier, da, dort einzel- wie übereinzelsprachlich vergleichend unter der Lupe zu betrachte. In keinem der genannten Forschungsprogramme ist es bisher zur Zusammenführung der beiden genannten Bereiche gekommen, im Falle der RI gibt es bislang noch überhaupt keine Eingliederung in die jeweiligen theoretischen Modelle. Zwar werden die Paradigmen in der einschlägigen Literatur zu relativ ausführlich beschriebenen Sprachen wie Deutsch thematisiert, aber eine grammatiktheoretische Evaluierung der Befunde steht weiterhin aus. Mein Beitrag versteht sich als morphologisch-orientierte Studie im sprachvergleichenden Sinne. Es bedarf allerdings der Klärung einer Reihe grundsätzlicher Fragen, die ich in dieser Einleitung im Rahmen der Diskussion des deutschen Befundes beantworte. Nachdem ich die für diese Studie relevanten Konzepte ausbuchstabiert habe, stelle ich in Abschnitt 2 einen allgemeinen Maßstab für den Sprachvergleich unter der Bezeichnung Kanon vor. In Abschnitt 3 erweitere ich in Rückkopplung zu diesem Maßstab schrittweise die Empirie innerhalb der Germania, um in Abschnitt 4 abschließend auf der Grundlage der im Sprachvergleich gemachten Beobachtungen zu vorläufigen Schlüssen zu gelangen, die ihrerseits den Grundriss eines künftigen sprachtypologischen Projekts skizzieren, aus dem auch andere Forschungsgebiete Gewinn ziehen können. Relevante Sekundärliteratur wird passim selektiv erwähnt, weil aus Platzgründen eine ausführliche Würdigung aller thematisch affinen Beiträge in Form eines klassischen Literaturberichts nicht möglich ist. Ebenfalls aus Platzgründen stelle ich ausschließlich synchrone Betrachtungen an.

Tabelle 10.1 enthält einen Aufriss der hier fokussierten Kategorien. Dieses tabellarische Schema bildet mit wenigen Modifikationen das Muster für die Präsentation der empirischen Fakten aller in diesem Beitrag herangezogenen Beispielsprachen. Was hinter dieser Darstellungsform steckt, wird nachstehend im Einzelnen erläutert.

Tab. 10.1: RI- und LD-Paradigmen im Deutschen.

\begin{tabular}{|c|c|c|c|c|}
\hline \multirow[t]{2}{*}{$\mathfrak{R}$} & \multirow[t]{2}{*}{$\mathbf{R I}$} & \multicolumn{3}{|c|}{ LD } \\
\hline & & D1 & D2 & D3 \\
\hline Place & $w o$ & hier & $d a$ & dort \\
\hline Goal & wohin & $\begin{array}{l}\text { hierhin } \\
\text { nach hier }\end{array}$ & $\begin{array}{l}\text { dahin } \\
\text { nach da }\end{array}$ & $\begin{array}{l}\text { dorthin } \\
\text { nach dort }\end{array}$ \\
\hline Source & $\begin{array}{l}\text { woher } \\
\text { von wo }\end{array}$ & $\begin{array}{l}\text { hierher } \\
\text { von hier }\end{array}$ & $\begin{array}{l}\text { daher } \\
\text { von da }\end{array}$ & $\begin{array}{l}\text { dorther } \\
\text { von dort }\end{array}$ \\
\hline
\end{tabular}


Berücksichtigt wird in Tabelle 10.1 nur ein ganz bestimmter Ausschnitt aus den ansonsten viel umfänglicheren Paradigmen der RI und LD des Deutschen, nämlich genau diejenigen Formen, die zum Ausdruck von drei der ,vier grundlegende[n] lokale[n] Relationen [...] LOCATIVE, SOURCE, PATH und GOAL“ (Goldap 1991: 22) dienen, die Bennett (1975) in seiner Analyse englischer Präpositionen unter der Rubrik der generellen Lokation (,general location“) postuliert. U.a. wegen der übereinzelsprachlich nur sehr spärlichen Dokumentation für die räumliche Relation $(=\mathfrak{R})$ Path konzentriere ich mich hier unter Beibehaltung der international etablierten anglisierenden Terminologie auf die empirisch wesentlich besser abgedeckte Trias aus Place (= Bennetts LocATIVE), Goal und Source.

In diesem Kategorien-Tripel bezieht sich Place auf die statische Situation der Befindlichkeit eines Trajektors ( bewegliche Entität) bezogen auf eine Landmarke ( unbeweglicher Hintergrund) (Goldap 1991: 14, Terminologie nach Langacker 1986). Goal und Source beziehen sich auf dynamische Situationen, in denen sich ein Trajektor auf eine Landmarke zu bewegt (= Goal) oder von dort wegbewegt (= Source). RI dienen dem Zweck, diese Landmarken zu erfragen. Die LD können als potenzielle Antworten auf die jeweiligen RI verstanden werden, bei denen wie in (1) keine lexikalische Identifikation der Landmarke erfolgt.

(1) Deutsch: RI-LD im Wechselspiel von Frage und Antwort ${ }^{1}$
a. Place: Wo ist sie?
Sie ist hier/da/dort.
b. Goal: Wohin geht sie?
Sie geht hierhin/dahin/dorthin.
c. Source: Woher kommt sie? Sie kommt hierher/daher/dorther.

Auch wenn sich die LD keineswegs auf diese Aufgabe reduzieren lassen, ist es doch offensichtlich, dass zwischen RI und LD ein funktionales Band besteht, das es nahelegt, die beiden Klassen von Ausdrücken miteinander vergleichend zu betrachten. Es überrascht in diesem Zusammenhang, dass im weiten Rahmen der nun auf mehrere Jahrzehnte intensiver Forschungen zurückblickenden „Raum-Linguistik“ (Vater 1991) oder Raumgrammatik (Svorou 1993) dieses spezifische Thema bisher noch nicht systematisch aufgegriffen wurde. ${ }^{2}$ Auch

1 Alle sentenziellen deutschen Beispiele beruhen auf meiner Introspektion als deutscher Muttersprachler. In Beispielsätzen werden RI und LD durch Fettdruck hervorgehoben.

2 Gelegentlich werden RI und LD in anderem Zusammenhang - z. B. Lautsymbolismus (Haase 1992) - punktuell zueinander in Beziehung gesetzt, ohne dass dabei jedoch systematische Zusammenhänge überzeugend dargestellt werden können. 
im Bereich der Linguistik der Frage wird die RI-LD-Beziehung eher nur am Rande erwähnt (Dixon 2012: 407-409). Die erste große sprachvergleichende Studie zu den RI (Stolz et al. 2017) ist jungst erschienen. Die LD sind in der reichhaltigen Literatur zur Deixis vor und seit Anderson \& Keenan (1985) immer wieder auch ein Thema, das in der kognitiv-anthropologischen Forschung aufgegriffen wird (Burenhult 2008); dennoch harren sie weiterhin einer großangelegten systematischen Bestandsaufnahme, nach deren Abschluss es erst möglich sein wird, das Beziehungsgeflecht zwischen RI und LD gründlich zu durchleuchten. An dieser Stelle kann ich daher nur einen Vorgeschmack auf das geben, was eventuell zukünftig noch folgen wird.

Die Daten in Tabelle 10.1 beruhen zum einen auf den Angaben in Zifonun, Hoffmann \& Strecker (1997: 332) und sind zum anderen durch mich um (zum Teil stilistisch markierte) Fundstücke introspektiv ergänzt. Diese Ergänzungen betreffen ausschließlich zweiwortige Bildungen, sodass im Deutschen die unter (2) aufgeführten Konstruktionsmuster nebeneinander vorkommen.

(2) Deutsch: Konstruktionsmuster zur Bildung von RI und LD

a. $[\mathrm{X}]_{\text {statisch }}$

b. $[\mathrm{X}=\text { Klitikon }]_{\text {dynamisch }}$

c. $\quad[\text { Präp } \mathrm{X}]_{\text {dynamisch }}$

$\mathrm{X}$ steht dabei als Variable für wo, hier, da, dort. Wie die Ausdrücke für Place zeigen, kann X alleine eine paradigmatische Zelle füllen. Im Einklang mit den Annahmen der Konstruktionsmorphologie (Booij 2010: 5-6) betrachte ich auch monomorphisch-einwortige Ausdrücke als Konstruktionen. Einwortige und mehrwortige Ausdrücke können zusammen ein Paradigma konstituieren. Im Falle von (2a) liegt zudem obligatorische Null-Markierung einer räumlichen Relation vor (Stolz, Lestrade \& Stolz 2014: 291-295), ${ }^{3}$ weil die Konstruktion keinen Exponenten umfasst, der die Relation expliziert, sondern nur das Element, das den Ausdruck als RI bzw. LD identifiziert und im restlichen Paradigma jeweils als Konstante („Stamm“) auftritt.

Bei den strukturell komplexen Konstruktionen ist eine weitere Leerstelle durch ein Klitikon =hin bzw. =her oder eine Präposition nach bzw. von gefüllt. ${ }^{4}$

3 Zur Null-Markierung räumlicher und anderer Relationen z. B. im Kiezdeutschen verweise ich auf Wiese \& Pohle (2016).

4 Die Mobilität der Klitika (wie in: Sie geht dorthin. Dort geht sie hin. Sie ist dort gestern hingegangen.) hat für den weiteren Argumentationszusammenhang dieses Beitrags keine Bedeutung. Siehe hierzu Fleischer (2002). 
Nur die dynamischen $\mathfrak{R}$ sind obligatorisch durch Relatoren spezifiziert. ${ }^{5}$ Der formale Parallelismus zwischen den RI- und LD-Paradigmen geht so weit, dass dort, wo die Ausdrücke morphologisch komplex sind, dies auch auf die entsprechenden LD zutrifft und umgekehrt. Mehrfach gefüllte (= doppelt belegte) Paradigmenzellen kommen nur dort vor, wo komplexe Ausdrücke auftreten, aber nicht jeder komplexe Ausdruck hat eine entsprechende Alloform neben sich. Die augenfälligen Komplexitätsunterschiede zwischen monomorphischeinwortigen Place-Ausdrücken und den polymorphen/zweiwortigen Ausdrücken für Goal und Source bieten sich für eine Interpretation im Sinne einer natürlichkeitstheoretischen Markiertheitshierarchie (Mayerthaler 1987: 48-49) wie in (3) an, die man heute im Lichte von Haspelmath (2006) eventuell eher als Frequenzeffekt werten würde, was in einer rein qualitativen Untersuchung wie dieser jedoch auf Grund fehlender statistisch belastbarer Daten nicht zu leisten ist.

(3) Deutsch: Markiertheitshierarchie

$\mathrm{m}<$ statisch; dynamisch $>$

Die Paradigmen sind also durch einen relativ hohen Grad an Symmetrie gekennzeichnet, was sie für die Schule der kanonischen Morphologie interessant macht, die verstärkt nach „morphological mismatches“ Ausschau hält und ihre Musterhaftigkeit sprachunabhängig erklären will (Corbett 2007: 30-31). Für diesen Ansatz ist beispielsweise die Koexistenz von synonymen Ausdrücken ein Beleg für ein Überangebot („overabundance“ gemäß Thornton (2012)) von Optionen. Gleichzeitig ist das Nebeneinander von einwortigen und mehrwortigen Ausdrücken im selben Paradigma ein Ausweis für das Phänomen der Periphrase (oder ihres Gegenstücks Antiperiphrase). Stets ist dabei Heterogenität der im Paradigma zusammengefassten Wortformen im Spiel.

Ein ganz wesentlicher Unterschied zwischen RI und LD besteht darin, dass letztere auf einer Dimension Differenzierungen aufweisen, die den RI fremd sind, nämliche die Stufen der deiktischen Distanz (= D1/2/3), die Zifonun, Hoffmann \& Strecker (1997: 328-331) als in Nah- und Fernbereich untergliederte Raumbereiche bezogen auf das deiktische Zentrum (= Origo) fassen. Im Deutschen spielt die deiktische Distanz für die RI keine Rolle, d.h. dass die bei den LD getroffenen Unterscheidungen bei den RI neutralisiert sind. In der einschlägigen Literatur zur Deixis wird gerade dem Aspekt der Differenzierung von Distanzstufen viel Prominenz zugebilligt. In einer entsprechenden typologischen

5 Die PP *nach wo ist für mich als RI-Konstruktion nicht akzeptabel. 
Studie nimmt Diessel (2005: 170) an, dass „[i]n general, it seems that all languages have at least two adverbial demonstratives (or deictic particles) that indicate a distance contrast between two referents or locations", sodass die stärkere Differenzierung der LD gegenüber den RI für allgemein gegeben gelten darf. Wegen des eng bemessenen Raums schenke ich im Folgenden dieser für die LD typischen Eigenschaft keine weitere Beachtung, um mich ausschließlich auf dasjenige zu konzentrieren, was sowohl für die RI als auch für die LD relevant ist. Es genügt demnach, das Paradigma der RI mit nur einer der verschiedenen Distanzstufen der LD zu vergleichen, sofern keine besonderen Gründe im Einzelfall dafür sprechen, den Vergleich auf mehrere Distanzstufen auszuweiten.

Aus der Sicht der kognitiv-orientierten Forschungen zum Komplex Sprache und Raum sind die strukturellen Parallelen der Paradigmen von RI und LD im Deutschen gewissermaßen erwartbar. Denn es ist intuitiv plausibel, dass die kognitiven Grundlagen der Raumgrammatik in einer Einzelsprache homogen sind und daher für funktional verwandte grammatische Teilbereiche denselben Satz von Konzepten mit vergleichbarer Manifestation auf der strukturellen Ebene anbieten (Goldap 1991: 6-12). Heißt dies dann auch, dass sich RI und LD in den Sprachen der Welt grundsätzlich, wenn auch nicht punktgenau dem Deutschen entsprechend, untereinander stets in ihrer paradigmatischen Ausgestaltung parallel verhalten? Dies zu überprüfen ist eine der Aufgaben des von mir hier skizzierten Beitrags.

\section{Ein vorläufiger Goldstandard}

Nach Eichinger (2012: xii) „erhellt sich [das Profil, der Sprachtyp] aus dem Ort des Deutschen im Rahmen der Optionen von Sprachen überhaupt“, d.h. dass $\mathrm{zu}$ überprüfen ist, wie sich die in Tabelle 10.1 erfassten Gegebenheiten des Deutschen in das Gesamtbild der Sprachen der Welt einordnen. Verhält sich das Deutsche nach einem majoritären Muster oder optiert es für eine Minderheitenlösung? Sind die Sprachen, die hinsichtlich von RI-LD-Beziehungen dem Deutschen gleichen, auch auf anderen Gebieten strukturell dem Deutschen ähnlich? Spielt sprachliche Verwandtschaft beim Gleichverhalten eine Rolle? Lassen sich Sprachkontaktphänomene für Ähnlichkeiten und Unähnlichkeiten verantwortlich machen? Keine dieser Fragen ist bisher in der Literatur thematisiert worden. Um hier Abhilfe zu schaffen, ist es angemessen, einen unabhängigen Referenzrahmen für den folgenden Sprachvergleich zu schaffen. Dabei bediene ich mich des Konstrukts des kanonischen Paradigmas (Corbett 2007: 22-24), demzufolge die logisch möglichen Distinktionen für ein Paradigma kal- 
Tab. 10.2: Kanonische RI- und LD-Paradigmen.

\begin{tabular}{lll}
\hline$\Re$ & Ausdrucksklasse & \\
\cline { 2 - 3 } & RI & LD \\
\hline Place & RI, $\mathrm{X}_{\text {LoKAtIV }}$ & $\mathrm{LD}_{1 / 2 / 3}, \mathrm{X}_{\text {LoKativ }}$ \\
Goal & RI, $\mathrm{Y}_{\text {ALLATIV }}$ & $\mathrm{LD}_{1 / 2 / 3}, \mathrm{Y}_{\text {ALLATIV }}$ \\
Source & RI, $\mathrm{Z}_{\text {ABLATIV }}$ & $\mathrm{LD}_{1 / 2 / 3}, \mathrm{Z}_{\text {ABLATIV }}$ \\
\hline
\end{tabular}

kuliert werden, um das Maximum an eindeutigen und expliziten Ausdrücken (= Konstruktionen) zu berechnen. Auf den Fall der RI-LD-Beziehungen berechnet ergibt sich das kanonische Paradigma in Tabelle 10.2.

Es liegen sechs distinkte Ausdrücke vor, die sich aus der einfachen Multiplikation von zwei Ausdrucksklassen (RI und LD) mal drei $\Re$ (Place, Goal, Source) ergeben. Jede Ausdrucksklasse muss distinkt gekennzeichnet werden, ebenso jede $\Re$. Keine zwei Ausdrücke dürfen völlig identisch miteinander sein. Keine Zelle soll mehrfach belegt sein. Diese Bedingungen sind mit insgesamt fünf morphologischen Einheiten zu leisten, nämlich einem RI-Morphem, einem LD-Morphem und je einem lokativischen, allativischen und ablativischen Morphem X/Y/Z. Ob es sich dabei um freie (z. B. Adpositionen) oder gebundene (z. B. Kasusaffixe) Morpheme handelt, ist in dem kanonischen Paradigma nicht festgelegt (daher die Kommanotation in Tab. 10.2). Bezogen auf den RI-LDKomplex bestehen gleich auf zwei Dimensionen paradigmatische Beziehungen. Zum einen gibt es intern-paradigmatische (oder vertikale) Beziehungen, die das Verhältnis der Konstruktionen verschiedener $\Re$ ein und derselben Ausdrucksklasse betreffen. Zum anderen liegen extern-paradigmatische (oder horizontale) Beziehungen vor, die Konstruktionen involvieren, die gleiche $\Re$ verschiedener Ausdrucksklassen betreffen.

Wie ein Abgleich der Tabellen 10.1 und 10.2 zeigt, stimmt das Deutsche nur teilweise mit dem Kanon überein. Die Null-Markierung von Place und die Ausdrucksalternativen bei den dynamischen $\Re$ können als nicht-kanonische Eigenschaften der RI- und LD-Paradigmen des Deutschen gewertet werden. Mit dem Kanon im Reinen ist das Deutsche jedoch hinsichtlich der maximalen Distinktivität der Konstruktionen bei gleichzeitig sparsamem Einsatz von morphologischen Exponenten. Extern-paradigmatisch gleichen sich RI und LD weitgehend. Der Parallelismus von RI und LD ist in diesem Bereich so stark, dass man versucht ist, von einem gemeinsamen Mega-Paradigma mit den entsprechenden vertikalen und horizontalen Dimensionen zu sprechen. Auf dieser noch etwas impressionistischen Basis lässt sich in (4) eine Arbeitshypothese formulieren, die in Abschnitt 3 auf ihre Stichhaltigkeit hin überprüft werden soll. 
(4) Arbeitshypothese

Jede Sprache besitzt RI und LD, deren Paradigmen (mit Ausnahme der deiktischen Distanzstufen) eine völlig parallele innere Organisation aufweisen.

Wie das Deutsche bereits andeutet, muss dabei die paradigmatische Organisation nicht notwendigerweise dem Kanon aus Tabelle 10.2 bis ins Detail entsprechen.

\section{Empirisches}

Die folgende Datenschau erhebt keinen Anspruch auf Vollständigkeit. Vielmehr hat sich die Identifikation von vollständigen LD-Paradigmen schon jetzt als äußerst frustationsanfällig erwiesen, weil in den konsultierten deskriptivlinguistischen Quellen dem Gegenstand häufig wenig Beachtung geschenkt wird. Als Folge davon sind bislang noch viele Paradigmen in meinem Korpus fragmentarisch und müssen nachträglich über Expertenbefragung und/oder aus Korpusmaterialien ergänzt werden. Ich beziehe mich daher hier ausschließlich auf vollständig gesicherte Daten, d.h. auf solche, die den entsprechenden Referenzgrammatiken und anderen deskriptiv-linguistischen Arbeiten entnommen sind. Den Umstand, dass auf diesem Wege die germanischen Sprachen Europas privilegiert werden, nehme ich als geeignete Gelegenheit wahr, Eichinger (2008) indirekt um ein paar weitere Fakten zu ergänzen. Man möge den gesamten Abschnitt 3 als Appetithäppchen verstehen, dem die Hauptmahlzeit später und separat noch folgen wird. Aus bekannten Gründen muss ich mich kurzfassen, weshalb ich zur Füllung einiger verbleibender Informationslücken zu den RI auf Stolz et al. (2017) und die dortige ausführliche Diskussion verweise.

Berücksichtigt werden sieben germanische Verwandte des Deutschen, nämlich Dänisch, Englisch, Färöisch, Isländisch, Niederländisch, Norwegisch (Bokmål) und Schwedisch, deren RI- und LD-Daten in den Unterabschnitten 3.1-3.7 in alphabetischer Reihenfolge der Glossonyme vorgestellt und in $3.8 \mathrm{mit}$ einer außereuropäischen Sprache (Palikur) verglichen werden. Für die Zwecke dieses Abschnitts werden tabellarisch jeweils das RI-Paradigma und das Paradigma des LD der Ferndeixis (= D2) nebeneinander angegeben. ${ }^{6}$

6 In dieser und den folgenden Tabellen gebe ich die Belege in genau der Form wieder, wie sie in den Quellen erscheinen, ohne dabei gelegentliche Allographien (wie Getrennt- und Zusammenschreibung) o. Ä. zu vereinheitlichen. 


\subsection{Dänisch}

Den ersten Blick werfe ich auf das Dänische. In Tabelle 10.3 finden sich die entsprechenden Paradigmen. Die Angaben basieren auf der Beschreibung von Christensen \& Christensen (2009: 142).

Tab. 10.3: RI- und LD-Paradigmen im Dänischen.

\begin{tabular}{lll}
\hline$\Re$ & RI & LD (D2) \\
\hline Place & $\begin{array}{l}\text { hvor } \\
\text { hvor henne }\end{array}$ & $\begin{array}{l}\text { der } \\
\text { derhenne }\end{array}$ \\
\hline Goal & hvor hen & derhen \\
\hline Source & hvor fra & derfra \\
\hline
\end{tabular}

Im Dänischen sind die Paradigmen von RI und LD völlig symmetrisch aufgebaut. Die komplexen Konstruktionen sind sämtlich morphologisch transparent und können als auf der Grundlage des null-markierten Place-Ausdrucks gebildet verstanden werden. Die Konstruktionen reflektieren in beiden Paradigmen gleiche strukturelle Muster. Diese Muster sind in (5) aufgeführt, wobei die Variable X für hvor „wo“ und der „dort“ steht.

(5) Dänisch: Konstruktionsmuster zur Bildung von RI und LD
a. $[\mathrm{X}]_{\text {statisch }}$
b. $[\mathrm{X}=\text { Klitikon }]_{\text {statisch/dynamisch }}$

Für jede räumliche Relation gibt es also wenigstens einen Ausdruck, bei dem sie durch einen Exponenten expliziert ist. Nur hinsichtlich der Doppelbelegung der Place-Zellen und der damit gegebenen Möglichkeit zur alternativen NullMarkierung von Place weicht das Dänische vom Kanon ab. Diese Doppelbelegung einschließlich der Möglichkeit, Place overt zu markieren, unterscheidet das Dänische zudem auch vom Deutschen. Die „langen“ Place-Ausdrücke hvor henne „wo“ und derhenne „dort“ sind darüber hinaus phonologisch komplexer als die entsprechenden Goal-Ausdrücke hvor hen „wohin“ und derhen „dorthin“. ${ }^{7}$ Keine andere der hier berücksichtigten germanischen Sprachen weist

7 Der Goal-Exponent $=$ hen $\left[\right.$ [fen $\left.{ }^{2}\right]$ ist einsilbig, wohingegen der Place-Exponent $=$ henne [he.nə] zweisilbig ist. 
einen gleich stark ausgeprägten RI-LD-Parallelismus wie das Dänische auf. Es darf festgehalten werden, dass das Dänische dem Kanon in besonders hohem Maße entspricht - und das heißt in höherem Maße als das Deutsche.

\subsection{Englisch}

Dem Kanon wesentlich weniger entspricht das Englische. Tabelle 10.4 zeigt durch Fettdruck, dass vertikal die Place- und Goal-Zellen jeweils durch gleiche Ausdrücke belegt sind. Hier tritt also Synkretismus auf, der erheblich zur nichtkanonischen Ausprägung von Paradigmen beiträgt (Baerman, Brown \& Corbett 2005: 13-36). Ich berufe mich hinsichtlich der Daten auf Quirk et al. (1985: 660).

Tab. 10.4: RI- und LD-Paradigmen im Englischen.

\begin{tabular}{lll}
\hline $\mathfrak{R}$ & RI & LD (D2) \\
\hline Place & where & there \\
Goal & where & there \\
Source & where from $\sim$ from where & from there \\
\hline
\end{tabular}

Dieser Befund weicht nicht nur wegen des Synkretismus, sondern auch wegen der konstruktionellen Unterschiede im Falle der Source-Ausdrücke vom Kanon ab. Das Englische unterscheidet sich sowohl vom Deutschen als auch vom Dänischen sehr deutlich. Aus den Angaben in (6) geht hervor, dass das Englische drei Konstruktionsmuster zur Bildung der Paradigmen heranzieht.

(6) Englisch: Konstruktionsmuster zur Bildung von RI und LD
a. $[\mathrm{X}]_{\text {Place }=\text { Goal }}$
b. $\left[\mathrm{X}_{\mathrm{RI}} \text { from }\right]_{\text {Source }} \sim\left[\text { from } \mathrm{X}_{\mathrm{RI}}\right]_{\text {Source }}$
c. $\left[\text { from } \mathrm{X}_{\mathrm{LD}}\right]_{\text {Source }}$

Die Konstruktionsmuster (6b) und (6c) unterscheiden sich darin, dass die ablativische Adposition from ,von“ bei LD grundsätzlich vor X zu stehen kommt. Beim RI folgt sie dem X, während sie beim LD wie eine gewöhnliche Präposition dem X vorangeht. Diese Art von Linearisierungsunterschied ist bisher in der Taxonomie der „types of deviation“ (Corbett 2007: 30) vom Kanon noch nicht aufgenommen worden. Es muss dessenungeachtet konstatiert werden, 
dass zwei Konstruktionen, die einander funktional sehr nahe stehen, formal unterschiedliche innere Strukturen aufweisen. Der Parallelismus der Paradigmen ist dadurch empfindlich gestört, weil für jede Source-Konstruktion zur Bildung eine eigene Regel aufgestellt werden muss.

Quirk et al. (1978: 660) vermerken allerdings, dass „in formal contexts“ das englische LD (D2) die dynamischen $\Re$ auch noch durch thither „dorthin“ und thence „dorther“ ausdrücken kann. Von RI-Äquivalenten wie whither „wohin“ und whence „woher“ wird auffälligerweise nicht gesprochen. Es handelt sich in jedem Fall um stilistisch markierte Überbleibsel aus der frühneuenglischen Periode. Integrierte man sie in Tabelle 10.4, würden automatisch mehrere Zellen doppelt besetzt, was den Grad der Kanonizität der Paradigmen noch weiter beschnitte.

\subsection{Färöisch}

Bezüglich des Färöischen ist es notwendig, gleich mehrere deskriptiv-linguistische Quellen zu konsultieren, um den Gesamtbestand an Konstruktionen für die zu analysierenden Paradigmen zusammenzustellen. Im Anschluss an Tabelle 10.5 wird in der Diskussion angegeben, aus welcher Quelle die Konstruktionen stammen.

Tab. 10.5: RI- und LD-Paradigmen im Färöischen.

\begin{tabular}{lll}
\hline$\Re$ & RI & LD (D2) \\
\hline Place & hvar & har \\
\hline Goal & $\begin{array}{l}\text { hvar } \\
\text { hvagar }\end{array}$ & hagar \\
\hline Source & $\begin{array}{l}\text { hvar frá } \\
\text { hvaðan } \\
\text { hvaðani }\end{array}$ & haðan \\
& & haðani \\
\hline
\end{tabular}

Das Nebeneinander der Kurz- und Langformen bei den Source-Ausdrücken hvaðan hvaðani „woher“ und haðan haðani „dorther“ wird in allen Quellen angegeben. Die übrigen Fälle von Mehrfachbelegung der Goal- und SourceZellen des RI resultieren hingegen aus Differenzen zwischen den Quellen. Für die rezente Referenzgrammatik von Thráinsson et al. (2004: 182 und 186) gibt es weder Place-Goal-Synkretismus noch eine periphrastische Source- 
Konstruktion, die jedoch beide als alternative Optionen bei Lockwood (1977: 60-61) verzeichnet werden. Lockwood (1977: 61) taxiert das Source-RI hvar frá „woher“ als „common in spoken Faroese“. Zwar wird diese Konstruktion im einsprachigen färöischen Wörterbuch von Poulsen et al. (1998: 500) nicht erwähnt, dafür aber der Place-Goal-Synkretismus bei hvar „wo(hin)“. Ebenso verfährt das dänisch-färöische Wörterbuch von Petersen/Staksberg (1995: 287), wo Dänisch hvorhen „wohin“ mit Färöisch hvar „wo(hin)“ hvagar „wohin“ übersetzt wird.

Der Place-Goal-Synkretismus und die Source-Periphrase tragen zur Heterogenität des Gesamt aus RI- und LD-Paradigmen bei, in denen die unter (7) aufgeführten Konstruktionsmuster koexistieren. Die Stammveränderung an der Variablen X (= hvar $\sim$ har) wird durch ein tiefgestelltes doppelt durchgestrichenes (r) angezeigt.

(7) Färöisch: Konstruktionsmuster zur Bildung von RI und LD
a. $\left[\mathrm{X}_{\mathrm{RI}}\right]_{\text {Place }=\text { Goal }}$
b. $\left[\mathrm{X}_{\mathrm{LD}}\right]_{\text {Place }}$
c. $\left[\mathrm{X}_{(\mathrm{F})}-\text { gar }\right]_{\text {Goal }}$
d. $\left[\mathrm{X}_{(\mathrm{f})}-ð a n\right]_{\text {Source }}$
e. $\left[\mathrm{X}_{(\mathrm{f})} \text { ðani }\right]_{\text {Source }}$
f. $\left[\mathrm{X}_{\mathrm{RI}} \text { frá }\right]_{\text {Source }}$

Mit sechs Konstruktionsmustern weist das Färöische eine viel größere paradigmatische Variation als Dänisch, Deutsch und Englisch auf. Der Parallelismus zwischen RI und LD ist im Färöischen stark reduziert, da auf der RI-Seite eine deutlich größere Formenvielfalt als auf der LD-Seite besteht. Konstruktionsmuster (7f) fällt zudem aus dem Rahmen des RI-Paradigmas, in dem die dynamischen $\Re$ ansonsten durch Suffigierung expliziert werden. Im Vergleich zu den bisher besprochenen germanischen Fällen zeichnet sich das Färöische durch einen besonders geringen Grad an Kanonizität aus.

\subsection{Isländisch}

Wie bei seiner inselskandinavischen Schwestersprache Färöisch erfordert das Isländische die Konsultation von mehreren Quellen, um zu dem abschließenden Ergebnis zu kommen, das in Tabelle 10.6 abgebildet ist. 
Tab. 10.6: RI- und LD-Paradigmen im Isländischen.

\begin{tabular}{lll}
\hline$\Re$ & RI & LD (D2) \\
\hline Place & hvar & $\begin{array}{l}\text { parna } \\
\text { par }\end{array}$ \\
\hline Goal & hvert & $\begin{array}{l}\text { par } \\
\text { pangað }\end{array}$ \\
\hline Source & hvaðan & paðan \\
\hline
\end{tabular}

Die Kurz- und Langformen in der Place-Zelle des LD-Paradigmas werden von allen Quellen angegeben. Was bei Kress (1982: 97) jedoch nicht angesprochen wird, ist die Möglichkeit, beim LD den Place-Ausdruck auch synkretistisch für die Goal- $\Re$ zu verwenden. Dass bar „dort(hin)“ synkretistisch ist, geht aus den Angaben im einsprachigen isländischen Wörterbuch von Böðvarsson (1985: 1190-1191) hervor.

Formal völlig parallel verhalten sich die Source-Ausdrücke, die morphologisch identischen Bau verraten. Die Parallelen sind für die morphologisch komplexen Goal-Ausdrücke hvert „wohin“ und pangað „dorthin“ opak, weil zum einen keine phonologisch übereinstimmenden „Endungen“ identifiziert (- $t$ ggü. - $a ð$ ) und zum anderen ihre formale Beziehung zum jeweiligen PlaceAusdruck synchron nicht mehr in Regelform gegossen werden können (hvar + $-t>$ hvert?, par + -að > pangað?). Es darf in beiden Fällen von schwachem Suppletivismus gesprochen werden. Außerdem hat die Langform des Place-LD parna „dort“ keine Langform als Entsprechung auf der Seite des RI. Es ergibt sich wiederum ein Nebeneinander mehrerer Konstruktionsmuster, das ich in (8) darstelle.

(8) Isländisch: Konstruktionsmuster zur Bildung von RI und LD
a. $\left[\mathrm{X}_{\mathrm{RI}}\right]_{\text {Place }}$
b. $\left[\mathrm{X}_{\mathrm{LD}}\right]_{\text {Place }=\text { Goal }}$
c. $\left[\mathrm{X}_{\mathrm{LD}}-n a\right]_{\text {Place }}$
d. $\left[\mathrm{X}_{\text {(r)LD }}-n g a ð\right]_{\text {Goal }}$
e. $\left[\mathrm{X}_{(\mathrm{a}>\mathrm{e}) \mathrm{RI}}-t\right]_{\mathrm{Goal}}$
f. $\left[\mathrm{X}_{(\mathrm{Y})}-\partial a n\right]_{\text {Source }}$

Die Divergenz vom Kanon ist relativ stark ausgeprägt. Die Null-Markierung bei Place und optional bei Goal trägt hierzu genauso bei wie die formalen Unter- 
schiede bei der Bildung der morphologisch komplexen Goal-Ausdrücke. Konstruktionsmuster (8c) kann man mit etwas gutem Willen als eine overte Markierung von Place werten (entsprechend den Bildungen mit =henne im Dänischen), die mit den Anforderungen des Kanons, der keine Null-Markierung vorsieht, konform geht. Nachteilig ist in diesem Zusammenhang, dass keine Entsprechung beim RI gegeben ist. Damit entfernt sich Isländisch weiter vom Kanon als Dänisch und Deutsch.

\subsection{Niederländisch}

Nur für das Niederländische war es nötig, zusätzlich zum LD (D2) noch das Paradigma des „neutralen“ Deiktikons er „hier, dort“ (Geerts et al. 1984: 392) aufzuführen, weil es von den übrigen LD derselben Sprache abweicht, indem es Place-Goal-Synkretismus aufweist. Tabelle 10.7 zeigt, dass unter den verschiedenen LD paradigmatische Unterschiede zu Tage treten.

Tab. 10.7: RI- und LD-Paradigmen im Niederländischen.

\begin{tabular}{llll}
\hline$\Re$ & RI & LD \\
\cline { 3 - 4 } & & D2 & neutral \\
\hline Place & waar & daar & er \\
\hline Goal & $\begin{array}{l}\text { waarheen } \\
\text { waar naartoe }\end{array}$ & $\begin{array}{l}\text { daarheen } \\
\text { daarnaartoe } \\
\text { naar daar }\end{array}$ & er \\
\hline Source & $\begin{array}{l}\text { waar vandaan } \\
\text { vanwaar }\end{array}$ & $\begin{array}{l}\text { daandaan } \\
\text { vandaar }\end{array}$ & ervandaan \\
\hline
\end{tabular}

Im niederländischen Fall treten fünf Abweichungen vom kanonischen Paradigma auf (Geerts et al. 1984: 382, 392).

1. Null-Markierung gilt für alle Place-Ausdrücke - und im neutralen Paradigma auf Grund des Place-Goal-Synkretismus ebenfalls für Goal.

2. Place und Goal werden im neutralen LD-Paradigma formal nicht unterschieden, sodass Synkretismus gegeben ist.

3. In den Zellen der dynamische $\Re$ von RI und LD (D2) herrscht ein Überangebot von synonymen Konstruktionen.

4. Die Bildungsweisen der Goal-Ausdrücke stimmen bei RI und LD (D2) nicht in jedem Fall überein.

5. Im neutralen LD-Paradigma gibt es keine Evidenz für „overabundance“. 
Es ist demnach nicht möglich, ein Paradigmen-übergreifendes Konstruktionsprinzip zu postulieren. Vielmehr stehen die in (9) angeführten sieben Konstruktionsmuster nebeneinander.

(9) Niederländisch: Konstruktionsmuster zur Bildung von RI und LD
a. $\left[\mathrm{X}_{\mathrm{RI} / \mathrm{LD}(\mathrm{D} 2)}\right]_{\text {Place }}$
b. $\left[\mathrm{X}_{\mathrm{LD}(\text { neutral })}\right]_{\text {Place }=\text { Goal }}$
c. $\left[\mathrm{X}_{\mathrm{RI} / \mathrm{LD}(\mathrm{D} 2)}=\text { heen }\right]_{\mathrm{Goal}}$
d. $\left[\mathrm{X}_{\mathrm{RI} / \mathrm{LD}(\mathrm{D} 2)}=\text { naartoe }\right]_{\mathrm{Goal}}$
e. $\left[\text { naar } \mathrm{X}_{\mathrm{LD}(\mathrm{D} 2)}\right]_{\mathrm{Goal}}$
f. $[\mathrm{X}=\text { vandaan }]_{\text {Source }}$
g. $\left[\text { van } \mathrm{X}_{\mathrm{RI} / \mathrm{LD}(\mathrm{D} 2)}\right]_{\text {Source }}$

Die Konstruktionsmuster (9c)-(9e) zeigen zudem ein weiteres Mal, dass Linearisierungsdifferenzen auftreten, die sowohl vertikal als auch horizontal die Paradigmen betreffen. Der RI-LD-Parallelismus ist recht starken Einschränkungen unterworfen. Dem Kanon nähert sich das Niederländische nur in Teilen. Das Niederländische ist im Vergleich zum Dänischen und zum Deutschen deutlich weniger kanonisch.

\subsection{Norwegisch (Bokmål)}

Norwegisch (Bokmål) bietet die in Tabelle 10.8 zusammengefassten RI- und LD-Paradigmen (Faarlund, Lie \& Vannebo 1997: 936-943 und 1179). Es sticht unmittelbar ins Auge, dass der Parallelismus zwischen den beiden Formenreihen nicht vollständig ist.

Tab. 10.8: RI- und LD-Paradigmen im Norwegischen (Bokmål).

\begin{tabular}{lll}
\hline$\Re$ & RI & LD (D2) \\
\hline Place & hvor & der \\
Goal & hvor hen & dit \\
Source & hvor fra & derfra \\
\hline
\end{tabular}

Die Konstruktionen ähneln denen des Dänischen aus Tabelle 10.3. Die Option eines overt markierten Place-Ausdrucks fehlt im Norwegischen (Bokmål), dessen Paradigmen frei von alternativen Optionen und Synkretismus sind. Der 
Gleichklang mit dem Dänischen erstreckt sich nicht auf alle Zellen der beiden Paradigmen. Im LD-Paradigma fällt die Goal-Konstruktion aus dem Rahmen und zwar sowohl vertikal als auch horizontal. Der Ausdruck dit „dorthin“ wird weder wie das Source-LD derfra „dorther“ durch Univerbierung mit einer Postposition noch wie das Goal-RI durch Hinzufügung des direktionalen Klitikons hen „hin“ gebildet. Man könnte daher von Anti-Periphrase sprechen, also vom Auftreten einer synthetischen Wortform in einem Paradigma, dessen dominante Konstruktionsform periphrastisch ist (Corbett 2007: 30). Dit „dorthin“ steht zudem in einem (schwach-)suppletiven Verhältnis zu der „dort“.

Es lassen sich insgesamt vier Konstruktionsmuster für den norwegischen Befund konstatieren, die ich in (10) aufliste.

(10) Norwegisch (Bokmål): Konstruktionsmuster zur Bildung von RI und LD

a. $[\mathrm{X}]_{\text {Place }}$

b. $\left[\mathrm{X}_{\mathrm{RI}}=h e n\right]_{\mathrm{Goal}}$

c. $[\text { dit }]_{\text {Goal }}$

d. $[\mathrm{X}=\text { fra }]_{\text {Source }}$

Vom Kanon distanziert sich das Norwegische (Bokmål) vor allem dadurch, dass Null-Markierung von Place vorliegt und zum Ausdruck von Goal unterschiedliche Kodierungsstrategien zum Einsatz kommen. Die paradigmatische Transparenz ist daher gestört, obwohl vier von insgesamt sechs Zellen der Paradigmen von Konstruktionen besetzt sind, die miteinander formal harmonieren. Fünf von sechs Zellen zeigen Ähnlichkeiten mit dem Dänischen.

\subsection{Schwedisch}

Ein Blick auf die schwedischen Gegebenheiten in Tabelle 10.9 (Teleman, Helberg \& Andersson 1999: 642 und 660) genügt, um festzustellen, dass die im vorangegangenen Abschnitt diskutierten norwegischen Daten eine Art Kompromiss zwischen dem Dänischen und dem Schwedischen darstellen.

Tab. 10.9: RI- und LD-Paradigmen im Schwedischen.

\begin{tabular}{lll}
\hline$\Re$ & RI & LD (D2) \\
\hline Place & var & där \\
Goal & vart & dit \\
Source & varifrån & därifrån \\
\hline
\end{tabular}


Synkretismus und Allomorphie treten im schwedischen Fall nicht auf. Während das norwegische RI-Paradigma sein dänisches Äquivalent reflektiert, ist gleichzeitig die Affinität des norwegischen LD-Paradigmas zu seiner schwedischen Entsprechung unverkennbar. Maßgeblich hierfür ist die Präsenz des Goal-LD dit „dorthin“ im Schwedischen und Norwegischen (Bokmål). Anders als im norwegischen Kontext ist das schwedische dit „dorthin“ nicht vollständig paradigmatisch isoliert. Zwar kann die vertikale Beziehung zu där „dort“ ebenfalls nur als schwach suppletiv eingestuft werden. Jedoch ist horizontal noch erkennbar, dass die beiden Goal-Ausdrücke vart „wohin“ und dit „dorthin“ in dem auslautenden /t/ eine Gemeinsamkeit aufweisen. Da vart „wohin“ durch Anfügung von - $t$ an var „wo“ als morphologisch transparente Ableitung aus dem Place-Ausdruck erkennbar ist, ergibt sich die Möglichkeit, auch für das Goal-LD eine innere morphologische Struktur $d_{\mathrm{LD}}$-it $t_{\text {Allativ }}$ anzusetzen. Diese Möglichkeit besteht im norwegischen Fall jedoch nicht.

Mit insgesamt nur drei Konstruktionsmustern (siehe (11)) ist Schwedisch recht ökonomisch, wenn es um den Regel-Aufwand geht, der betrieben werden muss, um die Paradigmen mit Ausdrücken zu füllen.

(11) Schwedisch: Konstruktionsmuster zur Bildung von RI und LD
a. $[\mathrm{X}]_{\text {Place }}$
b. $\left[\mathrm{X}_{(\mathrm{x})}-(i) t\right]_{\text {Goal }}$
c. $[\mathrm{X}=\text { ifrån }]_{\text {Source }}$

Als abweichend im Sinne des Kanons erweist sich die obligatorische NullMarkierung von Place, die gem. Tabelle 10.2 nicht vorgesehen ist. Die Ausdrücke der dynamischen $\Re$ werden zudem nach unterschiedlichen Prinzipien gebildet. Die Goal-Ausdrücke sind flexivischer Natur, während für die SourceAusdrücke auf eine klitische Postposition zurückgegriffen wird. Ungeachtet solcher Abweichungen vom kanonischen Ideal ist der Parallelismus der RIund LD-Paradigmen im Schwedischen ziemlich stark ausgeprägt.

\subsection{Blick über den germanischen Tellerrand}

Die acht germanischen Sprachen geben ein buntes Bild ab. Keine zwei von ihnen sind in dem hier interessierenden Bereich genau gleich strukturiert. Dieser Umstand lässt insofern aufhorchen, als selbst unter eng verwandten Sprachen ein als grundlegend für die menschliche Kognition erachtetes funktionales Feld wie das der räumlichen Konzepte starker Variation bei seiner Versprachlichung 
ausgesetzt zu sein scheint. Um wie viel stärker mag die Diversität sein, wenn der Germania fernliegende Sprachen in den Vergleich mit einbezogen werden?

Ich muss mich hier mit einem kurzen Schlaglicht auf die Daten aus der arawakischen Sprache Palikur, die beiderseits der Grenze von Französisch-Guiana und dem brasilianischen Bundesstaat Amapá gesprochen wird, begnügen. Launey (2003: 140) führt die in Tabelle 10.10 zusammengefassten Paradigmen auf.

Tab. 10.10: RI- und LD-Paradigmen im Palikur.

\begin{tabular}{lllll}
\hline$\Re$ & RI & \multicolumn{2}{c}{ LD } \\
\cline { 3 - 5 } & & D1 & D2 & D3 \\
\hline Place & kiney & ay & ayge & ayhte \\
\hline Goal & kit & atan & atere & $\begin{array}{l}\text { atere } \\
\text { ayhtetan }\end{array}$ \\
\hline Source & kitak & $\begin{array}{l}\text { ayteke } \\
\text { aytekihan }\end{array}$ & $\begin{array}{l}\text { aytohtak } \\
\text { aytohtihan }\end{array}$ & aytohtak \\
\hline
\end{tabular}

Um Synkretismen zu erfassen, sind die Paradigmen aller drei deiktischen Distanzstufen berücksichtigt worden. Der Fettdruck gibt zu erkennen, dass Synkretismus ausschließlich bei den dynamischen $\Re$ der LD der Distanzstufen D2 und D3 auftritt - und zwar so, dass die Goal-Ausdrücke bzw. die SourceAusdrücke beider Distanzstufen optional miteinander identisch sind. Overabundance ist ebenfalls nur bei den LD und dort wiederum nur bei den dynamischen $\Re$ belegt, wobei Source zweimal und Goal nur einmal von diesem Phänomen betroffen ist. RI und LD verhalten sich insofern unterschiedlich, als „mismatches“ ausschließlich bei den LD auftreten.

Darüber hinaus unterscheiden sich die RI- und LD-Paradigmen noch deutlich darin, wie sie die Ausdruckskomplexität über die Kategorien verteilen. NullMarkierung von Place begegnet ausschließlich bei den LD (dort allerdings in allen Distanzstufen). Völlig unmarkiert ist LD (D1) ay „hier“, während bei LD (D2) ay-ge „da“ und LD (D3) ay-hte „dort“ durch Suffixe die Distanzstufe zusätzlich overt explizieren. Die Ausdrücke der entsprechenden Source-LD sind durch die Affigierung von zwei- bis dreisilbigen Exponenten vom Place-LD (D1) abgeleitet: z. B. ay + teke $\rightarrow$ ayteke ,hierher“. Auch das nicht-synkretische Goal-LD (D3) ayhtetan „dorther“ ist morphologisch transparent und lässt sich auf ay-hte + tan $\rightarrow$ ayhtetan zurückführen. Die Prominenz von Place lässt sich für den RI-Bereich jedoch nicht aufrechterhalten, weil in diesem Paradigma das Goal-RI kit „wohin“ als einziger Ausdruck einsilbig ist. Demgegenüber ist das 
Place-RI kiney „wo“ zweisilbig und daher ausdrucksseitig komplexer als das Goal-RI. Kit „wohin“ trägt das Allativsuffix -t, ist also (wie ki-tak „woher“) morphologisch komplex. Dies trifft aber auch auf kiney „wo“ zu, das mit -ey einen deiktischen Marker involviert. Die Goal-Ausdrücke sind ggü. den PlaceAusdrücken (mit Ausnahme von ayhtetan „dorther“) schwach suppletiv. Die Distanzstufenmarker -ge und -hte treten bei den LD in den dynamischen $\Re$ nicht auf (Ausnahme: ayhtetan ,dorther“). Stattdessen begegnet bei den SourceLD -toh. Ebenfalls bei den Source-LD tritt das ablativische Suffix - $(t)$ ihan auf. Die Goal-LD weisen die allativischen Suffixe -(i)tan und -re auf, die allesamt im RI-Paradigma unbekannt sind.

Von einem ausgeprägten RI-LD-Parallelismus kann also auf keinen Fall die Rede sein. In der Summe ist das Palikur im RI-LD-Komplex sicher nicht näher am Kanon als dies für die acht germanischen Beispielsprachen der Fall ist. Nicht-kanonisches Verhalten ist mithin weder ein germanisches Vorrecht noch eine typologische Ausnahme.

\section{Schlussfolgerungen}

Auch wenn ich in dieser Studie nur ein wenig an der Oberfläche kratzen durfte, ergeben sich schon jetzt einige interessante Einsichten in das RI-LD-Netzwerk. Die Daten widerlegen die Arbeitshypothese (4) in ziemlich eindeutiger Weise. Die vermutete Gleichförmigkeit der RI- und LD-Paradigmen hat sich nämlich als Chimäre erwiesen. Unter den Beispielsprachen ist der formale Parallelismus zwischen RI und LD eine Seltenheit. Viel häufiger lässt sich beobachten, dass Idiosynkrasien wenigstens in einer paradigmatischen Zelle auftreten. Die gegenseitige Bindung von RI und LD ist mithin eine graduelle Eigenschaft. Da partielle Divergenz unter den Paradigmen gang und gäbe zu sein scheint, könnte angezweifelt werden, dass RI und LD überhaupt eine gemeinsame funktionale Einheit bilden. Dieser Gedanke kommt speziell dort auf, wo in einer Sprache im RI-Paradigma Distinktionen gemacht werden, die auf der LD-Seite zusammenfallen - und umgekehrt. Stellt dieses Ungleichverhalten funktional verwandter grammatischer Teilbereiche einer Einzelsprache ein Problem für die Annahme eines einheitlichen räumlichen Konzeptsystems dar? Dieser Frage sollte man typologisch nachgehen, indem man Sprachen danach klassifiziert, wie ähnlich sie ihre jeweiligen RI- und LD-Paradigmen gestalten. Von Forschungen dieser Art würde die Sprachtypologie genauso viel Nutzen ziehen wie die Raum-Linguistik. Nicht vergessen werden darf, dass auch in der diachronischen Entwicklung das Entstehen bzw. das Füllen von Lücken innerhalb eines Systems nachvollzogen werden kann. 
Die Datenschau hat zudem ergeben, dass der Kanon nicht sakrosankt ist, sondern in der Regel gegen ihn verstoßen wird. Zwar erwartet der kanonische Ansatz gerade nicht, dass der Kanon in der Sprachenwelt überwiegt; er muss noch nicht einmal empirisch belegt sein (Corbett 2011: 108). Dennoch fällt es auf, dass keine einzige der Beispielsprachen dem Kanon uneingeschränkt entspricht. Dies lässt vermuten, dass bei der Strukturbildung in den Sprachen andere Prinzipien Vorrang genießen als bei der Ausformung des kanonischen Konstrukts. Vom Kanon nicht vorgesehen ist die Null-Markierung von Kategorien. In allen Beispielsprachen einschließlich Palikur weisen die Paradigmen jedoch Null-Markierung wenigstens als fakultative Möglichkeit aus - und zwar überwiegend bei Place; Goal ist nur bei Place-Goal-Synkretismus null-markiert. Eine null-markierte paradigmatische Position zu haben, scheint eine attraktive Option für die Sprachen zu sein, die kanonische Prinzipien an Durchsetzungskraft übertrifft. Gleichzeitig verhalten sich die Beispielsprachen dahingehend gleich, dass sie für die dynamische $\Re$ Source sowohl für RI als auch für LD stets komplexe Konstruktionen verwenden. Ihre Komplexität ist dabei in der Regel größer als die der entsprechenden Goal- und Place-Konstruktionen. Auch hier gehen die germanischen Sprachen und das Palikur miteinander konform und bestätigen weitgehend die Markiertheitshierarchie (3).

Ob meine vorläufigen Beobachtungen auch bei Erweiterung des Samples und der Datengrundlage Bestand haben, müssen zukünftige Forschungen auf diesem Gebiet ergeben. Ich bin zuversichtlich, dass der mikroskopische RI-LDVergleich in crosslinguistischer Perspektive noch viele Überraschungen für die an diesem Gegenstand interessierten Disziplinen parat hält. Nicht zuletzt könnten die hier gemachten sprachtypologischen Beobachtungen für die am IDS entstehende „Grammatik des Deutschen in europäischen Vergleich (GDE-V)“ interessant sein, da sie zeigen, dass mit zunehmender Granularität der Betrachtung selbst in einer genetisch homogenen Gruppe von Sprachen deutliche strukturelle Unterschiede zu Tage treten. Wie mag es hinsichtlich der strukturellen Ähnlichkeit und Unähnlichkeit erst aussehen, wenn im Sprachvergleich die genetischen Grenzen überschritten werden?

\section{Abkürzungen}

$\begin{array}{ll}\text { D1/D2/D3 } & =\text { (Stufen der) Deixis } \\ \text { LD } & =\text { lokales Deiktikon } \\ \text { PP } & =\text { Präpositionalphrase } \\ \text { Präp } & =\text { Präposition } \\ \Re & =\text { (räumliche) Relation } \\ \text { RI } & =\text { räumliches Interrogativum }\end{array}$




\section{Literatur}

Anderson, Stephen R. \& Edward L. Keenan (1985): Deixis. In Timothy Shopen (Hrsg.), Language Typology and Syntactic Description. Volume III: Grammatical categories and the lexicon, 259-308. Cambridge: Cambridge University Press.

Baerman, Matthew \& Dunstan Brown \& Greville G. Corbett (2005): The syntax-morphology interface. A study of syncretism. Cambridge: Cambridge University Press.

Bennett, David C. (1975): Spatial and temporal uses of English prepositions. An essay in stratificational semantics. London: Longman.

Booij, Geert (2010): Construction morphology. Oxford: Oxford University Press.

Böðvarsson, Árni (1985): Íslensk orðabók handa skólum og almenningi. Reykjavík: Bókaútgáfa Menningarsjóds.

Bowern, Claire (2008): Linguistic fieldwork. New York: Palgrave Macmillan.

Burenhult, Niclas (2008): Spatial coordinate systems in demonstrative meaning. Linguistic Typology 12 (1), 99-142.

Christensen, Robert Zola \& Lisa Christensen. (2009): Dansk grammatik. Odense: Syddansk Universitetsforlag.

Corbett, Greville G. (2007): Deponency, syncretism, and what lies in between. In Matthew Baerman, Greville G.Corbett, Dunstan Brown \& Andrew Hippisley (Hrsg.), Deponency and morphological mismatches, 21-44. Oxford: Oxford University Press.

Corbett, Greville G. (2011): Higher order exceptionality in inflectional morphology. In Horst J. Simon \& Heike Wiese (Hrsg.), Expecting the unexpected: Exceptions in grammar, 107126. Berlin/New York: De Gruyter Mouton.

Diessel, Holger (2005): Distance contrasts in demonstratives. In Martin Haspelmath, Matthew S. Dryer, David Gil \& Bernard Comrie (Hrsg.), The world atlas of language structures, 170-173. Oxford: Oxford University Press.

Dixon, R. M. W (2012): Basic linguistic theory. Volume 3: Further grammatical topics. Oxford: Oxford University Press.

Eichinger, Ludwig M. (2008): Deutsch als europäische Sprache. In Ludwig M. Eichinger \& Albrecht Plewnia (Hrsg.), Das Deutsche und seine Nachbarn. Über Identitäten und Mehrsprachigkeit, 13-30. Tübingen: Narr.

Eichinger, Ludwig M. (2012): Deutsch im Sprachvergleich. Grammatische Kontraste und Konvergenzen. In Lutz Gunkel \& Gisela Zifonun (Hrsg.), Deutsch im Sprachvergleich. Grammatische Kontraste und Konvergenzen, vii-xvi. Berlin/Boston: De Gruyter.

Faarlund, Terje \& Svein Lie \& Kjell Ivar Vannebo (1997): Norsk Referanse-Grammatikk. Oslo: Universitetsforlaget.

Fleischer, Jürg (2002): Die Syntax von Pronominaladverbien in den Dialekten des Deutschen. Eine Untersuchung zu Preposition Stranding und verwandten Phänomenen. Wiesbaden: Franz Steiner.

Geerts, G., W. Haeseryn, J. de Rooij \& M. C. van den Toorn (1984): Algemene Nederlandse Spraakkunst. Groningen/Leuven: Wolters-Noordhoff.

Goldap, Christel (1991): Lokale Relationen im Yukatekischen. Frankfurt a. M./Bern/New York/ Paris: Lang.

Haase, Martin (1992): Pointing with sounds: Iconicity and deictic localization. In Thomas Müller-Bardey \& Werner Drossard (Hrsg.), Aspekte der Lokalisation, 105-119. Bochum: Brockmeyer.

Haspelmath, Martin (2006): Against markedness (and what to replace it with). Journal of Linguistics 42 (1), 25-70. 
Holm, Britta \& Elizabeth Nylund Lindgren (1977): Deskriptiv svensk grammatik. Stockholm:

Skriptor.

Kress, Bruno (1982): Isländische Grammatik. München: Hueber.

Langacker, Ronald W. (1986): An introduction to cognitive grammar. Cognitive Science 10, $1-40$.

Launey, Michel (2003) : Awna parikwaki. Introduction à la langue palikur de Guyane et de l'Amapá. Paris: IRD Éditions.

Lockwood, William B. (1977): An Introduction to modern Faroese. Tórshavn: Føroya Skúlabókagrunnur.

Mayerthaler, Willi (1987): System-independent morphological naturalness. In Wolfgang U. Dressler (Hrsg.), Leitmotifs in Natural Morphology, 25-58. Amsterdam/Philadelphia: Benjamins.

Petersen, Hjalmar P. \& Marius Staksberg (1995): Donsk-Føroysk Orðabók. Tórshavn: Føroya Fródskaparfelag.

Poulsen, Jóhann Hendrik W. et al. (1998): Føroysk Orðabók. Tórshavn: Føroya Fródskaparfelag.

Quirk, Randolph, Sidney Greenbaum, Geoffrey Leech \& Jan Svartvik (1985): A grammar of contemporary English. London: Longman.

Stolz, Thomas, Sander Lestrade \& Christel Stolz (2014): The crosslinguistics of zero-marking of spatial relations. Berlin/Boston: De Gruyter Mouton.

Stolz, Thomas, Nataliya Levkovych, Aina Urdze, Julia Nintemann, \& Maja Robbers (2017): Spatial interrogatives in cross-linguistic perspective: Where - Whither - Whence. Berlin/Boston: De Gruyter Mouton.

Svorou, Soteria (1993): The grammar of space. Amsterdam/Philadelphia: Benjamins.

Teleman, Ulf, Staffan Hellberg \& Erik Andersson (1999): Svenska Akademiens Grammatik 2: Ord. Stockholm: Norstedts Ordbok.

Thornton, Anna M. (2012): Overabundance in Italian verb morphology and its interactions with other non-canonical phenomena. In Thomas Stolz, Hitomi Otsuka, Aina Urdze \& Johan van der Auwera (Hrsg.), Irregularity in morphology and beyond, 251-270. Berlin: Akademie Verlag.

Thráinsson, Höskuldur, Hjalmar P. Petersen, Jógvan í Lon Jacobsen, \& Zakaris Svabo Hansen (2004): Faroese. An overview and reference grammar. Tórshavn: Føroya Fródskaparfelag. Vater, Heinz (1991): Einführung in die Raum-Linguistik. Hürth: Gabel.

Wiese, Heike \& Maria Pohle (2016): „Ich geh Kino“ oder „... ins Kino“? Zeitschrift für Sprachwissenschaft 35 (2), 171-216.

Zifonun, Gisela, Ludger Hoffmann \& Bruno Strecker (1997): Grammatik der deutschen Sprache. Band 1. Berlin/New York: De Gruyter. 


\title{
Jürg Fleischer \\ 11 Perspektiven der Grammatiktheorie: Sprachwandel
}

\begin{abstract}
Sprachwandel ist primär Objekt der diachronen Forschung, doch auch bei synchroner Betrachtung eines Sprachsystems treten Reflexe diachroner Entwicklungen als synchrone Variation historisch „älterer“ und „jüngerer“ Formen auf, was die synchrone Grammatikographie vor theoretische und praktische Probleme stellt. Dies wird am Genitiv und Dativ Singular der althochdeutschen -iz-/-az-Stämme, am Nebeneinander starker und schwacher Präterita und anhand des „Rückumlauts“ illustriert.
\end{abstract}

Keywords: Diachronie, Grammatikographie, Sprachwandel, Synchronie, Variation

Es ist eingewendet, dass es noch eine andere wissenschaftliche Betrachtung der Sprache gäbe, als die geschichtliche. Ich muss das in Abrede stellen. (Paul 1920: 20)

\section{Einleitung}

Vor etwa hundert Jahren erlebte die Sprachwissenschaft mit der 1916 erfolgten Publikation von de Saussures Cours de linguistique générale eine Revolution. In deren Folge wurde die synchrone Beschreibung eines Sprachsystems zu einem wissenschaftlich adäquaten Gegenstand. Für die philologisch orientierten Anfänge der Germanistik vor etwa zweihundert Jahren - der erste Band von Jacob Grimms Deutscher Grammatik wurde in der ersten Auflage 1819 als Buch publiziert - hatten dagegen historische Entwicklungen im Vordergrund gestanden.

Anmerkung: Für Unterstützung bei der Datananalyse danke ich Magnus Breder Birkenes, für Angaben und Diskussionen zum Althochdeutschen Brigitte Bulitta und Frank Heidermanns, für Diskussionen und Feedback Magnus Breder Birkenes, Canan Sertkaya sowie seitens der HerausgeberInnen besonders Mechthild Habermann und Angelika Wöllstein. Alle Fehler stehen natürlich in meiner Verantwortung.

Jürg Fleischer, Institut für Germanistische Sprachwissenschaft, Forschungszentrum Deutscher Sprachatlas, Pilgrimstein 16, D-35032 Marburg, E-Mail: jfleischer@staff.uni-marburg.de

Ә Open Access. (C) 2018 Jürg Fleischer, publiziert von De Gruyter. (c) BY Dieses Werk ist lizenziert unter der Creative Commons Attribution 4.0 Lizenz. 
Das gilt ebenso für die sich gegen Ende des 19. Jahrhunderts etablierenden Junggrammatiker, deren erstmals 1880 erschienenem wohl wichtigstem sprachtheoretischem Text das diesem Artikel vorangestellte Motto entnommen ist. Für die historisch arbeitende Germanistik in der Tradition von Jacob Grimm und Hermann Paul bildet die Tatsache des Sprachwandels die „Geschäftsgrundlage“. Dies gilt für den im Gefolge de Saussures stehenden synchronen Ansatz, der heute zweifellos dominiert, und gerade auch für die Beschäftigung mit der deutschen Standardsprache, wie sie auch am IDS betrieben wird, nicht gleichermaßen. Im folgenden Überblick sollen einige Reflexionen darüber angestellt werden, wie sich der synchrone und der diachrone Zugang unterscheiden, wo sie ineinander übergehen und wo sie ihre jeweiligen Stärken ausspielen. Dabei spielen Probleme der Grammatikographie eine wichtige Rolle: Die Grammatik, verstanden als ein Werk, in dem sprachliche Strukturen anhand bestimmter, als relevant erkannter Prinzipien beschrieben und analysiert werden, ist der Ort, an dem sich theoretische Konzepte erst bewähren müssen. Gerade die Arbeit mit elektronischen Korpora, wie sie am IDS schon seit der Gründung im Zentrum steht (vgl. Moser 1967: 13-14, Eichinger 2014: 38), führt für den synchronen wie für den diachronen Zugang zu neuen Perspektiven.

\section{Standortbestimmung}

Für das Deutsche besteht eine beachtenswerte grammatikographische Tradition seit der frühen Neuzeit (vgl. z. B. Polenz 2013: 144-192). Zwar scheinen viele dieser Grammatiken aus einer heutigen Perspektive „un-“ oder „vorwissenschaftlich“, doch muss man anerkennen, dass sie - in ihrer Zeit - den wissenschaftlichen Diskurs zu Fragen der Sprache reflektieren. Diese ältere Grammatikographie des Deutschen ist synchron ausgerichtet. Gegen Ende des 18. Jahrhunderts fand diese Tradition mit dem Werk Johann Christoph Adelungs (1732-1806) einen Höhepunkt und, wie Eichinger (2008b) ausführt, auch einen gewissen Abschluss.

Im 19. Jahrhundert, mit dem Aufstieg der historischen Sprachwissenschaft im Gefolge von Jacob Grimm, stand eine synchron ausgerichtete Grammatikschreibung, wie sie etwa Adelung betrieben hatte, nicht mehr im Fokus der wissenschaftlichen Aufmerksamkeit. Die hellsten grammatischen Köpfe - für manche von ihnen wurden im Lauf des 19. Jahrhunderts auch spezifisch germanistische Lehrstühle eingerichtet - leuchteten nun für die historische Sprachwissenschaft. Auf dem Gebiet der synchronen Grammatikschreibung, für das bis zum 18. Jahrhundert gilt, dass meist „Liebhaber, Amateure den wissenschaftlichen Diskurs bestimmten“ (Eichinger 2008b: 257), lässt sich nun eine 
zunehmende „Anwendungsorientierung“ beobachten, denn im Kontext der Schule, um nur den wichtigsten zu nennen, existierte natürlich im 19. Jahrhundert ein immer größer werdender Bedarf an Regelwerken, als deren Anfang Adelungs Deutsche Sprachlehre für Schulen (1781) gelten kann. Die synchrone Grammatikschreibung - und damit der synchrone Zugang zur Sprache überhaupt - steht nun aber erst einmal außerhalb der universitären Forschung und Lehre. Noch die zentralen grammatischen Werke zum Deutschen aus der ersten Hälfte des 20. Jahrhunderts - Hermann Pauls Deutsche Grammatik (1916-1920) und Behaghels monumentale Deutsche Syntax: eine geschichtliche Darstellung (1923-1932) - sind historisch ausgerichtet. Die erste unter dem (Marken-)Namen Duden erschienene Grammatik will „nicht mit sprachgeschichtlichem Wissen überbeschwert“ sein, dennoch wird ,,auf die ältere Sprache und auf die Sprachgeschichte innerhalb des grammatischen Bezirks immer erneut“ hingewiesen (Basler 1935: V). Erst mit der „Inneren Form des Deutschen“ (Glinz 1952) verkörperte sich „der Auftakt zu einer strukturalen Beschreibung der deutschen Sprache“ (Eichinger 2008a: 29). Diese wurde dann in der Folge auch und gerade im Umfeld des IDS weitergeführt.

Zwar ist die verbreitete Ansicht, dass der Strukturalismus in Deutschland verspätet rezipiert worden sei, wie Ehlers (2005) in seiner erhellenden Studie aufzeigt, sachlich falsch: Vielmehr bot dieses Narrativ einer Gruppe von Sprachwissenschaftlern ab den 1960er Jahren die Möglichkeit zur Abgrenzung und stiftete deren Identität (vgl. Ehlers 2005: 39). Der Mythos der verspäteten Strukturalismusrezeption hat seinerseits ,seinen wissenschaftsgeschichtlichen Ort - in den sechziger Jahren und danach.“ (Ehlers 2005: 41). Bis zu einem gewissen Grad kann auch die Entstehung des IDS in diesen Kontext gestellt werden. So wird im „Gründungsdokument“ des IDS ein gewisser Nachholbedarf artikuliert:

Bis weit ins 20. Jahrhundert hinein war die Sprachwissenschaft historisch ausgerichtet. Die Erkenntnis, daß der Sprache der Gegenwart besondere Aufmerksamkeit geschenkt werden müsse, brach sich bezeichnenderweise zuerst im Ausland Bahn. In Deutschland setzte, wenn man von der Mundartforschung absieht, nach vereinzelten Ansätzen vor allem zwischen den beiden Kriegen die intensive, systematische Erforschung der Sprache der Gegenwart erst nach dem Ende des zweiten Weltkriegs ein. (Moser et al. 1967: 7)

Moser (1967: 12) führt bei der Benennung der „Sondergebiete“, die bearbeitet werden sollen, nicht nur die „Dokumentation der deutschen Gegenwartssprache“ an, sondern auch explizit als dritten Punkt: „Strukturalistische Grammatik“. Insgesamt steht die „Erarbeitung einer wissenschaftlichen Grammatik des heutigen Deutsch“ (Moser 1967: 13) im Zentrum. Dem zentralen Auftrag, „die deutsche Gegenwartssprache $\mathrm{zu}$ untersuchen und $\mathrm{zu}$ dokumentieren“ (Eichinger 2014: 19), ist das IDS bis heute treu geblieben. 
Für Glinz (1952) ist kennzeichnend, dass er sich einerseits in den wissenschaftlich-grammatischen Diskurs einbringt, andererseits aber konstitutiv in die Schulgrammatik hineinwirkt und diese eben auch auf eine wissenschaftliche Grundlage stellen will (vgl. Eichinger 2008a). Hier kommt es nun also zu einer (Wieder-)Vereinigung der „schulgrammatischen“ und der „wissenschaftlichen“ Tradition, wie sie bis zum 18. Jahrhundert und speziell bei Adelung wenn man hier von einer „wissenschaftlichen“ Grammatik sprechen will schon einmal vorhanden war. In diesem Zusammenhang steht auch die nach dem Zweiten Weltkrieg erstmals 1959 wieder erschienene, aber gegenüber Basler (1935) völlig neu konzipierte Duden-Grammatik, die übrigens - in Fußnote 1 im „Vorwort des Herausgebers“ (Grebe 1959: unpaginiert) - explizit auf Glinz (1952) verweist (darüber hinaus wird Hans Glinz am Ende des Vorworts gedankt). Bis heute macht es sich das IDS zur Aufgabe, „die Ergebnisse der Forschung in unterschiedlicher Abstraktheit und Detailliertheit und damit in verschiedenen Formen an die Öffentlichkeit zu bringen.“ (Eichinger 2014: 26). Dies manifestiert sich etwa in den verschiedenen Auflagen der DudenGrammatik, an denen das IDS jeweils in zentraler Weise beteiligt war.

Die Diachronie geriet dagegen gerade in der zweiten Hälfte des 20. Jahrhunderts etwas aus dem Blickfeld. Dies war durchaus auch ein aktiver Prozess der Abgrenzung einer jungen Generation von Sprachwissenschaftlern ab den 1960er Jahren (vgl. Ehlers 2005: 21). Nicht nur schien „die historische Schule“ nun endgültig den berühmten „Muff von tausend Jahren“ auszuströmen, sie schien auch nicht viel zur synchronen Beschreibung, geschweige denn zu Problemen der anwendungsorientierten Grammatik, beitragen zu können (dass diese Darstellung allerdings sehr verkürzt und damit ein Zerrbild ist, wird im nächsten Abschnitt unter anderem an der Grammatik-Konzeption von Glinz gezeigt). Insofern ist es bemerkenswert, dass Moser (1967: 13) als zehntes und letztes der zu bearbeitenden Gebiete immerhin „Älteres Neuhochdeutsch“ anführt. Insgesamt wird man aber sicher nicht ungerecht urteilen, wenn man feststellt, dass der synchrone Zugang am IDS und in dessen wissenschaftlicher Umgebung dominiert.

\section{Diachrone und synchrone Beschreibung}

Zentral für den diachronen Zugang ist die Beschreibung und Analyse sprachlicher Veränderungen, d. h. sprachlicher Entwicklungen über die Zeit hinweg. Der synchrone Zugang beschreibt und analysiert dagegen sprachliche Systeme zu einem bestimmten Zeitpunkt. Allerdings kann das Verhältnis der beiden Zugänge durchaus so gesehen werden, dass Überschneidungen und Übergänge 
bestehen. Dazu sei Hans Glinz zitiert. In den „Vorbemerkungen“ zur zweiten Auflage der „Inneren Form des Deutschen“, die deswegen besonders aufschlussreich sind, weil darin eine Reflexion über die Position des vertretenen Ansatzes vorgenommen wird, führt Glinz aus,

\begin{abstract}
daß wohl die strenge SAUSSURE'sche Scheidung von Synchronie und Diachronie nur verstanden werden kann als ein Gegenschlag gegen die Verkennung der Synchronie in der vorangehenden Wissenschaftsepoche, und daß die volle synchrone Forschung mit Notwendigkeit auch zu Fragen der Entwicklung führt, zunächst indem sie einen gegebenen, geltenden Sprachzustand (ein «geltendes System») mit allen in ihm noch lebendigen, noch wirksamen «Tiefenschichten» überhaupt versteht [...], und dann indem sie diesen Zustand mit einem früheren (primär ebenso synchron erforschten) geltenden $\mathrm{Zu}$ stand vergleicht. Ja, dadurch wird erst eine wirkliche Sprachgeschichte, eine Sprachgeschichte als Systemgeschichte möglich, und alle Sprachgeschichte, die wir besitzen, ruht (teils unbewußt, teils durchaus bewußt) auf solchen Fundamenten [...] (Glinz 1961: 7: Kapitälchen, Sperrungen und Anführungszeichen im Original)
\end{abstract}

Für eine Erklärung des Wandels auf systemischer Ebene sind einzelne synchrone Beschreibungen die Grundvoraussetzung - die synchrone Betrachtung hat also auch und gerade für die Erforschung historischer Entwicklungen direkte Relevanz. Umgekehrt nimmt Glinz auch für das synchrone System an, dass „noch wirksame «Tiefenschichten» “, die für ältere Zustände stehen, existieren. Das heißt: Auch in einem synchronen System gibt es gewisse historisch bedingte „Irregularitäten“. Dies soll im folgenden Abschnitt anhand eines konkreten Beispiels aufgezeigt werden.

\title{
3.1 Ein Beispiel: -iz-/-az-Stämme im Althochdeutschen
}

Es stellt eine alte Erkenntnis dar, dass diachrone Entwicklungen zu synchronen Problemen im System führen können. Als ein bereits von Paul (1920) angeführtes morphologisches Beispiel kann auf den Singular der neutralen -iz-/ - $a z$-Stämme im Althochdeutschen, deren Kerngruppe fünf Tierbezeichnungen ausmachen (lamb ,Lamm', kalb ,Kalb', rind ,Rind', huon ,Huhn' und farh ,Ferkel'), verwiesen werden. Bei dieser Deklinationsklasse entstand zunächst das folgende (nach Paul 1920: 215 wiedergegebene) Paradigma: ${ }^{1}$

1 Paul (1920: 215) führt aus, dass der Nominativ Singular dieses Paradigmas aus der lautgesetzlichen Entwicklung hervorgegangen sei. Die übrigen Formen werden nicht detailliert diskutiert, allerdings wird darauf hingewiesen, dass das Paradigma „[u]nter der Einwirkung der vokalischen Deklination“ entstanden sei, Analogie hat also bereits hier eine Rolle gespielt. Als rein lautgesetzlich zu erwartende Formen im Genitiv und Dativ Singular setzt Schenker (1971: 55) - mit einem anderen Beispiel-Lexem - lembir an (für eine ältere Sprachstufe wäre ohne 


\begin{tabular}{lll}
\hline & Sg. & Pl. \\
\hline Nom. & kalb & kalbir \\
Gen. & kalbir-es & kalbir-o \\
Dat. & kalbir-e & kalbir-um \\
Akk. & kalb & kalbir \\
\hline
\end{tabular}

Dieses System ist insofern unregelmäßig, als bei den meisten anderen starken Neutra im Genitiv und Dativ Singular kein -ir- vor der „eigentlichen“ Kasusendung steht (vgl. etwa wort - wort-es - wort-e etc.). Paul kommentiert diesen Zustand wie folgt:

Im Gen. und Dat. Sg. war das -ir- jedenfalls unnötig und störend. Daher sind die betreffenden Formen schon in der Zeit, aus der unsere ältesten Quellen stammen, bis auf vereinzelte Reste verschwunden und durch kalbes, kalbe ersetzt [...] (Paul 1920: 215)

Hier manifestiert sich das vielleicht zentralste Element der junggrammatischen Sprach(wandel)theorie: Durch das Wirken der Lautgesetze verursachte synchrone Unregelmäßigkeiten können sekundär durch analogischen Ausgleich wieder „bereinigt“ werden:

Man kann sich schwer eine Vorstellung davon machen, bis zu welchem Grade der Zusammenhangslosigkeit, Verworrenheit und Unverständlichkeit die Sprache allmählich gelangen würde, wenn sie alle Verheerungen des Lautwandels geduldig ertragen müsste, wenn keine Reaktion dagegen möglich wäre. [...] Wo durch den Lautwandel eine unnötige und unzweckmässige Differenz entstanden ist, da kann dieselbe mit Hilfe der Analogie beseitigt werden [...] (Paul 1920: 198)

In einer diachronen Beschreibung würde die Entstehung und Beseitigung von Irregularitäten im Vordergrund stehen - so, wie Paul beschreibt, dass die „unnötigen und störenden“ Formen ersetzt wurden. Wichtig ist die Richtung der Entwicklung. Die einzelnen synchronen Zustände - die natürlich auch

Umlaut entsprechend lambir anzunehmen), dies entspräche den im Altenglischen tatsächlich belegten ältesten Formen (vgl. Schenker 1971: 54). Die durch Pauls Paradigma repräsentierte Stufe, die im Genitiv und Dativ Singular sowohl das auf -iz-/-az-zurückgehende -ir-, also das ursprünglich stammbildende Morphem, als auch die Kasusendungen, die aus der $a$-Deklination stammen, aufweist, ist also bereits eine Zwischenstufe, in der die lautgesetzlich zu erwartenden Formen nicht mehr als solche vorliegen. In dieser Perspektive stellen die älteren Formen kalb-ir-es, kalb-ir-e des Genitiv und Dativ Singular eine interessante Parallele zu ursprünglich schwach flektierenden Substantiven dar, die im Genitiv Singular das - $(e) s$ der starken Deklination übernommen haben, etwa Herz-en-s. 
bereits Paul konzeptuell voraussetzt - stehen dagegen nicht im Fokus des Interesses.

Das ist beim synchronen Zugang anders. Wenn sich eine synchrone Beschreibung auf einen homogenen Endzustand (oder auch Anfangszustand) beziehen kann, wird die Konsistenz des Systems mit Befriedigung konstatiert werden können. Wie aber ginge die synchrone Beschreibung mit nicht systemkonformen „Zwischenzuständen“ um, in denen sich, um Glinz (1961: 7) noch einmal zu zitieren, „noch wirksame «Tiefenschichten» “, die (eigentlich) für ältere Stufen gelten, manifestieren? Dieses Problem wird im Folgenden etwas vertieft.

\subsection{Zum Status von Variation}

Es ist schwer, wenn nicht unmöglich, Sprachwandel direkt zu beobachten. Wie hat sich „der“ Wandel von den älteren zu den jüngeren Formen des Genitiv und Dativ Singular bei den -iz-/-az-Stämmen abgespielt? Lief dieser Wandel sehr schnell (sozusagen von heute auf morgen) ab? Oder dauerte es mehrere Generationen, bis sich die neuen Formen durchsetzten?

In der Sprachwandelforschung ist die Ansicht verbreitet, dass es zwischen einem homogenen Anfangs- und einem ebensolchen Endzustand „Zwischenstufen“ gibt, die durch Variation, d. h. das Auftreten der alten und neuen Formen nebeneinander, geprägt sind. Die Frage, wie derartige „Zwischenstufen“ geartet sind (und wie lange sie andauern und ob sie langfristig persistieren können), ist für die Sprachwandelforschung zentral. Es gilt eigentlich als ausgemacht, dass durch Variation geprägte „Zwischenstufen“ nicht von Dauer sind (sofern die Varianten nicht mit einer neuen Funktion verbunden werden - s. u.):

Man kann als gegeben ansehen, dass sich die Sprache - und das heißt ihre Sprecher und Schreiber - auf Dauer kaum funktionslose Doppelungen leisten [...] (Eichinger 2011: 7)

Die Variation wird entweder aufgegeben (d. h.: eine Variante setzt sich durch das ist bei den -iz-/-az-Stämmen im Singular mit dem Verlust des ursprünglich stammbildenden Suffixes geschehen), oder aber sekundär re-funktionalisiert. In grammatischer Hinsicht kann dies etwa im Sinne einer „Exaptation“ (vgl. dazu Lass 1990) geschehen, wie sie bei den -iz-/-az-Stämmen in der Re-Interpretation des ursprünglich stammbildenden Suffixes als Pluralmorphem vorliegt. Auch eine sekundäre Funktionalisierung in Abhängigkeit von externen Faktoren kann auftreten (vgl. dazu den folgenden Abschnitt). Allerdings wird teilweise auch angenommen, dass es Variation gibt, die gänzlich funktionslos, d. h. weder intern noch extern bedeutsam ist. Kein Geringerer als Trubetzkoy 
ging offensichtlich davon aus, dass derartige „freie Variation“ (vgl. Trubetzkoy 1939: 44) existiert:

Den stilistisch irrelevanten fakultativen Varianten kommt überhaupt gar keine Funktion zu: sie ersetzen einander ganz willkürlich, ohne daß dabei die Kundgabefunktion der Rede irgendwie geändert würde. (Trubetzkoy 1939: 43)

Gerade für Übergangszustände könnte man sich freie Variation - die dann abgebaut wird - durchaus vorstellen. In einer synchronen Hinsicht ist man allerdings häufig etwas skeptischer, wie die folgende aktuelle Formulierung anhand des Adverbs „,tatsächlich“ erkennen lässt:

[G]ibt es tatsächlich freie Variation? (Konopka \& Wöllstein 2017: XI)

Die Frage, ob „freie“ Variation, die weder durch interne noch durch externe Faktoren determiniert ist, existiert, hat für den synchronen Ansatz nach wie vor Gretchen-Qualität (vgl. Dufter et al. 2009: 4-5). Wenn die Sprache ein System ist, „où tout se tient“, ${ }^{2}$ ist für (funktionslose) Variation an sich kein Platz. Für den diachronen Ansatz - sofern er nur dem Endpunkt und nicht dem „Zwischenzustand“ seine Aufmerksamkeit schenkt - steht dieses Problem dagegen nicht im Zentrum.

\subsection{Dimensionen von Variation}

Abgesehen von der freien Variation, die für die synchrone Beschreibung ein besonderes Problem darstellt, kann sprachliche Variation durch externe und interne Faktoren bedingt sein. Sofern externe Faktoren die Verteilung von Varianten bestimmen, kann - in einer systemischen Perspektive - durchaus dennoch von homogenen Systemen ausgegangen werden: Wenn eine Variante nur in einem bestimmten Gebiet, nur von einer bestimmten sozialen Schicht oder, auf schriftsprachlicher Ebene, nur in einer bestimmtem Textsorte verwendet wird, dort aber exklusiv, bedeutet dies, dass das entsprechende System nur diese eine Variante kennt und also Variation nur dann „vorliegt“, wenn verschiedene Systeme miteinander verglichen werden. Bei intern gesteuerter

2 Es stellt ein interessantes Faktum dar, dass die Herkunft dieses strukturalistischen Credos unklar ist: Koerner (1999: 183-202) nennt verschiedene Namen, denen es zugeschrieben wurde, unter anderem Ferdinand de Saussure und Antoine Meillet, doch kann der Ursprung letztlich nicht eruiert werden. Offensichtlich gibt diese Formulierung einen Kerngedanken des Strukturalismus dermaßen gut wieder, dass sie eher den Gesetzmäßigkeiten der mündlichen Tradierung unterliegt. 
Variation wird es dagegen für die Grammatikographie besonders interessant: Hier manifestieren sich die eigentlichen grammatisch relevanten sprachinternen Faktoren.

Methodisch ist es in manchen Fällen nicht ganz einfach, das Wirken sprachinterner und -externer Faktoren auseinanderzuhalten. Dies sei zunächst an einem historischen Beispiel, an der Voranstellung bzw. Nachstellung des attributiv verwendeten Genitivs im Frühneuhochdeutschen, illustriert (vgl. Fleischer \& Schallert 2011: 79-80). Noch im Neuhochdeutschen sind im Prinzip beide Abfolgen möglich (des Teufels Großmutter vs. die Großmutter des Teufels), allerdings hat die Voranstellung im modernen Deutsch - von Eigennamen abgesehen - eine antiquierte Konnotation. In älteren Sprachstufen war dagegen die Voranstellung noch wesentlich weiter verbreitet, wobei gerade in frühneuhochdeutscher Zeit beide Abfolgen nebeneinander auftreten und sich damit die Frage stellt, unter welchen Bedingungen welche Abfolge auftritt. In ihrer Untersuchung zu diesem Problem gliedert Fritze (1970) die Belege nach verschiedenen Textsorten (Fachprosa, Flugschrift, Chronik, Volksbuch, Reisebeschreibung), also anhand eines sprachexternen Kriteriums, verzichtet allerdings auf eine Unterteilung anhand sprachinterner Kriterien, etwa auf eine Unterscheidung von Substantiven in verschiedene semantische Gruppen. Nach Fritze (1970: 426-427) überwiegt die Nachstellung in der Fachprosa und in den Flugschriften, sie ist seltener in den Chroniken und noch weniger häufig in den Volksbüchern und Reisebeschreibungen. Die Stellung des Genitivattributs scheint also von der Textsorte und somit von einem externen Faktor determiniert zu sein.

Fritzes empirischer Befund kann allerdings auch anders interpretiert werden: Entscheidend ist wohl nicht die Textsorte, sondern die Tatsache, dass beispielsweise Eigennamen, die in der Regel vorangestellt werden, „in den Chroniken, Volksbüchern und Reisebeschreibungen häufiger vorkommen als in den Flugschriften und in der Fachprosa“ (Ebert 1999: 96). Wichtig ist also, wie Ebert (1999: 95-96) aufzeigt, die Semantik des Nomens im Genitiv (letztlich dürfte dahinter die Kategorie der Belebtheit stehen, deren erstmalige Formulierung auf Silverstein 1976 zurückgeht).

Ebert (1999: 19) leitet - von derartigen Beispielen ausgehend - ein methodologisches Postulat ab: Auch bei der Untersuchung sprachexterner Faktoren ist es notwendig, die wichtigsten sprachinternen Faktoren zu berücksichtigen. Nur wenn gezeigt werden kann, dass interne Faktoren - wie etwa im diskutierten Beispiel die Semantik des Nomens - keine Auswirkung auf eine bestimmte grammatische Struktur haben, kann der Effekt externer Faktoren zweifelsfrei festgestellt werden. Während es sich bei den internen Faktoren in der Regel um bekannte grammatische Größen handelt, kommen als externe Faktoren - 
im Gefolge der Labovschen Soziolinguistik - etwa „all six of the major independent variables in sociolinguistics: sex, age, social class, ethnicity, race, and community size“ (Labov 1994: 2) in Frage.

In sprachwandeltheoretischer Hinsicht erscheint hier eine zentrale Erkenntnis aus der Sprachgeographie - im deutschsprachigen Gebiet durch Georg Wenker (1852-1911) und seine Sprachatlanten, insbesondere den monumentalen, aber zu Wenkers Lebzeiten unpublizierten Sprachatlas des Deutschen Reichs verkörpert - besonders bedeutend: die „Gleichzeitigkeit des Ungleichzeitigen“ (so die Formulierung von Schmidt \& Herrgen 2011: 82). Synchrone areale Variation entpuppt sich in vielen Fällen als ein Nebeneinander historisch älterer und jüngerer Entwicklungsstufen. In grammatiktheoretischer Sicht bedeutet allerdings areale Variation das geringste Problem: Grundsätzlich konstituiert jeder Idiolekt und somit auch jeder Dialekt ein eigenes grammatisches System, areale Variation ist also ganz klar extern determiniert (vgl. z. B. Dufter et al. 2009: 8; Lenz i. d. Bd.).

Gerade in jüngster Zeit hat sich gezeigt, dass selbst bei „der“ neuhochdeutschen Norm areale Unterschiede bzw. verschiedene „Gebrauchsstandards“ (so der Begriff von Elspaß \& Dürscheid 2017) auftreten. Dass die neuhochdeutsche Norm nicht völlig einheitlich ist, ist an sich keine neue Erkenntnis. Schon in Hugo Mosers Formulierung der Ziele und Aufgaben des IDS heißt es:

Auch auf die regionalen Unterschiede der deutschen Hochsprache, besonders in Österreich und der deutschsprachigen Schweiz, soll das Augenmerk gerichtet werden. (Moser 1967: 13)

Aus einer heutigen Perspektive scheint es allerdings angezeigt, nicht mehr nur von einer Plurizentrik mit verschiedenen nationalen Normen auszugehen, sondern besser von einer „Pluriarealität“ (vgl. Elspaß \& Dürscheid 2017: 87-89): Nicht nur ist eine Unterteilung in verschiedene nationale Standards in manchen Fällen zu wenig feinkörnig; darüber hinaus lässt sich auch zeigen, dass es klare areale Strukturen gibt, die nationale Grenzen überschreiten. Dies zeigt sich etwa bei der „Zwischenstellung“ bei dreigliedrigen Verbalkomplexen im Nebensatz (vgl. Elspaß \& Dürscheid 2017: 96-99 und die dort zitierte Literatur): Diese Serialisierung - exemplifiziert etwa durch ... dass er immer wieder in den Hausgang hinaus verschwinden hat müssen (zit.n. Elspaß \& Dürscheid 2017: 96) - wird häufig mit „dem“ österreichischen Standarddeutsch in Verbindung gebracht. Wie nun allerdings die Karte von Elspaß \& Dürscheid (2017: 98) eindrücklich zeigt, ist dieses Muster außer in österreichischen durchaus auch in bundesdeutschen Zeitungen $\mathrm{zu}$ finden. Das gilt etwa für das zitierte Beispiel ... verschwinden hat müssen: Dieser Beleg stammt aus den „Passauer Neuen Nachrichten“. Im Wesentlichen korreliert die areale Verbrei- 
tung in den Gebrauchsstandards mit einer aus der Dialektologie gut bekannten Entität: dem bairischen Dialektverband. Tatsächlich ist diese Abfolge für die bairischen Dialekte belegt, daneben kommt sie allerdings - auf dialektaler Ebene - durchaus auch anderswo vor, etwa im Westmitteldeutschen (vgl. Fleischer \& Schallert 2011: 168).

\section{Variation im Korpus}

Aus der bisherigen Diskussion ergibt sich, dass synchroner Variation in grammatisch-theoretischer Hinsicht ein besonders wichtiger Status zukommt: Varianten können für historisch ältere bzw. historisch jüngere Formen stehen. Dabei ist die Frage zentral, durch welche - sprachinternen oder/und externen Faktoren das Auftreten der verschiedenen Varianten determiniert ist. In den folgenden Abschnitten zeigen einige Fallbeispiele auf, mit welchen theoretischen und praktischen Schwierigkeiten die grammatische Analyse hier verbunden ist. Dabei zeigt sich, dass die Art und Beschaffenheit des Korpus eine zentrale Rolle spielt.

\subsection{Ein althochdeutsches Lehrstück: -iz-/-az-Stämme revisited}

Es ist für die folgende Diskussion aufschlussreich, das in 3.1 im Gefolge von Paul (1920: 215) diskutierte Problem des Genitiv und Dativ Singular der -iz-/ - $a z$-Stämme auf die empirische Grundlage in der althochdeutschen Überlieferung zu befragen. Man muss sich klar machen, dass das „Korpus“, d.h. im konkreten Fall die gesamte Überlieferung des Althochdeutschen, sehr beschränkt ist: Die althochdeutsche Tatian-Übersetzung und Otfrids von Weißenburg Evangelienbuch, die beiden größten Texte des 9. Jahrhunderts, umfassen jeweils nur ca. 55.000 bzw. 82.200 tokens $^{3}$ - wer von der Textmenge, wie sie etwa das DeReKo mit zur Zeit über 31 Milliarden tokens bietet, verwöhnt ist, kann angesichts solcher Zahlen nur müde lächeln.

Insofern wundert es nicht, dass sich die empirische Situation zum Genitiv und Dativ Singular der -iz-/-az-Stämme im Althochdeutschen als prekär entpuppt: Braune \& Reiffenstein (2004: 188) verweisen - außerhalb der Überlieferung von Ortsnamen - auf genau je einen Beleg für den Genitiv und Dativ

3 Zahlen nach https://korpling.german.hu-berlin.de/annis3/ddd (letzter Zugriff 17.11. 2017). 
Singular mit dem alten Suffix, nämlich rindares und chalbire. Beide Belege stammen aus dem Zweiten Reichenauer Glossar (vgl. Steinmeyer \& Sievers 1879: 426 [Nr. 24] bzw. 409 [Nr. 9]), einem Zeugnis, das Meineke (2013: 422) als „die zweitgrößte Glossenhandschrift des 8. Jh.s.“ charakterisiert. Für eine bessere Einschätzung der Zahlen ist es allerdings sinnvoll, sie den entsprechenden ,jungen“ Formen ohne -ir-/-ar- gegenüberzustellen. Das wird in der folgenden Tabelle, die sich auf die veröffentlichten Artikel bzw. das Zettelmaterial des Althochdeutschen Wörterbuchs ${ }^{4}$ bezieht, für die fünf zentralen Lexeme dieser Gruppe getan:

Tab. 11.1: Anzahl Formen des Genitiv und Dativ Singular von -iz-/-az-Stämmen nach dem Althochdeutschen Wörterbuch.

\begin{tabular}{lrrrrrr}
\hline & \multicolumn{2}{c}{ mit -ir-/ar- } & & \multicolumn{2}{c}{ ohne -ir-/ar- } \\
\cline { 2 - 3 } \cline { 5 - 6 } & Gen. Sg. & Dat. Sg. & & Gen. Sg. & Dat. Sg. \\
\hline lamb & - & - & & 1 & - \\
kalb & - & 1 & & 2 & 1 \\
rind & 1 & - & & 1 & 1 \\
huon & - & - & & - & - \\
farh & - & - & & 4 & 2 \\
\hline Total: & 1 & 1 & & & \\
\hline
\end{tabular}

Es stehen also im gesamten althochdeutschen Korpus zwei Belege für die älteren Formen sechs Belegen für die jüngeren, durch analogischen Ausgleich entstandenen Formen gegenüber - die alten, von Paul (1920: 215) als „unnötig und störend" charakterisierten Formen machen also immerhin ein Viertel des gesamten Belegmaterials aus. ${ }^{6}$ Dabei ist zu berücksichtigen, dass die hier referierte Beleglage sich auf die gesamte althochdeutsche Sprachstufe, also immerhin ca. drei Jahrhunderte, bezieht. Bei genauerer Betrachtung ergeben

4 Die Zahlen für alle Lexeme außer rind stammen aus den entsprechenden Artikeln des Althochdeutschen Wörterbuchs (lamb: 5: 599; kalb: 5: 7; huon: 4: 1381; farh: 3: 570). Für das Zusammenstellen der Belege zu rind aus dem Zettelmaterial dieses noch nicht veröffentlichten Artikels danke ich Brigitte Bulita (Leipzig), Leiterin des Althochdeutschen Wörterbuchs, sehr herzlich.

5 Neben der unzweifelhaft althochdeutschen Form ríndes aus Notkers „Categoriae“ (die in beiden Handschriften in gleicher Weise belegt ist; vgl. Cod. Sang. 818: 81, Cod Sang. 825: 320b) findet sich im Zettelmaterial des Althochdeutschen Wörterbuchs auch die Form hrithas aus einer Glossenhandschrift (Düsseldorf, ULB Ms. F 1). Diese Glosse ist jedoch altniederdeutsch (und wird bei Tiefenbach 2010: 183 entsprechend gebucht), weshalb sie hier nicht mitgezählt wird.

6 Dagegen zeigt ei ,Ei', das von Braune \& Reiffenstein (2004: 188) als erstes Substantiv nach den fünf Tierbezeichnungen angeführt wird, wesentlich mehr Belege für den Genitiv und Dativ 
sich allerdings eher zwei „Zeitscheiben“: Von den zwei Belegen aus dem Zweiten Reichenauer Glossar abgesehen stammen die übrigen Belege erst aus dem 10./11. Jahrhundert, die meisten davon aus dem Notker-Korpus. Für die ältere - durch das Zweite Reichenauer Glossar repräsentierte - Stufe des späten 8. Jahrhunderts zeigen alle Belege für den Genitiv und Dativ Singular die historisch älteren Formen. Im 10./11. Jahrhundert finden wir dagegen ausschließlich die historisch jüngeren Formen. Zum 9. Jahrhundert haben wir keine Daten.

$\mathrm{Ob}$ es gerechtfertigt ist, bei den beiden Belegen für die historisch älteren Formen, wie Paul (1920: 215) dies tut, von „vereinzelten Resten“ zu sprechen, scheint zumindest diskussionswürdig: Wiewohl sich - angesichts der insgesamt sehr dünnen Datensituation - prozentuale Angaben eigentlich verbieten, kann darauf verwiesen werden, dass immerhin $25 \%$ aller althochdeutschen Belege noch die historisch älteren Formen aufweisen und dass sich umgekehrt vor dem 10./11. Jahrhundert kein einziger positiver Beleg für die historisch jüngeren Formen anführen lässt. In dieser Perspektive stehen die zwei Stellen für die historisch älteren Formen für $100 \%$ aller Belege vor dem 10. Jahrhundert. Die Belegsituation lässt keine Entscheidung der Frage zu, ob die beiden Belege aus dem Zweiten Reichenauer Glossar sozusagen die letzten Überreste der alten Formen zeigen oder ob diese noch das ganze 9. Jahrhundert hindurch persistierten.

Als Konsequenz für eine diachrone Beschreibung muss nun festgehalten werden: Wir können nicht genau nachvollziehen, wie der Wandel abgelaufen ist. In einem Denkmal, dem Zweiten Reichenauer Glossar, könnte der durch Pauls (1920: 215) Paradigma repräsentierte ältere Zustand tatsächlich fassbar sein - allerdings ist dort nur je eine Form zu je einem Lexem belegt. Alle übrigen Belege deuten darauf hin, dass der Wandel bereits abgeschlossen ist. Ein Übergangsstadium - das hieße etwa: mit Variation zwischen der alten und der jungen Form in einem Denkmal - lässt sich nicht belegen. Die prekäre Datensituation wird besonders deutlich, wenn man sich die Lexeme huon ,Huhn' und farh,Ferkel' vor Augen führt: Für diese ist in der gesamten althochdeutschen Überlieferung kein einziger Genitiv und Dativ Singular belegt. Für die Grammatikographie des Althochdeutschen zeigt sich hier - einmal mehr die insgesamt sehr beschränkte Datenbasis, die uns die älteste Sprachstufe des Deutschen bietet. ${ }^{7}$ Allzu konkrete Aussagen zur Art und Weise, wie die histo-

Singular, allesamt ohne -ir-/-ar- (vgl. Althochdeutsches Wörterbuch 3: 93-94). Allerdings gehört dieses Lexem nicht zur Kerngruppe der Tierbezeichnungen.

7 Eine Ursache dieses grammatikographischen Problems ist im konkreten Fall natürlich auch, dass die hier interessierenden Kasus - Genitiv und Dativ - schon an sich seltener sind als Nominativ und Akkusativ. 
risch älteren von den jüngeren Formen abgelöst wurden, verbieten sich angesichts der Beleglage.

Wer sich mit jüngeren Sprachstufen, zumal der Gegenwartssprache, beschäftigt, hat also einen klaren Vorteil. Wie nun an den folgenden Beispielen aufgezeigt werden soll, kann das synchrone Nebeneinander von „älteren“ und „jüngeren“ Formen auch im Neuhochdeutschen an verschiedenen Stellen beobachtet werden. Dabei fallen sowohl für die an der Diachronie wie auch für die an der Synchronie Interessierten einige interessante Beobachtungen ab.

\section{2 Übergang von starkem zu schwachem Präteritum}

Es ist eine bekannte Beobachtung, dass die starken Verben, die ihre Präteritalformen mit Ablaut bilden (nehmen - nahm), in der deutschen Sprachgeschichte - was die Anzahl der types betrifft - immer weniger werden. Nach Augst (1975: 255, Tabelle 4) existieren im Althochdeutschen 349 starke Verben, im Mittelhochdeutschen noch 339, im Neuhochdeutschen dann nur noch 169. Wiewohl die Abnahme von 349 auf 339 starke Verben auf den ersten Blick nicht dramatisch scheint, ist doch der Rückgang der starken Verben schon im Mittelhochdeutschen evident, wenn man bedenkt, dass das überlieferte Korpus des Althochdeutschen um ein Vielfaches kleiner ist als das des Mittelhochdeutschen: Die 349 starken Verben des Althochdeutschen verteilen sich auf viel weniger überliefertes Textmaterial als die 339 starken Verben des Mittelhochdeutschen.

Manche starke Verben sind im Lauf der Zeit vollständig verschwunden (z. B. mhd. wëllen ,rollen', mhd. tëlben ,graben'). Viele ursprünglich starke Verben sind aber zu den schwachen übergetreten (z. B. pflegen - pflog > pflegen pflegte, schmiegen - schmog > schmiegen - schmiegte). In synchroner Hinsicht bedeutsam ist, dass bei manchen Verben Konkurrenz zwischen stark und schwach gebildeten Formen besteht (vgl. Duden-Grammatik 2016: $463=\S 636$ ): backen - buk/backte, glimmen - glomm/glimmte, melken - molk/melkte. Diachron kann das Nebeneinander von stark und schwach gebildeten Präteritalformen als ein Zustand des Übergangs gedeutet werden. Wie aber stellt sich die synchrone Situation dar?

Die folgende Tabelle zeigt für diese drei Verben eine Übersicht anhand einer Suche im DeReKo (wobei eine Beschränkung auf Daten der Jahrzehnte von 1990 bis zum Zeitpunkt dieser Untersuchung vorgenommen wurde, da diese Periode im DeReKo dominiert). ${ }^{8}$ Es zeigt sich bei diesen drei Verben, dass areale

8 Beispielsweise Belege aus den Märchen der Brüder Grimm - die etwa bei backen die starken Formen verwenden - werden also nicht reflektiert. Dadurch werden die Daten in zeitlicher Hinsicht einheitlicher. 
Unterschiede auftreten. Dies wird hier anhand der im DeReKo gegebenen Gruppierung der Treffer nach Ländern aufgezeigt, allerdings nicht noch feiner aufgeschlüsselt, was durchaus sinnvoll wäre (vgl. 3.3). In den einzelnen Zellen stehen jeweils die absoluten Zahlen für die starke und die schwache Präteritalform (wobei sich der Wert auf sämtliche Numeri/Personen bezieht), der danach folgende Prozentwert gibt den Anteil der starken Präteritalform an.

Tab. 11.2: Präteritalformen von backen, glimmen und melken nach DeReKo.

\begin{tabular}{lrrrrr}
\hline & \multicolumn{1}{c}{ Total } & \multicolumn{1}{c}{ D } & \multicolumn{1}{c}{ A } & \multicolumn{1}{c}{ CH } & \multicolumn{1}{c}{ FL } \\
\hline backen & $1.165: 2.330$ & $833: 1.962$ & $179: 205$ & $151: 160$ & $2: 3$ \\
& $(33 \%)$ & $(30 \%)$ & $(47 \%)$ & $(49 \%)$ & $(40 \%)$ \\
\hline glimmen & $204: 278$ & $163: 237$ & $22: 18$ & $19: 23$ & - \\
& $(42 \%)$ & $(41 \%)$ & $(55 \%)$ & $(45 \%)$ & \\
\hline melken & $154: 92$ & $102: 47$ & $23: 11$ & $28: 34$ & $1: 0$ \\
& $(63 \%)$ & $(68 \%)$ & $(68 \%)$ & $(45 \%)$ & $(100 \%)$ \\
\hline Total & $1.523: 2.800$ & $1.098: 2.246$ & $224: 234$ & $198: 217$ & $3: 3$ \\
& $(36 \%)$ & $(33 \%)$ & $(49 \%)$ & $(48 \%)$ & $(50 \%)$ \\
\hline & & & & &
\end{tabular}

Wenn alle Resultate zusammen betrachtet werden, ergibt sich, dass die starken Formen etwas mehr als ein Drittel ausmachen. Diese globale Perspektive verwischt aber nicht nur Unterschiede zwischen den einzelnen Ländern, sondern auch zwischen den einzelnen Verben: Der Süden, d. h. Österreich, die Schweiz und Liechtenstein (bei dem sich die Aussagen allerdings insgesamt auf nur sechs Belege beziehen), zeigt gesamthaft höhere Werte für die starken Formen, um $50 \%$ gegenüber $33 \%$ bei Deutschland. Das Verb backen, für das insgesamt am meisten Belege vorhanden sind (und das somit am meisten zum Total beiträgt), entspricht dieser Verteilung recht genau. Auch bei glimmen, bei dem die starken Formen insgesamt eine größere Rolle spielen, erweist sich der Süden als etwas konservativer, was hier allerdings vor allem für Österreich gilt (die Abweichungen dürfen angesichts der kleineren Belegmengen nicht überinterpretiert werden). Eine Überraschung bietet dann aber melken: Hier sind Deutschland und Österreich mit identischen relativen Werten deutlich konservativer, wogegen die Schweiz eine Präferenz für die historisch jüngere Form hat. Damit zeigt die Schweiz auch bei allen drei Verben ähnliche Werte, wogegen Deutschland und Österreich zwischen den drei Verben jeweils deutliche Unterschiede aufweisen.

Aus der Perspektive der einzelnen Verben zeigt sich, dass zwar alle Variation aufweisen, dass aber melken mit $63 \%$ noch am meisten den starken Formen 
zuneigt, gefolgt von glimmen mit $42 \%$ und, nochmals niedriger, von backen mit $33 \%$. Es bestehen also auch deutliche lexikalische Unterschiede.

Es soll im Folgenden den Gründen für diese Verteilung nicht weiter nachgegangen werden. Festgehalten werden soll aber, dass sich hier auf der Ebene der Standardsprache - abhängig vom Verb - zum Teil deutliche areale Unterschiede zeigen, die auch in unterschiedliche Richtungen deuten. Dass gerade Österreich und die Schweiz (und, wenn wir die wenigen Belege gelten lassen wollen, Liechtenstein) zu den starken Präteritalformen neigen, überrascht insofern, als die oberdeutschen Dialekte das Präteritum bekanntermaßen weitgehend abgebaut haben (vgl. z. B. Fleischer \& Schallert 2011: 129-131). Dass sich dennoch gerade in Österreich und in der Schweiz die älteren Formen besser erhalten haben, ist also eine rein standardsprachliche Entwicklung.

\subsection{Verben mit sogenanntem „Rückumlaut“}

Bei den in der historischen Grammatik als „Verben mit sogenanntem Rückumlaut“ bezeichneten Verben ergab sich lautgesetzlich (neben der regelmäßigen schwachen Präteritalbildung mittels -t-) ein Schema mit Vokalwechsel, das etwa bei Verben wie rennen - rannte oder brennen - brannte in der neuhochdeutschen Standardsprache als einzig mögliche Präteritalbildung nach wie vor belegt ist. Daneben haben aber viele derartige Verben den „Rückumlaut“ aufgegeben oder es herrscht(e) Variation. Das folgende Schema zeigt für vier verschiedene Verben (denen jeweils analog gelagerte Beispiele hinzugefügt werden könnten), welche Präterialformen im Althochdeutschen und in der neuhochdeutschen Standardsprache auftreten (Angaben zum Althochdeutschen nach Braune \& Reiffenstein 2004: 292-294 = §356):

\begin{tabular}{lllll}
\hline & ,brennen & ,wenden“ & ,stellen & ,retten“ \\
\hline Prät.: Ahd. & branta & wanta & stalta & ratta, retita \\
Prät.: Nhd. & brannte & wandte, wendete & stellte & rettete \\
\hline
\end{tabular}

Die vier Verben sind von ,konservativ‘ nach ,innovativ‘ angeordnet: Das Verb brennen ist besonders konservativ, weil der Rückumlaut hier noch im Neuhochdeutschen vollständig erhalten ist und sich kaum Tendenzen zu einer Bildung *brennte feststellen lassen (die Duden-Grammatik 2016: $457=\S 627$ belegt brennte ausschließlich als - seltene - Konjunktiv-Form). Bei wenden ist im Althochdeutschen nur Rückumlaut belegt, im Neuhochdeutschen dagegen Variation zwischen beiden Bildungsweisen. Bei stellen scheint der Wandel dagegen 
vollendet: Während im Althochdeutschen nur die rückumlautende Präteritalbildung belegt ist, kommt im Neuhochdeutschen nur das Präteritum ohne Rückumlaut vor. Das letzte Beispiel, retten, zeigt, dass bestimmte Repräsentanten dieser Klasse schon im Althochdeutschen Varianz aufweisen, wobei sich dann hier, wie bei stellen, im Neuhochdeutschen nur noch die regelmäßig gebildete Form ohne Rückumlaut findet.

Für eine synchrone Beschreibung sind diejenigen Fälle, in denen Variation zwischen den verschiedenen Bildungsweisen auftritt, problematisch. Diese Zustände können als Übergangsstadium hin zu einem Zustand mit (ausschließlich) regulärer Präteritalbildung ohne Rückumlaut verstanden werden. Im Fall von retten scheint dies gerechtfertigt: Aus dem althochdeutschen Nebeneinander zweier Bildungsweisen hat sich im Neuhochdeutschen die reguläre Präteritalbildung durchgesetzt.

Wie stellt sich aber die Lage bei Variation im Neuhochdeutschen dar? Neben dem im obigen Schema angeführten wenden soll im Folgenden auch senden betrachtet werden: Die Duden-Grammatik (2016: $457=\S 627$ ) konstatiert für diese beiden Verben das Nebeneinander der Präteritalformen sandte/wandte und sendete/wendete. In einer diachronen Betrachtung treten hier alte und neue Formen nebeneinander auf. Die Erwartung wäre, dass sich langfristig die regelmäßig gebildeten Formen durchsetzen, wie dies Nübling (2000: 216) annimmt, wenn sie von den „noch (ko)existenten“ älteren Formen mit Rückumlaut spricht.

Die folgende Tabelle zeigt zunächst die Anzahl der beiden Präteritalformen für diese beiden Verben im DeReKo:

Tab. 11.3: Präteritalformen von senden und wenden im DeReKo.

\begin{tabular}{lrrr}
\hline & \multicolumn{1}{c}{ Total } & \multicolumn{1}{c}{ senden } & \multicolumn{1}{c}{ wenden } \\
\hline mit Rückumlaut & $103.456(79,4 \%)$ & $13.159(64,5 \%)$ & $90.297(82,2 \%)$ \\
regulär & $26.867(20,6 \%)$ & $7.254(35,5 \%)$ & $19.613(17,8 \%)$ \\
\hline
\end{tabular}

Die absoluten Zahlen zeigen zunächst, dass die Form mit Rückumlaut insgesamt wesentlich häufiger ist. Es kann also noch in keiner Weise davon die Rede sein, dass sich die regelmäßig gebildeten jüngeren Formen bereits durchgesetzt hätten. Die reinen Frequenzangaben verstellen allerdings den Blick eher. Bei senden hat eine Bedeutungsdifferenzierung stattgefunden (vgl. DudenGrammatik 2016: 457 = §627): Während das Präteritum mit Rückumlaut eher eine umfassende Bedeutung zu haben scheint, tritt das regulär gebildete Präteritum vor allem in Zusammenhang mit Funk und Fernsehen auf (die Duden- 
Grammatik umschreibt: „im Fernsehen ausstrahlen“). Eine exhaustive Analyse der über 20.000 Belege der Präteritalformen von senden kann an dieser Stelle natürlich nicht geleistet werden. Die Kookkurenz-Daten, die DeReKo bietet, deuten aber eindeutig in diese Richtung: Die Form sendete zeigt dominierend Kollokate aus dem Wortfeld „Fernsehen“, z. B. „Erstausstrahlung“, „live“, „Sender“; bei der Form „sandte“ hingegen finden sich Kollokate wie „Brief“, „Boten, „Botschaft“, „Schockwellen“, „Truppen“, „Grüße“ etc. Das Lexem „Signal“, das bei beiden Präteritalformen häufig auftritt, zeigt einen interessanten Überschneidungsbereich, was angesichts seiner Bedeutung (ein „Signal senden“ kann sowohl eine Rundfunkanstalt als auch - im übertragenen Sinn etwa die Politik) durchaus Sinn ergibt.

Auch bei wenden scheint eine Bedeutungsdifferenzierung eingetreten $\mathrm{zu}$ sein: Nach Duden-Grammatik (2016: $457=\S 627$ ) haben die regulär gebildeten Präteritalformen die Bedeutung „die Richtung ändern, umkehren“ oder „die andere Seite zeigen“. Eine exhaustive Analyse der über 100.000 Belege für Präteritalformen von wenden ist noch weniger realistisch als bei senden. Aber auch hier erlaubt ein Blick auf die Kookurrenz-Daten einige Aussagen: Bei wendete finden sich als Kollokate z. B. „Blatt“, „Fahrzeug“, „Wagen“, „fuhr“ („er wendete und fuhr“) und „drehte“ („er wendete und drehte“). Bei wandte treten hingegen „hilfesuchend“ (,er wandte sich hilfesuchend an ...“) oder „Brief“ (,er wandte sich in einem Brief an ...") besonders prominent auf.

\section{Schluss}

Die diskutierten Beispiele zeigen, dass das Einbeziehen diachroner Gesichtspunkte auch für die Beurteilung synchroner Fragestellungen relevant sein kann. Variation, die für die synchrone Beschreibung ein Problem darstellen kann, bedeutet in vielen Fällen das Nebeneinander historisch älterer und jüngerer Formen. Dabei leistet das DeReKo gerade auch den am Wandel Interessierten durch das Bereitstellen großer Datenmengen, in denen sich durch Wandel hervorgerufene Variation manifestiert, gute Dienste. Das am IDS angesiedelte Projekt „Korpusgrammatik - grammatische Variation im standardsprachlichen und standardnahen Deutsch“, das es sich zur Aufgabe macht, eine neue, „variationssensitive“ Grammatik des Standarddeutschen zu erarbeiten, ${ }^{9}$ wird hier in Zukunft sicher weitere interessante Befunde zur Variation erbringen und es auch erlauben, sprachlichen Wandel im Vollzug zu beobachten.

9 Vgl. http://www1.ids-mannheim.de/gra/projekte/korpusgrammatik.html (letzter Zugriff 17.11. 2017). 
Die sich aus einer diachronen Perspektive ergebende Erwartungshaltung, dass synchrone Variation im Lauf der Zeit verschwindet, kann sich in manchen Fällen als trügerisch erweisen, besonders, wenn Varianten funktionalisiert werden - was ja bedeutet, dass die Variation gar nicht mehr echt ist und den Variantenbegriff in dieser Hinsicht obsolet erscheinen lässt.

Das zu Beginn dieses Überblicks beschriebene Auseinandergehen des diachronen und des synchronen Ansatzes, das wissenschaftsgeschichtlich und in einem dialektischen Sinn vielleicht unumgänglich war, ist keine von der Sache her gegebene Notwendigkeit. Gerade bei auf Korpora beruhenden Analysen erweist sich die Trennung als wenig zielführend. Vielleicht ist nun, etwa zweihundert Jahre nach Grimm und einhundert Jahre nach de Saussure, eine Synthese der beiden Zugänge angezeigt.

\section{Literatur}

\section{Zitiertes Korpus}

Das Deutsche Referenzkorpus DeReKo, www.ids-mannheim.de/kl/projekte/korpora/ (letzter Zugriff: 17.11. 2017) am Institut für Deutsche Sprache, Mannheim.

\section{Zitierte Literatur}

Althochdeutsches Wörterbuch (1968-). Auf Grund der von Elias von Steinmeyer hinterlassenen Sammlungen im Auftrag der Sächsischen Akademie der Wissenschaften zu Leipzig. Begründet von Elisabeth Karg-Gasterstädt und Theodor Frings. Berlin: Akademie.

Augst, Gerhard (1975): Untersuchungen zum Morpheminventar der deutschen Gegenwartssprache (Forschungsberichte des Instituts für Deutsche Sprache Mannheim 25). Tübingen: Narr.

Basler, Otto (1935): Der Große Duden: Grammatik der deutschen Sprache. Eine Anleitung zum Verständnis des Aufbaus unserer Muttersprache. Leipzig: Bibliographisches Institut.

Braune, Wilhelm \& Ingo Reiffenstein (2004): Althochdeutsche Grammatik I: Laut- und Formenlehre. 15. Auflage, bearbeitet von Ingo Reiffenstein. Tübingen: Niemeyer.

Duden (2016): Duden. Die Grammatik. Unentbehrlich für richtiges Deutsch. Herausgegeben von der Dudenredaktion. 9., vollständig überarbeitete und aktualisierte Auflage (Duden 4). Berlin: Dudenverlag.

Dufter, Andreas, Jürg Fleischer \& Guido Seiler (2009): Introduction. In Andres Dufter, Jürg Fleischer \& Guido Seiler (Hrsg.), Describing and modeling variation in grammar (Trends in Linguistics Studies and Monographs 204), 1-18. Berlin/New York: De Gruyter.

Ebert, Robert Peter (1999): Historische Syntax des Deutschen II: 1300-1750. 2., überarbeitete Auflage (Germanistische Lehrbuchsammlung 6). Berlin: Weidler. [11986] 
Ehlers, Klaas-Hinrich (2005): Strukturalismus in der deutschen Sprachwissenschaft: die Rezeption der Prager Schuler zwischen 1926 und 1945 (Studia Linguistica Germanica 77). Berlin/New York: De Gruyter.

Eichinger, Ludwig M. (2008a): Hans Glinz 1.12. 1913-23.10. 2008. In Sprachreport 4/2008, 29. Mannheim: Institut für Deutsche Sprache.

Eichinger, Ludwig M. (2008b): Vom Glück, Regeln zu befolgen - Adelung im Stil des 18. Jahrhunderts. In Heidrun Kämper, Annette Klosa \& Oda Vietze (Hrsg.), Aufklärer, Sprachgelehrter, Didaktiker: Johann Christoph Adelung (1732-1806) (Studien zur Deutschen Sprache 45), 247-270. Tübingen: Narr.

Eichinger, Ludwig M. (2011): Normprobleme, oder: Variation ist sinnvoll: Überlegungen zum heutigen Deutsch (Akademie der Wissenschaften und der Literatur Mainz, Abhandlungen der Geistes- und Sozialwissenschaftlichen Klasse 6). Stuttgart: Steiner.

Eichinger, Ludwig M. (2014): Das Institut für Deutsche Sprache im fünfzigsten Jahr seines Bestehens. In: Institut für Deutsche Sprache (Hrsg.), Ansichten und Einsichten. 50 Jahre Institut für Deutsche Sprache. Redaktion: Melanie Steinle \& Franz Josef Berens, 19-46. Mannheim: Institut für Deutsche Sprache.

Elspaß, Stefan \& Christa Dürscheid (2017): Areale grammatische Variation in den Gebrauchsstandards des Deutschen. In Marek Konopka \& Angelika Wöllstein (Hrsg.), Grammatische Variation: empirische Zugänge und theoretische Modellierung (Institut für Deutsche Sprache Jahrbuch 2016), 85-104. Berlin/Boston: De Gruyter.

Fleischer, Jürg \& Oliver Schallert (2011): Historische Syntax des Deutschen: eine Einführung. Tübingen: Narr.

Fritze, Marie-Elisabeth (1970): Bezeichnungen für den Zugehörigkeits- und Herkunftsbereich beim substantivischen Attribut. In Hans-Gert Roloff (Hrsg.), Stilstudien zur Prosa des 14. Jahrhunderts. Die Melusine des Thüring von Ringoltingen, 417-476. Köln/Wien: Böhlau.

Glinz, Hans (1961): Die innere Form des Deutschen: eine neue deutsche Grammatik. Zweite, nachgeführte Auflage (Bibliotheca Germanica 4). Bern/München: Francke. [1952.]

Grebe, Paul (1959): Vorwort des Herausgebers. In Duden Grammatik der deutschen Gegenwartssprache. Herausgegeben von der Dudenredaktion unter Leitung von Dr. phil. habil. Paul Grebe unter Mitwirkung namhafter Fachgelehrter völlig neu bearbeitet (Der Große Duden 4), unpaginiertes Vorwort. Mannheim: Dudenverlag.

Koerner, Ernst F. K. (1999): Linguistic historiography: projects and prospects (Studies in the History of the Language Sciences 92). Amsterdam: Benjamins.

Konopka, Marek \& Angelika Wöllstein (2017): Einleitung. In Marek Konopka \& Angelika Wöllstein (Hrsg.), Grammatische Variation: empirische Zugänge und theoretische Modellierung (Institut für Deutsche Sprache Jahrbuch 2016), xi-xv. Berlin/Boston: De Gruyter.

Labov, William (1994): Principles of linguistic change. Volume 1: Internal factors (Language in Society 20). Oxford UK/Cambridge USA: Blackwell.

Lass, Roger (1990): How to do things with junk: Exaptation in language evolution. In Journal of Linguistics 26, 79-102.

Meineke, Eckhard (2013): ,Rb`. In Rolf Bergmann (Hrsg.), Althochdeutsche und altsächsische Literatur, 420-422. Berlin/New York: De Gruyter.

Moser, Hugo (1967): Ziele und Aufgaben des Instituts für deutsche Sprache Mannheim. In Hugo Moser, Hans Eggers, Johannes Erben \& Hans Neumann (Hrsg.), Satz und Wort im heutigen Deutsch: Probleme und Ergebnisse neuerer Forschung (Sprache 
der Gegenwart - Schriften des Institus für deutsche Sprache Mannheim 1), 9-14. Düsseldorf: Schwann.

Moser, Hugo, Hans Eggers, Johannes Erben \& Hans Neumann (1967): Geleitwort. In Hugo Moser, Hans Eggers, Johannes Erben \& Hans Neumann (Hrsg.), Satz und Wort im heutigen Deutsch: Probleme und Ergebnisse neuerer Forschung (Sprache der Gegenwart - Schriften des Instituts für deutsche Sprache Mannheim 1), 7-8. Düsseldorf: Schwann.

Nübling, Damaris (2000): Prinzipien der Irregularisierung: eine kontrastive Analyse von zehn Verben in zehn germanischen Sprachen (Linguistische Arbeiten 415). Tübingen: Niemeyer.

Paul, Hermann (1920): Prinzipien der Sprachgeschichte. 5. Auflage. Halle: Niemeyer. [11880.]

Polenz, Peter von (2013): Deutsche Sprachgeschichte vom Spätmittelalter bis zur Gegenwart. Band II: 17. und 18. Jahrhundert. 2. Auflage bearbeitet von Claudine Moulin unter Mitarbeit von Dominic Harion. Berlin/New York: De Gruyter. [11994].

Schenker, Walter (1971): es/os-Flexion und es/os-Stämme im Germanischen. In Beiträge zur Geschichte der deutschen Sprache und Literatur (Tübingen) 93, 46-58.

Schmidt, Jürgen Erich \& Joachim Herrgen (2011): Sprachdynamik: eine Einführung in die moderne Regionalsprachenforschung (Grundlagen der Germanistik 49). Berlin: Erich Schmidt.

Silverstein, Michael (1976): Hierarchy of features and ergativity. In R. M. W. Dixon (Hrsg.), Grammatical categories in Australian languages, 112-171. Canberra: Australian Institute of Aboriginal Studies.

Steinmeyer, Elias \& Eduard Sievers (1879): Die althochdeutschen Glossen. Erster Band: Glossen zu biblischen Schriften. Berlin: Weidmann.

Tiefenbach, Heinrich (2010): Altsächsisches Handwörterbuch/A concise Old Saxon dictionary. Berlin/New York: De Gruyter.

Trubetzkoy, N. S. (1939): Grundzüge der Phonologie (Travaux du Cercle Linguistique de Prague 7). Prague: Cercle Linguistique de Prague. 

V Grammatikographie 



\title{
Eva Breindl
}

\section{Grammatikographie: Deskriptive Grammatik}

\begin{abstract}
Der Beitrag versucht eine Bestandsaufnahme deskriptiver wissenschaftlicher Grammatiken des Deutschen, die den Anspruch einer Gesamtdarstellung erheben und einen Zeitrahmen von ca. 50 Jahren abdecken. Die Gegenstandsabgrenzung stützt sich auf einen weitreichenden Konsens in der Linguistik, welche Grammatiken in der universitären Lehre eingesetzt und metagrammatisch reflektiert werden. Es wird gezeigt, welche Variationsbreite diese Grammatiken in Bezug auf die Konzeption ihrer Gegenstände, Sprache, Grammatik und Standardsprache aufweisen und welche Folgen das hat für die grammatikographische Praxis, insbesondere die Art und Weise der Beispielgebung.
\end{abstract}

Keywords: Deskriptive Grammatik, Grammatikographie, Grammatiktheorie, Korpusgrammatik, Standardsprache

\section{Gegenstandsabgrenzung}

Beim Bemühen, den Gegenstand einer Bestandsaufnahme zur Deskriptiven Grammatik des Deutschen intensional und extensional abzugrenzen, stößt man in mehrfacher Hinsicht auf Schwierigkeiten.

Unter „Grammatik“, im Sinne eines disziplinären Fachbegriffs der Linguistik, wird bekanntlich recht Verschiedenes verstanden. Die Begriffsbestimmungen (s. z. B. Glück 2000; Helbig 1992, 1993, 2001; Hennig 2001; Linke, Nussbaumer \& Portmann 1994: 52 ff.; Thurmair 2010) lassen sich zwei Hauptverwendungsweisen zuordnen, die ihrerseits wiederum je zwei Unterverwendungen umfassen. Sie beziehen sich zum einen auf den sprachlichen Gegenstand selbst, wobei zwischen dem der Sprache innewohnenden Regelsystem, dem Baumuster der Sprache, und dessen kognitiver Repräsentation im menschlichen Geist unterschieden werden kann. Gegenbegriff zu dieser Konzeption ist das Lexikon. Zum anderen hebt man damit auf die forschende und lehrende, auf Erkenntnis-

Eva Breindl, Friedrich-Alexander-Universität Erlangen-Nürnberg, Bismarckstr. 1, D-91054 Erlangen, E-Mail: eva.breindl@fau.de

Ә Open Access. (c) 2018 Eva Breindl, publiziert von De Gruyter. (c) BY Dieses Werk ist lizenziert unter der Creative Commons Attribution 4.0 Lizenz. 
gewinn gerichtete Auseinandersetzung mit ebendiesem Gegenstand, seine Modellierung im Rahmen einer wissenschaftlichen Theorie und auf die Resultate dieser Bemühungen ab - Grammatik als wissenschaftliche Disziplin, als akademisches oder schulisches Lehrfach und als Kodex.

So einleuchtend diese Differenzierungen auf den ersten Blick erscheinen mögen, so wenig lassen sie sich für einen analytischen Zugang zur Grammatikographie einer Sprache fruchtbar machen. Die grammatikographischen Endprodukte repräsentieren ja nicht einen physikalischen Gegenstand Sprache an sich, sondern immer eine bestimmte Gegenstandskonzeption von Seiten des Grammatikers und der Grammatikerin - das umgreift das Verständnis vom Wesen der Sprache, ein Konzept von Grammatik und dem, was zum Regelsystem Grammatik gehört, und - insofern hier die Grammatik einer Einzelsprache zur Debatte steht - auch das Konstrukt einer deutschen Standardsprache. In Grammatiken sind deshalb immer der Gegenstand und der analytisch-systematische Zugriff darauf gleichzeitig präsent.

Mit dem Attribut deskriptiv wird in Grammatiktypologien eine bestimmte Art von Grammatiken dem Gegenmodell einer präskriptiven (oder normativen) Grammatik gegenübergestellt. Solche schematisierenden polaren Charakterisierungen sind typisch für die metagrammatische Literatur. Neben dem Gegensatz deskriptiv vs. präskriptiv finden sich wissenschaftliche Grammatik (oder linguistische Grammatik) vs. Gebrauchsgrammatik; Problemgrammatik vs. Resultatsgrammatik; didaktische (oder pädagogische) Grammatik vs. wissenschaftliche Grammatik; einzelsprachbezogene vs. kontrastive oder sprachvergleichende Grammatik; synchrone vs. diachrone Grammatik. Diese Dichotomien sind bestenfalls als Groborientierungen $\mathrm{zu}$ verstehen. So entgeht keine sich als deskriptiv präsentierende Grammatik - und welche Grammatik mit wissenschaftlichem Anspruch täte das nicht - dem Normativitätsdilemma, dass deskriptive Ist-Aussagen von den Benutzern als Soll-Aussagen, als Handlungsanweisungen bei gleichzeitigem Verbot der Nicht-Befolgung dieser Aussage gelesen werden können: Aus der Regelbeschreibung „Das Verb steht im Aussagesatz an zweiter Stelle“ darf der Leser aufgrund des Relevanzprinzips schließen, dass es eben nicht an erster oder dritter Stelle steht usw.

Auch von einer Gebrauchsgrammatik oder einer didaktischen Grammatik darf man als Nutzer ein linguistisches, im besten Fall zusätzlich ein spracherwerbstheoretisches oder didaktisches, jedenfalls ein wissenschaftliches Fundament erwarten. Umgekehrt möchte jede wissenschaftliche Grammatik, die für sich beansprucht, auf einer theoretischen Basis das grammatisch Mögliche vom grammatisch Unmöglichen zu scheiden, auch „gebrauchbar“ sein, wenn der Nutzer eine entsprechende Auskunft benötigt. Ob sich eine Grammatik mehr als wissenschaftliche Grammatik oder mehr als Gebrauchsgrammatik 
präsentiert, ob also Regeln nur präsentiert oder auch hergeleitet, funktional erklärt und problematisiert werden, ob die Darstellung in einen sprachtheoretischen Zusammenhang und in den Forschungsdiskurs eingebettet wird, in welcher Breite, Tiefe und Kondensation das Regelwerk dargestellt wird, das hängt in erster Linie von den anvisierten Zielgruppen und Nutzungssituationen ab. Die Zuschreibungen und Selbstauskünfte, letztere oft marktstrategischen Erwägungen geschuldet, müssen nicht übereinstimmen mit der grammatikographischen Praxis des Werks: Die Duden-Grammatik etwa offeriert sich als Gebrauchsgrammatik ${ }^{1}$ und wird von Laien als normative Instanz wahrgenommen, macht ihr wissenschaftliches Fundament aber mit Verweisen auf die Forschungsliteratur, Verwendung systematischer Fachterminologie und gelegentlicher Erörterung alternativer Konzepte (z. B. zur Systematik der Tempora, Duden 2016: 509) deutlich. Umgekehrt bezeichnet Eisenberg seinen „Grundriss“, der wohl in erster Linie im akademischen Kontext rezipiert wird, als „Gebrauchsbuch“2 . „Die Deutsche Grammatik“ von Hoffmann (2013) weist mit ihrem Konzept der „didaktischen Pfade“ wesentliche Merkmale didaktischer Grammatiken auf, ohne dass man ihr die wissenschaftliche Fundierung und Orientierung absprechen würde.

Am eindeutigsten scheint noch die Klassifikation als einzelsprachbezogene gegenüber einer sprachvergleichenden Grammatik, doch gibt es sehr wohl Abstufungen im Ausmaß, in dem auf andere Sprachen eingegangen wird. Neben vergleichenden Grammatiken, die zwei oder mehr Sprachsysteme in gleicher Breite und Tiefe zu erfassen und aufeinander $\mathrm{zu}$ beziehen trachten (wie etwa die am IDS entstandenen kontrastiven Grammatiken), ${ }^{3}$ die vergleichende Grammatik der Schulsprachen (Glinz 1994) oder die vierbändige vergleichende Grammatik der Sprachen Südtirols (Gallmann, Siller-Runggaldier \& Sitta 20082013) liegt mit der „Grammatik des Deutschen im europäischen Vergleich“/GDE (Gunkel et al. 2017) neuerdings eine Grammatik vor, die die Einzelsprache Deutsch systematisch und (bezogen auf den Gegenstand Nominale) vollständig auf der Folie mehrerer Vergleichssprachen zu profilieren sucht, ohne dass für letztere der gleiche Abdeckungsgrad angestrebt wird. Hoffmanns „Deutsche Grammatik“ kontrastiert punktuell und phänomenbezogen das Deutsche mit dem Türkischen, das als Kontrastsprache aufgrund der Adressierung dieser

1 Der Charakter als Gebrauchsgrammatik für eine kaum eingegrenzte Leserschaft wird schon im Untertitel „Die Grammatik. Unentbehrlich für richtiges Deutsch“ offensiv beansprucht.

2 „Eine Grammatik als Gebrauchsbuch soll Auskunft darüber geben, was richtig und was falsch ist.“ (Eisenberg 1999: 1).

3 Z. B. dt.-rumän. (Engel et al. 1993), dt.-poln. (Engel et al. 1999), dt.-span. (Cartagena \& Gauger 1989), dt.-frz. (Zemb 1978/1984). 
Grammatik an Lehrer bzw. Lehramtsstudierende des Deutschen als Mutter- und Zweitsprache ausgewählt wurde. Daraus ergibt sich aber keine konsistente Grammatik des Türkischen. Das „Handbuch der deutschen Grammatik“ schließlich (Hentschel \& Weydt 2003) streut - eher unsystematisch - Hinweise auf verschiedenste andere Sprachen ein mit dem Ziel, typologische Optionen des Deutschen zu profilieren. ${ }^{4}$

Abstufungen lassen sich auch in der Berücksichtigung der Diachronie in den aktuellen Grammatiken des Gegenwartsdeutschen finden, auch wenn diese sich - anders als im 19. Jh. - immer als Querschnittsaufgaben verstehen, als Präsentationen eines Status Quo. Gerade in ihren Erklärungskomponenten machen Grammatiken mit wissenschaftlichem Anspruch aber auch gern Gebrauch von sprachhistorischen Daten (ausgiebig etwa Hentschel \& Weydt 2003).

Der vorliegende Beitrag konzentriert sich auf Grammatiken, denen im linguistischen Fachdiskurs das Prädikat „wissenschaftlich“ zugeschrieben wird. Eine intensionale Abgrenzung eines Gegenstands „wissenschaftliche Grammatiken“ von anderen Typen von Grammatiken erweist sich, wie gezeigt werden wird, als nicht wirklich trennscharf. ${ }^{5}$ Dennoch scheint es im Kontext der germanistischen Sprachwissenschaft extensional einen weitreichenden Konsens darüber zu geben, welche Grammatiken man in der universitären Lehre einsetzt und/oder einer metagrammatischen Analyse für würdig befindet. So liefern etwa aktuelle Literaturlisten einschlägiger germanistischer Lehrveranstaltungen, Zusammenstellungen von Grundlagenliteratur oder die Rezensionsteile von Zeitschriften aus den letzten 3 Jahrzehnten eine Art kleinsten gemeinsamen Nenner. Darunter fallen mindestens (hier in chronologischer Ordnung) „Duden: Die Grammatik“ (in den verschiedenen Auflagen seit 1959), die DaF-ausgerichtete Grammatik von Helbig \& Buscha (Erstauflage 1972), die Akademie-Grammatik (1981), Engels „Deutsche Grammatik“ (1988, Neubearbeitung 2004), das „Handbuch der deutschen Grammatik“ von Hentschel \& Weydt (Erstauflage 1989), Eisenbergs „Grundriss“ in der einbändigen (1986) und den zweibändigen Versionen seit 1999, die Weinrichsche „Textgrammatik“ (1993), die IDS-Grammatik (= Zifonun, Hoffmann und Strecker 1997) und die

\footnotetext{
4 Beispielsweise wird im Tempuskapitel auf die dem Deutschen vergleichbare Verwendbarkeit des deutschen Präsens für vergangene und zukünftige Ereignisse im Französischen, Englischen, Türkischen und Serbischen hingewiesen (Hentschel \& Weydt 2003: 97), bei der Beschreibung des Artikels wird die typologische Variante eines gebundenen Morphems im Türkischen, Mazedonischen und Dänischen mit Beispielen angeführt (Hentschel \& Weydt 2003: 396).

5 Allenfalls didaktische Grammatiken mit einem Übungsteil lassen sich als sog. „Übungsgrammatiken“ (i. d.R. mit einer DaF-Orientierung) gut abgrenzen. S. dazu den Beitrag von Thurmair in diesem Band.
} 
„Deutsche Grammatik“ von Hoffmann (2013). ${ }^{6}$ Was diese Grammatiken jenseits ihres universitären Rezeptionszusammenhangs (und auch: universitären/ akademischen Entstehungskontexts) eint, ist ihr Anspruch, das Sprachsystem vollständig zu erfassen und ein wissenschaftlich abgesichertes Urteil darüber abzugeben, was im Deutschen grammatisch möglich und was nicht möglich ist.

Es sind vor allem (weder ausschließlich noch gleich gewichtet) die hier genannten Grammatiken, auf die sich diese Bestandsaufnahme stützt. Sie können in diesem Kontext nicht in extenso kritisch rezensiert, sondern nur exemplarisch zur Verdeutlichung der Bandbreite konzeptioneller Entwürfe und rezenter grammatikographischer Entwicklungstendenzen herangezogen werden. An ihnen soll die Variationsbreite der Grammatikographie des Deutschen seit ca. 1980 gezeigt werden: in Bezug auf die Konzeption der Gegenstände Sprache (Abschnitt 2.1), Grammatik (2.2) und das Konstrukt Standarddeutsch (2.3); Abschnitt 3 geht auf grammatikographische Praktiken ein: Von Interesse sind hier Form, Funktion und Legitimation der Beispielangaben (3.1) und der damit zusammenhängenden Grammatikalitätsurteile und die Art der Regelformulierung und -begründung (3.2). Abschnitt 4 versucht eine zusammenfassende Auswertung der Charakteristika, Probleme, Defizite und Entwicklungstendenzen gegenwärtiger Grammatikographie.

\section{Gegenstandskonzeption}

\subsection{Die Konzeption von Sprache}

Auf die kaum überschaubare Vielfalt der Vorstellungen vom Wesen der Sprache und der Art, wie die menschliche Spezies über Sprache verfügt und mit ihr kommuniziert, die in einer mehrtausendjährigen Geschichte von Philosophen, Psychologen und Sprachwissenschaftlern hervorgebracht wurden, kann hier nicht eingegangen werden. Relevant sind solche Vorstellungen hier nur insofern, als sie sich sprachreflexiven Einlassungen von Grammatikern (allenfalls in einem Vorwort) und der grammatischen Praxis entnehmen lassen, die sich nicht zuletzt im Stellenwert und der Auswahl sprachlicher Beispiele manifestiert.

6 Einige Grammatiken mit Erscheinungsdatum zwischen 1950 und 1980 werden bisweilen in sprachwissenschaftshistorischem Kontext genannt (so z. B. „Die innere Form des Deutschen“ von Hans Glinz und die darauf aufbauende mehrbändige „Deutsche Grammatik“ (1970-1975) oder Brinkmanns inhaltbezogene Grammatik (Brinkmann 1962); sie werden aber aktuell nicht mehr als Basisliteratur für die Vermittlung von Einsicht in das grammatische System des Deutschen genutzt. In zunehmendem Maße gilt das wohl auch für die Akademie-Grammatik. 
Eine grundlegende sprachtheoretische Maxime der Sprachwissenschaft des 20. Jhs. ist (spätestens seit de Saussure, aber auch bei Humboldt schon angelegt) die Dichotomie Sprachsystem als Regelwerk (zu diesem Pol auch die Termini langue, Kompetenz, Schema, internal language, Sprachkenntnis) vs. Sprachgebrauch als soziales Phänomen (wahlweise parole, Performanz, external language, Sprachverhalten). Generativistische Ansätze teilen die Überzeugung einer Zweiteilung, erachten aber allein den Pol Sprachsystem/Kompetenz als Untersuchungsgegenstand, konstruktionsgrammatische Ansätze lehnen die Unterscheidung generell ab. In einer Ringvorlesung an der FU Berlin wurde im Jahr 2000 diese Unterscheidung unter der Fragestellung „Gibt es eine Sprache hinter dem Sprechen?“ zur Diskussion gestellt. Die meisten Beiträger haben dies spontan bejaht (s. Krämer \& König (Hrsg.) 2002), wenn auch die Vorstellungen davon, wie diese „Sprache hinter dem Sprechen“ beschaffen ist, sehr verschieden sind. Im Konzept der generativen Grammatik ist sie als Regelwerk in Form einer angeborenen „Universalgrammatik“ ein kognitives Phänomen und Teil der genetischen Ausstattung. Bei einer derartigen Konzeption sind Korpusbelege als Sprachdaten irrelevant, da das Ziel ja gerade die Abbildung des grammatischen Regelapparats im Kopf ist. Auch auf die Gegenstandsabgrenzung hat dies Auswirkungen: Deskriptive Vollständigkeit, Erfassung von Gebrauchsaspekten oder lexembasierte Idiosynkrasien sind im Rahmen einer generativen Theorie keine anzustrebenden Ziele. Es ist daher wenig verwunderlich, wenn generative Ansätze sich kaum je in einer deskriptiven „großen“, auf Vollständigkeit abzielenden Grammatik einer Einzelsprache niedergeschlagen haben. ${ }^{7}$ Für das Deutsche stellt die Akademie-Grammatik (1981) einen Versuch dar, über das Prinzip der „Abwandlungen“ die transformationsgrammatischen Ansätze aus der Arbeitsstelle strukturelle Grammatik mit einem aussagenlogisch basierten Beschreibungssystem und marxistischen Positionen $\mathrm{zu}$ integrieren. So ist zwar das Sprachsystem durchaus konventionell als Zuordnung von Inhalt und Lautform aufgefasst, in der Betonung von „Bewußtseinsinhalten" und sprachlichen Fähigkeiten zeigen sich aber die Spuren der generativen Theorie. Von der Vorstellung, dass „der Gegenstand der linguistischen Theorie (hier: der Grammatik) die „immanente Sprachkompetenz“ eines idealisierten Sprechers/Hörers sei“ (Heidolph et al. 1981: 31), distanziert sich die Akademie-Grammatik allerdings wieder. Auch wird, ganz im Sinne de Saus-

$7 \mathrm{Zu}$ den wenigen Ausnahmen gehören die „Grande grammatica italiana di consultazione“ von Renzi et al. (1988). Ein generativer Ansatz kennzeichnet auch die „Syntax of Dutch“ (Broekhuis \& Corver 2017) und die „Deutsche Syntax“ von Sternefeld (2006). Durch die für theoriehomogene Darstellungen typische Konzentration auf die Syntax können sie aber nicht als Gesamtdarstellungen einer Einzelsprache gewertet werden. 
sures und entgegen der generativen Konzeption von Sprache, die Wechselwirkung zwischen Äußerungen und Sprachsystem betont: Äußerungen sind durch das Sprachsystem determiniert, umgekehrt „gibt es im genetischen Sinne eine Determination des Sprachsystems durch die Äußerungen, da die Individuen sich das System aus einzelnen Äußerungen einzelner Partner in einzelnen Situationen aneignen“ (Heidolph et al. 1981; 29). Schließlich ist auch das hybride Anliegen, „einen Überblick über den Aufbau des Systems der gegenwärtigen deutschen Literatursprache mit der Darstellung der internen Zusammenhänge dieses Systems zu verbinden“ (Heidolph et al. 1981: 5), also das Systematische hinter einer bestimmten Varietät des Sprachgebrauchs herauszuarbeiten, nicht im Einklang mit den Erkenntnisinteressen generativer Ansätze. ${ }^{8}$ Allerdings verfährt die Akademie-Grammatik in diesem Punkt wieder inkonsequent, insofern die Datenbasis größtenteils eben nicht literarische Belege sind - für die Grammatiken des 19. Jhs. noch gängige Praxis - sondern allenfalls davon inspirierte, kompetenzbasierte Beispiele, die im Rahmen der grammatischen Argumentation abgewandelt werden bis hin zur Erzeugung ungrammatischer Beispiele.

Auch in der „Deutschen Grammatik“ von Helbig \& Buscha (1998) wird der Zusammenhang von Kompetenz und Performanz thematisiert. Dem NichtMuttersprachler, an den diese Grammatik sich richtet, fehle das „Sprachgefühl, die ,Kompetenz““, die er sich erst „über explizite Regeln, die möglichst genau angeben, wie richtige deutsche Sätze gebildet, interpretiert und verwendet werden“ (Helbig \& Buscha 1998: 17), aneignen müsse. Die anvisierte Zielgruppe erzwingt hier also eine Orientierung am Sprachgebrauch, was aber - wie im Falle der Akademie-Grammatik - nicht durch authentische Beispiele unterstützt wird. Im Vorwort zählen die Autoren die der Zielgruppe geschuldeten Zugeständnisse dieser Grammatik auf - und machen damit indirekt ihr Verständnis einer deskriptiven Grammatik deutlich. Sie bezeichnen ihre Grammatik als „Resultatsgrammatik“, die keine Entscheidungswege darlegen und nur auf die „Norm der Standardsprache“ eingehen kann, weisen auf „ausführliche Listen“ bei Funktionswortklassen hin und bekennen sich zur Notwendigkeit einer Heterogenität der „linguistischen Verfahren und Methoden“ (Helbig \& Buscha 1998: 18).

8 Die kritische Rezeption (s. etwa die beiden OBST-Bände 27 (1984) und 30 (1985)) hat dieser Grammatik neben ihrer Inhomogenität auch das Fehlen einer pragmatischen Komponente vorgeworfen, vor allem aber, dass ihr theoretischer Ansatz zum Erscheinungstermin bereits veraltet war. Die rasche Weiterentwicklung der generativen Theorie in immer neue Versionen kann sicher als weiterer Grund für den Mangel an einzelsprachlichen generativen Grammatiken ins Feld geführt werden, s. hierzu auch Haider in diesem Band. 
Unter den wenigen Aussagen von Grammatikern zum Verhältnis von Sprache und Kognition findet sich Engel (2004). Er postuliert für seine Grammatik nicht, sie sei „identisch mit dem Regelwerk, das im Gehirn eines Deutschsprechers funktioniert. Wir wissen nicht sehr viel darüber, was beim Sprechen in unseren Köpfen abläuft. Was wir letzten Endes haben, sind Texte“ (Engel 2004: 10). Das Prinzip, aus dem Sprachgebrauch auf das Sprachsystem zu schließen, hat aber auch in dieser Grammatik keine Konsequenzen für die Datenbasis: Beispiele sind nicht als Belege ausgewiesen und selbst das Textsortenkapitel enthält keine authentischen Texte.

Von den hier berücksichtigten Grammatiken positionieren sich ohnehin nur wenige überhaupt explizit in Bezug auf aktuelle sprachtheoretische Auseinandersetzungen. Eisenberg etwa lehnt im Vorwort des zweiten Bands die Vorstellung von Sprache als Menge von Sätzen und Grammatik als diese Sätze erzeugenden Mechanismus klar ab und bekennt sich zur Orientierung am Sprachgebrauch, die Grammatik müsse „Sprache so beschreiben, wie sie heute gebraucht wird“, und „Auskunft darüber geben, was richtig und was falsch ist“, der „Grundriss“ sei ein „Gebrauchsbuch“ (Eisenberg 1999: 3). Auf die kontrovers diskutierten Fragen, ob das Sprachvermögen angeboren sei, wie grammatisches Wissen kognitiv verankert ist, ob es universalgrammatische Strukturen und Prinzipien gibt, wie das Verhältnis von Sprachsystem und Sprachgebrauch $\mathrm{zu}$ explizieren ist, lassen sich die meisten Grammatiker gar nicht ein. Im „Handbuch der deutschen Grammatik“ von Hentschel \& Weydt (2003) findet sich in einem Einleitungskapitel mit dem vielversprechenden Titel „Was ist Grammatik“ lediglich eine Aufzählung von Teilgebieten, für die sich die Verf. auf eine „Grammatik der primären Formen des Deutschen im traditionellen Sinn“ (Hentschel \& Weydt 2003: 10) berufen. Eine Antwort auf die Frage, welche Eigenschaften eine solche Grammatik auszeichnen, bleiben sie aber schuldig. Und auch die Duden-Grammatik nimmt in keiner der Auflagen seit $1995 \mathrm{zu}$ sprach- und grammatiktheoretischen Fragen Stellung, ${ }^{9}$ sondern adressiert klar einen Laien-Nutzer, der sich für solche Fragen nicht interessiert.

Konstruktionsgrammatische Ansätze verstehen Sprache weder als ein abstraktes System der Kombinatorik von kleineren zu größeren Einheiten noch als angeborenes, gar universales System von Prinzipien und Parametern. Vielmehr gehen sie davon aus, dass Grammatik als „Nebenprodukt des Sprechens“ (Haspelmath 2002) in der Interaktion emergiert, der Sprachgebrauch also das Regelsystem determiniert, das sich wiederum als Summe von Konstruktionen

9 In den älteren Auflagen der Duden-Grammatik ( ${ }^{2} 1966$; ${ }^{3} 1973$; Hrsg. Grebe; ${ }^{4} 1984$; Hrsg. Drosdowski) finden sich ausführlichere Stellungnahmen. 
unterschiedlichen Abstraktionsgrads darstellen lässt, wobei die lexikalische Füllung der Konstruktionen zu berücksichtigen ist. Der Konstruktionsbegriff wird in neueren Arbeiten eher weit gefasst: darunter fallen neben regelhaft konstruierten Einheiten wie der Subjekt-Prädikat-Konstruktion oder der Ditransitiv-Konstruktion auch idiomatische und syntaktisch nicht - oder nur eingeschränkt - produktive Einheiten wie geschweige denn (s. dazu Eggs 2011), anders/kurz/genauer gesagt/ausgedrückt/formuliert (s. dazu Breindl \& Schreiber i. Dr.) oder verblose Direktive vom Typ her/weg/raus mit X (s. dazu Jacobs 2009) und schließlich auch Lexeme. Ziel einer konstruktionsgrammatisch angelegten Grammatik ist die Erfassung dieser Konstruktionen in einer Art „Konstruktikon“, in dem die traditionelle Grenzziehung zwischen Lexikon und Grammatik aufgehoben ist. Jacobs (2008) hat auf eine Beschreibungsschwäche der konstruktionsgrammatischen Konzeption hingewiesen: auf der Basis von Konstruktionen lässt sich keine Aussage darüber treffen, was in einer Sprache nicht möglich ist, somit ist keine analytische Scheidung von grammatischen und ungrammatischen Strukturen möglich. Was die grammatikographische Praxis betrifft, gilt für konstruktionsgrammatische Ansätze cum grano salis dasselbe wie für die generativen Ansätze: Es gibt keine theoriehomogene konstruktionsgrammatische Gesamtdarstellung einer Einzelsprache, wohl aber ein wachsendes Aufkommen an konstruktionsgrammatischen Beschreibungen einzelner grammatischer Strukturen und Formen. Die Übersicht in Ziem \& Lasch (2013) zeigt, dass es für das Deutsche gerade die mit projektionistischen Grammatikmodellen schwer zu beschreibenden, (halb-)idiomatischen und teilschematischen Strukturen sind, die bevorzugte Gegenstände konstruktionsgrammatischer Beschreibung wurden. Jacobs Plädoyer für eine theorieintegrierende Grammatik, die projektionistische und konstruktionistische Angänge gegenstandsabhängig nutzt, könnte sich so durchaus als wegweisend für künftige Grammatikographie erweisen.

Die Rolle lexikalischer Einheiten im System ist in einer Konstruktionsgrammatik von ganz anderer Art, als sie sich in deskriptiven Grammatiken präsentiert, wo sie mitunter in Form von Listen bei geschlossenen Wortklassen (Präpositionen, Konjunktionen, Partikeln) oder in Form der „Satzbaupläne“ auch bei Verben ein eigentlich systemfremdes Einsprengsel bilden. Mit solchen Listen versuchen insbesondere Grammatiken mit einer starken Gebrauchsorientierung die grundsätzliche Lexemblindheit grammatischer Regeln (,primäre Präpositionen regieren den Akkusativ oder Dativ“ bzw. noch stärker: „Präpositionen regieren im Defaultfall den Dativ“); „Partikeln können nicht im Vorfeld stehen“ usw.) im Interesse der Nutzer aufzufangen. (S. dazu den nächsten Abschnitt.)

Eine andere Ausprägung einer „Sprache hinter dem Sprechen“ ist das Konzept von Sprache als extrakognitives soziales Phänomen, als Instrument 
der Kommunikation, das sozialen Regeln folgt, Variation und Veränderungen unterworfen ist. Zifonun (2009) weist darauf hin, dass diese Vorstellung auch bei de Saussure schon angelegt ist. Gegenstand der Sprachbetrachtung sind dann die konkreten Kommunikationsakte der Sprecher und das dahinter wirkende und die Kommunikation erst ermöglichende abstrakte Regelsystem der Einzelsprache. Für die meisten neueren Grammatiken ist dieser Ansatz leitend, auch wenn sie in Bezug auf die Gegenstandsabgrenzung und Datengrundlage unterschiedlich verfahren. Am konsequentesten wird Grammatik als Summe der Formen und Mittel sprachlichen Handelns in der IDS-Grammatik und in der Grammatik von Hoffmann (2013) dargestellt. Beide machen im Unterschied zu den meisten anderen Grammatiken ausgiebigen Gebrauch von Belegen einschließlich gesprochensprachlicher Beispiele. Hoffmanns „Deutsche Grammatik“ basiert auf dem Prinzip, Formen „nicht isoliert, sondern immer in der kommunikativen Funktion, die sie in einer Wortgruppe haben“, zu behandeln. Die IDS-Grammatik betrachtet „kommunikative Aufgaben und Zwecke“ als letztlich verantwortlich für grammatische Strukturen (ebd. S. 3). Dieser Ansatz wird konsequent umgesetzt in Form einer durchgehenden „Doppelperspektivik“, bei der das Sprachsystem einmal auf traditionelle Weise ausgehend von den konkreten Formen und Mitteln und dem Aufbau größerer sprachlicher Einheiten dargestellt wird, und einmal ausgehend von elementaren Funktionen, für die spezifische sprachliche Mittel ausgebildet sind (wie z. B. die Funktion Gegenstände zu entwerfen, thematisch fortzuführen oder Aspekte von Sachverhalten zu perspektivieren bis hin zu unmittelbar gesprächsbezogenen Funktionen wie Thematisierung oder Vergabe des Rederechts).

Auch in Eisenbergs „Grundriss“ spielt der Funktionsbegriff eine wichtige Rolle, ist aber etwas anders gelagert als in der IDS-Grammatik. Er versteht darunter in erster Linie die systeminterne Funktionalität von strukturellen Eigenschaften sprachlicher Einheiten, hinter der indirekt kommunikative Bedürfnisse wie Ökonomisierung eine Rolle spielen.

Unter den deskriptiven Grammatiken des Deutschen gibt es nur zwei, die auch im Aufbau von Funktionen ausgehen, denen Formen zugeordnet werden, die „Grammatik in Feldern“ (Buscha et al. 1998) und die „Kommunikative Grammatik“ von Engel \& Tertel (1993). Beide haben als Zielgruppe Lerner des Deutschen als Fremdsprache - und beide erkaufen die Abkehr von der traditionellen Gliederung mit erheblicher Lückenhaftigkeit und mangelnder Systematizität bei den grammatischen Formen und Strukturen.

Dass funktionale Angänge in der Grammatikographie des Deutschen - trotz der „pragmatischen Wende“ und der starken Kommunikationsorientierung in den 80er Jahren - im Vergleich zu formorientierten selbst unter den fremdsprachendidaktischen Grammatiken eher ein Schattendasein fristen, hat wohl 
auch damit zu tun, dass es für ein Inventar an Funktionskategorien keine etablierte Tradition und keine Terminologie gibt. Die IDS-Grammatik, die ein solches Inventar entwickelt hat, könnte hier Vorbildfunktion haben. In den nach 1997 erschienenen deutschen Grammatiken ist aber mit Ausnahme von Hoffmann (2013), der ja bereits an der IDS-Grammatik maßgeblich mitgewirkt hat, kein Niederschlag des funktionalen Ansatzes der IDS-Grammatik zu erkennen.

\subsection{Die Konzeption von Grammatik}

Eine Grammatik hinsichtlich des Kriteriums der Vollständigkeit zu charakterisieren, würde voraussetzen, dass es einen Konsens darüber gibt, was in eine Grammatik gehört. Eine Grammatik Chomsky'schen Zuschnitts sieht den Satz als Bezugsgröße, Grammatiken sind in diesem Sinne Mechanismen der Erzeugung von Sätzen. Für die deskriptiven Grammatiken des Deutschen existiert mit Wort, Wortgruppe und Satz zwar ein Kanon, an dem keine Grammatik vorbeikommt, darüber hinaus unterscheiden sie sich aber erheblich: Gegenstandsausweitungen finden sich „nach unten“ (Laut, Buchstabe, Silbe), „nach oben“ (Text und Diskurs) in die Lexik und die Pragmatik - und schließlich kann all das mit sehr unterschiedlicher Granularität behandelt werden. Der Inhaltsumfang von Grammatiken zeigt immer auch bestimmte zeit- und kulturspezifische Präferenzen. War die antike Grammatik, bedingt durch die typologische Zugehörigkeit ihres Gegenstands zum flektierend-fusionierenden Typ, vor allem eine Wort- und Formenlehre, gehört seit dem Mittelalter auch die Satzlehre zum Kern von Grammatiken (s. Ganslmayer 2010). Eine funktionale, bedeutungs- oder gebrauchsorientierte Betrachtungsweise ist der antiken Grammatik fremd.

Für die Grammatiken ab der zweiten Hälfte des 20. Jhs. lässt sich als kanonischer Bestandteil die Lehre von Wortarten und Wortformen, Satzgliedern, einfachem und komplexem Satz ausmachen. In welchem Umfang und wodurch dieser Kern ausgeweitet wird, hängt auch von der Forschungslage sowie den individuellen Interessen der Grammatiker ab. Die in großen Teams erarbeiteten Grammatiken (Akademie-Grammatik, IDS-Grammatik, „Duden“) sind deshalb in aller Regel nicht nur umfangreicher, sondern zeigen auch größere Diversifizierung im Gegenstand. So finden sich in der Duden-Grammatik und der IDS-Grammatik Kapitel zu Phonetik und Phonologie, zur Intonation und zur Graphematik, ${ }^{10}$ in der Akademie-Grammatik nimmt Phebys Kapitel

10 In der IDS-Grammatik findet sich die Beschreibung von Lauten, Silben und Intonation im Kapitel „Diskurs und Mündlichkeit“, die Graphematik unter „Schrift als Mittel der Textkonstitution“. 
zur Phonologie und Intonation fast ein Fünftel des Textumfangs ein. Wortbildung findet sich in „Duden“, Hentschel \& Weydt (2003), Weinrich (1993) und in Eisenbergs „Grundriss“, nicht aber in der Akademie-Grammatik, in der IDS-Grammatik, in Helbig \& Buscha und in Hoffmann (2013). Wortstellung wird nur in Eisenbergs „Grundriss“ nicht berücksichtigt.

Etwa ab Ende der 90er Jahre lässt sich als Trend eine Erweiterung von Grammatiken um den Bereich Text beobachten, nachdem sich die Textlinguistik als Forschungszweig und Bestandteil linguistischer Lehrveranstaltungen in Deutschland konsolidiert hat. Noch während der Arbeit an Harald Weinrichs Textgrammatik, die zwar traditionell nach Wortarten gegliedert ist, aber grammatische Strukturen durchgehend vom Text her $\mathrm{zu}$ denken verspricht, erscheint 1988 die „Deutsche Grammatik“ von Ulrich Engel mit einem deszendenten Aufbau Text - Satz - Wort. Die IDS-Grammatik widmet der „Grammatik von Text und Diskurs“ ein mehrere hundert Seiten starkes Kapitel, die Duden-Grammatik zieht mit der Auflage von 2005 mit einem separaten Textkapitel nach, das Bestandteil der nachfolgenden Auflagen bleibt. Auch die Grammatiken von Wellmann (2008) und Hoffmann (2013) widmen dem Text eigene Kapitel, die rezeptive Grammatik von Heringer (1987) und (1988) schreiten in Abbildung des Rezeptionsprozesses konsequent vom Text aus zu den kleineren Einheiten vor. Auf die Heterogenität dieser Textkapitel hat Hennig (2001) hingewiesen. Mitunter sind sie ein Sammelsurium von Phänomenen, die nicht recht in die Systematik der Wortarten, Satzglieder und Satzarten passen, sie können quer zum systematischen Angang wortartenübergreifend Kohäsionsund Kohärenzphänomene (Konnexion, referentielle Verkettung, Informationsstruktur) zusammenführen wie die Duden-Grammatik, oder wie die IDSGrammatik hier genau solche Phänomene subsumieren, die den spezifischen Bedingungen der Schriftlichkeit unterliegen, von der Graphematik und der Interpunktion über textstrukturelle Phänomene bis zu Formen der Themenentfaltung. Kein separates Textkapitel weisen die Akademie-Grammatik (Heidolph, Flämig \& Motsch 1981), Helbig \& Buscha (1998) und Hentschel \& Weydt (2003) auf und Eisenbergs „Grundriss“ verweigert sich auch in der neuesten Auflage dem Trend.

Neben dem Text finden zunehmend auch die gesprochene Sprache und der Diskurs Eingang in Grammatiken. Dabei geht es nicht um die Beschreibung von Laut, Silbe und Suprasegmentalia als Grundeinheiten des Sprechens, die sich in vielen Grammatiken findet, aber nur in der IDS-Grammatik konsequent unter „Grammatik von Text und Diskurs“ subsumiert wird. Das Charakteristische der Diskurskapitel ist vielmehr, dass sie zum einen grammatische Phänomene erfassen, die sich vorzugsweise oder mit auffällig hoher Frequenz in der gesprochenen Sprache finden (Ellipsen, Anakoluthe, Interjektionen), dass sie aber darüber hinaus auch weit in die Pragmatik und Gesprächs- 
forschung ausgreifen, indem sie Formen des Gesprächsaufbaus wie Sprecherwechsel und Gestaltung von Redebeiträgen thematisieren. Entsprechende Kapitel finden sich in den Grammatiken von Weinrich (1993) mit der „Syntax des Dialogs“, in der IDS-Grammatik, in „Duden“ ab 2005 und in Hoffmann (2013).

Die Variation in der Gegenstandskonzeption über den Kernbereich Wort, Satzglied, einfacher und komplexer Satz hinaus ist in Tabelle 12.1 für einige aktuelle Grammatiken des Deutschen zusammengefasst.

Die hier berücksichtigten Grammatiken unterscheiden sich ferner im Grad der Berücksichtigung lexemspezifischer Ausprägungen grammatischer Kategorisierungen wie z. B. der Rektion von Präpositionen, idiosynkratischen Stellungseigenschaften von Konjunktionen und Konjunktionaladverbien, Stammformen der unregelmäßigen Verben oder der Zuordnung von Verben zu Satzbauplänen. Vor allem die Duden-Grammatik mit ihrer breiten Zielgruppenadressierung und dem Anspruch einer Gebrauchsgrammatik, auch ein „praktischer Helfer“ in Nachschlage- und Zweifelsfallsituationen zu sein, zeichnet sich durch umfängliche Wortlisten aus, die nicht immer ganz konform mit dem systematischen Aufbau einer Grammatik zusammengestellt sein müssen, wie etwa das Kapitel „Zweifelsfälle“ (Duden 2016: 220-246), das Substantive mit Schwankungen in Genus-, Plural- und Genitivbildung in alphabetischer Reihenfolge zusammenstellt. Ausgiebige Lexemlisten finden sich auch in Helbig \& Buscha (1998), in Weinrich (1993) und in Engel (2004), wo die alphabetische Auflistung mitunter auch zu Lasten einer systematischen Darstellung der gemeinsamen grammatischen Eigenschaften der jeweiligen Wortart geht. Keine oder nur wenige Wortlisten enthalten die Grammatiken von Eisenberg, Hentschel \& Weydt (2003), die Akademie-Grammatik (1981) und Hoffmann (2013). Einen eigenwilligen Ansatz liefert die aus Materialien für das Lehramtsstudium entwickelte Grammatik von Wellmann (2008), die viel Lexemspezifisches enthält wie eine 10-seitige, nach Präpositionen sortierte Liste von Valenzträgern mit Präpositionsrektion oder ausführliche Angaben zu Idiomen, zur Wortsemantik und zu lexikalischen Kompatibilitäten, oft aber für lexematische Informationen einfach auf Langenscheidts Großwörterbuch Deutsch als Fremdsprache (aus der Werkstatt des Verf.) verweist. Ein durchgehendes Konzept ist hier nicht erkennbar und sofern es um rein technische Lösungen für die nutzerorientierte Vernetzung von grammatikalischer und lexikalischer Information geht, ist die mediale Lösung des grammatischen Informationssystems Grammis ${ }^{11}$ zweifellos überlegen. Grammis integriert eine Hypertext-

11 Grammis. Grammatisches Informationssystem. IDS Mannheim. http://hypermedia.idsmannheim.de/grammis/ (letzter Zugriff: 20.11. 2017). 


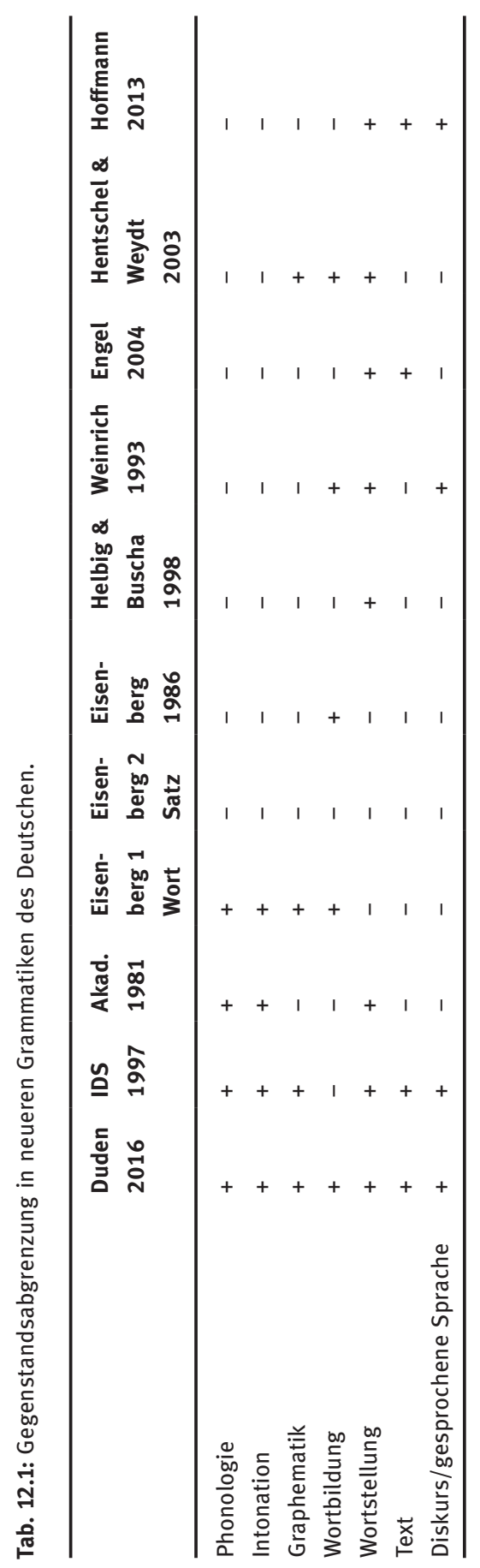


Grammatik auf der Basis der IDS-Grammatik und eine Hypertext-Version des Handbuchs der deutschen Konnektoren (Pasch, Brauße, Breindl \& Waßner 2003) mit mehreren elektronischen Wörterbüchern (z. B. das Valenzwörterbuch VALBU) und einem Glossar grammatischer Fachtermini.

\subsection{Das Konstrukt deutsche Standardsprache}

Alle hier erwähnten Grammatiken betrachten als ihren Gegenstand das gegenwärtige Standarddeutsche. Mitunter wird das ohne weitere Eingrenzung oder Erläuterung mit „der deutschen Sprache“ gleichgesetzt (so z. B. bei Wellmann 2008; Hentschel \& Weydt 2003). In den Angaben, und mehr noch in der grammatischen Praxis, wie dieses Standarddeutsch von anderen - diatopischen, diaphasischen, diastratischen oder auch zeitlichen - Varietäten des Deutschen abzugrenzen ist, gibt es aber doch Unterschiede. Die IDS-Grammatik steckt den zeitlichen Horizont bis zum Ende des 19. Jhs. ab, spricht von einem „Konstrukt der Standardsprache in ihrer schriftlichen und mündlichen Ausprägung“, die sich durch überregional gültige Regeln und „neutrale“ Stilhöhe auszeichne (ebd. S. 2). Sie versteht sich nicht als Variationsgrammatik, ist aber bemüht, die Variationsbreite innerhalb der Standardsprache durch die Nutzung von Korpora zur Phänomenillustration abzubilden. Auch regionale und Substandard-Varietäten werden gelegentlich angeführt. Die Belege entstammen einer großen Bandbreite von Texten und Diskursen (literarische Texte, Gebrauchstexte, Alltagsgespräche, formelle Gespräche). Ähnlich breit ist die Datenbasis der Grammatik von Hoffmann (2013), die damit ihrem Anspruch, sich „der Sprachwirklichkeit zu stellen“, nachkommt. Auch die „Duden-Grammatik“ betrachtet eine überregionale geschriebene und gesprochene Standardsprache der Gegenwart als ihren Gegenstand. Beispiele entstammen dem „Duden-Korpus“ ${ }^{12}$ und dem Internet, Belegquellen werden jedoch nie angegeben. Deutlich eingeschränkter definiert die Akademie-Grammatik ihren Gegenstand als die „gegenwärtige deutsche Literatursprache“, verwendet aber keine Belege aus literarischen Texten. Eisenberg (2013) will die deutsche Sprache „so beschreiben, wie sie heute verwendet wird“, beruft sich aber auch auf eine implizite Norm (ein Begriff, der in den meisten Grammatiken vermieden wird), die zu explizieren und zu kodifizieren Aufgabe des Grammatikers sei. Dabei trifft er immer eine Auswahl und verhilft einer bestimmten Sprachausprägung „zum

12 In der Grammatik selbst werden zur Korpusgrundlage keine Angaben gemacht. Unter www.duden.de/ueber_duden/Partner (letzter Zugriff: 27.11. 2017) findet sich eine sehr knappe Beschreibung. 
Ausdruck des Natürlichen“. Eisenberg macht allerdings nicht deutlich, auf welcher Basis diese Auswahl geschieht. In Band 1 (Eisenberg 2006) problematisiert er am Beispiel der Aussprache des ig-Auslauts das Konzept Standardlautung. Ist bei Aussprachevarianten eine Entscheidung zu treffen, wählt er den Weg, sich auf einen Kodex zu beziehen, den Ausspracheatlas König (1989). In allen anderen Fällen ist es aber wohl das Urteil des Grammatikers selbst, da Eisenberg keine authentischen Belege, sondern nur „Linguistenprosa“ verwendet. Die Bandbreite an Varietäten ist im „Grundriss“ deutlich geringer als in der IDS-Grammatik. Von der „Norm der Standardsprache“ sprechen auch Helbig \& Buscha (1998) und präzisieren diese als „Schriftsprache“.

Um die grammatikographische Praxis im Umgang mit Variation zu überprüfen, bieten sich Zweifelsfälle im Sinne von Klein (2003), (2010) an, für die ein hoher Konsultationsbedarf nachgewiesen ist, die aber keine reinen Distributionsvarianten innerhalb eines Systems Standardsprache sind, sondern in Bezug auf eine der Variationsdimensionen unterschiedlich zuordenbar sind. Vergleicht man etwa die Angaben zur Kasusrektion der Präpositionen wegen und trotz, zum Doppelperfekt, zur Distribution von haben und sein als Perfektauxiliare oder zu Eigennamen mit Artikel, stellt man nicht nur Unterschiede zwischen den einzelnen Grammatiken fest, sondern auch Inkonsistenzen in der Auszeichnung der Varianten, Vagheit in den Angaben und kaum je konkrete Frequenzangaben. Freilich muss hier zugestanden werden, dass der Stand der empirischen korpusbasierten Forschung die Ermittlung exakter Zahlen erst seit verhältnismäßig kurzer Zeit zulässt und dies zudem beträchtlichen Aufwand mit sich bringt.

Ein transparentes Auszeichnungssystem wie in Wörterbüchern haben Grammatiken in aller Regel nicht. Die Duden-Grammatik etwa verwendet für diaphasische Varianten umgangssprachlich und gehoben, aber auch gesprochene Sprache oder gesprochene Alltagssprache, ohne dass das Verhältnis zu umgangssprachlich geklärt würde. Die Angaben zu regionalen Varietäten spiegeln nicht die Systematik der Areallinguistik, der oberdeutsche Raum erscheint differenzierter als der niederdeutsche - neben schweiz., österr., schwäb., bayr., südd. gibt es nur noch nordd. An Fach- und Sondersprachen erscheinen neben der generellen Kennzeichnung fachspr. - nur med., techn., theol.: vermutlich ausgewählt als diejenigen Fachbereiche, mit denen der Laie am ehesten in Berührung kommt. Überprüft man exemplarisch die Angaben zum Doppelperfekt, einem sowohl unter normativem als auch diasystematischen Aspekt relevanten Phänomen, findet sich in der Duden-Grammatik (2009: 514), es komme „im wesentlichen in der gesprochenen Sprache und der mündlich gefärbten Schriftsprache, und zwar im ganzen Sprachraum“ vor. Sie schreibt ihm eine Funktionalität im Rahmen des Tempussystems zu, behauptet dann 
aber, dass es in der geschriebenen Standardsprache als nicht korrekt gelte. Als „(noch?) nicht korrekt“ wird es auch bei Hentschel \& Weydt (2003: 114) beschrieben, die es dagegen „im südlichen Raum“ verorten. Eisenberg erwähnt es weder in Bd. 1 noch in Bd. 2, Hoffmann (2013: 265) beschreibt es als Ersatz für „das regional bzw. umgangssprachlich vermiedene Präteritumperfekt“, ordnet es eher der Mündlichkeit $\mathrm{zu}$ und gesteht ihm keine standardsprachliche Geltung zu. Auch die IDS-Grammatik sieht es regional als Ersatzform des Präteritumperfekts und bezeichnet es als ,in der geschriebenen Hochsprache“ sehr selten (ebd. 1687), beruft sich dafür aber immerhin auf eine Korpusauszählung. ${ }^{13}$ Die Erklärung als Präteritumersatz in vom Präteritumschwund betroffenen Regionen wird in der Forschung zum Doppelperfekt heute eher abgelehnt (vgl. etwa Buchwald-Wargenau 2012); auch wäre stärker zu differenzieren zwischen indikativischen und konjunktivischen Doppelperfektformen: Gerade letztere füllen eine Lücke im Tempussystem, da der Konjunktiv II nicht zwischen abgeschlossenen Ereignissen und solchen im Verlauf differenziert. Die hier untersuchten Grammatiken sind also bereits explanativ inadäquat und leiten aus der falschen Erklärung dann normative Geltungsansprüche und falsche regionale Zuordnungen ab.

Sowohl die IDS-Grammatik (S. 2) als auch die Akademie-Grammatik (S. 45) thematisieren die empirischen Schwierigkeiten einer Varietätengrammatik angesichts einer noch schwach entwickelten Forschungslage zur regionalen und diasituativen grammatischen Variation. In der Zwischenzeit hat die Forschung hier jedoch aufgeholt. (Zur arealen Variation s. Lenz i.d.Bd.). Nicht nur zur Grammatik der gesprochenen Sprache liegen mittlerweile eine Vielzahl von Arbeiten vor, sondern auch zum Textsortenbezug grammatischer Phänomene (Fandrych \& Thurmair 2011) und neuerdings auch zur arealen grammatischen Variation. Mit Biber et al. (2006) gibt es nun auch ein Modell für eine Varietätengrammatik auf korpuslinguistischer Grundlage, die die oben beschriebenen diasystematischen Varietäten und die Textsortenspezifik grammatischer Phänomene berücksichtigt. Für das Deutsche sind hier zwei aktuelle Projekte besonders vielversprechend: Das Projekt zur arealen grammatischen Variation des Deutschen in Deutschland, Österreich und der Schweiz (Dürscheid, Elspaß \& Ziegler 2011, 2015) und zur grammatischen Variation das IDS-Projekt

13 Man muss hinzufügen, dass das in der IDS-Grammatik genutzte Korpus in seinem damaligen Zuschnitt heutigen Anforderungen an eine korpuslinguistische Fundierung grammatischer Regelformulierungen nicht mehr genügen würde, weder in Bezug auf die Korpusgröße (die IDS-Korpora sind im Zeitraum von 1997-2017 ca. um den Faktor 100 gewachsen) noch in Bezug auf die Variationsbreite bei den Textsorten. Das gilt natürlich erst recht für Grammatiken, die zeitlich vor der IDS-Grammatik erschienen sind. 
Korpusgrammatik - grammatische Variation im standardsprachlichen und standardnahen Deutsch, das eine neue, „variationssensitive“ Grammatik des Standarddeutschen korpuslinguistisch basierend erarbeiten will. Möglich wird dies auch durch die Nutzung neuer korpuslinguistischer Methoden wie der statistischen Berechnung von Signifikanzen und Effektstärken und Techniken des Data-Minings. Erste Ergebnisse liegen mit Konopka \& Fuß (2016), Konopka \& Wöllstein (2017) und Fuß, Konopka \& Wöllstein (im Ersch.) vor.

\section{Grammatische Praxis}

Unter dem Terminus „Grammatische Praxis“ wird hier mit Meyer (2006), der sich wiederum auf Wittgenstein beruft, ein Geflecht von Tätigkeiten, Urteilen und Methoden verstanden, die der Grammatiker zum Zwecke der Beschreibung einer Einzelsprache anwendet. Zur grammatischen Praxis gehören wesentlich die Kategorisierung, d. h. Etablierung von Klassen, und das Zuordnen von Einheiten zu einer Klasse auf der Basis ermittelter gemeinsamer Eigenschaften, die Strukturierung komplexer Äußerungen sowie das Urteilen über die Wohlgeformtheit sprachlicher Ausdrücke und die Art und Weise der Regelformulierung und -erklärung. Aufgabe jeder deskriptiven Grammatik ist letztlich, eine Grenze zwischen grammatischen und ungrammatischen Ausdrücken zu ziehen und diese Grenze über geeignete Generalisierungen in die Form von Regeln zu überführen. Ein weiterer Schritt, den reine Resultatsgrammatiken in der Regel nicht gehen, ist es, die Regeln durch Einbettung in einen übergeordneten $\mathrm{Zu}$ sammenhang auch zu begründen. Auf dem Weg zu diesem Ziel stehen Grammatiker vor mehreren Entscheidungen:

1. Grenzziehung: Wird die Grenze scharf gezogen oder werden Abstufungen von Grammatikalität zugelassen? Ist der Abstand zwischen grammatisch und ungrammatisch immer gleich groß? (s. dazu Abschnitt 3.1)

2. Regelformulierung: Wie und ggf. mit Hilfe welcher Formalismen werden Regeln formuliert? Wie einheitlich sind die Regelformulierungen?

3. Regelbasis: Auf welche Evidenz stützt sich das Urteil des Grammatikers? Hier besteht ein enger Zusammenhang mit 2., insofern Angaben wie

- „x kommt (nicht/selten/häufig/nur in bestimmten Varietäten) vor“,

- „X ist (nicht) gebräuchlich“ „X ist richtig/zulässig/falsch/ungrammatisch",

- „X gilt als nicht standardsprachlich“

über die Formulierung oft auch auf eine Urteilsquelle schließen lassen.

4. Regelzusammenhang: Werden Regeln ad hoc formuliert oder erscheinen sie durch Einordnung in ein übergeordnetes Konzept begründet? Ein solcher Zusammenhang kann 
- innersystematischer Natur sein (so lassen sich z. B. Stellungseigenschaften der Argumente im Mittelfeld durch die typologische Eigenschaft der grundlegenden SOV-Stellung des Deutschen erklären),

- in einer diachronen Perspektive liegen (z. B. können Flexionserscheinungen innerhalb der NP auf eine Tendenz zur Monoflexion zurückgeführt werden),

- in einem Bezug auf Bedingungen des sprachlichen Handelns bestehen (das gilt $\mathrm{u}$. a. für viele informationsstrukturell determinierte grammatische Phänomene),

- auf sprachliche Universalien Bezug nehmen, hinter denen wiederum kognitive und biologische Eigenschaften des Menschen stehen, oder

- „universalgrammatisch“ mit Rekurs auf übereinzelsprachliche Strukturprinzipien begründet sein.

Diese Entscheidungen müssen vor dem Hintergrund von Erwartungen an Grammatiken getroffen werden, auf deren Unvereinbarkeit Gisela Zifonun im Vorwort der IDS-Grammatik (S. 3) aufmerksam gemacht hat.

1. „Vollständigkeitsparadox“: Grammatiken sollen eine Einzelsprache deskriptiv vollständig, möglichst auch in Hinsicht auf regionale, mediale und andere Varietäten erfassen. Das ist nach Zifonun nur sehr schwer vereinbar mit dem Anspruch auf ein einheitliches theoretisches Fundament. In der Tat ist nicht vorstellbar, dass mit einem einheitlichen grammatiktheoretischen Format nicht nur der kompositionale Aufbau auf den Ebenen Silbe, Morphem, Wort, Phrase, Satz, Intonation, Text modelliert werden kann, sondern auch lexemspezifische Idiosynkrasien und varietätenspezifische Ausprägungen. Das Vollständigkeitsparadox ist ein prinzipielles und kein praktisches, das mit der Weiterentwicklung grammatischer Theoriebildung zu beheben wäre. Zifonun weist in diesem Zusammenhang auch auf die „vornehme Abstinenz mancher Ansätze“ hin, die die Bewährungsprobe, die Validität ihrer Theorie an den systematischen Erfordernissen einer deskriptiven Grammatik zu überprüfen, gar nicht erst eingehen. Hinzuzufügen ist, dass die grammatische Theoriebildung der letzten Jahrzehnte sich auf die Syntax konzentrierte, in einer Grammatik erwartbare Teilbereiche wie Flexionsmorphologie, Intonation, Graphematik aber ganz andere Beschreibungsinstrumentarien benötigen. Eine Referenzgrammatik mit Anspruch auf Vollständigkeit kann also nur eine „pluralistische“ Grammatik sein (so auch Buscha 1995).

2. „Hase-Igel-Paradox“: Sind konzeptionelle Grundentscheidungen einmal getroffen, kann für die Einzelphänomene kaum mehr der aktuelle Forschungsstand in einer theoretisch konsistenten Weise integriert werden. 
3. Das „Präzisierungsdilemma“: ein höherer Präzisionsgrad geht zulasten der Verständlichkeit. Dieses Dilemma ist für didaktische Grammatiken wie Helbig \& Buscha (1998) und für die Duden-Grammatik in ihrem Selbstverständnis als Gebrauchsgrammatik mit nahezu uneingeschränkter Zielgruppenadressierung deutlich schärfer ausgeprägt als für Grammatiken mit einer überwiegend akademischen Zielgruppe wie die IDS-Grammatik oder die Akademie-Grammatik. Verlagsstrategisch bedingte Umfangsbeschränkungen setzen in dieser Hinsicht zusätzlich Weichen. Einige Grammatiken richten sich in erster Linie an Studierende der Germanistik, so z. B. Eisenbergs „Grundriss“, Wellmann (2008), eine Grammatik, die aus Materialien für Lehramtsstudierende zusammengestellt wurde, oder Hoffmann (2013) mit einer speziellen DaZ-Orientierung. Diese Grammatiken unterscheiden sich dennoch sehr stark sowohl in ihrem Präzisionsgrad als auch in der Gewichtung der Teile.

4. Das „Normativitätsdilemma“ (bereits in Kap. 2 erwähnt), dass ein in einer Grammatik kodifizierter Sprachgebrauch vom Nutzer als verbindliche Norm interpretiert werden kann.

Diese Dilemmata addieren sich zum grundlegenden Konflikt wissenschaftlicher Grammatikographie: Nutzer wollen eine schnelle Orientierung in Zweifelsfällen und deshalb eine Grammatik „zum Nachschlagen“, die Verfasser wissenschaftlicher Grammatiken erwarten eher eine durchgehende Lektüre, ohne die sich dem Nutzer der systematische Zusammenhang nicht wirklich erschließt. Dieser Nutzeraspekt bleibt im Konzept der „grammatische(n) Praxis“ bei Meyer (2006) ausgeblendet.

\subsection{Formen und Funktionen von sprachlichen Beispielen}

Kompetenzbasierte Beispiele haben in einer Grammatik die Funktion, die Grenzen des Grammatischen sichtbar zu machen. Mit ihrer Hilfe können u. a. die Klassenzugehörigkeit von Einheiten, ihre Distribution und Selektionsrestriktionen bei der Bildung größerer Einheiten sowie Stellungsrestriktionen gezeigt werden, die sich aus authentischen Beispielen nicht erschließen. Zur Grenzziehung gehört auch die Verwendung ungrammatischer Beispiele, die symbolisch mit einem Asterisk (im Falle scharfer Grenzziehung) und ein bis mehreren Fragezeichen bei gradueller Abstufung von Grammatikalitätsurteilen gekennzeichnet wird. Kaum eine der hier berücksichtigten Grammatiken verzichtet auf solche Beispiele, sosehr sie sich auch der Sprachwirklichkeit verpflichtet fühlt. Das liegt auch an der Funktion dieser Beispiele, Hypothesen und Argumente des Grammatikers aufzuzeigen, wofür sie unverzichtbar sind. 
Die Urteilsbasis ist hier letztlich die individuelle Sprachkompetenz des Grammatikers. Meyer (2006) hat am Beispiel der IDS-Grammatik und des „Grundrisses“ auf damit zusammenhängende Probleme hingewiesen. Die als ungrammatisch oder fragwürdig gekennzeichneten Beispiele sind keineswegs immer gleich schlecht bzw. fragwürdig und in Beispielreihen mit abgestuften Grammatikalitätsurteilen sind die Abstände nicht äquidistant. So werden etwa in der Akademie-Grammatik (S. 318) die Imperativformen *Sei blond!, *Sei müde! und ${ }^{\star} H u s t e$ ! durch identische Auszeichnung als gleich schlecht eingestuft, was für den Laien, dem die ärztliche Anweisung „Husten Sie mal“ geläufig ist, zumindest irritierend ist. Feinkörnig abgestufte Grammatikalitätsurteile, die durch geschickte Umfragen bei einer großen Zahl von Sprechern erhoben werden, könnten hier Abhilfe schaffen, sind aber wegen des hohen Aufwands kaum systematisch in die grammatikographische Praxis $\mathrm{zu}$ integrieren.

Bei dieser Art der Beispielgebung handelt es sich um eine Technik, die dem Normalsprecher nicht vertraut ist und die ihn mit Formen konfrontiert, die er abweichend vom Urteil des Grammatikers akzeptabel findet oder die umgekehrt dem Grammatiker als wohlgeformt gelten, in der Sprachwirklichkeit aber nicht vorkommen und deshalb vom Laien abgelehnt werden. Im Vorwort der IDS-Grammatik (S.12) werden Sätze wie Entweder hat Peter sich einen Trabant oder seiner Frau ein Auto gekauft als „Linguisten-Prosa“ bezeichnet. Im Verhältnis von authentischen Belegen zu Beispielen, die im Rahmen einer Argumentation einen wichtigen Stellenwert haben, aber allein durch die Kompetenz des Grammatikers legitimiert sind, und zur Verwendung von ungrammatischen Beispielen unterscheiden sich die hier berücksichtigten Grammatiken in erstaunlichem Maße. So verzichtet etwa Weinrichs „Textgrammatik“ komplett auf ungrammatische Beispiele und kommt selbst im Kapitel „Restriktionen bei der Vorfeldbesetzung“ ohne den Asterisk aus. Neben authentischen Texten werden Beispielsätze verwendet, die oft auf der Basis der Texte und meist in einem lockeren inhaltlichen Zusammenhang formuliert sind. Ganz ohne Belege arbeiten die Grammatiken von Helbig \& Buscha (1998), Hentschel \& Weydt (2003), Engel (2004), Eisenbergs „Grundriss“ und die Akademie-Grammatik. Das ist nur zum Teil mit dem Erscheinungsdatum erklärbar: In der Benutzung von Korpora hat die IDS-Grammatik 1997 Maßstäbe gesetzt, hinter die später erschienene Grammatiken, die auf Korpusdaten verzichten, dann zurückfallen.

Als ungrammatisch ausgezeichnete Beispiele werden in den Grammatiken von Helbig \& Buscha (1998), Hentschel \& Weydt (2003), Engel (2004), Hoffmann (2013) und auch in der Duden-Grammatik sehr sparsam verwendet, während die Akademie-Grammatik, die IDS-Grammatik und der „Grundriss“ ausgiebigen Gebrauch davon machen, wobei der „Grundriss“ als einzige Grammatik keine Abstufungen vornimmt. Charakteristisch für diese drei Grammati- 
ken ist, dass ihre Rezeption weitgehend auf eine linguistisch geschulte Leserschaft beschränkt ist.

Auch in den Erläuterungen der Symbole * und ? werden unterschiedliche Bezüge deutlich (s. Tabelle 12.2).

Tab. 12.2: Auszeichnung ungrammatischer Beispiele.

\begin{tabular}{|c|c|c|}
\hline & * & $?$ \\
\hline Hoffmann 2013 & ungrammatisch, nicht wohlgeformt & Grammatikalität fragwürdig \\
\hline Duden 2016 & $\begin{array}{l}\text { nicht grammatischer } \\
\text { Sprachgebrauch }\end{array}$ & $\begin{array}{l}\text { standardsprachlich nicht } \\
\text { akzeptierter Sprachgebrauch }\end{array}$ \\
\hline $\begin{array}{l}\text { Helbig \& Buscha } \\
1998\end{array}$ & ungrammatischer Satz & $\begin{array}{l}\text { (*) halbgrammatischer } \\
\text { (unüblicher) Satz }\end{array}$ \\
\hline Grundriss & ungrammatisch & \\
\hline IDS-Grammatik & $\begin{array}{l}\text { grammatisch nicht akzeptabel; } \\
\text { dialektale und standardsprachlich } \\
\text { nicht akzeptable Verwendungen }\end{array}$ & $\begin{array}{l}\text { fragliche, für manche (?) bzw. } \\
\text { für viele (??) Sprecher oder } \\
\text { Verwendungszusammenhänge } \\
\text { nicht gegebene Akzeptabilität }\end{array}$ \\
\hline $\begin{array}{l}\text { Akademie- } \\
\text { Grammatik }\end{array}$ & $\begin{array}{l}\text { Form falsch (in sich widersprüchlich, } \\
\text { nicht interpretierbar, falsch gebildet, } \\
\text { unter den angegebenen Bedingungen } \\
\text { nicht zulässig) }\end{array}$ & $\begin{array}{l}\text { Form ungewöhnlich, } \\
\text { nicht üblich, } \\
\text { möglicherweise falsch }\end{array}$ \\
\hline
\end{tabular}

Während die meisten Grammatiken den Asterisk lapidar als „ungrammatisch“ erklären, versuchen die IDS-Grammatik und die Akademie-Grammatik, über den Bezug zu Sprechern und zu Verwendungskontexten hier eine genauere Erläuterung. Bei den Abstufungssymbolen ? und ?? (letzteres nur in der IDSGrammatik) zeigen sich die Unterschiede noch deutlicher. In der IDSGrammatik und der Akademie-Grammatik wird hypothetisch über Sprecherurteile quantifiziert, wofür im Grunde Sprecherbefragungen nach der Methode des „Thermometer judgements“ (Featherston 2008) nötig wären, die Duden-Grammatik nimmt auf das Konstrukt Standardsprache Bezug und in der Grammatik von Helbig \& Buscha wird „halbgrammatisch“ mit unüblich gleichgesetzt.

Die Basis für Grammatikalitätsurteile ist also in den betrachteten deskriptiven Grammatiken des Deutschen teils unklar, teils inkonsistent. Zwar werden auch künftige Grammatiken nicht auf kompetenzbasierte und ungrammatische Beispiele verzichten können, gerade die Abstufungen sollten aber klarer definiert und methodisch besser abgesichert werden. 


\subsection{Regelformulierung und -erklärung}

Wie unterschiedlich Grammatiken in Bezug auf die Formulierung von Regeln und deren Begründung verfahren, soll hier an einem Testfall gezeigt werden, der eine Schnittstelle zwischen verschiedenen grammatischen Ebenen darstellt, also möglicherweise an verschiedenen Stellen in einer Grammatik behandelt wird und der gleichzeitig eine Variationsdimension involviert, nämlich die Verbzweitstellung nach Subjunktoren (Terminus und Konzept nach dem Handbuch der deutschen Konnektoren, Breindl, Volodina \& Waßner 2014) wie weil und obwohl/obschon/obgleich oder während. Hier besteht nicht nur ein hoher Konsultationsbedarf seitens der Nutzer, sondern der Gegenstand ist auch in Bezug auf die grammatische Norm umstritten.

Eisenberg geht im zweiten Band des „Grundriss“ (1999: 325) im Kapitel Adverbialsätze auf Subjunktoren mit Verbzweitstellung kaum ein. Er führt weil mit Verbzweitstellung ohne jegliche stilistische Einschränkung als äquivalent mit da-Sätzen in replikativen Schlüssen wie Ein Baum liegt auf der Straße, weil Karl hat gebremst an. Engel (2004: 407) beschränkt sich auf ein Beispiel mit Sprechaktbezug (Trinken Sie noch was? Weil ich muss jetzt Schluss machen), kommentiert aber weder die Verbzweitstellung noch den Bezug zum Standard. Helbig \& Buscha (1998) und die Akademie-Grammatik erwähnen es überhaupt nicht, Hentschel \& Weydt (2003: 288) geben an, dass weil-Sätze „im modernen gesprochenen Deutsch zunehmend zu Hauptsatzstellung tendieren“, was auf eine fließende Grenze zwischen Haupt- und Nebensatz hindeute: In dieser Allgemeinheit ist das nicht zutreffend und beschreibt auch nicht die Besonderheit der weil-Verbzweitsätze, die auf der epistemischen oder der Sprechaktebene verknüpfen (s. Breindl, Volodina \& Waßner 2014). Weinrichs „Textgrammatik“ (1993: 758) gibt ein Beispiel für weil mit Verbzweitstellung und bemerkt dazu ähnlich allgemein wie Hentschel \& Weydt (2003), dass „heute in der mündlichen Umgangssprache auch bei der Konjunktion weil manchmal die Endstellung des Verbs“ unterbleibe, dass dieser Sprachgebrauch in der Schriftsprache aber nicht als normgerecht gilt. Bei Hoffmann (2013: 355) wird die Verbzweitstellung für die Subjunktoren weil, obwohl, trotzdem und während angegeben: Diesen Gebrauch führt Hoffmann auf eine in den süddeutschen Varietäten stets bewahrte Stellungsvariante, die sich seit ca. 100 Jahren zunehmend von Süden her ausbreite. Er geht auch auf die besondere Intonation ein und schreibt diesen Verbzweitsätzen eigenständiges Illokutionspotenzial zu. Eine stilistische Eingrenzung trifft er nicht. Am ausführlichsten werden Verbzweit-Subjunktoren in der IDS-Grammatik behandelt. Hier werden sie in die Systematik unterschiedlicher Kausalzusammenhänge Realgründe, Beweggründe, Moduskommentare - eingeordnet, die auch für die 
Beschreibung anderer kausal fundierter Adverbialsätze (konzessive, konditionale, konsekutive) fruchtbar gemacht wird. Die Verbzweitstellung bei weil wird dann als Variante zur Verbletztstellung bei Begründungszusammenhängen, insbesondere bei reduktiven Schlüssen, angeführt, mit der Einschränkung, dass sich diese Variante „häufig im Diskurs“ finde (ebd. 2305). In der Duden-Grammatik wird die Verbzweitstellung weder im Wortartenkapitel noch im Textkapitel erwähnt, aber im Kapitel zum zusammengesetzten Satz (Duden 2016: 1066) und im Kapitel zur Gesprochenen Sprache (Duden 2016: 1222). Die Verbzweitstellung nach weil sei „,bisher sehr weitgehend auf die gesprochene Sprache beschränkt“. Epistemische und sprechhandlungsbezogene weil-Sätze würden mit Verbzweitstellung verwendet, faktisches weil könne beide Stellungsvarianten aufweisen.

Es zeigt sich, dass Grammatiken, wenn sie über Einzelbeobachtungen und ad-hoc-Erklärungen hinausgehen wollen, eine Systematik der Beschreibungskategorien aufbauen müssen, die erstens einen gewissen Raum benötigt und zweitens dem Leser auch die zusammenhängende Lektüre nicht erspart. Die Einordnung der Varietät - unkommentiert als Standard oder der gesprochenen Sprache zugeordnet, ist in keinem Fall empirisch gestützt.

\section{Fazit und Ausblick}

Es sollte deutlich geworden sein, wie groß die Variationsbreite in der Gegenstandsbestimmung und -abgrenzung und in den Darstellungsformen der deskriptiven Grammatiken des Deutschen der letzten Jahrzehnte ist. Soweit sich Trends feststellen lassen, betreffen sie nur bedingt eine zunehmende Empirisierung, insofern sich nicht alle Grammatiken der letzten drei Jahrzehnte auf Belege oder gar korpusbasierte Frequenzangaben stützen. Grammatiken, die vor der IDS-Grammatik erschienen sind, nutzen in aller Regel nahezu ausschließlich kompetenzbasierte Beispiele zur Illustration von Hypothesen und Regeln. Das kann man insofern kaum kritisch sehen, als zum damaligen Zeitpunkt sowohl der Aufbau elektronischer Korpora als auch deren Erschließung durch korpus- und computerlinguistische Tools noch in den Kinderschuhen steckte. Aber auch für viele Grammatiken der letzten zwei Jahrzehnte gilt, dass das Urteil des Grammatikers zu einem Konstrukt „Standardsprache“ verabsolutiert wird. Verzichtbar sind „künstliche“ Beispiele aber ohnehin nicht, ebensowenig wie ungrammatische Beispiele: Sie werden benötigt, da über ein Korpus nicht die negative Evidenz ermittelt werden kann, was nicht möglich ist, und da die systematische Abwandlung einer sprachlichen Struktur über die Bildung von Minimalpaaren als Instrument des Hypothesentestens unabdingbar ist. 
Eine stärkere Varietätendifferenzierung zeigt sich in der Berücksichtigung gesprochensprachlicher Formen. Von einer echten Varietätengrammatik kann jedoch hier nicht gesprochen werden; das IDS-Projekt einer Korpusgrammatik des Deutschen könnte hier wegweisend sein. Was sich an den grammatischen Neuerscheinungen der letzten Jahre aber zeigt, ist - abgesehen von der „DudenGrammatik“ - ein Trend zu einer stärkeren Zielgruppenfestlegung, die dann auch Neuerungen erlaubt, wie im Falle der Grammatik von Hoffmann (2013) etwa die punktuelle Darstellung grammatischer Phänomene des Deutschen vor der typologischen Folie anderer Sprachen. Auch in diesem Punkt könnte wiederum ein IDS-Projekt, die Grammatik des Deutschen im europäischen Vergleich, richtunggebend sein.

\section{Literatur}

\section{Grammatiken des Deutschen}

Brinkmann, Hennig (1962): Die deutsche Sprache. Gestalt und Leistung. Düsseldorf: Schwann. Cartagena, Nelson \& Hans-Martin Gauger (1989): Vergleichende Grammatik Spanisch - Deutsch. Mannheim u. a.: Duden.

Broekhuis, Hans \& Norbert Corver (2017): Syntax of Dutch. Verbs and verb phrases. 3 Vols. Amsterdam: Amsterdam University Press.

Buscha, Joachim, Renate Freudenberg-Findeisen, Eike Forstreuter, Herrmann Koch \& Lutz Kuntsch (1998): Grammatik in Feldern. Ein Lehr- und Übungsbuch für Fortgeschrittene. Ismaning: Verlag für Deutsch.

Duden (1959): Grammatik der deutschen Gegenwartssprache. Neu bearb. von Paul Grebe. Mannheim u. a.: Duden.

Duden (1995): Grammatik der deutschen Gegenwartssprache. 5., völlig neu bearb. und erw. Aufl. Mannheim u. a.: Duden.

Duden (1984): Die Grammatik. Unentbehrlich für richtiges Deutsch. 4., völlig neu bearb. und erw. Aufl. Mannheim u. a.: Duden.

Duden (2009): Die Grammatik. Unentbehrlich für richtiges Deutsch. 8., völlig neu bearb. und erw. Aufl. Mannheim u. a.: Duden.

Duden (2016): Die Grammatik. Unentbehrlich für richtiges Deutsch. 9. Auflage. Mannheim u. a.: Duden.

Duden (2007): Richtiges und gutes Deutsch. Wörterbuch der sprachlichen Zweifelsfälle. 6., vollst. überarb. Aufl. Mannheim u. a.: Duden.

Eisenberg, Peter (1999): Grundriß der deutschen Grammatik. Band 2: Der Satz. Stuttgart, Weimar: Metzler.

Eisenberg, Peter (2013): Grundriss der deutschen Grammatik. Band 2: Der Satz. 4., aktual. und überarb. Aufl. Stuttgart, Weimar: Metzler.

Eisenberg, Peter (2006): Grundriss der deutschen Grammatik. Band 1: Das Wort. 3., aktual. und überarb. Aufl. Stuttgart, Weimar: Metzler.

Engel, Ulrich (1991): Deutsche Grammatik. Heidelberg: Groos. 
Engel, Ulrich (2004): Deutsche Grammatik. Neubearb. München: ludicium.

Engel, Ulrich, Mihai Isbasescu, Speranta Stanescu \& Octavian Nicolae (1993): Kontrastive Grammatik deutsch-rumänisch. Heidelberg: Groos.

Engel, Ulrich, Danuta Rytel-Kuc, Leslaw Cirko, Antoni Debski et al. (1999): Deutsch-polnische kontrastive Grammatik. Heidelberg: Groos.

Engel, Ulrich \& Rozemaria K. Tertel (1993): Kommunikative Grammatik Deutsch als Fremdsprache. München: ludicium.

Gallmann, Peter, Heidi Siller Runggaldier \& Horst Sitta (2008-2013): Sprachen im Vergleich: Deutsch - Ladinisch - Italienisch. 4 Bände. Bozen: Istitut Pedagogich Ladin.

Glinz, Hans (1952): Die innere Form des Deutschen. Bern: Francke.

Glinz, Hans (1970): Deutsche Grammatik. 1: Satz, Verb, Modus, Tempus. Wiesbaden: Athenaion.

Glinz, Hans (1975): Deutsche Grammatik 2: Kasusyntax, Nominalstrukturen, Wortarten, Kasus. Wiesbaden: Athenaion.

Glinz, Hans (1975): Deutsche Grammatik 3: Zusammengesetzter Satz und äquivalente Strukturen. Wiesbaden: Athenaion.

Glinz, Hans (1994): Grammatiken im Vergleich: Deutsch - Französisch - Englisch - Latein; Formen - Bedeutungen - Verstehen. Tübingen: Niemeyer.

Gunkel, Lutz, Adriano Murelli, Susan Schlotthauer, Bernd Wiese \& Gisela Zifonun (2017): Grammatik des Deutschen im europäischen Vergleich. Das Nominal. Berlin/Boston: De Gruyter.

Heidolph, Karl-Erich, Walter Flämig \& Wolfgang Motsch (1981): Grundzüge einer deutschen Grammatik. Berlin: Akademie-Verlag.

Helbig, Gerhard \& Joachim Buscha (1998): Deutsche Grammatik: Ein Handbuch für den Ausländerunterricht. Leipzig u. a.: Langenscheidt.

Helbig, Gerhard \& Joachim Buscha (2001): Deutsche Grammatik: Ein Handbuch für den Ausländerunterricht. Berlin u. a.: Langenscheidt.

Hentschel, Elke \& Harald Weydt (2003): Handbuch der deutschen Grammatik. 3., völlig neu bearb. Aufl. Berlin/New York: De Gruyter.

Heringer, Hans Jürgen (1987): Wege zum verstehenden Lesen. Lesegrammatik für Deutsch als Fremdsprache. Ismaning: Hueber.

Heringer, Hans Jürgen (1988): Lesen lehren lernen: Eine rezeptive Grammatik des Deutschen. Tübingen: Niemeyer.

Hoffmann, Ludger (2013): Deutsche Grammatik. Grundlagen für Lehrerausbildung, Schule, Deutsch als Zweitsprache und Deutsch als Fremdsprache. Berlin: Erich Schmidt.

Renzi, Lorenzo et al. (Hrsg.) (1988): Grande grammatica italiana di consultazione. Bologna: Il Mulino.

Sternefeld, Wolfgang (2006): Deutsche Syntax. Eine morphologisch motivierte generative Beschreibung des Deutschen. Bd. 1-2 Tübingen: Stauffenburg.

Weinrich, Harald (1993): Textgrammatik der deutschen Sprache. Mannheim u. a.: Duden.

Wellmann, Hans (2008): Deutsche Grammatik. Laut. Wort. Satz. Text. Heidelberg: Winter.

Zemb, Jean-Marie (1978): Vergleichende Grammatik Französisch-Deutsch. Mannheim: Bibliographisches Institut.

Zifonun, Gisela, Ludger Hoffmann \& Bruno Strecker (1997): Grammatik der deutschen Sprache. Berlin/New York: De Gruyter. 


\section{Sekundärliteratur}

Ágel, Vilmos \& Rita Brdar-Szabo (Hrsg.) (1995): Grammatik und deutsche Grammatiken. Budapester Grammatiktagung 1993. Tübingen: Niemeyer.

Biber, Douglas, Edward Finegan, Stig Johansson, Susan Conrad \& Geoffrey Leech (2006): Longman grammar of spoken and written English. Harlow: Longman.

Buchwald-Wargenau, Isabel (2012): Die doppelten Perfektbildungen im Deutschen. Berlin/ Boston: De Gruyter.

Buscha, Joachim (1995): Referenzgrammatiken als theoretische Mischgrammatiken. In Vilmos Ágel \& Rita Brdar-Szabó (Hrsg.), 175-185.

Breindl, Eva und Jonas Schreiber (i. Dr.): Metakommunikative Konnektoren als Konstruktionsfamilie. In Anne Erhard-Macris (Hrsg.), Die kommentative Funktion. Tübingen: Stauffenburg

Breindl, Eva, Anna Volodina \& Ulrich H. Waßner (2014): Handbuch der deutschen Konnektoren. Teil 2: Semantik der deutschen Satzverknüpfer. Berlin/New York: De Gruyter.

Dürscheid, Christa, Stephan Elspaß \& Arne Ziegler (2015): Variantengrammatik des Standarddeutschen. Konzeption, methodische Fragen, Fallanalysen. In Alexandra N. Lenz \& Manfred Glauninger (Hrsg.), Standarddeutsch im 21. Jahrhundert. Theoretische und empirische Ansätze mit einem Fokus auf Österreich. Wien: Vienna University Press.

Dürscheid, Christa, Stephan Elspaß \& Arne Ziegler (2011): Grammatische Variabilität im Gebrauchsstandard: das Projekt „Variantengrammatik des Standarddeutschen“. In Marek Konopka et al. (Hrsg.), Grammatik und Korpora 2009/Grammar \& Corpora 2009, 123-140. Tübingen: Narr.

Eggs, Frederike (2011): Zur Funktionalität des Konnektors „geschweige denn“. In Eva Breindl, Gisella Ferraresi \& Anna Volodina (Hrsg.), Satzverknüpfungen. Zur Interaktion von Form, Bedeutung und Diskursfunktion, 229-262. Berlin: De Gruyter.

Fandrych, Christian \& Maria Thurmair (2011): Textsorten im Deutschen. Tübingen: Stauffenburg.

Featherston, Sam (2008): Thermometer judgements as linguistic evidence. In Claudia Riehl \& A. Rothe (Hrsg.), Was ist linguistische Evidenz?, 69-89. Aachen: Shaker.

Fuß, Eric, Marek Konopka \& Angelika Wöllstein (Hrsg.) (im Ersch.): Grammatik im Korpus. Tübingen: Narr.

Ganslmayer, Christine (2010): Tradition und Entwicklung einer deutschen Grammatik. In Mechthild Habermann (Hrsg.), Grammatik wozu? Vom Nutzen des Grammatikwissens in Alltag und Schule, 31-46. Mannheim u. a.: Duden.

Glück, Helmut (2000): Metzler Lexikon Sprache. Stuttgart: Metzler. Habermann, Mechthild (Hrsg.): Grammatik wozu? Vom Nutzen des Grammatikwissens in Alltag und Schule. Mannheim u. a.: Duden.

Haspelmath, Martin (2002): Grammatikalisierung: von der Performanz zur Kompetenz ohne angeborene Grammatik. In: Sybille Krämer \& Ekkehard König (Hrsg.): Gibt es eine Sprache hinter dem Sprechen? Frankfurt a. M.: Suhrkamp, 262-286.

Helbig, Gerhard (1992): Grammatiken und ihre Benutzer. In Vilmos Ágel \& Regina Hessky (Hrsg.), Offene Fragen - offene Antworten in der Sprachgermanistik, 135-151. Tübingen: Niemeyer.

Helbig, Gerhard (1993): Wieviel Grammatik braucht der Mensch? In Theo Harden \& Cliona Marsh (Hrsg.), Wieviel Grammatik braucht der Mensch?, 19-29. München: Iudicium. 
Helbig, Gerhard (2001): Arten und Typen von Grammatiken. In Gerhard Helbig, Lutz Götze, Gert Henrici \& Hans-Jürgen Krumm (Hrsg.), Deutsch als Fremdsprache.

Ein internationales Handbuch, 175-186. Berlin/New York: De Gruyter.

Hennig, Mathilde (2001): Welche Grammatik braucht der Mensch? Grammatikenführer für Deutsch als Fremdsprache. München: ludicium.

Jacobs, Joachim (2008): Wozu Konstruktionen? In: Linguistische Berichte 213, 3-44. Jacobs, Joachim (2009): Valenzbindung oder Konstruktionsbindung? Eine Grundfrage der Grammatiktheorie. In: Zeitschrift für Germanistische Linguistik 37/3, 490-513.

Klein, Wolf Peter (2003): Sprachliche Zweifelsfälle als linguistischer Gegenstand. Zur Einführung in ein vergessenes Thema der Sprachwissenschaft. In: Linguistik Online 16, https://bop.unibe.ch/linguistik-online/article/view/793/1365 (letzter Zugriff: 16. 5. 2018).

Klein, Wolf Peter (2010): Grammatik zwischen Deskription und Präskription. In Mechthild Habermann (Hrsg.), Grammatik wozu? Vom Nutzen des Grammatikwissens in Alltag und Schule, 97-111. Mannheim u. a.: Duden.

König, Werner (1989): Atlas zur Aussprache des Schriftdeutschen in der Bundesrepublik Deutschland. 2 Bände. Ismaning: Hueber.

Konopka, Marek \& Eric Fuß (2016): Genitiv im Korpus. Untersuchungen zu starken Flexion des Nomens im Deutschen. Tübingen: Narr.

Konopka, Marek \& Bruno Strecker (Hrsg.) (2008): Deutsche Grammatik - Regeln, Normen, Sprachgebrauch. Berlin/New York: De Gruyter.

Konopka, Marek \& Angelika Wöllstein (Hrsg.) (2017): Grammatische Variation: Empirische Zugänge und theoretische Modellierung. Berlin/Boston: De Gruyter.

Krämer, Sybille \& Ekkehard König (Hrsg.) (2002): Gibt es eine Sprache hinter dem Sprechen? Frankfurt a. M.: Suhrkamp.

Linke, Angelika, Markus Nussbaumer, Markus \& Paul R. Portmann (1994): Studienbuch Linguistik. 2. Aufl. Tübingen: Niemeyer.

Meyer, Markus (2006): Grammatische Praxis. Probleme der grammatischen Theoriebildung und der Grammatikschreibung. Tübingen: Stauffenburg.

Osnabrücker Beiträge zur Sprachtheorie (1985) Heft 30. Deutsche Grammatik II.

Osnabrücker Beiträge zur Sprachtheorie (1984) Heft 27: Die „Grundzüge einer deutschen Grammatik" in der Diskussion.

Pasch, Renate, Ursula Brauße, Eva Breindl \& Ulrich Hermann Waßner (2003): Handbuch der deutschen Konnektoren. Linguistische Grundlagen der Beschreibung und syntaktische Merkmale der deutschen Satzverknüpfer (Konjunktionen, Satzadverbien und Partikeln). Berlin/New York: De Gruyter

Thurmair, Maria (2010): Grammatiken. In Hans-Jürgen Krumm, Christian Fandrych, Claudia Riemer \& Britta Hufeisen (Hrsg.), Deutsch als Fremd- und Zweitsprache. Ein internationales Handbuch, 293-304. Berlin/Boston: De Gruyter.

Ziem, Alexander \& Alexander Lasch (2013): Konstruktionsgrammatik. Konzepte und Grundlagen gebrauchsbasierter Ansätze. Berlin/New York: De Gruyter.

Zifonun, Gisela (1995): Beitrag zur Podiumsdiskussion: Grammatik - ja aber meine. In Vilmos Ágel \& Rita Brdar-Szabó (Hrsg.), Grammatik und deutsche Grammatiken. Budapester Grammatiktagung 1993, 253-260. Tübingen: Niemeyer.

Zifonun, Gisela (2009): Zum Sprachverständnis der Grammatikographie: System, Norm und Korpusbezug. In Marek Konopka \& Bruno Strecker (Hrsg.), Deutsche Grammatik Regeln, Normen, Sprachgebrauch, 333-354. Berlin/New York: De Gruyter. 


\title{
Mathilde Hennig 13 Wie funktional sind Grammatiken des Deutschen?
}

\begin{abstract}
In Grammatiken des Deutschen werden systematisch verschiedene funktionalgrammatische Möglichkeiten ausgelotet. Im Sinne der dafür grundlegenden Modellierung des Verhältnisses von Form und Funktion stellt der Beitrag Grammatiken vor, die funktionalgrammatische Ansätze ausgehend von der Form, ausgehend von der Funktion oder in der Wechselwirkung von Form und Funktion ausarbeiten. Der Umgang mit der Funktionalität erweist sich als ein zentraler Prüfstein der modernen Grammatikschreibung.
\end{abstract}

Keywords: Funktionale Grammatik, Grammatikschreibung

\section{Einleitung}

Die Frage nach einem angemessenen Verhältnis von Form und Funktion kann insbesondere seit den diversen sprach- und grammatiktheoretischen Entwicklungen des letzten Drittels des 20. Jahrhunderts als eine Gretchenfrage für Grammatiktheorie und Grammatikschreibung verstanden werden. Nachdem sich die Hinwendung zu Funktionsfragen zunächst quasi automatisch aus Abgrenzungsbestrebungen zur Generativen Grammatik ergab (vgl. Welke 1992: $9 \mathrm{ff}$.), führt - nicht zuletzt auch in Folge der sogenannten pragmatischen Wende die starke Ausdifferenzierung sprachgebrauchsbezogener Forschungsrichtungen in der Linguistik zu einer stärkeren Konzentration auf funktionale Fragen. Und schließlich bieten auch die Entwicklungen in der Korpuslinguistik in jüngerer Zeit sehr gute Möglichkeiten zur Rückbindung der Analyse grammatischer Formen an den Kontext ihres Gebrauchs.

Dabei gilt aber - wie so häufig als Folge der jüngeren Ausdifferenzierungsentwicklungen in der Wissenschaft -, dass sich hinter dem Stichwort ,funktionale Grammatik' keine einheitliche Theorie verbirgt, sondern ,funktionale Grammatik‘ ist vielmehr „ein Sammelname für sehr vielfältige Richtungen innerhalb der Grammatik“ (Welke 1992: 13). Gemeinsam ist verschiedenen funk-

Mathilde Hennig, Justus-Liebig-Universität Gießen, Institut für Germanistik, Otto-BehaghelStraße 10 B, D-35394 Gießen, E-Mail: Mathilde.Hennig@germanistik.uni-giessen.de

○ Open Access. (c) 2018 Mathilde Hennig, publiziert von De Gruyter. (๔) BY Dieses Werk ist lizenziert unter der Creative Commons Attribution 4.0 Lizenz. 
tionalen Theorien hingegen der Grundgedanke, „dass die Sprache nicht isoliert, sondern nur in Beziehung zu ihrer Rolle in der zwischenmenschlichen Kommunikation erforscht werden kann“ (Smirnova \& Mortelmans 2010: 13): „Sprache ist das Medium der Verständigung zwischen Menschen. Die Verständigung beruht auf der Funktionalität sprachlicher Formen.“ (Hoffmann 2013: 25).

Ziel des vorliegenden Beitrags ist kein allgemeiner Überblick über Modelle der funktionalen Grammatik - mit dem Studienbuch von Elena Smirnova und Tanja Mortelmans (2010) liegt eine sehr gute Übersicht vor (vgl. auch Helbig 1970; Hoffmann 1999; Wildgen 2010). Im Folgenden soll es vielmehr um die Rolle der funktionalen Grammatik in der germanistischen Linguistik bzw. in der Grammatikschreibung des Gegenwartsdeutschen gehen. In Bezug auf die germanistische Linguistik kann man nicht unbedingt von Schulen der funktionalen Grammatik sprechen. Zwar spielen Funktionsfragen in jüngerer Zeit in vielen Forschungsrichtungen eine zentrale Rolle - zu nennen ist hier vor allem die interaktionale Linguistik, aber - wie auch bei Smirnova \& Mortelmans - ebenso die Konstruktionsgrammatik und die Grammatikalisierungstheorie. Dabei handelt es sich aber quasi um Funktionalisierungen des Funktionsbegriffs für die jeweiligen Interessen und nicht um eine funktionale Schule i. e.S., wie sie etwa mit der jeweiligen Variante der funktionalen Grammatik nach Dik (1978) oder Halliday (1985) (vgl. Smirnova \& Mortelmans 2010: $17 \mathrm{ff}$., $49 \mathrm{ff}$.) vorliegen. In der DDR wurde zwar der Versuch einer eigenen funktionalen Theorie unternommen (insbesondere mit Schmidt 1973; vgl. dazu Helbig 1970: $162 \mathrm{ff} .$, 1984: $221 \mathrm{ff}$.), diese ist aber ohne langfristige Wirkung geblieben.

Während man also nicht von einer i.e.S. eigenständigen funktionalgrammatischen Schule in der germanistischen Linguistik sprechen kann, hat sich die Grammatikschreibung in den letzten 20-30 Jahren aber bezüglich des Form-Funktions-Verhältnisses sehr innovationsfreudig gezeigt. $\mathrm{Zu}$ nennen seien an dieser Stelle exemplarisch die IDS-Grammatik (1997) mit dem Grundprinzip der Doppelperspektivik, die Ausweitung der Gegenstände der Dudengrammatik auf Text und gesprochene Sprache seit der 7. Auflage (2005) sowie die funktional-pragmatische Ausrichtung der Grammatik von Ludger Hoffmann (2013). Dass es sich dabei um teilweise sehr unterschiedliche Typen von Innovationen handelt, liegt sicherlich an der sehr eigenen Dynamik der deutschen Grammatikenlandschaft.

Das Ziel des vorliegenden Beitrags besteht in einem Überblick über den Beitrag der deutschen Grammatikschreibung zur funktionalen Grammatik. Es geht also darum, aufzuarbeiten, inwiefern Grammatiken des Gegenwartsdeutschen als funktionale Grammatiken betrachtet werden können. Als Grundlage dafür wird in Abschnitt 2 zunächst ein Überblick über mögliche Funktionsbegriffe erarbeitet, der in Abschnitt 4 die Grundlage für die Einordnung 
der verschiedenen Zugänge zur Funktion in einschlägigen Grammatiken des Gegenwartsdeutschen bieten wird. Als weitere Grundlage dafür gibt Abschnitt 3 einen Überblick über die gegenwärtige Grammatikenlandschaft. In Abschnitt 5 werden zaghafte Schlussfolgerungen aus den Befunden zu den Aufgaben der künftigen Grammatikschreibung gezogen.

\section{Funktionsbegriff}

Wie viele andere Grundbegriffe der Linguistik ist auch der Begriff der Funktion mindestens genauso diffus wie schillernd. Helbig nennt ihn einen „Joker“ (1999: 108) und warnt vor einer undifferenzierten Verwendung. So unterscheidet er

- „eine syntaktische Funktion (entweder asemantisch-distributionell [...] oder funktional im Sinne der Satzglieder),

- eine semantische Funktion (entweder im Sinne eines innersprachlichen Inhalts oder im Sinne der Bezeichnung von außersprachlichen Sachverhalten $[. .]$.$) ,$

- $\quad$ eine kommunikative Funktion (entweder im Sinne von Sprecherintention und/oder kommunikativem Effekt oder im Sinne der Thema-RhemaGliederung), [...]

- $\quad$ eine logische Funktion (als Rolle im logischen Urteil - als Bezugspunkt des Satzes.“ (Helbig 1999: 108; meine Hervorhebungen, M. H.).

Smirnova \& Mortelmans arbeiten aus ihrer Übersicht über funktionale Theorien die folgenden drei Funktionsbegriffe heraus:

- eine soziale Funktion: Diese nimmt ,in allen besprochenen Modellen eine vorrangige Rolle ein“ (2010: 213): „Die Auffassung von Sprache als Kommunikationsmittel liegt allen funktionalen Sprachmodellen zugrunde [...].“ (2010: 214) Halliday etwa nennt die soziale Funktion interpersonale Funktion;

- $\quad$ eine symbolische bzw. kognitive Funktion: Für Langacker ist als symbolische Funktion die „Fähigkeit, Konzeptualisierungen durch Wörter (und Gesten) zu symbolisieren“ zentral (2010: 214); Smirnova \& Mortelmans sehen darin eine Verbindung zur von der Konstruktionsgrammatik und der Grammatikalisierungstheorie betonten kognitiven Funktion. Aber auch seit Halliday ist die kognitive Funktion als ideationelle Funktion präsent (2010: 215);

- eine textuelle Funktion: Diese ist nach Smirnova \& Mortelmans spezifisch für Halliday. So beginnt etwa die dritte Auflage seiner „Introduction to functional grammar“ mit dem Satz „When people speak or write, they produce text.“ (Halliday 2004: 3). 
Wie die kurze Zusammenschau bereits zeigt, sind die Möglichkeiten der Begriffsbestimmung vielfältig und dabei jeweils in die Zusammenhänge eines breiteren theoretischen Kontextes eingebettet. Hinzu kommt, dass für vergleichbare Funktionen teilweise unterschiedliche Termini verwendet werden so dürften wohl die kommunikative Funktion bei Helbig und die soziale Funktion bei Smirnova \& Mortelmans in etwa das Gleiche bezeichnen; wahrscheinlich würde Helbig die textuelle Funktion mit unter die kommunikative Funktion subsummieren. Smirnova \& Mortelmans kognitive Funktion ist bei Helbig in (außersprachlich-)semantische und logische Funktion untergliedert. Dass sich bei Smirnova \& Mortelmans kein Hinweis auf die syntaktische Funktion findet, dürfte daran liegen, dass diese aufgrund ihrer langen Tradition gerade keine zentrale Aufmerksamkeit der innovativen funktionalen Theorien auf sich zieht.

Da der Begriff der Funktion (unabhängig davon, ob er in der Grammatik Anwendung findet oder in einer anderen linguistischen Disziplin) immer auch in engem Zusammenhang mit den jeweiligen sprachtheoretischen Grundannahmen steht, erscheint es mir sinnvoll, anstelle einer weiteren Auflistung möglicher Funktionsbegriffe Zusammenhänge zwischen Sprachtheorie und Funktion zum Ausgangspunkt für einen Versuch der Systematisierung von Funktionsbegriffen zu machen. Auch hierin folge ich gewissermaßen Smirnova \& Mortelmans, die ihrem Überblick über Theorien der funktionalen Grammatik eine kurze Übersicht über zentrale Sprachbegriffe der letzten zwei Jahrhunderte voranstellen (2010: $1 \mathrm{ff}$.). Von den dort genannten fünf Sprachbegriffen - Sprache als Organismus, Sprache als Organ, Sprache als Werkzeug, Sprache als Tätigkeit und Sprache als System - sind sicherlich die Begriffe ,Sprache als Werkzeug' und ,als Tätigkeit‘ diejenigen, denen man am ehesten eine Affinität zu funktionalen Ansätzen zusprechen würde. Aber der Hinweis von Helbig auf die syntaktische Funktion macht bereits deutlich, dass durchaus auch die Vorstellung von Sprache als System einen Funktionsbegriff impliziert. Bei Eisenberg heißt es dazu: „Wenn das Sprachsystem insgesamt funktionieren soll, dann müssen seine Teilsysteme bis hin $\mathrm{zu}$ den einzelnen Einheiten im Sinne des Gesamtsystems funktionieren.“ (2010: 7). Eisenberg bezeichnet diese Art der Funktionalität als sprachimmanent und grenzt sie damit ab von einer sprachfunktionalen Funktionalität.

Besonders ausgeprägt ist der Zusammenhang zwischen System und Funktion bei Hallidays Variante der funktionalen Grammatik. Sie wird aus diesem Grund auch als „systemisch-funktionale Grammatik“ bezeichnet: „Das Attribut ,systemisch` weist auf eine allgemeine Sprachauffassung hin, der zufolge die Sprache als ein Netzwerk verstanden wird, das seinen Benutzern alternative Wahlmöglichkeiten beim Erzeugen und Kommunizieren unterschiedlicher 
Bedeutungen bereitstellt.“ (Smirnova \& Mortelmans 2010: 49). Zentral für Hallidays Systembegriff ist also die Auffassung, dass das (paradigmatische) System Wahlmöglichkeiten für die Realisierung von (syntagmatischen) Strukturen bereitstellt: „What that means is that each system - each moment of choice - contributes to the formation of the structure. [...] Structural operations inserting elements, ordering elements and so on - are explained as realizing systemic choices.“ (2004: 24). Hallidays Theorie ist laut Smirnova \& Mortelmans funktional, „insofern sie sich auf den Gebrauchsaspekt der Sprache in unterschiedlichen Kommunikationssituationen konzentriert“ (Smirnova \& Mortelmans 2010: 49): „[...] the grammar has to interface with what goes on outside language: with the happenings and conditions of the world, and with the social process we engage in.“ (2004: 24). Daraus lässt sich folgender Zusammenhang von System und Funktion ableiten: Das System stellt Wahlmöglichkeiten zur Verfügung, die im Rahmen der jeweiligen Kommunikationssituationen entsprechend funktionalisiert werden können (vgl. dazu auch allerdings ohne Bezug auf Halliday - Hennig 2017).

Doch zurück zu den Sprachbegriffen ,Sprache als Werkzeug' und ,Sprache als Tätigkeit‘. Eisenberg bezeichnet die Sprachtheorie von Bühler als das „wohl einflussreichste funktionale Sprachmodell“ (Eisenberg 2013: 9). Das liegt sicherlich nicht nur an der Werkzeugmetapher (siehe dazu Smirnova \& Mortelmans 2013: $4 \mathrm{f}$.), sondern vor allem daran, dass das Organonmodell funktional nicht eindimensional ist, sondern mit der Modellierung von drei Dimensionen, die laut Bühler dazu „berufen“ sind, das konkrete Schallphänomen „zum Rang eines Zeichens zu erheben“ (Bühler 1999: 28) eine mehrdimensionale Betrachtung der Funktionalität sprachlicher Zeichen erlaubt. Folglich lassen sich im Grunde genommen die meisten der mit Rückgriff auf Helbig und Smirnova \& Mortelmans genannten Funktionen mit dem Organonmodell in Beziehung setzen: Die soziale bzw. kommunikative Funktion korreliert mit Bühlers Ausdruck- und Appellfunktion, die kognitive bzw. logische und semantische Funktion mit Bühlers Darstellungsfunktion. Für die syntaktische Funktion hingegen wird über das Organonmodell von Bühler hinaus die von Morris (1988) modellierte syntaktische Dimension der Semiose (Beziehung mehrerer Zeichenträger untereinander) benötigt.

Während das Organonmodell Bühlers aufgrund seiner Mehrdimensionalität eine Verortung verschiedener Funktionsbegriffe erlaubt, legt die Humboldtsche Definition von Tätigkeit als „die sich ewig wiederholende Arbeit des Geistes, den artikulierten Laut zum Ausdruck des Gedanken fähig zu machen“ (Humboldt 1836: 418) zunächst eine kognitive Funktion von Sprache nahe. Indem - so Smirnova \& Mortelmans - es sich um eine Tätigkeit handelt, „in die sowohl Sprecher als auch Hörer involviert sind“ (2010: 6), kann auch der Bezug zur kommunikativen bzw. sozialen Funktion nicht ausgeschlossen werden. 
Funktionsbegriffe

\section{Sprachbegriffe}

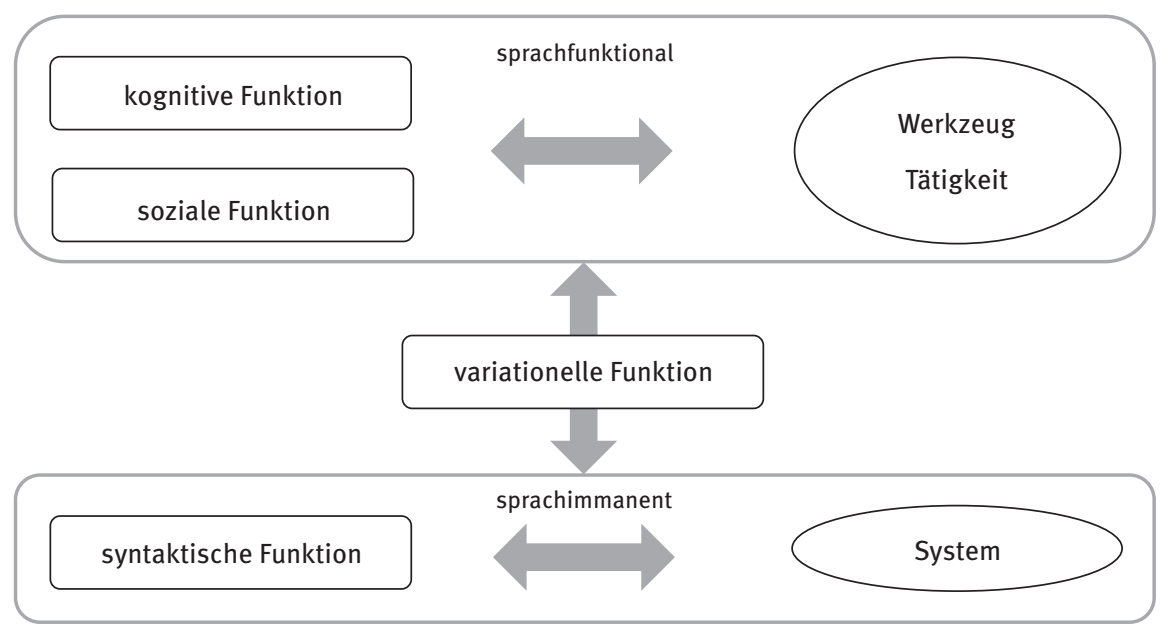

Abb. 13.1: Funktionsbegriff.

Abbildung 13.1 fasst die vorgestellten Überlegungen zum Funktionsbegriff zusammen.

Die Übersicht erhebt keinen Anspruch auf Vollständigkeit. Sie soll vielmehr eine grobe Orientierung bieten. Mit der Eisenbergschen Unterscheidung zwischen sprachimmanenter und sprachfunktionaler Funktionalität lässt sich m. E. eine erste Unterscheidung von zwei wesentlichen Klassen von Funktionsbegriffen vornehmen: Es ist ein zentraler Unterschied, ob mit ,Funktion' das Funktionieren innerhalb des sprachlichen Systems gemeint ist, oder ob der Begriff der ,Funktion“ auf Funktionen von Sprache im Verwendungszusammenhang abzielt. Innerhalb des Bereichs sprachexterner Funktionen - in der Terminologie Eisenbergs „sprachfunktional“ - können die kognitive und die soziale Funktion sicherlich relativ klar abgegrenzt werden. Da das umgekehrt nicht bedeutet, dass sie jeweils eindeutig auf den Begriff von Sprache als Werkzeug oder Tätigkeit bezogen werden können, werden diese in der Darstellung zusammengefasst. Der gegenüber Helbig und Smirnova \& Mortelmans neue Begriff der variationellen Funktion greift die vorgestellten Ideen Hallidays auf und ist hier quasi als Vermittler zwischen sprachimmanenter und sprachfunktionaler Funktion angelegt: Welche „systemic choices“ wir in einer konkreten Kommunikationssituation treffen, hängt vom komplexen Beziehungsgefüge der Kommunikationspartner ab, soziale und systemische Funktion werden 
also im Rahmen variationslinguistisch definierbarer Parameter aufeinander bezogen (vgl. Hennig 2017).

\section{Grammatiken des Gegenwartsdeutschen}

Das Deutsche verfügt über eine lange Tradition der Grammatikschreibung (vgl. Moulin 1988; Moulin-Fankhänel 1994; Eichinger 1984). Im 20. Jahrhundert erlebte die Grammatikschreibung insbesondere in den 80er Jahren einen Aufschwung - etwa mit der Akademie-Grammatik der DDR („Grundzüge“ 1980), der Erstausgabe von Peter Eisenbergs „Grundriß“ (1984), der vierten Auflage der Dudengrammatik (1984), der Grammatik von Ulrich Engel (1986) sowie der rezeptiven Grammatik von Hans Jürgen Heringer (1989). In den 90er Jahren folgte Harald Weinrichs „Textgrammatik“ (1993) sowie die IDS-Grammatik (1997). Der Grammatikenboom der 80er Jahre veranlasste Ulrich Engel zur Herausgabe des Themenheftes „Grammatiken eines Jahrzehnts“ im Jahrbuch für Deutsch als Fremdsprache (1990) sowie Vilmos Ágel und Rita Brdar-Szabó zur Durchführung einer Tagung mit dem Titel „Grammatik und deutsche Grammatiken“ 1993 (Tagungsband 1995). In ihrem Vorwort heißt es: „Anlaß zu der Tagung gab der erfreuliche Umstand, daß in den 80er Jahren eine Reihe neuer deutscher Grammatiken erschienen ist, aber auch der Umstand, daß die wachsende Zahl verschiedener grammatischer Konkurrenzunternehmen Inlands- wie Auslandsgermanisten vor immer größere Rezeptionsschwierigkeiten stellt.“ (1995: VII) Diese Einschätzung verleitete mich einige Jahre später zum Wagnis eines „Grammatikenführers für Deutsch als Fremdsprache“ (Hennig 2001). Aber auch nach der Jahrtausendwende sind neue bzw. stark überarbeitete Neuauflagen erschienen: Die radikale Neufassung der Dudengrammatik (siebente Auflage, 2005), die Grammatik von Ludger Hoffmann (2013), die „Grammatische Textanalyse“ von Vilmos Ágel (2017). Dabei wurden bislang nur diejenigen Grammatiken genannt, die im Sinne der Helbigschen Grammatikentypologie (2001) „linguistische“ Grammatiken sind, also solche, die „der wissenschaftlichen Abbildung [...] dienen“ (2001: 137) - als Gegenbegriff nennt Helbig die „didaktische“ Grammatik. Ein handhabbares Kriterium für die Wissenschaftlichkeit einer Grammatik dürfte ihre Zitationsfähigkeit in wissenschaftlicher Fachliteratur sein. Der Bereich der didaktischen Grammatiken für den Mutter-, Fremd- und Zweitsprachbereich ist sicherlich noch einmal um einiges umfangreicher und wächst gerade in jüngerer Zeit aufgrund der aktuellen gesellschaftspolitisch bedingten Aufwertung des Bereichs Deutsch als Zweitsprache erneut an. 
Wie kann die große Grammatikenfreudigkeit der Deutschen erklärt werden? Ganz offensichtlich ergibt sich diese zunächst aus der Einsicht, dass es „die Grammatik des Deutschen nicht gibt und nicht geben kann“ (Hennig 2001: 8), da unterschiedliche Benutzergruppen unterschiedliche Arten von Darstellungen verlangen. Helbig formuliert das folgendermaßen: ,jede wissenschaftliche Abbildung eines Objektbereichs [ergibt sich] nicht nur von den Objekten selbst und von unserem Kenntnisstand über den abzubildenden Objektbereich, sondern auch vom gesellschaftlichen Zweck und von „Konsumenten“ (Leser) der Abbildung“ (Helbig 1992: 136). Zugespitzt ausgedrückt: „Grammatiken werden geschrieben, um benutzt zu werden.“ (Hennig 2010: 20). In seinem Aufsatz „Grammatiken und ihre Benutzer“ unterscheidet Helbig (1992) in binären Oppositionen: Linguistische und pädagogische/didaktische Grammatiken, Lerner- und Lehrergrammatiken, Grammatiken für den Mutter- und Fremdsprachenunterricht, Resultats- und Problemgrammatiken, deskriptive und normativpräskriptive Grammatiken, konfrontative und nichtkonfrontative Grammatiken, Produktions- und Rezeptionsgrammatiken (interessanterweise findet sich hier nicht die Opposition ,formale vs. funktionale Grammatiken'). Das sind natürlich idealtypische Gegenüberstellungen, „weil eine Grammatik - schon aus praktischen Gründen - in der Regel mehreren Typen von Benutzern und Benutzungssituationen dienen muß“ (Helbig 1992: 137). In Hennig \& Löber (2010) plädieren wir deshalb für ein offeneres Benutzertypenmodell ausgehend von den Benutzerdimensionen ,beruflicher, sprachlicher und situativer Hintergrund‘.

Die ausdifferenzierte Benutzerorientierung ist aber sicherlich nicht der alleinige Grund für die breit gefächerte Grammatikenlandschaft des Gegenwartsdeutschen. Gerade die hier genannten wissenschaftlichen Grammatiken unterscheiden sich ja nicht wesentlich in ihrer Zielgruppenorientierung: Sie richten sich vor allem an Germanistikstudenten, aber auch an die Fachkollegen. Hier zeigt sich, dass der Textsorte Grammatik (im Sinne einer umfassenden Überblicksdarstellung zum grammatischen System einer Einzelsprache) eine hohe Bedeutung in der germanistischen Grammatikforschung beigemessen wird: Im Gegensatz zu Veröffentlichungen zu grammatischen Einzelphänomenen bieten diese die Möglichkeit, das jeweilige Grammatikverständnis in seiner gesamten Breite auszubuchstabieren.

\section{Funktionale Ansätze in Grammatiken des Gegenwartsdeutschen}

Im Folgenden wird nun der Frage nachgegangen, welche Ansätze zur Berücksichtigung von Funktionalem in Grammatiken des Gegenwartsdeutschen aus- 
gemacht werden können. Bei der Auswahl der berücksichtigten Grammatiken ist also entscheidend, ob eine dezidierte offenbarte oder zumindest klar erkennbare Funktionsorientierung vorliegt. Die getroffene Auswahl erhebt aber keinen Anspruch auf Vollständigkeit (vgl. auch Hoffmann 1999).

Im Grunde genommen ist die Gretchenfrage ,Wie hältst du's mit der Funktion?“ immer eine Frage nach dem Verhältnis von Form und Funktion. Da das Form-Funktions-Zusammenspiel ein Grundanliegen der DDR-Variante der funktionalen Grammatik war, bringt Schmidt das in seiner Einführung in die funktionale Sprachlehre gut auf den Punkt:

Das Wesen der funktionalen Sprachbetrachtung besteht darin, daß sie grundsätzlich immer beide Pole der Form-Funktion-Korrelation im Auge behält. Dabei kann die funktionale Betrachtung sowohl von der Form wie auch von der Funktion ausgehen, sie kann die Akzente in der Darstellung unterschiedlich setzen, je nach der besonderen Zielsetzung der Untersuchung. Entscheidend ist nur, daß sie nicht bei der isolierten Betrachtung der einen Seite der Wechselbeziehung stehenbleibt und daß sie den sprachlichen Kommunikationsakt immer in der Absicht untersucht, die Gesetzmäßigkeiten des Funktionierens der sprachlichen Mittel und ihre funktional bedingte Ordnung aufzudecken. (Schmidt 1973: 32)

Die Einschätzung, dass die funktionale Betrachtung sowohl von der Form als auch von der Funktion ausgehen könne, soll hier zum Ausgangspunkt für eine Sortierung der funktionalen Ansätze deutscher Gegenwartsgrammatiken gemacht werden. Prinzipiell ergeben sich die folgenden Möglichkeiten:

1. Form $\rightarrow$ Funktion

2. Funktion $\rightarrow$ Form

3. Form $\leftrightarrow$ Funktion

Die Möglichkeiten werden potenziert, wenn man jeweils die verschiedenen in Kapitel 2 zusammengefassten Funktionsbegriffe einbezieht. Die Frage, was für ein Funktionsbegriff der jeweiligen Darstellung zugrunde liegt, wird deshalb eine wichtige Rolle bei der Auswertung der Grammatiken spielen.

\subsection{Form $\rightarrow$ Funktion}

Der Weg von der Form zur Funktion kann sicherlich als die klassische Herangehensweise beschrieben werden. Bereits die übliche Gliederung in Abschnitte wie „Wort“ und „Satz“ (so der Titel der beiden Bände von Eisenbergs Grundriss), „die einzelnen Wortarten“ und „Satz“ (Helbig \& Buscha 2001) oder „Phonem und Graphem“, „das Wort“, „der Satz“, „der Text“, „die gesprochene Sprache“ (Dudengrammatik 2005/2016) dokumentiert das Ausgehen von der Form. Dabei wird meist ein Weg von der kleineren zur größeren Form be- 
schritten. Die umgekehrte Richtung vom Text zum Satz zum Wort findet sich bspw. bei Engel (1988) sowie Ágel (2017).

Als Grammatiken, die bei einem Ausgehen von der Form einen dezidierten funktionalen Ansatz verfolgen, seien hier Eisenbergs „Grundriss der deutschen Grammatik“ (2013), Ágels „Grammatische Textanalyse“ (2017) sowie die Dudengrammatik (seit 2005) genannt.

Auf Eisenbergs Unterscheidung zwischen sprachimmanenter und sprachfunktionaler Funktionalität sowie seinen Hinweis auf die Bedeutung der Sprachtheorie von Bühler für funktionale Ansätze wurde bereits in Abschnitt 2 hingewiesen. Eisenberg geht dabei davon aus, dass „die Sprachfunktionen nicht gleichgewichtig sind, sondern dass die Darstellungsfunktion grundlegend ist. Die Darstellungsfunktion [...] bestimmt im Wesentlichen die Sprachstruktur“ (Eisenberg 2013: 10). Folglich steht die soziale Funktion nicht im Mittelpunkt seines funktionalen Ansatzes. Zur Frage, ob die variationelle Funktion Gegenstand der Grammatikschreibung sein solle, äußert er sich kritisch in seinem Aufsatz „Sollen Grammatiken die gesprochene Sprache beschreiben?“ (Eisenberg 2007). Was Eisenberg mit seiner Konzentration auf die Darstellungsfunktion meint, erläutert er am Beispiel kurzer, aus einem Nomen und einem Verb bestehender Sätze. So werden Nomen und Verb „als Formkategorien etabliert, denen man nun eine je spezifische Leistung zuschreiben möchte.“ (Eisenberg 2013: 10) Als grundlegende Leistung des Nomens beschreibt er die Referenz, als die des Verbs die Prädikation; das Zusammenspiel der beiden im Satz bildet die Proposition. Als weitere Beispiele für zentrale Funktionen von syntaktischen Strukturen benennt er die Klassifizierung von Sachverhalten in Zustand und Vorgang (Ereignis und Handlung) sowie den Satzmodus. Ausgehend davon formuliert er das folgende Ziel für seine Grammatik:

Unsere Grammatik soll die Form sprachlicher Einheiten so beschreiben, dass der Zusammenhang von Form und Funktion deutlich wird. Alle Formmerkmale, die funktional von Bedeutung sein können, soll die Grammatik erfassen. Das heißt aber nicht, dass alles Funktionale ein Formkorrelat hat, noch heißt es, dass jeder Formunterschied funktional relevant ist. Es interessiert der Zusammenhang, seine Explikation ist das Ziel einer funktionalen Grammatik. (Eisenberg 2013: 11)

Neben der Darstellungsfunktion im Sinne Bühlers, die von den in Abschnitt 2 genannten grundlegenden Funktionsbegriffen sicherlich am ehesten der kognitiven Funktion zugeordnet werden kann, spielt die syntaktische Funktion bei Eisenberg eine gewichtige Rolle. Sein Ansatz der syntaktischen Relationen trägt dem Umstand Rechnung, dass sich „die Bedeutung eines Satzes [...] allein aufgrund der Bedeutungen der elementaren Einheiten und des semantischen Gehalts der syntaktischen Relationen“ ergibt (Eisenberg 2013: 45). Eisenberg 
sieht die Leistung der „relationalen Redeweise“ darin, dass man mit ihr „auf eine einfache Weise ausdrücken [kann], dass syntaktische Einheiten mit einer bestimmten Form [...] in unterschiedlicher syntaktischer Funktion vorkommen“ (Eisenberg 2013: 41). Die wichtigsten relationalen Begriffe sind auch bei Eisenberg die Satzgliedbegriffe, die ,eine Konstituente nicht für sich selbst und unabhängig von der Umgebung [kennzeichnen], sondern [...], welche Funktion die Konstituente innerhalb einer größeren Einheit hat“ (Eisenberg 2013: 39). Im Sinne der Teuberschen (2005) Unterscheidung zwischen Schwesterrelationen (= Komplement- und Adjunktrelationen) und Tochter-Mutter-Relationen (= Kopf- und Kernrelationen) als Typen von Konstituenzrelationen integriert Eisenberg auch letztere konsequent in seine Darstellung syntaktischer Funktionen. Dem Status beider Typen von Relationen als Konstituenzrelationen wird Eisenberg gerecht, indem er in seiner Darstellung von Konstituentenstrukturen systematisch syntaktische Kategorien und Relationen aufeinander bezieht (vgl. exemplarisch die zusammenfassenden Beispiele in Eisenberg 2013: 54 f.).

Durch die Berücksichtigung der beiden genannten Typen syntaktischer Relationen erhalten bei Eisenberg nicht nur die Satzglieder den Status von syntaktischen Funktionen, sondern auch die unterhalb der Satzgliedebene liegenden Teile von Wortgruppen. In seiner „Grammatischen Textanalyse“ spricht Ágel deshalb von „Wortgruppengliedern“. Er plädiert aber vor allem dafür, „die begriffliche Basis der Satzgliedlehre“ nicht nur „nach unten - in Richtung Wortgruppenglieder“, sondern auch nach oben, in Richtung Text auszudehnen (Ágel 2017: 24). So bedeutet „Textorientierung“ für ihn, „dass die grammatische Analyse auf der Textebene - und nicht erst auf der Satzebene ansetzt und dass auch Sätze als grammatische Einheiten der Textebene begriffen werden.“ (Ágel 2017: 3). Als Textglieder nimmt er Sätze, Nichtsätze und Kohäsionsglieder an (Ágel 2017: 13 ff., 24 ff., 61 ff.).

Ágel betrachtet die konkreten Realisierungen der Textglieder, Satzglieder und Wortgruppenglieder nicht als Funktionen, sondern als Werte. Die zentrale funktionalgrammatische Grundlage seiner Grammatik bildet die folgende aus der Logik stammende Formel:

$$
\mathrm{F}(\mathrm{A})=\mathrm{W}
$$

„Diese Formel besagt, dass die Anwendung einer bestimmten Funktion (= F) auf ein bestimmtes Argument (= A) einen bestimmten Wert (= W) ergibt.“ (2017: 18) Als „allgemeine grammatische Formel“ (Ágel 2017: 22) ergibt sich daraus:

grammatische Funktion (grammatische Form) = grammatischer Wert (2017: 23) 
Angewendet auf die Satzglieder bedeutet das:

satzgrammatische Funktion (grammatische Form) = Satzglied (Ágel 2017: 23)

Eine Anwendung dieser Formel auf das aus der Eisenberg-Grammatik bekannte Beispiel

Sie besteht auf der neuen Startbahn.

ergibt

Objekt (Präpositionalgruppe) = Präpositionalobjekt

Die Funktion-Argument-Wertformel wird in der Grammatischen Textanalyse auf die drei Ebenen Makro, Meso und Mikro angewendet: „Textglieder sind grammatische Werte auf der Makroebene, Satzglieder grammatische Werte auf der Mesoebene und Wortgruppenglieder grammatische Werte auf der Mikroebene.“ (Âgel 2017: 23)

Als ein weiteres zentrales funktionalgrammatisches Anliegen der „Grammatischen Textanalyse“ kann der systematische Bezug syntaktischer Grundstrukturen auf semantische Grundstrukturen gewertet werden. So kann beispielsweise

„eine grammatische Grundstruktur wie

Subjekt $\quad$ - Prädikat - Akkusativobjektnebensatz

eine semantische Grundstruktur wie

HANDLUNGSTRÄGER - HANDLUNG - HANDLUNGSSACHVERHALT indizieren“ (Ágel 2017: 5).

Ágel verfolgt dabei einen signifikativ-semantischen Ansatz, d. h., beschrieben werden hier „einzelsprachlich perspektivierte Sachverhalte“ (Ágel 2017: 5). Das bedeutet, dass nicht im denotativ-semantischen Sinn von einer Abbildung von außersprachlichen Situationen ausgegangen wird, sondern dass die jeweilige formale Realisierung stets ernst genommen wird. Insofern zeigt sich hier, dass eine funktionale Orientierung keineswegs bedeuten muss, dass die Formseite vernachlässigt wird. Die auf diese Weise erfasste Funktion lässt sich als semantische Funktion einordnen. Aufgrund des signifikativ-semantischen Ansatzes gilt für diese aber nicht die Begriffsbestimmung von Helbig (1999: 108; vgl. 
Abschnitt 2) und es gilt auch nicht die Einschätzung, dass die semantische Funktion als Teilbereich der kognitiven Funktion aufgefasst werden kann. Vielmehr handelt es sich bei dem Ágelschen Verständnis der semantischen Funktion um eine sprachimmanente Funktion, die also im Modell der Funktionsbegriffe (Abb. 13.1) neben der syntaktischen Funktion in diesem Bereich zu verorten wäre.

Wenn nun die Dudengrammatik als ein weiteres Beispiel für einen vorrangig von der Form ausgehenden funktionalen Ansatz vorgestellt wird, so soll dabei vor allem die variationelle Funktion in den Blick genommen werden. Das bedeutet nicht, dass die Grammatik keine anderen funktionalen Aspekte enthält. Bspw. bietet die „Übersicht über die Satzglieder“ (Duden 2016: 807 f.) einen systematischen Bezug syntaktischer Formen und Funktionen. Die seit der 7. Auflage (Duden 2005) bestehenden großen Kapitel „der Text“ und „die gesprochene Sprache“ bilden aber sicherlich den herausragendsten funktionalen Ansatz dieser Grammatik. Mit den genannten beiden Kapiteln geht die Dudengrammatik über den traditionellen Gegenstand der Grammatik hinaus. In der Auflage von 2005 wird das im Vorwort relativ lapidar wie folgt begründet: „Sie kann damit [mit der Erläuterung der Eigenschaften von Texten sowie dem Kapitel zur Grammatik der gesprochenen Sprache, M. H.] auch zur Klärung von Normunsicherheiten herangezogen werden, die sich aus der Differenz zwischen geschriebener und gesprochener Sprache ergeben." (Duden 2005: 5 f.). Gerade das würde allerdings eine systematischere Bezugnahme der dem traditionellen Schema folgenden Teile sowie des Kapitels zur gesprochenen Sprache aufeinander erfordern, die bislang nicht erfolgt (vgl. Hennig 2006: 83).

Das Kapitel „Der Text“ kann eigentlich im Sinne der IDS-Grammatik (siehe 4.3) als doppelperspektivisch eingeordnet werden. So weist insbesondere der Teil zur Textkohäsion sowohl formale als auch funktionale Gliederungsaspekte auf: Zwar erfolgt zunächst eine Unterteilung nach textkohäsiven Mitteln, insbesondere die Darstellung zur Textkohäsion durch Konnektoren ist aber eher funktional perspektiviert: Den zentralen Ausgangspunkt der Bestandsaufnahme bilden die Bedeutungsrelationen von Konnektoren (Duden 2016: $1091 \mathrm{ff}$.), hier werden den semantischen Relationen die verschiedenen formalen Realisierungsmöglichkeiten zugeordnet.

Während man in Bezug auf das von Thomas Fritz verfasste Textkapitel von einem ausgewogenen Verhältnis der Perspektiven Form und Funktion sprechen kann, ist das von Reinhard Fiehler stammende Kapitel zur gesprochenen Sprache stärker funktional ausgerichtet. Es liest sich wie eine Einführung in die gesprochene Sprache; grammatische Formen im engeren Sinne finden sich im Unterkapitel „Besondere syntaktische Konstruktionen“ - mit den aus der Gesprochene-Sprache-Forschung bekannten Themen wie Verbzweitsätze nach 
weil, obwohl, wobei, während und Strukturen am linken und rechten Satzrand wie Referenz-Aussage-Strukturen und Expansionen. Die weiterführenden Teile etwa zum Verhältnis gesprochener und geschriebener Sprache, zu den Grundbedingungen mündlicher Verständigung sowie zum Gespräch illustrieren, inwiefern ein radikaler funktionaler Perspektivwechsel auch dazu führt, dass das Verständnis von ,Grammatik‘ neu ausgelotet werden muss (vgl. Hennig 2002, 2018).

\subsection{Funktion $\rightarrow$ Form}

Mit den Grammatiken von Peter Eisenberg sowie der Dudengrammatik wurden Beispiele für Schwerpunktsetzungen auf die syntaktische und die variationelle Funktion im Sinne von Abschnitt 2 vorgestellt. In der Grammatik von Ágel spielt neben der syntaktischen Funktion auch die semantische Funktion als sprachimmanente Funktion eine wichtige Rolle. Mit dem Perspektivwechsel zur Funktion als Ausgangspunkt ist automatisch auch eine stärkere Fokussierung der sprachfunktionalen Funktionalität im Sinne von Eisenberg verbunden. Dabei sind die Übergänge zwischen kognitiver und sozialer Funktion häufig fließend, sodass im Folgenden nicht der Versuch einer eindeutigen Zuordnung zu dem ohnehin groben Raster unternommen wird.

Wenn hier im Folgenden insgesamt vier Grammatiken diskutiert werden, die den Weg von der Funktion zur Form einschlagen, so mag dadurch der Eindruck entstehen, dass es sich dabei inzwischen um eine gleichberechtigte Strategie neben der in 4.1 vorgestellten handelt. Dieser Eindruck trügt aber sicherlich, denn für die Ausführungen in 4.1 wurden ja nur wenige Grammatiken ausgewählt, die ausgehend von der Form bestimmte funktionale Schwerpunkte setzen. Außerdem handelt es sich bei den im Folgenden vorzustellenden Ansätzen nicht um den Standardtyp „einzelsprachliche wissenschaftliche Grammatik“, sondern drei der Grammatiken haben einen starken didaktischen Schwerpunkt, eine ist kontrastiv.

Dass sich gerade die didaktischen Grammatiken besonders innovationsfreudig zeigen, indem sie die Perspektive umkehren und von der Funktion ausgehen, ist sicherlich kein Zufall: „Für unser Verständnis einer funktionalen Grammatik für Lernende des Deutschen als Fremdsprache ist die entscheidende Frage, welche Funktion in der Kommunikation eine bestimmte Struktur bzw. eine Form X hat.“ (Götze 1999: 83). Dem kann man sicherlich leicht zustimmen. Ob daraus umgekehrt folgt, dass der Weg von der Funktion zur Form der sinnvollere ist, kann hingegen kaum als unstrittig angesehen werden. Vor allem aber stellt sich die Frage, was eigentlich die Funktionen sind, die den Ausgangspunkt einer funktionsorientierten Grammatikdarstellung 
bieten sollen. Während sich Grammatiken, die den Weg von der Form zur Funktion bestreiten, auf ein teilweise über Jahrhunderte bzw. sogar Jahrtausende etabliertes Set an formalen Kategorien stützen können, kann von einem vergleichbaren Inventar an einschlägigen Funktionen keine Rede sein. So konstatiert Freudenberg-Findeisen zu Recht: „Eine von Funktionen ausgehende Sicht auf grammatische Gegenstände als Ausgangspunkt der Sprachbeschreibung ist aber keine schlichte Umkehrung bisheriger Darstellungsarten, sondern birgt neue Fragestellungen in sich.“ (Freudenberg-Findeisen 2000: 22).

$\mathrm{Zu}$ was für unterschiedlichen Ergebnissen die Aufbereitung grammatischer Themen nach Funktionen führen kann, zeigt eindrücklich der Vergleich der „Kommunikativen Grammatik“ von Ulrich Engel und Rozemaria Tertel (1993) sowie der „Grammatik in Feldern“ von einem Autorenkollektiv und Joachim Buscha und Renate Freudenberg-Findeisen (1998). Eine Vergleichbarkeit ist gegeben, weil beide sich an fortgeschrittene DaF-Lerner richten. „Grammatik in Feldern“ ist als Lehr- und Übungsbuch konzipiert; in ähnlicher Form bieten Engel \& Tertel ihre Grammatik als Basiswerk oder als Ergänzung zu anderen Lehrwerken an.

Die insgesamt 30 Kapitel in der Grammatik von Engel \& Tertel tragen Titel wie „Mitteilen und Fragen“, „Personen und Gegenstände benennen“, „Abgeschlossenheit“ aber auch „Determination“, „Valenz“, „Steigerung und Vergleich“, „Textwiedergabe“ und „Benennen von Sachverhalten“. Im Vorwort nennen die Autoren diese Themen „kommunikative Bedürfnisse“:

Wir [...] gehen davon aus, daß die Menschen insgemein nicht reden, um einfach und irgendwie miteinander zu reden, sondern daß sie sich etwas zu sagen haben, daß sie etwas mitteilen oder etwas erfahren, etwas veranlassen oder verhindern wollen und vieles andere mehr. Das aber sind allesamt keine grammatischen Kategorien, sondern Kategorien der Kommunikation: man verständigt sich, weil man im Hinblick auf den/die anderen bestimmte Bedürfnisse hat, und man spricht miteinander, weil man diese Bedürfnisse nur mit sprachlichen Mitteln ausdrücken und letzten Endes erfüllen kann. (Engel \& Tertel 1993: 7)

Ob nun etwa Valenz oder Determination gleichermaßen ein Bedürfnis ist wie „Mitteilen und Fragen“ und „Personen und Gegenstände benennen“, sei hier erst einmal dahingestellt. Die entscheidende Frage für die Beurteilung des vorliegenden funktionalen Ansatzes ist hingegen, in welchem Verhältnis die postulierten „kommunikativen Bedürfnisse“ zu den grammatischen Formen stehen. So finden sich bspw. im Kapitel „Möglichkeit, Notwendigkeit, Einschätzung“ Angaben zu Modalverben, Modalitätsverben und modalen Partikeln; in den beiden Kapiteln zu „geschehensbezogener Ausdrucksweise“ geht es im Grunde genommen um Deagentivierung (also Passiv und andere Formen der Agensvermeidung). M. a. W.: Die Grammatik enthält durchaus die üblichen und erwartbaren grammatischen Themen, nur sind sie eben anders angeordnet als 
in formbezogenen Darstellungen. Ob das tatsächlich im Sinne der Adressatenorientierung funktioniert, ob also bspw. die Aufteilung verschiedener Nebensatztypen auf Kapitel wie „Sprechen mit Vorbehalt“ (Konditionalsätze) und „Einen Zweck benennen“ (Finalsätze) und „Personen, Gegenstände u.ä. näher bestimmen“ (Relativsätze) im Sinne der „kommunikativen Bedürfnisse“ den Nutzerinteressen entgegenkommt, kann hier nicht beurteilt werden. Aufschlussreich ist allerdings, dass die Autoren es doch als notwendig erachtet haben, in einem Anhang ein Verzeichnis der Fachausdrücke, Flexionstabellen sowie Listen der geschlossenen Wortklassen zur Verfügung zu stellen.

In „Grammatik in Feldern“ erfolgt eine Aufarbeitung grammatischer Phänomene nach „Inhaltsbereichen“. Das Konzept der funktional-semantischen Felder hat seinen Ursprung in der russischen Linguistik (zu einer deutschen Fassung vgl. Bondarko 2007). Bei Bondarko findet sich die folgende Begriffsbestimmung: „Das funktional-semantische Feld (= FSF) wird von uns als eine semantische Kategorie betrachtet, die zusammen mit dem System ihrer Ausdrucksmittel in der zu analysierenden Sprache zu untersuchen ist (vgl. solche Felder wie Temporalität, Taxis, Qualität, Quantität, Lokalität u. dgl.).“ (Bondarko 2007: 23). Auch Buscha \& Freudenberg-Findeisen sehen das Potenzial des Ansatzes darin, dass den funktional-semantischen Feldern bestimmte Sprachmittel entsprechen: „Damit wird ein semantisch-pragmatischer Ausgangspunkt gewählt, der es gestattet, sprachliche Mittel aller Sprachebenen zu erfassen: Neben grammatischen treten auch lexikalische, wortbildende, idiomatische, textgrammatische wie prosodische Mittel in den Beschreibungsblickwinkel.“ (Buscha \& Freudenberg-Findeisen 2007: 9). Der Feldergrammatikansatz stelle somit „,Sprachmittelreservoirs“ zusammen, die dem Sprecher bzw. Schreiber für die Versprachlichung einer bestimmten Intention zur Verfügung stehen" (Buscha \& Freudenberg-Findeisen 2007: 9). Die zentrale Aufgabe der Feldergrammatik besteht aber nicht nur darin, die sprachlichen Mittel zusammenzustellen, die aus funktional-semantischer Perspektive zusammengefasst werden können, sondern sie muss auch zeigen,

wodurch sich die einzelnen Ausdrucksmöglichkeiten im Feld unterscheiden, d. h. welche syntaktischen Eigenschaften sie besitzen, welche semantisch-pragmatischen Nuancierungen durch die einzelnen Sprachmittel erzielt werden können, welchen konnotativen Mehrwert sie u. U. einzubringen vermögen und vor allem, welchen kommunikativen Verwendungsbedingungen die einzelnen Sprachmittel unterliegen (Buscha \& FreudenbergFindeisen 2007: 10).

Die in der „Grammatik in Feldern“ berücksichtigten Felder sind: Feld der Person, Feld der Begründung, Feld der Bedingung, Feld der Absicht, Feld der Folge, Feld des Widerspruchs, Feld des Vergleichs, Feld der Aufforderung, Feld des 
Wunsches und Feld der Vermutung. Am Beispiel des Feldes der Absicht sei die Darstellungsform der „Grammatik in Feldern“ illustriert: Zunächst werden als Teilfelder der Absicht die „Zielgerichtetheit von Handlungen“ sowie die „Zweckbestimmtheit von Handlungen“ bestimmt. Als grammatische Sprachmittel des Teilfeldes A werden Satzgefüge mit damit, um zu etc. sowie Satzverbindungen mit dafür, dazu, hierfür etc. bestimmt, schließlich auch präpositionale Wortgruppen mit Präpositionen wie zugunsten, zwecks, um .... willen. Als grammatische Sprachmittel für Teilfeld B werden Präpositionalgruppen mit Präpositionen wie als, für, zu benannt. Lexikalische Sprachmittel sind hier Komposita mit einem den Zweck angebenden Bestimmungswort wie Bindfaden und Studienreise sowie Substantive wie Aufgabe, Funktion, Zweck und Verben wie dienen, geeignet sein, bestimmt sein.

Die zentrale Frage, die an diesen Ansatz genauso gerichtet werden kann wie an den von Engel \& Tertel, ist, wie die Auswahl der „Felder“ resp. „kommunikativen Bedürfnisse“ begründet werden kann. Die Zusammenstellung in der „Grammatik in Feldern“ weist interessanterweise einen großen Anteil an Überschneidungen mit dem Kapitel „Textkohäsion durch Konnektoren“ der Dudengrammatik auf. Neben den für die Darstellung von Satzverknüpfungen häufig genutzten semantischen Relationen ist die Kategorie Satzmodus hier noch eine zentrale Größe. Hingegen finden sich gerade nicht die von Bondarko genannten Felder wie Temporalität und Quantität.

Auch die „Deutsche Grammatik“ von Ludger Hoffmann (2013) kann als ein funktionalgrammatischer Ansatz beschrieben werden, der neben den damit verbundenen sprachtheoretischen Grundannahmen auch stark sprachdidaktisch motiviert ist. Dabei ist - wie der Untertitel „Grundlagen für Lehrerausbildung, Schule, Deutsch als Zweitsprache und Deutsch als Fremdsprache“ bereits erkennen lässt - die Zielgruppe aber breiter als bei den beiden zuvor vorgestellten, auf Deutsch als Fremdsprache konzentrierten Ansätzen. Die didaktische Perspektivierung ist unmittelbar daran erkennbar, dass in den in der Einleitung formulierten Prinzipien funktionalgrammatische Grundannahmen stets mit didaktischen Prämissen verknüpft werden. Offenbar geht es dabei Hoffmann nicht zuletzt auch darum, zu einer Überwindung der „Krise des Grammatikunterrichts“ (2013: 13) beizutragen.

Ludger Hoffmann betrachtet Grammatik als „das Formensystem einer Sprache, das ausgebildet ist, um die Zwecke der Handelnden zu erfüllen“ (2013: 14). Indem Hoffmann davon ausgeht, dass „die Bedürfnisse menschlicher Praxis fundamental für die Ausbildung des Formensystems sind“ (2013: 14), ergibt sich trotz aller Unterschiede auch eine Vergleichbarkeit mit den beiden zuvor vorgestellten Grammatiken. In dem Kapitel „Vorüberlegungen zu einer funktional-pragmatischen Grammatik“ in Hoffmann (1999) hat er u. a. die folgenden Grundsätze einer funktionalen Grammatik vorgestellt: 
Grundsatz 1: Gegenstand der Syntax ist nicht ein Moment des sprachlichen Wissens, das Grammatikalitätsurteile erlaubt, sondern die Frage, in welcher Weise die Struktur von Äußerungen [...] ihren Beitrag zum Verständigungshandeln zwischen Sprechern und Hörern bestimmt.

Grundsatz 2: Der Aufbau einer Äußerung ist durch die in ihr enthaltenen Sprachmittel mit ihren Funktionen und durch das Zusammenwirken der Mittel (funktionale Synergetik) bestimmt. [...]

Grundsatz 4: Jede Form bestimmt sich durch die von ihr realisierte (einfache oder komplexe) Funktion im Rahmen von Wissensprozessierung und Verständigungshandeln. Insofern ist Syntax nicht autonom.

Grundsatz 5: Das Verstehen einer Äußerung setzt das Verstehen aller funktionalen Beziehungen in der Äußerung voraus. (Hoffmann 1999: 52)

Die funktional-pragmatische Grundlegung seiner Grammatik ist bereits in der Benennung der zentralen Teile sichtbar: C Redegegenstände, D Gedanken formulieren, E Ausbau von Gedanken, F Gedanken verknüpfen und erweitern, G Abfolge und Gewichtung, H Zweckbereiche des Handelns (ein Überblick über den Aufbau der Grammatik findet sich als Übersicht auf S. 23). Im Sinne der bereits angesprochenen engen Verknüpfung von handlungstheoretischem funktionalgrammatischem Ansatz und didaktischer Zielsetzung beschreibt Hoffmann den Aufbau seiner Grammatik als „durch eine Sachlogik - das Konzept Didaktischer Pfade - bestimmt“ (Hoffmann 2013: 22). Die Orientierung am sprachlichen Handeln manifestiert sich auch in Unterkapiteln wie „Themen einführen, fortführen, entwickeln“, „Transfer von Wissen“, „Koordination von Handlungen“. Den folglich insgesamt stark durch den funktional-pragmatischen Ansatz Hoffmanns geprägten Funktionen sind durchaus klassische grammatische Formbereiche zugeordnet wie Perspektive - Aktiv, Passiv; Koordination Konjunktoren und Präpositionalgruppen; Spezifizieren - Adverbien und Adverbialsätze. Der grundständige schulgrammatische Apparat - also vor allem Wortarten und Satzglieder - findet sich hingegen im den funktional-pragmatisch strukturierten Kapiteln vorgelagerten Kapitel B „Grundbegriffe“.

Die großen Unterschiede in den skizzierten Beispielen für einen Perspektivwechsel von der Form zur Funktion zeigen, dass es offenbar keineswegs auf der Hand liegt, was eigentlich die zentralen Funktionen sind, die zum Ausgangspunkt einer funktional motivierten Gruppierung grammatischer Formen gemacht werden sollen. Dennoch dürfte die Innovationsfreudigkeit der vorgestellten Ansätze wichtige Impulse für die weitere Profilierung funktionalgrammatischer Ansätze bieten.

Als ein letztes Beispiel für eine Grammatik, die den Weg von der Funktion zur Form wählt, sei hier der kürzlich erschienene Teil zum Nominal der „Grammatik des Deutschen im europäischen Vergleich“ (Gunkel et al. 2017) genannt. 
Hier ist das Ausgehen von der Funktion nicht didaktisch motiviert, sondern durch den Sprachvergleich:

\begin{abstract}
In sprachtypologischen Untersuchungen und sprachvergleichenden Grammatiken ist der Rekurs auf die Funktion grammatischer Einheiten notwendigerweise bedeutsamer als in einer einzelsprachlichen: Während in einer deutschen Grammatik - für den Muttersprachler - die Funktionalität grammatischer Konstruktionen und Kategorien ggf. als bekannt vorausgesetzt und implizit bleiben kann, ist der Sprachvergleich - ob auf den universalen Vergleich oder auf die Kontrastierung weniger Sprachen ausgerichtet - auf die explizite Klarstellung von Funktionen angewiesen. (Gunkel et al. 2017: 3)
\end{abstract}

Die Autoren sehen im typologisch fundierten Sprachvergleich „die einmalige Chance [...], a) funktionale Konstanten $\mathrm{zu}$ identifizieren und $\mathrm{zu}$ bestimmen, b) die Varianzbreite und die Differenzmerkmale zu ermitteln“ (Gunkel et al. 2017: 3).

Als solche funktionalen Konstanten betrachten die Autoren so genannte „funktionale Domänen“ (u. a. mit Rückgriff auf Lehmann o. J.). Diese fungieren als tertium comparationis, also als übergeordnete Größe für einen bilateralen Sprachvergleich. Den Vorteil des Begriffs der Domäne sehen die Autoren darin, dass dieser „anders als die Zuweisung einer eindeutig benannten Funktion, per se einen Spielraum in der Ausdifferenzierung“ zulässt (Gunkel et al. 2017: 19). Außerdem sei „auch insofern von ,Domänen“ zu sprechen, als es nicht um eine einfache Liste einzelner Funktionen geht, sondern um komplex strukturierte Aufgabenbereiche.“ (Gunkel et al. 2017: 20). So setzt bspw. „die ,Referenz“ als übergeordnete Aufgabe der NP im Normalfall voraus, dass die Teilaufgaben der ,Nomination“ und ,Identifikation' erfüllt sind [...]. Optional kann auch ,Modifikation“ und ,Quantifikation' gegeben sein“ (Gunkel et al. 2017: 20). Als funktionale Domänen der NP betrachten die Autoren: Referenz, Identifikation, Nomination, Modifikation und nominale Quantifikation. Den funktionalen Domänen ordnen sie Subdomänen zu wie etwa die klassifikatorische, qualifikative, referentielle und assertorische Modifikation.

Eine solche funktionale Perspektivierung setzt natürlich ein systematisch ausgearbeitetes Verständnis von Form und Funktion voraus. Von übergreifender Relevanz erscheint mir hier insbesondere die Warnung vor teleologischen Trugschlüssen wie „R liege vor, weil F. Oder auch: Es gäbe R zum Zwecke von F.“ (Gunkel et al. 2017: 29). Als Negativbeispiel führen die Autoren die Erklärung des Erhalts der pränominalen Stellung des Genitivs bei Eigennamen etwa mit dem „Bedarf nach einem optimalen thematischen Anknüpfungspunkt und referentiellen Anker für die NP“ an (Gunkel et al. 2017: 29). Für zulässig halten sie hingegen „korrelative Aussagen, bei denen das Zusammentreffen einer bestimmten Funktion bzw. eines funktionalen Vorteils mit einem grammatischen 
Phänomen oder einer grammatischen Regel konstatiert wird.“ Gunkel et al. (2017: 30)

Wichtig für die hier erfolgende Diskussion funktionalgrammatischer Ansätze ist außerdem, dass dieser gerade nicht zu einer Abkehr von der Aufgabe der Beschreibung grammatischer Formen als zentraler Aufgabe der Grammatikschreibung führt, im Gegenteil: „Sie [die grammatischen Formen, M. H.] stellen den eigentlichen Differenzbereich zwischen den Sprachen dar und damit auch den Schwerpunkt dieser sprachvergleichenden Grammatik.“ (Gunkel et al. 2017: 4) So enthält die kontrastive Grammatik neben den Kapiteln zu den genannten funktionalen Domänen auch wiederum Teile mit formalem Ausgangspunkt: Wort und Wortklassen, Nominalflexion, Nominale Syntagmen. Diese machen sogar den weitaus größeren Teil des zweibändigen Monumentalwerks aus. Aber auch zunächst formal anmutende Kapitel enthalten viel Funktionales, wie am Beispiel ,Kasus` erkennbar: Kasus wird hier über syntaktische Funktionen und semantische Rollen funktional perspektiviert und auch mit „Reichweite“ der Kasussysteme werden einzelsprachliche Anwendungsfelder von Kasus in den Blick genommen.

Eine angemessene Würdigung des umfassenden sprachvergleichenden Werks würde den Rahmen des vorliegenden Beitrags sprengen. Zumindest aber kann die Hoffnung ausgedrückt werden, dass der hier systematisch ausgearbeitete Ansatz der funktionalen Domänen eine wichtige Basis für die Weiterentwicklung funktionalgrammatischer Ansätze bilden wird.

\subsection{Form $\leftrightarrow$ Funktion}

Es sei an dieser Stelle sicherheitshalber noch einmal betont, dass die Einordnung der Grammatiken in die drei Typen von Form-Funktions-Verhältnissen nicht suggerieren soll, dass insbesondere die Typen „,von der Form zur Funktion“ (4.1) und „reziprokes Verhältnis von Form und Funktion“ (4.3) völlig eindeutig abgrenzbar seien. So haben beispielsweise die Ausführungen zu den Kapiteln „Text“ und „Gesprochene Sprache“ in der Dudengrammatik gezeigt, dass innerhalb dieser Kapitel durchaus teilweise ein Perspektivwechsel von der Form zur Funktion stattfindet. Zentraler Grund für die Zuordnung der Dudengrammatik zu 4.1 war der streng formale Aufbau von Laut und Buchstabe über Wort und Satz bis hin zu Text und gesprochener Sprache. Die IDS-Grammatik hingegen wird hier als Beispiel für ein wechselseitiges Verhältnis von Form und Funktion eingeführt, weil dort die „Doppelperspektive“ zum Leitprinzip erhoben wird:

Einerseits wird ausgegangen von den elementaren Funktionen, für die sprachliche Mittel ausgebildet sind (etwa der Funktion, Sachverhalte oder Gegenstände zu entwerfen, zu 
thematisieren oder thematisch fortzuführen). Dabei kommen nicht beliebige Funktionen in den Blick, sondern nur solche, für die spezifische sprachliche Formen und Mittel ausgebildet sind. (IDS-Grammatik 1997: 7)

Dass diesem Ansatz auch ein doppelperspektivisches Funktionsverständnis zugrunde liegt, macht Zifonun bereits 10 Jahre vor dem Erscheinen in den „Vor-Sätzen“ deutlich:

Zum einen kann kommunikative Funktion sprachlicher Einheiten eher prozedural gedeutet werden. Das heißt, sie wird jeweils als die Menge von Verfahren aufgefaßt, die Sprecher bzw. Hörer anwenden, um sich miteinander zu verständigen, indem sie Äußerungen in ganz bestimmter, grammatisch relevanter Weise bauen bzw. diesen Bau in ganz bestimmter Weise interpretieren. Bei dieser prozeduralen Sehweise ist die kommunikative Funktion grammatischer Einheiten eine Art Plan für die Versprachlichung kommunikativer Intentionen. [...] Zum anderen kann kommunikative Funktion stärker im Sinne des Potentials an sprachlichen Handlungen gesehen werden, das mit sprachlichen Einheiten verbunden ist. Dieser Ansatz untersucht also sprachliche Einheiten unter dem Aspekt, welche sprachlichen Handlungsmöglichkeiten im Sinne der Sprechhandlungstheorie diese Einheiten aufgrund der in ihnen gebrauchten Sprachmittel regelhaft eröffnen, zu welchen Handlungen sie geeignet sind. (Zifonun 1986: $35 \mathrm{ff}$.)

Als Beispiel für eine prozedurale Deutung kommunikativer Funktionen sei hier die Ellipsendefinition von Ludger Hoffmann angeführt, der bislang sicherlich prominenteste Versuch einer autonomen Begriffsbestimmung. Er definiert die Ellipse als Produkt der elliptischen Prozedur, die wiederum ein „Verbalisierungsverfahren für kommunikative Minimaleinheiten“ bildet, „bei dem der Sprecher systematisch nicht versprachlicht, was [...] mitverstanden werden kann“ (IdS-Grammatik 1997: 413).

Bei der sprachhandlungsbezogenen Deutung kommunikativer Funktionen geht es u. a. um die Funktionen sprachlicher Einheiten, die „möglichst klar, wirkungsvoll, abgestimmt auf Kontext und Interaktionspartner zur Geltung kommen“ sollen (Zifonun 1986: 39). In diesem Sinne kommt es bei grammatischen Strukturierungen funktional darauf an, ,zu verdeutlichen, worauf der Sprecher bei einer Sequenz von kommunikativen Akten oder bei ihren Teilhandlungen besonderes Gewicht legt“ (Zifonun 1986: 39). Als Beispiel für diese Perspektivierung von grammatischen Funktionen sei hier das Kapitel zu den „aufbauenden Operationen“ („Diktumserweiterungen“) im Teil D „funktionale Analyse von kommunikativen Minimaleinheiten und ihren Teilen“ genannt: Hier wird bspw. unter dem Stichwort ,Diktumsgradierung“ der funktionale Wert von Gradpartikeln herausgearbeitet.

Doppelperspektivik bedeutet aber auch, dass immer wieder Entscheidungen bezüglich des Ausgangspunkts der Betrachtung getroffen werden müssen. Die Autoren der IDS-Grammatik sprechen von „zwei perspektivische[n] Bewe- 
gungen - von der Funktion zur Form und von der Form zur Funktion“ (IdSGrammatik 1997: 8). Die erste Perspektive wird im bereits genannten Teil D verfolgt, die zweite in den Teilen „E Kompositionaler Aufbau kommunikativer Minimaleinheiten“, „F Verbale Gruppen“, „G Nicht-verbale Gruppen“, „H Subordinierte und koordinierte Strukturen“. In C „Zur Grammatik von Text und Diskurs“ gehen die Kapitel „Sprache und Illokution“, „Diskursaufbau: Die Organisation des Sprecherwechsels“ und „Thematische Organisation von Text und Diskurs“ von der Funktion aus, die Teile „Diskurs und Mündlichkeit“, „Text und Schriftlichkeit“ sowie „Diskurs- und Textsensitivität von Formen“ von der Form. Bereits diese Zuweisung der Teilkapitel zu den beiden Bewegungen zeigt, dass keine Pauschalisierungen vorgenommen werden im Sinne von „Text- und Diskursgrammatik repräsentieren eine funktionale Perspektive“. Zwar gilt Funktionalität „selbstverständlich für grammatische Großformen wie den Text oder Diskurs oder den Satz. [...] Andererseits [...] sind auch die Bausteine solcher Großformen, insbesondere des Satzes [...] funktional“ (Zifonun 1995: 254).

\section{Quo vadis deutsche Grammatikschreibung?}

Der zugegebenermaßen kursorische Überblick über wichtige funktionalgrammatische Ansätze in der modernen deutschen Grammatikschreibung hat gewisse Tendenzen in der Relevanz einschlägiger Funktionsbegriffe für die auf der Basis des Form-Funktions-Verhältnisses erfolgte Typisierung der Grammatiken erkennen lassen: Grammatiken, die die Funktion zum Ausgangspunkt ihrer Beschreibung machen, meinen damit sprachfunktionale Funktionalität, also die kognitive und/oder soziale Funktion. Wenn hingegen eine formbezogene Grammatik einen expliziten funktionalen Ansatz verfolgt, steht dabei gerne die sprachinterne syntaktische Funktion im Mittelpunkt des Interesses. Die Ausweitung des Gegenstandes einer formbezogenen Grammatik auf die Großformen Text und Gespräch führt zu einer Berücksichtigung der variationellen Funktion. Maximale Offenheit für verschiedene Funktionsbegriffe ergibt sich als logische Folge aus einem doppelperspektivischen Ansatz. Daraus nun aber abzuleiten, dass dies der Königsweg der Grammatikschreibung sei, wäre aus meiner Sicht ein übereifriger Eingriffsversuch in die Dynamik der Grammatikschreibung. So war es auch ein Anliegen des Überblicks, zu zeigen, dass mit den jeweiligen funktionalgrammatischen Ansätzen ein breites Spektrum an Schwerpunktsetzungen und Theoriebildungen erfolgt - all das könnte wohl kaum in einen einzigen doppelperspektivischen Ansatz der Grammatikschreibung integriert werden. 
Ulrich Engel hat in seiner Einführung in die Übersicht zu „Grammatiken eines Jahrzehnts“ im Jahrbuch Deutsch als Fremdsprache (1990) aus der Entwicklung in den 80er Jahren Zukunftsvisionen für künftige Grammatiken abgeleitet. So ging er davon aus, dass künftige Grammatiken adressatenorientierter, didaktischer, kontrastiver, umfassender, präziser und stärker kommunikativ fundiert sein werden. Knapp dreißig Jahre später kann festgestellt werden, dass die Entwicklung nicht in dem gleichen Maße vorangeschritten ist, wie Engel es offenbar auf der Basis des Grammatikenbooms der 80er vermutet hatte. Das kann man positiv oder negativ deuten: Positiv gedeutet erweist sich die Textsorte Grammatik als so robust, dass eine langfristige Nutzbarkeit möglich ist - man denke etwa an die ungebrochen starke Rezeption der nun mehr 20 Jahre alten IDS-Grammatik in der linguistischen Forschung oder die breite Anwendung, die die Grammatik von Helbig \& Buscha (Erstauflage 1972) nach 45 Jahren immer noch erfährt. Negativ gewendet ließe sich auch vermuten, dass gerade die von Engel formulierten Anforderungen an künftige Grammatiken dokumentieren, dass die Aufgabe immer schwieriger wird.

Es wäre nun ein Leichtes, aus dem hier vorgestellten Überblick über funktionale Ansätze in Grammatiken des Gegenwartsdeutschen Forderungen abzuleiten wie: Grammatiken müssen funktional sein, Grammatiken sollten möglichst doppelperspektivisch vorgehen und Grammatiken sollten das Spektrum aufgezeigter möglicher grammatischer Funktionen möglichst breit abdecken. Die Liste ließe sich ja beliebig erweitern etwa durch: Grammatiken sollen die gesprochene Sprache berücksichtigen, Grammatiken sollen korpuslinguistisch fundiert sein. Wenn das Ergebnis solcher Listen aber ist, dass keine Grammatiken mehr geschrieben werden, weil die Aufgabe zu komplex ist, sollte man darauf lieber verzichten. Vielmehr ist damit zu rechnen, dass die quer zu eher linguistisch motivierten Anforderungen der genannten Art liegende Aufgabe der Adressatenorientierung weiterhin dazu führen wird, dass sehr unterschiedliche Grammatiken mit sehr unterschiedlichen Zielgruppen und dementsprechend auch unterschiedlichen linguistischen Akzenten geschrieben werden. Gerade auch die jüngeren Beiträge zur deutschen Grammatikenlandschaft illustrieren, dass vor allem das jeweilige Grammatikmodell des Autors und nicht zuletzt auch seine Leidenschaft für den Gegenstand eine wichtige Grundlage für die Konzeption neuer Grammatiken bilden. So bleibt vor allem zu hoffen, dass die Textsorte Grammatik weiterhin sowohl dafür genutzt wird, für die jeweiligen Adressatengruppen Grundlagen der deutschen Grammatik aufzubereiten, als auch als Plattform für die linguistische Diskussion - nicht zuletzt gerade auch in Bezug auf das Verhältnis von grammatischen Formen und Funktionen. 


\section{Literatur}

\section{Grammatiken}

Ágel, Vilmos (2017): Grammatische Textanalyse. Textglieder, Satzglieder, Wortgruppenglieder. Berlin/Boston: De Gruyter.

Buscha, Joachim, Renata Freudenberg-Findeisen, Eike Forstreuter, Hermann Koch \& Lutz Kuntzsch (1998): Grammatik in Feldern. Ein Lehr- und Übungsbuch für Fortgeschrittene. Ismaning: Verlag für Deutsch.

Dudengrammatik = Duden (2005/2016): Die Grammatik. Unentbehrlich für richtiges Deutsch.

7., völlig neu erarbeitete und erweiterte Auflage. Hrsg. von der Dudenredaktion/

9., vollständig überarbeitete und aktualisierte Auflage. Herausgegeben von Angelika Wöllstein und der Dudenredaktion. Mannheim/Berlin: Dudenverlag (Duden 4).

Eisenberg, Peter (1986): Grundriß der deutschen Grammatik. Stuttgart u. a.: Metzler.

Eisenberg, Peter (2013): Grundriss der deutschen Grammatik. Der Satz. 4., überarbeitete und aktualisierte Auflage. Stuttgart et al.: Metzler.

Engel, Ulrich (1988): Deutsche Grammatik. Heidelberg: Groos.

Engel, Ulrich \& Rozemaria K. Tertel (1993): Kommunikative Grammatik Deutsch als Fremdsprache. Die Regeln der deutschen Gebrauchssprache in 30 gemeinverständlichen Kapiteln - mit Texten und Aufgaben. München: iudicum.

Grundzüge $=$ Heidolph, Karl Erich, Walter Flämig \& Wolfgang Motsch (1980): Grundzüge einer deutschen Grammatik. Berlin: Akademie-Verlag.

Gunkel, Lutz, Adriano Murelli, Susan Schlotthauer, Bernd Wiese \& Gisela Zifonun (2017): Grammatik des Deutschen im Europäischen Vergleich. Das Nominal. Teilband 1: Funktionale Domänen, Wort und Wortklassen (Schriften des Instituts für deutsche Sprache 14.1). Berlin/Boston: De Gruyter.

Helbig, Gerhard \& Joachim Buscha (2001): Deutsche Grammatik. Ein Handbuch für den Ausländerunterricht. Leipzig u. a.: Langenscheidt.

Heringer, Hans Jürgen (1989): Lesen lehren lernen. Eine rezeptive Grammatik des Deutschen. Tübingen: Niemeyer.

Hoffmann, Ludger (2013): Deutsche Grammatik. Grundlagen für Lehrerausbildung, Schule, Deutsch als Zweitsprache und Deutsch als Fremdsprache. Berlin: Schmidt.

IDS-Grammatik = Zifonun, Gisela et al. (Hrsg.) (1997): Grammatik der deutschen Sprache (= Schriften des Instituts für deutsche Sprache 7). Berlin/New York: De Gruyter.

Weinrich, Harald (1993): Textgrammatik der deutschen Sprache. Mannheim u. a.:

Dudenverlag.

\section{Sekundärliteratur}

Ágel, Vilmos \& Rita Brdar-Szabó (Hrsg.) (1995): Grammatik und deutsche Grammatiken. Budapester Germanistentagung 1993 (Linguistische Arbeiten 330). Tübingen: Niemeyer.

Bondarko, Aleksandr V. (2007): Funktional-semantische Felder. In Joachim Buscha \& Renate Freudenberg-Findeisen (Hrsg.), Feldergrammatik in der Diskussion (Sprache und Tätigkeit 56), 23-34. Frankfurt u. a.: Lang.

Bühler, Karl (1999) [1934]: Sprachtheorie. Die Darstellungsfunktion der Sprache. Stuttgart: Lucius \& Lucius. 
Buscha, Joachim \& Renate Freudenberg-Findeisen (2007): Zur Einführung: Feldergrammatik in der Diskussion. In Joachim Buscha \& Renate Freudenberg-Findeisen (Hrsg.), Feldergrammatik in der Diskussion (Sprache und Tätigkeit 56), 7-19. Frankfurt u. a.: Lang.

Dik, Simon (1978): Functional grammar. Amsterdam: North-Holland.

Eichinger, Ludwig M. (1984): Die großen historischen Grammatiken des Deutschen. In Werner Besch, Oskar Reichmann \& Stefan Sonderegger (Hrsg.), Sprachgeschichte. Ein Handbuch zur Geschichte der deutschen Sprache und ihrer Erforschung (Handbücher zur Sprach- und Kommunikationswissenschaft 2.1), 433-440. Berlin/New York: De Gruyter.

Eisenberg, Peter (2007): Sollen Grammatiken die gesprochene Sprache beschreiben? Sprachmodalität und Sprachstandard. In Vilmos Ágel \& Mathilde Hennig, Zugänge zur Grammatik der gesprochenen Sprache (Reihe Germanistische Linguistik), 275-295. Tübingen: Niemeyer.

Engel, Ulrich (1990): Grammatiken eines Jahrzehnts. Einführung in den thematischen Teil. In Jahrbuch Deutsch als Fremdsprache 16, 141-146.

Freudenberg-Findeisen, Renate (2000): Feldergrammatik als inhaltlich orientierte (Lerner)Grammatik dargestellt am Feld des Wunsches. In Deutsch als Fremdsprache, 22-29.

Götze, Lutz (1999): Eine funktionale Grammatik für Deutsch als Fremdsprache. In Bernd Skibitzki \& Barbara Wotjak (Hrsg.), Linguistik und Deutsch als Fremdsprache. Festschrift für Gerhard Helbig zum 70. Geburtstag, 81-94. Tübingen: Niemeyer.

Halliday, M. A. K. (1985/2004): An introduction to functional grammar. 2004: Third Edition. Revised by Christian M. I. M. Matthiessen. London: Hodder education.

Helbig, Gerhard (1970): Geschichte der neueren Sprachwissenschaft. Leipzig: VEB Bibliographisches Institut.

Helbig, Gerhard (1984): Entwicklung der Sprachwissenschaft seit 1970. Leipzig: VEB Bibliographisches Institut.

Helbig, Gerhard (1992): Grammatiken und ihre Benutzer. In Vilmoa Ágel \& Regina Hessky (Hrsg.), Offene Fragen, offene Antworten in der Sprachgermanistik (Reihe Germanistische Linguistik 128), 135-150. Tübingen: Niemeyer.

Helbig, Gerhard (1999): Was ist und was soll eine Lern(-er)-Grammatik? In Deutsch als Fremdsprache, 103-112.

Helbig, Gerhard (2001): Arten und Typen von Grammatiken. In Heinrich Anz, Lutz Götze, Gerhard Helbig, Gerd Henrici, Hans-Jürgen Krumm \& Walter Veit (Hrsg.), Deutsch als Fremdsprache (Handbücher zur Sprach- und Kommunikationswissenschaft 19.1), 175-186. Berlin: De Gruyter.

Hennig, Mathilde (2001): Welche Grammatik braucht der Mensch? Grammatikenführer für Deutsch als Fremdsprache [Unter Mitarbeit von Carsten Hennig]. München: iudicium.

Hennig, Mathilde (2002): Wie kommt die gesprochene Sprache in die Grammatik? In Deutsche Sprache 30, 307-327.

Hennig, Mathilde (2006): Rezension zu: Duden. Die Grammatik. Unentbehrlich für richtiges Deutsch. In Zeitschrift für Dialektologie und Linguistik, 82-86.

Hennig, Mathilde (2010): Plädoyer für eine Grammatikbenutzungsforschung: Anliegen, Daten, Perspektive. In Deutsche Sprache 38, 19-42.

Hennig, Mathilde (2017): Grammatik und Variation im Spannungsfeld von Sprachwissenschaft und öffentlicher Sprachreflexion. In Marek Konopka \& Angelika Wöllstein (Hrsg.), Grammatik und Variation. Empirische Zugänge und theoretische Modellierung (= Jahrbuch des Instituts für deutsche Sprache 2016), 23-45. Berlin/Boston: De Gruyter. 
Hennig, Mathilde (2018): Grammatik und Variation in Grammatikforschung und Grammatikschreibung. In Eric Fuß \& Angelika Wöllstein (Hrsg.), Grammatiktheorie und Grammatikographie. Tübingen: Narr.

Hennig, Mathilde \& Melanie Löber (2010): Benutzung und Benutzbarkeit von Grammatiken. In Iris Bons, Thomas Gloning \& Dennis Kaltwasser (Hrsg.), Fest-Platte für Gerd Fritz. Gießen 23.5. 2010. www.festschrift-gerd-fritz.de/files/hennig_loeber_2010_benutzungund-benutzbarkeit-von-grammatiken.pdf (letzter Zugriff: 18.5. 2017).

Hoffmann, Ludger (1999): Grammatiken und die Funktionalität von Sprache. In Renate Freudenberg-Findeisen (Hrsg.), Ausdrucksgrammatik versus Inhaltsgrammatik. Linguistische und didaktische Aspekte der Grammatik, 23-38. München: iudicium.

Humboldt, Wilhelm von (1836): Über die Verschiedenheit des menschlichen Sprachbaues und ihren Einfluß auf die geistige Entwicklung des Menschengeschlechts. Berlin: Akademie der Wissenschaften.

Lehmann, Christian (o. J.): Funktionale Domäne. www.christianlehmann.eu/ling/lg_system/ sem/index.html?http://www.christianlehmann.eu/ling/lg_system/sem/funktionale_ domaene.php (letzter Zugriff: 18. 5. 2017).

Moulin, Claudine (1988): Deutsche Grammatiken vom Humanismus bis zur Aufklärung. Ausstellung der Forschungsstelle für deutsche Sprachgeschichte der Universität Bamberg in Zusammenarbeit mit der Staatsbibliothek Bamberg. Bamberg: Fränkischer Tag.

Moulin-Fankhänel, Claudine (1994): Bibliographie der deutschen Grammatiken und Orthographielehren. I. Von den Anfängen bis zum Ende des 16. Jahrhunderts. Unter Mitarbeit von Ursula Götz. Heidelberg: Winter.

Morris, Charles (1988): Grundlagen der Zeichentheorie. Frankfurt a. M.: Fischer.

Schmidt, Wilhelm (1973): Grundfragen der deutschen Grammatik. Eine Einführung in die funktionale Sprachlehre. Berlin: Volk und Wissen.

Smirnova, Elena \& Tanja Mortelmans (2010): Funktionale Grammatik. Konzepte und Theorien. Berlin/New York: De Gruyter (De Gruyter Studium).

Teuber, Oliver (2005): Analytische Verbformen im Deutschen. Syntax - Semantik Grammatikalisierung (= Germanistische Linguistik Monographien 18). Hildesheim u.a.: Olms.

Welke, Klaus (1992): Funktionale Satzperspektive. Ansätze und Probleme der funktionalen Grammatik. Münster: Nodus.

Wildgen, Wolfgang (2010): Die Sprachwissenschaft des 20. Jahrhunderts. Versuch einer Bilanz. Berlin/New York: De Gruyter.

Zifonun, Gisela (1986): Eine neue Grammatik des Deutschen. Konzept zu Inhalt und Struktur. In Gisela Zifonun (Hrsg.), Vor-Sätze zu einer neuen deutschen Grammatik (Forschungsberichte des Instituts für deutsche Sprache 63), 11-75. Tübingen: Narr.

Zifonun, Gisela (1995): Grammatik ja, aber meine. In Vilmos Ágel \& Rita Brdar-Szabó (Hrsg.), Grammatik und deutsche Grammatiken. Budapester Grammatiktagung 1993

(Linguistische Arbeiten 330), 253-260. Tübingen: Niemeyer. 


\title{
14 An der Schnittstelle von DaF und Germanistischer Sprachwissenschaft
}

\author{
Bestandsaufnahme und Perspektiven
}

\begin{abstract}
Der Artikel befasst sich mit der Schnittstelle zwischen DaF und Germanistischer Sprachwissenschaft, er thematisiert die Berührungspunkte zwischen beiden, und zwar sowohl in der unterrichtlichen Praxis und der Ausbildung als auch in der Forschung; dabei werden zwei Bereiche, Text(sorten)linguistik und Gesprochene Sprache, besonders beleuchtet. Neben einer Bestandsaufnahme zentraler Fragestellungen sollen auch Desiderate benannt werden.
\end{abstract}

Keywords: Deutsch als Fremdsprache, gesprochene Sprache, linguistisches Wissen, Textsortenlinguistik, Variation

\section{Einleitung}

Der folgende Beitrag beschäftigt sich rückblickend und perspektivisch mit der Schnittstelle zwischen Didaktik und Germanistischer Sprachwissenschaft. Dabei konzentriere ich mich auf den Bereich Deutsch als Fremdsprache (DaF) unter teilweiser Berücksichtigung von Deutsch als Zweitsprache (DaZ). Was die muttersprachliche Didaktik betrifft, die wiederum heute in der Regel auch DaZ mit einschließt, gibt es mittlerweile eine lebhafte Diskussion zum Thema Grammatik (bzw. auch Sprachreflexion) im Unterricht, die hier nicht nachgezeichnet werden kann (vgl. in jüngerer Zeit etwa die Beiträge in Köpcke \& Ziegler 2007, 2011, 2013, 2015; Hoffmann \& Ekinci-Kock 2010; Habermann 2010; Rothstein 2010, 2011; Rödel 2014). Vorausgeschickt sei noch, dass die Trennung zwischen Germanistischer Sprachwissenschaft und DaF nicht immer ganz strikt zu ziehen ist, weder inhaltlich noch institutionell oder personell: es gibt viele Konstellationen, bei denen beides in den Blick genommen wird. Nicht zuletzt hat

Maria Thurmair, Universität Regensburg, Fakultät für Sprach-, Literatur- und Kulturwissenschaften, Universitätsstraße 31, D-93053 Regensburg,

E-Mail: maria.thurmair@sprachlit.uni-regensburg.de

Ә Open Access. (c) 2018 Maria Thurmair, publiziert von De Gruyter. (c) BY Dieses Werk ist lizenziert unter der Creative Commons Attribution 4.0 Lizenz. https://doi.org/10.1515/9783110490992-015 
ja auch Ludwig M. Eichinger eine kurze DaF-Vergangenheit, und auch das IDS hat den DaF-Aspekt, der unter anderem auch von einer Auslandsgermanistik immer wieder speziell thematisiert wird, nie aus dem Blick verloren.

\section{Was ist DaF? Konturen des Faches}

Das Fach Deutsch als Fremdsprache ist - im Vergleich mit der Germanistischen Sprachwissenschaft - ein junges Fach, das nach lebhaft geführten Strukturdiskussionen mittlerweile als konsolidiert und als wissenschaftliche Disziplin gelten kann. ${ }^{1}$ Ungeachtet der unterschiedlichen Ausprägungen gilt als Kern des Faches das, was führende Vertreter zu unterschiedlichen Zeiten folgendermaßen formulieren: „Der spezifische Gegenstand von DaF ist die Theorie und Praxis des Lernens und Lehrens von Deutsch als Fremdsprache“ (so wiederholt Gerhard Helbig, s. ausführlich Götze, Helbig, Henrici \& Krumm 2001/2010) und in einer Bestimmung jüngeren Datums: „die Forschungsperspektive des Faches $\mathrm{DaF} / \mathrm{DaZ}$ ist wesentlich durch die auf das Deutsche bezogene (Fremd-)Perspektive bestimmt und durch seine Genese und Verpflichtung zur Verzahnung mit der Anwendung, der Praxis konturiert“ (Fandrych, Hufeisen, Krumm \& Riemer 2010: 5).

Bei einem breiten Fachverständnis werden Fragen der Sprachwissenschaft, der Sprachdidaktik, der Lehr-/Lernforschung sowie der Literatur- und Kulturwissenschaft aus der Perspektive des Deutschen als Fremdsprache (und mittlerweile meistens auch Zweitsprache) erforscht. Für die Sprachwissenschaft heißt das, dass eine DaF- und DaZ-spezifische Sprachwissenschaft die deutsche Sprache aus dem Blickwinkel des Fremden unter der Perspektive der Vermittlung betrachtet. Mit ersterem ist eine kontrastive bzw. mehrsprachige Perspektive impliziert. Letzteres umfasst zentral spracherwerbliche und didaktische Fragestellungen und kann für $\mathrm{DaF}$ und $\mathrm{DaZ}$ zu unterschiedlichen Fokussierungen führen. Die besondere Perspektive bedingt auch, dass Sprachwissenschaft im Bereich DaF immer anwendungsbezogen ist - Theorie- und Modellbildung steht nicht im Fokus. Bei einem engeren Fachverständnis von DaF und DaZ lassen sich Engführungen auf linguistische und sprachdidaktische oder lehr-/lernwissenschaftliche oder kultur- und literaturwissenschaftliche Fragestellungen feststellen. In diesen Fällen kann die sprach(wissenschaft)liche Seite weitgehend ausgeblendet sein.

1 Vgl. dazu die positionierenden Beiträge verschiedener Fachvertreter anlässlich des 50. Geburtstags der Zeitschrift „Deutsch als Fremdsprache“ in den Heften 2/2015 und 3/2015 und die Publikationen zur Strukturdebatte in Götze, Helbig, Henrici \& Krumm 2001/2010. 
Was im Fach DaF allgemein unter „Grammatik“ (im Sinne sprachbezogener Herangehensweise) verstanden wird, ist heute in jedem Fall ein weites Verständnis von Grammatik. Fandrych (2016: 34) unterscheidet in diesem Zusammenhang (mit Bezug auf Ehlich) systematisch folgende sieben Bereiche des „Lehrund Lerngegenstands Sprache“, die (mindestens weitgehend) enge Berührungspunkte mit einer entsprechenden Germanistischen Sprachwissenschaft aufweisen: Phonetik, Lexiko-Semantik, Morpho-Syntax, alltagsbezogene Pragmatik, mündlicher Diskurs, Literalität und Textkompetenz sowie gesellschaftsund deutungsbezogene Pragmatik. Für Spracherwerbs- und -vermittlungskontexte, mit denen DaF und DaZ zentral beschäftigt sind, ist also eine strikte Trennung zwischen Sprachsystem und Sprachgebrauch nicht förderlich.

Eine DaF-Linguistik ist also inhaltlich, methodisch und perspektivisch anders gelagert als eine Germanistische Sprachwissenschaft, insofern sie die deutsche Sprache aus dem Blickwinkel des Fremden unter der Perspektive des Erwerbs und der Vermittlung betrachtet. Bezüglich des Verhältnisses zwischen Sprachwissenschaft und Sprachdidaktik macht Fandrych (2016: 35 f.) zwei Auffassungen aus: Einmal gilt die Sprachwissenschaft als eine Art Bezugswissenschaft, aus deren Ergebnissen die Didaktik auswählt und sie ihren Bedürfnissen anpasst, sie also importiert und mit lernpsychologischen, didaktischen und medienwissenschaftlichen Erkenntnissen anreichert. Zum anderen gibt es die Auffassung, die fremdsprachenphilologisch orientierten Fächer sollten stärker eigenständige Perspektiven und eigenständige Forschungsinteressen in Bezug auf Sprache entwickeln und diese aus der Perspektive der Lernenden untersuchen.

Darüber hinaus können auch andere Bereiche von linguistischen Herangehensweisen profitieren: das gilt gleichermaßen für Formen der Textarbeit, auch der Arbeit mit literarischen Texten sowie für Formen der Kulturvermittlung (vgl. dazu etwa die Beiträge in Dobstadt, Fandrych \& Riedner 2015).

\section{Sprachwissenschaft in der (DaF-)didaktischen Praxis?}

Die Frage nach dem Stellenwert der Sprachwissenschaft (meist verkürzt als „Grammatik“ bezeichnet) in der DaF-/DaZ-didaktischen Praxis muss für zwei unterschiedliche Seiten gestellt werden: für LernerInnen und damit für den Unterricht und für Lehrende $u$. a. und damit in der Ausbildung.

Für DaF-Unterricht gilt, dass der Stellenwert von Grammatik im Fremdsprachenunterricht „seit undenklichen Zeiten das beliebteste Streitobjekt der 
Praktiker, Didaktiker und Linguisten“ (Rall 2001: 880) ist. Diskutiert wurde dabei vor allem die Frage, wie Lernende am besten zielsprachliche Kompetenz erwerben, ob dafür expliziter Grammatikunterricht und der Erwerb metasprachlichen Wissens nötig ist, ob er sinnvoll ist und Erwerbsprozesse optimieren kann, für wen unter welchen Bedingungen welche Art von Grammatikunterricht adäquat ist und vieles mehr. Antworten auf diese Fragen werden in verschiedenen Forschungszusammenhängen gesucht, etwa der Spracherwerbsforschung und der Sprachlehr- und -lernforschung. Heute kann man als common sense feststellen, dass sich die Frage nach der Rolle metasprachlichen Wissens nicht pauschal beantworten lässt, da die Bedingungen des Fremdspracherwerbs zu komplex und unterschiedlich sind; Faktoren wie Lernkontexte, die Lern- und Lehrvoraussetzungen, die Lernmotivationen, die Lernziele, Ausgangs- und Zielsprache etc. spielen alle eine Rolle (s. dazu genauer Fandrych 2010). Tatsache ist, dass seit einigen Jahrzehnten expliziter Grammatikunterricht (wieder) eine wichtigere Rolle erlangt hat; gestützt wird dies durch verschiedene Erkenntnisse aus Forschungen zum Erst-, Zweit- und Fremdspracherwerb. Eine wesentliche und förderliche Rolle in diesem Zusammenhang spielt die Entwicklung von Sprachaufmerksamkeit (language awareness), für die das noticing, das Bewusstmachen und Fokussieren der Aufmerksamkeit des Lerners auf bestimmte grammatische Phänomene eine zentrale Komponente darstellt (vgl. dazu etwa Portmann-Tselikas 2001; grundlegend und zusammenfassend Oomen-Welke 2016). Explizite Grammatikerklärungen können demnach den Spracherwerbsprozess verkürzen und optimieren, und somit kann auch deklaratives Grammatikwissen einen positiven Effekt auf den Grammatikerwerb und den Spracherwerb haben (vgl. Fandrych 2010).

Die Frage nach dem Stellenwert von linguistischem Wissen beim Fremdspracherwerb wird aber nur selten für die ,andere Seite', die Lehrenden und andere im weiteren Bereich der Sprachmittlung Tätigen gestellt. Einerseits wird hier wohl stillschweigend davon ausgegangen, dass Grammatikwissen unabdingbar ist, zum anderen macht man sich leider oft (vor allem außerhalb des engeren fachlichen Zirkels) nicht bewusst, dass es einen Unterschied zwischen Sprachwissen und Sprachkönnen gibt und dass Muttersprachler in der Regel ein Defizit in Ersterem haben. Für Lehrende, Lehrwerkautoren und andere ist metasprachliches Wissen, also explizites, deklaratives Wissen über Strukturen, Regularitäten, Regeln einer Sprache und ihren Gebrauch essentiell, „gute linguistische Kenntnisse [...] über die zu vermittelnde Sprache [sind] zentrales Handwerkszeug von Lehrenden sowie allen weiteren professionellen Akteuren im Kontext Fremdsprachenunterricht (Textentwickler, Curriculums-Entwickler, Autoren von Lehrmaterialien, Fortbilder etc.)“ (Fandrych 2016: 37). Dies gilt unabhängig davon, wie man zum Thema ,Grammatik für Lerner`steht, ob man dieses Wissen etwa im Unterricht thematisiert oder nicht. 
Lehrende und andere Sprachmittler brauchen explizites Sprachwissen bzw. deklaratives Metawissen, das systematisch vorhanden und möglichst jederzeit weitgehend verfügbar sein sollte, aus den nachfolgend aufgeführten Gründen: Sie sollten Regularitäten und Regeln der Sprache, die sie unterrichten, kennen; sie sollten Regeln einschätzen, bewerten und auswählen können; sie müssen sprachbezogene Fragen systematisch und fundiert beantworten können; sie sollten eigenständig adäquaten Input bereitstellen können, also entsprechendes Sprachmaterial und Beispiele finden, auswählen oder auch konstruieren; sie müssen ihren Unterrichtsprozess strukturieren und Fragen der Progression klären, was immer auch sprachbezogen erfolgt; sie müssen Lerneräußerungen in vielfältigsten Kontexten bewerten und etwa Abweichungen erklären bzw. begründen (will man über allgemeine Bewertungen hinaus zu Erklärungen kommen, dann ist das fundiert ohne grammatische Kenntnisse nicht möglich); sie müssen Tests und Prüfungen unterschiedlichster Art erstellen und auswerten, darunter fallen auch die im Bereich DaZ so wichtigen Sprachstandserhebungen (fundiert ist dies ohne grammatische Kenntnisse und ohne explizites Metawissen nicht möglich - unabhängig davon, was bei den Lernern getestet wird). Lehrende müssen in der Lage sein, metasprachliche Werke unterschiedlichster Art zu rezipieren - auch dafür braucht man Grammatikwissen; und schließlich ist, um im Kontext von Mehrsprachigkeit adäquat (z. B. sprachreflexiv) agieren zu können, ebenfalls umfangreiches sprachstrukturelles Wissen erforderlich.

Es sollte also offensichtlich sein, dass in diesem Sinne die Germanistische Sprachwissenschaft in der DaF-Ausbildung (wie in der DaZ-Ausbildung) eine gewichtige Rolle spielen sollte; umso überraschender (und bedauerlicher) ist es, dass sie dies nicht immer tut.

\section{Sprachwissenschaft und DaF in der Forschung}

Im Folgenden sollen exemplarisch zwei Bereiche genauer beleuchtet werden, in denen die Interessen einer Germanistischen Sprachwissenschaft mit DaF besonders gut zusammengehen und wo vielfältige Verflechtungen und Beeinflussungen festgestellt werden können, nämlich die Text(sorten)linguistik (4.1) und die gesprochene Sprache/Mündlichkeit (4.2); weitere wichtige Bereiche werden anschließend (4.3) kurz gestreift. 


\subsection{Textsortenbezogene Grammatik}

Im Bereich des Fremdsprachenunterrichts und der Sprachdidaktik wird seit längerem eine stärkere Berücksichtigung textbezogener Phänomene gefordert (vgl. dazu etwa die Beiträge in Adamzik \& Krause 2009; Scherner \& Ziegler 2006; Schmölzer-Eibinger \& Weidacher 2007; Foschi Albert, Hepp \& Neuland 2006; Foschi Albert, Hepp, Neuland \& Dalmas 2010; Dalmas, Foschi Albert, Hepp \& Neuland 2015; Bachmann \& Feilke 2014; Freudenberg-Findeisen 2016). Diese Forderungen konkretisieren sich auch in dem Konzept einer textsortenbezogenen Grammatik, wie sie unter anderem von Fandrych \& Thurmair propagiert und an verschiedenen grammatischen Aspekten analytisch vorgeführt wird (vgl. Fandrych \& Thurmair 2011a, 2011b, 2016; Thurmair 2016). Ähnliche Ansätze finden sich etwa auch in vielen Beiträgen in Freudenberg-Findeisen (2016) oder bei Freudenberg-Findeisen (2013). Hier wirken Germanistische Sprachwissenschaft (als Textsortenlinguistik) und die Perspektive von DaF in einer vielversprechenden Weise zusammen.

Konkret wird dabei davon ausgegangen, dass Textsorten eine Klasse von Texten darstellen, die als konventionell geltende Muster bestimmten (komplexen) sprachlichen Handlungen zuzuordnen sind, Textsorten sind musterhafte Ausprägungen zur Lösung wiederkehrender kommunikativer Aufgaben (vgl. u. a. Brinker 2005: 144; Fandrych \& Thurmair 2011a: 13-16). Textsortenanalysen sollten dementsprechend verschiedene Beschreibungsdimensionen kombinieren, nämlich: 1. die Kommunikationssituation, die situativ und kontextuell relevante Merkmale berücksichtigt wie: die Welt, in der Texte angesiedelt sind (s. Adamzik 2004: 61-68), den Kommunikationsbereich (etwa Medizin, Wissenschaft), den medialen Aspekt, Textproduzent und -rezipient und ihr Verhältnis zueinander, Aspekte von Raum und Zeit sowie die kulturräumliche Gebundenheit; 2. die Textfunktion: in Fandrych \& Thurmair werden die Funktionen in drei großen Textgruppen mit entsprechenden Unterdifferenzierungen gefasst: wissensbezogene, handlungsbeeinflussende und handlungspräformierende sowie expressiv-soziale, sinnsuchende Texte (s. genauer Fandrych \& Thurmair 2011a: 29-33); 3. die Ebene der sprachlichen Merkmale, die die thematischstrukturelle sowie die grammatisch-stilistische Ebene umfasst und die schließlich die konkrete sprachliche Ausgestaltung von Textsorten, die syntaktischen Muster, die lexikalische Gestaltung und anderes betrifft. Auf dieser Ebene zeigt sich die Musterhaftigkeit von Textsorten am besten.

Eine textsortenbezogene Grammatik setzt bei den Kennzeichen der dritten Ebene an und kann deren sprachliche und grammatische Phänomene funktional adäquat beschreiben, indem Aspekte der ersten und zweiten Ebene, also Parameter der Kommunikationssituation und die Textfunktion strukturiert und 
systematisch herangezogen werden. So lassen sich viele ,klassische“ grammatische Themen anhand von Textsortenanalysen funktional adäquat(er) und durchaus noch erkenntnisgewinnend analysieren.

Zum Thema Tempus z.B. gibt es schon länger entsprechende Text- und auch textsortenbezogene Ansätze (s. Weinrich 1964/1985, 2003; Marschall 1995; Hennig 2000; Willkop 2003).

Auch das Thema Passiv wird zwar nicht zuletzt in der Didaktik oft mit Verweis auf Textsorten behandelt; systematische textsortenbezogene Herangehensweisen konnten allerdings zeigen, dass Passiv in einer wesentlich größeren Vielfalt von Textsorten auftritt, dass z. B. sowohl Vorgangs- als auch Zustandspassiv eine Affinität zu bewertenden Texten aufweisen und nicht nur - wie in der Didaktik üblich (dazu Steinhoff 2011) - in fachlich angehauchten Textsorten (wie Lexikonartikel, Vorgangsbeschreibungen) vorkommen. Bewertende Texte, seien es Bewertungen von Dienstleistungen, bestimmten Produkten oder kulturellen Erzeugnissen, sind heute - bedingt durch die Neuen Medien und Kommunikationsformen - weit verbreitet und werden von einer wesentlich größeren Anzahl von Schreibern verfasst; sie bieten schon alleine deshalb einen ansprechenden Ausgangspunkt für den Sprachunterricht (s. dazu genauer Thurmair 2016).

Textsortenorientierte Analysen konnten z. B. auch zum komplexen Thema Attribution zeigen, wie die formal unterschiedlichen Attributsformen, die ja typischerweise ganz unterschiedliche „nähere Bestimmungen“ bzw. Spezifikationen liefern (s. genauer Fabricius-Hansen 2010), auch funktional in verschiedenen Textsorten unterschiedlich auftreten, insofern etwa in wissensbezogenen Textsorten (wie z. B. Reiseführern) gehäuft Partizipialattribute auftreten, die beiläufig recht komplexe Szenen im Nominalen einbetten: so liefert das Partizip II vor allem Vorgeschichten, das Modalpartizip oft bestimmte Modalisierungsszenarien. In anderen Textsorten dagegen dienen Attribute wesentlich dazu, die Bezugsausdrücke zu modifizieren und die mit den Nomina bezeichneten Referenzobjekte zu spezifizieren (wie z. B. in Audioguides). Anderswo treten vermehrt Attribute in wertender Funktion auf, dann häufig formal als evaluative Adjektive (vgl. dazu genauer Fandrych 2011; Thurmair 2007, 2011, 2013). Ausgehend von den Attributsanalysen propagieren Fandrych \& Thurmair (2016) das in der Didaktik weitgehend unbeachtete Konzept der Linksdeterminierung im Deutschen und übertragen dieses auch in den verbalen Bereich, wo sie an einfachen und leicht zugänglichen Textsorten (wie öffentliche Verbote/Gebote, kurze Planungstexte, z. B. Einfahrt freihalten! Bad putzen) die Strukturen vorstellen und didaktisch fruchtbar machen und auch zeigen, wie sich die typischen Illokutionen dieser elliptischen Strukturen aus der jeweiligen Funktion der Text(sorten) ergeben. Ähnlich lässt sich im Fall von grammatischen Struk- 
turen, die bestimmte Modalitäten zum Ausdruck bringen, wie etwa das Modalpartizip (zu erwerbende Kompetenzen) oder das sogenannte Modalpassiv (etwas ist vorzulegen), die Modalität durch die jeweilige Textsorte, in der die Strukturen auftreten, genauer bestimmen. Eine wiederum etwas anders perspektivierte Herangehensweise einer textsortenorientierten Grammatik, nämlich stärker funktional, ist die Analyse z. B. bestimmter Sprachhandlungen in verschiedenen Textsorten. Im Rahmen des Konzepts einer textsortenbezogenen Grammatik kann man also stärker formorientiert oder funktionsorientiert vorgehen beide Vorgehensweisen haben ihre rein linguistische wie auch ihre fremdsprachendidaktische Berechtigung.

Die Vorteile, die die Arbeit mit authentischen Textsorten für den Fremdsprachenunterricht bietet, können hier nicht ausführlich diskutiert werden (s. dazu Fandrych \& Thurmair 2011a: 350-355, 2011b), nur soviel: Sie gewährleistet die Berücksichtigung authentischer Sprache, sie öffnet den Blick für sprachliche Variation und Vielfalt, sie zeigt sprachliche Formen wirklich in Funktion; die Arbeit mit authentischen Textsorten kann auch gezielt für spezifisches Fertigkeitstraining eingesetzt werden. Darüber hinaus weisen Textsorten eine kulturelle und landeskundliche Dimension auf. Didaktisch nicht ganz unerheblich ist auch, dass das Vorkommen bestimmter sprachdidaktisch unverzichtbarer Themen in überraschender Umgebung nachgewiesen werden konnte. ${ }^{2}$ Dass nicht nur größere, komplexere und eventuell schwierigere Textsorten, sondern dass auch kleine Texte in dieser Hinsicht sehr ergiebig sein können, diskutieren etwa Dürscheid (2016) und auch Fandrych \& Thurmair (2016); aus anderer Perspektive wird ebenfalls vermehrt für die Beschäftigung mit kleinen Texten plädiert, wie sie in den Neuen Medien vorkommen, also etwa E-Mail und SMS (Dittmann 2006; Topalovic 2009) oder Twitter (Moraldo 2015).

Voraussetzung für eine derartige textsortenbezogene Grammatik ist natürlich, dass es entsprechende Analysen verschiedenster Textsorten ${ }^{3}$ gibt. Hier ist in den letzten Jahren viel entstanden, was als Basis für eine textsortenbezogene Grammatik dienen kann, aber es lassen sich natürlich immer noch verschiedene Desiderate benennen: Neben erforderlichen Analysen weiterer Text- und

2 In der Fremdsprachendidaktik werden in der letzten Zeit zwar an manchen Stellen durchaus einschlägige Textsorten verwendet (z.B. Anzeigen bei der starken Adjektivdeklination oder fachliche Texte beim Passiv), dies könnte und sollte aber systematischer geschehen. Dabei ist natürlich zu beachten, dass manche grammatischen Themen besser und eindeutiger Textsorten zuzuordnen sind als andere.

3 Da sich das Konzept einer textsortenbezogenen Grammatik idealerweise mit einer korpuslinguistischen Herangehensweise verbindet, ist es erforderlich, dass die zur Verfügung stehenden Korpora auch eine breite Vielfalt an Textsorten aufweisen. 
Diskurssorten ${ }^{4}$ (und anderer grammatischer Themen, wie etwa Wortstellung, Vorfeldbesetzung o. ä.) lassen sich auch andere vielversprechende Herangehensweisen vorschlagen: so könnte man stärker auch Textsorten unter verschiedenen Parametern bündeln (ergiebig erscheint unter didaktisch-grammatischem Aspekt zum Beispiel die Textfunktion); es könnte auch stärker die Vernetzung verschiedener Textsorten in einer Handlungsabfolge in den Blick genommen werden (vgl. Adamzik 2011). Während also im Textsortenbereich schon viele Erkenntnisse für Sprachwissenschaft wie für DaF gewonnen wurden, besteht für den Diskurs-Bereich diesbezüglich ein deutliches Desiderat.

\subsection{Grammatik der gesprochenen Sprache bzw. Mündlichkeit}

Das Themenfeld gesprochene Sprache und Mündlichkeit berührt - was den DaF-Bereich betrifft - sehr viele unterschiedliche Facetten. Unterscheiden lassen sich mindestens folgende vier Forschungsrichtungen, nämlich Phonetikforschungen zur medialen Mündlichkeit, Forschungen zur konzeptionellen Mündlichkeit, Forschungen zur gesprochenen Sprache sowie Gesprächsanalyse und ihr verwandte Disziplinen (s. Hirschfeld, Rösler \& Schramm 2016: 133 f.). Im Zusammenhang mit der hier behandelten Fragestellung sollen vor allem die Forschungen zur Grammatik der gesprochenen Sprache im Fokus stehen. Der Einbezug der „Grammatik der gesprochenen Sprache“ hat sich in den DaF-Diskussionen mittlerweile zu einem ähnlichen Dauerbrenner entwickelt, wie die Frage nach Grammatik generell (vgl. dazu u.a. die Sammelbände von Bachmann-Stein \& Stein 2009; Reeg, Gallo \& Moraldo 2012; Moraldo \& Missaglia 2013; Imo \& Moraldo 2015; Grundler \& Spiegel 2014; Handwerker, Bäuerle \& Sieberg 2016 und schon Günthner 2000, 2002).

Festhalten lässt sich dabei zunächst, dass die linguistischen Forschungen zur gesprochenen Sprache und ihren spezifischen Strukturen eine Fülle von Erkenntnissen zu relevanten Erscheinungen erbracht haben; von den auch für Spracherwerbskontexte relevanten sollen im Folgenden (strukturiert nach verschiedenen sprachlichen Ebenen) die wichtigsten genannt werden.

Auf der phonetischen Ebene lassen sich insbesondere Elisionen, Klitisierung, Verschmelzungen sowie stärker regional bedingte Erscheinungen als spezifisch gesprochen- und nähesprachlich anführen (s. dazu Schwitalla 2012: 37-42).

4 Ergiebig ist dies nicht zuletzt auch deshalb, weil es in diesem Bereich seit einiger Zeit große Veränderung gibt, vor allem bedingt durch die Neuen Medien und Kommunikationsformen (s. dazu z. B. Marx \& Weidacher 2014; Fandrych \& Thurmair 2010). 
Spezifische Kennzeichen der gesprochenen Sprache auf der lexikalischen Ebene sind einmal alle deiktischen Ausdrücke, deren Verwendung Kopräsenz der Kommunikationspartner voraussetzt, dann typischerweise Ausdrücke, die stärker dem umgangssprachlichen Register zugeordnet werden (d.h. gesprochene Sprache verstanden als Nähekommunikation) und schließlich die Mittel, die mit der spezifischen dialogischen Kommunikationssituation zu tun haben (also Modalpartikeln, Diskurs- bzw. Gesprächspartikeln, die u.a. als Bestätigungs-, Fortsetzungs-, Rückmelde-, Beendigungssignale eingesetzt werden können, die der Reparatur bzw. Korrektur dienen oder Verstehen und Nicht-Verstehen signalisieren, und schließlich die neu etablierte Gruppe der Diskursmarker ${ }^{5}$ (grundlegend Imo 2012; Blühdorn et al. 2017)). Hierzu gehören auch umfangreichere Formulierungsroutinen wie ich mein, sag mal, weißt du (Imo 2009), ich sag mal, ehrlich gesagt, die wie Partikeln funktionieren. Die Klasse dieser Ausdrücke mit zentral dialogbezogener Funktion steht besonders im Fokus der Forschung und wird laufend durch neue Ausdrücke erweitert; sie wird besonders oft als Desiderat im DaF-Unterricht benannt und auch immer wieder für die Didaktisierung vorgeschlagen (vgl. z. B. Fiehler 2015; Weidner 2015; Günthner 2015).

Auf der morphosyntaktischen Ebene ist für die gesprochene Sprache zum einen der geringere Gebrauch verschiedener Strukturen und Formen typisch (wenig Konjunktiv in der indirekten Rede, wenig Passiv, andere Tempusformen); typische gesprochensprachliche Formen sind v. a. der am-Progressiv, Dativ-Passiv mit kriegen als Passiv-Hilfsverb, von-Phrasen oder Dativ statt attributivem Genitiv, Possessiva ohne Fernkongruenz (Qualität hat seinen Preis), daneben auch vielfältige Wortbildungsoperationen, wie etwa Konversionen.

Die Erscheinungen auf der syntaktischen Ebene, die als typisch gesprochensprachlich genannt werden und die besonders viel forscherische Aufmerksamkeit erfahren haben, sind ganz wesentlich auf die spezifischen Bedingungen mündlicher Kommunikation zurückzuführen; das ist nach Auer (2000, 2007) die Linearität in der Zeit, die Synchronisierung der Handlungsabläufe zwischen den Kommunikationspartnern und der an Mustern (constructions) orientierte Ablauf, die „online-Syntax“. Stärker formal-grammatisch betrachtet zeigt sich diese an spezifischen Stellungserscheinungen insbesondere an den Rändern von „Sätzen“ bzw. Äußerungen: so die - mit unterschiedlichen Begriffen belegten - Erscheinungen vor Sätzen (wie etwa Linksversetzungen, Freies Thema, Referenz-Aussage-Strukturen bzw. Operator-Skopus-Strukturen), die z. B. der Präsentation eines Themas dienen können, dem Gesprächspartner die

5 Die Kategorien und die terminologischen Unterschiede sind wie immer groß, im Bereich der gesprochenen Sprache und der Partikeln ganz besonders. 
thematische Orientierung erleichtern oder seine Aufmerksamkeit steuern. Hierher gehören auch die gesprochensprachlichen Fälle von sogenannter doppelter Vorfeldbesetzung (bestimmte Adverbien bzw. Diskursmarker, wenn-Sätze, um-Infinitive o. ä. besetzen zusammen mit einem anderen Satzglied (meist dem Subjekt) ohne Pause und intonatorisch integriert das Vorfeld; vgl. dazu Auer 1997). Am Ende von „Sätzen“ bzw. Äußerungen sind für die gesprochene Sprache verschiedene Formen der Nachfeldbesetzung (wie Nachträge oder Rechtsversetzungen) typisch, die etwa Informationen nachliefern, expandieren, präzisieren, spezifizieren oder auch reparieren (s. dazu u.a. Imo 2015), daneben auch Ausklammerungen. Eine weitere Erscheinung der gesprochensprachlichen Syntax sind „Ellipsen“ - ein besonders in der Forschung zur gesprochenen Sprache sehr kontrovers diskutierter Begriff, da damit meist ein Konzept der Reduktion bzw. Ergänzbarkeit und somit ein durchaus als problematisch angesehener Vollständigkeitsbegriff zugrunde liegt. Ellipsen sind dann gesprochensprachliche Erscheinungen, denen kein im schriftsprachlichen Sinne verstandener vollständiger „Satz“ zugrunde liegt (wie etwa Her mit den Bonbons! Da runter! Nicht den!). Als syntaktisches Kennzeichen ist hier schließlich noch der Klassiker zu nennen: weil-Sätze mit Verb-Zweit und ihre Schwestern mit obwohl, wobei u.ä. Dass hier keine syntaktische Veränderung vorliegt, sondern semantisch-lexikalische Differenzierung, ist in der einschlägigen Literatur (vgl. etwa schon Wegener 1993; Günthner 2002) hinreichend belegt. Unter normativem Gesichtspunkt und aus der DaF-Perspektive ist dies eine der besonders intensiv problematisierten Erscheinungen der (vor allem) gesprochenen Sprache (vgl. Thurmair 2005), sie wird mittlerweile in den neueren Lehr- und Lernmaterialien durchgehend erwähnt. Die letzte Gruppe von typisch gesprochensprachlichen Erscheinungen sind v. a. Performanzerscheinungen wie Neubearbeitung, Wiederholung, Umformung, die etwa zu Anakoluthformen, d. h. Konstruktionsabbrüchen und Konstruktionswechsel, zu Korrekturen oder zu Apokoinu-Konstruktionen führen (vgl. genauer Schwitalla 2012: 117-129).

Auch auf der diskurspragmatischen Ebene lassen sich spezifische gesprochensprachliche Erscheinungen feststellen, wobei diese vermutlich in den verschiedenen Diskursarten je spezifisch eingesetzt werden. Es handelt sich hier einmal um spezifische Formulierungsverfahren wie etwa Wiederholungen, die der Bekräftigung, der Verständnissicherung, der Hörerbestätigung, als Aufmerksamkeitssignal u.a. dienen können (vgl. Schwitalla 2012: 173-191), oder Ankündigungen, Paraphrasen oder auch das Code-Switchen (z. B. zwischen dialektnäheren und weniger nahen Formen). Zum anderen lassen sich hier auch Fälle von typischen festen Ausdrücken, formelhaften Routinen anführen, die insbesondere in der gesprochenen Sprache diskurspragmatisch zu verschiedenen Zwecken eingesetzt werden; etwa: na und ob! schön/nett/super/ 
Mist, dass ...! Der und freundlich/verständnisvoll/sparsam! Für diese verschiedenen Formulierungsroutinen lässt sich ein umfangreiches Reservoir für die gesprochene Sprache feststellen, das seit längerem etwa auch im Rahmen der construction grammar/Konstruktionsgrammatik intensiver erforscht wird.

Diese Fülle an Charakteristika der gesprochenen Sprache, die die Forschungen mittlerweile herausgearbeitet haben (vgl. auch die einschlägigen Kapitel in den Grammatiken von Zifonun 1997; Weinrich 2003; Duden 2016), sind selbstverständlich auch für Lernende, die eine echte mündliche Kompetenz erwerben sollen und damit in einem fremdsprachendidaktischen Kontext, von Relevanz. Ihre Einbeziehung wird auch, gerade aus Sicht einer Auslandsgermanistik, immer wieder gefordert (vgl. etwa Durell 2006, 2012; Moraldo 2012). Die oben angeführten Charakteristika unterscheiden sich aber durchaus zum einen darin, wie sehr sie von einer (eher an der Schriftsprache orientierten) Standardvarietät ${ }^{6}$ abweichen (das berührt Fragen der Norm), ob sie für Lernende rezeptiv oder auch produktiv relevant sind und ob sie überhaupt explizit vermittelt werden können bzw. müssen.

In der Forschung kommen auch zunehmend Formen einer konzeptionellen Mündlichkeit in den Blick, die zum Teil vergleichbare Kennzeichen aufweisen wie die vorher genannten der gesprochenen Sprache. ${ }^{7}$ So werden die einschlägigen Charakteristika von neuen Textsorten in den Neuen Medien analysiert und zum Teil auch für den DaF-Unterricht aufbereitet, die konzeptionell mündlicher Natur sind, wie etwa Chat (vgl. Kilian 2005), E-Mail/SMS (Dittmann 2006; Topalovic 2009), Internetforen (Ehrhardt 2012) und Twitter (Moraldo 2015). Imo (2009: 54) meint zutreffend: „Der Aspekt, dass für computervermittelte Kommunikation eine ,emulierte Mündlichkeit‘ (...) typisch ist, kann für den DaF-Bereich überhaupt nicht ernst genug genommen werden“. Für DaF-Lerner sei das Internet einer der ersten Kontakte mit der deutschen Umgangssprache und biete die Chance schlechthin, sich auf die Sprachrealität in Deutschland vorzubereiten. Allerdings wird hier im Bereich der konzeptionellen Mündlichkeit das sprachdidaktisch grundsätzliche Problem der ungeheuren Bandbreite der Varianz von authentischen Vorkommen ${ }^{8}$ noch viel

\footnotetext{
6 Die Frage, ob und wie weit eine solche Standardvarietät, wie sie sich auch in Grammatiken oder im Zweifelsfälle-Duden (Duden 2016) finden lässt, Grundlage des Fremdsprachenunterrichts sein kann/soll, wird diskutiert.

7 Rösler (2016: 138-140) weist allerdings darauf hin, dass hier noch Forschungsbedarf besteht und beklagt für den DaF-Bereich die fehlende Differenzierung zwischen medialer vs. konzeptioneller Mündlichkeit und Schriftlichkeit.

8 Diese Variationsbreite hat zum einen damit zu tun, dass sich eine grundsätzliche Veränderung von Kommunikationsformen, genauer ein kommunikationsstruktureller Wandel durchsetzt (Linke 2000 bezeichnet entsprechende Tendenzen als „Ent-Distanzierung“ und „Familiarisierung“),
} 
virulenter, da diese Texte eben nicht flüchtig sind. Entsprechende authentische Belege machen überzeugend deutlich, wie nötig aus der DaF-Perspektive eine reflektierte Diskussion zu Fragen von Standard, Norm und Variation ist (s. auch Breindl 2017: 53). Aus der Vermittlungsperspektive ist eine Einhegung der Varianz unerlässlich.

Unter dem Aspekt der Beziehung zwischen Germanistischer Sprachwissenschaft und DaF lässt sich also zusammenfassend feststellen, dass viele Phänomene der gesprochenen Sprache auf den unterschiedlichsten Ebenen mittlerweile sehr gut erforscht sind, dass sehr viel Erkenntnis akkumuliert wurde und dass DaF hier von den sprachwissenschaftlichen Forschungen sehr viel profitieren kann. Auch wird in vielen Fällen mit den einzelnen Analysen schon die Relevanz für DaF mit thematisiert. Die Entscheidungen aber, wie die Fülle von Erkenntnissen in einen DaF-Unterrichtskontext einzubringen ist, ist eine fremdsprachendidaktische und keine linguistische, meint Rösler (2016: 139) zutreffend, und weiter: „Die Tatsache, dass die Linguistik zunehmend ausdifferenziert die gesprochene Sprache analysiert, ist für die Fremdsprachendidaktik ein wichtiger Hinweis darauf, dass sie einen Aspekt ihrer Zielsprache zu wenig im Auge gehabt hat und dass sie sich Gedanken über dessen Integration machen muss. Diese Tatsache bestimmt jedoch nicht das Ob und schon gar nicht das Wie“. Damit macht er auch die Grenzen der Sprachwissenschaft im Zusammenhang mit den Forschungen zur gesprochenen Sprache sehr deutlich.

Selbstverständlich müssen Lernende mit sprachlicher Variation bekannt gemacht werden (das betrifft nicht nur die gesprochene Sprache, sondern auch andere Parameter wie regionale/nationale Varianz), aber andere, fremdsprachendidaktische Parameter sind ebenso wesentlich: Zielgruppe, Sprachniveau der Lernenden, angestrebte Zielnorm, Frequenz und Verbreitung einer sprachlichen Struktur (s. dazu Rösler 2016).

Was die Forschungen zur gesprochenen Sprache bzw. auch zur konzeptionell mündlichen Sprache betrifft, so werden aber immer noch eine Reihe von Desideraten benannt, die auch aus DaF-Sicht von großem Interesse sind: So fehlen systematische Untersuchungen von unterschiedlichen Diskursarten (bzw. Genres bzw. kommunikativen Gattungen), ähnlich den oben (4.1) erwähnten Textsortenanalysen, deren unterschiedliche Charakteristika und Konventionen aus fremdsprachendidaktischer Sicht eine Basis für den Umgang mit sprachlicher Variation bieten könnten, vielleicht auch deutlicher in Richtung

und zum anderen, dass es durch die Ausdehnung der schriftbasierten Kommunikation auf das Internet zu einem ungeheuren ,Variationsschub im Textsortenbereich gekommen ist, weil digitale Texte für Autoren wie Produzenten räumlich und zeitlich immer umfassender zugänglich sind. 
eines gesprochenen Standards gehen könnten und ein Reservoir an spezifischen Gesprächsroutinen bereitstellen könnten. „Anders als bei der Textsortenlinguistik, die die Schreibdidaktik seit langem orientiert und inspiriert, steht aber die systematische Nutzung [der] Mündlichkeitsforschung für DaFLehr- und Lernkontexte weitgehend noch aus“, meint Schramm (2017: 5) in diesem Zusammenhang zutreffend.

\subsection{Weitere Schnittstellen zwischen DaF und Germanistischer Sprachwissenschaft in der Forschung}

Neben den oben dargestellten zentralen Schnittstellen im Bereich der Text(sorten)linguistik und der Gesprochenen Sprache lassen sich natürlich noch eine Reihe weiterer Schnittstellen zwischen DaF und Germanistischer Sprachwissenschaft ausmachen. Diese können stärker inhaltlich oder auch stärker methodisch sein.

Korpuslinguistische Zugänge sind für die DaF-bezogene Forschung essentiell. Zum einen - naheliegenderweise - für alle Formen der Forschungen zum Spracherwerb. Hier sind mittlerweile eine Reihe von Untersuchungen vorgelegt worden, insbesondere auch an Lernerkorpora wie FALKO. ${ }^{9}$ Korpuslinguistische Herangehensweisen können aber auch die Grammatikvermittlung besser fundieren, indem sie auf der Basis des tatsächlichen Sprachgebrauchs helfen, adäquate(re) Regeln für die Vermittlung $\mathrm{zu}$ formulieren ${ }^{10}$, Regeln nach ihrer Zuverlässigkeit und Reichweite stärker zu gewichten, Grammatikerklärungen und Grammatikurteile besser zu überprüfen, sprachliche Variationen angemessener zu beurteilen oder Konstruktionen (bzw. chunks) zu bestimmen (zu Korpuslinguistik und DaF siehe etwa Fandrych \& Tschirner 2007; Schmidt 2008).

Konstruktionsgrammatische Ansätze können aus DaF-Sicht die Zusammenhänge zwischen Grammatik und Lexikon bzw. zwischen grammatischem und lexikalischem Lernen besser fundieren. In neueren DaF-Ansätzen wird u. a. auf Forschungen zur Konstruktionsgrammatik aufbauend - vermehrt mit chunks unterschiedlicher Struktur und Komplexität gearbeitet (vgl. schon Ellis 2002; daneben Haberzettl 2006; Handwerker \& Madlener 2013; Handwerker

\footnotetext{
9 https://www.linguistik.hu-berlin.de/de/institut/professuren/korpuslinguistik/forschung/ falko/standardseite, letzter Zugriff: 20.11. 2017.

10 Ein in diesem Zusammenhang wiederholt erwähntes Beispiel ist etwa die in Lehrmaterialien durchgehend als „Wechselpräposition“ beschriebene Präposition über, die nach Korpusanalysen aber zu 99\% mit dem Akkusativ verwendet wird, hinter dagegen zu $80 \%$ mit Dativ (s. Schmidt 2008: 75, die hier Jones zitiert).
} 
2015). Auch hier ist natürlich eine korpuslinguistische Herangehensweise unerlässlich. Stärker konstruktionsgrammatische Ansätze bieten sich im grammatischen Bereich an etwa für den Absentiv (Stollhans 2015), für Verbklassen und Partizipien (Handwerker \& Madlener 2009/2013), für Formen von machen + Adjektiv (Fehrmann \& Möller 2012), Orts- und Zustandsveränderungen (Handwerker 2015); didaktisch ergiebig könnte auch das Zustandspassiv sein, Satzglieder und Valenz, sekundäre Prädikate und anderes mehr. Hier ließe sich aus DaF-Sicht also mithilfe konstruktionsgrammatischer Ansätze, kombiniert mit korpuslinguistischen Methoden besser, weil fundierter, an den einzelnen Phänomenen entscheiden, wo regelbasierter oder lexembasierter Spracherwerb ergiebiger ist.

Eine ganz zentrale Schnittstelle zwischen DaF und Sprachwissenschaft besteht natürlich in allen variationslinguistischen Ansätzen. Die Variationsbreite der Sprache wurde (und wird) schließlich nicht nur im Zusammenhang mit der Medialität, also vor allem der gesprochenen Sprache diskutiert, sondern schon früher im Konzept der nationalen Varietäten; dieses fand relativ schnell Eingang in den DaF-Bereich (übrigens nicht nur im linguistischen Bereich, sondern auch im Bereich der Landeskundevermittlung angesichts der aufkommenden Forderungen nach einem breiteren Ansatz, der sich im sogenannten DACH-Konzept niederschlug). ${ }^{11}$ Während aus DaF-Sicht der Umgang mit nationalen Varianten, wie sie sich vor allem im lexikalischen Bereich zeigen, nicht so problematisch ist (in Lehrwerken werden dann einfach die österreichischen oder schweizerdeutschen Varianten genannt und als solche markiert), ist die medial bedingte Varianz für die Sprachvermittlung herausfordernder, da hier Fragen von Standard, Norm und Register u. ä. virulent werden. Hennig (2017: 24) sieht eine Reihe von Indizien dafür, dass ,grammatische Variation als ein Zentralthema der germanistischen Linguistik des 21. Jahrhunderts aufgefasst werden kann“. Der Bereich DaF ist davon ganz besonders betroffen: Gerade im Sprachvermittlungskontext ist eine Einhegung der Varianz unerlässlich, und ein Bedürfnis nach mehr als reiner Deskription sprachlicher Variation ist in vielen DaF-Kontexten, insbesondere im nicht-deutschsprachigen Ausland nicht zu unterschätzen. Die Germanistische Sprachwissenschaft, die normative Fragen ja gerne ausgeblendet hat, öffnet sich neuerdings diesen Aspekten: so werden in den einzelnen Beiträgen zur gesprochenen Sprache und zur konzeptionellen Mündlichkeit Normfragen immer wieder berührt (s. z. B. Schneider 2015, 2016), genauso in variationslinguistischen Publikationen

11 Vgl. dazu etwa das Kapitel V „Variation und Sprachkontakt“ in Krumm, Fandrych, Hufeisen \& Riemer 2010, 343-457; zum DACH-Konzept (= Deutschland (D) - Österreich (A) Schweiz (CH)) s. Fischer, Frischherz \& Noke (2010). 
(s. z. B. jüngst die Beiträge in Konopka \& Wöllstein (2017), besonders auch Eichinger 2017, oder in Günthner, Imo, Meer \& Schneider 2012), und neuerdings werden auch Sprachkodexfragen diskutiert (vgl. die Beiträge in Klein \& Staffeldt 2016) - ein Aspekt, der für DaF bisher keine große Rolle gespielt hat, dabei kann die Rolle von Lehrwerkautoren, Lehrkräften und Muttersprachlern in dieser Hinsicht nicht hoch genug eingeschätzt werden (sie geben unweigerlich Normen vor, wenn auch nicht immer klar ist, auf welcher Basis das geschieht). Ein weiterer Aspekt, von dem auch DaF profitieren wird, sind Angebote der Sprachberatung, insbesondere auch im Internet, die für DaF zunehmend wichtiger werden (s. dazu Breindl 2016). Von einer Professionalisierung vieler dieser Sprachberatungsangebote würden die im DaF-Bereich Tätigen ganz sicher profitieren.

Unter einer ganz anderen Perspektive gibt es schließlich breite Schnittstellen zwischen DaF und der Fachsprachenforschung und neuerdings zwischen DaF und der Wissenschaftssprachforschung. Hier scheint sich die Beeinflussungsrichtung fast umgedreht zu haben: auch die frühen Fachsprachenforschungen scheinen wesentliche Impulse aus der (fach- wie fremdsprachlichen) Didaktik erhalten zu haben. Für die Wissenschaftssprachforschung jedenfalls lässt sich konstatieren, dass alle wesentlichen Forschungen und die Impulse zu Forschungen aus dem Bereich Deutsch als Fremdsprache bzw. von in diesem Bereich tätigen Personen kommt (vgl. etwa die frühen Publikationen von Weinrich 1989, 1995, etwas später Ehlich 1993, 1995, 1999 und andere, etwa Fandrych 2006, 2014; Graefen 2001, 2004; Moll \& Thielmann 2017). Hier sind eine ganze Reihe von sprachlichen Kennzeichen herausgearbeitet worden, neben idiomatischen Strukturen, metaphorischen Verfahren oder Sprechhandlungskommentaren (in letzter Zeit auch im gesprochenen Wissenschaftsdeutsch) auch verschiedene Verfahren der Kondensation, die für das Deutsche ja vielfältig ausgeprägt und besonders typisch sind. All das sind eher Erscheinungen einer konzeptionell schriftlichen Sprache, und möglicherweise entwickelt sich im Zusammenhang mit diesen Forschungen so etwas wie eine „Grammatik der Schriftlichkeit“, was sich wiederum mit Diskussionen (aus dem eher muttersprachlichen bzw. DaZ-Bereich) zur Bildungssprache trifft.

Eine sehr umfangreiche Schnittstelle lässt sich auch zwischen DaF und einer Kontrastiven Linguistik ausmachen: Gerade für die Sprachvermittlung sind kontrastive Ansätze außerordentlich hilfreich (s. dazu Tekin 2012), ${ }^{12}$ nicht nur - wie in frühen Zeiten - als Sprachvergleich zwischen der Erstsprache der

12 Natürlich ist die Anwendung der Kontrastiven Linguistik im Zusammenhang mit Spracherwerb und -vermittlung nur eines ihrer Felder; zur Standortbestimmung der Kontrastiven Linguistik s. ausführlich Tekin 2012; Eichinger 2012; König 2012. 
Lernenden und dem Deutschen, und auch nicht nur - wie in der Tertiärsprachendidaktik - auf das Englische ausgerichtet, sondern es sollten möglichst viele Sprachen mit einbezogen werden. ${ }^{13}$ Zum einen ist eine kontrastiv ausgerichtete Linguistik auch im Sinne der Mehrsprachigkeitsüberlegungen grundlegend: Sprachreflexionen erfordern wenigstens grundsätzliche Kenntnisse von Strukturunterschieden verschiedener Sprachen; eine kontrastive Ausrichtung ist aber auch im Sinne der internationalen Forschungslandschaft relevant, denn die Germanistik in den nicht-deutschsprachigen Ländern beschäftigt sich naturgemäß meist auch mit kontrastiven Fragestellungen.

Ein etwas anderer, aber letztlich auch aus Sprachvergleich resultierender Beitrag der Sprachwissenschaft, von dem DaF erheblich profitieren kann, geht in den Bereich des bewertenden Sprachvergleichs. Deutsch gilt als schwere Sprache, als „Latein des 20. Jhdts“, und was der (eher negativen) Bewertungen mehr sind. Hier ist es ein großes Verdienst verschiedener sprachwissenschaftlicher Forschungen, diese Ansichten auf wissenschaftliche Füße zu stellen (vgl. dazu die Beiträge in Stickel 2003, insbesondere Fabricius-Hansen 2003 oder in Wolff \& Winters-Ohle 2000, auch Wegener 2007), indem sie bestimmte als schwer geltende (Be-)Sonderheiten der deutschen Sprache (Genus, Klammer, Großschreibung) in ihrer Funktionaliät analysieren und auf dieser Basis differenziert bewerten: So entsteht etwa das schöne Konzept des Deutschen als „reife Sprache“ (Fabricius-Hansen 2003) oder die Vorstellung von Sprachen, die aufgrund ihrer morphologischen Ausdifferenziertheit eine Reihe von Gestaltungsmöglichkeiten in der Anordnung bieten (auch ein Vorteil) oder von Sprachen, die eher sprecher- bzw. produzentenfreundlich oder hörerbzw. rezipientenfreundlich sind (wie eben das Deutsche). Wer das Deutsche vermitteln muss (bzw. darf), ist froh über solche wissenschaftlich fundierten positiven Bewertungen!

\section{Germanistische Sprachwissenschaft und DaF: eine Einbahnstraße?}

Im Vorangegangenen wurden vielfältige Schnittstellen zwischen Germanistischer Sprachwissenschaft und DaF gezeigt, und es wurde deutlich gemacht, wo und wie DaF von der Sprachwissenschaft beeinflusst wurde und wird und

13 In diesem Sinne wird die am IdS erarbeitete „Grammatik des Deutschen im europäischen Vergleich“ einen willkommenen Ausgangspunkt bieten auch für entsprechende sprachvermittelnde Umsetzungen. 
gegebenenfalls profitieren kann. Zum Abschluss soll kurz die Frage erörtert werden, ob diese Beeinflussungen unidirektional sind - anders gefragt: Kann denn DaF auch der Germanistischen Sprachwissenschaft etwas bieten? Grundsätzlich lässt sich hervorheben, dass der für eine DaF-Linguistik konstituierende fremde Blick und der Vermittlungsaspekt für viele Themen oder auch für die methodische Herangehensweise in ganz anderer Weise sensibilisieren. Einige wenige kurze Schlaglichter mögen hier genügen: So gibt es Themen, die zunächst überhaupt erst aus einer fremden, in irgendeiner Weise sprachvergleichenden Perspektive in den forscherischen Blick geraten sind: Dies gilt z. B. für die Abtönungs- bzw. Modalpartikeln (vgl. Krivonosow 1963 oder Weydt 1969); die Problematik der erweiterten Partizipialgruppen und der Nominalklammer ist wesentlich auch durch DaF-Didaktiker (Rall \& Rall 1983) bzw. durch kontrastiv wahrnehmende Forscher (z. B. Weinrich 1986) beleuchtet worden. Auch die Beschreibung bestimmter Phänomene gerade in der als schwierig geltenden Morphologie des Deutschen hat durch einen vermittlungsrelevanten Blick ganz sicher gewonnen: So z. B. die Adjektivdeklination, die mit dem Konzept der Monoflexion (nach Admoni ${ }^{4} 1982$ ) heute eigentlich Standard in allen Lehrwerken ist (was sich aber offensichtlich in der sprachwissenschaftlichen Literatur nicht überall durchgesetzt hat, vgl. Wegener 2016: 116); auch die veränderte Darstellung der Deklination im Bereich des Nomens (wie sie sich etwa in der Duden-Grammatik ab der 7. Auflage 2005, $182 \mathrm{ff}$. findet im Gegensatz zu den Darstellungen in früheren Auflagen) könnte didaktisch beeinflusst sein. Es ließen sich ganz sicher noch eine Reihe weiterer Phänomene anführen, bei denen eine Beeinflussung der Germanistischen Sprachwissenschaft durch DaF stattgefunden hat. Ganz grundsätzlich lässt sich deshalb konstatieren, dass der fremde Blick und die didaktische Perspektive durchaus für die Germanistische Sprachwissenschaft von Vorteil sein können.

\section{Literatur}

Adamzik, Kirsten (2004): Textlinguistik. Eine einführende Darstellung. Tübingen: Niemeyer. Adamzik, Kirsten (2011): Textsortennetze. In Stephan Habscheid (Hrsg.), Textsorten, Handlungsmuster, Oberflächen. Typologien der Kommunikation, 367-385. Berlin/ Boston: De Gruyter.

Adamzik, Kirsten \& Wolf-Dieter Krause (Hrsg.) (2009): Text-Arbeiten. Textsorten im fremdund muttersprachlichen Unterricht an Schule und Hochschule. 2. Aufl. Tübingen: Narr. Admoni, Wladimir (1982): Der deutsche Sprachbau, 4. Aufl. München: Beck. Auer, Peter (1997): Formen und Funktionen der Vor-Vorfeldbesetzung im gesprochenen Deutsch. In Peter Schlobinski (Hrsg.), Syntax des gesprochenen Deutsch, 55-92. Opladen. 
Auer, Peter (2000): On line-Syntax - Oder: was es bedeuten könnte, die Zeitlichkeit der mündlichen Sprache ernst zu nehmen. Sprache und Literatur 85 (2000), 43-56.

Auer, Peter (2007): Syntax als Prozess. In Heiko Hausendorf (Hrsg.), Gespräch als Prozess, 95-124. Tübingen: Narr.

Bachmann, Thomas \& Helmut Feilke (Hrsg.) (2014): Werkzeuge des Schreibens. Beiträge zu einer Didaktik der Textprozeduren. Stuttgart.

Bachmann-Stein, Andrea \& Stephan Stein (Hrsg.) (2009): Mediale Varietäten: Analysen von gesprochener und geschriebener Sprache und ihre fremdsprachendidaktischen Potenziale. Landau: VEP.

Blühdorn, Hardarik, Arnulf Deppermann, Henrike Helmer \& Thomas Spranz-Fogasy (Hrsg.) (2017): Diskursmarker im Deutschen, Reflexionen und Analysen. Verlag für Gesprächsforschung.

Breindl, Eva (2016): Sprachberatung im interaktiven Web. In Klein \& Staffeldt (Hrsg.), 85-109.

Breindl, Eva (2017): Rezension von Gabriella Gárgyán: Der am-Progressiv im heutigen Deutsch, Deutsch als Fremdsprache 54 (1), 52-53.

Brinker, Klaus (2005): Linguistische Textanalyse. Eine Einführung in Grundbegriffe und Methoden. 6. Aufl. Berlin: Erich Schmidt.

Dalmas, Martine, Marina Foschi Albert, Marianne Hepp \& Eva Neuland (Hrsg.) (2015): Texte im Spannungsfeld von medialen Spielräumen und Normorientierung. München: iudicium.

Dittmann, Jürgen (2006): Konzeptionelle Mündlichkeit in E-Mail und SMS. In Ulrike Reeg (Hrsg.), Interkultureller Fremdsprachenunterricht. Grundlagen und Perspektiven, 79-97. Bari.

Dobstadt, Michael, Christian Fandrych \& Renate Riedner (Hrsg.) (2015): Linguistik und Kulturwissenschaft: Zu ihrem Verhältnis aus der Perspektive des Faches Deutsch als Fremd- und Zweitsprache und anderer Disziplinen. Frankfurt a. M.: Peter Lang.

Duden (2016): Die Grammatik. 9. Auflage. Mannheim u. a.: Duden-Verlag.

Duden (2016): Richtiges und gutes Deutsch: das Wörterbuch der sprachlichen Zweifelsfälle. 8. Auflage. Mannheim u. a.: Duden-Verlag.

Durrell, Martin (2006): Deutsche Standardsprache und Registervielfalt im DaF-Unterricht. In Eva Neuland (Hrsg.), Variation im heutigen Deutsch: Perspektiven für den Sprachunterricht, 111-122. Frankfurta. M.: Peter Lang.

Durrell, Martin (2012): Zur Relativierung von hochsprachlichen Normen in der deutschen Sprache der Gegenwart. Der Blick von außen. In Günthner, Imo, Meer \& Schneider (Hrsg.), 85-102.

Dürscheid, Christa (2016): Reflexion über Sprache im DaF-Untericht - am Beispiel von kleinen Texten. In Freudenberg-Findeisen (Hrsg), 167-183.

Ehlich, Konrad (1993): Deutsch als fremde Wissenschaftssprache. Jahrbuch Deutsch als Fremdsprache 19, 13-42.

Ehlich, Konrad (1995): Die Lehre der deutschen Wissenschaftssprache: sprachliche Strukturen, didaktische Desiderate. In Heinz L. Kretzenbacher \& Harald Weinrich (Hrsg.), Linguistik der Wissenschaftssprache, 325-351. Berlin/New York: De Gruyter.

Ehlich, Konrad (1999): Alltägliche Wissenschaftssprache. Info DaF 26 (1), 3-24.

Ehrhardt, Claus (2012): Internetforen: Eine Kommunikationsform zwischen Mündlichkeit und Schriftlichkeit. In Reeg, Gallo \& Moraldo (Hrsg.), 73-97.

Eichinger, Ludwig M. (2012): Deutsch im Sprachvergleich. Grammatische Kontraste und Konvergenzen. In Lutz Gunkel \& Gisela Zifonun (Hrsg.), Deutsch im Sprachvergleich, VII-XVI Berlin/New York: De Gruyter. 
Eichinger, Ludwig M. (2017): Standarddeutsch - die beste aller möglichen Sprachen. In Konopka \& Wöllstein (Hrsg.), 3-17.

Ellis, Nick C. (2002): Frequency effects in lanugage acquisition. Studies in Second Language Acquisition 24, 143-188.

Fabricius-Hansen, Cathrine (2003): Deutsch - eine ,reife‘ Sprache. Ein Plädoyer für die Komplexität. In Stickel (Hrsg.), 99-112.

Fabricius-Hansen, Cathrine (2010): Adjektiv-/Partizipialattribute im diskursbezogenen Kontrast (Deutsch - Englisch/Norwegisch). Deutsche Sprache 38 (2), 175-192.

Fandrych, Christian (2006): Bildhaftigkeit und Formelhaftigkeit in der allgemeinen Wissenschaftssprache als Herausforderung für Deutsch als Fremdsprache. In Konrad Ehlich \& Dorothee Heller (Hrsg.), Die Wissenschaft und ihre Sprachen, 39-61. Bern.

Fandrych, Christian (2010): Grammatikerwerb und -vermittlung. In Krumm, Fandrych, Hufeisen \& Riemer (Hrsg.), 1008-1021.

Fandrych, Christian (2011): ... die auf Sockeln stehenden Monumentalfiguren: Verschachtelung und Entschachtelung im Deutsch als Fremdsprache-Unterricht. In Barbara Schmenk \& Nicola Würffel (Hrsg.), Drei Schritte vor und manchmal auch sechs zurück, 49-58. Tübingen: Narr.

Fandrych, Christian (2014): Metakommentierungen in wissenschaftlichen Vorträgen. In Christian Fandrych, Cordula Meißner \& Adriana Slavcheva (Hrsg.), Gesprochene Wissenschaftssprache: Korpusmethodische Fragen und empirische Analysen, 95-111. Heidelberg: Synchron.

Fandrych, Christian (2016): Fokus Sprache. In Eva Burwitz-Melzer et al. (Hrsg.), Handbuch Fremdsprachenunterricht. 6. Aufl., 34-38, Tübingen: Narr.

Fandrych, Christian, Britta Hufeisen, Hans-Jürgen Krumm \& Claudia Riemer (2010): Perspektiven und Schwerpunkte des Faches Deutsch als Fremd- und Zweitsprache. In Krumm, Fandrych, Hufeisen \& Riemer (Hrsg.), 1-18.

Fandrych, Christian \& Maria Thurmair (2010): Textsortenvariationen im Zeitalter des Internets. In Foschi Albert, Hepp, Neuland \& Dalmas (Hrsg), 381-400.

Fandrych, Christian \& Maria Thurmair (2011a): Textsorten im Deutschen. Linguistische Analysen aus didaktischer Perspektive. Tübingen: Stauffenburg.

Fandrych, Christian \& Maria Thurmair (2011b): Plädoyer für eine textsortenbezogenen Sprachdidaktik. Deutsch als Fremdsprache 48 (2), 84-93.

Fandrych, Christian \& Maria Thurmair (2016): Grammatik an und mit Textsorten lernen: Das Prinzip der „Linksdeterminierung“ im Deutschen. In Freudenberg-Findeisen (Hrsg.), 185-200.

Fandrych, Christian \& Erwin Tschirner (2007): Korpuslinguistik und Deutsch als Fremdsprache. Ein Perspektivenwechsel. Deutsch als Fremdsprache 44 (4), 195-204.

Fehrmann, Ingo \& Max Möller (2012): Verwendungen von machen + Adjektiv als Beispiel für eine musterbezogene DaF-Vermittlung. Deutsch als Fremdsprache 49 (1), 12-19.

Fiehler, Reinhard (2012): Gesprochene Sprache - gehört sie in den DaF-Unterricht? In Reeg, Gallo \& Moraldo (Hrsg.), 13-28.

Fiehler, Reinhard (2015): Die Vielfalt der Besonderheiten gesprochener Sprache - und zwei Beispiele, wie sie für den DaF-Unterricht geordnet werden kann:

Gesprächspartikeln und Formulierungsverfahren. In Imo \& Moraldo (Hrsg.), 23-44.

Fischer, Roland, Bruno Frischherz \& Knuth Noke (2010): DACH-Landeskunde. In Krumm, Fandrych, Hufeisen \& Riemer (Hrsg.), 1500-1510.

Foschi Albert, Marina, Marianne Hepp \& Eva Neuland (Hrsg.) (2006): Texte in Sprachforschung und Sprachunterricht. München. 
Foschi Albert, Marina, Marianne Hepp, Eva Neuland \& Martine Dalmas (Hrsg.) (2010): Text und Stil im Kulturvergleich. München.

Freudenberg-Findeisen, Renate (2013): Netze - Felder - Texte. Für eine text(sorten)fundierte Grammatikarbeit. In Marta Fernandez Bueno et al. (Hrsg.), Rückblicke und neue Perspektiven, 351-363. Frankfurta. M.: Peter Lang.

Freudenberg-Findeisen, Renate (Hrsg.) (2016): Auf dem Weg zu einer Textsortendidaktik. Linguistische Analysen und text(sorten)didaktische Bausteine nicht nur für den fremdsprachlichen Deutschunterricht. Hildesheim: Olms.

Götze, Lutz, Gerhard Helbig, Gert Henrici \& Hans-Jürgen Krumm (2001/2010): Die Strukturdebatte als Teil der Fachgeschichte. In Krumm, Fandrych, Hufeisen \& Riemer (Hrsg.), 19-34.

Graefen, Gabriele (2001): Einführung in den Gebrauch der Wissenschaftssprache. Materialien Deutsch als Fremdsprache 58, 191-210. Regensburg: FaDaF.

Graefen, Gabriele (2004): Aufbau idiomatischer Kenntnisse in der Wissenschaftssprache. Materialien Deutsch als Fremdsprache 73, 293-309. Regensburg: FaDaF.

Grundler, Elke \& Carmen Spiegel (Hrsg.) (2014): Konzeptionen des Mündlichen wissenschaftliche Perspektiven und didaktische Konsequenzen. Bern: hep.

Günthner, Susanne (2000): Grammatik der gesprochenen Sprache - eine Herausforderung für Deutsch als Fremdsprache? Info DaF 27, 352-366.

Günthner, Susanne (2002): Konnektoren im gesprochenen Deutsch: Normverstoß oder funktionale Differenzierung? Deutsch als Fremdsprache 39, 67-74.

Günthner, Susanne (2015): Diskursmarker in der Interaktion - zum Einbezug alltagssprachlicher Phänomene in den DaF-Unterricht. In: Imo \& Moraldo (Hrsg.), 135-164.

Günthner, Susanne, Wolfgang Imo, Dorothee Meer \& Jan Georg Schneider (Hrsg) (2012): Kommunikation und Öffentlichkeit. Sprachwissenschaftliche Potenziale zwischen Empirie und Norm. Berlin: De Gruyter.

Habermann, Mechthild (Hrsg.) (2010): Grammatik wozu? Mannheim u. a.: Duden-Verlag.

Haberzettl, Stefanie (2006): Konstruktionsgrammatik im Zweitspracherwerb. In Kerstin Fischer \& Anatol Stefanowitsch (Hrsg.), Konstruktionsgrammatik: Von der Anwendung zur Theorie, 55-77. Tübingen: Stauffenburg.

Handwerker, Brigitte (2015): Konstruktionen im L2-Lernformat: Orts- und Zustandsveränderungen in der Rezeption und Produktion des Deutschen als Fremdsprache. In Stefan Engelberg et al. (Hrsg.), Argumentstruktur zwischen Valenz und Konstruktion, 127-153. Tübingen: Narr.

Handwerker, Brigitte \& Karin Madlener (2013): Chunks für Deutsch als Fremdsprache. Theoretischer Hintergrund und Prototyp einer multimedialen Lernumgebung, 2. Aufl. Baltmannsweiler: Schneider.

Handwerker, Brigitte, Rainer Bäuerle \& Bernd Sieberg (Hrsg.) (2016): Gesprochene Fremdsprache Deutsch. Baltmannsweiler: Schneider.

Hennig, Mathilde (2000): Tempus und Temporalität in geschriebenen und gesprochenen Texten. Tübingen: Niemeyer.

Hennig, Mathilde (2017): Grammatik und Variation im Spannungsfeld von Sprachwissenschaft und öffentlicher Sprachreflexion. In Konopka \& Wöllstein (Hrsg.), 23-46.

Hennig, Mathilde \& Christoph Müller (Hrsg.) (2010): Wie normal ist die Norm? Sprachliche Normen im Spannungsfeld von Sprachwissenschaft, Sprachdidaktik und Sprachöffentlichkeit. Kassel: University Press. 
Hirschfeld, Ursula, Dietmar Rösler \& Karen Schramm (2016): Facetten der Mündlichkeit im DaF-Unterricht. Deutsch als Fremdsprache 53 (3), 131-134.

Hoffmann, Ludger \& Yüksel Ekinci-Kocks (Hrsg.) (2010): Sprachdidaktik in mehrsprachigen Lerngruppen. Vermittlungspraxis Deutsch als Zweitsprache. Baltmannsweiler: Schneider. Imo, Wolfgang (2009): Welchen Stellenwert sollen und können Ergebnisse der GesprocheneSprache-Forschung für den DaF-Unterricht haben? In Bachmann-Stein \& Stein (Hrsg.), 39-62.

Imo, Wolfgang (2012): Wortart Diskursmarker? In Björn Rothstein (Hrsg.), Nicht-flektierende Wortarten, 48-88. Berlin: De Gruyter.

Imo, Wolfgang (2015): Nachträge im Spannungsfeld von Medialität, Situation und interaktionaler Funktion. In Hélène Vinckel-Roisin (Hrsg.), Das Nachfeld im Deutschen: Theorie und Empirie, 231-253. Berlin: De Gruyter.

Imo, Wolfgang \& Sandro M. Moraldo (Hrsg.) (2015): Interaktionale Sprache und ihre Didaktisierung im DaF-Unterricht. Tübingen: Stauffenburg.

Kilian, Jörg (2005): DaF im Chat. Zur Grammatik geschriebener Umgangssprachen als Ergänzung zum Erwerb standardsprachlichen Wissens. In Michael Beißwenger \& Angelika Storrer (Hrsg.), Chat-Kommunikation in Beruf, Bildung und Medien: Konzepte - Werkzeuge - Anwendungsfelder, 201-220. Stuttgart.

Klein, Wolf Peter \& Sven Staffeldt (Hrsg.) (2016): Die Kodifizierung der Sprache. Strukturen, Funktionen, Konsequenzen. Würzburger elektronische sprachwissenschaftliche Arbeiten (WespA), 17.

Konopka, Marek \& Angelika Wöllstein (Hrsg.) (2017): Grammatische Variation. Empirische Zugänge und theoretische Modellierung. Berlin/Boston: De Gruyter.

König, Ekkehard (2012): Zur Standortbestimmung der Kontrastiven Linguistik innerhalb der vergleichenden Sprachwissenschaft. In: Lutz Gunkel \& Gisela Zifonun (Hrsg.), Deutsch im Sprachvergleich, 13-40. Berlin/New York: De Gruyter.

Köpcke, Klaus-Michael \& Arne Ziegler (Hrsg.) (2007): Grammatik in der Universität und für die Schule. Tübingen: Niemeyer.

Köpcke, Klaus-Michael \& Arne Ziegler (Hrsg.) (2011): Grammatik verstehen lernen - Zugänge zur Grammatik der deutschen Gegenwartssprache. Berlin/New York: De Gruyter.

Köpcke, Klaus-Michael \& Arne Ziegler (Hrsg.) (2013): Schulgrammatik und Sprachuntericht im Wandel. Berlin/Boston: De Gruyter.

Köpcke, Klaus-Michael \& Arne Ziegler (Hrsg.) (2015): Deutsche Grammatik in Kontakt. Deutsch als Zweitsprache in Schule und Unterricht. Berlin/Boston: De Gruyter.

Krivonosov, Alexej (1963): Die modalen Partikeln in der deutschen Gegenwartssprache. Berlin (masch. Diss.).

Krumm, Hans-Jürgen, Christian Fandrych, Britta Hufeisen \& Claudia Riemer (Hrsg.) (2010): Deutsch als Fremd- und Zweitsprache. Ein internationales Handbuch. Berlin/New York: De Gruyter.

Linke, Angelika (2000): Informalisierung? Ent-Distanzierung? Familiarisierung? Sprach(gebrauchs)wandel als Indikator soziokultureller Entwicklungen. Der Deutschunterricht 52 (3), 66-77.

Marschall, Matthias (1995): Textfunktionen der deutschen Tempora. Genf: Slatkine. Marx, Konstanze \& Georg Weidacher (2014): Internetlinguistik. Tübingen: Narr.

Moll, Melanie \& Winfried Thielmann (2017): Wissenschaftliches Deutsch. Konstanz: utb.

Moraldo, Sandro M. (2012): Gesprochene Sprache. Eine Herausforderung für den DaFUnterricht. In Andrea M. Birk \& Claudia Buffagni (Hrsg.), Linguistik und Sprachdidaktik im universitären DaF-Unterricht, 185-200. Münster u. a. 
Moraldo, Sandro M. (2015): Sprachliche Interaktion im Zeitalter der Neuen Medien. Die Kommunikationsplattform Twitter und ihre Bedeutung im Unterricht Deutsch als Fremdsprache. In Imo \& Moraldo (Hrsg.), 301-320.

Moraldo, Sandro M. \& Federica Missaglia (Hrsg.) (2013): Gesprochene Sprache im DaFUnterricht. Grundlagen - Ansätze - Praxis. Heidelberg: Winter.

Oomen-Welke, Ingelore (2016): Mehrsprachigkeit - Language Awareness Sprachbewusstheit. Eine persönliche Einführung. Zeitschrift für Interkulturellen Fremdsprachenunterricht, 21, H 2, 5-12.

Portmann-Tselikas, Paul (2001): Sprachaufmerksamkeit und Grammatiklernen. In Paul Portmann-Tselikas \& Sabine Schmölzer-Eibinger (Hrsg.), Grammatik und Sprachaufmerksamkeit, 9-48. Innsbruck u. a.

Rall, Dietrich \& Marlene Rall (1983): Gegen den Strich lesen: Das erweiterte Partizipialattribut als Lernschwierigkeit für Hispanophone. Jahrbuch Deutsch als Fremdsprache 9, 132-146.

Rall, Marlene (2001): Grammatikvermittlung. In Gerhard Helbig, Lutz Götze, Gert Henrici \& Hans-Jürgen Krumm (Hrsg.), Deutsch als Fremdsprache, 880-886 Berlin: De Gruyter.

Reeg, Ulrike, Pasquale Gallo \& Sandro M. Moraldo (Hrsg.) (2012): Gesprochene Sprache im DaF-Unterricht. Zur Theorie und Praxis eines Lerngegenstandes. Münster u. a.: Waxmann.

Rödel, Michael (Hrsg.) (2014): Deutschunterricht am Gymnasium - Was kann die Sprachwissenschaft leisten? Baltmannsweiler: Schneider.

Rösler, Dietmar (2016): Nähe und Distanz zur Mündlichkeit in der fremdsprachendidaktischen Diskussion. Deutsch als Fremdsprache 53 (3), 135-149.

Rothstein, Björn (2010): Sprachintegrativer Grammatikunterricht. Zum Zusammenspiel von Sprachwissenschaft und Sprachdidaktik im Mutter- und Fremdsprachenunterricht. Tübingen: Stauffenburg.

Rothstein, Björn (Hrsg.) (2011): Sprachvergeich in der Schule. Baltmansweiler: Schneider. Scherner, Maximilan \& Arne Ziegler (Hrsg.) (2006): Angewandte Textlinguistik: Perspektiven für den Deutsch- und Fremdsprachenunterricht. Tübingen.

Schmidt, Claudia (2008): Grammatik und Korpuslinguistik. Überlegungen zur Unterrichtspraxis DaF. Deutsch als Fremdsprache 45 (2), 74-80.

Schmölzer-Eibinger, Sabine \& Georg Weidacher (Hrsg.) (2007): Textkompetenz. Eine Schlüsselkompetenz und ihre Vermittlung. Tübingen: Narr.

Schneider, Jan Georg (2015): Gesprochenes Standarddeutsch und DaF. Begriffe - Empirie didaktische Implikationen. In Imo \& Moraldo (Hrsg.), 45-66.

Schneider, Jan Georg (2016): Syntax der gesprochenen Sprache und Kodifizierung. In Klein \& Staffeldt (Hrsg.), 272-284.

Schramm, Karen (2017): Pragmalinguistische Aspekte einer Mündlichkeitsdidaktik. Deutsch als Fremdsprache 54 (1), 3-9.

Schwitalla, Johannes (2012): Gesprochenes Deutsch. Eine Einführung. 4. Aufl. Berlin: Erich Schmidt.

Spiegel, Carmen \& Rüdiger Vogt (Hrsg.) (2006): Vom Nutzen der Textlinguistik für den Unterricht. Baltmannsweiler: Schneider.

Steinhoff, Annette (2011): Lernmedien Deutsch als Fremdsprache und die Vermittlung des Passivs. München: iudicium.

Stickel, Gerhard (Hrsg.) (2003): Deutsch von außen. Berlin/New York: De Gruyter.

Stollhans, Sascha (2015): „Ich bin dann mal Deutsch lernen!“: Der Absentiv im DaFUnterricht. GFL-Journal 1, 44-71. 
Tekin, Özlem (2012): Grundlagen der Kontrastiven Linguistik in Theorie und Praxis. Tübingen: Stauffenburg.

Thurmair, Maria (2005): „Aber man spricht doch ganz anders heute!“? Wortstellungsvariationen der gesprochenen Sprache im DaF-Unterricht. Fremdsprache Deutsch 32, 42-48.

Thurmair, Maria (2007): Ihre katzengrünen Augen blickten auf das mit edlem Buchenholz getäfelte Parkett. Zur Textsortenspezifik von Attributen. In Joachim Buscha \& Renate Freudenberg-Findeisen (Hrsg.), Feldergrammatik in der Diskussion. Funktionaler Grammatikansatz in Sprachbeschreibung und Sprachvermittlung, 165-183. Frankfurt a. M.: Peter Lang.

Thurmair, Maria (2011): Grammatik verstehen lernen - mithilfe von Textsorten. In Köpcke \& Ziegler (Hrsg.), 411-429.

Thurmair, Maria (2013): Von schwer zu schließenden Lücken und erweiterten Kompetenzen. Attribute mit Partizip und Modalpartizip. In Köpcke \& Ziegler (Hrsg.), 329-352.

Thurmair, Maria (2016): Textsortenlinguistik für den Fremdsprachenunterricht. In Thomas Tinnefeld (Hrsg.), Fremdsprachenvermittlung zwischen Anspruch und Wirklichkeit, 379-395. Saarbrücken.

Topalovic, Elvira (2009): Sprache, Grammatik und Emotion. E-Mails und SMS im DaFUnterricht. In Bachmann-Stein \& Stein (Hrsg.), 269-294.

Wegener, Heide (1993): weil - das hat schon seinen Grund. Zur Verbstellung in Kausalsätzen mit weil im gegenwärtigen Deutsch. Deutsche Sprache 21, 289-305.

Wegener, Heide (2007): Entwicklungen im heutigen Deutsch: Wird Deutsch einfacher? Deutsche Sprache 35 (1), 35-62.

Wegener, Heide (2016): Rezension von Hilke Elsen: Grundzüge der Morphologie des Deutschen. Deutsch als Fremdsprache 53 (2), 115-117.

Weidner, Beate (2015): Das funktionale Spektrum von ja im Gespräch - Ein Didaktisierungsvorschlag für den DaF-Unterricht. In Imo \& Moraldo (Hrsg.), 165-196.

Weinrich, Harald (1964/1985): Tempus. Besprochene und erzählte Welt. Stuttgart: Kohlhammer.

Weinrich, Harald (1986): Klammersprache Deutsch. In Sprachnormen in der Diskussion. Beiträge vorgelegt von Sprachfreunden, 116-145. Berlin.

Weinrich, Harald (1989): Formen der Wissenschaftssprache. Jahrbuch 1988 der Akademie der Wissenschaften, 119-158. Berlin.

Weinrich, Harald (1995): Wissenschaftssprache, Sprachkultur und die Einheit der Wissenschaft. In Heinz L. Kretzenbacher \& Harald Weinrich (Hrsg.), Linguistik der Wissenschaftssprache, 155-174. Berlin/New York: De Gruyter.

Weinrich, Harald (2003): Textgrammatik der deutschen Sprache. Unter Mitarbeit von Maria Thurmair, Eva Breindl, Eva-Maria Willkop, 2. rev. Aufl. Hildesheim u. a.: Olms.

Weydt, Harald (1969): Abtönungspartikel. Bad Homburg.

Willkop, Eva-Maria (2003): Perfekte Geschichten - Tempuswahl in Erzähltexten. In Maria Thurmair \& Eva-Maria Willkop (Hrsg.), Am Anfang war der Text. 10 Jahre „Textgrammatik der deutschen Sprache“, 235-258. München: iudicium.

Wolff, Armin \& Elmar Winters-Ohle (Hrsg.) (2000): Wie schwer ist die deutsche Sprache wirklich? Regensburg: FadaF.

Zifonun, Gisela, Ludger Hoffmann \& Bruno Strecker (1997): Grammatik der deutschen Sprache. Berlin, New York: De Gruyter. 
VI Grammatik an den Schnittstellen 



\title{
15 Textstrukturen: Was bleibt
}

\section{Zu Phänomenen und Theorien des Textaufbaus}

\begin{abstract}
Der Beitrag spannt einen Bogen von den zentralen Fragestellungen der traditionellen Textlinguistik hin zu den aktuellen Themen der Textstrukturanalyse. Nach einer kurzen Problematisierung des Textbegriffes befasst sich der Beitrag zunächst mit referentiellen Strukturen und diskursanaphorischen Beziehungen, von denen bekannt ist, dass sie einen wesentlichen Beitrag zur Textkonstitution leisten. Sie bilden zugleich die Basis für komplexere semantisch-pragmatische Diskursstrukturen, die die thematische Struktur im Text markieren und die Gewichtung der dargestellten Information steuern. Dieses Themenfeld wird unter Berücksichtigung einschlägiger neuerer Theorien der Diskursmodellierung vorgestellt. Insgesamt zielt der Beitrag darauf, die textstrukturellen Phänomene und Theorien herauszustellen, hinter die auch künftige Forschung nicht mehr zurückfallen kann, ohne auf bereits erzielte Erkenntnisse und Generalisierungen zu verzichten. Insofern versteht sich der Aufsatz als ein Beitrag zur wissenschaftlichen Nachhaltigkeit im Bereich der Textmodellierung.
\end{abstract}

Keywords: Diskursanaphorik, Diskursrelationen, Informationsgewichtung, Kohärenz, Kohäsion, Textkonstitution, Textmodellierung

\section{Zum Textbegriff}

Texte bestimmen unseren kommunikativen Alltag. Sie begegnen uns in mannigfachen Formen und Funktionen. Sie können gedruckt sein, auf einem Display erscheinen oder vorgelesen werden. Wir produzieren Texte aus vielerlei

Anmerkung: Ich möchte den Herausgeber_innen sowie Cathrine Fabricius Hansen, Annika Hübl und Edgar Onea für wertvolle Kommentare und Korrekturen zu früheren Versionen dieses Textes danken. Alle verbliebenen Mängel habe ich selbst zu verantworten.

Anke Holler, Seminar für Deutsche Philologie, Courant-Forschungszentrum „Textstrukturen“, Universität Göttingen, Käte-Hamburger-Weg 3, D-37073 Göttingen,

E-Mail: anke.holler@phil.uni-goettingen.de

Ә Open Access. (c) 2018 Anke Holler, publiziert von De Gruyter. (c) BY Dieses Werk ist lizenziert unter der Creative Commons Attribution 4.0 Lizenz. 
Gründen, zum Beispiel, um zu erzählen, zu argumentieren oder zu werben, und wir rezipieren und verstehen Texte äußerst routiniert - dank unserer sprachlichen Kompetenz. Vor diesem Hintergrund ist es nur folgerichtig, dass Texte und ihre Charakteristika sowie die Prozesse der Texterzeugung und des Textverstehens $\mathrm{zu}$ einem zentralen Gegenstand der theoretischen und angewandten sprachwissenschaftlichen Forschung geworden sind. Bereits in den frühen siebziger Jahren des vergangenen Jahrhunderts hat sich mit der Textlinguistik eine eigene Subdisziplin herausgebildet, die sich dem Text als dem „sprachlich manifeste[n] Teil der Äußerung in einem Kommunikationsakt“ (Große 1976: 13) widmet. Was einen Text genau ausmacht, welche Eigenschaften konstitutiv sind und wie er adäquat $\mathrm{zu}$ analysieren ist, wird seither kontrovers diskutiert und ganz unterschiedlich beantwortet. Dies liegt sicher an der intrinsischen Komplexität des Textes, aber auch an den verschiedenen Perspektiven, unter denen Texte untersucht werden: Aus grammatischer Perspektive wird der Text zumeist als eine regelhaft gebildete sprachliche Entität oberhalb der Satzebene betrachtet, aus semantischer Perspektive als eine sinnstiftende thematische Einheit und aus pragmatischer Perspektive als eine kommunikative Einheit, die in Bezug auf einen konkreten Kontext mit einer bestimmten illokutiven Funktion in einer bestehenden Kommunikationssituation geäußert wird. Hinzu kommen handlungsorientierte, soziokulturelle und kognitiv-affektive Aspekte, die in die Textanalyse einbezogen werden (müssen). All diese Perspektiven berühren konstitutive Bereiche des Textaufbaus und begründen zusammengenommen das breite Spektrum der textlinguistischen Gegenstände, die von den sprachlichen Mitteln zur Textkonstitution über die Textmuster und Textfunktionen bis hin zu den Texttypen oder Textsorten reichen. Mit jeweils etwas anderer Schwerpunktsetzung führen unter anderem Vater (2001); Brinker (2005, jetzt: Brinker, Cölfen \& Pappert $\left.{ }^{8} 2014\right)$; Stede (2007); Schwarz-Friesel \& Consten (2014); Heringer (2015) sowie Adamzik (2016) in dieses umfangreiche Forschungsthema ein.

Im Sinne des etymologischen Ursprungs des Wortes - lateinisch textum bedeutet „Gewebe“ - wird der Text üblicherweise als ein verwobenes, präziser: multidimensionales Gebilde angesehen, das sich aus dem prinzipiengeleiteten Zusammenwirken verschiedener Textebenen ergibt (Brandt \& Rosengren 1992; Motsch 1996; Heinemann \& Heinemann 2002; Stede 2007). Dabei werden in der Regel textinterne von textexternen Ebenen getrennt. Obwohl die einzelnen Ansätze die Ebenen im Detail verschieden aufbauen, lässt sich verallgemeinern, dass in allen Fällen auf die eine oder andere Weise textintern eine formal-grammatische, die Äußerungsform betreffende Ebene und eine inhaltlich-thematische, die Textinterpretation betreffende Ebene unterschieden werden sowie dass textextern mindestens eine funktionale und eine situa- 
tive Ebene angesetzt werden, die je verschiedene Aspekte der Textpragmatik, der rhetorischen und der illokutiven Struktur umfassen. Allgemein akzeptiert ist zudem, dass Textproduktion und Textverstehen kognitiv verankerte Prozesse sind, die Bezüge zu den mentalen Wissensbeständen der Kommunikationspartner und zur jeweiligen Kommunikationssituation herstellen. Mitunter wird in Textmodellen explizit eine kognitiv-konzeptuelle Ebene hinzugefügt, die unter anderem bestimmte kognitive Schemata und Frames einbezieht.

Allerdings existiert bisher keine übergreifende Texttheorie, die alle genannten Aspekte bündelt und integriert. Dazu wäre es nicht nur erforderlich, alle einzelnen Ebenen des Textes angemessen zu modellieren, sondern es müssten auch die Prinzipien ihres Zusammenwirkens adäquat herausgearbeitet werden. Das Zusammenspiel der verschiedenen Ebenen ist bis heute in seiner Regelhaftigkeit aber alles andere als verstanden und auch die Modellierung der einzelnen textstrukturellen Ebenen kann keineswegs als abgeschlossen gelten.

Ludwig M. Eichinger hat auf die wandelbaren Eigenschaften des Textbegriffes hingewiesen und den Text zu Recht als „Proteus“ und „Baldanders“ bezeichnet, „der sich dem jeweils Untersuchenden in anderer Gestalt zeigt“ (Eichinger 2006: 4). Dies ist vermutlich der wichtigste Grund, warum eine einheitliche textlinguistische Bestimmung des Untersuchungsgegenstandes Text bisher nicht gelungen ist und womöglich auch nicht abschließend gelingen kann. Die traditionelle textlinguistische Forschung hat dennoch wertvolle Einsichten und Konzepte hervorgebracht, auf die sich die sprachtheoretische Textanalyse bis dato stützt. Prominent sind die sieben Textualitätskriterien, die seit de Beaugrande \& Dressler (1981) regelmäßig zur Bestimmung der Texthaftigkeit einer Zeichenfolge genutzt werden. Einmütig werden die folgenden Eigenschaften eines Textes als konstitutiv betrachtet: Kohäsion, Kohärenz, Intentionalität, Akzeptabilität, Informativität, Situationalität und Intertextualität. Diese Faktoren adressieren insgesamt neben sprachlichen Mitteln und Strukturen des Textes situative Aspekte sowie Sprecherintentionen und Einstellungen der Rezipienten und verdeutlichen zudem, dass Textualität auch als eine kognitiv-affektive Größe aufgefasst werden muss, da Textproduktion und -rezeption letztlich immer an mentale Prozesse zur Sprachverarbeitung gebunden sind. Kohäsion betrifft die sprachliche Oberfläche eines Textes und erfasst die Menge aller Einheiten und Strukturen, die dazu dienen, grammatisch markierte Bezüge und Verknüpfungen zwischen aufeinanderfolgenden Sätzen herzustellen. Traditionell sind Wiederaufnahme und Konnexion die sprachlichen Mittel par excellence, um kohäsive Textstrukturen zu erzeugen. Sie bilden die sprachliche Grundlage zur Herstellung von Kohärenz, worunter im Allgemeinen der „semantisch-kognitive Sinnzusammenhang eines Textes“ (Bußmann 42008: 344) verstanden wird. Kohäsion und Kohärenz nehmen Bezug auf text- 
interne Eigenschaften. Die weiteren Textualitätskriterien beziehen sich eher auf die äußeren Umstände, die sich aus der jeweiligen Kommunikationssituation ergeben. Durch die Intentionalität wird beschrieben, dass ein Text immer mit einer bestimmten Absicht verfasst wird. Diese Sprecherintention muss vom Rezipienten rekonstruiert werden können, was im Textualitätsmerkmal Akzeptabilität seinen Widerhall findet. Informativität und Situationalität erfassen die Anforderung, dass ein Text einen informativen Gehalt hat und situativ angemessen ist. Dass jeder Text in Bezug zu anderen Texten steht und von diesen in seiner Interpretation abhängt, wird schließlich durch das Merkmal der Intertextualität erfasst.

Es hat sich inzwischen gezeigt, dass die durch die Textualitätskriterien beschriebenen Bedingungen weder hinreichend noch notwendig für die Textkonstitution sind, weswegen sie sich nur bedingt dafür eignen, einen Text von einem Nicht-Text zu unterscheiden. ${ }^{1}$ Dies hat sicher verschiedene Gründe, liegt aber auch daran, dass die Ausprägungsformen von Texten in der Kommunikation äußerst vielfältig sind - man denke nur an die Entwicklung der Hypertexte oder an Text-Bild-Verschränkungen - und dass daher ihre Abgrenzung hin zu anderen Kommunikationsformen wie zum Beispiel dem Dialog am besten prototypisch erfolgt, wie zum Beispiel Vater (2001) vorschlägt. Allein die Frage, inwieweit die neuen internetbasierten Texttypen als klassische Texte anzusehen sind, hat keine einfache Antwort. Dies demonstriert anhand des Hypertextes exemplarisch die Tabelle 15.1, in der nach Storrer (2004) die wesentlichen Merkmale der drei Kommunikationsformen Text, Hypertext und Dialog gegenübergestellt sind. ${ }^{2}$ In Holler (2003) wird ausführlich diskutiert, welche Auswirkungen die Besonderheiten des Hypertextes auf die Modellierung (ko-)referentieller Strukturen haben und welche eklatanten Schwierigkeiten hinsichtlich der Übertragbarkeit klassischer textbezogener Konzepte auf neue (textnahe) Kommunikationsformen auftauchen.

Die Schwierigkeiten bei der Festlegung des Textbegriffes schlagen sich nicht zuletzt in der Grenzziehung zwischen Text und Diskurs nieder, was selbstredend nicht nur mit dem Textbegriff, sondern auch mit dem ebenso schillernden Diskursbegriff zu tun hat. Während es neuerlich in der Textlinguistik modern ist, im Foucault'schen Sinne vom Diskurs zu sprechen und somit vor allem sozio-

1 Stede (2007: 28) sieht Textualität daher als graduelles Maß, „dem Texte mehr oder weniger Genüge tun“.

2 Die Netzkommunikation hat darüber hinaus noch andere Formen hervorgebracht, wie beispielsweise die zeitversetzte E-Mail- und Forenkommunikation oder die Chat-Kommunikation, die üblicherweise als Mischform zwischen medialer Schriftlichkeit und mündlichem Gespräch analysiert wird. Siehe hierzu auch die Beiträge in Beißwenger (2001). 
Tab. 15.1: Merkmale der Kommunikationsformen Text, Hypertext und Dialog (nach Storrer 2004).

\begin{tabular}{|c|c|c|}
\hline Text & Hypertext & Dialog \\
\hline linear & nicht-linear & direktional \\
\hline schriftlich & digital & mündlich \\
\hline $\begin{array}{l}\text { bewusst planerische } \\
\text { Produktion }\end{array}$ & $\begin{array}{l}\text { durch Verlinkung strukturierte } \\
\text { Dokumentenbasis }\end{array}$ & $\begin{array}{l}\text { situativ, thematisch und } \\
\text { intentional gesteuert }\end{array}$ \\
\hline dauerhafte Existenz & $\begin{array}{l}\text { zyklische Produktion, } \\
\text { flexibel veränderbar }\end{array}$ & $\begin{array}{l}\text { beständig in } \\
\text { der Äußerungssituation }\end{array}$ \\
\hline $\begin{array}{l}\text { klar definierte } \\
\text { Textgrenzen }\end{array}$ & $\begin{array}{l}\text { Wegfall sichtbarer } \\
\text { Textgrenzen }\end{array}$ & abgrenzbare Turns \\
\hline $\begin{array}{l}\text { visuelle Rezeption, } \\
\text { losgelöst von } \\
\text { der Äußerungssituation }\end{array}$ & $\begin{array}{l}\text { interaktive Rezeption, losgelöst } \\
\text { von der Äußerungssituation }\end{array}$ & $\begin{array}{l}\text { auditive Rezeption in } \\
\text { der Äußerungssituation }\end{array}$ \\
\hline $\begin{array}{l}\text { sequenzielle, i. d. R. } \\
\text { vollständige Rezeption }\end{array}$ & $\begin{array}{l}\text { partielle und selektive Rezeption } \\
\text { durch Generierung von variablen } \\
\text { "Sichten“ }\end{array}$ & $\begin{array}{l}\text { Abhängigkeit } \\
\text { der Vollständigkeit } \\
\text { der Rezeption von } \\
\text { der Vollständigkeit } \\
\text { der Wahrnehmung }\end{array}$ \\
\hline $\begin{array}{l}\text { vorgegebene } \\
\text { Rezeptionsabfolge }\end{array}$ & $\begin{array}{l}\text { ohne antizipierbare } \\
\text { Rezeptionsabfolge }\end{array}$ & $\begin{array}{l}\text { wechselseitige } \\
\text { Rezeption, dialogisch }\end{array}$ \\
\hline $\begin{array}{l}\text { thematisch } \\
\text { kontinuierlich }\end{array}$ & $\begin{array}{l}\text { thematisch } \\
\text { diskontinuierlich }\end{array}$ & $\begin{array}{l}\text { thematisch kontinuierlich } \\
\text { mit angekündigten } \\
\text { Themenwechseln }\end{array}$ \\
\hline
\end{tabular}

kulturelle und intertextuelle Bezüge herzustellen (vgl. Warnke 2000), wird in der angelsächsischen Forschung Diskurs oft als Oberbegriff für die unterschiedlichen oben thematisierten Aspekte des Textbegriffes verwendet (vgl. Schiffrin 1994). Daran anknüpfend scheint es für die Zwecke des vorliegenden Beitrags zielführend, Text und Diskurs weitgehend synonym zu verwenden und nur, wo notwendig, Diskurs als einen übergeordneten Begriff zu nutzen, der Texte und Dialoge subsumiert.

In einem Band, der sich der Grammatik des Deutschen widmet, ist es angezeigt, vor allem die Textphänomene in den Vordergrund zu stellen, die durch grammatische Mittel und Prinzipien markiert oder induziert werden, wohl wissend, dass eine vollständige Charakterisierung eines Textes darauf nicht reduziert werden kann. Letzteres folgt schon aus den oben angeführten Textualitätskriterien. Ohne in Abrede zu stellen, dass der Text „das sprachliche Korrelat 
eines Kommunikationsaktes im Kommunikationsprozeß“" (Rosengren 1980: 275 f.) ist und somit eine kommunikative Einheit darstellt, werden hier vor allem die „Vertextungsmittel“ thematisiert (Isenberg 1968), d.h. die sprachlichen Mittel, die Folgen von Sätzen miteinander verknüpfen, so dass sich verschiedenartige Textstrukturen herausbilden.

Die Phänomene, die hier herausgegriffen werden, sind allesamt an der Grammatik-Pragmatik-Schnittstelle angesiedelt. Konsequenterweise dürfen auch die Analyseverfahren, die angewendet werden, nicht auf die Morphosyntax beschränkt werden, sondern müssen gleichermaßen logisch-semantische und pragmatische Zusammenhänge erfassen können. ${ }^{3}$ Betrachtet man die sprachlichen Strukturen im Text aus dieser Perspektive, so rücken frühe, semiotisch motivierte Annahmen vom Zeichencharakter des Textes wieder stärker ins Blickfeld. So schreibt zum Beispiel schon Viehweger (1976: 197): „Der Text ist als Resultat der kommunikativen Tätigkeit des Menschen - ein komplexes sprachliches Zeichen, eine nach einem Handlungsplan erfolgte und durch die Regeln des Sprachsystems realisierte Zuordnung von Bewußtseinsinhalten und Lautfolgen“. Aktuell erlebt diese Sichtweise in der Bestimmung des Textes als komplexes indexikalisches Zeichen (vgl. Antos 2009) eine Renaissance.

Die Linguistik hat seit den 1990er Jahren neue analytische Werkzeuge und theoretische Modelle entwickelt, die es erlauben, den Text in all seinen grammatisch-pragmatischen Facetten als ein komplexes sprachliches Zeichen zu begreifen, das - wie die Duden-Grammatik ( $\left.{ }^{9} 2016: 1076\right)$ sinngemäß formuliert von den Kommunizierenden zusammenhängend kodiert bzw. dekodiert wird, wobei Schreiber und Leser syntaktischen, semantischen und pragmatischen Regeln folgen. Die neueren texttheoretischen Ansätze zeichnet aus, dass sie gestützt auf die frühen Einsichten der Textlinguistik - satzübergreifende Phänomene und textuelle Strukturen empirisch fundiert und formal adäquat zu erfassen suchen, indem sie einerseits an aktuelle, eher satzbezogene Grammatikmodelle und Theorien der formalen Semantik und Pragmatik anschließen und andererseits die modernen experimentellen und korpuslinguistischen Methoden der Datengewinnung adaptieren. Die nachfolgenden Abschnitte sollen anhand einiger ausgewählter textstruktureller Phänomene und Modelle einen Einblick in dieses derzeit äußerst dynamische Forschungsfeld geben. Dabei sollen drei zentrale Probleme der Textkonstitution im Vordergrund stehen, und zwar (i) welche sprachlichen Prinzipien daran teilhaben, eine Reihung von

3 Daraus folgt auch, dass das ältere Konzept der Textgrammatik, wonach der Text rein oberflächenstrukturell beschrieben wird, untauglich ist, um den Text in seiner Komplexität zu erfassen und ihm als semantisch-pragmatisches Objekt bzw. Resultat kognitiv-affektiver Prozesse gerecht zu werden, vgl. dazu schon Halliday \& Hasan (1985). 
Sätzen in einen Text zu überführen, (ii) welche Inferenzen durch unterschiedliche strukturelle Merkmale von Texten ausgelöst werden und (iii) wie der Text und die Struktur des Textes die Interpretation der einzelnen Sätze beeinflussen. Vor dem Hintergrund dieser Fragestellungen befasst sich der nachfolgende Abschnitt 2 mit der referentiellen Struktur in Texten und rekapituliert den aktuellen empirischen und theoretischen Erkenntnisstand hinsichtlich der Bedingungen der Auflösung diskursanaphorischer Bezüge. Darauf aufbauend thematisiert Abschnitt 3 einschlägige theoretische Ansätze zur Beschreibung diskursrelationaler Strukturen in Texten. Der sich anschließende Abschnitt 4 versucht eine Bestandsaufnahme bezüglich der thematischen Struktur in Texten anhand fragebasierter Diskursmodelle. Nachdem diese drei Abschnitte jeweils die Erträge der empirischen und theoretischen Forschung zur Textstruktur in den Vordergrund stellen und damit zusammenfassen, was wir in Bezug auf die Textkonstitution zu wissen glauben, benennt der Abschnitt 5, was wir noch nicht wissen und was demnach noch zu tun ist.

\section{Diskursanaphorik und referentielle Bezüge}

In der aktuellen Texttheorie gilt die Etablierung von referentiellen Bezügen zwischen Entitäten, die im Text verhandelt werden, als wesentlicher Bestandteil des Textaufbaus. Typischerweise werden dazu anaphorische Ausdrücke verwendet, deren Referenz sich erst durch die Verknüpfung mit einer bereits im Vortext erwähnten Entität, dem Antezedens, ergibt. ${ }^{4}$ Entsprechend gilt die Diskursanaphorik als klassisches kohäsives Mittel.

Anaphorische Beziehungen belegen aber auch, dass der Diskurs auf eine essentielle Weise die Satzbedeutung wahrheitsfunktional beeinflusst. Ein charakteristisches Beispiel hierfür ist der Kontrast zwischen (1a) versus (1b):

(1) a. Eine Anwältin jodelt. Sie ist heiser.

b. Eine Politikerin jodelt. Sie ist heiser.

Während im ersten Satzpaar ausgesagt wird, dass eine Anwältin heiser ist, wird im zweiten ausgesagt, dass eine Politikerin heiser ist, obwohl sich die jeweils zweiten Sätze buchstäblich nicht unterscheiden. Die Auflösung von

4 Kataphorische Bezüge, die zur Textkonstitution (abgesehen von der Verweisrichtung) in vergleichbarer Weise beitragen wie anaphorische Bezüge, werden hier aus Platzgründen ausgespart. Sie haben aber auch wissenschaftshistorisch weitaus weniger Aufmerksamkeit erlangt als die entsprechenden anaphorischen Beziehungen im Text. 
solchen anaphorischen Bezügen ist eines der Kernprobleme der SemantikPragmatik-Schnittstelle (siehe auch Gutzmann \& Schumacher in diesem Band zu einer Reihe von weiteren Aspekten dieser Schnittstelle), weil die Resolutionsprozesse den wechselseitigen Einfluss von semantischem Gehalt und textstruktureller Konstitution offenlegen. Es geht also nicht nur darum, wie etwas im Diskurs ausgedrückt wird (sprich: ,Inhalt beeinflusst Textstrukturen'), sondern auch darum, was gesagt wird (sprich: ,Textstrukturen beeinflussen Inhalt').

Die Wiederaufnahme von bereits Erwähntem wird in der Regel durch pronominale oder nominale Ausdrücke realisiert, kann aber auch von bestimmten elliptischen ${ }^{5}$ oder temporalen Ausdrücken geleistet werden, wie die Beispiele in (2) illustrieren.

(2) a. Andy Warhol war überzeugt, dass [er] ein Genie ist.

b. Neo Rauchs Gemälde erreichen Spitzenpreise. [Der Leipziger Künstler] versucht aber, öffentlichen Auftritten zu entgehen.

c. Oskar Kokoschka wurde von den Nazis als entartet diffamiert und [_] war deswegen gezwungen, nach Großbritannien $\mathrm{zu}$ fliehen.

d. Mark Rothko saß stundenlang vor den Bildern von Matisse. [Dann] begann er zu malen.

Wie ein anaphorischer Ausdruck interpretiert wird, hängt von verschiedenen Faktoren ab, die sowohl sprachlich-struktureller als auch kognitiver oder situativer Natur sein können. Diese Faktoren begrenzen einerseits die Menge der sprachlichen Ausdrücke, die in einem Text überhaupt als Antezedentien einer Diskursanapher infrage kommen, und restringieren andererseits, welche der prinzipiell möglichen anaphorischen Bezüge schließlich realisiert werden. Ob eine Beziehung zwischen einer potentiellen Bezugsgröße und einem anaphorischen Ausdruck etabliert wird oder nicht, ist aber nicht nur eine Frage sprachlicher Gesetzmäßigkeiten, sondern hängt gleichermaßen von den beim Textverstehen involvierten mentalen Prozessen ab sowie von den Dispositionen der Kommunikationspartner, zum Beispiel hinsichtlich ihrer individuellen Wissensbestände, auf die sie zurückgreifen können. Dies wird besonders sinnfällig bei Phänomenen der indirekten Anaphorik (Clark 1977; Schwarz 2000; Irmer 2011), die dadurch gekennzeichnet sind, dass der anaphorische Bezug nicht unmittel-

5 Allerdings existieren auch Ellipsen, die nicht kohäsionsstiftend wirken, weil sie keinen Ausdruck wiederaufnehmen, sondern zum Beispiel rein syntaktisch motiviert sind. 
bar, sondern erst nach einem zusätzlichen Inferenzschritt hergestellt werden kann, weswegen metaphorisch auch vom Bridging ${ }^{6}$ gesprochen wird:

(3) a. Es war nichts los im Restaurant. Die Kellner gähnten. (Schwarz 2000)

b. Der Fürst heiratete wieder. Sie war eine Schauspielerin. (Hoffmann 1997)

Angesichts der Komplexität der möglichen diskursanaphorischen Beziehungen ist es dringend geboten, die ihnen zugrundeliegenden Prinzipien und Strategien aus einer interdisziplinären Perspektive zu untersuchen. Die Grammatiktheorie stellt dabei eher die formalen Bedingungen für die Strukturierung von Textinformation und die Auflösung von Diskursanaphern in den Vordergrund, während psycholinguistische Ansätze das Hauptaugenmerk auf die prozessualen Aspekte legen, die bei der Herstellung referentieller Bezüge involviert sind. Darüber hinaus ist die algorithmische Auflösung anaphorischer Beziehungen ein klassisches computerlinguistisches Thema, da für die maschinelle Verarbeitung der Textbedeutung eine effiziente Zuordnung passender Bezugsausdrücke $\mathrm{zu}$ anaphorischen Größen im Text essentiell ist. Ohne dieses sind angewandte Aufgaben wie die maschinelle Übersetzung, die Textzusammenfassung oder die Dialogverarbeitung nicht vorstellbar. Die genannten drei großen disziplinären Forschungsstränge, i. e. Grammatiktheorie sowie psycholinguistische und maschinelle Verarbeitung, befruchten sich wechselseitig auch weil sie mehrere gemeinsame empirische und theoretische Schnittpunkte haben, und zwar sowohl hinsichtlich der Faktoren der Anaphernauflösung als auch in Bezug auf die Analyse diskursstruktureller Beziehungen.

Auf theoretischer Seite findet dies seinen Ausdruck in der breiten Akzeptanz der Diskursrepräsentationstheorie (kurz: DRT) (Kamp 1984; Kamp \& Reyle 1993; Kamp, van Genabith \& Reyle 2011), die inzwischen als Standardtheorie zur Modellierung diskursanaphorischer Beziehungen bezeichnet werden kann. Der Erfolg der DRT verdankt sich vor allem zwei Grundideen: erstens dem von Karttunen (1969) übernommenen Konzept des Diskursreferenten und zweitens der Annahme, dass die Textbedeutung Ergebnis eines dynamischen Informationsverarbeitungsprozesses ist und daher über eine einfache Spezifikation von Wahrheitsbedingungen einzelner propositionaler Strukturen hinausgeht.

Diskursreferenten werden in der DRT als abstrakte konzeptuelle Entitäten aufgefasst, die Entitäten der realen oder vorgestellten Welt, wie zum Beispiel Individuen, Ereignisse, Sachverhalte, Situationen etc., repräsentieren. Sie fun-

6 Bridging befördert wie andere Formen des kognitiven Schließens die Kohärenzbildung im Text. 
gieren als mentale Stellvertreter für die Entitäten, über die der Text spricht. Die Diskursreferenten unterliegen bestimmten Bedingungen, die im Text ausgedrückt werden und entweder Eigenschaften der Diskursreferenten selbst oder Beziehungen zwischen ihnen betreffen. Die Bedingungen werden grob gesprochen im Laufe eines Diskurses durch die zu verarbeitende Textinformation erweitert und konkretisiert und durch sogenannte Diskursrepräsentationsstrukturen (kurz: DRS) satzweise inkrementell erfasst.

Eine DRS besteht aus einer Menge von Diskursreferenten und einer Menge von Bedingungen, die diese Referenten erfüllen müssen. Man kann sagen, dass eine DRS die Textinformation so abbildet, wie sie sich im Geiste von Sprecher und Hörer „entspinnt“. Jede DRS wird selbst modelltheoretisch interpretiert: Eine DRS ist wahr in Bezug auf ein totales Modell M, wenn das zu der DRS korrespondierende partielle Modell in M eingebettet werden kann, d.h. Teil von $\mathrm{M}$ ist.

Wegen ihres dynamischen Charakters und ihres kognitiven Anspruchs ist die DRT besonders gut geeignet, die Information, die sich im Text entfaltet, zu erfassen und die sich dabei ergebenden anaphorischen Beziehungen zu modellieren. Die Grundannahme hierbei ist, dass Diskursanaphern in der DRS vorhandene Diskursreferenten wieder aufgreifen, sofern diese erreichbar sind. Im typischen Fall werden Diskursreferenten von im Text erwähnten (in-)definiten Nominalphrasen oder Eigennamen eingeführt, aber auch andere Entitäten, wie zum Beispiel Ereignisse, Fakten und Sachverhalte etc., können entsprechende Diskursreferenten zur Repräsentation beisteuern (vgl. Asher 1993; Holler 2005):

(4) a. Niemand hat die Pop Art so geprägt wie Andy Warhol. Er hat Kunst und Kommerz geschickt verbunden.

b. Beim Verkauf haben Warhols 32 Suppenkonservendosenbilder zusammen 15 Millionen Dollar eingebracht, was selbst Beuys neidlos anerkennen musste.

In der Computerlinguistik wird - wenn auch nicht immer konsequent zwischen Anaphorik und Koreferenz unterschieden, vgl. van Deemter \& Kibble (2001); Holler (2003). Auf den Punkt gebracht, stellt Anaphorik eine irreflexive und asymmetrische Beziehung zwischen sprachlichen Ausdrücken auf Textebene her. Ein Ausdruck $\alpha$ bezieht sich anaphorisch auf einen Ausdruck $\beta$ genau dann, wenn $\beta$ Antezedens von $\alpha$ ist und $\alpha$ in seiner Interpretation von $\beta$ abhängig ist. Koreferenz hingegen beschreibt eine Äquivalenzbeziehung zwischen mindestens zwei Referenten in der Welt, d.h. die Kommunikationspartner nutzen zwei oder mehrere sprachliche Ausdrücke, um auf ein und 
denselben Referenten in der realen oder einer fiktiven Welt Bezug zu nehmen. ${ }^{7}$ Dass die Differenzierung zwischen anaphorischen und referentiellen Bezügen generell notwendig ist, illustrieren auch Daten wie (5), die wahrheitsfunktional nicht identisch sind:

(5) a. Jeder Künstler denkt, dass er ein Genie ist.

b. Jeder Künstler denkt, dass jeder Künstler ein Genie ist.

Die Beispiele (5a) und (5b) haben unterschiedliche Bedeutungen, weil die komplexe Nominalphrase jeder Künstler, die in (5a) das Antezedens des Pronomens er bildet, einen Quantor enthält und somit nicht referentiell gedeutet werden kann. Das Pronomen kann daher nicht ohne Bedeutungsveränderung durch die Nominalphrase ersetzt werden. So wie in diesem Fall Anaphorik ohne Koreferenz vorkommen kann, belegen über Dokumentgrenzen hinweg gebildete Koreferenzketten, wie sie beispielsweise in Hypertexten $\mathrm{zu}$ finden sind, dass es auch Koreferenz ohne Anaphorik geben kann, vgl. z. B. Holler (2003).

In der modelltheoretischen Semantik ist die adäquate Interpretation anaphorischer Pronomen ebenfalls ein anhaltend untersuchtes Thema, vgl. Cooper (1979); Reinhart (1983); Heim (1990); Elbourne (2005) u. v. a. m. Allerdings wird hier von den Strategien und Bedingungen des Resolutionsprozesses weitgehend abstrahiert. Abweichend vom computerlinguistischen Herangehen wird Anaphorik häufig auch als eine übergeordnete Beziehung angesehen. Dies ist möglich, weil im Rahmen der Semantik mit einer anaphorischen Relation nur verbunden wird, dass ein Ausdruck in interpretatorischer Beziehung zu einem weiteren Ausdruck steht, der links von ihm realisiert ist. Diese weite Auffassung von Anaphorik trifft letztlich auch auf koreferentielle Beziehungen zu. Koreferenz setzt aber zusätzlich voraus, dass beide involvierten Ausdrücke referieren, und zwar auf dasselbe Individuum. Mit anderen Worten, Koreferenz ist ohne Referenz nicht möglich, Anaphorik hingegen schon. Für eine ausführliche Diskussion dieser terminologischen Unterscheidung und ihrer analytischen Konsequenzen sei auf Heim \& Kratzer (1998: 239 ff.) verwiesen.

In Bezug auf anaphorische Pronomen wird in der formalen Semantik seit Geach (1962) angenommen, dass diese mindestens zwei Verwendungsweisen

7 In der Computerlinguistik wird zumeist auch zwischen Anaphernauflösung und Koreferenzauflösung unterschieden. Im ersten Fall wird das Antezedens der Anapher gesucht, wodurch die Anapher auf den Diskursreferenten verweist, den der Antezedensausdruck eingeführt hat. Im zweiten Fall geht es darum, die in einem Text erwähnten Diskursreferenten in Klassen zusammenzufassen, wodurch Mengen koreferenter Ausdrücke entstehen, die sogenannte Referenzketten bilden. 
haben. Sie werden entweder referentiell gebraucht und verweisen in diesem Fall auf ein Individuum, das kontextuell bereits eingeführt ist, oder sie müssen nicht-referentiell gedeutet werden, weil sie beispielsweise auf einen quantifizierenden oder elliptischen Ausdruck Bezug nehmen. Im ersten Fall werden anaphorische Pronomen oftmals als freie Variablen analysiert und im zweiten Fall als semantisch gebundene Variablen. Siehe hierfür ebenfalls Heim \& Kratzer (1998). Hinzu kommt aber eine weitere Form anaphorischer Beziehungen, auf die erstmals Evans (1977) aufmerksam gemacht hat und die durch Beispiele wie (6) illustriert wird.

(6) a. Nur ein Ölgemälde wurde versteigert. Ein reicher Sammler hat es gekauft.

b. Jeder Künstler dachte, dass nur ein Ölgemälde von ihm versteigert wurde und dass es ein reicher Sammler gekauft hat.

Das anaphorische Pronomen es kann in (6) weder über Koreferenz noch über Variablenbindung adäquat gedeutet werden, da - etwas verkürzt ausgedrückt das Antezedens quantifiziert ist (was gegen Koreferenz spricht) und das Pronomen in einem separaten bzw. eingebetteten Satz realisiert ist (weswegen Variablenbindung ausscheidet). Um dieses Problem zu lösen, stipuliert Evans (1977) eine dritte Klasse anaphorischer Pronomen, die sogenannten E-TypePronomen. Dabei handelt es sich nach Evans um ungebundene anaphorische Pronomen, die die Entität bezeichnen, die eine aus dem deskriptiven Material des Antezedenssatzes gewonnene definite Kennzeichnung designiert, zum Beispiel das Ölgemälde, das versteigert wurde. ${ }^{8}$

Die Existenz von E-Type-Pronomen wird zudem durch die sogenannten Eselsätze wie (7) begründet, die beide eine universale Interpretation haben. Auch hier scheidet eine referentielle Deutung des anaphorischen Pronomens er bzw. ihn aus, weil es jeweils kein Individuum gibt, das als Referent fungieren könnte. Als gebundene Variable kann das Pronomen ebenfalls nicht interpretiert werden, weil es nicht im Skopus des quantifizierenden Ausdrucks steht, auf den es sich bezieht.

(7) a. Wenn ein Bauer einen Esel besitzt, dann schlägt er ihn.

b. Jeder Bauer, der einen Esel besitzt, schlägt ihn.

8 E-Type-Pronomen sind nach wie vor Gegenstand der Diskussion in der formalen Semantik. Einen aktuellen Überblick dazu gibt Nouwen (i.E.). 
Für die Analyse von Sätzen wie (6) und (7) ist die DRT vergleichsweise gut gerüstet, weil durch die Einführung der Diskursreferenten im Rahmen dieser Theorie auch eine Bezugnahme auf Entitäten der mentalen Repräsentation, also auf nur gedachte Entitäten, möglich ist. Dies gestattet zugleich eine Analyse des anaphorischen Bezugs in Sätzen wie (8), denn auch hier entspricht das Antezedens des Pronomens er keiner realen Entität.

(8) Kein Künstler lehnt einen Auftrag ab, wenn er pleite ist.

Formt man den komplexen Satz (8) aber in die Satzfolge (9) um, ist der anaphorische Bezug offenbar nicht mehr ohne weiteres herstellbar.

(9) Kein Künstler lehnt einen Auftrag ab. \#Er ist pleite.

Der Kontrast zwischen (8) und (9) illustriert, dass Diskursreferenten eine gewisse Lebensspanne im Text haben. Beispielsweise führt die Nominalphrase kein Künstler keinen Diskursreferenten ein, der als potenzielles Antezedens für das Pronomen er auch über die Satzgrenze hinaus zugänglich wäre. Ohne in die Details zu gehen, lässt sich sagen, dass der Negationsoperator den Diskursreferenten gewissermaßen einkapselt und damit verhindert, dass er satzübergreifend wieder aufgenommen werden kann.

Die Zugänglichkeit potenzieller Antezedentien eines anaphorischen Pronomens im Text wird von verschiedenen grammatischen und kognitiven Faktoren bestimmt. Im Deutschen zählt die morphologische Genus- und NumerusKongruenz, die zwischen Antezedens und Diskursanapher notwendig gegeben sein muss, als grammatischer Faktor dazu. Erfüllen diese grammatische Bedingung aber mehrere Ausdrücke im Text, kommen weitere linguistische und kognitive Strategien ins Spiel, die die Präferenzen bei der Auswahl eines passenden Antezedenskandidaten mitbestimmen. Beispielsweise scheint der syntagmatische Abstand zwischen Antezedenskandidat und anaphorischem Ausdruck für die Zugänglichkeit eine Rolle zu spielen (recency effect) oder auch die Ersterwähnung eines potenziellen Antezedensausdrucks (first mention effect) im vorangehenden Text, vgl. z. B. Sanford \& Garrod (1981); Garnham (2001) u.v. a.m.

Die verschiedenen Bedingungen zusammengenommen führen $\mathrm{zu}$ einer Rangordnung unter den Antezedenskandidaten, die die Menge der potenziellen Antezedentien danach hierarchisiert, wie gut die Diskursreferenten, die sie jeweils repräsentieren, für einen anaphorischen Ausdruck „sichtbar“ bzw. erreichbar sind. Ein Antezedenskandidat mit einem höheren Gewicht erhält einen höheren Rang, was bedeutet, dass er leichter zugänglich ist als ein 
Kandidat mit niedrigerem Gewicht. Lappin \& Leass (1994) und Mitkov, Evans \& Orasan (2002) haben inzwischen als klassisch geltende regelbasierte maschinelle Verfahren zur Pronomenresolution implementiert, die auf solchen Hierarchien gewichteter Antezedenskandidaten aufbauen. ${ }^{9}$

Die Hervorhebung eines potenziellen Antezedens aus der jeweiligen Kandidatenmenge wird üblicherweise mit dem kognitionspsychologischen Konzept der Salienz beschrieben, wonach ein Ausdruck eine höhere Salienz als ein anderer aufweist, wenn seine Repräsentation im Arbeitsgedächtnis der Kommunikationspartner ein höheres Aktivationsniveau erreicht. Die linguistischen Reflexe der Salienz und ihre genaue Rolle bei der Sprachverarbeitung sind allerdings notorisch unklar, was übrigens in gleicher Weise für den neuerdings wieder verwendeten, aber ähnlich vagen Begriff der Prominenz gilt. ${ }^{10}$

Das Gefälle im Salienzniveau potenzieller Antezedentien bildet die Grundlage für sogenannte Zugänglichkeits- oder Gegebenheitshierarchien (Gundel, Hedberg \& Zacharski 1993; Ariel 2001). Normalerweise wird dabei angenommen, dass hoch saliente und damit leicht zugängliche Antezedenskandidaten aufgrund ihres hohen Aktivationsgrades mit einfachen Ausdrucksmitteln wie zum Beispiel Pronomen aufgegriffen werden können, während nicht saliente, nicht aktivierte und daher kaum zugängliche Diskursreferenten nur mit komplexen Ausdrucksmitteln wie zum Beispiel definiten Kennzeichnungen wieder aufgenommen werden können. Auch wenn diese Hierarchien in wichtigen Einzelaspekten durch neuere experimentelle und korpuslinguistische Ergebnisse in Zweifel stehen, prägen sie nach wie vor die allgemeinen Vorstellungen darüber, wie Resolutionsprozesse verlaufen. Entsprechend hat sich besonders die empirische Forschung, vor allem im Bereich der Psycholinguistik und der Texttechnologie, für die Faktoren interessiert, die die Salienz eines Ausdrucks beeinflussen. Für Überblicke dazu vgl. z. B. Garnham (2001) oder auch die Beiträge in von Heusinger \& Kaiser (2010) und Holler \& Suckow (2016). Zu den Faktoren zählen neben grammatischen (wie zum Beispiel der syntaktischen Funktion $^{11}$ ), lexikalisch-semantischen (wie zum Beispiel der Verbkausalität ${ }^{12}$ ) und allgemein-kognitiven (wie zum Beispiel der Animatheit ${ }^{13}$ ) auch informa-

\footnotetext{
9 Auch die automatische Anaphernresolution ist inzwischen dem allgemeinen computerlinguistischen Trend hin zu statistischen Verfahren gefolgt. Aktuelle Systeme sind aber modular aufgebaut und integrieren nach wie vor ein linguistisches Modul als Filter für nachfolgende Verarbeitungsschritte, vgl. Mitkov (2002) und Stede (2012) für kurze Überblicksdarstellungen. 10 Für eine kritische Auseinandersetzung mit dem Salienzbegriff vgl. auch Auer (2014).

11 Vgl. Crawley, Stevenson \& Kleinman 1990; Smyth 1994 u. a.m.

12 Vgl. Brown \& Fish 1983; Bott \& Solstad 2014 u. a. m.

13 Vgl. Fukumura \& van Gompel 2010 u. a.m.
} 
tionsstrukturelle Faktoren (wie zum Beispiel die Topikalität ${ }^{14}$ ) und diskursrelationale Gegebenheiten (wie zum Beispiel die rechte Diskursgrenze ${ }^{15}$ ). In einer Reihe von Experimenten wird von Kehler et al. (2008) zudem gezeigt, dass die Qualität der Diskursrelation beim Leser bestimmte Erwartungen hinsichtlich einer mehr oder minder präferierten Pronomenauflösung erzeugt. Das passt auch zu Ergebnissen von Ellert \& Holler (2011), die den strukturellen und semantischen Einfluss von Konnektoren auf die Pronomenauflösung untersuchen.

Wichtig ist für alle Ansätze, dass sich die Salienz eines Diskursreferenten im fortschreitenden Text dynamisch verändert, denn verschiedene sprachliche Markierungen (cues) im Text können den Fokus der Aufmerksamkeit verschieben und damit Veränderungen im Salienzgefälle der Diskursreferenten auslösen. Grosz \& Sidner (1986) entwickeln einen der ersten algorithmischen Ansätze, der eine Verschiebung des Aufmerksamkeitsfokus beschreibt, wobei sie neben einer linguistischen (structure of the sequence of utterances, aggregation of utterances) und einer intentionalen Ebene (intentional structure, structure of purposes) explizit eine separate Ebene für die Aufmerksamkeitssteuerung (attentional state, state of focus of attention) ansetzen.

Dieser Ansatz von Grosz \& Sidner (1986) bildet eine wesentliche Grundlage für die spätere Ausarbeitung der Centering-Theorie (Brennan, Friedman \& Pollard 1987; Grosz, Joshi \& Weinstein 1995), die ihrerseits darauf zielt, Präferenzen bei der Etablierung anaphorischer Bezüge in Abhängigkeit vom Salienzniveau der beteiligten Diskursreferenten $\mathrm{zu}$ erklären. Dazu werden lokale Übergangsrelationen zwischen Sätzen definiert, die den Erhalt oder den Wechsel des Diskursgegenstandes (center) auf Grundlage einer Hierarchie syntaktischer Funktionen (grob: Subjekt < Objekt < Adjunkt) regelbasiert beschreiben, wodurch Vorhersagen über die Art und Weise der anaphorischen Wiederaufnahme einzelner Entitäten im folgenden Satz getroffen werden, die auch in verschiedenen psycholinguistischen Studien (Hudson-D'Zmura 1989; Gordon, Grosz \& Gilliom 1993) überprüft wurden. Beaver (2004) hat die CenteringTheorie optimalitätstheoretisch ausbuchstabiert und damit versucht, die präferenzgesteuerte Auflösung anaphorischer Bezüge formal als Optimierungsprozess zu begreifen.

Das Verdienst der Centering-Theorie ist zweifellos, dass sie beobachtbare Gesetzmäßigkeiten der satzübergreifenden Anaphernresolution mit der dynamischen Änderung der Salienz von Diskursreferenten zusammengebracht hat. Da der Centering-Algorithmus aber nur die grammatischen Funktionen als möglichen Salienzfaktor berücksichtigt, deckt die Theorie viele Vorkommen

14 Vgl. Tanenhaus et al. 1995; Bosch \& Umbach 2007 u. a. m.

15 Vgl. Poesio \& di Eugenio 2001; Holler \& Irmen 2007; Afantenos \& Asher 2010 u. a.m. 
anaphorischer Wiederaufnahme nicht ab. Insbesondere übersieht die CenteringTheorie die Bedeutung anderer Einflussgrößen auf die Auflösung anaphorischer Ausdrücke, wie beispielsweise der Wortstellung (siehe auch Rambow 1993), der Informationsstruktur (siehe auch Strube \& Hahn 1999) und der diskursrelationalen Struktur (siehe auch Kehler 1997). Letzterer Aspekt wird im nächsten Abschnitt aufgegriffen. Dabei wird auch erläutert, wie die Diskursstruktur auf die Textbedeutung rückwirkt.

\section{Diskursrelationen und rhetorische Struktur}

Wie im vorherigen Abschnitt ausführlich dargelegt, leisten diskursanaphorische Beziehungen einen zentralen Beitrag zur Textkonstitution. Minimalpaare wie (10) belegen jedoch, dass eine Satzfolge auch ohne pronominale Wiederaufnahme als zusammenhängend interpretiert werden kann, und zwar auch dann, wenn keine anderen expliziten kohäsiven Mittel realisiert sind:

(10) a. Arp schaltete die Lampe ein. Das Atelier war dunkel.

b. Arp schaltete die Lampe aus. Das Atelier war dunkel.

c. Arp schaltete die Lampe aus. Das Atelier war frisch gestrichen.

In der Regel wird (10a) derart interpretiert, dass Arp die Lampe eingeschaltet hat, weil das Atelier dunkel war; (10b) wird präferiert so verstanden, dass das Atelier dunkel war, nachdem Arp die Lampe ausgeschaltet hat. Offenbar werden beim Lesen/Hören semantische Relationen ergänzt, um einen sinnvollen Zusammenhang zwischen beiden Sätzen herzustellen. ${ }^{16}$ Wenn dies jedoch nicht gelingt, wird die Satzfolge als inkohärent wahrgenommen. Dies kann in (10c) der Fall sein, es sei denn, Leser/Hörer reichern die Satzfolge pragmatisch derart an, dass sie für eine spezifische Situation eine beide Sätze einschließende übergreifende Interpretation konstruieren können. Wird (10c) in einen Text eingebettet, ist zudem vorstellbar, dass der zweite Satz der Folge einen Themenwechsel anzeigt. Damit illustriert (10c) die inzwischen gängige Sichtweise, dass Leser/Hörer unter Zuhilfenahme bestimmter zusätzlicher, ggf. auch extralinguistischer Information eine scheinbar inkohärente Satzfolge letztlich immer kohärent verstehen können.

16 Dies entspricht bereits frühen Annahmen von Lang (1977), wonach die Satzkoordination einer gemeinsamen Einordnungsinstanz (Common Integrator) bedarf. 
Aus Beispielen wie (10) ist geschlossen worden, dass Konnektivität eine konstituierende Texteigenschaft ist. Wenn man Beispiele wie (11) hinzunimmt, wird weiterhin deutlich, dass die Anordnung von Sätzen in einem Text nicht beliebig ist, sondern die Textbedeutung unmittelbar beeinflusst: Die präferierte Lesart von (11a) ist, dass der Unfall passierte, nachdem die Autobahn gesperrt war, während (11b) überwiegend so verstanden wird, dass der Unfall die Ursache für die Sperrung darstellt. ${ }^{17}$

(11) a. Die Autobahn wurde gesperrt. Zwei Autos rasten ineinander.

b. Zwei Autos rasten ineinander. Die Autobahn wurde gesperrt.

Die Beispiele (10) und (11) zusammengenommen veranschaulichen die allgemein akzeptierte Auffassung, dass Textverstehen ,als Wechselspiel zwischen der Aufnahme von Information aus dem Text und dem Herantragen von Vorwissen an den Text aufzufassen ist“ [Hervorhebungen im Original] (Stede 2007: 132).

Auf frühe Arbeiten zur Textkohärenz, insbesondere auf Halliday \& Hasan (1976) und Hobbs (1979), geht die Idee zurück, dass mittels einer festgelegten Menge von sogenannten Diskursrelationen über einem Text eine weitere Struktur aufgebaut werden kann, indem implizite semantisch-pragmatische Relationen, die zwischen Sätzen im Text bestehen, expliziert werden. Angesichts ihrer Funktion werden diese Relationen seither auch als Kohärenzrelationen, rhetorische Relationen oder Diskursrelationen bezeichnet. ${ }^{18}$

Zwei einschlägige Theorien bestimmen sowohl in der Linguistik als auch in der Computerlinguistik die formale Analyse diskursrelationaler Strukturen in Texten: (i) die Rhetorische Strukturtheorie (kurz: RST) von Mann \& Thompson (1988) und (ii) die Segmentierte Diskursrepräsentationstheorie (kurz: SDRT) von Asher (1993) und Asher \& Lascarides (2003). ${ }^{19}$ Beide Theorien haben ihre

17 Die Auflösung anaphorischer Beziehungen und informationsstrukturelle Gegebenheiten sind zwei weitere Indikatoren dafür, dass die Interpretation von Texten nicht unabhängig von der Reihenfolge der präsentierten Sätze ist.

18 In der Segmentierten Diskursrepräsentationstheorie (SDRT) wird Diskursrelation als Oberbegriff für zwei Arten von Relationen verstanden: rhetorische Relationen, wie zum Beispiel Parallel, Comment, Narration oder Explanation, die Propositionen mit unterschiedlicher rhetorischer Funktion verknüpfen, und Kohärenzrelationen, wie zum Beispiel Cause oder Temporal, die Ereignisse/Zustände, Ereignistypen, Fakten etc., die in den Diskurseinheiten ausgedrückte semantische Objekte miteinander in Beziehung setzen.

19 Dass zwischen den einzelnen Sätzen im Text netzwerkartige Kohärenzbeziehungen etabliert sein können, ist schon früh von de Beaugrande \& Dressler (1981) erkannt worden. Allerdings sehen sie Kohärenzrelationen als Verkettung von Konzepten. Sie trennen diese nicht von Koreferenzrelationen, Quantitätsbeziehungen, Teil-Ganzes-Relationen etc. 
Wurzeln in der maschinellen Sprachverarbeitung und teilen die Annahme, dass sich Texte rekursiv segmentieren lassen..$^{20}$ Diese einzelnen Segmente (oder auch: Diskurskonstituenten, elementare Diskurseinheiten) sind durch die entsprechenden Diskursrelationen miteinander verknüpft, wodurch sich eine hierarchische Textstruktur ergibt, die in der RST als Baum und in der SDRT als gerichteter Graph repräsentiert wird. RST und SDRT unterscheiden sich weiterhin dahingehend, welche Segmente verknüpft werden. Die RST verfolgt einen lokalen und oft als statisch bezeichneten Ansatz, wonach der Text als bestehende Einheit beschrieben wird und Diskursrelationen nur zwischen zwei adjazenten Diskurseinheiten etabliert werden können. Die SDRT, die auf die DRT aufbaut und wie diese auf kognitive Plausibilität zielt, ist dynamisch und modelliert bottom-up den inkrementellen Prozess des Textverstehens. Daraus resultiert, dass Diskurssegmente im Prinzip an jedes bereits in die Struktur eingeführte Segment (unterschiedlicher Komplexität) anschließen können. Die Wahl einer geeigneten Anknüpfungsstelle (i.e. der entsprechende Knoten im Diskursgraphen) wird allerdings vom Right Frontier Constraint (kurz: RFC) (Polanyi 1988; Webber 1988; Asher \& Lascarides 2003) restringiert. Der RFC besagt, dass ein neues Segment entweder an den zuletzt in die Struktur eingeführten Knoten angebunden werden darf oder an einen Knoten, der diesen dominiert. Daraus resultiert, dass alle Knoten, die als Anknüpfungsstelle zur Verfügung stehen, de facto an der rechten Grenze des Diskursgraphen liegen. Es ist inzwischen vielfach gezeigt worden, dass der RFC auch für die Pronomenresolution relevant ist, vgl. hierzu auch die Hinweise in Abschnitt 2.

Das Inventar der Diskursrelationen ist bis dato nicht abschließend festgelegt, was möglicherweise Grosz \& Sidner (1986) bestätigt, die sogar grundsätzlich bezweifeln, dass die unendliche Menge möglicher Sinnzusammenhänge überhaupt durch eine endliche Menge von Relationen zu beschreiben ist. Gleichwohl ist dieser Versuch vielfach unternommen worden: Neben einem ersten taxonomischen Ansatz von Sanders, Spooren \& Noordman (1992) und dem späteren psycholinguistisch geprägten Ansatz von Knott \& Sanders (1998), die merkmalsbasiert zwölf Relationen ableiten, ist vor allem Kehler (2002) einschlägig, der in Anlehnung an die Philosophie (unter Berufung auf David Humes Relationen der Ideenassoziation) drei Gruppen mit insgesamt vierzehn Relationen ansetzt: Ähnlichkeits- und Parallelismusrelationen (zum Beispiel

20 Der Vollständigkeit halber sei erwähnt, dass neben der RST und der SDRT das Linguistic Discourse Model (kurz: LDM) von Polanyi (1988) und die Discourse Lexicalized Tree-Adjoining Grammar (kurz: D-LTAG) von Forbes et al. (2003) einschlägige Diskursmodelle sind, die beide auch von einer hierarchischen Diskursstrukturierung ausgehen und wie die SDRT den inkrementellen Prozess des Diskursaufbaus erfassen wollen. 
Elaboration, Contrast), kausale Relationen (zum Beispiel Explanation, Effect) und Kontiguitätsrelationen (zum Beispiel Okkasion, Narration), die räumliche oder zeitliche Nachbarschaft ausdrücken. Deutlich umfangreicher, aber auch unrestringierter ist die Menge der Relationen, die die RST anhand konkreter Textanalysen als hinreichend ansieht (wobei sie keinen Anspruch auf Vollständigkeit erhebt). Auch die SDRT postuliert eine recht umfassende Menge von Diskursrelationen, wobei diese bottom-up anhand bestimmter cues (Diskursmarker) axiomatisch abgeleitet werden. Anders als die RST nimmt die SDRT an, dass Diskursrelationen nur semantische Entitäten, genauer abstrakte Objekte im Sinne von Asher (1993), verknüpfen und nicht, wie in der RST möglich, auf Sprecherintentionen operieren. ${ }^{21}$

Für eine umfassende Diskussion des möglichen Inventars (semantischer und pragmatischer) Diskursrelationen sei auf Zeevat (2011) verwiesen. Darin wird auch der Frage nach dem semantischen Status der Argumente von Diskursrelationen nachgegangen, denn die einzelnen Diskurstheorien treffen dazu sehr unterschiedliche Annahmen. Neben Propositionen sind Ereignisse oder Zustände, Ereignistypen bzw. Mengen von Propositionen und Sprechakte etc. prinzipiell als diskursrelationale Argumente vorstellbar. Wichtig ist, dass die Argumente nicht syntaktisch, sondern semantisch-pragmatisch festgelegt sind, was auch bedeutet, dass sie durch Einheiten unterhalb (beispielsweise Teilsätze oder Prädikative) oder oberhalb der Satzebene (beispielsweise bereits verknüpfte Sätze) ausgedrückt werden können. ${ }^{22}$

Trotz der Abweichungen hinsichtlich des Inventars teilen RST und SDRT die Annahme, dass die Klasse der Diskursrelationen in unterordnende und nebenordnende Relationen untergliedert werden muss, um realen (a)symmetrischen Textstrukturen gerecht zu werden. Unterordnende Relationen sind in dem Sinne asymmetrisch, dass sie weniger bedeutsame und ggf. entbehrliche Information mit Information höherer Relevanz im Text verknüpfen, während die Argumente symmetrischer und somit nebenordnender Relationen von gleicher Bedeutung im Text sind. In RST bildet sich dies auf die Unterscheidung

21 Dies hängt letztlich auch damit zusammen, dass die SDRT darauf zielt, Diskursbedeutungen aufbauend auf die DRT in einem logikbasierten Formalismus zu erfassen. Die SDRT kombiniert eine Variante der Standardprädikatenlogik zur Erfassung des semantischen Gehalts der Diskurssegmente mit einer propositionalen nicht-monotonen Logik für die Modellierung der diskursrelationalen Bedeutung.

22 Bei der computerlinguistischen Umsetzung der diskursrelationalen Annotation großer Textmengen werden entgegen der theoretischen Annahmen meistens Oberflächenformen im Text als Argumente der Diskursrelationen verwendet. Dies hat oft rein praktische Gründe, um die entsprechenden Etiketten bei der Annotation adäquat verankern zu können. Siehe hierzu auch Stede (2004). 
zwischen Nukleus-Satellit-Relationen einerseits und multinuklearen Relationen andererseits ab. In der SDRT ist mit Asher \& Vieu (2005) von subordinierenden und koordinierenden Relationen die Rede, ${ }^{23}$ was (gewollte) Parallelen zur Satzstruktur wachruft. Allerdings sei angemerkt, dass die Strukturierung auf Diskursebene weitaus weniger eindeutig analysierbar ist als auf Satzebene, und zwar wegen der Unschärfe der zugrundeliegenden Regeln und der möglichen Mehrdeutigkeit der involvierten Diskursmarker.

Diskursrelationen werden im Deutschen typischerweise von Konnektoren und Diskurspartikeln markiert, aber auch andere grammatische Mittel wie zum Beispiel bestimmte Funktionswörter (wie zum Beispiel Präpositionen) oder syntaktische Konstruktionen (wie zum Beispiel $w$-Relativsätze) können rhetorische Relationen explizieren. Allen diesen Einheiten und Strukturen gemeinsam ist, dass sie die jeweiligen Relationen nicht eindeutig markieren: Entweder weil sie selbst polysem sind oder weil die Relation trotz Markierung unterspezifiziert bleibt. Maximale Unterspezifikation würde in Satzfolgen wie oben in (10) vorliegen, bei denen keine formale Einheit vorhanden ist, die eine Diskursrelation anzeigt. ${ }^{24}$

Seit Erscheinen des Handbuchs der deutschen Konnektoren (Pasch et al. 2003; Breindl, Volodina \& Waßner 2014) ist gesetzt, dass die Klasse der Konnektoren nicht nur die klassischen Subjunktionen wie zum Beispiel da und Konjunktionen wie zum Beispiel oder umfasst, sondern auch Postponierer wie zum Beispiel sodass, Verbzweiteinbetter wie zum Beispiel vorausgesetzt und relationale Adverbien wie zum Beispiel zudem mit einschließt. Die zirka 350 Konnektoren des Deutschen sind über ihre Form (unflektiert, nicht kasusregierend) und ihre Konnexionsfunktion als Klasse definiert. Semantisch steuern sie zur Interpretation eine zweistellige Relation bei, auf deren Basis die entsprechenden Diskursrelationen inferiert werden können. Dass hierfür große Interpretationsspielräume existieren, liegt vor allem an der hochgradigen Mehrdeutigkeit der Konnektoren selbst, die nicht zuletzt dadurch zustande kommt, dass als Argumente der Konnektorenbedeutungen nicht nur Propositionen fungieren können, sondern auch epistemische Minimaleinheiten und Illokutionen. Beispielsweise argumentiert Sweetser (1990) für eine solche generelle Ambiguität in Bezug auf kausale und adversative Konnektoren. Diese Sachlage hat

23 Asher \& Vieu (2005) argumentieren, dass Diskurssubordination bzw. -koordination als Strukturmerkmal der Diskursrepräsentationen modelliert werden müssen, weil sie keine semantischen Eigenschaften der Diskursinformation sind und daher auch nicht modelltheoretisch erfasst werden können.

24 Für eine explorative Studie zur Explikation von Diskursrelationen durch Konnektoren siehe Fabricius Hansen (2005). 
die Konnektorenprojektgruppe am Institut für Deutsche Sprache dazu veranlasst, zwischen der Grundbedeutung eines Konnektors und seinem kognitivkommunikativen Potenzial zu unterscheiden. Die Grundbedeutung beschreibt den minimalen Bedeutungsbestandteil eines Konnektors, der lexikalisch festgelegt ist. Auf dieser Grundlage wird im Einklang mit kognitiv verankerten Prinzipien und Schemawissen - so die Annahme - in einem konkreten Kontext eine Äußerungsbedeutung des Konnektors abgeleitet, die ggf. auch unterspezifiziert bleiben kann. Siehe zu diesem Themenkomplex insbesondere auch Breindl \& Waßner (2006) sowie Wöllstein (2008). ${ }^{25}$

Es ist offensichtlich, dass die Klasse der Konnektoren als Bindeglied zwischen syntaktischer Strukturierung und Diskurstrukturierung fungiert. Daher ist naheliegend, nach dem Verhältnis beider Domänen zueinander zu fragen: Geht syntaktische Subordination/Koordination stets mit Unterordnung/Nebenordnung im Diskurs einher? Blühdorn (2008) verneint diese Frage unter anderen anhand von Beispielen wie (12):

(12) Maria ging zu McDonald’s, und sie bekam Hunger. (Blühdorn 2008)

Blühdorn argumentiert, dass beide Teilsätze zwar syntaktisch koordiniert sind, aber diskurssemantisch auch asymmetrische Relationen inferiert werden können, denn Beispiel (12) weist mindestens drei Diskursbedeutungen auf, und zwar (i) ,außerdem bekam sie Hunger', (ii) ,dann bekam sie Hunger‘ und (iii) ,deswegen bekam sie Hunger‘. Blühdorn nimmt an, dass nur (i) symmetrisch und damit wie in der Syntax koordinierend ist, (ii) und (iii) stuft er als asymmetrisch ein, weil sie jeweils das zweite Konjunkt diskursstrukturell subordinieren würden. Inwiefern man im Rahmen der SDRT zu der gleichen Einschätzung käme, sei dahingestellt. Es ist aber zweifellos richtig, dass die hierarchischen Strukturen der syntaktischen Domäne und der Diskursdomäne nicht direkt aufeinander abbildbar sind, wie auch verschiedene Beiträge in Fabricius Hansen \& Ramm (2008) zeigen.

Diese Einsicht passt schließlich auch zu aktuellen Ergebnissen hinsichtlich der Annotation von Diskursrelationen in großen Textmengen. Dabei hat sich

25 In Wöllstein (2008) wird eine semantische Dependenzrelation zwischen zwei Konnekten etabliert, die auf einem intrinsisch asymmetrischen, kontrafaktisch kausalen Schlussverfahren basiert und weitere Annahmen lizenziert wie etwa: a) eine asymmetrische, binär verzweigende und von einem funktionalen Kopf projizierte Konnexionsphrase, b) eine Konnektorenfunktion, die die semantische Defaultinterpretation entweder modifiziert, spezifiziert oder überschreibt, und c) dass konnektorlose Konnexionen sich in das vorgeschlagene Erklärungsmuster fügen, indem sie die semantische Defaultrelation unmodifiziert abbilden. 
ebenfalls gezeigt, dass syntaktische Konnektoren simultan mehrere Diskursrelationen evozieren können. Oder anders ausgedrückt, obwohl ein Konnektor eine Diskursrelation zwischen zwei Diskurssegmenten markiert, können weitere implizite Relationen inferiert werden. Infolgedessen ist es möglich, dass zwischen zwei Diskursegmenten simultan mehrere semantische und pragmatische Diskursrelationen etabliert sind, vgl. hierzu auch die experimentellen und korpuslinguistischen Ergebnisse von Webber (2016) in Bezug auf die Penn Discourse TreeBank (Miltsakaki et al. 2004) sowie die von ihr diskutierten Konsequenzen für die automatische Annotation von Diskursrelationen.

Die Schnittstelle zwischen Syntax und Diskurs hat gleichzeitig auch im Bereich der Grammatiktheorie neue Aufmerksamkeit erlangt. Im Zuge der Untersuchung komplexer Satzstrukturen hat sich gezeigt, dass syntaktische Verknüpfung und semantische Interpretation sensitiv für diskursrelationale Strukturen sein können. Kehler (2002) hat dies beispielsweise anhand von VP-Ellipsen im Englischen eindrucksvoll demonstriert. Dass der Diskursstatus in der Grammatik bedeutsam ist, wird zudem durch komplexe nicht-kanonische Satzkonstruktionen wie (13) belegt, die unter Rückgriff auf diskursstrukturelle Beziehungen von ihren kanonischen Pendants abgegrenzt werden können (vgl. auch Holler 2009). (13a) ist ein Beispiel für einen weiterführenden $d$-Relativsatz und (13b) für einen weiterführenden $w$-Relativsatz. ${ }^{26}$ Beide teilen mit dem weil-V2-Adverbialsatz in (13c), dass sie syntaktisch (und auch prosodischsemantisch) als unintegriert gelten.

(13) a. Max gibt seiner Mitarbeiterin ein Buch, das sie dann gleich in die Bibliothek in der Melanchthonstraße bringt und dort abgibt.

b. Anton schreibt kein neues Kochbuch, was den Verlag wegen der bevorstehenden Buchmesse maßlos ärgert.

c. Hans hat seit Jahren Schulden, weil - er hat ein teures Haus direkt am Meer gekauft.

Nicht-kanonische Konstruktionen wie (13) sind in der sprachtheoretischen Literatur zum Deutschen vielschichtig ausgeleuchtet worden, was dennoch nicht $\mathrm{zu}$ einer konsensuellen Vorstellung davon geführt hat, wie der nichtkanonische Satz an den (vermeintlichen) Matrixsatz anzubinden ist. Eine denkbare, wenngleich extreme Position ist, dass die Verknüpfungsbeziehung nicht

26 Ein weiterführender Relativsatz ist eine Variante eines nicht-restriktiven Relativsatzes, die dadurch gekennzeichnet ist, dass im Relativsatz die Narration des Bezugssatzes fortgesetzt wird, vgl. Holler (2005). 
auf syntaktischer, sondern nur auf diskursrelationaler Ebene etabliert wird, z. B. Holler (2007). Unabhängig davon, ob diese konkrete Analyse von Bestand sein wird, lässt sich feststellen, dass die Auffassung, dass der Diskursstatus sprachlicher Ausdrücke auch grammatische und semantische Reflexe hat, wird inzwischen breit geteilt, was nicht zuletzt auch die Arbeiten von Doherty (2002); Holler (2005, 2009); Schlenker (2010); Koev (2013); Ott \& Onea (2015); Fabricius Hansen (2016) und Jasinskaja (2016, 2017) belegen.

Das anhaltend große bzw. teils neu entfachte grammatiktheoretische Interesse am Diskurs hat einen weiteren Grund. Es hat sich herausgestellt, dass die diskursrelationale Struktur in Bezug auf das information packaging (Chafe 1976; Vallduvi \& Engdahl 1996; Krifka 2007) Effekte zeigt und dazu beiträgt, sprachliche Entitäten sowohl im komplexen Satz als auch im Text zu gewichten, d.h. entweder durch Fokussierung die Aufmerksamkeit auf diese Entitäten $\mathrm{zu}$ richten oder sie in den Hintergrund zu versetzen. Dieser Aspekt ist eng verwoben mit anderen grammatischen Mitteln der Topik- und Fokusmarkierung, die zusammengenommen Kohärenz im Text stiften.

Die Funktion der Fokus-Hintergrund-Gliederung und ihre Sichtbarmachung durch Frage-Antwort-Heuristiken ist schon von Hermann Paul (1880) bemerkt worden und wurde später auch in der germanistischen Sprachwissenschaft für die Beschreibung der sogenannten Reliefgebung (Weinrich 1964, Hartmann 1984) herangezogen, um beispielsweise pragmatische Angemessenheitsunterschiede semantisch äquivalenter komplexer Satzkonstruktionen zu erfassen. Die aktuellen diskurssemantischen und pragmatischen Theorien zur fragegesteuerten Informationsgewichtung im Diskurs, die im nächsten Abschnitt thematisiert werden, haben im weitesten Sinne dort ihren Ursprung.

\section{Fragen im Diskurs}

Was macht aufeinanderfolgende Sätze zum Text? Dies ist nach wie vor die Gretchenfrage für jede formale Texttheorie. Eine naheliegende Antwort ist, dass das Thema eine Folge von Sätzen zu einem Text verbindet, was wiederum die Frage aufwirft, wie das Thema zu bestimmen ist. Die Diskussion über den Thema-Begriff wird seit Jahrzehnten geführt und hat eine schier unüberschaubare Literatur hervorgebracht. ${ }^{27}$

Die verschiedenen Konzeptionen unterscheiden sich unter anderem darin, ob das Thema eher aus semantischer, pragmatischer oder kognitiver Perspektive

27 Für eine neuere Zusammenstellung vgl. beispielsweise Bärenfänger (2011). 
bestimmt wird. Auch in der Begrifflichkeit gibt es eine große Variation, so wird beispielsweise auch vom Topic oder Diskurstopik gesprochen, wenn das Thema eines Textes gemeint ist. Die frühen Arbeiten von Daneš (1970), in denen er die bis dahin eher satzbezogene Thema-Rhema-Gliederung der Prager Schule auf den Text überträgt, legen den Grundstein für die Vorstellung des Themas als zentralem Textgegenstand, der fortlaufend konstituiert wird, vgl. z. B. auch Hoffmann (2000). Zwar ist seine Festlegung von lokalen thematischen Übergangsmustern im Rahmen der sogenannten thematischen Progression im Detail nicht ausreichend, die verfolgte Grundidee hat aber einiges für sich und steckt letztlich sowohl im Topic-Continuity-Ansatz von Givon (1992) als auch in der Centering-Theorie (Grosz, Joshi \& Weinstein 1995). Anders als Daneš (1970) versteht Givon aber thematische Progression als mentale Kontinuität, die durch entsprechende Diskursreferenten repräsentiert und durch geeignete sprachliche Mittel angezeigt wird.

Eine zweite große Gruppe von Thema-Konzeptionen, wonach das Thema eines Textes als Antwort auf eine (ggf. implizite) Frage bestimmt wird, ist gewissermaßen auch schon bei Daneš (1970) und dem von ihm verwendeten Fragetest zur Thema-Bestimmung angelegt, wird aber vor allem auf Keenan \& Schieffelin (1976) zurückgeführt, die den Blick auf den Dialog und die dabei beteiligten Kommunikationspartner richten. Sie argumentieren, dass der Sprecher mit seiner Äußerung eine implizite question of immediate concern (Keenan \& Schieffelin 1976: 344) beantwortet, die der Hörer erschließen muss. Die Proposition oder die Menge von Propositionen, die diese implizite Frage präsupponiert, definieren sie entsprechend als das Diskurstopik bzw. das Thema. Beispielsweise würde die Frage Was für Bilder malte Robert Rauschenberg? präsupponieren, dass es Bilder gibt, die Robert Rauschenberg malte, wodurch das Thema festgelegt ist.

In der germanistischen Forschungstradition ist diese Sichtweise prominent mit dem Quaestio-Ansatz (vgl. von Stutterheim 1994, 1997; Klein \& von Stutterheim 1987, 1992 und von Stutterheim \& Klein 2002) aufgegriffen und in Bezug auf die Prinzipien der Textproduktion weiterentwickelt worden. Nach Klein \& von Stutterheim orientiert sich der Textaufbau an einer einleitenden (impliziten) Frage, der Quaestio, die das Thema des Textes abhängig von der jeweiligen Intention bestimmt. Wie diese Leitfrage im Einzelfall lautet, ist vom jeweiligen Texttyp abhängig. ${ }^{28}$ Die Quaestio gibt einen Referenzrahmen vor, der durch den Text gefüllt wird, wobei beispielsweise referentielle, temporale, modale und räumliche Zusammenhänge spezifiziert werden. Die Leistung des

28 Für einen narrativen Text setzen Klein \& von Stutterheim (1992) beispielsweise die Quaestio „Was geschah (dir) zum Zeitpunkt x am Ort y?“ an. 
Quaestio-Ansatzes besteht darin, dass die Prinzipien der Textkonstitution systematisch zu den informationsstrukturellen Gegebenheiten im Text und den mentalen Wissensstrukturen der Kommunikationspartner in Beziehung gesetzt werden. Auf diese Weise steuert die Quaestio sowohl den lokalen als auch den globalen Informationsaufbau und -fortgang. Aus der Quaestio lassen sich texttypbezogene Vorgaben für die propositionale Fokus-Hintergrund-Gliederung, die referentielle Bewegung, die Linearisierung der Information sowie ihre Gewichtung in Haupt- und Nebenstrukturen ableiten, wodurch insgesamt eine pragmatische Informationsstruktur evoziert wird, die den kommunikativen Interessen und Zielen der Kommunikationspartner entspricht.

Der Quaestio-Ansatz bzw. Derivate dieses Ansatzes haben nicht nur die korpusgestützte Textanalyse maßgeblich beeinflusst, sondern auch Eingang in die Grammatiktheorie gefunden. Insbesondere im Umfeld der theoretischen Diskussion zur kommunikativen Gewichtung im Deutschen - z. B. Reis (1993) in Bezug auf die implikative Koordination, Brandt (1994) in Bezug auf Parenthesen und Holler (2005) in Bezug auf weiterführende Relativsätze bzw. Holler (2009) in Bezug auf nicht-kanonische Satzkonstruktionen - hat sich gezeigt, dass bestimmte Linearisierungseffekte mit Hilfe des Quaestio-Ansatzes erklärbar sind. (Teil-)Sätze, die zur Beantwortung der Textfrage beitragen, werden der Hauptstruktur des Textes zugeordnet und damit als Hauptinformation eingestuft, während (Teil-)Sätze, die nicht durch die Quaestio des Textes gedeckt sind und daher weniger relevante Information beitragen, Nebenstrukturen bilden. Wichtig ist, dass die Unterscheidung zwischen Haupt- und Nebenstrukturen nicht generell mit dem Haupt- bzw. Nebensatzstatus der entsprechenden (Teil-)Sätze korreliert. Mit anderen Worten, es gibt durchaus Nebensatztypen, die quaestiobezogene Information ausdrücken, was ein weiterer Hinweis dafür ist, dass syntaktische Subordination und diskursstrukturelle Nebenordnung nicht Eins-zu-Eins aufeinander abgebildet werden können. In Holler (2008, 2009) wird zudem argumentiert, dass sich die genannten Zusammenhänge im Rahmen eines constraintbasierten Grammatikmodells unter Rückgriff auf die diskursrelationale Struktur modellieren lassen.

Die Sicht, dass Texte durch implizite Fragen strukturiert werden, findet sich auch in formalen Theorien, die dem Konzept der question under discussion (kurz: QUD) (z.B. Roberts 1996; Büring 2003; Beaver \& Clark 2008; Benz \& Jasinskaja 2017) verpflichtet sind. Ohne in die formalen Details zu gehen, lässt sich sagen, dass QUD-Ansätze mit Quaestio-Ansätzen die Annahme teilen, dass die implizite(n) Textfrage(n) aus den jeweiligen Sprecherintentionen abgeleitet werden. Anders als in der Quaestio-Theorie, wo eine Satzsequenz die zugrundeliegende Datenstruktur bildet, werden aber in QUD-Ansätzen Dialoge analysiert. Diese Annahme ermöglicht es, den Informationsfluss zwischen den Kom- 
munikationspartnern zu modellieren. Der Diskurs wird durch eine Frage wie „Worüber sprechen wir?“ initiiert und die allgemeinste QUD lautet „Was ist der Fall und was nicht?“. Ein weiterer Unterschied betrifft den Formalisierungsgrad, der in QUD-Ansätzen höher ist als in der Quaestio-Theorie. QUD-Ansätze beziehen sich in der Regel auf dynamische Semantikmodelle, in denen QUDs partiell geordnete Mengen von Fragen darstellen, die ein Diskurssegment partitionieren, also kriterienbezogen zerlegen bzw. unterteilen. Dadurch, dass Subund Superfragen repräsentiert werden, entsteht eine hierarchische Diskursstruktur. Wenn eine aktuelle Äußerung an diese Struktur angebunden wird, so ist die Anknüpfungsstelle davon abhängig, welche Frage die Sprecherin intendiert zu beantworten. Ein Diskurssegment ist abgeschlossen und spätestens dann auch für anaphorische Bezüge nicht mehr zugänglich, wenn alle offenen Fragen beantwortet sind. Ähnlich wie die Quaestiones lenken auch die QUDs die Informationsgewichtung im Diskurs. Hauptinformation beantwortet die QUD und ist at-issue-Gehalt; Nebeninformation hingegen ist not-at-issue, weil sie die jeweilige QUD nicht auflöst. Typischerweise wird hierzu der durch Appositionen, Parenthesen und Präsuppositionen projizierte Gehalt gerechnet. ${ }^{29}$

Ein fragebasiertes Diskursmodell wird auch von einer zweiten Klasse von QUD-basierten Theorien vorausgesetzt, die die Hypothese vertreten, dass sich ein Text oder Dialog ausgehend von einer linguistischen oder nichtlinguistischen Begebenheit in Frage-Antwort-Sequenzen entwickelt und aufbaut. Stark vereinfachend und im Detail nicht vollkommen präzise ausgedrückt, triggern die angenommenen impliziten Fragen bottom-up satzweise den Textaufbau, was - gedacht in Textorientierung - bedeutet, dass diese Ansätze kataphorisch agieren, indem sie auf die schrittweise Erzeugung des Diskursbaumes zielen, während Quaestio- und QUD-Ansätze à la Roberts (1996) eher top-down und anaphorisch ausgerichtet sind, indem sie die Zugänglichkeit bzw. Anknüpfbarkeit im bereits erzeugten (in Teilen aber unterspezifizierten) Diskursbaum modellieren.

Ein prominenter Vertreter der zweiten Sicht ist van Kuppevelt (1995), der Topikalität als das entscheidende Organisationsprinzip für den Diskurs ansieht. Die zentrale Annahme ist, dass jede kontextuell induzierte (implizite) Frage, die im Diskurs beantwortet wird, zugleich ein Topik konstituiert, d. h. das Topik wird implizit erfragt und die Menge der möglichen Antworten auf diese Frage wird mit dem erfragten Topik identifiziert. Die vom Sprecher aus

29 Potts (2005) hat argumentiert, dass at-issue und not-at-issue-Gehalt logisch und kompositional voneinander zu trennen sind. Diese Annahme hat bis heute einen großen Einfluss auf die Forschungsdiskussion. Zahlreiche theoretische und experimentelle Arbeiten haben diese Hypothese inzwischen geprüft und kommen naturgemäß zu einem etwas differenzierteren Bild. 
der Menge der möglichen Antworten tatsächlich ausgewählte Antwort auf die implizite Frage wird mit dem Kommentar identifiziert. Solange diese Antwort noch nicht befriedigend ist, werden weitere Subfragen erzeugt, andernfalls gilt das Topik als abgeschlossen, wodurch eine hierarchische Diskursstruktur erzeugt wird. Zudem sieht van Kuppevelt vor, dass einzelne Aussagen - neben ihrer Funktion, eine Frage zu beantworten - auch in der Lage sind, neue Fragen im Diskurs aufzuwerfen und dadurch ein neues Topik zu etablieren. Damit erfüllen sie eine kataphorische Funktion. Diese Idee wird in Onea (2016) mit dem Konzept der potenziellen Fragen neu aufgegriffen und in dem allgemeinen Rahmen der Inquisitiven Semantik ${ }^{30}$ (Groenendijk \& Roelofsen 2009) formalisiert. Grob gesagt sind potenzielle Fragen alle diejenigen Fragen, deren Präsuppositionen durch die bereits geäußerten Assertionen erfüllt sind. Potenzielle Fragen können Äußerungen im Diskurs aufwerfen und dadurch als potenzielle Anknüpfungspunkte für neue Diskursstrategien dienen. Wichtig ist in diesem Zusammenhang, dass Onea (2016) für eine Reihe von grammatischen Phänomenen, u. a. die Markierung von Indefinitheit in unterschiedlichen Sprachen, zeigt, dass sie mit diesem kataphorischen Potenzial von Äußerungen grammatisch interagieren.

Auch wenn es hier dahingestellt bleiben muss, welcher der diskutierten Ansätze überdauern wird, so ist wohl deutlich geworden, dass der frühe Gedanke, dass explizite oder implizite Fragen den Textaufbau steuern und aus einer Satzfolge Texte erzeugen, einige Erklärungskraft besitzt.

\section{Was noch zu tun ist}

Der vorliegende Aufsatz hat sich vor allem mit der Frage befasst, wie textuelle Strukturen aufgespürt, beschrieben und modelliert werden können, wobei der aktuelle Stand der wissenschaftlichen Diskussion zur Textanalyse schlaglichtartig rekapituliert wurde. Die aus verschiedenen Gründen recht vertrackte Beziehung zwischen einzelnen Sätzen und dem Text ist dabei sichtbar geworden. Dies schließt ein, dass der Diskurs die Interpretation der Sätze, die in ihm vorkommen, teilweise mitbestimmt, und die Textstruktur zusätzliche Inferenzen auslöst, deren Informationsbeitrag über die Bedeutung der einzelnen Sätze

30 In der Inquisitiven Semantik wird die Bedeutung eines Satzes dynamisch als Differenz zwischen zwei Informationszuständen (Mengen von Welten) charakterisiert, wodurch es unter anderem möglich wird, den Informationszuwachs eines Individuums durch eine sprachliche Äußerung zu modellieren. 
hinausgeht. Entsprechend beruht das Erstellen und Verstehen von Texten auf einem komplexen Zusammenspiel von Textmaterial, Wissensstrukturen sowie sprachlichen und kommunikativen Prinzipien. Um textuelle Einheiten und Strukturen möglichst vollständig aufzudecken, adäquat zu analysieren und die Mechanismen ihrer kognitiven Verarbeitung zu verstehen, bedarf es sowohl einer empirisch wie theoretisch ausgeprägten sprachwissenschaftlichen Forschung zu allen Ebenen der Grammatik und Pragmatik als auch einer Intensivierung des interdisziplinären Austausches. Dies betrifft neben der Psychologie und Informatik vor allem auch andere textbezogen arbeitende Fächer, allen voran die Literaturwissenschaft.

Die Probleme, die insgesamt noch zu lösen sind, sind nicht trivial. Auf theoretischer Seite geht es erstens darum, die formalen Theorien zur Modellierung satzübergreifender sprachlicher Phänomene, die an der GrammatikPragmatik-Schnittstelle angesiedelt sind, so weiterzuentwickeln, dass auch die dynamischen und kognitiven Aspekte des textbasierten Informationsaustausches zwischen den Kommunikationspartnern besser erklärt werden können. Hier sind neuere Theorieansätze wie die Commitment-Space-Semantik (Krifka 2015) und die Mental-State-DRT (Kamp, Reyle \& van Genabith 2011) besonders vielversprechend. Damit wird auch deutlich, dass die Entstehung und Verarbeitung kohärenter Strukturen im Diskurs nicht ohne Bezug zur aktuellen Theoriebildung im Bereich der formalen Semantik und Pragmatik beschrieben werden kann, vgl. auch Gutzmann \& Schumacher in diesem Band. Auf der anderen Seite müssen diskursstrukturelle Gegebenheiten offenbar in die Beschreibung komplexer Satzkonstruktionen einbezogen werden. Wann genau und in welchem Ausmaß dies geschieht und welchen Restriktionen es unterliegt, ist zum jetzigen Zeitpunkt alles andere als geklärt. Insofern werden die Prinzipien, die an der Syntax-Diskurs-Schnittstelle wirksam sind, künftig stärker in den Forschungsfokus rücken.

Zweitens bedarf es einer stärkeren Berücksichtigung der Spezifik unterschiedlicher Texttypen, wie beispielsweise argumentativer, explanativer oder narrativer Texte. ${ }^{31}$ In Bezug auf den literarischen Text wäre zum Beispiel interessant, wie narrative Effekte aus linguistischen Strukturen hervorgehen und umgekehrt sprachliche Bedeutungen von narrativen Mustern abhängen und welche Rolle kognitive Aspekte und Lesereinstellungen für die Textinterpretation spielen. Wie einige Ergebnisse der Arbeiten zu diversen mikro- und makrostrukturellen Ebenen des narrativen Textes am Göttinger Courant-Forschungszentrum „Textstrukturen“ zeigen, lässt sich auch grundsätzlich in Frage

31 In der Forschungsliteratur zum Thema stehen vor allem instruktive und deskriptive Texte im Vordergrund. 
stellen, ob ein geschichtetes Ebenenmodell, wie es in der Textstrukturanalyse üblicherweise vorausgesetzt wird, überhaupt für alle Zwecke tauglich ist. Die Eindimensionalität der Abhängigkeit zwischen sprachlicher Form und textuellen Effekten, die ein solches Modell nahegelegt, erweist sich bei der Untersuchung narrativer Texte nicht als haltbar. Vielmehr scheinen synchrone Veränderungen an verschiedenen Stellen der Textstruktur zu bestimmten Effekten führen zu können. Beispielsweise wirken oftmals gattungsspezifische Muster und sprachliche Marker zusammen. Sofern das Verstehen literarischer Texte strukturgetrieben ist, ist es jedenfalls komplexer als ursprünglich angenommen, da einzelne sprachliche Mittel erst im Kontext bestimmter anderer sprachlicher Konstellationen und narrativer Gegebenheiten eine bestimmte Wirkung entfalten. Das schließt ein, dass sie in einer anderen narrativen Umgebung andere Effekte auslösen können, weswegen der Zusammenhang zwischen Form und ästhetischem Effekt nicht zwingend eineindeutig ist. Das kontextabhängige Wechselspiel zwischen sprachlichen und narrativen Mitteln kann vermutlich durch das Ebenenmodell nicht ausreichend erfasst werden. Die sprachlichen Mittel im Text haben eher ein bestimmtes Informationspotenzial, das in Abhängigkeit vom strukturellen Kontext und weiteren narrativen und autor-/leserabhängigen Bedingungen wirksam wird.

Drittens kann die Vielfalt der Textstrukturphänomene ohne einen Methodentransfer zwischen den theoretisch-analytischen Ansätzen der Linguistik und Literaturwissenschaft und der experimentell-empirischen Forschung in Psychologie und Informatik nicht vollständig ergründet werden. In dieser Hinsicht unterscheidet sich die Textanalyse nicht von der Satzanalyse.

\section{Literatur}

Adamzik, Kirsten (2016): Textlinguistik. Eine einführende Darstellung. 2. Aufl. Tübingen: Niemeyer.

Afantenos, Stergos \& Nicholas Asher (2010): Testing SDRT's right frontier. Proceedings of the 23rd International Conference on Computational Linguistics (CoLing 2010), 1-9.

Antos, Gerd (2009): Semiotik der Text-Performanz. Symptome und Indizien als Mittel der Bedeutungskonstitution. In Angelika Linke \& Helmuth Feilke (Hrsg.), Oberfläche und Performanz. Untersuchungen zur Sprache als dynamischer Gestalt, 407-427. Tübingen: Niemeyer.

Ariel, Mira (2001): Accessibility theory: An overview. In Ted Sanders, Joost Schilperoord \& Wilbert Spooren (Hrsg.), Text representation. Linguistic and psycholinguistic aspects, 29-87. Amsterdam/Philadelphia: Benjamins.

Asher, Nicholas (1993): Reference to abstract objects in discourse. Dordrecht: Kluwer.

Asher, Nicholas \& Alex Lascarides (2003): Logics of conversation. Cambridge, UK: Cambridge University Press. 
Asher, Nicholas \& Laura Vieu (2005): Subordinating and coordinating discourse relations. Lingua 115, 591-610.

Auer, Peter (2014): Anmerkungen zum Salienzbegriff in der Soziolinguistik. Linguistik Online 66 (4). http://dx.doi.org/10.13092/lo.66.1569 (letzter Zugriff: 29. 9. 2017).

Bärenfänger, Maja (2011): Ebenen des Themas. Zur Interaktion von Thema, Text und Wissen. Dissertation, Universität Gießen.

Beaugrande, Robert de \& Wolfgang Ulrich Dressler (1981): Einführung in die Textlinguistik. Tübingen: Niemeyer.

Beaver, David (2004): The optimization of discourse anaphora. Linguistics and Philosophy 27 (1), 3-56.

Beaver, David \& Brady Clark (2008): Sense and sensitivity. How focus determines meaning. Oxford: Blackwell.

Beißwenger, Michael (Hrsg.) (2001): Chat-Kommunikation. Sprache, Interaktion, Sozialität \& Identität in synchroner computervermittelter Kommunikation. Perspektiven auf ein interdisziplinäres Forschungsfeld. Stuttgart: ibidem.

Benz, Anton \& Katja Jasinskaja (2017): Questions Under Discussion: From Sentence to Discourse. Discourse Processes 54 (3), 177-186, DOI: 10.1080/0163853X.2017.1316038

Blühdorn, Hardarik (2008): Subordination and coordination in syntax, semantics, and discourse: Evidence from the study of connectives. In Cathrine Fabricius Hansen \& Wiebke Ramm (Hrsg.), "Subordination" vs. "coordination" in sentence and text, 59-85. Amsterdam: Benjamins.

Bosch, Peter \& Carla Umbach (2007): Reference determination for demonstrative pronouns. In Dagmar Bittner \& Natalia Gagarina (Hrsg.), Intersentential pronominal reference in child and adult language: Proceedings of the Conference on Intersentential Pronominal Reference in Child and Adult Language (ZAS Papers in Linguistics 48), 39-51. Berlin: ZAS.

Bott, Oliver \& Torgrim Solstad (2014): From verbs to discourse: A novel account of implicit causality. In Barbara Hemforth, Barbara Mertins \& Cathrine Fabricius Hansen (Hrsg.), Psycholinguistic approaches to meaning and understanding across languages, 213-251. Cham: Springer International.

Brandt, Margareta (1994): Subordination und Parenthese als Mittel der Informationsstrukturierung in Texten. In Wolfgang Motsch (Hrsg.), Ebenen der Textstruktur: sprachliche und kommunikative Prinzipien, 211-240. Tübingen: Niemeyer.

Brandt, Margareta \& Inger Rosengren (1992): Zur Illokutionsstruktur von Texten. Zeitschrift für Literaturwissenschaft und Linguistik 22 (86), 9-51.

Breindl, Eva \& Ulrich H. Waßner (2006): Syndese vs. Asyndese. Konnektoren und andere Wegweiser für die Interpretation semantischer Relationen in Texten. In Hardarik Blühdorn, Eva Breindl \& Ulrich H. Waßner (Hrsg.), Text - Verstehen: Grammatik und darüber hinaus, 46-70. Berlin: De Gruyter.

Breindl, Eva, Anna Volodina \& Ulrich H. Waßner (Hrsg.) (2014): Handbuch der deutschen Konnektoren 2. Semantik der deutschen Satzverknüpfer. Berlin/New York: De Gruyter.

Brennan, Susan E., Marilyn W. Friedman \& Carl J. Pollard (1987): A centering approach to pronouns. In Proceedings of the 25th Meeting of the Association for Computational Linguistics, 155-162.

Brinker, Klaus (2005): Linguistische Textanalyse: Eine Einführung in Grundbegriffe und Methoden. Berlin: Erich Schmidt.

Brinker, Klaus, Herrmann Cölfen \& Steffen Pappert (2014): Linguistische Textanalyse. Eine Einführung in Grundbegriffe und Methoden. 8., neu bearb. und erw. Aufl. Berlin: Erich Schmidt. 
Brown, Roger \& Deborah Fish (1983): The psychological causality implicit in language. Cognition 14, 237-273.

Büring, Daniel (2003): On D-trees, beans and B-accents. Linguistics and Philosophy 26 (5), 511-545.

Bußmann, Hadumod (2008): Lexikon der Sprachwissenschaft. 4. Aufl. Stuttgart: Alfred Kröner.

Chafe, Wallace L. (1976): Givenness, contrastiveness, definiteness, subjects, topics and point of view. In Charles N. Li (Hrsg.), Subject and topic, 27-55. New York: Academic Press.

Clark, Herbert (1977): Bridging. In Philip N. Johnson-Laird \& Peter C. Wason (Hrsg.), Thinking: Readings in Cognitive Science, 411-420. Cambridge, UK: Cambridge University Press.

Cooper, Robin (1979): The interpretation of pronouns. In Frank Heny \& Helmut S. Schnelle (Hrsg.), Syntax and semantics, volume 10, 61-92. New York u. a.: Academic Press.

Crawley, Rosalind, Rosemary Stevenson \& David Kleinman (1990): The use of heuristic strategies in the interpretation of pronouns. Journal of Psycholinguistic Research 4, 245-264.

Daneš, František (1970): Zur linguistischen Analyse der Textstruktur. Folia Linguistica 4, 72-78.

Deemter, Kee van \& Roger Kibble (2001): On coreferring: Coreference in MUC and related annotation schemes. Journal of Computational Linguistics 26 (4), 629-637.

Doherty, Monika (2002): Language processing in discourse. London: Routledge.

Duden 4: Die Grammatik (2016): Hg. v. Cathrine Fabricius Hansen, Peter Gallmann, Peter Eisenberg \& Reinhard Fiehler. 9., vollst. überarb.und aktual. Aufl. Mannheim: Bibliographisches Institut.

Eichinger, Ludwig M. (2006): Wege und Umwege zum Verstehen, oder: Wie man zu verstehen gibt, dass man jemanden willkommen heißt. In Hardarik Blühdorn, Eva Breindl \& Ulrich H. Waßner (Hrsg.), Text - Verstehen: Grammatik und darüber hinaus, 1-4. Berlin: De Gruyter.

Elbourne, Paul (2005): Situations and individuals. Cambridge, MA: MIT Press.

Ellert, Miriam \& Anke Holler (2011): Semantic and structural constraints on the resolution of ambiguous personal pronouns - A psycholinguistic study. In Iris Hendrickx, Sobha Lalitha Devi, Antonio Branco \& Ruslan Mitkov (Hrsg.), Anaphora processing and applications. LNAI 7099, 157-170. Heidelberg: Springer.

Evans, Gareth (1977): Pronouns, quantifiers and relative clauses. Canadian Journal of Philosophy 7, 467-536.

Fabricius Hansen, Cathrine (2005): Elusive connectives. A case study on the explicitness dimension of discourse coherence. Linguistics 43 (1), 17-48.

Fabricius Hansen, Cathrine (2016): Vorangestellte Attribute und Relativsätze im Deutschen: Wettbewerb und Zusammenspiel. In Mathilde Hennig (Hrsg.), Komplexe Attribution. Ein Nominalstilphänomen aus sprachhistorischer, grammatischer, typologischer und funktionalstilistischer Perspektive, 135-168. Berlin/Boston: De Gruyter Mouton.

Fabricius Hansen, Cathrine \& Wiebke Ramm (2008): Editor's introduction: Subordination and coordination from different perspectives. In Cathrine Fabricius Hansen \& Wiebke Ramm (Hrsg.), "Subordination" vs. "coordination" in sentence and text, 1-30. Amsterdam: Benjamins.

Forbes, Katherine, Eleni Miltsakaki, Rashmi Prasad, Anoop Sarkar, Aravind Joshi \& Bonnie Webber (2003): D-LTAG System: Discourse parsing with a lexicalized tree-adjoining grammar. Journal of Logic, Language, and Information 12 (3), Special issue on discourse and information structure, 261-279. 
Fukumura, Kumiko, Roger P. G. van Gompel (2010): The effects of animacy in the choice of referring expressions. Language and Cognitive Processes 26 (10), 1472-1504.

Garnham, Alan (2001): Mental models and the interpretation of anaphora. Hove: Psychology Press.

Geach, Peter (1962): Reference and generality. Ithaca, NY: Cornell University Press.

Givon, Talmy (1992): The grammar of referential coherence as mental processing Instructions. Linguistics 30, 5-55.

Gordon, Peter C., Barbara J. Grosz \& Laura A. Gilliom (1993): Pronouns, names and the centering of attention in discourse. Cognitive Science 17 (3), 311-347.

Groenendijk, Jereon \& Floris Roelofsen (2009): Inquisitive semantics and pragmatics. In Jesus M. Larrazabal \& Larraitz Zubeldia (Hrsg.), Meaning, content and argument: Proceedings of the ILCLI International Workshop on Semantics, Pragmatics and Rhetoric, 41-72. San Sebastián: Universidad del País Vasco, Servicio Editorial.

Große, Ernst U. (1976): Text und Kommunikation. Eine linguistische Einführung in die Funktionen der Texte. Stuttgart u. a.: Kohlhammer.

Grosz, Barbara J., Aravind K. Joshi \& Scott Weinstein (1995): Centering: A framework for modeling the local coherence of discourse. Computational Linguistics 21 (2), 203-225.

Grosz, Barbara \& Candace Sidner (1986): Attention, intention and the structure of discourse. Computational Linguistics 12 (3), 175-204.

Gundel, Jeanette, Nancy Hedberg \& Ron Zacharski (1993): Cognitive status and the form of referring expressions in discourse. Language 69 (2), 274-307.

Halliday, Michael A. K. \& Ruqaiya Hasan (1976): Cohesion in English. London: Longman.

Halliday, Michael A. K. \& Ruqaiya Hasan (1985): Language, context, and text: Aspects of language in a social-semiotic perspective. Oxford: Oxford University Press.

Hartmann, Dieter (1984): Reliefgebung: Informationsvordergrund und Informationshintergrund in Texten als Problem von Textlinguistik und Stilistik. Wirkendes Wort 34, 305-323.

Heim, Irene (1990): E-type Pronouns and Donkey Anaphora. Linguistics and Philosophy 13, 137-177.

Heim, Irene \& Angelika Kratzer (1998): Semantics in generative grammar. Malden, MA: Blackwell.

Heinemann, Margot \& Wolfgang Heinemann (2002): Grundlagen der Textlinguistik. Interaktion - Text - Diskurs. Tübingen: Niemeyer.

Heringer, Hans Jürgen (2015): Linguistische Texttheorie. Eine Einführung. Tübingen:

A. Francke.

Heusinger, Klaus von \& Elsi Kaiser (Hrsg.) (2010): Reference and discourse structure. Special Issue of International Review of Pragmatics 2 (2).

Hobbs, Jerry (1979): Coherence and coreference. Cognitive Science 3 (1), 67-82.

Hoffmann, Ludger (1997): Thematische Organisation von Text und Diskurs. In Gisela Zifonun et al. (Hrsg.), Grammatik der deutschen Sprache, 507-594. Berlin/New York: De Gruyter.

Hoffmann, Ludger (2000): Thema, Themenentfaltung, Makrostruktur. In Klaus Brinker, Gerd Antos, Wolfgang Heinemann \& Sven F. Sager (Hrsg.), Text- und Gesprächslinguistik. Ein internationales Handbuch zeitgenössischer Forschung. 1. Halbband (= Handbücher zur Sprach- und Kommunikationswissenschaft (HSK), 16.1), 344-356. Berlin/New York: De Gruyter.

Holler, Anke (2003): Koreferenz in Hypertexten: Anforderungen an die Annotation. Osnabrücker Beiträge zur Sprachtheorie 68, 9-29. 
Holler, Anke (2005): Weiterführende Relativsätze. Empirische und theoretische Aspekte. Berlin: Akademie.

Holler, Anke (2007): Uniform oder different? Zum syntaktischen Status nicht-restriktiver Relativsätze. Deutsche Sprache 3, 250-270.

Holler, Anke (2008): German dependent clauses from a constraint-based perspective. In Cathrine Fabricius Hansen \& Wiebke Ramm (Hrsg.), "Subordination" vs. "coordination" in sentence and text, 187-216. Amsterdam: Benjamins.

Holler, Anke (2009): Informationsreliefs in komplexen Sätzen: eine diskursrelationale Analyse. In Veronika Ehrich, Christian Fortmann, Ingo Reich \& Marga Reis (Hrsg.), Koordination und Subordination im Deutschen (Linguistische Berichte, Sonderheft 16), 135-159. Hamburg: Buske.

Holler, Anke \& Lisa Irmen (2007): Empirically assessing the effects of the right frontier constraint. In Antonio Branco (Hrsg.), Anaphora: Analysis, algorithms and applications. LNAI Lecture Notes in Artificial Intelligence, 15-27. Berlin/Heidelberg: Springer.

Holler, Anke \& Katja Suckow (Hrsg.) (2016): Empirical perspectives on anaphora resolution. Berlin: De Gruyter.

Hudson-D'Zmura, Susan (1989): The structure of discourse and anaphor resolution: The discourse center and the roles of nouns and pronouns. Ph.D. thesis, University of Rochester.

Irmer, Matthias (2011): Bridging inferences. Constraining and resolving underspecification in discourse interpretation. Berlin: De Gruyter.

Isenberg, Horst (1968): Überlegungen zur Texttheorie. In ASG-Bericht Nr. 2, 1-18. Berlin: Akademie der Wissenschaften, Arbeitsstelle Strukturelle Grammatik.

Jasinskaja, Katja (2016): Salience and (not-)at-issue status of subordinate clauses. In Proceedings of SuB 2016, 111-112.

Jasinskaja, Katja (2017): Not at issue anymore. Mskr. Universität Köln.

Kamp, Hans (1984): A theory of truth and semantic representation. In Jeroen Groenendijk, Theo M. V. Janssen \& Martin Stokhof (Hrsg.), Truth, interpretation and information. Selected papers from the Third Amsterdam Colloquium, 1-42. Berlin: De Gruyter.

Kamp, Hans \& Uwe Reyle (1993): From discourse to logic. Kluwer: Dordrecht.

Kamp, Hans, Joseph van Genabith \& Uwe Reyle (2011): Discourse representation theory. In Gabbay Dov \& Franz Guenthner (Hrsg.), Handbook of philosophical logic. Band 15, 2. Aufl., 125-394. Dordrecht: Springer Netherlands.

Karttunen, Lauri (1969): Discourse referents. In International Conference on Computational Linguistics COLING 70,1-37. Stockholm: Research Group For Quantitative Linguistics. Reprinted in James D. McCawley (Hrsg.) (1976), Syntax and semantics, 363-385. New York: Academic Press.

Keenan, Elinor O. \& Bambi B. Schieffelin (1976): Topics as a discourse notion: A study of topic in the conversations of children and adults. In Charles N. Li (Hrsg.), Subject and topic, 335-384. New York: Academic Press.

Kehler, Andrew (1997): Current theories of centering for pronoun interpretation: A critical evaluation. Computational Linguistics (Squibs and Discussions) 23 (3).

Kehler, Andrew (2002): Coherence, reference, and the theory of grammar. Stanford: CSLI.

Kehler, Andrew, Laura Kertz, Hannah Rohde \& Jeffrey L. Elman (2008): Coherence and coreference revisited. Journal of Semantics 25, 1-44.

Klein, Wolfgang \& Christiane von Stutterheim (1987): Quaestio und referentielle Bewegung in Erzählungen. Linguistische Berichte 108, 163-183. 
Klein, Wolfgang \& Christiane von Stutterheim (1992): Textstruktur und referentielle Bewegung. Zeitschrift für Literaturwissenschaft und Linguistik 22 (86), 67-92.

Knott, Alistair \& Ted Sanders (1998): The classification of coherence relations and their linguistic markers: An exploration of two languages. Journal of Pragmatics 30, 135-175.

Koev, Todor K. (2013): Apposition and the structure of discourse. Ph.D. thesis, University of Rutgers.

Krifka, Manfred (2007): Basic notions of information structure. In Caroline Féry, Gisbert Fanselow \& Manfred Krifka (Hrsg.), The notions of information structure. Working Papers of the SFB 632, 6, 13-55. Potsdam: Universitätsverlag.

Krifka, Manfred (2015): Bias in commitment space semantics. Declarative questions, negated questions, and question tags. In Sarah D'Antonio, Mary Moroney \& Carol Rose Little (Hrsg.), Semantics and linguistic theory (SALT) 25, 328-345. Washington, D.C.: LSA Open Journal Systems.

Kuppevelt, Jan van (1995): Discourse structure, topicality and questioning. Journal of Linguistics 31, 109-114.

Lang, Ewald (1977): Zur Semantik der koordinativen Verknüpfung. Berlin: Akademie.

Lappin, Shalom \& Herbert J. Leass (1994): An algorithm for pronominal anaphora resolution. Computational Linguistics 20 (4), 535-561.

Mann, William C. \& Sandra A. Thompson (1988): Rhetorical Structure Theory: Towards a functional theory of text organisation. Text 8 (3), 243-281.

Miltsakaki, Eleni, Rashmi Prasad, Aravind Joshi \& Bonnie Webber (2004): The Penn Discourse Treebank. In Proceedings of LREC. www.lrec-conf.org/proceedings/Irec2004/ (letzter Zugriff: 28. 9. 2017).

Mitkov, Ruslan (2002): Anaphora resolution. Upper Saddle River, NJ: Pearson Education.

Mitkov, Ruslan, Richard Evans \& Constantin Orasan (2002): A new, fully automatic version of Mitkov's knowledge-poor pronoun resolution method. In Proceeedings of the 3rd International Conference on Intelligent Text Processing and Computational Linguistics. http://rgcl.wlv.ac.uk/papers/ciclingAR19.pdf (letzter Zugriff: 29. 9. 2017).

Motsch, Wolfgang (1996): Ebenen der Textstruktur. Begründung eines Forschungsprogramms. In Wolfgang Motsch (Hrsg.), Ebenen der Textstruktur: sprachliche und kommunikative Prinzipien, 3-36. Tübingen: Niemeyer.

Nouwen, Rick (i. E.): E-type Pronouns: Congressmen, sheep and paychecks. In Lisa Matthewson, Cécile Meier, Hotze Rullmann \& Ede Zimmermann (Hrsg.), The companion to semantics. Oxford: Wiley.

Onea, Edgar (2016): Potential questions at the semantics-pragmatics interface. Leiden: Brill.

Ott, Dennis \& Edgar Onea (2015): On the form and meaning of appositives. In Proceedings of NELS 45, 203-212.:

Pasch, Renate, Ursula Brauße, Eva Breindl \& Ulrich H. Waßner (Hrsg.) (2003): Handbuch der deutschen Konnektoren. Linguistische Grundlagen der Beschreibung und syntaktische Merkmale der deutschen Satzverknüpfer (Konjunktionen, Satzadverbien, Partikel).

Berlin/New York: De Gruyter.

Paul, Herrmann (1880): Prinzipien der Sprachgeschichte. Halle: Niemeyer.

Poesio, Massimo \& Barbara di Eugenio (2001): Discourse structure and anaphoric accessibility. In Proceedings of the ESSLLI Workshop on Information Structure, Discourse Structure, and Discourse Semantics, 129-144.

Polanyi, Livia (1988): A formal model of the structure of discourse. Journal of Pragmatics 12, 601-638. 
Potts, Chris (2005): The logic of conventional implicatures. New York: Oxford University Press.

Rambow, Owen (1993): Pragmatic aspects of scrambling and topicalization in German. In Proceedings of the IRCS Workshop on Centering Theory in Naturally-Occurring Discourse. www.cs.columbia.edu/ rambow/papers/german-centering.ps (letzter Zugriff: 29. 9. 2017).

Reinhart, Tanya (1983): Anaphora and semantic interpretation. London: Croom Helm.

Reis, Marga (1993): Satzfügung und kommunikative Gewichtung. Zur Grammatik und Pragmatik von Neben- vs. Unterordnung am Beispiel ,implikativer‘ und-Konstruktionen im Deutschen. In Marga Reis (Hrsg.), Wortstellung und Informationsstruktur, 203-250. Tübingen: Niemeyer.

Roberts, Craige (1996): Information structure: Towards an integrated theory of formal pragmatics. In Jae H. Yoon \& Andreas Kathol (Hrsg.), OSU Working Papers in Linguistics 48, 91-136.

Rosengren, Inger (1980): Texttheorie. In Hans Peter Althaus, Helmut Henne \& Herbert Ernst Wiegand (Hrsg.), Lexikon der germanistischen Linguistik, 275-286. Tübingen: Niemeyer.

Sanders, Ted, Wilbert Spooren \& Leo Noordman (1992): Toward a taxonomy of coherence relations. Discourse Processes 24, 119-147.

Sanford, Antony J. \& Simon C. Garrod (1981): Understanding written language: Explorations of comprehension beyond the sentence. Chichester: Wiley.

Schiffrin, Deborah (1994): Approaches to discourse. Oxford: Wiley-Blackwell.

Schlenker, Philippe (2010): Supplements within a unidimensional semantics I: Scope. In Maria Aloni, Harald Bastiaanse, Tikitu de Jager \& Katrin Schulz (Hrsg.), Logic, language and meaning. 17th Amsterdam Colloquium, Amsterdam, The Netherlands, December 1618, 2009, revised selected papers, 74-83. Berlin/Heidelberg: Springer.

Schwarz, Monika (2000): Indirekte Anaphern in Texten. Studien zur domänengebundenen Referenz und Kohärenz im Deutschen. Tübingen: Niemeyer.

Schwarz-Friesel, Monika \& Manfred Consten (2014): Einführung in die Textlinguistik. Darmstadt: Wissenschaftliche Buchgesellschaft.

Smyth, Ron (1994): Grammatical determinants of ambiguous pronoun resolution. Journal of Psycholinguistic Research 23, 197-229.

Stede, Manfred (2004): The Potsdam Commentary Corpus. In Proceedings of the ACL Workshop on Discourse Annotation, Barcelona, 96-102.

Stede, Manfred (2007): Korpusgestützte Textanalyse. Grundzüge der Ebenen-orientierten Textlinguistik. Tübingen: Narr.

Stede, Manfred (2012): Discourse processing. San Rafael: Morgan \& Claypool.

Storrer, Angelika (2004): Text und Hypertext. Mit einem Exkurs zu XLink und XPointer von Eva Anna Lenz. In Lothar Lemnitzer \& Hennig Lobin (Hrsg.), Texttechnologie, 13-50. Tübingen: Stauffenburg.

Strube, Michael \& Udo Hahn (1999): Functional centering: Grounding referential coherence in information structure. Computational Linguistics 25 (3), 309-344.

Stutterheim, Christiane von (1994): Quaestio und Textaufbau. In Hans-Joachim Kornadt, Joachim Grabowski \& Roland Mangold-Allwinn (Hrsg.), Sprache und Kognition. Perspektiven moderner Sprachpsychologie, 251-272. Heidelberg: Spektrum Akademischer Verlag.

Stutterheim, Christiane von (1997): Einige Prinzipien des Textaufbaus. Empirische Untersuchungen zur Produktion mündlicher Texte. Tübingen: Niemeyer. 
Stutterheim, Christiane von \& Wolfgang Klein (2002): Quaestio und l-perspectivation. In Carl Friedrich Graumann \& Werner Kallmeyer (Hrsg.), Perspectivity and perspectivation in discourse, 59-88. Amsterdam: Benjamins.

Sweetser, Eve (1990): From etymology to pragmatics: Metaphorical and cultural aspects of semantic structure. Cambridge, UK: Cambridge University Press.

Tanenhaus, Michael K., Michael J. Spivey-Knowlton, Kathlee M. Eberhard \& Julie C. Sedivy (1995): Integration of visual and linguistic information in spoken language comprehension. Science 268 (5217), 1632-1634.

Vallduvi Enric \& Elisabeth Engdahl (1996): The linguistic realization of information packaging. Linguistics 34, 459-519.

Vater, Heinz (2001): Einführung in die Textlinguistik. Struktur und Verstehen von Texten. München: Fink.

Viehweger, Dieter (1976): Semantische Merkmale und Textstruktur. In František Daneš \& Dieter Viehweger (Hrsg.), Probleme der Textgrammatik, 195-206. Berlin: Akademie.

Warnke, Ingo (Hrsg.) (2000): Schnittstelle Text: Diskurs. Frankfurt am Main u. a.: Peter Lang.

Webber, Bonnie (1988): Discourse deixis and discourse processing. Technical report MS-CIS-88-75, University of Pennsylvania, Department of Computer and Information Science. http://repository.upenn.edu/cgi/viewcontent.cgi $?$ article $=1642 \&$ context $=$ cis_ reports (letzter Zugriff: 29. 9. 2017).

Webber, Bonnie (2016): Exploring for concurrent discourse relations. In Proceedings of the Fifth Joint Conference on Lexical and Computational Semantics, Berlin 2016, ix.

Weinrich, Harald (1964): Tempus. Besprochene und erzählte Welt. Stuttgart: Kohlhammer.

Wöllstein, Angelika (2008): Konzepte der Satzkonnexion. (= Studien zur deutschen Grammatik 70). Tübingen: Stauffenburg.

Zeevat, Henk (2011): Rhetorical relations. In Claudia Maienborn, Klaus von Heusinger \& Paul Portner (Hrsg.), Semantics: An international handbook of natural language and meaning, 946-970. Berlin: De Gruyter. 


\title{
Daniel Gutzmann und Petra B. Schumacher 16 Schnittstelle Semantik-Pragmatik
}

\begin{abstract}
Ausgehend vom klassischen Grice'schen Ansatz zum Verhältnis von Semantik und Pragmatik werden zunächst Grenzfälle für die SemantikPragmatik-Schnittstelle vorgestellt, die sich durch die Unbestimmtheit gewisser Bedeutungsaspekte auszeichnen und die Vielfältigkeit der Schnittstellenthematik illustrieren. Im Folgenden werden neuere theoretische Ansätze zur Semantik-Pragmatik-Schnittstelle gegenübergestellt und der Beitrag der experimentellen Forschung zum Verhältnis der Bedeutungsebenen gewürdigt.
\end{abstract}

Keywords: Äußerungsbedeutung, Bedeutungsebenen, Linguistische Bedeutung, Sprecherbedeutung, Unbestimmtheit

\section{Einleitung}

Semantik und Pragmatik befassen sich mit Bedeutung, jedoch erweist sich eine klare Aufteilung der Aufgaben dieser beiden Bereiche als äußerst schwierig. Während die Unterteilung in wörtliche Bedeutung und Sprecherbedeutung einen ersten Abgrenzungsversuch illustriert, wird sich zeigen, dass dieser noch $\mathrm{zu}$ grob ist. Insbesondere fällt die Äußerungsbedeutung in das Grenzgebiet zwischen Semantik und Pragmatik, da es viele unterschiedliche Formen der Unbestimmtheit in Äußerungen gibt. So wird die Debatte darüber, welche Prozesse zur Bedeutungskonstitution beitragen und auf welcher Ebene diese zu verorten sind, nicht ohne Grund als Border Wars bezeichnet (Horn 2006). Welche Bedeutungsaspekte sollen der Semantik zugeordnet werden und welche der Pragmatik? Wo hört semantische Bedeutung auf und wo fängt pragmatische Bedeutungskonstitution an? In welcher Ebene und mit welchen Mechanismen wird die Unbestimmtheit aufgelöst?

Anmerkung: Dieser Beitrag stellt eine überarbeitete und erweiterte Fassung von Gutzmann 2010 dar.

Daniel Gutzmann, Institut für deutsche Sprache und Literatur I, Universität zu Köln, Albertus-Magnus-Platz, D-50923 Köln, E-Mail: mail@danielgutzmann.com

Petra B. Schumacher, Institut für deutsche Sprache und Literatur I, Universität zu Köln, Albertus-Magnus-Platz, D-50923 Köln, E-Mail: petra.schumacher@uni-koeln.de

Ә Open Access. () 2018 Daniel Gutzmann und Petra B. Schumacher, publiziert von De Gruyter. (c) BY Dieses Werk ist lizenziert unter der Creative Commons Attribution 4.0 Lizenz. https://doi.org/10.1515/9783110490992-017 
Im Folgenden stellen wir ausgehend vom klassischen Grice'schen Ansatz zum Verhältnis von Semantik und Pragmatik eine Reihe von Grenzfällen für die Semantik-Pragmatik-Schnittstelle vor, die sich durch die Unbestimmtheit gewisser Bedeutungsaspekte auszeichnen und die Vielfältigkeit der Herausforderungen im Grenzgebiet von Semantik und Pragmatik illustrieren sollen. Anschließend versuchen wir die Unterschiede neuerer theoretischer Ansätze zur Semantik-Pragmatik-Schnittstelle, die die Grenze zwischen Semantik und Pragmatik auf sehr unterschiedliche Weise ziehen, herauszuarbeiten. Der Beitrag der experimentellen Forschung zum Verhältnis der Bedeutungsebenen wird in der abschließenden Diskussion gewürdigt.

\section{Das klassische (oder naive) Bild}

Die Unterscheidung zwischen Semantik und Pragmatik hat eine lange Tradition, jedoch wird das erste ausformulierte Modell der Semantik-PragmatikSchnittstelle, das expliziten Bezug auf die beteiligten Prozesse nimmt, die von der Semantik zur pragmatischen Bedeutung führen, gemeinhin Grice zugeschrieben. ${ }^{1}$ Grice unterteilt die effektiv von der Sprecherin kommunizierte Bedeutung (die sogenannte Sprecherbedeutung, speaker meaning) in das Gesagte (what is said) und das Implikatierte (what is implicated) und führt dadurch eine Unterscheidung ein zwischen der wörtlichen Bedeutung einer Äußerung und dem, was eine Sprecherin darüber hinaus kommuniziert. Hieraus ergibt sich die klassische Grice'sche Bedeutungstaxonomie in Abbildung 16.1.

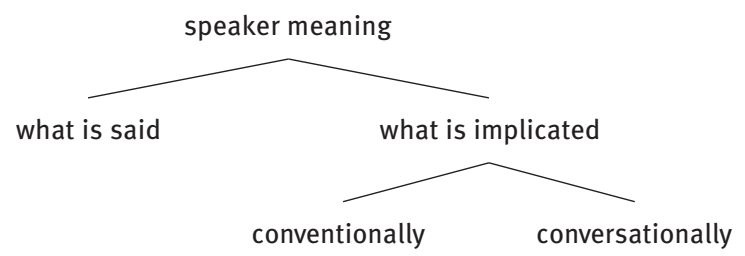

Abb. 16.1: Speaker Meaning nach Grice 1975.

1 Die Unterscheidung lässt sich beispielsweise bereits im semiotischen System von Morris finden: „One may study the relations of signs to the objects to which the signs are applicable. [...] [T]he study of this dimension will be called semantics. Or the subject of study may be the relation of signs to interpreters. [...] [T] he study of this dimension will be named pragmatics“ (Morris 1938: 6). 
Grice selbst äußerte sich nicht zum Verhältnis zwischen Semantik und Pragmatik, aber trotzdem wurde diese Unterteilung in der nachfolgenden Forschung als Vorschlag für eine Abgrenzung zwischen Semantik und Pragmatik interpretiert. Die Ebene des what is said entspräche demnach der Semantik einer Äußerung, während what is implicated den pragmatischen Teil stellen würde. ${ }^{2}$ Eine solche Parallelsetzung von Grices Bedeutungsebenen mit Semantik bzw. Pragmatik stößt natürlich schnell an ihre Grenzen und sie lässt viele pragmatische Aspekte wie z. B. Sprechakte komplett außer Acht. Dennoch ist Grices Modell sehr einflussreich und bildet den Ausgangspunkt für die gesamte Debatte zur Semantik-Pragmatik-Unterscheidung. Vor allem Grices primäres Ziel, die logische Semantik vor allzu gebrauchsorientierten Strömungen zu retten, indem er einer bedeutungsminimalistischen Semantik (vgl. Posner 1980) einen pragmatischen Apparat zur Seite stellt, hat gezeigt, dass sich semantische und pragmatische Bedeutungstheorien nicht zwangsläufig widersprechen, sondern eine Arbeitsteilung zwischen ihnen auch aus theoretischer, sprachphilosophischer Sicht durchaus sinnvoll sein kann.

Eine weitere Unterscheidung, die in der Bedeutungsklassifikation in Abbildung 16.1 nicht vorgenommen wird, aber mehr oder weniger implizit in Grices Arbeiten vorkommt, ist die Unterscheidung zwischen dem Gesagten und der rein sprachlich kodierten, konventionellen Bedeutung der Äußerung. Für Grice 1975: 25 ist what is said eng mit der Bedeutung des linguistischen Materials verbunden:

In the sense in which I am using the word say, I intend what someone has said to be closely related to the conventional meaning of the words (the sentence) he has uttered. (Grice 1975: 44, unsere Hervorhebung)

Grice nimmt also insgesamt drei verschiedene Bedeutungsebenen an:

(1) a. die linguistische Bedeutung

b. das Gesagte (what is said)

c. das Gemeinte (Sprecherbedeutung)

2 Dabei bleibt es jedoch unklar, welcher Status den konventionellen Implikaturen zugesprochen wird, da diese eine Art Doppelleben führen. Zwar haben sie keinen Einfluss auf den wahrheits-konditionalen Inhalt eines Satzes (zumindest nach Grice), doch sind sie fest mit der wörtlichen Bedeutung eines Ausdrucks verbunden. Die Frage ist also, auf welches Kriterium man bei der Unterscheidung zwischen Semantik und Pragmatik mehr Gewicht legt: auf Relevanz für die Wahrheitsbedingungen oder auf Konventionalität. Für diesbezügliche Diskussionen vgl. u. a. Gutzmann (i. E.); Horn (2008); Neale (1992); Potts (2005). 
Für Grice ist der Abstand zwischen der linguistischen Bedeutung und dem Gesagten also relativ gering (vgl. Carston 2002: 21). Allerdings erwähnt Grice selbst zwei pragmatische Prozesse, die stattfinden müssen, bevor what is said spezifiziert werden kann:

Suppose someone to have uttered the sentence He is in the grip of a vice. Given a knowledge of the English language, but no knowledge of the circumstances of the utterance, one would know something about what the speaker had said. [...] But for a full understanding of what the speaker had said, one would need to know the identity of $x$, the time of utterance, and the meaning, on the particular occasion of utterance, of the phrase in the grip of a vice. (Grice 1975: 44, unsere Hervorhebung)

Die Referenz eines indexikalischen Ausdrucks und die konkrete Lesart ambiger Ausdrücke ist nicht in dem linguistischen Material verankert. Um diese Unbestimmtheiten aufzulösen, sind also die Prozesse der Disambiguierung und Referenzbestimmung notwendig. Folgendes Beispiel aus Grices klassischem Text illustriert diese beiden Prozesse.

(2) Ambiguitäten $\rightarrow$ Disambiguierung: He is in the grip of a vice.

a. He is unable to rip himself of a certain kind of bad character trait.

b. Some part of him is caught in a certain kind of tool.

(3) Deixis $\rightarrow$ Referenzbestimmung: He is in the grip of a vice.

a. Peter is in the grip of a vice.

b. John is in the grip of a vice.

c. $\quad \ldots$

Disambiguierung und Referenzbestimmung sind somit zwei pragmatische Prozesse, die oft im Zusammenspiel mit der syntaktischen und semantischen Analyse erforderlich werden, damit das Gesagte bestimmt werden kann. Diese Art der Unbestimmtheit wird in der Literatur meist als unproblematisch erachtet, da es sich hier um offensichtliche Fälle handelt. In der Debatte um die theoretische Modellierung der Semantik-Pragmatik-Schnittstelle spielen diese zwei Arten folglich nur eine untergeordnete Rolle. ${ }^{3}$

3 Allerdings wird ihr gemeinhin akzeptierter Status als ein „präsemantischer“ pragmatischer Prozess oft als Argument dafür herangezogen, dass auch andere pragmatische Prozesse aktiv sind, bevor die Berechnung der semantischen Bedeutung abgeschlossen ist. Vergleiche hierzu vor allem Levinson (2000: §3) Diskussion zum präsemantischen Status von generalisierten konversationellen Implikaturen. 


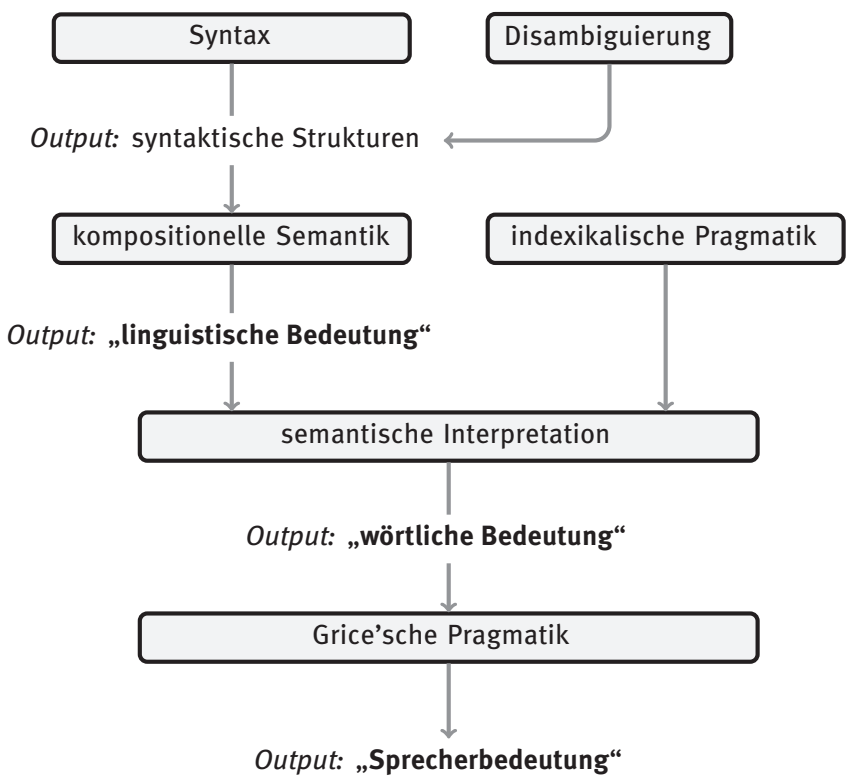

Abb. 16.2: Klassisches Modell der Semantik-Pragmatik-Schnittstelle (nach Levinson 2000: 173).

Zusammengefasst ergibt sich also das in Abbildung 16.2 illustrierte Bild der Schnittstelle zwischen Semantik und Pragmatik, das als das klassische Modell bezeichnet werden kann: Eine wie auch immer geartete Syntax generiert linguistisches Material. Ist dieses Material ambig, muss zunächst disambiguiert werden, wozu auch Pragmatik notwendig ist. Die sprachliche Bedeutung (zum Beispiel in Form von logischen Formen oder semantischen Repräsentationen) wird kompositionell aus dem sprachlichen Material berechnet. Das Gesagte kann jedoch immer noch aufgrund von indexikalischen Ausdrücken unterbestimmt sein, sodass ein weiterer pragmatischer Prozess diese Leerstellen füllen muss. Erst dann kann die wörtliche Bedeutung (what is said) eines Satzes berechnet werden. Diese dient dann als Input für weitergehende pragmatische Prozesse, durch die die tatsächlich kommunizierte Sprecherbedeutung erschlossen wird und die Implikaturen, Ironie, Metaphern, etc. umfassen.

In diesem klassischen Grice'schen Modell gibt es also nur die zwei erwähnten Arten von Unbestimmtheit, die durch Ambiguitäten und Indexikalität eingeführt werden. Für beide gilt, dass sie noch vor der Ebene von what is said aufgelöst werden, sodass eine unterschiedliche Auflösung der Unbestimmtheit auch zu einem anderen Gesagten führt. Die an der Auflösung beteiligten Prozesse sind präsemantische, pragmatische Prozesse, die das jeweilig Gesagte im 
Kontext auswählen. Darüber, welche Mechanismen bei diesen pragmatischen Prozessen eine Rolle spielen, sagt Grice (1975) selbst nichts. Der Großteil der modernen Ansätze geht allerdings davon aus, dass dafür die gleichen pragmatischen Prinzipien verantwortlich sind wie in der postsemantischen, Grice'schen Pragmatik, auch wenn Uneinigkeit darüber besteht, welche diese genau sind (vgl. u. a. Blutner 1998; Carston 2002; Levinson 2000).

\section{Grenz- und Problemfälle}

Im Folgenden gehen wir zunächst auf einige Grenzfälle an der SemantikPragmatik-Schnittstelle ein, die sich durch die Unbestimmtheit des sprachlichen Materials auszeichnen und damit eine Herausforderung für die Frage nach der Ebene der Auflösung der Unbestimmtheit darstellen. Die einzelnen Problemfelder unterscheiden sich dabei hinsichtlich der möglichen sprachlichen Explizierung des impliziten Gehalts, ihrer kontextuellen Erschließbarkeit bzw. lexikalischen Verankerung und ihrem Beitrag zu den Wahrheitsbedingungen.

\subsection{Unartikulierte Konstituenten}

Ein mittlerweile selbst schon klassisch zu nennendes Problemfeld für die simple Arbeitsteilung zwischen Semantik und Pragmatik stellen fehlende oder sogenannte unartikulierte Konstituenten dar (vgl., unter vielen anderen, Carston 2002; Recanati 2002). Dieser Begriff bezieht sich auf Äußerungen, denen obwohl sie syntaktisch vollständig zu sein scheinen - kein vollständiger, propositionaler Gehalt zugewiesen werden kann. Die folgenden Beispiele illustrieren dies.

(4) a. Paracetamol ist besser. Als was?

b. Das ist das gleiche. Wie was?

c. Sie ist bereit. Wofür?

d. Er ist zu jung. Wozu?

e. Es regnet. Wo?

Wichtig ist hierbei, dass die Sätze in (4a)-(4e) syntaktisch vollständig zu sein scheinen, sodass die Unvollständigkeit nicht eindeutig auf syntaktische Ellip- 
sen zurückzuführen ist. ${ }^{4}$ Zumindest sind die Beispiele in (4) alle satzwertig. Und dennoch - so die Argumentation in der Literatur - wird in keinem der Beispiele eine vollständige Proposition ausgedrückt. Um die Sätze jeweils danach zu bewerten, ob sie wahr oder falsch sind, müssen sie um die fehlenden Konstituenten erweitert werden, die eine Antwort auf die kursiv angegebenen Fragen darstellen. ${ }^{5}$ Folgt man nun Grice (1975) in seiner Annahme, dass what is said voll-propositional sein muss, dann stellen die Sätze in (4a)-(4e) folglich Fälle dar, in denen die Bedeutung des sprachlichen Materials selbst nicht nur das Gemeinte, sondern bereits das Gesagte unterspezifiziert.

\subsection{Genitivkonstruktionen}

Ein etwas anderer Fall liegt beim possessiven Gebrauch von Genitivattributen vor. Auch hier bleibt ein Teil der intuitiven Semantik durch das sprachliche Material unterbestimmt: Die Relation, die zwischen der nominalen Bezugskonstituente und dem Genitivattribut vorliegt, wird - ähnlich wie bei den unartikulierten Konstituenten - nicht explizit sprachlich ausgedrückt, sondern muss kontextuell inferiert werden. Wie das folgende Beispiel illustriert, kann diese Relation in Abhängigkeit vom Kontext sehr unterschiedlich ausfallen (Asher 2011; Kolkmann 2016).

(5) Danielas Auto ist schnell.

a. Das Auto, das Daniela besitzt, ist schnell.

b. Das Auto, das Daniela fährt, ist schnell.

c. Das Auto, das Daniela gemietet hat, ist schnell.

d. Das Auto, auf das Daniela gewettet hat, ist schnell.

e. Das Auto, das Daniela entwickelt hat, ist schnell.

f. $\quad .$.

Auch wenn attributive Genitive oft als possesive Genitive bezeichnet werden, kann es sich bei der tatsächlich possessiven Relation in (5a) höchstens um eine DefaultInterpretation handeln. Je nach Kontext sind beliebige andere Relationen wie in (5b)-(5e) möglich, solange diese im Kontext lizenziert sind.

4 Auch wenn in der semantisch-pragmatischen Literatur die Beispiele in (4) alle gleich behandelt werden, sei darauf hingewiesen, dass sie unterschiedlichen syntaktischen Status haben. So fehlen in (4a)-(4c) Komplemente im Sinne der IDS-Grammatik (Zifonun, Hoffmann \& Strecker 1997), während dies für (4d) und (4e) nicht der Fall ist.

5 Für einige der Fälle wird dies allerdings kontrovers diskutiert. Siehe dazu u. a. Cappelen \& Lepore (2005); Recanati (2007). 
Die Genitivrelation hat mit den unartikulierten Konstituenten gemeinsam, dass die Relation kontextuell bestimmt werden muss, um zu einer wahrheitswertfähigen Aussage zu gelangen. Die Relation zwischen dem genitivischen Attribut und dem Bezugsnomen kann entscheidend sein für die Referenz der gesamten NP. ${ }^{6}$ So kann das Auto, das Daniela gehört, ein anderes sein als das Auto, das sie fährt. Um zu bestimmen, auf welches Auto die Sprecherin mit (5) referiert, muss die Hörerin die Genitivrelation erschließen. Erst wenn sie weiß, auf welches Auto referiert wird, kann sie entscheiden, ob das Auto schnell ist oder nicht. In einem Kontext, in dem Daniela einen Porsche besitzt, aber immer nur den benzinsparenden Kleinwagen ihrer Partnerin fährt, kann einer Äußerung wie (5) ein jeweils anderer Wahrheitswert zukommen, je nachdem, welche Genitivrelation die Hörerin erschließt.

Während die attributiven Genitive in dieser Hinsicht den unartikulierten Konstituenten gleichen, gibt es aber einen entscheidenden Unterschied. Die in Abschnitt 3.1 diskutierten Bedeutungsaspekte können ohne weiteres sprachlich realisiert werden; deshalb spricht man ja überhaupt von „unartikulierten“ oder „fehlenden“ Konstituenten: es sind letztlich sprachlich vorgesehene Konstituenten.

(6) a. Paracetamol ist besser als Antibiotikum.

b. Es regnet in Hamburg.

Im Gegensatz dazu kann die Relation zwischen Genitivattribut und Bezugsnomen nicht ohne strukturell zusätzliches Material explizit gemacht werden, da die Genitivkonstruktion keine strukturelle Leerstelle dafür vorsieht. Nur durch parenthetisch eingeschobene Erläuterungen wie in (7a) oder wenn man die Struktur der NP ändert wie in (7b), lässt sich die Genitivrelation sinnvoll explizieren. In (7b) liegt dann allerdings keine attributive Genitivkonstruktion mehr vor, in die die Relation eingefügt wird. Explikation der Genitivrelation bei gleichzeitiger Strukturerhaltung ist entweder ungrammatisch (7c) oder der Genitiv wirkt redundant (7d).

(7) Danielas Auto ist schnell.

a. Danielas Auto - also das, das sie fährt - ist schnell.

b. Das Auto (das Daniela fährt) ist schnell.

c. *Danielas (fährt) Auto ist schnell.

d. ?Danielas Auto, das sie fährt, ist schnell.

6 Für eine Diskussion von Fällen, in denen die Inferenz der Genitivrelation nicht unbedingt notwendig zu sein scheint, vgl. Kolkmann 2016. 
Zusammenfassend lässt sich festhalten, dass attributive Genitive zwar eine semantische „Lücke“ aufweisen, diese jedoch keiner syntaktischen Leerstelle entspricht, wie es bei den unartikulierten Konstituenten der Fall ist.

\subsection{Nominalkomposita}

Einen ähnlichen Fall wie die attributiven Genitive stellen determinative Nominalkomposita dar, bei denen die Relation zwischen dem Kopfnomen und dem spezifizierenden Erstglied ebenfalls sprachlich nicht explizit ausgedrückt wird und im Kontext von der Hörerin realisiert werden muss (Fanselow 1981). Dabei weisen sie eine noch viel größere Bandbreite an leicht zugänglichen Interpretationsmöglichkeiten auf, wie das folgende Beispiel illustriert. ${ }^{7}$

(8) Peter geht in das Holzhaus.

a. Peter geht in das Haus, das aus Holz besteht.

b. Peter geht in das Haus, in dem Holz gelagert ist.

c. Peter geht in das Haus, in dem Holz verkauft wird.

d. Peter geht in das Haus, ...

Wie bei den Genitivkonstruktionen ist es bei Nominalkomposita ebenfalls nicht möglich, die Relation zwischen Kopfnomen und determinierendem Teil explizit $\mathrm{zu}$ realisieren. Es handelt sich also nicht um lediglich unartikulierte Bestandteile, die prinzipiell realisierbar wären, denn dies ist nicht der Fall, wie (9) veranschaulicht.

(9) *Peter geht in das Holzbestehendhaus.

Natürlich könnte man argumentieren, dass man die Leerstelle ja auch außerhalb des Kompositums explizit machen kann. Doch dies ist nur möglich, wenn man die Struktur des Kompositums auflöst, wie in (8) illustriert. Ließe man das Kompositum bestehen, würde die resultierende Form redundant wirken.

(10) Peter geht in das Holzhaus, das aus Holz besteht.

7 Ein weiteres Beispiel, auf das uns Mechthild Habermann stieß: Heringer (1984) gibt 11 Bedeutungen für das Kompositum Fischfrau an. Eine wichtige, noch relativ unerforschte Frage ist, warum Komposita überhaupt zum Einsatz kommen (anstelle syntaktischer Verbindungen). Siehe hierzu beispielsweise Härtl 2016; Peschel 2002. 
Da die Art und Weise, wie das Erstglied den Kopf des Kompositums näher bestimmt, entscheidend für die Referenz des gesamten Kompositums ist, muss diese erschlossen werden, um eine vollständige Proposition $\mathrm{zu}$ erhalten. Abgesehen von der Tatsache, dass es sich bei Nominalkomposita um morphologische Einheiten handelt und nicht um syntaktische Strukturen, verhalten sie sich also analog zu den Genitivkonstruktionen.

\subsection{Adjektive}

Eine weitere Klasse von sprachlich regelmäßig unterbestimmten Ausdrücken stellen Adjektive dar. Dabei können unterschiedliche Klassen ausgemacht werden, die sich in der Hinsicht unterscheiden, in der das Adjektiv im Kontext näher bestimmt werden muss. Die drei wichtigsten Gruppen stellen skalare, teilbezogene und relative Adjektive dar (Terminologie nach Gross 1998; zitiert in Carston 2002: 23).

Skalare Adjektive wie groß in (11) sind insofern semantisch unbestimmt, als es nicht sprachlich kodiert ist, ab welchem Punkt auf der Größenskala ein Objekt als groß definiert wird, da dies immer nur in Bezug auf einen kontextuellen Vergleichsstandard möglich ist (vgl. neben vielen anderen Bierwisch 1984; Kamp 1975; Kennedy \& McNally 2005; von Stechow 1984).

\section{(11) Bob ist groß.}

Die kontextuelle Abhängigkeit vom einem Vergleichsstandard zeigt folgende Überlegung. Vergleicht man den zehnjährigen Bob mit seinen Klassenkameraden, dann kann er als groß gelten, auch wenn er im Vergleich zu allen Schülern der Schule (oder gar Erwachsenen) als klein gelten würde. Doch selbst wenn Bob ein erwachsener Mann von 1,87 m ist und im Vergleich zu den meisten Menschen als groß gelten würde, ist er nicht groß im Vergleich zu den anderen Spielern in seiner Basketballmannschaft. Im Gegensatz zu den Nominalkomposita und Genitivkonstruktionen lässt sich die Bezugsskala bei solchen Adjektiven problemlos elaborieren, wie die Beispiele in (12a)-(12c) zeigen (vgl. Pinkal 1979).

(12) a. Bob ist groß für einen Fünftklässler.

b. Bob ist groß für einen Basketballspieler.

c. Bob ist groß für einen Menschen.

d. ... 
Teilbezogene Adjektive stellen eine zweite Klasse von generell sprachlich nicht voll bestimmten Adjektiven dar. $\mathrm{Zu}$ diesen gehören beispielsweise die Farbadjektive. $^{8}$

\section{(13) Der Stift ist rot.}

Um die durch (13) ausgedrückte Proposition $\mathrm{zu}$ erhalten und die Äußerung nach ihrer Wahrheit zu beurteilen, muss festgelegt werden, auf welchen Teil des Stiftes sich das Adjektiv bezieht. Auch dies lässt sich wieder ergänzen und dadurch explizit machen.

(14) a. Der Stift ist hinsichtlich seiner Oberfläche rot.

b. Der Stift ist hinsichtlich seiner Tinte rot.

c. Der Stift ist hinsichtlich seiner Kappe rot.

d. $\quad$...

Je nach Kontext kann dies ein unterschiedlicher Teil sein und je nach erzielter Interpretation kann eine Äußerung von (13) dann wahr oder falsch sein.

Relative Adjektive wie schwer in (15) haben immer nur Gültigkeit in Bezug auf etwas anderes.

(15) Das Matheproblem ist schwer.

Ein Problem ist nicht schwer an sich, sondern immer schwer für jemanden. Wie in (16a) und (16b) zu sehen, lässt sich auch bei dieser letzten Gruppe die Bezugsklasse sprachlich explizit machen, ohne die adjektivische Grundstruktur aufzubrechen.

(16) a. Das Problem ist schwer für einen Schüler.

b. Das Problem ist schwer für einen Matheleher.

c. Das Problem ist schwer für einen Matheprofessor.

d. $\quad$...

Ein Großteil der relativen Adjektive ist außerdem auch skalar. Ein Beispiel für ein relatives, aber nicht-skalares Prädikat wäre zum Beispiel letzt-. Ein Objekt

8 Die Vagheit von Farbadjektiven ist ein Problem, das unabhängig von ihrer Teilbezogenheit gilt und ebenfalls zu semantischer Unbestimmtheit führen kann, worauf wir im Rahmen dieses Artikels jedoch nicht eingehen können. Zum Problem der Vagheit, siehe u. a. Pinkal 1985 und Williamson 1994. 
ist immer nur das letzte in Bezug auf etwas, während letzt- offensichtlich nicht skalar ist.

(17) Paul ist der letzte.

a. Paul ist der letzte mit der Abgabe seiner Hausarbeit.

b. Paul ist der letzte auf der Namensliste.

c. Paul ist der letzte, der in der Prüfung dran kommt.

Bei allen drei Adjektiven stellt sich die Frage, ob wir es hier tatsächlich mit semantischer Unbestimmtheit zu tun haben oder ob es sich eher um eine pragmatische Modulation einer Grundbedeutung handelt, beispielsweise, dass gro $\beta$ sich per Default auf normal-gewachsene Erwachsene bezieht und die Schülerbzw. Basketballspieler-Lesarten nur pragmatische Anreicherungen sind, um zu einer pragmatisch gesehen wahren Äußerung zu gelangen. Hält man an der Prämisse fest, dass what is said voll propositional ist, stellt sich also die Frage, ob Sätze wie (11), (13) und (15) auch ohne Explikation eine volle Proposition ausdrücken oder nicht. Auf die unterschiedlichen Positionen zu dieser Frage kommen wir in Abschnitt $4 \mathrm{zu}$ sprechen.

\subsection{Offene Zitate}

Sogenannte offene Zitate (Recanati 2001) werden nicht oft im Zusammenhang mit Unbestimmtheitsphänomenen diskutiert. Dabei können sie die Problematik für die Semantik-Pragmatik-Schnittstelle gut illustrieren (Gutzmann 2007). Offene Zitate sind die Formen von Anführung, in denen der angeführte Ausdruck dieselbe Rolle im Satz spielt, die er auch ohne die Anführungszeichen spielen würde. Dies umfasst also alle Formen von Anführungen mit Ausnahme der direkten Zitate und der rein metasprachlichen Anführung. ${ }^{9}$

Peters Brötchen sind „frisch“.

Die Verwendung der Anführungszeichen markiert hier, dass eine pragmatisch angereicherte oder abweichende Interpretation des Ausdrucks frisch vorliegt. Die konkrete Interpretation jedoch bleibt unbestimmt und muss erschlossen werden, sodass (18) je nach Kontext unterschiedlich interpretiert werden kann.

9 Für einen Überblick über die Debatten zur Anführung, vgl. die Bände von Brendel, Meibauer \& Steinbach $(2007,2011)$ oder de Brabanter (2005). 
Die folgenden Beispiele, in denen ein zugegebenermaßen sehr minimaler Kontext für (18) angegeben wird, illustrieren die Kontextabhängigkeit von offener Anführung und geben in Klammern jeweils an, um welche „Art von Anführung“ es sich dabei jeweils handelt. ${ }^{10}$

(19) A: Was hat die Zeitschrift Kaffee \& Frühstück über Peters Brötchen geschrieben?

B: Peters Brötchen sind „frisch“.

$\sim$ Peters Brötchen sind das, was Kaffee \& Frühstück als frisch bezeichnet hat.

(gemischtes Zitat)

(20) A: Peters Brötchen sind steinhart!

B: Peters Brötchen sind „frisch“.

$\sim$ Peters Brötchen sind nicht frisch.

(modalisierendes Zitat)

(21) Auf einer Reklametafel: Peters Brötchen sind „frisch“.

$\sim$ Peters Brötchen sind sehr frisch.

(emphatisches Zitat)

Auch im Falle von Anführung wird debattiert, auf welcher Ebene die Unbestimmtheit aufgelöst werden muss. Dass dies geschehen muss, um zur Sprecherbedeutung zu gelangen, ist natürlich unumstritten. Die Streitfrage ist jedoch, ob es sich bei den verschiedenen Interpretationen um Ambiguitäten bereits auf der Ebene des Gesagten handelt (z. B. Cappelen \& Lepore 2008), ob die Semantik der Anführung das Gesagte nicht hinreichend bestimmt (z. B. Geurts \& Maier 2005; Recanati 2001) oder ob das Gesagte durch die Anführung nicht beeinflusst wird, sondern die Anführungszeichen ihren Effekt erst auf der Ebene der Sprecherbedeutung entfalten (z. B. Gutzmann 2007; Gutzmann \& Stei 2011; Klockow 1980; Stei 2007). Anführung und die offene Variante im Speziellen stellen somit einen guten Testfall für die Diskussion um die Semantik-PragmatikSchnittstelle dar, weshalb es umso verwunderlicher ist, dass diese in den meisten Abhandlungen dazu kaum Erwähnung finden.

10 In Anlehnung an die Arbeiten von Klockow $(1978,1980)$ argumentieren Gutzmann \& Stei (2011a, b), dass es sich bei allen Arten von Anführung um ein und dasselbe Phänomen handelt was die Anführung an sich betrifft und dass die verschiedenen Anführungsarten letztendlich nur Labels für verschiedene Interpretationen und syntaktische Kontexte sind. Für einen ähnlichen Ansatz siehe Meibauer 2007. 


\subsection{Geschmacksprädikate}

Ein weiterer Fall, in dem das sprachliche Material nicht hinreichend zu sein scheint, um das Gesagte vollständig zu bestimmen, sind sogenannte Geschmacksprädikate (predicates of personal taste) wie lecker, lustig und macht Spaß.

(22) a. Tofu ist lecker.

b. Böhmermann ist lustig.

c. Horrorfilme machen Spaß.

Die Intuition ist, dass wir, um Sätze wie in (22) bewerten zu können, wissen müssen, für wen das ausgedrückte Geschmacksurteil gilt. Während Tofu lecker für manche ist, ist er das nicht für andere. Ähnliches gilt für (22b) und (22c). Das heißt, um zu einer vollen Proposition zu kommen, müssen wir wissen, wer der sogenannte kontextuelle judge (Lasersohn 2005) ist. In vielen Fällen ist der judge die Sprecherin. Dass dies nicht immer der Fall sein muss, zeigt folgendes in der Literatur viel diskutierte Beispiel.

(23) Das neue Katzenfutter ist lecker. (Unsere Katze Elsa isst immer ganz viel davon.)

Höchstwahrscheinlich gibt die Sprecherin von (23) kein Urteil über ihren eigenen Geschmack ab, sondern beurteilt das Katzenfutter aus der Sicht ihrer Katze Elsa, was sich durch eine für-Präpositionalphrase explizit machen lässt.

(24) Das Katzenfutter ist lecker für unsere Katze Elsa.

Das spezifische Problem der Verortung der Unbestimmtheit bei Geschmacksprädikaten ist, dass es sich nicht ohne weiteres auf die Füllung des judgeArguments zurückführen lässt, da sich zwei Sprecherinnen mit einem Geschmacksurteil widersprechen können, auch wenn sie intuitiv beide etwas Wahres sagen.

(25) A: Tofu ist lecker.

B: Tofu ist nicht lecker.

Würde man den judge-Bezug explizieren wie in (26), ergibt sich eine andere Intuition: die Sprecherinnen haben zwar einen unterschiedlichen Geschmack in Bezug auf Tofu, sie widersprechen sich jedoch nicht. 
(26) A: Tofu ist lecker für mich.

B: Tofu ist nicht lecker für mich.

Das Problem, dass in (25) beide Sprecherinnen etwas Wahres sagen, sich aber im Gegensatz zu (26) dennoch widersprechen, ist als faultless disagreement in der Literatur bekannt und führt $\mathrm{zu}$ verschiedensten Lösungsvorschlägen (u. a. Lasersohn 2005). Für die Zwecke dieses Aufsatzes ist vor allem die Feststellung wichtig, dass der judge-Bezug von Geschmacksprädikaten nicht von der gleichen Art ist, wie es bei fehlenden Konstituenten der Fall ist, auch wenn er sich explizieren lässt: die explizite Nennung des judges verändert die Bedeutung der Aussage insofern, als nun keine subjektive Aussage vorliegt.

\subsection{Expressiva}

Ein weiteres Phänomen, das ähnlich wie Geschmacksprädikate mit einer Evaluation verbunden ist, sind sogenannte Expressiva, wobei es sich hierbei um Ausdrücke handelt, die eine Sprechereinstellung oder -emotion kommunizieren (Gutzmann 2013). Wichtig dabei ist, dass die Einstellung oder das Gefühl nicht beschrieben wird, sondern direkt zum Ausdruck gebracht wird (deskriptiver vs. expressiver Modus). So kommunizieren sowohl (27a) als auch (27b), dass die Sprecherin erzürnt über den Kater ist, doch nur in (27b) geschieht dies durch expressive Mittel.

(27) a. Ich bin wütend auf den Kater. Er hat heute auf das Sofa gepinkelt.

b. Der verdammte Kater hat auf das Sofa gepinkelt.

In (27a) wird das Gefühl beschrieben, während in (27b) es durch das Adjektiv verdammt expressiv zum Ausdruck gebracht wird, was unter anderem Einfluss auf die Rolle hat, die Information im Diskurs spielt und weiteres. Einer der grundlegenden Unterschiede zwischen deskriptiver und expressiver Bedeutung scheint dabei zu sein, dass sich letztere nicht direkt durch Wahrheitsbedingungen erfassen lässt, sondern viel mehr durch Gebrauchsbedingungen. ${ }^{11}$

11 Neben der semiotischen Unterscheidung zwischen deskriptiver und expressiver Sprachfunktion, die mindestens auf Bühler (1999 [1934]) und explizit auf Jakobson (1960) zurückgeht, ist hier als Klassiker das einflussreiche Manuskript von Kaplan (1999) zu nennen, das zahlreiche weitere Arbeiten beeinflusst hat (siehe u. a. Gutzmann 2015; Kratzer 2004; McCready 2010; Potts 2005 und viele mehr). 
Was Expressiva neben der Tatsache, dass expressive Bedeutungsaspekte nicht zu den Wahrheitsbedingungen eines Satzes beitragen, interessant für die Semantik-Pragmatik-Schnittstelle macht, ist die Beobachtung, dass sich viele Expressiva - ähnlich wie die Geschmacksprädikate - auf eine Person beziehen, die die Evaluation vornimmt (Potts 2007). In vielen Fällen ist dies die Sprecherin, jedoch kann es auch ein anderes salientes Individuum sein, wie das folgende Beispiel aus Kratzer 1999 zeigt.

(28) My father screamed that he would never allow me to marry that bastard Webster.

In (28) bezieht sich das durch den expressiven Ausdruck bastard vermittelte Gefühl nicht auf die Sprecherin von (28), sondern auf den Vater.

Ähnlich wie für die Geschmacksprädikate wurde für einige Expressiva vorgeschlagen, diese Abhängigkeit durch einen judge-Parameter zu modellieren (Potts 2007). Im Unterschied $\mathrm{zu}$ den Geschmacksprädikaten lässt sich dieser bei Expressiva jedoch nicht ohne Weiteres explizit machen.

(29) ??Der für Peter verdammte Kater hat auf das Sofa gepinkelt.

Expressiva weisen also eine ähnliche Unbestimmtheit wie die Geschmacksprädikate auf, die sich jedoch nicht einfach explizit machen lässt. Darüber hinaus unterscheiden sie sich durch ihren nicht wahrheitskonditionalen Charakter.

\subsection{Perspektivierung}

Die judge-Abhängigkeit von Expressiva und Geschmacksprädikaten lässt sich zu dem allgemeineren Phänomen der Perspektivierung zählen. Genauso lässt sich der vollständige propositionale Gehalt von Sätzen mit unartikulierten Konstituenten zum Teil unter Bezugnahme auf das perspektivische Zentrum bestimmen. So zeigt etwa (30), dass der Blickwinkel, aus dem eine Situation betrachtet wird, im Satz nicht explizit artikuliert ist, aber aus dem Kontext abgeleitet wird (vgl. Recanati 2010). Die Aussage ist wahr, wenn sie auf Basis der Perspektive der Rednerin wahr ist.

(30) a. Es regnet (hier/bei mir).

b. Das Buch liegt links neben dem Weinglas (aus meiner Perspektive). 
Der zugrunde liegende Prozess wird an der Semantik-Pragmatik-Schnittstelle ganz unterschiedlich verortet, was in Abschnitt 4 nochmals aufgegriffen wird: Wetterprädikate nehmen zum Beispiel bei Recanati (2010) eine Sonderrolle ein, indem er sich gegen eine generelle Unterspezifikation ausspricht und dafür plädiert, dass sie kein implizites Argument besitzen, sondern es im Kontext zu einer optionalen Bedeutungsspezifikation durch freie Anreicherung kommen kann.

Ein weiteres Beispiel für Perspektivierung ist (31), mit dem Travis (1997) die Kontextabhängigkeit von Wahrheitswerten illustriert. Pias Aussage Die Blätter an meinem Baum sind grün ist wahr im Kontext von (31a) und falsch in (31b). Dieser Kontrast kann auf eine semantische Ambiguität des Adjektivs (z. B. Graduierbarkeit bei Kennedy \& McNally 2009: grün mit seinen Farbstufen vs. grün als Chlorophyll-Lieferant) oder des Nomens (z. B. Vicente 2015: Struktureigenschaften vs. Erscheinungsbild des Blattes) zurückgeführt werden oder basiert auf der Berücksichtigung von individuellen Anforderungen und Erwartungen (Perspektiven) an Blätter (Travis 1997; siehe auch Predelli 2005).

(31) Pia hat einen Japanischen Ahorn, der voll roter Blätter ist. Da Pia glaubt, dass Blätter grün sein sollten, färbt sie die Blätter ihres Japanischen Ahornbaums grün.

a. Als sie sich das Ergebnis anschaut, sagt sie: „Die Blätter an meinem Baum sind grün.“

b. Kurze Zeit später ruft ein befreundeter Botaniker an, der grüne Blätter für eine Chlorophyll-Studie sucht. Pia sagt zu ihm: „Die Blätter an meinem Baum sind grün. Du kannst sie gerne für Deine Studie verwenden.“

Perspektivenabhängige Bedeutungsaspekte können hierbei zum Wahrheitsgehalt einer Aussage beitragen, was wie bei den Geschmacksprädikaten auch explizit gemacht werden kann:

(32) Diese Blätter sind für Pia grün.

Expressiva (siehe Abschnitt 3.7) , Diskurspartikeln (ja, überhaupt) und indexikalische Ausdrücke wie hier oder heute in (33) können ebenfalls einen kontextabhängigen Bedeutungswechsel erfahren, wenn sie zum Beispiel in erlebter Rede auftreten und damit weder auf den Zeitpunkt des Lesens noch des Schreibens verweisen, sondern bei der Perspektiventrägerin verankert sind. Die wahrheitskonditionale Bewertung erfordert dabei den Wechsel von der Welt der Erzählerin zur gedanklichen Welt der Protagonistin (cf. Banfield 1973; Eckardt 2012; Maier 2015; Schlenker 2004). 
(33) Sina schaute in die Ferne. Heute war ihr Jubiläum und in den nächsten Stunden würden hier viele Gratulanten vorbeikommen.

Perspektive beeinflusst somit den Wahrheitsgehalt eines Satzes, was eine Herausforderung für die Zuordnung zur Semantik oder Pragmatik darstellen kann. Der Bezug zu einem perspektivischen Anker schlägt sich in zahlreichen Konstruktionen nieder, für die es bisher noch keine einheitliche Modellierung gibt. Einen vielversprechenden Ansatz hierfür stellt beispielsweise die Verankerung in der Diskursrepräsentationsstruktur dar, die es ermöglicht unterschiedliche Einstellungen und Standpunkte (Meinungen, Wünsche, Intentionen, etc.) zu modellieren (für einen Überblick siehe Maier 2016).

\subsection{Bedeutungsverschiebungen}

Unter den Begriff der Bedeutungsverschiebung zählen wir Fälle wie in (34), die sich typischerweise dadurch auszeichnen, dass ein Ausdruck eine Kernbedeutung hat, z. B. Kafka als Person (a), Löwe als Tier (b), Becher als Behälter (c) oder Omelett als Lebensmittel (d) und die im Kontext intendierte Bedeutung aus den Qualia-Eigenschaften des Konzeptes abgeleitet werden kann. ${ }^{12}$

(34) a. Henriette liest Kafka. - ein Buch von Kafka

b. Jana fotografiert einen steinernen Löwen. - ein Objekt, das die Form eines Löwen hat

c. Simon verspeiste den ganzen Becher. - den Inhalt des Bechers

d. Das Omelett bittet den Kellner um die Rechnung. - die Person, die das Omelett bestellt hat

Ein rein kombinatorischer Ansatz der Bedeutungskonstitution würde hier zu morphosemantischen Konflikten führen, da das Komplement von lesen vom Typ Objekt/Inhalt sein sollte, nicht jedoch vom Typ Person. Ein Merkmalskonflikt stellt jedoch keine notwendige Voraussetzung für Bedeutungsverschiebung dar, wie Henriette hört Beethoven illustriert, bei dem die Bedeutungsverschiebung nicht durch einen Merkmalskonflikt zwischen Prädikat und Komplement initiiert wird.

Durch die Notwendigkeit der kontextuellen Lizensierung sind Bedeutungsverschiebungen den unartikulierten Konstituenten sehr ähnlich, unterscheiden

12 Unter Qualia-Eigenschaften versteht man Informationen über Form, Zweck, Entstehung einer Entität (vgl. Pustejovsky 1995). 
sich jedoch dahingehend, dass sie hinsichtlich möglicher Bedeutungsintentionen lexikalisch-konzeptuell beschränkt sind. Die Sprecherbedeutung wird unter Hinzunahme lexikalisch-konzeptueller Information inferiert - ein Omelett hat den Zweck (telische Qualia-Rolle) verspeist zu werden und so kann auf einen Omelett-Esser geschlossen werden, ein Löwe hat eine bestimmte Form (formale Qualia-Eigenschaft), die zur Statuen-Lesart beiträgt, etc. Im sprachlichen Material bleibt ein Teil der Sprecherbedeutung unterdeterminiert und die intendierte Bedeutung muss kontextuell inferiert werden.

Die Zugänglichkeit der einzelnen Bedeutungen und somit die zugrunde liegende semantische Repräsentation variiert in (34a)-(34d) (für psycholinguistische Evidenz siehe Frisson \& Frazier 2005; Schumacher 2013). Einige Bedeutungsvariationen sind konventionalisiert und als solche im Lexikon verankert. In diesen Fällen ist die Bedeutung zunächst unterspezifiziert und wird kontextuell erschlossen (Kafka als Person oder als Werk). In anderen Fällen tritt eine bestimmte Verschiebung nur in sehr spezialisierten Kontexten auf (Omelett im Restaurantkontext), die eine Verschiebung von der Kern- zur intendierten Bedeutung erforderlich machen. Im Bedeutungswandel wird hier auf unterschiedliche Stufen der Bedeutungsverschiebung hingewiesen (cf. Koch 2001; Traugott \& Dasher 2001 für eine diachrone Modellierung). Eine zusätzliche Bedeutung wird zunächst von einer kleinen Sprechergruppe entwickelt, die das Bedürfnis hat, auf einen bestimmten Referenten so kurz und präzise wie möglich $\mathrm{zu}$ verweisen, und so eine Bedeutung ad hoc konstruiert. Die Beachtung dieses pragmatischen Ökonomieprinzips führt $\mathrm{zu}$ Bedeutungsverschiebungen wie in (34d). In der nächsten Entwicklungsstufe breitet sich diese Bedeutung innerhalb einer größeren Sprechergruppe aus und wird konventionalisiert. Hier entstehen entweder Verschiebungsregeln (z. B. Autor für Werk für (34a) oder Tier für visuelle Repräsentation für (34b)) oder es kommt zu einer Unterspezifikation der lexikalischen Repräsentation (cf. Bierwisch 1983; Copestake \& Briscoe 1995; Jackendoff 1997). In späteren Entwicklungsstufen kann es dann zu einer Lexikalisierung der Bedeutungsverschiebung kommen, die gelegentlich auch zur Verdrängung der Ursprungsbedeutung führt. ${ }^{13}$

13 Ein historisches Beispiel hierfür ist das mittelhochdeutsche berille, das ursprünglich einen Halbedelstein bezeichnete und der um 1300 für die Brillenherstellung geschliffen wurde. In der Gegenwartssprache hat die Produktbedeutung die Edelsteinbedeutung (heute Beryll) verdrängt. 


\section{Komplexere Ansätze zur Semantik-Pragmatik- Schnittstelle}

Der vorangegangene Abschnitt hat klar gemacht, dass es eine Vielzahl von sprachlich sehr unterschiedlichen Phänomenen gibt, die sich nicht leicht in das klassische Bild der Semantik-Pragmatik-Schnittstelle einordnen lassen, da das sprachlich explizit ausgedrückte Material nicht nur die (pragmatische) Sprecherbedeutung unterbestimmt, sondern auch bereits das Gesagte nicht vollständig durch die Äußerung determiniert wird. Die eingangs erwähnten Fälle von Ambiguitäten und Deixis sind also bei weitem nicht die einzigen „Ausnahmen“ zu dem Ideal, dass das sprachlich realisierte Material das „Gesagte“ bestimmt. Dieser Abschnitt widmet sich verschiedenen Typen von Ansätzen zur Semantik-Pragmatik-Schnittstelle, die eine solche semantische Unbestimmtheit nicht als Ausnahme betrachten und in ihr einen festen Platz in dem jeweiligen Modell vorsehen. Wie wir sehen werden, führt dies zu verschiedenen Konzeptionen des Gesagten.

Die erste grobe Unterscheidung der zahlreichen vorgeschlagenen Ansätze besteht darin, in welche „Richtung“ das Problem der semantischen Unbestimmtheit gelöst wird. Das heißt, es gibt Ansätze, die versuchen, das ganze bereits in der Semantik zu lösen, während andere Ansätze die Pragmatik „früher“ einsetzen lassen. Wir können dies einfach als semantische und pragmatische Ansätze bezeichnen (Gutzmann 2010).

\section{(35) Semantische Ansätze}

Wörter haben eine feste, wörtliche Bedeutung, nur wird ihre Bedeutung wesentlich komplexer und reichhaltiger, genauso wie der Prozess der Bedeutungskomposition. Es wird mit Techniken wie Coercion, Unifikation, und Type-Shifting gearbeitet (vgl. u. a. Asher 2011; Del Pinal 2018; Pustejovsky 1995).

\section{(36) Pragmatische Ansätze}

Die konkrete Wortbedeutung eines Ausdrucks wird im Kontext generiert. Dazu gibt es verschiedene Mechanismen und Techniken (Modulation, Freie Anreicherung, Explikaturen, Implizituren ...) (vgl. u. a. Bach 1994a; Carston 2002; Recanati 2004, 2007).

Im folgenden geben wir einen knappen Einblick in die Grundidee aber auch Probleme von semantischen Ansätzen, bevor wir uns etwas detailierter verschiedenen pragmatischen Ansätzen zuwenden. 


\subsection{Semantische Ansätze}

Semantische Ansätze haben das Ziel, die verschiedenen Bedeutungen, die ein Ausdruck annehmen kann, durch die Interaktion mit den anderen Ausdrücken zu berechnen, mit denen er kombiniert wird. Dadurch, dass solche Ansätze reichhaltige Bedeutungsrepräsentationen annehmen, kann gleichzeitig die Flexibilität von Wortbedeutungen erfasst werden und es können notwendige konkrete Beschränkungen für mögliche Bedeutungen formuliert werden (etwas, das oft eine Schwachstelle von pragmatischen Ansätzen ist).

Die in Abschnitt 3.2 besprochene Genitivrelation ist ein gutes Beispiel, um die Grundideen, aber auch die Grenzen der semantischen Ansätze aufzuzeigen. Ein semantischer Ansatz wie der des Generativen Lexikons (Pustejovsky 1995) nimmt komplexe lexikalische Merkmalsstrukturen für Wörter an, die unter anderem sogenannte Qualia-Merkmale umfassen. Diese Merkmale beinhalten Informationen über den Zweck eines Objektes (telische Rolle), dessen Entstehung (agentivische Rolle) usw. Die Genitivrelation wird mit einem solchen QualiaMerkmal assoziiert. ${ }^{14}$

$$
\left[\begin{array}{l}
\text { Auto } \\
\text { QUALIA }=\left[\begin{array}{l}
\text { TELISCH } \\
\cdots \\
\text { AGENTIVISCH }=\text { entwickeln }(x)(y)
\end{array}\right]
\end{array}\right]
$$

Die Grundidee ist, dass sprachliche Ausdrücke oder Strukturen Zugang zu verschiedenen Teilen der semantischen Repräsentation anderer Ausdrücke haben. So kann die Genitivrelation zum Beispiel sowohl auf die telische als auch die agentivische Qualia-Rolle zugreifen.

$$
\begin{aligned}
\text { Danielas Auto } & \sim \text { a. Das Auto, das Daniela fährt } \\
& \sim \text { b. Das Auto, das Daniela entwickelt } \\
& \sim \ldots
\end{aligned}
$$

(agentivisch)

14 Im Generativen Lexikon sind Qualia-Merkmale Eigenschaften oder Ereignisse, die mit einem Ausdruck assoziiert sind und am besten erklären, was der Ausdruck bedeutet (Pustejovsky 1995: 77). Es sei darauf hingewiesen, dass telisch und agentivisch nur entfernt mit den Aktionsarten in Verbindung stehen. So ist das agentivische Merkmal beispielsweise etwas, wodurch das Objekt zustande kommt oder das telische, wofür es gemacht wurde. Bei Kuchen beispielsweise wäre das telische Qualia-Merkmal essen und das agentivische backen. 
Ein Ansatz, der die Unbestimmtheit der Genitivrelation auf semantische Art zu lösen versucht, hat zwei Probleme. Erstens muss festgestellt werden, dass auch eine reichere lexikalische Semantik wie in (37) zusammen mit einer entsprechenden kompositionalen Semantik die Unbestimmtheit nicht vollständig auflöst. Sobald, wie im Falle von Auto, mehr als ein Qualia-Merkmal vorhanden ist, kann die Semantik alleine nicht entscheiden, auf welchen Aspekt sich die Genitivrelation bezieht; also ob eine Lesart wie in (38a) oder (38b) vorliegt, sodass wieder kontextuelle Inferenzen herangezogen werden müssen, um zu bestimmen, ob Danielas Auto nun das Auto, das Daniela fährt, bezeichnet oder das Auto, das sie entwickelt hat. Es ist also wieder Pragmatik nötig.

Während ein semantischer Ansatz einerseits nicht restriktiv genug ist, da er immer noch auf kontextuelle Informationen angewiesen ist, um aus der Vielzahl an möglichen Interpretationen auszuwählen, ist er in anderer Hinsicht $z u$ restriktiv, da er nur Relationen lizenziert, die sich auf Teile der semantischen Repräsentation zurückführen lassen. Das heißt, rein kontextuell lizenzierte Lesarten der Genitivrelation werden durch einen rein semantischen Ansatz ausgeschlossen (vgl. Asher 2011; Blutner 1998). Das folgende Beispiel illustriert eine rein kontextuell lizenzierte Relation.

(39) [Kontext: Daniela leidet unter Spielsucht und wettet auf alles, was man sich vorstellen kann. Heute ist die Formel 1 dran und Daniela hat Glück.] Ihr Auto ist schnell. $\sim$ Das Auto, auf das Daniela wettet, ist schnell.

Ein spezieller Kontext wie dieser ermöglicht es, dass die Genitivrelation, die zwischen Daniela und dem Auto besteht, als wetten auf interpretiert wird, obwohl wetten offensichtlich nicht Bestandteil der Qualia-Struktur von Auto ist. Qualia-Relationen können also höchstens Defaultinterpretationen der Genitivrelation sein, während im Kontext durchaus sehr spezielle Lesarten generiert werden können, die in keiner der semantischen Repräsentationen der beteiligten Ausdrücke enthalten ist.

Ein semantischer Ansatz wie der des Generativen Lexikons ist also sehr gut dazu geeignet, sprachlich bedingte Standardinterpretationen vorherzusagen. Es wird trotzdem eine Form von semantischer Unbestimmtheit bleiben. Pragmatische Inferenz- oder Anreicherungsprozesse sind auch bei einer reichhaltigeren Semantik notwendig, um die Bedeutung einer Äußerung zu bestimmen.

\subsection{Pragmatische Ansätze}

Wie wir in Abschnitt 2 dargestellt haben, gibt es im klassischen Grice'schen Modell der Semantik-Pragmatik-Schnittstelle drei Bedeutungsebenen: 
(40) a. die linguistische Bedeutung: rein sprachlich kodierte Bedeutung

b. das Gesagte (what is said): näher bestimmte Bedeutung

c. das Gemeinte (Sprecherbedeutung): effektiv kommunizierte Bedeutung

Die Erkenntnis, dass die semantische Unbestimmtheit nicht gänzlich in der Semantik aufgelöst werden kann, führt dann (mindestens) zu den folgenden zwei Fragestellungen:

(41) a. Auf welcher Bedeutungsebene kann und soll Unbestimmtheit aufgelöst werden?

b. Welcher Status soll Grices Ebene von what is said zugeschrieben werden?

Die verschiedenen theoretischen Ansätze zur Semantik-Pragmatik-Schnittstelle geben jeweils unterschiedliche Antworten auf diese beiden Fragestellungen, was zu unterschiedlichen Konzeptionen der Interaktion zwischen Semantik und Pragmatik führt.

Im Folgenden geben wir einen Überblick über die verschiedenen Antwortstrategien und die daraus entstehenden Architekturen der Semantik-PragmatikSchnittstelle. Wir wollen hier bereits die wesentlichen Unterschiede der drei Hauptpositionen vorstellen, die wir diskutieren werden: den (semantischen) Minimalismus, den Synkretismus und den Kontextualismus.

Die Unterschiede zwischen den drei Modellen der Semantik-PragmatikSchnittstelle lassen sich wie folgt zusammenfassen. Zunächst unterscheidet sich der Synkretismus von den anderen beiden Richtungen dadurch, dass er im Unterschied zu (40) eine zusätzliche Bedeutungsebene annimmt, da er von zwei verschiedenen Ebenen des Gesagten ausgeht.

\section{(42) Anzahl der Bedeutungsebenen}
a. Minimalismus: 3 Ebenen
b. Synkretismus: 4 Ebenen
c. Kontextualismus: 3 Ebenen

Ein entscheidender Unterschied zwischen den drei Ausrichtungen liegt in der Beantwortung der Frage nach dem Status des Gesagten (41b).

\section{(43) Konzeption des Gesagten}

a. Minimalismus: minimales what is said min $_{\text {man }}$

b. Kontextualismus: pragmatisch angereichertes what is said prag

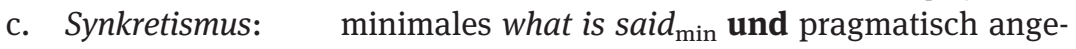
reichertes what is said prag 
Alle drei Ansätze unterscheiden zwischen sogenannten primären und sekundären pragmatischen Prozessen. Konsens besteht darüber, dass Referenzfixierung und Disambiguierung primäre pragmatische Prozesse sind, während beispielsweise die Erschließung konversationeller Implikaturen im Allgemeinen als ein sekundärer pragmatischer Prozess verstanden wird. Unterschiedliche Auffassungen vertreten die drei Ausrichtungen aber bezüglich der Auflösung von semantischen Unbestimmtheitsphänomenen wie den in Abschnitt 3 besprochenen. Im Minimalismus gelten diese als sekundärer Prozess und finden erst auf dem Weg zur Sprecherbedeutung statt, während sie im Kontextualismus bereits auf der Ebene des Gesagten aufgelöst sind. Im Synkretismus findet dies zwischen den beiden unterschiedlichen Ebenen des Gesagten statt.

\section{(44) Auflösung von semantischer Unbestimmtheit}

a. Minimalismus: what is said ${ }_{\text {min }} \rightarrow$ Sprechbedeutung

b. Synkretismus: what is said $d_{\min } \rightarrow$ what is said prag $_{\text {a }}$

c. Kontextualismus: linguistische Bedeutung $\rightarrow$ what is said prag

Die hier nur kurz angerissenen Unterschiede werden wir im Folgenden genauer ausarbeiten. Es sei darauf hingewiesen, dass wir hier eine sehr grobe Perspektive einnehmen und die möglichen Ansätze in die drei genannten Ausrichtungen unterteilen - in Abhängigkeit von ihrer Antwort auf die formulierten Fragen und dabei über die durchaus vorhandenen, feineren Unterschiede zwischen den verschiedenen Ansätzen innerhalb einer Position hinwegsehen.

\subsubsection{Semantischer Minimalismus}

Unter dem Begriff semantischer Minimalismus fassen wir alle Ansätze zusammen, in denen die Diskrepanz zwischen der sprachlich kodierten und kompositionell berechenbaren Bedeutung und der Ebene des Gesagten möglichst minimal gehalten wird. Minimalistische Ansätze unterschiedlicher Ausprägung werden unter anderem von Cappelen \& Lepore (2005) oder Borg (2004) verfochten und ausführlich in Preyer \& Peter (2008) diskutiert.

Damit dies möglich ist, rücken minimalistische Ansätze die Ebenen von what is said und die linguistisch kodierte Bedeutung konzeptuell näher zusammen. Der Minimalismus betont somit die Nähe von what is said zur linguistischen Bedeutung. ${ }^{15}$ Die wörtliche Bedeutung (literal meaning) einer Äußerung

15 Da auch Grice (1975) diese Nähe betont - vgl. das Zitat aus Abschnitt 2 - ließe sich ihm unter Umständen auch eine minimalistische Position zuschreiben. 
sollte im semantischen Minimalismus möglichst frei von pragmatischen Inferenzen sein - abgesehen von Referenzbestimmung und Disambiguierung. Das bedeutet also, dass im Minimalismus das Gesagte und die linguistische Bedeutung eng zusammen gehören und der Sprecherbedeutung gegenüberstehen, wie folgende Skizze illustriert (Recanati 2004: 6).

\section{(45) Semantischer Minimalismus}

wörtliche Bedeutung $\left\{\begin{array}{l}\text { linguistische Bedeutung } \\ \text { what is said }\end{array}\right.$ $v s$.

kommunikative Bedeutung $=$ Sprecherbedeutung Pragmatik

Um diese konzeptuelle Annäherung zwischen what is said und der linguistischen Bedeutung umzusetzen, gibt es zwei unterschiedliche Strategien, die in einer „Absenkung“ von what is said bzw. in einer „Anhebung“ der linguistischen Bedeutung bestehen.

Die erste Strategie, die what is said näher an die linguistische Bedeutung bringen will, ist der semantische Minimalismus im eigentlichen Sinne. Ansätze aus dieser Gruppe lehnen die Annahme ab, dass die in Abschnitt 3 diskutierten Phänomene zu einer Unbestimmtheit auf der Ebene der Semantik führen. Stattdessen wird die Unbestimmtheit hier erst auf der Ebene des Gemeinten bzw. des Sprechakts angesiedelt. So verfechten beispielsweise Cappelen \& Lepore (2005) die Ansicht, dass ein Sprecher des Satzes in (46) sagt (im Sinne von what is said), dass Danielas Auto schnell ist und dass (46) genau dann wahr ist, wenn Danielas Auto schnell ist. Das heißt, die explizierte Genitivrelation findet sich gar nicht auf der Ebene des Gesagten wieder.

Danielas Auto ist schnell.

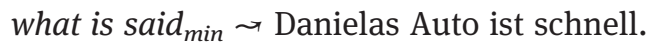

Die semantische Repräsentation des Gesagten wird in einem solchen Ansatz minimal gehalten und die Auflösung der Unbestimmtheit auf die rein pragmatische Ebene verschoben. Diese Verschiebung führt allerdings zu einem oft kritisierten Sprechaktpluralismus (Cappelen \& Lepore 2005), dem zufolge alle möglichen Arten von Sprechakten von der Sprecherin vollzogen werden, die mit allen möglichen Erweiterungen der einfachen Proposition in (46) kompatibel sind. Nach dieser Idee werden mit (46) unter anderem die Behauptungen vollzogen, dass das Auto, das Daniela fährt, schnell für ein normales Auto ist, oder auch die Empfehlung, auf Danielas Auto zu wetten, weil dieses schnell im Vergleich zu anderen Rennwagen ist, oder aber auch die Warnung, nicht 
über die Straße zu gehen, da sich das Auto, das Daniela fährt, mit $50 \mathrm{~km} / \mathrm{h}$ in einer verkehrsberuhigten Zone nähert.

Es ist dann Aufgabe der Hörerin, den Sprechakt herauszufiltern, der im Kontext tatsächlich kommuniziert werden soll. Eine einfache, minimale Semantik zieht also eine reiche und komplexe kognitiv-pragmatische Komponente nach sich.

Eine nicht so radikale minimalistische Variante nimmt keinen Sprechaktpluralismus an, sondern geht davon aus, dass das „minimale Gesagte“ erst auf der Ebene der Sprecherbedeutung angereichert wird. Also auch hier wird die Unbestimmtheit erst in der Pragmatik aufgelöst; allerdings wird trotzdem nur ein Sprechakt tatsächlich vollzogen. Wichtig für die Zwecke unseres Überblicks ist, dass in diesen minimalistischen Varianten das Gesagte oft nicht vollständig propositional ist. Das bedeutet, dass Wahrheitswertfähigkeit, die klassischerweise als ein genuin semantischer Begriff betrachtet wird, letztendlich keine semantische Eigenschaft ist, sondern oft erst in der Pragmatik erreicht wird.

Eine andere Strategie, die Distanz zwischen der linguistischen Bedeutung und dem Gesagten zu minimieren, besteht darin, dass das sprachliche Material selbst als reichhaltiger aufgefasst wird, als es oberflächlich zu sein scheint, beispielsweise indem versteckte indexikalische Variablen im linguistischen Material postuliert werden. Da diese Variablen ihren Wert im Äußerungskontext zugewiesen bekommen, sind sie deshalb für die Kontextabhängigkeit von what is said verantwortlich. In einem solchen Hidden Indexicals Ansatz, wie er unter anderem von Stanley (2007) vertreten wird, wird die Unbestimmtheit also direkt in der Repräsentation der linguistischen Bedeutung verankert. Dadurch wird die Auflösung von semantischen Unbestimmtheiten zu einem einfachen Sättigungsprozess (siehe Abschnitt 4.2.4 weiter unten), wie z. B. die Bestimmung der Referenz von gewöhnlichen indexikalischen Ausdrücken.

Selbst wenn es keine Gründe a priori gegen die Annahme solcher versteckter Variablen gibt - es gibt viele Fälle wie beispielsweise die Zeitvariable des einfachen Präsens, in denen dies sehr plausibel ist - stößt ein solcher Ansatz schnell auf Probleme. Unbestimmtheit, die durch sprachliche Ausdrücke wie die im letzten Abschnitt diskutierten Phänomene ausgelöst wird, grenzt sich ja gerade von der auf Deixis zurückgehenden Unbestimmtheit ab, weil sie nicht durch einfache semantische Regeln aufgelöst werden kann, die sich auf konkrete, grammatisch verankerte kontextuelle Parameter beziehen (wie Sprecherin, Ort und Zeit). Stattdessen können sie zumeist nur durch inferentielle Prozesse und Bezugnahme auf den weiten Kontext bestimmt werden (Recanati 2005: 453). Während die Bedeutung eines indexikalischen Ausdrucks wie ich zwar auch nur im Bezug auf den kontextuellen Parameter der Sprecherin ermittelt werden kann, so wird dies dennoch durch eine semantische Regel ge- 
steuert, nämlich, dass sich ich auf die Sprecherin bezieht. ${ }^{16}$ Auf die Unbestimmtheitsphänomene aus Abschnitt 3 trifft dies nicht zu, da es für sie keine semantische Regel gibt, die allein durch Bezugnahme auf einen kontextuellen Parameter die semantische Repräsentation vervollständigen würde (Recanati 2004: 56). Natürlich ließe es sich technisch bewerkstelligen, einen Parameter für die Relation einzuführen, die zwischen Daniela und ihrem Auto besteht. Dieser würde dann von der unsichtbaren indexikalischen Variable herausgegriffen, die für das possessive Genitivattribut postuliert wird. Auch wenn wir dadurch eine semantische Regel für die Genitivkonstruktion hätten, so wäre klar, dass man „philosophisch schummeln“ würde (Recanati 2005: 453): wir hätten lediglich einer pragmatischen Inferenz die Tarnung einer semantischen Regel gegeben. Eine andere Frage ist darüber hinaus die nach der psychologischen Realität solcher versteckter indexikalischer Variablen. ${ }^{17}$

\subsubsection{Synkretismus}

Eine andere ebenfalls in gewisser Hinsicht als minimalistisch $\mathrm{zu}$ bezeichnende Position gibt ebenfalls die Grice'sche Prämisse auf, dass what is said notwendigerweise voll-propositional ist. Als wichtigster Ansatz sei hier die Theorie von Bach genannt. ${ }^{18}$ In einer Reihe von Aufsätzen (Bach 1994a, b, 2001, 2005) verteidigt Bach eine minimale Auffassung von what is said, die sich recht strikt an dem tatsächlich geäußerten sprachlichen Material orientiert. Diese Ebene ist nicht notwendigerweise propositional, sondern besteht oftmals nur aus einem subpropositionalen propositional radical (Bach 1994a: 127). In dieser Hinsicht gleicht Bachs Ansatz dem Minimalismus. Abweichend von einer rein minimalistischen Position nimmt Bach jedoch eine zweite Ebene des Gesagten an, die

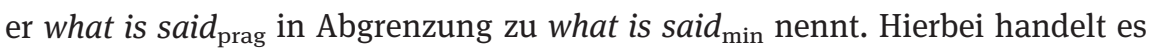
sich um eine pragmatisch bereits angereicherte „höhere“ Ebene des Gesagten, auf der semantische Unbestimmtheiten durch die Prozesse der Kompletion und Expansion aufgelöst werden, um eine voll-propositionale Repräsentation des

16 Die semantische Regel, die die Bedeutung eines rein indexikalischen Ausdrucks liefert, entspricht Kaplans (1989) character, die konkrete Bedeutung, die ein solcher Ausdruck im Kontext erhält (also die konkrete Sprecherin), entspricht seinem content.

17 Für einen vehementen Angriff auf Ansätze, die Indexikalität über die Standardfälle hinaus postulieren, vgl. Cappelen \& Lepore 2005. Für weitere Argumente gegen den Indexikalismus, siehe auch Carston 2002: §2.7 und Recanati 2004: §7.

18 Weitere Vertreter als synkretisch anzusehender Ansätze wären u.a. Salmon (1991) oder Soames (2002), vgl. Recanati 2004: §4. 
Gesagten - Bach nennt dies Implizitur des (minimal) Gesagten (also das, was „implizit gesagt“ wird) - zu erreichen. Diese Strategie, what is said in eine minimale semantische und eine pragmatisch angereicherte Ebene zu zerlegen, lässt sich als Synkretismus bezeichnen (Recanati 2004).

Während dem Minimalismus zufolge (47) bereits eine vollwertige Proposition auf der Ebene des Gesagten ausdrückt (oder - je nach Analyse - semantisch komplett unbestimmt bleiben wird), so schreibt der Synkretismus einer Äußerung von (47) zunächst eine subpropositionale Struktur zu, die erst auf der weiteren Ebene der Implizitur, also what is said prag $_{\mathrm{zu}}$ einer vollwertigen Proposition erweitert wird.

(47) Danielas Auto ist schnell.

a. what is said $\min \sim$ Danielas Auto ist schnell.

b. what is said prag $\sim$ Das Auto, das Daniela gehört, ist schnell für normale Autos.

Durch die Einführung der zusätzlichen bereits pragmatisch angereicherten Ebene der Implizitur zu dem minimalen Gesagten, das sehr nah an der sprachlichen Bedeutung bleibt, betont der synkretische Ansatz sowohl die Rolle des Gesagten für die wörtliche Bedeutung, als auch den Zusammenhang zwischen dem Gesagten und der Sprecherbedeutung.

\section{(48) Synkretismus}

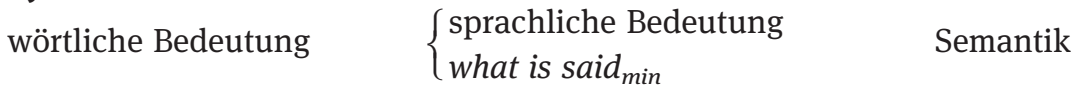
vs.

kommunikative Bedeutung $\left\{\begin{array}{l}\text { what is said } \text { prag } \\ \text { Sprecherbedeutung }\end{array}\right.$

Pragmatik

Durch diese Aufteilung des Gesagten in zwei Ebenen kann der synkretische Ansatz die Unbestimmtheiten weiterhin als semantisch behandeln und deren

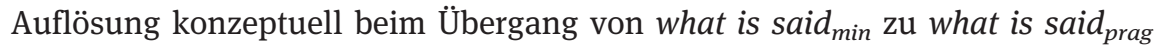
verorten. Somit muss der Synkretismus die Auflösung von Unbestimmtheiten nicht auf die rein pragmatische Ebene verschieben, was beispielsweise den Sprechaktpluralismus von Cappelen \& Lepore (2005) vermeiden kann.

Auch wenn die synkretische Sicht zwei Ebenen des Gesagten (eine minimale und eine pragmatisch angereicherte) und mit der linguistischen Bedeutung und der Sprecherbedeutung insgesamt vier Ebenen annimmt, gehen sowohl Synkretismus als auch Minimalismus von der Existenz einer minimalen Ebene des Gesagten aus, die eng an der rein linguistisch kodierten Bedeutung angelehnt ist. Deshalb werden beide Sichtweisen gelegentlich unter dem Begriff 
Literalismus zusammengefasst, der im Gegensatz zum im Folgenden vorgestellten Kontextualismus steht.

\subsubsection{Kontextualismus}

Grundsätzlich haben die Positionen, die wir hier unter dem Label Kontextualismus zusammenfassen, gemeinsam, dass sie die Annahme einer minimalen Ebene des Gesagten, wie sie von den beiden literalistischen Ansätzen vertreten wird, ablehnen. ${ }^{19}$ Nach Recanati (2004: 90) gibt es aus kontextualistischer Sicht keine Bedeutungsebene, die zugleich (i) propositional ist (d.h. die der Wahrheit nach beurteilt werden kann) und (ii) in dem Sinne minimalistisch ist, dass sie nicht von sogenannten pragmatischen top-down Prozessen beeinflusst wird, die im Gegensatz zu bottom-up Prozessen, wie z. B. Referenzzuweisung, nicht linguistisch ausgelöst sind, sondern vielmehr kognitiv-konzeptuell bedingt sind. Dadurch betont der Kontextualismus den pragmatischen Charakter des Gesagten und rückt diese Ebene näher an die Sprecherbedeutung heran.

\section{(49) Kontextualismus}

sprachliche Bedeutung

Semantik

vs.

kommunikative Bedeutung $\left\{\begin{array}{l}\text { what is said } \text { prag } \\ \text { Sprecherbedeutung }\end{array}\right.$

Pragmatik

In einem kontextualistischen Modell gibt es folglich keine wörtliche Bedeutung eines Satzes mehr, die über das rein sprachlich kodierte „Skelett“ hinausgeht. Nur in einem konkreten Kontext haben Äußerungen eine bestimmte Bedeutung. Während im Minimalismus auf der Ebene des Gesagten also noch keine Auflösung der Unbestimmtheitsphänomene aus Abschnitt 3 stattgefunden hat und diese im synkretischen Modell zwischen what is said min $_{\text {und }}$ what is said prag $_{\text {and }}$ verortet wird, ist das Gesagte im Kontextualismus frei von semantischer Unbestimmtheit. Hier haben die pragmatischen Prozesse bereits stattgefunden. In einem kontextualistischen Ansatz enthält die semantische Repräsentation einer Äußerung folglich alle konkreten Spezifizierungen der unbestimmten Ausdrücke, selbst wenn sie nur durch rein pragmatische kontextuelle Prozesse erschlossen werden können.

19 Als kontextualistische Ansätze lassen sich u. a. die Ansätze von Searle (1978, 1983), Travis (1975, 1981) und Recanati (2004) zählen, ebenso wie die Relevanztheorie (Carston 2002; Sperber \& Wilson 1996; Wilson \& Sperber 2005) und eventuell Levinsons (2000) Modell. 
(50) Danielas Auto ist schnell. what is said prag $\sim$ Danielas Auto ist schnell.

Der Kontextualismus geht somit von einer sehr reichhaltigen, bereits durch kognitive Prozesse angereicherten semantischen Repräsentation des Gesagten aus, wodurch der Unterschied zwischen Semantik und der rein pragmatischen („Grice'schen“) Komponente, in der die Sprecherbedeutung berechnet wird, geringer wird. Wie (49) darstellt bedeutet dies, dass es im Kontextualismus keine wörtliche Bedeutung mehr gibt, die über die rein sprachliche Bedeutung hinausgeht, da das Gesagte bereits der Sprecherbedeutung zugerechnet wird. Es ist überraschend, dass nur im Kontextualismus, der oft als radikal pragmatische Sichtweise kritisiert wird (Cappelen \& Lepore 2005), die Ebene des Gesagten immer voll-propositional und somit wahrheitswertfähig ist.

\subsubsection{Vergleich der drei Positionen}

Die drei skizzierten Modelle zur Semantik-Pragmatik-Schnittstelle unterscheiden sich insbesondere darin, welche Rolle sie dem Gesagten zuschreiben. Dass die linguistische Bedeutung (per Definition) rein sprachlich bedingt ist und dass die Sprecherbedeutung durch eine Vielzahl pragmatischer Effekte bereichert oder modifiziert sein kann, die über die konventionelle Bedeutung des sprachlich Kodierten und des (wie auch immer aufgefassten) Gesagten hinausgeht, darin sind sich die verschiedenen Ansätze weitgehend einig. ${ }^{20}$ Als Streitpunkt bleibt also vor allem die konzeptuelle Verortung des Gesagten. Der Minimalismus betont die Nähe des Gesagten zur linguistisch kodierten Bedeutung, der Kontextualismus die Nähe zur Sprecherbedeutung, während der Synkretismus beide Aspekte berücksichtigen will und zwei distinkte Ebenen des Gesagten annimmt.

Wie aus der vorangegangenen Diskussion klar geworden sein sollte, korreliert ein unterschiedliches theoretisches Verständnis von what is said (i) mit einer unterschiedlichen Auffassung von semantischer Unterbestimmtheit und (ii) mit einer unterschiedlichen Lokalisierung der Auflösungsprozesse.

Unterschiedliche Ansichten vertreten die drei Ausrichtungen aber bezüglich der Auflösung von semantischen Unbestimmtheitsphänomenen wie den in Abschnitt 3 besprochenen. Im semantischen Minimalismus findet dies erst nach der semantischen Repräsentation statt, weshalb die Auflösung seman-

$20 \mathrm{Zu}$ einer kritischen Diskussion der Annahme, dass viele rein pragmatische Prozesse nicht durch Konventionen bestimmt sind, siehe Lepore \& Stone 2014. 
tischer Unbestimmtheit dort als ein sekundärer pragmatischer Prozess verstanden wird.

In Bachs (1994a) synkretischem Modell hingegen werden zwei Arten von primären Prozessen unterschieden: Sättigungsprozesse finden auf der Ebene des minimal Gesagten statt, während weitere Prozesse wie Kompletion und Erweiterung die minimale Repräsentation des Gesagten von dem pragmatisch

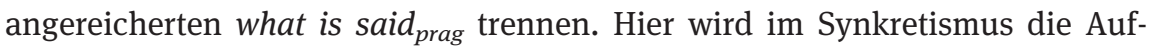
lösung der semantischen Unbestimmtheit verortet: das pragmatische oder, wie Bach es nennt, maximale Gesagte enthält keine semantische Unbestimmtheit mehr und wird nur durch die rein pragmatischen sekundären Prozesse von der Sprecherbedeutung getrennt.

Der Kontextualismus unterscheidet zwar auch zwischen Sättigungsprozessen und anderen primären pragmatischen Prozessen, siedelt jedoch beide auf dem Weg von der linguistischen Bedeutung hin zum Gesagten an und nimmt keine minimale Zwischenebene des Gesagten an. ${ }^{21}$

Die Unterschiede zwischen den drei Modellen sind in Abbildung 16.3 nochmals schematisch dargestellt.

\section{Diskussion und Ausblick}

Wir haben hier gezeigt, dass das klassische Bild zur Abgrenzung von Semantik und Pragmatik nicht ausreicht und durch vielfältige Unbestimmtheitsphänomene an seine Grenzen stößt. Die sprachtheoretische Reaktion hierauf hat zu einer Reihe von Positionierungen geführt, die sich durch sehr feine Unterschiede in der Definition von what is said und der Grenzziehung zwischen einzelnen Bedeutungsebenen auszeichnen. Die unterschiedlichen Modellierungen der Semantik-Pragmatik-Schnittstelle illustrieren die Komplexität der Unbestimmtheit von Bedeutung und fordern uns auf, zwischen ihnen zu wählen. Konzeptuelle Abwägungen stoßen hier an ihre Grenzen. Das Feld der Experimentellen Pragmatik könnte uns hier voranbringen: es verfolgt das Ziel, sich auf Basis empirischer Befunde dem kognitiv plausibelsten Modell anzunähern.

Die oben skizzierten Unterschiede der theoretischen Traditionen lassen sich jedoch auch aus psycholinguistischer Perspektive nur bedingt lösen. Experimentelle Methoden leisten einen wichtigen Beitrag dazu, zugrunde liegende Verarbeitungsprozesse $\mathrm{zu}$ identifizieren und ihren Zeitverlauf zu beschrei-

21 Recanati (2004: § 4.5) argumentiert gegen die psychologische Realität des minimalen Gesagten im Synkretismus. 


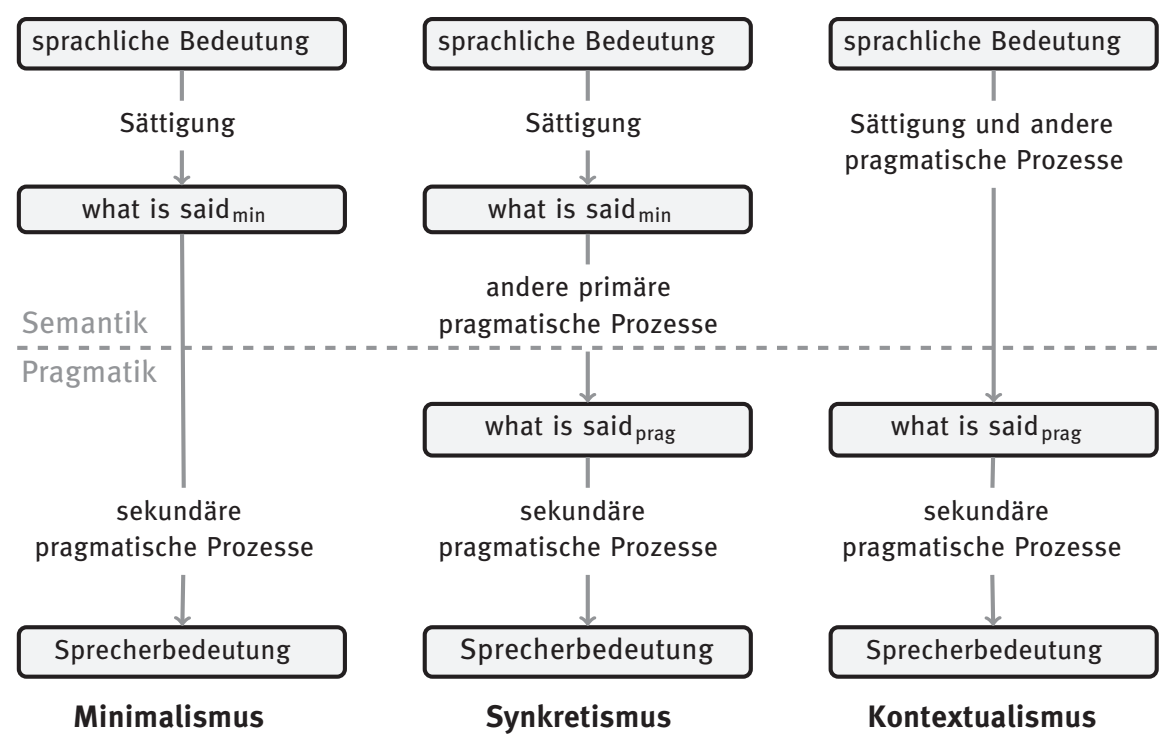

Abb. 16.3: Unterschiedliche Modelle der Semantik-Pragmatik-Schnittstelle im Vergleich (zusammengestellt aus Recanati 2004).

ben oder „Verarbeitungskosten“ für Inferenz- und Anreicherungsprozesse zu messen. Es gibt für einige der oben skizzierten Problemfelder für die SemantikPragmatik-Schnittstelle bereits klare Evidenz dafür, dass ihre Verarbeitung zu Prozessierungsmehraufwand bzw. -unterschieden führt, so etwa zu beobachten bei bestimmten Bedeutungsverschiebungen (Frisson \& Frazier 2005; Schumacher 2013 - jeweils im Vergleich von zwei Bedeutungsintentionen) oder beim Rekurs auf den Vergleichsstandard bei skalaren Adjektiven (z. B. Sedivy, Tanenhaus, Chambers \& Carlson 1999 - gemessen an guten und schlechten Vergleichskontexten). Bedeutungskonstitution verursacht demnach unter bestimmten Bedingungen „Kosten“, allerdings bleibt es eine Herausforderung, diese der Semantik oder Pragmatik zuzuordnen und zum Beispiel die Frage zu klären, ob sich die Verarbeitungskosten auf semantische Unterspezifikation oder pragmatische Prozesse zurückführen lassen. Möglicherweise kommen wir diesem Ziel durch eine systematische Untersuchung von Grenzfällen wie in Abschnitt 3 skizziert näher.

Was die experimentelle Forschung leisten kann, ist, Kontexteffekte zu isolieren. Kontexteffekte treten sehr früh auf und beeinflussen prädiktive Verarbeitungsschritte (vgl. z. B. Schumacher 2012; Sedivy, Tanenhaus, Chambers \& Carlson 1999). Würde man also die Annahme akzeptieren, dass Kontext ein entscheidender Faktor zur Differenzierung von Semantik und Pragmatik ist, so 
könnte man hier mit Hilfe experimenteller Verfahren den Grenzverlauf aufzeigen. Betrachtet man Konventionalität als ein Grenzziehungskriterium, könnten auch hier Verarbeitungsunterschiede zwischen unterspezifizierten konventionalisierten Bedeutungen und nicht konventionalisierten Bedeutungen herausgearbeitet werden (siehe Abschnitt 3.9).

Idealerweise würde man jedoch gerne auf ein Korrelat für Semantik vs. Pragmatik verweisen, um eine klare Abgrenzung vorzunehmen und zwischen den einzelnen theoretischen Positionen wählen zu können. Dies erweist sich als schwierig, da Bedeutungskonstitution auf sich überlagernde Prozesse zugreift und zum Beispiel kontextuelle Effekte aus Satz- und Äußerungskontext herrühren. Potentielle Hinweise zur Abgrenzung von Semantik und Pragmatik finden sich allerdings in der Spracherwerbs- und Sprachstörungsforschung. Von besonderem Interesse ist hierbei Theory-of-Mind-Fähigkeit, die dazu beiträgt, Intentionen, Gefühle, Perspektivierungen von sich und von anderen zu identifizieren und die somit für pragmatische Verarbeitungsprozesse herangezogen wird. Kinder erwerben diese Fähigkeit im Alter von ca. 4 Jahren. Selektive Defizite hinsichtlich der Theory-of-Mind-Fähigkeiten werden bei Sprechern im autistischen Spektrum beobachtet (Baron-Cohen, Leslie \& Frith 1985). Neben einer Beeinträchtigung von Theory-of-Mind Fähigkeiten werden Patienten mit einer erworbenen rechtshemisphärischen Läsion eingeschränkte Inferenzleistungen attestiert (Tompkins 1995). Allerdings gibt es mit diesen Populationen noch kaum Untersuchungen zu den oben genannten Problemfeldern (mit Ausnahme von Bedeutungsverschiebungen, vgl. MacKay \& Shaw 2004; Rundblad \& Annaz 2010).

An anderer Stelle gibt es ebenfalls Evidenz für eine Differenzierung der zugrunde liegenden Prozesse. Für skalare Implikaturen, die typischerweise der Pragmatik zugeordnet werden, gibt es zahlreiche experimentelle Studien, die belegen, dass es für Beispiele wie in (51a), die basierend auf Weltwissen als unterinformativ gelten, zwei Gruppen von Urteilen gibt, wenn Versuchsteilnehmer den Wahrheitsgehalt von (51a) bestimmen sollen. Die einen kommen zur „logisch-semantischen“ Lesart (51b) und akzeptieren (51a), während die anderen die Implikatur in (51c) ziehen und (51a) ablehnen (Noveck 2001; Noveck \& Posada 2003).

(51) a. Einige Elefanten haben Rüssel.

b. Einige, vielleicht auch alle Elefanten haben Rüssel.

c. Einige, aber nicht alle Elefanten haben Rüssel.

Der Zeitverlauf des Verstehens skalarer Implikaturen kann darüber hinaus Aufschluss geben über die (zeitliche) Trennung von wahrheitskonditionaler 
Verarbeitung und pragmatischer Inferenz. Zur Unterstützung der Differenzierung von Semantik und Pragmatik berichten Noveck \& Posada (2003), dass die pragmatische Interpretation von (51c) längere Reaktionszeiten evoziert als die logisch-semantische Interpretation (51b). Weitere Studien zur Verarbeitung skalarer Implikaturen bestätigen, dass die zusätzliche Inferenzziehung zu Verzögerungen in der Sprachverarbeitung führt (s. z. B. Huang \& Snedeker 2011). In Abhängigkeit von kontextuellen Faktoren und Sprecher-HörerInteraktion sind jedoch auch frühe Inferenzprozesse $\mathrm{zu}$ verzeichnen (z. B. Breheny, Ferguson \& Katsos 2013; Degen \& Tanenhaus 2016).

Insgesamt kann ein empirisches Vorgehen Unterschiede in den zugrunde liegenden Prozessen herausarbeiten und zur Fundierung und Verfeinerung unserer Modelle führen, die wiederum in der Lage sein sollten, diese Prozesse zu modellieren. Eine systematische Überprüfung der Vorhersagen der einzelnen theoretischen Positionen für Unbestimmheitsphänomene könnte so ein erster Schritt zu einem besseren Verständnis der Semantik-Pragmatik-Schnittstelle sein.

\section{Literatur}

Asher, Nicholas (2011): Lexical meaning in context. A web of words. Cambridge: Cambridge University Press.

Bach, Kent (1994a): Conversational impliciture. Mind \& Language 9 (2), 124-162. doi: 10.1111/j.1468-0017.1994.tb00220.x.

Bach, Kent (1994b): Semantic slack. What is said and more. In Savas L. Tsohatzidis (Hrsg.), Foundations of speech act theory, 267-91. London: Routledge.

Bach, Kent (2001): You don’t say? Synthese 128 (1-1), 15-44. doi: 10.1023/A:1010353722852.

Bach, Kent (2005): Context ex machina. In Zoltán Gendler Szabó (Hrsg.), Semantics vs. pragmatics, 15-44. Oxford: Oxford University Press. doi: 10.1093/acprof:oso/ 9780199251520.003.0002

Banfield, Ann (1973): Narrative style and the grammar of direct and indirect speech. Foundations of Language 10 (1), 1-39. http://www.jstor.org/stable/25000702 (letzter Zugriff: 14. 5. 2018).

Baron-Cohen, Simon, Alan M. Leslie \& Uta Frith (1985): Does the autistic child have a „theory of mind"? Cognition 21 (1), 37-46. doi: 10.1016/0010-0277(85)90022-8

Bierwisch, Manfred (1983): Semantische und konzeptuelle Repräsentation lexikalischer Einheiten. In Rudolf Růžička \& Wolfgang Motsch (Hrsg.), Untersuchungen zur Semantik, 61-99. Berlin: Akademie.

Bierwisch, Manfred (1984): Dimensionsadjektive: Semantische Struktur und begriffliche Interpretation. Sprachtypologie und Universalienforschung 37 (4), 490-512. doi: 10.1524/stuf.1984.37.16.664

Blutner, Reinhard (1998): Lexical pragmatics. Journal of Semantics 15 (2), 115-162. doi: 10.1093/jos/15.2.115 
Borg, Emma (2004): Minimal semantics. Oxford: Oxford University Press. doi: 10.1093/ 0199270252.001.0001

Breheny, Richard, Heather J. Ferguson \& Napoleon Katsos (2013): Investigating the timecourse of accessing conversational implicatures during incremental sentence interpretation. Language and Cognitive Processes 28 (4), 443-467. doi: 10.1080/ 01690965.2011.649040

Brendel, Elke, Jörg Meibauer \& Markus Steinbach (Hrsg.) (2007): Zitat und Bedeutung. (Linguistische Berichte Sonderheft 15). Hamburg: Buske.

Bühler, Karl (1999 [1934]): Sprachtheorie: Die Darstellungsfunktion der Sprache. Stuttgart: Fischer.

Cappelen, Herman \& Ernie Lepore (2005): Insensitive semantics. A defense of semantic minimalism and speech act pluralism. Oxford: Blackwell. doi: 10.1002/9780470755792

Cappelen, Herman \& Ernie Lepore (2008): Language turned on itself. The semantics and pragmatics of metalingustic discourse. Oxford: Oxford University Press. doi: 10.1093/ acprof:0so/9780199231195.001.0001

Carston, Robyn (2002): Thoughts and utterances. The pragmatics of explicit communication. Oxford: Blackwell. doi: 10.1002/9780470754603

Copestake, Ann \& Ted Briscoe (1995): Semi-productive polysemy and sense extension. Journal of Semantics 12, 15-67. doi: 10.1093/jos/12.1.15

De Brabanter, Philippe (Hrsg.) (2005): Hybrid quotation. (Belgian Journal of Linguistics 17). Amsterdam: Benjamins.

Degen, Judith \& Michael K. Tanenhaus (2016): Availability of alternatives and the processing of scalar implicatures: A visual world eye-tracking study. Cognitive Science 40 (1), 172-201. doi: 10.1111/cogs.12227

Del Pinal, Guillermo (2018): Meaning, modulation, and context. A multidimensional semantics for truth-conditional pragmatics. Linguistics and Philosophy 41 (2), 165-207. doi: 10.1007/s10988-017-9221-z

Eckardt, Regine (2012): Particles as speaker indexicals in free indirect discourse. Sprache und Datenverarbeitung 36 (1), 1-21.

Fanselow, Gisbert (1981): Neues von der Kompositafront oder Zu den drei Paradigmata in der Kompositagrammatik. Studium Linguistik 11, 43-57.

Frisson, Steven \& Lyn Frazier (2005): Carving up word meaning: Portioning and grinding. Journal of Memory and Language 53 (2), 277-291. doi: 10.1016/j.jml.2005.03.004

Geurts, Bart \& Emar Maier (2005): Quotation in context. In Philippe de Brabanter (Hrsg.), Hybrid quotation. (Belgian Journal of Linguistics 17), 109-128. Amsterdam: Benjamins. doi: 10.1075/bjl.17.07geu.

Grice, H. Paul (1975): Logic and conversation. In Peter Cole \& Jerry L. Morgan (Hrsg.), Syntax and semantics 3. Speech acts, 41-58. New York: Academic Press.

Gross, S. (1998): Essays on linguistic context-sensitivity and its philosophical significance. Diss. Cambridge, MA: Harvard.

Gutzmann, Daniel (2007): Zitate und die Semantik/Pragmatik-Schnittstelle. In Elke Brendel, Jörg Meibauer \& Markus Steinbach (Hrsg.), Zitat und Bedeutung. (Linguistische Berichte Sonderheft 15), 111-133. Hamburg: Buske.

Gutzmann, Daniel (2010): Unbestimmtheit und die Semantik/Pragmatik-Schnittstelle. In Inge Pohl (Hrsg.), Semantische Unbestimmtheit im Lexikon, 19-44. Frankfurt: Lang.

Gutzmann, Daniel (2013): Expressives and beyond. An introduction to varieties of use-conditional meaning. In Daniel Gutzmann \& Hans-Martin Gärtner (Hrsg.), Beyond expressives. Explorations in use-conditional meaning. (Current Research in 
the Semantics Pragmatics-Interface (CRiSPI) 28), 1-58. Leiden: Brill. doi: 10.1163/ 9789004183988_002. http://www.danielgutzmann.com/work/expressives-and-beyond/ (letzter Zugriff: 18.12. 2017).

Gutzmann, Daniel (2015): Use-conditional meaning. Studies in multidimensional semantics. (Oxford Studies in Semantics and Pragmatics 6). Oxford: Oxford University Press.

Gutzmann, Daniel (i. E.): Semantics vs. pragmatics. In Daniel Gutzmann, Lisa Matthewson, Cécile Meier, Hotze Rullmann \& Thomas Ede Zimmermann (Hrsg.), The companion to semantics, Oxford: Wiley.

Gutzmann, Daniel \& Erik Stei (2011): How quotation marks what people do with words. Journal of Pragmatics 43 (10), 2650-2663. doi: 10.1016/j.pragma.2011.03.010.

Härtl, Holden (2016): Normality at the boundary between word-formation and syntax. In Franz d'Avis \& Horst Lohnstein (Hrsg.), Normalität in der Sprache. (Linguistische Berichte Sonderheft 22), 71-98. Hamburg: Buske.

Heringer, Hans J. (1984): Gebt endlich die Wortbildung frei! Sprache und Literatur in Wissenschaft und Unterricht 15, 43-53.

Horn, Laurence R. (2006): The border wars: A neo-Gricean perspective. In Klaus von Heusinger \& Ken Turner (Hrsg.), Where semantics meets pragmatics, 21-48. Amsterdam: Elsevier.

Horn, Laurence R. (2008): On F-implicature. Myth-analysis and rehabilitation. Manuskript. New Haven, CT. http://www.eecs.umich.edu/ rthomaso/lpw08/ Horn_LPW.pdf (letzter Zugriff: 18. 12. 2017).

Huang, Yi Ting \& Jesse Snedeker (2011): Logic and conversation revisited: Evidence for a division between semantic and pragmatic content in real-time language comprehension. Language and Cognitive Processes 26 (8), 1161-1172. doi: 10.1080/ 01690965.2010 .508641

Jackendoff, Ray (1997): The architecture of the language faculty. Cambridge, MA: MIT Press. Jakobson, Roman (1960): Linguistik und Poetik. Ausgewählte Aufsätze 1921-1971. In Roman Jakobson (Hrsg.), Poetik, 83-121. Frankfurt: Suhrkamp.

Kamp, Hans (1975): Two theories about adjectives. In Edward Keenan (Hrsg.), Formal semantics of natural language, 123-155. Cambridge: Cambridge University Press.

Kaplan, David (1989): Demonstratives. In Howard Almog, Howard Wettstein \& John Perry (Hrsg.), Themes from Kaplan, 481-563. Oxford: Oxford University Press.

Kaplan, David (1999): The meaning of ouch and oops. Explorations in the theory of meaning as use. 2004 version. Manuskript. Los Angeles.

Kennedy, Christopher \& Louise McNally (2005): Scale structure, degree modification, and the semantics of gradable predicates. Language 81 (2), 345-381. doi: 10.1353/ lan.2005.0071.

Kennedy, Christopher \& Louise McNally (2009): Color, context, and compositionality. Synthese 174 (1), 79-98. doi: 10.1007/s11229-009-9685-7.

Klockow, Reinhard (1978): Anführungszeichen, Norm und Abweichung. Linguistische Berichte 57, 14-24.

Klockow, Reinhard (1980): Linguistik der Gänsefüßchen. Frankfurt: Haag und Herchen.

Koch, Peter (2001): Metonymy: Unity in diversity. Journal of Historical Pragmatics 2, 201244. doi: $10.1075 /$ jhp.2.2.03koc

Kolkmann, Julia (2016): The pragmatics of possession: Issues in the interpretation of prenominal possessives in English. Diss. The University of Manchester. https:// www.research.manchester.ac.uk/portal/files/54586947/FULL_TEXT.PDF (letzter Zugriff: 14. 5. 2018). 
Kratzer, Angelika (1999): Beyond ouch and oops. How descriptive and expressive meaning interact. Talk given at the Cornell Conference on Theories of Context Dependency. Ithaca, NY. 26. März 1999. http://semanticsarchive.net/Archive/WEwNGUyO/ (letzter Zugriff: 18. 12. 2017).

Kratzer, Angelika (2004): Interpreting focus: Presupposed or expressive meanings? A comment on Geurt and van der Sandt. Theoretical Linguistics 30 (1), 123-136. doi: 10.1515/thli.2004.002.

Lasersohn, Peter (2005): Context dependence, disagreement, and predicates of personal taste. Linguistics and Philosophy 28 (6), 643-686. doi: 10.1007/s10988-0050596-x.

Lepore, Ernie \& Matthew Stone (2014): Imagination and convention. Distinguishing grammar and inference in language. Oxford: Oxford University Press. doi: 10.1093/acprof:oso/ 9780198717188.001 .0001

Levinson, Stephen C. (2000): Presumptive meanings. The theory of generalized conversational implicature. Cambridge, MA: MIT Press.

Mackay, Gilbert \& Adrienne Shaw (2004): A comparative study of figurative language in children with autistic spectrum disorders. Child Language Teaching and Therapy 20, 13-32. doi: 10.1191/0265659004ct2610a

Maier, Emar (2015): Quotation and unquotation in free indirect discourse. Mind \& Language 30 (3), 345-373. doi: 10.1111/mila.12083

Maier, Emar (2016): Attitudes and mental files in discourse representation theory. Review of Philosophy and Psychology 7 (2), 473-490. doi: 10.1007/s13164-015-0296-6

McCready, Eric (2010): Varieties of conventional implicature. Semantics \& Pragmatics 3 (8), 1-57. doi: 10.3765/sp.3.8.

Meibauer, Jörg (2007): Linguistik und Psychologie. Experimentelle Pragmatik. In Katja Kessel \& Sandra Reimann (Hrsg.), Wissenschaften im Kontext. Kooperationsfelder der Deutschen Sprachwissenschaft, 361-374. Tübingen: Narr.

Morris, Charles (1938): Foundations of the theory of science. Bd. 1.2: Foundation of a theory of signs. Chicago: University of Chicago Press, 1-59.

Neale, Stephen (1992): Paul Grice and the philosophy of language. Linguistics and Philosophy 15 (5), 509-59. doi: 10.1007/BF00630629.

Noveck, Ira A. (2001): When children are more logical than adults: Experimental investigations of scalar implicature. Cognition 78 (2), 165-188. doi: 10.1016/S00100277(00)00114-1

Noveck, Ira A. \& Andres Posada (2003): Characterizing the time course of an implicature: An evoked potentials study. Brain and Language 85 (2), 203-210. doi: 10.1016/S0093934X(03)00053-1

Peschel, Corinna (2002): Zum Zusammenhang von Wortneubildung und Textkonstitution. Tübingen: Niemeyer.

Pinkal, Manfred (1979): Zur semantischen Analyse von Adjektiven. In Joachim Ballweg \& Hans Glinz (Hrsg.), Grammatik und Logik. Jahrbuch 1979 des Instituts für deutsche Sprache, 231-259. Düsseldorf: Schwann.

Pinkal, Manfred (1985): Logik und Lexikon. Die Semantik des Unbestimmten. Berlin: De Gruyter.

Posner, Roland (1980): Semantics and pragmatics of sentence connectives in natural language. In John R. Searle, Ferenc Kiefer \& Manfred Bierwisch (Hrsg.), Speech act theory and pragmatics, 169-203. Dordrecht: Kluwer. doi: 10.1007/978-94-009-8964-1_8

Potts, Christopher (2005): The logic of conventional implicature. (Oxford Studies in Theoretical Linguistics 7). Oxford: Oxford University Press. 
Potts, Christopher (2007): The expressive dimension. Theoretical Linguistics 33 (2), 165-197. doi: 10.1515/TL.2007.011.

Predelli, Stefano (2005): Contexts. Oxford University Press. doi: 10.1093/0199281734. 001.0001.

Preyer, Gerhard \& Georg Peter (Hrsg.) (2008): Context-sensitivity and semantic minimalism. New essays on semantics and pragmatics. Oxford: Oxford University Press. doi: 10.1093/acprof:oso/9780199273829.001.000

Pustejovsky, James (1995): The generative lexicon. Cambridge, MA: MIT Press.

Recanati, François (2001): Open quotation. Mind 110, 637-687. doi: 10.1093/mind/ 110.439.637.

Recanati, François (2002): Unarticulated constituents. Linguistics and Philosophy 25, 299-345. doi: 10.1023/A:1015267930510.

Recanati, François (2004): Literal meaning. Cambridge: Cambridge University Press. doi: 10.1093/acprof:oso/9780199273829.001.000 10.1017/CB09780511615382

Recanati, François (2005): Pragmatics and semantics. In Laurence R. Horn \& Gregory Ward (Hrsg.), The handbook of pragmatics, 442-462. Oxford: Oxford University Press. doi: 10.1002/9780470756959.ch20

Recanati, François (2007): It is raining (somewhere). Linguistics and Philosophy 30 (1), 123-146. doi: 10.1007/s10988-006-9007-1.

Recanati, François (2010): Truth-conditional pragmatics. Oxford: Oxford University Press. doi: 10.1093/acprof:oso/9780199226993.001.0001

Rundblad, Gabriella \& Dagmara Annaz (2010): Development of metaphor and metonymy comprehension: Receptive vocabulary and conceptual knowledge. British Journal of Developmental Psychology 28 (3), 547-563. doi: 10.1348/026151009X454373

Salmon, Nathan (1991): The pragmatic fallacy. Philosophical Studies 63 (1), 83-97. doi: $10.1007 /$ BF00375998.

Schlenker, Phillippe (2004): Context of thought and context of utterance: a note on free indirect discourse and the historical present. Mind \& Language 19 (3), 279-304.

Schumacher, Petra B. (2012): Context in neurolinguistics: Time-course data from electrophysiology. In Rita Finkbeiner, Jörg Meibauer \& Petra B. Schumacher (Hrsg.), What is a context? Theoretical and empirical perspectives, 33-53. Amsterdam/ Philadelphia: Benjamins.

Schumacher, Petra B. (2013): When combinatorial processing results in reconceptualization: towards a new approach to compositionality. Frontiers in Psychology 4, 677. doi: 10.3389/fpsyg.2013.00677

Searle, John R. (1978): Literal meaning. Erkenntnis 13, 207-224. doi: 10.1007/BF00160894

Searle, John R. (1983): The background of meaning. In John R. Searle, Ferenc Kiefer \& Manfred Bierwisch (Hrsg.), Speech act theory and pragmatics, 221-231. Dordrecht: Reidel. doi: 10.1007/978-94-009-8964-1_10

Sedivy, Julie C., Michael K. Tanenhaus, Craig G. Chambers \& Gregory N. Carlson (1999): Achieving incremental semantic interpretation through contextual representation. Cognition 71 (2), 109-147. doi: 10.1016/S0010-0277(99)00025-6

Soames, Scott (2002): Beyond Rigidity. The unfinished agenda of ,Naming and Necessity“. Oxford: Oxford University Press. doi: 10.1093/0195145283.001.0001

Sperber, Dan \& Deirdre Wilson (1996): Relevance. Communication and cognition. 2. Aufl. Oxford: Blackwell.

Stanley, Jason (2007): Language in context. Selected essays. Oxford: Oxford University Press. 
von Stechow, Arnim (1984): Comparing semantic theories of comparison. Journal of Semantics 3 (1-2), 1-77. doi: 10.1093/jos/3.1-2.1.

Stei, Erik (2007): Über Gebrauch und „Bedeutung“ von Anführungszeichen. In Elke Brendel, Jörg Meibauer \& Markus Steinbach (Hrsg.), Zitat und Bedeutung. (Linguistische Berichte Sonderheft 15), 215-235. Hamburg: Buske.

Tompkins, Connie A. (1995): Right hemisphere communication disorders: Theory and management. San Diego/London: Singular Publishing Group.

Traugott, Elizabeth Closs \& Richard B. Dasher (2001): Regularity in semantic change. (Cambridge Studies in Linguistics 97). Cambridge: Cambridge University Press. doi: 10.1017/CB09780511486500

Travis, Charles (1975): Saying and understanding. Oxford: Blackwell.

Travis, Charles (1981): The true and the false. The domain of the pragmatic. Amsterdam: Benjamins.

Travis, Charles (1997): Pragmatics. In Bob Hale \& Crispin Wright (Hrsg.), A companion to the philosophy of language, 87-107. Oxford: Blackwell.

Vicente, Agustin (2015): The green leaves and the expert: Polysemy and truth-conditional variability. Lingua 157, 54-65. doi: 10.1016/j.lingua.2014.04.013

Williamson, Timothy (1994): Vagueness. London: Routledge.

Wilson, Deirdre \& Dan Sperber (2005): Relevance theory. In Laurence R. Horn \& Gregory Ward (Hrsg.), The handbook of pragmatics, 607-632. Oxford: Blackwell. doi: 10.1002/ 9780470756959.ch27

Zifonun, Gisela, Ludger Hoffmann \& Bruno Strecker (1997): Grammatik der deutschen Sprache. Berlin: De Gruyter. 



\title{
Artemis Alexiadou und Gereon Müller 17 Externe Argumente und quantifikationale Variabilität im deutschen Passiv
}

\begin{abstract}
Im Hinblick auf die Frage der syntaktischen Zugänglichkeit von externen Argumenten im Passiv lassen sich vier verschiedene Theorietypen (A-D) identifizieren. Die derzeit verfügbare empirische Evidenz zum Deutschen erweist sich als noch nicht konklusiv. Angesichts dieser Ausgangslage präsentieren wir neue Daten, die quantifikationale Variabilitätseffekte involvieren, eine wenigstens partielle Zugänglichkeit im Passiv nahelegen und so zwischen den verschiedenen Passivtheorien zu diskriminieren erlauben.
\end{abstract}

Keywords: externes Argument, Medium, Passiv, quantifikationale Variabilität, von-Phrase

\section{Hintergrund: Dunkle Materie}

Den Hintergrund der vorliegenden Untersuchung bildet die Frage, inwieweit nicht sichtbare (,leere“) Elemente in syntaktischen Repräsentationen enthalten sind, d.h., wieviel dunkle Materie syntaktische Repräsentationen durchzieht und welchen Status sie hat. Wie die etablierte dunkle Materie in der Astrophysik ist auch sprachliche dunkle Materie allein dadurch zu rechtfertigen, dass durch sie Effekte erklärt werden, für die keine andere, sichtbare Quelle identifiziert werden kann.

Anmerkung: Für hilfreiche Hinweise und Diskussion bedanken wir uns bei Benjamin Bruening, Terje Lohndal, Florian Schäfer, Wolfgang Sternefeld und den HerausgeberInnen, insbesondere Peter Gallmann und Angelika Wöllstein. Die vorliegende Studie wurde gefördert durch die DFGProjekte AL554/8-1 (Artemis Alexiadou) und MU1444/14-1 (Gereon Müller).

Artemis Alexiadou, Humboldt Universität Berlin, Englische Sprachwissenschaft, Unter den Linden 6, D-10099 Berlin, \& Leibniz-ZAS, E-Mail: artemis.alexiadou@hu-berlin.de Gereon Müller, Universität Leipzig, Institut für Linguistik, Beethovenstr. 15, D-04107 Leipzig, E-Mail: gereon.mueller@uni-leipzig.de

○ Open Access. (C) 2018 Artemis Alexiadou und Gereon Müller, publiziert von De Gruyter. (c) BY Dieses Werk ist lizenziert unter der Creative Commons Attribution 4.0 Lizenz.

https://doi.org/10.1515/9783110490992-018 
In der Geschichte der modernen Syntaxforschung gibt es wechselnde Einschätzungen zu dunkler Materie in syntaktischen Repräsentationen. In der klassischen Transformationsgrammatik (Chomsky 1957, 1965; Ross 1967) war man davon ausgegangen, dass syntaktische Tiefenstrukturen diverse Elemente enthalten, die im Laufe der Derivation verloren gehen können und in diesem Sinne abstrakt sind; syntaktische Oberflächenstrukturen sind allerdings in diesem Modell grundsätzlich frei von dunkler Materie. Mit der Einführung der Spurentheorie zur Erfassung von Bewegung (vgl. Wasow 1972; Fiengo 1977; Chomsky 1977) hat sich dies radikal geändert: Spuren sind eine prototypische Instanz von dunkler Materie; sie sind gerechtfertigt worden dadurch, dass ohne sie Phänomene nicht erklärt werden können (z. B. Kontraktion, Rekonstruktion, Lokalitätsrestriktionen für Bewegung, Überkreuzungseffekte), und es sind im Rahmen der Rektions- und Bindungstheorie spezielle Beschränkungen für diese abstrakten Elemente postuliert worden (vgl. z. B. Chomsky (1981) zum Prinzip der leeren Kategorien („Empty Category Principle“; ECP)). Spuren haben dann nach anfänglichen Widerständen (vgl. etwa Pullum (1979)) auch Einzug gehalten in sehr viele andere Grammatikmodelle wie z. B. die Generalisierte Phrasenstrukturgrammatik (GPSG; vgl. Gazdar et al. 1985) oder die HeadDriven Phrase Structure Grammar (HPSG; vgl. Pollard \& Sag 1994; Levine \& Sag 2003; Müller, St. (2007)). Spuren werden ebenfalls standardmäßig angenommen in der optimalitätstheoretischen Syntax (vgl. Grimshaw 1997; Legendre et al. 2006; sowie Müller 2015 zu einem Überblick). Dies heißt jedoch nicht, dass Spuren komplett unkontrovers sind. In der HPSG gibt es diverse Ansätze, in denen auf diese unsichtbaren Elemente verzichtet wird (vgl. z. B. Sag \& Wasow 1999). Ebenso vermeiden konstruktionsgrammatische Ansätze üblicherweise die Postulierung von Spuren (vgl. etwa Fischer \& Stefanowitsch 2006; Goldberg 2006). Die Evidenz aus kognitiver Perspektive ist in diesem Bereich inkonklusiv: Bever \& McElree (1988), Grodzinsky (2000) und Muckel (2002) präsentieren Argumente für Spuren; aber Pickering \& Barry (1991) und viele der KommentatorInnen von Grodzinsky (2000) können keine psycho- oder neurolinguistische Evidenz für Spuren finden.

Dieselben Kontroversen bzgl. der Existenz von dunkler Materie durchziehen auch viele andere Phänomenbereiche in der Syntax. Dies gilt z. B. für Kontrollkonstruktionen, wo manche Ansätze ein leeres Element wie PRO im abhängigen Infinitiv postulieren (vgl. Chomsky 1981; Landau 2013), oder sogar eine Bewegungsspur (vgl. Boeckx \& Hornstein 2006), andere Analysen aber keine Rechtfertigung von leeren Elementen sehen (vgl. etwa Bresnan 2001; Stiebels 2015). Analog liegt der Fall auch bei Pro-Drop, bei impliziten Argumenten, bei Ellipsen und bei vielen weiteren Konstruktionen.

Instruktiv ist an diesem Punkt eine mögliche Erweiterung der aus der Astrophysik entlehnten Metaphorik: Dunkle Materie kann weiter unterteilt wer- 
den in baryonische und nicht-baryonische Materie. Baryonische dunkle Materie ist ontologisch vom selben Typ wie sichtbare Materie; der einzige Unterschied ist, dass sie nicht der direkten Beobachtung zugänglich ist. Nicht-baryonische dunkle Materie ist demgegenüber von einer ganz anderen Art als sichtbare Materie; sie ist anders gestaltet, sie verhält sich anders, und sie ruft andere Effekte hervor. Interessanterweise ist der Großteil der bisher angeführten vorgeschlagenen unsichtbaren Elemente in der Syntax von letzterem Typ: Spuren sind besondere Elemente, und es gelten spezielle Beschränkungen für sie (z. B. das bereits erwähnte ECP); Analoges gilt für PRO in Kontrollkonstruktionen (vgl. etwa Chomskys (1981) PRO-Theorem), für die für Pro-Drop-Konstruktionen vorgeschlagene leere Kategorie pro (vgl. Rizzis (1986) spezielle Beschränkungen der Lizensierung und Interpretation), usw. Im Minimalistischen Programm (Chomsky 1995, 2001, 2013) wird demgegenüber explizit vorausgesetzt, dass sämtliche dunkle Materie in der Syntax strikt baryonisch ist; d.h., sie darf ontologisch keinen besonderen Status haben, und es dürfen keine speziellen Beschränkungen für sie gelten. Im Einklang hiermit sind z. B. (nicht-baryonische) Spuren durch (baryonische) Kopien ersetzt; Kopien unterscheiden sich nicht substantiell von sichtbaren Elementen, und sie unterliegen exakt denselben Beschränkungen. Pro-Drop-Analysen sind nicht mehr über designierte (nichtbaryonische) pro-Elemente $\mathrm{zu}$ formulieren, sondern nur unter Rekurs auf vollkommen reguläre Pronomina ohne phonologische Realisierung (vgl. z. B. Holmberg 2005); usw.

Vor diesem Hintergrund wenden wir uns in diesem Beitrag einem weiteren möglichen Stück dunkler Materie zu, nämlich externen Argumenten in deutschen Passivsätzen (d.h., Argumenten, die in Aktivsätzen basisgeneriert sind außerhalb der Projektion des Prädikats, von dem sie ein Argument sind, und die die höchste Position auf Argumentlisten einnehmen, die mit Prädikaten im Lexikoneintrag verbunden sind). Wir möchten der Frage nachgehen, ob diese (nicht sichtbar auftretenden, aber mitverstandenen) externen Argumente in syntaktischen Repräsentationen vorhanden sind und, falls sie das sind, inwiefern sie für andere syntaktische Operationen zugänglich sind.

\section{2 Überblick}

Die in den letzten Jahrzehnten entwickelten Theorien des Passivs unterscheiden sich im Hinblick auf die Frage, ob die externen Argumente der passivierten Verben in Passivkonstruktionen syntaktisch zugänglich sind, ob also z. B. das externe Agens-Argument, das im Aktivsatz in (1a) als Subjekt realisiert wird (hier die Leute), auch im entsprechenden (Vorgangs-) Passivsatz in (1b) in ir- 
gendeiner Weise syntaktisch repräsentiert ist und, falls das der Fall ist, ob seine Repräsentation dann für andere syntaktische Prozesse zugänglich ist. ${ }^{1}$

(1) a. dass die Leute ein Buch gelesen haben.

b. dass ein Buch gelesen wurde.

Dabei lassen sich grob vier Analysetypen unterscheiden, auf die wir uns im Folgenden mit Typ A, Typ B, Typ C und Typ D beziehen werden. Typ-AAnalysen sind lexikalische Ansätze; vgl. hier Höhle (1978), Chomsky (1981), Bresnan (1982), Wunderlich (1993) und Kiparsky (2013), neben vielen anderen Arbeiten. Diese Analysen haben gemeinsam, dass Passiv als eine lexikalische Operation betrachtet wird, die das externe Argument aus der Argumentstruktur eines Verbs entfernt. Dies hat zur Folge, dass das externe Argument nicht in der Syntax repräsentiert sein kann; damit ist klar, dass es dann auch von anderen syntaktischen Operationen nicht gesehen werden kann.

Typ-B-Analysen sind demgegenüber syntaktische Ansätze. Hier wird postuliert, dass Passiv eine Operation ist, die in der Syntax stattfindet, und die zur Folge hat, dass ein externes Argument systematisch, und ohne Einschränkung, syntaktisch zugänglich ist. Typ-B-Analysen sind entwickelt worden u.a. von Chomsky (1957), Baker, Johnson \& Roberts (1989), Sternefeld (1995), Stechow (1998), Collins (2005), Harley (2013), Merchant (2013) und Georgi (2014).

Typ-C-Analysen sind wie Typ-B-Analysen syntaktische Ansätze: Auch hier wird postuliert, dass Passiv eine in der Syntax applizierende Operation ist. Im Gegensatz zu Typ-B-Analysen ist aber bei Typ C-Analysen das externe Argument, obschon grundsätzlich repräsentiert, für syntaktische Operationen nicht zugänglich. Solche Analysen sind verfolgt worden u.a. von Bach (1980), Keenan (1980), Stechow (1987, 1992), Bruening (2013, 2014), Hole (2014),

1 Hier und im Folgenden sind wir fast ausschließlich mit dem Vorgangspassiv befasst; wir blenden das Zustandspassiv im Deutschen größtenteils (abgesehen von einer kurzen Bemerkung in Abschnitt 4) aus. Eine klassische Annahme ist, dass das Zustandspassiv (,adjectival passive“ im Englischen; vgl. Wasow 1977; Williams 1981; Borer 1984) keine Evidenz für syntaktische Zugänglichkeit des externen Arguments aufweist. Für die wenigen Fälle, wo es auf den ersten Blick so aussieht, als könnte ein externes Argument im Zugangspassiv doch selektiv zugänglich sein (und z.B. eine von-Phrase erlauben) wird üblicherweise angenommen, dass es sich letztlich nicht um echt externe Argumente handelt; vgl. Zifonun et al. (1997, Band 3), Eisenberg (1999) und Maienborn (2007, 2011) zum Deutschen; ebenso Eichinger (1987) zum Althochdeutschen. Aber vgl. auch Alexiadou, Gehrke \& Schäfer (2014), wo Argumente für die selektive Zugänglichkeit des externen Arguments im Zustandspassiv vorgebracht werden (auf der Grundlage u. a. von morphologischer Evidenz bei kontrollierten Adjektiven und von Fällen disjunkter Referenz). 
Schäfer (2012b), Alexiadou \& Doron (2013) und Alexiadou, Anagnastopoulou \& Schäfer (2015).

Typ-D-Analysen schließlich setzen ebenfalls voraus, dass Passiv eine Operation ist, die in der Syntax erfolgt. Hier wird aber im Gegensatz zu Typ-BAnalysen und Typ-C-Analysen angenommen, dass das externe Argument am Anfang der syntaktischen Derivation zwar zugänglich ist, im Laufe der syntaktischen Derivation aber systematisch unzugänglich wird. Die im Rahmen der Relationalen Grammatik entwickelte Analyse von Perlmutter \& Postal (1983a, b) ist von dieser Art, letztlich auch frühe transformationsgrammatische Analysen wie die in Chomsky (1975), und dasselbe gilt für die in Müller $(2016,2017)$ und Murphy (2016) entwickelte Analyse per Strukturabbau.

Wir setzen voraus, dass die Kernfrage der Zugänglichkeit externer Argumente im Passiv eine empirische ist. Hier und im Folgenden werden wir uns dabei hauptsächlich auf das Deutsche konzentrieren. Die Ausgangslage ist, dass die empirische Evidenz, die bisher in der Literatur zum Thema präsentiert worden ist, nicht konklusiv ist. Einerseits gibt es einige Evidenz für die syntaktische Unzugänglichkeit externer Argumente in deutschen Passivkonstruktionen. Andererseits gibt es widerstreitende Evidenz für die syntaktische Zugänglichkeit externer Argumente in deutschen Passivkonstruktionen; diese ist allerdings nicht ganz unkontrovers. Vor diesem Hintergrund ist es das Hauptziel des vorliegenden Beitrags, ein neues Argument für die syntaktische Zugänglichkeit von externen Argumenten in Passivkonstruktionen des Deutschen vorzubringen. Dieses Argument beruht auf dem Effekt quantifikationaler Variabilität (vgl. Heim 1982; Diesing 1992), der in diesen Umgebungen zu betrachten ist, und der darin besteht, dass eine indefinite DP, die zunächst einmal lediglich als ein offener Satz mit einer freien Individuenvariable interpretiert wird, von einem Quantifikationsadverb unselektiv gebunden werden kann (und damit dann der Default-Interpretation als existentiell quantifiziert entgeht).

Wir werden wie folgt vorgehen. In Abschnitt 3 skizzieren wir grundsätzliche Charakteristika der vier Analysetypen auf der Grundlage von einigen wenigen ausgewählten Ansätzen. Abschnitt 4 präsentiert die bisher verfügbare, miteinander konfligierende empirische Evidenz bzgl. syntaktischer Zugänglichkeit von externen Argumenten im deutschen Passiv. Abschnitt 5 entwickelt das zentrale Argument auf der Basis des Effekts quantifikationaler Variabilität im deutschen Passiv. Im letzten Abschnitt 6 schließlich zeigen wir die Konsequenzen des Effekts der quantifikationalen Variabilität für die unterschiedlichen Passivtheorien im Deutschen auf. Die Konklusion wird sein, dass lexikalische Typ-A-Analysen und syntaktische Typ-B-Analysen mit der empirischen Evidenz bzgl. der Zugänglichkeit externer Argumente im deutschen Passiv 
unverträglich sind, während syntaktische Typ-C-Analysen (wie die in Alexiadou, Anagnastopoulou \& Schäfer 2015) und syntaktische Typ-D-Analysen (wie die in Müller 2016) im Prinzip mit den Daten zurechtkommen können. ${ }^{2}$

\section{Passivanalysen}

\subsection{Typ-A-Analysen: Chomsky (1981)}

Die in Chomsky (1981) entwickelte, mehr oder weniger kanonische Analyse des Passivs im Rahmen der Rektions-Bindungs-Theorie ist inhärent lexikalisch (auch wenn sie vielerorts fälschlicherweise als syntaktisch charakterisiert worden ist). Es gibt in dieser Analyse vier Schritte, von denen nur der letzte (und am wenigsten den Kern des Passivs betreffende) ein syntaktischer ist, und zwar: (i) Argumentreduktion, (ii) Kasusabsorption, (iii) morphologischer Reflex sowie (iv) Bewegung in eine spezielle Subjektposition.

Der erste und wichtigste Schritt bei Chomskys (1981) Passivanalyse ist die Argumentreduktion. Eine lexikalische Regel generiert eine Passivversion eines Verbs, die sich von der zugrundeliegenden Aktivversion nur dadurch unterscheidet, dass die externe $\theta$-Rolle der Aktivform getilgt wird; vgl. (2) für transitive Verben (mit x als absorbierter und y als zweiter, nicht affizierter $\theta$-Rolle).

(2) $\mathrm{V}(\mathrm{x}, \mathrm{y}) \rightarrow \mathrm{V}(-, \mathrm{y})$

Im zweiten Schritt erfolgt Kasusabsorption: Ein gemäß (2) im Lexikon erzeugtes passiviertes Verb kann keinen Akkusativ zuweisen, selbst wenn die korrespondierende Aktivform dies kann; vgl. (3). ${ }^{3}$

2 Auch wenn längst nicht alle diskutierten Analysen darunter fallen, wählen wir als unmarkierten Bezugsrahmen im Folgenden grundsätzlich das Minimalistische Programm (vgl. Chomsky (2001)), mit einer Satzstruktur CP-TP-vP-VP für das Deutsche (aber vgl. Haider (2010) zu Argumenten gegen eine separate TP-Projektion), die möglicherweise noch um weitere funktionale Projektionen zu ergänzen ist.

3 Dies muss vielleicht nicht separat stipuliert werden, sondern folgt u. U. daraus, wie Burzios Generalisierung (derzufolge Akkusativzuweisung an die Vergabe einer externen $\theta$-Rolle gekoppelt ist) abgeleitet wird (vgl. Burzio 1986). In einer Kasustheorie, wo Akkusativ nicht von einem syntaktischen Kopf zugewiesen wird, sondern direkt die Präsenz eines höheren Arguments signalisiert (sog. „abhängiger Kasus“), folgt dies unmittelbar (vgl. Marantz 1991; Bittner \& Hale 1996; Wunderlich 1997; Stiebels 2000; McFadden 2004; Schäfer 2012a; Preminger 2014; Baker 2015 und Bobaljik 2015); in diesem Fall müsste die Kasusabsorption dann kein lexikalischer Prozess mehr sein. Diese Fragen sind für unsere gegenwärtigen Zwecke aber orthogonal, und wir sehen im Folgenden von der Option von abhängigem Kasus ab. 
(3) $\mathrm{V}_{\text {acc }}(-, \mathrm{y}) \rightarrow \mathrm{V}_{-}(-, \mathrm{y})$

Drittens hat Passivierung in den meisten Sprachen und Konstruktionen einen morphologischen Reflex: Ein Verb [V $(-, y)]$, das durch die ersten beiden Schritte generiert worden ist, wird von Passivmorphologie begleitet. Und viertens schließlich erfolgt in Passivkonstruktionen in Sprachen wie dem Englischen, in denen es eine designierte vP-externe Subjektposition SpecT gibt, die besetzt werden muss (die sogenannte „EPP-Eigenschaft“), noch Bewegung des zugrundeliegenden Objekts nach SpecT. ${ }^{4}$ Eine Beispielderivation für einen englischen Passivsatz findet sich in (4).

(4) Beispielableitung: ${ }^{5}$
a. $\operatorname{kiss}_{a c c}\left(\underline{\theta_{1}}, \theta_{2}\right) \rightarrow \operatorname{kiss}_{a c c}\left(-, \theta_{2}\right)$
(Lexikon)
b. $\operatorname{kiss}_{\text {acc }}\left(-, \theta_{2}\right) \rightarrow$ kiss $_{-}\left(-, \theta_{2}\right)$
(Lexikon)
c. kiss $_{-}\left(-, \theta_{2}\right) \rightarrow$ kiss-ed_ $\left(-, \theta_{2}\right)$
(Lexikon)
d. Verkettung (kiss-ed $\left(-, \theta_{2}\right)$, John $) \rightarrow$ [vP kiss-ed John ]
e. $[\ldots]$
f. Bewegung $\left(\left[_{\mathrm{T}^{\prime}}\left[{ }_{\mathrm{T}}\right.\right.\right.$ was $]$ [vp kissed John $\left.]\right]$, John $) \rightarrow$ [ ${ }_{\mathrm{TP}}$ John $_{1}\left[{ }_{\mathrm{T}^{\prime}}\left[{ }_{\mathrm{T}}\right.\right.$ was $]\left[\mathrm{VP}_{\mathrm{V}}\right.$ kissed $\left.\left.\mathrm{t}_{1}\right]\right]$
(Syntax)

Der wesentliche Schritt bei der Passivierung - das, was Passiv in letzter Instanz ausmacht - ist hier klarerweise die Argumentreduktion, und dies ist bei Chomsky (1981) eine strikt Lexikon-interne Operation, die zunächst einmal nichts mit der Syntax zu tun hat. Alle anderen Schritte sind sekundär. Manche Sprachen können Burzios Generalisierung verletzen und zeigen keine Kasusabsorption im Passiv (vgl. Baker, Johnson \& Roberts 1989, neben vielen ande-

\footnotetext{
4 „EPP“ steht ursprünglich für Erweitertes Projektionsprinzip („Extended Projection Principle“). Es wurde in Chomsky (1982) als Erweiterung des Projektionsprinzips aus Chomsky (1981) eingeführt, demzufolge die lexikalisch festgelegte Argumentstruktur von Prädikaten auf jeder syntaktischen Repräsentationsebene beibehalten werden muss; die Erweiterung besagt dann, dass jeder Satz eine designierte abgeleitete Subjektposition haben muss, die durch ein syntaktisches Element besetzt ist. Es ist hin und wieder festgestellt worden, dass diese beiden Forderungen recht disparat sind; und tatsächlich wird in der Nachfolge von Chomsky (1982) mit EPP in der Regel nur der zweite Teil (also die Erweiterung) des Prinzips gemeint, dass also Sätze eine Füllung einer abgleiteten Subjektposition aufweisen. Im Einklang damit hat etwa David Pesetsky (Facebook-Eintrag, 6. 8. 2015) vorgeschlagen, EPP als „Extra-Periphere Position“ zu verstehen und die ursprüngliche Auflösung des Akronyms aufzugeben.
}

5 Die Konvention ist hier, dass Unterstreichung $\theta$-Rollen für externe Argumente anzeigt. 
ren). Manche Sprachen zeigen keinen morphologischen Reflex der Passivierung (vgl. etwa Perlmutter \& Postal 1983b); tatsächlich hat ja auch das Deutsche in lassen-Passiven beim eingebetteten Verb die Möglichkeit zur Passivierung, die nicht von einem sichtbaren morphologischen Reflex auf dem passivierten Prädikat (oder sonstwo) begleitet wird; vgl. (5a) (Aktiv) vs. (5b) (Passiv). Das lassen-Passiv erfüllt sonst wesentliche Kriterien für echte Passivierung (vgl. zu dieser Konstruktion u. a. Höhle 1978; Gunkel 2003; Pitteroff 2014). Z. B. kann das externe Argument auch mit einer von-Phrase wieder aufgenommen werden; vgl. (5c).

(5) a. dass der König [ die Sklaven den Wein reinbringen ] lässt.

b. dass der König [ den Wein reinbringen ] lässt.

c. dass der König [ von den Sklaven den Wein reinbringen ] lässt.

Und schließlich ist die Bewegung des internen Arguments in eine externe Subjektposition SpecT offensichtlich auf einen komplett unabhängigen Faktor zurückzuführen (eben die EPP-Eigenschaft). Sprachen wie das Deutsche, die keine obligatorische EPP-Eigenschaft aufweisen (vgl. Grewendorf 1988; Haider 1993, 2010) und Nominativ auch innerhalb der VP realisieren können, müssen in Passivkonstruktionen keine Bewegung des internen Arguments in eine externe Subjektposition durchführen; vgl. (6), wo die unmarkierte Wortstellung vom Aktiv (in (6a)) zum Passiv (in (6b)) vollständig erhalten bleibt und das Nominativ tragende interne (Thema-) Argument durchweg dem (Ziel-) Ko-Argument und den zwei adverbialen Bestimmungen nachfolgt. ${ }^{6}$

(6) a. dass der Karl der Maria in der Kneipe wahrscheinlich eine Rose gegeben hat.

b. dass der Maria in der Kneipe wahrscheinlich eine Rose gegeben wurde.

Zusammenfassend lässt sich damit festhalten, dass gemäß Chomskys (1981) Analyse (die u. a. in Fanselow 1985; Grewendorf 1988; Stechow \& Sternefeld 1988 auf das Deutsche übertragen worden ist) externe Argumente in der Syntax von Passivkonstruktionen an keinem Punkt vorhanden sind (im Einklang damit

6 Davon abgesehen ist die Vorstellung, dass in der Passivanalyse Chomskys (1981) Bewegung ein wesentliches Element sei, auch für das Englische spätestens mit der Postulierung vP-interner Subjekte hinfällig geworden, denn damit müssen sich in dieser Sprache (im Regelfall) alle Nominativ tragenden Elemente nach SpecT bewegen, unabhängig von ihrer Basisverkettung als Spezifikator von v (externes Argument) oder als Tochter von VP (internes Argument). 
ist auch der Status von von-Phrasen in Passivkonstruktionen in diesem Modell notorisch unklar). Die Vorhersage dieses lexikalischen Ansatzes (wie auch anderer, für die er in dieser Beziehung als repräsentativ gelten kann) ist damit klar: Erwartet wird vollständige syntaktische Unzugänglichkeit von externen Argumenten im Passiv.

\subsection{Typ-B-Analysen: Sternefeld (1995)}

Wie Baker, Johnson \& Roberts (1989) nimmt Sternefeld (1995) an, dass Passiv ein genuin syntaktisches (und nicht lexikalisches) Phänomen ist: Das externe Argument ist in der Syntax vorhanden und systematisch zugänglich. Allerdings unterscherscheidet sich Sternefelds Analyse von der in Baker, Johnson \& Roberts (1989) dadurch, dass nicht der morphologische Reflex des Passivs (also verbales Material) als das externe Argument angesehen wird; vielmehr ist das externe Argument syntaktisch repräsentiert als leere Kategorie pro, die in der kanonischen Position für externe Argumente (bei Sternefeld: dem höchsten Spezifikator von VP) verkettet wird und sich dann in den Spezifikator einer designierten VoiceP bewegen muss; dieses pro trägt Akkusativ. Ein einfaches Beispiel aus dem Englischen illustriert das in (7).

(7)

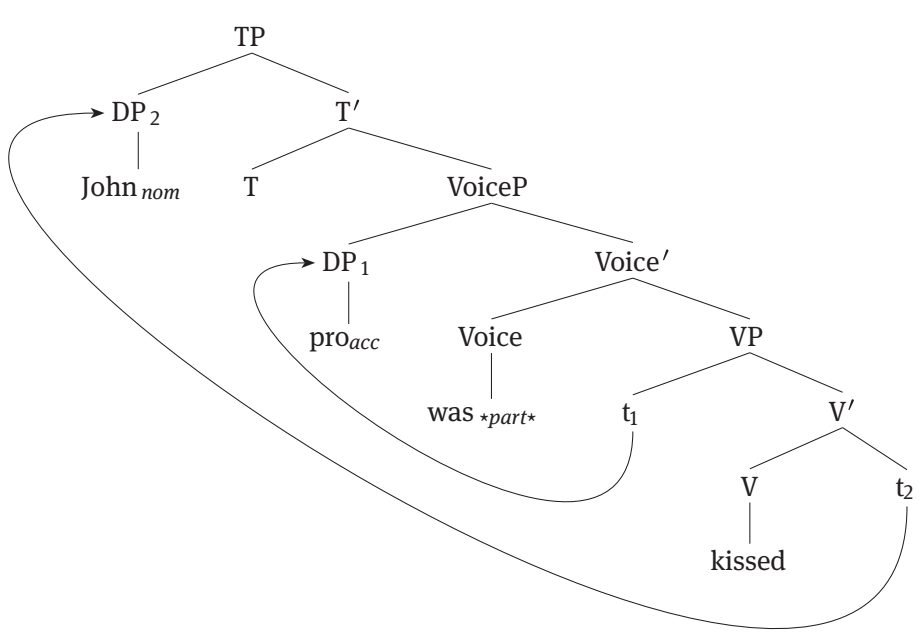

Die theoretische Kernnahme ist, dass der Kopf einer passivischen VoiceP über Spezifikator-/Kopf-Kongruenz ein pro lizensieren muss, das die $\theta$-Rolle für das externe Argument trägt. Dabei sind Passiv-Auxiliare Voice-Köpfe, die spezielle abhängige Verbformen selegieren (bzw. status-regieren). Die Analyse erfordert 
eine etwas komplexere, zweistufige Kasustheorie, denn es muss ja gewährleistet werden, dass ein externes Argument eines finiten Satzes im Prinzip Akkusativ tragen kann: Struktureller Kasus wird zunächst zugewiesen und in der Folge noch einmal lizensiert. Bzgl. Kasuszuweisung wird angenommen, dass in der VP (i) Nominativ per Default zugewiesen werden kann, (ii) Akkusativ zugewiesen werden kann, wenn Nominativ zugewiesen worden ist, und (iii) Dativ zugewiesen werden kann, wenn Akkusativ zugewiesen worden ist. Dabei kann jeder Kasus nur einmal zugewiesen werden (wenn zwei Kasus in einer VP gleich sind, dann muss einer davon somit ein lexikalischer Kasus sein). Für Kasuslizensierung gilt demgegenüber, dass (i) finites T Nominativ in seinem Spezifikator lizensiert; dass (ii) Akkusativ in der VP auf einem direkten Objekt lizensiert ist; dass (iii) Dativ in der VP auf einem indirekten Objekt lizensiert ist; dass (iv) der Voice-Kopf werden $_{* \text { part* }}$ Akkusativ auf einem externen Argument lizensiert; und dass (v) der Voice-Kopf kriegen *part* (bzw. bekom$m e n_{* \text { part* }}$ ) im Rezipientenpassiv Dativ auf einem externen Argument lizensiert. ${ }^{7}$

Es sei kurz skizziert, wie in dieser Analyse die Kerneigenschafen des Passivs folgen. Zunächst einmal ist festzuhalten, dass es keine wie auch immer geartete Argumentreduktion gibt: Das externe Argument ist syntaktisch präsent und aktiv. Wenn ein passivischer Voice-Kopf auftritt, braucht er ein pro, das die Subjekt- $\theta$-Rolle trägt. Daher können hier nur Ableitungen erfolgreich sein, in denen pro in der Subjektposition der VP basisverkettet wird. Zweitens gilt, dass es ebensowenig wie zu einer Argumentreduktion zu einer Kasusabsorption kommt. Vielmehr wird in kanonischen Passiven struktureller Akkusativ an ein pro in SpecVoice zugewiesen (s. o.); daher ist Akkusativ nicht mehr für eine Objekt-DP verfügbar, und eine solche DP muss somit Nominativ von T lizensiert bekommen. Der typische morphologische Reflex des Passivs ergibt sich aus einer Kombination der Form des Voice-Kopfes (d.h., des Passivauxiliars in Sprachen wie Deutsch oder Englisch) und der Form des abhängigen Verbs, das dadurch selegiert wird (z. B. ein Partizip II). Was schließlich die Bewegung des Objekts in die Subjektposition betrifft, so gilt hier tatsächlich zunächst einmal bei Sternefeld (1995), dass dies auch im Deutschen notwendig sein muss, denn nur in SpecT kann per Annahme Nominativ lizensiert werden. Man sieht aber leicht, dass diese Annahme nicht zentral für die Analyse ist: Wenn es einen anderen, von Bewegung nach SpecT unabhängigen Weg gibt, wie Nominativ durch T lizensiert werden kann, dann sollte Nominativlizensierung auch ohne Bewegung möglich sein (der naheliegende Ausweg auf der Basis von Chomsky (2001) ist die Postulierung einer Abgleichsrelation

7 Ein Eintrag wie werden $_{* \text { part* }}$ signalisiert, dass das Passivauxiliar werden eine Partizipform des Hauptverbs status-regiert. 
(„Agree“)). Die Analyse erfasst auf einfache Weise die zwei möglichen Passivierungen von Doppelobjektkonstruktionen wie (8a) im Deutschen, nämlich das reguläre Vorgangspassiv mit werden in (8b) und das Rezipientenpassiv mit bekommen (kriegen) in (8c).

(8) a. dass der Fritz der Maria den Film geschenkt hat.

b. dass der Film der Maria geschenkt wird.

c. dass die Maria den Film geschenkt bekommt (kriegt).

(9) zeigt, wie in (8b) mit dem primären Passivauxiliar werden $_{* \text { part* }}$ das ThemaArgument Nominativ erhält (und das nicht sichtbare externe Argument Akkusativ). ${ }^{8}$

(9)

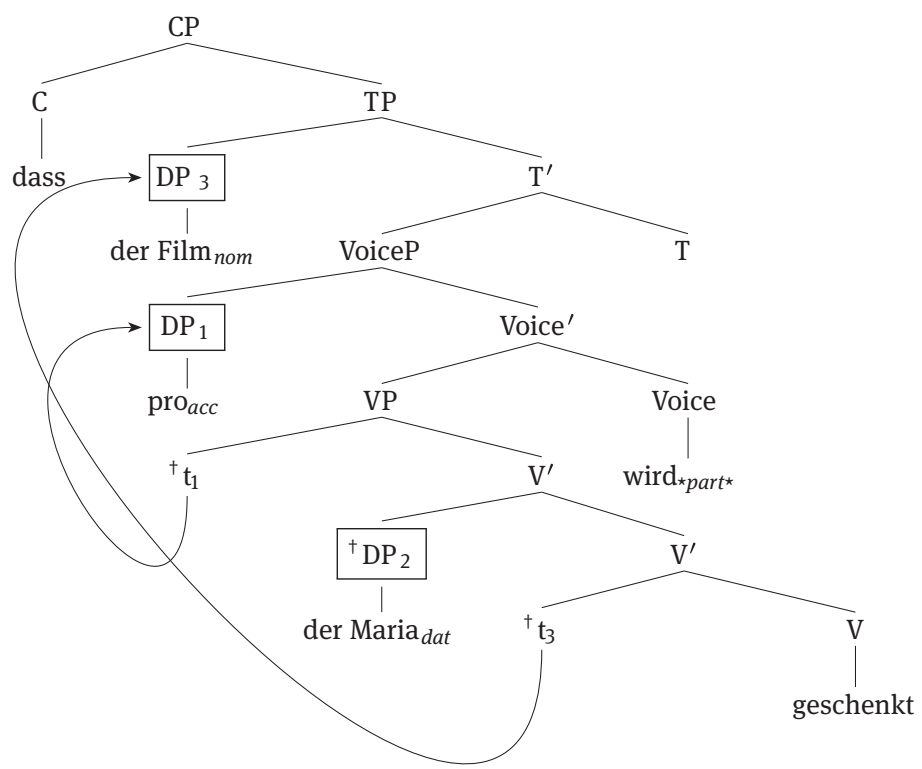

Wie man sich leicht klarmachen kann, führen unter Sternefelds Annahmen alle anderen Verteilungen von Kasus an die drei DP-Argumente notwendigerweise zu Ungrammatikalität.

8 Eine Bemerkung zur Notation: ${ }^{\dagger} \mathrm{DP}_{n}$ steht für eine $\mathrm{DP}_{n}$, der Kasus zugewiesen worden ist; $\mathrm{DP}_{n}$ dagegen steht für eine $\mathrm{DP}$, deren Kasus lizensiert ist. 
Das Rezipientenpassiv in (8c) dagegen hat eine Ableitung wie in (10). Weil das Ziel-Argument statt Dativ nun Nominativ erhält, muss pro als externes Argument nun Dativ tragen, nicht Akkusativ wie beim Passiv mit werden. Wiederum sind alle anderen Kombinationen von Kasus und Argumenttyp als unmöglich vorhergesagt.

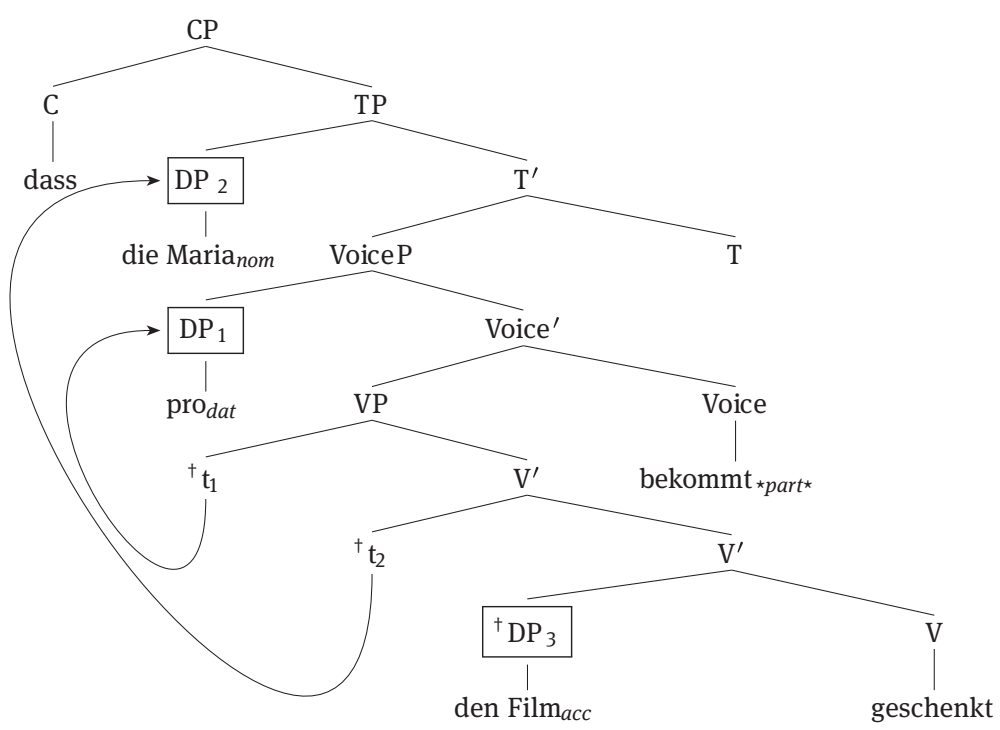

Die Analyse hat noch viele weitere interessante Konsequenzen, und sie wirft auch ein paar offensichtliche Fragen auf. ${ }^{9}$ Der im gegenwärtigen Zusammenhang entscheidende Punkt ist aber, dass das externe Argument als im Passiv systematisch vorhanden (als leere Kategorie pro) und für syntaktische Operationen (z. B. Kasuszuweisung und Kasuslizensierung sowie Bewegung) zugänglich postuliert wird; die Präsenz des externen Arguments im Passiv ist dabei für die Analyse absolut unabdingbar (z. B. könnten sonst die Kasusabsorptionseffekte nicht erfasst werden).

9 Z.B. ist zunächst einmal nicht klar, wie das unpersönliche Passiv, wie es in (i) dargestellt ist, analysiert werden kann.

(i) dass nicht [VoiceP $\operatorname{pro}_{1}\left[\mathrm{vp}_{1} \mathrm{t}_{1}\right.$ getanzt $]$ wurde ].

Das leere externe Argument pro kann ja hier keinen Akkusativ haben, weil der entsprechende Aktivsatz intransitiv ist. Wenn pro hier Nominativ tragen könnte, würden falsche Vorhersagen für die Kasusverteilung in transitiven Passiven gemacht werden. Sternefelds Konklusion ist hier, dass externe Argumentpros im Deutschen nicht nur mit Akkusativ (werden) und Dativ (bekommen), sondern auch kasuslos möglich sind. 


\subsection{Typ-C-Analysen: Bruening (2013), Alexiadou et al. (2015), Stechow (1992)}

\subsubsection{Analyse}

Stellvertretend für die Typ-C-Analysen werden wir im Folgenden den in Bruening (2013) und Alexiadou, Anagnastopoulou \& Schäfer (2015) entwickelten Ansatz betrachten. Eine zentrale Annahme ist, dass Passiv wie in (11) zu verstehen ist.

(11) Definition des Passivs:

Das Passiv ist eine morpho-syntaktische Operation, die die Realisierung des externen Arguments verhindert.

Die Merkmale, die ein externes Argument im Passiv identifizieren, können dann wie in (12a) oder wie in (12b) aussehen.

(12) Identifizierende Merkmale des Passivs:

a. Das externe Argument fehlt und wird existentiell interpretiert.

b. Das externe Argument wird als Adjunkt realisiert.

Der Passiv-Kopf selegiert in dieser Analyse eine Projektion eines an sich ganz normalen aktivischen Voice-Kopfes, die ihr externes Argument aber noch nicht projiziert hat. Daraus ergibt sich, dass Passiv das externe Argument von Voice saturieren muss. Für das externe Argument im Passiv (beim Fehlen einer vonPhrase) wird angenommen, dass es existentiell abquantifiziert wird. Daraus folgt, dass es nicht anderweitig kontrolliert oder gebunden werden kann (s. u.) - es erweist sich als syntaktisch vorhanden, aber für syntaktische Operationen nicht zugänglich. Diese Annahmen sind implementiert in der Charaktierisierung des primären Passiv-Kopfes bei Bruening (2013) in (13).

(13) Bruenings primärer Passiv-Kopf:

$\llbracket$ Pass $\rrbracket=\lambda \mathrm{f}<\mathrm{e}, \mathrm{st}>\lambda \mathrm{e} . \exists \mathrm{x}: \mathrm{f}(\mathrm{x}, \mathrm{e})$

Der Passiv-Kopf in (13) nimmt ein Prädikat mit noch nicht saturiertem externen Argument (die Voice-Projektion) und quantifiziert dieses externe Argument existentiell ab, so dass es nicht mehr zugänglich ist. Dies illustriert (15), als Teil eines Passivsatzes wie (14). 
(14) dass der Minister bestochen wurde.

(15) Voice verbindet sich mit VP, Pass verbindet sich mit VoiceP

a. [PassP(ii) Pass [VoiceP(i) Voice [vP den Minister bestochen ]]]

b. (i) $\lambda x \lambda$ e.bestechen(e,der Minister) \& Initiator(e,x)

(ii) $\lambda$ e. $\exists x$ :bestechen(e,der Minister) \& Initiator(e,x)

Wenn nun allerdings eine von-Phrase auftritt, ist das externe Argument als Teil davon ja offen realisiert, und weil das externe Argument in diesen Fällen natürlich nicht existentiell abquantifiziert werden muss (es kann ja, wie z. B. (16a, b) zeigen, mit einem anderen Quantor erscheinen) und überdies systematisch syntaktisch zugänglich ist (s.u.), braucht man, wie es zumindest zunächst einmal aussieht, mindestens noch einen zweiten Pass-Kopf, der alternativ gewählt werden kann und an der Voice-Projektion semantisch im Prinzip gar nichts verändert, so dass ein später hinzukommendes von-Phrasen-Adjunkt die externe $\theta$-Rolle des Verbs saturieren kann; vgl. (17).

(16) a. dass der Minister [ ${ }_{P P}$ von niemandem ] bestochen wurde.

b. dass der Minister [ ${ }_{P P}$ von allen Lobbyisten ] bestochen wurde.

(17) Bruenings sekundärer Passiv-Kopf:

$\llbracket$ Pass $\rrbracket=\lambda \mathrm{f}<\mathrm{st}>\lambda$ e. $\mathrm{f}(\mathrm{e})$

Die Annahme zweier semantisch komplett unterschiedlicher Passiv-Köpfe scheint uns zwar im Wesentlichen den Stand der Kunst in Typ-C-Analysen zu reflektieren; sie ist jedoch nicht ganz unproblematisch. Zum einen ist aus dieser Perspektive unklar, warum die postulierten zwei Passiv-Morpheme immer morphologisch identisch realisiert $\mathrm{zu}$ werden scheinen. Zum anderen ist noch eine zusätzliche Stipulation notwendig, um sicherzustellen, dass der sekundäre Passiv-Kopf, der ja im Prinzip die Identitätsfunktion denotiert, niemals mit einer aktiven Voice-Projektion kombiniert werden kann, und generell eine vonPhrase voraussetzt. Wir kommen zum Ende dieses Unterabschnitts noch einmal kurz auf dieses potenzielle Problem zurück.

\subsubsection{Das griechische Passiv: Medium}

Embick (2004) und Alexiadou, Anagnastopoulou \& Schäfer (2015) haben argumentiert, dass externe Argumente im griechischen Passiv nicht syntaktisch 
projiziert werden. Tatsächlich lässt sich darüber hinaus vor dem Hintergrund der soeben skizzierten Passivanalyse zeigen, dass das griechische Passiv gar keine echte Passivkonstruktion involviert, sondern der Diathese Medium („middle voice“) subsumierbar ist. Wie das Passiv, so führt auch das Medium die externe Argumentstelle ein und bindet sie zugleich existentiell ab; anders als dem Passiv fehlt dem Medium aber die Präsupposition disjunkter Interpretation. (18) hat damit die Struktur in (19) und die semantische Ableitung in (20).

(18) O Janis plithike.

der Janis waschen.NONAKT.3SG

„Janis wusch sich.“

„Janis wurde gewaschen.“

(19) [MiddleVoiceP MiddleVoice [ ${ }_{\mathrm{vP}} \mathrm{V}$ [ Vpli [DP O Janis]]]]

(20) a. $\llbracket v P \rrbracket=\lambda$ e. $\operatorname{waschen}(\mathrm{e}) \&$ Thema(Janis)(e)

b. $\llbracket$ Medium $\rrbracket=\lambda g_{s, t} \lambda e \exists x \cdot g(e) \& \operatorname{Agens}(x)(e)$

c. $\quad \llbracket$ MediumP $\rrbracket=\lambda e \exists x$. waschen(e) \& Thema(Janis)(e) \& Agens(x)(e)

(20c) denotiert die Menge der Ereignisse, in denen jemand Janis wäscht; diese Bedeutung ist möglich sowohl bei Ereignissen, wo eine andere Person als Janis das Agens ist, als auch bei Ereignissen, wo Janis selbst das Agens ist. (Genau dies ist im Passiv normalerweise nicht möglich.) Da eine disjunktive Interpretation nicht erzwungen wird, wird die letztendliche Interpretation des existentiell gebundenen externen Arguments durch konzeptuelles Wissen oder durch Modifikatoren wie von-Phrasen oder sog. antiassistive Intensifikatoren (wie afto-, paraphrasierbar als „ohne Hilfe“) determiniert.

Diese Modellierung des Unterschieds von Passiv und Medium ist allerdings nicht die einzig mögliche. Eine alternative Option könnte wie folgt aussehen: Man könnte annehmen, dass ein wesentlicher Unterschied zwischen Passiv und Medium der ist, dass Passiv syntaktisch einen Spezifikator von Voice projiziert, der das implizite externe Argument enthält, während das Medium syntaktisch intransitiv bleibt. Wir werden diese Fragen aber hier nicht weiter verfolgen, da sie die zentrale Charakterisierung von Typ-C-Analysen nur indirekt berühren.

Bevor wir uns dem vierten und letzten Analysetyp zuwenden, wollen wir noch kurz auf eine mögliche Alternative zur Doppelcharakterisierung von PassMorphemen eingehen, die zwar einerseits wie erwähnt eine Standardannahme 
in Typ-C-Analysen ist, andererseits aber wie gesehen auch weder konzeptuell noch empirisch vollständig unproblematisch ist.

\subsubsection{Eine Alternative: Stechow $(1987,1992)$}

Stechow (1987, 1992) hat eine Analyse des deutschen Passivs entworfen, die den hier angeführten Typ-C-Analysen im Kern ganz ähnlich ist (und wie diese letztlich auf Bach (1980) und Keenan (1980) zurückführbar ist), die es aber möglich macht, auf Mehrfacheinträge für das Pass-Morphem (einen für Passivsätze ohne von-Phrase, einen für Passivsätze mit von-Phrase) komplett zu verzichten. Der einzige von Stechow postulierte Passiv-Kopf ist der in (21). ${ }^{10}$

(21) Stechows Passiv-Kopf:

$\llbracket \mathrm{Pass} \rrbracket=\lambda \mathrm{P}_{<e t\rangle} \exists \mathrm{x} . \mathrm{P}(\mathrm{x})$

Dieser Passiv-Kopf führt existentielle Quantifikation über das externe Argument herbei, auf grundsätzlich genau dieselbe Art wie auch sonst in Typ-CAnalysen. Was Stechows (1987, 1992) Analyse aber besonders macht, ist die Behandlung der Präposition von, wie sie in von-Phrasen im Passiv auftritt: $v o n_{\text {pass }}$ wird betrachtet als ein Operator höherer Stufe, der zunächst auf ein Individuum angewendet wird (y in (22), das interne Argument der Präposition), und dann auf ein Prädikat (Q in (22), die VP), und der dann wieder ein Prädikat ergibt; vgl. (22).

(22) Stechows von pass $: \lambda y \lambda \mathrm{Q}_{<e t>} \lambda \mathrm{z} \cdot \mathrm{Q}(\mathrm{z}) \& \mathrm{z}=\mathrm{y}$

Wesentlich ist, dass dieser Operator die Identität der externen Argumentvariable, über die später (durch den Passiv-Kopf in (21)) existentiell quantifiziert wird ( $z$ in (22)), auf der einen Seite und der neuen Variable, die durch die Präposition $v_{\text {pass }}$ eingeführt wird (y in (22)) auf der anderen Seite konstatiert. Damit macht diese Analyse auf indirekte Art und Weise beim Auftreten einer vonPhrase die „eigentlich“ (als z) durch den Passiv-Kopf existentiell abgebundene Variable wieder (als y) zugänglich. Genau dies wird benötigt in Fällen wie denen in (16), hier wiederholt als (23a, b):

10 Der lexikalische Eintrag hier ist leicht vereinfacht, im Hinblick auf Faktoren, die im gegenwärtigen Zusammenhang irrelevant sind. 
(23) a. dass der Minister [ ${ }_{P P}$ von niemandem ] bestochen wurde.

b. dass der Minister [ ${ }_{\mathrm{PP}}$ von allen Lobbyisten ] bestochen wurde.

Das externe Argument des Verbs ist hier immer noch existentiell abgebunden, aber das interne Argument der Präposition von, das gemäß (22) damit identisch sein muss, ist frei für Bindung von außen. ${ }^{11}$

Insgesamt ergibt sich damit, dass Typ-C-Analysen sehr wohl mit einem einzigen Lexikoneintrag für das Passiv-Morphem auskommen können. Ungeachtet dieser Frage bleibt als Vorhersage dieser Art von Ansatz, dass externe Argumente in einfachen Passivsätzen (also ohne von-Phrase) aufgrund der festverdrahteten existentiellen Abbindung durch den Passiv-Kopf systematisch syntaktisch unzugänglich sein sollten.

\subsection{Typ-D-Analysen: Perlmutter \& Postal (1983a, b), Chomsky (1975), Müller (2016, 2017)}

Im Rahmen der Relationalen Grammatik haben Perlmutter \& Postal 1983a,b eine sprachübergreifende Analyse des Passivs entwickelt, die (i) eine Promotion des zugrundeliegenden Objekts zum Subjekt vorsieht (mit zunächst einmal möglichen Problemen bei der Ableitung unpersönlicher Passivkonstruktionen, wo genau dies nicht passiert) und (ii) ganz explizit aber auch eine syntaktische

11 Nebenbei bemerkt hat dies zur Folge, dass damit Aktivsätze und entsprechende Passivsätze mit von-Phrasen leicht unterschiedliche Bedeutungszuweisungen involvieren. (ia) bedeutet klassisch (iia), während (ib) (= (23a)) als (iib) paraphrasierbar ist.

(i) a. dass den Minister niemand bestochen hat.

b. dass der Minister von niemandem bestochen wurde.

(ii) a. Es gibt niemanden, der den Minister bestochen hat.

b. Es gibt niemanden, der mit jemandem identisch ist, der den Minister bestochen hat.

Da die Sätze in (iiab) logisch äquivalent sind, ist diese Konsequenz unproblematisch.

Man beachte im Übrigen, dass technisch gesehen in diesem System die Hinzufügung von $v n_{\text {pass }}$-Phrasen iteriert werden könnte, mit immer neuen Argumenten für die Präposition. Der

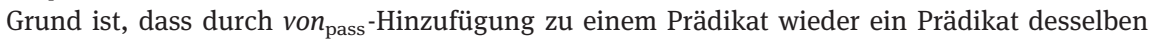
Typs generiert wird. Allerdings ist davon auszugehen, dass derartige Mehrfachagensaufnahmen durch die Identititätsbedingung in (22) normalerweise entweder direkt zu Kontradiktionen führen und damit unmöglich sind (wie etwa bei *dass der Minister von Karl von Maria bestochen wurde oder bei ${ }^{*}$ dass der Minister von jemandem von niemandem bestochen wurde), oder aber durch pragmatische Prinzipien ausgefiltert werden (wie z. B. bei ${ }^{*}$ dass der Minister von jemandem von vier Leuten bestochen wurde). 
Demotion des externen Arguments postuliert. Für die genaue Charakterisierung dieses zweiten Aspekts werden verschiedene Möglichkeiten erwogen. Die vielleicht bekannteste ist die, dass Passiv aus einem zugrundeliegenden Subjekt in der Syntax einen sog. Chômeur macht, mit stark reduzierter Zugänglichkeit für syntaktische Prozesse. Die ursprünglich von Perlmutter und Postal im Rahmen der Relationalen Grammatik ins Auge gefasste Version der Abstufung von Subjekten im Passiv, das Gesetz der Relationalen Vernichtung (,Relational Annihilation Law“; vgl. Perlmutter \& Postal (1983a, 62)), involviert aber ein noch radikaleres Konzept: Passivierung führt demzufolge bei einem ursprünglichen Subjekt dazu, dass dieses Argument überhaupt keine grammatische Relation mehr eingeht, also im Prinzip nicht mehr zugänglich ist.

Ein solcher Ansatz unterscheidet sich damit systematisch von den bisher betrachten Analysen: Passiv ist hier nicht lexikalisch (wie bei Typ-A-Analysen), sondern syntaktisch, und das externe Argument ist im Passiv (Fehlen einer von-Phrase vorausgesetzt) weder grundsätzlich in der Syntax zugänglich (wie in Typ-B-Analysen), noch grundsätzlich in der Syntax unzugänglich (wie in Typ-C-Analysen). Vielmehr ist das externe Argument in der Syntax zunächst zugänglich, und dann, nach der Anwendung der Passivoperation, unzugänglich.

Letztlich gilt dieselbe Konklusion auch für klassische transformationsgrammatische Analysen. Der Ansatz in Chomsky (1957) war oben den Typ-BAnalysen subsumiert worden, und dies ist insofern sicherlich gerechtfertigt, als (i) Passiv mit Hilfe einer designierten syntaktischen Transformation erfasst wird, und (ii) das externe Argument vor der Anwendung der PassivTransformation (als Subjekt) und nach der Anwendung der PassivTransformation (als obligatorisch generierter von-Phrase) im Prinzip syntaktisch zugänglich ist. Tatsächlich wird aber z. B. in Chomsky (1975, 455) (mit Erstzirkulation 1955) zur Ableitung von Passivsätzen ohne von-Phrase eine weitere, folgende Transformation eingeführt („By-Phrase Deletion“), die ein externes Argument optional vollständig aus der syntaktischen Struktur entfernt. Auch in dieser Analyse wird somit zunächst syntaktische Zugänglichkeit und später syntaktische Unzugänglichkeit von externen Argumenten postuliert.

Die in Müller (2016, 2017) entwickelte Analyse fügt sich ebenfalls in diese Tradition ein. Die zentrale Behauptung ist, dass syntaktische Derivationen zwei elementare Operationen vorsehen, die syntaktische Repräsentationen in ihrer Größe modifizieren können: Neben der Operation, die Struktur aufbaut nämlich Verkettung („Merge“; vgl. Chomsky 2001, 2013) -, gibt es hier eine komplementäre Operation Abbau („Remove“), die Struktur entfernt. Wenn Abbau als Spiegelbild von Verkettung existiert, dann sollte die Nullhypothese sein, dass die beiden Operationen exakt denselben Beschränkungen unterliegen. Die folgenden Annahmen werden für Verkettung gemacht: 
(24) Annahmen über Verkettung:

a. Verkettung ist merkmalsgetrieben. Die Operation wird ausgelöst durch designierte Merkmale $[\bullet \mathrm{F} \bullet$, die auf lexikalischen Elementen geordnet erscheinen und der Reihe nach abgearbeitet (und gelöscht) werden.

b. Verkettung unterliegt der Bedingung des Strikten Zyklus in (25) (vgl. Chomsky 1973, 1995, 2001, 2008).

c. Verkettung kann Phrasen (XP) oder Köpfe $\left(\mathrm{X}^{0}\right)$ betreffen.

(25) Bedingung des Strikten Zyklus („Strict Cycle Condition“):

In der gegenwärtigen XP $\alpha$ kann sich eine syntaktische Operation nicht exklusiv auf ein Element $\delta$ im Bereich einer anderen XP $\beta$ beziehen, wenn $\beta$ selbst im Bereich von $\alpha$ ist.

(Der Bereich eines Kopfes X umfasst die Menge von Knoten, die von XP dominiert werden und weder $\mathrm{X}$ enthalten noch mit X identisch sind; vgl. Chomsky 1995.)

Die Bedingung des Strikten Zyklus hat zur Folge, dass Verkettung immer nur in der höchsten aktuellen Projektion möglich ist (vgl. auch die sog. Erweiterungsbedingung („Extension Condition“) aus Chomsky 1995).

Wie (26) zeigt, unterliegt Abbau denselben Beschränkungen:

(26) Annahmen über Abbau:

a. Abbau ist merkmalsgetrieben. Die Operation wird ausgelöst durch designierte Merkmale [-F-], die auf lexikalischen Elementen geordnet erscheinen und der Reihe nach abgearbeitet (und gelöscht) werden.

b. Abbau unterliegt der Bedingung des Strikten Zyklus.

c. Abbau kann Phrasen (XP) oder Köpfe $\left(\mathrm{X}^{0}\right)$ betreffen.

Aus (26b) ergibt sich, dass Material, das im Laufe der Derivation von Abbau betroffen sein soll, immer nur einen kurzen Lebenszyklus haben kann - wenn die Derivation $\mathrm{zu}$ weit vorangeschritten ist und das abzubauende Element in einer Phrase eingebettet ist, die nicht der derzeitige Wurzelknoten ist, kann es wegen der Bedingung des Strikten Zyklus nicht mehr entfernt werden.

In (27) ist abstrakt illustriert, wie ein Komplement YP, das vorher aufgrund eines entsprechenden Merkmals $[\bullet \mathrm{Y} \bullet$ ] auf dem Kopf $\mathrm{X}$ mit $\mathrm{X}$ verkettet worden ist, danach durch das Abbaumerkmal [-Y-] auf X wieder entfernt wird. Man beachte, dass ZP und WP hier nicht von X entfernt werden könnten (selbst 
wenn X die geeigneten Abbaumerkmale dafür hätte), weil diese Phrasen zu tief eingebettet sind und ihre Entfernung somit die Bedingung des Strikten Zyklus verletzen würde.

(27) Abbau: Komplemente

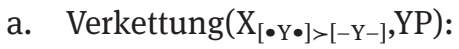

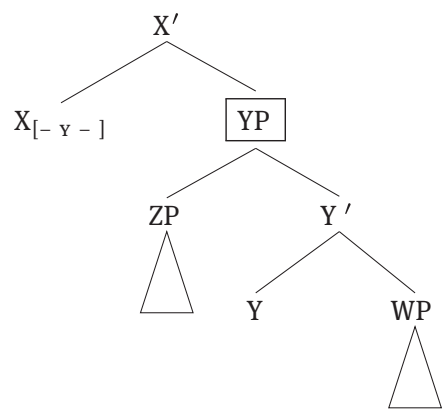

b. $\operatorname{Abbau}\left(\mathrm{X}_{[-\mathrm{Y}-]}, \mathrm{YP}\right)$ :

$\mathrm{X}$

(28) zeigt analog den Abbau von Spezifikatoren. Wiederum können ZP und WP aufgrund der Bedingung des Strikten Zyklus in dieser Konfiguration nicht entfernt werden.

Grundsätzlich solltes es allerdings möglich sein, UP hier erfolgreich von Abbau affizieren zu lassen. ${ }^{12}$

(28) Abbau: Spezifikatoren

a. Verkettung $\left(\mathrm{X}_{\left[\bullet \mathrm{Y}_{\bullet}^{\prime}\right]>[-\mathrm{Y}-]}, \mathrm{YP}\right)$ :

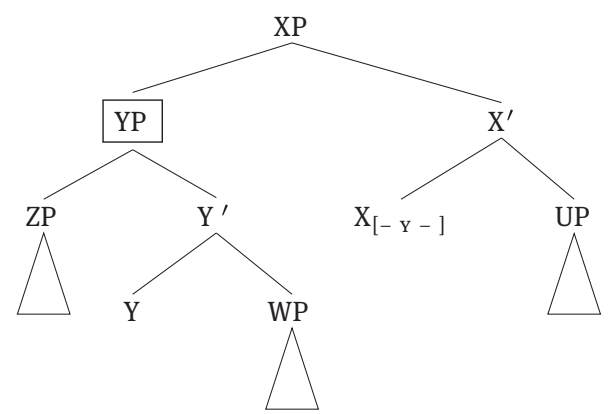

12 Richards (2001) argumentiert, dass genau dies auch bei Verkettung erlaubt ist; vgl. sein Konzept des „tucking in“. 
b. $\operatorname{Abbau}\left(\mathrm{X}_{[-\mathrm{Y}-]}^{\prime}, \mathrm{YP}\right)$ :

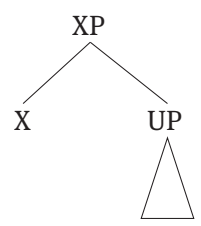

Vor diesem Hintergrund wird in Müller (2016, 2017) eine Analyse des Passivs im Deutschen entwickelt, die im Kern wie folgt funktioniert: Passiv wird ausgelöst durch die optionale Hinzufügung eines Merkmals [-D-] zum funktionalen Kopf $\mathrm{v}$ in der präsyntaktischen Numeration, also dem Bereich, in den während der Derivation zu benutzende Lexikon-Elemente zunächst platziert werden, bevor die syntaktische Derivation anläuft. Damit kommt das Abbau-Merkmal gerade auf jenem funktionalen Kopf zu liegen, der auch das externe Argument einführt. Dieses Merkmal muss (um Effekte zeitigen zu können) unter dem strukturaufbauenden Merkmal geordnet sein, das zur Verkettung des externen Arguments als Spezifikator von v führt. Und sobald das Abbaumerkmal [-D-] auf v an der Reihe ist, entfernt es ein externes Argument in dieser Position; denn auch wenn das strukturabbauende Merkmal nicht inhärent auf externe Argumente abzielt, sondern einfach auf nominale Argumente (DPs), so ist doch in der vP eine DP im Spezifikator von v die einzig zugängliche DP (interne Argumente in der VP sind zu tief eingebettet, um im Einklang mit der Bedingung des Strikten Zyklus Strukturabbau zu ermöglichen). Kasusabsorption kann so analysiert werden, dass die Berechnung der Kasuszuweisung etwa gemäß des Konzepts des abhängigen Kasus (vgl. die Literaturangaben in Fußnote 3 auf Seite 516) nach der Entfernung des externen Arguments durchgeführt wird. Eine Derivation einer passivischen vP, wie sie unter diesen Annahmen im Deutschen erfolgt, ist in (29) skizziert.

(29) Ableitung einer passivischen vP:
a. $\mathrm{V}_{[\bullet \mathrm{V} \bullet]>[\bullet \mathrm{D} \bullet]>[-\mathrm{D}-]},[\mathrm{VP}$ das Buch gelesen ]
b. $\left[\mathrm{v}^{\prime} \mathrm{v}_{[\bullet \mathrm{D} \bullet]>[-\mathrm{D}-]}[\mathrm{vP}\right.$ das Buch gelesen $\left.]\right]$
c. $\left[{ }_{v P} \mathrm{DP}_{\text {ext }}\left[\mathrm{v}^{\prime} \mathrm{V}_{[-\mathrm{D}-]}[\mathrm{vP}\right.\right.$ das Buch gelesen $\left.\left.]\right]\right]$
d. .... Syntaktische Zugänglichkeit von $\mathrm{DP}_{e x t} \ldots$
e. [ $\mathrm{vP}_{\mathrm{V}}[\mathrm{vP}$ das Buch gelesen ]]

Man sieht, dass das externe Argument - $\mathrm{DP}_{\text {ext }}$ - nach diesen Annahmen nur eine knapp bemessene Lebensspanne hat: Es gelangt beim Aufbau der vP in 
die Struktur (vgl. (29c)), ist dann zunächst einmal zugänglich für andere Operationen (vgl. (29d)), und wird schließlich wieder entfernt, bevor die Derivation zur nächsthöheren Phrase (TP) voranschreitet (vgl. (29e)).

Diese Analyse sagt u.a. voraus, dass unpersönliches Passiv von intransitiven Verben im Deutschen nur mit unergativischen Verben gebildet werden kann (bei denen das einzige Argument extern ist, also im Spezifikator von vP verkettet wurde), aber nicht mit unakkusativischen Verben (bei denen das einzige Argument intern ist, also innerhalb der VP verkettet wurde und somit aufgrund der Bedingung des Strikten Zyklus von v nicht dem Abbau ausgesetzt werden kann). Klassischerweise wird angenommen, dass diese Konsequenz erwünscht ist; vgl. (30a) gegenüber (30b). ${ }^{13}$

(30) a. dass dann gearbeitet wurde.

b. *dass dann gefallen wurde.

Abschließend sei noch vermerkt, dass optionale von-Phrasen in dieser Analyse so modelliert werden, dass angenommen wird, dass eine durch [-D-] auf v aus der Struktur entfernte $\mathrm{DP}_{\text {ext }}$ in den Arbeitsbereich der Derivation zurückgeschoben wird (aus dem sie auch ursprünglich kam), und dann optional auf die einzige Art und Weise, die ohne merkmalsgetriebene Verkettung möglich ist, wieder in die Struktur integriert werden kann, nämlich als Adjunkt. Diese Detailaspekte der Analyse sind allerdings im vorliegenden Zusammenhang nicht von entscheidender Bedeutung; entscheidend ist die Konsequenz bzgl. der Zugänglichkeit externer Argumente in deutschen Passivsätzen: Es wird vorhergesagt, dass externe Argumente im Passiv zunächst zugänglich sind, und später aber aufgrund der vollständigen, spurlosen Entfernung aus der Struktur unzugänglich sind. Weil in dem hier allgemein zugrundegelegten derivationellen Ansatz syntaktische Strukturen von unten nach oben wachsen, lässt sich diese zeitliche Asymmetrie bzgl. der Sichtbarkeit in eine strukturelle Asymmetrie übersetzen: Externe Argumente sollten innerhalb ihrer minimalen XP sicht-

13 Vgl. aber auch Primus (2010, 2011), Kiparsky (2013) zu gegensätzlichen Einschätzungen, sowie Müller, St. (2007) (Kap. 17) und Alexiadou, Anagnostopoulou \& Schäfer (2018) zu weiterer Diskussion. Generell lässt sich argumentierten, dass Fälle scheinbarer Passivierung von unakkusativischen Verben im Deutschen typischerweise einen meta-grammatischen Gebrauch einer an sich ungrammatischen Konstruktion involvieren, oder eine Reinterpretation von unakkusativischen Verben als unergativische Verben (dadurch, dass dem einzigen DP-Argument eine Agens-artige Interpretation gegeben wird). Manche agentivischen Verben der Bewegung, die Passivierung erlauben, aber wie unakkusativische Verben sein im Perfekt nehmen, sind in dieser letzteren Beziehung eine Ausnahme; vgl. Grewendorf (1989). 
bar sein (Abwärtszugänglichkeit), außerhalb ihrer minimalen XP jedoch nicht (Aufwärtsunzugänglichkeit).

Insgesamt ergeben sich somit für die vier Analysetypen die folgenden Vorhersagen im Hinblick auf die Zugänglichkeit externer Argumente in deutschen Passivsätzen (immer vorausgesetzt, dass keine von-Phrase auftritt):

(31) Vorhersagen bzgl. Zugänglichkeit von DPext:

\begin{tabular}{lllll}
\hline & Typ-A-Analysen & Typ-B-Analysen & Typ-C-Analysen & Typ D-Analysen \\
\hline $\begin{array}{l}\text { Zugänglichkeit } \\
\text { von DP }\end{array}$ & - & + & - & + \\
\hline $\begin{array}{l}\text { Unzugänglich- } \\
\text { keit von DP }\end{array}$ & + & - & + & + \\
\hline
\end{tabular}

Im folgenden Abschnitt tragen wir den bisherigen Stand der Forschung zur Frage der Zugänglichkeit externer Argumente im deutschen Passiv zusammen.

\section{Zugänglichkeit externer Argumente im deutschen Passiv}

Was die Frage der Zugänglichkeit externer Argumente im deutschen Passiv betrifft, so ist die bisher verfügbare empirische Evidenz nicht konklusiv. Auf der einen Seite gibt es Evidenz für die Annahme einer syntaktischen Unzugänglichkeit externer Argumente in deutschen Passivkonstruktionen. Hierzu zählt etwa die fehlende Verfügbarkeit von Bindung durch eine quantifizierte DP in einem höheren Satz (vgl. Alexiadou, Anagnastopoulou \& Schäfer 2015). Wie (32a) zeigt, kann das externe Argument eines passivierten Verbs im eingebetteten Satz nicht von einem quantifizierten Matrix-Subjekt gebunden werden. ${ }^{14}$ Ist das externe Argument dagegen mit Hilfe einer von-Phrase syntaktisch reaktiviert, ist derartige lange Bindung unproblematisch; vgl. (32b). ${ }^{15}$

14 Die fehlende syntaktische Zugänglichkeit des externen Arguments wird hier und im Folgenden signalisiert durch die Notation $\mathrm{PP}_{\text {ext }}$.

15 Dieselbe Asymmetrie tritt z. B. auch im Englischen auf; vgl. (ia) gegenüber (ib).

(i) a. *Every journalist $t_{1}$ wants the president to be $\mathrm{DP}_{\text {ext }_{1}}$ interviewed.

b. Every journalist ${ }_{1}$ wants the president to be interviewed by him $_{1}$.

Nur (ib) kann die Lesart repräsentatieren, dass für alle Journalisten gilt, dass sie selbst den Präsidenten interviewen möchten. 
(32) a. *Kein Student $t_{1}$ sagt $\left[\mathrm{CP}\right.$ dass $\mathrm{DP}_{\text {ext }}$ schlecht gearbeitet wurde ].

b. Kein Student ${ }_{1}$ sagt $\left[\mathrm{CP}\right.$ dass $\mathrm{DP}_{\text {ext }_{1}}$ schlecht $\left[{ }_{\mathrm{PP}}\right.$ von $\left.\mathrm{ihm}_{1}\right]$ gearbeitet wurde ].

Ein zweites Argument für syntaktische Unzugänglichkeit von externen Argumenten im deutschen Passiv ergibt sich aus der Unmöglichkeit, in einem Infinitivsatz von einem Argument des übergeordneten Satzes kontrolliert zu werden (vgl. Stechow \& Sternefeld 1988; Wunderlich 1989; Stechow 1989 sowie Haider 2010): Kontroll-Infinitive müssen ein für Kontrolle von außen zugängliches Subjekt haben, und das externe Argument von intransitiven infinitven Verben im Passiv kann diese Bedingung im Deutschen offensichtlich nicht erfüllen; vgl. (33a, b). Der Fall liegt anders bei internen Argumenten von transitiven infiniten Verben im Passiv; diese sind (als PRO-Argumente) von außen kontrollierbar; vgl. (33c, d).

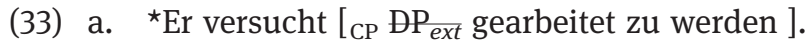

b. *weil [ $\mathrm{CP}$ bald $\mathrm{DP}_{\text {ext }}$ geschlafen zu werden ] gewünscht wird.

c. Er versucht $\left[{ }_{\mathrm{CP}}\left[{ }_{\mathrm{DP}_{\text {int }}} \mathrm{PRO}\right]\right.$ geliebt zu werden $\left.]\right]$.

d. weil $\left[{ }_{\mathrm{CP}}\left[\mathrm{DP}_{\text {int }} \mathrm{PRO}\right]\right.$ geliebt zu werden $\left.]\right]$ gewünscht wird.

Ein drittes Argument dafür, dass externe Argumente im deutschen Passiv syntaktisch nicht zugänglich sind, ergibt sich aus der Unmöglichkeit, dass durch $\mathrm{DP}_{\text {ext }}$ ein kriterialer Auslöser für Bewegung erfüllt werden kann, wie z. B. die obligatorische Vorfeldbesetzung in deutschen Hauptsätzen; vgl. das ungrammatische Beispiel (34a) (ohne von-Phrase) mit dem wohlgeformten Satz (34b) (in dem die von-Phrase das Vorfeldkriterium erfüllt).

(34) a. *Ich denke [ ${ }_{\mathrm{CP}} \mathrm{DP}_{\text {ext }}$ ist gut gearbeitet worden ].

b. Ich denke $\left[\mathrm{CP}\left[\mathrm{PP}\right.\right.$ von $\left.\mathrm{ihr}_{1}\right]$ ist gut gearbeitet worden ].

Viertens würde man, wie Collins (2005) zuerst für das Englische festgestellt hat, eigentlich erwarten, dass in einem Passivsatz ein externes Argument in Specv die Bewegung eines internen Arguments in die abgeleitete Subjektposition SpecT aufgrund eines Minimalitätseffekts blockieren sollte. Es kommt jedoch keine solche fatale Intervention zustande. Für das Deutsche ist das Ausbleiben eines Minimalitätseffekts etwas schwieriger nachzuweisen, weil es in dieser Sprache keine obligatorische EPP-getriebene Bewegung in die Subjektposition gibt (s. o.). In Müller (2001) wird argumentiert, dass eine nominati- 
vische DP, die schwachtonigen Objektpronomina im Mittelfeld vorangeht, zeigt, dass es eine optional zu besetzende abgeleitete Subjektposition SpecT im Deutschen gibt, und dass nur Subjekte (bzw. nominativisch kasusmarkierte DPs) diese Position ansteuern können; vgl. die Beispiele in (35) (mit nominativischen Subjekten in (35a, b) und dativischen Objekten in (35c, d)).

(35) a. dass die Maria es gelesen hat.

b. dass es die Maria gelesen hat.

c. *dass der Maria es gefallen hat.

d. dass es der Maria gefallen hat.

Aus dieser Perspektive zeigen dann Beispiele wie das in (36a), dass die optionale Bewegung eines internen Arguments (hier: der Karl) nach SpecT im Passiv keine Verletzung der Minimalitätsbedingung nach sich zieht; und dies folgt dann auf einfache Weise aus der Annahme, dass gar kein externes Argument im Passiv vorhanden ist, das zwischen der VP-internen Basisposition des internen Arguments und der Zielposition SpecT intervenieren könnte. (In (36b) ist von vornherein die Bewegung des internen Arguments unterblieben.) ${ }^{16}$

(36) a. dass der $\operatorname{Karl}_{2} \operatorname{ihr}_{3}\left[{ }_{v P} D P_{\text {ext, },}\left[v_{v^{\prime}}\left[{ }_{v P} t_{3} t_{2}\right.\right.\right.$ vorgestellt $] v$ ] $]$ wurde.

b. dass $\operatorname{ihr}_{3}\left[{ }_{v P} D P_{\text {ext, } 1}\left[v_{v^{\prime}}\left[{ }_{v P} t_{3}\right.\right.\right.$ der $\operatorname{Karl}_{2}$ vorgestellt $\left.\left.] v\right]\right]$ wurde.

Ein fünftes und im gegenwärtigen Zusammenhang letztes Argument für die Unzugänglichkeit von externen Argumenten im deutschen Passiv betrifft lassen-Passive, wie sie oben in (5) bereits angeführt wurden als Indikator dafür, dass nicht alle Passive notwendigerweise morphologische Reflexe nach sich ziehen müssen. Das Argument beruht auf der Transparenz von lassen-Passiven für Reflexivierung (vgl. Pitteroff 2014). Man betrachte zunächst die aktivische lassen-Einbettung in (37b), eine reguläre AcI-Konstruktion im Deutschen. Hier kann sich das eingebette Objekt-Reflexivpronomen nicht auf den König beziehen, sondern nur auf die Diener. Der Grund ist, dass das akkusativisch markierte Subjekt des Infinitivs die Diener strukturell interveniert zwischen dem Reflexivum sich und dem Matrix-Subjekt der König; das eingebettete externe Argument ist für das Reflexivum das nächste Subjekt und blockiert somit lange

16 Bei genauerem Hinsehen erweist sich, dass für die vollständige Gültigkeit dieses Arguments noch ein paar weitere Annahmen gemacht werden müssen, z. B. zur Irrelevanz von Minimalitätseffekten für vP-interne Bewegungen im Deutschen. Siehe hierzu Müller (2016). 
Bindung. ${ }^{17}$ Anders liegt der Fall beim lassen-Passiv in (37a). Hier ist die Koreferenz von sich und der König problemlos möglich. ${ }^{18}$ Dies legt nahe, dass das (kontra-indizierte) externe Argument des Infinitivs in (37a) nicht vorhanden ist; somit kann der König für sich hier als nächstes Subjekt fungieren.
a. Der König 1 lässt [pass $\mathrm{DP}_{\text {ext }_{2}} \operatorname{sich}_{1 / 2}$ rasieren ].
b. Der König 1 lässt [akt die $_{\text {Diener }} \operatorname{sich}_{* 1 / 2}$ rasieren ].

So viel zu den Argumenten gegen die syntaktische Sichtbarkeit von externen Argumenten im deutschen Passiv. Auf der anderen Seite gibt es nun jedoch auch widerstreitende Evidenz, die die syntaktische Zugänglichkeit externer Argumente in deutschen Passivkonstruktionen nahelegen könnte. Ein erstes mögliches Argument involviert Kontrolle aus passivierten übergeordneten Sätzen in adverbiale Nebensätze hinein (vgl. Roberts 1987), wie in (38). Hier sieht es zunächst einmal so aus, als sei die syntaktische Präsenz von $\mathrm{DP}_{\text {ext }}$ im Hauptsatz notwendig, um das eingebettete PRO-Subjekt in seiner Referenz zu determinieren - der/diejenige, der/die den Reifen aufpumpt, ist auch der/diejenige, der/die die Fahrt fortsetzen möchte.

(38) Der Reifen wurde $\mathrm{DP}_{\text {ext }}$ aufgepumpt [CP $\mathrm{PRO}_{1}$ um die Fahrt fortzusetzen ].

Ebenso ist, wie Müller, St. (2002) beobachtet (und sorgfältig empirisch absichert), Kontrolle auch in sekundäre Prädikate hinein möglich; vgl. (39a, b). ${ }^{19}$ Erneut suggeriert das die Präsenz des externen Arguments im passivierten Matrixsatz.

17 Tatsächlich sind, wie Reis (1976) gezeigt hat, AcI-Konstruktionen im Deutschen manchmal trotz Präsenz eines eingebetteten Subjekts für lange Reflexivierung transparent; vgl. auch Grewendorf (1983), Gunkel (2003) und Barnickel (2014), neben anderen. Eine derartige Transparenz tritt jedoch immer nur mit Reflexivpronomina auf, die in PPs eingebettet sind; und eine solche Situation liegt in (37) nicht vor, weil hier die Reflexivpronomina direkt Argumente des eingebetteten Verbs sind. Im Einklang damit scheint die Ungrammatikalität von (37b) (bei Index 1 auf dem Reflexivpronomen) für die meisten SprecherInnen des Deutschen völlig unkontrovers.

18 Die alternative Lesart, derzufolge es wieder die Diener sind, die sich rasieren, ist ebenfalls hier verfügbar. Für den Moment sehen wir von dieser Option ab; wir werden darauf zurückkommen.

19 SC steht hier für „small clause“, also die Kategorie, die der Projektion des sekundären Prädikats zukommt. Möglicherweise ist dies schlicht die Kategorie des Adjektivs selbst. 
(39) a. Das Handout wurde $\mathrm{DP}_{\text {ext }}\left[\mathrm{SC} \mathrm{PRO}_{1}\right.$ übermüdet ] verfasst.

b. Auf dem Land wird $\mathrm{DP}_{\text {ext }}\left[\mathrm{sC}_{\mathrm{SC}} \mathrm{PRO}_{1}\right.$ betrunken $]$ gefahren.

In Sprachen, die Vissers Generalisierung umgehen können (vgl. van Urk 2013), ist Kontrolle aus einem passivierten übergeordneten Satz nicht nur in einen infiniten Adverbialsatz hinein möglich (wie in (38)), sondern auch in einen infiniten Objektsatz; vgl. (40). Auch dies legt zunächst einmal nahe, dass das externe Argument des passivierten Matrixverbs syntaktisch präsent ist (vgl. Müller (2016); Pitteroff \& Schäfer (2017)).

(40) Es wurde $\mathrm{DP}_{\text {ext }}$ versucht [CP $\mathrm{PRO}_{1} \mathrm{zu}$ schlafen ].

Viertens kann ein externes Argument im Passiv im Deutschen auch ObjektReflexivpronomina und Objekt-Reziprokpronomina lizensieren; vgl. (41a, b) (aus Müller 1993, 2016), sowie auch schon die alternative Lesart von (37a), die in Fußnote 18 erwähnt wurde. Wenn Reflexivpronomina im Deutschen immer ein syntaktisch vorhandendes Antezedens brauchen, ist auch dies ein Argument für (i) die Präsenz und (ii) die Zugänglichkeit von $\mathrm{DP}_{\text {ext }}$ im deutschen Passiv.

(41) a. Hier wurde $\mathrm{DP}_{\text {ext }_{1}}$ sich $_{1}$ nicht geprügelt.

b. Es wurde $\mathrm{DP}_{\text {ext }}$ einander ${ }_{1}$ gedankt.

Es ergibt sich somit konfligierende Evidenz bzgl. der Zugänglichkeit von externen Argumenten im deutschen Passiv. Die Schlussfolgerung in Müller (2016) ist, dass beide Typen von Evidenz gültig sind, und dass der relevante Unterschied ist, dass externe Argumente im deutschen Passiv für Prozesse, die tiefere (genauer: in der minimalen XP enthaltene) Struktur involvieren, zugänglich sind, aber für Prozesse, die höhere (genauer: außerhalb der minimalen XP liegende) Struktur unzugänglich sind.

Eine andere mögliche Schlussfolgerung ist die, einen der beiden Typen von konfligierender Evidenz als nicht gültig zu erweisen. So ist insbesondere argumentiert worden, dass die existierenden Argumente für die Zugänglichkeit von externen Argumenten im Passiv in Sprachen wie dem Deutschen oder Englischen nicht ganz unkontrovers sind; vgl. insbesondere Schäfer (2012b), Landau (2013, Kap. 6), Williams (2015, Kap. 12) und Alexiadou, Anagnastopoulou \& Schäfer (2015) zur Infragestellung der Relevanz der Kontroll- und Reflexivierungsevidenz für syntaktische Zugänglichkeit. Konkret wird in Schäfer (2012b) und Alexiadou, Anagnastopoulou \& Schäfer (2015) vorgebracht, dass Reflexivierung wie in (41) sprachübergreifend selten ist und außerdem auch noch 
unerwartete Einschränkungen und Eigenschaften aufweist. Landau (2013, Kap. 6) und Williams (2015, Kap. 12) haben argumentiert, dass Kontrolle in adverbiale Infinitive hinein kein gutes Diagnostikum für die Präsenz von nicht sichtbaren externen Argumenten ist. Wenn vergleichbare Vorbehalte auch gegen die Evidenz auf der Grundlage von sekundären Prädikaten (vgl. (39)) und Kontrolle in Objektsätze hinein (vgl. (40)) vorgebracht werden und als stichhaltig erwiesen werden können, ist die Annahme syntaktischer Sichtbarkeit von externen Argumenten im deutschen Passiv nicht mehr sehr stark gestützt; aber vgl. auch Müller (2016), wo versucht wird, alle diese Gegenargumente gegen selektive Zugänglichkeit von externen Argumenten im Passiv zu entkräften. ${ }^{20}$

Vor dem Hintergrund solcherart kontroverser Evidenz möchten wir im folgenden Abschnitt ein weiteres Argument für die Zugänglichkeit von externen Argumenten im deutschen Passiv vorbringen, eines, das in der bisherigen Diskussion zum deutschen Passiv noch keine Rolle gespielt hat. Das Argument beruht auf dem Auftreten von Effekten quantifikationaler Variabilität. ${ }^{21}$

\section{Effekte quantifikationaler Variabilität}

Effekte quantifikationaler Variabilität, wie sie mit indefiniten DPs im Englischen auftreten, sind in (42) illustriert (vgl. Heim 1982; Diesing 1992).

20 Ein weiteres mögliches Argument gegen die Zugänglichkeit von externen Argumenten im deutschen Passiv für tiefer lokalisierte Elemente geht auf Haider (2010) zurück. Wie Koster \& May (1982) für das Isländische beobachtet hatten, liefert die Kasuskongruenz im sekundären Prädikat in Fällen wie (ia) ein Argument für die Postulierung eines leeren Subjekts PRO im Infinitiv, von dem der Kasus für das abhängige Element (hier: als erster) kommt; denn die kontrollierende DP im Matrixsatz (hier: $i \mathrm{hm}$ ) hat ja einen anderen Kasus. Haider zeigt nun, dass das externe Argument in deutschen Passivsätzen diese Aufgabe nicht leisten kann; vgl. (ib).

(i) a. Wir empfahlen ihm [CP PRO als erster zu streiken ].

b. *Damals wurde $\mathrm{DP}_{\text {ext }}$ als erster gestreikt.

Es ist aber nicht ganz klar, ob (ib) als Hinweis auf die Unzugänglichkeit des externen Arguments betrachtet werden sollte. Alle uns bekannten Passivanalysen, die in der ein oder anderen Form ein syntaktisch vorhandenes externes Argument im Passiv vorsehen, konvergieren in der Annahme, dass dieses Element keinen Nominativ erhält (denn der muss ja in transitiven Kontexten für das interne Argument verfügbar sein). Damit entfällt aber die potenzielle Quelle für die Kasuskongruenz mit als erster in Satz (ib) (der im Übrigen auch nicht besser wird, wenn das externe Argument in einer von-Phrase wieder aufgenommen wird.)

21 Das vorliegende Material haben wir zum ersten Mal im Herbst 2015 präsentiert; in der Zwischenzeit haben wir bemerkt, dass Malamud (2004, 107-108) in ganz anderem Kontext für das Russische im Wesentlichen dieselbe Beobachtung gemacht hat, und eine analoge Schlussfolgerung zieht. 
(42) A cat is usually smart. $\cong$ Most cats are smart.

(43b) zeigt den gleichen Effekt für eingebettete Fragesätze (genauer, indirekte Ergänzungsfragen) (vgl. Berman 1991).

(43) John partly remembers who cheated.

Eine Standardannahme, die wir hier übernehmen, ist die, dass eine indefinite DP (die auch eine W-Phrase sein kann, wie in (43)), einen offenen Satz denotiert, mit einer freien Individuenvariable, die von einem Quantifikationsadverb unselektiv gebunden werden kann. Im Einklang damit ist existentielle Quantifikation keine inhärente Eigenschaft einer indefiniten DP; es handelt sich hierbei lediglich um eine Default-Operation, die im Prinzip durch einen im Satz vorhandenen anderen quantifikationalen Ausdruck (wie die Adverbien usually und partly in (42) und (43)) außer Kraft gesetzt werden kann. ${ }^{22}$

Die zentrale Beobachtung ist nun, dass das externe Argument in deutschen Passivkonstruktionen auch Effekten quantifikationaler Variabilität unterworfen sein kann. Dies zeigen die Beispiele in (44a, b, c), mit den Quantifikationsadverbien größtenteils (in (44a)), zum Teil (in (44b)) und normalerweise (in (44c)). Die aus den Paraphrasen ersichtlichen Interpretationen setzen Lesarten mit unselektiver Bindung des nicht sichtbaren externen Arguments voraus (das hier wieder als $\mathrm{DP}_{\text {ext }}$ notiert wird). Diese Lesarten sind für SprecherInnen problemlos zu bekommen und vollkommen natürlich. ${ }^{23}$

(44) a. Es wurde größtenteils $\mathrm{DP}_{\text {ext }}$ geschlafen beim Vortrag.

„Die meisten Leute schliefen beim Vortrag.“

b. Dann wurde der Sprecher zum Teil DP $\mathrm{P}_{\text {ext }}$ ausgebuht. „Dann buhten einige Leute den Sprecher aus.“

c. Damals wurde normalerweise $\mathrm{DP}_{\text {ext }}$ mehr als fünf Tage in der Woche gearbeitet.

„Damals arbeiteten die meisten Leute mehr als fünf Tage in der Woche.“

22 Auf der Basis von Effekten quantifikationaler Variabilität mit definiten DPs stellt Hinterwimmer (2005) diese Analyse in Frage; aber vgl. Zobel (2014) zu Argumenten dafür, die Standardanalyse beizubehalten für indefinite DPs von dem Typ, an dem wir hier interessiert sind. 23 Daneben sind natürlich auch Lesarten ohne Bindung des externen Arguments durch das Quantifikationsadverb möglich, denen gemäß über Zeitspannen, Situationen oder Ähnliches quantifiziert wird. 
Damit die Bindung durch das Quantifikationsadverb in (44a, b, c) möglich ist, muss $\mathrm{DP}_{\text {ext }}$ in der syntaktischen Struktur (i) vorhanden und (ii) zugänglich (also nicht bereits existentiell abquantifiziert worden) sein. Außerdem muss das Quantifikationsadverb $\mathrm{DP}_{\text {ext }}$ c-kommandieren.

Der Effekt quantifikationaler Variabilität mit externen Argumenten in Passivkonstruktionen ist in seiner Distribution stark restringiert. Zunächst einmal ist zu bemerken, dass es zwischensprachliche Variation gibt. Wie z. B. (45) zeigt, gibt es keinen derartigen Effekt mit externen Argumenten in griechischen Passivkonstruktionen (die wie oben vermerkt Instanzen des Genus Verbi Medium sind).

(45) O omilitis $\mathrm{DP}$ ext giuharistike $\quad$ en meri.
der Sprecher $\quad$ ausbuhen.NONAKT.3sG zum Teil
„ ${ }^{\star}$ Manche Leute buhten den Sprecher aus.“

Andererseits ist das Phänomen aber auch keineswegs auf das Deutsche beschränkt. So weist neben dem Russischen (vgl. Fußnote 21) etwa auch das Norwegische Effekte quantifikationaler Variabilität bei Passivierung auf, und zwar sowohl im analytischen Passiv (vgl. (46a)), als auch im synthetischen Passiv (vgl. (46b)). ${ }^{24}$

(46) a. I Spania ble sangeren stort sett $\mathrm{DP}_{\text {ext }}$ buet ut. in Spanien wurde Sänger-DEF größtenteils ausgebuht „In Spanien buhten die meisten Leute den Sänger aus.“

b. I Spania bues sangeren stort sett $\mathrm{DP}_{\text {ext }}$ ut. in Spanien buhen.PASs Sänger-DEF größtenteils aus „In Spanien buhen die meisten Leute den Sänger aus.“

Darüber hinaus tritt z. B. auch im deutschen Zustandspassiv offenbar kein Effekt quantifikationaler Variabilität auf; vgl. (47a) (Vorgangspassiv, mit Effekt quantifikationaler Variabilität) mit (47b) (Zustandspassiv, ohne solchen Effekt).

(47) a. Der Aufsatz wurde größtenteils $\mathrm{DP}_{\text {ext }}$ gelesen.

b. Der Aufsatz war größtenteils DP ext gelesen.

24 Wir bedanken uns bei Terje Lohndal für Daten und Diskussion. 
(47a) kann die Lesart haben, dass der größte Teil des Aufsatzes gelesen wurde, aber auch die - im gegenwärtigen Zusammenhang interessantere - Lesart, dass die meisten Leute (z. B. kontextuell restringiert: die meisten SeminarteilnehmerInnen) den Aufsatz gelesen haben. (47b) kann diese zweite Lesart nicht haben; nur die erste Lesart ohne Effekt quantifikationaler Variabilität ist möglich. 25

Weiterhin lässt sich festhalten, dass $\mathrm{DP}_{\text {ext }}$ von einen Quantifikationsadverb nicht außerhalb des minimalen Satzes gebunden werden kann. Dies zeigen die Beispiele in (48a, b, c), wo nur Lesarten möglich sind, denen zufolge das Adverb über Zeitspannen oder Situationen quantifiziert. ${ }^{26}$

(48) a. Es war größtenteils so [ ${ }_{\mathrm{CP}}$ dass $\mathrm{DP}_{\text {ext }}$ geschlafen wurde beim Vortrag ]. „ ${ }^{\star}$ Die meisten Leute schliefen beim Vortrag.“ „Die meiste Zeit schliefen die Leute beim Vortrag.“

b. Es war zum Teil so [ ${ }_{\mathrm{CP}}$ dass der Sprecher $\mathrm{DP}_{\text {ext }}$ ausgebuht wurde ]. „ ${ }^{\star}$ Einige Leute buhten den Sprecher aus.“ „An einigen Stellen des Vortrags wurde der Sprecher ausgebuht.“

c. Es war normalerweise so $\left[_{\mathrm{CP}}\right.$ dass $\mathrm{DP}_{\text {ext }}$ mehr als fünf Tage in der Woche gearbeitet wurde ].

„?^Die meisten Leute haben mehr als fünf Tage in der Woche gearbeitet."

„Zu den meisten Zeiten wurde mehr als fünf Tage in der Woche gearbeitet."

Das Fehlen des Effekts quantifikationaler Variabilität in (48) zeigt, dass dieser Effekt bei nicht sichtbaren externen Argumenten in deutschen Passivsätzen ein extrem lokales Phänomen ist. Interessanterweise sieht es so aus, als befänden sich die hier involvierten Quantifikationsadverbien immer in einer relativ tiefen syntaktischen Position - konkret: als seien sie in der vP enthalten. So können diese Quantifikationsadverbien z. B. an vP-Topikalisierung teilnehmen,

25 Zumindest gilt dies wohl für die meisten SprecherInnen. Wenn sich auch eine liberalere Varietät des Deutschen substantiieren lässt, mit SprecherInnen, die quantifikationale Variabilität auch beim Zustandspassiv erlauben, dann könnte dies dafür sprechen, Vorgangs- und Zustandspassiv im Deutschen noch etwas mehr anzugleichen (vgl. Alexiadou, Anagnastopoulou \& Schäfer (2015) zur Annahme, dass auch das Zustandspassiv im Deutschen über eine VoiceProjektion verfügt).

26 Hier liegt der Fall im Übrigen ganz anders bei offen realisierten indefiniten DPs; diese können nicht-lokal von einem Quantifikationsadverb gebunden werden (vgl. Heim (1982)). 
im Gegensatz etwa zu Satzadverbien, die in einer höheren, vP-externen Position basisverkettet werden. Die Sätze in (49a, b, c) haben jeweils ein Quantifikationsadverb, das bei externen Argumenten im Passiv einen Effekt der quantifikationalen Variabilität auslösen kann; Topikalisierung der komplexen verbalen Konstituente ist problemlos möglich. ${ }^{27}$

(49) a. [ [vP1 Größtenteils DP ${ }_{\text {ext }}$ geschlafen ] wurde beim Vortrag $t_{1}$. „Die meisten Leute schliefen beim Vortrag.“

b. [ ${ }_{v P 1}$ Zum Teil DP ${ }_{\text {ext }}$ ausgebuht ] wurde der Sprecher gestern $t_{1}$. „Einige Leute buhten den Sprecher aus.“

c. [ ${ }_{\mathrm{vP}}$ Normalerweise $\mathrm{DP}_{\text {ext }}$ mehr als fünf Tage in der Woche gearbeitet ] wurde damals nicht.

„Damals war es nicht der Fall, dass die meisten Leute mehr als fünf Tage in der Woche gearbeitet haben.“

Umgekehrt zeigt (50) die Unmöglichkeit der Topikalisierung eines Satzadverbs zusammen mit dem Passiv-Partizip; das Satzadverb wird in einer vP-externen Position generiert, kann also bei vP-Topikalisierung nicht mitmachen.

(50) $*\left[_{\mathrm{TP}}\right.$ Wahrscheinlich $\mathrm{DP}_{\text {ext }}$ geschlafen ] wurde beim Vortrag.

„Die Leute schliefen wahrscheinlich beim Vortrag.“

Insgesamt bietet sich damit als korrekte empirische Generalisierung an, dass das nicht sichtbare externe Argument in deutschen Passivsätzen syntaktisch aktiv ist, und zugänglich ist für unselektive Bindung durch ein Quantifikationsadverb, dass dies aber nur in seinem minimalen syntaktischen Bereich gilt. Dieser minimale syntaktische Bereich kann als Phase identifiziert werden (wobei Phasen den minimalen vollständigen Prädikatsbereich vP und den minimalen vollständigen Satz CP umfassen; vgl. Chomsky 2001).

27 Vgl. Haider (1990, 2010) und Frey \& Tappe (1991) zu unabhängiger Evidenz dafür, dass vP (d.h., eine verbale Konstituente, die ein externes Argument enthält) im Deutschen topikalisiert werden kann; sowie Geilfuß (1988) und Abels (2012) zu unabhängiger Evidenz dafür, dass höhere Projektionen im deutschen Satz (wie TP) eine solche Topikalisierung nicht erlauben. 


\section{Konsequenzen}

Abschließend möchten wir die Konsequenzen beleuchten, die die Existenz von Effekten quantifikationaler Variabilität in deutschen Passivkonstruktionen hat für die unterschiedlichen Typen von Passivanalysen, wie sie in Abschnitt 2 diskutiert worden sind.

Erstens kann man festhalten, dass Typ-A-Analysen mit der Evidenz unverträglich sind. Wenn das externe Argument im Passiv gar nicht in der Syntax zugänglich ist, in welcher Form auch immer, dann kann es nicht durch ein Quantifikationsadverb in der Syntax gebunden werden. Man beachte auch, dass es keine Möglichkeit gibt, die Bindung durch ein solches Adverb bereits im Lexikon vorzusehen (denn das Quantifikationsadverb bildet ja ganz offensichtlich nicht zusammen mit dem Verb so etwas wie ein komplexes Prädikat). Somit ergibt sich gemäß der im ersten Abschnitt gemachten Vorgaben in jedem Fall ein Argument für dunkle Materie in der Syntax.

Zweitens ist das externe Argument im Passiv in Typ-B-Analysen in der Syntax durchweg zugänglich. Dies bedeutet, dass Effekte quantifikationaler Variabilität wie in (44) an sich erfasst werden können. Probleme ergeben sich allerdings nicht nur im Hinblick auf die in Abschnitt 3 angeführte empirische Evidenz gegen die Zugänglichkeit von externen Argumenten im deutschen Passiv; es ist darüber hinaus auch zunächst einmal ganz unklar, wie die systematische Unzugänglichkeit von externen Argumenten im Passiv für unselektive Bindung aus einem Matrixsatz heraus (vgl. (48)) abgeleitet werden kann.

Was drittens Typ-C-Analysen betrifft, so ist hier Passiv eine syntaktische Operation; diese Operation wird ausgelöst durch einen funktionalen Kopf wie $\mathrm{v}_{\text {pass }}$ oder Voice $_{\text {pass }}$, der mit einer verbalen Projektion verkettet wird und das externe Argument direkt existentiell abbindet. Das externe Argument im Passiv sollte folglich in Typ-C-Analysen nicht für syntaktische Operationen zugänglich sein. Auf der Grundlage von empirischer Evidenz wie der in (44) und (48) lässt sich somit schließen, dass die syntaktische Zugänglichkeit des externen Arguments in (44) für Typ-C-Analysen zunächst einmal ein Problem darstellt, wenigstens solange, wie Passivierung (bei Abwesenheit einer von-Phrase) notwendigerweise ein Passivmorphem wie in (13) (vgl. Bruening (2013)) involviert. Eine mögliche Lösung dieses Problems für Typ-C-Analysen könnte nun darin bestehen, dass man annimmt, dass Quantifikationsadverbien einen Effekt auslösen können, der genau dem entspricht, was in der Analyse von Stechow (1992), wie sie in Abschnitt 2.3.3 dargestellt wurde, bei (quantifizierten) vonPhrasen geschieht; vgl. (22); hier ergibt sich ja die Option, eine bereits abgebundene Variable auf indirekte Art und Weise (nämlich über die Konstatierung der Identität mit einer freien Variable) wieder zugänglich zu machen. Angesichts der strikten Lokalität, die einem Lexikoneintrag wie (22) inhärent ist (der 
Operator hier muss sich mit einem Prädikat verbinden, das eine offene Argumentstelle hat), könnte man so auch die Satzgebundenheit von Effekten quantifikationaler Variabilität mit externen Argumenten im deutschen Passiv erfassen (vgl. (48)). ${ }^{28}$

Die Konsequenz für Typ-D-Analysen, in denen das externe Argument in der Syntax selektiv zugänglich ist, wäre schließlich, dass sowohl das Auftreten von Effekten quantifikationaler Variabilität in Fällen wie in (44), als auch das Fehlen solcher Effekte in Fällen wie (48) ableitbar ist, wenn sichergestellt werden kann, dass das externe Argument innerhalb seiner minimalen Phase zugänglich ist, und außerhalb seiner minimalen Phase unzugänglich ist.

\section{Literatur}

Abels, Klaus (2012): Phases. An essay on cyclicity in syntax. Berlin: De Gruyter.

Alexiadou, Artemis, Elena Anagnastopoulou \& Florian Schäfer (2015): External arguments in transitivity alternations. Oxford: Oxford University Press.

Alexiadou, Artemis, Elena Anagnostopoulou \& Florian Schäfer (2018): Passive. In Norbert Hornstein, Howard Lasnik, Pritty Patel-Grosz \& Charles Yang (Hrsg.), 'Syntactic Structures' 60 years on, 403-425. Berlin: Mouton de Gruyter.

Alexiadou, Artemis \& Edit Doron (2013): The syntactic construction of two non-active voices: Passive and middle. Journal of Linguistics 48, 1-34.

Alexiadou, Artemis, Berit Gehrke \& Florian Schäfer (2014): The argument structure of adjectival passives revisited. Lingua 119, 118-138.

Bach, Emmon (1980): In defense of passive. Linguistics and Philosophy 3, 297-341.

Baker, Mark (2015): Case. Its principles and parameters. Cambridge: Cambridge University Press.

Baker, Mark, Kyle Johnson \& Ian Roberts (1989): Passive arguments raised. Linguistic Inquiry 20, 219-251.

Barnickel, Katja (2014): Opazität in Acl-Konstruktionen. Universität Leipzig MA-Arbeit.

Berman, Stephen (1991): The semantics of open sentences. Amherst: University of Massachusetts Dissertation.

Bever, Thomas G. \& Brian McElree (1988): Empty categories access their antecedents during comprehension. Linguistic Inquiry 19, 35-43.

Bittner, Maria \& Ken Hale (1996): The structural determination of case and agreement. Linguistic Inquiry 27, 1-68.

28 Eine alternative Möglichkeit wäre, auf der Grundlage einer „klassischen“ Typ-C-Analyse wie in Bruening (2013) und Alexiadou, Anagnastopoulou \& Schäfer (2015) anzunehmen, dass die sekundäre Passivvariante ohne direkte existentielle Quantifikation bei Bruening (2013) bzw. die Annahme der Einführung einer nicht direkt gebundenen Argumentvariable durch Voice bei Alexiadou, Anagnastopoulou \& Schäfer (2015) noch eine größere Distribution haben kann als nur Umgebungen mit von-Phrasen.

Letztendlich wäre man damit bei einer Mischung von Typ-B- und Typ-C-Analysen. 
Bobaljik, Jonathan (2015): Some differences between case and agreement. Manuskript, Unversity of Connecticut.

Boeckx, Cedric \& Norbert Hornstein (2006): The virtues of control as movement. Syntax 9, 118-130.

Borer, Hagit (1984): Parametric syntax. Dordrecht: Foris.

Bresnan, Joan (1982): The passive in lexical theory. In Joan Bresnan (Hrsg.), The mental representation of grammatical relations, Cambridge, Mass.: MIT Press.

Bresnan, Joan (2001): Lexical-functional syntax. Oxford: Blackwell.

Bruening, Benjamin (2013): By-phrases in passives and nominals. Syntax 16, 1-41.

Bruening, Benjamin (2014): Depictive secondary predicates, light verb give, and theories of double object constructions. Manuskript, University of Delaware.

Burzio, Luigi (1986): Italian syntax. Dordrecht: Kluwer.

Chomsky, Noam (1957): Syntactic structures. The Hague / Paris: Mouton.

Chomsky, Noam (1965): Aspects of the theory of syntax. Cambridge, MA: MIT Press.

Chomsky, Noam (1973): Conditions on transformations. In Stephen Anderson \& Paul Kiparsky (Hrsg.), A Festschrift for Morris Halle, 232-286. New York: Academic Press.

Chomsky, Noam (1975): The logical structure of linguistic theory. New York: Plenum Press.

Chomsky, Noam (1977): On wh-movement. In Peter Culicover, Tom Wasow \& Adrian Akmajian (Hrsg.), Formal Syntax, 71-132. New York: Academic Press.

Chomsky, Noam (1981): Lectures on government and binding. Dordrecht: Foris.

Chomsky, Noam (1982): Some concepts and consequences of the theory of government and binding. Cambridge, Mass.: MIT Press.

Chomsky, Noam (1995): The Minimalist Program. Cambridge, Mass.: MIT Press.

Chomsky, Noam (2001): Derivation by phase. In Michael Kenstowicz (Hrsg.), Ken Hale. A life in language, 1-52. Cambridge, Mass.: MIT Press.

Chomsky, Noam (2008): On phases. In Robert Freidin, Carlos Otero \& Maria Luisa Zubizarreta (Hrsg.), Foundational issues in linguistic theory, 133-166. Cambridge, Mass.: MIT Press.

Chomsky, Noam (2013): Problems of projection. Lingua 130, 33-49.

Collins, Chris (2005): A smuggling approach to the passive in English. Syntax 8, 81-120.

Diesing, Molly (1992): Indefinites. Cambridge, Mass: MIT Press.

Eichinger, Ludwig M. (1987): Zum Passiv im althochdeutschen Isidor. Versuch einer valenzgrammatischen Beschreibung. In Centre de Recherche en Linguistique Germanique (Nice) (Hrsg.), Das Passiv im Deutschen, 129-145. Tübingen: Niemeyer.

Eisenberg, Peter (1999): Grundriß der deutschen Grammatik. Band 2: Der Satz. Stuttgart: Metzler.

Embick, David (2004): Unaccusative syntax and verbal alternations. In Artemis Alexiadou, Elena Anagnostopoulou \& Martin Everaert (Hrsg.), The unaccusativity puzzle:

Explorations of the syntax-lexicon interface, 137-158. Oxford: Oxford University Press.

Fanselow, Gisbert (1985): Deutsche Verbalprojektionen und die Frage der Universalität konfigurationaler Sprachen. Passau: Universität Passau Dissertation.

Fiengo, Robert (1977): On trace theory. Linguistic Inquiry 8, 35-61.

Fischer, Kerstin \& Anatol Stefanowitsch (2006): Konstruktionsgrammatik: Ein Überblick. In Kerstin Fischer \& Anatol Stefanowitsch (Hrsg.), Konstruktionsgrammatik I: Von der Anwendung zur Theorie, 3-17. Tübingen: Stauffenberg.

Frey, Werner \& Thilo Tappe (1991): Zur Interpretation der X-bar-Theorie und zur Syntax des Mittelfeldes. Grundlagen eines GB-Fragmentes. Manuskript, Universität Stuttgart.

Gazdar, Gerald, Ewan Klein, Geoffrey Pullum \& Ivan Sag (1985): Generalized Phrase Structure Grammar. Oxford: Blackwell. 
Geilfuß, Jochen (1988): Reanalyse? Universität Tübingen MA-Arbeit.

Georgi, Doreen (2014): A reformulation of Baker et al. (1989) in terms of ordering.

Manuskript, Universität Leipzig.

Goldberg, Adele E. (2006): Constructions at work. Oxford: Oxford University Press.

Grewendorf, Günther (1983): Reflexivierung in deutschen Acl-Konstruktionen - Kein

transformationsgrammatisches Dilemma mehr. Groninger Arbeiten zur Germanistischen

Linguistik 23, 120-196.

Grewendorf, Günther (1988): Aspekte der deutschen Syntax. Narr.

Grewendorf, Günther (1989): Ergativity in German. Dordrecht: Foris.

Grimshaw, Jane (1997): Projection, heads, and optimality. Linguistic Inquiry 28, 373-422.

Grodzinsky, Yosef (2000): The neurology of syntax: Language use without Broca's area.

Behavioral and Brain Sciences 23, 1-71.

Gunkel, Lutz (2003): Infinitheit, Passiv und Kausativkonstruktionen im Deutschen. Tübingen: Stauffenburg Verlag.

Haider, Hubert (1990): Topicalization and other puzzles of German syntax. In Günther Grewendorf \& Wolfgang Sternefeld (Hrsg.), Scrambling and barriers, 93-112. Amsterdam: Benjamins.

Haider, Hubert (1993): Deutsche Syntax - generativ. Tübingen: Narr.

Haider, Hubert (2010): The syntax of German. Cambridge: Cambridge University Press.

Harley, Heidi (2013): External arguments and the mirror principle: On the distinctness of voice and v. Lingua 125 , 34-57.

Heim, Irene (1982): The semantics of definite and indefinite noun phrases. Amherst: University of Massachusetts Dissertation.

Hinterwimmer, Stefan (2005): Q-adverbs as selective binders: The quantificational variability of free relatives and definite DPs. Berlin: Humboldt-Universität Dissertation.

Höhle, Tilman (1978): Lexikalistische Syntax: Die Aktiv-Passiv-Relation und andere Infinitkonstruktionen im Deutschen. Tübingen: Niemeyer.

Hole, Daniel (2014): Dativ, Bindung und Diathese. Berlin: Akademie-Verlag.

Holmberg, Anders (2005): Is there a little pro? Evidence from Finnish. Linguistic Inquiry 36 (4), 533-564.

Keenan, Ed (1980): Passive is phrasal (not sentential or lexical). In Teun Hoekstra, Harry van der Hulst \& Michael Moortgat (Hrsg.), Lexical Grammar, 181-214. Dordrecht: Foris.

Kiparsky, Paul (2013): Towards a null theory of the passive. Lingua 125, 7-33.

Koster, Jan \& Robert May (1982): On the constituency of infinitives. Language 58, 117-143.

Landau, Idan (2013): Control in generative grammar. Cambridge: Cambridge University Press.

Legendre, Géraldine, Colin Wilson, Paul Smolensky, Kristin Homer \& William Raymond (2006): Optimality in syntax II: Wh-questions. In Paul Smolensky \& Géraldine Legendre (Hrsg.), The Harmonic Mind, Band II, Kap. 14, 183-230. Cambridge, Mass.: MIT Press.

Levine, Robert \& Ivan Sag (2003): Wh-nonmovement. Manuskript, Stanford University. To appear in Gengo Kenkyu.

Maienborn, Claudia (2007): Das Zustandspassiv. Zeitschrift für germanistische Linguistik 35, 83-114.

Maienborn, Claudia (2011): Strukturausbau am Rande der Wörter: Adverbiale Modifikatoren beim Zustandspassiv. In Stefan Engelberg, Anke Holler \& Kristel Proost (Hrsg.), Sprachliches Wissen zwischen Lexikon und Grammatik, 317-343. Berlin: De Gruyter. Malamud, Sophia (2004): Arbitrariness: A definite account. In G. Gardina \& M. Tsujimura (Hrsg.), WCCFL 23 Proceedings, 101-114. Somerville, Mass.: Cascadilla Press. 
Marantz, Alec (1991): Case and licensing. In German Westphal, Benjamin Ao \& Hee-Rahk Chae (Hrsg.), Proceedings of the Eigth Eastern States Conference on Linguistics, 234- 253. University of Maryland.

McFadden, Thomas (2004): The position of morphological case in the derivation: A study on the syntax-morphology interface: University of Pennsylvania Dissertation.

Merchant, Jason (2013): Voice and ellipsis. Linguistic Inquiry 44, 77-108.

Muckel, Sandra (2002): Wortstellungseffekte beim Satzverstehen: Zur Rolle syntaktischer, verbspezifischer und prosodischer Informationen. Wiesbaden: DUV.

Müller, Gereon (1993): On deriving movement type asymmetries: Universität Tübingen Dissertation.

Müller, Gereon (2001): Order preservation, parallel movement, and the emergence of the unmarked. In Géraldine Legendre, Jane Grimshaw \& Sten Vikner (Hrsg.), OptimalityTheoretic Syntax, 279-313. Cambridge, Mass.: MIT Press.

Müller, Gereon (2015): Optimality-theoretic syntax. In Tibor Kiss \& Artemis Alexiadou (Hrsg.), Syntax. An international handbook, Band 2, 875-936. Berlin: De Gruyter.

Müller, Gereon (2016): The short life cycle of external arguments in German passive derivations. Manuskript, Universität Leipzig.

Müller, Gereon (2017): Structure removal: An argument for feature-driven Merge. Glossa 2 (1).

Müller, Stefan (2002): Complex predicates. Stanford, California: CSLI Publications.

Müller, Stefan (2007): Head-Driven Phrase Structure Grammar: Eine Einführung. Tübingen: Stauffenburg.

Murphy, Andrew (2016): Double passivization in Turkish: A structure removal approach. In K. Bellamy, L. Karvovskaya, M. Kohlberger \& G. Saad (Hrsg.), Proceedings of ConSOLE 23, 316-339.

Perlmutter, David \& Paul Postal (1983a): The relational succession law. In David Perlmutter (Hrsg.), Studies in Relational Grammar 1, 30-80. Chicago: Chicago University Press.

Perlmutter, David \& Paul Postal (1983b): Toward a universal characterization of passivization. In David Perlmutter (Hrsg.), Studies in Relational Grammar 1, 3-29. Chicago: Chicago University Press.

Pickering, Martin \& Guy Barry (1991): Sentence processing without empty categories. Language and Cognitive Processes 6 (3), 229-259.

Pitteroff, Marcel (2014): Non-canonical lassen-middles: Universität Stuttgart Dissertation.

Pitteroff, Marcel \& Florian Schäfer (2017): Implicit control crosslinguistically. https://ling.auf.net/lingbuzz/003493 (letzter Zugriff: 23. 01. 2018).

Pollard, Carl J. \& Ivan A. Sag (1994): Head-Driven Phrase Structure Grammar. Chicago: University of Chicago Press.

Preminger, Omer (2014): Agreement and its failures. Cambridge, Mass.: MIT Press.

Primus, Beatrice (2010): Event-structure and individuation in impersonal passives. In Patrick Brandt \& Marco García García (Hrsg.), Transitivity, 209-233. Amsterdam: Benjamins.

Primus, Beatrice (2011): Das unpersönliche Passiv - ein Fall für die Konstruktionsgrammatik. In Stefan Engelberg, Anke Holler \& Kristel Proost (Hrsg.), Sprachliches Wissen zwischen Lexikon und Grammatik, 285-313. Berlin: De Gruyter.

Pullum, Geoffrey (1979): The nonexistence of the trace-binding algorithm. Linguistic Inquiry 10, 356-362.

Reis, Marga (1976): Reflexivierung in deutschen A.c.I.-Konstruktionen. Ein transformationsgrammatisches Dilemma. Papiere zur Linguistik 9, 5-82.

Richards, Norvin (2001): Movement in language. Oxford: Oxford University Press. 
Rizzi, Luigi (1986): Null objects in Italian and the theory of 'pro'. Linguistic Inquiry 17, 501- 557.

Roberts, Ian (1987): The representation of implicit and dethematized subjects. Dordrecht: Foris.

Ross, John (1967): Constraints on variables in syntax. Cambridge, Mass.: MIT Dissertation.

Sag, Ivan \& Thomas Wasow (1999): Syntactic theory. A formal introduction. Stanford University: CSLI Publications.

Schäfer, Florian (2012a): Local case, cyclic agree, and the syntax of truly ergative verbs. In Artemis Alexiadou, Tibor Kiss \& Gereon Müller (Hrsg.), Local modelling of non-local dependencies in syntax, 273-304. Berlin: De Gruyter.

Schäfer, Florian (2012b): The passive of reflexive verbs and its implications for theories of binding and case. Journal of Comparative Germanic Linguistics 15, 213-268.

Stechow, Arnim von (1987): Rektionsblockierung: Passivkonstruktionen. Handgeschriebenes Handout, DGfS Conference, Augsburg.

Stechow, Arnim von (1989): Distinguo. Linguistische Berichte 122, 330-339.

Stechow, Arnim von (1992): Kompositionsprinzipien und grammatische Struktur. In Peter Suchsland (Hrsg.), Biologische und soziale Grundlagen der Sprache, 175-248. Tübingen: Niemeyer.

Stechow, Arnim von (1998): German participles II in Distributed Morphology. Manuskript, Universität Tübingen.

Stechow, Arnim von \& Wolfgang Sternefeld (1988): Bausteine syntaktischen Wissens. Opladen: Westdeutscher Verlag.

Sternefeld, Wolfgang (1995): Voice phrases and their specifiers. Manuskript, Universität Tübingen. (SfS-Report 05-95).

Stiebels, Barbara (2000): Linker inventories, linking splits and lexical economy. In Barbara Stiebels \& Dieter Wunderlich (Hrsg.), Lexicon in focus, 211-245. Berlin: Akademie.

Stiebels, Barbara (2015): Control. In Tibor Kiss \& Artemis Alexiadou (Hrsg.), Syntax - Theory and analysis, 412-446. Berlin: de Gruyter.

van Urk, Coppe (2013): Visser's generalization: The syntax of control and the passive. Linguistic Inquiry 44, 168-178.

Wasow, Tom (1972): Anaphoric relations in English. Cambridge, Mass.: MIT Dissertation. Wasow, Tom (1977): Transformations and the lexicon. In Peter Culicover, Tom Wasow \& Adrian Akmajian (Hrsg.), Formal syntax, New York: Academic Press.

Williams, Alexander (2015): Arguments in syntax and semantics. Cambridge: Cambridge University Press.

Williams, Edwin (1981): Argument structure and morphology. The Linguistic Review 1, 81-114. Wunderlich, Dieter (1989): Arnim von Stechow, das Nichts und die Lexikalisten. Linguistische Berichte 122, 321-329.

Wunderlich, Dieter (1993): Diathesen. In Joachim Jacobs, Arnim von Stechow, Wolfgang Sternefeld \& Theo Vennemann (Hrsg.), Syntax. Ein internationales Handbuch zeitgenössischer Forschung, Band 1, Berlin: de Gruyter.

Wunderlich, Dieter (1997): Cause and the structure of verbs. Linguistic Inquiry 28, 27-68. Zifonun, Gisela, Ludger Hoffmann, Bruno Strecker et al. (1997): Grammatik der deutschen Sprache. Berlin: de Gruyter.

Zobel, Sarah (2014): Impersonally interpreted personal pronouns: Universität Göttingen Dissertation. 


\title{
Renate Raffelsiefen \\ 18 Phonologische Abstraktheit und symbolische Repräsentation
}

\begin{abstract}
Symbolische Repräsentation sprachlicher Lautstruktur beinhaltet die Zergliederung kontinuierlicher Rede in diskrete Einheiten, die mit einem finiten Inventar von Zeichen assoziiert werden. Die Grundidee hinter dieser Abstraktion ist, „wiederkehrendes“ Material, das trotz phonetischer Unterschiede als gleich aufgefasst wird, mit jeweils gleichen Zeichen zu assoziieren. Die Entwicklung geeigneter Verfahren zur Ermittlung einheitlicher und empirisch adäquater Abstraktionsgrade wurde in strukturalistischen Arbeiten vehement diskutiert, scheint aber allgemein seltsam vernachlässigt. In vorliegendem Beitrag wird ein solches im Rahmen der Optimalitätstheorie entwickeltes Verfahren anhand der sogenannten Vokalopposition im Deutschen vorgestellt. Verschiedene Typen konvergierender empirischer Evidenz untermauern die Annahme einer einzigen phonologisch relevanten Abstraktionsebene mit fünfzehn qualitativ unterschiedlichen Vollvokalen.
\end{abstract}

Keywords: Abstraktheit, Markiertheitsbeschränkungen, symbolische Repräsentation, Vokalismus, Wortphonologie

\section{Einleitung}

Jegliche Auseinandersetzung mit sprachlicher Lautstruktur, sei sie eher anwendungsorientiert (z.B. die Erstellung eines Aussprachewörterbuchs, Reformierung bestehender Schreibkonventionen) oder verbunden mit dem Ziel, eine phonologische Grammatik zu modellieren, rührt unmittelbar an die Frage der Abstraktheit. Diesbezügliche Vorstellungen reichen von hochabstrakten zugrundeliegenden Formen, von denen diverse Oberflächenformen durch ein komplexes Regelwerk abgeleitet werden (Chomsky \& Halle 1968), bis hin zu der Auffassung, dass konkret erfahrene Wortformen mit sämtlichen wahrgenommenen phonetischen Details im Gedächtnis abgespeichert werden und Lexikoneinträge aus „Wolken“ solcher erinnerten Formen bestehen (Johnson

Renate Raffelsiefen, Institut für Deutsche Sprache, R5, 6-13, D-68161 Mannheim, E-Mail: raffelsiefen@ids-mannheim.de

○ Open Access. (๑) 2018 Renate Raffelsiefen, publiziert von De Gruyter. (๔) BY Dieses Werk ist lizenziert unter der Creative Commons Attribution 4.0 Lizenz.

https://doi.org/10.1515/9783110490992-019 
2005). Auch im Vergleich symbolischer Repräsentationen in Form aufeinanderfolgender diskreter Zeichen zeigen sich unterschiedliche Vorstellungen hinsichtlich anzunehmender Abstraktionsgrade. Sogenannte „enge“ Transkriptionen, in denen phonetische Eigenschaften möglichst detailliert kodiert werden, kennzeichnen das eine Ende des Spektrums (vgl. (1a)). Repräsentationen, die durch den Verzicht auf Symbole für „,vorhersagbare“ Lautstruktur bestimmt sind, lassen sich dem anderen Ende zuordnen (vgl. (1d)).

(1) a. Rues et al. (2007)

['?a:ße]

['kьøаßə] b. Krech et al. (1982)

['a:be]

['krabə]

$\begin{array}{ll}\text { c. } & \begin{array}{l}\text { Duden } \\ \text { (2015) }\end{array} \\ \text { ['a:be] } \\ \text { ['krabə] }\end{array}$

d. Viëtor (1912) [a:bər] <aber> [krabə] <Krabbe>

Während in (1d) alle vier für die Umschrift von aber verwendeten Segmentsymbole auch in der Umschrift von Krabbe erscheinen, wird in (1b) nur das Symbol $[b]$ in beiden Wörtern verwendet. In (1b, c) kommt das Symbol [e], nicht aber das Symbol [?], zur Verwendung, während in (1a) beide, in (1d) keins von beiden erscheinen. Wie lässt sich ermitteln, ob hier jeweils ein einheitlicher Abstraktionsgrad vorliegt? Ist es angemessen, inhärent diskrete Symbole zur Repräsentation gradienter Realisierungserscheinungen zu verwenden (z. B. die Verwendung des Symbols [ $\beta]$ für die Repräsentation der möglichen Spirantisierung stimmhafter Plosive in (1a))? Sollte symbolische Repräsentation idealerweise nur einer Abstraktionsebene zugeordnet werden, die gleichzeitig die Spezifizierung lexikalischer Einträge bestimmt?

Das Problem, das sich hier stellt, liegt im Erstellen klarer Kriterien zur Feststellung geeigneter und einheitlicher Abstraktionsgrade. Das Ringen um solche Kriterien ist kennzeichnend für die strukturalistische Phonemtheorie, die auf einer grundlegenden Dichotomie zwischen den Kategorien „dasselbe“ versus „nicht dasselbe“ beruht. Dabei geht es um die Bedingungen, unter denen (i) diverse Sprachlaute demselben Phonem sowie (ii) diverse Phonempaare derselben, „proportionalen“ Opposition zuzuordnen sind. Phonemische Verschiedenheit setzt dabei das Vorkommen von Sprachlauten in derselben Umgebung in Form sogenannter Minimalpaare voraus. Die Vorstellung ist dabei, dass eine nicht-überlappende, exhaustive Zerlegung von Sprache in Form solcher Phoneme möglich ist und einen Abstraktionsgrad aufweist, der sowohl einheitlich ist als auch eine geeignete Grundlage für die Ableitung phonetischer Form bildet.

Die hier zugrundeliegenden Intuitionen finden sich im Kern bereits im Ersten Grammatischen Traktat, einer im zwölften Jahrhundert anonym verfassten Abhandlung mit Vorschlägen zu einer grundlegenden Reformierung der isländischen Rechtschreibung. In dieser Schrift wird das Fehlen vieler notwendiger 
Symbole moniert, ein Mangel, der dem Umstand geschuldet ist, dass die Grapheme meist aus dem lateinischen Alphabet übernommen wurden. So weist der Verfasser $^{1}$ darauf hin, dass die bestehenden Vokalgrapheme nicht genügend differenziert und zunächst um die Symbole $\langle$ ę $\rangle,\langle\underline{\langle}\rangle,\langle\emptyset\rangle,\langle\mathrm{y}\rangle$ zu erweitern seien. In der Begründung wird die Relevanz des Vorkommens von Lauten in derselben Umgebung hervorgehoben.

Ich nehme nun acht von diesen Buchstaben ... und stelle jeden der Reihe nach zwischen dieselben Konsonanten, und ich werde anhand von Beispielen zeigen, wie jeder von ihnen, wenn er von denselben Buchstaben gestützt wird und in derselben Position steht, ein anderes Wort bewirkt, und ich werde in derselben Weise in meinem ganzen Büchlein Beispiele geben für die sehr genauen Unterschiede, die durch diese Buchstaben bewirkt werden: sar, sq̨r, ser, sęr, sor, sør, sur, syr. (Arens 1969: 52).

Die Beispiele erfolgen in Form von in Sätzen eingebauten Minimalpaaren, in denen minimal kontrastierende Vokale in derselben Umgebung erscheinen (z. B. „Sur eru augu syr“ ,Trüb sind die Augen der Sau“2). Ein besonderes Interesse an der Proportionalität von Oppositionen kommt etwa in den folgenden Anmerkungen zum Ausdruck, wo die graphemische Repräsentation von als gleichartig aufgefassten Vokalrelationen durch einheitliche Diakritika vorgeschlagen wird:

$<0>$ erhält seinen Haken vom $<\mathrm{a}>$ und seinen Kreis vom $<0>$, denn es ist eine Mischung der beiden Laute und wird ausgesprochen mit geringerer Mundöffnung als $<\mathrm{a}>$, aber mit weiterer als $\langle 0\rangle$. $\langle$ ę $\rangle$ ist geschrieben mit dem Häkchen des $\langle a\rangle$, im Übrigen aber ganz in der Form des $\langle\mathrm{e}\rangle$, denn es ist eine Mischung der beiden, ausgesprochen mit geringerer Mundöffnung als <a>, aber mit weiterer als <e>. (Arens 1969: 51).

Der Bezug auf proportionale Oppositionen rückt im Folgenden noch stärker in den Mittelpunkt, wenn der Verfasser eine durchgehende Opposition von Oralund Nasalvokalen und schließlich für jeden dieser Vokale eine weitere Quantitätsopposition feststellt. Für die Repräsentation dieser Kontraste schlägt er Diakritika vor: ein Punkt unterscheidet Nasal- von entsprechenden Oralvokalen, ein Strich unterscheidet Lang- von entsprechenden Kurzvokalen. Insgesamt ergeben sich auf diese Weise 36 verschiedene Zeichen für Vokale, wobei die Proportionalität von Oppositionen in Form einheitlicher Diakritika zum Ausdruck kommt. Der Verfasser kommentiert wie folgt:

1 Historiker gehen davon aus, dass diese Schrift von einem Mann verfasst wurde; daher die Referenz auf eine männliche Person.

2 Vgl. Arens (1969: 52). 
Wenn irgendeine dieser 36 Distinktionen wegfallen kann und in unserer Sprache nie gebraucht wird, dann habe ich mich sehr geirrt, was ja durchaus möglich ist; desgleichen, falls es noch mehr als diese in der Rede der Leute geben sollte. (Arens 1969: 51).

In diesen Bemerkungen schwingt ein verhaltener Optimismus mit, dass das vorgestellte Inventar von Symbolen geeignet ist, die Sprache in angemessener Weise zu repräsentieren. Zumindest wird suggeriert, dass eine solche systematische und eindeutige Zuordnung von Symbolen und Sprachlauten möglich ist. Der Verfasser wäre daher wohl verwundert angesichts der Vielfalt der über achthundert Jahre später für das heutige Deutsch vorgeschlagenen Repräsentationen. Wie lässt sich erklären, dass die Anzahl der für das moderne Standarddeutsch angenommenen Vokale zwischen acht und siebzehn schwankt, obwohl die Relevanz von Minimalpaaren wie auch von proportionalen Oppositionen für die Bestimmung solcher Inventare $\mathrm{zu}$ guter Letzt von den meisten Autoren anerkannt werden? Auf welcher Basis kann hier entschieden werden?

Der vorliegende Beitrag zielt auf eine im Rahmen der Optimalitätstheorie entwickelte Vorgehensweise zur Bestimmung phonologischer Repräsentationen, angewendet auf den Vokalismus des Deutschen. ${ }^{3}$ In Abschnitt 2 werden zunächst Daten und Analysen zur sogenannten Vokalopposition im Deutschen vorgestellt. In Abschnitt 3 erfolgt eine Beschreibung der Daten in Form von geordneten Beschränkungen. Das Ziel ist dabei, eine Vorstellung davon zu vermitteln, wie eine solche Grammatik Aufschluss über Phoneme, phonologische Oppositionen und eine geeignete Abstraktionsebene gewähren kann. In Abschnitt 4 geht es um Möglichkeiten der empirischen Verifizierung der Analyse durch experimentalphonetische Untersuchungen. In Abschnitt 5 erfolgt eine kurze Zusammenfassung der Ergebnisse und ein Ausblick auf mögliche Anwendungen.

\section{Daten und Analysen zur Vokalopposition}

Studien zur Phonologie des Deutschen zeigen Übereinstimmung dahingehend, dass die in (2) gezeigten Wortpaare kontrastieren, wobei der Kontrast in (2d) nur in unbetonten Silben besteht. Die Vokale in stählen und stehlen kontrastieren nicht in allen Regionen; selbst wo dieser Kontrast besteht, ist offen, ob der Vokal in stellen in beiden Fällen als Oppositionsglied fungiert oder für den Vokal in stählen hier eine Lücke anzunehmen ist.

3 Detailliertere und weitere Analysen erscheinen in Raffelsiefen (in Vorbereitung). 
(2) a. /'m[i:]nə/ <Mine> /'d[y:]nə/ <Düne> /'b[u:]lə/ <Buhle> /'d[o:]lə/ <Dohle> /'h[ø:]lə/<Höhle> /'Jt[e:]lən/ <stehlen>

b. /'pR[a:]lən/ <prahlen>

c. /'ft[æ:]lən/ <stählen>

d. /'od[ə]]/<Ode>
/'m[I]nə/ <Minne>

/'d[Y]nə/ <Dünne>

/'b[v]lə/ <Bulle>

/'d[J]la/ <Dolle>

/'h[œ]lə/ <Hölle>

/'St[E]lən/ <stellen>

/'pr[a]lən/ <prallen>

?/'St[ $[\varepsilon]$ lən/ <stellen>

/'od[e]/ <oder>

Die qualitativen Unterscheidungen der in (2a, b, c) verwendeten Symbole sollen im Folgenden phonologisch motiviert werden. Phonetische Korrelate zeigen sich in den in (3a) gezeigten unterschiedlichen Positionen der Vokale im F1/F2Raum. ${ }^{4}$ Die in (2) markierten Längenunterschiede sind durch die in (3b) gezeigten Messungen der entsprechenden Vokaldauern bedingt.

(3) a. F1/F2-Werte deutscher Vokale (in Bark).

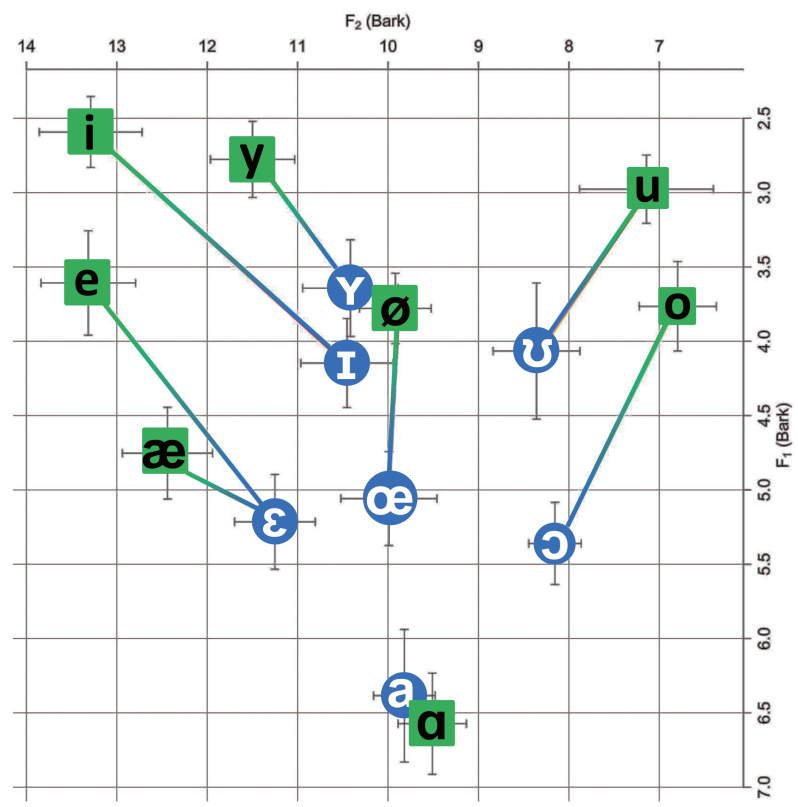

4 Die in (3) gezeigten Messwerte beruhen auf Studien ausgesuchter Minimalpaare aus der Wortliste Deutsch Heute (Brinckmann et al. 2008), beschränkt auf die Aufnahmen von fünfzig 
b. Dauern deutscher Vokale (in ms).

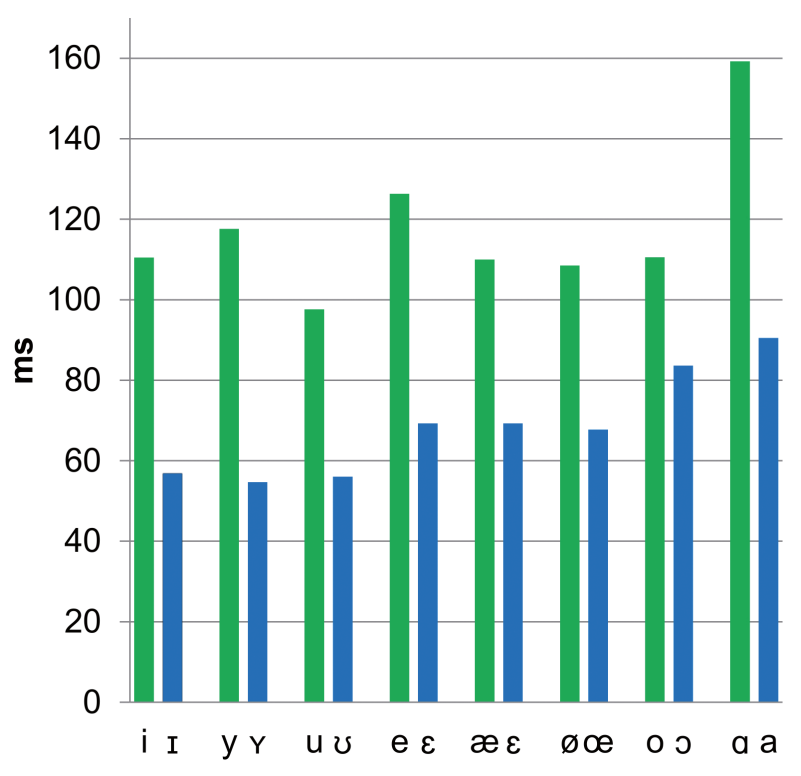

Gravierende Unterschiede zeigen sich in den Analysen der Kontraste. So veranschaulichen sämtliche Paare in $(2 a, b, c)$ für manche Autoren eine einheitliche proportionale Opposition, für andere trifft dies nur auf die Paare in (2a) zu. Letztere Sicht geht meist mit der Auffassung einher, dass die Fälle in (2b, c) eine reine Quantitätsopposition verkörpern, manifestiert in der Verwendung jeweils gleicher Vokalsymbole (vgl. ['pra:lən] <prahlen> vs. ['pralən] <prallen>, [' $\left.\int \varepsilon: l ə n\right]<$ stählen> vs. [' $\left.\int t \varepsilon l ə n\right]<$ stellen> in Viëtor 1912). Die phonetische Motivation dieser Entscheidung ist nicht offensichtlich. Zwar liegen die beiden offenen Vokale relativ dicht beieinander, aber dies trifft auch auf die Vokale /ø/ /y/ zu (vgl. Höhle - Hülle), die dennoch in allen Beschreibungen wie selbstverständlich mit verschiedenen Symbolen repräsentiert werden. Noch fragwürdiger erscheint die Wahl desselben Symbols für die betonten Vokale in stählen versus stellen, die sich qualitativ recht deutlich unterscheiden (s. 3a). Aufgrund welcher Kriterien wurde hier entschieden?

männlichen Sprechern aus Nordwestdeutschland. Es handelt sich um die Wörter $s[\mathrm{i}:]$ ben $<$ sieben> : $d r[\mathrm{I}]$ bbeln $<$ dribbeln>, Fl[y:]che <Flüche> : $K[\mathrm{y}]$ che $<$ Küche>, g[u:]te <gute $>: M[v]$ tter $<$ Mutter>, Schw[e:]fel <Schwefel> : Pf[c]ffer <Pfeffer, Gr[ø:]ße <Größe>:R[œ]sser <Rösser>, $V[\mathrm{o}:$ ]gel $<$ Vogel $>$ : $R[$ ] ]ggen $<$ Roggen $>$, Spr[a:]che $<$ Sprache $>$ : $R[\mathrm{a}]$ che $<$ Rache $>$. Die Formantenkarte zeigt die Mittelwerte bei 55\% der akustischen Vokaldauer. Für die Durchführung der Messungen und Auswertungen danke ich meinem früheren Mitarbeiter Fabian Brackhane. 
Die Klassifikation der fraglichen Paare als reine Quantitätsopposition steht vermutlich in Zusammenhang mit dem in (4a) gezeigten Vokalsystem des Internationalen Phonetischen Alphabets (IPA) von 1908 und dessen Einfluss auf Wilhelm Viëtor, Autor eines 1912 erschienenen deutschen Aussprachewörterbuchs.

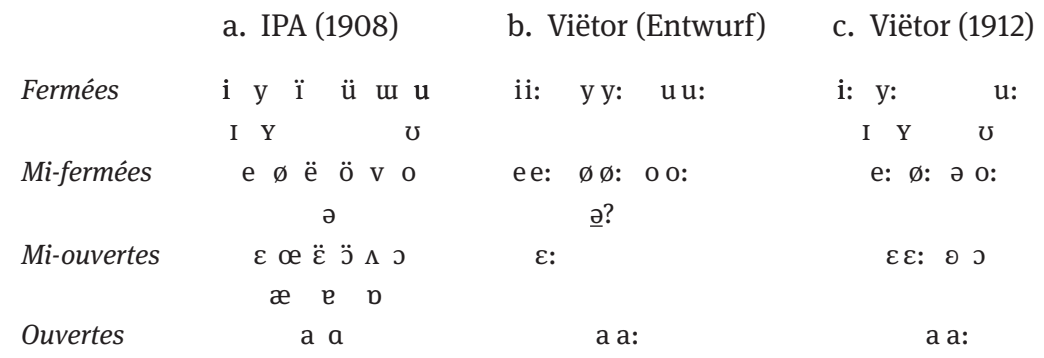

IPA-Vokalsysteme beruhen auf der Annahme sogenannter Kardinalvokale, die extreme Positionen des potenziellen Vokalraums markieren und als Referenzpunkte für gleichmäßig abgestufte Zwischenpositionen dienen. Im in (4a) gezeigten System von 1908 werden sieben Öffnungsgrade angenommen, von denen die für das Französische als relevant angesehenen Höhenstufen mit Bezeichnungen versehen sind ((Voyelles) fermées, mi-fermées, mi-ouvertes, ouvertes).

Viëtors Haltung gegenüber den IPA-Konventionen wird im Vorwort seines Aussprachewörterbuchs deutlich: „Als Lautschrift konnte für ein Aussprachewörterbuch, das auch weitergehenden Zwecken dienen will, allein die Lautschrift der Association phonétique internationale in Betracht kommen." (Viëtor 1912: X). In der Tat lässt sowohl die Wahl seiner Symbole als auch deren Anordnung einen engen Bezug auf das 1908 erschienene IPA-System erkennen. In einem im Vorwort erwähnten ersten Entwurf ging Viëtor noch davon aus, dass nur Längenunterschiede („lang, bzw. halblang, kurz“) zu markieren seien bei „i, u, ü, usw.“ (ebd.). Das mutmaßlich vollständige für die Repräsentation der deutschen Vokale verwendete Inventar ist in (4b) dargestellt. Hinsichtlich der „offenen Frage“ nach dem angemessenen „Grad der Genauigkeit“ entscheidet Viëtor, relevante Qualitätsunterschiede „im Interesse einer möglichst einfachen Darstellung“ (ebd.) außer Acht zu lassen. Sein späteres Abrücken von dieser Entscheidung begründet er wie folgt: „Der Widerspruch meiner ausländischen Freunde war aber so allgemein, dass ich die genauere Bezeichnung nachträglich eingeführt habe.“ (Viëtor 1912: X).

Viëtors Vorgehensweise bei den daraufhin in Angriff genommenen Änderungen ist mit Blick auf das in (4a) gezeigte IPA-System leicht zu rekonstruie- 
ren. Anstelle der Symbole für die kurzen Vokale in seinem ersten Entwurf verwendet er die passenden Symbole der jeweils unmittelbar darunterliegenden Höhenstufe aus dem IPA-System. Die jeweils kurzen Oppositionsglieder in den Paaren $\{\mathrm{i}, \mathrm{i}:\} ;\{\mathrm{y}, \mathrm{y}:\} ;\{\mathrm{u}, \mathrm{u}:\}$ werden somit durch $\mathrm{I}, \mathrm{y}, \mathrm{v}$ ersetzt, statt der kurzen Vokale in $\{e, e:\},\{\varnothing \emptyset:\},\{0,0:\}$ erscheinen $\varepsilon$, ø, ว. ${ }^{5}$ Diese Vorgehensweise stößt an ihre Grenze, wenn man zu dem Paar $\{\mathrm{a}, \mathrm{a}:\}$ und dem Vokal $\varepsilon$ : kommt. Im ersten Fall gibt es keine darunterliegende Höhenstufe. Im Fall $\varepsilon$ : gibt es kein kurzes Oppositionsglied. ${ }^{6}$ An diesem Punkt scheint Viëtor schlicht aufzugeben und es einfach bei seiner ursprünglich gewählten Notation zu belassen. Über die genauen Hintergründe, die zu der spezifischen Zusammensetzung des in (4c) abgebildeten Viëtorschen Mischsystems geführt haben, lässt sich natürlich nur spekulieren; mit Gewissheit festgestellt werden kann nur zum einen die empirische Inadäquatheit des Ergebnisses und zum anderen sein durchschlagender Erfolg.

Die Inadäquatheit zeigt sich schon im Vergleich des Systems in (4c) mit den Daten in (3). Qualitätsunterschiede werden in manchen Fällen durch die Wahl unterschiedlicher Vokalsymbole repräsentiert (z. B. [i:] vs. [I]), in anderen nicht (z. B. [ع:] vs. [ $\varepsilon]$ ). Die mit Blick auf das IPA-System postulierten Höhenunterschiede haben oft keine Entsprechung (vgl. die Anordnung der Vokale [I] und [e:] in (4c) mit den Messergebnissen in (3a)). Auch aus typologischer Sicht ist Viëtors System in mehrfacher Hinsicht unplausibel. Typologische Studien von Vokalsystemen haben gezeigt, dass vier Öffnungsgrade schon rar sind, die Annahme von fünf Öffnungsgraden ist zu bezweifeln (vgl. Becker-Kristal 2010). Alternierende Höhenstufen sind in Viëtors System zudem auf eine unerklärliche Weise mit phonetischer Länge assoziiert (vgl. Fourquet 1961). Die hier genannten Ungereimtheiten lassen vermuten, dass die Paare in (2a, b, c) eine andersartige Opposition (z. B. gespannt versus ungespannt) verkörpern, deren phonetische Korrelate zwar auch Höhenunterschiede einschließen, aber von phonologischen Höhenoppositionen strikt $\mathrm{zu}$ trennen sind. ${ }^{7}$

Der durchschlagende Erfolg des Viëtorschen Systems zeigt sich unter anderem in seiner Verwendung in den wichtigsten aktuellen Aussprachekodizes des Deutschen, das Duden Aussprachewörterbuch und das Deutsche Aussprache-

5 Seine Entscheidung statt des naheliegenden Symbols œ das Symbol ə einzuführen ist nicht nachzuvollziehen und wird von ihm selbst nicht kommentiert.

6 Das Symbol $\varepsilon$ ersetzt selbst das kurze [e] aus dem Paar \{e, e:\}, lässt sich also nicht seinerseits einfach weiter ersetzen.

7 Die Warnung Sievers’ (1901) vor einer Verwechslung des „Gespanntheitskontrasts“, den er für die Paare in (2) annimmt (Sievers 1901: 99), mit Höhenstufenkontrasten müsste Viëtor eigentlich bekannt gewesen sein. 
wörterbuch (Krech et al. 2009). ${ }^{8}$ (Lediglich Viëtors offensichtlicher Missgriff, die Wahl des Symbols [ø] statt [œ] für das kurze Oppositionsglied zu [ø], wurde berichtigt). Auch der von Kohler (1999) verfasste aktuelle Beitrag zur Repräsentation des deutschen Vokalsystems im IPA-Handbuch (s. (5)) lässt die Beibehaltung des Kerns des Viëtorschen Mischsystems deutlich erkennen. ${ }^{9}$ Kohler tilgt lediglich die Diakritika für Länge in den Fällen, wo die Opposition bereits durch unterschiedliche Vokalsymbole markiert ist. Sein Anpassen der Vokale an das unterdessen offiziell auf vier Höhenstufen beschränkte Vokalschema des IPA wirft Fragen auf. Soll hier ausgedrückt werden, dass der Unterschied in Fällen wie /'d[o:]lə/ <Dohle> versus /'d[o]lə/ <Dolle> ein genuiner Höhenunterschied ist, der Kontrast in /'b[u:]lə/ <Buhle> versus /'b[v]lə/ <Bulle> hingegen nicht? ${ }^{10}$ Eindeutig scheint nur, dass die Vokalunterschiede in (2b, c) als reine Quantitätskontraste aufzufassen sind, aber selbst hier fragt man sich nach der Signifikanz der Repräsentation der /a/-Qualitäten durch einen einzigen Punkt im Raster, im Gegensatz zu den zwei distinkten mit den / $\varepsilon /$ Qualitäten assoziierten Punkten.

(5) Das Vokalinventar des Deutschen nach Kohler (1999: 87).

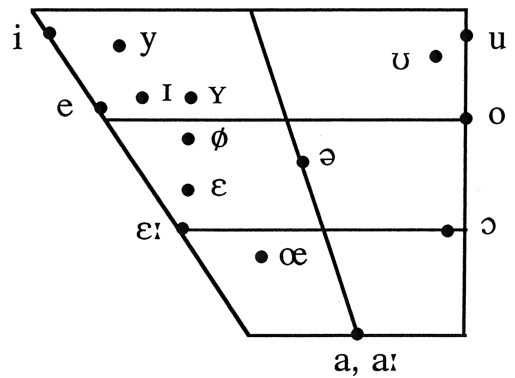

Expliziter ist die Darstellung Mayers (2010: 43), dessen Inventar (s. (6)) in besonderem Maße den Kern des Viëtorschen Systems zur Geltung bringt. So zeigt Mayers Klassifikation die charakteristische Beschränkung der Quantitäts-

8 Noch im Vorläufer des Deutschen Aussprachewörterbuchs wurden die tiefen Vokale in prahlen und prallen auch qualitativ unterschieden (['pra:lən] vs. ['pralən]) (Krech et al 1982).

9 Das von Kohler (1977: 173) angenommene System stimmt noch völlig mit dem ersten Entwurf Viëtors in (4b) überein.

10 Die Möglichkeit, dass hier einfach nur Messwerte in das Schema übertragen wurden, erscheint unwahrscheinlich in Anbetracht der genauen Verortung der meisten Vokale auf den vorgegebenen Linien. 
opposition auf zwei Vokalpaare (/a/: /a:/, / $/$ / : / $\varepsilon$ :/), wie auch die Analyse der Vokalopposition mit Bezug auf Höhenstufen.

\begin{tabular}{|c|c|c|c|c|}
\hline & \multicolumn{2}{|c|}{ Vorne } & zentral & hinten \\
\hline & nicht-rund & rund & nicht-rund & rund \\
\hline hoch & /i/ & $/ \mathrm{y} /$ & & $/ \mathrm{u} /$ \\
\hline halbhoch & /I/ & $/ \mathrm{Y} /$ & & $|v|$ \\
\hline obermittelhoch & /e/ & $|\varnothing|$ & & /o/ \\
\hline $\begin{array}{l}\text { mittel } \\
\text { untermittelhoch }\end{array}$ & $/ \varepsilon /,(/ \varepsilon: /)$ & $/ œ /$ & & $/ 0 /$ \\
\hline $\begin{array}{l}\text { halbtief } \\
\text { tief }\end{array}$ & & & $\begin{array}{c}/ \mathrm{e} / \\
/ \mathrm{a} /, / \mathrm{a}: /\end{array}$ & \\
\hline
\end{tabular}

Die beharrliche Beibehaltung des Viëtorschen Systems lässt sich vielleicht am ehesten wissenschaftssoziologisch erklären. In anderen Umfeldern ausgebildete AutorInnen gehen jedenfalls eher von einer einheitlichen Vokalopposition im Deutschen aus (z. B. die Gespanntheitsopposition in Moulton 1962, die Längenopposition in Hall 1992). Alternativ zu segmentalen Oppositionen werden auch prosodische Oppositionen vorgeschlagen, wobei Energieverläufe (Vennemann 1991) oder Silbenstrukturen (Becker 1998) kontrastieren. Eine einfache Analyse als Silbenstrukturkontrast ist anhand des Minimalpaares Mine : Minne in (7) veranschaulicht, wo die Organisation des intervokalischen Konsonanten als ausschließlicher Ansatz mit einer ambisilbischen Organisation kontrastiert. In diesen Ansätzen werden unter der Annahme jeweils identischer segmentaler Repräsentationen (s. $\mathrm{V}_{\mathrm{i}}$; in $(7 \mathrm{a}, \mathrm{b})$ ) sowohl Qualitäts- als auch Quantitätsunterschiede gleichermaßen als allophonische Erscheinungen gewertet: im Nukleus offener Silben erfolgen eher periphere, länger anhaltende Realisationen der Vokale, während geschlossene Silben zentralisierte, kürzere Artikulationen von Vokalen begünstigen.

(7) a.

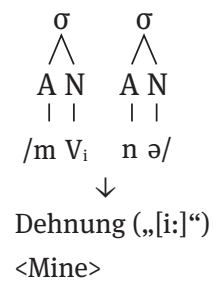

b.

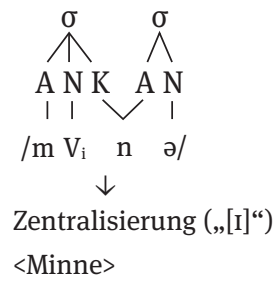

Eine solche Analyse geht folglich mit einem drastisch reduzierten Vokalinventar einher. Vennemann (1991) nimmt nur acht Vokalphoneme an, für deren Repräsentation er konventionelle Grapheme des Deutschen wählt: 
(8)

\begin{tabular}{lcccc} 
& \multicolumn{2}{c}{ vorne } & \multicolumn{2}{c}{ hinten } \\
hoch & nicht-rund & rund & nicht-rund & rund \\
mittel & $\mathrm{i}$ & $\ddot{\mathrm{u}}$ & & $\mathrm{u}$ \\
tief & $\mathrm{e}$ & $\ddot{0}$ & & $\mathrm{o}$
\end{tabular}

Aus typologischer Sicht ist das von Vennemann (1991) vorgeschlagene Inventar weitaus plausibler als etwa das von Mayer (2010) vorgeschlagene System, da die durch die Parameter Zungenhöhe, Zungenstellung (vorne, zentral, hinten) und Lippenrundung bestimmten Basissysteme in den Sprachen der Welt zwischen drei und neun Vokale umfassen (Becker-Kristal 2010: 7). Darüber hinausgehende Zahlen weisen auf andersartige Oppositionen hin, wie z. B. die im Ersten Grammatischen Traktat erwähnten Quantitäts- und Nasalitätsoppositionen. Im Folgenden möchte ich Argumente für eine Analyse vorstellen, die zwar auf unterschiedliche Silbenstrukturen wie in (7) Bezug nimmt, aber eine qualitative Vokalopposition mit einem Gesamtinventar von fünfzehn Vollvokalen für das Deutsche beinhaltet. ${ }^{11}$

\section{Eine beschränkungsbasierte Analyse der Vokalopposition}

In diesem Abschnitt soll zunächst die Grundidee einer beschränkungsbasierten Analyse kurz vorgestellt werden. Darauf folgen Erklärungen der Relevanz einer solchen Analyse für die Feststellung von Proportionalität, der anzunehmenden prosodischen Organisation und des Wesens der Opposition (z. B. segmental vs. prosodisch). Auch der Phonemstatus von [e] soll aus dieser Perspektive kurz erörtert werden.

\subsection{Eine phonologische Grammatik in Form geordneter Beschränkungen}

Eine phonologische Grammatik im Sinne der Optimalitätstheorie besteht aus einer sprachspezifischen Ordnung universeller Beschränkungen (Prince und Smolensky 2004). Der zentrale Konflikt betrifft das Streben nach einer Maxi-

11 Van Oostendorp (1995) analysiert eine Vokalopposition im Niederländischen, die auf ähnliche Weise umstritten ist wie die hier behandelte Opposition im Deutschen, als phonologische Qualitätsopposition. Botma \& van Oostendorp (2012) stellen diese Analyse wieder in Frage. 
mierung des Kontrastpotenzials einerseits, erzielt durch die Zulässigkeit möglichst vieler Strukturen zwecks Unterscheidung von Morphemen, und dem Streben nach einer Minimierung phonologischer Markiertheit andererseits. Weitere Beschränkungen betreffen die Übereinstimmung von phonologischer Struktur zusammenhängender Wörter, sowohl zur Stärkung von Kohäsion auf der syntagmatischen Ebene (z. B. Reim, Alliteration) als auch zur Minimierung von phonemischer Variation auf der paradigmatischen Ebene (Paradigmenuniformität).

Die Relevanz eines solchen Grammatikmodells für die Bestimmung des Abstraktionsgrads soll im Folgenden anhand der deutschen Vokalopposition veranschaulicht werden. Als Ausgangspunkt für die Analyse dient Evidenz für eine aktive Treuebeschränkung in Form von Minimalpaaren. Die in (2) gezeigten Fälle motivieren die Annahme einer solchen Treuebeschränkung; auf den Kontrast zwischen den betonten Vokalen in der linken Spalte in (2a, b, c) und den entsprechenden Vokalen in der rechten Spalte wird hier zunächst mit den Bezeichnungen „ $A$ “ versus „ $B$ “ referiert:

\section{(9) TREUE(A:B)}

Die Beschränkung in (9) soll zunächst so verstanden werden, dass in Inputs angenommene $A$ - versus $B$-Vokale auch im Output als solche erscheinen. Der Schlüssel $\mathrm{zu}$ den Fragen, ob es sich in den Fällen in (2) immer um echte Minimalpaare handelt, welche Fälle eine proportionale Opposition bilden, wie die Opposition beschaffen ist (Qualität, Quantität, Prosodie) und wie die fraglichen Phonemketten prosodisch organisiert sind, liegt in der Interaktion der Treuebeschränkung mit Markiertheitsbeschränkungen.

Die Relevanz einer solchen Interaktion für die Bewertung „echter“ Minimalpaare soll vorab kurz an einem Beispiel erläutert werden. Der Kontrast in Paaren wie Po[?] ét < Poet> - Pro[1]ét < Prolet $>$ wird unterschiedlich gewertet. Für manche Autoren belegt er den Phonemstatus des Glottalverschlusses (Maas 1999: 225). Andere betrachten den Glottalverschluss als subphonemisch, bedingt durch das Erscheinen des Vokals in fußinitialer Position (Krech 1968).

Die Adäquatheit der Bewertung solcher Minimalpaare lässt sich aufgrund messphonetischer Studien kaum entscheiden. Phonologische Untersuchungen von Neutralisationserscheinungen hingegen liefern Aufschluss. Es zeigt sich, dass Vokalkombinationen im Deutschen beschränkt sind: zwei adjazente Nuklei sind möglich (s. (10a)), drei hingegen nicht (s. (10b)). ${ }^{12}$

12 Das vielleicht einzige Gegenbeispiel ist /maoam/ <Maoam>, eine lautmalerische Warenbezeichnung für ein Kaubonbon. Es ist somit eine regelbestätigende Ausnahme, da ungewöhnliche Verletzungen phonologischer Markiertheitsbeschränkungen in der Werbesprache bewusst eingesetzt werden, um Aufmerksamkeit zu erregen. 


$$
\begin{aligned}
& \text { a. } \quad \stackrel{\sigma}{\wedge} \stackrel{\sigma}{\wedge}^{\sigma} \\
& \text { A N A N N } \\
& \text { l l } 11 \text { । } \\
& \text { /n i ve a/ }
\end{aligned}
$$

b.

$\begin{array}{cccc}* & \sigma & \sigma \\ \bigwedge_{A N} & \mid & \mid \\ 1 & 1 & 1 & \frac{N}{l} \\ / n & \text { i } & \text { e } & \text { a } /\end{array}$

c.

$$
\begin{aligned}
& \wedge^{\sigma} \quad \sigma \quad \sigma \\
& \wedge \wedge 1 \\
& \text { A N A N N } \\
& \begin{array}{lll|l}
1 & 1 & 1 & 1
\end{array} \\
& \text { [n i ? e: a] }
\end{aligned}
$$

Diese Einschränkung des Kontrastpotenzials ließe sich in der phonologischen Grammatik des Deutschen leicht durch die Annahme einer undominierten Markiertheitsbeschränkung *NNN (Keine drei adjazenten Nuklei) erfassen, solange die Grammatik die phonemische Ebene beschreibt wie in (10a, b). Wird der Glottalverschluss hingegen als Phonem gewertet oder erfolgt die Spezifizierung der phonologischen Grammatik mit Bezug auf die phonetische Ebene wie in (10c) ließe sich die Ungrammatikalität der fraglichen Lautfolge im Vergleich zu der wohlgeformten Sequenz in (10a) nur schwerlich erklären. ${ }^{13}$

Das Beispiel veranschaulicht somit die Relevanz (universell begründeter) Markiertheitsbeschränkungen für die Festlegung einer spezifischen Abstraktheitsebene sowie die Vorzüge des ausschließlichen Bezugs auf die phonemische Ebene für die Ausarbeitung der phonologischen Grammatik.

\subsection{Proportionalität von Oppositionen}

Die Annahme einer proportionalen Opposition stützt sich auf Evidenz für parallele Neutralisationskontexte. Die Daten in (11) zeigen, dass am Wortende oder in der Erstposition im Hiatus jeweils nur die $A$-Vokale vorkommen. Wichtig ist hier, dass die Neutralisation sämtliche hier betrachteten Fälle betrifft, einschließlich der in (11b) veranschaulichten Vokale. Der vorangesetzte Asterisk markiert ungrammatische Formen.

$$
\begin{aligned}
& \text { a. } / \int[\mathrm{i}:] /<\text { Ski }>\quad * / \int[\mathrm{I}] / \\
& / \int[\mathrm{u}:] /\langle\text { Schuh }\rangle \quad * / \int[\mathrm{v}] / \\
& \left./ \mathrm{fR}_{\mathrm{R}}[\mathrm{y}:] /<\mathrm{früh}\right\rangle \quad * / \mathrm{fR}[\mathrm{y}] / \\
& / \mathrm{s}[\mathrm{e}[\mathrm{e}] /<\text { Schnee }>\quad * / \mathrm{s}[\mathrm{n}] / \\
& / \mathrm{t}^{\mathrm{s}}[\mathrm{0}: \mathrm{s}] /\langle\mathrm{ZOO}\rangle \quad * / \mathrm{t}^{\mathrm{s}}[\mathrm{s}] / \\
& / \mathrm{b}[\varnothing:] /<\mathrm{Bö}>\quad * / \mathrm{b}[\propto] /
\end{aligned}
$$

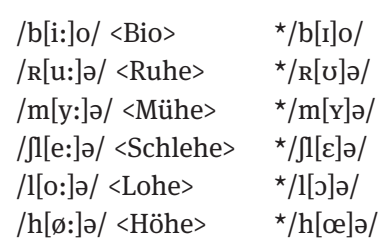

13 Für eine Analyse des Glottalverschlusses als Silbenansatz siehe Alber (2001). Vorausgesetzt, dass silbische Organisation ausschließlich die phonemische Ebene betrifft, ist Albers Analyse des Glottalverschlusses zur Optimierung der Silbenstruktur von vorneherein anzuzweifeln. Stattdessen ist diese Erscheinung auch als artikulatorische Stärkung des linken Wortoder Fußrands erklärbar, erreicht durch die Glottalisierung des initialen Vokals (Krech 1968). Die Verwendung eines Symbols ([?]) zur Beschreibung dieser gradienten Erscheinung ist wie in allen solchen Fällen fragwürdig. 

b. $/ \mathrm{t}^{\mathrm{s}}[æ:] /<z a ̈ h>\quad * / \mathrm{t}^{\mathrm{s}}[\varepsilon] /$
/kR[æ:]ə/ <Krähe> */kR[ع]ə/
$/ \mathrm{n}[\mathrm{a}:] /<\mathrm{nah}>\quad \star / \mathrm{n}[\mathrm{a}] /$
/bl[a: $]$ ə/ <Blahe $>\quad * / \mathrm{bl}[\mathrm{a}] ə /$

In der Optimalitätstheorie deutet die Aufhebung eines Kontrasts auf die Dominierung einer Treuebeschränkung durch eine phonologische Markiertheitsbeschränkung hin. Die in (11) veranschaulichten Kontexte legen einen Bezug auf Silbenstruktur nahe. Eine vorläufige Fassung dieser Beschränkung ist in (12) gegeben (, ${ }^{\star}{ }{ }^{\text {offen}} / B^{\text {“ }}$ heißt: In offenen Silben dürfen keine $B$-Vokale stehen):

$$
{ }^{\star} \sigma^{\text {offen }} / \mathrm{B} \gg \text { > TREUE(A:B) }
$$

Die Ordnung der Beschränkungen in (12) besagt, dass die fragliche phonologische Markiertheitsbeschränkung (das Verbot von $B$-Vokalen in offenen Silben) im Deutschen wichtiger ist als die Maximierung des Kontrastpotenzials (das Zulassen von $A$ - und $B$-Vokalen zwecks potenzieller Bedeutungsunterscheidung).

Ein weiterer Neutralisationskontext ist in (13) gezeigt. Vor postvokalischen Konsonantenverbindungen wie $/ \mathrm{nts} /$ und $/ \mathrm{lk} /$, die notwendigerweise die Schließung der Erstsilbe bewirken, erscheinen nur $B$-Vokale. Hier ist wieder zu beachten, dass sämtliche $A$-Vokale ausgeschlossen sind, einschließlich der mit Sternchen versehenen Formen in (13b).
a. $/ \mathrm{m}[\mathrm{I}] \mathrm{nt} \mathrm{t}^{\mathrm{s}} \mathrm{\rho} /<$ Minze $>$
$/ \mathrm{m}[\mathrm{Y}] \mathrm{nt}^{\mathrm{s}} \partial /<$ Münze $>$ $/[v] \mathrm{nt}^{\mathrm{s}} \mathrm{s} /<$ Unze $>$
$/ \mathrm{b}[\mathrm{J}] \mathrm{nt} \mathrm{s}^{\mathrm{s}} /<$ <Bonze>
$\star / \mathrm{m}[\mathrm{i}:] \mathrm{nt} \mathrm{t}^{\mathrm{s}} \mathrm{a}$
$\star / \mathrm{m}[\mathrm{y}:] \mathrm{nt} \mathrm{s}^{\mathrm{s}} /$
$* /[\mathrm{u}:] \mathrm{nt}^{\mathrm{s}} \mathrm{s} /$
$\star / \mathrm{b}[0:] n t^{5} \partial /$
b. $/ \mathrm{gR}[\varepsilon] \mathrm{nt} \mathrm{s}^{\mathrm{s}} /<\mathrm{Grenze}>$ $\mid \mathrm{gR}[\varepsilon] \mathrm{nt} \mathrm{t}^{\mathrm{s}} /<$ <Grenze $>$ $/ /[a] n t^{\mathrm{s}} \partial /<$ Lanze $>$
*/gr[e:]nts ${ }^{\mathrm{s}} /$
*/gR[æ:]nt ${ }^{\mathrm{s} \partial /}$
$\star /\left[1[a:] n t^{s} \partial /\right.$

\begin{tabular}{|c|c|}
\hline 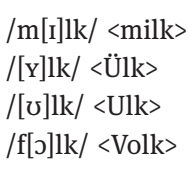 & $\begin{array}{l}\star / \mathrm{m}[\mathrm{i}:] \mathrm{k} / \\
* /[\mathrm{y}:] \mathrm{k} / \\
\star /[\mathrm{u}:] \mathrm{k} / \\
* / \mathrm{f}[\mathrm{o}:] \mathrm{k} /\end{array}$ \\
\hline $\begin{array}{l}/ \mathrm{v}[\varepsilon] 1 \mathrm{k} /<\text { <elk } \\
/ \mathrm{v}[\varepsilon] \mathrm{k} /<\text { welk }\end{array}$ & $\begin{array}{l}\star / / \mathrm{v}[\mathrm{e}:] \mathrm{k} / \\
\star / \mathrm{v}[æ:] \mathrm{k} /\end{array}$ \\
\hline
\end{tabular}

Eine vorläufige Fassung dieser Beschränkung ist in (14) gegeben („, ${ }^{\text {geschlossen }} / \mathrm{A}^{\text {“ }}$ heißt: In geschlossenen Silben dürfen keine $A$-Vokale stehen):

$$
{ }^{\star} \sigma^{\text {geschlossen }} / \mathrm{A} \gg \text { TREUE(A:B) }
$$

Die Wichtigkeit von Neutralisationserscheinungen für die phonologische Bewertung von Oppositionen lässt sich anhand der in Abschnitt 1 vorgestellten alternativen Phonemanalysen veranschaulichen. Die Annahme des z. B. von Mayer vorgeschlagenen Vokalsystems hätte zur Folge, dass etwa für die Klasse der A-Vokale unterschiedliche Markiertheitsbeschränkungen anzunehmen wären, wobei zum einen auf spezifische Höhenstufen, zum anderen 
auf Quantitätseigenschaften Bezug genommen würde ( ${ }^{\star} \sigma^{\text {geschlossen }} /[$ hoch], ${ }^{\star} \sigma^{\text {geschlossen }} /\left[\right.$ obermittelhoch], ${ }^{\star} \sigma^{\text {geschlossen }} /\{\varepsilon$ :, a: $\left.\}\right)$. Der jeweils gleiche Bezug auf spezifische Silbenstrukturen erschiene dabei als Zufall. Wichtiger noch ist der Kritikpunkt, dass es keine unabhängige Evidenz für die fraglichen Markiertheitsbeschränkungen gibt: Aus den Sprachen der Welt sind keine Fälle belegt, in denen Vokale aufgrund von Zungenhöhenmerkmalen in bestimmten Silbenstrukturen ausgeschlossen sind. Die Parallelität der Opposition in $(2 \mathrm{a}, \mathrm{b}, \mathrm{c})$ wird im Folgenden noch anhand weiterer Neutralisierungskontexte belegt.

\subsection{Prosodische Struktur}

Der wesentliche Bezug auf offene versus geschlossene Silben in den vorläufigen Grammatikfragmenten in (12) und (14) deutet auf die Relevanz von Markiertheitsbeschränkungen für die Repräsentation prosodischer Struktur hin. Die diesbezügliche Annahme lässt sich wie folgt zusammenfassen: Wenn eine Markiertheitsbeschränkung die Neutralisation eines Kontrasts bewirkt und auf prosodische Struktur Bezug nimmt, so ist die fragliche Struktur Teil der phonologischen Repräsentation. Diese Annahme hat unmittelbare Auswirkungen auf die Repräsentation der Vokalkontraste wie in Mine versus Minne. Die in (13) gezeigte Evidenz für die Markiertheitsbeschränkung, die das Erscheinen von $B$-Vokalen auf geschlossene Silben begrenzt, indiziert die notwendige Assoziation des auf einen $B$-Vokal folgenden Phonems mit der Silbenkoda. Einzelne intervokalische Konsonanten wie in Minne sind infolgedessen ambisilbisch zu organisieren (s. 15b). ${ }^{14}$
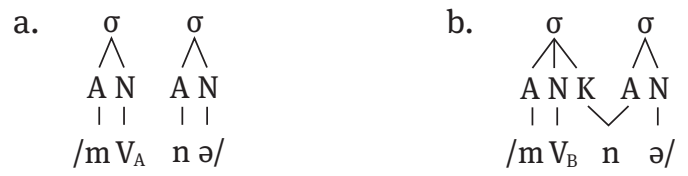

Neben den in (2) dargestellten Fällen zeigt sich die Vokalopposition auch in wortfinalen Silben, die auf einen Konsonanten enden. Dieser Kontext, der die Proportionalität sämtlicher in (2a, b, c) aufgeführten Fälle stützt, ist in (16) veranschaulicht.

14 Der Unterschied zu der in (7) veranschaulichten Analyse wird noch eingehend erläutert. 
(16)

a. $\begin{array}{ll}/ \int[\mathrm{I}] \mathrm{f} / & <\text { Schiff }> \\ / \mathrm{R}[\mathrm{v}] \mathrm{m} /<\text { Rum }> \\ / \mathrm{d}[\mathrm{y}] \mathrm{n} /<\text { dünn }> \\ / \int \mathrm{R}[\mathrm{\jmath}] \mathrm{t} /<\text { Schrott }> \\ / \mathrm{g}[œ] \mathrm{n} /<\text { gönn }> \\ / \mathrm{b}[\varepsilon] \mathrm{t} /<\text { Bett }> \\ / \mathrm{b}[\varepsilon] \mathrm{t} /<\text { Bett }> \\ / \int[\mathrm{a}] \mathrm{l} /<\text { Schall }>\end{array}$

b. $/ \int[\mathrm{i}:] \mathrm{f} /\langle$ schief $>$

/R[u:]m/ <Ruhm>

/k[y:]n/ <kühn>

$/ \int \mathrm{R}[\mathrm{o}:] \mathrm{t} /<$ Schrot $>$

/ $[\varnothing:] \mathrm{n} /<$ schön $>$

/b[e:]t/ <Beet>

$/$ pp[æ:]t/ <spät $>$

$/ \int[a:] 1 /<$ Schal $>$

Auch die Repräsentation dieses zweiten Kontrastkontexts ergibt sich aus den relevanten Markiertheitsbeschränkungen. Die Beschränkung gegen $A$-Vokale in geschlossenen Silben indiziert die notwendige Offenheit der betonten Silben in (16b). Die prosodischen Repräsentationen sind in (17) veranschaulicht, wo der $B$-Vokal in einer geschlossenen Silbe, der $A$-Vokal hingegen in einer offenen Silbe erscheint. Der wortfinale Konsonant bildet den Ansatz einer sogenannten katalektischen Silbe mit leerem Nukleus. ${ }^{15}$

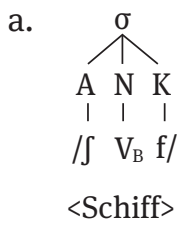

b.

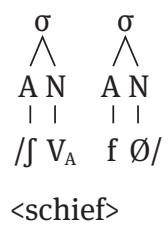

Das spezifische Kontrastpotenzial der deutschen Vokalopposition deutet somit auf die Rangordnung in (18) hin, wo die Treuebeschränkung TREUE A:B in den oben erwähnten Markiertheitsbeschränkungen eingebettet ist ( ${ }^{\star} \mathrm{AMB}$ (Ambisilbizität ist verboten), ${ }^{\star} \mathrm{N} / \varnothing$ (Leere Nuklei sind verboten)). Das Verbot von $A$ - bzw. $B$-Vokalen aufgrund der Silbenstruktur ist im Deutschen kategorisch, Ambisilbizität und leere Nuklei am Wortende sind der „Preis“, der gezahlt wird, um den Kontrast dennoch zu ermöglichen.

$$
{ }^{\star} \sigma^{\text {geschlossen }} / \mathrm{A},{ }^{\star} \sigma^{\text {offen }} / \mathrm{B}>>\text { TREUE(A:B) }>{ }^{\star} \mathrm{AMB},{ }^{\star} \mathrm{N} / \varnothing
$$

Die Grammatik in (18) drückt die Möglichkeit eines Kontrasts wie in /koma/ : /kэma/ im Deutschen aus, vorausgesetzt, dass /m/ im zweiten Fall ambi-

15 Die Beschränkung des Vokalkontrasts auf die Position vor dem wortfinalen Konsonanten

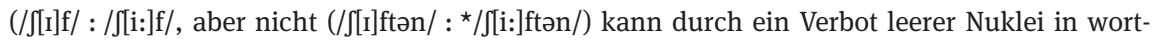
internen Positionen ausgedrückt werden. Das Erscheinen zusätzlicher Konsonanten am rechten Wortrand in Fällen wie Herbst, Obst, Arzt, Vogt, Biest, Mond deutet auf die mögliche Organisation alveolarer Konsonanten als Appendix hin, der auf einen leeren Nukleus folgen kann. 
silbisch organisiert wird. Ebenso setzt die Möglichkeit des Kontrasts in $/ \int \mathrm{R}$ t/ : / $\mathrm{R}$ Rot/ die Silbifizierung des finalen Konsonanten in Ansatzposition voraus. Die Vokalopposition ist somit vor / $\mathrm{h} /$ ausgeschlossen, das im Deutschen ausschließlich im Ansatz erscheinen kann und daher nur vorangehende $A$-Vokale erlaubt (s. 19a). Ebenso ist sie vor /y/ ausgeschlossen, das im Deutschen mit der Koda assoziieren muss und daher nur vorangehende $B$-Vokale erlaubt (s. 19b). Auch in diesen Fällen zeigt sich ein klarer Parallelismus zwischen nicht-tiefen und tiefen Vokalen, was die Annahme einer einheitlichen proportionalen Opposition bestärkt.
a. /'[o:]haim/ <Oheim $>\quad \star /[$ ] $]$ haim/
$/ /[\mathrm{e}:]$ hek/ $\langle\text { Ehec }\rangle^{16} \quad \star / /[\varepsilon]$ hek/
/'[a:]hวRn/ <Ahorn> */'[a]hวRn/

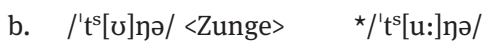

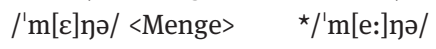
/'z[a:]hel/ <Sahel $>\quad \star / ' z[a] h \varepsilon l /$

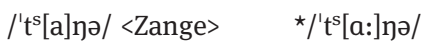

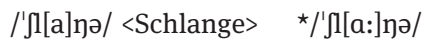

Die Neutralisationserscheinungen in (19), (s. auch (11) und (13)), veranschaulichen die Annahme, dass es sich bei den in Aufhebungspositionen erscheinenden Segmenten um „dieselben“ Phoneme handelt, die auch in den Kontrastpositionen erscheinen (z. B. die relevanten A-Vokale in Oheim, Zoo, Dohle). Die alternative Auffassung, dass in Aufhebungspositionen sogenannte Archiphoneme oder andere unterspezifizierte Segmente erscheinen, die sich von den Segmenten in Kontrastpositionen unterscheiden, wird somit abgelehnt. Phonetische Studien bestätigen die Auffassung, dass Aufhebungspositionen eine Beschränkung des Inventars der Phoneme, nicht zusätzliche Arten von Phonemen, bedingen (s. Abschnitt 3).

Weitere Evidenz für die Annahme, dass das mögliche Auftreten von $A$ versus $B$-Vokalen im Deutschen von den Silbifizierungsmöglichkeiten des Folgesegments abhängt, findet sich in der Restriktion vor Plosiv-LiquidVerbindungen in (20). ${ }^{17}$ Wie erwartet verhalten sich nicht-offene und offene Vokale wieder parallel.
a. /'l[e:]pra/ <Lepra $>$
*/'l[c]pra/
$\star / ' k[0]$ bra/
/'k[o:]bra/ <Kobra >
$/ /[i:] g l u /<$ Iglu $>$
$\star /[$ IIglu/
b. /'k[a:]pRi/ <Capri $>$
/'p[a:]dRə/ <Padre $>$
/'m[a: $] \mathrm{kro} /<$ Makro $>$

*/'k[a]pri/

*/'p[a]dRə/

*/'m[a]kro/

16 Dieses Akronym steht für Enterohämorrhagische Escherichia coli.

17 Alle hier behandelten Beschränkungen gelten nur für Simplizia. Abweichungen in Ableitungen wie Kuppler, wacklig lassen sich durch Paradigmenuniformität bewirkende Korrespondenzbeschränkungen erklären. 
Die Ungrammatikalität der mit Asterisk markierten Formen deutet darauf hin, dass Plosiv-Liquid-Verbindungen als komplexer Ansatz zu organisieren sind. Wie aber lässt sich erklären, dass die ambisilbische Organisation des Folgekonsonanten in (21b) erzwungen werden kann, um den Kontrast zu gewährleisten, nicht aber in (21d)?

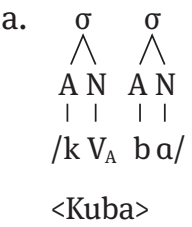

b.

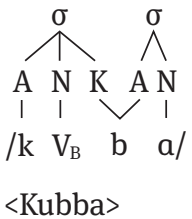

c.

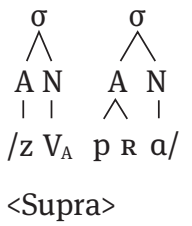

d. *

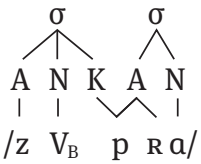

Eine mögliche Antwort liegt in der Beobachtung, dass die ungrammatische Form in (21d) gleich zwei Markiertheitsbeschränkungen verletzt, *AMB und *KOMPLEXER ANSATZ (Komplexe Ansätze sind verboten). Die Beobachtung, dass Verletzungen einzelner Beschränkungen zwar toleriert werden, eine „Häufung“ von Verletzungen diverser Beschränkungen, bezogen auf dasselbe sprachliche Material, aber die Ungrammatikalität der fraglichen Struktur zur Folge haben kann, wird in der Optimalitätstheorie mithilfe „lokaler Beschränkungsverknüpfungen“ erfasst (Smolensky 2006). Solche Verknüpfungen werden nur dann verletzt, wenn sämtliche verknüpfte Einzelbeschränkungen verletzt werden. Die Ordnung einer entsprechenden Verknüpfung (d.h. \{ ${ }^{\star} A M B$, *KOMPLEXER ANSATZ\}) über TREUE A:B in der Grammatik in (18) würde das Ausscheiden der ungrammatischen Form in (21d) bewirken.

Untersuchungen weiterer Neutralisationskontexte lassen auf die Notwendigkeit bestimmter Modifikationen der Treuebeschränkung in (18) sowie auf zusätzliche prosodische Struktur schließen. Die Daten in (22) zeigen, dass der Kontrast in unbetonten Endsilben neutralisiert ist, selbst wenn ein einzelner Obstruent oder /m/ am Wortende steht. ${ }^{18}$ Hier erscheinen nur $B$-Vokale.

a. /t $\mathrm{t}^{\mathrm{s}} \mathrm{y}^{\prime} \mathrm{kl}[\mathrm{o}:] \mathrm{p} /<$ Zyklop> $/ \mathrm{ka}$ d $[\mathrm{u}:] \mathrm{k} /<$ kaduk $>$ /ko'm[e:]t/ <Komet $>$ /o'p[a:]k/ <opak> /i'm[a:]m/ <Imam> $\left./ \mathrm{a}^{\prime} \mathrm{k}[\mathrm{u}:] \mathrm{t} /<\mathrm{akut}\right\rangle$ /a'n[i:]s/ <Anis>

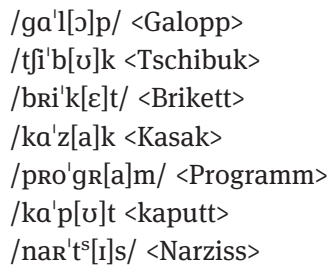

/ga'l[3]p/ <Galopp> $/ \mathrm{t} f \mathrm{i} \mathrm{b}[\mathrm{v}] \mathrm{k}<\mathrm{Tschibuk}>$ han'tris/ $<$ Narziss $>$ b. /'bosk[j]p/ <Boskop $>$ /'kaut [u]k/ <Kautschuk> /'deb[c]t/ <Debet> /'koni[a]k/ <Kognak> /'biz[a]m/ <Bisam> /'mam[v]t/ <Mammut> /'kyRb[I]s/ <Kürbis>

18 Fälle mit finalem $\{\mathrm{R}, 1, \mathrm{n}\}$ sind gesondert zu behandeln (s. 3.5 und Raffelsiefen i. Vorb.). 
Das unterschiedliche Kontrastpotenzial in (22a) versus (22b) lässt sich durch eine Begrenzung der Treuebeschränkung auf betonte Silben erfassen $(\Sigma-\mathrm{K}=$ Kopfposition innerhalb eines Fußes):

$$
{ }^{\star} \sigma^{\text {geschlossen }} / \mathrm{A},{ }^{\star} \sigma^{\text {offen }} / \mathrm{B}>>\operatorname{TREUE}(\mathrm{A}: \mathrm{B})_{\Sigma-\mathrm{K}}>>{ }^{\star} \mathrm{AMB},{ }^{\star} \mathrm{N} / \varnothing
$$

Die zusätzliche Spezifizierung in (23) deutet auf die Präsenz von Fußstruktur in phonologischen Repräsentationen des Deutschen hin. Die Gruppierung von Silben zu Füßen, wie auch die Organisation von Phonemen in Form von Silben, finden innerhalb spezifischer prosodischer Domänen, sogenannter phonologischer Wörter $(\omega)$, statt, deren Grenzen notwendigerweise mit Morphemgrenzen zusammenfallen. Die vollständigen Repräsentationen der Wörter in (22), bis auf die Spezifizierung der relevanten Vokalkontraste, sind in (24) veranschaulicht:

(24) a.

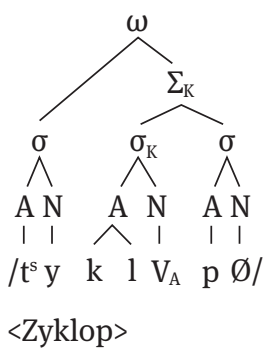

b.

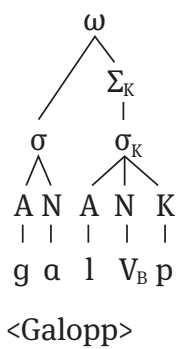

c.

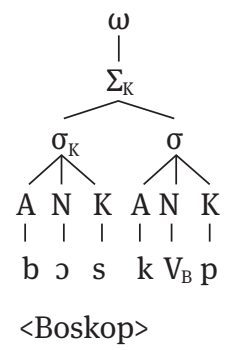

Die Begrenzung des Kontrasts auf betonte Silben ( $\Sigma-\mathrm{K}=$ Kopfposition innerhalb eines Fußes) ist im Einklang mit der universellen Tendenz zur Begrenzung von Kontrasten auf prominente Positionen, die die Wahrnehmung lautlicher Unterschiede begünstigen (vgl. das Konzept „Positional Faithfulness“ von Beckmann 1998). ${ }^{19}$ Allgemein gilt, dass Treue in prominenten Positionen höher geordnet ist als entsprechende Treuebeschränkungen in weniger prominenten Positionen.

Die Grammatik in (23) drückt aus, dass leere Nuklei am Wortende zwar in Kauf genommen werden, um das Kontrastpotenzial in betonten Silben zu gewährleisten, nicht aber in unbetonten Silben. Eine Organisation des letzten Konsonanten als Ansatz einer katalektischen Silbe in Fällen wie (22b) wäre daher ungrammatisch im Deutschen. Gleichzeitig drückt die Grammatik aus,

19 Die Beschaffenheit prominenter Positionen hängt von dem jeweiligen Kontrast ab. Eigenschaften von Vokalen werden am besten in betonten Silben wahrgenommen, Stimmhaftigkeitskontraste von Konsonanten werden am besten in fußinterner intervokalischer Position wahrgenommen (vgl. die in Steriade 1997 vorgestellten Perzeptionshierarchien). 
dass Ambisilbizität nicht möglich ist, um den Kontrast in prätonischen Silben zu gewährleisten. Hier kommen, wie in (22a) veranschaulicht, nur $A$-Vokale in Frage. ${ }^{20}$ Allerdings besteht der Einwand, dass in wortinitialen prätonischen Silben zumindest eine marginale Kontrastmöglichkeit im Deutschen besteht, zu sehen in Fällen wie K[o]lónne ,Kolonne“ versus K[ว]llége ,Kollege‘. Ein solcher Kontrast scheint allerdings die Schreibung des nachfolgenden Lauts als Einzel- versus Doppelkonsonant vorauszusetzen, wobei dieser Unterschied in der Graphemstruktur in der Regel bereits schriftsprachlich entlehnt ist (vgl. lat. co<ll>ega gegenüber frz. co<l>onne). Kohler (1977: 149) urteilt entsprechend, dass es sich um eine „typische Schriftaussprache“ handelt, an die sich einige wenige Sprecher „mit viel Mühe halten“, die aber in der „wirklich gesprochenen Sprache“ nicht vorkomme (vgl. auch Moulton 1962: 63, Becker 1998: 86). Aus Sicht des hier verwendeten Grammatikmodells lässt sich sagen, dass der K[o]lónne - K[ว]llége-Kontrast nicht abwegig wäre, da auch die wortinitiale Position als Bezug für positionelle Treuebeschränkungen infrage käme (Walker 2011: 136). Eine Grammatik, die einen $A: B$-Kontrast wie in (25a, b) zuließe, wäre entsprechend durch eine Modifikation der Treuebeschränkung leicht $\mathrm{zu}$ beschreiben. Interessant in diesem Zusammenhang ist die notwendige Aufhebung des fraglichen Kontrasts für alle Sprecher, wenn der folgende fußinitiale Ansatz aus /s/ besteht. Hier gehen ausschließlich $B$-Vokale voran (vgl. Gl[ग]ssár, $F[\jmath]$ ssíl, $P[\varepsilon]$ ssár, $\operatorname{Dr}[\varepsilon]$ ssúr, $T[\varepsilon]$ ssín, $p l[\mathrm{I}]$ ssieren), was auf die regulär ambisilbische Organisation von /s/ und somit auf eine Markiertheitsbeschränkung gegen dessen Silbifizierung als strikter Ansatz ( $\left.{ }^{\star} \mathrm{A} / \mathrm{s}\right)$ hindeutet, s. $(25 \mathrm{c}) .^{21}$

(25) a.

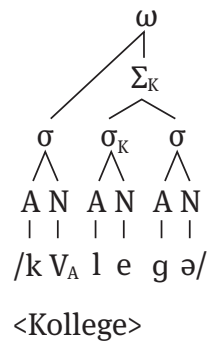

b.

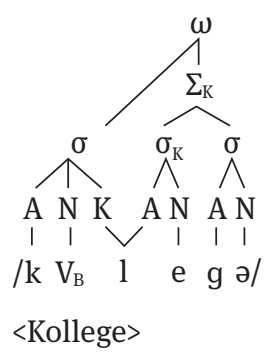

c.

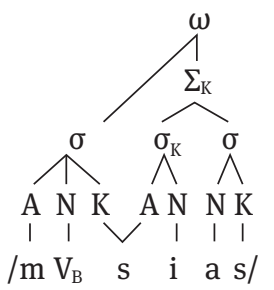

$<$ Messias $>$

20 Die Beobachtung, dass diese $A$-Vokale nicht lang sind, wohl aber eine von den $B$-Vokalen in den unbetonten Silben in (22c) unterschiedliche Qualität aufweisen (vgl. die Vokale in K[o]mét $<$ Komet> vs. Slál[ $[0] m<$ Slalom>), ist ein erster Hinweis darauf, dass es sich bei der Vokalopposition um eine Qualitäts- und keine Quantitätsopposition handelt (Moulton 1962). Dieser Punkt wird in Abschnitt 2.3. durch unabhängige Argumente untermauert.

21 Hier mag ein Zusammenhang zu der Abwesenheit von /s/ in wortinitialer Position im Standarddeutschen bestehen. Aber auch vor fußinitialem /S/ zeigt sich zumindest für manche Sprecher eine Neutralisation zugunsten von $B$-Vokalen (z. B. $M[0]$ schée, Br[0]schüre). 
Eine weitere Differenzierung der positionellen Treuebeschränkung ergibt sich aufgrund der Neutralisation des $A: B$-Kontrasts auch in nebentonigen Endsilben wie in Hérkulès. Die Beobachtung, dass der $A$ :B-Kontrast auch in dieser Position nicht möglich ist, erfordert eine weitere Modifizierung der Treuebeschränkung in der Grammatik in (23). Das Ersetzen des Subskripts ${ }_{\Sigma-\mathrm{K}}$ durch $\omega$-K (Kopf des phonologischen Wortes) bewirkt die notwendige Silbifizierung des finalen Konsonanten in der Silbenkoda. ${ }^{22}$ Folglich erscheinen in den nebentonigen Silben konsonantfinaler Wörter nur B-Vokale, s. (26).

(26)

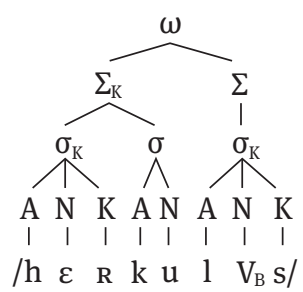

$<$ Herkules $>$

Eine genauere Untersuchung von Wörtern des Typs Herkules ergibt, dass auch die hauptbetonte Silbe zumindest keinen soliden $A$ :B-Kontrast zulässt. Stattdessen zeigt sich Variation, wobei viele Sprecher eine klare Präferenz für $B$-Vokale zeigen.

(27) a.

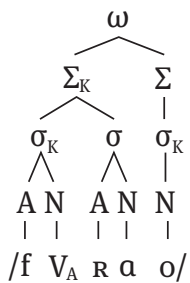

b.

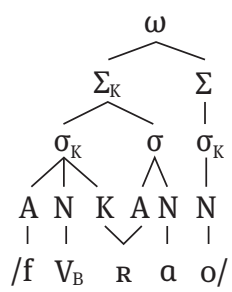

$<$ Pharao $>$

Weitere Beispiele sind in (28) gegeben. Die Neigung, in solchen Fällen nur $B$-Vokale zuzulassen, unterscheidet sich jeweils deutlich von den Beispielen in der rechten Spalte in (28), die die Stabilität von $A$-Vokalen in wortfinalen Füßen veranschaulichen. Auch hier zeigt sich im Übrigen wieder die Parallelität nichtoffener und offener Vokale (vgl. (28a) und (28b)).

22 Auch hier haben auf $\{\mathrm{R}, \mathrm{l}, \mathrm{n}\}$ endende Wörter einen Sonderstatus, vgl. 3.5. 
(28)
a. /'m[o]lo,tof/ <Molotow>
/'[v]ni, $t^{\mathrm{s}} \mathrm{\varepsilon f} /\langle\mathrm{UNICEF}\rangle$
/'g[c]ne, zIs/ <Genesis>
/'k[I]mo,no/ <Kimono>
/'b[v]mə, Ray/ <Bumerang>
b. /'[a]na,nas/<Ananas>
/'p[a]pri,ka/ <Paprika>
/'k[a]ba, Ret/ <Kabarett>
/as'p[a]Ra,gus/ <Asparagus>
/'[a]di,das/ <Adidas>
/'[a]no,Rak/ <Anorak>
/'z[o:]lo/ <Solo $>$
/'i[u:]ni/ <Juni>
/'b[e:]ne/ <bene>
/'kl[i:]ma/ <Klima>
/'p[u:]ma/ <Puma >
/maRihu'[a:]na/ <Marihuana>
/'k[a:]pri/ <Capri >
/'R[a:]bə/ <Rabe>
/mas'k[a:]Ra/ <Mascara>
/'k[a:]di/ <Kadi >
/pi'[a:]no/ <Piano>

Die Idee, den $A$ :B-Kontrast auf rechtsbündige Köpfe zu beschränken, ließe sich formal nicht ohne Weiteres ausdrücken und scheint ohnehin unplausibel. Warum sollte der wortinitiale Akzent die Wahrnehmung des Vokalkontrasts weniger begünstigen als andere Positionen? Eine entsprechende Modifizierung der Treuebeschränkung würde zudem das zweite bemerkenswerte Merkmal der fraglichen Neutralisationserscheinung nicht erklären, nämlich die Beschränkung auf $B$-Vokale und die somit indizierte Ambisilbizität.

Da eine Analyse mit Bezug auf positionelle Treue unplausibel ist, bleibt nur die Suche nach relevanten Markiertheitsbeschränkungen. Angesichts der Evidenz für die phonologische Schwere geschlossener Silben im Deutschen, im Gegensatz zu offenen und leichten Silben (Vennemann 1994: 17), bietet sich ein Bezug auf die Markiertheitsbeschränkung STRESS-TO-WEIGHT (Wenn betont, dann schwer) (Kager 1999: 268) an. Die Generalisierung, dass diese Markiertheitsbeschränkung im Deutschen nur dann aktiv wird, wenn auf den fraglichen Kopffuß ein weiterer Fuß folgt, ${ }^{23}$ lässt sich mithilfe lokaler Beschränkungsverknüpfungen erfassen. Die in (27a) gezeigte Struktur verletzt sowohl STRESS-TO-WEIGHT als auch ALIGN-FT-RIGHT (Jeder Fuß steht am rechten Ende des phonologischen Worts) ${ }^{24}$ (Kager 1999: 268) und ist daher unstabil. Die Struktur in (27b) hingegen verletzt nur ALIGN-FT-RIGHT, die Formen in der rechten Spalte in (28) verletzen nur STRESS-TO-WEIGHT. In beiden Fällen sind die fraglichen Lautmuster im Deutschen weitgehend stabil.

23 Eine denkbare phonetische Erklärung des in (28) veranschaulichten Unterschieds aufgrund einer Kürzung der Vokaldauer in mehrsilbigen Wörtern ist durch die Stabilität langer Vokale in der Pänultima mehrsilbiger Wörter widerlegt (z. B. Makka'r[o:]ni, Pano'r[a:]ma). Wenn auch betonte Vokale in mehrsilbigen Wörtern wie Pyjáma oder Panoráma etwas kürzer als der entsprechende Vokal in zweisilbigem Láma sein mögen, so tendieren sie keinesfalls dazu, mit dem entsprechenden B-Vokal zusammenzufallen (vgl. die betonten Vokale in Panoráma und Gámma). 24 Markiertheitsbeschränkungen wie ALIGN-FT-RIGHT oder ALIGN-FT-LEFT bringen zum Ausdruck, dass Fußbildung universell entweder rechts- oder linksbündig ist. Im Deutschen ist sie rechtsbündig. 
Zusammenfassend lässt sich sagen, dass Neutralisationserscheinungen spezifische Interaktionen von Markiertheits- und Treuebeschränkungen motivieren, die ihrerseits Licht auf anzunehmende prosodische Strukturen liefern. Dazu gehören in der deutschen Grammatik nicht nur bestimmte Silbenstrukturen, sondern auch übergeordnete Fußstruktur, einschließlich der Markierung von Kopffüßen. Unabhängige empirische Evidenz für solche prosodischen Strukturen betrifft phonetische Realisationserscheinungen, d. h. mögliche Stärkung bzw. Abschwächung der auf der phonemischen Ebene vorgegebenen Segmente in Abhängigkeit von der prosodischen Position (s. Abschnitt 4).

\subsection{Evidenz für die Art der Opposition (Qualität, Quantität, Prosodie)}

Neutralisationserscheinungen werfen nicht nur Licht auf prosodische Organisation oder die Frage der Proportionalität von Oppositionen, sondern geben auch Aufschluss über das Wesen der Opposition. Hier liegt der Schlüssel in den spezifischen Markiertheitsbeschränkungen, die Treuebeschränkungen dominieren und damit die Aufhebung von Kontrasten verursachen.

Hinsichtlich der im Deutschen aktiven Markiertheitsbeschränkungen in (23) stellt sich die Frage, ob ein solcher Aufhebungskontext eher mit einem Bezug auf Quantität (29a) oder Qualität (29b) einhergeht.
a. ${ }^{\star} \sigma^{\text {geschlossen }} /[+$ lang $],{ }^{\star} \sigma^{\text {offen }} /[$-lang $]$
b. ${ }^{\star} \sigma^{\text {geschlossen }} /[+$ peripher $],{ }^{\star} \sigma^{\text {offen }} /[$-peripher $]$

Zunächst sind beide Fassungen der Markiertheitsbeschränkungen in (29) als natürlich anzusehen, da eine geschlossene gegenüber einer offenen Silbe eher eine kürzere und stärker zentralisierte Vokalartikulation begünstigt. Bekannte sprachübergreifende Erscheinungen wie Open Syllable Lengthening und Closed Syllable Shortening stützen die Annahme der Spezifikation in (29a), aber es wäre zunächst zu prüfen, inwieweit diese Regeln die Aufhebung vokalischer Kontraste verursachen. Aufschlussreich ist in diesem Zusammenhang das sogenannte Loi de Position im Französischen. Die Daten in (30a) veranschaulichen das Bestehen eines dem $A: B$-Kontrast im Deutschen ähnelnden Kontrasts für mittlere Vokale im Französischen. Die Kontexte für die Aufhebung des Kontrasts in (30b) weisen auf die Relevanz von Silbenstrukturen hin (nur gerundete „periphere“ Vokale in offenen Silben, nur ungerundete „zentralisierte“ Vokale in geschlossenen Silben), wobei eindeutig auf Qualität und nicht Vokallänge Bezug genommen wird. 
(30)

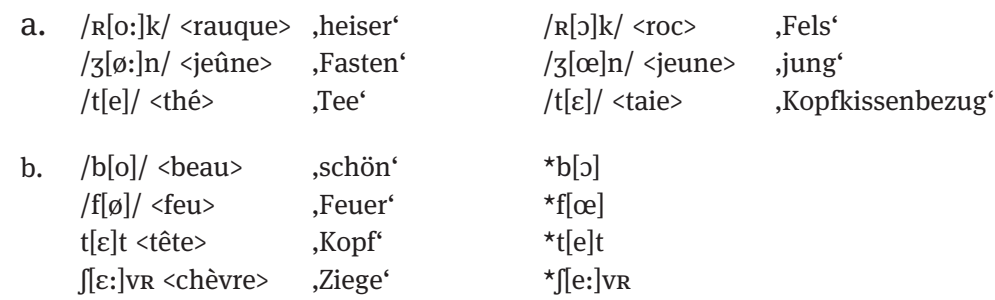

Die Daten sind somit mit der Annahme konsistent, dass die Markiertheitsbeschränkung in (29b) in beiden Sprachen aktiv ist. Die Sprachen unterscheiden sich unabhängig hinsichtlich der phonetischen Vokaldehnungsregeln, die im Französischen auf bestimmte geschlossene Silben begrenzt sind (Tranel 1987).

Eine wenig bekannte Neutralisierungserscheinung, die sehr stark für die Annahme einer Qualitätsopposition im Deutschen spricht, ist in (31a) veranschaulicht. Es zeigt sich, dass auch in betonten Silben der $A: B$-Kontrast weitgehend aufgehoben ist und nur $B$-Vokale erscheinen, wenn die Folgesilbe geschlossen ist und sich auf diese Weise zwei gleiche $B$-Vokale ergeben. ${ }^{25}$ Auch dieses Phänomen gilt gleichermaßen für offene und nicht-offene Vokale und untermauert somit weiter die Parallelität der relevanten Opposition.
a. /'f $[\varepsilon] \mathrm{n} \varepsilon \mathrm{k} /<$ Fenek $>$ /'b[o]trop/ <Bottrop $>$ /'n[v]bvk/ <Nubuk $>$ /'v[I]tip/ <Witib> /'t[a]bak/ <Tabak > /'m[a]dRas/ <Madras $>$ /'t[a]kRaf/ <TAKRAF>
b. /'l[e:]nə/<Lehne> : : /'h[c]nə/ <Henne > /'l[o:]tRInən/ <Lothringen>
/'k[u:]ba/ <Kuba $>\quad$ : /'k[v]ba/ $<$ Kubba $>$
/'v[i:]ta/ <Vita $\quad:$ : me'l[I]ta/ <Melitta $>$
$/$ 'ks[a:]bi/ <Ksabi $>\quad: /$ R[a]bi/ $<$ Rabbi $>$
/'p[a:]dRə/ <Padre $>$
/'n[a:]gRa/ $\langle\text { NAGRA }\rangle^{26}$

Die besondere Signifikanz dieser Neutralisation liegt darin, dass es sich offensichtlich um eine Harmonieregel handelt, die universell nur auf segmentale Merkmale zugreifen kann. Quantitäts- oder gar Silbenstrukturharmonien sind aus den Sprachen der Welt nicht bekannt. Die Daten in (31a) legen nahe, dass sogar die Neutralisation zu $A$-Vokalen vor Plosiv-Liquid-Verbindungen (z. B. /'l[o:]tRInən/ <Lothringen>) außer Kraft gesetzt ist: Um die Harmonie zu gewährleisten, erscheinen auch vor solchen Verbindungen nur zentralisierte Vokale (s. /'b[j]tRop/ <Bottrop>, 't[a]kRaf/ <TAKRAF>). Nur vor /h/, das unter keinen Umständen ambisilbisch organisiert werden kann, kann sich die Harmoniebeschränkung nicht durchsetzen (s. (32b)).

25 In anderen Fällen zeigen sich auch derartige Harmonieeffekte, allerdings weniger systematisch (z. B. /'gulaf/ <Gulasch>, /'amık/ <Amok>, /'patRik/ <Patrick>).

26 TAKRAF: Tagebauausrüstungen, Krane und Förderanlagen, NAGRA: Nationale Genossenschaft für die Lagerung radioaktiver Abfälle. 
(32) a.

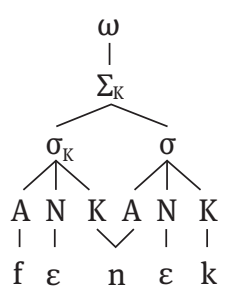

$<$ Fenek> b.

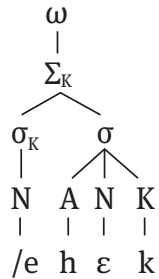

$<$ EHEC $\rangle^{27}$

Da sich die Vokalopposition nunmehr als eine segmentale Qualitätsopposition $\mathrm{zu}$ erkennen gegeben hat, erscheinen von nun an nur noch entsprechende Symbole in den phonemischen Repräsentationen. Die Vokaldehnung in offener betonter Silbe in (32b) sowie die Glottalisierung des Vokals werden gleichermaßen als kontextuell bedingte Realisationserscheinungen aufgefasst, die nicht in die phonologische Repräsentation gehören.

Ein abschließendes Argument gegen die Annahme einer Quantitätsopposition im Deutschen betrifft die Stabilität des Wortakzents auf der geschlossenen Pänultima wie in (33a), im Vergleich zu der möglichen Variation in (33b, c) (vgl. Visch \& Kager 1984; ${ }^{28}$ Vennemann 1994). Hier zeigt sich, dass der Akzent im Deutschen keineswegs von Vokallänge angezogen wird (s. die phonetische Dehnung des Vokals in der Pänultima in (33b)), sondern von geschlossenen Silben (vgl. auch Kommándo, Nirósta). Die Vokaldehnung ist somit zwingend als Folge der Position des Wortakzents anzusehen, nicht als dessen Ursache.

(33) a.

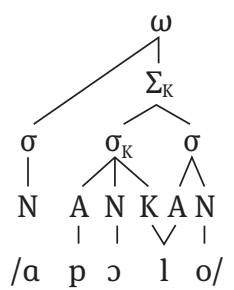

<Apollo> b.

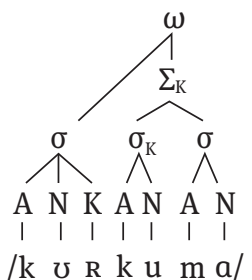

Dehnung c.

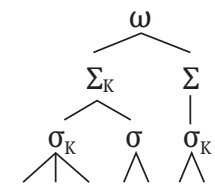

A N K A NA N

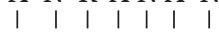

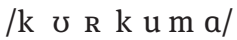

$<$ Kurkuma $>$

27 S. Fußnote 16.

28 Die früheste Veröffentlichung dieses Arguments findet sich in dieser Publikation zum Holländischen, wo der gleiche Zusammenhang wie im Deutschen besteht. 
Wie zu erwarten, besteht der gleiche Zusammenhang für offene Vokale: auch hier ist der Akzent auf zentralisierten Vokalen in der Pänultima stabil (Havánna), auf peripheren, phonetisch gelängten Vokalen hingegen schwankt er (Dynámo Dýnamo). Die Parallelität sämtlicher Vokale in der deutschen Vokalopposition ist somit weiter untermauert. Auch dieses Argument hat mit Kontrast und Neutralisation zu tun, da in Fällen wie (33b, c) ein Bedeutungsunterschied zumindest möglich wäre (vgl. /'eta/ <Eta> vs. /e'ta/ <Etat>), in (33a) aufgrund der Fixierung des Akzents auf der Pänultima aber ausgeschlossen ist.

\subsection{Der Status von [e]}

Abschließend soll noch kurz auf die Frage des phonemischen Status von [e] im Deutschen aus Sicht des hier vorgestellten Grammatikmodells eingegangen werden. Der Hinweis auf Minimalpaare wie /'od[ə]/ <Ode> vs. /'od[e]/ <oder> (s. 2d) mag in den Augen mancher Autoren genügen, um den Phonemstatus für beide Vokale abzusegnen. Aus Sicht der hier vorgestellten Herangehensweise stellt aber die Begrenzung dieses Kontrasts auf unbetonte Silben ein starkes Gegenargument dar, da allgemein gilt, dass ein phonemischer Kontrast das Bestehen des Kontrasts in prominenteren Positionen voraussetzt. Die Beschränkung eines einzelnen merkmallosen Reduktionsvokals auf unbetonte Silben scheint hingegen mit der fraglichen Präferenz vereinbar.

Die eingangs behandelten Wörter Krabbe und aber werden entsprechend beide mit Schwa in der unbetonten Silbe repräsentiert. Die Silbifizierung des wortfinalen /R/ im Ansatz beruht auf der Annahme einer aktiven Markiertheitsbeschränkung *Koda/ $\{\mathrm{R}, 1, \mathrm{n}\}$ (Keine Assoziation von $\{\mathrm{R}, 1, \mathrm{n}\}$ mit der Koda), auf die an dieser Stelle nicht näher eingegangen werden kann. ${ }^{29}$

(34) a.

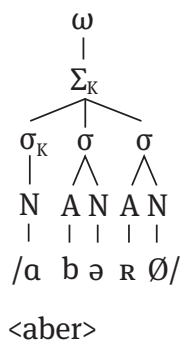

b.

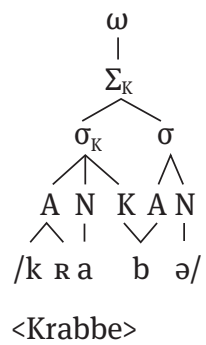

29 Im Deutschen bewirkt diese Beschränkung die Organisation sämtlicher wortfinaler Sonoranten $\{\mathrm{R}, \mathrm{l}, \mathrm{n}\}$ im Silbenansatz, sofern Schwa oder nicht hauptbetonte hohe Vokale vorausgehen. S. Raffelsiefen (i. Vorb.). 
Diverse Probleme, die sich aus der Annahme eines Phonems /e/ im Deutschen ergeben, zeigen sich in den Umschriften in deutschen Aussprachewörterbüchern. Die Repräsentation des Kontrasts in Wörtern wie <eher> und <er> durch ein Diakritikum für silbisches versus unsilbisches /e/, wie von Mangold (Duden 2005) vorgeschlagen (i.e. ['e:e] <eher> vs ['e:e् ] <er>), ist aus markiertheitstheoretischer Sicht unplausibel. Sprachübergreifend gilt, dass Vokale desto eher in Silbenrandpositionen zugelassen sind, je höher sie sind (Prince \& Smolensky 2004: 160). Für /i/ und / $\mathrm{u} /$ wird eine Silbifizierung im Silbenrand entsprechend oft angenommen, für das „halbtiefe“ [e] (s. (6)) wäre sie völlig unerwartet. Die Repräsentationen dieser Wörter in dem hier vorgestellten System sind in (35a, b) gezeigt.

Die Silbifizierung von / $\mathrm{R} /$ in der Koda in (35c) ist dadurch bedingt, dass die Treuebeschränkung TREUE( \pm peripher) ${ }_{\omega-\mathrm{K}}$ die Markiertheitsbeschränkung ${ }^{\star} \mathrm{Koda} /\{\mathrm{R}, \mathrm{l}, \mathrm{n}\}$ dominiert.

(35) a.

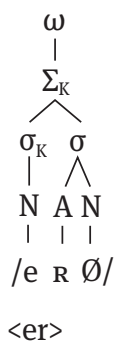

b.

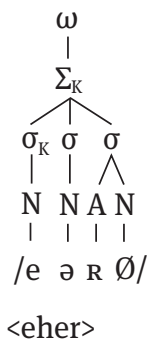

c.

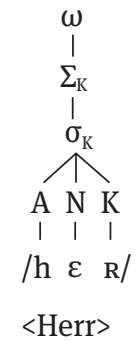

In den Umschriften der deutschen Aussprachewörterbücher wird gewöhnlich dasselbe Symbol [e] (mit Diakritika für die silbische Organisation) für die Repräsentation der Wortenden in $(35 \mathrm{a}, \mathrm{b})$ verwendet. Aus phonetischer Sicht erscheint diese Gleichstellung zweifelhaft. ${ }^{30}$ Für das Wortende in (35c) wird meist ein anderes Symbol verwendet, dessen Auswahl keineswegs offensichtlich ist. Hier zeigen sich typische Unzulänglichkeiten im Zusammenhang mit der symbolischen Repräsentation phonetischer Form. Aus phonemischer Sicht ist wesentlich, dass in allen drei Fällen in (35) das Phonem /R/, das einen uvularen Approximanten bezeichnet, erscheint. Phonetische Unterschiede ergeben sich bei der Annahme dieses jeweils selben artikulatorischen Ziels aufgrund unterschiedlicher Kontexte und entsprechend unterschiedlicher Bedingungen für die Artikulation (vgl. Schiller \& Mooshammer 1995).

30 Kohler nähme in (35a) einen seiner insgesamt dreizehn „,[e]-Diphthonge“ an (/ee/) (1999: 88). Seine Anmerkung „the ending -er is realized as [e] (e.g. ['bute] <Butter> “ (1999: 88)) lässt darauf schließen, dass er in (35b) einen Monophthong / $\mathrm{e} /$ annimmt. Die intendierten Ebenen 


\section{Empirische Voraussagen}

Die hier vorgestellte Analyse macht diverse empirisch überprüfbare Voraussagen, die im Folgenden mit einigen Beispielen erläutert werden. ${ }^{31}$

Eine Voraussage betrifft phonetische Korrelate für phonemische Kontraste. Die Annahme eines phonologischen Qualitätskontrasts in Fällen wie prahlen: prallen lässt signifikante qualitative Unterschiede zwischen diesen Vokalen erwarten. Insbesondere artikulatorische Studien bestätigen eine eher zentralisierte Artikulation des kurzen Vokals gegenüber einer mehr peripheren, weiter hinten gelegenen Artikulation des langen Vokals (vgl. Wängler 1964; Bohn 1992; Hoole 2002). Dieser spezifische Unterschied rührt an eine weitere Voraussage, nämlich dass proportionale Oppositionen ein konsistentes phonetisches Korrelat aufweisen sollten. ${ }^{32}$ Die in (3a) gezeigten Messergebnisse zeigen dass eine stärker periphere versus stärker zentralisierte Artikulation ein plausibles phonetisches Korrelat für die phonologischen $A: B$-Kontraste sind. (Lindau 1978). Für die offenen Vokale ist dieses Korrelat am umstrittensten, lässt sich aber neben den artikulatorischen Studien auch in akustischen Studien bestätigen. Die Abbildung in (36) zeigt die dynamischen Verläufe von $25 \%$ bis $75 \%$ (Pfeilende) der beiden offenen Vokale wie in prallen (links) versus prahlen (rechts), gesprochen von 26 Sprecherinnen im Kielkorpus. ${ }^{33}$ Das Merkmal [+peripher] sagt eine größere Distanz der jeweiligen Vokale von einer berechneten Zentralposition voraus, das Merkmal [-peripher] eine entsprechend kürzere Distanz. Diese Voraussage wird durch die spezifischen Längenunterschiede der relevanten durch gepunktete Linien markierten Distanzen in (36)

der Abstraktheit sind dabei nicht erklärt. Schwebt ihm vor, dass aufgrund der „[e]-Diphthonge“ das Vokalsystem zu verdoppeln ist? Wie ist es zu verstehen, dass er das Symbol [e] in seinen Beispielwörtern verwendet, es aber nicht in sein Inventar der Monophthonge aufnimmt?

31 Ich danke den jetzigen und früheren MitarbeiterInnen im Projekt Wortphonologie am IDS Mannheim, Fabian Brackhane, Anja Geumann und Sandra Hansen-Morath, für die Durchführung und Auswertung der hier vorgestellten Messungen.

32 Hier stellt sich die wichtige Frage, auf welcher Basis sich die relevanten Oppositionsglieder einander zuordnen lassen. Aufschluss bieten hier regelhafte phonologisch bedingte Alternationen wie etwa /'tibst/ <Tibet $>$ - /ti'betif/ <tibetisch>, /'dæmon/ <Dämon > - /dæ'monIJ/ $<$ dämonisch>, die die Zuordnung der Vokale $/ \varepsilon /: / e /$ und / /://o/ stützen. Andere Evidenz für solche Zuordnungen zeigt sich in Abweichungen von strikter lautlicher Übereinstimmung in Assonanz- oder Reimmustern. So stützt etwa die Assonanz in <treffen $>-<$ Lehrer $>$ sowie $<$ Messer $>-<$ Tränen> in Brentanos Gedicht Romanzen vom Rosenkranze ebenfalls die Zuordnung der Vokale $/ \varepsilon /: / \mathrm{e} /$, eventuell auch / $\varepsilon /: / æ /$ sofern Brentano /e/ und /æ/ unterscheidet. 33 Für weitere relevante Studien s. Hansen-Morath, Geumann \& Raffelsiefen (2018). 
bestätigt. ${ }^{34}$ Insgesamt wird die Annahme unterschiedlicher Vokalqualitäten durch die signifikant unterschiedlichen dynamischen Verläufe gestützt.

(36) Unterschiedliche dynamische Verläufe (25\%-75\%) der beiden offenen Vokale im Deutschen

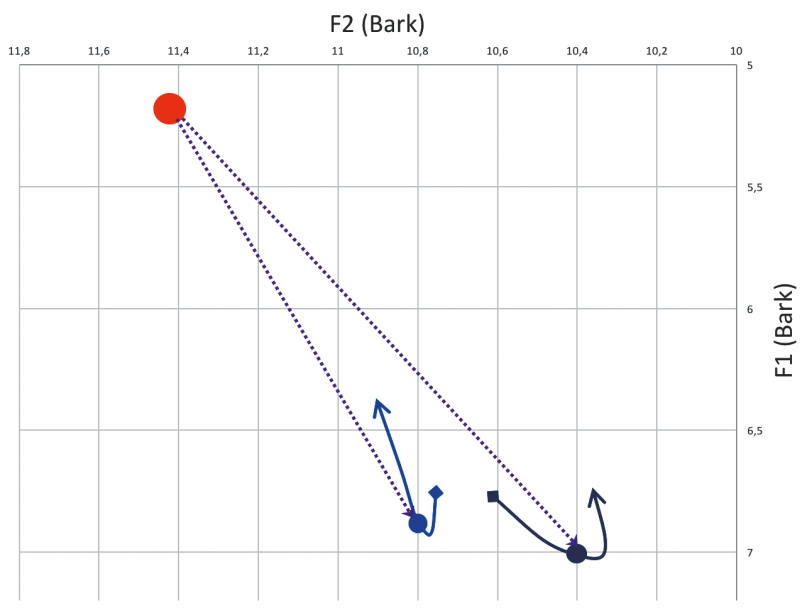

Die Voraussagen der hier vorgestellten Analyse betreffen nicht nur die Kontrast-, sondern auch die Aufhebungspositionen. So wird etwa erwartet, dass $A$-Vokale gegenüber $B$-Vokalen auch in Aufhebungspositionen qualitativ unterschiedene Gruppen bilden, wobei für Dauer keinerlei Gruppenbildung vorausgesagt wird. Die Ergebnisse einer hier relevanten Studie, basierend auf Deutsch-Heute-Daten, sind in (37) gezeigt. Die grünen Markierungen in der Formantenkarte in (37a) markieren die Positionen hinterer mittlerer Vokale in offenen unbetonten Silben (z. B. Áut[O], K[O]píe), während die blauen Markierungen die Positionen der entsprechenden Oppositionsglieder in geschlossenen unbetonten Silben markieren (z. B. [O]któber, Poseid[O]n). Es zeigt sich jeweils eine Gruppenbildung, wobei offene Silben (Áut[O], K[O]píe) mit mehr peripheren und geschlossene Silben ([0]któber, Poseid[O]n) mit eher zentralisierten Positionen einhergehen. Wichtig ist nun, dass sich für die jeweiligen Vokaldauern keine solche Gruppenbildung zeigt (vgl. (37b)). Stattdessen ist die Dauer ausschließlich von der Position des Vokals innerhalb der prosodischen Struktur determiniert.

34 Der große Punkt links oben markiert die berechnete Zentralposition, die Distanzen beziehen sich auf die 50-Prozent-Marken der jeweiligen Vokalverläufe. 
(37) a. Vokalqualitäten in Aufhebungspositionen

(Aufhebungspositionen: unbetonte offene versus geschlossene Silben)

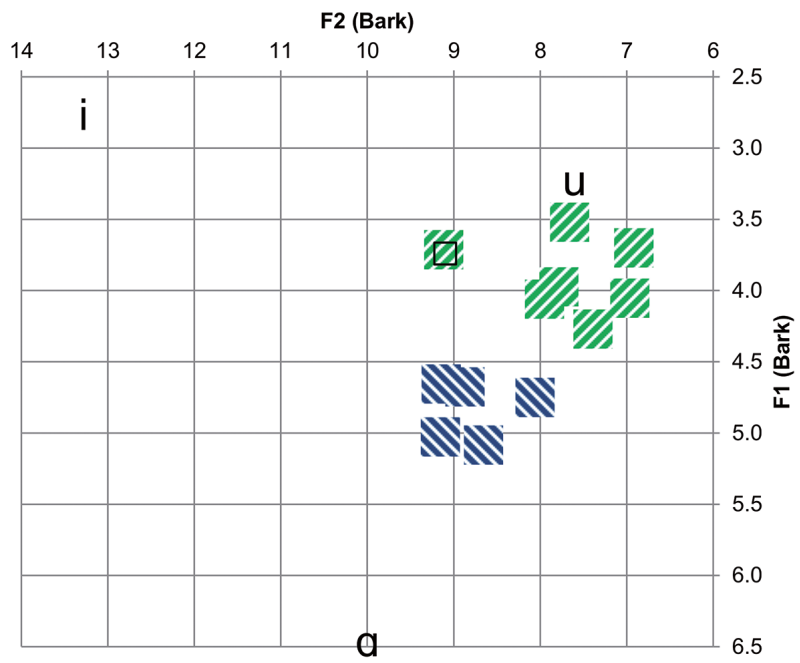

b. Vokaldauern in Aufhebungspositionen

(Aufhebungspositionen: unbetonte offene versus geschlossene Silben)

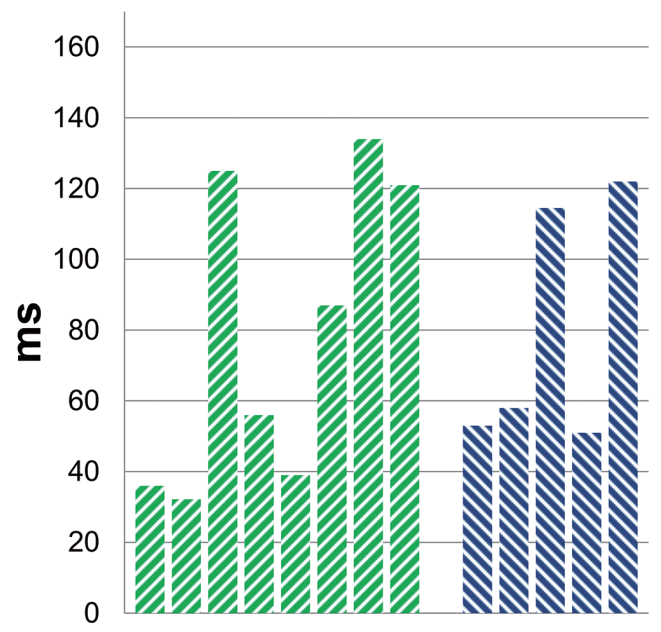

Die Dauerunterschiede in (37b) weisen auf eine weitere Voraussage hinsichtlich der phonetischen Realisation von Phonemen in unterschiedlichen Kontexten. Hier wird erwartet, dass dieselben Phoneme in unterschiedlichen Kontexten durchaus unterschiedlich realisiert werden, wobei die fraglichen Unterschiede 
durch den Kontext determiniert sind. So wird längere Vokaldauer in Kopffüßen gegenüber anderen Füßen erwartet, ebenso stärkere Glottalisierung oder Aspiration in fußinitialer Position im Vergleich zu unbetonten Silben.

Die in (37) gezeigten Ergebnisse passen zu Ergebnissen weiterer Studien zu Paradigmenuniformitätseffekten, basierend auf einem eigens erstellten Korpus von 20 Sprechern. Unter der Annahme, dass Korrespondenzbeschränkungen immer nur auf phonemische Struktur Zugriff haben können, wird vorausgesagt, dass qualitätsbezogene Paradigmenuniformitätseffekte im Deutschen existieren können, quantitätsbezogene hingegen nicht. Die erste Voraussage wird in den Messungen der Vokale in (38) bestätigt. Das <i > in der Pluralform Yetis zeigt die gleichen Formantenwerte wie das $<\mathrm{i}>$ in der dazugehörigen Singularform Yeti, nicht wie das $<\mathrm{i}>$ in gratis. Ähnliche Effekte zeigen sich auch für die mit <a> geschriebenen Vokale in Plural Omas, Singular Oma und der Vergleichsform Thomas. $^{35}$

(38) Beeinflussung von Vokalqualität durch Korrespondenzbeschränkungen a.

\section{F2 (Bark)}

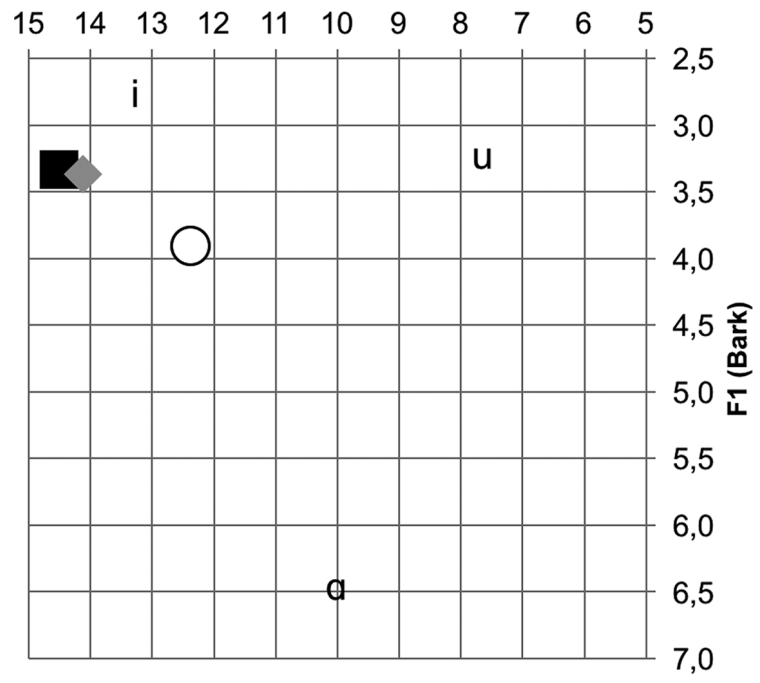

- Yeti $>$ Yetis $O$ gratis

35 Paradigmenuniformitätseffekte sind nicht unbedingt so kategorisch wie in (38), sondern zeigen sich oft nur in bestimmten systematischen Abweichungen von Vergleichsformen. 
b. F2 (Bark)

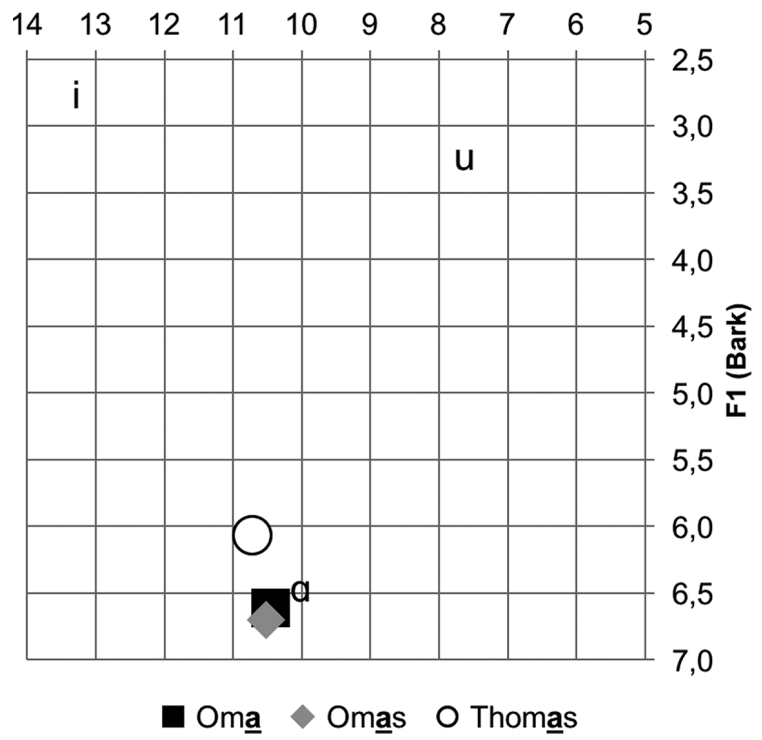

Die Daten in (39) zeigen die völlige Abwesenheit vergleichbarer Paradigmenuniformitätseffekte für die Vokaldauer. Das betonte $<\mathrm{a}>$ in Plato ist relativ lang. Das $<\mathrm{a}>$ in platonisch scheint von der Länge dieses korrespondierenden Vokals aber vollkommen unbeeinflusst und ist nicht länger als das <a> in der ersten Silbe in der Vergleichsform Platane. Ein ähnlicher Mangel an paradigmatischer Beeinflussung der <a>-Dauern lässt sich auch in (39b) beobachten. ${ }^{36}$

(39) Keine Beeinflussung von Vokaldauer durch Korrespondenzbeschränkungen

a.

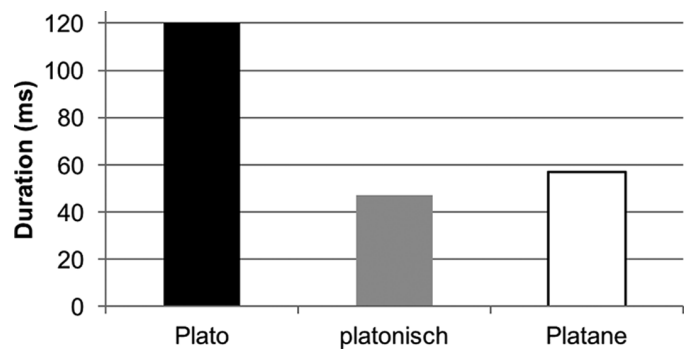

36 Für weitere Analysen dieser Art, s. Raffelsiefen (2016). Dort werden u. a. die Abwesenheit von Paradigmenuniformitätseffekten für phonetische Realisationserscheinungen wie Auslautverhärtung, /R/-Vokalisierung, und der [ç]-[x]-Allophonie im Deutschen behandelt. 
b.

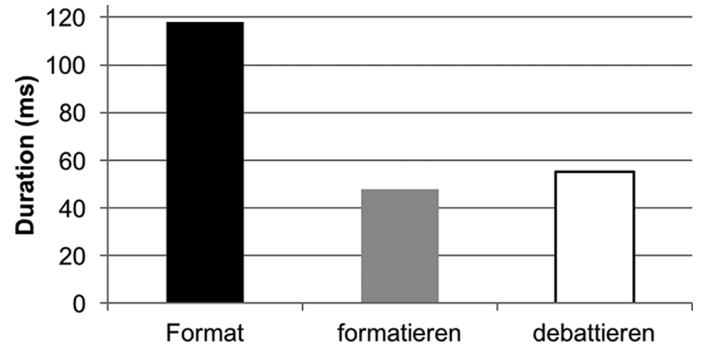

Paradigmenuniformitätseffekte werden auch für phonemische Struktur nicht zwangsläufig erwartet, da übergeordnete Markiertheitsbeschränkungen phonemische Alternationen verursachen können (z. B. Tib/ع/t - Tib/e/ter). Die strikte Voraussage betrifft die notwendige Abwesenheit solcher Effekte mit Bezug auf subphonemische Lautstruktur.

\section{Zusammenfassung und Ausblick}

Das zentrale Anliegen des vorliegenden Artikels ist die Bestimmung symbolischer Repräsentationen für Simplizia des Deutschen. Das Ziel ist dabei, klare Kriterien zu entwickeln, die zu einer angemessenen und einheitlichen Abstraktionsstufe führen.

Die hier vorgestellte Vorgehensweise zielt zunächst auf die Herausarbeitung einer phonologischen Grammatik in Form geordneter Beschränkungen im Rahmen der Optimalitätstheorie. Die Grundidee ist dabei, ausgehend von Minimalpaaren vorläufige Treuebeschränkungen anzunehmen, die potenzielle Kontraste repräsentieren. Die Untersuchung der relevanten Neutralisationserscheinungen wirft Licht auf aktive Markiertheitsbeschränkungen, die auf sprachvergleichender Grundlage zu identifizieren sind. Deren Eigenschaften lassen dann Schlüsse sowohl auf die Beschaffenheit der Phoneme und der relevanten Oppositionen als auch auf die prosodische Organisation der Phonemverbindungen in Wörtern $\mathrm{zu}^{37}$

37 Anzumerken ist hier, dass diese Herangehensweise in der Optimalitätstheorie keineswegs üblich ist. Im Allgemeinen besteht auch unter Vertretern dieser Richtung wenig Interesse an Abstraktheitsfragen; die Theorie wird gewöhnlich auf Beschreibungen phonetischer Strukturen angewendet. Auch dieser Umstand dürfte am ehesten wissenschaftssoziologisch zu erklären sein. Die Begründer und viele Anwender entstammen dem Umfeld der Generativen Phonologie, die die dem Strukturalismus zugeordnete Phonemebene vehement ablehnte. 
Diese Herangehensweise wurde hier anhand der sogenannten Vokalopposition im Deutschen veranschaulicht. Das Ergebnis ist ein System von vierzehn bzw. fünfzehn Vollvokalen, die eine durchgehende Qualitätsopposition (peripher versus zentralisiert) bilden. Das Inventar erscheint zwar aus sprachtypologischer Sicht ausgesprochen groß, ist aber von drastischen Neutralisierungserscheinungen geprägt. Ein Simplex im Deutschen weist maximal eine potenzielle Kontrastposition für das Merkmal [ \pm peripher] auf (die Kopfsilbe im Kopffuß), in vielen Fällen aber besteht aufgrund aktiver Markiertheitsbeschränkungen nicht einmal eine solche Position.

Die Analyse wird empirisch gestützt durch konvergierende Typen von Evidenz, einschließlich messphonetischer Studien. Die Evidenz widerspricht der Annahme des Mischsystems wie auch eines reinen Quantitätssystems. Auch die vielversprechendste der alternativen Analysen, die den phonologischen Kontrast ausschließlich in der prosodischen Struktur verankert, passt nicht mit allen Befunden zusammen. $\mathrm{Zu}$ nennen wäre hier die Vokalharmonie, die einen segmentalen Qualitätsunterschied voraussetzt, sowie die phonetische Beschaffenheit der Vokale in diversen Kontrastaufhebungskontexten. Hier macht die hier vorgeschlagene Analyse konkrete Voraussagen darüber, welches Oppositionsglied in der jeweiligen Aufhebungsposition erscheint. Eine Analyse, die Qualitäts- und Längenunterschiede gleichermaßen als allophonische Realisationserscheinungen begreift, kann diesen Mustern kaum Rechnung tragen. Darüber hinaus ist die Beschränkung möglicher Paradigmeneffekte auf segmentale Qualitätsmerkmale zu nennen, die in der in (7) veranschaulichten prosodischen Analyse nicht erfasst wird.

Vorausgesetzt, dass die phonologische Grammatik einer Sprache und die entsprechenden Repräsentationen empirisch adäquat sind, sind sie auch für Aussprachewörterbücher und orthographische Studien relevant. Hinsichtlich der Aussprachewörterbücher ergäbe sich mit Blick auf die hier vorgestellten hierarchischen prosodischen Repräsentationen die praktische Frage, wie diese sich durch eine lineare Abfolge von Symbolen repräsentieren lassen. Es stellt sich heraus, dass die Markierung der linken Fußgrenzen im Deutschen ausreicht, wobei Kopffüße traditionell durch ein hochgestelltes, andere Füße durch ein tiefgestelltes Zeichen markiert werden. Einige Beispiele sind aufgeführt.

(40) 'RJgən <Roggen>, 'Rogən <Rogen>, /ka'ot/ <Chaot>, /'kaəs/ <Chaos>, /ROR/ <Rohr $>$

Phonetische Erscheinungen wie Vokaldehnung, Glottalisierung, oder/R/-Vokalisierung gehören nicht in symbolische phonologische Repräsentationen. Sie 
gehören in die separaten Beschreibungen der „Ausspracheregeln“, die ja ohnehin einen festen Bestandteil bestehender Aussprachewörterbücher bilden.

In Fällen, in denen Varianz besteht, hat eine der Varianten in vielen Fällen eher den Charakter eines unassimilierten Lehnworts, während die andere eher mit der phonologischen Grammatik des Deutschen im Einklang steht. Hier wäre $\mathrm{zu}$ überlegen, letztere Varianten entsprechend $\mathrm{zu}$ kennzeichnen. Einige Beispiele, in denen regelkonforme Varianten unterstrichen sind, sind in (41) aufgeführt: ${ }^{38}$

'tiflıs, 'tifllis <Tiflis>, bi'stro, 'bIstro $<$ Bistro>, 'teta,nus, 'teta,nus <Tetanus>

Hinsichtlich orthographischer Studien gilt, dass der Bezug auf angemessene phonologische Repräsentationen essentiell für die Erforschung des relevanten Regelapparats ist, unabhängig davon, ob es sich um eine Silben- oder um eine Alphabetschrift wie im Deutschen handelt. Als Beispiel mit Bezug auf die oben vorgestellten Analysen sei die orthographische Markierung zentralisierter Vokale durch nachfolgende Doppelkonsonanz erwähnt, die die ambisilbische Organisation widerspiegelt. Interessant ist hier, dass die Doppelkonsonanz nur dann systematisch erscheint, wenn der Vokal in einer Kontrastposition erscheint (s. 42a). Erscheint ein zentralisierter Vokal in einer Aufhebungsposition, folgt oft selbst in betonten Silben ein einfacher Konsonant (s. (42b)).
a. /'krabə/ <Krabbe >
: $\quad /$ 'Rabə/ <Rabe $>$
/'bet/ <Bett $>$
: $\quad$ /'bet/ <Beet $>$
/'moka/ <Mokka >
: $\quad /$ 'koka/ <Koka $>$
b. /'kaba, Ret/ <Kabarett>, /'tabak/ <Tabak>, /e'liza,bet/ <Elisabeth>, /'moka $\sin /<$ Mokassin>

\begin{abstract}
Abschließend sei noch darauf hingewiesen, dass die symbolische Repräsentation der hier festgestellten fünfzehn deutschen Vokalphoneme Schwierigkeiten bereitet. Das hier verwendete Symbol /a/ ist eigentlich zur Bezeichnung eines vorderen Vokals gedacht, auch das Symbol /æ/ passt nicht sonderlich zur Bezeichnung des Vokals in Wörtern wie zäh.
\end{abstract}

38 Der Begriff der Regelkonformität rührt natürlich an schwierige empirische Fragen. Grundsätzlich gilt, dass die Stabilität oder gar Neuentstehung vermeintlicher Regelabweichungen die empirische Inadäquatheit der Beschreibung nahelegt. Wenn etwa die Verletzung der Harmonieregel in Fällen wie /'iRIs/ <Iris>, /'ibıs/ <Ibis> stabil ist, gilt zu prüfen, ob es unabhängige Evidenz für übergeordnete Beschränkungen bzw. lokale Verknüpfung solcher Beschränkungen gibt, die die Regelhaftigkeit dieser Ausnahmen zum Ausdruck bringen. 
Das Problem ist schlicht, dass nicht genügend passende Symbole für die Vokale des Deutschen von den IPA-Kommissionen bereitgestellt wurden. Dieses Problem sollte nicht durch die Verwendung gleicher Symbole für kontrastierende Vokale gelöst werden. Eher sollte man sich an der Kühnheit des Ersten Grammatikers ein Beispiel nehmen und zusätzliche Zeichen einführen.

\section{Literatur}

Alber, Birgit (2001): Regional variation and edges: Glottal stop epenthesis and dissimilation in standard and southern varieties of German. Zeitschrift für Sprachwissenschaft 20 (1), 3-41.

Arens, Hans (1969): Sprachwissenschaft. Der Gang ihrer Entwicklung von der Antike bis zur Gegenwart. Zweite Auflage. Freiburg/München: Verlag Karl Alber.

Becker, Thomas (1998): Das Vokalsystem der deutschen Standardsprache. Frankfurt am Main: Peter Lang.

Becker-Kristal, Roy (2010): Acoustic typology of vowel inventories and dispersion theory. Ph.D. Dissertation. University of California Los Angeles.

Beckman, Jill (1998): Positional faithfulness. Ph.D. Dissertation. University of Massachusetts, Amherst.

Bohn, Ocke-Schwen, James Emil Flege, Paul A. Dagenais \& Samuel G. Fletcher (1992): Differenzierung und Variabilität der Zungenpositionen bei der Artikulation deutscher Vokale. Zeitschrift für Dialektologie und Linguistik, Beiheft 72, 1-26.

Chomsky, Noam \& Halle, Morris (1968): The sound pattern of English. Cambridge, MA: The MIT Press.

Botma, Bert \& Marc van Oostendorp (2012): A propos of the Dutch vowel system 21 years on. In Bert Botma \& Roland Noske (Hrsg.), Phonological explorations: Empirical, theoretical, and diachronic issues. Berlin: Mouton de Gruyter. 135-154.

Brinckmann, Caren, Stefan Kleiner, Ralf Knöbl \& Nina Berend (2008): GERMAN Today. An areally extensive corpus of spoken Standard German. In Nicoletta Calzolari, Khalid Choukri, Bente Maegaard et al. (Hrsg.), Proceedings 6th International Conference on Language Resources and Evaluation (LREC), 3185-3191. Marrakesch, Marokko.

Duden (2005) = Mangold, Max (2005): Duden. Bd. 6. Das Aussprachewörterbuch. Berlin/ Mannheim: Dudenverlag.

Fourquet, Jean (1961): Der Vokalismus nichthaupttoniger Silben im deutschen Fremdwort. Phonetica 6, 65-77.

Hall, Tracy Alan (1992): Syllable structure and syllable-related processes in German. Tübingen: Niemeyer.

Hansen-Morath, Sandra, Anja Geumann \& Renate Raffelsiefen (2018): Vergleich der Quantität, Qualität und Dynamik in den deutschen <a>-Laute. In Proceedings of Phonetik und Phonologie im deutschsprachigen Raum. Berlin.

Hoole, Phil \& Christine Mooshammer (2002): Articulatory analysis of the German vowel system. In Peter v. Auer, Peter Gilles \& Helmut Spiekermann (Hrsg.), Silbenschnitt und Tonakzente, 129-153. Tübingen: Niemeyer. 
International Phonetic Association (1908): Exposé des principes de l'Association phonétique internationale. Paris.

Johnson, Keith (2005): Decisions and mechanisms in exemplar-based phonology. UC Berkeley, phonology lab annual report.

Kager, René (1999): Optimality Theory. Cambridge: Cambridge University Press.

Kohler, Klaus J. (1977): Einführung in die Phonetik des Deutschen. Berlin: Erich Schmidt.

Kohler, Klaus (1999): German. In: International Phonetic Association: Handbook of the International Phonetic Association, 86-89. Cambridge: Cambridge University Press.

Krech, Eva Maria (1968): Sprechwissenschaftlich-phonetische Untersuchungen zum Gebrauch des Glottissschlageinsatzes in der allgemeinen deutschen Hochlautung. Basel: Karger.

Krech, Eva-Maria, Eduard Kurka, Helmut Stelzig, Eberhard Stock, Ursula Stötzer \& Rudi Teske (1982): Großes Wörterbuch der deutschen Aussprache (GWDA). Leipzig: VEB Bibliographisches Institut.

Krech, Eva-Maria, Eberhard Stock, Ursula Hirschfeld \& Lutz Christian Anders (2009): Deutsches Aussprachewörterbuch. New York: De Gruyter.

Lindau, Mona (1978): Vowel features. Language 54, 541-563.

Maas, Utz (1999): Phonologie. Einführung in die funktionale Phonetik des Deutschen. Opladen/Wiesbaden: Westdeutscher Verlag.

Mayer, Jörg (2010): Linguistische Phonetik. Monographie. Universität Stuttgart.

Moulton, William G. (1962): The sounds of English and German. Chicago: Chicago University Press.

Oostendorp, Marc van (1995): Vowel quality and phonological projection. Dissertation. Universiteit van Tilburg.

Prince, Alan \& Paul Smolensky (2004): Optimality Theory. Constraint interaction in generative grammar. Oxford: Blackwell.

Raffelsiefen, Renate (2016): Allomorphy and the question of abstractness. Evidence from German. Morphology 26 (3), 235-267.

Raffelsiefen, Renate (in Vorb.): Prosodie und Kontrastivität. Mannheim: IDS.

Rues, Beate, Beate Redecker, Evelyn Koch, Uta Wallraff \& Adrian P. Simpson (2007): Phonetische Transkription des Deutschen. Ein Arbeitsbuch. Tübingen: Narr.

Schiller, Niels O. \& Christine Mooshammer (1995): The character of /r/-sounds: Articulatory evidence for different reduction processes with special reference to German. Proceedings of the XIIIth International Congress of Phonetic Sciences, 452-455.

Sievers, Eduard (1901): Grundzüge der Phonetik zur Einführung in das Studium der Lautlehre der indogermanischen Sprachen. Leipzig: Breitkopf \& Härtel.

Smolensky, Paul (2006): Optimality in phonology II. Harmonic completeness, local constraint conjunction, and feature domain markedness. In Paul Smolensky, Géraldine Legendre (Hrsg.), The harmonic mind. From neural computation to optimality-theoretic grammar 2, 453-535. Cambridge, MA: MIT Press.

Tranel, Bernard (1987): The sound of French. An introduction. Cambridge: University Press.

Steriade, Donca (1997): Phonetics in phonology. The case of laryngeal neutralization. University of California Los Angeles.

Vennemann, Theo (1991): Skizze der deutschen Wortprosodie. Zeitschrift für Sprachwissenschaft 10, 86-111.

Vennemann, Theo (1994): Universelle Nuklearphonologie mit epiphänomenaler Silbenstruktur. In Karl Heinz Ramers, Heinz Vater \& Henning Wode (Hrsg.), Universale phonologische Strukturen und Prozesse, 7-54. Tübingen: Niemeyer.

Viëtor, Wilhelm (1912): Die Aussprache des Schriftdeutschen. Leipzig: O. R. Reisland. 
Visch, Ellis \& René Kager (1984): Syllable weight and Dutch word stress. In Hans Bennis \& Wus van Lessen Kloeke (Hrsg.), Linguistics in the Netherlands, 197-205. Amsterdam: Benjamins.

Walker, Rachel (2011): Vowel patterns in language. Cambridge: Cambridge University Press. Wängler, Hans-Heinrich (1964): Atlas deutscher Sprachlaute. 3. Aufl. Berlin: Akademie-Verlag. 


\title{
19 Graphematik des Deutschen im europäischen Vergleich
}

\begin{abstract}
Eichinger (2012) beschrieb verschiedene Schritte der vergleichenden Grammatik, die sich in den Jahrestagungen des Instituts für Deutsche Sprache widerspiegeln: die kontrastive Grammatik der 1950er Jahre, die insbesondere im Fremdsprachenunterricht eingesetzt werden sollte, die typologische Forschung und anschließend ein „typologisch basierter Vergleich“ (2012: XIII). In der soeben erschienenen „Grammatik des Deutschen im europäischen Vergleich - Das Nominal“ vom Institut für Deutsche Sprache (Gunkel et al. 2017) wird deutlich, wie augenöffnend der Sprachvergleich sein kann, auch um das Deutsche besser zu verstehen - was das Deutsche mit anderen Sprachen teilt, was das Deutsche gegenüber anderen Sprachen auszeichnet und was das Deutsche nicht hat. In diesem letzten Punkt verbirgt sich eine Besonderheit, die zu einem neuen Thema überleiten könnte: Als eines von drei Phänomenen, das im Deutschen fehlt, wird exemplarisch „das Fehlen einer formalen Unterscheidung zwischen restriktiven und nicht-restriktiven Relativsätzen“ (Gunkel et al. 2017: 5) genannt. Diese fehlende formale Unterscheidung betrifft auch das Setzen oder Nicht-Setzen des Kommas und somit einen in der Graphematik zu beschreibenden Unterschied (Zifonun in Gunkel et al. 2017: 1797). Womit wir beim Thema wären: Die Zeit ist reif für eine ,Graphematik des Deutschen im europäischen Vergleich‘.
\end{abstract}

Keywords: Kontrastive Graphematik, Prinzipien der Wortschreibung, Morphologische Schreibung, Wortzeichen, Verbformschreibung

\section{Das Deutsche als Ausgangspunkt}

Wie wohl kaum ein anderes Schriftsystem eignet sich das deutsche als Ausgangspunkt für einen Vergleich der Graphematiken, denn kein anderes

\footnotetext{
Anmerkung: Für zahlreiche Hinweise danke ich den Herausgebern/Herausgeberinnen, insbesondere Angelika Wöllstein und Peter Gallmann.
}

Nanna Fuhrhop, Universität Oldenburg, Fakultät III - Sprach- und Kulturwissenschaften, Institut für Germanistik, D-26111 Oldenburg, E-Mail: nanna.fuhrhop@uni-oldenburg.de

Ә Open Access. (c) 2018 Nanna Fuhrhop, publiziert von De Gruyter. (c) BY Dieses Werk ist lizenziert unter der Creative Commons Attribution 4.0 Lizenz.

https://doi.org/10.1515/9783110490992-020 
Schriftsystem ist so gut erforscht wie das Deutsche. Freilich kann in der Graphematik heftig gestritten werden, man denke nur an die vielen widersprüchlichen Beschreibungen zur Schreibung von Doppelkonsonanten (Ramers 1999; Eisenberg 2017: 3 ff.) oder in jüngster Zeit die Diskussion darüber, ob das Komma syntaktisch oder über Pausen geregelt sei (Sappok \& Naumann 2016: 122 ff.; Bredel \& Hlebec 2015). Doch eines ist trotz solcher Streitigkeiten festzuhalten: Für das Deutsche steckt eine umfangreiche Forschung dahinter, die sich losgelöst hat von dem Gedanken der ,Orthographie‘, also das System erforscht und nicht primär festlegt, wie es denn richtig sei.

\section{Graphematische Typologie bisher}

Es gibt einige Überblicke zu den Schriftsystemen der Welt und viele von ihnen sind absolut lesenswert. Es werden Einblicke in verschiedene Alphabetschriften, verschiedene eher logographische Schriften, Silbenschriften usw. gegeben (so zum Beispiel Rogers 2005). Beim hier vorgestellten Projekt einer Graphematik des Deutschen im europäischen Vergleich geht es jedoch um etwas ziemlich Anderes: Schriftsysteme sollen im Einzelnen verglichen werden, zunächst auch nur solche, die mit dem lateinischen Alphabet operieren, wie es die Zeitschrift WLL in einzelnen Beiträgen tut (so zum Beispiel Neef \& Primus 2004). Ohne Zweifel hat Meisenburg (1996) dies im Zusammenhang für die romanischen Schriftsysteme getan. Vergleichssprachen, die ich hier exemplarisch erwähnen möchte, sind Englisch, ${ }^{1}$ Französisch und Niederländisch. Auf einem Parameter der Tiefe sind diese unterschiedlich zu verorten. Die Tiefenskala unterscheidet dabei, wie nah ein Schriftsystem an der Lautstruktur verschriftet wird - bei großer Nähe handelt es sich um ein flaches System. Niederländisch ist von den vier Sprachen die mit dem flachsten Schriftsystem; es gibt noch sehr viel flachere wie Spanisch, Tschechisch oder Türkisch. ${ }^{2}$ Englisch und Französisch sind beide sehr viel tiefer als das Deutsche; sie sind aber auf sehr unterschiedliche Weise tief und genau das gilt es herauszuarbeiten. ${ }^{3}$ Neben der unter-

1 Eine wichtige Analyse der letzten 50 Jahre ist Chomsky \& Halle (1968) mit ausdrücklichem Einbezug der Graphematik; allerdings nicht typologisch ausgerichtet.

2 Programmatisch wäre es großartig, das Türkische mit aufzunehmen. Denn im Jahr 2028 jährt sich die Umstellung auf das lateinische Alphabet. Es wäre überaus interessant, die hundert Jahre Schriftgeschichte genauer zu betrachten - vermutlich ist das Schriftsystem tiefer (,reifer nach Fabricius-Hansen 2003) geworden.

3 Die Tiefe kann dabei auch durchaus diachron verstanden werden. 
schiedlichen Verortung in der Tiefe spricht für diese vier Sprachen, dass vergleichsweise viel über ihre Graphematik bekannt ist.

\section{Vorschläge zum Vorgehen}

Dieser Artikel entwirft programmatisch und exemplarisch eine Forschung, die jetzt angegangen werden kann und angegangen werden sollte. Ich nutze die Gelegenheit, programmatisch zu schreiben - einiges ist gut abgesichert, einiges ist ein wenig spekulativ. Varianten innerhalb der einzelnen Schriftsysteme werden hier explizit weggelassen, es wird in jeder Sprache von einer standardisierten Verschriftung ausgegangen, was keineswegs so bleiben muss. Im Gegenteil - die Parameter könnten durchaus dazu dienen, auch Varianten zu beschreiben. Auch historische Systeme können damit beschrieben werden. Im Folgenden schlage ich vor, wie eine Beschreibung funktionieren könnte: Sie kann 1. an den verschiedenen Einheiten festgemacht werden, 2. an bereits bekannten oder angenommenen Schreibprinzipien, 3. an bestimmten Phänomenen, 4. an (graphematischen) Teilsystemen und 5. an bestimmten grammatischen (also nicht primär graphematischen) Teilbereichen, wie zum Beispiel an der Schreibung der Verbflexion.

- $\quad \mathrm{Zu}$ 1.: Die verschiedenen Einheiten können sich grundsätzlich unterscheiden. So wird die Schreibsilbe in den unterschiedlichen Sprachen unterschiedlich realisiert - zum Beispiel an der Silbengrenze: Das Deutsche markiert Silbengrenzen in bestimmten Fällen (nach Vokalbuchstaben, vor $<\mathrm{e}>$ ) mit $<\mathrm{h}>$, im Französischen und Niederländischen hingegen mit einem Trema: deutsch Ruhe, frz. noël, ndl. poëzie. Das Deutsche hat auch ein Trema, verwendet es aber grundsätzlich anders.

- $\quad \mathrm{Zu}$ 2.: Wichtige Schreibprinzipien kennen wir schon, so beispielsweise die Stammkonstanz im Deutschen. Im Vergleich zeigt sich, dass die anderen Sprachen viel eher ein Prinzip der Affixkonstanz haben und deutlich weniger Stammkonstanz. Für das Englische könnte es auch sein, dass es ein systematisch wirkendes Homographievermeidungsprinzip gibt (break brake, Berg 2017b: 192 ff.). Die Schreibprinzipien werden daran gemessen, ob man von einer Lautkette zu der Zielschreibung mit den immer gleichen Prinzipien innerhalb eines Schriftsystems kommt und damit einen Großteil der Schreibungen erfassen kann. So finden sich zwar einzelne Beispiele im Deutschen für eine Homographievermeidung bei Lied - Lid; es ist aber kein durchgängiges Prinzip: Diele, Fliege, Niete, Kiefer haben jeweils wenigstens zwei Bedeutungen, werden aber gleich geschrieben. 
- $\mathrm{Zu}$ 3.: Herausgelöste Phänomene können interessant sein. So sind die Schreibdiphthonge (s. 6.2) in den verschiedenen Sprachen unterschiedlich organisiert und zeigen doch erstaunliche Ähnlichkeiten, und zwar nicht in ihrer Phonographie, sondern in der Organisation der Erst- und Zweitbestandteile. So sind $<\mathrm{i}>$ und $<\mathrm{u}>$ über die Sprachen hinweg typische Zweitbestandteile. Auch die Doppelkonsonanten sind in den verschiedenen Sprachen unterschiedlich organisiert und zeigen doch auch Ähnlichkeiten. Im Französischen sind sie offenbar in den Fällen je jette, nous jetons nicht Silbengelenk; die genaue Funktion ist noch offen, aber vermutlich hängt es mit Betonungshinweisen zusammen und wäre dann so etwas wie ein Schweremarker. Dies könnte auch eine sekundäre Funktion des Doppelkonsonanten im Deutschen sein, wie Augst (1987) für die Fremdwortschreibung herausgearbeitet hat (frz. ballet - dt. Ballett mit Endbetonung). Diese herausgelösten Phänomene finden sich auf allen Ebenen, so auch die Beobachtung, welche (homophonen) Wörter gleich geschrieben werden und welche unterschiedlich: So wird offenbar nur im Deutschen die Konjunktion dass von anderen Wörtern mit der gleichen Lautung unterschieden (vs. that im Englischen, dat im Niederländischen usw.). Grundsätzlich findet sich aber auch in diesen Sprachen die Möglichkeit der Homographievermeidung bei Funktionswörtern wie engl. to - too, frz. $a-\grave{a}$ usw.

- $\mathrm{Zu}$ 4.: Insgesamt interessante (graphematische) Teilbereiche können herausgenommen werden, so zum Beispiel ein System der Wortzeichen. Diese sind im Deutschen der Abkürzungspunkt, der Divis und der Apostroph; sie operieren alle auf der Wortebene. Im Detail unterscheiden sich aber die Systeme - ein Divis wie im französischen avez-vous ist im Deutschen auf gänzlich andere Kontexte wie bei Durchkoppelung in Phraseologismen beschränkt: sein Haben-Sie-heute-Zeit-für-Mich-Geschwätz, aber nicht *haben-Sie.

- $\quad$ Zu 5.: Bezüglich grammatischer Teilbereiche hat sich die Verbflexion in verschiedenen Sprachen als besonders interessant erwiesen - im Deutschen und Englischen findet sich das gesamte Spektrum von recht systematisch (singen - sang - gesungen, sing - sang - sung) bis ganz unregelmäßig (bin - war - gewesen, am - was - been); verschiedene Schreibprinzipien können hier beobachtet werden, s. auch Abschnitt 5 und 8. Das Französische ist ein Paradebeispiel für graphematisch höhere Eindeutigkeit bei phonologisch höherer Mehrdeutigkeit; eine Endung / $\varepsilon /$ kann ais, ait, aber auch aient geschrieben werden und diese Schreibweisen schränken die Interpretationsmöglichkeiten stark ein.

Wie genau die Parameter aussehen, ist Gegenstand der Forschung. 


\section{Die Einheiten}

Im Folgenden werde ich anhand einiger Einheiten (Segmente, Buchstaben/ Grapheme, Silben, Füße, Wörter, Sätze, Absätze) beispielhaft zeigen, welche Fragen sich speziell für das jeweilige Schriftsystem ergeben.

\subsection{Die graphematische Silbe: Wie allgemein ist das Graphematische Silbenbaugesetz?}

In Fuhrhop \& Buchmann (2009) ist zunächst die Längenhierarchie erarbeitet worden, die die Buchstabenformen auf einer Skala einordnet: Sie reicht von Buchstaben mit einer Ober- oder Unterlänge wie $|\mathrm{b}|$ und $|\mathrm{p}|$ (,lange“ Buchstaben) bis zu den kompakten wie $|a|$. Mit Hilfe der Längenhierarchie konnte ein Allgemeines Graphematisches Silbenbaugesetz formuliert worden: Die Länge nimmt zum Silbenkern hin kontinuierlich ab bzw. die Kompaktheit der Buchstabenformen nimmt zum Silbenkern hin kontinuierlich zu (z. B. frech, derb). ${ }^{4}$ Das Vorgehen war analog zum Allgemeinen Phonologischen Silbenbaugesetz (Vennemann 1982); bei Vennemann wird die Anordnung der Phoneme auch zunächst nur strukturalistisch und an einer Sprache ermittelt. Dass dies mit der Sonoritätshierarchie ,erklärt‘ wurde, war methodisch sekundär! Beide Gesetze sind als Präferenzgesetze formuliert („Eine graphematische Silbe, die nach dem Graphematischen Silbenbaugesetz gebaut ist, ist präferiert gegenüber einer Silbe, die nicht nach dem Graphematischen Silbenbaugesetz gebaut ist.“) Damit sind Verstöße möglich, aber letztendlich ist es gerade interessant, diese Verstöße in den verschiedenen Sprachen zu betrachten. Die Längenhierarchie ist am Deutschen erarbeitet worden und so greift sie Besonderheiten des Deutschen auf, nämlich zum Beispiel die Tatsache, dass $|c|^{5}$, $|\mathrm{y}|$ und $|\mathrm{q}|$ nicht im nativen System des Deutschen alleine vorkommen, aber $|ß|$ ein Buchstabe des Deutschen ist. $|\mathrm{q}|$ kommt im Deutschen nur in Kombination mit $|\mathrm{u}|$ vor, $|c|$ im nativen Wortschatz nur in Verbindung mit $|\mathrm{h}|$ oder $|\mathrm{k}|$ und $|\mathrm{y}|$ aus-

4 Die Längenhierarchie bezieht sich zunächst nur auf Minuskeln. Die Tatsache, dass Wörter mit einer Majuskel anfangen, stärkt allerdings den Wortrand durchaus im Sinn der Grenzmarkierung. Dennoch ist die zugrundeliegende Zerlegung in Kopf und Koda so nur für Minuskeln durchzuführen. Bei den Majuskeln spielen andere Kriterien, wie zum Beispiel Symmetrie, eine Rolle.

5 Die geraden Striche beschreiben schriftliche Einheiten ohne eine Aussage darüber, ob es Grapheme in einer Sprache sind oder nicht. Sie stehen also in dem gleichen Verhältnis zu den spitzen Klammern wie die eckigen Klammern zu den Schrägstrichen in der Lautstruktur. 
schließlich in Eigennamen und Fremdwörtern. Das besondere Verhalten insbesondere von $|c|$ und $|y|$ kann ganz im Sinne des Allgemeinen Graphematischen Silbenbaugesetzes interpretiert werden, denn $|c|$ hat alleine keine Länge und ist daher kein ,guter' Silbenrandbuchstabe, |y| hingegen hat eine Länge und ist daher kein ,guter' Silbenkernbuchstabe. Als Buchstabe ist $|y|$ trotzdem der einzige, der sich weder eindeutig auf Silbenrand oder Silbenkern festlegen lässt - so findet sich Yoga neben System, Rhythmus, Handy. Diachron zeigt sich, dass weder $|\mathrm{y}|$ noch $|\mathrm{c}|$ immer diese Randstellung im System hatten, sondern dass sie erst in den Rand gedrängt wurden (Fuhrhop \& Schmidt 2014). Daher ist es meines Erachtens interessant, genau diese Buchstaben in den einzelnen Sprachen abzuprüfen - als erster schriftsystem-übergreifender Geltungsbereich. Ein weiterer wäre, die Verstöße in den jeweiligen Systemen herauszuarbeiten. Hier wird dann im Wesentlichen die Kombinatorik in den Silbenrändern geprüft. Drittens kann überprüft werden, wie sich speziell das $|1|$ verhält: In Fuhrhop \& Buchmann (2009) wurde es als ,zweiteilig mit einem kurzen Kopf interpretiert, in Primus (2006) als ,eine Länge - allerdings gibt es Hinweise, dass es sich kombinatorisch zwitterhaft verhält.

Im Folgenden werden die drei Buchstaben $|y|,|c|,|1|$ kontrastiv betrachtet mit ihren jeweiligen Auffälligkeiten - einerseits mit der Frage, ob sie Verstöße gegen das Silbenbaugesetz zeigen, wie die Form und die Distribution zunächst nahelegen könnten, und andererseits wie ,normal' sie sich verhalten. Wie im Deutschen findet sich $|\mathrm{y}|$ auch im Englischen, Französischen und Niederländischen in griechisch-stämmigen Wörtern wie system. ${ }^{6}$ Im Niederländischen hat $\langle y>$ eine ähnliche Stellung wie im Deutschen - es kommt in Fremdwörtern und Eigennamen vor, $\mathrm{zu}<\mathrm{j}>$ und $<\mathrm{ij}>\mathrm{s}$. 6.2.

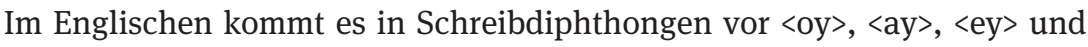
entwickelt hier die gleiche Lautqualität wie $<\mathrm{i}>$ boiler - boy. Analog dazu verhält sich die Alternation $\langle\mathrm{u}\rangle-\langle\mathrm{w}\rangle$ neuter - new. $\langle\mathrm{y}\rangle$ alterniert außerdem mit $<\mathrm{i}>$ am Wortende, und zwar durchaus auch am Wortformenende wie in lady ladies, s. Fuhrhop, Buchmann \& Berg (2011); Franke (2008). Insofern markiert $<\mathrm{y}\rangle$ das Wortende gut. Im Englischen ist $<\mathrm{i}>$ als Wortende marginalisiert. Allerdings kommt es als Wort vor, aber standardmäßig nur in Majuskelschreibung $<\mathrm{I}>$.

Im Französischen sind bisher erst die Alternationen bei den Verbformen gut untersucht, s. auch 8.1. $\langle\mathrm{y}\rangle$ scheint hier bevorzugt intersyllabisch aufzutreten (j'essaie - nous essayons) und fungiert als Marker für die Silbengrenze, die phonologisch interpretiert wird, seine Länge wird hier also genutzt. ${ }^{7}$ Außer-

6 Im Französischen heißt der Buchstabe sogar, i grec' (,griechisches $i^{\star}$ ).

7 In Verben ist es gut untersucht, aber crayon, moyen usw. lassen eine analoge Interpretation vermuten. 
dem ist auffällig, dass $\langle\mathrm{y}\rangle$ im Französischen alleine eine Wortform bilden kann; <i> nicht. Das alleinige Vorkommen als Wort ist unabhängig von der Silbe zu sehen, aber vermutlich abhängig von der konkreten Form. Auch das wäre also eine Untersuchungsebene.

Als nächster Buchstabe wird das $|c|$ behandelt: Im Englischen kommen sowohl $|c|$ als auch $|\mathrm{k}|$ einzeln vor, neben der Kombination $|\mathrm{ck}|$ wie im Deutschen. Ansonsten sind die romanischen und die germanischen Sprachen geradezu danach aufzuteilen, ob $|\mathrm{c}|$ oder $|\mathrm{k}|$ vorkommt - in den romanischen tritt regelmäßig $|c|$ auf und $|\mathrm{k}|$ nur in Fremdwörtern, in den germanischen hingegen im nativen Wortschatz $|\mathrm{k}|$ und im fremden $|\mathrm{c}|$. Diese sprachübergreifende Komplementarität ${ }^{8}$ wird im Folgenden interpretiert: Der Buchstabe $|c|$ ist auffällig, weil er einerseits keine Länge aufweist und andererseits von seinem Aussehen eher ein Silbenkernbuchstabe (kompakt) sein könnte, phonographisch aber nicht mit Vokalen sondern mit Konsonanten (Obstruenten) korrespondiert. Außerdem lässt er sich nicht in Kopf und Koda zerlegen. Überspitzt könnte die Frage also heißen, ob $|c|$ der Kopf oder die Koda fehlt. Primus (2006) zerlegt $|\mathrm{k}|$ in den Kopf $|1|$ und die Koda $|c|$, die Koda sieht also aus wie der Buchstabe $|c|$. Möglicherweise hat $|\mathrm{k}|$ dem $|c|$ eine Länge ,gegeben“ (Fuhrhop \& Schmidt 2014). Damit ist auch erklärbar, warum $|\mathrm{k}|$ aus den germanischen Sprachen $|c|$ verdrängen konnte. Aber auch in den romanischen Sprachen finden sich Reaktionen auf die Form von $|c|$ : Im Französischen gibt die Cedille dem $|c|$ Länge $(<c ̧>)$. Außerdem ist im Französischen $|q|$ häufig, und zwar mit weiterem graphematischen ,Aufwand': Außer in cinq und coq folgt dem |q| |ue| wie in baroque, clinique usw. Beide Besonderheiten können als Ausweichtendenzen für das längenlose bzw. kopflose $|c|$ gelten.

Kommen wir zum |1|: In Fuhrhop \& Buchmann 2011 haben wir gezeigt, dass sich die Bestandteile von |1| (,Kopf wie Koda') aus der Kombinatorik von Kopf und Koda ergeben und es sich im Gegensatz zu Primus (2006) nicht um einen kodalosen Buchstaben handelt; es sind zwei kurze gerade Striche, die sich berühren. Nichtsdestotrotz sieht |1| aus, als hätte es nur eine Länge. Am Anfangsrand kombiniert |l| ausschließlich mit langen Buchstaben: bl, pl, gl, kl, fl mit der Ausnahme Vlies ${ }^{9}$ und Fremdwörtern mit <sl> und <cl> (Slang, Slip, Slum, Slogan, Club, Clown, Clan). Im Endrand kombiniert es neben den langen Buchstaben wesentlich mit $|\mathrm{m}|$ wie Alm, Film, Helm, Halm, Schelm, Qualm, (Malm, Mulm, Palm, Psalm, walm); Wörter mit der Buchstabenfolge |ln| am Ende sind

8 Die Besonderheit des Englischen erscheint dann konsequent: Das Englische besteht sowohl aus einem romanischen als auch aus einem germanischen Wortschatz.

9 Die Majuskelschreibung ist syntaktisch motiviert. Bei Adjektiven wie vliesartig wird deutlich, dass das Silbenbaugesetz hier verletzt wird. 
wesentlich Verben, in denen vor $-n$ eine morphologische Grenze ist; wenn |1| also eine Länge hätte, würde der vermeintliche Verstoß gegen das Silbenbaugesetz eine morphologische Grenze verdeutlichen. CELEX (eine Datenbank mit rund 50.000 Einträgen zum Deutschen) listet jenseits der Verben ausschließlich Köln auf. In morphologisch komplexen Formen kann |1| noch mit $|\mathrm{s}|$ und |t| kombinieren: falls, Stuhls, fehlt, fehlst; mit |s| finden sich im System viele Verletzungen, der Verstoß gegen das Silbenbaugesetz zeigt die morphologische Grenze besonders deutlich (wie tags, Lobs, Halts, hältst). |1| kombiniert für seine ,Zwitterstellung im Deutschen also insgesamt angemessen. ${ }^{10}$ Trotz seiner vermeintlichen Länge sieht es nicht aus wie die anderen langen Buchstaben, die durch ihre Koda immer auch eine gewisse Mindestbreite aufweisen; es ist nur lang, die anderen sind auch breit. Hier steht die Untersuchung der anderen Sprachen noch aus. Es ist aber offensichtlich, dass Englisch und Französisch die gleichen Kombinationen zulassen, zusätzlich natürlich $\mathrm{cl}, \mathrm{sl}$. Das ,stumme' $l$ im Englischen ist selten, wäre aber entsprechend zu kommentieren (zu should, could, would s. 8.2, verstößt hier aber nicht gegen das Silbenbaugesetz, weil ein langer Buchstabe folgt; abhängig von der Interpretation von |1|, wäre calm zu diskutieren).

Als besondere Buchstaben (also Buchstaben mit verbundenen Diakritika) gibt es auffallenderweise mit der französischen Cedille <ç>, dem tschechischen Haček $<\check{c}>$ und dem polnischen $<\nmid>$ gerade Varianten von den Buchstaben mit einer für die Längenhierarchie problematischen Form: Das $|c|$ hat ,zu wenig Länge, das |1| zu wenig Breite.

Die bisher gestellten Fragen orientieren sich an der Längenhierarchie und dem Allgemeinen Graphematischen Silbenbaugesetz - sie setzen die Gültigkeit voraus, können aber letztendlich auch die Gültigkeit widerlegen oder bestätigen. Die Art der Verletzungen ist dabei ebenso interessant wie die Art der Verletzungen beim Allgemeinen Phonologischen Silbenbaugesetz. Mit der Untersuchung dieser und weiterer Phänomenbereiche können zwei Dinge gezeigt werden: Erstens wird untersucht, wie allgemeingültig das Gesetz ist und zweitens kann dieses Gesetz als tertium comparationis genutzt werden, um Gemeinsamkeiten und Unterschiede in den Sprachen herauszufinden. Grundlegende quantitative Korpuserhebungen zur Buchstabendistribution (wie in Berg 2012, 2017b) würden hierfür eine solide Voraussetzung schaffen.

\subsection{Der Fuß}

Den graphematischen Fuß haben zunächst Evertz \& Primus (2013) und anschließend Evertz (2016) ausführlich für das Deutsche und Englische beschrie-

10 Persönliche Mitteilung von Kristian Berg. 
ben. Der phonologisch unmarkierte Fuß ist der Trochäus; in sehr vielen Fällen wird er gekennzeichnet durch einen graphematischen Zweisilber mit <e> als Silbenkern der zweiten Silbe.

Im Französischen ist der Trochäus nicht der unmarkierte Fuß; allerdings steht auch hier <e> häufig in unbetonten (Neben-)Silben, die offen oder einfach geschlossen sind. Das Französische verdeutlicht die Hauptsilbe mit $<\mathrm{e}>$ durch ein Akzentzeichen sowohl am Wortanfang <défense> als auch am Wortende $<$ regardé> oder durch Doppelkonsonanten; Doppelkonsonanten und Akzentzeichen sind geradezu komplementäre Schwerekennzeichner wie in $<$ je jette>, $<$ je regrette $>$ vs. $<$ j'achète $>$.

Diese Schwerekennzeichner finden sich zum Teil bei Gallizismen im Deutschen; so wird frz. ballet zu dt. Ballett, frz. carrousel zu dt. Karussell ${ }^{11}$ (s. Augst 1987): Eine Markierung wird gewählt, wenn es sich um einen anderen Fuß als den unmarkierten handelt. Im Französischen ist der unmarkierte Fuß auf der Endsilbe betont, im Deutschen ist der unmarkierte Fuß der Trochäus. Entsprechend ,verschiebt‘ sich hier die Doppelkonsonantenschreibung.

Für die Beschreibung der Einheiten soll das an dieser Stelle reichen. Es ist ganz offensichtlich, dass sich sowohl die ,untere' Ebene (Grapheme, Diakritika) als auch die obere Ebene (graphematische Wörter, s. 7.1, zum Divis und die unterschiedliche Schreibung von Komposita im Deutschen und Englischen) in den einzelnen Sprachen stark unterscheiden. Das alles sollte systematisch beschrieben werden.

\section{Prinzipien der Wortschreibung}

Für das Deutsche nehmen wir neben phonographischen Prinzipien, worunter auch silbische Prinzipien zu fassen sind, Prinzipien der Schemakonstanz (morphologische Schreibungen) und verschiedene syntaktische Prinzipien, die zum Beispiel die Substantivgroßschreibung und die Getrennt- und Zusammenschreibung beschreiben, an. Die Prinzipien sind im Einzelnen zu vergleichen und zu ergänzen. So wirkt in vielen Sprachen die Stammkonstanzschreibung (als ein Typ von morphologischer Schreibung) weniger streng, andere Prinzipien sind stärker. Die nächste Frage wäre dann, ob es andere morphologische Schreibungen gibt. Bei den morphologischen Schreibungen geht es einerseits um Flexionsformen, andererseits um Wortbildungsformen. Grundsätzlich gehe

11 Damit hätte die Doppelkonsonantenschreibung primär bei (nicht-nativen Wörtern) am Fuß-/Wortende eine andere Funktion als die im Silbengelenk; s. Stammkonstanz unten. 
ich davon aus, dass eine gewisse Morphemkonstanz immer vorzufinden ist, die so genannte ,implizite“ Morphemkonstanz (s. Fuhrhop \& Barghorn 2012). Im Sprachvergleich interessanter scheint aber die ,explizite‘ Morphemkonstanz kurz gesagt: Die Schriftstruktur zeigt mehr Morphologie als die Lautstruktur. ${ }^{12}$

\subsection{Stammkonstanz}

Im Deutschen herrscht ein recht strenges Prinzip der Stammkonstanz. Exemplarisch und im Vergleich besonders deutlich sind im Deutschen die Doppelkonsonanten. Diese werden zunächst phonographisch für die Silbengelenke geschrieben - also intersyllabisch nach ungespanntem, betonten Vokal. Aufgrund von Morphemkonstanz werden sie auch im Einsilber geschrieben (schwimmen - schwimmt). Im Englischen gilt eine analoge Regel für den Zweisilber (also der phonographische Teil), aber die Übertragung auf den Einsilber findet weitgehend nicht statt (swimming - swim). Doppelkonsonanten im Einsilber gibt es schriftsystemspezifisch nur bei bestimmten Buchstaben, nämlich $f f$, ss, $l l$, außerdem <ck> wie im Deutschen.

Im Französischen findet sich oft einWechsel innerhalb der Paradigmen, im Verb bei j'appelle - nous appelons; bei den Adjektiven gentil - gentille; auch hier also keine Stammkonstanz. Der Doppelkonsonantwechsel innerhalb der Paradigmen könnte ein Hinweis darauf sein, dass die Stammkonstanz im Französischen eher von anderen Prinzipien überschrieben wird als beispielsweise im Deutschen; in der Terminologie der Optimalitätstheorie wäre das Prinzip weniger hoch gerankt.

\subsection{Affixkonstanz}

Sowohl das Englische als auch das Französische zeigen statt der Stammkonstanz eher Affixkonstanz. Im Englischen wird die Präteritumendung der schwachen Verben immer -ed geschrieben - unabhängig von der Aussprache: stimmhaft und silbisch repeated, stimmhaft und nicht-silbisch begged, stimmlos und nicht-silbisch looked. Allerdings hat das Prinzip der Affixkonstanz im Englischen durchaus seine Grenzen im Flexionssystem; die Endung -s (als Pluralendung bei Substantiven und als Verbendung) wird ab-

12 Theoretisch wäre auch der umgekehrte Fall denkbar: Die Lautstruktur zeigt mehr Morphologie als Schriftstruktur; so ist der Trochäus lautlich deutlicher gekennzeichnet als schriftlich, s. auch 4.2. 
hängig von der Aussprache silbisch oder nicht-silbisch geschrieben (silbisch in kiss-es), graphematisch spielt allerdings die Stimmhaftigkeit auch hier keine Rolle.

Im Französischen ist das Prinzip der graphematischen Affixkonstanz sehr weit ausgebildet. Gerade für die Flexionsmorphologie hat das Französische eine stumme Morphologie herausgebildet, also eine Morphologie, die geschrieben, aber nicht unbedingt gesprochen wird. Die Pluralformen von Substantiven werden bis auf wenige Ausnahmen ${ }^{13}$ mit $-s$ geschrieben. Der Plural ist also einheitlich markiert. In der Verbflexion werden bestimmte Endungen immer gleich geschrieben wie zum Beispiel die Endung für die 3. Person Plural -ent, auch ,nach' der Präteritumendung (-ai-), dann -aient usw.

Beim Rückschluss auf die Graphematik des Deutschen im europäischen Vergleich stellt sich die Frage, ob das Deutsche auch Affixkonstanz zeigt: Viele Affixe sind graphematisch konstant im Deutschen, so zum Beispiel die Derivationssuffixe - das Adjektivsuffix -bar immer < bar> usw. Explizit zeigt sich Affixkonstanz aber erst bei einer ,Überformung“ einer phonographischen Schreibung, so für das Adjektivsuffix -ig (einzig - einzige vs. /aıntzıç/ - /aıntzıgə/). Bei den schwachen Verben richtet sich die Variantenschreibung nach der Phonologie (arbeit-ete vs. leg-te, * arbeit-te, ${ }^{\star}$ leg-ete). Allerdings scheint die Adjektivflexion konstant silbisch - also blau-en, auch wenn [blaun] gesprochen wird. In Tabelle 19.1 sind einige Fälle von Konstanz und Nicht-Konstanz von Stämmen und Affixen in den verschiedenen Sprachen aufgelistet.

Tab. 19.1: Morphologische Schreibkonstanzen in den verschiedenen Sprachen.

\begin{tabular}{|c|c|c|c|c|}
\hline & \multicolumn{2}{|l|}{ Stamm: konstant? } & \multicolumn{2}{|l|}{ Affix: konstant? } \\
\hline & $\begin{array}{l}\text { Explizite } \\
\text { Stammkonstanz }\end{array}$ & $\begin{array}{l}\text { Keine explizite } \\
\text { Stammkonstanz }\end{array}$ & Affixkonstanz & $\begin{array}{l}\text { Keine } \\
\text { Affixkonstanz }\end{array}$ \\
\hline Deutsch & $\begin{array}{l}\text { älter, glatt, blöd, } \\
\text { heiiß, roh }\end{array}$ & nimmt (Doppel-m?) & $\begin{array}{l}|ı c ̧|-|ı g|-\langle i g\rangle \\
\text { Adjektivsuffixe } \\
e, e s, \text { en, ... }\end{array}$ & $\begin{array}{l}\text { arbeitete - legte } \\
\text { Hauses - Stuhls }\end{array}$ \\
\hline Englisch & & $\begin{array}{l}\text { lady - ladie(s), } \\
\text { run - running, } \\
\text { beg - begged }\end{array}$ & $\begin{array}{l}\text { ed: begged, } \\
\text { talked, repeated }\end{array}$ & knows - kisses \\
\hline
\end{tabular}

13 Ausnahmen sind zum Beispiel Pluralformen auf - $x$ wie in château - châteaux und die Nichtmarkierung, wenn der Stamm auf $<\mathrm{z}>$ oder $<$ s $>$ endet wie in un pays - des pays, un nezdes nez. 
Tab. 19.1 (fortgesetzt)

\begin{tabular}{|c|c|c|c|c|}
\hline & \multicolumn{2}{|c|}{ Stamm: konstant? } & \multicolumn{2}{|l|}{ Affix: konstant? } \\
\hline & $\begin{array}{l}\text { Explizite } \\
\text { Stammkonstanz }\end{array}$ & $\begin{array}{l}\text { Keine explizite } \\
\text { Stammkonstanz }\end{array}$ & Affixkonstanz & $\begin{array}{l}\text { Keine } \\
\text { Affixkonstanz }\end{array}$ \\
\hline Franz. & & $\begin{array}{l}\text { essaie - essayons } \\
\text { appelle - appelons } \\
\text { caduc - caduque }\end{array}$ & $\begin{array}{l}\text { Plural -s } \\
\text { bei Substantiven } \\
\text { (x als Ausnahme) } \\
\text { Verbflexion } \\
\text { ais, ait, aient }\end{array}$ & $(x)$ \\
\hline Niederl. & & $\begin{array}{l}\text { man - mannen } \\
\text { hoop - hopen } \\
\text { leef - leven }\end{array}$ & word-t, vind-t & $\begin{array}{l}\text { broers vs. } \\
\text { camera's } \\
\text { maakte - hoorde }\end{array}$ \\
\hline
\end{tabular}

\subsection{Affixeindeutigkeit}

Daneben ist auch Affixeindeutigkeit ein mögliches typologisches Kriterium, das für jedes Affix einzeln überprüft werden kann. Nach Berg (2017b: 215) geht es darum, wie „zuverlässig die schriftliche Form das Affix repräsentiert“. So ist zum Beispiel -ed im Englischen ziemlich wahrscheinlich als Präteritumendung für schwache Verben zu erkennen, andere Wörter mit -ed (z. B. hundred), sind sehr selten. Analog kann frz. -aient interpretiert werden, unter 170.000 morphologisch einfachen Einträgen in Lexique findet sich kein einziges Wort, das so endet; ein anderes Suffix mit dieser Form gibt es nicht. Das ist also eine Affixeindeutigkeit über das gesamte Lexikon. Es kann aber auch sein, dass ein Affix als Affix eindeutig oder eindeutiger ist: Die Endung -s kann im Deutschen und Englischen den Plural oder den Genitiv markieren, aber im Englischen wird hier graphematisch unterschieden, und zwar mit Apostroph. Im Deutschen ist das eine Möglichkeit für die kleine Gruppe der Personennamen (Hel$g a$ 's). Damit wäre die Affixeindeutigkeit im direkten Vergleich dieser Suffixe im Englischen höher (teacher's - teachers, Autos - Autos). Das Verbsuffix -s ist im Englischen allerdings nicht von dem Pluralsuffix zu unterscheiden. Demnach ergibt sich, dass -s sowohl bei Substantiven als auch bei Verben im Englischen eindeutig zu identifizieren ist - vorausgesetzt man weiß, welche Wortart vorliegt. Aber auch hier kann die Schleife zu dem obigen Kriterium gezogen werden: Im Englischen ist $z u$ beobachten, dass einfaches $-s$ beim morphologisch einfachen Einsilber verhindert wird, und zwar durch $\langle$ ss $>$ oder $\langle$ se $\rangle$ wie in glass, class, kiss und house. Durch diese Ausweichformen wird gesichert, dass -s als Suffix erkannt wird; etwas Vergleichbares ist im Deutschen nicht bekannt. 
Um noch auf das Französische einzugehen: Auch hier sind einfache Wörter auf -s rar; es wird also in den meisten Fällen als Suffix erkannt werden. Welches Suffix vorliegt, ist dann offen, es sei denn, man kennt die Wortart je/tu prends (Verb), bei Adjektiven und Substantiven ist es ein Pluralmarker gentils (Adjektiv), jardins (Substantiv).

Es geht hier also um die Verzahnung von ,eindeutiges Erkennen einer sprachlichen Einheit als Affix‘ und ,ein- oder mehrdeutige Interpretation eines Affixes‘. Ersteres funktioniert über die Gesamtheit der lexikalischen Einträge (-aient kommt im Französischen nur als Verbendung vor), zweiteres über ein ,erkanntes ‘ Affix (wenn -em im Deutschen ein Affix ist, dann steht es in Adjektivund Artikelformen für den Dativ, Maskulinum/Neutrum, wenn - $t$ ein Flexionsaffix ist und am Ende der Wortform steht, dann steht es für die 3. Ps., Sg., im Präs., Ind. oder die 2. Ps., Pl., Ind., Präs. oder Prät. bzw. den Imperativ Plural) und beides kann, aber muss nicht interagieren: -em ist im Deutschen sonst auch relativ selten (Atem), - $t$ hingegen ist eine häufige Endung auch bei morphologisch einfachen Wörtern. Einen interessanten Fall liefert hier das Niederländische mit <'s>: Durch den Apostroph ist es - in der Standardschreibung eindeutig ein Flexionsaffix, es kann aber Genitiv (Maria's) oder Plural (camera's $)^{14}$ sein.

\subsection{Morphographematische Schemata}

In Vorgriff auf Abschnitt 8.1 zur Schreibung in der Verbflexion zeigen sich morphographematische Schemata. Hier könnten sich Fragen ergeben - wie sieht eine gute Pluralform aus und wie eine gute Verbform? Zum Teil überschneidet sich das mit der Affixkonstanz - eine gute Pluralform (Substantiv oder Adjektiv) im Französischen endet mit -s. Aber was hier gemeint ist, spielt sich jenseits der Suffixe ab. So finden sich in der Flexion der Modalverben die Paare can - could, will - would, shall - should, die Formen could, would, should scheinen ein graphematisches Muster zur Kennzeichnung des Konditional zu bilden, das sich synchron nicht aus den Präsensformen ergibt. Übertragen auf das Deutsche könnte eine Form wie nimmt ${ }^{15}$ analog betrachtet werden, der Doppelkonsonant macht hier eine ,bessere' Verbform, weil er die Morphemgrenze ,zeigt‘. Solche Muster ergeben sich erst aus einer Gesamtbetrachtung

14 Der Plural wird nur mit Apostroph geschrieben bei vokalisch auslautenden Wörtern mit unbetonter Letztsilbe.

15 Sehr viele Verbformen in der 3. Person haben diese Form: kommt, schwimmt, nennt, lallt usw. Bei allen diesen ist aber eine Herleitung des Doppelkonsonanten über den Zweisilber möglich. 
(für nicht-schwache Verben im Deutschen und Englischen: Fuhrhop 2017); wie weit das Prinzip der grammatischen Muster jeweils trägt, muss gezeigt werden, siehe dazu auch 8.2 (Modalverben).

\section{Einzelphänomene herausgegriffen}

Es kann interessante Phänomene geben, die herausstechen und die gesondert interpretiert werden können. Dabei kann es selbstredend zu Überschneidungen kommen. Aber gerade im Vergleich kann das sehr erhellend sein.

\subsection{Doppelkonsonanten}

In 2.1 wurden bereits die Doppelkonsonanten thematisiert - als Beispiel dafür, wie deutlich Stammkonstanzschreibung in den Sprachen eingehalten ist. Im Englischen (und auch im Niederländischen) ist es eine Silbengelenkschreibung - das heißt, sie kommt intersyllabisch vor. Im Einsilber bis auf wenige Ausnahme (ss, ll, ff im Englischen) kommen Doppelkonsonanten nicht vor. Morphologisch wird die Schreibung also nicht beibehalten. Auch im Französischen findet sich die Aufgabe der Doppelkonsonantschreibung im Paradigma - so zum Beispiel j'appelle - nous appelons. Offenbar ist der Doppelkonsonant im Französischen in solchen Fällen kein Zeichen für ein Silbengelenk, sondern er gibt Hinweise zur Betonung. Im Niederländischen findet sich auch in der Verbflexion ein Phänomen, welches im Deutschen auf Komposita beschränkt ist: Die Doppelkonsonantschreibung an der Morphemgrenze wie in zucht-te, antwoord-de; die Doppelkonsonantschreibung deutet hier auf eine morphologische Grenze hin. Das Mittel der Doppelkonsonantschreibung kann also schriftsystemspezifisch unterschiedlich genutzt werden.

\subsection{Schreibdiphthonge}

In Berg \& Fuhrhop (2012, 2017) haben wir die Diphthonge in verschiedenen Sprachen verglichen. Herausstechend ist die Beobachtung, dass es bestimmte Erst- und Zweitbestandteile gibt (so auch Evertz \& Primus 2013). Besonders erstaunlich sind die Beobachtungen für das Französische, da im Französischen keine Sprechdiphthonge angenommen werden (Pustka 2011: 95). So sind <u> und $<\mathrm{i}>$ über die Sprachen hinweg bevorzugte Zweitbestandteile und entsprechend sind $\langle\mathrm{a}\rangle,\langle 0\rangle$, $\langle\mathrm{e}\rangle$ gute Erstbestandteile, auch wenn sie nicht alle in 
allen Sprachen genutzt werden - im Deutschen spielt zum Beispiel <0 $>$ in Schreibdiphthongen keine Rolle. Es wurden die phonographische Verschriftung und die innergraphematische verglichen.

Im Deutschen ist die Graphik beispielsweise so zu lesen: Phonologisch gibt es die drei Diphthonge/ai/, /oi/, /au/, die verschriftet werden mit <ai>, <ei>, $\langle\mathrm{eu}\rangle^{16}$ und $<\mathrm{au}>$. Phonographisch meint hier also, wie die vorhandenen Sprechdiphthonge verschriftet werden. Innergraphematisch hingegen meint, welche Vokalbuchstabenkombinationen häufig sind, unabhängig von der Lautung; hier ergibt sich, dass <ai> selten ist und als Schreibdiphthong zusätzlich $<$ ie ${ }^{17}$ hinzukommt. Es sind damit zwei unterschiedliche Herangehensweisen.

Deutsch, phonographisch

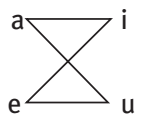

Deutsch, innergraphematisch

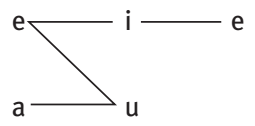

Im Englischen gibt es die Sprechdiphthonge / $\varepsilon \mathrm{c} /$, /ju/, /av/, /JI/, /aI/, /ov/, die

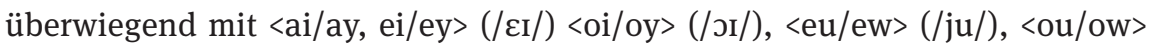
(/av/), /ov/ mit <oa> verschriftet werden. Der Diphthong/aI/ wird in den allermeisten Fällen mit i_e (side) ${ }^{18}$, der Diphthong /ov/ mit <oa > verschriftet. Daraus ergeben sich als große Unterschiede zum Deutschen einerseits $\langle 0\rangle$ als Erstbestandteil und $\langle\mathrm{y}, \mathrm{w}\rangle$ als Alternativen $\mathrm{zu}<\mathrm{i}, \mathrm{u}>$, s. auch 5.1. $\left\langle 0 \mathrm{oa}{ }^{19}\right.$ ist in der linken Graphik noch nicht erfasst. Die Systematik von <a> als zweitem Bestandteil erschließt sich noch deutlicher, wenn man die Schreibdiphthonge an sich betrachtet, unabhängig davon, ob sie auch mit einem Sprechdiphthong korrespondieren: <ea> vervollständigt die Kombinatorik von <a $>$; $<\mathrm{a}>$ korrespondiert mit allen anderen Vokalbuchstaben und richtet sich in seiner Position nach dem Kombinationspartner; mit den Erstbestandteilen <e, o> wird es Zweitbestandteil, mit den Zweitbestandteilen $<\mathrm{i}, \mathrm{u}>$ Erstbestandteil.

$16<a ̈ u>$ ist in den allermeisten Fällen morphologisch bedingt, es wird geschrieben, weil es eine morphologisch eng verwandte Form mit <au> gibt (Haus - Häuser, Maus - Mäuse usw.). 17 ie ist im Deutschen innergraphematisch nicht als Graphem zu lesen - es ist insgesamt keine kleinste bedeutungsunterscheidende Einheit, wie bieten - bitten und Sieben - Silben zeigen, s. Fuhrhop \& Peters (2013: 211).

18 Auch für andere Diphthonge gibt es die diskontinuierlichen Schreibungen, s. nächste Fußnote. Für /aI/ gibt es nur diese.

19 Die Systematik von Schreibdiphthongen mi <a> als zweitem Bestandteil erschließt sich phonographisch im Vergleich zu den diskontinuierlichen Schreibungen mit <...e >, denn <oa $>$ entspricht $\langle 0 . . . \mathrm{e}\rangle \mid\langle 0 \mathrm{Ce}\rangle$, so wie $\langle\mathrm{e} . . . \mathrm{e}\rangle \mid\langle\mathrm{eCe}\rangle$ und $\langle\mathrm{ea}\rangle$ gleiche Korrespondenzen haben können, Berg \& Fuhrhop 2011: 455, s. auch 8.2. 
Englisch, phonographisch

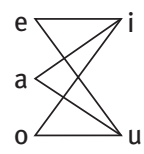

Englisch, innergraphematisch

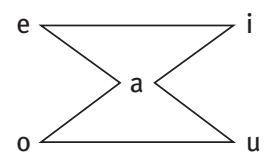

Weil das Französische keine Sprechdiphthonge hat, fällt die phonographische Betrachtung weg. Im Französischen sind zunächst nur die Schreibdiphthonge und nicht die Schreibtriphthonge (oder die Viererkombinationen) betrachtet. Damit könnte eine Aussage über die Segmentierung längerer Verbindungen getroffen werden: <eau $>$ wäre dann eine Verbindung aus $\langle$ e $>$ und $\langle a u>$, weil $<$ ea $>$ nicht als Diphthong vorkommt. Die Schreibdiphthonge im Französischen in der Abbildung entsprechen alle Sprechmonophthongen.

Französisch, innergraphematisch

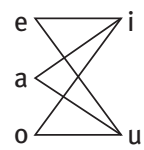

Für das Niederländische nennt Nunn (1998: 10) /عi/, /๕E/, /au/ als Sprechdiphthonge mit den folgenden Korrespondenzen: $/ \varepsilon \mathrm{i} /-\langle\mathrm{ei}\rangle,\langle\mathrm{ij}\rangle$, nach CELEX <ei> in 117 Fällen (65\%), mit <ij> in 63 (35\%) Fällen, / ๕Ey/ - <ui >, /au/ - <au>, $<$ ou> (van Megen \& Neijt 1998: 196). Alle Vokalbuchstaben können also Erstbestandteil sein, sie kombinieren alle jeweils genau mit einem anderen Buchstaben.

Niederländisch, phonographisch

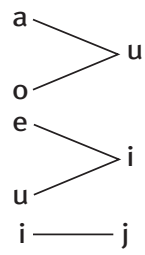

Niederländisch, innergraphematisch Teilsystem

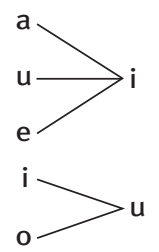

Vollsystem

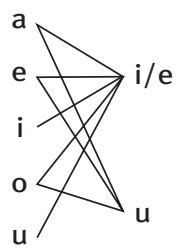

Daneben gibt es aber mit <oe>, <ie $>$ und den überaus häufigen Doppelvokalen $<$ aa $>$, <ee $>,\langle 00\rangle$ weitere Vokalbuchstabenkombinationen, die nicht mit Sprechdiphthongen korrespondieren. Betrachten wir also die Kombinatorik von Vokalbuchstaben, ergibt sich, dass als Zweitbestandteile <e, i, u $>$ vorkommen, <e> und $<\mathrm{i}>$ dabei komplementär. 


\subsection{Graphematische Kennzeichnung einer Konjunktion: das vs. dass}

Die Kommasetzung im Deutschen scheint in einem Punkt sehr speziell: Das Deutsche kommatiert grundsätzlich Nebensätze; es hat ein striktes Satzgrenzenkomma. Damit trennt es satzwertige Strukturen im Ganzsatz voneinander.

Im Englischen, Französischen und Niederländischen werden Nebensätze dann kommatiert, wenn sie vorangestellt sind. Es gibt hier einige Besonderheiten der einzelnen Sprachen, die sehr interessant sind. Im Folgenden möchte ich mich aber auf dass-Sätze und ihre Entsprechungen konzentrieren. Für die Konjunktion dass hat das Deutsche eine besondere Schreibung ${ }^{20}$ herausgebildet. Lautlich unterscheidet sich die Konjunktion nicht von dem Demonstrativpronomen, dem Relativpronomen und dem Artikel das. ${ }^{21}$ In den anderen Sprachen findet sich jeweils nur eine Schreibung (s. Tabelle 19.2).

Tab. 19.2: Überschneidung von Artikel, Subjunktion und Pronomen im Vergleich.

\begin{tabular}{lllllll}
\hline & Subjunktion & Artikel & $\begin{array}{l}\text { Demons.- } \\
\text { Pronomen }\end{array}$ & $\begin{array}{l}\text { Relativ- } \\
\text {,wort }\end{array}$ & $\begin{array}{l}\text { Frage- } \\
\text { pronomen }\end{array}$ & Sonstiges \\
\hline Deutsch & dass/das & + & + & + & & \\
\hline Englisch & that & & + & + & + Intensifier $^{23}$ \\
\hline Französisch & que & & + & Vergleich $^{24}$ \\
& & & & & Teil von $^{25}$ \\
\hline Niederländ. & dat & & + & + & & \\
\hline
\end{tabular}

Der größte Unterschied scheint erstens zu sein, dass die Konjunktion lautlich nur im Deutschen mit einem unmarkierten bestimmten Artikel zusammenfällt. Im Sprachvergleich sind Demonstrativ- und Fragepronomen komplementär

20 Allgemeines zur Schreibdifferenzierung homophoner Funktionswörter siehe oben.

21 Mechthild Habermann weist mich darauf hin, dass es im Süddeutschen hier zu falsch praktizierten Leseaussprachen käme mit /a:/ für das und /a/ für dass, und Peter Gallmann, dass das Pronomen in manchen oberdeutschen Dialekten des heiße, z. B. Schee is des, dass de do sand.

22 Diese ungewöhnliche Bezeichnung ist dem geschuldet, dass sich das Verhalten dieser Wörter in den verschiedenen Sprachen sehr unterscheidet.

23 She's that clever (Quirk et al. 1994: 447).

24 Je suis plus grande que toi.

25 Konjunktionen usw. puisque, parce que. 
verteilt - im Französischen fungiert que als Fragepronomen, in den anderen Sprachen als Demonstrativpronomen. Relativpronomen bzw. Relativsubjunktion können sie in allen Sprachen sein. Das ist auch die Stelle, in denen potenziell ein Komma stehen kann. Nach Auskunft der Grammatiken kann ein Komma gesetzt werden, wenn es sich um nicht-restriktive Relativsätze handelt. Wenn also ein Komma steht, muss es ein Relativsatz sein in den genannten nichtdeutschen Sprachen. Wenn kein Komma steht, kann es ein Relativsatz oder ein konjunktional eingeleiteter Nebensatz sein. Hingegen steht im Deutschen in jedem Fall ein Komma. Ob der Relativsatz restriktiv ist oder nicht, wird im Deutschen nicht markiert. Dagegen ist der Unterschied zwischen Relativsatz und eingeleitetem Konjunktionalsatz eindeutig markiert, nämlich durch die das/dass-Schreibung. Während also in allen anderen Sprachen restriktive Relativsätze und Konjunktionalsätze nicht unterschieden werden, wird im Deutschen zwischen Relativsätzen und Konjunktionalsätzen deutlich unterschieden. Das könnte also die Funktion der das/dass-Schreibung sein. In einer didaktischen Untersuchung zum Deutschen hat Feilke (2011) allerdings gezeigt, dass das als Relativpronomen in Texten die seltenste Verwendung der vier Möglichkeiten von /das/ ist; in der Nichtunterscheidung liegt nicht die große Fehlerquelle für die dass-Schreibung im Deutschen. Sie liegt vielmehr in der Verwechslung mit dem Demonstrativpronomen - das wird in den anderen Sprachen (Englisch und Niederländisch; Französisch que ist kein Demonstrativpronomen) nicht unterschieden. Wenn man dies zugrunde legt, verhält sich das Deutsche geradezu redundant. Es sind zwei Markierungen (Komma und s-Verdopplung), wo die anderen Sprachen keine haben.

Es ist natürlich Spekulation, warum das Deutsche zwei Schreibungen hat, wo andere Sprachen nur eine haben. Aber wie gesagt ist das Deutsche die einzige Sprache, in der die Konjunktion lautlich mit dem Artikel zusammenfällt, in den anderen Sprachen tut sie das nicht. Im Vergleich liegt es also nahe, dass Sprachen Konjunktion und Artikel unterscheiden.

\section{Graphematische Teilbereiche: Das System der Wortzeichen}

Für das Deutsche können drei Wortzeichen angenommen werden, der Abkürzungspunkt, der Divis und der Apostroph. Sie bilden auch graphisch ein System, indem sie im Liniensystem gut verteilt sind: der Abkürzungspunkt auf der Grundlinie, der Divis im Mittelband und der Apostroph im Oberband; Interpunktionszeichen besetzen offenbar grundsätzlich nicht das Unterband 
(Bredel 2008), so auch nicht die Wortzeichen. Auch in der Form unterscheiden sie sich maximal: der Abkürzungspunkt ohne Ausdehnung, der Divis horizontal, der Apostroph vertikal (Buchmann 2015: 90). Damit sind sie sowohl in der Form als auch in der Platzierung jeweils eindeutig. Sie sehen also überraschend systematisch aus, was die Vermutung nahelegt, dass andere Sprachen möglicherweise als Wortzeichen das gleiche Inventar nutzen. Die ersten Fragen sind also: Nutzen die anderen Sprachen das gleiche Inventar? Sind die Zeichen in den anderen Sprachen auch Wortzeichen? In welcher Systematik werden sie jeweils gesetzt?

\subsection{Der Divis}

Mit dem Divis werden drei Funktionen zusammengefasst - der Bindestrich, der Trennstrich und der Ergänzungsstrich. In den vier betrachteten Sprachen finden sich alle drei Funktionen. Im Folgenden wird die Funktion als Bindestrich thematisiert. Mit ,Bindestrich“ ist hier die Nutzung vom Divis ohne Leerzeichen bzw. Zeilenumbruch links und rechts gemeint, in Abgrenzung zum Trennstrich am Zeilenende (rechts vom Trennstrich ist das Zeilenende) und zum Ergänzungsstrich (be- und entladen, Verkehrssteuerung und -überwachung).

Im Deutschen wird er erstens genutzt, wenn eine normale Zusammenschreibung nicht ,möglich“ ist - das heißt bei Einheiten, die nicht aus Minuskeln bestehen wie einerseits ABM-Stelle, H-Milch und andererseits 100-jährig. Zweitens ist bei Syntagmen, die im konkreten Kontext als syntaktisches Wort benutzt werden, die Bindestrichsetzung zumindest eine mögliche Wahl (das ständige Den-Job-Wechseln, zu anderen Möglichkeiten s. auch Buchmann 2015). Mitunter wird der Bindestrich auch genutzt, um die Struktur eines Wortes deutlich zu machen - das kann bei neugebildeten Komposita oder Komposita mit Fremdwörtern wie Software-Preis, Autobahnhonig-Verkauf der Fall sein. Der Bindestrich ist in diesen Fällen eine Alternative zur Zusammenschreibung. In den anderen Fällen ,ersetzt‘ er die Zusammenschreibung. Der Bindestrich zeigt tendenziell eine ,losere' Verbindung.

Im Englischen hingegen steht der Bindestrich primär nicht wie im Deutschen als Alternativschreibung zur Zusammenschreibung (eines der wenigen Wörter ist girlfriend vs. girl-friend), sondern er steht als Alternative zur Getrenntschreibung. Er macht aus getrennten graphematischen Wörtern jeweils ein graphematisches Wort. Einigermaßen regulär zeigt sich der Bindestrich bei dreigliedrigen Komposita; hierbei muss man allerdings eine Hierarchie ,Zusammenschreibung - Bindestrich - Getrenntschreibung، (K. Berg et al. 2014) bedenken; die tiefer verschachtelte morphologische Verzweigung wird durch die engere graphematische Verbindung gezeigt. Insbesondere in rechtsverzwei- 
genden Komposita, so eines der Ergebnisse einer größeren Untersuchung von Dybiec (2014), wird die Verzweigung häufig deutlich angezeigt wie air vicemarshall ,Vize-General der Luftstreitkräfte' (Bindestrich ist enger als Getrenntschreibung), class-warfare ,Klassenkampf‘ (Zusammenschreibung ist enger als Bindestrich) (K. Berg et al. 2014). Daneben in der Konversion: So wird die ,Ausgangsform' getrennt geschrieben, bei der abgeleiteten Form wird zumindest zu einem Bindestrich geraten, so bei der Verbalisierung von Substantivkomposita in an ice skate - to ice-skate, a spot check - to spot-check bzw. umgekehrt bei der Substantivierung von ,phrasal verbs' to break in - a break-in, ebd. Oxford Dictionary (https://en.oxforddictionaries.com/punctuation/hyphen). Bei den Anglizismen im Deutschen wird wiederum die Bindestrichschreibung gerade für die ,phrasal verbs‘, die substantivisch verwendet werden, häufig übernommen: ein Add-on, ein Kick-off, aber auch Kickoff und Knockout. Jenseits der Anglizismen ist das im Deutschen so nicht denkbar: *vor werfen - *ein VorWurf, * ein brechen - *ein Ein-Bruch. Im Vergleich hat das Englische weniger eine morphologische Bindestrichsetzung (also zum Beispiel für Komposita) als vielmehr eine bei der syntaktischen Umkategorisierung. Das hat möglicherweise auch mit der Tatsache $\mathrm{zu}$ tun, dass die englischen Komposita auch in morphologischer und syntaktischer Hinsicht weniger eng zusammengehören als im Deutschen (Th. Berg 2012).

Im Niederländischen finden sich ähnliche typische Bindestrichstellen wie im Deutschen, so bei den graphematisch markierten Wörtern wie 80-jarige, tv-kijken, IQ-test. Daneben wird der Bindestrich im Niederländischen offenbar öfter gesetzt als im Deutschen wie in Oost-Vlaanderen, Zuid-Frankrijk, nietroker (Ostflandern, Südfrankreich, Nichtraucher, Beispiele aus mijnwoordenboek 2017) und dia-avond, mede-inzittende, auto-ongeluk (dt. Diaabend, Miteinsasse, Autounfall, Megen \& Neijt 1998).

Im Französischen kann der Bindestrich genutzt werden, wenn eine Struktur ,kompositumsähnlich wird‘, wie in après midi ,nachmittags“ vs. un aprèsmidi ,ein Nachmittag‘, zur aktuellen Diskussion s. auch Académie française (1990: 10). Wenn im Französischen Substantiv-Substantiv-Verbindungen auftauchen, sind Bindestriche möglich wie in frz. homme-sandwich (,Sandwichmann'). Im Französischen zeigt der Bindestrich in gewisser Weise, dass ein Wort vorliegt. Daneben gibt es auch ,syntaktische“ Bindestriche, also zwischen eigentlich selbständigen Wörtern bei bestimmter Wortstellung. Vous avez ,sie haben“ vs. avez-vous ,haben sie', il dit ,er sagt‘ vs. dit-il, tu vas ,du gehst‘ vs. vas-tu. Der Divis hat hier also eine andere Funktion als im Deutschen, syntaktisch selbständige Einheiten (Subjekte und Prädikate) werden miteinander graphisch verbunden. Bei il $a$ wird bei Umstellung außerdem ein $t$ eingefügt, das komplett von Divis umgeben ist, also $a$-t-il. Hier hat der Divis offenbar 
die Funktion, das eingefügte - $t$ - isoliert zu halten; im Schriftlichen ist die Ausgangsstruktur sehr viel deutlicher als im Mündlichen. Die Bestandteile -ci, -là, même werden in bestimmten Konstruktionen (celui-ci, cette maison-là, luimême) klitisch. Der Divis führt im Französischen selbständige Bestandteile zusammen - es scheint in keinem Fall Zusammenschreibung die Alternative zu sein, sondern immer eher Getrenntschreibung.

Der Divis als Bindestrich unterscheidet sich in den vier Sprachen also deutlich. Im Deutschen und Niederländischen ist er eine Alternative zur Zusammenschreibung, im Englischen und Französischen eine Alternative zur Getrenntschreibung. Damit ist er im Deutschen und Niederländischen mehr ein ,Trennzeichen' und im Englischen und Französischen eher ein ,Verbindezeichen‘.

\subsection{Der Apostroph}

Der Apostroph ist im Deutschen ein Elisionsapostroph (gibt's) und entwickelt sich zu einer morphologischen Kennzeichnung (Peter's) (s. auch Gallmann 1989; Klein 2002; Bunčić 2004; Scherer 2010, 2013; Buchmann 2015).

Im Englischen steht der Apostroph bei genitivartigen Konstruktionen wie in my father's house ,Haus meines Vaters' (Bunčić 2004: 188), aber auch als ,alleinige' graphematische Genitivmarkierung wie in the fathers' (fathers als Plural, fathers' als Genitiv). Hier ist der Apostroph Anzeiger morphologischer Strukturen. Er steht aber auch bei vermeintlichen Wortgrenzen, und zwar bei klitisierten Verben mit is in that's ,das ist', it's ,es ist', mit are in we're ,wir sind“ oder dem Pronomen us in let's ,lass uns' (Bunčić 2004: 190). Zumindest diese Formen wirken ähnlich lexikalisiert wie das deutsche geht's. Besonders interessant ist der Apostrophgebrauch bei_n’t für not ,nicht' mit einem Elisionsapostroph mitten im Wort, allerdings mit der Konsequenz, dass ein ansonsten vorhandenes Spatium nicht mehr geschrieben wird: isn't ,ist nicht', mustn't ,darf nicht‘. Ohne Apostroph ist die Zusammenschreibung nicht möglich ( ${ }^{\star}$ mustnot aber mustn't und nicht *must n't) außer bei can't - cannot.

Im Französischen findet sich wohl nur der Elisionsapostroph. Er ist im Allgemeinen obligatorisch und tritt an Wortgrenzen auf, insofern ist er auch morphologisch (und syntaktisch) zu interpretieren: l'ami ,der Freund' qu'il ,dass er'.

Im Niederländischen wird das $s$-Flexiv bei (Fremd-)Wörtern, die mit einem $|\mathrm{a}, \mathrm{o}, \mathrm{u}|$ enden, mit dem Apostroph segmentiert: Plural <auto's> ,Autos', <paprika's> ,Paprikas', <paraplu's> ,Regenschirme', aber nicht <garages> ,Garagen‘; Genitiv Anna's moeder vs. Peters moeder. Verhindert wird damit die Lesung als Kurzvokal, er kann also ein Elisionsapostroph sein für die drei Vokal- 
buchstaben (Shetter \& Van der Cruysse-Van Antwerpen 2002: 23); er zeigt aber ohne Zweifel auch eine morphologische Struktur.

Während also der Apostroph im Englischen eine Unterscheidung zwischen Genitiv und Plural systematisch ermöglicht, tut er das im Niederländischen in einigen Fällen gerade nicht. Aber er steht in beiden Fällen bei Flexionssuffixen; im Französischen steht er bei Wortgrenzen. Im Deutschen hingegen ist er bei keinem Flexionssuffix und an keiner Wortgrenze obligatorisch, sondern lediglich statt einer Genitivendung bei Eigennamen, deren Stamm auf $<\mathrm{s}>$ endet, sehr sinnvoll und daher möglicherweise zwingend.

\subsection{Der Abkürzungspunkt}

Der Abkürzungspunkt ist das unklarste Wortzeichen, weil er in der Form und der Lage zunächst nicht vom Satzpunkt zu unterscheiden ist, in keiner der vier Sprachen. Ein möglicher formaler Unterschied ist, dass der Abkürzungspunkt direkt zwischen Buchstaben stehen könnte (Buchmann 2015: 67) wie <d. h.>, ${ }^{26}$ mit Bredels Kriterien wäre er damit ,symmetrisch ${ }^{27}$ - ein Kennzeichen, das auch für die anderen Wortzeichen gilt, aber nicht für den Satzpunkt. ${ }^{28}$ Folgende mögliche Beispiele: dt. <z. B.>, engl. <J. R.> (für einen Namen), frz. <sc. nat.> (,sciences naturelles‘), nd. $<$ Z. Am.> (,Zuid Amerika‘). Daneben ist ohne Zweifel die Systematik des Abkürzungspunktes interessant. Für das Deutsche liegt hier mit Buchmann (2015) eine ausführliche Untersuchung vor, die zwischen ,Abbrüchen' und ,Zusammenziehungen“ unterscheidet; beim Abbruch erscheint die Buchstabenkette von Anfang bis zum Abbruch vollständig.

Zum Abkürzungspunkt stehen sprachvergleichende Untersuchungen noch aus. Ein Blick in die Abkürzungsverzeichnisse von Wörterbüchern zeigt aber folgendes: Im Englischen scheint es eine Tendenz zu geben, ihn gar nicht zu setzen (Langenscheidt \& Collins 2008: Umschlagseiten). Im Französischen scheint die Regulierung relativ vergleichbar mit dem Deutschen $\mathrm{zu}$ sein (Lange-Kowal 1991: $11 \mathrm{ff}$.); im Niederländischen hingegen scheint der Abkürzungspunkt ausschließlich möglich zu sein beim Abbruch, bei Zusammenziehungen - wenn also der letzte Buchstabe des ursprünglichen Wortes Teil der

26 Typographisch gilt dies als Fehler; es wird ein geschütztes Leerzeichen verlangt, was es aber eben deutlich vom Satzpunkt unterscheidet, zwischen $<$ d. h.> wird der Zeilenumbruch verhindert.

27 Das Zeichen steht „in beide Richtungen in Kontakt mit Elementen derselben Segmentklasse“ (Bredel 2008: 27).

28 Während ${ }^{\star}$ Hanna liest.Peter nicht. (Bredel 2008: 67) ungrammatisch ist, kommt <z. B.> im Sprachgebrauch vor und wird nicht unbedingt als Fehler betrachtet. 
Abkürzung ist - wird er offenbar nicht gesetzt (van der Linden 1989: 7 f.); die Regularität, die sich im Niederländischen findet, wird zum Teil typographisch auch im Deutschen verlangt, hat sich aber zumindest konsequent nicht durchgesetzt.

\subsection{Zusammenfassung Wortzeichen}

In dem kurzen Abriss finden sich Gemeinsamkeiten hinsichtlich des Inventars und der Kombinatorik mit Leerzeichen. Für jedes Zeichen finden sich aber auch deutliche Unterschiede. Zum Beispiel kann die Bindestrichsetzung danach typologisiert werden, ob sie eher mit der Zusammenschreibung oder eher mit der Getrenntschreibung konkurriert. Für das Französische und Englische kann die Nutzung des Bindestrichs als ,weniger Getrenntschreibung“ beschrieben werden; und das obwohl sich die beiden Sprachen hinsichtlich der Kompositabildung fundamental unterscheiden; im Deutschen und Niederländischen eher als ,weniger Zusammenschreibung'. Die Sprachen scheinen in dieser Hinsicht durchaus vergleichbar, auch wenn das Niederländische häufiger einen Bindestrich hat. In der Übersicht wurde der Trennstrich am Zeilenende noch nicht behandelt, aber es ist ja bekannt, dass er zum Beispiel im Englischen ,morphologischer' als im Deutschen ist, die Stellen, an denen getrennt wird, sind also andere.

Der phonologische Gebrauch des Apostrophs scheint für alle untersuchten Sprachen möglich. Im Deutschen, Englischen und Niederländischen bildet sich auch ein morphologischer Apostroph heraus. Im Französischen ist der Apostroph sowohl phonographisch (es wird bei l'ami der Vokal des Artikels nicht gesprochen) als auch morphosyntaktisch (im Geschriebenen zeigt es die Grenze zwischen den Wörtern) zu interpretieren. Für den Apostroph sind im Deutschen die nicht-standardisierten Verwendungen (Klein 2002; Scherer 2010, 2013) die interessanteren; der regelkonforme Gebrauch ist doch stark eingeschränkt. Insofern wäre auch für die anderen Sprachen hier eine Untersuchung des nicht-standardisierten Gebrauchs besonders interessant. Allerdings ist im Deutschen - bis auf den Typ Carlos' - der Apostroph nicht obligatorisch; es könnte sein, dass genau deswegen der Apostroph im Deutschen kreativ genutzt wird.

Beim Abkürzungspunkt liegt die Vermutung nahe, dass ein Unterschied zwischen Abbruch (Abt.) und Zusammenziehung (Abtg., jeweils für Abteilung) ${ }^{29}$ in verschiedenen Sprachen zu finden ist.

29 Nach Steinhauer (2005: 19). 


\section{Grammatische Teilbereiche - Besonderheiten bei der Schreibung}

\subsection{Verbflexion}

Alle erwähnten Sprachen haben eine ausgeprägte Verbflexion. Dabei haben Deutsch, Englisch und Niederländisch eine große Klasse der schwachen Verben, die regelmäßig und vorhersagbar flektiert werden und daneben eine Restmenge von starken und unregelmäßigen Verben, die es auch graphematisch im Detail zu untersuchen lohnt. Im Französischen gibt es mehrere Verbklassen, die unterschiedlich groß sind. Auch wenn sich auf der Formebene vergleichbare Wechsel in den verschiedenen Sprachen finden wie der zwischen $\langle\mathrm{i}\rangle$ und $\langle\mathrm{y}\rangle$ sowohl im Französischen (j'essaie - essayer) als auch im Englischen (say said), bleibt die Bewertung doch eine andere - im Französischen ist dieser Wechsel regelhaft, im Englischen ein isolierter Einzelfall. Solche ,Wechsel' und die graphematischen Flexionsmuster sind in der Verbflexion gut zu beobachten; Zusammenhänge werden deutlich. In 5.4 wurde bereits angedeutet, dass es möglicherweise auch graphematische Flexionsmuster gibt. Fuhrhop (2017) untersucht die starken und unregelmäßigen Verben im Deutschen und Englischen. Im Englischen sind die Verben zum Teil graphematisch recht regelmäßig wie sing - sang, ring - rang, zum Teil aber auf den ersten Blick eher unverständlich unregelmäßig wie speak - spoke statt *speke - spoke oder speak - *spoak. In den beiden nicht realisierten Paaren wäre mehr Morphemkonstanz. Die systematische Untersuchung dieser Formen hat zu der Annahme von ,graphematischen Mustern' geführt. Wir finden folgende vergleichbare Verben im Englischen: break - broke, speak - spoke, steal - stole; bear - bore, swear - swore, tear - tore, wear - wore.

Obwohl sie sich in der Aussprache unterscheiden, könnten alle Paare in beide Richtungen mehr Morphemkonstanz zeigen. Sie werden aber alle nach dem gleichen Muster geschrieben.

Im Niederländischen ist bereits die schwache Verbflexion sehr interessant. Auf der einen Seite ist die Schreibung der Präteritumendung offenbar lautlich beeinflusst - nach stimmlosen Vokalen folgt -te, sonst - de (s. auch 5.2). Hier gibt es also zwei Allomorphe. Wenn allerdings ein Stamm auf $-d$ oder $-t$ endet, wird dennoch -de, -te angehängt - es ergeben sich Schreibungen wie zuchtte, antwoordde, die Identifikation als Präteritumform wird also in Schriftform gesichert. 


\subsection{Modalverben}

Die erwähnten graphematischen Flexionsmuster zeigen sich besonders prägnant bei den Modalverben im Englischen. Die ,Präteritumformen` der drei Modalverben can, shall und will sind could, should, would - eine naheliegende Schreibung von $/ \mathrm{vd} /$ wäre $<\mathrm{ud}>$ oder $<\mathrm{ood}>$ wie in $<$ wood, shood $>$. Das vierte Modalverb ist may - might, das mit /ait/ entweder <ite> oder <ight > geschrieben werden könnte, might ist also eine regelmäßige normale Schreibung. Could, should, would sind es nicht. Dennoch ist auffällig, dass sie alle gleich geschrieben werden, und zwar mit einem Muster, das sonst nur noch in mould (Schimmel im Britischen Englisch, Amerikanisch: mold) zu finden ist, mit einer völlig anderen Aussprache. Es scheint also ein nahezu exklusives Muster zu geben für die ,Präteritumform ' von Modalverben - sie hat ja auch eine besondere Funktion und Semantik.

Im Deutschen gibt es neben vielen Formen mit Flexionsendungen eine Klasse von flektierten Formen ohne Endungen, und zwar die Präteritumform von starken Verben in der 1. und 3. Person, Indikativ (grub, log, bot) und entsprechend die Präteritopräsentia mit den Modalverben. Nach der Untersuchung in Fuhrhop (2017) ergab sich die Beobachtung, dass zwei Drittel dieser Formen eben doch zeigen, dass sie in ein flektierendes Paradigma gehören mit einem graphematischen Ende, das auf Zweisilbigkeit hinweist wie die Endungen $-b,-d,-g$ (wegen der nicht-realisierten Auslautverhärtung), die Endung auf $-\beta$, Doppelkonsonanten oder $-h$, die phonographisch ausschließlich in Zweisilbern herzuleiten sind. Das ist eine interessante Beobachtung und kann durchaus wie viele andere Phänomene in der Schreibung als Lesehilfe verstanden werden. Kommen wir aber auch hier auf die Modalverben: Die Modalverben sind Präteritopräsentia, für sie gilt Endungslosigkeit also bereits im Präsens:

a) kann, muss, soll will

b) $\mathrm{mag}$

c) möchte

d) darf

In (a) deuten die Doppelkonsonanten auf zweisilbige Formen hin, in (b) auf eine in der Schrift nicht-wiedergegebene Auslautverhärtung. In (c) sieht die Endung aus wie eine schwache Präteritumendung, im Zusammenhang mit den Präteritopräsentia ist die Form also nicht wirklich endungslos. Damit bleibt darf die einzige Form, die keinen Hinweis auf Flexion gibt. Sechs der sieben Modalverbformen zeigen also auch ohne Endung ihre Zugehörigkeit zu Flexionsparadigmen. 
Die Schreibungen ergeben sich in gewisser Weise logisch, da sie sich ja aus zweisilbigen Formen ergeben. Allerdings kann man schon anführen, dass ja Formen wie ${ }^{\star}$ kannen, ${ }^{\star}$ willen, ${ }^{\star}$ mussen (und magen) nicht vorkommen. Besonders beim letzten ist die Verwandtschaft $\mathrm{zu}$ möchten (als hypothetischer Infinitiv) ja durch die Schreibung eher verdeckt. Genau das meint aber ,graphematisches Muster' - offenbar ist es besser, wenn eine Verbform kann geschrieben wird als wenn sie kan geschrieben wird. Allein durch das Prinzip der Stammkonstanz ist das nicht zu erfassen, aber als sehr typische verbale Schreibweise.

Als Modalverben im Französischen gelten vouloir und pouvoir. In der 1. Ps., Sg., Ind., Präs. flektieren französische Verben entweder mit -s (je prends) oder endungslos (je parle). Die einzigen Verben, die Flexionsformen mit $-x$ haben, sind die Modalverben (je peux, je veux). ${ }^{30}$

Es ist ein kleiner Fund - aber es ist auffällig, dass Modalverben in ihrer Schreibung auf sehr unterschiedliche Weise aus dem System ,ausbrechen' und markiert sind.

\section{Zusammenfassung und Ausblick}

Der Vergleich von verschiedenen Schriftsystemen zeigt Gemeinsamkeiten und Unterschiede und in beiden Fällen sowohl Erwartbares als auch Unerwartetes. Ziel meiner Ausführungen war es zu zeigen, dass wir für einen Vergleich der Schriftsysteme bereit sind: Wir können sowohl sporadisch vergleichen als auch sehr viel systematischer entlang verschiedener Fragestellungen. Einige habe ich entwickelt.

Mitunter ist zu erkennen, dass das Deutsche einen Sonderweg geht. So ist es unter den vorgestellten Sprachen die einzige Sprache mit einer strengen Stammkonstanzschreibung. Bei der Unterscheidung von das - dass liegt die Vermutung nahe, dass zwischen dem definiten Artikel und der Konjunktion unterschieden wird; eine Tatsache, die in den anderen Sprachen sowieso gegeben ist, weil die Wörter in den anderen Sprachen gar nicht homophon sind.

Das Deutsche zeigt seine Morphologie, aber es zeigt sie unspezifisch. Das Englische hat weniger (Flexions-)Morphologie, zeigt sie aber spezifischer; das Französische zeigt graphematisch seine vielfältige Morphologie, und zwar im Allgemeinen recht spezifisch.

30 Nach <eu> ist sowohl eine Flexionsendung -s als auch - $x$ möglich, so im Plural bei Substantiven dieu - dieux neben pneu - pneus. Allerdings kommt bei der Form eus (Form von avoir für das Passé antérieur in der 1. und 2. Person Singular) sehr wohl ein -s nach eu. 
Im Inventar scheinen sich die Systeme der Wortzeichen sehr zu ähneln, sie funktionieren recht analog in den verschiedenen Sprachen. Allerdings reagiert die Graphematik offenbar auf die unterschiedlichen Systeme, sowohl deutlich an der unterschiedlichen Komposition (Bindestrich) in den Sprachen als auch in dem Hang oder Zwang zur Elision (Apostroph). Beim Abkürzungspunkt hingegen zeigen sich interessante Unterschiede - das Deutsche unterscheidet nicht (oder nicht konsequent) zwischen Abbruch (die ersten Buchstaben sind die gleichen und es wird abgebrochen) und dem Typ der Zusammenziehung, in der der letzte Buchstabe vorhanden ist, andere Sprachen offenbar doch.

Insgesamt zeigt sich an vielen Stellen, wie erhellend der Vergleich der Schriftsysteme sein kann. Welche interessanten Phänomene haben die anderen Sprachen zu bieten und wo ist das Deutsche speziell, auch jenseits der Substantivgroßschreibung? Für Antworten auf diese Frage brauchen wir weitere Forschung zu einer Graphematik im europäischen Vergleich.

\section{Literatur}

Académie française (1990): http://academie-francaise.fr/sites/academie-francaise.fr/files/ rectifications.pdf

Augst, Gerhard (1987): Zur graphischen Bezeichnung der Vokalqualität bei Fremdwörtern. In Hermann Zabel (Hrsg.), Fremdwortorthographie, 94-110 Tübingen: Niemeyer.

Berg, Kristian (2012): Identifying graphematic units: Vowel and consonant letters. In Written Language and Literacy 15 (1), 26-45.

Berg, Kristian (2017a): Sichtbare Flexionsmorphologie im Englischen und Deutschen. In Nanna Fuhrhop, Renata Szczepaniak \& Karsten Schmidt (Hrsg.), Sichtbare und hörbare Morphologie, 9-41. Berlin: De Gruyter.

Berg, Kristian (2017b): Wortschreibung im Deutschen und Englischen. Habilitationsschrift. Universität Oldenburg.

Berg, Kristian \& Mark Aronoff (2017): Self-organization in the spelling of English suffixes: The emergence of culture out of anarchy. Language 93, 37-64.

Berg, Kristian, Franziska Buchmann, Katharina Dyebic \& Nanna Fuhrhop (2014): Morphological spellings in English. Written Language and Literacy 14 (2), 282-307.

Berg, Kristian \& Nanna Fuhrhop (2011): „Komplexe Silbenkernschreibungen im Englischen im Vergleich mit dem Deutschen“. In Linguistische Berichte 228, 443-466.

Berg, Thomas (2012): The cohesiveness of English and German compounds. In The Mental Lexicon 7 (2), 1-33.

Bredel, Ursula (2008): Die Interpunktion des Deutschen. Ein kompositionelles System zur Online-Steuerung des Lesens. Tübingen: Niemeyer.

Bredel, Ursula \& Hrvoje Hlebec (2015): Kommasetzung im Prozess. In Praxis Deutsch 254, 36-43.

Buchmann, Franziska (2015): Die Wortzeichen im Deutschen. Heidelberg: Winter.

Bunčić, Daniel (2004): The apostrophe. A neglected and misunderstood reading aid. In Written Language and Literacy 7 (2), 185-204. 
Catach, Nina (1978): L'orthographe. Paris: Presses Universitaires de France.

Dybiec, Katharina (2014): Bindestrichschreibung im Deutschen und Englischen.

Eine korpusbasierte Analyse zwei- und dreigliedriger Substantivkomposita. https:// www.uni-oldenburg.de/fileadmin/user_upload/germanistik/personen/Dybiec_ Bindestrichschreibung_im_Deutschen_und_Englischen.pdf (letzter Zugriff: 8. 8. 2017)

Eichinger, Ludwig M. (2012): Deutsch im Sprachvergleich. Grammatische Kontraste und Konvergenzen. In Lutz Gunkel \& Gisela Zifonun (Hrsg.), Deutsch im Sprachvergleich, VII-XVI. Berlin: De Gruyter.

Eisenberg, Peter (2017): Deutsche Orthografie. Regelwerk und Kommentar. Berlin: De Gruyter. Evertz, Martin (2016): Graphematischer Fuß und graphematisches Wort. In Ulrike Domahs \& Beatrice Primus (Hrsg.), Handbuch Laut, Gebärde, Buchstabe, 377-397. Berlin: De Gruyter.

Evertz, Martin \& Beatrice Primus (2013), The graphematic foot in English and German. In Written Language and Literacy 5 (1), 1-23.

Fabricius-Hansen, Cathrine (2003): Deutsch - eine ,reife‘ Sprache. Ein Plädoyer für die Komplexität. In Gerhard Stickel (Hrsg.), Deutsch von außen, 99-112. Berlin: De Gruyter.

Feilke, Helmuth (2011): Der Erwerb der das/dass-Schreibung. In Ursula Bredel \& Tilo Reißig (Hrsg.), Weiterführender Orthographieerwerb, 340-354. Baltmannsweiler: Schneider Hohengehren.

Franke, Marlene (2008): Die graphematische Silbe des Deutschen und Englischen. Carl von Ossietzky Universität Oldenburg. https://www.uni-oldenburg.de/nanna-fuhrhop/ zitierte-bachelor-und-masterarbeiten/ (letzer Zugriff: 10.6. 2017).

Fuhrhop, Nanna (2017): Sichtbare Morphologie in der Flexion der starken und unregelmäßigen Verben im Deutschen und Englischen. In Nanna Fuhrhop, Renata Szczepaniak \& Karsten Schmidt (Hrsg.), Sichtbare und hörbare Morphologie, 43-76. Berlin: De Gruyter.

Fuhrhop, Nanna \& Rebecca Barghorn (2012): Prinzipien der Wortschreibung im Deutschen und Englischen am Beispiel der Schreibdiphthonge und der Doppelkonsonanten. In Lutz Gunkel \& Gisela Zifonun (Hrsg.), Deutsch im Sprachvergleich. Grammatische Kontraste und Konvergenzen, 135-160. Mannheim.

Fuhrhop, Nanna \& Kristian Berg (2017): Schreibdiphthonge und graphematische Silbenkerne - modalitätsspezifisch und modalitätsübergreifend. Mskr. Eingereicht bei Martin Evertz \& Frank Kirchhof (Hrsg), Geschriebene und gesprochene Sprache als Modalitäten eines Sprachsystems.

Fuhrhop, Nanna \& Franziksa Buchmann (2009): Die Längenhierarchie: Zum Bau der graphematischen Silbe. In Linguistische Berichte 218, 127-155.

Fuhrhop, Nanna \& Franziska Buchmann (2011): Buchstabenformen und ihre Relevanz für eine Schriftgrammatik. Erwiderung auf einige Thesen von Oliver Rezec. In Linguistische Berichte 225, 77-88.

Fuhrhop, Nanna, Franziska Buchmann \& Kristian Berg (2011): The length hierarchy and the graphematic syllable: Evidence from German and English. In Written Language and Literacy 14 (2), 275-292.

Fuhrhop, Nanna \& Jörg Peters (2013) Einführung in die Phonologie und Graphematik. Stuttgart/Weimar: Metzler.

Fuhrhop, Nanna \& Karsten Schmidt (2014): Die zunehmende Profilierung der Schreibsilbe in der Geschichte des Deutschen. In PBB 136 (4), 538-568.

Gallmann, Peter (1989): Syngrapheme an und in Wortformen. Bindestrich und Apostroph im Deutschen. In Peter Eisenberg \& Hartmut Günther (Hrsg.), Schriftsystem und Orthographie, 85-110. Tübingen: Niemeyer. 
Gunkel, Lutz, Adriano Murelli, Susan Schlotthauer, Bernd Wiese \& Gisela Zifonun (2017): Grammatik des Deutschen im europäischen Sprachenvergleich. Das Nominal. Berlin: De Gruyter.

Klein, Wolf-Peter (2002): Der Apostroph in der deutschen Gegenwartssprache. Logographische Gebrauchserweiterungen auf phonographischer Basis. In Zeitschrift für Germanistische Linguistik 30 (2), 169-197.

Lange-Kowal, Ernst Erwin (1991): Langenscheidts großes Schulwörterbuch DeutschFranzösisch. 10. Auflage. München: Langenscheidt.

Langenscheidt/Collins (2008): Großwörterbuch Englisch. 4. Auflage. München: Langenscheidt.

Lexique www.lexique.org/ (letzter Zugriff: 15. 9. 2017).

Linden, G. A. M. M. van der (1989): Nederlands - Duits. 14. Auflage. Utrecht.

Megen, Jan van \& Anneke Neijt (1998): Niederländische und deutsche Orthographie im Vergleich. In Deutsche Sprache 26, 193-217.

Meisenburg, Trudel (1996): Romanische Schriftsysteme im Vergleich. Eine diachrone Studie. Tübingen: Narr.

Mijnwoordenboek (2017): www.mijnwoordenboek.nl/spelling-samenstellings-streepje.php (letzter Zugriff: 30.5. 2017).

Neef, Martin \& Beatrice Primus (Hrsg.) (2004): From letters to sounds. New perspectives on writing systems. Special issue of Written Language \& Literacy 7 (2).

Nunn, Anneke Marijke (1998): Dutch orthography. A systematic investigation of the spelling of Dutch words. The Hague: Holland Academic Graphics.

Quirk, Randolph, Sidney Greenbaum, Geoffrey Leech \& Jan Svartvik (1994): A Comprehensive Grammar of the English Language. 12. Auflage. New York: Longman.

Primus, Beatrice (2006): Buchstabenkomponenten und ihre Grammatik. In Ursula Bredel \& Hartmut Günther (Hrsg.), Orthographietheorie und Rechtschreibunterricht, 5-43. Tübingen: Niemeyer.

Pustka, Elissa (2011): Einführung in die Phonetik und Phonologie des Französischen. Berlin: Schmidt.

Ramers, Karl-Heinz (1999): Vokalquantität als orthographisches Problem: Zur Funktion der Doppelkonsonanzschreibung im Deutschen. In Linguistische Berichte 177, 52-64.

Rogers, Henry (2005): Writing systems. A linguistic approach. Malden: Blackwell.

Sappok, Christopher \& Carl Ludwig Naumann (2016): Die ,Kommabrille“ - historische, psycholinguistische und didaktische Perspektiven. In Ralph Olsen, Christiane Hochstadt, und Simona Colombo-Scheffold (Hrsg.), Ohne Punkt und Komma ... Beiträge zu Theorie, Empirie und Didaktik der Interpunktion, 99-136. Berlin: RabenStück.

Scherer, Carmen (2010): Das Deutsche und die dräuenden Apostrophe. Zur Verbreitung von 's im Gegenwartsdeutschen. In Zeitschrift für germanistische Linguistik 38, 1-24.

Scherer, Carmen (2013): Kalb’s Leber und Dienstag's Schnitzeltag. Zur Funktionalisierung des Apostrophs im Deutschen. In Zeitschrift für Sprachwissenschaft 32, 75-112.

Shetter, William Z. \& Inge Van der Cruysse-Van Antwerpen (2002): Dutch. An essential Grammar. London/New York: Routledge.

Steinhauer, Anja (2005): Das Wörterbuch der Abkürzungen. 4. Auflage. Mannheim: Duden. Vennemann, Theo (1982): Zur Silbenstruktur der deutschen Standardsprache. In Theo Vennemann (Hrsg.), Silben, Segmente, Akzente, 261-305. Tübingen: Niemeyer. 



\section{Register}

Abstraktheit 4, 12, 334, 549, 576

Analyse, kontrastive 281, 283, 292, 294, 304-305

Areallinguistik 3, 241-252, 258, 260-262, 272-273, 370

Argument, externes 5, 511, 513-515, 517$528,531-539,542-544$

Assoziation 225-226, 229, 231, 233, 235237, 563, 574

Äußerungsbedeutung 455, 471

Bedeutungsebene 5, 471-473, 492-493, 499, 501

Deixis 3, 287-288, 309-310, 312-313, 316, 326-328, 418, 474, 490, 496

Dependenzgrammatik 2, 117-118, 123, 129 , 141-142

Deutsch als Fremdsprache (DaF) 4, 358, 364, 389, 396-397, 399, 405, 409-411, 413-414, 417-426

Diachronie 3-4, 20, 151, 187, 246, 262, 285, $331-332,334-336,338,343-344,347-$ $349,356,358,373,489,588$

Diskursanaphorik 435, 441, 443, 450

Diskursrelationen 435, 449-456

Dispersion 225, 235-237

First Conjunct Agreement (FCA) 185, 193194, 199, 204, 206, 210, 212-214, 216, 218, 220-221

Generalized Phrase Structure Grammar (GPSG) 2, 72, 117, 119, 126-129, 132, $140,142,512$

Government \& Binding (GB) 117, 122, 140, 142,512

Grammatik, deskriptive 176, 361, 372

Grammatik, frequenzbasierte 2, 9, 19-29

Grammatik, funktionale 383-384, 386, 391392, 396, 399

Grammatik, generative 2, 9, 14, 47, 51, 58, $88,95,105,360$

Grammatikographie/Grammatikschreibung 1-2, 4, 151, 154, 176-181, 243, 247-
$248,257,273,331-333,339,343,355-$ $357,359,363-364,370,374-375,383-$ $385,389,392,402,404$

Grammatiktheorie 1-4, 9-13, 15, 18-20, 2728, 35-36, 47-48, 54, 58, 76, 87, 117, $119,142,151-153,158,165-166,175$, $177,180,186,331,355,383,443,456$, 459

Häufigkeit 156, 173, 196, 210, 225, 229, 235-237, 259

Head-driven Phrase Structure Grammar (HPSG) 2, 69, 117, 119, 128-137, 139142,512

Informationsgewichtung 435, 457 Intension 2, 95-104, 110-113 Intersprechervariation 188, 213-214 Intrasprechervariation 185, 188-190, 214215,221

kognitive Sprachverarbeitung 2, 38

Kohärenz 60, 65, 67-68, 81-82, 137, 179, 291-293, 366, 435, 437, 450, 457, 462

Kohäsion 435, 437, 560

Kompetenz 1, 3, 10, 12, 14, 62-63, 98, 151, $166,175,178-179,186,212,216,294$, 299, 303-304, 360-361, 375, 412, 420, 436

Kongruenz 11, 185, 189, 192-197, 199, 201, 203-204, 206, 208-213, 215, 217-218, 220-222, 447, 519

Konnektionismus 2, 9, 15-19, 37

Konstituentenstellung 117

Konstruktionsgrammatik 2, 47-48, 60, 88, $117,129,139,142,227,360,363,384-$ $385,420,422-423,512$

Korpus 1-3, 14, 21-25, 27, 30, 32-33, 35, 38, 151-168, 174-176, 180, 189, 199, 204, 214-215, 220, 225-226, 228-229, 231-233, 235-236, 241, 246, 250-258, 260-261, 264, 268, 272-273, 316, 332, 341-344, 349, 369-371, 375, 378, 416, 579 
Korpusanalyse 2, 9, 21-25, 40, 155, 422

Korpusgrammatik 173, 243, 348, 355, 372, 379

Korpuslinguistik 2-4, 10, 21, 30, 151-155, $158,161-162,165,167,174,176,180-$ 181, 225-227, 234, 236-237, 243, 371$372,383,405,422-423,440,448,456$

\section{Lemma 229}

Lexikalisch-funktionale Grammatik (LFG) 2, 117, 119, 126, 135-137, 140, 142

Markiertheitsbeschränkung 549, 560-564, 566, 570-571, 574-575, 581-582

Medium 168, 172, 178, 196, 384, 415-417, $420,511,524-525,540$

Mehrwortausdruck 225-226, 229, 232-234, 237

Minimalismus $2,12,15,18,36,117,140-$ 142, 493-495, 497-500

Minimalistisches Programm (MP) 47-52, 54-56, 58-59, 62-63, 87-88, 139, 516, 566

Modell 1-2, 10, 15-16, 19, 22, 24-25, 35, 48, 51, 68-69, 80, 95-96, 108-111, 113, $122,140,159-160,163,172,186,232$, 234, 310, 371, 384, 395, 440, 444, 463, 472-473, 475, 490, 492, 499-502, 504. 512, 519

Montague-Grammatik 95

Morphologie 3, 73, 77, 128-130, 163, 185, 192, 195-197, 200, 207, 221-222, 243244, 282-283, 286-287, 309-310, 313, $315,317,321-322,325-327,425-426$, 447, 514, 516-520, 524, 535, 594-598, 600-601, 605-609, 612

Passiv 4, 81, 83-84, 117, 119-123, 125, 132, $135,137,142,170-171,241,247-248$, 256, 258-261, 268, 397, 400, 415-416, 418, 511, 514-515, 517-519, 522-528, $531-538,540,542-544$

Performanz 3, 10, 12, 14, 20, 151, 166, 175, 178-179, 186, 233, 360-361

Phrasenstrukturgrammatik 117, 121, 123 , 126-127, 512

Psycholinguistik 2, 9-10, 12-15, 20, 28, 31$32,34-37,50,54,62,122-123,140$,
$142,189,215,237,281,290,298,443$, 448-449, 452, 501

Raum-Linguistik 3, 309, 311, 327

Repräsentation, symbolische 4-5, 549-550, 575, 581

Resolutionsregeln 185, 190, 195, 218-219, 222

Schreibung, morphologische 595

Semantik, formale 2, 95-96, 104, 108-111, 440, 445-446, 462

Sprache, gesprochene $177,248,366,368$, 370-371, 378, 384, 391-392, 395, 402, 405, 409, 413, 417-421, 423, 568

Sprache und Kognition 281, 362

Sprachtypologie 3, 14, 286, 288, 309-310, 327-328, 401

Sprachvergleich 281-282, 284, 286-290, 292, 294, 296, 309-310, 312, 314, 328, 356-357, 401-402, 424-426, 581, 587, 596, 603, 608

Sprachwandel 3-4, 80, 175, 187, 189, 236, 331, 337

Sprecherbedeutung 471-473, 475, 483, 489-490, 493-496, 498-501

Standardsprache $4,177,188,242,245$, 247-248, 263-264, 268, 272-273, 332, 346, 348, 355-356, 361, 369-372, 376, 378

Subjektreihung 185, 189-190, 192-203, 205-211, 213-222

Synchronie 3-4, 151, 246, 249, 262, 281, 283, 285, 310, 321, 331-338, 341, 344, 347-349, 356, 463, 599

Syntax 2-3, 5, 12, 15-17, 20, 26, 30, 36-37, 49-50, 52-54, 56, 63-65, 68, 72, 78, 82-83, 87, 105-107, 119, 124, 130-133, $157,167,169,171,185,187,189,192$, 196, 207, 212, 217, 222, 241-251, 253, 257, 262-264, 267, 272-273, 282-283, 288-289, 292, 294-295, 333, 360, 363, $367,373,385-387,392-396,398,400$, 402, 404, 411, 414, 418-419, 440, 442, 448 , 453-457, 459, 462, 474-477, 479480, 483, 511-520, 522-525, 527-529, 531-534, 536-538, 540-544, 588, 593, $595,605-607$ 
tertium comparationis 281-282, 286-290, 294, 296, 304-305, 401, 594

Textkonstitution 5, 365, 435-436, 438, 440-441, 450, 459

Textmodellierung 5, 435

Textsortenlinguistik 409, 414

Transformation 117-118, 121-123, 126, 128, $132,135,139,142,528$

Tree Adjoining Grammar (TAG) 2, 117, 126, 134-137, 452

Typen 2, 5, 23, 73, 95-98, 101, 104-105, 107, 130, 132, 180, 187-188, 206, 214, 230, 295, 301, 358, 363, 365, 384, 390, $393,402,488,490,513-516,519,523-$ $528,533,537,539,543-544,549,582$, $595,609,613$

Unbestimmtheit 139, 471-472, 474-476, 481-484, 486, 490, 492-496, 499, 501
V2 $69,72,79,117,138,142,188,456$

Variabilität, quantifikationale 4, 511, 515, 538-544

Variation 1-4, 12, 64, 85, 109, 111, 151, 163, 166-169, 171-172, 174-176, 178-181, 185-192, 196, 208, 214-218, 222, 241246, 251, 263-264, 272-273, 320, 325, 331, 337-341, 343, 345-349, 364, 367, 370-372, 409, 416, 421, 423, 458, 540, $560,569,573$

Verbformschreibung 587

Vokalismus 549, 552

Wissenschaftstheorie 2, 47

Wortphonologie 549

Wortschreibung, Prinzipien 587, 595

Wortzeichen 587, 590, 604-605, 608-609, 613 



\section{Autorinnen und Autoren}

Artemis Alexiadou, Humboldt Universität Berlin, Englische Sprachwissenschaft, Unter den Linden 6, D-10099 Berlin \& Leibniz-ZAS, E-Mail: artemis.alexiadou@hu-berlin.de

Eva Breindl, Friedrich-Alexander-Universität Erlangen-Nürnberg, Bismarckstr. 1, D-91054 Erlangen, E-Mail: eva.breindl@fau.de

Jürg Fleischer, Institut für Germanistische Sprachwissenschaft, Forschungszentrum Deutscher Sprachatlas, Pilgrimstein 16, D-35032 Marburg, E-Mail: jfleischer@staff.uni-marburg.de

Nanna Fuhrhop, Universität Oldenburg, Fakultät III - Sprach- und Kulturwissenschaften, Institut für Germanistik, D-26111 Oldenburg, E-Mail: nanna.fuhrhop@uni-oldenburg.de

Eric Fuß, Institut für Deutsche Sprache, R5, 6-13, D-68161 Mannheim, E-Mail: fuss@ids-mannheim.de

Peter Gallmann, Friedrich-Schiller-Universität Jena, Philosophische Fakultät, Institut für Germanistische Sprachwissenschaft, Fürstengraben 30, D-07743 Jena,

E-Mail: peter.gallmann@uni-jena.de

Stefan Th. Gries, University of California,

Santa Barbara; Department of Linguistics, Santa Barbara, CA 93106-3100; U.S.A.,

E-Mail: stgries@linguistics.ucsb.edu

Daniel Gutzmann, Institut für deutsche Sprache und Literatur I, Universität zu Köln, Albertus-Magnus-Platz, D-50923 Köln, E-Mail: mail@danielgutzmann.com

Mechthild Habermann, Lehrstuhl für Germanistische Sprachwissenschaft, Friedrich-Alexander-Universität Erlangen-Nürnberg, Bismarckstraße 1, D-91054 Erlangen, E-Mail: mechthild.habermann@fau.de

Hubert Haider, Universität Salzburg, Fachbereich Linguistik \& Centre for Cognitive Neuroscience UNIPARK Nonntal, A-5020 Salzburg, E-Mail: hubert.haider@sbg.ac.at

Mathilde Hennig, Justus-Liebig-Universität Gießen, Institut für Germanistik, Otto-Behaghel-Straße 10 B, D-35394 Gießen,

E-Mail: Mathilde.Hennig@germanistik.uni-giessen.de

Anke Holler, Seminar für Deutsche Philologie, Courant-Forschungszentrum „Textstrukturen“, Universität Göttingen, Käte-Hamburger-Weg 3, D-37073 Göttingen,

E-Mail: anke.holler@phil.uni-goettingen.de

Marek Konopka, Institut für Deutsche Sprache, R5, 6-13, D-68161 Mannheim, E-Mail: konopka@ids-mannheim.de

Manfred Krifka, Institut für deutsche Sprache und Linguistik, Humboldt-Universität zu Berlin, Unter den Linden 6, D-10099 Berlin \& Leibniz-ZAS, E-Mail: krifka@rz.hu-berlin.de

Alexandra N. Lenz, Universität Wien, Institut für Germanistik, Universitätsring 1, A-1010 Wien, E-Mail: alexandra.lenz@univie.ac.at

Gereon Müller, Universität Leipzig, Institut für Linguistik, Beethovenstr. 15, D-04107 Leipzig, E-Mail: gereon.mueller@uni-leipzig.de

Ә Open Access. (C) 2018 publiziert von De Gruyter. (c) BY Dieses Werk ist lizenziert unter der Creative Commons Attribution 4.0 Lizenz.

https://doi.org/10.1515/9783110490992-022 
Stefan Müller, Institut für deutsche Sprache und Linguistik, Sprach- und literaturwissenschaftliche Fakultät, Humboldt Universität Berlin, E-Mail: st.mueller@hu-berlin.de

Beatrice Primus, Universität zu Köln, Institut für deutsche Sprache und Literatur I Sprachwissenschaft, Albertus Magnus Platz, D-50923 Köln, E-Mail: primus@uni-koeln.de

Renate Raffelsiefen, Institut für Deutsche Sprache, R5, 6-13, D-68161 Mannheim, E-Mail: raffelsiefen@ids-mannheim.de

Petra B. Schumacher, Institut für deutsche Sprache und Literatur I, Universität zu Köln, Albertus-Magnus-Platz, D-50923 Köln, E-Mail: petra.schumacher@uni-koeln.de

Thomas Stolz, Universität Bremen, FB 10: Linguistik, Universitäts-Boulevard 13, D-28359 Bremen, E-Mail: stolz@uni-bremen.de

Christiane von Stutterheim, Universität Heidelberg, Institut für Deutsch als Fremdsprachenphilologie, Plöck 55, D-69117 Heidelberg, E-Mail: cvs@idf.uni-heidelberg.de

Maria Thurmair, Universität Regensburg, Fakultät für Sprach-, Literatur- und Kulturwissenschaften, Universitätsstraße 31, D-93053 Regensburg,

E-Mail: maria.thurmair@sprachlit.uni-regensburg.de

Angelika Wöllstein, Institut für Deutsche Sprache, R 5, 6-13, D-68161 Mannheim, E-Mail: woellstein@ids-mannheim.de

Thomas Ede Zimmermann, Goethe-Universität Frankfurt, Institut für Linguistik, Norbert-Wollheim-Platz 1, D-60323 Frankfurt, E-Mail: T.E.Zimmermann@lingua.uni-frankfurt.de 